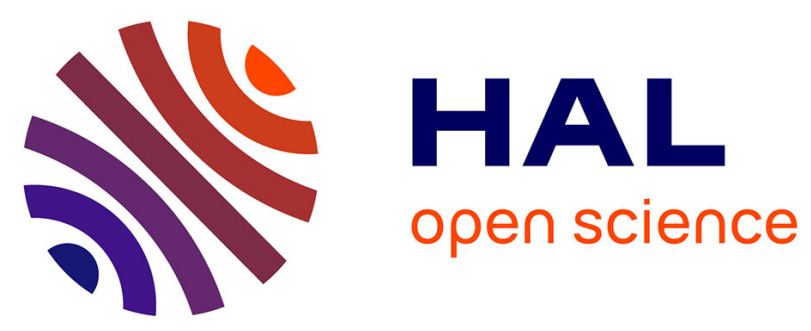

\title{
Identification of the artifact contribution to two urban Technosols by coupling a sorting test, chemical analyses, and a least absolute residual procedure
}

\author{
Thomas Lenoir, Myriam Duc, Laurent Lassabatère, Katia Bellagh
}

\section{- To cite this version:}

Thomas Lenoir, Myriam Duc, Laurent Lassabatère, Katia Bellagh. Identification of the artifact contribution to two urban Technosols by coupling a sorting test, chemical analyses, and a least absolute residual procedure. Journal of Soils and Sediments, 2019, 19 (2), pp. 683-701. 10.1007/s11368-0182074-4 . hal-02877805

\section{HAL Id: hal-02877805 \\ https://hal.science/hal-02877805}

Submitted on 2 Jun 2021

HAL is a multi-disciplinary open access archive for the deposit and dissemination of scientific research documents, whether they are published or not. The documents may come from teaching and research institutions in France or abroad, or from public or private research centers.
L'archive ouverte pluridisciplinaire HAL, est destinée au dépôt et à la diffusion de documents scientifiques de niveau recherche, publiés ou non, émanant des établissements d'enseignement et de recherche français ou étrangers, des laboratoires publics ou privés. 
SOILS, SEC 2 • GLOBAL CHANGE, ENVIRON RISK ASSESS, SUSTAINABLE LAND USE •

\section{RESEARCH ARTICLE}

Identification of the artefact contribution to two urban Technosols by coupling a sorting test, chemical analyses and a least-absolute residuals procedure

Thomas Lenoir $^{1}$ - Myriam Duc ${ }^{2}$ Laurent Lassabatere ${ }^{3} \cdot$ Katia Bellagh $^{1,2}$

Received: 1 March 2018 / Accepted: 27 June 2018

(c) Springer-Verlag GmbH Germany, part of Springer Nature 2018

Responsible editor: Maxine J. Levin

${ }^{1}$ IFSTTAR, GERS, GMG, 44344 Bouguenais, France

2 Université Paris-Est, IFSTTAR, GERS, SRO, 77447 Marne-la-Vallée, France

3 Univ Lyon, Université Claude Bernard Lyon 1, CNRS, ENTPE, UMR5023 LEHNA, 69518, Vaulx-enVelin, France

$\triangle$ Thomas Lenoir

Thomas.lenoir@ifsttar.fr 


\section{Abstract}

Purpose: In the context of urban extension, the depletion of natural resources for construction constitutes a crucial issue. Specifically, in the field of earthworks, the amounts of materials can be massive and pose the crucial problem of resource shortage. Therefore, the reuse of excavated urban soils from foundation layers as new earthwork construction materials appears to be a sustainable and promising solution. Before repurposing, a better knowledge of urban soils and their potential pollutant load is compulsory.

Materials and methods: In this paper, two soils excavated from the town of Paris are studied. After the stripping of their surface, to remove organic matter and surface pollution, the matrices were submitted to elemental analyses using ICP-OES, C/S measurements and XRF techniques. The elemental analyses were carried out on the whole materials, on three granulometric fractions $(<80 \mu \mathrm{m}, 80-400 \mu \mathrm{m}$, $>400 \mu \mathrm{m}$ ) and on the families of artefacts (i.e. construction and demolition wastes, natural gravel, industrial wastes, magnetic and non-magnetic slags) found in the soils. The combination of elemental analyses and a least absolute residuals procedure (LAR) were used to quantify artefacts contributions in all granulometric fractions.

Results and discussion: The soils exhibit evidence of anthropic inputs with high contents of pollutants under the forms of carbonaceous, sulfur mineral and metallic alloys. Carbonaceous and trace pollutions are concentrated in magnetic and non-magnetic slags, while sulfur and strontium pollutions are concentrated in gypsum components. In both cases, all the granulometric fractions (including whole material) can be retrieved as a combination of artefacts composition, suggesting that the Technosoils mainly result from the mixture of these artefacts. The amounts of natural material, gypsum components and magnetic slags increase with the fineness of the fractions. In contrary, amount of non-magnetic slags decrease with the fineness of the fractions and suggests that the processes of slags weathering are not similar: carbonaceous slags are more stable than iron-enriched slags.

Conclusions: The elemental analyses of granulometric fractions of soils and the artefacts using LAR analyses help in identifying the compositions of each granulometric fraction and give insight into the evolution of artefacts in the soil. These tools are also promising regarding the assessment of geoenvironmental characteristics of urban soils, which in turns provides relevant information regarding management and reuse.

Keywords Carbon $\cdot$ Construction and demolition wastes $\cdot$ Excavated soils $\cdot$ Least-absolute regression - Sulfur • Trace elements • Urban soils 


\section{Introduction}

Growth of cities leads to significant increases in demand for resources and construction materials (Rivas et al. 2006; Huang et al. 2010; Magnusson et al. 2015). In metropolitan areas, because of land-use changes, this leads to depletion of natural resources for construction (Rivas et al. 2006), and materials having to be imported and transported over long distances (loannidou et al. 2015). This increases global projects costs (Magnusson et al. 2015). Specifically, in the field of earthworks, the amounts of materials needed for the construction of the subgrade layers of structures can be huge (Cabello Eras et al. 2013; Capony et al. 2013).

In this context, (re-)using excavated urban soils from foundation layers as new earthwork construction materials in urban areas appears much more environmentally sustainable and promising. For civil engineering projects, it improves resource efficiency and reduces both costs and climate impacts (Magnusson et al. 2015). The management of excavated urban soil (re-)use in earthwork is governed by geotechnical and geo-environmental soil properties. Geotechnical properties determine whether the material has the required mechanical specifications before use in construction projects (Lenoir et al. 2016; Preteseille and Lenoir 2016). Besides, the geo-environmental properties assess the toxic potential of these materials (Mantis et al. 2005; Jean-Soro et al. 2015).

As urban soils were already used as base and subgrade materials, it is logical to suppose that they have adequate mechanical properties to support new construction projects. The geo-environmental properties of urban soils have been studied for several decades (Solomon and Harford 1976; Chen et al. 2005; Lorenz and Kandeler 2005; Rossiter 2007; Morel et al. 2015). Deeply impacted in their composition by human activities (Séré et al. 2010), they are composed of natural and anthropic materials (Morel et al. 2015) and may exhibit several types of pollution, such as construction and demolition waste (C\&DW), metal pollution, or organic pollution (Chen et al. 2005; Lorenz and Kandeler 2005; Norra et al. 2008; Magnusson et al. 2015).

Nevertheless, because they are more closely related to biomass production or human activities, only top soils (i.e. about the first $50 \mathrm{~cm}$ ) of urban matrixes are generally studied (Salvagio Manta et al. 2002; Chen et al. 2005; Hanna et al. 2009; Sun et al. 2010; Wei and Yang 2010; Bech et al. 2011; Jean-Soro et al. 2015; Pariente et al. 2015; Zhao and Hazelton 2016; Cai et al. 2017; Nezat et al. 2017). During earthwork operations, the surface layer is removed and landfilled to be sure to remove potential surface organic matter and potential surface pollution. Earthmoving programs concern depths beyond 1 meter (Cabello Eras et al. 2013). There is clearly a need for complete characterization of the geotechnical and geo-environmental properties of the urban soils underneath the first horizons. Some studies on urban and industrial soils have attempted such a characterization using pedological pits (Lu et al. 2003; Lorenz and Kandeler 2005; Douay et al. 2008; Norra et al. 2008; Luo et al. 2009; Huot et al. 2014a, 2014b; Burlakovs et al. 2016). However, though the pit depths were large enough to encompass earthwork depths, the excavated volumes were relatively small, with surface areas around $1 \mathrm{~m}^{2}$, and with discrete sampling on the earthwork operations scale. Such approaches appeared too limited to allow any conclusions whether using the urban soils was viable. Few studies have dealt with both pedological and 
representative statistical approaches (Lu et al. 2003; Douay et al. 2008; Luo et al. 2009) and lower

\section{Materials and methods}

\subsection{Sampling}

The first material, denoted SB, originates from the subgrade of a former industrial site located in the south west of the town of Boulogne-Billancourt, near Paris, France, along the Seine River (S 110,000 $\mathrm{m}^{2}$ - Fig. 1). The second material, denoted $\mathrm{SI}$, originates from a partially plant-covered area including an embankment of the Paris ring road, near the Seine River (S 20,000 $\mathrm{m}^{2}$ - Fig. 1).

Samples were taken using power shovels at a depth between $1.5 \mathrm{~m}$ and $11 \mathrm{~m}$ during earthwork operations. Several $50 \mathrm{~kg}$ samples were taken randomly. In all cases, between $1 \mathrm{~T}$ and $1.5 \mathrm{~T}$ of material were selected. Visual observations in the field allow forecasting for coarse-grained matrixes. After transportation to the laboratory, materials were dried at $40^{\circ} \mathrm{C}$. Soil matrixes were then sieved and the 
fraction $>20 \mathrm{~mm}$ ( $9 \%$ for $\mathrm{SB}$ and $10 \%$ for $\mathrm{SI}$ ) was crushed and reintroduced in each matrix as is done in the field of earthworks. Finally, they were manually homogenized with a shovel and stored at room temperature in a protected tank (Fig. 1).

\subsection{Characterization}

\subsubsection{Geotechnical identification}

To foresee the materials that could be present on the site, geological and historical research was done, completed by geotechnical identification. That included grain size distribution (AFNOR 1992a, 1992b, 1996), determination of liquid limit (WL), plastic limit (WP), plasticity index (IP), estimation of the clay activity $A=I_{P} / C_{2 \mu m}(A F N O R 1993,1998)$. In addition, loss on ignition (LOI) at $550^{\circ} \mathrm{C}$ (AFNOR 2007) and particle densities $\left(\rho_{d}\right)$ before and after heating at $T=550{ }^{\circ} \mathrm{C}$ were measured for the $<2 \mathrm{~mm}$ granulometric fraction (AFNOR 2008).

\subsubsection{Sorting test}

The materials were sorted into four granular fractions: 1 - Whole material: 0- $D_{\max }$, 2- Fine fraction: 0$80 \mu \mathrm{m}$, 3- Intermediate fraction: 80-400 $\mu \mathrm{m}$ and 4- Coase fraction: $400 \mu \mathrm{m}-\mathrm{D}_{\max }$. Artefacts were then sorted following standards (AFNOR 2009a, 2012), considering a threshold of $4 \mathrm{~mm}$ for particle diameter. We considered 6 artefact families: $R_{c}$ contains concretes, concrete products (including hydraulically bound material), mortars, concrete masonry units; $R_{u}$ contains unbound gravel, natural stones; $R_{b}$ contains terracotta units such as bricks and tiles, and non-floating cellular concretes; SF contains magnetic slags; SNF contains non-magnetic slags; $G_{y}$ contains gypsum components; and $X_{1}$ contains aggregated fine-grained material. Before further analysis, each family was washed quickly on a 4-mm sieve, and $\mathrm{LOI}$ at $\mathrm{T}=450^{\circ} \mathrm{C}$ and $\mathrm{T}=550^{\circ} \mathrm{C}$ (AFNOR 2013) and $\rho_{\mathrm{d}}$ were determined.

\subsubsection{Chemical analyses}

Elemental occurrences were obtained using X-ray fluorescence (XRF). A Brucker M4 TORNADO laboratory instrument equipped with an Rh X-ray tube operated at $50 \mathrm{keV}$ and $300 \mu \mathrm{A}$ was used. X-ray fluorescence was measured using a silicon drift detector with a spot size of $25 \mu \mathrm{m}$ (Jean-Soro et al. 2014). XRF spectra were measured under vacuum on the granular fractions and the artefact families ground to a diameter $<40 \mu \mathrm{m}$. For each fraction, 96 spectra were collected, and for each artefact, 30 . They were normalized on the Compton peak (i.e. peak maximum set to 1) and limited at the Rayleigh peak ( 20 keV). To study chemical correlation, the elemental composition of artefact families was analyzed semi-quantitatively by applying a semi-automatic method provided by the software 'Esprit' coupled with M4 TORNADO. Mean XRF spectra were then determined. 
We accurately quantified elements on both granular fractions and artefacts with Inductively Coupled Plasma-Optical Emission Spectroscopy - ICP-OES (AFNOR, 2001, 2009b) using a Varian 720 ES, after mineralization with a mixture of $\mathrm{HF}$ and $\mathrm{HClO}_{4}$ and alkali fusion with lithium tetraborate for silica content. Carbon (Total Carbon and Total Organic Carbon) and sulfur contents were determined using a LECO SC144 C/S analyzer.

All the chemical analyses, LOI, $\rho_{d}$, correlations from XRF analyses and mean XRF spectra are provided in an accompanying Electronic Supplementary Material (ESM).

\subsection{Least absolute residuals procedure (LAR)}

Based on measurements from the chemical analyses (ICP, C/S measurements and LOI), a least absolute regression approach was applied to quantify the content of the six artefacts in the four granular fractions. This approach is known to be less sensitive to outliers than other fitting methods. In addition, to make sure of equal weight between data, each absolute difference was normalized.

We named the sets of experimental measurements as follows:

- on the three granular fractions and the whole material, Meas.f (i.e., Meas.[ $[80 \mu \mathrm{m}]$, Meas. $[80-400 \mu \mathrm{m}]$, Meas.[ $>400 \mu \mathrm{m}]$, and Meas.wm)

- $\quad$ on each family of artefact, Meas.i (i.e., Meas.sNF, Meas.sF, Meas.Ru, Meas.Gy, Meas.Rc, Meas.rb)

Considering the $\mathrm{j}$ measurements in each set, the aim of the approach is to find, for each fraction $\mathrm{f}$, a set of six coefficients $q_{i}$ that minimizes the difference between predicted and observed values:

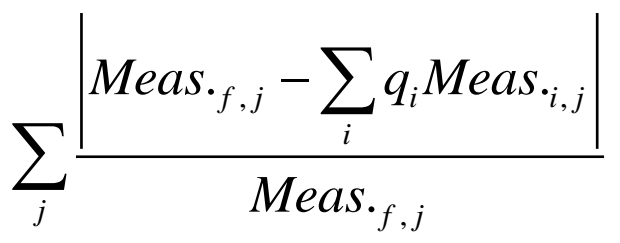

Calculations have been done using Microsoft Excel Solver.

\section{Results and discussion}

\subsection{Materials characterization}

\subsubsection{Geological origin}

In both cases, the materials originated from a geological deposit that is defined as old alluvium. It was deposited by the river at different stages of the morphological evolution of the site and consists of millstone grit, flint, limestone, granitic rocks and jurassic cherts. Since then, anthropic activities have impacted the natural deposit for several millenniums, leading to a material defined as backfill (Fig. 1). It 
consists of original soil wastes (e.g., demolition waste, gypsum from plaster) and excavated materials from quarries (e.g., sand, chalk, natural gypsum) mixed eventually with original soil (BRGM 1973).

Material SB originates from a site used for tannery and laundry, since the industrial period of the $18^{\text {th }}$ century. In the $20^{\text {th }}$ century, automotive industries operated on the site (Hatry and Mercier 1991). That stopped at the end of the century. Deconstruction and depollution operations were conducted in accordance with the French Environment Code on classified facilities for environmental protection.

Material SI originates from the subgrade of the $19^{\text {th }}$ century wall that surrounded Paris. After it was deconstructed in the beginning of the $20^{\text {th }}$ century, the site became a slum. Since the 1970 s, part of it was used as a subgrade layer for an embankment of the ring road, and the rest was converted into a private park. Several industrial sites have been operating in its vicinity.

\subsubsection{Geotechnical classification}

Both materials SB and SI can be considered as silty sand and silty gravel (Fig. 2). They are classified at the edge of A-6 and A-2-6 according the ASHTOO classification (ASTM 2009), SM according to Unified Soil Classification System (ASTM 2011) and at the edge between C1A1 and C1B5 according to the French soils classification (AFNOR 1992a). In accordance with literature (De Kimpe and Morel 2000; Biasoli et al. 2005; Sun et al. 2013), clay contents are low (<15\%) and the clays tend to be inactive in SB and have normal activity in SI.

Loss on ignition at $550^{\circ} \mathrm{C}$ are $14.6 \%$ for SB and $6.8 \%$ for SI. Such high levels have already been noticed in the depth of Technosols. They are related to previous human activities (e.g., industrial operations) and result from the presence of ash, coal and coke (Huot et al. 2014b; Lorenz and Kandeler 2004). Because of compaction and partial sealing of these deep layers (Wei et al. 2014), the microbial activity is too poor to degrade organic matter, which results in stable organic matter stocks at these depths. C\&DW are also known to be sensitive to heating (Asakura et al. 2010) and especially plaster and gypsum materials that bind water loosely or even degrade at low temperature (Chindaprasirt et al. 2011).

Particle densities are $\rho_{d}=2.52$ (standard deviation std $=0.01$ ) $\mathrm{g} / \mathrm{cm}^{3}$ for SB and $\rho_{d}=2.57(\mathrm{std}=0.02$ ) $\mathrm{g} / \mathrm{cm}^{3}$ for SI. Values are lower than the usual particle densities found in natural soils of around 2.6-2.8 $\mathrm{g} / \mathrm{cm}^{3}$ (Blanco-Canqui et al. 2005), and slightly higher than C\&DW particle densities (around 2.3-2.6 $\mathrm{g} / \mathrm{cm}^{3}$ given by Arulrajah et al. (2013), Asakura et al. (2010), Silva et al. (2014). In accordance with observations from LOI, after calcination at $550^{\circ} \mathrm{C}$, densities reach $2.7-2.8 \mathrm{~g} / \mathrm{cm}^{3}$.

\subsubsection{Sorting test}


After sorting the granular fraction (particle size above $4 \mathrm{~mm}$ ) in class of artefacts (Table 1), both matrixes

\subsection{Chemical analyses}

\subsubsection{Granulometric fractions of the two materials $S B$ and $S I$}

For both materials, all peaks measured on the whole material were found for the three granular fractions with similar intensities as illustrated for material SB in Fig. 3. In addition, the mean spectra from the two whole materials are very similar (Fig. 4). Magnesium (Mg), aluminum (Al), silicon (Si), sulfur (S), potassium $(\mathrm{K})$, calcium $(\mathrm{Ca})$, titanium $(\mathrm{Ti})$, chromium $(\mathrm{Cr})$, iron $(\mathrm{Fe})$, manganese $(\mathrm{Mn})$, nickel $(\mathrm{Ni})$, copper $(\mathrm{Cu})$, zinc $(\mathrm{Zn})$, lead $(\mathrm{Pb})$ and strontium $(\mathrm{Sr})$ were detected. Presence of phosphorous $(\mathrm{P})$, sodium $(\mathrm{Na})$, chlorine $(\mathrm{Cl})$, barium $(\mathrm{Ba})$, rhenium $(\mathrm{Re})$, rubidium $(\mathrm{Rb})$, yttrium $(\mathrm{Y})$, zirconium $(\mathrm{Zr})$ and molybdene $(\mathrm{Mo})$ can also be suspected.

Figure 5 and Fig. 6 summarize the contents of main major elements ( $\mathrm{Si}, \mathrm{Al}, \mathrm{Ca}, \mathrm{C}$ and $\mathrm{S}$ ) and trace elements ( $\mathrm{Ni}, \mathrm{Ba}, \mathrm{Cr}, \mathrm{Cu}, \mathrm{Mn}, \mathrm{Sr}, \mathrm{Zn}, \mathrm{Pb})$ in the different granulometric fractions and on the whole material (WM) measured with ICP-OES.

-Major elements 
For both materials, approaches with ICP-OES and XRF show that $\mathrm{SiO}_{2}$ contents vary with granulometric is not enough to explain these differences. Increasing $\mathrm{Si}$ in the intermediate and coarsest fractions may originate from the original matrixes (e.g. Si-rich minerals like quartz) but it could also be due to the introduction of technogenic material (Norra et al. 2006; El Khalil et al. 2008, 2013).

Total carbon ( $\left.\mathrm{CO}_{2 \text { tot }}\right)$ is the second main component of the two materials. Amounts of $\mathrm{CO}_{2 \text { org }}$ and $\mathrm{CO}_{2 \text { tot }}$ are highly correlated, with a determination coefficient $\mathrm{R}^{2}=0.94$. In both cases, the amounts of $\mathrm{CO}_{2}$ org are lower in the finest fraction.

For both SI and SB materials, $\mathrm{Ca}$ is the third main component of soils. Measures from ICP-OES are in agreement with XRF semi-quantification. The amount of $\mathrm{CaO}$ is the highest in the fine fraction, and similar in the intermediate and coarse ones. It represents $18 \%$ and $21 \%$ of the whole fraction of SB and $\mathrm{SI}$ respectively. Calcium carbonates, such as calcite $\mathrm{CaCO}_{3}$, are one of its main speciations in soils (Hillel 2005). Moreover, in C\&DW, calcium is also found as sulfate $\left(\mathrm{CaSO}_{4}, \mathrm{xH} 2 \mathrm{O}\right)$, like gypsum in plaster/drywall (Jimenez-Rivero and Garcia-Navarro, 2017; Lee et al. 2006). This hypothesis is plausible for these two materials, $\mathrm{SO}_{2}$ contents can be estimated reasonably around $1-2 \%$ for SB and $3-4 \%$ for SI.

In accordance with analyses by ICP-OES, XRF spectra suggest that both materials contain significant amounts of $\mathrm{Fe}$. Its occurrence seems to be independent of the granulometric fractions. In both cases, Fe concentrations are above $3,500 \mathrm{mg} / \mathrm{kg} \mathrm{dm}$, which is the common average for soils (Kabata-Pendias 2010). Part of Fe in SB and SI can be imputed to anthropogenic contributions (El Khalil et al. 2011).

To complete the analysis of major elements, for both the materials, XRF showed that $[\mathrm{Al}]<80 \mu \mathrm{m}>[\mathrm{Al}]>400$

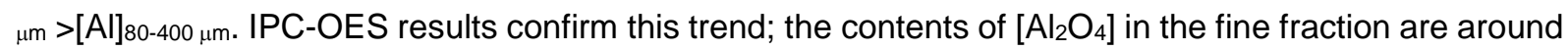
$1 \%$ higher than in the coarsest fraction and $2 \%$ higher than in the intermediate fraction. This is usual, considering that Al comes from clay minerals in the clay fraction $(<2 \mu \mathrm{m})$. However, further investigations must be done to determine if this noteworthy occurrence of $\mathrm{Al}$ in the coarsest fraction is natural or can be attributed to anthropic contributions.

-Intermediate elements

Elements $\mathrm{K}, \mathrm{Na}, \mathrm{P}, \mathrm{Mg}, \mathrm{Ti}$ and $\mathrm{Mn}$ are considered to have an intermediate occurrence in between the major and trace elements. Except for Ti, XRF analyses cannot give relevant information about these elements. The method is not sensitive to $\mathrm{Na}$, and backgrounds (from $\mathrm{Fe}, \mathrm{Ca}$ and $\mathrm{Si}$ ) can lead to interference and poor estimations of their contents (Fig. 3 and Fig. 4).

The amounts of $\mathrm{K}$ are around $6,000-8,000 \mathrm{mg} / \mathrm{kg} \mathrm{dm}$ regardless of the material origin and granulometric fraction. The $\mathrm{Na}$ contents seem also to be separated from granulometric fraction but are higher in $\mathrm{SI}$ than in SB. Never found as free solids in nature, $\mathrm{K}$ and $\mathrm{Na}$ can be associated with several minerals or brought by several anthropogenic activities (Hammond 2003). The difference between the matrixes could be attributed to salt used as a de-icing agent for roads in the case of SI (Bryson and Barker 2002). 
However, $\mathrm{Na}$ or $\mathrm{K}$ occurrence in soils is highly variable and their concentrations in both materials remain far from extreme contents (Reimann and de Caritat 2002; Alekseenko and Alekseenko 2014).

Levels of $\mathrm{P}$ are similar to regular standards for SB and slightly higher for material SI (Reimann and de Caritat 2002; Alekseenko and Alekseenko 2014). In SB, the P content is twice as high in the finest fraction than in the coarsest fraction. Measurements are relevant regarding the phosphorous cycle. After alteration of primary minerals, $\mathrm{P}$ may be adsorbed on clay minerals, $\mathrm{Fe}$ or $\mathrm{Al}$ oxy-hydroxides, or $\mathrm{CaCO}_{3}$ surfaces (Hillel 2005). This may explain why the $P$ levels increase in finest fraction of SB. This tendency is not observed for $\mathrm{SI}$, where the content of the coarse fraction is higher than the content of the two other fractions. This distribution is hard to explain. Roads are sources of $P$ (Withers and Jarvie, 2008) but it is then adsorbed to small particles in the road structure (Wu et al. 2015).

Content of $\mathrm{Mg}$ is around two times higher in the fine fraction than in the two others for both materials. It is also twice as high in $\mathrm{SI}$ as in SB. This is relevant with the geochemical processes that govern $\mathrm{Mg}$ geochemical behavior. After alteration of primary mineral, $\mathrm{Mg}$ is found in secondary silicate clay minerals like chlorite, vermicullite, illite, and montmorillonite, in organic matter as exchangeable cations, and in soil solutions (Hillel 2005). For SB, measured levels are not critically high. By contrast, for SI, they are a bit high in comparison with regular standards (Reimann and de Caritat 2002; Alekseenko and Alekseenko 2014). Magnesium chloride $\left(\mathrm{MgCl}_{2}\right)$ is a well-known road de-icing agent (Nelson et al. 2009) while $\mathrm{Mg}$ alloys are also commonly used in cars.

Interestingly, magnesium, phosphorus and potassium are often considered as secondary ( $\mathrm{Mg}$ ) and major ( $\mathrm{P}$ and $\mathrm{K})$ crop nutrients. Their occurrences show that the soils may have recreational/ecological potential (Hillel, 2005) despite their previous use as subgrade layer.

XRF and ICP-OES show that both materials contain Ti with [Ti] $<80 \mu \mathrm{m} \sim[\mathrm{Ti}]>400 \mu \mathrm{m}>[\mathrm{Ti}] 80-400 \mu \mathrm{m}$, regardless of the materials. Ti minerals are very resistant to weathering and occur practically undecomposed in soils. When Ti-bearing silicates are dissolved, the element is readily transformed into Ti oxide-aquate, which is transformed to anatase or rutile (Cornu et al. 1999, Kabata-Pendias 2010). However, anthropic use as Ti-alloys or Ti-paints can also be a significant source of this element in anthropogenic soils (Bech et al. 2011). As for Al, the observed uncommon distribution in the three granulometric fractions could reflect that titanium in the fine fraction originates from natural processes whereas these two elements may originate from anthropic sources in the coarsest fraction. Levels are similar for both soils and do not exceed critical concentrations (Reimann and de Caritat 2002; LopezGarcia et al. 2004; Kabata-Pendias 2010; El Khalil et al. 2013; Alekseenko and Alekseenko 2014).

Finally, the results tend to show that the Mn content does not depend on the granulometric fraction. This element is relatively mobile in soils and is known to readily concentrate under the forms of secondary $\mathrm{Mn}$ minerals after alteration. Colloidal $\mathrm{Mn}$ oxides reveal a great affinity for adsorption of cationic and anionic forms of elements as well as inorganic and organic substances (Kabata-Pendias, 2010). In both materials, measured levels are typical of most natural and anthropogenic soils (Reimann and de Caritat 2002; Kabata-Pendias 2010; Alekseenko and Alekseenko 2014). 


\section{-Trace elements}

Metalloids $\mathrm{Zn}, \mathrm{Cu}, \mathrm{Ni}$ and $\mathrm{Pb}$ are major pollutants of roadside soils (Munch 1993; Hanna et al. 2009). $\mathrm{XRF}$ analysis shows that their concentrations are clearly higher in the finest fraction. Such finding is quite common in soils and may be attributed to sorption processes in relation with higher specific areas (Hillel, 2005). Concentrations also seem to be higher in the 80-400 mm fraction than in the coarsest one but the ranges are similar (Fig. 3 and Fig. 4). For SB, those observations are confirmed by the chemical analysis (Fig. 6), except for copper (Cu). The Cu concentration in the coarsest fraction is around 2.5 times higher than in the finest fraction. For SI, XRF observations are confirmed by chemical

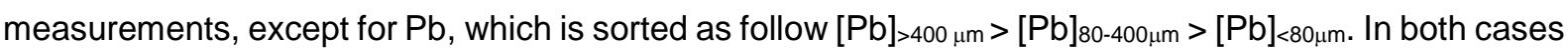
( $\mathrm{Cu}$ in $\mathrm{SB}$ and $\mathrm{Pb}$ in $\mathrm{SI}$ ), ICP-OES results have to be considered cautiously. They are not consistent with the chemical measurements on the whole fractions and the granulometric fractions. We suggest that " $\mathrm{Cu} / \mathrm{Pb}$ hot spots" may have been present in the blend of the samples.

In both matrixes, the $\mathrm{Zn}$ content was around ten times higher than the general values for average total Zn contents in soils (Reimann and de Caritat 2002; Kabata-Pendias 2010). Higher levels have been measured for urban soils (El Khalil et al. 2013; Alekseenko and Alekseenko 2014), but chemical results clearly highlight anthropic soil contamination (Jean-Soro et al. 2015).

Amounts of $\mathrm{Pb}$ are around $100-300 \mathrm{mg} / \mathrm{kg}_{\mathrm{dm}}$ in both whole materials. The concentrations usually encountered in areas remote from human activity are in the range of $3-90 \mathrm{mg} / \mathrm{kg}_{\mathrm{dm}}$, with $27 \mathrm{mg} / \mathrm{kg} \mathrm{dm}$ as overall mean value (Kabata-Pendias 2010). Even for urban soils, the levels are high (Lu et al. 2003; Cachada et al. 2013; Jean-Soro et al. 2015). Similar levels have been recorded in urban road dusts (Nazzal et al. 2013). Considering the geological origin of the soil and the history of the site, it is probable that the major part of $\mathrm{Pb}$ in the matrix originates from human activities.

Concentrations of $\mathrm{Cu}$ in the whole fraction of both materials are around 6 to 8 times higher than median total copper concentrations found in uncontaminated soils (WHO 1998). These levels are high, even for urban soils (Lu 2003; Alekseenko and Alekseenko 2014; Jean-Soro et al. 2015) and also higher than the levels found in urban road dusts (Nazzal et al. 2013). As for Pb, suggesting an anthropogenic origin is reasonable.

For $\mathrm{SI}$, loads of $\mathrm{Ni}$ are in the range of amounts measured from non-contaminated soils (Kabata-Pendias 2010) and are in line with previous Ni measurements in urban soils (Reimann and de Caritat 2002; EI Khalil et al. 2012; Alekseenko and Alekseenko 2014) and in soils near roads (Münch 1993; Duong and Lee 2011). For SB, with around $200 \mathrm{mg} / \mathrm{kg}_{\mathrm{dm}}$ levels are critically high (Lu et al. 2003; Herselman et al. 2005; Islam et al. 2015). It is clear that the processes of Ni-contamination are different for the two soils.

For $\mathrm{Cr}$, XRF analyses and IPC-OES measurements show that the occurrence of $\mathrm{Cr}$ is lower in the finest fraction than in the two others. As previous metalloids, there is a positive relationship between $\mathrm{Cr}$ and the fine granulometric fraction in soils (Kabata-Pendias 2010). For this reason, the unusual chromium content sorting in the two matrixes could be interpreted as the result of anthropic sources. Moreover, the measured amount of $\mathrm{Cr}$ in the whole SB fraction is high for the measured levels in the three 
granulometric fractions, this could be interpreted as the occurrence of $\mathrm{Cr}$-rich compounds. Chromium

\subsubsection{Artefact families}

-Gravels $\left(R_{\mathrm{u}}\right)$

Ignition does not have a significant impact on gravels in terms of LOI and particle density (Table 2). XRF and ICP-OES analyses show that $\mathrm{R}_{\mathrm{u}}$ families from both soils are very close (Fig. 7 and Fig. 8). Major constituents are $\mathrm{Si}, \mathrm{Ca}$ and $\mathrm{Fe}$. The occurrence of $\mathrm{Mg}, \mathrm{Al}, \mathrm{S}, \mathrm{K}, \mathrm{Ti}, \mathrm{Mn}, \mathrm{Ni}$ and $\mathrm{Sr}$ is also observed. These results are in accordance with the history of both sites. Imported gravels for backfilling are siliceous phases and calcium carbonate phases. FeO minerals constitute around $1 \%$ for both soils. Analyses with ICP-OES (Fig. 8) also highlight the occurrence of $\mathrm{Al}, \mathrm{Mg}, \mathrm{Na}, \mathrm{P}, \mathrm{S}$ and $\mathrm{Ti}$ in similar ranges 
for both matrixes. Considering their relative contents, $\mathrm{Al}$ and $\mathrm{Na}$ may come from feldspar minerals. $\mathrm{Ti}$ may be in the form of $\mathrm{TiO}_{2}$ and sulfur may be in the form of gypsum.

In $\mathrm{SB}$, elemental correlations from XRF analyses (see ESM) highlight that $\mathrm{P}$ and $\mathrm{Si}$ are negatively correlated, $R_{(S i, P)}=-0.81$. This means that $P$ is not linked to silicate. They also show that, $R_{(S i, C a)}=-0.77$. That means that $\mathrm{Ca}$ and $\mathrm{Si}$ tend to be independent, as suggested by the occurrence of siliceous phases and calcium carbonate phases. But the correlation is small, which suggests the occurrence of $\mathrm{Ca}-\mathrm{Si}$ minerals like Ca-feldspars (e.g. anorthite $\mathrm{CaAl}_{2} \mathrm{Si}_{2} \mathrm{O}_{8}$ ). In SI, no elemental correlations could be found based on XRF analyses. Amounts of chromium and strontium are higher than $100 \mathrm{mg} / \mathrm{kgdm}$. Other metalloids ( $\mathrm{Ba}, \mathrm{Cu}, \mathrm{Ni}, \mathrm{Pb}$ and $\mathrm{Zn}$ ) are present with trace amounts.

-Slags

- Non-magnetic slags (SNF)

XRF signal from the SNF families' exhibit some similarities (Fig. 7). For both soils, Mg, Al, Si, K, Ca, Ti, $\mathrm{Cr}, \mathrm{Mn}, \mathrm{Fe}, \mathrm{Ni} \mathrm{Cu}, \mathrm{Sr}$ are found in this family. This artefact is also the main carrier of carbon and all carbon is organic (Fig. 8). This explains the high value of LOI and the significant increase in particle density with ignition (Table 2). Despite the similarities between the two soils, numerous differences remain. Firstly, chemical analyses show that the amounts of major and intermediate elements are not similar in the soils. Specifically, the amount of $\mathrm{CO}_{2 \text { TOт }}$ in $\mathrm{SB}$ is highly higher than in SI. Secondly, for SI, the SNF family carries a high amount of $\mathrm{Zn}$ and $\mathrm{Pb}$ while it is not the case for $\mathrm{SB}$. In a similar way, amounts of barium, copper and vanadium are lower in the SNF family of SB. On the contrary, nickel is more present in the SNF family of SB.

In SB, elemental correlations from XRF analyses highlight that $\mathrm{Si}, \mathrm{Fe} \mathrm{Al}, \mathrm{K}, \mathrm{Ti}$ and $\mathrm{Ni}$ are correlated with

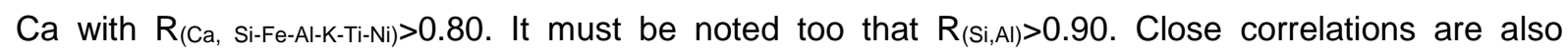

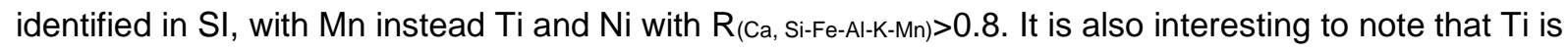

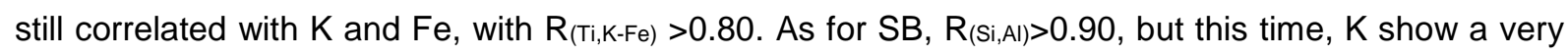

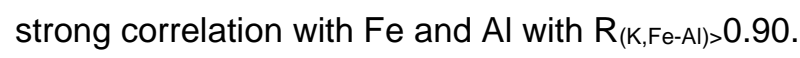

All these results show that the source of these slags is not the same for the two matrixes. Nevertheless, they are quite close and these analyses suggest that they contain at least one common organo-mineral phase, with mineralogy composed of a Ca-Si-Al-Fe-K-Ti cluster. This kind of relationship is commonly found in biomass ash (e.g. municipal solid wastes, refuse-derived fuel, coal, sewage sludge). Fe-Si-Al$\mathrm{Ti}, \mathrm{Ca}-\mathrm{Mg}-\mathrm{Mn}$ and $\mathrm{K}-\mathrm{P}-\mathrm{S}-\mathrm{Cl}$ complexes are usually considered as end-members in ash classification (Ollila et al. 2006; Adnan et al. 2013; Vassilev et al. 2013; Folgueras et al. 2017). These phases are often enriched with pollutants such as trace elements and sulfur (Vassilev et al. 2009, 2013). Note that barium is regularly observed in landfill sites (Burlakovs et al. 2016; Jani et al. 2016) and can be linked as barium sulfate to bottom ash (Rigo et al. 2009). Vanadium is also commonly found in slags (Chaurand et al. 2006, Tossavainen et al. 2007). 
.XRF signals from the SF families' exhibit (Fig. 7) some similarities between the two materials. Amounts of iron are very high. Some elements are also common for both soils: $\mathrm{Al}, \mathrm{Si}, \mathrm{S}, \mathrm{K}, \mathrm{Ca}, \mathrm{Ti}, \mathrm{Cr}, \mathrm{Mn}, \mathrm{Ni}, \mathrm{Cu}$, $\mathrm{Rb}, \mathrm{Sr}$ and $\mathrm{Zr}$. For both cases, a structure at $12.8 \mathrm{keV}$ near the $\mathrm{Pb} \mathrm{L}_{\beta}$ peak is not identified. It could be the thorium $L_{\alpha}$ peaks but other distinctive features $\left(L_{\beta}\right.$ and $\left.M\right)$ are not observed or are lost in other signals (e.g., Th $\mathrm{L}_{\beta}$ in $\mathrm{Zr} \mathrm{K}_{\alpha}$ ). The amounts of elements are different. Calcium and copper contents seem higher in the SF family of SI and the SNF family contains $\mathrm{Zn}$ and $\mathrm{Pb}$, yet $\mathrm{SB}$ does not. Chemical analyses confirm that iron is the major element but its content is $12 \%$ higher in the SF family from SB. On the contrary, the amounts of $\mathrm{CO}_{2 \text { Tот }}$ are $8 \%$ higher in the SF family from SI. These results are in accordance with observations on LOI and $\rho_{d}$ (Table 2). Amounts of major and intermediate elements in the two SF families are different too, so there are similarities but the accurate speciation of SF in the two materials is different.

On the other hand, the SNF family and the SF family seem to be very similar in the in two materials. First, in both a very large part of the carbon is organic. Second, they carry the main part of trace elements. Finally, for the SI, elemental correlations from XRF analyses highlight that $\mathrm{Ca}, \mathrm{Si}, \mathrm{Fe}, \mathrm{Al}, \mathrm{K}$, $\mathrm{Ti}$ and $\mathrm{Ni}$ are strongly correlated with correlation coefficients $\mathrm{R}$ around 0.90 regardless of element

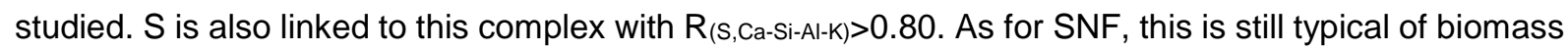
ash mineralogy (Vassilev et al. 2009, 2013). It suggests that the origins of slags are similar, regardless of their magnetic behavior. Unfortunately, elemental correlations from XRF analyses were not performed for the SF family in material SB.

\section{-Concrete products $\left(\mathrm{R}_{\mathrm{c}}\right)$}

XRF spectra of $R_{c}$ are similar for the two materials. The following elements are present in both matrixes: $\mathrm{Al}, \mathrm{Si}, \mathrm{S}, \mathrm{K}, \mathrm{Ca}, \mathrm{Ti}, \mathrm{Cr}, \mathrm{Fe}, \mathrm{Fr}$ and $\mathrm{Zr}$. Additionally, $\mathrm{Cu}$ and $\mathrm{Zn}$ are found in the $\mathrm{R}_{\mathrm{c}}$ family of $\mathrm{SI}$. Chemical analyses of major elements (Fig. 8) show that this family exhibits similar features for the two materials. Specifically, relative contents of $\mathrm{SiO}_{2}$ and $\mathrm{CaO}$ are around $60 \%$ and $15 \%$. In most cements, percentage of $\mathrm{CaO}$ is around $60 \%$, while percentage of $\mathrm{SiO}_{2}$ is around $20 \%$. The cement represents around $15-20 \%$ of the concrete dry matter, it appears that used materials in concrete were highly siliceous. Considering the calcareous geology of the Paris Basin (BRGM 1973), silica probably comes from sand-lime materials from the Seine River. Since the eighties, it has been forbidden to use the river material for concrete production. This point highlights that in both cases, concrete products date from earlier.

Moreover, for both materials, the amounts of $\mathrm{CO}_{2}$ org, $\mathrm{SO}_{2}, \mathrm{FeO}$ (and $\mathrm{CO}_{2}$ inorg for $\mathrm{R}_{\mathrm{c}}$ in material $\mathrm{SI}$ ) are a bit high for concrete products. $\mathrm{SO}_{2}$ and $\mathrm{FeO}$ could come from cement phases. However, the amounts of intermediate elements show that $\mathrm{S}$ and Fe have another origin than cement (Saiz Matrinez et al. 2016). In accordance with the excess of $\mathrm{CO}_{2}$ and the occurrence of $\mathrm{Zn}$ and $\mathrm{Cu}$ in $\mathrm{SI}$ and $\mathrm{Ba}$ in both $\mathrm{SB}$ and $\mathrm{SI}$ (Fig. 9), we can suggest that these occurrences result from the presence of slags in this family. This supposition is confirmed by correlation from XRF analyses. In the Rc family of material $\mathrm{SI}, \mathrm{Si}, \mathrm{Al}$ and $\mathrm{S}$

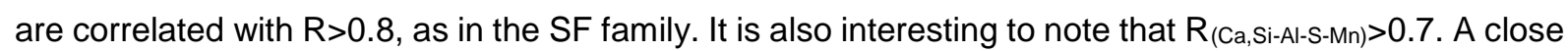


correlation of 0.8 between these elements is found in the SNF family. This mixing probably comes from the sorting process. In practice, it is visually difficult to distinguish the families of small particles. This is especially noticeable for $\mathrm{SI}$, with low $\mathrm{R}_{\mathrm{c}}$. It may have been complicated to exclusively select particles of this family, without any contamination from the dust of the other families. This is confirmed by LOI measurements (Table 2). It cannot be explain only with the loss generally attributed to non-evaporable water for this kind of material (Haecker et al. 2005; Asaruka et al. 2008).

$$
\text { -Gypsum components }\left(G_{y}\right)
$$

Ignition has a clear impact on these artefacts in terms of loss and densities, as does drying temperature (Table 2). They gain more than $0.4 \mathrm{~g} / \mathrm{cm}^{3}$ after calcination. These results are in accordance with the knowledge on the relationship between LOI and gypsum material (Santisteban et al. 2004; Chindaprasirt et al. 2011). As for previous families, the two XRF spectra are very close (Fig. 7). Major elements in the spectra are $\mathrm{S}, \mathrm{Ca}, \mathrm{Si}, \mathrm{Fe}$ and $\mathrm{Sr}$. Traces of $\mathrm{Ti}, \mathrm{Cr}, \mathrm{Mn}, \mathrm{Ni}$ and $\mathrm{Zn}$ were detected. In accordance with these results, chemical analyses on major elements show that $\mathrm{Ca}$ and $\mathrm{S}$ are the major components (Fig. 8). In addition, elemental correlations show that they are highly correlated ( $\left.R_{\text {ca-s }}>0.9\right)$. The amounts of $\mathrm{CO}_{\text {2inorg }}$ are not negligible and it shows that calcareous materials can be found in $\mathrm{G}_{\mathrm{y}}$. This is also confirmed by the amount of $\mathrm{Mg}$. This suggests the occurrence of a dolomite-like mineral $\left(\mathrm{CaMg}\left(\mathrm{CO}_{3}\right)_{2}\right)$ in both matrixes. Sr levels are high (Fig. 9): 1,155 and 1,310 mg/kgdm in the Gy families of SB and SI respectively. This association between gypsum and strontium in brine media, such as the Paris Basin, is common (Kushnir 1980; Fontes and Matray 1993; Rosell et al. 1998). Therefore, the occurrence of this element can be considered as resulting from the geological background.

-Terracotta units $\left(R_{b}\right)$

In both cases, bricks amounts are very small. XRF analyses show the noticeable occurrence of $\mathrm{Mg}, \mathrm{Al}$, $\mathrm{Si}, \mathrm{P}, \mathrm{S}, \mathrm{K}, \mathrm{Ca}, \mathrm{Ti}$ and $\mathrm{Fe}$ (Fig. 7). These elements are commonly found in ceramics. In terms of major and intermediate elements, amounts are in accordance with chemical composition of bricks (Cultrone et al. 2001; Lin et al. 2010) but they show that bricks do not have the same origin depending on the material (Fig. 8). XRF analyses also highlight the occurrence of trace elements such as $\mathrm{Cr}, \mathrm{Mn}, \mathrm{Ni}, \mathrm{Cu}$, $\mathrm{Zn}, \mathrm{Pb}, \mathrm{Sr}$ and $\mathrm{Zr}$. Chemical analyses show the presence of barium and vanadium (see ESM). In both materials, $\mathrm{Ba}, \mathrm{Cr}$ and $\mathrm{V}$ show noticeable amounts. SI also has noticeable zinc and lead contents (Fig. 9). These elements occur because of a small contribution of the dust from other artefacts, especially slags and gypsum.

\section{-Aggregated fine soil $\left(X_{1}\right)$}

For the two materials, the $X R F$ signal from the $X_{1}$ family is superimposed onto the $X R F$ signal of the whole material (Figs. 3, 4 and Fig. 7). For SB, on average, the elemental content ratio of the whole material over this artefact scores 1.20 (standard deviation $=0.78$ ) versus $1.07(0.51), 0.86(0.35)$ and 1.08 (0.55), when considering the ratio between the whole material and its fine, intermediate and coarse fractions, respectively. For soil SI, the ratios become $1.02(0.37)$ against $1.05(0.34), 1.15(0.65)$ and 1.18 (0.89). These results suggest that $X_{1}$ is close to the fine fraction. High levels of trace elements and 
LOI and $\rho_{d}$ measurements, which are characteristic of artefact families (Fig. 9), highlight that this fraction (Jain et al. 2005; Jani et al. 2016).

-Summary of the sorting test

The sorting test shows that both materials are a mix between natural gravels and anthropic material such as slags (magnetic and non-magnetic) and concrete products. Gypsum material present may be natural and/or anthropic. Linked with XRF and ICP-OES analyses, the test also shows that slags carry metallic and carbonaceous pollution and that gypsum components carry sulfur and strontium. Those results can be linked with physical considerations. Trends of $\mathrm{Sr}$ and $\mathrm{Ni}$ increasing with the amounts of fine grains (Fig. 6) should indicate that amounts of $G_{y}$ and SF are higher in the intermediate and fine fractions than in the coarsest one. On the contrary, the trend of total carbon being lower in the finest fractions than in others may indicate that the amount of SNF in the whole material is lower than in the coarsest fraction. Finally, the artefacts defined as aggregated fine soils are a mix of all kind of artefact families. This $X_{1}$ family is also close to the fine fraction original materials.

Interestingly, it has to be noted, that pollutant-bearing phases (SNF, SF and $\mathrm{G}_{\mathrm{y}}$ ) exhibit noteworthy physical properties. With $\rho_{d}=2.4 \mathrm{~g} / \mathrm{cm}^{3}$, the densities of SNF and Gy are the lowest, while, in addition of its magnetic behavior, with $\rho_{d}=3.7-4.0 \mathrm{~g} / \mathrm{cm}^{3}$, the densities of $S F$ are the highest.

\section{Quantification of the contributions of artefacts to the granulometric fractions}

Herein, we report how we tried to retrieve the percentage (in mass) of the artefacts that constitute the materials. To do so, we established a statistical link between the measurements for element content that characterize the artefacts and those that characterize the whole matrixes fractions to build a linear model using least absolute residual error (LAR) procedure. These represent the contribution (in mass) of the artefacts to the whole mixture and are discussed below. We address data consistency by comparing the measure values and the predicted values using LAR procedure for the whole matrixes.

\subsection{Determination of outliers in measured data}

As fitting approaches are very sensitive to outliers, they must be identified at first to be discarded for the statistical procedure. To do so, we firstly computed the equivalent value for the whole matrixes

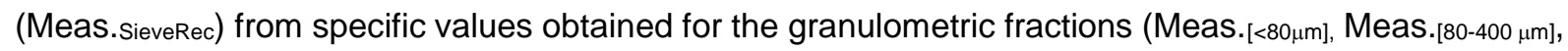
Meas.[>400 $\mu \mathrm{m}])$ and considering their mass ratios:

Meas.SieveRec $=37 \%$ Meas. $[<80 \mu \mathrm{m}]+16 \%$ Meas. $[80-400 \mu \mathrm{m}]+47 \%$ Meas. $[>400 \mu \mathrm{m}]$ (mat. SB)

Meas.SieveRec $=32 \%$ Meas. $[<80 \mu \mathrm{m}]+19 \%$ Meas. $[80-400 \mu \mathrm{m}]+49 \%$ Meas. $[>400 \mu \mathrm{m}]$ (mat. SI) 
Theoretically, Meas.sieverec should match the set of measurements done on the whole fraction (Meas.wm). For both materials SB and SI, the relative errors between Meas. SieveRec and Meas.wm were computed to assess the consistency between the two measures and are depicted in Fig. 10. Any given measurement is considered as an outlier when its ratio exceeds $20 \%$ in absolute value.

For SB, thirteen measurements out of twenty-seven were of concern, specifically trace elements such as $\mathrm{Ba}, \mathrm{Cd}, \mathrm{Cr}, \mathrm{Cu}, \mathrm{Mn}, \mathrm{Mo}, \mathrm{Pb}, \mathrm{V}$ and $\mathrm{Zn}$. These results highlight previous observations about the occurrence of rich spots. Measurements for silicon content and LOI at $450^{\circ} \mathrm{C}$ and $550{ }^{\circ} \mathrm{C}$ also had inconsistency. Carbon-rich slags were probably present in the sample of the whole material. The $S$ measurement must also be considered as outlier. This is a bit surprising because $S$ is mainly borne by gypsum as strontium and calcium and these two last give consistent measurements. The sum of percentages of the rest (i.e. the data that ensures a relative error lower than $20 \%$ ) scores $38 \%$, which is indicative of the correctness of the data.

For $\mathrm{SI}$, fewer measurements are inconsistent. Trace metallic elements such as $\mathrm{Ba}, \mathrm{Mo}, \mathrm{Pb}$ and $\mathrm{Zn}$ were still found as outliers. LOI at $450{ }^{\circ} \mathrm{C}$ was also of concern, but it is close to the boundary, with $21 \%$. Two intermediate elements Ti and P can be defined as outliers. The correctness of the data is $153 \%$.

\subsection{Results of least absolute residuals (LAR) procedure}

For both materials, $\mathrm{X}_{1}$ is close to the original material. To avoid problems linked to redundant data and related non-uniqueness of estimated parameters, we decided to avoid the implementation of this family in the statistical procedure. Initial calculation conditions were the amount of each artefact family in the granular fraction (Table 1) weighted by the lack of $X_{1}$. They are respectively for SNF, SF, $R_{u}, G_{y}, R_{b}$ and $\mathrm{R}_{\mathrm{c}}$ :

- $\quad$ In SB: $30.5 \%, 5 \%, 36.5 \%, 4.7 \%, 2.2 \%$ and $21.2 \%$.

- $\quad$ In SI: $20 \%, 2.1 \%, 65.4 \%, 7.4 \%, 2 \%$ and $3.2 \%$

\subsubsection{Calculations without constraints}

Results of mathematical regression without constraint are summarized in Table 3. TOT is the sum of the artefact weights. Exact. is the sum of distances between the model and the experimental measurements (expressed in percent). It quantifies the exactness or sensitivity of the model to compare with the correctness of the data.

Not all the results are physically relevant, but some interesting information can be tapped. In the case of SB, the exactness of the model is between 90 and $280 \%$. For SI, the exactness is around $300 \%$ with an exception for the fine fraction that exhibits a value of $650 \%$. It is higher than the correctness of the data but the order of magnitude is the same (less than 10 times higher). Interestingly, in both cases, the 
sum of artefact weights are relevant for a non-constrained approach. And even more, 5 reconstructions on 8 give sums of artefacts that are less than $10 \%$ far from $100 \%$.

\section{- Reconstruction of granulometric fractions of SB}

In all the granulometric fractions, determined amounts of SNF and SF are relevant with initial conditions of calculations (i.e. the amount of each artefact family in the granular fraction).

With extreme values, amounts of $R_{c}, R_{u}, G_{y}$ and $R_{b}$ in the coarsest fraction ( $>400 \mu \mathrm{m}$ ) are clearly not relevant. In the three other fractions, calculated amounts of $R_{u}$ are satisfying. Amounts of $R_{c}$ are also satisfying in the intermediate fraction but they are too far from initial conditions of calculations in the fine fraction and in the whole material. Amounts of $G_{y}$ are relevant in the whole material and intermediate fraction, but clearly too high in the fine fraction. For $\mathrm{R}_{\mathrm{b}}$, amounts are relevant in the whole material and in the fine fraction.

- Reconstruction of granular fractions of material SI

In the whole material, amounts of SNF, SF and $\mathrm{R}_{u}$ are relevant with initial conditions, even if occurrence of $R_{u}$ is a bit low. However, the amounts of $R_{c}, G_{y}$ and $R_{b}$ are clearly wrong. $R_{c}$ is far too high in the granular fraction. By contrast, the reconstruction of the $<80 \mu \mathrm{m}$ fraction is satisfying for all artefacts. In the $80-400 \mu \mathrm{m}$ fraction, the amounts of SNF, SF are relevant with initial conditions. The amount of $R_{u}$ is a bit low, while the amounts of $G_{y}, R_{c}, R_{b}$ are too high. In the coarsest fraction, except for $R_{u}$, which is a bit low, all amounts are relevant with $\mathrm{Cl}$.

\subsubsection{Calculations with constraints}

For this computation, we considered for the new initial conditions the results of the LAR procedure without constraints, which are detailed in Table 3. The first constraint is that all total sums (TOT) have been set to $100 \%$. Then, based on previous observations and chemical results, other constraints have been determined for calculations. Results are detailed in Table 4.

After calculations, in the case of SB, the exactness is around 1 to 1.5 times higher than the exactness of the modeling without constraints, except for the coarse fraction, 2.4 times higher. For SI, the exactness is very close to that of the modeling without constraint.

- Reconstruction of granular fractions of SB;

For the whole material, WM, the results from simulation without constraint were satisfying, except the amount of $R_{c}$ that was too high. Therefore, for this new simulation, the amount of $R_{c}$ has been set less than $25 \%$, which is consistent with the amount of $R_{c}$ in the granular fraction. Constrained results are very close to non-constrained results (Tables 3 and 4 ) with less than $4 \%$ of difference. This shows that for the whole material, running the LAR procedure with constraint did not significantly change the procedure. 
For the reconstruction of the $<80 \mu \mathrm{m}$ fraction, based on the good results for WM, the amounts of SNF, organic carbon. The ratio of this element between the fraction WM and the fraction $<80 \mu \mathrm{m}$ is 1.7 . Consequently, the amount of $C_{\text {org }}$ in SNF was set at $12.2 \%$. This value is close to that obtained with unconstrained LAR, i.e. $10.1 \%$. Likewise, for the SF family, the chosen chemical marker was nickel. The ratio between the two fractions is 0.7 . The amount of SF was consequently fixed at $13.5 \%$, which is also in agreement with the previous results from unconstrained LAR (i.e. $6.6 \%$ ). For the Gy family, the chosen chemical marker was $\mathrm{Sr}$. The ratio is 0.7 . The amount of $\mathrm{G}_{\mathrm{y}}$ was then set at $14.3 \%$, which is also close to the previous results (i.e. $19.1 \%$ ). Finally, to avoid divergence of calculation, the amount of $R_{b}$ was set $<5 \%$. The amounts of $R_{u}$ and $R_{c}$ are then $37.5 \%$ and $17.2 \%$. For $R_{u}$ the result is close to the previous unconstrained LAR results.

For the reconstruction of the $80-400 \mu \mathrm{m}$ fraction, following the same approach applied to the case of the fraction $<80 \mu \mathrm{m}$, the amounts of SNF, SF and $\mathrm{G}_{y}$ were set at respectively $23.2 \%, 6.4 \%$ and $8.3 \%$. All set amounts are close to the results obtained with the unconstrained LAR. The amounts of $R_{u}, R_{b}$ and $R_{c}$ are predicted at $32.1 \%, 0 \%$, and $29.2 \%$. About $R_{b}$, as previously mentioned, the null value obtained results from the lack of sensitivity of the approach. The amount of $29.2 \%$ for $R_{c}$ on the $<80 \mu \mathrm{m}$ fraction is a bit high but can be considered as satisfying, regarding the respect of the threshold of $25 \%$ set for the whole material. Lastly, the result is relevant for the $R_{c}$ family.

For the reconstruction of the $>400 \mu \mathrm{m}$ fraction, still following the same approach, the amounts of SNF, SF and $\mathrm{G}_{\mathrm{y}}$ were set at respective values of $23.2 \%, 6.4 \%$ and $8.3 \%$. As previously, the amounts of SF and SNF are close to that predicted by the unconstrained LAR. To avoid divergence, the amounts of $R_{c}$ were set at a maximum value of $25 \%$. The amounts of $R_{u}$ and $R_{b}$ are then $30.1 \%$ and $7.0 \%$. $R_{u}$ is in line with the previous results. Amount of $R_{b}$ is also relevant, even if a bit high.

\section{- Reconstruction of granular fractions of SI}

For the whole material, the previous results of unconstrained LAR exhibit levels of $R_{b}$ and $R_{c}$ that are too high. So for this new reconstruction these two levels were set at less than $5 \%$. The new results on SF, SNF and $G_{y}$ families are close to previous ones. $R_{u}$ increases to $62.6 \%$ from $44.1 \%$. This value is much more logical than the previous one, given the level of $R_{u}$ in the granular fraction (65.4\%).

For the $<80 \mu \mathrm{m}$ fraction, considering the satisfying reconstruction obtained without constraint, no constraint other than the total sum of $100 \%$ was set. Results are really close to previous results with unconstrained LAR. The determination of the SNF amount by following organic carbon as chemical marker would lead to a value of $4.4 \%$. In the same way, the determination of the SF amount by following $\mathrm{Mn}$ and $\mathrm{Cu}$ (Fig. 6) as chemical markers would lead to a value of $8.6 \%$. Finally, the determination of $\mathrm{G}_{\mathrm{y}}$ amount by following $\mathrm{Sr}$ and $\mathrm{S}$ as chemical markers would lead to a value of $11.2 \%$. All these relative contents are really close to the values predicted by the constrained LAR procedure. These shows that results from constrained LAR are relevant with chemical analysis. 
For the $80-400 \mu \mathrm{m}$ fraction, as for the WM, previous results of unconstrained LAR are too high for $\mathrm{R}_{\mathrm{b}}$

\subsubsection{Artefact distribution and limitations of the LAR approach}

The unconstrained LAR procedure gives some relevant results (e.g. the sum of the amounts) but it must be constrained to allow discriminating analyses. For SB and SI, in addition to unconstrained procedure and chemical analyses, the LAR procedure shows that the SNF family is more present in the coarsest fraction than in the intermediate and fine fractions. The high interaction of $\mathrm{Al}$ and Ti with this family confirms that the specific distributions of these elements in the granulometric fraction of original material result from anthropic input. In comparison, the levels of SF exhibit opposite behaviors. Considering that primarily, the two artefacts could have a similar origin, we can state that after break-up, in term of weathering, SF are less stable than SNF. The LAR procedure also shows that levels of $R_{u}$ increase with the fineness of the fractions as expected for a natural material. The tendencies are similar with gypsum that is known to be easily weathered with water (Eighmy et al. 1994).

Unfortunately, it is difficult to draw conclusions about the distribution of the families $R_{b}$ and $R_{c}$. There is no dedicated chemical marker and only threshold can be used to constrain values. Moreover, the low levels of $R_{b}$ and $R_{c}$ in $S I$ and $R_{b}$ in SB clearly limit the effectiveness of the LAR procedure.

\section{Conclusions}


Both Technosols have a similar geological origin and were used as backfill for industrial purposes in the

Acknowledgements This work was supported by the French Ministry of Ecology, Energy, Sustainable Development and Spatial Planning and the French National Federation of Public Works. The manuscript benefited from comments and suggestions by two anonymous reviewers.

\section{References}


Adnan SNSBM, Yusoff S, Chua YP (2013) Soil chemistry and pollution study of a closed landfill site at Ampar Tenang, Selangor, Malaysia. Waste Manage Res 31(6):599-612

AFNOR (1992a) Earthworks Classification of materials for use in the construction of embankments and capping layers of road infrastructures. NF P11-300. AFNOR Ed

AFNOR (1992b) Soils: investigation and testing. Granulometric analysis. Hydrometer method. NF P94057. AFNOR Ed

AFNOR (1993) Soils: investigation and testing. Determination of Atterberg's limits. Liquid limit test using cassagrande apparatus. Plastic limit test on rolled thread. NF P94-051. AFNOR Ed

AFNOR (2001) Soil quality - Dissolution for the determination of total element content - Part 1: Dissolution with hydrofluoric and perchloric acids. NF ISO 14869-1. AFNOR Ed

AFNOR (2007) Characterization of waste - Determination of loss on ignition in waste, sludge and sediments. NF EN 15169. AFNOR Ed

AFNOR (2008) Tests for mechanical and physical properties of aggregates - Part 7: Determination of the particle density of filler - Pycnometer method. NF EN 1097-7. AFNOR Ed

AFNOR (2009a) Tests for geometrical properties of aggregates - Part 11 : classification test for the constituents of coarse recycled aggregate. NF EN 933-11. AFNOR Ed

AFNOR (2009b) Water quality - Determination of selected elements by inductively coupled plasma optical emission spectrometry (ICP-OES) NF ISO 11885. AFNOR Ed

AFNOR (2012) Tests for chemical properties of aggregates - Part 8: Sorting test to determine metal content of Municipal Incinerator Bottom Ash (MIBA) Aggregates. NF EN 1744-8. AFNOR Ed

AFNOR (2013) Sludge, treated biowaste, soil and waste - Determination of loss on ignition. NF EN 15935. AFNOR Ed

Alekseenko V, Alekseenko A (2014) The abundances of chemical elements in urban soils. J Geochem Explor 147(B):245-249

Arulrajah A, Piratheepan J, Disfani MM, Bo MW (2013) Geotechnical and Geoenvironmental Properties of Recycled Construction and Demolition Materials in Pavement Subbase Applications. J Mater Civ Eng 25(8):1077-1088

Asakura H, Yamada M, Inoue Y, Watanabe Y, Ono Y (2010) Investigation on the components removed in loss on ignition test of sandy crushed construction and demolition waste. Waste Manage Res 28(1):11-19

ASTM (2009) Standard Practice for Classification of Soils and Soil-Aggregate Mixtures for Highway Construction Purposes. D3282-09. ASTM International, West Conshohocken, PA 
ASTM (2011) Standard Practice for Classification of Soils for Engineering Purposes (Unified Soil Classification System). D2487-11. ASTM International, West Conshohocken, PA

Bech J, Reverter F, Tume P, Sanchez P, Longan L, Bech J, Olivier T (2011) Pedogeochemical mapping of $\mathrm{Al}, \mathrm{Ba}, \mathrm{Pb}, \mathrm{Ti}$ and $\mathrm{V}$ in surface soils of Barcelona Province (Catalonia, NE Spain): Relationships with soil physicochemical properties. J Geochem Explor 109(1-3):26-37

Bianchini G, Marrocchino E, Tassinari R, Vaccaro C (2005) Recycling of construction and demolition waste materials : a chemical-mineralogical appraisal. Waste Manage 25(2):149-159

Biasoli M, Barberis R, Ajmone-Marsan F (2006) The influence of a large city on some soil properties and metals content. Sci Total Environ 356(1-3):154-164

Blanco-Canqui H, Lal R, Post WM, Izaurralde RC, Shipitalo MJ (2005) Organic Carbon Influences on Soil Particle Density and Rheological Properties. Soil Sci Soc Am J 70(4):1407-1414

Bonde TA, Christensen BT, Cerri CC (1992) Dynamics of soil organic-matter as reflected by natural ${ }^{13} \mathrm{C}$ abundance in particle-size fractions of forested and cultivated oxisols. Soil Biol Biochem 24(3):275277

BRGM (1973) Geological map Paris n¹83. Bureau de Recherches Géologiques et Minières Ed

Bryson GM, Barker AV (2002) Sodium accumulation in soils and plants along Massachussetts roadsides. Commun Soil Sci Anal 33(1-2):67-78

Burlakovs J, Kaczala F, Vincevica-Gaile Z, Rudovica V, Orupold K, Stapkevica M, Bhatnagar A, Kriipsalu M, Hogland M, Klavins M, Hogland W (2016) Mobility of Metals and Valorization of Sorted Fine Fraction of Waste After Landfill Excavation. Waste Biomass Valor 7(3):593-602

Cai M, McBride MB, Li K, Li Z (2017) Bioaccessibility of As and Pb in orchard and urban soils amended with phosphate, Fe oxide and organic matter. Chemosphere 173:153-159

Cabello Eras JJ, Sagastume Gutierrez A, Hernandez Capote D, Hens L, Vandecasteele C (2013) Improving the environmental performance of an earthwork project using cleaner production strategies. J Clean Prod 47 :368-376

Cachada A, Dias AC, Pato P, Mieiro C, Rocha-Santos T, Pereira ME, Ferreira da Silva E, Duarte AC (2013) Major inputs and mobility of potentially toxic elements contamination in urban areas. Environ Monit Assess 185(1):279-294

Capony A, Muresan B, Dauvergne M, Auriol JC, Ferber V, Jullien A (2013) Monitoring and environmental modeling of earthworks impacts: A road construction case study. Resour Conserv Recy 74:124133 
Carter MR, Angers DA, Gregorich EG, Bolinder MA (2003) Characterizing organic matter retention for surface soils in eastern Canada using density and particle size fractions. Can J Soil Sci 83(1):1123

Chaurand P, Rose J, Briois V, Olivi L, Hazemann JL, Proux O, Domas J, Bottero JY (2007) Environmental impacts of steel slag reused in road construction: A crystallographic and molecular (XANES) approach. J Hazard Mater 139(3):537-542

Chen TB, Zheng YM, Lei M, Huang ZC, Wu HT, Chen H, Fan KK, Yu K, Wu X, Tian QZ (2005) Assessment of heavy metal pollution in surface soils of urban parks in Beijing, China. Chemosphere 60(4):542-551

Chindaprasirt P, Boonserm K, Chairuangsri T, Vichit-Vadakan W, Eaimsin T, Sato T, Pimraksa K (2011) Plaster materials from waste calcium sulfate containing chemicals, organic fibers and inorganic additives. Constr Build Mater 25(8):3193-3203

Cornu S, Lucas Y, Lebon E, Ambrosi JP, Luizão F, Rouiller J, Bonnay M, Neal C (1999) Evidence of titanium mobility in soil profiles, Manaus, central Amazonia. Geoderma 91(3-4):281-295

Coronado M, Dorsal E, Coz A, Viguri JR, Andrés A (2011) Estimation of Construction and Demolition Waste (C\&DW) Generation and Multicriteria analysis of C\&DW Management Alternatives: A case Study in Spain. Waste Biomass Valor 2(2):209-225

Cultrone G, Rodriguez-Navarro C, Sebastian E, Cazalla O, De la Torre MJ (2001) Carbonate and silicate phase reactions during ceramic firing. Eur J Mineral 13(3):621-634

De Kimpe CR, Morel JL (2000) Urban soil management: A growing concern. Soil Sci 165(1):31-40

Ding T, Xiao J (2014) Estimation of building-related construction and demolition waste in Shanghai. Waste Manage 34(11):2327-2334

Douay F, Pruvot C, Roussel H, Ciesielski H, Fourrier H, Proix N, Waterlot C (2008) Contamination of urban soils in an area of northern France polluted by dust emissions of tws Smelters. Water Air Soil Pollut 188(1-4):247-260

Duong TTT, Lee BK (2011) Determining contamination level of heavy metals in road dust from busy traffic areas with different characteristics. J Environ Manage 92(3):554-562

Eighmy TT, Eusden Jr JD, Marselle K, Hogan J, Domingo D, Krzanowski JE, Stampfli D (1994) Particle Petrogenesis ans Speciation of Elements in MSW incineration Bottom Ashes. Stud Environ Science 60:111-136

El Khalil H, Schwartz C, El Hamiani O, Kubiniok J, Morel JL, Boularbah A (2008) Contribution of Technic Materials to the Mobile Fraction of Metals in Urban Soils in Marrakech (Morocco). J Soils Sediments $8(1): 17-22$ 
El Khalil H, Schwartz C, El Hamiani O, Kubiniok J, Morel JL, Boularbah A (2013) Distribution of major elements and trace metals as indicators of technosolisation of urban and suburban soils. J Soils Sediments 13(3):519-530

Folgueras MB, Alonso M, Fernandez FJ (2017) Coal and sewage sludge ashes as sources of rare earth elements. Fuel 192:128-139

Fontes JC, Matray JM (1993) Geochemistry and origin of formation brines from Paris Basin, France. 2. Saline solutions associated with oil fields. Chem Geol 109(1-4):177-200

Haecker CJ, Garboczi EJ, Bullard JW, Bohn RB, Sun Z, Shah SP, Voigt T (2005) Modeling the linear elastic properties of Portland cement paste. Cem Concr Res 35(10):1948-1960

Hammond CR (2003) Handbook of chemistry and Physics. 84 ${ }^{\text {th }}$ ed. Lide DR (ed) CRC Press, ISBN 9780-849-30484-2, $2620 p$

Hanna K, Lassabatere L, Bechet B (2009) Zinc and lead transfer in a contaminated roadside soil: Experimental study and modeling. J Hazard Mater 161(2-3):1499-1505

Hatry G, Mercier P (1991) L'île Seguin. JCM Ed. Paris, ISBN 2-902667-14-0, 110 p

Herselman JE, Steyn CE, Fey MV (2005) Baseline concentration of Cd, Co, Cr, Cu, Pb, Ni and Zn in surface soils of South Africa. South African J Sci 101(11-12):509-512

Hillel D (2004) Encyclopedia of soils in the environment, $1^{\text {st }}$ ed. Academic Press

Huang SL, Yeh CT, Chang LF (2010) The transition to an urbanizing world and the demand for natural resources. Curr Opin Environ Sustain 2(3):136-143

Huot H, Simmonot MO, Watteau F, Marion P, Yvon J, De Donato P, Morel JL (2014a) Early transformation and transfer processes in a Technosol developing on iron industry deposits. Eur $\mathrm{J}$ Soil Sci 65(4):470-484

Huot H, Faure P, Biache C, Lorgeoux C, Simonnot MO, Morel JL (2014b) A Technosoil as archives of organic matter related to past industrial activities. Sci Total Environ 487:389-398

Ioannidou D, Vasileios N, Briere R, Zerbi S, Habert G (2015) Land-cover-based indicator to assess the accessibility of resources used in the construction sector. Resour Conserv Recy 94:80-91

Islam MS, Ahmed MK, Habibullah-Al-Mamun M, Raknuzzaman M (2015) Trace elements in different land use soils of Bangladesh and potential ecological risk. Envrion Monit Assess 187:587-597

IUSS Working Group WRB (2015) World Reference Base for Soil Resources 2014, update 2015International soil classification system for naming soils and creating legends for soil maps. World Soil Resources Reports No. 106. FAO, Rome 
Jain P, Kim H, Townsend TG (2005) Heavy metal content in soil reclaimed from a municipal solid waste landfill. Waste Manage 25(1):25-35

Jani Y, Kaczala F, Marchand C, Hogland M, Kriipsalu M, Hogland W, Kihl A (2016) Characterisation of excavated fine fraction and waste composition from Swedish landfill. Waste Manage Res 34(12):1292-1299

Jean-Soro L, Le Guern C, Bechet B, Lebeau T, Ringeard MF (2015) Origin of trace elements in an urban garden in Nantes, France. J Soils Sediments 15(8):1802-1812

Jimenez-Rivero A, Garcia-Navarro J (2017) Exploring factors influencing post-consumer gypsum recycling and landfilling in the European Union. Resour Conserv Recy 116:116-123

Kabata-Pendias A (2010) Trace Elements in Soils and Plants, $4^{\text {th }}$ ed. CRC Press, Boca Raton

Kushnir J (1980) The coprecipitation of strontium, magnesium, sodium and chloride ions with gypsum. An experimental study. Geochim Cosmochim Ac 44(10):1471-1482

Lee S, Xu Q, Booth M, Townsend TG, Chadik P, Bitton G (2006) Reduced sulfur compounds in gas from construction and demolition debris landfills. Waste Manage 26(5):526-533

Leharne S, Charlesworth D, Chodhry B (1992) A survey of metals levels in street dusts in an inner London neighbourhood. Environ Int 18(3):263-270

Leguedois S, Séré G, Auclerc A, Cortet J, Huot H, Ouvrard S, Watteau F, Schwartz C, Morel JL (2016) Modelling pedogenesis of Technosols. Geoderma 262:199-212

Lenoir T, Preteseille M, Ricordel S (2016) Contribution of the fiber reinforcement on the fatigue behavior of two cement-modified soils. Int J Fatigue 93(1):71-81

Lin KL, Wu HH, Shie JL, Hwang CL, Cheng A (2010) Recycling waste brick from construction and demolition of buildings as pozzolanic materials. Waste Manage Res 28(7):653-659

Lopez-Garcia I, Arnau-Jerez I, Campillo N, Hernandez-Cordoba M (2004) Determination of tin and titanium in soils, sediments ans sludges using electrothermal atomic spectrometry with slurry sample introduction. Talanta 62(2):413-419

Lorenz K, Kandeler E (2005) Biochemical characterization of urban soil profiles from Stuttgart, Germany. Soil Biol Biochem 37(7):1373-1385

Lu Y, Gong Z, Zhang G, Burghardt W (2003) Concentrations and chemical speciations of $\mathrm{Cu}, \mathrm{Zn}, \mathrm{Pb}$ and $\mathrm{Cr}$ of urban soils in Nanjing, China. Geoderma 115(1-2):101-111

Luo W, Lu Y, Wang B, Tong X, Wang G, Shi Y, Wang T, Giesy JP (2009) Distribution and sources of mercury in soils from former industrialized urban areas of Beijing, China. Environ Monit Assess 158:507-517 
Magnusson S, Lundberg K, Svedberg B, Knutsson S (2015) Sustainable management of excavated soil and rock in urban areas - A literature review. J Clean Prod 93:18-25

Mantis I, Voutsa D, Samara C (2005) Assessment of the environmental hazard from municipal and industrial wastewater treatment sludge by employing chemical biological methods. Ecotox Environ Safe 62(3):397-407

Morel JL, Chenu C, Lorenz K (2015) Ecosystem services provided by soils of urban, industrial, traffic, mining, and military areas (SUITMAs). J Soils Sediments 15(8):1659-1666

Münch D (1993) Concentration profiles of arsenic, cadmium, chromium, copper, lead, mercury, nickel, zinc, vanadium and polynuclear aromatic hydrocarbons $(\mathrm{PAH})$ in forest soil beside an urban road. Sci Total Environ 138(1-3):47-55

Nazzal Y, Rosen MA, Al-Rawabdeh AM (2013) Assessment of metal pollution in urban road dusts from selected highways of the Greater Toronto Area in Canada. Environ Monit Assess 185(2):18471858

Nelson SS, Yonge DR, Barber ME (2009) Effects of road salts on heavy metal mobility in two eastern Washington soils. J Environ Eng - ASCE 137(7):505-510

Nezat CA, Hatch SA, Uecker T (2017) Heavy metal content in urban residential and park soils: A case study in Spokane, Washington, USA. Appl Geochem 78:186-193

Norra S, Lanka-Panditha M, Kramar U, Stüben D (2006) Mineralogical and geochemical patterns of urban surface soils, the example of Pforzheim, Germany. Appl Geochem 21(12):2064-2081

Norra S, Fjer N, Li F, Chu X, Xie X, Stüben D (2008) The influence of different land uses on mineralogical and chemical composition and horizonation of urban soil profiles in Quingdao, China. J Soils Sediments 8(1):4-16

Ollila HJ, Moilanen A, Tiainen MS, Laitinen RS (2006) SEM-EDS characterization of inorganic material in refuse-derived fuels. Fuel 85(17-18):2586-2592

Pariente S, Zhevelev HM, Oz A (2016) Human activities modify soil properties in urban parks : a case study of Tel Aviv-Jaffa. J Soils Sediments 16(11):2538-2547

Poon CS, Yu ATW, Ng LH (2001) On-site sorting of construction and demolition waste in Hong Kong, Resour Conserv Recy 32(2):157-172

Preteseille M, Lenoir T (2016) Structural test at the laboratory scale for the utilization of stabilized finegrained soils in the subgrades of High Speed Rail infrastructures: experimental aspects. Int $J$ Fatigue 82(3):505-513

Reimann C, de Caritat P (1998) Chemical Elements in the Environment: Factsheets for the Geochemist and Environmental Scientist, $1^{\text {st }}$ Ed. Springer-Verlag Berlin Heidelberg. ISBN 978-3-642-72018-5 
Rigo C, Zamengo L, Rampazzo G, Argese E (2009) Characterization of a former dump site in the Laggoon of Venice contaminated by municipal solid waste incerator bottom ash, and estimation of possible environmental risk. Chemosphere 77(4):510-517

Rivas V, Cendrero A, Hurtado M, Cabral M, Gimenez J, Forte L, del Rio L, Cantù M, Becker A (2006) Geomorphic consequences of urban development and mining activities; an analysis of study areas in spain and Argentina. Geomorphology 73(3-4):185-206

Rosell L, Orti F, Kasprzyk A, Playa E, Peryt TM (1998) Strontium geochemistry of Miocene primary gypsum: Messinian of southestern Spain and Sicily and Bedenian of Poland. J Sediment Res 68(1):63-79

Rossiter GG (2007) Classification of urban and industrial soils in the world reference base for soil resources. J Soils Sediments 7(2):96-100

Saiz Martinez P, Gonzalez Cortina M, Fernandez Martinez F, Rodriguez Sanchez A (2016) Comparative study of three types of fine recycled aggregates from construction and demolition waste (CDW), and their use in masonry mortar fabrication. J Clean Prod 118:162-169

Salvagio Manta D, Angelone M, Bellanca A, Neri R, Sprovieri M (2002) Heavy metals in urban soils: a case study from the city of Palermo (Sicily), Italy. Sci Total Environ 300(1-3):229-243

Santisteban, J.I., Mediavilla, R., Lopez-Pamo, E., Dabrio, C.J., Blanca Ruiz Zapata, M., Jose Gil Garcia, M., Castano, S., Martinez-Alfaro, P.E., 2004. Loss on ignition: a qualitative or quantitative method for organic matter and carbonate mineral content in sediments? J. Paleolimnol. 32(3), 287-299.

Séré G, Schwarz C, Ouvrard S, Renat JC, Watteau F, Villemin G, Morel JL (2010) Early pedogenic evolution of constructed Technosols. J Soils Sediments 10(7):1246-1254

Silva RV, de Brito J, Dhir RK (2014) Properties and composition of recycled aggregates from construction and demolition waste suitable for concrete production. Constr Build Mat 65:201-217

Solomon RL, Harford JW (1976) Lead and cadmium in dusts and soils in a small urban community. Environ Sci Technol 10(8):773-777

Sun XL, Wu SC, Wang HL, Zhao YG, Zhang GL, Man YB, Wong MH (2013) Dealing with spatial outliers and mapping uncertainty for evaluating the effects of urbanization on soil: $\mathrm{A}$ case study of soil $\mathrm{pH}$ and particle fractions in Hong Kong. Geoderma 195-196:220-233

Sun Y, Zhou Q, Xie X, Liu R (2010) Spatial, sources and risk assessment of heavy metal contamination of urban soils in typical regions of Shenyang, China. J Hazard Mater 174(1-3):455-462

Tossavainen M, Engstrom F, Yang Q, Menad N, Lidstrom Larsson M, Bjorkman B (2007) Characteristic of steel slag under different cooling conditions. Waste Manage 27(10):1335-1344 
Vassilev SV, Vassileva CG, Baxter D, Andersen LK (2009) A new approach for the combined chemical and mineral classification of the inorganic matter in coal. 2. Potential applications of the classification systems. Fuel 88(2):246-254

Vassilev SV, Baxter D,Andersen LK, Vassileva CG (2013) An overview of the composition and applications of biomass ash. Part 1. Phase-mineral and chemical composition and classification. Fuel 105:40-76

Vodyanitskii YN, Savichev AT, Vasil'ev AA, Lobanova ES, Chashchin AN, Prokopovich EV (2010) Contents of heavy alkaline-earth $(\mathrm{Sr}, \mathrm{Ba})$ and rare-earth $(\mathrm{Y}, \mathrm{La}, \mathrm{Ce})$ metals in technogenically contamined soils. Eurasian Soil Sci+ 43(7):822-832

Wei B, Yang L (2010) A review of heavy metal contaminations in urban soils, urban road dusts and agricultural soils from China. Microchem J 94(2):99-107

Wei ZQ, Wu SH, Zhou SL, Li JT, Zhao QG (2014) Soil Organic Carbon transformation and Related Properties in Urban Soil Under Impervious surfaces. Pedosphere 24(1):56-64

WHO (1990) Barium, Environmental Health Criteria 107, World Health Organization, Vammala Finland

WHO (1998) Copper, Environmental Health Criteria 200, World Health Organization, Vammala Finland

WHO (2010) Strontium and Strontium compounds, Concise International Chemical Assessment Document 77, Vammala Finland

Withers PJA, Jarvie HP (2008) Delivery and cycling of phosphorus in rivers: A review. Sci Total Environ 400(1-3):379-395

Wu J, Ren Y, Wang X, Wang X, Chen L, Liu G (2015) Nitrogen and phosphorus associating with different size suspended solids in roof and road runoff in Beijing, China. Environ Sci Pollut Res 22(20):15788-15795

Zhao Z, Hazelton P (2016) Evaluation of accumulation and concentration of heavy metals in different urban roadside soil types in Miranda Park, Sydney. J Soils Sediments 16(11):2548-2556 


\section{FIGURE CAPTIONS}

Fig. 1 Sampling sites (top) and soils after excavation in the field (bottom). SB left and SI right

Fig. 2 Particle size distribution and geotechnical identification parameters of the two materials

Fig. 3 Mean XRF spectra in the whole material SB and in three granular fractions

Fig. 4 Mean XRF spectra in the whole materials SB and SI

Fig. 5 Occurrence of major chemical elements expressed in \% of oxides in both materials, in the whole material and the three granular fractions

Fig. 6 Occurrence of trace elements expressed in $\mathrm{mg} / \mathrm{kg}$ dry matt. in both materials, in the whole material and in three granular fractions

Fig. 7 Mean XRF spectra of the artefacts of materials SB (solid line) and SI (dashed line)

Fig. 8 Occurrence of major chemical elements expressed in \% of oxides in artefacts from both studied materials

Fig. 9 Occurrence of trace elements expressed in $\mathrm{mg} / \mathrm{kg}_{\mathrm{dm}}$ in artefacts from both soils

Fig. 10 Ratios between the measurements on the whole material (Meas.wm) and the combination of measurements from granulometric fractions (Meas.sieveRec). SB, in blue, SI in brown 


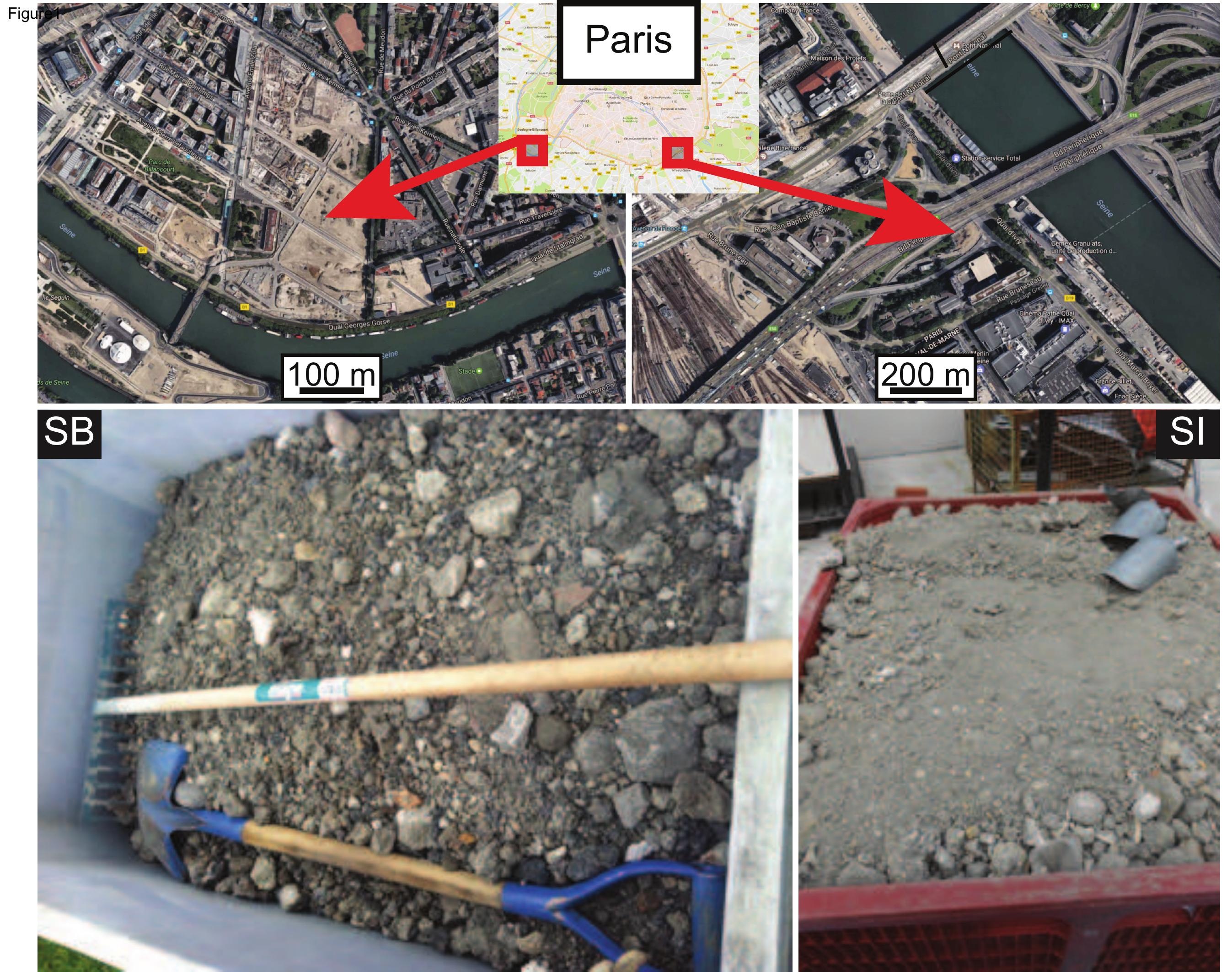




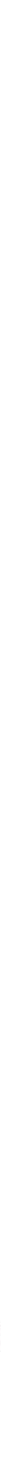




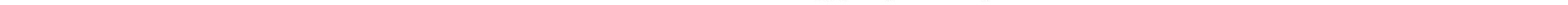




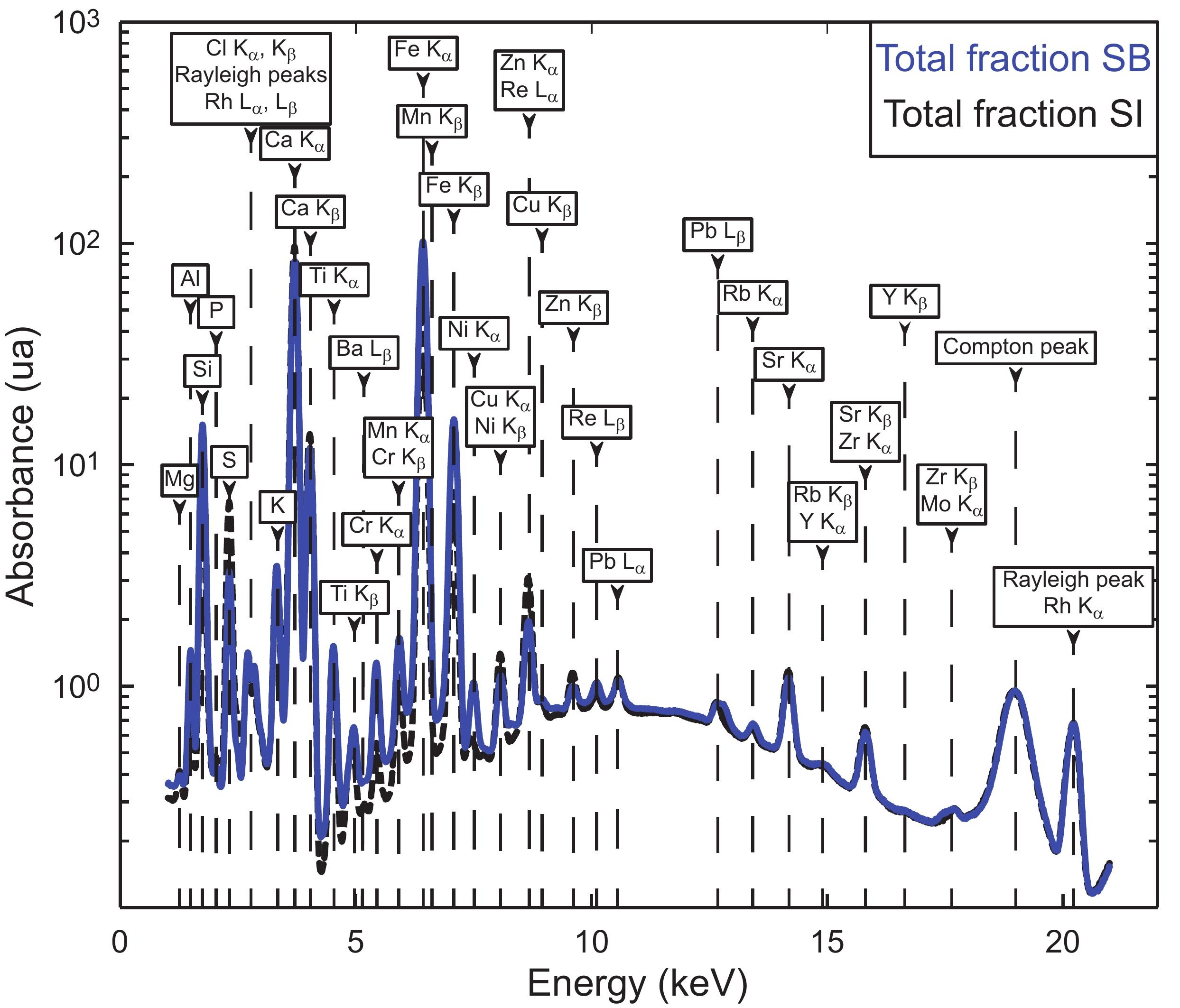



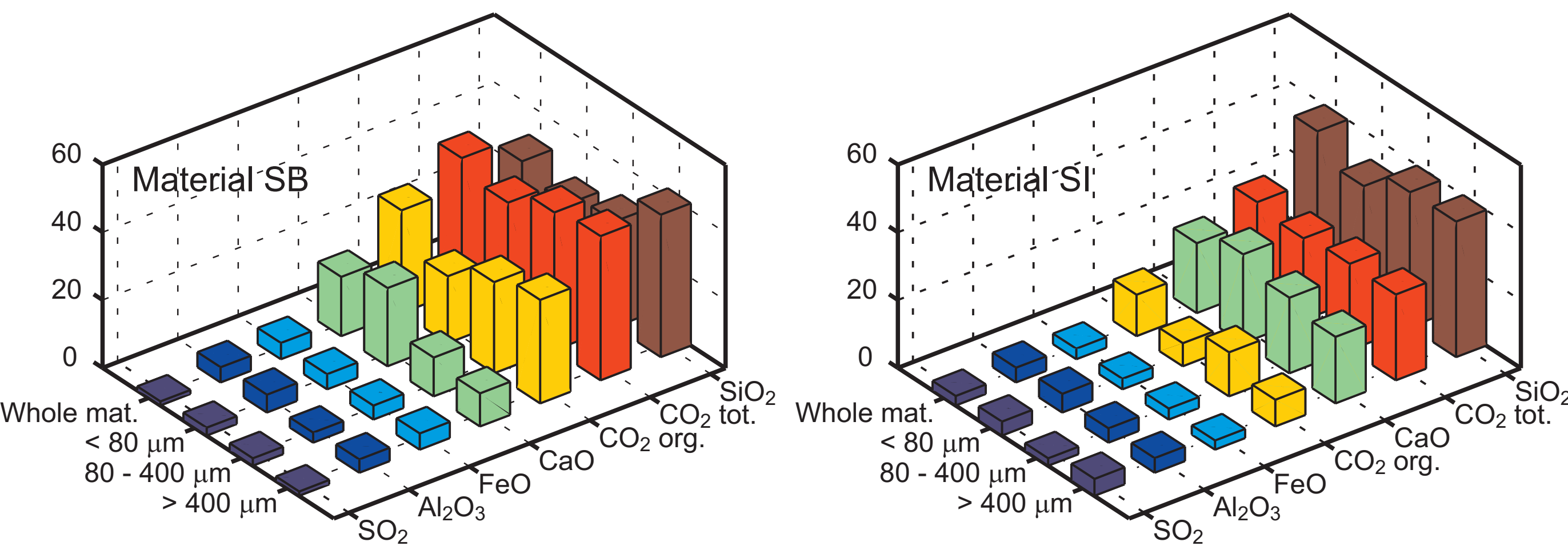


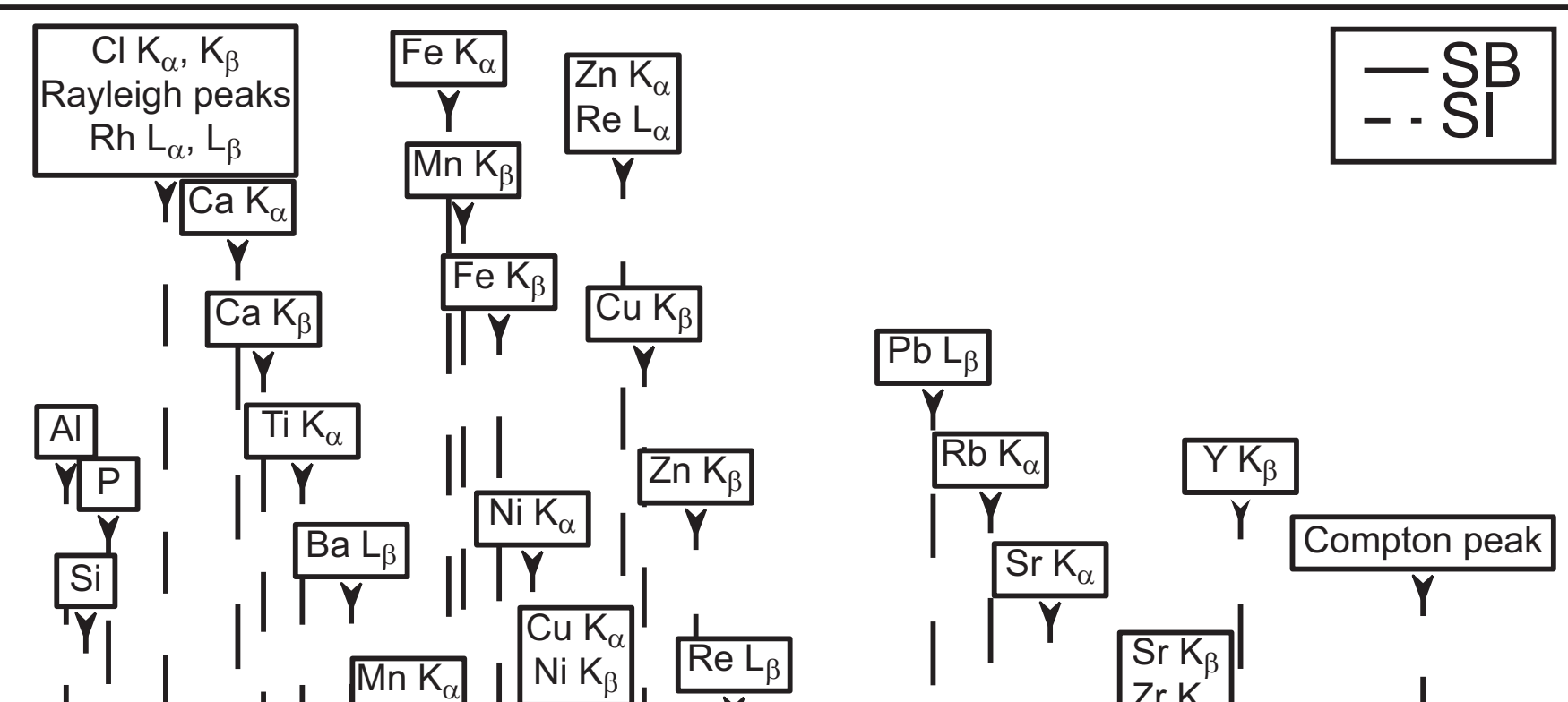

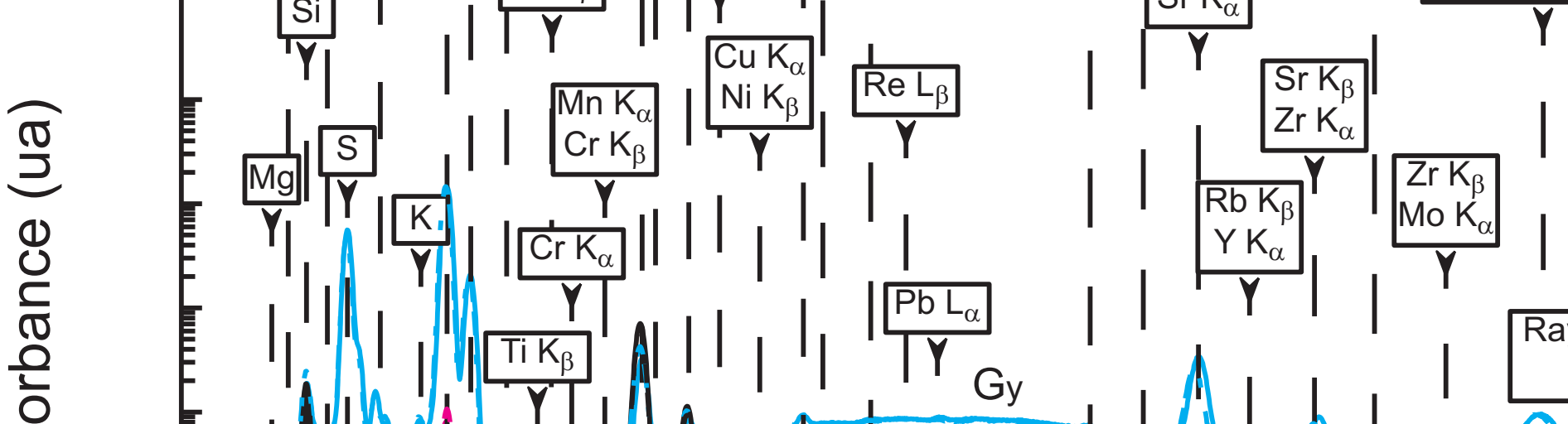

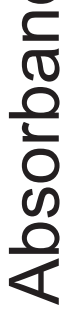

100 悲 +p ply

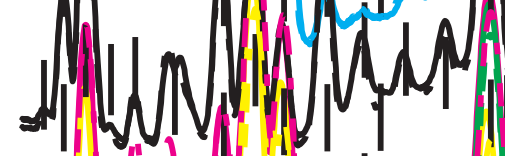

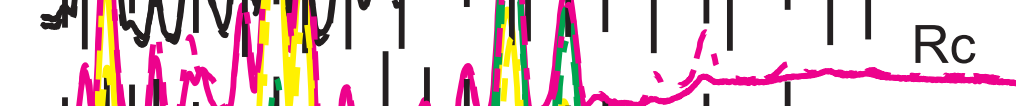

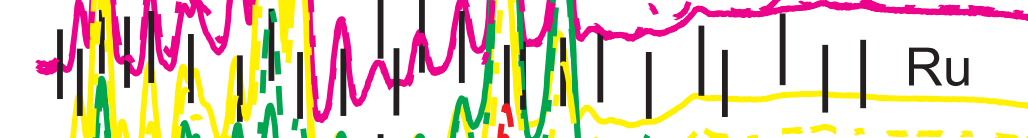

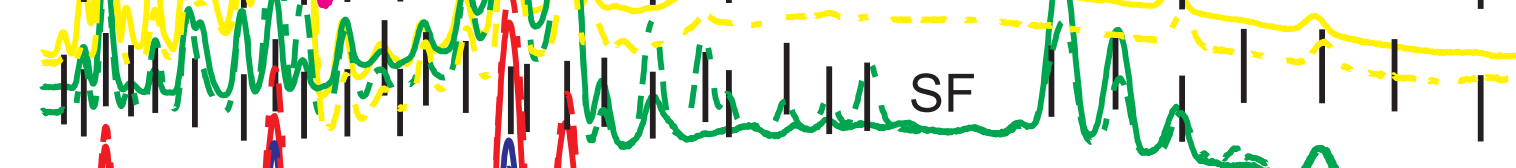

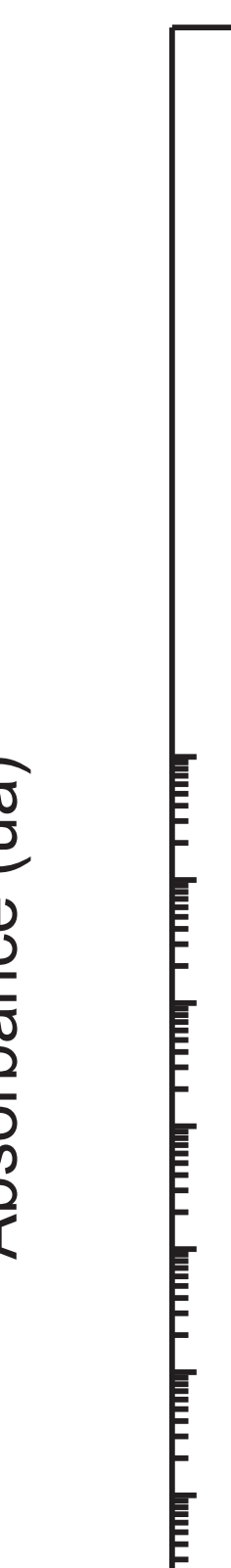

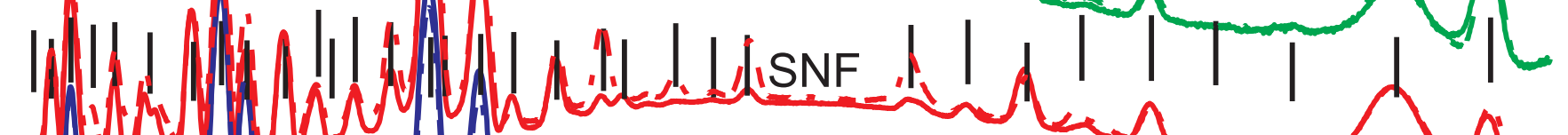
ry Now Mond

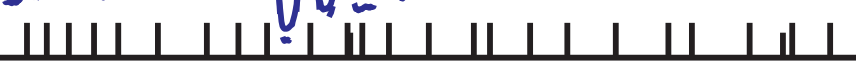

5

10

15

20

Energy (keV) 

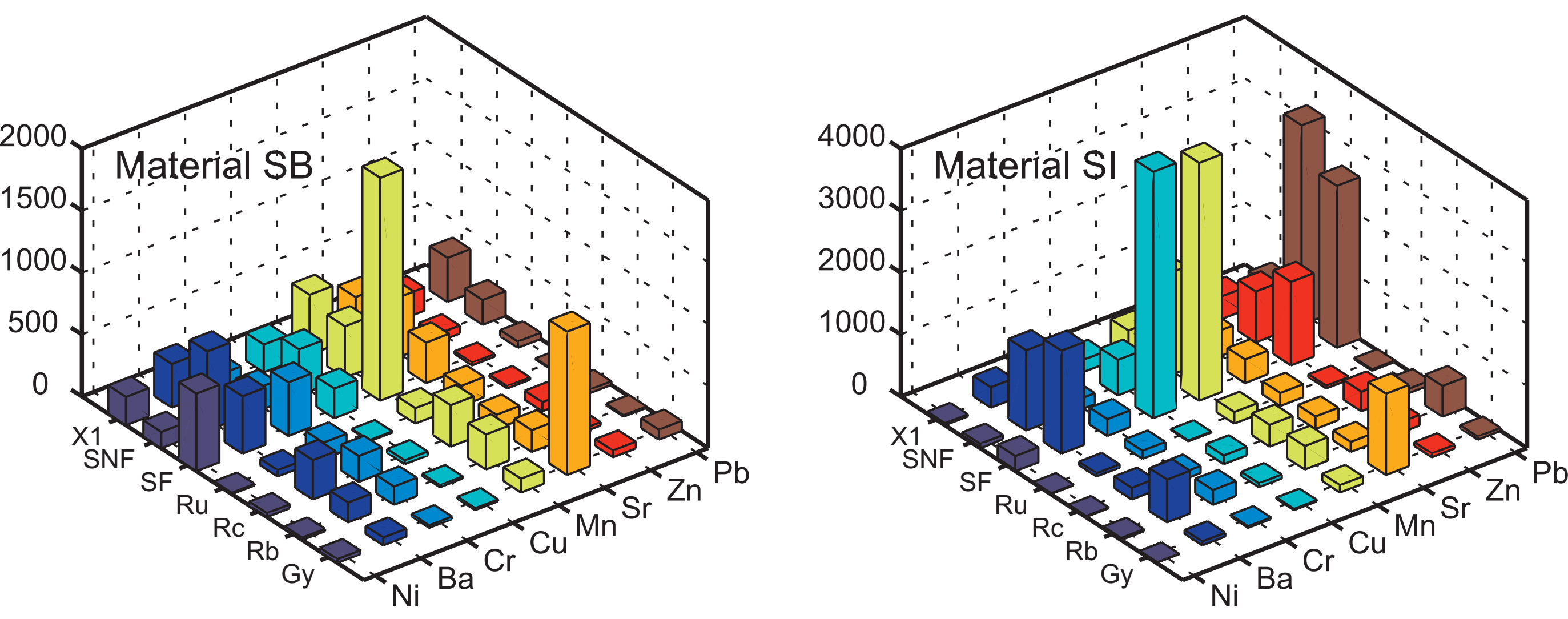


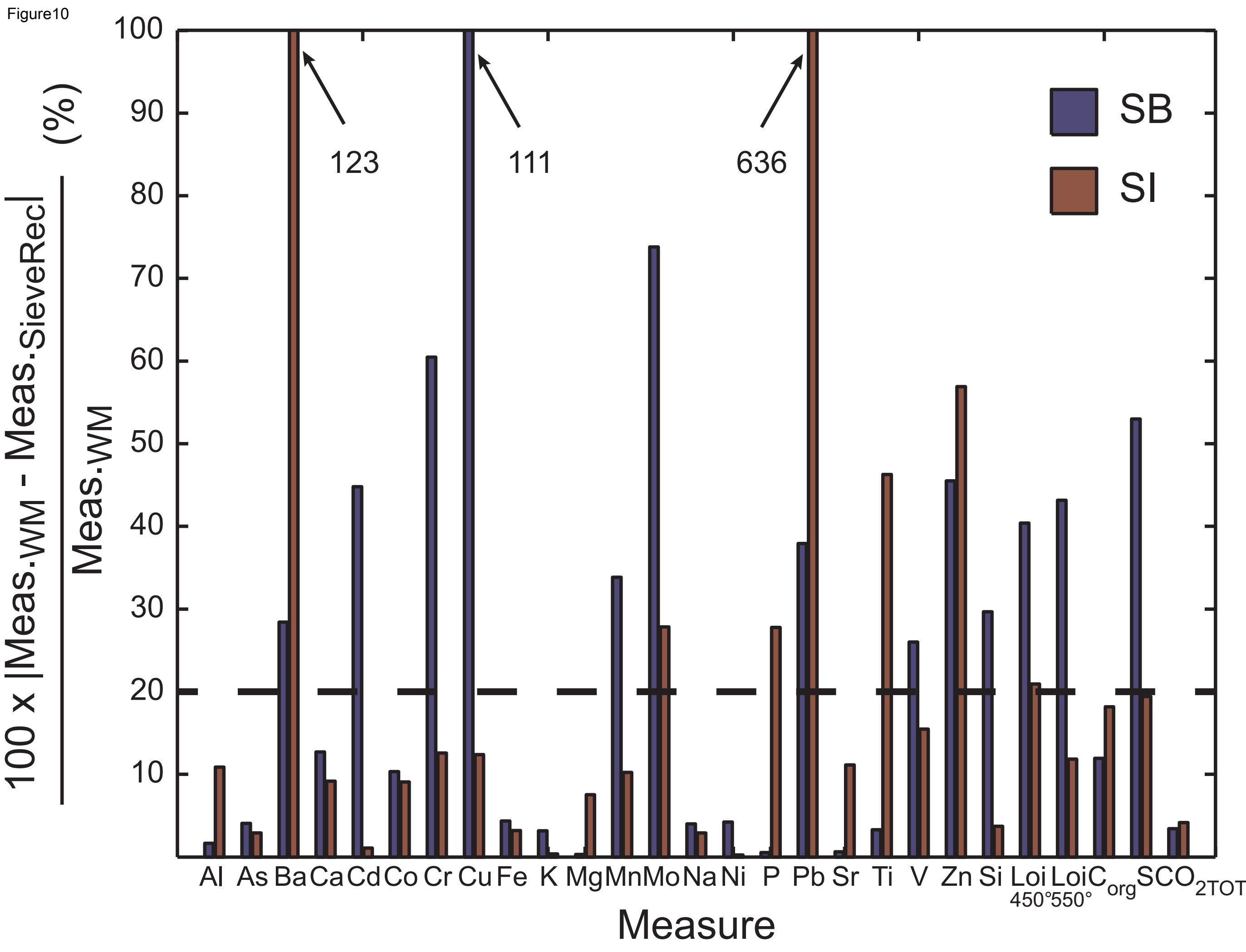


Table 1 Classification of artefacts in the granular fraction of SB and SI

\begin{tabular}{|l|l|l|l|l|l|l|l|}
\hline & $\mathbf{X}_{\mathbf{1}}$ & $\mathbf{S N F}$ & $\mathbf{S F}$ & $\mathbf{R}_{\mathbf{u}}$ & $\mathbf{R}_{\mathbf{c}}$ & $\mathbf{R}_{\mathbf{b}}$ & $\mathbf{G}_{\mathbf{y}}$ \\
\hline SB & 17.9 & 24.9 & 4.1 & 29.8 & 17.3 & 1.8 & 3.8 \\
\hline SI & 32.8 & 13.3 & 1.4 & 43.5 & 2.1 & 1.3 & 4.9 \\
\hline
\end{tabular}


Table 2 Loss On Ignition measurements at $450^{\circ} \mathrm{C}$ and $550^{\circ} \mathrm{C}\left(\mathrm{LOl}_{450^{\circ}}, \mathrm{LO}_{550^{\circ}}\right)$ expressed in \%, particle density $\left(\rho_{d}\right)$ in $\mathrm{g} / \mathrm{cm}^{3}$ and particle density after ignition at $550^{\circ} \mathrm{C}\left(\rho_{\mathrm{dLO}}\right)$ in $\mathrm{g} / \mathrm{cm}^{3}$. For LOI, the drying temperature $\left(T_{d}\right)$ was $105^{\circ} \mathrm{C}$, except underlined values for which $T_{d}=50^{\circ} \mathrm{C}$

\begin{tabular}{|c|c|c|c|c|c|c|c|c|c|c|c|c|c|c|}
\hline & \multicolumn{2}{|l|}{$X_{1}$} & \multicolumn{2}{|l|}{ SNF } & \multicolumn{2}{|l|}{ SF } & \multicolumn{2}{|l|}{$\mathbf{R}_{\mathrm{u}}$} & \multicolumn{2}{|l|}{$\overline{\mathbf{R}_{\mathrm{c}}}$} & \multicolumn{2}{|l|}{$\mathbf{R}_{\mathbf{b}}$} & \multicolumn{2}{|l|}{$\mathbf{G}_{\mathbf{y}}$} \\
\hline & SB & $\mathrm{SI}$ & SB & $\mathrm{SI}$ & SB & $\mathrm{SI}$ & SB & $\mathrm{SI}$ & SB & $\mathrm{SI}$ & SB & $\mathrm{SI}$ & SB & SI \\
\hline $\begin{array}{l}\text { LOI } \\
450^{\circ} \mathrm{C}\end{array}$ & 4 & 5.5 & 0.6 & 7.8 & .1 & 5.4 & .6 & 1.1 & 4.3 & .2 & .9 & 0.8 & 1.8 & 4.3 \\
\hline $\begin{array}{l}\text { LOI } \\
550^{\circ} \mathrm{C}\end{array}$ & $\underline{8.6}$ & 7.3 & $\underline{22.6}$ & 7 & 1.1 & 6. & 1 & 2.0 & 5. & 4 & $\underline{1.3}$ & 1. & 15.3 & $\begin{array}{l}5.0 / \\
17.5 \\
\end{array}$ \\
\hline$\rho_{\mathrm{d}}$ & 2.62 & 2.6 & 2.40 & 2.4 & 3.73 & 4. & 2.67 & 2. & 2.62 & 2.65 & 2.63 & 2. & 2.42 & 2.43 \\
\hline & 71 & 2.67 & 2.50 & 3 & 3.85 & $T . L I$ & 2.64 & 267 & 67 & 2.67 & 65 & 2.65 & .86 & 2.88 \\
\hline
\end{tabular}


Table 3 Results of LAR without constraint: estimated percentages of the artefacts that constitute the whole material and the granulometric fractions

\begin{tabular}{|l|l|l|l|l|l|l|l|l|}
\hline & \multicolumn{9}{|l}{ SB } & SI & \multicolumn{2}{l|}{ ( } \\
\hline$\%$ & WM & $<80 \mu \mathrm{m}$ & $\begin{array}{l}80-400 \\
\mu \mathrm{m}\end{array}$ & $>400 \mu \mathrm{m}$ & WM & $<80 \mu \mathrm{m}$ & $\begin{array}{l}80-400 \\
\mu \mathrm{m}\end{array}$ & $>400 \mu \mathrm{m}$ \\
\hline SNF & 18.0 & 10.1 & 17.6 & 19.6 & 15.3 & 7.3 & 10.1 & 22.5 \\
\hline SF & 7.8 & 6.6 & 8.0 & 10.4 & 2.8 & 7.3 & 3.7 & 2.4 \\
\hline $\mathbf{R}_{\mathbf{u}}$ & 42.0 & 37.8 & 45.3 & 0.0 & 44.1 & 60.2 & 40.5 & 55.1 \\
\hline $\mathbf{G}_{\mathbf{y}}$ & 6.2 & 19.1 & 6.1 & 0.5 & 7.6 & 11.7 & 16.3 & 5.8 \\
\hline $\mathbf{R}_{\mathbf{b}}$ & 2.1 & 5.8 & 0.0 & 0.0 & 8.5 & 3.3 & 12.7 & 2.2 \\
\hline $\mathbf{R}_{\mathbf{c}}$ & 53.2 & 62.2 & 15.3 & 76.5 & 24.5 & 4.2 & 22.0 & 3.3 \\
\hline TOT & 129.3 & 141.6 & 92.3 & 107.1 & 102.8 & 74.3 & 105.2 & 91.4 \\
\hline Exact. & 155 & 274 & 250 & 94 & 307 & 652 & 343 & 337 \\
\hline
\end{tabular}


Table 4 Results of LAR with constraints. Constrained parameters are bolded and highlighted; see text for details

\begin{tabular}{|c|c|c|c|c|c|c|c|c|}
\hline & \multicolumn{4}{|l|}{ SB } & \multicolumn{4}{|l|}{ SI } \\
\hline$\%$ & WM & $<80 \mu \mathrm{m}$ & $\begin{array}{l}80-400 \\
\mu \mathrm{m}\end{array}$ & $>400 \mu \mathrm{m}$ & WM & $<80 \mu \mathrm{m}$ & $\begin{array}{l}80-400 \\
\mu \mathrm{m}\end{array}$ & $>400 \mu \mathrm{m}$ \\
\hline SNF & 20.8 & 12.2 & 20.8 & 23.2 & 16.1 & 7.3 & 10.6 & 20.6 \\
\hline SF & 9.6 & 13.5 & 9.6 & 6.4 & 3.4 & 7.3 & 4.1 & 2.6 \\
\hline $\mathrm{R}_{\mathrm{u}}$ & 34.6 & 37.5 & 32.1 & 30.1 & 62.6 & 66.1 & 63.2 & 61.1 \\
\hline $\mathrm{G}_{\mathrm{y}}$ & 10.0 & 14.3 & 8.3 & 8.3 & 7.9 & 11.6 & 12.0 & 5.7 \\
\hline $\mathrm{R}_{\mathrm{b}}$ & 0.0 & 5 & 0 & 7.0 & 5.0 & 3.4 & 5.0 & 5.0 \\
\hline $\mathrm{R}_{\mathrm{c}}$ & 25.0 & 17.2 & 29.2 & 25.0 & 5.0 & 4.3 & 5.0 & 5.0 \\
\hline exact & 204 & 432 & 302 & 232 & 375 & 664 & 458 & 310 \\
\hline
\end{tabular}


ELECTRONIC SUPPLEMENTARY MATERIAL

SOILS, SEC 2 • GLOBAL CHANGE, ENVIRON RISK ASSESS, SUSTAINABLE LAND USE •

RESEARCH ARTICLE

Identification of the artefact contribution to two urban Technosols by coupling a sorting test, chemical analyses and a least-absolute residuals procedure

Thomas Lenoir $^{1}$ Myriam Duc ${ }^{2}$ - Laurent Lassabatere ${ }^{3}$ Katia Bellagh ${ }^{1,2}$

Received: 1 March 2018 / Accepted: 27 June 2018

(C) Springer-Verlag GmbH Germany, part of Springer Nature 2018

Responsible editor: Maxine J. Levin

1 IFSTTAR, GERS, GMG, 44344 Bouguenais, France

2 Université Paris-Est, IFSTTAR, GERS, SRO, 77447 Marne-la-Vallée, France

3 Univ Lyon, Université Claude Bernard Lyon 1, CNRS, ENTPE, UMR5023 LEHNA, 69518, Vaulx-en-

Velin, France

$\triangle$ Thomas Lenoir

Thomas.lenoir@ifsttar.fr 


\section{Data}

This electronic annex presents data measured on two technosoils excavated at depths higher than one meter. After excavation and preparation, they have been split into four granulometric fractions (whole material, $<80 \mu \mathrm{m}, 80-400 \mu \mathrm{m}$ and $>400 \mu \mathrm{m}$ ). In addition, the artefacts composing the granular fraction ( $>4 \mathrm{~mm}$ ) have been sorted in relevant Construction and Demolition Waste (CD\&W) families (AFNOR 2009a; 2012). Six artifact families have been considered: Rc contains concretes, concrete products (including hydraulically bound material), mortars, concrete masonry units; Ru contains unbound gravel, natural stones; Rb contains terracotta units such as bricks and tiles, and non-floating cellular concretes; SF contains magnetic slags; SNF contains nonmagnetic slags; Gy contains gypsum components; and X1 contains aggregated fine-grained material.

For all these materials, after measurements of loss on ignition (LOI) and particle densities ( $\left.\rho_{\mathrm{d}}\right)$, elemental occurrence and elemental correlations were determined using X-ray fluorescence spectroscopy (XRF). Then, an accurate elemental quantification of trace, intermediate and major chemical elements were done using Inductively Coupled Plasma Optical Emission Spectroscopy (ICPOES) and Carbon/Sulfur measurements.

\section{Experimental Design, Materials, and Methods}

\section{A - Loss on ignition, particles densities and chemical data}

Methodology for LOI and $\rho_{d}$ measurements are fully described in standards (AFNOR 2008, 2013). Quantification of elements have been performed with Inductively Coupled Plasma Optical Emission Spectroscopy - ICP-OES (AFNOR 2001, 2009b, Jean-Soro et al. 2015) by using a Varian $720 \mathrm{ES}$, after mineralization with a mixture of $\mathrm{HF}$ and $\mathrm{HClO} 4$ and alkali fusion with lithium tetraborate for silica content. Carbon (Total Carbon and Total Organic Carbon) and sulfur contents were determined using a LECO SC144 C/S analyzer.

+ Chemical analyses on the material SI 
Table A1 Chemical analyses on each fraction selected from sieve analyses - material SB. Units are $\mathrm{mg} / \mathrm{kg}$ of dry matter $\left(\mathrm{mg} / \mathrm{kg}_{\mathrm{dm}}\right)$ for chemical elements

\begin{tabular}{|l|c|c|c|c|}
\hline & \multicolumn{4}{|c|}{ Particles size } \\
\hline $\mathrm{mg} / \mathrm{kg}_{\mathrm{dm}}$ & Whole mat. & $<80 \mu \mathrm{m}$ & $80-400 \mu \mathrm{m}$ & $>400 \mu \mathrm{m}$ \\
\hline $\mathrm{Al}$ & 26393 & 28489 & 19281 & 28047 \\
\hline $\mathrm{As}$ & 14 & 23 & 14 & 8.9 \\
\hline $\mathrm{B}$ & $/$ & 26 & 27 & $/$ \\
\hline $\mathrm{Ba}$ & 341 & 164 & 297 & 290 \\
\hline $\mathrm{Ca}$ & 139926 & 164980 & 100710 & 94931 \\
\hline $\mathrm{Cd}$ & 0.64 & 0.6 & 0.3 & 0.21 \\
\hline $\mathrm{Co}$ & 16 & 17 & 20 & 15.9 \\
\hline $\mathrm{Cr}$ & 351 & 75 & 97 & 204 \\
\hline $\mathrm{Cu}$ & 212 & 258 & 207 & 679 \\
\hline $\mathrm{Fe}$ & 41473 & 36638 & 37968 & 50419 \\
\hline $\mathrm{K}$ & 6988 & 6710 & 5697 & 8120 \\
\hline $\mathrm{Li}$ & $/$ & 28 & 21 & $/$ \\
\hline $\mathrm{Mg}$ & 4053 & 6043 & 2916 & 2862 \\
\hline $\mathrm{Mn}$ & 418 & 480 & 376 & 685 \\
\hline $\mathrm{Mo}$ & 26 & 3 & 2 & 11 \\
\hline $\mathrm{Na}$ & 1281 & 1237 & 1040 & 1507 \\
\hline $\mathrm{Ni}$ & 192 & 292 & 201 & 127 \\
\hline $\mathrm{P}$ & 542 & 757 & 460 & 404 \\
\hline $\mathrm{Pb}$ & 265.1 & 638 & 322 & 161 \\
\hline $\mathrm{Sr}$ & 347 & 465 & 282 & 279 \\
\hline $\mathrm{Ti}$ & 943 & 942 & 641 & 978 \\
\hline $\mathrm{V}$ & 65 & 47 & 36 & 53.4 \\
\hline $\mathrm{Zn}$ & 400 & 273 & 173 & 189 \\
\hline $\mathrm{Si}$ & 163008 & 135041 & 230116 & 266439 \\
\hline $\mathrm{S}$ & 6600 & 12900 & 12300 & 7100 \\
\hline $\mathrm{Corg}$ & 92600 & 56100 & 93900 & 97800 \\
\hline $\mathrm{LOI}{ }_{450^{\circ}}(\%)$ & 11.0 & 8.9 & 8.1 & 4.1 \\
\hline $\mathrm{LOI}{ }_{550^{\circ}}(\%)$ & 14.6 & 10.4 & 10.2 & 6.0 \\
\hline & & & & \\
\hline & & & & \\
\hline
\end{tabular}


Table A2 Major elements expressed in terms of oxides percentages - material SB - Weighted values

\begin{tabular}{|l|c|c|c|c|}
\hline$(\%)$ & Whole Mat & $<80 \mu \mathrm{m}$ & $80-400 \mu \mathrm{m}$ & $>400 \mu \mathrm{m}$ \\
\hline $\mathrm{SiO}_{2}$ & 31.2 & 27.9 & 38.4 & 41.7 \\
\hline $\mathrm{Al}_{2} \mathrm{O}_{3}$ & 4.5 & 5.2 & 2.8 & 3.9 \\
\hline $\mathrm{FeO}$ & 4.8 & 4.6 & 3.8 & 4.7 \\
\hline $\mathrm{MgO}$ & 0.6 & 1.0 & 0.4 & 0.4 \\
\hline $\mathrm{K}{ }_{2} \mathrm{O}$ & 0.8 & 0.8 & 0.5 & 0.7 \\
\hline $\mathrm{CaO}$ & 17.5 & 22.3 & 11.0 & 9.7 \\
\hline $\mathrm{CO}_{2 \text { оrg }}$ & 30.3 & 19.9 & 26.9 & 25.2 \\
\hline $\mathrm{CO}_{2 \text { тот }}$ & 38.9 & 34.9 & 40.6 & 36.5 \\
\hline $\mathrm{SO}_{2}$ & 1.2 & 2.5 & 1.9 & 1.8 \\
\hline
\end{tabular}

+ Chemical analyses on the soil SI 
Table A3 Chemical analyses on each fraction selected from sieve analyses - material SI - Units are $\mathrm{mg} / \mathrm{kg}$ of dry matter $\left(\mathrm{mg} / \mathrm{kg}_{\mathrm{dm}}\right)$ for chemical elements

\begin{tabular}{|l|c|c|c|c|}
\hline & \multicolumn{4}{|c|}{ Particles size } \\
\hline $\mathrm{mg} / \mathrm{kg}_{\mathrm{dm}}$ & Whole mat. & $<80 \mu \mathrm{m}$ & $80-400 \mu \mathrm{m}$ & $>400 \mu \mathrm{m}$ \\
\hline $\mathrm{Al}$ & 22989 & 27999 & 22073 & 25175 \\
\hline $\mathrm{As}$ & 19 & 21 & 19 & 16 \\
\hline $\mathrm{B}$ & $/$ & $/$ & $/$ & $/$ \\
\hline $\mathrm{Ba}$ & 126 & 162 & 299 & 352 \\
\hline $\mathrm{Ca}$ & 150037 & 181084 & 160128 & 153826 \\
\hline $\mathrm{Cd}$ & 0.6 & 0.8 & 0.2 & 0.4 \\
\hline $\mathrm{Co}$ & 10 & 12 & 10 & 11 \\
\hline $\mathrm{Cr}$ & 102 & 43 & 92 & 119 \\
\hline $\mathrm{Cu}$ & 245 & 348 & 249 & 237 \\
\hline $\mathrm{Fe}$ & 24255 & 23407 & 25024 & 22914 \\
\hline $\mathrm{K}$ & 7491 & 7857 & 6778 & 7470 \\
\hline $\mathrm{Li}$ & $/$ & $/$ & $/$ & $/$ \\
\hline $\mathrm{Mg}$ & 9602 & 12904 & 6615 & 7114 \\
\hline $\mathrm{Mn}$ & 370 & 482 & 336 & 387 \\
\hline $\mathrm{Mo}$ & 9 & 3 & 7 & 9 \\
\hline $\mathrm{Na}$ & 2439 & 2094 & 2593 & 2460 \\
\hline $\mathrm{Ni}$ & 31 & 37 & 28 & 29 \\
\hline $\mathrm{P}$ & 882 & 1035 & 1001 & 1236 \\
\hline $\mathrm{Pb}$ & 159 & 358 & 1224 & 1678 \\
\hline $\mathrm{Sr}$ & 369 & 534 & 313 & 368 \\
\hline $\mathrm{Ti}$ & 769 & 1210 & 717 & 1230 \\
\hline $\mathrm{V}$ & 42 & 46 & 42 & 53 \\
\hline $\mathrm{Zn}$ & 822 & 516 & 324 & 260 \\
\hline $\mathrm{Si}$ & 190629 & 147505 & 187088 & 205846 \\
\hline $\mathrm{S}$ & 14300 & 20000 & 27700 & 11000 \\
\hline $\mathrm{Corg}$ & 33800 & 18000 & 23600 & 35600 \\
\hline $\mathrm{LOI}{ }_{450^{\circ}}(\%)$ & 3.5 & 4.3 & 6.1 & 3.4 \\
\hline $\mathrm{LOI}{ }_{550^{\circ}}(\%)$ & 6.8 & 5.3 & 6.4 & 6.3 \\
\hline & & & & \\
\hline & & & & \\
\hline
\end{tabular}


Table A4 Major elements expressed in terms of oxides percentages - material SI - Weighted values

\begin{tabular}{|l|c|c|c|c|}
\hline$(\%)$ & Whole Mat & $<80 \mu \mathrm{m}$ & $80-400 \mu \mathrm{m}$ & $>400 \mu \mathrm{m}$ \\
\hline $\mathrm{SiO}_{2}$ & 40.0 & 32.7 & 37.7 & 42.2 \\
\hline $\mathrm{Al}_{2} \mathrm{O}_{3}$ & 4.3 & 5.5 & 3.9 & 4.6 \\
\hline $\mathrm{FeO}$ & 3.1 & 3.1 & 3.0 & 2.8 \\
\hline $\mathrm{MgO}$ & 1.6 & 2.2 & 1.0 & 1.1 \\
\hline $\mathrm{K}{ }_{2} \mathrm{O}$ & 0.9 & 1.0 & 0.8 & 0.9 \\
\hline $\mathrm{CaO}$ & 20.6 & 26.2 & 21.1 & 20.6 \\
\hline $\mathrm{CO}_{2 \text { org }}$ & 12.2 & 6.8 & 8.2 & 12.5 \\
\hline $\mathrm{CO}_{2 \text { тот }}$ & 25.9 & 24.1 & 26.2 & 24.5 \\
\hline $\mathrm{SO}_{2}$ & 2.8 & 4.1 & 5.2 & 2.1 \\
\hline
\end{tabular}

+ Chemical analyses on artefacts from the material SB 
Table A5 Chemical analyses on artefacts - material SB - Units are $\mathrm{mg} / \mathrm{kg}$ of dry matter $\left(\mathrm{mg} / \mathrm{kg}_{\mathrm{dm}}\right)$ for chemical elements

\begin{tabular}{|c|c|c|c|c|c|c|c|}
\hline & \multicolumn{7}{|c|}{ SB } \\
\hline $\mathrm{mg} / \mathrm{kg}_{\mathrm{dm}}$ & $X_{1}$ & SNF & SF & $\mathrm{R}_{\mathrm{u}}$ & $\mathrm{R}_{\mathrm{c}}$ & $R_{b}$ & $\mathrm{G}_{\mathrm{y}}$ \\
\hline $\mathrm{Al}$ & 24010 & 66437 & 32767 & 5577 & 15174 & 53354 & 4824 \\
\hline As & 16 & 10.8 & 14.7 & 4 & 6.77 & 12.6 & 2.68 \\
\hline B & 1 & 26.7 & 281 & 5 & 8.25 & 31.8 & 2.47 \\
\hline $\mathrm{Ba}$ & 351 & 638 & 462 & 51 & 160 & 317 & 62.8 \\
\hline $\mathrm{Ca}$ & 111628 & 25541 & 6663 & 149842 & 115413 & 13042 & 164968 \\
\hline $\mathrm{Cd}$ & 0.3 & I & I & I & I & I & I \\
\hline Co & 17 & 30 & 61 & 3 & 5 & 10 & 1 \\
\hline $\mathrm{Cr}$ & 119 & 185 & 431 & 131 & 147 & 208 & 18.6 \\
\hline $\mathrm{Cu}$ & 224 & 370 & 239 & 7.56 & 15.2 & 30.9 & 15.0 \\
\hline $\mathrm{Fe}$ & 51929 & 29294 & 356180 & 5805 & 9986 & 24043 & 1760 \\
\hline $\mathrm{K}$ & 7245 & 12175 & 7408 & 1954 & 6055 & 6369 & 714 \\
\hline $\mathrm{Li}$ & 1 & 100 & 38 & 5 & 16 & 42 & 3 \\
\hline $\mathrm{Mg}$ & 3428 & 4431 & 2515 & 2976 & 2252 & 4367 & 6902 \\
\hline $\mathrm{Mn}$ & 483 & 414 & 1797 & 125 & 282 & 334 & 111 \\
\hline Mo & 7 & 1 & 1 & I & I & I & 1 \\
\hline $\mathrm{Na}$ & 1344 & 2644 & 1196 & 925 & 1150 & 2180 & 214 \\
\hline $\mathrm{Ni}$ & 228 & 131 & 625 & 11 & 18 & 32 & 30 \\
\hline$P$ & 514 & 1736 & 682 & 115 & 245 & 293 & 176 \\
\hline $\mathrm{Pb}$ & 355 & 200 & 49.9 & 6 & 11 & 29 & 80 \\
\hline $\mathrm{Sr}$ & 322 & 482 & 326 & 163 & 174 & 114 & 1155 \\
\hline $\mathrm{Ti}$ & 784 & 2475 & 1458 & 200 & 519 & 2742 & 188 \\
\hline $\mathrm{V}$ & 43.6 & 111 & 99 & 18 & 33.0 & 73.9 & 10 \\
\hline $\mathrm{Zn}$ & 188 & 64 & 21 & 17 & 27.8 & 73.7 & 54 \\
\hline $\mathrm{Si}$ & 205531 & 146926 & 139972 & 216518 & 262407 & 341680 & 40230 \\
\hline$S$ & 6900 & 4600 & 1300 & 1700 & 2500 & 1000 & 6900 \\
\hline$C_{\text {org }}$ & 74900 & 407500 & 16200 & 2300 & 20400 & 500 & 74900 \\
\hline${ }^{*} \mathrm{LOI}_{450^{\circ}}(\%)$ & 8.4 & 16.6 & 1.1 & 1.6 & 4.3 & 0.9 & 4.8 \\
\hline${ }^{*} \mathrm{LOI}_{550^{\circ}}(\%)$ & 8.6 & 22.6 & 1.1 & 1.5 & 5.1 & 1.3 & 15.3 \\
\hline$\rho_{d}\left(T / m^{3}\right)$ & 2.62 & 2.40 & 3.73 & 2.67 & 2.62 & 2.63 & 2.42 \\
\hline$\rho_{d L O I}\left(T / m^{3}\right)$ & 2.71 & 2.50 & 3.85 & 2.64 & 2.67 & 2.65 & 2.86 \\
\hline
\end{tabular}

*For $\mathrm{LOI}_{450^{\circ}}$ temperature of drying was $105^{\circ} \mathrm{C}$, for $\mathrm{LOI}_{550^{\circ}}$ temperature of drying was $50{ }^{\circ} \mathrm{C}$ 
Table A6 Major elements expressed in terms of oxides percentages - artefacts from material SB - Weighted values

\begin{tabular}{|c|c|c|c|c|c|c|c|}
\hline & \multicolumn{7}{|c|}{ SB } \\
\hline & $X_{1}$ & SNF & SF & $\mathrm{R}_{\mathrm{u}}$ & $\mathrm{R}_{\mathrm{c}}$ & $R_{b}$ & $\mathrm{G}_{\mathrm{y}}$ \\
\hline $\mathrm{SiO}_{2}$ & 39.5 & 15.2 & 32.8 & 50.2 & 57.5 & 79.7 & 12.0 \\
\hline $\mathrm{Al}_{2} \mathrm{O}_{3}$ & 4.1 & 6.1 & 6.8 & 1.1 & 2.9 & 11.0 & 1.3 \\
\hline $\mathrm{FeO}$ & 6.0 & 1.8 & 50.2 & 0.8 & 1.3 & 3.4 & 0.3 \\
\hline $\mathrm{MgO}$ & 0.5 & 0.4 & 0.5 & 0.5 & 0.4 & 0.8 & 1.6 \\
\hline $\mathrm{K}_{2} \mathrm{O}$ & 0.8 & 0.7 & 1.0 & 0.3 & 0.7 & 0.8 & 0.1 \\
\hline $\mathrm{CaO}$ & 14.0 & 1.7 & 1.0 & 22.7 & 16.6 & 2.0 & 32.2 \\
\hline $\mathrm{CO}_{2 \mathrm{org}}$ & 24.7 & 72.4 & 6.5 & 0.9 & 7.7 & 0.2 & 1.1 \\
\hline $\mathrm{CO}_{2 \mathrm{TOT}}$ & 33.2 & 72.9 & 6.3 & 23.8 & 19.6 & 1.0 & 14.4 \\
\hline $\mathrm{SO}_{2}$ & 1.2 & 0.4 & 0.3 & 0.4 & 0.5 & 0.2 & 37.7 \\
\hline
\end{tabular}

+ Chemical analyses on artefacts from the material SI 
Table A7 Chemical analyses on artefacts - material SI

\begin{tabular}{|c|c|c|c|c|c|c|c|}
\hline & \multicolumn{7}{|c|}{$\mathrm{SI}$} \\
\hline $\mathrm{mg} / \mathrm{kg}_{\mathrm{dm}}$ & $X_{1}$ & SNF & SF & $\mathrm{R}_{\mathrm{u}}$ & $\mathrm{R}_{\mathrm{c}}$ & $\mathrm{R}_{\mathrm{b}}$ & $\mathrm{G}_{\mathrm{y}}$ \\
\hline $\mathrm{Al}$ & 31928 & 66437 & 47237 & 4189 & 8801 & 74253 & 3466 \\
\hline As & 16 & 26 & 167 & 4 & 6 & 16 & 1 \\
\hline$B$ & & & & & & & \\
\hline $\mathrm{Ba}$ & 326 & 1293 & 1657 & 53 & 187 & 691 & 62 \\
\hline $\mathrm{Ca}$ & 139705 & 56316 & 59067 & 220789 & 86882 & 20892 & 252145 \\
\hline $\mathrm{Cd}$ & 0.6 & 0.2 & 0.6 & 0.2 & 0.5 & 0.2 & 0.2 \\
\hline Co & 10 & 31 & 64 & 2 & 5 & 19 & 3 \\
\hline $\mathrm{Cr}$ & 110 & 177 & 271 & 130 & 113 & 238 & 23 \\
\hline $\mathrm{Cu}$ & 198 & 571 & 3977 & 11 & 139 & 61 & 20 \\
\hline $\mathrm{Fe}$ & 22246 & 56589 & 299689 & 7713 & 4387 & 30284 & 1861 \\
\hline $\mathrm{K}$ & 9311 & 14150 & 9735 & 2218 & 3613 & 14340 & 959 \\
\hline $\mathrm{Li}$ & & & & & & & \\
\hline $\mathrm{Mg}$ & 9931 & 8624 & 9289 & 3434 & 3032 & 6717 & 10257 \\
\hline $\mathrm{Mn}$ & 388 & 1653 & 3837 & 188 & 352 & 380 & 119 \\
\hline Mo & 8 & 13 & 24 & 10 & 9 & 12 & 2 \\
\hline $\mathrm{Na}$ & 1785 & 8319 & 6238 & 527 & 2138 & 2855 & 297 \\
\hline $\mathrm{Ni}$ & 32 & 69 & 243 & 10 & 12 & 32 & 3 \\
\hline $\mathrm{P}$ & 866 & 2527 & 3230 & 133 & 286 & 484 & 481 \\
\hline $\mathrm{Pb}$ & 309 & 3219 & 2612 & 23 & 71 & 493 & 47 \\
\hline $\mathrm{Sr}$ & 354 & 413 & 380 & 209 & 200 & 160 & 1310 \\
\hline $\mathrm{Ti}$ & 1212 & 2378 & 1791 & 130 & 532 & 3452 & 99 \\
\hline $\mathrm{V}$ & 50 & 123 & 115 & 19 & 28 & 92 & 7 \\
\hline $\mathrm{Zn}$ & 365 & 823 & 1346 & 42 & 318 & 172 & 59 \\
\hline $\mathrm{Si}$ & 194356 & 173260 & 107607 & 174294 & 222876 & 315867 & 36950 \\
\hline$S$ & 14600 & 4300 & 5800 & 1200 & 3500 & 1400 & 156900 \\
\hline Corg & 18600 & 200600 & 32900 & 1200 & 6600 & 900 & 400 \\
\hline $\mathrm{LOI}_{450^{\circ}}(\%)$ & 5.5 & 7.8 & 5.4 & 1.1 & 2.2 & 0.8 & 4.3 \\
\hline $\mathrm{LOI}_{550^{\circ}}(\%)$ & 7.3 & 19.7 & 6.3 & 2.0 & 4.7 & 1.2 & 5.0 \\
\hline$* \mathrm{LOI}_{550^{\circ}}(\%)$ & 9.1 & 20.3 & 7.4 & 1.9 & 4.5 & 1.4 & 17.5 \\
\hline$\rho_{d}\left(T / m^{3}\right)$ & 2.60 & 2.49 & 4.00 & 2.67 & 2.65 & 2.65 & 2.43 \\
\hline$\rho_{\mathrm{d} L O I}\left(T / \mathrm{m}^{3}\right)$ & 2.6 & 2.73 & 4.21 & 2.67 & 2.67 & 2.65 & 2.88 \\
\hline
\end{tabular}

*temperature of drying was $50{ }^{\circ} \mathrm{C}$ 
Table A8 Major elements expressed in terms of oxides percentages - artefacts from material SI - Weighted values

\begin{tabular}{|c|c|c|c|c|c|c|c|}
\hline & \multicolumn{7}{|c|}{$\mathrm{SI}$} \\
\hline & $X_{1}$ & SNF & SF & $\mathrm{R}_{\mathrm{u}}$ & $\mathrm{R}_{\mathrm{c}}$ & $R_{b}$ & $\mathrm{G}_{\mathrm{y}}$ \\
\hline $\mathrm{SiO}_{2}$ & 40.7 & 25.9 & 22.8 & 39.0 & 57.9 & 71.8 & 9.3 \\
\hline $\mathrm{Al}_{2} \mathrm{O}_{3}$ & 5.9 & 8.8 & 8.8 & 0.8 & 2.0 & 14.9 & 0.8 \\
\hline $\mathrm{FeO}$ & 2.8 & 5.1 & 38.2 & 1.0 & 0.7 & 4.1 & 0.3 \\
\hline $\mathrm{MgO}$ & 1.6 & 1.0 & 1.5 & 0.6 & 0.6 & 1.2 & 2.0 \\
\hline $\mathrm{K}_{2} \mathrm{O}$ & 1.1 & 1.2 & 1.2 & 0.3 & 0.5 & 1.8 & 0.1 \\
\hline $\mathrm{CaO}$ & 19.1 & 5.5 & 8.2 & 32.3 & 14.8 & 3.1 & 41.6 \\
\hline $\mathrm{CO}_{\text {2org }}$ & 6.7 & 51.4 & 12.0 & 0.5 & 2.9 & 0.4 & 0.2 \\
\hline $\mathrm{CO}_{2 \mathrm{TOT}}$ & 25.0 & 49.8 & 14.4 & 25.5 & 21.9 & 1.3 & 8.5 \\
\hline $\mathrm{SO}_{2}$ & 2.9 & 0.6 & 1.1 & 0.3 & 0.8 & 0.3 & 36.9 \\
\hline
\end{tabular}

\section{B - XRF analyses}

Elemental occurrences were obtained using X-ray fluorescence (XRF). A Brucker M4 TORNADO laboratory instrument equipped with an Rh X-ray tube operated at $50 \mathrm{keV}$ and $300 \mu \mathrm{A}$ was used. The X-ray fluorescence was measured using a silicon drift detector with a spot size of 25 $\mu \mathrm{m}[8]$. XRF spectra were measured under vacuum on the granular fractions and the artifact families grinded at a diameter $<40 \mu \mathrm{m}$. 96 spectra were collected for each fraction and 30 for each artefact. They were normalized on the Compton peak (i.e maximum of the peak set to 1 ) and limited at the Rayleigh peak ( $20 \mathrm{keV})$. In order to study chemical correlation, a semiquantification of elemental composition of artifacts families was realized by applying a semiautomatic method provided by the software 'Esprit' coupled with M4 TORNADO. Mean normalized XRF spectra with standard deviation have been then determined. They are provided in supplementary material in ASCII format

+ Elemental correlations 
Table B1 Elemental correlations from xrf analyses on $\mathrm{R}_{\mathrm{u}}$ (aggregates) - material SB

\begin{tabular}{|c|c|c|c|c|c|c|c|c|c|c|c|c|c|c|c|c|c|}
\hline & $\mathrm{Si}$ & $\mathrm{Fe}$ & $\mathrm{Al}$ & K & S & $\mathrm{Mg}$ & $\mathrm{Ti}$ & $\mathrm{Na}$ & $\mathrm{Sr}$ & $\mathrm{Pb}$ & $\mathrm{Zn}$ & $\mathrm{Mn}$ & $\mathrm{Cu}$ & $\mathrm{Cr}$ & P & $\mathrm{Ba}$ & $\mathrm{Ni}$ \\
\hline $\mathrm{Ca}$ & -0.78 & -0.47 & -0.13 & -0.15 & -0.45 & 0.04 & -0.37 & 0.21 & -0.33 & -0.27 & -0.45 & -0.35 & -0.14 & -0.24 & 0.63 & -0.54 & -0.03 \\
\hline & \multirow[t]{16}{*}{$\mathrm{Si}$} & 0.19 & 0.19 & 0.05 & 0.43 & -0.01 & 0.43 & -0.17 & 0.19 & 0.01 & 0.34 & 0.29 & 0.10 & 0.01 & -0.81 & 0.66 & 0.10 \\
\hline & & $\mathrm{Fe}$ & 0.28 & 0.27 & -0.06 & -0.15 & 0.08 & 0.19 & -0.07 & 0.03 & 0.03 & 0.08 & 0.19 & 0.63 & -0.35 & 0.36 & -0.05 \\
\hline & & & $\mathrm{Al}$ & 0.59 & -0.11 & 0.28 & 0.34 & 0.05 & -0.03 & -0.16 & -0.05 & 0.12 & 0.24 & 0.09 & -0.15 & 0.38 & -0.11 \\
\hline & & & & K & -0.17 & 0.08 & 0.32 & -0.06 & -0.03 & -0.03 & 0.00 & 0.41 & 0.31 & 0.02 & -0.05 & 0.38 & -0.14 \\
\hline & & & & & S & 0.18 & -0.16 & -0.09 & 0.33 & 0.19 & 0.38 & 0.21 & 0.03 & -0.17 & -0.23 & 0.09 & 0.03 \\
\hline & & & & & & $\mathrm{Mg}$ & -0.03 & -0.14 & 0.32 & -0.13 & 0.08 & 0.19 & 0.07 & 0.11 & -0.10 & 0.13 & 0.01 \\
\hline & & & & & & & $\mathrm{Ti}$ & -0.20 & -0.08 & -0.28 & 0.25 & 0.56 & 0.30 & -0.04 & -0.37 & 0.75 & -0.12 \\
\hline & & & & & & & & $\mathrm{Na}$ & -0.29 & -0.12 & -0.31 & 0.00 & 0.09 & 0.02 & 0.15 & -0.15 & 0.10 \\
\hline & & & & & & & & & $\mathrm{Sr}$ & 0.46 & 0.20 & -0.05 & -0.04 & 0.03 & -0.09 & 0.06 & 0.19 \\
\hline & & & & & & & & & & $\mathrm{Pb}$ & 0.11 & -0.21 & 0.03 & -0.07 & 0.10 & -0.27 & -0.11 \\
\hline & & & & & & & & & & & $\mathrm{Zn}$ & 0.56 & 0.48 & -0.24 & -0.37 & 0.33 & -0.44 \\
\hline & & & & & & & & & & & & $\mathrm{Mn}$ & 0.62 & -0.13 & -0.34 & 0.58 & -0.35 \\
\hline & & & & & & & & & & & & & $\mathrm{Cu}$ & -0.08 & -0.08 & 0.21 & -0.72 \\
\hline & & & & & & & & & & & & & & $\mathrm{Cr}$ & -0.17 & 0.13 & 0.10 \\
\hline & & & & & & & & & & & & & & & P & -0.64 & -0.10 \\
\hline & & & & & & & & & & & & & & & & $\mathrm{Ba}$ & 0.07 \\
\hline
\end{tabular}


Table B2 Elemental correlations from xrf analyses on $\mathrm{R}_{\mathrm{u}}$ (aggregates) - material SI

\begin{tabular}{|c|c|c|c|c|c|c|c|c|c|c|c|c|c|c|c|c|c|}
\hline & Si & $\mathrm{Fe}$ & $\mathrm{Al}$ & K & S & $\mathrm{Mg}$ & $\mathrm{Ti}$ & $\mathrm{Na}$ & $\mathrm{Sr}$ & $\mathrm{Pb}$ & $\mathrm{Zn}$ & $\mathrm{Mn}$ & $\mathrm{Cu}$ & $\mathrm{Cr}$ & P & $\mathrm{Ba}$ & $\mathrm{Ni}$ \\
\hline $\mathrm{Ca}$ & 0.43 & 0.46 & 0.13 & 0.02 & 0.57 & 0.07 & 0.25 & 0.11 & 0.55 & 0.10 & 0.15 & 0.62 & 0.35 & 0.17 & I & I & 0.13 \\
\hline & Si & -0.13 & 0.36 & 0.10 & 0.51 & 0.11 & -0.03 & -0.09 & 0.38 & -0.03 & 0.28 & 0.57 & -0.03 & 0.41 & I & I & 0.54 \\
\hline & & $\mathrm{Fe}$ & 0.04 & -0.29 & 0.22 & 0.21 & 0.37 & 0.00 & -0.20 & -0.04 & -0.06 & 0.12 & 0.17 & 0.19 & / & / & -0.33 \\
\hline & & & $\mathrm{Al}$ & 0.42 & 0.19 & 0.21 & -0.04 & -0.22 & 0.10 & 0.09 & 0.12 & 0.22 & -0.21 & 0.00 & I & I & -0.13 \\
\hline & & & & K & 0.00 & -0.04 & -0.26 & -0.24 & 0.22 & -0.19 & -0.07 & -0.07 & -0.25 & 0.10 & / & / & -0.31 \\
\hline & & & & & S & 0.10 & 0.00 & -0.05 & 0.34 & 0.10 & -0.12 & 0.30 & 0.04 & 0.27 & / & / & 0.16 \\
\hline & & & & & & $\mathrm{Mg}$ & 0.10 & 0.01 & -0.12 & 0.17 & 0.28 & 0.15 & -0.28 & 0.21 & / & / & -0.24 \\
\hline & & & & & & & $\mathrm{Ti}$ & 0.27 & 0.01 & 0.15 & 0.19 & 0.30 & 0.19 & -0.10 & I & I & 0.08 \\
\hline & & & & & & & & $\mathrm{Na}$ & 0.10 & -0.05 & 0.11 & -0.03 & -0.14 & -0.01 & / & / & 0.14 \\
\hline & & & & & & & & & $\mathrm{Sr}$ & 0.01 & 0.09 & 0.45 & 0.23 & -0.01 & / & I & 0.17 \\
\hline & & & & & & & & & & $\mathrm{Pb}$ & 0.36 & 0.15 & -0.06 & -0.16 & / & I & 0.00 \\
\hline & & & & & & & & & & & $\mathrm{Zn}$ & 0.61 & -0.07 & -0.12 & / & I & 0.21 \\
\hline & & & & & & & & & & & & $\mathrm{Mn}$ & 0.17 & -0.08 & / & / & 0.27 \\
\hline & & & & & & & & & & & & & $\mathrm{Cu}$ & 0.15 & / & I & 0.35 \\
\hline & & & & & & & & & & & & & & & I & I & 0.22 \\
\hline & & & & & & & & & & & & & & & $r$ & / & I \\
\hline & & & & & & & & & & & & & & & & $\mathrm{Ba}$ & I \\
\hline
\end{tabular}


Table B3 Elemental correlations from xrf analyses on SNF (non-magnetic slags) - material SB

\begin{tabular}{|c|c|c|c|c|c|c|c|c|c|c|c|c|c|c|c|c|c|}
\hline & $\mathrm{Si}$ & $\mathrm{Fe}$ & Al & K & S & $\mathrm{Mg}$ & $\mathrm{Ti}$ & $\mathrm{Na}$ & $\mathrm{Sr}$ & $\mathrm{Pb}$ & $\mathrm{Zn}$ & $\mathrm{Mn}$ & $\mathrm{Cu}$ & $\mathrm{Cr}$ & P & $\mathrm{Ba}$ & $\mathrm{Ni}$ \\
\hline \multirow[t]{17}{*}{$\mathrm{Ca}$} & 0.86 & 0.81 & 0.83 & 0.88 & 0.58 & 0.13 & 0.83 & -0.18 & 0.54 & 0.63 & 0.75 & 0.47 & 0.33 & 0.60 & 0.33 & 0.33 & 0.81 \\
\hline & $\mathrm{Si}$ & 0.60 & 0.96 & 0.86 & 0.54 & 0.24 & 0.73 & 0.04 & 0.43 & 0.51 & 0.60 & 0.39 & 0.30 & 0.37 & 0.31 & 0.18 & 0.65 \\
\hline & & $\mathrm{Fe}$ & 0.55 & 0.69 & 0.46 & 0.04 & 0.78 & -0.16 & 0.46 & 0.58 & 0.63 & 0.50 & 0.27 & 0.69 & 0.24 & 0.38 & 0.79 \\
\hline & & & $\mathrm{Al}$ & 0.90 & 0.47 & 0.30 & 0.75 & 0.05 & 0.47 & 0.53 & 0.59 & 0.36 & 0.36 & 0.40 & 0.37 & 0.16 & 0.65 \\
\hline & & & & K & 0.49 & 0.18 & 0.89 & -0.12 & 0.57 & 0.62 & 0.71 & 0.34 & 0.36 & 0.59 & 0.27 & 0.27 & 0.76 \\
\hline & & & & & S & -0.12 & 0.46 & 0.10 & 0.19 & 0.28 & 0.27 & 0.47 & 0.37 & 0.52 & 0.07 & -0.11 & 0.54 \\
\hline & & & & & & $\mathrm{Mg}$ & 0.08 & 0.13 & 0.16 & 0.17 & 0.19 & 0.03 & 0.34 & -0.05 & 0.31 & 0.04 & 0.16 \\
\hline & & & & & & & $\mathrm{Ti}$ & -0.24 & 0.52 & 0.61 & 0.68 & 0.37 & 0.37 & 0.66 & 0.27 & 0.29 & 0.76 \\
\hline & & & & & & & & $\mathrm{Na}$ & -0.04 & -0.15 & -0.38 & -0.12 & -0.01 & -0.09 & 0.19 & -0.33 & 0.02 \\
\hline & & & & & & & & & $\mathrm{Sr}$ & 0.55 & 0.64 & 0.26 & 0.25 & 0.44 & 0.52 & 0.50 & 0.61 \\
\hline & & & & & & & & & & $\mathrm{Pb}$ & 0.77 & 0.41 & 0.30 & 0.46 & 0.26 & 0.33 & 0.59 \\
\hline & & & & & & & & & & & $\mathrm{Zn}$ & 0.29 & 0.29 & 0.34 & 0.40 & 0.34 & 0.58 \\
\hline & & & & & & & & & & & & $\mathrm{Mn}$ & 0.25 & 0.55 & 0.15 & 0.09 & 0.38 \\
\hline & & & & & & & & & & & & & $\mathrm{Cu}$ & 0.25 & -0.06 & -0.30 & 0.42 \\
\hline & & & & & & & & & & & & & & $\mathrm{Cr}$ & 0.15 & 0.30 & 0.76 \\
\hline & & & & & & & & & & & & & & & P & 0.31 & 0.20 \\
\hline & & & & & & & & & & & & & & & & $\mathrm{Ba}$ & 0.27 \\
\hline
\end{tabular}


Table B4 Elemental correlations from xrf analyses on SNF (non-magnetic slags) - material SI

\begin{tabular}{|c|c|c|c|c|c|c|c|c|c|c|c|c|c|c|c|c|c|}
\hline & $\mathrm{Si}$ & $\mathrm{Fe}$ & $\mathrm{Al}$ & $\mathrm{K}$ & S & $\mathrm{Mg}$ & $\mathrm{Ti}$ & $\mathrm{Na}$ & $\mathrm{Sr}$ & $\mathrm{Pb}$ & $\mathrm{Zn}$ & $\mathrm{Mn}$ & $\mathrm{Cu}$ & $\mathrm{Cr}$ & P & $\mathrm{Ba}$ & $\mathrm{Ni}$ \\
\hline \multirow[t]{17}{*}{$\mathrm{Ca}$} & 0.79 & 0.83 & 0.86 & 0.93 & 0.52 & 0.28 & 0.66 & 0.04 & 0.40 & 0.47 & 0.64 & 0.83 & 0.45 & 0.51 & 0.61 & 0.28 & 0.28 \\
\hline & $\mathrm{Si}$ & 0.76 & 0.95 & 0.83 & 0.70 & 0.30 & 0.64 & -0.10 & 0.25 & 0.30 & 0.36 & 0.67 & 0.41 & 0.54 & 0.68 & 0.08 & 0.29 \\
\hline & & $\mathrm{Fe}$ & 0.84 & 0.92 & 0.64 & 0.26 & 0.81 & 0.11 & 0.50 & 0.58 & 0.50 & 0.75 & 0.54 & 0.65 & 0.55 & 0.12 & 0.40 \\
\hline & & & Al & 0.91 & 0.64 & 0.30 & 0.71 & -0.03 & 0.35 & 0.35 & 0.42 & 0.71 & 0.44 & 0.56 & 0.68 & 0.13 & 0.33 \\
\hline & & & & K & 0.59 & 0.24 & 0.81 & 0.02 & 0.47 & 0.57 & 0.63 & 0.83 & 0.49 & 0.62 & 0.58 & 0.22 & 0.36 \\
\hline & & & & & S & 0.19 & 0.53 & -0.17 & 0.02 & 0.37 & 0.27 & 0.44 & 0.55 & 0.55 & 0.53 & -0.06 & 0.51 \\
\hline & & & & & & $\mathrm{Mg}$ & 0.15 & 0.46 & -0.16 & 0.07 & 0.22 & 0.17 & 0.18 & 0.35 & 0.34 & 0.05 & 0.10 \\
\hline & & & & & & & $\mathrm{Ti}$ & -0.11 & 0.40 & 0.70 & 0.55 & 0.59 & 0.40 & 0.57 & 0.44 & 0.11 & 0.20 \\
\hline & & & & & & & & $\mathrm{Na}$ & 0.20 & -0.04 & 0.03 & 0.12 & -0.15 & 0.14 & -0.15 & -0.04 & 0.02 \\
\hline & & & & & & & & & $\mathrm{Sr}$ & 0.27 & 0.27 & 0.43 & 0.06 & 0.09 & 0.07 & 0.10 & 0.14 \\
\hline & & & & & & & & & & $\mathrm{Pb}$ & 0.71 & 0.53 & 0.44 & 0.45 & 0.23 & 0.24 & 0.32 \\
\hline & & & & & & & & & & & $\mathrm{Zn}$ & 0.64 & 0.37 & 0.37 & 0.18 & 0.60 & 0.13 \\
\hline & & & & & & & & & & & & $\mathrm{Mn}$ & 0.24 & 0.58 & 0.31 & 0.16 & 0.22 \\
\hline & & & & & & & & & & & & & $\mathrm{Cu}$ & 0.28 & 0.69 & 0.15 & 0.55 \\
\hline & & & & & & & & & & & & & & $\mathrm{Cr}$ & 0.21 & 0.02 & 0.23 \\
\hline & & & & & & & & & & & & & & & P & 0.07 & 0.42 \\
\hline & & & & & & & & & & & & & & & & $\mathrm{Ba}$ & -0.07 \\
\hline
\end{tabular}


Table B5 Elemental correlations from xrf analyses on SF (magnetic slags) - material SI

\begin{tabular}{|c|c|c|c|c|c|c|c|c|c|c|c|c|c|c|c|c|c|}
\hline & $\mathrm{Si}$ & $\mathrm{Fe}$ & $\mathrm{Al}$ & $\mathrm{K}$ & s & $\mathrm{Mg}$ & $\mathrm{Ti}$ & $\mathrm{Na}$ & $\mathrm{Sr}$ & $\mathrm{Pb}$ & $\mathrm{Zn}$ & $\mathrm{Mn}$ & $\mathrm{Cu}$ & $\mathrm{Cr}$ & P & $\mathrm{Ba}$ & $\mathrm{Ni}$ \\
\hline $\mathrm{Ca}$ & 0.94 & 0.94 & 0.94 & 0.95 & 0.84 & 0.27 & 0.91 & 0.11 & 0.26 & 0.34 & 0.41 & 0.61 & 0.08 & 0.64 & 0.72 & 0.65 & 0.81 \\
\hline & $\mathrm{Si}$ & 0.94 & 0.97 & 0.96 & 0.87 & 0.27 & 0.89 & 0.05 & 0.15 & 0.30 & 0.40 & 0.63 & 0.18 & 0.60 & 0.66 & 0.60 & 0.83 \\
\hline & & $\mathrm{Fe}$ & 0.93 & 0.91 & 0.87 & 0.28 & 0.86 & 0.04 & 0.19 & 0.19 & 0.38 & 0.67 & 0.11 & 0.72 & 0.65 & 0.63 & 0.82 \\
\hline & & & Al & 0.93 & 0.87 & 0.34 & 0.90 & 0.08 & 0.16 & 0.29 & 0.39 & 0.57 & 0.16 & 0.59 & 0.74 & 0.60 & 0.83 \\
\hline & & & & K & 0.85 & 0.22 & 0.90 & 0.03 & 0.23 & 0.39 & 0.38 & 0.59 & 0.12 & 0.57 & 0.67 & 0.57 & 0.83 \\
\hline & & & & & $S$ & 0.42 & 0.78 & 0.11 & 0.08 & 0.27 & 0.35 & 0.57 & 0.22 & 0.66 & 0.70 & 0.56 & 0.74 \\
\hline & & & & & & Mg & 0.28 & 0.08 & -0.03 & 0.29 & 0.12 & 0.06 & 0.05 & 0.11 & 0.29 & 0.36 & 0.33 \\
\hline & & & & & & & $\mathrm{Ti}$ & 0.07 & 0.22 & 0.26 & 0.35 & 0.58 & 0.15 & 0.49 & 0.62 & 0.45 & 0.80 \\
\hline & & & & & & & & $\mathrm{Na}$ & -0.20 & 0.08 & 0.17 & -0.05 & 0.31 & 0.25 & -0.07 & 0.03 & 0.06 \\
\hline & & & & & & & & & $\mathrm{Sr}$ & -0.02 & 0.14 & 0.02 & -0.32 & 0.01 & 0.07 & 0.02 & 0.30 \\
\hline & & & & & & & & & & $\mathrm{Pb}$ & 0.21 & 0.03 & -0.07 & 0.11 & 0.38 & 0.30 & 0.16 \\
\hline & & & & & & & & & & & $\mathrm{Zn}$ & 0.30 & 0.32 & 0.38 & 0.28 & 0.20 & 0.18 \\
\hline & & & & & & & & & & & & $\mathrm{Mn}$ & 0.10 & 0.61 & 0.38 & 0.43 & 0.49 \\
\hline & & & & & & & & & & & & & $\mathrm{Cu}$ & 0.02 & 0.18 & 0.10 & 0.15 \\
\hline & & & & & & & & & & & & & & $\mathrm{Cr}$ & 0.48 & 0.52 & 0.53 \\
\hline & & & & & & & & & & & & & & & P & 0.57 & 0.52 \\
\hline & & & & & & & & & & & & & & & & $\mathrm{Ba}$ & 0.41 \\
\hline
\end{tabular}


Table B6 Elemental correlations from xrf analyses on $R_{c}$ (concrete products) - material SB

\begin{tabular}{|c|c|c|c|c|c|c|c|c|c|c|c|c|c|c|c|c|c|}
\hline & $\mathrm{Si}$ & $\mathrm{Fe}$ & Al & K & S & $\mathrm{Mg}$ & $\mathrm{Ti}$ & $\mathrm{Na}$ & $\mathrm{Sr}$ & $\mathrm{Pb}$ & $\mathrm{Zn}$ & $\mathrm{Mn}$ & $\mathrm{Cu}$ & $\mathrm{Cr}$ & P & $\mathrm{Ba}$ & $\mathrm{Ni}$ \\
\hline $\mathrm{Ca}$ & 0.61 & 0.20 & 0.65 & 0.39 & 0.61 & 0.47 & 0.70 & 0.00 & 0.37 & 0.16 & -0.31 & 0.53 & -0.03 & 0.12 & 0.48 & -0.03 & -0.29 \\
\hline & $\mathrm{Si}$ & 0.07 & 0.75 & 0.53 & 0.66 & 0.35 & 0.53 & -0.02 & 0.22 & 0.00 & -0.14 & 0.36 & 0.03 & 0.16 & -0.05 & 0.23 & -0.42 \\
\hline & & $\mathrm{Fe}$ & 0.23 & 0.25 & 0.10 & 0.09 & 0.31 & -0.03 & -0.34 & 0.09 & -0.10 & 0.27 & 0.14 & 0.22 & -0.04 & 0.12 & -0.07 \\
\hline & & & $\mathrm{Al}$ & 0.73 & 0.68 & 0.46 & 0.67 & 0.05 & 0.12 & 0.31 & -0.20 & 0.35 & -0.02 & 0.23 & -0.02 & 0.42 & -0.43 \\
\hline & & & & K & 0.51 & 0.24 & 0.38 & 0.13 & 0.05 & 0.39 & -0.31 & 0.23 & 0.11 & 0.25 & -0.10 & 0.58 & -0.25 \\
\hline & & & & & o & 0.21 & 0.64 & 0.15 & 0.49 & 0.08 & -0.35 & 0.50 & 0.13 & 0.09 & 0.04 & 0.07 & -0.30 \\
\hline & & & & & & $\mathrm{Mg}$ & 0.35 & 0.17 & 0.11 & -0.08 & -0.06 & 0.35 & -0.19 & 0.28 & 0.09 & -0.07 & -0.30 \\
\hline & & & & & & & $\mathrm{Ti}$ & -0.01 & 0.28 & 0.17 & -0.34 & 0.68 & 0.30 & 0.14 & -0.07 & 0.00 & -0.23 \\
\hline & & & & & & & & $\mathrm{Na}$ & -0.07 & 0.01 & -0.13 & 0.15 & -0.20 & 0.08 & -0.21 & -0.04 & -0.02 \\
\hline & & & & & & & & & $\mathrm{Sr}$ & -0.11 & -0.16 & 0.37 & 0.32 & -0.27 & 0.23 & -0.10 & 0.02 \\
\hline & & & & & & & & & & $\mathrm{Pb}$ & -0.38 & -0.12 & -0.05 & -0.10 & 0.03 & 0.41 & 0.02 \\
\hline & & & & & & & & & & & $\mathrm{Zn}$ & -0.49 & -0.16 & -0.25 & 0.00 & -0.07 & -0.19 \\
\hline & & & & & & & & & & & & $\mathrm{Mn}$ & 0.34 & -0.05 & 0.04 & -0.09 & -0.05 \\
\hline & & & & & & & & & & & & & $\mathrm{Cu}$ & -0.15 & -0.08 & 0.12 & 0.13 \\
\hline & & & & & & & & & & & & & & $\mathrm{Cr}$ & -0.15 & -0.07 & -0.20 \\
\hline & & & & & & & & & & & & & & & $P$ & -0.11 & -0.06 \\
\hline & & & & & & & & & & & & & & & & $\mathrm{Ba}$ & 0.04 \\
\hline
\end{tabular}


Table B7 Elemental correlations from xrf analyses on $R_{c}$ (concrete products) - material SI

\begin{tabular}{|c|c|c|c|c|c|c|c|c|c|c|c|c|c|c|c|c|c|}
\hline & $\mathrm{Si}$ & $\mathrm{Fe}$ & $\mathrm{Al}$ & K & S & $\mathrm{Mg}$ & $\mathrm{Ti}$ & $\mathrm{Na}$ & $\mathrm{Sr}$ & $\mathrm{Pb}$ & $\mathrm{Zn}$ & $\mathrm{Mn}$ & $\mathrm{Cu}$ & $\mathrm{Cr}$ & P & $\mathrm{Ba}$ & $\mathrm{Ni}$ \\
\hline $\mathrm{Ca}$ & 0.76 & 0.64 & 0.76 & 0.49 & 0.77 & 0.20 & 0.40 & -0.17 & 0.68 & 0.23 & 0.43 & 0.71 & 0.13 & 0.48 & I & 1 & 0.10 \\
\hline & \multirow[t]{16}{*}{$\mathrm{Si}$} & 0.66 & 0.85 & 0.53 & 0.87 & 0.38 & 0.56 & 0.00 & 0.72 & 0.22 & 0.31 & 0.73 & 0.06 & 0.38 & I & I & 0.16 \\
\hline & & $\mathrm{Fe}$ & 0.71 & 0.73 & 0.59 & 0.37 & 0.58 & -0.06 & 0.72 & 0.30 & 0.28 & 0.68 & -0.06 & 0.61 & I & I & -0.22 \\
\hline & & & Al & 0.68 & 0.83 & 0.36 & 0.68 & -0.07 & 0.69 & 0.33 & 0.47 & 0.68 & -0.03 & 0.47 & I & I & 0.15 \\
\hline & & & & K & 0.61 & 0.17 & 0.71 & 0.05 & 0.52 & 0.18 & 0.31 & 0.37 & -0.17 & 0.37 & / & / & -0.01 \\
\hline & & & & & S & 0.27 & 0.58 & -0.08 & 0.61 & 0.12 & 0.33 & 0.61 & 0.03 & 0.39 & / & / & 0.35 \\
\hline & & & & & & $\mathrm{Mg}$ & 0.25 & -0.01 & 0.36 & 0.10 & -0.16 & 0.53 & 0.17 & 0.09 & I & I & -0.14 \\
\hline & & & & & & & $\mathrm{Ti}$ & 0.08 & 0.51 & 0.24 & 0.40 & 0.46 & 0.11 & 0.27 & / & I & -0.07 \\
\hline & & & & & & & & $\mathrm{Na}$ & 0.24 & 0.34 & 0.03 & -0.33 & -0.13 & -0.19 & / & / & -0.26 \\
\hline & & & & & & & & & $\mathrm{Sr}$ & 0.40 & 0.25 & 0.56 & 0.00 & 0.45 & I & / & -0.27 \\
\hline & & & & & & & & & & $\mathrm{Pb}$ & 0.35 & 0.14 & -0.06 & 0.12 & / & / & -0.06 \\
\hline & & & & & & & & & & & $\mathrm{Zn}$ & 0.23 & -0.09 & -0.02 & I & / & -0.03 \\
\hline & & & & & & & & & & & & $\mathrm{Mn}$ & 0.19 & 0.49 & / & / & 0.04 \\
\hline & & & & & & & & & & & & & $\mathrm{Cu}$ & -0.09 & 1 & / & -0.06 \\
\hline & & & & & & & & & & & & & & & f & / & 0.04 \\
\hline & & & & & & & & & & & & & & & P & 7 & 7 \\
\hline & & & & & & & & & & & & & & & & Ba & I \\
\hline
\end{tabular}


Table B8 Elemental correlations from xrf analyses on $\mathrm{R}_{\mathrm{b}}$ (terracotta units) - material SB

\begin{tabular}{|c|c|c|c|c|c|c|c|c|c|c|c|c|c|c|c|c|c|}
\hline & $\mathrm{Si}$ & $\mathrm{Fe}$ & $\mathrm{Al}$ & K & S & $\mathrm{Mg}$ & $\mathrm{Ti}$ & $\mathrm{Na}$ & $\mathrm{Sr}$ & $\mathrm{Pb}$ & $\mathrm{Zn}$ & $\mathrm{Mn}$ & $\mathrm{Cu}$ & $\mathrm{Cr}$ & P & $\mathrm{Ba}$ & $\mathrm{Ni}$ \\
\hline $\mathrm{Ca}$ & 0.53 & 0.68 & 0.74 & 0.81 & 0.67 & 0.25 & 0.45 & -0.24 & 0.17 & -0.16 & 0.08 & 0.01 & 0.16 & 0.38 & 0.21 & 0.05 & -0.01 \\
\hline & $\mathrm{Si}$ & 0.29 & 0.77 & 0.52 & 0.59 & 0.19 & 0.26 & 0.01 & 0.14 & 0.18 & 0.34 & 0.53 & 0.11 & 0.19 & 0.10 & 0.25 & -0.25 \\
\hline & & $\mathrm{Fe}$ & 0.45 & 0.52 & 0.49 & 0.33 & 0.44 & -0.03 & 0.36 & -0.11 & 0.27 & -0.05 & 0.33 & 0.48 & 0.08 & -0.20 & 0.01 \\
\hline & & & Al & 0.76 & 0.76 & 0.24 & 0.39 & -0.17 & 0.28 & -0.08 & 0.07 & 0.20 & -0.02 & 0.26 & 0.15 & 0.25 & -0.21 \\
\hline & & & & K & 0.59 & 0.05 & 0.50 & -0.28 & 0.08 & -0.29 & 0.06 & 0.01 & 0.16 & 0.46 & 0.39 & -0.01 & -0.08 \\
\hline & & & & & S & 0.24 & 0.34 & -0.04 & 0.24 & -0.15 & -0.08 & 0.00 & 0.01 & 0.17 & -0.01 & 0.15 & -0.11 \\
\hline & & & & & & $\mathrm{Mg}$ & -0.08 & 0.31 & -0.06 & 0.41 & 0.31 & 0.14 & 0.07 & -0.26 & -0.22 & 0.09 & 0.06 \\
\hline & & & & & & & $\mathrm{Ti}$ & -0.08 & 0.16 & -0.26 & 0.26 & -0.03 & 0.13 & 0.52 & 0.02 & -0.34 & 0.03 \\
\hline & & & & & & & & $\mathrm{Na}$ & -0.29 & 0.23 & 0.19 & -0.02 & 0.12 & -0.14 & -0.17 & -0.01 & -0.25 \\
\hline & & & & & & & & & $\mathrm{Sr}$ & -0.13 & 0.09 & 0.00 & 0.10 & 0.15 & -0.18 & 0.08 & -0.22 \\
\hline & & & & & & & & & & $\mathrm{Pb}$ & 0.44 & 0.63 & 0.14 & -0.16 & -0.19 & -0.06 & 0.10 \\
\hline & & & & & & & & & & & $\mathrm{Zn}$ & 0.47 & 0.69 & 0.12 & -0.09 & -0.24 & 0.00 \\
\hline & & & & & & & & & & & & $\mathrm{Mn}$ & 0.10 & -0.01 & 0.01 & 0.13 & -0.16 \\
\hline & & & & & & & & & & & & & $\mathrm{Cu}$ & 0.23 & -0.07 & -0.28 & -0.07 \\
\hline & & & & & & & & & & & & & & $\mathrm{Cr}$ & 0.30 & -0.17 & -0.06 \\
\hline & & & & & & & & & & & & & & & P & 0.16 & -0.04 \\
\hline & & & & & & & & & & & & & & & & $\mathrm{Ba}$ & -0.20 \\
\hline
\end{tabular}


Table B9 Elemental correlations from xrf analyses on $\mathrm{R}_{\mathrm{b}}$ (terracotta units) - material SI

\begin{tabular}{|c|c|c|c|c|c|c|c|c|c|c|c|c|c|c|c|c|c|}
\hline & $\mathrm{Si}$ & $\mathrm{Fe}$ & $\mathrm{Al}$ & K & S & $\mathrm{Mg}$ & $\mathrm{Ti}$ & $\mathrm{Na}$ & $\mathrm{Sr}$ & $\mathrm{Pb}$ & $\mathrm{Zn}$ & $\mathrm{Mn}$ & $\mathrm{Cu}$ & $\mathrm{Cr}$ & P & $\mathrm{Ba}$ & $\mathrm{Ni}$ \\
\hline \multirow{17}{*}{\multicolumn{2}{|c|}{0.71}} & 0.87 & 0.75 & 0.85 & 0.48 & 0.10 & 0.60 & -0.05 & -0.27 & 0.27 & 0.27 & 0.54 & -0.01 & 0.28 & / & 0.56 & 0.33 \\
\hline & & 0.56 & 0.95 & 0.68 & 0.65 & 0.20 & 0.37 & 0.20 & -0.16 & 0.14 & 0.15 & 0.17 & 0.06 & 0.22 & / & 0.29 & 0.18 \\
\hline & & $\mathrm{Fe}$ & 0.63 & 0.65 & 0.49 & -0.12 & 0.59 & -0.05 & -0.09 & 0.36 & 0.25 & 0.46 & 0.25 & 0.41 & / & 0.62 & 0.45 \\
\hline & & & $\mathrm{Al}$ & 0.73 & 0.65 & 0.16 & 0.45 & 0.10 & -0.18 & 0.18 & 0.09 & 0.18 & 0.15 & 0.13 & / & 0.24 & 0.29 \\
\hline & & & & K & 0.44 & 0.28 & 0.52 & -0.05 & -0.46 & 0.17 & 0.31 & 0.58 & -0.27 & 0.17 & / & 0.47 & 0.19 \\
\hline & & & & & S & 0.12 & 0.17 & -0.05 & -0.21 & 0.27 & 0.34 & 0.02 & 0.19 & 0.32 & / & 0.15 & 0.15 \\
\hline & & & & & & $\mathrm{Mg}$ & -0.21 & -0.02 & -0.19 & -0.02 & 0.41 & 0.17 & -0.44 & 0.06 & / & -0.08 & -0.15 \\
\hline & & & & & & & $\mathrm{Ti}$ & 0.09 & -0.12 & 0.18 & 0.10 & 0.29 & 0.25 & 0.03 & / & 0.17 & -0.02 \\
\hline & & & & & & & & $\mathrm{Na}$ & 0.43 & -0.07 & 0.10 & -0.30 & 0.22 & 0.30 & / & 0.17 & -0.30 \\
\hline & & & & & & & & & $\mathrm{Sr}$ & -0.33 & -0.06 & -0.35 & 0.33 & -0.01 & / & -0.19 & 0.30 \\
\hline & & & & & & & & & & $\mathrm{Pb}$ & 0.39 & 0.33 & 0.13 & 0.48 & / & 0.28 & 0.08 \\
\hline & & & & & & & & & & & $\mathrm{Zn}$ & 0.37 & -0.15 & 0.42 & / & 0.08 & 0.05 \\
\hline & & & & & & & & & & & & $\mathrm{Mn}$ & -0.43 & 0.14 & / & 0.51 & 0.10 \\
\hline & & & & & & & & & & & & & $\mathrm{Cu}$ & 0.01 & / & -0.13 & 0.02 \\
\hline & & & & & & & & & & & & & & $\mathrm{Cr}$ & I & 0.43 & 0.06 \\
\hline & & & & & & & & & & & & & & & P & / & / \\
\hline & & & & & & & & & & & & & & & & $\mathrm{Ba}$ & 0.05 \\
\hline
\end{tabular}


Table B10 Elemental correlations from xrf analyses on $\mathrm{G}_{\mathrm{y}}$ (Gypsum products) - material SB

\begin{tabular}{|c|c|c|c|c|c|c|c|c|c|c|c|c|c|c|c|c|c|}
\hline & Si & $\mathrm{Fe}$ & $\mathrm{Al}$ & K & S & $\mathrm{Mg}$ & $\mathrm{Ti}$ & $\mathrm{Na}$ & $\mathrm{Sr}$ & $\mathrm{Pb}$ & $\mathrm{Zn}$ & $\mathrm{Mn}$ & $\mathrm{Cu}$ & $\mathrm{Cr}$ & P & $\mathrm{Ba}$ & $\mathrm{Ni}$ \\
\hline $\mathrm{Ca}$ & 0.68 & 0.72 & 0.59 & 0.24 & 0.99 & 0.51 & 0.15 & 0.18 & 0.71 & 0.69 & 0.87 & 0.75 & 0.28 & 0.35 & / & / & 0.61 \\
\hline & \multirow[t]{16}{*}{$\mathrm{Si}$} & 0.56 & 0.57 & 0.31 & 0.68 & 0.32 & 0.18 & 0.20 & 0.53 & 0.51 & 0.54 & 0.61 & 0.07 & 0.49 & I & I & 0.42 \\
\hline & & $\mathrm{Fe}$ & 0.49 & 0.24 & 0.70 & 0.44 & 0.05 & -0.05 & 0.56 & 0.45 & 0.51 & 0.59 & 0.18 & 0.38 & / & / & 0.31 \\
\hline & & & $\mathrm{Al}$ & 0.18 & 0.61 & 0.37 & -0.02 & 0.08 & 0.36 & 0.24 & 0.53 & 0.54 & 0.00 & 0.02 & / & / & 0.27 \\
\hline & & & & K & 0.25 & -0.15 & -0.09 & 0.06 & 0.19 & -0.19 & 0.22 & 0.37 & 0.06 & 0.06 & / & / & 0.24 \\
\hline & & & & & S & 0.54 & 0.16 & 0.17 & 0.70 & 0.68 & 0.85 & 0.79 & 0.27 & 0.36 & I & I & 0.59 \\
\hline & & & & & & $\mathrm{Mg}$ & 0.08 & 0.13 & 0.44 & 0.53 & 0.37 & 0.57 & -0.02 & 0.01 & / & / & 0.07 \\
\hline & & & & & & & $\mathrm{Ti}$ & -0.14 & 0.03 & 0.05 & 0.14 & 0.07 & -0.22 & 0.08 & / & / & 0.14 \\
\hline & & & & & & & & $\mathrm{Na}$ & 0.19 & 0.23 & 0.01 & 0.14 & -0.06 & 0.13 & / & / & 0.28 \\
\hline & & & & & & & & & Sr & 0.70 & 0.44 & 0.41 & 0.21 & 0.16 & I & I & 0.55 \\
\hline & & & & & & & & & & $\mathrm{Pb}$ & 0.50 & 0.36 & 0.43 & 0.26 & / & / & 0.52 \\
\hline & & & & & & & & & & & $\mathrm{Zn}$ & 0.72 & 0.37 & 0.22 & / & I & 0.49 \\
\hline & & & & & & & & & & & & $\mathrm{Mn}$ & 0.20 & 0.25 & / & / & 0.33 \\
\hline & & & & & & & & & & & & & $\mathrm{Cu}$ & 0.04 & / & / & 0.19 \\
\hline & & & & & & & & & & & & & & $\mathrm{Cr}$ & / & / & 0.26 \\
\hline & & & & & & & & & & & & & & & $r$ & / & / \\
\hline & & & & & & & & & & & & & & & & $\mathrm{Ba}$ & / \\
\hline
\end{tabular}


Table B11 Elemental correlations from xrf analyses on $\mathrm{G}_{\mathrm{y}}$ (Gypsum products) - material SI

\begin{tabular}{|c|c|c|c|c|c|c|c|c|c|c|c|c|c|c|c|c|c|}
\hline & $\mathrm{Si}$ & $\mathrm{Fe}$ & $\mathrm{Al}$ & K & S & $\mathrm{Mg}$ & $\mathrm{Ti}$ & $\mathrm{Na}$ & $\mathrm{Sr}$ & $\mathrm{Pb}$ & $\mathrm{Zn}$ & $\mathrm{Mn}$ & $\mathrm{Cu}$ & $\mathrm{Cr}$ & P & $\mathrm{Ba}$ & $\mathrm{Ni}$ \\
\hline $\mathrm{Ca}$ & 0.45 & 0.17 & 0.43 & 0.16 & 0.91 & 0.19 & -0.27 & -0.23 & 0.00 & 0.40 & 0.22 & 0.48 & 0.06 & 0.27 & 0.53 & -0.15 & -0.14 \\
\hline & $\mathrm{Si}$ & 0.02 & 0.37 & 0.04 & 0.62 & 0.30 & -0.19 & -0.29 & -0.32 & 0.09 & 0.16 & 0.08 & 0.11 & 0.04 & 0.28 & -0.01 & -0.41 \\
\hline & & $\mathrm{Fe}$ & 0.02 & -0.15 & -0.07 & -0.26 & 0.24 & -0.16 & 0.06 & 0.04 & -0.11 & 0.06 & 0.04 & 0.89 & 0.25 & -0.27 & -0.19 \\
\hline & & & $\mathrm{Al}$ & 0.21 & 0.45 & 0.48 & -0.13 & -0.14 & -0.24 & 0.21 & 0.26 & -0.21 & -0.07 & -0.06 & 0.40 & 0.04 & -0.16 \\
\hline & & & & K & 0.19 & 0.13 & -0.19 & -0.08 & 0.10 & -0.07 & -0.15 & -0.03 & 0.01 & -0.06 & -0.07 & -0.19 & -0.11 \\
\hline & & & & & S & 0.28 & -0.28 & -0.30 & -0.07 & 0.32 & 0.21 & 0.39 & -0.03 & 0.06 & 0.53 & -0.09 & -0.19 \\
\hline & & & & & & $\mathrm{Mg}$ & -0.20 & -0.12 & -0.03 & 0.12 & 0.05 & -0.18 & -0.08 & -0.16 & 0.09 & 0.20 & -0.14 \\
\hline & & & & & & & $\mathrm{Ti}$ & 0.06 & -0.08 & 0.14 & -0.32 & -0.05 & -0.05 & 0.00 & 0.22 & -0.08 & -0.12 \\
\hline & & & & & & & & $\mathrm{Na}$ & -0.03 & -0.11 & -0.05 & -0.17 & 0.11 & -0.15 & -0.20 & 0.19 & 0.01 \\
\hline & & & & & & & & & $\mathrm{Sr}$ & -0.01 & -0.15 & 0.34 & 0.14 & 0.10 & -0.28 & 0.16 & 0.21 \\
\hline & & & & & & & & & & $\mathrm{Pb}$ & 0.06 & 0.45 & 0.14 & 0.00 & 0.29 & 0.09 & 0.17 \\
\hline & & & & & & & & & & & $\mathrm{Zn}$ & -0.07 & -0.05 & -0.07 & 0.15 & 0.04 & -0.19 \\
\hline & & & & & & & & & & & & $\mathrm{Mn}$ & 0.11 & 0.06 & 0.01 & -0.13 & 0.06 \\
\hline & & & & & & & & & & & & & $\mathrm{Cu}$ & 0.12 & -0.10 & 0.26 & 0.12 \\
\hline & & & & & & & & & & & & & & $\mathrm{Cr}$ & 0.25 & -0.22 & -0.12 \\
\hline & & & & & & & & & & & & & & & P & -0.18 & -0.18 \\
\hline & & & & & & & & & & & & & & & & $\mathrm{Ba}$ & 0.42 \\
\hline
\end{tabular}


Table B12 Elemental correlations from xrf analyses on $\mathrm{X}_{1}$ (Aggregated fine material) - material SB

\begin{tabular}{|c|c|c|c|c|c|c|c|c|c|c|c|c|c|c|c|c|c|}
\hline & $\mathrm{Si}$ & $\mathrm{Fe}$ & $\mathrm{Al}$ & K & $S$ & $\mathrm{Mg}$ & $\mathrm{Ti}$ & $\mathrm{Na}$ & $\mathrm{Sr}$ & $\mathrm{Pb}$ & $\mathrm{Zn}$ & $\mathrm{Mn}$ & $\mathrm{Cu}$ & $\mathrm{Cr}$ & $P$ & $\mathrm{Ba}$ & $\mathrm{Ni}$ \\
\hline \multirow[t]{17}{*}{$\mathrm{Ca}$} & 0.70 & 0.10 & 0.80 & 0.75 & 0.74 & 0.46 & 0.50 & -0.33 & 0.31 & -0.12 & -0.03 & 0.62 & 0.06 & 0.04 & 0.30 & 0.20 & 0.48 \\
\hline & $\mathrm{Si}$ & 0.29 & 0.83 & 0.73 & 0.72 & 0.55 & 0.61 & -0.32 & -0.07 & -0.22 & -0.05 & 0.65 & 0.01 & 0.25 & 0.11 & -0.03 & 0.35 \\
\hline & & $\mathrm{Fe}$ & 0.18 & 0.39 & 0.07 & 0.15 & 0.16 & -0.01 & -0.49 & -0.25 & -0.18 & 0.58 & -0.14 & 0.23 & 0.08 & 0.16 & 0.06 \\
\hline & & & $\mathrm{Al}$ & 0.73 & 0.88 & 0.62 & 0.38 & -0.34 & -0.01 & -0.24 & 0.08 & 0.63 & 0.17 & 0.10 & 0.33 & 0.31 & 0.30 \\
\hline & & & & K & 0.66 & 0.28 & 0.59 & -0.21 & -0.05 & -0.11 & -0.04 & 0.78 & 0.00 & 0.35 & 0.31 & 0.11 & 0.38 \\
\hline & & & & & S & 0.38 & 0.28 & -0.33 & -0.02 & -0.21 & 0.01 & 0.50 & 0.11 & 0.11 & 0.58 & 0.29 & 0.30 \\
\hline & & & & & & $\mathrm{Mg}$ & 0.19 & -0.34 & -0.03 & -0.18 & 0.09 & 0.32 & 0.11 & 0.04 & -0.16 & 0.17 & 0.14 \\
\hline & & & & & & & $\mathrm{Ti}$ & -0.25 & 0.01 & -0.02 & -0.01 & 0.70 & 0.09 & 0.09 & -0.04 & -0.31 & 0.18 \\
\hline & & & & & & & & $\mathrm{Na}$ & -0.03 & 0.44 & 0.12 & -0.30 & 0.09 & 0.30 & -0.03 & -0.03 & -0.07 \\
\hline & & & & & & & & & $\mathrm{Sr}$ & 0.16 & -0.07 & -0.20 & -0.08 & -0.13 & -0.09 & 0.09 & 0.32 \\
\hline & & & & & & & & & & $\mathrm{Pb}$ & -0.11 & -0.14 & -0.09 & -0.07 & -0.12 & -0.08 & 0.13 \\
\hline & & & & & & & & & & & $\mathrm{Zn}$ & -0.13 & 0.94 & -0.10 & -0.01 & -0.14 & -0.10 \\
\hline & & & & & & & & & & & & $\mathrm{Mn}$ & -0.04 & 0.12 & 0.23 & 0.09 & 0.26 \\
\hline & & & & & & & & & & & & & $\mathrm{Cu}$ & -0.11 & 0.10 & -0.08 & -0.10 \\
\hline & & & & & & & & & & & & & & $\mathrm{Cr}$ & 0.10 & 0.00 & 0.14 \\
\hline & & & & & & & & & & & & & & & $\mathrm{P}$ & 0.40 & 0.16 \\
\hline & & & & & & & & & & & & & & & & $\mathrm{Ba}$ & 0.08 \\
\hline
\end{tabular}


Table B13 Elemental correlations from xrf analyses on $\mathrm{X}_{1}$ (Aggregated fine material) - material SI

\begin{tabular}{|c|c|c|c|c|c|c|c|c|c|c|c|c|c|c|c|c|c|}
\hline & $\mathrm{Si}$ & $\mathrm{Fe}$ & $\mathrm{Al}$ & K & S & $\mathrm{Mg}$ & $\mathrm{Ti}$ & $\mathrm{Na}$ & $\mathrm{Sr}$ & $\mathrm{Pb}$ & $\mathrm{Zn}$ & $\mathrm{Mn}$ & $\mathrm{Cu}$ & $\mathrm{Cr}$ & $\mathrm{P}$ & $\mathrm{Ba}$ & $\mathrm{Ni}$ \\
\hline \multirow[t]{17}{*}{$\mathrm{Ca}$} & 0.16 & 0.23 & 0.54 & 0.32 & 0.21 & 0.50 & 0.18 & 0.16 & -0.09 & 0.12 & 0.18 & 0.27 & 0.11 & 0.08 & 0.26 & -0.17 & -0.06 \\
\hline & $\mathrm{Si}$ & 0.18 & 0.70 & 0.54 & 0.27 & 0.56 & 0.66 & -0.08 & -0.13 & 0.12 & 0.18 & 0.20 & 0.41 & 0.52 & -0.06 & 0.07 & -0.07 \\
\hline & & $\mathrm{Fe}$ & 0.43 & 0.55 & 0.71 & 0.30 & 0.41 & 0.09 & 0.09 & 0.43 & 0.51 & 0.64 & 0.66 & 0.04 & 0.38 & 0.02 & 0.05 \\
\hline & & & $\mathrm{Al}$ & 0.75 & 0.48 & 0.89 & 0.60 & 0.09 & 0.10 & 0.31 & 0.46 & 0.42 & 0.50 & 0.23 & 0.06 & 0.00 & -0.09 \\
\hline & & & & K & 0.35 & 0.61 & 0.54 & 0.14 & 0.24 & 0.60 & 0.62 & 0.72 & 0.46 & 0.06 & 0.13 & 0.24 & -0.03 \\
\hline & & & & & S & 0.34 & 0.27 & -0.16 & 0.04 & 0.15 & 0.21 & 0.16 & 0.52 & 0.05 & -0.05 & -0.21 & 0.39 \\
\hline & & & & & & $\mathrm{Mg}$ & 0.54 & 0.16 & 0.22 & 0.28 & 0.44 & 0.39 & 0.48 & 0.27 & -0.06 & 0.05 & -0.12 \\
\hline & & & & & & & $\mathrm{Ti}$ & 0.26 & 0.02 & 0.14 & 0.12 & 0.42 & 0.29 & 0.39 & 0.06 & 0.13 & 0.02 \\
\hline & & & & & & & & $\mathrm{Na}$ & 0.02 & 0.18 & -0.02 & 0.24 & 0.04 & 0.10 & 0.18 & -0.01 & -0.26 \\
\hline & & & & & & & & & $\mathrm{Sr}$ & 0.48 & 0.54 & 0.32 & 0.08 & -0.12 & -0.08 & 0.28 & 0.20 \\
\hline & & & & & & & & & & $\mathrm{Pb}$ & 0.76 & 0.55 & 0.55 & 0.11 & -0.01 & -0.03 & -0.25 \\
\hline & & & & & & & & & & & $\mathrm{Zn}$ & 0.66 & 0.59 & -0.01 & 0.12 & 0.19 & -0.12 \\
\hline & & & & & & & & & & & & $\mathrm{Mn}$ & 0.42 & 0.01 & 0.38 & 0.30 & -0.17 \\
\hline & & & & & & & & & & & & & $\mathrm{Cu}$ & 0.18 & -0.01 & -0.16 & -0.34 \\
\hline & & & & & & & & & & & & & & $\mathrm{Cr}$ & -0.06 & 0.03 & -0.21 \\
\hline & & & & & & & & & & & & & & & $\mathrm{P}$ & 0.12 & -0.13 \\
\hline & & & & & & & & & & & & & & & & $\mathrm{Ba}$ & 0.08 \\
\hline
\end{tabular}

+ XRF spectra

See accompanying .xrf file.

\section{References}

AFNOR (2001) Soil quality - Dissolution for the determination of total element content - Part 1: Dissolution with hydrofluoric and perchloric acids. 2001. NF ISO 14869-1. AFNOR Ed

AFNOR (2008) Tests for mechanical and physical properties of aggregates - Part 7: Determination of the particle density of filler - Pycnometer method. 2008. NF EN 1097-7. AFNOR Ed

AFNOR (2009a) Tests for geometrical properties of aggregates - Part 11 : classification test for the constituents of coarse recycled aggregate. 2009. NF EN 933-11. AFNOR Ed

AFNOR (2009b) Water quality - Determination of selected elements by inductively coupled plasma optical emission spectrometry (ICP-OES). 2009. NF ISO 11885. AFNOR Ed

AFNOR (2012) Tests for chemical properties of aggregates - Part 8: Sorting test to determine metal content of Municipal Incinerator Bottom Ash (MIBA) Aggregates. 2012. NF EN 17448. AFNOR Ed 
AFNOR (2013) Sludge, treated biowaste, soil and waste - Determination of loss on ignition. 2013. NF EN 15935. AFNOR Ed

Jean-Soro L, Le Guern C, Bechet B, Lebeau T, Ringeard MF (2015) Origin of trace elements in an urban garden in Nantes, France. J Soils Sediments 15(8):1802-1812 


\begin{tabular}{|c|c|c|c|c|c|c|}
\hline & SB WM & & $\mathrm{SB}<80 \mu \mathrm{m}$ & & $80 \mu \mathrm{m}<\mathrm{SB}<40$ & \\
\hline $\mathrm{E}(\mathrm{keV})$ & mean & std & mean & std & mean & std \\
\hline 1.0075 & 0.355107 & 0.0376594 & 0.381873 & 0.0451926 & 0.287061 & 0.0521488 \\
\hline 1.0175 & 0.355862 & 0.0392194 & 0.383248 & 0.0527283 & 0.282271 & 0.0508933 \\
\hline 1.0275 & 0.361439 & 0.0370037 & 0.380587 & 0.0454798 & 0.28223 & 0.0451912 \\
\hline 1.0375 & 0.356922 & 0.0388802 & 0.378815 & 0.0484536 & 0.284055 & 0.0518202 \\
\hline 1.0475 & 0.353138 & 0.035798 & 0.382326 & 0.0483799 & 0.2792 & 0.0516598 \\
\hline 1.0575 & 0.35213 & 0.0408871 & 0.377129 & 0.0465784 & 0.284525 & 0.0515342 \\
\hline 1.0675 & 0.349765 & 0.0368127 & 0.379615 & 0.0496447 & 0.279146 & 0.0511656 \\
\hline 1.0775 & 0.34985 & 0.033993 & 0.378507 & 0.0476951 & 0.276903 & 0.0497673 \\
\hline 1.0875 & 0.350064 & 0.0387156 & 0.376278 & 0.0468922 & 0.277277 & 0.0470911 \\
\hline 1.0975 & 0.352398 & 0.0365149 & 0.374251 & 0.0479163 & 0.277769 & 0.0484087 \\
\hline 1.1075 & 0.349488 & 0.0349309 & 0.37556 & 0.0457723 & 0.277902 & 0.0483425 \\
\hline 1.1175 & 0.35084 & 0.0327768 & 0.370083 & 0.0459784 & 0.276235 & 0.0482069 \\
\hline 1.1275 & 0.349796 & 0.0385719 & 0.374467 & 0.0455516 & 0.279427 & 0.0499173 \\
\hline 1.1375 & 0.34652 & 0.037075 & 0.374004 & 0.0462161 & 0.274431 & 0.0500929 \\
\hline 1.1475 & 0.350302 & 0.0359478 & 0.376598 & 0.0513535 & 0.274624 & 0.0491421 \\
\hline 1.1575 & 0.350272 & 0.0360908 & 0.379187 & 0.0496586 & 0.275582 & 0.0506263 \\
\hline 1.1675 & 0.348967 & 0.0377382 & 0.380983 & 0.0487965 & 0.27763 & 0.0525208 \\
\hline 1.1775 & 0.351466 & 0.0328768 & 0.380455 & 0.0446271 & 0.279822 & 0.0488033 \\
\hline 1.1875 & 0.356999 & 0.0356013 & 0.38758 & 0.0456826 & 0.281541 & 0.0528866 \\
\hline 1.1975 & 0.359975 & 0.037152 & 0.394428 & 0.0465862 & 0.286045 & 0.0519804 \\
\hline 1.2075 & 0.364112 & 0.033261 & 0.402391 & 0.0468169 & 0.289781 & 0.0498972 \\
\hline 1.2175 & 0.372246 & 0.0387636 & 0.415875 & 0.052624 & 0.294872 & 0.0496238 \\
\hline 1.2275 & 0.378989 & 0.0357447 & 0.424083 & 0.0508483 & 0.302034 & 0.0506722 \\
\hline 1.2375 & 0.384125 & 0.0364917 & 0.432605 & 0.0561858 & 0.304251 & 0.0518911 \\
\hline 1.2475 & 0.389614 & 0.0374028 & 0.437119 & 0.0606616 & 0.309435 & 0.05383 \\
\hline 1.2575 & 0.385235 & 0.0379249 & 0.442052 & 0.0575235 & 0.31398 & 0.0539902 \\
\hline 1.2675 & 0.390816 & 0.0350435 & 0.442979 & 0.0550323 & 0.313228 & 0.0529003 \\
\hline 1.2775 & 0.389681 & 0.0380541 & 0.43512 & 0.0594494 & 0.308548 & 0.0563976 \\
\hline 1.2875 & 0.385908 & 0.0362197 & 0.423474 & 0.0529552 & 0.303397 & 0.055309 \\
\hline 1.2975 & 0.377933 & 0.0392337 & 0.419796 & 0.0513018 & 0.297941 & 0.0518076 \\
\hline 1.3075 & 0.371062 & 0.0358826 & 0.405813 & 0.0494599 & 0.290452 & 0.0513099 \\
\hline 1.3175 & 0.36574 & 0.033994 & 0.393002 & 0.044722 & 0.288839 & 0.0526203 \\
\hline 1.3275 & 0.359256 & 0.0373004 & 0.388081 & 0.0496341 & 0.282154 & 0.0503527 \\
\hline 1.3375 & 0.354088 & 0.0381069 & 0.384377 & 0.0459628 & 0.282214 & 0.0512427 \\
\hline 1.3475 & 0.355478 & 0.0352832 & 0.381648 & 0.0490687 & 0.279059 & 0.0496765 \\
\hline 1.3575 & 0.362136 & 0.0316549 & 0.385161 & 0.0431973 & 0.282467 & 0.0505247 \\
\hline 1.3675 & 0.363387 & 0.0341079 & 0.393043 & 0.0448996 & 0.288402 & 0.0475815 \\
\hline 1.3775 & 0.375979 & 0.0365287 & 0.412377 & 0.0472549 & 0.297314 & 0.0530736 \\
\hline 1.3875 & 0.395995 & 0.037932 & 0.444195 & 0.0511168 & 0.31516 & 0.0524356 \\
\hline 1.3975 & 0.427037 & 0.0392373 & 0.498151 & 0.0637309 & 0.346338 & 0.0579818 \\
\hline 1.4075 & 0.482725 & 0.0438048 & 0.571784 & 0.0767376 & 0.390149 & 0.0650325 \\
\hline 1.4175 & 0.554268 & 0.0457005 & 0.679048 & 0.0996944 & 0.45355 & 0.0798009 \\
\hline 1.4275 & 0.655774 & 0.0505158 & 0.81738 & 0.116893 & 0.53425 & 0.0935592 \\
\hline 1.4375 & 0.78428 & 0.0641301 & 0.979893 & 0.162041 & 0.639934 & 0.107672 \\
\hline 1.4475 & 0.925649 & 0.0709437 & 1.15875 & 0.215285 & 0.75255 & 0.130241 \\
\hline
\end{tabular}




\begin{tabular}{|c|c|c|c|c|c|c|}
\hline 1.4575 & 1.08317 & 0.079964 & 1.34624 & 0.249 & 0.868049 & 0.159211 \\
\hline 1.4675 & 1.23253 & 0.0911347 & 1.50548 & 0.291181 & 0.970228 & 0.180736 \\
\hline 1.4775 & 1.34791 & 0.103436 & 1.61854 & 0.315983 & 1.04486 & 0.195795 \\
\hline 1.4875 & 1.42288 & 0.106371 & 1.67764 & 0.327342 & 1.08313 & 0.202816 \\
\hline 1.4975 & 1.44411 & 0.104487 & 1.66286 & 0.321028 & 1.08641 & 0.200838 \\
\hline 1.5075 & 1.40724 & 0.108994 & 1.58941 & 0.299792 & 1.03935 & 0.190327 \\
\hline 1.5175 & 1.319 & 0.0967119 & 1.44499 & 0.270692 & 0.958296 & 0.175297 \\
\hline 1.5275 & 1.19822 & 0.0898974 & 1.27692 & 0.223008 & 0.860895 & 0.157898 \\
\hline 1.5375 & 1.05974 & 0.0806408 & 1.10206 & 0.18848 & 0.758461 & 0.138949 \\
\hline 1.5475 & 0.915876 & 0.0728223 & 0.933223 & 0.152441 & 0.648631 & 0.114563 \\
\hline 1.5575 & 0.787631 & 0.057481 & 0.786528 & 0.117237 & 0.560277 & 0.102478 \\
\hline 1.5675 & 0.682459 & 0.0506282 & 0.673729 & 0.0883767 & 0.488377 & 0.0857852 \\
\hline 1.5775 & 0.5996 & 0.0520666 & 0.587154 & 0.0750564 & 0.435716 & 0.0758437 \\
\hline 1.5875 & 0.548498 & 0.0445008 & 0.535012 & 0.0617667 & 0.404069 & 0.0705101 \\
\hline 1.5975 & 0.524382 & 0.0442851 & 0.513425 & 0.0613113 & 0.39279 & 0.0672536 \\
\hline 1.6075 & 0.542811 & 0.0440128 & 0.530292 & 0.0619221 & 0.417347 & 0.0669073 \\
\hline 1.6175 & 0.608855 & 0.0496285 & 0.587658 & 0.0742219 & 0.475923 & 0.0786262 \\
\hline 1.6275 & 0.756756 & 0.0570315 & 0.714487 & 0.10475 & 0.59763 & 0.106738 \\
\hline 1.6375 & 1.01714 & 0.0838383 & 0.941866 & 0.159229 & 0.818519 & 0.149908 \\
\hline 1.6475 & 1.44873 & 0.117207 & 1.29787 & 0.230483 & 1.15766 & 0.211539 \\
\hline 1.6575 & 2.1114 & 0.175385 & 1.82333 & 0.356329 & 1.67129 & 0.316394 \\
\hline 1.6675 & 3.0765 & 0.25626 & 2.55471 & 0.524708 & 2.38447 & 0.461112 \\
\hline 1.6775 & 4.37337 & 0.368552 & 3.50082 & 0.738843 & 3.32222 & 0.643132 \\
\hline 1.6875 & 5.99447 & 0.518126 & 4.64845 & 1.00634 & 4.46983 & 0.88181 \\
\hline 1.6975 & 7.86923 & 0.683453 & 5.89416 & 1.28815 & 5.7492 & 1.14497 \\
\hline 1.7075 & 9.88478 & 0.868773 & 7.16991 & 1.57117 & 7.05376 & 1.41627 \\
\hline 1.7175 & 11.8503 & 1.02044 & 8.29567 & 1.78889 & 8.26993 & 1.68108 \\
\hline 1.7275 & 13.5044 & 1.16335 & 9.16808 & 1.95979 & 9.20618 & 1.85237 \\
\hline 1.7375 & 14.6481 & 1.2911 & 9.65018 & 2.08276 & 9.75085 & 1.96212 \\
\hline 1.7475 & 15.1083 & 1.31894 & 9.6468 & 2.08177 & 9.82758 & 1.98436 \\
\hline 1.7575 & 14.8161 & 1.29995 & 9.17584 & 1.96127 & 9.44142 & 1.91145 \\
\hline 1.7675 & 13.8297 & 1.2092 & 8.33313 & 1.78688 & 8.63619 & 1.74629 \\
\hline 1.7775 & 12.2868 & 1.08165 & 7.2561 & 1.53543 & 7.52807 & 1.53006 \\
\hline 1.7875 & 10.4553 & 0.926039 & 6.03132 & 1.2501 & 6.28275 & 1.2528 \\
\hline 1.7975 & 8.52284 & 0.749765 & 4.84605 & 0.988073 & 5.0382 & 1.00544 \\
\hline 1.8075 & 6.69553 & 0.582581 & 3.78119 & 0.748577 & 3.92211 & 0.781436 \\
\hline 1.8175 & 5.10808 & 0.441515 & 2.89082 & 0.555264 & 2.96979 & 0.583689 \\
\hline 1.8275 & 3.83002 & 0.33034 & 2.20684 & 0.395799 & 2.23158 & 0.425189 \\
\hline 1.8375 & 2.88581 & 0.236261 & 1.70415 & 0.270932 & 1.69318 & 0.314197 \\
\hline 1.8475 & 2.14743 & 0.178789 & 1.33788 & 0.200176 & 1.28327 & 0.233265 \\
\hline 1.8575 & 1.63137 & 0.128421 & 1.06356 & 0.143757 & 0.999169 & 0.178119 \\
\hline 1.8675 & 1.28047 & 0.101445 & 0.865644 & 0.106616 & 0.792963 & 0.139824 \\
\hline 1.8775 & 1.02635 & 0.0789982 & 0.725226 & 0.0829064 & 0.645841 & 0.11111 \\
\hline 1.8875 & 0.844685 & 0.0677062 & 0.624587 & 0.0727768 & 0.536655 & 0.0937568 \\
\hline 1.8975 & 0.714833 & 0.0582742 & 0.54399 & 0.0591528 & 0.453599 & 0.0787014 \\
\hline 1.9075 & 0.615505 & 0.0505378 & 0.4916 & 0.0584572 & 0.39922 & 0.0725226 \\
\hline 1.9175 & 0.546325 & 0.0512119 & 0.466635 & 0.062177 & 0.359542 & 0.0659289 \\
\hline
\end{tabular}




\begin{tabular}{|c|c|c|c|c|c|c|}
\hline 1.9275 & 0.492394 & 0.0526085 & 0.455613 & 0.0560667 & 0.33362 & 0.0619497 \\
\hline 1.9375 & 0.457896 & 0.0500376 & 0.455479 & 0.0650331 & 0.325846 & 0.0643942 \\
\hline 9475 & 0.429935 & 0.0477574 & 0.485316 & 0.0711879 & 0.334166 & 0.0638847 \\
\hline 9575 & 0.411124 & 0.0436332 & 0.5119 & 0.0774324 & 0.336433 & 0.0612954 \\
\hline .9675 & 0.400667 & 0.0467377 & 0.531345 & 0.0758338 & 0.34716 & 0.0647139 \\
\hline .9775 & 0.397999 & 0.045856 & 0.553116 & 0.0824222 & 0.361037 & 0.0689638 \\
\hline 9875 & 0.397877 & 0.0469935 & 0.575117 & 0.0812282 & 0.372121 & 0.0729625 \\
\hline 1.9975 & 0.406028 & 0.0436418 & 0.587416 & 0.0784253 & 0.3788 & 0.075241 \\
\hline .0075 & 0.409506 & 0.0463274 & 0.594295 & 0.0840987 & 0.388653 & 0.0747237 \\
\hline 2.0175 & 0.420658 & 0.0447384 & 0.589311 & 0.0859312 & 0.389652 & 0.0732183 \\
\hline 2.0275 & 0.425806 & 0.0446998 & 0.575846 & 0.081853 & 0.383561 & 0.071385 \\
\hline 2.0375 & 0.426747 & 0.0451879 & 0.560238 & 0.0812416 & 0.375355 & 0.0703605 \\
\hline 2.0475 & 0.424507 & 0.0442853 & 0.540794 & 0.0715278 & 0.36647 & 0.0677828 \\
\hline 2.0575 & 0.416337 & 0.0421837 & 0.51807 & 0.0661035 & 0.351507 & 0.066912 \\
\hline 2.0675 & 0.406814 & 0.0417106 & 0.488291 & 0.064055 & 0.334689 & 0.0633267 \\
\hline 2.0775 & 0.393518 & 0.0391961 & 0.468383 & 0.0574169 & 0.319956 & 0.0612306 \\
\hline 2.0875 & 0.380823 & 0.0378146 & 0.450552 & 0.0551862 & 0.308935 & 0.058454 \\
\hline 2.0975 & 0.369458 & 0.0373257 & 0.436074 & 0.0520082 & 0.300212 & 0.0550042 \\
\hline 2.1075 & 0.358411 & 0.0392621 & 0.424367 & 0.0520885 & 0.292739 & 0.0525166 \\
\hline 2.1175 & 0.35068 & 0.0384962 & 0.418135 & 0.0533216 & 0.286182 & 0.0533485 \\
\hline 2.1275 & 0.345363 & 0.0345282 & 0.416744 & 0.0525743 & 0.283545 & 0.0548856 \\
\hline 2.1375 & 0.345269 & 0.0344165 & 0.419354 & 0.052881 & 0.284023 & 0.0536223 \\
\hline 2.1475 & 0.347414 & 0.0342327 & 0.428677 & 0.0572747 & 0.286831 & 0.0526793 \\
\hline 2.1575 & 0.351408 & 0.0334328 & 0.447519 & 0.0638716 & 0.295022 & 0.0539867 \\
\hline 2.1675 & 0.361503 & 0.03292 & 0.483861 & 0.0705896 & 0.308859 & 0.0574331 \\
\hline 2.1775 & 0.381998 & 0.0384134 & 0.536063 & 0.0780863 & 0.335672 & 0.0633655 \\
\hline 2.1875 & 0.411044 & 0.0417337 & 0.62526 & 0.104234 & 0.381651 & 0.0698411 \\
\hline 2.1975 & 0.460773 & 0.044962 & 0.773597 & 0.15192 & 0.450959 & 0.0780548 \\
\hline 2.2075 & 0.537129 & 0.0450533 & 0.984622 & 0.22773 & 0.553879 & 0.0983058 \\
\hline 2.2175 & 0.654793 & 0.0528487 & 1.28641 & 0.325727 & 0.698713 & 0.125608 \\
\hline 2.2275 & 0.820188 & 0.065171 & 1.70577 & 0.464945 & 0.903313 & 0.163014 \\
\hline 2.2375 & 1.03635 & 0.0836838 & 2.23397 & 0.650653 & 1.17074 & 0.212219 \\
\hline 2.2475 & 1.31124 & 0.106027 & 2.88007 & 0.87518 & 1.49517 & 0.272431 \\
\hline 2.2575 & 1.64037 & 0.135126 & 3.62278 & 1.13461 & 1.87244 & 0.336502 \\
\hline 2.2675 & 2.00874 & 0.165882 & 4.39172 & 1.38073 & 2.27834 & 0.415147 \\
\hline 2.2775 & 2.38073 & 0.193299 & 5.14259 & 1.63094 & 2.66376 & 0.493437 \\
\hline 2.2875 & 2.7345 & 0.221919 & 5.77516 & 1.85752 & 3.00646 & 0.554893 \\
\hline 2.2975 & 3.03161 & 0.244251 & 6.21233 & 2.01174 & 3.26615 & 0.603618 \\
\hline 2.3075 & 3.22076 & 0.258383 & 6.41488 & 2.08586 & 3.40742 & 0.631511 \\
\hline 2.3175 & 3.28421 & 0.261026 & 6.35527 & 2.06979 & 3.39854 & 0.62388 \\
\hline 2.3275 & 3.22847 & 0.259368 & 6.04928 & 1.9739 & 3.25961 & 0.600501 \\
\hline 2.3375 & 3.05242 & 0.249924 & 5.52838 & 1.78465 & 3.00132 & 0.556826 \\
\hline 2.3475 & 2.76686 & 0.221997 & 4.84911 & 1.55634 & 2.65639 & 0.492784 \\
\hline 2.3575 & 2.41695 & 0.198788 & 4.10024 & 1.30693 & 2.26903 & 0.416533 \\
\hline 2.3675 & 2.0437 & 0.170591 & 3.35854 & 1.05379 & 1.88188 & 0.348862 \\
\hline 2.3775 & 1.67453 & 0.139874 & 2.68643 & 0.84018 & 1.51618 & 0.28256 \\
\hline 2.3875 & 1.35785 & 0.108897 & 2.11518 & 0.659003 & 1.20643 & 0.223317 \\
\hline
\end{tabular}




\begin{tabular}{|c|c|c|c|c|c|c|}
\hline 2.3975 & 1.09355 & 0.0912403 & 1.67934 & 0.525691 & 0.964237 & 0.175132 \\
\hline 2.4075 & 0.89268 & 0.0759264 & 1.35857 & 0.430169 & 0.787073 & 0.140956 \\
\hline .4175 & 0.749818 & 0.0677561 & 1.14347 & 0.367938 & 0.664511 & 0.117946 \\
\hline .4275 & 0.653307 & 0.057658 & 1.01374 & 0.339105 & 0.588294 & 0.104499 \\
\hline 2.4375 & 0.600651 & 0.0523824 & 0.938368 & 0.316486 & 0.545388 & 0.10068 \\
\hline 2.4475 & 0.571417 & 0.0506815 & 0.896086 & 0.294611 & 0.521492 & 0.095067 \\
\hline 2.4575 & 0.55313 & 0.0484179 & 0.863869 & 0.272 & 0.50688 & 0.0920645 \\
\hline 2.4675 & 0.540104 & 0.0477304 & 0.838411 & 0.250353 & 0.493385 & 0.0921069 \\
\hline 2.4775 & 0.527706 & 0.046744 & 0.79945 & 0.224864 & 0.477154 & 0.086521 \\
\hline 2.4875 & 0.512394 & 0.0449959 & 0.751639 & 0.196752 & 0.458037 & 0.084281 \\
\hline 2.4975 & 0.494353 & 0.0452328 & 0.703583 & 0.168707 & 0.430569 & 0.0805991 \\
\hline 2.5075 & 0.470009 & 0.0439045 & 0.647962 & 0.140546 & 0.401351 & 0.0725539 \\
\hline 2.5175 & 0.444446 & 0.0410706 & 0.593186 & 0.117057 & 0.37599 & 0.0661368 \\
\hline 2.5275 & 0.422598 & 0.0385837 & 0.546699 & 0.09544 & 0.351992 & 0.063183 \\
\hline 2.5375 & 0.401766 & 0.0350146 & 0.50761 & 0.0740875 & 0.332589 & 0.0623916 \\
\hline 2.5475 & 0.389364 & 0.0348233 & 0.479284 & 0.062506 & 0.319179 & 0.0586605 \\
\hline 2.5575 & 0.382283 & 0.035774 & 0.463069 & 0.0603286 & 0.314054 & 0.0558678 \\
\hline 2.5675 & 0.381021 & 0.0346771 & 0.463762 & 0.0574569 & 0.317615 & 0.0579661 \\
\hline 2.5775 & 0.392502 & 0.0365627 & 0.482064 & 0.0609617 & 0.330146 & 0.0595246 \\
\hline 2.5875 & 0.413911 & 0.0406966 & 0.507453 & 0.0700092 & 0.352939 & 0.0633128 \\
\hline 2.5975 & 0.446585 & 0.0426494 & 0.549581 & 0.0764285 & 0.384653 & 0.0674108 \\
\hline 2.6075 & 0.49639 & 0.0439655 & 0.610541 & 0.0861259 & 0.42887 & 0.0758529 \\
\hline 2.6175 & 0.56443 & 0.0524978 & 0.68546 & 0.0985263 & 0.486205 & 0.0890613 \\
\hline 2.6275 & 0.650485 & 0.0638511 & 0.780316 & 0.113712 & 0.557106 & 0.102566 \\
\hline 2.6375 & 0.750973 & 0.0750152 & 0.887819 & 0.126558 & 0.639269 & 0.120686 \\
\hline 2.6475 & 0.864133 & 0.0890821 & 0.996972 & 0.137976 & 0.732021 & 0.139592 \\
\hline 2.6575 & 0.984383 & 0.106543 & 1.1184 & 0.156943 & 0.825525 & 0.16122 \\
\hline 2.6675 & 1.10563 & 0.123689 & 1.22323 & 0.17766 & 0.910227 & 0.183495 \\
\hline 2.6775 & 1.22539 & 0.138047 & 1.3069 & 0.194716 & 0.984958 & 0.196234 \\
\hline 2.6875 & 1.32003 & 0.152369 & 1.37071 & 0.210884 & 1.04149 & 0.206912 \\
\hline 2.6975 & 1.38179 & 0.160052 & 1.39871 & 0.221867 & 1.06888 & 0.215605 \\
\hline 2.7075 & 1.40973 & 0.16474 & 1.39152 & 0.217952 & 1.06963 & 0.215842 \\
\hline 2.7175 & 1.40243 & 0.162566 & 1.35433 & 0.209261 & 1.04754 & 0.206066 \\
\hline 2.7275 & 1.36073 & 0.152654 & 1.30049 & 0.19945 & 1.00601 & 0.194573 \\
\hline 2.7375 & 1.29867 & 0.14539 & 1.23894 & 0.186477 & 0.951163 & 0.185408 \\
\hline 2.7475 & 1.22748 & 0.133345 & 1.17846 & 0.179528 & 0.896083 & 0.176409 \\
\hline 2.7575 & 1.15607 & 0.11878 & 1.13449 & 0.182334 & 0.851731 & 0.171259 \\
\hline 2.7675 & 1.09769 & 0.113616 & 1.11357 & 0.191366 & 0.823293 & 0.178201 \\
\hline 2.7775 & 1.06695 & 0.119054 & 1.11748 & 0.215747 & 0.814213 & 0.190347 \\
\hline 2.7875 & 1.06069 & 0.127593 & 1.1442 & 0.247077 & 0.820311 & 0.212519 \\
\hline 2.7975 & 1.07378 & 0.143817 & 1.18643 & 0.27598 & 0.846323 & 0.238834 \\
\hline 2.8075 & 1.10802 & 0.162411 & 1.23868 & 0.311152 & 0.881695 & 0.25982 \\
\hline 2.8175 & 1.15057 & 0.176149 & 1.29218 & 0.348508 & 0.913909 & 0.282463 \\
\hline 2.8275 & 1.19122 & 0.19056 & 1.33021 & 0.368773 & 0.936065 & 0.300857 \\
\hline 2.8375 & 1.22058 & 0.201695 & 1.33918 & 0.380446 & 0.946907 & 0.308495 \\
\hline 2.8475 & 1.22672 & 0.204501 & 1.32785 & 0.381578 & 0.942491 & 0.304296 \\
\hline 2.8575 & 1.20559 & 0.19867 & 1.29081 & 0.366647 & 0.916809 & 0.293181 \\
\hline
\end{tabular}




\begin{tabular}{|c|c|c|c|c|c|c|}
\hline 2.8675 & 1.16458 & 0.187243 & 1.23219 & 0.34816 & 0.875144 & 0.273212 \\
\hline 2.8775 & 1.10855 & 0.16868 & 1.16658 & 0.322815 & 0.825625 & 0.243518 \\
\hline .8875 & 1.03805 & 0.146672 & 1.09353 & 0.295249 & 0.772053 & 0.214848 \\
\hline 2.8975 & 0.964663 & 0.125049 & 1.01602 & 0.261553 & 0.718559 & 0.190058 \\
\hline 2.9075 & 0.894509 & 0.099833 & 0.941738 & 0.22334 & 0.670124 & 0.162361 \\
\hline 2.9175 & 0.826791 & 0.0850017 & 0.877519 & 0.194534 & 0.626559 & 0.137735 \\
\hline 2.9275 & 0.770343 & 0.0727716 & 0.830925 & 0.172804 & 0.590715 & 0.121957 \\
\hline 2.9375 & 0.7283 & 0.0595277 & 0.790804 & 0.15666 & 0.563305 & 0.110783 \\
\hline 2.9475 & 0.697384 & 0.0569674 & 0.755711 & 0.145259 & 0.545517 & 0.101686 \\
\hline 2.9575 & 0.674348 & 0.0568496 & 0.73106 & 0.136818 & 0.532149 & 0.0966274 \\
\hline 2.9675 & 0.65425 & 0.0520594 & 0.709985 & 0.131776 & 0.51937 & 0.0919664 \\
\hline 2.9775 & 0.634439 & 0.051717 & 0.692832 & 0.133265 & 0.505986 & 0.0886304 \\
\hline 2.9875 & 0.61959 & 0.0521274 & 0.676935 & 0.133887 & 0.494153 & 0.0866346 \\
\hline 2.9975 & 0.606116 & 0.0491009 & 0.659404 & 0.134178 & 0.481409 & 0.0853978 \\
\hline 3.0075 & 0.591902 & 0.0491779 & 0.642869 & 0.13105 & 0.466335 & 0.083116 \\
\hline 3.0175 & 0.571093 & 0.0506016 & 0.621973 & 0.126754 & 0.449168 & 0.0794335 \\
\hline 3.0275 & 0.552371 & 0.0501477 & 0.602487 & 0.124443 & 0.431976 & 0.0782898 \\
\hline 3.0375 & 0.531929 & 0.0478189 & 0.583581 & 0.11968 & 0.414901 & 0.0743434 \\
\hline 3.0475 & 0.510352 & 0.0428152 & 0.567413 & 0.112864 & 0.398422 & 0.0722996 \\
\hline 3.0575 & 0.492859 & 0.0443658 & 0.555119 & 0.104379 & 0.387186 & 0.0700777 \\
\hline 3.0675 & 0.478493 & 0.042928 & 0.543743 & 0.0943363 & 0.37806 & 0.069264 \\
\hline 3.0775 & 0.466132 & 0.042 & 0.53 & 0.0891383 & 0.36901 & 0.0693777 \\
\hline 3.0875 & 0.45477 & 0.0422887 & 0.533565 & 0.0846873 & 0.36379 & 0.0688656 \\
\hline 3.0975 & 0.447686 & 0.0410831 & 0.532011 & 0.0828175 & 0.361214 & 0.0690131 \\
\hline 3.1075 & 0.440177 & 0.0376569 & 0.538139 & 0.0804119 & 0.361225 & 0.067579 \\
\hline 3.1175 & 0.440293 & 0.0375774 & 0.544186 & 0.0786812 & 0.362172 & 0.067455 \\
\hline 3.1275 & 0.445163 & 0.039015 & 0.551842 & 0.0774753 & 0.365002 & 0.066805 \\
\hline 3.1375 & 0.451523 & 0.0384694 & 0.56166 & 0.0768046 & 0.371612 & 0.0658574 \\
\hline 3.1475 & 0.458486 & 0.0384468 & 0.575998 & 0.0783215 & 0.380105 & 0.0688902 \\
\hline 3.1575 & 0.471615 & 0.0417643 & 0.596353 & 0.0786205 & 0.393277 & 0.0715675 \\
\hline 3.1675 & 0.488256 & 0.0426299 & 0.627497 & 0.0789071 & 0.413968 & 0.0770537 \\
\hline 3.1775 & 0.513552 & 0.0462002 & 0.673296 & 0.0851265 & 0.442435 & 0.0826182 \\
\hline 3.1875 & 0.555879 & 0.0499787 & 0.738763 & 0.0950013 & 0.482972 & 0.0911198 \\
\hline 3.1975 & 0.611099 & 0.0536602 & 0.840032 & 0.118099 & 0.543949 & 0.10251 \\
\hline 3.2075 & 0.697232 & 0.0644045 & 0.981688 & 0.15028 & 0.630613 & 0.125494 \\
\hline 3.2175 & 0.819062 & 0.0781567 & 1.1655 & 0.197577 & 0.749257 & 0.155041 \\
\hline 3.2275 & 0.979553 & 0.0984782 & 1.41036 & 0.263463 & 0.906317 & 0.194665 \\
\hline 3.2375 & 1.18847 & 0.124049 & 1.70746 & 0.34897 & 1.10498 & 0.247071 \\
\hline 3.2475 & 1.45295 & 0.158267 & 2.06091 & 0.447526 & 1.33346 & 0.30488 \\
\hline 3.2575 & 1.762 & 0.19258 & 2.45203 & 0.553195 & 1.5913 & 0.378428 \\
\hline 3.2675 & 2.10128 & 0.230676 & 2.85716 & 0.667895 & 1.8738 & 0.455891 \\
\hline 3.2775 & 2.4575 & 0.279364 & 3.23308 & 0.771084 & 2.14767 & 0.523754 \\
\hline 3.2875 & 2.80608 & 0.322188 & 3.55863 & 0.858845 & 2.38868 & 0.587059 \\
\hline 3.2975 & 3.09478 & 0.367187 & 3.79901 & 0.929303 & 2.56357 & 0.636289 \\
\hline 3.3075 & 3.32694 & 0.38895 & 3.93366 & 0.968882 & 2.6877 & 0.669202 \\
\hline 3.3175 & 3.45523 & 0.395435 & 3.94262 & 0.95778 & 2.72866 & 0.67875 \\
\hline 3.3275 & 3.47443 & 0.406471 & 3.82628 & 0.917537 & 2.67396 & 0.665162 \\
\hline
\end{tabular}




\begin{tabular}{|c|c|c|c|c|c|c|}
\hline 3.3375 & 3.38374 & 0.400126 & 3.59593 & 0.858198 & 2.5304 & 0.627515 \\
\hline 3.3475 & 3.19901 & 0.374066 & 3.27887 & 0.776982 & 2.32241 & 0.574135 \\
\hline 3.3575 & 2.91735 & 0.336796 & 2.91733 & 0.667209 & 2.07804 & 0.512703 \\
\hline 3.3675 & 2.58931 & 0.292484 & 2.53325 & 0.547598 & 1.80504 & 0.43782 \\
\hline 3.3775 & 2.24351 & 0.246884 & 2.14622 & 0.446413 & 1.53553 & 0.362615 \\
\hline 3.3875 & 1.90095 & 0.207215 & 1.80538 & 0.352675 & 1.28836 & 0.299215 \\
\hline 3.3975 & 1.58791 & 0.169331 & 1.50788 & 0.269041 & 1.07327 & 0.240692 \\
\hline 3.4075 & 1.31919 & 0.133921 & 1.27043 & 0.203509 & 0.897492 & 0.19071 \\
\hline 3.4175 & 1.09158 & 0.108083 & 1.09469 & 0.160506 & 0.762087 & 0.15508 \\
\hline 3.4275 & 0.926234 & 0.0856387 & 0.973492 & 0.134283 & 0.669846 & 0.132214 \\
\hline 3.4375 & 0.811915 & 0.0753456 & 0.905752 & 0.119404 & 0.611463 & 0.118307 \\
\hline 3.4475 & 0.739295 & 0.0654429 & 0.879492 & 0.118273 & 0.580903 & 0.109664 \\
\hline 3.4575 & 0.701097 & 0.0582997 & 0.884787 & 0.120759 & 0.577351 & 0.107823 \\
\hline 3.4675 & 0.693546 & 0.0593843 & 0.930985 & 0.130709 & 0.594654 & 0.109126 \\
\hline 3.4775 & 0.716661 & 0.0605865 & 1.00743 & 0.146323 & 0.630992 & 0.116108 \\
\hline 3.4875 & 0.765213 & 0.0607901 & 1.12491 & 0.168051 & 0.693362 & 0.129438 \\
\hline 3.4975 & 0.840386 & 0.0690205 & 1.30801 & 0.203678 & 0.79134 & 0.147872 \\
\hline 3.5075 & 0.957788 & 0.08108 & 1.56485 & 0.251934 & 0.931845 & 0.17388 \\
\hline 3.5175 & 1.13264 & 0.095137 & 1.95147 & 0.321577 & 1.14364 & 0.210844 \\
\hline 3.5275 & 1.38993 & 0.115001 & 2.53912 & 0.426576 & 1.45951 & 0.265772 \\
\hline 3.5375 & 1.77302 & 0.146116 & 3.43374 & 0.601238 & 1.93939 & 0.357453 \\
\hline 3.5475 & 2.34879 & 0.18969 & 4.78703 & 0.860446 & 2.66799 & 0.490005 \\
\hline 3.5575 & 3.21193 & 0.26259 & 6.78801 & 1.23359 & 3.74088 & 0.686801 \\
\hline 3.5675 & 4.49817 & 0.363966 & 9.70571 & 1.79842 & 5.30714 & 0.979051 \\
\hline 3.5775 & 6.35615 & 0.5126 & 13.8063 & 2.59095 & 7.55003 & 1.39692 \\
\hline 3.5875 & 8.99184 & 0.739128 & 19.3767 & 3.63072 & 10.628 & 1.97697 \\
\hline 3.5975 & 12.6265 & 1.04303 & 26.6399 & 5.01233 & 14.6999 & 2.74121 \\
\hline 3.6075 & 17.395 & 1.42249 & 35.7199 & 6.70922 & 19.8877 & 3.71621 \\
\hline 3.6175 & 23.4152 & 1.91076 & 46.6253 & 8.69214 & 26.1595 & 4.90693 \\
\hline 3.6275 & 30.7052 & 2.53321 & 59.0027 & 10.9659 & 33.4077 & 6.29151 \\
\hline 3.6375 & 39.0924 & 3.21951 & 72.222 & 13.3595 & 41.3588 & 7.79074 \\
\hline 3.6475 & 48.2373 & 3.95677 & 85.6032 & 15.7238 & 49.5448 & 9.34578 \\
\hline 3.6575 & 57.5091 & 4.73788 & 97.985 & 17.9168 & 57.3359 & 10.8549 \\
\hline 3.6675 & 66.3172 & 5.47406 & 108.263 & 19.6955 & 64.1309 & 12.1583 \\
\hline 3.6775 & 73.9257 & 6.1269 & 115.57 & 20.8973 & 69.2622 & 13.1514 \\
\hline 3.6875 & 79.5363 & 6.618 & 119.023 & 21.4456 & 72.1661 & 13.7633 \\
\hline 3.6975 & 82.6366 & 6.90152 & 118.273 & 21.2876 & 72.5697 & 13.865 \\
\hline 3.7075 & 82.8298 & 6.92312 & 113.431 & 20.4344 & 70.4508 & 13.4616 \\
\hline 3.7175 & 80.1448 & 6.71861 & 104.977 & 18.8976 & 65.9723 & 12.6298 \\
\hline 3.7275 & 74.8571 & 6.30547 & 93.7486 & 16.811 & 59.5976 & 11.4285 \\
\hline 3.7375 & 67.4238 & 5.69972 & 80.7938 & 14.4749 & 51.9749 & 9.9744 \\
\hline 3.7475 & 58.6408 & 4.9967 & 67.2168 & 12.0949 & 43.747 & 8.43462 \\
\hline 3.7575 & 49.243 & 4.18792 & 53.9906 & 9.76141 & 35.5585 & 6.87657 \\
\hline 3.7675 & 39.9055 & 3.39071 & 41.9366 & 7.58038 & 27.9249 & 5.40802 \\
\hline 3.7775 & 31.2469 & 2.68454 & 31.4989 & 5.70828 & 21.2007 & 4.11881 \\
\hline 3.7875 & 23.6671 & 2.03122 & 22.8778 & 4.16635 & 15.5751 & 3.02118 \\
\hline 3.7975 & 17.3672 & 1.49518 & 16.1336 & 2.93807 & 11.0971 & 2.149 \\
\hline
\end{tabular}




\begin{tabular}{|c|c|c|c|c|c|c|}
\hline 3.8075 & 12.3625 & 1.06301 & 11.0938 & 2.01863 & 7.68957 & 1.49223 \\
\hline 3.8175 & 8.57245 & 0.731156 & 7.48905 & 1.34871 & 5.21544 & 1.0171 \\
\hline 3.8275 & 5.83338 & 0.503616 & 5.02724 & 0.893499 & 3.50662 & 0.685265 \\
\hline 3.8375 & 3.92525 & 0.34144 & 3.43148 & 0.592905 & 2.3773 & 0.462483 \\
\hline 3.8475 & 2.67787 & 0.236483 & 2.48296 & 0.415323 & 1.68192 & 0.323947 \\
\hline 3.8575 & 1.9187 & 0.168314 & 1.99878 & 0.333642 & 1.29811 & 0.248711 \\
\hline 3.8675 & 1.49471 & 0.133618 & 1.83659 & 0.303162 & 1.13391 & 0.215511 \\
\hline 3.8775 & 1.32094 & 0.11695 & 1.92344 & 0.318437 & 1.13505 & 0.212891 \\
\hline 3.8875 & 1.33644 & 0.112185 & 2.23292 & 0.38118 & 1.27334 & 0.23733 \\
\hline 3.8975 & 1.50445 & 0.120491 & 2.75434 & 0.486891 & 1.53697 & 0.286741 \\
\hline 3.9075 & 1.81445 & 0.147557 & 3.49063 & 0.615987 & 1.92796 & 0.357429 \\
\hline 3.9175 & 2.2654 & 0.186464 & 4.46717 & 0.774235 & 2.4579 & 0.455961 \\
\hline 3.9275 & 2.8706 & 0.245612 & 5.68801 & 0.989238 & 3.13119 & 0.579952 \\
\hline 3.9375 & 3.65355 & 0.313663 & 7.1368 & 1.24367 & 3.93353 & 0.733415 \\
\hline 3.9475 & 4.59186 & 0.38011 & 8.77223 & 1.53729 & 4.85703 & 0.912825 \\
\hline 3.9575 & 5.66624 & 0.461131 & 10.5208 & 1.84214 & 5.88633 & 1.10002 \\
\hline 3.9675 & 6.85418 & 0.559408 & 12.3094 & 2.12766 & 6.95727 & 1.29172 \\
\hline 3.9775 & 8.08321 & 0.673384 & 14.0129 & 2.40513 & 7.9905 & 1.4854 \\
\hline 3.9875 & 9.26819 & 0.783721 & 15.4536 & 2.63038 & 8.90539 & 1.67437 \\
\hline 3.9975 & 10.3147 & 0.882382 & 16.5193 & 2.80157 & 9.63052 & 1.81375 \\
\hline 4.0075 & 11.1613 & 0.952932 & 17.1168 & 2.89987 & 10.0965 & 1.90111 \\
\hline 4.0175 & 11.709 & 0.991058 & 17.1772 & 2.89922 & 10.2517 & 1.93529 \\
\hline 4.0275 & 11.8671 & 0.998896 & 16.6792 & 2.82379 & 10.0784 & 1.90372 \\
\hline 4.0375 & 11.6562 & 0.990479 & 15.6431 & 2.64845 & 9.57925 & 1.8113 \\
\hline 4.0475 & 11.0918 & 0.939182 & 14.2055 & 2.38604 & 8.82125 & 1.67091 \\
\hline 4.0575 & 10.2182 & 0.8654 & 12.503 & 2.09801 & 7.86623 & 1.49758 \\
\hline 4.0675 & 9.11243 & 0.77247 & 10.6642 & 1.79893 & 6.7892 & 1.30103 \\
\hline 4.0775 & 7.85998 & 0.665576 & 8.79311 & 1.47944 & 5.67551 & 1.08909 \\
\hline 4.0875 & 6.58599 & 0.562885 & 7.01638 & 1.1742 & 4.59257 & 0.87772 \\
\hline 4.0975 & 5.34718 & 0.456237 & 5.44563 & 0.913638 & 3.60708 & 0.689429 \\
\hline 4.1075 & 4.20078 & 0.359611 & 4.0991 & 0.680769 & 2.75331 & 0.520503 \\
\hline 4.1175 & 3.21756 & 0.281135 & 3.00778 & 0.491422 & 2.04555 & 0.390408 \\
\hline 4.1275 & 2.39758 & 0.209325 & 2.15402 & 0.344639 & 1.48521 & 0.284595 \\
\hline 4.1375 & 1.75512 & 0.14927 & 1.51427 & 0.228902 & 1.06377 & 0.202567 \\
\hline 4.1475 & 1.26984 & 0.110111 & 1.05538 & 0.154483 & 0.755261 & 0.144892 \\
\hline 4.1575 & 0.909739 & 0.0788295 & 0.734104 & 0.105278 & 0.538846 & 0.102182 \\
\hline 4.1675 & 0.656914 & 0.0614459 & 0.522055 & 0.0707184 & 0.390122 & 0.0743163 \\
\hline 4.1775 & 0.484954 & 0.047822 & 0.382411 & 0.0500783 & 0.293421 & 0.0603005 \\
\hline 4.1875 & 0.37274 & 0.0375684 & 0.297387 & 0.0422604 & 0.229563 & 0.0487612 \\
\hline 4.1975 & 0.301687 & 0.0312128 & 0.245643 & 0.0403201 & 0.191573 & 0.0423882 \\
\hline 4.2075 & 0.256421 & 0.029052 & 0.216813 & 0.0377648 & 0.171006 & 0.0414836 \\
\hline 4.2175 & 0.230051 & 0.0266892 & 0.203319 & 0.036367 & 0.158723 & 0.0403513 \\
\hline 4.2275 & 0.217035 & 0.0254582 & 0.194656 & 0.0367866 & 0.152014 & 0.0373176 \\
\hline 4.2375 & 0.209666 & 0.0280772 & 0.188088 & 0.0368141 & 0.148134 & 0.0371587 \\
\hline 4.2475 & 0.205626 & 0.0288596 & 0.186665 & 0.0368223 & 0.147471 & 0.0378385 \\
\hline 4.2575 & 0.203807 & 0.0277602 & 0.188931 & 0.0385954 & 0.147244 & 0.0377353 \\
\hline 4.2675 & 0.204941 & 0.0279511 & 0.189486 & 0.0381789 & 0.147047 & 0.0373109 \\
\hline
\end{tabular}




\begin{tabular}{|c|c|c|c|c|c|c|}
\hline 4.2775 & 0.207152 & 0.0267879 & 0.190203 & 0.0375882 & 0.148517 & 0.0373036 \\
\hline 4.2875 & 0.208546 & 0.0269344 & 0.193682 & 0.0388675 & 0.149738 & 0.0376048 \\
\hline 2975 & 0.207545 & 0.0276951 & 0.196586 & 0.040146 & 0.150287 & 0.0358895 \\
\hline 4.3075 & 0.209622 & 0.0260113 & 0.199252 & 0.0395684 & 0.152615 & 0.0368418 \\
\hline 4.3175 & 0.213989 & 0.0269615 & 0.204922 & 0.0412158 & 0.156498 & 0.0363346 \\
\hline 4.3275 & 0.217247 & 0.0284831 & 0.212458 & 0.047329 & 0.160339 & 0.0365952 \\
\hline 4.3375 & 0.223329 & 0.0318301 & 0.225992 & 0.0538011 & 0.166089 & 0.0389123 \\
\hline 4.3475 & 0.230656 & 0.0311411 & 0.245448 & 0.0643821 & 0.174879 & 0.0386132 \\
\hline 4.3575 & 0.240555 & 0.0307228 & 0.271597 & 0.0808132 & 0.186844 & 0.0395325 \\
\hline 4.3675 & 0.25649 & 0.0359277 & 0.307992 & 0.105252 & 0.204459 & 0.042972 \\
\hline 4.3775 & 0.280069 & 0.0395492 & 0.35612 & 0.146097 & 0.227339 & 0.0469538 \\
\hline 4.3875 & 0.310655 & 0.0435974 & 0.423975 & 0.199883 & 0.2558 & 0.0535769 \\
\hline 4.3975 & 0.351992 & 0.0494516 & 0.510125 & 0.271896 & 0.298893 & 0.0625052 \\
\hline 4.4075 & 0.405587 & 0.0645529 & 0.619478 & 0.369223 & 0.350958 & 0.0743812 \\
\hline 4.4175 & 0.477227 & 0.0855907 & 0.753071 & 0.489652 & 0.414055 & 0.0891591 \\
\hline 4.4275 & 0.561549 & 0.108653 & 0.905953 & 0.638358 & 0.488635 & 0.10644 \\
\hline 4.4375 & 0.658846 & 0.137971 & 1.08172 & 0.815875 & 0.575395 & 0.128642 \\
\hline 4.4475 & 0.773466 & 0.174823 & 1.26187 & 0.99421 & 0.666447 & 0.149331 \\
\hline 4.4575 & 0.900772 & 0.208619 & 1.44791 & 1.18172 & 0.759815 & 0.175078 \\
\hline 4.4675 & 1.03253 & 0.248262 & 1.62812 & 1.37669 & 0.848242 & 0.200331 \\
\hline 4.4775 & 1.16297 & 0.2925 & 1.77757 & 1.53673 & 0.931283 & 0.219543 \\
\hline 4.4875 & 1.28 & 0.336847 & 1.89332 & 268 & 1.00157 & 0.238997 \\
\hline 4.4975 & 1.37962 & 0.367636 & 1.96925 & 1.74779 & 1.04714 & 0.25012 \\
\hline 4.5075 & 1.45197 & 0.403289 & 1.98585 & 1.79241 & 1.07362 & 0.257149 \\
\hline 4.5175 & 1.49625 & 0.417616 & 1.94754 & 1.76571 & 1.06821 & 0.259788 \\
\hline 4.5275 & 1.49875 & 0.417551 & 1.87319 & 1.70615 & 1.03452 & 0.251889 \\
\hline 4.5375 & 1.46529 & 0.410278 & 1.75213 & 1.59209 & 0.977252 & 0.238367 \\
\hline 4.5475 & 1.39872 & 0.393608 & 1.58752 & 1.42135 & 0.904257 & 0.217863 \\
\hline 4.5575 & 1.29389 & 0.361897 & 1.40866 & 1.24952 & 0.820694 & 0.196192 \\
\hline 4.5675 & 1.17339 & 0.321352 & 1.22454 & 1.05231 & 0.718275 & 0.167914 \\
\hline 4.5775 & 1.05618 & 0.277051 & 1.04286 & 0.860725 & 0.628824 & 0.144342 \\
\hline 4.5875 & 0.93204 & 0.237084 & 0.872645 & 0.688639 & 0.540824 & 0.123769 \\
\hline 4.5975 & 0.811023 & 0.201299 & 0.720833 & 0.530047 & 0.459195 & 0.0996021 \\
\hline 4.6075 & 0.692522 & 0.16721 & 0.599045 & 0.393443 & 0.395528 & 0.0852319 \\
\hline 4.6175 & 0.597207 & 0.134287 & 0.49932 & 0.282799 & 0.342945 & 0.073949 \\
\hline 4.6275 & 0.522003 & 0.11378 & 0.425011 & 0.203666 & 0.296824 & 0.06651 \\
\hline 4.6375 & 0.454969 & 0.100244 & 0.369095 & 0.138799 & 0.263538 & 0.059814 \\
\hline 4.6475 & 0.405104 & 0.0842092 & 0.328926 & 0.10255 & 0.244211 & 0.054592 \\
\hline 4.6575 & 0.367421 & 0.0760745 & 0.305298 & 0.0749836 & 0.227094 & 0.0505717 \\
\hline 4.6675 & 0.343019 & 0.0687293 & 0.291291 & 0.0589376 & 0.216919 & 0.0461967 \\
\hline 4.6775 & 0.323083 & 0.0630952 & 0.278175 & 0.0528695 & 0.209799 & 0.0499794 \\
\hline 4.6875 & 0.307756 & 0.063604 & 0.271461 & 0.0524519 & 0.204351 & 0.0486995 \\
\hline 4.6975 & 0.294662 & 0.0548461 & 0.270441 & 0.0515647 & 0.204733 & 0.0478756 \\
\hline 4.7075 & 0.288291 & 0.0492627 & 0.27133 & 0.051464 & 0.206795 & 0.0499194 \\
\hline 4.7175 & 0.285285 & 0.0462183 & 0.278716 & 0.0548145 & 0.206375 & 0.0471623 \\
\hline 4.7275 & 0.282013 & 0.040604 & 0.285992 & 0.0598668 & 0.208289 & 0.046733 \\
\hline 4.7375 & 0.286282 & 0.0387714 & 0.290707 & 0.0643521 & 0.210252 & 0.0471376 \\
\hline
\end{tabular}




\begin{tabular}{|c|c|c|c|c|c|c|}
\hline 4.7475 & 0.288612 & 0.0354217 & 0.301941 & 0.0718993 & 0.216092 & 0.0488428 \\
\hline 4.7575 & .294378 & 0.0369188 & 0.31492 & 0.0860212 & 0.225551 & 0.0515005 \\
\hline .7675 & 0.302484 & 0.0394258 & 0.33178 & 0.0948332 & 0.232467 & 0.0512134 \\
\hline 4.7775 & .315232 & 0.0366163 & 0.348961 & 0.111931 & 0.241345 & 0.0505835 \\
\hline 4.7875 & 0.327121 & 0.0354927 & 0.365078 & 0.125699 & 0.252853 & 0.0542183 \\
\hline 4.7975 & 0.346476 & 0.0382647 & 0.386647 & 0.143272 & 0.26655 & 0.057997 \\
\hline 4.8075 & 0.361189 & 0.0391038 & 0.409159 & 0.153854 & 0.277262 & 0.0609021 \\
\hline 4.8175 & 0.376864 & 0.0435177 & 0.429879 & 0.163965 & 0.290406 & 0.0626005 \\
\hline 4.8275 & 0.398561 & 0.0463045 & 0.453725 & 0.173666 & 0.302151 & 0.0615546 \\
\hline 4.8375 & 0.417992 & 0.0490057 & 0.480343 & 0.183669 & 0.315527 & 0.0674221 \\
\hline 4.8475 & 0.437693 & 0.0525427 & 0.501322 & 0.190232 & 0.332032 & 0.0708647 \\
\hline 4.8575 & 0.456224 & 0.058755 & 0.524186 & 0.196798 & 0.346273 & 0.0741072 \\
\hline 4.8675 & 0.475672 & 0.0673244 & 0.548085 & 0.206069 & 0.36405 & 0.0805927 \\
\hline 4.8775 & 0.503663 & 0.075451 & 0.571014 & 0.213972 & 0.375362 & 0.0842698 \\
\hline 4.8875 & 0.531017 & 0.0826098 & 0.595995 & 0.232639 & 0.389307 & 0.0881151 \\
\hline 4.8975 & 0.55374 & 0.0874958 & 0.614852 & 0.244739 & 0.405269 & 0.0925711 \\
\hline 4.9075 & 0.576932 & 0.0998398 & 0.630287 & 0.265252 & 0.420311 & 0.101735 \\
\hline 4.9175 & 0.599075 & 0.108855 & 0.639379 & 0.276473 & 0.430775 & 0.108294 \\
\hline 4.9275 & 0.619722 & 0.112707 & 0.643418 & 0.280264 & 0.437842 & 0.115254 \\
\hline 4.9375 & 0.632077 & 0.117291 & 0.637186 & 0.27766 & 0.436691 & 0.120389 \\
\hline 4.9475 & 0.641651 & 0.12214 & 0.626187 & 0.267684 & 0.438136 & 0.127043 \\
\hline 4.9575 & 0.644961 & 0.123457 & 0.610383 & 0.260397 & 0.436306 & 0.137049 \\
\hline 4.9675 & 0.635246 & 0.118428 & 0.586408 & 0.234888 & 0.424408 & 0.136584 \\
\hline 4.9775 & 0.619025 & 0.114496 & 0.55459 & 0.203512 & 0.409529 & 0.144561 \\
\hline 4.9875 & 0.601476 & 0.104695 & 0.527117 & 0.186109 & 0.394073 & 0.149919 \\
\hline 4.9975 & 0.569934 & 0.103861 & 0.488045 & 0.165507 & 0.376124 & 0.1554 \\
\hline 5.0075 & 0.541258 & 0.0878967 & 0.450796 & 0.135919 & 0.361231 & 0.157829 \\
\hline 5.0175 & 0.512275 & 0.081074 & 0.421473 & 0.115614 & 0.345724 & 0.164405 \\
\hline 5.0275 & 0.486338 & 0.0766446 & 0.39161 & 0.0977266 & 0.328022 & 0.16453 \\
\hline 5.0375 & 0.458783 & 0.0654236 & 0.368307 & 0.0843119 & 0.312229 & 0.163279 \\
\hline 5.0475 & 0.428533 & 0.0515106 & 0.348561 & 0.0701046 & 0.30306 & 0.160443 \\
\hline 5.0575 & 0.414131 & 0.0477457 & 0.33378 & 0.0679058 & 0.294689 & 0.16472 \\
\hline 5.0675 & 0.396312 & 0.048081 & 0.32635 & 0.0654374 & 0.285321 & 0.161613 \\
\hline 5.0775 & 0.381499 & 0.0455656 & 0.319463 & 0.0601415 & 0.277843 & 0.152691 \\
\hline 5.0875 & 0.368707 & 0.042571 & 0.31785 & 0.0637808 & 0.274891 & 0.144698 \\
\hline 5.0975 & 0.36254 & 0.0404706 & 0.317128 & 0.063841 & 0.276773 & 0.148931 \\
\hline 5.1075 & 0.362498 & 0.0421704 & 0.318043 & 0.0627633 & 0.274107 & 0.141341 \\
\hline 5.1175 & 0.356866 & 0.0415498 & 0.318955 & 0.0685441 & 0.272024 & 0.129637 \\
\hline 5.1275 & 0.35841 & 0.0415084 & 0.325226 & 0.0692876 & 0.273035 & 0.125257 \\
\hline 5.1375 & 0.360309 & 0.0435132 & 0.3283 & 0.0716988 & 0.273084 & 0.117453 \\
\hline 5.1475 & 0.361127 & 0.0424765 & 0.329374 & 0.0743558 & 0.274762 & 0.117484 \\
\hline 5.1575 & 0.363888 & 0.0414109 & 0.332671 & 0.0709414 & 0.272451 & 0.109214 \\
\hline 5.1675 & 0.366167 & 0.0432015 & 0.335824 & 0.0739725 & 0.271612 & 0.105411 \\
\hline 5.1775 & 0.365027 & 0.0437191 & 0.334968 & 0.074559 & 0.27422 & 0.0995638 \\
\hline 5.1875 & 0.366468 & 0.0430763 & 0.336102 & 0.0721661 & 0.27361 & 0.0975461 \\
\hline 5.1975 & 0.367238 & 0.04138 & 0.335229 & 0.0693031 & 0.273813 & 0.0918808 \\
\hline 5.2075 & 0.367037 & 0.0399159 & 0.334488 & 0.0714257 & 0.270167 & 0.0855005 \\
\hline
\end{tabular}




\begin{tabular}{|c|c|c|c|c|c|c|}
\hline 5.2175 & 0.370231 & 0.0436594 & 0.334574 & 0.0695267 & 0.265353 & 0.0794399 \\
\hline 5.2275 & 0.367847 & 0.0410166 & 0.326071 & 0.062974 & 0.265255 & 0.0786636 \\
\hline 2375 & .372872 & 0.0423036 & 0.324456 & 0.0614279 & 0.266 & 0.0777768 \\
\hline 2475 & 0.374688 & 0.0428487 & 0.325741 & 0.0616854 & 0.265634 & 0.0717454 \\
\hline 2575 & 0.379244 & 0.0419763 & 0.327622 & 0.0624611 & 0.264558 & 0.0677667 \\
\hline 2675 & 0.386557 & 0.0454354 & 0.327029 & 0.0613394 & 0.266193 & 0.070638 \\
\hline .2775 & 0.39651 & 0.0497864 & 0.331309 & 0.0603511 & 0.273448 & 0.0714066 \\
\hline 5.2875 & 0.411572 & 0.0492007 & 0.333912 & 0.0638758 & 0.281259 & 0.069883 \\
\hline 5.2975 & 0.433517 & 0.0561009 & 0.340011 & 0.0682246 & 0.292837 & 0.0770825 \\
\hline 5.3075 & 0.463766 & 0.0659486 & 0.347638 & 0.0733835 & 0.30723 & 0.082831 \\
\hline 5.3175 & 0.502901 & 0.071084 & 0.358796 & 0.0757315 & 0.325935 & 0.0940626 \\
\hline 5.3275 & 0.550133 & 0.0834154 & 0.37 & 0.0804714 & 0.34845 & 0.0999695 \\
\hline 5.3375 & 0.611084 & 0.103028 & 0.387446 & 0.0916461 & 0.371967 & 0.115346 \\
\hline 5.3475 & 0.684709 & 0.128517 & 0.405067 & 0.102438 & 0.408593 & 0.140471 \\
\hline 5.3575 & 0.764169 & 0.151618 & 0.423775 & 0.114432 & 0.440213 & 0.163064 \\
\hline 5.3675 & 0.852732 & 0.184958 & 0.445448 & 0.122513 & 0.475125 & 4108 \\
\hline 5.3775 & 0.940999 & 0.21209 & 0.464292 & 0.134236 & 0.505139 & 0.204581 \\
\hline 5.3875 & 1.03182 & 0.236353 & 0.473752 & 0.140332 & 0.536735 & 1226 \\
\hline 5.3975 & 1.11702 & 0.26773 & 0.488336 & 0.147672 & 0.557961 & 0.243899 \\
\hline 5.4075 & 1.18195 & 0.291801 & 0.505691 & 0.153175 & 0.578804 & 0.247375 \\
\hline 5.4175 & 1.23175 & 0.30524 & 0.50667 & 0.153969 & 0.590176 & 0.264409 \\
\hline 5.4275 & 1.25924 & 0.312062 & 0.506654 & 0.155738 & 0.589099 & 0.255928 \\
\hline 5.4375 & 1.26874 & 0.310606 & 0.507581 & 0.145979 & 0.577755 & 0.245431 \\
\hline 5.4475 & & 0.300658 & & & & 5051 \\
\hline 5.4575 & 1.20755 & 0.281026 & 0.488294 & 0.133742 & 0.547189 & 0.214419 \\
\hline 5.4675 & 1.14366 & 0.257584 & 0.480946 & 0.125742 & 0.51717 & 0.193369 \\
\hline & & & & & 0.490966 & 7047 \\
\hline 5.4875 & 0.985049 & 0.209456 & 0.453975 & 0.112408 & 0.461521 & 0.153323 \\
\hline 5.4975 & 0.895394 & 0.179039 & 0.443094 & 0.104465 & 0.435395 & 0.134644 \\
\hline 5.5075 & 0.814668 & 0.152975 & 0.434908 & 0.100495 & 0.414907 & 0.129373 \\
\hline 5.5175 & 0.74611 & 0.124751 & 0.423839 & 0.0963463 & 0.390773 & 0.111588 \\
\hline 5.5275 & 0.669337 & 0.107502 & 0.420409 & 0.0916729 & 0.372346 & 0.0989682 \\
\hline 5.5375 & 0.613754 & 0.0908819 & 0.415873 & 0.0916714 & 0.357903 & 0.0955207 \\
\hline 5.5475 & 0.568623 & 0.0772382 & 0.407792 & 0.0905892 & 0.347917 & 0.0889824 \\
\hline 5.5575 & 0.527648 & 0.0695395 & 0.409768 & 0.0902326 & 0.342723 & 0.0919873 \\
\hline 5.5675 & 0.504381 & 0.0620832 & 0.406258 & 0.0905917 & 0.337352 & 0.08869 \\
\hline 5.5775 & 0.482898 & 0.0629064 & 0.400268 & 0.0916897 & 0.325728 & 0.0904083 \\
\hline 5.5875 & 0.464337 & 0.0590538 & 0.398857 & 0.089925 & 0.32735 & 0.0871069 \\
\hline 5.5975 & 0.457149 & 0.0542647 & 0.39674 & 0.085628 & 0.327058 & 0.0902516 \\
\hline 5.6075 & 0.452747 & 0.0563697 & 0.395223 & 0.0871706 & 0.325391 & 0.0887988 \\
\hline 5.6175 & 0.45439 & 0.0555631 & 0.398868 & 0.0887857 & 0.327515 & 0.087393 \\
\hline 5.6275 & 0.449323 & 0.0572165 & 0.398595 & 0.0865011 & 0.326082 & 0.091318 \\
\hline 5.6375 & 0.446283 & 0.05515 & 0.394059 & 0.0877652 & 0.325433 & 0.0904512 \\
\hline 5.6475 & 0.447178 & 0.0585498 & 0.395002 & 0.0793557 & 0.32887 & 0.0934386 \\
\hline 5.6575 & 0.449732 & 0.0560776 & 0.397734 & 0.0853936 & 0.331175 & 0.0936959 \\
\hline 5.6675 & 0.452263 & 0.0588292 & 0.401395 & 0.0861664 & 0.335995 & 0.0956677 \\
\hline 5.6775 & 0.457291 & 0.0538073 & 0.402151 & 0.0837958 & 0.339024 & 0.096608 \\
\hline
\end{tabular}




\begin{tabular}{|c|c|c|c|c|c|c|}
\hline 5.6875 & 0.465435 & 0.0573193 & 0.41065 & 0.0878067 & 0.347357 & 0.0989492 \\
\hline 5.6975 & 0.470954 & 0.0572358 & 0.413921 & 0.0840695 & 0.351634 & 0.107029 \\
\hline .7075 & 0.471803 & 0.0603649 & 0.413861 & 0.0807098 & 0.356282 & 0.111557 \\
\hline .7175 & 0.477202 & 0.0545416 & 0.428347 & 0.0854135 & 0.368725 & 0.116435 \\
\hline 5.7275 & 0.484286 & 0.0627126 & 0.440493 & 0.0910912 & 0.374425 & 0.119134 \\
\hline 5.7375 & 0.499673 & 0.0637306 & 0.461766 & 0.0980528 & 0.386045 & 0.129119 \\
\hline 5.7475 & 0.512426 & 0.063837 & 0.486264 & 0.107266 & 0.400452 & 0.127931 \\
\hline 5.7575 & 0.530077 & 0.0627409 & 0.519079 & 0.119211 & 0.421683 & 0.147399 \\
\hline 5.7675 & 0.553157 & 0.0664665 & 0.552589 & 0.143928 & 0.449444 & 0.151239 \\
\hline 5.7775 & 0.582508 & 0.0757418 & 0.598637 & 0.164069 & 0.4732 & 0.153066 \\
\hline 5.7875 & 0.621797 & 0.0761416 & 0.665005 & 0.203053 & 0.510469 & 0.167328 \\
\hline 5.7975 & 0.663122 & 0.0891975 & 0.741956 & 0.24546 & 0.553066 & 0.175148 \\
\hline 5.8075 & 0.71885 & 0.0977917 & 0.828151 & 0.308441 & 0.606825 & 0.186208 \\
\hline 5.8175 & 0.790263 & 0.105313 & 0.928055 & 0.375973 & 0.66601 & 0.19893 \\
\hline 5.8275 & 0.865945 & 0.119508 & 1.03902 & 0.434928 & 0.733549 & 0.217834 \\
\hline 5.8375 & 0.955027 & 0.13363 & 1.15559 & 0.496086 & 0.803113 & 0.234097 \\
\hline 5.8475 & 1.05942 & 0.153306 & 1.27054 & 0.592571 & 0.876736 & 0.251632 \\
\hline 5.8575 & 1.1663 & 0.189115 & 1.38049 & 0.665402 & 0.952125 & 0.267454 \\
\hline 5.8675 & 1.27711 & 0.210039 & 1.47897 & 0.730315 & 1.02136 & 0.283144 \\
\hline 5.8775 & 1.37303 & 0.224778 & 1.54692 & 0.766332 & 1.07531 & 0.289307 \\
\hline 5.8875 & 1.46412 & 0.244899 & 1.59865 & 0.798911 & 1.1176 & 0.30861 \\
\hline 5.8975 & 1.54323 & 0.261597 & 1.61661 & 0.795417 & 1.14218 & 0.310818 \\
\hline 5.9075 & 1.60129 & 0.278538 & 1.61702 & 0.791602 & 1.15182 & 0.313675 \\
\hline 5.9175 & 1.62062 & 0.28234 & 1.58155 & 0.773239 & 1.14614 & 0.308114 \\
\hline 5.9275 & 1.61985 & 0.27165 & 1.52531 & 0.725615 & 1.11065 & 0.292074 \\
\hline 5.9375 & 1.6065 & 0.27273 & 1.43738 & 0.661226 & 1.06563 & 0.285826 \\
\hline 5.9475 & 1.55277 & 0.258012 & 1.34015 & 0.586699 & 1.0075 & 0.264702 \\
\hline 5.9575 & 1.4824 & 0.239441 & 1.23463 & 0.508033 & 0.942692 & 0.252426 \\
\hline 5.9675 & 1.40285 & 0.218256 & 1.13276 & 0.445122 & 0.879774 & 0.235876 \\
\hline 5.9775 & 1.31516 & 0.200258 & 1.03225 & 0.384175 & 0.816369 & 0.21176 \\
\hline 5.9875 & 1.22741 & 0.175806 & 0.939289 & 0.308204 & 0.753689 & 0.198031 \\
\hline 5.9975 & 1.12894 & 0.156948 & 0.866793 & 0.260493 & 0.693049 & 0.179901 \\
\hline 6.0075 & 1.04479 & 0.142078 & 0.802007 & 0.215119 & 0.64025 & 0.166698 \\
\hline 6.0175 & 0.965677 & 0.128257 & 0.740633 & 0.17702 & 0.603801 & 0.15141 \\
\hline 6.0275 & 0.908391 & 0.120272 & 0.711285 & 0.164683 & 0.572313 & 0.147874 \\
\hline 6.0375 & 0.84843 & 0.102039 & 0.677437 & 0.14052 & 0.548173 & 0.142397 \\
\hline 6.0475 & 0.807591 & 0.0993857 & 0.663028 & 0.134197 & 0.532948 & 0.141096 \\
\hline 6.0575 & 0.777791 & 0.101019 & 0.648522 & 0.132453 & 0.520572 & 0.142134 \\
\hline 6.0675 & 0.758935 & 0.0963871 & 0.644783 & 0.126909 & 0.514172 & 0.142307 \\
\hline 6.0775 & 0.742765 & 0.100925 & 0.652988 & 0.13164 & 0.51376 & 0.135636 \\
\hline 6.0875 & 0.743353 & 0.0987349 & 0.656271 & 0.134075 & 0.514794 & 0.137442 \\
\hline 6.0975 & 0.743634 & 0.103081 & 0.658801 & 0.141005 & 0.520203 & 0.141834 \\
\hline 6.1075 & 0.745177 & 0.102723 & 0.672628 & 0.146635 & 0.528233 & 0.144793 \\
\hline 6.1175 & 0.757203 & 0.109475 & 0.689469 & 0.155662 & 0.540345 & 0.147225 \\
\hline 6.1275 & 0.775729 & 0.110811 & 0.712841 & 0.157996 & 0.558314 & 0.156168 \\
\hline 6.1375 & 0.795291 & 0.120052 & 0.733821 & 0.174114 & 0.577872 & 0.166838 \\
\hline 6.1475 & 0.817582 & 0.124131 & 0.761682 & 0.185743 & 0.601795 & 0.174985 \\
\hline
\end{tabular}




\begin{tabular}{|c|c|c|c|c|c|c|}
\hline 6.1575 & 0.857066 & 0.127179 & 0.811148 & 0.196866 & 0.631296 & 0.192501 \\
\hline 6.1675 & 0.895776 & 0.141543 & 0.867842 & 0.218332 & 0.675984 & 0.207417 \\
\hline 6.1775 & 0.9463 & 0.149992 & 0.953434 & 0.248117 & 0.730577 & 0.227772 \\
\hline 6.1875 & 1.02547 & 0.169785 & 1.07767 & 0.294334 & 0.809261 & 0.264395 \\
\hline 6.1975 & 1.13393 & 0.187612 & 1.26274 & 0.351806 & 0.936462 & 0.306425 \\
\hline 6.2075 & 1.29559 & 0.22551 & 1.54035 & 0.454407 & 1.13203 & 0.384332 \\
\hline 6.2175 & 1.53095 & 0.272525 & 1.95532 & 0.601242 & 1.40113 & 0.484124 \\
\hline 6.2275 & 1.88029 & 0.350656 & 2.56506 & 0.816339 & 1.8162 & 0.641783 \\
\hline 6.2375 & 2.40258 & 0.446152 & 3.42279 & 1.12003 & 2.41336 & 0.863121 \\
\hline 6.2475 & 3.17771 & 0.612091 & 4.65672 & 1.55082 & 3.26684 & 1.1901 \\
\hline 6.2575 & 4.27328 & 0.831021 & 6.32035 & 2.14535 & 4.44875 & 1.65399 \\
\hline 6.2675 & 5.82907 & 1.1525 & 8.59299 & 2.93168 & 6.06123 & 2.27552 \\
\hline 6.2775 & 7.97098 & 1.57876 & 11.5369 & 3.95952 & 8.17516 & 3.08901 \\
\hline 6.2875 & 10.8485 & 2.18639 & 15.1844 & 5.22758 & 10.9213 & 4.12884 \\
\hline 6.2975 & 14.5785 & 2.9574 & 19.6939 & 6.7876 & 14.3196 & 5.45573 \\
\hline 6.3075 & 19.3114 & 3.94243 & 25.0397 & 8.62213 & 18.4193 & 7.05271 \\
\hline 6.3175 & 25.1193 & 5.09918 & 31.1001 & 10.7131 & 23.1751 & 8.95235 \\
\hline 6.3275 & 32.0449 & 6.55022 & 37.8063 & 13.0418 & 28.5552 & 11.029 \\
\hline 6.3375 & 39.9764 & 8.22174 & 44.8269 & 15.4313 & 34.3627 & 13.3243 \\
\hline 6.3475 & 48.6996 & 9.98278 & 51.9071 & 17.9054 & 40.3773 & 15.7352 \\
\hline 6.3575 & 57.8951 & 11.872 & 58.63 & 20.1687 & 46.2833 & 18.0931 \\
\hline 6.3675 & 67.2599 & 13.8326 & 64.5834 & 22.3151 & 51.7469 & 20.2647 \\
\hline 6.3775 & 76.2458 & 15.7229 & 69.4996 & 24.013 & 56.4269 & 22.1482 \\
\hline 6.3875 & 84.2553 & 17.3966 & 72.8352 & 25.2316 & 60.0271 & 23.6009 \\
\hline 6.3975 & 90.9239 & 18.7401 & 74.4404 & 25.7654 & 62.2705 & 24.624 \\
\hline 6.4075 & 95.5409 & 19.7439 & 74.2162 & 25.7454 & 62.9776 & 24.9957 \\
\hline 6.4175 & 97.9757 & 20.2335 & 72.1465 & 25.0923 & 62.1533 & 24.6926 \\
\hline 6.4275 & 97.9601 & 20.2822 & 68.3705 & 23.8311 & 59.7428 & 23.8355 \\
\hline 6.4375 & 95.5627 & 19.7916 & 63.1607 & 22.1136 & 56.0337 & 22.409 \\
\hline 6.4475 & 90.7711 & 18.7883 & 56.9756 & 19.9207 & 51.2655 & 20.5217 \\
\hline 6.4575 & 84.218 & 17.4608 & 50.0977 & 17.5354 & 45.6862 & 18.3042 \\
\hline 6.4675 & 76.1104 & 15.8146 & 42.9536 & 15.0235 & 39.7873 & 16.0146 \\
\hline 6.4775 & 67.1414 & 13.9706 & 35.9233 & 12.6883 & 33.7557 & 13.6103 \\
\hline 6.4875 & 57.7032 & 12.0405 & 29.3798 & 10.3516 & 27.966 & 11.2518 \\
\hline 6.4975 & 48.4559 & 10.1053 & 23.4187 & 8.29232 & 22.5837 & 9.1066 \\
\hline 6.5075 & 39.6955 & 8.21901 & 18.3364 & 6.49177 & 17.8517 & 7.19251 \\
\hline 6.5175 & 31.7542 & 6.59948 & 14.011 & 4.96498 & 13.8188 & 5.56203 \\
\hline 6.5275 & 24.8246 & 5.1151 & 10.4523 & 3.67287 & 10.4431 & 4.16743 \\
\hline 6.5375 & 18.9927 & 3.94854 & 7.70326 & 2.69371 & 7.7644 & 3.09017 \\
\hline 6.5475 & 14.2228 & 2.92954 & 5.58361 & 1.9162 & 5.67855 & 2.22811 \\
\hline 6.5575 & 10.4752 & 2.14609 & 4.03274 & 1.36839 & 4.10258 & 1.58348 \\
\hline 6.5675 & 7.58072 & 1.55453 & 2.90356 & 0.934638 & 2.94683 & 1.1006 \\
\hline 6.5775 & 5.41893 & 1.07479 & 2.11128 & 0.640688 & 2.13641 & 0.771894 \\
\hline 6.5875 & 3.87877 & 0.747474 & 1.56658 & 0.439719 & 1.56641 & 0.532638 \\
\hline 6.5975 & 2.79202 & 0.522708 & 1.20748 & 0.311428 & 1.18104 & 0.361596 \\
\hline 6.6075 & 2.04429 & 0.362836 & 0.973882 & 0.229509 & 0.931447 & 0.270677 \\
\hline 6.6175 & 1.53796 & 0.255469 & 0.826598 & 0.170404 & 0.764923 & 0.202182 \\
\hline
\end{tabular}




\begin{tabular}{|c|c|c|c|c|c|c|}
\hline 6.6275 & 1.21575 & 0.190497 & 0.733783 & 0.142981 & 0.666448 & 0.168946 \\
\hline 6.6375 & 1.0048 & 0.147646 & 0.679688 & 0.118357 & 0.598825 & 0.137371 \\
\hline 6.6475 & 0.870576 & 0.120709 & 0.648136 & 0.105387 & 0.562253 & 0.125487 \\
\hline 6.6575 & 0.790384 & 0.100843 & 0.6276 & 0.103315 & 0.541142 & 0.121441 \\
\hline 6.6675 & 0.745735 & 0.0971234 & 0.611922 & 0.0939584 & 0.528458 & 0.119052 \\
\hline 6.6775 & 0.717558 & 0.0852256 & 0.614885 & 0.0943618 & 0.521869 & 0.118357 \\
\hline 6.6875 & 0.699097 & 0.0828196 & 0.614509 & 0.0897042 & 0.51526 & 0.111662 \\
\hline 6.6975 & 0.6905 & 0.0812036 & 0.617302 & 0.093121 & 0.517952 & 0.113429 \\
\hline 6.7075 & 0.687996 & 0.0871343 & 0.625398 & 0.0930195 & 0.521055 & 0.114243 \\
\hline 6.7175 & 0.686863 & 0.0857161 & 0.627718 & 0.0942546 & 0.522251 & 0.116013 \\
\hline 6.7275 & 0.693572 & 0.0823656 & 0.633928 & 0.0922639 & 0.524014 & 0.120624 \\
\hline 6.7375 & 0.694585 & 0.0808557 & 0.642637 & 0.102485 & 0.528226 & 0.118105 \\
\hline 6.7475 & 0.699563 & 0.0866129 & 0.649474 & 0.0969355 & 0.536522 & 0.118798 \\
\hline 6.7575 & 0.70512 & 0.0860496 & 0.66175 & 0.101669 & 0.542067 & 0.121781 \\
\hline 6.7675 & 0.709942 & 0.0857431 & 0.673866 & 0.101272 & 0.54935 & 0.123277 \\
\hline 6.7775 & 0.725158 & 0.086691 & 0.680477 & 0.10613 & 0.562301 & 0.127383 \\
\hline 6.7875 & 0.741997 & 0.0881939 & 0.704678 & 0.115194 & 0.576544 & 0.130083 \\
\hline 6.7975 & 0.753318 & 0.0976054 & 0.720981 & 0.115558 & 0.583863 & 0.131053 \\
\hline 6.8075 & 0.76886 & 0.0957483 & 0.745992 & 0.11891 & 0.601391 & 0.146654 \\
\hline 6.8175 & 0.790611 & 0.101817 & 0.767745 & 0.125473 & 0.623783 & 0.147328 \\
\hline 6.8275 & 0.820368 & 0.106987 & 0.808572 & 0.142927 & 0.641247 & 0.150987 \\
\hline 6.8375 & 0.849729 & 0.114733 & 0.84657 & 0.154505 & 0.673289 & 0.163335 \\
\hline 6.8475 & 0.891326 & 0.121741 & 0.896789 & 0.164984 & 0.710272 & 0.171547 \\
\hline 6.8575 & 0.935653 & 0.129792 & 0.970664 & 0.193562 & 0.755703 & 0.181001 \\
\hline 6.8675 & 0.994669 & 0.147545 & 1.06223 & 0.221593 & 0.81937 & 0.202834 \\
\hline 6.8775 & 1.07339 & 0.161175 & 1.17519 & 0.254407 & 0.908032 & 0.234658 \\
\hline 6.8875 & 1.18162 & 0.178379 & 1.34342 & 0.312025 & 1.02234 & 0.277789 \\
\hline 6.8975 & 1.32524 & 0.217127 & 1.551 & 0.375524 & 1.16018 & 0.33466 \\
\hline 6.9075 & 1.51215 & 0.247597 & 1.81649 & 0.461309 & 1.35882 & 0.404292 \\
\hline 6.9175 & 1.75541 & 0.305572 & 2.16332 & 0.562453 & 1.60073 & 0.481823 \\
\hline 6.9275 & 2.07786 & 0.368295 & 2.60232 & 0.704463 & 1.92836 & 0.59328 \\
\hline 6.9375 & 2.49587 & 0.44244 & 3.14358 & 0.876111 & 2.32503 & 0.758017 \\
\hline 6.9475 & 3.03028 & 0.554934 & 3.79468 & 1.09167 & 2.81919 & 0.931057 \\
\hline 6.9575 & 3.68758 & 0.701102 & 4.55253 & 1.32465 & 3.3976 & 1.1578 \\
\hline 6.9675 & 4.48131 & 0.867832 & 5.41176 & 1.5966 & 4.06891 & 1.40434 \\
\hline 6.9775 & 5.43788 & 1.04154 & 6.35991 & 1.90159 & 4.81625 & 1.66982 \\
\hline 6.9875 & 6.51044 & 1.27887 & 7.32194 & 2.21686 & 5.64143 & 1.97718 \\
\hline 6.9975 & 7.71707 & 1.53478 & 8.3634 & 2.54861 & 6.50628 & 2.31215 \\
\hline 7.0075 & 9.01916 & 1.80493 & 9.34096 & 2.85839 & 7.33157 & 2.60607 \\
\hline 7.0175 & 10.3504 & 2.09078 & 10.2294 & 3.14347 & 8.13094 & 2.91708 \\
\hline 7.0275 & 11.6289 & 2.37394 & 10.9557 & 3.38245 & 8.84887 & 3.22708 \\
\hline 7.0375 & 12.8827 & 2.60155 & 11.5235 & 3.56801 & 9.43109 & 3.44759 \\
\hline 7.0475 & 13.8911 & 2.82218 & 11.8726 & 3.66365 & 9.83875 & 3.58225 \\
\hline 7.0575 & 14.7169 & 3.01557 & 11.9351 & 3.68962 & 10.0595 & 3.67911 \\
\hline 7.0675 & 15.2622 & 3.09139 & 11.7633 & 3.64706 & 10.0237 & 3.68305 \\
\hline 7.0775 & 15.4656 & 3.14898 & 11.3476 & 3.48341 & 9.79994 & 3.59404 \\
\hline 7.0875 & 15.3494 & 3.13026 & 10.6864 & 3.30149 & 9.37064 & 3.46975 \\
\hline
\end{tabular}




\begin{tabular}{|c|c|c|c|c|c|c|}
\hline 7.0975 & 14.9037 & 3.05941 & 9.84521 & 3.03296 & 8.73959 & 3.19194 \\
\hline 7.1075 & 14.0971 & 2.86856 & 8.89539 & 2.71131 & 8.02744 & 2.94711 \\
\hline 7.1175 & 13.101 & 2.65198 & 7.85428 & 2.40335 & 7.1682 & 2.61468 \\
\hline 7.1275 & 11.8829 & 2.39156 & 6.80336 & 2.05171 & 6.29398 & 2.31725 \\
\hline 7.1375 & 10.555 & 2.10895 & 5.80225 & 1.74718 & 5.42351 & 1.93953 \\
\hline 7.1475 & 9.20874 & 1.84668 & 4.8512 & 1.42442 & 4.58284 & 1.64207 \\
\hline 7.1575 & 7.84163 & 1.57038 & 3.98113 & 1.15202 & 3.79078 & 1.33199 \\
\hline 7.1675 & 6.55859 & 1.28085 & 3.22244 & 0.903249 & 3.0912 & 1.06313 \\
\hline 7.1775 & 5.38453 & 1.04414 & 2.58857 & 0.704823 & 2.48447 & 0.826861 \\
\hline 7.1875 & 4.35152 & 0.813081 & 2.06156 & 0.528902 & 1.98702 & 0.635627 \\
\hline 7.1975 & 3.46822 & 0.636168 & 1.64604 & 0.388028 & 1.58548 & 0.488645 \\
\hline 7.2075 & 2.73806 & 0.478179 & 1.3245 & 0.280879 & 1.25319 & 0.356511 \\
\hline 7.2175 & 2.14632 & 0.367433 & 1.08812 & 0.21198 & 1.01719 & 0.273812 \\
\hline 7.2275 & 1.68824 & 0.256281 & 0.907735 & 0.150581 & 0.821313 & 0.196917 \\
\hline 7.2375 & 1.33342 & 0.191255 & 0.785481 & 0.110133 & 0.692926 & 0.15396 \\
\hline 7.2475 & 1.06094 & 0.136898 & 0.693025 & 0.0777165 & 0.593021 & 0.119385 \\
\hline 7.2575 & 0.877334 & 0.101898 & 0.639078 & 0.0667665 & 0.526901 & 0.103966 \\
\hline 7.2675 & 0.739586 & 0.0742831 & 0.609449 & 0.0628953 & 0.490022 & 0.0920435 \\
\hline 7.2775 & 0.647576 & 0.0596715 & 0.602386 & 0.0666375 & 0.463367 & 0.0828096 \\
\hline 7.2875 & 0.589825 & 0.0544532 & 0.600894 & 0.0654464 & 0.447344 & 0.0781838 \\
\hline 7.2975 & 0.558173 & 0.0423667 & 0.615192 & 0.0764644 & 0.449803 & 0.082353 \\
\hline 7.3075 & 0.5316 & 0.0450636 & 0.635833 & 0.0793359 & 0.451746 & 0.0772271 \\
\hline 7.3175 & 0.524651 & 0.0415354 & 0.667999 & 0.0969221 & 0.455728 & 0.0739676 \\
\hline 7.3275 & 0.529714 & 0.0435468 & 0.70598 & 0.116101 & 0.477237 & 0.0834643 \\
\hline 7.3375 & 0.54144 & 0.0450063 & 0.745255 & 0.138862 & 0.496733 & 0.0828166 \\
\hline 7.3475 & 0.54991 & 0.0484562 & 0.799284 & 0.175438 & 0.525016 & 0.0903204 \\
\hline 7.3575 & 0.572124 & 0.0446858 & 0.859316 & 0.224444 & 0.556875 & 0.0951053 \\
\hline 7.3675 & 0.594453 & 0.0480326 & 0.930293 & 0.27985 & 0.587587 & 0.106045 \\
\hline 7.3775 & 0.627188 & 0.0508989 & 1.01452 & 0.363351 & 0.627583 & 0.111609 \\
\hline 7.3875 & 0.664213 & 0.0623696 & 1.08717 & 0.4172 & 0.665014 & 0.125494 \\
\hline 7.3975 & 0.706485 & 0.0621975 & 1.17508 & 0.512333 & 0.720177 & 0.138419 \\
\hline 7.4075 & 0.739404 & 0.0716222 & 1.26099 & 0.604395 & 0.769421 & 0.151988 \\
\hline 7.4175 & 0.787514 & 0.0762703 & 1.33556 & 0.691501 & 0.818055 & 0.163589 \\
\hline 7.4275 & 0.835353 & 0.0889603 & 1.41949 & 0.787825 & 0.862907 & 0.182577 \\
\hline 7.4375 & 0.876753 & 0.100172 & 1.48573 & 0.866106 & 0.912811 & 0.199986 \\
\hline 7.4475 & 0.921432 & 0.109431 & 1.53828 & 0.938614 & 0.948844 & 0.20364 \\
\hline 7.4575 & 0.960524 & 0.110531 & 1.57559 & 0.983126 & 0.976023 & 0.218949 \\
\hline 7.4675 & 0.996542 & 0.124304 & 1.58521 & 1.02891 & 0.990594 & 0.220717 \\
\hline 7.4775 & 1.01836 & 0.132781 & 1.57299 & 1.01464 & 0.994213 & 0.234578 \\
\hline 7.4875 & 1.03336 & 0.13919 & 1.54274 & 1.00153 & 0.991833 & 0.225069 \\
\hline 7.4975 & 1.03791 & 0.14052 & 1.50045 & 0.957238 & 0.968208 & 0.222819 \\
\hline 7.5075 & 1.03229 & 0.142142 & 1.4171 & 0.894379 & 0.941348 & 0.199656 \\
\hline 7.5175 & 1.01213 & 0.136356 & 1.34323 & 0.822372 & 0.904921 & 0.203091 \\
\hline 7.5275 & 0.976104 & 0.13426 & 1.24898 & 0.759384 & 0.8588 & 0.188693 \\
\hline 7.5375 & 0.940994 & 0.120267 & 1.15309 & 0.65229 & 0.809132 & 0.176557 \\
\hline 7.5475 & 0.897053 & 0.109049 & 1.06126 & 0.575753 & 0.753678 & 0.155438 \\
\hline 7.5575 & 0.851336 & 0.108242 & 0.971987 & 0.474598 & 0.708209 & 0.138028 \\
\hline
\end{tabular}




\begin{tabular}{|c|c|c|c|c|c|c|}
\hline 7.5675 & 0.805327 & 0.100013 & 0.880446 & 0.404519 & 0.657291 & 0.12544 \\
\hline 7.5775 & 0.754841 & 0.0889819 & 0.811727 & 0.327407 & 0.614742 & 0.114009 \\
\hline .5875 & 0.711213 & 0.0831416 & 0.750772 & 0.254574 & 0.574289 & 0.100604 \\
\hline .5975 & 0.668171 & 0.0763687 & 0.699 & 0.210658 & 0.548734 & 0.0955317 \\
\hline 6075 & 0.63531 & 0.067605 & 0.656217 & 0.160653 & 0.52148 & 0.0939989 \\
\hline 6175 & 0.613561 & 0.0688344 & 0.624713 & 0.126784 & 0.500253 & 0.0869292 \\
\hline 6275 & 0.581004 & 0.0645406 & 0.601739 & 0.102087 & 0.485575 & 0.0862412 \\
\hline 6375 & 0.566557 & 0.0585973 & 0.583523 & 0.0919164 & 0.481949 & 0.087301 \\
\hline 6475 & 0.554828 & 0.0553818 & 0.574346 & 0.0769836 & 0.469806 & 0.0837073 \\
\hline 6575 & 0.546698 & 0.0519341 & 0.568644 & 0.073219 & 0.460841 & 0.0843448 \\
\hline 6675 & 0.535533 & 0.0526142 & 0.564411 & 0.0662374 & 0.458474 & 0.0853001 \\
\hline 7.6775 & 0.533387 & 0.0496972 & 0.559643 & 0.0653191 & 0.463315 & 0.0875517 \\
\hline 7.6875 & 0.529016 & 0.0459545 & 0.560572 & 0.0585853 & 0.461992 & 0.0866369 \\
\hline 7.6975 & 0.528549 & 0.0466848 & 0.554305 & 0.0600104 & 0.456591 & 0.0867196 \\
\hline 7.7075 & 0.527364 & 0.0444032 & 0.561743 & 0.0646721 & 0.459162 & 0.093537 \\
\hline 7.7175 & 0.525279 & 0.0443662 & 0.55755 & 0.0677084 & 0.456297 & 0.0933542 \\
\hline 7.7275 & 0.520252 & 0.043625 & 0.553785 & 0.0567254 & 0.458919 & 0.0925218 \\
\hline 7.7375 & 0.522219 & 0.0427622 & 0.549222 & 0.0638233 & 0.457058 & 0.0899406 \\
\hline 7.7475 & 0.51973 & 0.0431499 & 0.551898 & 0.0618779 & 0.459155 & 0.0941531 \\
\hline 7.7575 & 0.517 & 0.0424196 & 0.548995 & 0.0625014 & 0.454916 & 0.0894451 \\
\hline 7.7675 & 0.519924 & 0.0430705 & 0.544755 & 0.0619501 & 304 & 0.0894856 \\
\hline 7.7775 & 0.516523 & 0.0413625 & 0.540851 & 0.0631766 & 0.447956 & 0.0901101 \\
\hline 7.7875 & 0.513367 & 0.0423076 & 0.540656 & 0.0591226 & 0.451023 & 0.0916783 \\
\hline 7.7975 & 0.514332 & 0.0416412 & 0.539321 & 0.0668232 & 0.451953 & 0.0837906 \\
\hline 7.8075 & 0.508025 & 0.0405694 & 0.536043 & 0.0655293 & 0.444915 & 0.0836665 \\
\hline 7.8175 & 0.511303 & 0.0382439 & 0.536838 & 0.0726167 & 0.446018 & 0.0846428 \\
\hline 7.8275 & 0.511002 & 0.0449235 & 0.543334 & 0.0800616 & 0.445838 & 0.0855152 \\
\hline 7.8375 & 0.508042 & 0.0431225 & 0.540133 & 0.0850729 & 0.448061 & 0.0853163 \\
\hline 7.8475 & 0.508718 & 0.0402395 & 0.551659 & 0.103163 & 784 & 0.0836167 \\
\hline 7.8575 & 0.507077 & 0.0410211 & 0.560205 & 0.113342 & 0.452736 & 0.0785441 \\
\hline 7.8675 & 0.509682 & 0.0394306 & 0.573939 & 0.15112 & 0.456925 & 0.0837699 \\
\hline 7.8775 & 0.514125 & 0.0444462 & 0.598024 & 0.208152 & 0.465036 & 0.082554 \\
\hline 7.8875 & 0.523676 & 0.0385116 & 0.61864 & 0.265822 & 0.478752 & 0.0824785 \\
\hline 7.8975 & 0.525489 & 0.0394464 & 0.65015 & 0.355255 & 0.492515 & 0.0796022 \\
\hline 7.9075 & 0.539292 & 0.041249 & 0.689662 & 0.448372 & 0.513683 & 0.0835366 \\
\hline 7.9175 & 0.550611 & 0.0390403 & 0.744784 & 0.588092 & 0.535806 & 0.0912477 \\
\hline 7.9275 & 0.568833 & 0.0401839 & 0.79881 & 0.73282 & 0.562493 & 0.10055 \\
\hline 7.9375 & 0.591879 & 0.0419305 & 0.87117 & 0.920398 & 0.601423 & 0.108157 \\
\hline 7.9475 & 0.62461 & 0.0518865 & 0.951167 & 1.14935 & 0.641529 & 0.126782 \\
\hline 7.9575 & 0.659239 & 0.048726 & 1.03927 & 1.36456 & 0.684016 & 0.12987 \\
\hline 7.9675 & 0.699312 & 0.0579294 & 1.1342 & 1.59751 & 0.739373 & 0.153775 \\
\hline 7.9775 & 0.750846 & 0.0604589 & 1.22959 & 1.8633 & 0.796697 & 0.167014 \\
\hline 7.9875 & 0.79908 & 0.0651143 & 1.32472 & 2.08897 & 0.855676 & 0.192085 \\
\hline 7.9975 & 0.85074 & 0.0728982 & 1.40495 & 2.35377 & 0.907164 & 0.21251 \\
\hline 8.0075 & 0.903014 & 0.0797657 & 1.47687 & 2.55124 & 0.955581 & 0.231882 \\
\hline 8.0175 & 0.953626 & 0.0908614 & 1.55197 & 2.73363 & 0.992696 & 0.244194 \\
\hline 8.0275 & 1.0037 & 0.0874596 & 1.59213 & 2.88294 & 1.03286 & 0.26497 \\
\hline
\end{tabular}




\begin{tabular}{|c|c|c|c|c|c|c|}
\hline 8.0375 & 1.05151 & 0.0991072 & 1.61996 & 2.91669 & 1.05453 & 0.265749 \\
\hline 8.0475 & 1.07797 & 0.104645 & 1.60991 & 2.87845 & 1.06421 & 0.267043 \\
\hline 8.0575 & 1.10243 & 0.106086 & 1.60307 & 2.87044 & 1.06454 & 0.266752 \\
\hline 8.0675 & 1.11335 & 0.109028 & 1.55452 & 2.68903 & 1.0444 & 0.254282 \\
\hline 8.0775 & 1.11462 & 0.105013 & 1.49653 & 2.56133 & 1.02273 & 0.253493 \\
\hline 8.0875 & 1.09768 & 0.0970694 & 1.42484 & 2.3663 & 0.982902 & 0.235714 \\
\hline 8.0975 & 1.0753 & 0.104236 & 1.3427 & 2.10396 & 0.946129 & 0.226255 \\
\hline 8.1075 & 1.04407 & 0.102996 & 1.24618 & 1.88625 & 0.899127 & 0.194667 \\
\hline 8.1175 & 1.00618 & 0.0933949 & 1.16218 & 1.60619 & 0.845643 & 0.183238 \\
\hline 8.1275 & 0.967699 & 0.0861452 & 1.07365 & 1.3292 & 0.797538 & 0.162022 \\
\hline 8.1375 & 0.920238 & 0.0873343 & 0.998526 & 1.1249 & 0.753094 & 0.136933 \\
\hline 8.1475 & 0.867283 & 0.0765861 & 0.929988 & 0.918328 & 0.711032 & 0.130279 \\
\hline 8.1575 & 0.833678 & 0.0681517 & 0.874422 & 0.727125 & 0.675383 & 0.118741 \\
\hline 8.1675 & 0.790515 & 0.0650577 & 0.82718 & 0.555731 & 0.649085 & 0.104274 \\
\hline 8.1775 & 0.754377 & 0.063701 & 0.794015 & 0.44254 & 0.621984 & 0.0986972 \\
\hline 8.1875 & 0.727429 & 0.0606255 & 0.768071 & 0.338514 & 0.609482 & 0.0993402 \\
\hline 8.1975 & 0.701955 & 0.0583148 & 0.752676 & 0.27107 & 0.595024 & 0.0877137 \\
\hline 8.2075 & 0.688175 & 0.0555954 & 0.744501 & 0.218801 & 0.590557 & 0.0937508 \\
\hline 8.2175 & 0.681062 & 0.0567282 & 0.737675 & 0.18286 & 0.589551 & 0.0920478 \\
\hline 8.2275 & 0.665792 & 0.0571801 & 0.739583 & 0.171615 & 0.589791 & 0.0933219 \\
\hline 8.2375 & 0.662487 & 0.0580817 & 0.746728 & 0.165825 & 0.591448 & 0.0939514 \\
\hline 8.2475 & 0.663151 & 0.0555235 & 0.741989 & 0.156796 & 0.598052 & 0.0966664 \\
\hline 8.2575 & 0.663491 & 0.0561146 & 0.744601 & 0.157695 & 0.59913 & 0.09736 \\
\hline 8.2675 & 0.663956 & 0.0580868 & 0.741632 & 0.160858 & 0.605267 & 0.0996335 \\
\hline 8.2775 & 0.671101 & 0.0645568 & 0.732545 & 0.15593 & 0.609032 & 0.102027 \\
\hline 8.2875 & 0.678742 & 0.0588588 & 0.73565 & 0.149528 & 0.60653 & 0.104537 \\
\hline 8.2975 & 0.672426 & 0.0609401 & 0.729441 & 0.144756 & 0.611275 & 0.110428 \\
\hline 8.3075 & 0.674403 & 0.0570426 & 0.721525 & 0.136033 & 0.611729 & 0.112797 \\
\hline 8.3175 & 0.670933 & 0.0584847 & 0.711878 & 0.120201 & 0.60861 & 0.11405 \\
\hline 8.3275 & 0.675665 & 0.0502171 & 0.703235 & 0.117738 & 0.60753 & 0.126048 \\
\hline 8.3375 & 0.670823 & 0.0517668 & 0.686879 & 0.106715 & 0.604208 & 0.126708 \\
\hline 8.3475 & 0.671141 & 0.0564834 & 0.685928 & 0.102281 & 0.606912 & 0.125561 \\
\hline 8.3575 & 0.667603 & 0.0514633 & 0.676871 & 0.0877296 & 0.602294 & 0.130945 \\
\hline 8.3675 & 0.664838 & 0.0537437 & 0.670257 & 0.0824748 & 0.603026 & 0.137853 \\
\hline 8.3775 & 0.660974 & 0.0537853 & 0.663711 & 0.0838301 & 0.601126 & 0.141364 \\
\hline 8.3875 & 0.661255 & 0.0522806 & 0.659525 & 0.0757379 & 0.60578 & 0.14718 \\
\hline 8.3975 & 0.660816 & 0.0531984 & 0.656385 & 0.0681358 & 0.60972 & 0.14537 \\
\hline 8.4075 & 0.672613 & 0.0556614 & 0.655966 & 0.0695959 & 0.608357 & 0.151063 \\
\hline 8.4175 & 0.665661 & 0.0524594 & 0.65818 & 0.0663572 & 0.613819 & 0.154937 \\
\hline 8.4275 & 0.673361 & 0.0499248 & 0.658569 & 0.0624283 & 0.614631 & 0.149126 \\
\hline 8.4375 & 0.674262 & 0.053868 & 0.669742 & 0.0629897 & 0.618134 & 0.153419 \\
\hline 8.4475 & 0.678621 & 0.0522891 & 0.685069 & 0.0677185 & 0.627385 & 0.150128 \\
\hline 8.4575 & 0.694201 & 0.0491632 & 0.695561 & 0.0658264 & 0.631677 & 0.149799 \\
\hline 8.4675 & 0.706719 & 0.053873 & 0.720651 & 0.0685434 & 0.645198 & 0.151318 \\
\hline 8.4775 & 0.72121 & 0.0559543 & 0.749258 & 0.0708501 & 0.658482 & 0.146032 \\
\hline 8.4875 & 0.741679 & 0.0570991 & 0.783007 & 0.0776272 & 0.682846 & 0.141617 \\
\hline 8.4975 & 0.769018 & 0.059633 & 0.824674 & 0.0816889 & 0.700701 & 0.140025 \\
\hline
\end{tabular}




\begin{tabular}{|c|c|c|c|c|c|c|}
\hline 8.5075 & 0.803181 & 0.0684675 & 0.881191 & 0.102266 & 0.728724 & 0.140341 \\
\hline 8.5175 & 0.847257 & 0.0755818 & 0.94204 & 0.106545 & 0.762533 & 0.140306 \\
\hline 8.5275 & 0.90004 & 0.088493 & 1.01651 & 0.120352 & 0.791647 & 0.141689 \\
\hline 8.5375 & 0.966011 & 0.09285 & 1.10041 & 0.138394 & 0.83968 & 0.142692 \\
\hline 8.5475 & 1.05193 & 0.114025 & 1.18623 & 0.165244 & 0.894516 & 0.157782 \\
\hline 8.5575 & 1.13424 & 0.13326 & 1.26695 & 0.169373 & 0.947454 & 0.168824 \\
\hline 8.5675 & 1.23763 & 0.156634 & 1.35626 & 0.199476 & 1.00409 & 0.172454 \\
\hline 8.5775 & 1.33666 & 0.183319 & 1.45312 & 0.221632 & 1.06093 & 0.187881 \\
\hline 8.5875 & 1.44133 & 0.201614 & 1.53868 & 0.235624 & 1.10307 & 0.200623 \\
\hline 8.5975 & 1.55405 & 0.237455 & 1.61155 & 0.255828 & 1.1519 & 0.202376 \\
\hline 8.6075 & 1.66331 & 0.268424 & 1.66821 & 0.273952 & 1.2028 & 0.215521 \\
\hline 8.6175 & 1.74865 & 0.287029 & 1.70892 & 0.27698 & 1.23055 & 0.226154 \\
\hline 8.6275 & 1.8323 & 0.310527 & 1.72698 & 0.282205 & 1.25383 & 0.229929 \\
\hline 8.6375 & 1.89601 & 0.322382 & 1.72764 & 0.271358 & 1.25945 & 0.237313 \\
\hline 8.6475 & 1.94118 & 0.335619 & 1.70982 & 0.261246 & 1.25422 & 0.234594 \\
\hline 8.6575 & 1.95625 & 0.33695 & 1.67869 & 0.264138 & 1.24128 & 0.228947 \\
\hline 8.6675 & 1.95986 & 0.347177 & 1.61988 & 0.251173 & 1.21221 & 0.217479 \\
\hline 8.6775 & 1.928 & 0.328276 & 1.55006 & 0.231638 & 1.1711 & 0.209264 \\
\hline 8.6875 & 1.87229 & 0.32282 & 1.48042 & 0.218611 & 1.12716 & 0.19951 \\
\hline 8.6975 & 1.80573 & 0.295365 & 1.39473 & 0.194771 & 1.07872 & 0.179464 \\
\hline 8.7075 & 1.71925 & 0.279054 & 1.3127 & 0.174347 & 1.03108 & 0.172623 \\
\hline 8.7175 & 1.62619 & 0.250691 & 1.23526 & 0.163819 & 0.977298 & 0.154551 \\
\hline 8.7275 & 1.51504 & 0.220968 & 1.15785 & 0.139895 & 0.933814 & 0.142359 \\
\hline 8.7375 & 1.41364 & 0.198407 & 1.08875 & 0.13724 & 0.887249 & 0.141122 \\
\hline 8.7475 & 1.3126 & 0.175258 & 1.02934 & 0.128798 & 0.849599 & 0.124552 \\
\hline 8.7575 & 1.2214 & 0.151256 & 0.984918 & 0.118558 & 0.812145 & 0.123157 \\
\hline 8.7675 & 1.13667 & 0.125362 & 0.946721 & 0.130633 & 0.786945 & 0.118698 \\
\hline 8.7775 & 1.07275 & 0.120821 & 0.917374 & 0.131503 & 0.765302 & 0.114001 \\
\hline 8.7875 & 1.00637 & 0.0990881 & 0.898476 & 0.146558 & 0.752042 & 0.108055 \\
\hline 8.7975 & 0.958668 & 0.0930139 & 0.887372 & 0.166089 & 0.736022 & 0.113473 \\
\hline 8.8075 & 0.922181 & 0.0985648 & 0.87463 & 0.18705 & 0.731608 & 0.111473 \\
\hline 8.8175 & 0.894899 & 0.0942834 & 0.873305 & 0.21548 & 0.726894 & 0.109643 \\
\hline 8.8275 & 0.867978 & 0.103594 & 0.8829 & 0.257858 & 0.728834 & 0.113588 \\
\hline 8.8375 & 0.85825 & 0.110729 & 0.890311 & 0.277876 & 0.733271 & 0.109315 \\
\hline 8.8475 & 0.854371 & 0.123498 & 0.900985 & 0.308988 & 0.74009 & 0.112801 \\
\hline 8.8575 & 0.857934 & 0.122359 & 0.906329 & 0.358481 & 0.745191 & 0.114022 \\
\hline 8.8675 & 0.852986 & 0.129927 & 0.916961 & 0.368103 & 0.753085 & 0.114713 \\
\hline 8.8775 & 0.861536 & 0.141743 & 0.926016 & 0.38842 & 0.761587 & 0.125677 \\
\hline 8.8875 & 0.864882 & 0.149833 & 0.935415 & 0.40403 & 0.769192 & 0.118135 \\
\hline 8.8975 & 0.866337 & 0.158736 & 0.941333 & 0.410054 & 0.771569 & 0.115142 \\
\hline 8.9075 & 0.872441 & 0.168299 & 0.935305 & 0.40611 & 0.776683 & 0.117637 \\
\hline 8.9175 & 0.881128 & 0.176472 & 0.93966 & 0.412553 & 0.772538 & 0.11031 \\
\hline 8.9275 & 0.878113 & 0.179842 & 0.934868 & 0.407363 & 0.766075 & 0.109165 \\
\hline 8.9375 & 0.873098 & 0.178363 & 0.927684 & 0.383534 & 0.770131 & 0.110302 \\
\hline 8.9475 & 0.881974 & 0.185134 & 0.920459 & 0.356036 & 0.760192 & 0.103736 \\
\hline 8.9575 & 0.877465 & 0.19391 & 0.902294 & 0.318297 & 0.752579 & 0.104388 \\
\hline 8.9675 & 0.869769 & 0.194335 & 0.889502 & 0.294056 & 0.747355 & 0.105463 \\
\hline
\end{tabular}




\begin{tabular}{|c|c|c|c|c|c|c|}
\hline 8.9775 & 0.871138 & 0.201402 & 0.889267 & 0.257954 & 0.737383 & 0.0969135 \\
\hline 8.9875 & 0.860201 & 0.200993 & 0.870985 & 0.235906 & 0.727528 & 0.0910117 \\
\hline 9975 & 0.85559 & 0.203618 & 0.857721 & 0.191208 & 0.723367 & 0.093806 \\
\hline .0075 & 0.846397 & 0.204824 & 0.848377 & 0.164548 & 0.722018 & 0.0937989 \\
\hline 9.0175 & 0.838245 & 0.2107 & 0.83096 & 0.13406 & 0.707217 & 0.0880278 \\
\hline 9.0275 & 0.835992 & 0.197223 & 0.828007 & 0.126925 & 0.709569 & 0.0916916 \\
\hline 9.0375 & 0.826996 & 0.203457 & 0.824912 & 0.11141 & 0.704219 & 0.0889208 \\
\hline 9.0475 & 0.825871 & 0.198865 & 0.81998 & 0.0939084 & 0.698948 & 0.090859 \\
\hline 9.0575 & 0.810985 & 0.186341 & 0.814138 & 0.0873436 & 0.691065 & 0.0874672 \\
\hline 9.0675 & 0.802509 & 0.185569 & 0.817288 & 0.0863374 & 0.700064 & 0.0959657 \\
\hline 9.0775 & 0.805964 & 0.189313 & 0.810358 & 0.0781685 & 0.695914 & 0.0895504 \\
\hline 9.0875 & 0.802477 & 0.178201 & 0.807423 & 0.076811 & 0.693712 & 0.0897185 \\
\hline 9.0975 & 0.797137 & 0.172691 & 0.816978 & 0.078129 & 0.691797 & 0.0895956 \\
\hline 9.1075 & 0.795638 & 0.168904 & 0.814169 & 0.0749552 & 0.697832 & 0.0892663 \\
\hline 9.1175 & 0.792176 & 0.158447 & 0.815308 & 0.0797096 & 0.695957 & 0.0943891 \\
\hline 9.1275 & 0.790358 & 0.150947 & 0.817754 & 0.0825655 & 0.701555 & 0.0932389 \\
\hline 9.1375 & 0.794203 & 0.151291 & 0.820425 & 0.0819028 & 0.707595 & 0.0985526 \\
\hline 9.1475 & 0.789109 & 0.140262 & 0.825617 & 0.0898001 & 0.706894 & 0.0961395 \\
\hline 9.1575 & 0.793105 & 0.129748 & 0.829302 & 0.0896735 & 0.708063 & 0.0966937 \\
\hline 9.1675 & 0.795033 & 0.122371 & 0.833156 & 0.0881591 & 0.713076 & 0.0936125 \\
\hline 9.1775 & 0.796925 & 0.11814 & 0.836015 & 0.0936996 & 0.714744 & 0.0993921 \\
\hline 9.1875 & 0.79 & 0.113081 & 0.831926 & 7866 & 1803 & 0.100194 \\
\hline 9.1975 & 0.793982 & 0.103791 & 0.836204 & 0.0936861 & 0.722056 & 0.0957335 \\
\hline 9.2075 & 0.792452 & 0.10281 & 0.841757 & 0.0930055 & 0.719985 & 0.0972359 \\
\hline 9.2175 & 0.795473 & 0.0951458 & 0.839334 & 0.0927627 & 0.724575 & 0.106467 \\
\hline 9.2275 & 0.797546 & 0.0926531 & 0.840832 & 0.0885825 & 0.72377 & 0.101698 \\
\hline 9.2375 & 0.802346 & 0.0893741 & 0.835451 & 0.0874796 & 0.729878 & 0.107273 \\
\hline 9.2475 & 0.803049 & 0.0875548 & 0.837076 & 0.0861403 & 0.731183 & 0.104401 \\
\hline 9.2575 & 0.793111 & 0.0884942 & 0.835072 & 0.0807297 & 0.729692 & 0.105749 \\
\hline 9.2675 & 0.799911 & 0.0800888 & 0.826731 & 0.0854835 & 0.723333 & 0.101459 \\
\hline 9.2775 & 0.803509 & 0.0823904 & 0.828245 & 0.0751257 & 0.726702 & 0.105331 \\
\hline 9.2875 & 0.79692 & 0.0796258 & 0.829869 & 0.0705916 & 0.723281 & 0.109887 \\
\hline 9.2975 & 0.800551 & 0.0814034 & 0.818809 & 0.0698753 & 0.723472 & 0.104819 \\
\hline 9.3075 & 0.798281 & 0.0733895 & 0.818309 & 0.0658032 & 0.723343 & 0.105736 \\
\hline 9.3175 & 0.794852 & 0.0791728 & 0.815591 & 0.0697896 & 0.723262 & 0.106265 \\
\hline 9.3275 & 0.79425 & 0.0776611 & 0.811694 & 0.064331 & 0.723209 & 0.109261 \\
\hline 9.3375 & 0.792483 & 0.0723909 & 0.802393 & 0.0631082 & 0.72699 & 0.115517 \\
\hline 9.3475 & 0.789995 & 0.0798345 & 0.807141 & 0.0602812 & 0.72428 & 0.1091 \\
\hline 9.3575 & 0.792128 & 0.0719696 & 0.805773 & 0.0576159 & 0.729478 & 0.113021 \\
\hline 9.3675 & 0.791671 & 0.074244 & 0.806614 & 0.0577744 & 0.730516 & 0.121924 \\
\hline 9.3775 & 0.788571 & 0.0698585 & 0.805668 & 0.0545859 & 0.730242 & 0.115956 \\
\hline 9.3875 & 0.796 & 0.0714865 & 0.810016 & 0.0618984 & 0.735262 & 0.115326 \\
\hline 9.3975 & 0.784014 & 0.0668824 & 0.801659 & 0.0566815 & 0.736997 & 0.125065 \\
\hline 9.4075 & 0.794277 & 0.062162 & 0.813558 & 0.0570061 & 0.741901 & 0.132667 \\
\hline 9.4175 & 0.793713 & 0.0619876 & 0.810899 & 0.0594214 & 0.744325 & 0.13038 \\
\hline 9.4275 & 0.793882 & 0.0660501 & 0.822462 & 0.0625121 & 0.742886 & 0.129659 \\
\hline 9.4375 & 0.797825 & 0.0641136 & 0.824183 & 0.0602573 & 0.75322 & 0.134148 \\
\hline
\end{tabular}




\begin{tabular}{|c|c|c|c|c|c|c|}
\hline 9.4475 & 0.805129 & 0.0618361 & 0.836171 & 0.0567653 & 0.758528 & 0.138992 \\
\hline 9.4575 & 0.814814 & 0.0585604 & 0.84644 & 0.0614732 & 0.769661 & 0.141075 \\
\hline 9.4675 & 0.820708 & 0.0594159 & 0.859722 & 0.0659271 & 0.772665 & 0.138987 \\
\hline .4775 & 0.832186 & 0.0600834 & 0.869412 & 0.067314 & 0.780263 & 0.142698 \\
\hline 9.4875 & 0.846062 & 0.0578457 & 0.884254 & 0.0719766 & 0.78955 & 0.138959 \\
\hline 9.4975 & 0.858033 & 0.0658385 & 0.898791 & 0.0624155 & 0.803986 & 0.147351 \\
\hline 9.5075 & 0.87921 & 0.062326 & 0.916407 & 0.066108 & 0.813399 & 0.151325 \\
\hline 9.5175 & 0.889704 & 0.0651804 & 0.932261 & 0.0689216 & 0.818251 & 0.15435 \\
\hline 9.5275 & 0.906788 & 0.0733674 & 0.93413 & 0.0622847 & 0.8332 & 0.157449 \\
\hline 9.5375 & 0.928508 & 0.0691435 & 0.946283 & 0.0702172 & 0.838389 & 0.160838 \\
\hline 9.5475 & 0.937718 & 0.0749826 & 0.963417 & 0.0789704 & 0.849444 & 0.165772 \\
\hline 9.5575 & 0.96519 & 0.0749026 & 0.964949 & 0.0715315 & 0.857438 & 0.169621 \\
\hline 9.5675 & 0.971364 & 0.08557 & 0.968375 & 0.074034 & 0.857423 & 0.163202 \\
\hline 9.5775 & 0.982122 & 0.0801829 & 0.974126 & 0.07361 & 0.860382 & 0.16594 \\
\hline 9.5875 & 0.998833 & 0.0842269 & 0.965591 & 0.0702374 & 0.865348 & 0.166355 \\
\hline 9.5975 & 0.997176 & 0.0815635 & 0.961363 & 0.0677083 & 0.86249 & 0.171242 \\
\hline 9.6075 & 0.995459 & 0.078835 & 0.957227 & 0.0722108 & 0.858184 & 0.174217 \\
\hline 9.6175 & 0.999762 & 0.0836646 & 0.950332 & 0.0623562 & 0.857074 & 0.16953 \\
\hline 9.6275 & 0.998027 & 0.0811017 & 0.938222 & 0.0637458 & 0.849527 & 0.176085 \\
\hline 9.6375 & 0.98056 & 0.083534 & 0.932901 & 0.0608979 & 0.843339 & 0.171557 \\
\hline 9.6475 & 0.977554 & 0.0819194 & 0.920909 & 0.0615464 & 0.837463 & 0.172068 \\
\hline 9.6575 & 0.964513 & 0.0791852 & 0.913568 & 0.060472 & 0.837696 & 0.168257 \\
\hline 9.6675 & 0.952602 & 0.0810306 & 0.898678 & 0.0547762 & 0.824026 & 0.172875 \\
\hline 9.6775 & 0.94035 & 0.0816329 & 0.890342 & 0.0608413 & 0.820998 & 0.167242 \\
\hline 9.6875 & 0.921995 & 0.070 & 0.878345 & 0.0593193 & & 0.165874 \\
\hline 9.6975 & 0.912283 & 0.0777646 & 0.870693 & 0.0574584 & 0.813905 & 0.159042 \\
\hline 9.7075 & 0.90331 & 0.0701138 & 0.87074 & 0.059479 & 0.806598 & 0.171596 \\
\hline 9.7175 & 0.89131 & 0.0712774 & 0.858244 & 0.0554151 & 0.796084 & 0.169211 \\
\hline 9.7275 & 0.884489 & 0.0711072 & 0.856291 & 0.0581113 & 0.798954 & 0.161827 \\
\hline 9.7375 & 0.876777 & 0.0661164 & 0.853991 & 0.058714 & 0.797501 & 0.164526 \\
\hline 9.7475 & 0.865415 & 0.0646217 & 0.85538 & 0.0593088 & 0.789306 & 0.161581 \\
\hline 9.7575 & 0.859385 & 0.0661794 & 0.850386 & 0.0579974 & 0.793446 & 0.159998 \\
\hline 9.7675 & 0.854071 & 0.0619555 & 0.845551 & 0.0515515 & 0.790209 & 0.162507 \\
\hline 9.7775 & 0.848992 & 0.0652034 & 0.842954 & 0.0516147 & 0.789687 & 0.159331 \\
\hline 9.7875 & 0.848287 & 0.0605204 & 0.849385 & 0.0515399 & 0.786021 & 0.152291 \\
\hline 9.7975 & 0.848173 & 0.0565079 & 0.844976 & 0.0527768 & 0.788328 & 0.156695 \\
\hline 9.8075 & 0.848027 & 0.0559615 & 0.845439 & 0.0575742 & 0.779408 & 0.155712 \\
\hline 9.8175 & 0.845247 & 0.0560311 & 0.849573 & 0.0558205 & 0.790383 & 0.149168 \\
\hline 9.8275 & 0.840021 & 0.0555216 & 0.848233 & 0.058873 & 0.780374 & 0.147117 \\
\hline 9.8375 & 0.845341 & 0.0561632 & 0.846783 & 0.0578648 & 0.779896 & 0.147491 \\
\hline 9.8475 & 0.836107 & 0.0557917 & 0.853484 & 0.0552143 & 0.78182 & 0.139406 \\
\hline 9.8575 & 0.837411 & 0.0517033 & 0.856492 & 0.0591133 & 0.78515 & 0.14148 \\
\hline 9.8675 & 0.840425 & 0.0550067 & 0.858015 & 0.0601533 & 0.788438 & 0.136411 \\
\hline 9.8775 & 0.842169 & 0.0502905 & 0.864529 & 0.0511068 & 0.786545 & 0.132744 \\
\hline 9.8875 & 0.849614 & 0.0513636 & 0.861442 & 0.0519282 & 0.793824 & 0.133284 \\
\hline 9.8975 & 0.844944 & 0.047505 & 0.873798 & 0.0553949 & 0.786379 & 0.124477 \\
\hline 9.9075 & 0.852235 & 0.0527656 & 0.879727 & 0.0644008 & 0.793665 & 0.12397 \\
\hline
\end{tabular}




\begin{tabular}{|c|c|c|c|c|c|c|}
\hline 9.9175 & 0.851913 & 0.0528987 & 0.881154 & 0.0596466 & 0.797917 & 0.120511 \\
\hline 9.9275 & 0.856031 & 0.049057 & 0.89255 & 0.0605935 & 0.799001 & 0.119568 \\
\hline 9.9375 & 0.85941 & 0.0532722 & 0.896569 & 0.0620674 & 0.797411 & 0.117531 \\
\hline 9.9475 & 0.865345 & 0.0489952 & 0.923974 & 0.0608092 & 0.804132 & 0.116097 \\
\hline 9.9575 & 0.870552 & 0.051998 & 0.930938 & 0.0660084 & 0.810605 & 0.111355 \\
\hline 9.9675 & 0.885582 & 0.0493542 & 0.93382 & 0.0719283 & 0.817047 & 0.111985 \\
\hline 9.9775 & 0.886712 & 0.0561537 & 0.94927 & 0.0719037 & 0.830048 & 0.111625 \\
\hline 9.9875 & 0.893842 & 0.0514775 & 0.964408 & 0.0702737 & 0.833532 & 0.111336 \\
\hline 9.9975 & 0.916531 & 0.0532081 & 0.988397 & 0.0795982 & 0.842066 & 0.111576 \\
\hline 10.0075 & 0.929031 & 0.0484285 & 0.993634 & 0.0865001 & 0.854984 & 0.109598 \\
\hline 10.0175 & 0.937612 & 0.0584895 & 1.01774 & 0.0801763 & 0.860101 & 0.116839 \\
\hline 10.0275 & 0.958106 & 0.0575512 & 1.02956 & 0.0901164 & 0.867059 & 0.115942 \\
\hline 10.0375 & 0.967834 & 0.0525539 & 1.0412 & 0.0969893 & 0.874489 & 0.117231 \\
\hline 10.0475 & 0.980016 & 0.0594884 & 1.04628 & 0.0934966 & 0.888349 & 0.121735 \\
\hline 10.0575 & 0.996523 & 0.0638519 & 1.05839 & 0.0962736 & 0.888602 & 0.123947 \\
\hline 10.0675 & 1.00878 & 0.0623161 & 1.06065 & 0.103326 & 0.884916 & 0.119524 \\
\hline 10.0775 & 1.0185 & 0.0702633 & 1.06494 & 0.0971774 & 0.890868 & 0.120733 \\
\hline 10.0875 & 1.02174 & 0.06815 & 1.06394 & 0.11272 & 0.898364 & 0.122728 \\
\hline 10.0975 & 1.03046 & 0.0667059 & 1.05176 & 0.106554 & 0.896843 & 0.128148 \\
\hline 10.1075 & 1.03132 & 0.0675632 & 1.04774 & 0.0972951 & 0.89237 & 0.126707 \\
\hline 10.1175 & 1.03575 & 0.0691628 & 1.03655 & 0.101355 & 0.885866 & 0.122907 \\
\hline 10.1275 & 1.02326 & 0.0686486 & 1.03212 & 0.100581 & 0.878936 & 0.117836 \\
\hline 10.1375 & 1.01992 & 0.0710016 & 1.01301 & 0.0959799 & 0.872559 & 0.116401 \\
\hline 10.1475 & 1.0093 & 0.0679757 & 0.996046 & 0.0874509 & 0.865013 & 0.11495 \\
\hline 10.1575 & 0.996799 & 0.0710563 & 0.975783 & 0.0807713 & 0.855068 & 0.113258 \\
\hline 10.1675 & 0.986551 & 0.0711849 & 0.967922 & 0.0788572 & 0.840471 & 0.111333 \\
\hline 10.1775 & 0.971019 & 0.0615332 & 0.951275 & 0.0766581 & 0.839687 & 0.1114 \\
\hline 10.1875 & 0.950427 & 0.058571 & 0.932457 & 0.079981 & 0.826369 & 0.101202 \\
\hline 10.1975 & 0.934674 & 0.0685486 & 0.92391 & 0.0738384 & 0.812854 & 0.105683 \\
\hline 10.2075 & 0.919694 & 0.0572607 & 0.91438 & 0.0759657 & 0.805363 & 0.106236 \\
\hline 10.2175 & 0.909736 & 0.0558707 & 0.906227 & 0.0824122 & 0.801735 & 0.100331 \\
\hline 10.2275 & 0.897773 & 0.0564313 & 0.898601 & 0.0714432 & 0.796085 & 0.099408 \\
\hline 10.2375 & 0.886672 & 0.0551841 & 0.904678 & 0.0764801 & 0.786752 & 0.096562 \\
\hline 10.2475 & 0.873205 & 0.0571963 & 0.889489 & 0.0713108 & 0.783009 & 0.0990614 \\
\hline 10.2575 & 0.864632 & 0.051994 & 0.893163 & 0.0768475 & 0.783919 & 0.101722 \\
\hline 10.2675 & 0.860904 & 0.0516376 & 0.884093 & 0.0814475 & 0.779177 & 0.0987347 \\
\hline 10.2775 & 0.852472 & 0.0510642 & 0.892111 & 0.0815486 & 0.783841 & 0.0986836 \\
\hline 10.2875 & 0.84593 & 0.0501283 & 0.892813 & 0.0804853 & 0.777449 & 0.0955032 \\
\hline 10.2975 & 0.849101 & 0.0558999 & 0.897999 & 0.0794258 & 0.78296 & 0.0977121 \\
\hline 10.3075 & 0.851031 & 0.0514832 & 0.903199 & 0.0872306 & 0.788563 & 0.0974647 \\
\hline 10.3175 & 0.850446 & 0.0529008 & 0.914889 & 0.093782 & 0.79057 & 0.100518 \\
\hline 10.3275 & 0.85123 & 0.0534266 & 0.92189 & 0.0985558 & 0.792717 & 0.0956865 \\
\hline 10.3375 & 0.854116 & 0.0538052 & 0.928752 & 0.102038 & 0.799658 & 0.0931541 \\
\hline 10.3475 & 0.854369 & 0.0563261 & 0.943577 & 0.123918 & 0.803078 & 0.0992494 \\
\hline 10.3575 & 0.859841 & 0.0595843 & 0.959696 & 0.137342 & 0.807759 & 0.0999378 \\
\hline 10.3675 & 0.863161 & 0.0511305 & 0.982873 & 0.164579 & 0.814672 & 0.102152 \\
\hline 10.3775 & 0.862911 & 0.0541446 & 1.00476 & 0.184217 & 0.821848 & 0.102547 \\
\hline
\end{tabular}




\begin{tabular}{|c|c|c|c|c|c|c|}
\hline 10.3875 & 0.868958 & 0.056019 & 1.03149 & 0.216576 & 0.838001 & 0.105194 \\
\hline 10.3975 & 0.873692 & 0.0553873 & 1.06989 & 0.264422 & 0.85455 & 0.103881 \\
\hline 0.4075 & 0.888931 & 0.056672 & 1.10272 & 0.306331 & 0.871363 & 0.101308 \\
\hline 0.4175 & 0.895136 & 0.0567036 & 1.14326 & 0.353179 & 0.879066 & 0.111556 \\
\hline 10.4275 & 0.898381 & 0.0569745 & 1.18302 & 0.417629 & 0.895178 & 0.113475 \\
\hline 10.4375 & 0.913446 & 0.0525497 & 1.23401 & 0.472365 & 0.918607 & 0.117905 \\
\hline 0.4475 & 0.92202 & 0.056264 & 1.29342 & 0.553 & 0.93693 & 0.117505 \\
\hline 10.4575 & 0.934186 & 0.0522403 & 1.34354 & 0.626817 & 0.963488 & 0.125941 \\
\hline 10.4675 & 0.947053 & 0.0573663 & 1.39787 & 0.714331 & 0.981701 & 0.131978 \\
\hline 10.4775 & 0.950851 & 0.0536879 & 1.4487 & 0.785407 & 1.01 & 0.142065 \\
\hline 10.4875 & 0.974224 & 0.0644851 & 1.50441 & 0.853792 & 1.0312 & 0.152269 \\
\hline 10.4975 & 0.983168 & 0.061576 & 1.55195 & 0.916616 & 1.05306 & 0.160643 \\
\hline 10.5075 & 1.00588 & 0.0615461 & 1.59129 & 0.994706 & 1.0783 & 0.170325 \\
\hline 10.5175 & 1.01605 & 0.0597267 & 1.62589 & 1.05299 & 1.10092 & 0.175531 \\
\hline 10.5275 & 1.03261 & 0.0632485 & 1.64924 & 1.09541 & 1.10846 & 0.182537 \\
\hline 10.5375 & 1.04312 & 0.0689705 & 1.66118 & 1.10403 & 1.11722 & 0.190401 \\
\hline 10.5475 & 1.0545 & 0.0591391 & 1.65821 & 1.11404 & 1.12168 & 0.187847 \\
\hline 10.5575 & 1.06106 & 0.0666118 & 1.6459 & 1.09704 & 1.11775 & 0.194042 \\
\hline 10.5675 & 1.06392 & 0.0632957 & 1.62193 & 1.07 & 1.10999 & 0.185984 \\
\hline 10.5775 & 1.06245 & 0.0624372 & 1.59125 & 1.03593 & 1.09371 & 0.181755 \\
\hline 10.5875 & 1.05927 & 0.0645001 & 1.54151 & 0.963505 & 1.08309 & 0.181675 \\
\hline 10.5975 & 1.04868 & 0.0656524 & 1.4804 & 0.915505 & 1.05859 & 0.173086 \\
\hline 10.6075 & 1.03704 & 0.0599363 & 1.42418 & 0.838111 & 1.03594 & 0.155905 \\
\hline 10.6175 & 1.02294 & 0.0614218 & 1.36829 & 0.754533 & 1.00344 & 0.144482 \\
\hline 10.6275 & 1.0084 & 0.0677555 & 1.30351 & 0.665313 & 0.981074 & 0.14904 \\
\hline 10.6375 & 0.997973 & 0.0536959 & 1.23551 & 0.582288 & 0.948517 & 0.13659 \\
\hline 10.6475 & 0.971608 & 0.0585591 & & 0.494975 & 0.926825 & 0.130235 \\
\hline 10.6575 & 0.955375 & 0.057091 & 1.13602 & 0.447436 & 0.898922 & 0.120061 \\
\hline 10.6675 & 0.940887 & 0.05629 & 1.08176 & 0.365103 & 0.882189 & 0.115075 \\
\hline 10.6775 & 0.921622 & 0.0516174 & 1.04161 & 0.297344 & 0.851856 & 0.105418 \\
\hline 10.6875 & 0.911429 & 0.0543217 & 1.00445 & 0.24818 & 0.831316 & 0.0951057 \\
\hline 10.6975 & 0.895925 & 0.054759 & 0.968341 & 0.198818 & 0.816017 & 0.0975353 \\
\hline 10.7075 & 0.876347 & 0.0521723 & 0.949079 & 0.169095 & 0.804081 & 0.0942409 \\
\hline 10.7175 & 0.865694 & 0.0510803 & 0.924214 & 0.131318 & 0.790218 & 0.0885789 \\
\hline 10.7275 & 0.859734 & 0.0491118 & 0.905215 & 0.108923 & 0.783669 & 0.0895001 \\
\hline 10.7375 & 0.851762 & 0.0469278 & 0.896908 & 0.0932158 & 0.773394 & 0.0837909 \\
\hline 10.7475 & 0.841319 & 0.0501546 & 0.876607 & 0.0796833 & 0.76602 & 0.0861839 \\
\hline 10.7575 & 0.837423 & 0.044464 & 0.870979 & 0.0754505 & 0.759969 & 0.084684 \\
\hline 10.7675 & 0.835913 & 0.0483502 & 0.861055 & 0.064338 & 0.757469 & 0.0816942 \\
\hline 10.7775 & 0.826177 & 0.0466982 & 0.858678 & 0.0651026 & 0.753335 & 0.0820678 \\
\hline 10.7875 & 0.823526 & 0.0471568 & 0.850384 & 0.0614973 & 0.75549 & 0.0791857 \\
\hline 10.7975 & 0.818955 & 0.0461234 & 0.843798 & 0.0521362 & 0.746596 & 0.0770291 \\
\hline 10.8075 & 0.818861 & 0.0514832 & 0.841296 & 0.063882 & 0.745786 & 0.0785219 \\
\hline 10.8175 & 0.820468 & 0.0470431 & 0.836694 & 0.0524787 & 0.745161 & 0.0785549 \\
\hline 10.8275 & 0.808374 & 0.0463291 & 0.833484 & 0.0564695 & 0.74154 & 0.0780169 \\
\hline 10.8375 & 0.810553 & 0.0467335 & 0.835121 & 0.0553274 & 0.734643 & 0.0776283 \\
\hline 10.8475 & 0.800934 & 0.0493602 & 0.831067 & 0.0507662 & 0.736867 & 0.076293 \\
\hline
\end{tabular}




\begin{tabular}{|c|c|c|c|c|c|c|}
\hline 10.8575 & 0.803558 & 0.0491248 & 0.828423 & 0.0523813 & 0.735163 & 0.0744281 \\
\hline 10.8675 & 0.804561 & 0.0476558 & 0.830359 & 0.0542523 & 0.72761 & 0.0795406 \\
\hline 10.8775 & 0.798432 & 0.0498245 & 0.826492 & 0.0507721 & 0.723525 & 0.0723287 \\
\hline 10.8875 & 0.800075 & 0.0492215 & 0.824748 & 0.0595682 & 0.732888 & 0.0749818 \\
\hline 10.8975 & 0.795832 & 0.0486338 & 0.826597 & 0.0529767 & 0.734137 & 0.0736761 \\
\hline 10.9075 & 0.794173 & 0.047181 & 0.824988 & 0.0509287 & 0.727867 & 0.0755601 \\
\hline 10.9175 & 0.796853 & 0.04919 & 0.818482 & 0.0490617 & 0.729941 & 0.0752471 \\
\hline 10.9275 & 0.79327 & 0.0545177 & 0.822197 & 0.0519561 & 0.728989 & 0.0754207 \\
\hline 10.9375 & 0.790204 & 0.0460476 & 0.821818 & 0.0507618 & 0.728998 & 0.0732863 \\
\hline 10.9475 & 0.792455 & 0.0485882 & 0.820195 & 0.0519042 & 0.730603 & 0.0794749 \\
\hline 10.9575 & 0.794694 & 0.0527517 & 0.818772 & 0.0500887 & 0.72587 & 0.0762019 \\
\hline 10.9675 & 0.789182 & 0.0518498 & 0.819759 & 0.0516628 & 0.723493 & 0.0798588 \\
\hline 10.9775 & 0.793352 & 0.0486472 & 0.822492 & 0.0518916 & 0.727033 & 0.0764566 \\
\hline 10.9875 & 0.794104 & 0.0594204 & 0.818238 & 0.0501644 & 0.725751 & 0.0731887 \\
\hline 10.9975 & 0.793315 & 0.0560171 & 0.815844 & 0.0507662 & 0.728748 & 0.0750216 \\
\hline 11.0075 & 0.791534 & 0.0562734 & 0.816172 & 0.0547746 & 0.72861 & 0.0769308 \\
\hline 11.0175 & 0.791951 & 0.0560601 & 0.822554 & 0.0507794 & 0.734026 & 0.0754631 \\
\hline 11.0275 & 0.791736 & 0.0557378 & 0.818499 & 0.0535799 & 0.726589 & 0.078787 \\
\hline 11.0375 & 0.796557 & 0.0548643 & 0.823709 & 0.0541966 & 0.726993 & 0.0757095 \\
\hline 11.0475 & 0.795144 & 0.0554523 & 0.826432 & 0.0492836 & 0.730694 & 0.0812062 \\
\hline 11.0575 & 0.792178 & 0.0563309 & 0.817062 & 0.048637 & 0.73149 & 0.0774945 \\
\hline 11.0675 & 0.7928 & 0.0567921 & 0.823418 & 0.051054 & 0.733203 & 0.0820319 \\
\hline 11.0775 & 0.790737 & 0.0593391 & 0.821704 & 0.0505011 & 0.725665 & 0.0732877 \\
\hline 11.0875 & 0.789563 & 0.056773 & 0.820789 & 0.0497932 & 0.730746 & 0.0810774 \\
\hline 11.0975 & 0.792115 & 0.0620392 & 0.816694 & 0.0531584 & 0.728663 & 0.0767721 \\
\hline 11.1075 & 0.794054 & 0.0569385 & 0.81684 & 0.0496776 & 0.730247 & 0.0833569 \\
\hline 11.1175 & 0.792883 & 0.0630078 & 0.819454 & 0.0491607 & 0.728197 & 0.0837579 \\
\hline 11.1275 & 0.794136 & 0.0590799 & 0.820876 & 0.0538185 & 0.735486 & 0.0819514 \\
\hline 11.1375 & 0.791565 & 0.0577967 & 0.819326 & 0.0495839 & 0.732062 & 0.0843702 \\
\hline 11.1475 & 0.792111 & 0.0571432 & 0.818098 & 0.0483163 & 0.72994 & 0.086525 \\
\hline 11.1575 & 0.786684 & 0.0570317 & 0.82021 & 0.0509362 & 0.725337 & 0.0823477 \\
\hline 11.1675 & 0.790261 & 0.0625084 & 0.816549 & 0.0473122 & 0.733966 & 0.0850578 \\
\hline 11.1775 & 0.79281 & 0.0537726 & 0.820277 & 0.0519025 & 0.734299 & 0.0833455 \\
\hline 11.1875 & 0.793277 & 0.0517425 & 0.810159 & 0.0508354 & 0.731802 & 0.0859079 \\
\hline 11.1975 & 0.786697 & 0.0588738 & 0.816567 & 0.0559819 & 0.730242 & 0.0865414 \\
\hline 11.2075 & 0.794318 & 0.0569768 & 0.818745 & 0.0483314 & 0.736408 & 0.0828183 \\
\hline 11.2175 & 0.791703 & 0.05153 & 0.817802 & 0.04753 & 0.733903 & 0.087024 \\
\hline 11.2275 & 0.787793 & 0.0510582 & 0.821122 & 0.0483061 & 0.738071 & 0.0847086 \\
\hline 11.2375 & 0.788196 & 0.0546202 & 0.818665 & 0.0525564 & 0.735238 & 0.0839835 \\
\hline 11.2475 & 0.790055 & 0.0519077 & 0.820285 & 0.048322 & 0.738347 & 0.0845398 \\
\hline 11.2575 & 0.783587 & 0.0513297 & 0.81584 & 0.0549282 & 0.737078 & 0.0832964 \\
\hline 11.2675 & 0.787054 & 0.050676 & 0.821396 & 0.0522769 & 0.730695 & 0.0781758 \\
\hline 11.2775 & 0.784768 & 0.0528701 & 0.815928 & 0.0528357 & 0.732256 & 0.082408 \\
\hline 11.2875 & 0.786183 & 0.0545083 & 0.816477 & 0.0513961 & 0.740468 & 0.0860518 \\
\hline 11.2975 & 0.787088 & 0.0501279 & 0.813343 & 0.0456221 & 0.735832 & 0.085986 \\
\hline 11.3075 & 0.787124 & 0.0493567 & 0.811556 & 0.0469375 & 0.737404 & 0.0848781 \\
\hline 11.3175 & 0.785539 & 0.0525914 & 0.821316 & 0.0490005 & 0.73878 & 0.0819955 \\
\hline
\end{tabular}




\begin{tabular}{|c|c|c|c|c|c|c|}
\hline 11.3275 & 0.784365 & 0.0495757 & 0.820184 & 0.0548487 & 0.740273 & 0.0816653 \\
\hline 1.3375 & 0.784006 & 0.0457547 & 0.819582 & 0.0533716 & 0.73852 & 0.0869568 \\
\hline 1.3475 & 0.78722 & 0.0467151 & 0.815099 & 0.0486725 & 0.734374 & .0822843 \\
\hline .3575 & 0.78538 & 0.0443066 & 0.810248 & 0.0475268 & 0.731519 & 0.0855302 \\
\hline .3675 & 0.782479 & 0.0476387 & 0.811299 & 0.0534634 & 0.728336 & 0.0779975 \\
\hline 1.3775 & 0.784478 & 0.0471214 & 0.813765 & 0.0508411 & 0.729804 & 0.0787387 \\
\hline 1.3875 & 0.782648 & 0.0486638 & 0.811757 & 0.0465589 & 0.730994 & 0.0810158 \\
\hline 11.3975 & 0.776799 & 0.0434448 & 0.812568 & 0.0502468 & 0.731397 & 0.0801188 \\
\hline 11.4075 & 0.783404 & 0.0483725 & 0.806628 & 0.0468209 & 0.732533 & 0.0800847 \\
\hline 1.4175 & 0.782267 & 0.0473112 & 0.808127 & 0.0458267 & 0.728654 & 0.0801857 \\
\hline 11.4275 & 0.779062 & 0.0451907 & 0.804604 & 0.0485592 & 0.723448 & 0.0800845 \\
\hline 11.4375 & 0.775876 & 0.0449118 & 0.799356 & 0.0475207 & 0.729744 & 0.0739339 \\
\hline 11.4475 & 0.777401 & 0.0453202 & 0.800517 & 0.0453334 & 0.729187 & 0.0749665 \\
\hline 11.4575 & 0.774827 & 0.0445123 & 0.800024 & 0.0438243 & 0.722867 & 0.0763726 \\
\hline 11.4675 & 0.781138 & 0.0448858 & 0.798572 & 0.0458335 & 0.727125 & 0.0820999 \\
\hline 11.4775 & 0.776674 & 0.0453026 & 0.80196 & 0.0461711 & 0.728879 & 0.0735887 \\
\hline 11.4875 & 0.77934 & 0.0437379 & 0.799611 & 0.0486977 & 0.724758 & 0.0804163 \\
\hline 11.4975 & 0.776143 & 0.0509051 & 0.790318 & 0.0475722 & 0.724076 & 0.0750453 \\
\hline 11.5075 & 0.770295 & 0.04 & 0.799022 & 0.04 & 0.727272 & 0.0795554 \\
\hline 11.5175 & 0.773248 & 0.0453887 & 0.799254 & 0.049543 & 0.720483 & 0.0734297 \\
\hline 11.5275 & 0.77389 & 0.0454071 & 0.79619 & 0.0486349 & 0.72159 & 0.0804844 \\
\hline 11.5375 & 0.769612 & 152 & 0.799209 & 0.04 & 0.717218 & 739915 \\
\hline 11.5475 & 0.76956 & 0.0468057 & 0.800743 & 0.0434214 & 0.716111 & 0.0761314 \\
\hline 11.5575 & 0.771602 & 0.0440178 & 0.797891 & 0.0481231 & 0.721963 & 0.0793617 \\
\hline 11.5675 & 0.770485 & 0.0466259 & 0.795822 & 0.0428775 & 0.71979 & 0.0749865 \\
\hline 11.5775 & 0.77534 & 0.0450578 & 0.797397 & 0.0446198 & 0.717374 & 0.0768788 \\
\hline 11.5875 & 0.772785 & 0.0475539 & 0.793471 & 0.0448436 & 0.722345 & 0.0786453 \\
\hline 11.5975 & 0.774839 & 0.0437215 & 0.80044 & 0.042176 & 0.715096 & 0.0783435 \\
\hline 11.6075 & 0.769677 & 0.049243 & 0.792306 & 0.035961 & 0.718596 & 0.0767693 \\
\hline 11.6175 & & 0.04 & 0.796311 & 194 & 0.720606 & 0.0763343 \\
\hline 11.6275 & 0.769077 & 0.0419792 & 0.799541 & 0.042698 & 0.723915 & 0.0794506 \\
\hline 11.6375 & 0.768108 & 0.0454985 & 0.800749 & 0.0465734 & 0.725305 & 0.0759302 \\
\hline 11.6475 & 0.777525 & 0.0451018 & 0.809247 & 0.0467566 & 0.724048 & 0.0734211 \\
\hline 11.6575 & 0.77252 & 0.041885 & 0.805652 & 0.0459444 & 0.727758 & 0.078023 \\
\hline 11.6675 & 0.769151 & 0.0471919 & 0.804464 & 0.0460586 & 0.725636 & 0.0744804 \\
\hline 11.6775 & 0.768913 & 0.046427 & 0.796278 & 0.0470281 & 0.728792 & 0.0817493 \\
\hline 11.6875 & 0.774465 & 0.0408586 & 0.803369 & 0.0466533 & 0.726432 & 0.0803343 \\
\hline 11.6975 & 0.765835 & 0.0404068 & 0.798854 & 0.0426567 & 0.722952 & 0.0778534 \\
\hline 11.7075 & 0.768509 & 0.041883 & 0.806908 & 0.0503609 & 0.726036 & 0.0781683 \\
\hline 11.7175 & 0.766865 & 0.0460049 & 0.801984 & 0.0451519 & 0.727236 & 0.0771695 \\
\hline 11.7275 & 0.775484 & 0.0417109 & 0.803984 & 0.0425368 & 0.723588 & 0.0759059 \\
\hline 11.7375 & 0.775252 & 0.0399239 & 0.795989 & 0.0458717 & 0.72577 & 0.0781275 \\
\hline 11.7475 & 0.775633 & 0.0402032 & 0.802911 & 0.0437629 & 0.723722 & 0.0692781 \\
\hline 11.7575 & 0.779372 & 0.0425642 & 0.798658 & 0.0482386 & 0.728454 & 0.0766564 \\
\hline 11.7675 & 0.776566 & 0.0448945 & 0.798718 & 0.0426586 & 0.725257 & 0.0796019 \\
\hline 11.7775 & 0.76814 & 0.0419654 & 0.805703 & 0.0475378 & 0.721287 & 0.0792571 \\
\hline 11.7875 & 0.773401 & 0.0452698 & 0.800212 & 0.0435175 & 0.720645 & 0.077647 \\
\hline
\end{tabular}




\begin{tabular}{|c|c|c|c|c|c|c|}
\hline 11.7975 & 0.774727 & 0.0443088 & 0.797589 & 0.0413558 & 0.71574 & 0.0743204 \\
\hline 1.8075 & 0.775138 & 0.0442403 & 0.797063 & 0.0434745 & 0.715781 & 0.070183 \\
\hline 1.8175 & 0.772109 & 0.0422454 & 0.793299 & 0.045289 & 0.720633 & 0.0723627 \\
\hline .8275 & 0.765601 & 0.045476 & .798591 & 0.0445364 & 0.720599 & 0.0754654 \\
\hline .8375 & 0.77024 & 0.045607 & 0.7994 & 0.0468326 & 0.716439 & 0.0716732 \\
\hline 1.8475 & 0.769009 & 0.0436245 & 0.795769 & 0.0449025 & 0.718278 & 0.0707824 \\
\hline .8575 & 0.771535 & 0.0434684 & 0.793755 & 0.0472354 & 0.715293 & 0.0728109 \\
\hline 11.8675 & 0.768531 & 0.0384548 & 0.790963 & 0.0455706 & 0.716706 & 0.0738084 \\
\hline 1.8775 & 0.76707 & 0.0395556 & 0.794478 & 0.043047 & 0.711797 & 0.0708877 \\
\hline 1.8875 & 0.763337 & 0.0424559 & 0.784732 & 0.0398243 & 0.715584 & 0.0712914 \\
\hline 11.8975 & 0.767328 & 0.0443728 & 0.789794 & 0.0454905 & 0.713774 & 0.0688338 \\
\hline 1.9075 & 0.768258 & 0.0481804 & 0.790272 & 0.046103 & 0.711945 & 0.0665018 \\
\hline 11.9175 & 0.765562 & 0.0425093 & 0.796739 & 0.0409383 & 0.71292 & 0.073999 \\
\hline 11.9275 & 0.764646 & 0.0442966 & 0.789537 & 0.0386008 & 0.705728 & 0.0700926 \\
\hline 11.9375 & 0.763197 & 0.042231 & 0.790961 & 0.0463507 & 0.707153 & 0.0726315 \\
\hline 11.9475 & 0.759154 & 0.0410979 & 0.788226 & 0.0437279 & 0.706718 & 0.0715602 \\
\hline 11.9575 & 0.759469 & 0.0433091 & 0.782205 & 0.0435764 & 0.706561 & 0.0705912 \\
\hline 11.9675 & 0.761731 & 0.0417018 & 0.786338 & 0.0467887 & 0.705574 & 0.0677752 \\
\hline 11.9775 & 0.754877 & 0.0410968 & 0.790686 & 6611 & 0.704303 & 8667 \\
\hline 11.9875 & 0.754634 & 0.0437563 & 0.775913 & 0.046123 & 0.707764 & 0.070919 \\
\hline 11.9975 & 0.755687 & 0.0380896 & 0.78736 & 0.0463901 & 0.700042 & 0.06499 \\
\hline 12.0075 & 0.750391 & 0.0424873 & 0.776663 & 0.0422038 & 0.69588 & 0.0652602 \\
\hline 12.0175 & 0.749881 & 0.0443389 & 0.783451 & 0.0477134 & 0.693701 & 0.0671177 \\
\hline 12.0275 & 0.753492 & 0.0462259 & 0.77 & 7309 & 0.702078 & 096 \\
\hline 12.0375 & 0.749941 & 0.0463629 & 0.779617 & 0.0472201 & 0.698938 & 0.0695269 \\
\hline 12.0475 & 0.747849 & 0.0424573 & 0.782966 & 0.0476116 & 0.694371 & 4963 \\
\hline 12.0 & 0.746326 & 76837 & 811 & 0.05 & & 797 \\
\hline 12.0675 & 0.744044 & 0.0473997 & 0.776225 & 0.0443 & 0.691239 & 0.067528 \\
\hline 12.0775 & 0.748953 & 0.0386165 & 0.773367 & 0.0525046 & 0.695434 & 0.069594 \\
\hline 12.0875 & 0.743267 & 0.0432825 & 0.777236 & 0.0458248 & 0.689041 & 2575 \\
\hline 12.0975 & 0.742194 & 0.0432065 & 0.781129 & 0.0566457 & 0.690648 & 0.0684383 \\
\hline 12.1075 & 0.74188 & 0.0378259 & 0.77246 & 0.0504136 & 0.692573 & 0.0680457 \\
\hline 12.1175 & 0.74271 & 0.0372686 & 0.773515 & 0.0527794 & 0.685841 & 4922 \\
\hline 12.1275 & 0.740518 & 0.0434652 & 0.778524 & 0.0544928 & 0.691527 & 0.0653033 \\
\hline 12.1375 & 0.743017 & 0.0431073 & & 0.0525773 & 0.692432 & 0.0643386 \\
\hline 12.1475 & 0.737661 & 0.0403176 & 0.774968 & 0.05576 & 0.690302 & 0.0669352 \\
\hline 12.1575 & 0.736863 & 0.0430972 & 0.775994 & 0.0614005 & 0.685827 & 0.0660815 \\
\hline 12.1675 & 0.736233 & 0.0422995 & 0.773845 & 0.0537765 & 0.68312 & 0.0671594 \\
\hline 12.1775 & 0.735321 & 0.0431173 & 0.780721 & 0.0594079 & 0.685025 & 0.0663427 \\
\hline 12.1875 & 0.732953 & 0.0397676 & 0.771226 & 0.0644243 & 0.681176 & 0.0686028 \\
\hline 12.1975 & 0.730822 & 0.0473642 & 0.777069 & 0.0564087 & 0.690435 & 0.0653628 \\
\hline 12.2075 & 0.736955 & 0.042791 & 0.777979 & 0.063419 & 0.683471 & 0.0695055 \\
\hline 12.2175 & 0.733159 & 0.0452416 & 0.780479 & 0.0655534 & 0.684781 & 0.0680261 \\
\hline 12.2275 & 0.731016 & 0.0450128 & 0.780398 & 0.064962 & 0.682173 & 0.0650608 \\
\hline 12.2375 & 0.728539 & 0.0431955 & 0.779137 & 0.0662783 & 0.676951 & 0.0651604 \\
\hline 12.2475 & 0.732946 & 0.0437401 & 0.777535 & 0.058057 & 0.68498 & 0.0669692 \\
\hline 12.2575 & 0.73561 & 0.0416731 & 0.780489 & 0.0682601 & 0.678759 & 0.0677311 \\
\hline
\end{tabular}




\begin{tabular}{|c|c|c|c|c|c|c|}
\hline 12.2675 & 0.730457 & 0.0469497 & 0.770894 & 0.0750544 & 0.678222 & 0.065759 \\
\hline 12.2775 & 0.732333 & 0.0459747 & 0.777359 & 0.0758925 & 0.677354 & 0.0659866 \\
\hline 12.2875 & 0.729175 & 0.0438195 & 0.769943 & 0.0646192 & 0.678732 & 0.06455 \\
\hline 12.2975 & 0.725322 & 0.0457278 & 0.771488 & 0.0782008 & 0.677428 & 0.0676717 \\
\hline 12.3075 & 0.718143 & 0.0413188 & 0.77104 & 0.0719237 & 0.671935 & 0.0673057 \\
\hline 12.3175 & 0.721614 & 0.0421627 & 0.770272 & 0.0696916 & 0.674922 & 0.0671852 \\
\hline 12.3275 & 0.72277 & 0.0407296 & 0.768976 & 0.0668848 & 0.670877 & 0.0659371 \\
\hline 12.3375 & 0.725551 & 0.0375026 & 0.770101 & 0.0732184 & 0.669411 & 0.0644368 \\
\hline 12.3475 & 0.717344 & 0.0423311 & 0.763565 & 0.0740044 & 0.66923 & 0.0656827 \\
\hline 12.3575 & 0.720422 & 0.0400462 & 0.766531 & 0.0731832 & 0.667551 & 0.0640453 \\
\hline 12.3675 & 0.718507 & 0.0411155 & 0.764719 & 0.0757885 & 0.665551 & 0.0656303 \\
\hline 12.3775 & 0.713973 & 0.0407415 & 0.764837 & 0.0744686 & 0.661972 & 0.0627078 \\
\hline 12.3875 & 0.70894 & 0.0383681 & 0.756958 & 0.0732029 & 0.663726 & 0.0654776 \\
\hline 12.3975 & 0.712162 & 0.0411331 & 0.7614 & 0.0823972 & 0.661741 & 0.0625851 \\
\hline 12.4075 & 0.708111 & 0.0478577 & 0.759601 & 0.0861475 & 0.666095 & 0.0647983 \\
\hline 12.4175 & 0.704441 & 0.0358494 & 0.773882 & 0.096696 & 0.660347 & 0.0627561 \\
\hline 12.4275 & 0.700303 & 0.0405724 & 0.78126 & 0.106657 & 0.665646 & 0.0585787 \\
\hline 12.4375 & 0.702398 & 0.0365783 & 0.792929 & 0.121635 & 0.667396 & 0.0650661 \\
\hline 12.4475 & 0.704751 & 0.039743 & 0.80706 & 0.160988 & 0.670676 & 0.067728 \\
\hline 12.4575 & 0.702503 & 0.0408468 & 0.827341 & 0.183955 & 0.680162 & 0.0698404 \\
\hline 12.4675 & 0.702769 & 0.035556 & 0.844492 & 0.214245 & 0.68239 & 0.0688697 \\
\hline 12.4775 & 0.703854 & 0.0398898 & 0.87173 & 0.259562 & 0.690214 & 0.072451 \\
\hline 12.4875 & 0.707501 & 0.0431185 & 0.89567 & 0.291713 & 0.702837 & 0.0712993 \\
\hline 12.4975 & 0.711096 & 0.038363 & 0.935791 & 0.356751 & 0.714072 & 0.0821679 \\
\hline 12.5075 & 0.713264 & 0.0396173 & 0.973305 & 0.394893 & 0.725697 & 0.0926507 \\
\hline 12.5175 & 0.723151 & 0.0396264 & 1.00336 & 0.459998 & 0.736037 & 0.0980878 \\
\hline 12.5275 & 0.731191 & 0.0395669 & 1.04577 & 0.526167 & 0.76088 & 0.107532 \\
\hline 12.5375 & 0.737548 & 0.043418 & 1.08385 & 0.586823 & 0.776577 & 0.119698 \\
\hline 12.5475 & 0.752013 & 0.0411013 & 1.12711 & 0.661854 & 0.791851 & 0.122551 \\
\hline 12.5575 & 0.750988 & 0.0400233 & 1.1625 & 0.713871 & 0.811541 & 0.136802 \\
\hline 12.5675 & 0.769324 & 0.0440326 & 1.19146 & 0.765131 & 0.824449 & 0.152149 \\
\hline 12.5775 & 0.775533 & 0.0417277 & 1.20786 & 0.797561 & 0.837946 & 0.157943 \\
\hline 12.5875 & 0.783402 & 0.0414781 & 1.23754 & 0.840481 & 0.844984 & 0.160625 \\
\hline 12.5975 & 0.798143 & 0.0455832 & 1.25722 & 0.859043 & 0.855541 & 0.169595 \\
\hline 12.6075 & 0.804783 & 0.0456372 & 1.26679 & 0.884341 & 0.861009 & 0.171505 \\
\hline 12.6175 & 0.81592 & 0.0463505 & 1.26707 & 0.89529 & 0.867849 & 0.174356 \\
\hline 12.6275 & 0.82071 & 0.0470171 & 1.25338 & 0.878038 & 0.872058 & 0.180931 \\
\hline 12.6375 & 0.82389 & 0.0436313 & 1.2415 & 0.847344 & 0.866677 & 0.173702 \\
\hline 12.6475 & 0.830505 & 0.0498503 & 1.22052 & 0.804172 & 0.859774 & 0.170917 \\
\hline 12.6575 & 0.835379 & 0.0468189 & 1.18994 & 0.758879 & 0.853908 & 0.164937 \\
\hline 12.6675 & 0.836097 & 0.0462655 & 1.15574 & 0.727991 & 0.84112 & 0.162551 \\
\hline 12.6775 & 0.835671 & 0.0449627 & 1.12505 & 0.659875 & 0.834951 & 0.151106 \\
\hline 12.6875 & 0.834832 & 0.0477749 & 1.0909 & 0.602115 & 0.825059 & 0.147701 \\
\hline 12.6975 & 0.835373 & 0.0444732 & 1.05707 & 0.551195 & 0.806221 & 0.135642 \\
\hline 12.7075 & 0.830904 & 0.0458691 & 1.02302 & 0.501751 & 0.795512 & 0.122294 \\
\hline 12.7175 & 0.82588 & 0.0478097 & 0.995856 & 0.446442 & 0.78089 & 0.121252 \\
\hline 12.7275 & 0.824252 & 0.0545475 & 0.965816 & 0.402691 & 0.764812 & 0.108232 \\
\hline
\end{tabular}




\begin{tabular}{|c|c|c|c|c|c|c|}
\hline 12.7375 & 0.819304 & 0.0531401 & 0.934476 & 0.346198 & 0.755878 & 0.102793 \\
\hline 12.7475 & 0.816403 & 0.0494271 & 0.909153 & 0.297548 & 0.74591 & 0.102581 \\
\hline 2.7575 & 0.815648 & 0.0568053 & 0.88639 & 0.254687 & 0.731732 & 0.0987985 \\
\hline 2.7675 & 0.815855 & 0.0608704 & 0.863468 & 0.238791 & 0.723343 & 0.0960721 \\
\hline 12.7775 & 0.809668 & 0.0631797 & 0.852396 & 0.21607 & 0.716435 & 0.0913902 \\
\hline 2.7875 & 0.806319 & 0.0577773 & 0.830452 & 0.182885 & 0.711517 & 0.0929904 \\
\hline 2.7975 & 0.811158 & 0.0580677 & 0.815039 & 0.16371 & 0.702334 & 0.0901823 \\
\hline 12.8075 & 0.800066 & 0.0555468 & 0.803366 & 0.145893 & 0.691569 & 0.0853117 \\
\hline 12.8175 & 0.793056 & 0.0691608 & 0.792067 & 0.133705 & 0.682304 & 0.0877085 \\
\hline 12.8275 & 0.785911 & 0.057954 & 0.776895 & 0.114251 & 0.680464 & 0.0821453 \\
\hline 12.8375 & 0.780076 & 0.0634839 & 0.758199 & 0.110371 & 0.671963 & 0.0781087 \\
\hline 12.8475 & 0.773957 & 0.054884 & 0.748064 & 0.0990969 & 0.670524 & 0.077676 \\
\hline 12.8575 & 0.762192 & 0.0542376 & 0.741558 & 0.0946808 & 0.658132 & 0.071689 \\
\hline 12.8675 & 0.75662 & 0.0559559 & 0.741241 & 0.0808839 & 0.650704 & 0.0697394 \\
\hline 12.8775 & 0.743885 & 0.0480127 & 0.728619 & 0.0846674 & 0.643542 & 0.0683996 \\
\hline 12.8875 & 0.73527 & 0.0505327 & 0.715358 & 0.0719402 & 0.642021 & 0.0654426 \\
\hline 12.8975 & 0.723803 & 0.046368 & 0.712152 & 0.0670676 & 0.638593 & 0.0651572 \\
\hline 12.9075 & 0.7165 & 0.0474436 & 0.704645 & 0.0646925 & 0.62659 & 0.0606907 \\
\hline 12.9175 & 0.700765 & 0.0441268 & 0.70002 & 13769 & 0.625237 & 0.0580473 \\
\hline 12.9275 & 0.695463 & 0.0446624 & 0.689442 & 0.0629895 & 0.620018 & 0.0593522 \\
\hline 12.9375 & 0.68584 & 0.0425895 & 0.686333 & 0.0636736 & 0.614191 & 0.052725 \\
\hline 12.9475 & 0.67 & 0.0384427 & 0.688256 & 0.0601585 & 0.615252 & 0.0549249 \\
\hline 12.9575 & 0.674036 & 0.0358357 & 0.679442 & 0.0599647 & 0.610266 & 0.0558769 \\
\hline 12.9675 & 0.66 & 0.0393041 & 0.6771 & 0.0588442 & 968 & 0.0511323 \\
\hline 12.9775 & 0.657924 & 0.0347745 & 0.674461 & 0.0579668 & 0.603853 & 0.0544604 \\
\hline 12.9875 & 0.653898 & 0.0336174 & 0.670066 & 0.0631448 & 0.597552 & 0.0565906 \\
\hline 12.9975 & 0.65 & 0.036431 & & 126 & & 325 \\
\hline 13.0075 & 0.645044 & 0.0367123 & 0.66675 & 0.0582516 & 0.593572 & 0.0509142 \\
\hline 13.0175 & 0.63544 & 0.0320798 & 0.665638 & 0.0586725 & 0.591547 & 0.0551068 \\
\hline 13.0275 & 0.632166 & 0.0367013 & 0.658137 & 0.0582925 & 0.588303 & 0.0541329 \\
\hline 13.0375 & 0.635033 & 0.0359392 & 0.658347 & 0.0552597 & 0.58495 & 0.0531391 \\
\hline 13.0475 & 0.626593 & 0.0330578 & 0.651472 & 0.0513754 & 0.584004 & 0.0528374 \\
\hline 13.0575 & 0.622386 & 0.0368476 & 0.642894 & 0.0457873 & 0.582759 & 0.0521291 \\
\hline 13.0675 & 0.619066 & 0.0377116 & 0.641883 & 0.0501273 & 0.582778 & 0.0527801 \\
\hline 13.0775 & 0.617295 & 0.0375835 & 0.638063 & 0.0443028 & 0.580133 & 0.05174 \\
\hline 13.0875 & 0.620077 & 0.0390525 & 0.6385 & 0.0469117 & 0.57372 & 0.0520364 \\
\hline 13.0975 & 0.609382 & 0.0356786 & 0.62534 & 0.0434001 & 0.570363 & 0.0554862 \\
\hline 13.1075 & 0.607811 & 0.0353501 & 0.625424 & 0.0437053 & 0.570244 & 0.0552146 \\
\hline 13.1175 & 0.607336 & 0.0352784 & 0.625133 & 0.0385281 & 0.567072 & 0.0552915 \\
\hline 13.1275 & 0.608119 & 0.0353346 & 0.624256 & 0.0373326 & 0.570479 & 0.0497258 \\
\hline 13.1375 & 0.602498 & 0.0332422 & 0.616844 & 0.035025 & 0.571478 & 0.0540773 \\
\hline 13.1475 & 0.597759 & 0.0353426 & 0.620903 & 0.0399508 & 0.562305 & 0.0551465 \\
\hline 13.1575 & 0.594383 & 0.0344503 & 0.615005 & 0.0392646 & 0.563982 & 0.0548146 \\
\hline 13.1675 & 0.597919 & 0.0378278 & 0.620011 & 0.0380576 & 0.565032 & 0.0554261 \\
\hline 13.1775 & 0.59677 & 0.0337453 & 0.616202 & 0.0352368 & 0.563146 & 0.0553087 \\
\hline 13.1875 & 0.597813 & 0.0334247 & 0.614097 & 0.0340805 & 0.563684 & 0.0504486 \\
\hline 13.1975 & 0.596311 & 0.0341597 & 0.621366 & 0.0334658 & 0.566456 & 0.054527 \\
\hline
\end{tabular}




\begin{tabular}{|c|c|c|c|c|c|c|}
\hline 13.2075 & 0.597124 & 0.0344335 & 0.621448 & 0.0363397 & 0.56598 & 0.0550609 \\
\hline 13.2175 & 0.595165 & 0.0325656 & 0.614777 & 0.0395894 & 0.56612 & 0.0569751 \\
\hline 13.2275 & 0.600251 & 0.0346998 & 0.625087 & 0.0380718 & 0.56614 & 0.0547079 \\
\hline 3.2375 & 0.598124 & 0.0310324 & 0.633151 & 0.0342652 & 0.570098 & 0.0568344 \\
\hline 3.2475 & 0.598204 & 0.0328631 & 0.632212 & 0.0401234 & 0.571559 & 0.0543724 \\
\hline 13.2575 & 0.602293 & 0.0351055 & 0.640879 & 0.0375952 & 0.573042 & 0.0528424 \\
\hline 13.2675 & 0.606518 & 0.0359934 & 0.642242 & 0.036701 & 0.580325 & 0.0573582 \\
\hline 13.2775 & 0.608511 & 0.0329398 & 0.650144 & 0.0349276 & 0.583322 & 0.0563666 \\
\hline 13.2875 & 0.615542 & 0.0302259 & 0.659697 & 0.0390163 & 0.586896 & 0.0566787 \\
\hline 13.2975 & 0.61382 & 0.0348411 & 0.664502 & 0.0334856 & 0.58961 & 0.0583796 \\
\hline 13.3075 & 0.620212 & 0.0333979 & 0.665425 & 0.0356665 & 0.593177 & 0.0563982 \\
\hline 13.3175 & 0.622732 & 0.0346071 & 0.671862 & 0.0402398 & 0.596035 & 0.0585833 \\
\hline 13.3275 & 0.630509 & 0.0335134 & 0.67767 & 0.0412083 & 0.603057 & 0.0614956 \\
\hline 13.3375 & 0.638128 & 0.0378851 & 0.674893 & 0.0413629 & 0.607888 & 0.0578837 \\
\hline 13.3475 & 0.644895 & 0.0345362 & 0.67945 & 0.0443589 & 0.610739 & 0.056727 \\
\hline 13.3575 & 0.649753 & 0.0343244 & 0.687982 & 0.0396762 & 0.607524 & 0.0572407 \\
\hline 13.3675 & 0.651639 & 0.0348326 & 0.681882 & 0.0369274 & 0.607249 & 0.0590085 \\
\hline 13.3775 & 0.657905 & 0.0371677 & 0.682589 & 0.0402701 & 0.607804 & 0.058677 \\
\hline 13.3875 & 0.665249 & 0.0352141 & 0.684553 & 0.0372349 & 0.61243 & 0.0586014 \\
\hline 13.3975 & 0.670188 & 0.0340793 & 0.676472 & 0.0400753 & 0.605238 & 0.0561826 \\
\hline 13.4075 & 0.667485 & 0.038564 & 0.681037 & 0.0345162 & 0.607743 & 0.0616011 \\
\hline 13.4175 & 0.67399 & 0.0371237 & 0.679446 & 0.0405363 & 0.610582 & 0.059764 \\
\hline 13.4275 & 0.66529 & 0.0358588 & 0.666345 & 0.0413908 & 0.601087 & 0.0620263 \\
\hline 13.4375 & 0.665871 & 0.0373357 & 0.657946 & 0.0440649 & 0.595649 & 0.0587271 \\
\hline 13.4475 & 0.66686 & 0.0391493 & 0.655401 & 0.036965 & 0.59312 & 0.0569629 \\
\hline 13.4575 & 0.663163 & 0.0320649 & 0.639148 & 0.0399415 & 0.586771 & 0.059373 \\
\hline 13.4675 & 0.654078 & 0.03989 & 0.635453 & 0.0440452 & 0.585927 & 0.0564191 \\
\hline 13.4775 & 0.652361 & 0.0367009 & 0.631202 & 0.0379622 & 0.576085 & 0.0553419 \\
\hline 13.4875 & 0.649152 & 0.0354328 & 0.627266 & 0.0377449 & 0.567659 & 0.0524593 \\
\hline 13.4975 & 0.638604 & 0.0321258 & 0.613138 & 0.0405907 & 0.560389 & 0.048704 \\
\hline 13.5075 & 0.63495 & 0.0333414 & 0.608128 & 0.0359873 & 0.562448 & 0.0511728 \\
\hline 13.5175 & 0.62157 & 0.0361052 & 0.597121 & 0.035017 & 0.552225 & 0.0511004 \\
\hline 13.5275 & 0.617229 & 0.034671 & 0.591056 & 0.0310171 & 0.548797 & 0.0486974 \\
\hline 13.5375 & 0.610413 & 0.0344267 & 0.588578 & 0.0340143 & 0.541439 & 0.0485761 \\
\hline 13.5475 & 0.602322 & 0.0363174 & 0.582456 & 0.033294 & 0.538465 & 0.0482296 \\
\hline 13.5575 & 0.597274 & 0.0347469 & 0.57882 & 0.0354304 & 0.534269 & 0.0444298 \\
\hline 13.5675 & 0.587682 & 0.0322832 & 0.576957 & 0.0325095 & 0.531936 & 0.0443373 \\
\hline 13.5775 & 0.584122 & 0.0329755 & 0.574456 & 0.0291139 & 0.531369 & 0.0426317 \\
\hline 13.5875 & 0.579508 & 0.0333262 & 0.569501 & 0.0268543 & 0.52574 & 0.0421066 \\
\hline 13.5975 & 0.569773 & 0.0314857 & 0.564899 & 0.0310935 & 0.523395 & 0.0471613 \\
\hline 13.6075 & 0.561523 & 0.031434 & 0.564056 & 0.0326462 & 0.521795 & 0.0472778 \\
\hline 13.6175 & 0.562769 & 0.030841 & 0.565016 & 0.0284896 & 0.520399 & 0.0500323 \\
\hline 13.6275 & 0.556745 & 0.030518 & 0.561612 & 0.032351 & 0.522186 & 0.0427689 \\
\hline 13.6375 & 0.552901 & 0.0322545 & 0.559546 & 0.0338222 & 0.513519 & 0.0438085 \\
\hline 13.6475 & 0.55384 & 0.0311074 & 0.557762 & 0.0280375 & 0.515181 & 0.0457527 \\
\hline 13.6575 & 0.547347 & 0.0315468 & 0.556622 & 0.0282238 & 0.516572 & 0.0438589 \\
\hline 13.6675 & 0.552202 & 0.0316068 & 0.553859 & 0.0347808 & 0.509118 & 0.0424357 \\
\hline
\end{tabular}




\begin{tabular}{|c|c|c|c|c|c|c|}
\hline 13.6775 & 0.549294 & 0.0314645 & 0.546811 & 0.0320673 & 0.511104 & 0.0470942 \\
\hline 13.6875 & 0.545279 & 0.0317966 & 0.557075 & 0.0288545 & 0.51209 & 0.0458092 \\
\hline 13.6975 & 0.542851 & 0.0329616 & 0.550209 & 0.0296352 & 0.509721 & 0.0451238 \\
\hline 13.7075 & 0.541401 & 0.0342537 & 0.550119 & 0.0311967 & 0.509114 & 0.046236 \\
\hline 13.7175 & 0.537681 & 0.0320962 & 0.546759 & 0.0299065 & 0.508436 & 0.0430864 \\
\hline 13.7275 & 0.541305 & 0.0323831 & 0.55101 & 0.0283676 & 0.508559 & 0.0440896 \\
\hline 13.7375 & 0.539037 & 0.0325864 & 0.54912 & 0.0295412 & 0.505616 & 0.0424319 \\
\hline 13.7475 & 0.543667 & 0.0319088 & 0.548886 & 0.0287417 & 0.504353 & 0.0452133 \\
\hline 13.7575 & 0.536896 & 0.027729 & 0.546125 & 0.0334017 & 0.511673 & 0.0466622 \\
\hline 13.7675 & 0.536563 & 0.0297973 & 0.544782 & 0.031464 & 0.505082 & 0.0467284 \\
\hline 13.7775 & 0.53065 & 0.0332996 & 0.547951 & 0.0328881 & 0.505113 & 0.0425821 \\
\hline 13.7875 & 0.530478 & 0.0279789 & 0.543395 & 0.0334369 & 0.50227 & 0.041717 \\
\hline 13.7975 & 0.532068 & 0.031178 & 0.543871 & 0.0303872 & 0.506406 & 0.0422619 \\
\hline 13.8075 & 0.53102 & 0.0279785 & 0.543739 & 0.0333995 & 0.501917 & 0.0459514 \\
\hline 13.8175 & 0.528469 & 0.0300559 & 0.549942 & 0.0336088 & 0.503653 & 0.0429438 \\
\hline 13.8275 & 0.527667 & 0.0297856 & 0.54256 & 0.0348687 & 0.499107 & 0.0393361 \\
\hline 13.8375 & 0.528806 & 0.0316138 & 0.546639 & 0.0333058 & 0.501553 & 0.044468 \\
\hline 13.8475 & 0.531142 & 0.0316497 & 0.547267 & 0.037551 & 0.50173 & 0.0447044 \\
\hline 13.8575 & 0.534768 & 0.0276108 & 0.554674 & 0.0462333 & 0.505355 & 0.0440603 \\
\hline 13.8675 & 0.53209 & 0.0320015 & 0.562024 & 0.0473445 & 0.502261 & 0.0420632 \\
\hline 13.8775 & 0.525002 & 0.0288012 & 0.563065 & 0.0528453 & 0.501993 & 0.0445205 \\
\hline 13.8875 & 0.533279 & 0.0311931 & 0.569771 & 0.0613851 & 0.509116 & 0.046449 \\
\hline 13.8975 & 0.534668 & 0.029271 & 0.577307 & 0.0729838 & 0.516663 & 0.0431265 \\
\hline 13.9075 & 0.533004 & 0.0294436 & 0.588547 & 0.0761891 & 0.513637 & 0.0438039 \\
\hline 13.9175 & 0.534319 & 0.0327847 & 0.602386 & 0.095608 & 0.522902 & 0.0422717 \\
\hline 13.9275 & 0.535725 & 0.029605 & 0.625702 & 0.113504 & 0.523769 & 0.044134 \\
\hline 13.9375 & 0.545391 & 0.0300971 & 0.642535 & 0.135632 & 0.538124 & 0.0449229 \\
\hline 13.9475 & 0.552672 & 0.0351394 & 0.673226 & 0.172932 & 0.542513 & 0.0480645 \\
\hline 13.9575 & 0.556674 & 0.0320274 & 0.707957 & 0.210364 & 0.559486 & 0.0490361 \\
\hline 13.9675 & 0.566803 & 0.0335339 & 0.74114 & 0.239863 & 0.573808 & 0.0550299 \\
\hline 13.9775 & 0.573266 & 0.0366488 & 0.781393 & 0.2937 & 0.592347 & 0.0503903 \\
\hline 13.9875 & 0.586891 & 0.0369582 & 0.836387 & 0.347023 & 0.610938 & 0.0526318 \\
\hline 13.9975 & 0.603701 & 0.0413489 & 0.882118 & 0.397751 & 0.636171 & 0.0599066 \\
\hline 14.0075 & 0.620998 & 0.045542 & 0.945243 & 0.474544 & 0.664381 & 0.0635733 \\
\hline 14.0175 & 0.64411 & 0.0529252 & 1.00566 & 0.533338 & 0.686539 & 0.0668999 \\
\hline 14.0275 & 0.669961 & 0.0631504 & 1.07722 & 0.603788 & 0.725516 & 0.0740798 \\
\hline 14.0375 & 0.690419 & 0.0666313 & 1.14452 & 0.676998 & 0.752441 & 0.0773144 \\
\hline 14.0475 & 0.721484 & 0.0694388 & 1.2075 & 0.754616 & 0.784588 & 0.083149 \\
\hline 14.0575 & 0.763068 & 0.0850861 & 1.27605 & 0.813674 & 0.81781 & 0.0872607 \\
\hline 14.0675 & 0.800591 & 0.0861977 & 1.34958 & 0.879673 & 0.851711 & 0.0921413 \\
\hline 14.0775 & 0.833046 & 0.0977673 & 1.40742 & 0.962339 & 0.892107 & 0.105135 \\
\hline 14.0875 & 0.874494 & 0.117201 & 1.45758 & 1.00607 & 0.914615 & 0.106764 \\
\hline 14.0975 & 0.911007 & 0.124883 & 1.51198 & 1.06176 & 0.94771 & 0.104505 \\
\hline 14.1075 & 0.93906 & 0.133971 & 1.53989 & 1.08933 & 0.972794 & 0.115654 \\
\hline 14.1175 & 0.985862 & 0.154553 & 1.56967 & 1.13285 & 0.989124 & 0.111602 \\
\hline 14.1275 & 1.01491 & 0.156298 & 1.58436 & 1.14871 & 1.00425 & 0.121861 \\
\hline 14.1375 & 1.03727 & 0.164102 & 1.58473 & 1.12941 & 1.00745 & 0.123629 \\
\hline
\end{tabular}




\begin{tabular}{|c|c|c|c|c|c|c|}
\hline 14.1475 & 1.06564 & 0.170282 & 1.56557 & 1.10057 & 1.01236 & 0.129639 \\
\hline 14.1575 & 1.07935 & 0.163815 & 1.53599 & 1.09138 & 1.00503 & 0.125029 \\
\hline 14.1675 & 1.09354 & 0.178614 & 1.50707 & 1.04072 & 0.989182 & 0.118863 \\
\hline 14.1775 & 1.09336 & 0.177051 & 1.45983 & 1.02007 & 0.97729 & 0.118407 \\
\hline 14.1875 & 1.09433 & 0.169178 & 1.39982 & 0.940575 & 0.952927 & 0.112546 \\
\hline 14.1975 & 1.08052 & 0.173836 & 1.33945 & 0.871143 & 0.922999 & 0.110698 \\
\hline 14.2075 & 1.05891 & 0.169307 & 1.28019 & 0.814417 & 0.898759 & 0.106937 \\
\hline 14.2175 & 1.03277 & 0.15736 & 1.19151 & 0.740506 & 0.865101 & 0.0997458 \\
\hline 14.2275 & 1.01235 & 0.153469 & 1.12332 & 0.668635 & 0.828114 & 0.0918659 \\
\hline 14.2375 & 0.981451 & 0.139014 & 1.05354 & 0.592433 & 0.787323 & 0.0861403 \\
\hline 14.2475 & 0.938368 & 0.142738 & 0.983078 & 0.505832 & 0.753965 & 0.0807869 \\
\hline 14.2575 & 0.899711 & 0.121553 & 0.923531 & 0.450814 & 0.713947 & 0.0742683 \\
\hline 14.2675 & 0.864875 & 0.113863 & 0.856592 & 0.38885 & 0.676596 & 0.0703966 \\
\hline 14.2775 & 0.819071 & 0.107111 & 0.79444 & 0.322945 & 0.652067 & 0.0571923 \\
\hline 14.2875 & 0.778905 & 0.0926059 & 0.744331 & 0.273253 & 0.615858 & 0.0586227 \\
\hline 14.2975 & 0.739455 & 0.0830006 & 0.697367 & 0.224339 & 0.584917 & 0.0544655 \\
\hline 14.3075 & 0.706122 & 0.0775909 & 0.657939 & 0.185441 & 0.565695 & 0.0532687 \\
\hline 14.3175 & 0.676827 & 0.0588702 & 0.62498 & 0.15087 & 0.540122 & 0.0471244 \\
\hline 14.3275 & 0.636942 & 0.060383 & 0.59461 & 0.121317 & 0.525798 & 0.0481686 \\
\hline 14.3375 & 0.607782 & 0.0446278 & 0.568924 & 0.0991367 & 0.511876 & 0.0441616 \\
\hline 14.3475 & 0.582737 & 0.0454162 & 0.548626 & 0.0785711 & 0.496885 & 0.0451995 \\
\hline 14.3575 & 0.566267 & 0.0379423 & 0.539114 & 0.0680769 & 0.481441 & 0.0429488 \\
\hline 14.3675 & 0.544537 & 0.0350785 & 0.521041 & 0.0506333 & 0.47653 & 0.0434769 \\
\hline 14.3775 & 0.524942 & 0.0377584 & 0.514381 & 0.0503531 & 0.46847 & 0.0420876 \\
\hline 14.3875 & 0.516973 & 0.0336214 & 0.497884 & 0.042335 & 0.462193 & 0.0417199 \\
\hline 14.3975 & 0.508909 & 0.0336032 & 0.493355 & 0.0347028 & 0.457769 & 0.0378616 \\
\hline 14.4075 & 0.492953 & 0.028316 & 0.487619 & 0.0302743 & 0.453813 & 0.0400384 \\
\hline 14.4175 & 0.490575 & 0.0297954 & 0.479728 & 0.0305036 & 0.4492 & 0.039321 \\
\hline 14.4275 & 0.48614 & 0.0292605 & 0.483572 & 0.0298185 & 0.445405 & 0.0377041 \\
\hline 14.4375 & 0.478417 & 0.0278531 & 0.475687 & 0.0288326 & 0.443511 & 0.0392485 \\
\hline 14.4475 & 0.474712 & 0.0251017 & 0.477877 & 0.0292809 & 0.441892 & 0.0382661 \\
\hline 14.4575 & 0.469313 & 0.0317439 & 0.471103 & 0.0292991 & 0.436919 & 0.0381487 \\
\hline 14.4675 & 0.469593 & 0.02816 & 0.470431 & 0.0277712 & 0.434544 & 0.0375176 \\
\hline 14.4775 & 0.466039 & 0.0278257 & 0.470919 & 0.0286553 & 0.434652 & 0.0409314 \\
\hline 14.4875 & 0.463538 & 0.0283759 & 0.466447 & 0.0289841 & 0.434477 & 0.03885 \\
\hline 14.4975 & 0.459141 & 0.0302314 & 0.468581 & 0.0269177 & 0.429346 & 0.0360879 \\
\hline 14.5075 & 0.460564 & 0.0272976 & 0.464614 & 0.0289384 & 0.436401 & 0.0390882 \\
\hline 14.5175 & 0.460645 & 0.0275779 & 0.465224 & 0.0282307 & 0.429979 & 0.0377761 \\
\hline 14.5275 & 0.456597 & 0.0277944 & 0.465687 & 0.0287003 & 0.432236 & 0.0339173 \\
\hline 14.5375 & 0.456339 & 0.0292335 & 0.466656 & 0.0278746 & 0.427476 & 0.0348018 \\
\hline 14.5475 & 0.454638 & 0.0287111 & 0.461221 & 0.0278101 & 0.425907 & 0.0376498 \\
\hline 14.5575 & 0.457378 & 0.0287708 & 0.462382 & 0.0275587 & 0.424131 & 0.0396073 \\
\hline 14.5675 & 0.450338 & 0.0295322 & 0.465638 & 0.0322394 & 0.426358 & 0.0386156 \\
\hline 14.5775 & 0.448381 & 0.0298452 & 0.463914 & 0.0325677 & 0.422169 & 0.038032 \\
\hline 14.5875 & 0.450084 & 0.0260467 & 0.46772 & 0.0391948 & 0.425057 & 0.0386521 \\
\hline 14.5975 & 0.450251 & 0.0301743 & 0.468434 & 0.0378319 & 0.423836 & 0.0342584 \\
\hline 14.6075 & 0.447595 & 0.0274466 & 0.468868 & 0.0402598 & 0.422573 & 0.0351764 \\
\hline
\end{tabular}




\begin{tabular}{|c|c|c|c|c|c|c|}
\hline 14.6175 & 0.449296 & 0.0260999 & 0.471339 & 0.0408168 & 0.424005 & 0.0374173 \\
\hline 14.6275 & 0.443322 & 0.0289656 & 0.473263 & 0.0505016 & 0.42437 & 0.0358215 \\
\hline 7.6375 & 0.447156 & 0.0287228 & 0.475864 & 0.0579051 & 0.425175 & 0.0350531 \\
\hline 4.6475 & 0.442427 & 0.0317886 & 0.483764 & 0.064049 & 0.4203 & 0.0361839 \\
\hline 4.6575 & 0.44778 & 0.0251231 & 0.487594 & 0.0694234 & 0.429248 & 0.0350275 \\
\hline 4.6675 & 0.444024 & 0.0281823 & 0.488134 & 0.0759156 & 0.426644 & 0.0385861 \\
\hline 4.6775 & 0.442875 & 0.0277673 & 0.491621 & 0.0811232 & 0.430332 & 0.0346292 \\
\hline 14.6875 & 0.444807 & 0.0247434 & 0.503159 & 0.0967041 & 0.430669 & 0.0382846 \\
\hline 14.6975 & 0.44713 & 0.02639 & 0.502141 & 0.0912061 & 0.434711 & 0.0378149 \\
\hline 14.7075 & 0.441877 & 0.0297936 & 0.507607 & 0.109308 & 0.434101 & 0.0369938 \\
\hline 14.7175 & 0.438664 & 0.0252171 & 0.512748 & 0.10399 & 0.432139 & 0.0380555 \\
\hline 14.7275 & 0.447144 & 0.0231645 & 0.516259 & 0.118871 & 0.434981 & 0.0416476 \\
\hline 14.7375 & 0.447868 & 0.0254113 & 0.514286 & 0.111656 & 0.436973 & 0.0413349 \\
\hline 14.7475 & 0.442713 & 0.0296054 & 0.519129 & 0.115155 & 0.433404 & 0.0412754 \\
\hline 14.7575 & 0.445067 & 0.0262186 & 0.511875 & 0.109516 & 0.43235 & 0.0424353 \\
\hline 14.7675 & 0.445575 & 0.0262211 & 0.517692 & 0.114411 & 0.434299 & 0.0401282 \\
\hline 14.7775 & 0.445129 & 0.0256409 & 0.514077 & 0.11122 & 0.43509 & 0.0432783 \\
\hline 14.7875 & 0.447557 & 0.0260013 & 0.515465 & 0.110843 & 0.4354 & 0.0407969 \\
\hline 14.7975 & 0.444914 & 0.0265763 & 185 & 0.104392 & 6682 & 1888 \\
\hline 14.8075 & 0.448569 & 0.0272522 & 0.504045 & 0.0964163 & 0.436709 & 0.0423819 \\
\hline 14.8175 & 0.449477 & 0.0297506 & 0.50335 & 0.0905528 & 0.43278 & 0.0394781 \\
\hline 14.8275 & 0.446196 & 0.0270536 & 0.499957 & 0.0888607 & 0.426101 & 0.036539 \\
\hline 14.8375 & 0.4466 & 0.0261577 & 0.495574 & 0.0874744 & 0.427562 & 0.0400488 \\
\hline 14.8475 & 0.446879 & 0.0258201 & $0.4 \mathrm{~S}$ & 0.0755444 & 0.424216 & 129 \\
\hline 14.8575 & 0.445413 & 0.0283422 & 0.490023 & 0.0641512 & 0.430108 & 0.0423523 \\
\hline 14.8675 & 0.445408 & 0.0266933 & 0.48788 & 0.0690037 & 0.423442 & 0.0324474 \\
\hline 14.8775 & 0.445631 & 579 & 572 & 2832 & 986 & 5331 \\
\hline 14.8875 & 0.445775 & 0.0269341 & 0.475902 & 0.0513435 & 0.422841 & 0.0368088 \\
\hline 14.8975 & 0.445198 & 0.0243744 & 0.473184 & 0.047572 & 0.421653 & 0.0326147 \\
\hline 14.9075 & 0.44078 & 0.0291911 & 0.471576 & 0.0383819 & 0.418172 & 0.0402234 \\
\hline 14.9175 & 0.444434 & 0.0270689 & 0.467906 & 0.0374446 & 0.41497 & 0.0327863 \\
\hline 14.9275 & 0.446918 & 0.0267999 & 0.468843 & 0.0333847 & 0.413954 & 0.0335927 \\
\hline 14.9375 & 0.44579 & 0.0297045 & 0.466189 & 0.0338392 & 0.410736 & 0.0347881 \\
\hline 14.9475 & 0.4382 & 0.0292136 & 0.462228 & 0.0347791 & 0.414516 & 0.0348037 \\
\hline 14.9575 & 0.438251 & 0.0309326 & 0.461715 & 0.0312895 & 943 & 0.0343771 \\
\hline 14.9675 & 0.438639 & 0.0259838 & 0.453636 & 0.0343574 & 0.407223 & 0.0311151 \\
\hline 14.9775 & 0.440718 & 0.0262049 & 0.457133 & 0.0319095 & 0.407091 & 0.0322955 \\
\hline 14.9875 & 0.437394 & 0.0303478 & 0.451954 & 0.0327394 & 0.400246 & 0.0351362 \\
\hline 14.9975 & 0.439256 & 0.0250472 & 0.44903 & 0.0317786 & 0.401713 & 0.0315523 \\
\hline 15.0075 & 0.43531 & 0.0252135 & 0.448089 & 0.0285397 & 0.398695 & 0.0313354 \\
\hline 15.0175 & 0.429124 & 0.026007 & 0.43952 & 0.0279849 & 0.397676 & 0.0339511 \\
\hline 15.0275 & 0.43223 & 0.0269975 & 0.441379 & 0.0341046 & 0.392671 & 0.0321137 \\
\hline 15.0375 & 0.42827 & 0.0263463 & 0.441427 & 0.0313546 & 0.389956 & 0.0313361 \\
\hline 15.0475 & 0.422207 & 0.0214947 & 0.433832 & 0.0345711 & 0.390242 & 0.0300136 \\
\hline 15.0575 & 0.419594 & 0.0298087 & 0.434889 & 0.0349725 & 0.388109 & 0.0329239 \\
\hline 15.0675 & 0.416362 & 0.0279325 & 0.425568 & 0.0334138 & 0.386585 & 0.0297905 \\
\hline 15.0775 & 0.415409 & 0.0239613 & 0.428413 & 0.0379015 & 0.384028 & 0.029957 \\
\hline
\end{tabular}




\begin{tabular}{|c|c|c|c|c|c|c|}
\hline 15.0875 & 0.413016 & 0.02496 & 0.426918 & 0.0393906 & 0.380203 & 0.0330493 \\
\hline 5.0975 & 0.411547 & 0.0244813 & 0.42454 & 0.0377933 & 0.382237 & 0.0344294 \\
\hline 5.1075 & 0.406614 & 0.0235741 & 0.420943 & 0.0442435 & 0.375828 & 0.030248 \\
\hline 5.1175 & 0.405015 & 0.0226855 & 0.421031 & 0.0460518 & 0.376861 & 0.0308584 \\
\hline 5.1275 & 0.400994 & 0.0255206 & 0.417738 & 0.0398908 & 0.378152 & 0.0307576 \\
\hline 15.1375 & 0.40149 & 0.0230239 & 0.419138 & 0.0394607 & 0.373549 & 0.0312906 \\
\hline 15.1475 & 0.398568 & 0.0242085 & 0.412604 & 0.0399915 & 0.370744 & 0.0282979 \\
\hline 15.1575 & 0.39626 & 0.0242233 & 0.413707 & 0.0430659 & 0.372524 & 0.0290919 \\
\hline 15.1675 & 0.396462 & 0.0241224 & 0.410564 & 0.0420666 & 0.371074 & 0.0285905 \\
\hline 15.1775 & 0.39142 & 0.0246863 & 0.413817 & 0.0440864 & 0.368769 & 0.0303426 \\
\hline 15.1875 & 0.387565 & 0.0240634 & 0.409365 & 0.0415953 & 0.363689 & 0.0299448 \\
\hline 15.1975 & 0.38554 & 0.0229253 & 0.407604 & 0.0437111 & 0.365983 & 0.0312828 \\
\hline 15.2075 & 0.38663 & 0.0230237 & 0.401497 & 0.0456478 & 0.364479 & 0.0287312 \\
\hline 15.2175 & 0.389748 & 0.0222217 & 0.399383 & 0.0350614 & 0.363334 & 0.0277175 \\
\hline 15.2275 & 0.381857 & 0.0232823 & 0.40234 & 0.0426814 & 0.357176 & 0.0287675 \\
\hline 15.2375 & 0.37978 & 0.0226774 & 0.398534 & 0.034078 & 0.35833 & 0.026048 \\
\hline 15.2475 & 0.378676 & 0.0256442 & 0.395835 & 0.0362704 & 0.360408 & 0.0290246 \\
\hline 15.2575 & 0.375824 & 0.0219862 & 0.395901 & 0.0356491 & 0.357027 & 0.0283246 \\
\hline 15.2675 & 0.375067 & 0.0220442 & 0.390193 & 0.0290651 & 0.355474 & 0.0285012 \\
\hline 15.2775 & 0.372553 & 0.0226359 & 0.385414 & 0.0257363 & 0.354186 & 0.0277346 \\
\hline 15.2875 & 0.371872 & 0.0216595 & 0.385973 & 0.0269256 & 0.348767 & 0.028014 \\
\hline 15.2975 & 0.368891 & 0.0235706 & 0.38464 & 0.0278482 & 0.348871 & 0.0269556 \\
\hline 15.3075 & 0.370045 & 0.0239826 & 0.379778 & 0.0294962 & 0.353428 & 0.0271069 \\
\hline 15.3175 & 0.365776 & 0.0176353 & 0.377447 & 0.0247761 & 0.347594 & 0.029256 \\
\hline 15.3275 & 0.370676 & 0.0200517 & 0.373104 & 0.0259697 & 0.345321 & 0.0260056 \\
\hline 15.3375 & 0.367014 & 0.0193907 & 0.371704 & 0.0233852 & 0.345425 & 0.0278569 \\
\hline 15.3475 & 0.367076 & 0.021343 & 0.373042 & 0.0254275 & 0.343734 & 0.0263443 \\
\hline 15.3575 & 0.362791 & 0.0216276 & 0.368937 & 0.0274016 & 0.341083 & 0.0274449 \\
\hline 15.3675 & 0.36399 & 0.022589 & 0.369775 & 0.0232533 & 0.340705 & 0.0277662 \\
\hline 15.3775 & 0.360061 & 0.0204737 & 0.372166 & 0.025386 & 0.339026 & 0.0270598 \\
\hline 15.3875 & 0.360163 & 0.0210265 & 0.371805 & 0.0231234 & 0.336735 & 0.0274012 \\
\hline 15.3975 & 0.358562 & 0.0216152 & 0.370474 & 0.0223422 & 0.338051 & 0.0293416 \\
\hline 15.4075 & 0.355408 & 0.0216113 & 0.368944 & 0.0237275 & 0.336511 & 0.0244736 \\
\hline 15.4175 & 0.356704 & 0.0235517 & 0.363374 & 0.0237462 & 0.335283 & 0.0279106 \\
\hline 15.4275 & 0.358737 & 0.0231407 & 0.366327 & 0.0252734 & 0.33744 & 0.0278306 \\
\hline 15.4375 & 0.35135 & 0.0213968 & 0.365072 & 0.0209679 & 0.335613 & 0.025902 \\
\hline 15.4475 & 0.352832 & 0.0224371 & 0.369165 & 0.0254391 & 0.336102 & 0.0288922 \\
\hline 15.4575 & 0.354034 & 0.0221455 & 0.371273 & 0.0267967 & 0.337813 & 0.0281494 \\
\hline 15.4675 & 0.351447 & 0.0209922 & 0.373843 & 0.0240802 & 0.339513 & 0.0254282 \\
\hline 15.4775 & 0.353468 & 0.023426 & 0.374778 & 0.0282461 & 0.333631 & 0.0256416 \\
\hline 15.4875 & 0.352449 & 0.021749 & 0.379889 & 0.0314658 & 0.33783 & 0.0263317 \\
\hline 15.4975 & 0.351019 & 0.0223977 & 0.37536 & 0.0349625 & 0.342125 & 0.0292932 \\
\hline 15.5075 & 0.352487 & 0.0232956 & 0.379463 & 0.0374807 & 0.338519 & 0.0315339 \\
\hline 15.5175 & 0.35245 & 0.0230427 & 0.393224 & 0.0392278 & 0.347047 & 0.0366557 \\
\hline 15.5275 & 0.354081 & 0.0222254 & 0.396694 & 0.0523533 & 0.348987 & 0.0408226 \\
\hline 15.5375 & 0.354768 & 0.0221619 & 0.409967 & 0.0602946 & 0.355865 & 0.0469433 \\
\hline 15.5475 & 0.355872 & 0.0232894 & 0.417043 & 0.0711297 & 0.360946 & 0.0563505 \\
\hline
\end{tabular}




\begin{tabular}{|c|c|c|c|c|c|c|}
\hline 15.5575 & 0.359787 & 0.0218016 & 0.425917 & 0.0787039 & 0.371019 & 0.0626296 \\
\hline 15.5675 & 0.367759 & 0.0241605 & 0.444099 & 0.0897084 & 0.372705 & 0.0636491 \\
\hline .5775 & 0.371503 & 0.0197158 & 0.451376 & 0.120156 & 0.383902 & 0.0771993 \\
\hline 5.5875 & 0.375977 & 0.0247425 & 0.471537 & 0.127106 & 0.39391 & 0.0917936 \\
\hline 5.5975 & 0.383603 & 0.022599 & 0.491133 & 0.141127 & 0.3992 & 0.104587 \\
\hline 5.6075 & 0.386341 & 0.0287331 & 0.509874 & 0.158835 & 0.416958 & 0.120951 \\
\hline .6175 & 0.391495 & 0.0263577 & 0.529779 & 0.182024 & 0.426692 & 0.13842 \\
\hline 15.6275 & 0.407055 & 0.0314877 & 0.558153 & 0.198285 & 0.445107 & 0.158598 \\
\hline 5.6375 & 0.414768 & 0.0300954 & 0.575632 & 0.209892 & 0.459627 & 0.170049 \\
\hline 5.6475 & 0.427078 & 0.03387 & 0.604217 & 0.24116 & 0.471149 & 0.183385 \\
\hline 15.6575 & 0.440287 & 0.0331563 & 0.633306 & 0.257162 & 0.491844 & 0.203366 \\
\hline 15.6675 & 0.452953 & 0.039072 & 0.658988 & 0.280277 & 0.505954 & 0.228297 \\
\hline 15.6775 & 0.469662 & 0.0412047 & 0.675305 & 0.289713 & 0.523411 & 0.254354 \\
\hline 15.6875 & 0.486119 & 0.0488755 & 0.705998 & 0.31163 & 0.539339 & 0.255243 \\
\hline 15.6975 & 0.498051 & 0.0442069 & 0.721748 & 0.331366 & 0.555613 & 0.261 \\
\hline 15.7075 & 0.510237 & 0.0529717 & 0.745073 & 0.337386 & 0.571535 & 0.287258 \\
\hline 15.7175 & 0.528347 & 0.0548966 & 0.76354 & 0.347442 & 0.581624 & 0.289241 \\
\hline 15.7275 & 0.540359 & 0.0572077 & 0.777867 & 0.352421 & 0.591747 & 0.301393 \\
\hline 15.7375 & 0.55 & 0766 & 0078 & 2897 & 216 & 0.29681 \\
\hline 15.7475 & 0.572747 & 0.0640668 & 0.800001 & 0.352886 & 0.608852 & 0.302731 \\
\hline 15.7575 & 0.583503 & 0.0686003 & 0.800022 & 0.349863 & 0.610479 & 0.308878 \\
\hline 15.7675 & 0.592054 & 0.0604881 & 0.804469 & 0.347608 & 0.617782 & 0.304412 \\
\hline 15.7775 & 0.606358 & 0.0676033 & 0.791676 & 0.334798 & 0.614727 & 0.293908 \\
\hline 15.7875 & 0.610315 & 0.0689401 & 0.788753 & 0.324402 & 0.608326 & 0.278468 \\
\hline 15.7975 & 0.622364 & 0.0701208 & 0.771153 & 0.310258 & 0.603138 & 0.269712 \\
\hline 15.8075 & 0.61999 & 0.0691133 & 0.758307 & 0.302317 & 0.594248 & 0.255992 \\
\hline 15.8175 & 0.61 & 0.0641152 & & & & 3895 \\
\hline 15.8275 & 0.609256 & 0.0630257 & 0.719551 & 0.265672 & 0.567401 & 0.219944 \\
\hline 15.8375 & 0.609018 & 0.0652065 & 0.688623 & 0.255811 & 0.557004 & 0.203479 \\
\hline 15.8475 & 0.597612 & 0.0608382 & 0.66342 & 0.231372 & 0.535716 & 0.192244 \\
\hline 15.8575 & 0.587186 & 0.0592394 & 0.638676 & 0.213229 & 0.517036 & 0.168522 \\
\hline 15.8675 & 0.575527 & 0.0575193 & 0.609943 & 0.197273 & 0.49666 & 0.145447 \\
\hline 15.8775 & 0.561084 & 0.051549 & 0.58236 & 0.174113 & 0.475455 & 0.125436 \\
\hline 15.8875 & 0.543365 & 0.0490409 & 0.552338 & 0.161452 & 0.460967 & 0.108902 \\
\hline 15.8975 & 0.527552 & 0.048883 & 0.522184 & 0.145388 & 0.446661 & 0.101229 \\
\hline 15.9075 & 0.508206 & 0.0505697 & 0.503124 & 0.127226 & 0.428881 & 0.0804791 \\
\hline 15.9175 & 0.492632 & 0.0427419 & 0.479186 & 0.113548 & 0.411683 & 0.0710043 \\
\hline 15.9275 & 0.472311 & 0.0408635 & 0.453902 & 0.0977701 & 0.393769 & 0.0609901 \\
\hline 15.9375 & 0.458368 & 0.0393843 & 0.435509 & 0.0894278 & 0.380678 & 0.0568721 \\
\hline 15.9475 & 0.439435 & 0.0354102 & 0.414597 & 0.0812468 & 0.371836 & 0.0485603 \\
\hline 15.9575 & 0.422903 & 0.0337734 & 0.399545 & 0.0684192 & 0.35398 & 0.0407174 \\
\hline 15.9675 & 0.40808 & 0.0269838 & 0.393807 & 0.0646699 & 0.347411 & 0.0332036 \\
\hline 15.9775 & 0.396674 & 0.0299004 & 0.378943 & 0.0572858 & 0.339655 & 0.0329332 \\
\hline 15.9875 & 0.382091 & 0.0287363 & 0.369852 & 0.048527 & 0.332893 & 0.0302174 \\
\hline 15.9975 & 0.373072 & 0.0267926 & 0.363111 & 0.048435 & 0.324185 & 0.0298154 \\
\hline 16.0075 & 0.362963 & 0.0251691 & 0.355898 & 0.0416138 & 0.322281 & 0.0245175 \\
\hline 16.0175 & 0.351629 & 0.0248229 & 0.350923 & 0.0411859 & 0.317581 & 0.0239209 \\
\hline
\end{tabular}




\begin{tabular}{|c|c|c|c|c|c|c|}
\hline 16.0275 & 0.345402 & 0.0201729 & 0.346448 & 0.0393496 & 0.313496 & 0.0268135 \\
\hline 16.0375 & 0.337618 & 0.023624 & 0.342084 & 0.0352263 & 0.309056 & 0.022486 \\
\hline 16.0475 & 0.335743 & 0.0203443 & 0.340829 & 0.0380982 & 0.305309 & 0.021475 \\
\hline 16.0575 & 0.326329 & 0.0218457 & 0.33752 & 0.036616 & 0.306428 & 0.0227255 \\
\hline 16.0675 & 0.330475 & 0.0199928 & 0.332175 & 0.0333308 & 0.304902 & 0.0242362 \\
\hline 16.0775 & 0.325228 & 0.0206609 & 0.332505 & 0.0298338 & 0.300759 & 0.0226671 \\
\hline 16.0875 & 0.322405 & 0.0210432 & 0.330572 & 0.0326466 & 0.304381 & 0.0225452 \\
\hline 16.0975 & 0.320576 & 0.0179505 & 0.327231 & 0.0329702 & 0.300844 & 0.0221117 \\
\hline 16.1075 & 0.318151 & 0.0187631 & 0.326091 & 0.0318167 & 0.297234 & 0.0237927 \\
\hline 16.1175 & 0.314439 & 0.0209502 & 0.32284 & 0.0319573 & 0.29615 & 0.0222871 \\
\hline 16.1275 & 0.31357 & 0.0216355 & 0.324869 & 0.0293285 & 0.293435 & 0.0222574 \\
\hline 16.1375 & 0.312103 & 0.0205923 & 0.323134 & 0.027623 & 0.292691 & 0.0219048 \\
\hline 16.1475 & 0.310159 & 0.0182929 & 0.320681 & 0.027619 & 0.296299 & 0.0227966 \\
\hline 16.1575 & 0.310084 & 0.0190017 & 0.314728 & 0.0265253 & 0.289656 & 0.0202856 \\
\hline 16.1675 & 0.309456 & 0.019839 & 0.31541 & 0.0270859 & 0.289224 & 0.0207048 \\
\hline 16.1775 & 0.304621 & 0.0200475 & 0.311152 & 0.0240114 & 0.289008 & 0.0215503 \\
\hline 16.1875 & 0.307121 & 0.0190152 & 0.31178 & 0.0223989 & 0.287076 & 0.0219014 \\
\hline 16.1975 & 0.305796 & 0.0197506 & 0.309929 & 0.0260858 & 0.284353 & 0.0216005 \\
\hline 16.2075 & 0.303591 & 0.0184865 & 0.30491 & 0.0192502 & 0.28222 & 0.0208358 \\
\hline 16.2175 & 0.298524 & 0.0196678 & 0.300414 & 0.0203772 & 0.284282 & 0.0218608 \\
\hline 16.2275 & 0.299697 & 0.0189759 & 0.300525 & 0.0228267 & 0.282186 & 0.020609 \\
\hline 16.2375 & 0.29826 & 0.0193413 & 0.295978 & 0.0195879 & 0.282492 & 0.0191697 \\
\hline 16.2475 & 0.296351 & 0.0192995 & 0.297296 & 0.0228157 & 0.278296 & 0.0222515 \\
\hline 16.2575 & 0.294137 & 0.021891 & 0.295037 & 0.0197183 & 0.277518 & 0.0197785 \\
\hline 16.2675 & 0.294441 & 0.0201971 & 0.294881 & 0.0192663 & 0.278636 & 0.020985 \\
\hline 16.2775 & 0.292602 & 0.0192441 & 0.292708 & 0.0183553 & 0.27866 & 0.0196016 \\
\hline 16.2875 & 0.289894 & 0.020703 & 0.293622 & 0.0214725 & 0.274466 & 0.0210264 \\
\hline 16.2975 & 0.292781 & 0.0187712 & 0.291923 & 0.0215875 & 0.275717 & 0.021272 \\
\hline 16.3075 & 0.289275 & 0.0199148 & 0.294345 & 0.0210013 & 0.272384 & 0.0189452 \\
\hline 16.3175 & 0.288258 & 0.0172173 & 0.290675 & 0.0196158 & 0.273247 & 0.021131 \\
\hline 16.3275 & 0.285957 & 0.0171743 & 0.291641 & 0.0193854 & 0.272538 & 0.0185074 \\
\hline 16.3375 & 0.288382 & 0.0181643 & 0.286292 & 0.018757 & 0.2699 & 0.0188439 \\
\hline 16.3475 & 0.284794 & 0.0201716 & 0.289884 & 0.0192212 & 0.272985 & 0.021182 \\
\hline 16.3575 & 0.285507 & 0.018563 & 0.289834 & 0.0213808 & 0.268936 & 0.0209543 \\
\hline 16.3675 & 0.286425 & 0.0183139 & 0.286265 & 0.0216105 & 0.270702 & 0.0215083 \\
\hline 16.3775 & 0.285407 & 0.0194566 & 0.286298 & 0.0198798 & 0.272525 & 0.0196948 \\
\hline 16.3875 & 0.2822 & 0.0177497 & 0.284317 & 0.0193391 & 0.270362 & 0.0213994 \\
\hline 16.3975 & 0.281562 & 0.0160486 & 0.286644 & 0.0195456 & 0.268494 & 0.0189299 \\
\hline 16.4075 & 0.28116 & 0.0197755 & 0.285532 & 0.0198504 & 0.266148 & 0.0165848 \\
\hline 16.4175 & 0.282305 & 0.0193782 & 0.2798 & 0.0206015 & 0.267202 & 0.0210997 \\
\hline 16.4275 & 0.27986 & 0.0205823 & 0.281681 & 0.0173279 & 0.265498 & 0.0222488 \\
\hline 16.4375 & 0.276215 & 0.0191323 & 0.282387 & 0.0183184 & 0.267526 & 0.0216327 \\
\hline 16.4475 & 0.279056 & 0.0179315 & 0.283008 & 0.0169326 & 0.266833 & 0.0205188 \\
\hline 16.4575 & 0.276025 & 0.0182963 & 0.28371 & 0.0203424 & 0.266588 & 0.0196831 \\
\hline 16.4675 & 0.278834 & 0.0181147 & 0.27891 & 0.0202715 & 0.265097 & 0.0194404 \\
\hline 16.4775 & 0.276475 & 0.0181497 & 0.280019 & 0.0207455 & 0.264326 & 0.0202031 \\
\hline 16.4875 & 0.279724 & 0.0171204 & 0.285212 & 0.0185039 & 0.26562 & 0.0214943 \\
\hline
\end{tabular}




\begin{tabular}{|c|c|c|c|c|c|c|}
\hline 16.4975 & 0.275766 & 0.0190212 & 0.282174 & 0.0233243 & 0.264341 & 0.0195286 \\
\hline 16.5075 & 0.272975 & 0.0178642 & 0.282531 & 0.0185154 & 0.263633 & 0.019585 \\
\hline 16.5175 & 0.276513 & 0.0182184 & 0.277766 & 0.0186296 & 0.265137 & 0.0215771 \\
\hline 6.5275 & 0.275495 & 0.0192233 & 0.282243 & 0.0170226 & 0.260997 & 0.0202336 \\
\hline 16.5375 & 0.275396 & 0.0177811 & 0.280307 & 0.0220708 & 0.265739 & 0.0191316 \\
\hline 16.5475 & 0.278736 & 0.0211859 & 0.281353 & 0.019731 & 0.267763 & 0.0203208 \\
\hline 16.5575 & 0.27438 & 0.0186839 & 0.281475 & 0.0192825 & 0.263138 & 0.0201757 \\
\hline 16.5675 & 0.273774 & 0.0185012 & 0.28174 & 0.0201559 & 0.262779 & 0.018917 \\
\hline 16.5775 & 0.278223 & 0.0166068 & 0.279493 & 0.0219258 & 0.26303 & 0.0226158 \\
\hline 16.5875 & 0.270088 & 0.0167298 & 0.279453 & 0.0190862 & 0.263182 & 0.0212646 \\
\hline 16.5975 & 0.272405 & 0.019025 & 0.27898 & 0.0182241 & 0.263594 & 0.0191047 \\
\hline 16.6075 & 0.275605 & 0.0205178 & 0.275827 & 0.0197037 & 0.258318 & 0.0202088 \\
\hline 16.6175 & 0.27301 & 0.018344 & 0.276743 & 0.0183233 & 0.26421 & 0.0209325 \\
\hline 16.6275 & 0.272541 & 0.0190935 & 0.277847 & 0.0186473 & 0.259943 & 0.0173218 \\
\hline 16.6375 & 0.275776 & 0.0174907 & 0.273705 & 0.0212396 & 0.258848 & 0.0201796 \\
\hline 16.6475 & 0.274754 & 0.0176322 & 0.276014 & 0.0188749 & 0.262863 & 0.0191487 \\
\hline 16.6575 & 0.271479 & 0.0176345 & 0.276592 & 0.0175401 & 0.26078 & 0.0183288 \\
\hline 16.6675 & 0.271407 & 0.0172081 & 0.274796 & 0.0159544 & 0.260256 & 0.0193598 \\
\hline 16.6775 & 0.274621 & 0.0210569 & 0.27436 & 0.0213793 & 0.258437 & 0.0180009 \\
\hline 16.6875 & 0.27059 & 0.0192708 & 0.272056 & 0.0187968 & 0.256874 & 0.0193177 \\
\hline 16.6975 & 0.27091 & 0.0199787 & 0.272019 & 0.0197834 & 0.257523 & 0.0199796 \\
\hline 16.7075 & 0.270245 & 0.0180874 & 0.272326 & 0.0166853 & 0.258442 & 0.0189664 \\
\hline 16.7175 & 0.267723 & 0.0208757 & 0.27374 & 0.0204055 & 0.257504 & 0.0191558 \\
\hline 16.7275 & 0.26813 & 0.01888 & 0.271149 & 0.0166357 & 0.254604 & 0.0193902 \\
\hline 16.7375 & 0.267232 & 0.0174678 & 0.267906 & 0.0181 & 0.255168 & 0.0179771 \\
\hline 16.7475 & 0.268978 & 0.0194051 & 0.269848 & 0.019048 & 0.256429 & 0.0168498 \\
\hline 16.7575 & 0.266732 & 0.0189014 & 0.267216 & 0.0173224 & 0.256538 & 0.0183219 \\
\hline 16.7675 & 0.26648 & 0.0191945 & 0.264543 & 0.018333 & 0.250527 & 0.0177094 \\
\hline 16.7775 & 0.2657 & 0.017461 & 0.268908 & 0.0182031 & 0.251633 & 0.0194704 \\
\hline 16.7875 & 0.263464 & 0.0176161 & 0.261553 & 0.0178552 & 0.249539 & 0.0180146 \\
\hline 16.7975 & 0.260815 & 0.0181135 & 0.265082 & 0.017069 & 0.25062 & 0.0193116 \\
\hline 16.8075 & 0.263617 & 0.0185805 & 0.262983 & 0.0186789 & 0.249421 & 0.0166931 \\
\hline 16.8175 & 0.26448 & 0.0185879 & 0.263612 & 0.0188748 & 0.252658 & 0.0173382 \\
\hline 16.8275 & 0.262585 & 0.0179445 & 0.259642 & 0.0139873 & 0.250234 & 0.0202801 \\
\hline 16.8375 & 0.258285 & 0.0191066 & 0.256972 & 0.0188298 & 0.25022 & 0.0195479 \\
\hline 16.8475 & 0.262025 & 0.0188291 & 0.25934 & 0.0191473 & 0.250896 & 0.0171231 \\
\hline 16.8575 & 0.260125 & 0.0144454 & 0.261762 & 0.016828 & 0.245854 & 0.0176492 \\
\hline 16.8675 & 0.261471 & 0.0172875 & 0.257621 & 0.0191314 & 0.246022 & 0.0182986 \\
\hline 16.8775 & 0.258359 & 0.0195811 & 0.254833 & 0.0195248 & 0.245749 & 0.0181568 \\
\hline 16.8875 & 0.260711 & 0.0196244 & 0.254523 & 0.0172509 & 0.246011 & 0.0184547 \\
\hline 16.8975 & 0.255388 & 0.0166953 & 0.254469 & 0.0207757 & 0.249692 & 0.0186865 \\
\hline 16.9075 & 0.251277 & 0.0187212 & 0.259621 & 0.0191631 & 0.246874 & 0.0162025 \\
\hline 16.9175 & 0.255408 & 0.0172576 & 0.255953 & 0.0160439 & 0.243325 & 0.0164336 \\
\hline 16.9275 & 0.255134 & 0.0159702 & 0.255817 & 0.0173838 & 0.244615 & 0.0176519 \\
\hline 16.9375 & 0.253229 & 0.0199265 & 0.255291 & 0.0176212 & 0.239507 & 0.0194591 \\
\hline 16.9475 & 0.253832 & 0.0178775 & 0.254854 & 0.0169945 & 0.243179 & 0.0186704 \\
\hline 16.9575 & 0.251576 & 0.0173378 & 0.258764 & 0.0182878 & 0.24234 & 0.0191875 \\
\hline
\end{tabular}




\begin{tabular}{|c|c|c|c|c|c|c|}
\hline 16.9675 & 0.253737 & 0.0185062 & 0.255927 & 0.0198262 & 0.242637 & 0.0190396 \\
\hline 16.9775 & 0.25082 & 0.0183549 & 0.251626 & 0.0164246 & 0.241708 & 0.0183037 \\
\hline .9875 & 0.252201 & 0.0184409 & 0.253477 & 0.0185888 & 0.241415 & 0.0181092 \\
\hline 5.9975 & 0.250263 & 0.0184415 & 0.250666 & 0.0159346 & 0.242437 & 0.0181385 \\
\hline 7.0075 & 0.252235 & 0.016562 & 0.249154 & 0.01572 & 0.243015 & 0.0180195 \\
\hline 7.0175 & 0.250198 & 0.0157392 & 0.25263 & 0.01769 & 0.244505 & 0.0163521 \\
\hline 7.0275 & 0.252162 & 0.0173244 & 0.252144 & 0.0182581 & 0.239748 & 0.0182511 \\
\hline 17.0375 & 0.248333 & 0.0189152 & 0.252497 & 0.018793 & 0.239784 & 0.0170979 \\
\hline 7.0475 & 0.246183 & 0.0163501 & 0.252767 & 0.0186461 & 0.236673 & 0.0170627 \\
\hline 7.0575 & 0.250339 & 0.0171161 & 0.250732 & 0.0197785 & 0.238332 & 0.018977 \\
\hline 17.0675 & 0.248383 & 0.0168454 & 0.250084 & 0.0175716 & 0.238036 & 0.0166722 \\
\hline 7.0775 & 0.248798 & 0.0168981 & 0.251012 & 0.0192224 & 0.23576 & 0.0164082 \\
\hline 17.0875 & 0.246179 & 0.0192742 & 0.248254 & 0.0193326 & 0.237939 & 0.0161793 \\
\hline 17.0975 & 0.247517 & 0.0185762 & 0.249015 & 0.0183449 & 0.239181 & 0.0164302 \\
\hline 17.1075 & 0.244862 & 0.017791 & 0.251157 & 0.0157159 & 0.237514 & 0.0174949 \\
\hline 17.1175 & 0.248798 & 0.0188493 & 0.245753 & 0.0182423 & 0.237198 & 0.0189722 \\
\hline 17.1275 & 0.245642 & 0.0173775 & 0.250536 & 0.0161765 & 0.237761 & 0.0161223 \\
\hline 17.1375 & 0.244369 & 0.0167314 & 0.246709 & 0.0171606 & 0.237613 & 0.0187071 \\
\hline 17.1475 & 0.242935 & 0.0179341 & 0.243188 & 0.0185058 & 0.238723 & 3904 \\
\hline 17.1575 & 0.24607 & 0.0175682 & 0.246467 & 0.0165349 & 0.232832 & 0.0173411 \\
\hline 17.1675 & 0.245277 & 0.0172887 & 0.244092 & 0.0170499 & 0.235795 & 7737 \\
\hline 17.1775 & 0.24 & 0.0188596 & 9 & 0.0157027 & 8777 & 62657 \\
\hline 17.1875 & 0.24284 & 0.0146972 & 0.244233 & 0.0193572 & 0.237671 & 0.0178286 \\
\hline 17.1975 & 0.24337 & 0.0177641 & 0.2404 & 0.0190156 & 0.235673 & 613 \\
\hline 17.2075 & 0.244849 & 0.0164791 & 0.245812 & 0.0196436 & 0.235341 & 0.0161116 \\
\hline 17.2175 & 0.241748 & 0.0164307 & 0.24295 & 0.0166081 & 0.235538 & 0.0157746 \\
\hline 17.2275 & 0.240888 & 0.0165795 & 0.244935 & 0.0195375 & 166 & 188 \\
\hline 17.2375 & 0.243218 & 0.01811 & 0.242443 & 0.0211288 & 0.233829 & 0.0157984 \\
\hline 17.2475 & 0.245335 & 0.0171753 & 0.245198 & 0.0179103 & 0.235667 & 0.016331 \\
\hline 17.2575 & 0.24395 & 0.0161155 & 0.242851 & 0.018417 & 0.235511 & 0.0173542 \\
\hline 17.2675 & 0.244399 & 0.0190538 & 0.246586 & 0.0176636 & 0.236596 & 0.0164843 \\
\hline 17.2775 & 0.248472 & 0.0177925 & 0.241588 & 0.0188011 & 0.23405 & 0.0161389 \\
\hline 17.2875 & 0.244531 & 0.0161003 & 0.245708 & 0.0166466 & 0.236786 & 0.0188483 \\
\hline 17.2975 & 0.251701 & 0.015794 & 0.241248 & 0.0167676 & 0.236137 & 0.0173306 \\
\hline 17.3075 & 0.247742 & 0.0173965 & 0.241456 & 0.0179354 & 0.234176 & 0.0153612 \\
\hline 17.3175 & 0.250585 & 0.016494 & 0.242635 & 0.0184367 & 0.242586 & 0.0161757 \\
\hline 17.3275 & 0.248787 & 0.0203925 & 0.245018 & 0.0181911 & 0.23988 & 0.0189996 \\
\hline 17.3375 & 0.253402 & 0.0185511 & 0.244353 & 0.0189912 & 0.237018 & 0.0174323 \\
\hline 17.3475 & 0.251198 & 0.0165877 & 0.245648 & 0.0161904 & 0.238132 & 0.0157986 \\
\hline 17.3575 & 0.254509 & 0.0180576 & 0.243899 & 0.0186123 & 0.238419 & 0.0174616 \\
\hline 17.3675 & 0.255121 & 0.0183877 & 0.244203 & 0.0171037 & 0.237986 & 0.018557 \\
\hline 17.3775 & 0.257471 & 0.0160632 & 0.245339 & 0.018494 & 0.237542 & 0.0162574 \\
\hline 17.3875 & 0.26038 & 0.018929 & 0.243386 & 0.0161591 & 0.240726 & 0.0187539 \\
\hline 17.3975 & 0.260333 & 0.0168728 & 0.246378 & 0.0190773 & 0.243892 & 0.0197907 \\
\hline 17.4075 & 0.261386 & 0.0178182 & 0.24614 & 0.0190306 & 0.244554 & 0.0179031 \\
\hline 17.4175 & 0.264474 & 0.0185444 & 0.250696 & 0.0177116 & 0.241213 & 0.0176892 \\
\hline 17.4275 & 0.263284 & 0.015427 & 0.248553 & 0.0174447 & 0.240813 & 0.0165581 \\
\hline
\end{tabular}




\begin{tabular}{|c|c|c|c|c|c|c|}
\hline 17.4375 & 0.266036 & 0.0171004 & 0.248295 & 0.0187373 & 0.242234 & 0.0171402 \\
\hline 17.4475 & 0.265061 & 0.0161018 & 0.251876 & 0.0184031 & 0.242837 & 0.0184437 \\
\hline 7.4575 & 0.269188 & 0.0156663 & 0.250976 & 0.0211519 & 0.246763 & 0.0195768 \\
\hline 7.4675 & .270186 & 0.016168 & 0.249591 & 0.0201695 & 0.24787 & 0.0197276 \\
\hline 17.4775 & 0.264925 & 0.0169838 & 0.253702 & 0.02187 & 0.249188 & 0.0172015 \\
\hline 7.4875 & 0.269222 & 0.0181633 & 0.256938 & 0.0245898 & 0.250576 & 0.0171961 \\
\hline 7.4975 & 0.271085 & 0.0186603 & 0.259336 & 0.0273502 & 0.251423 & 0.0243994 \\
\hline 17.5075 & 0.269683 & 0.0182795 & 0.262904 & 0.0262665 & 0.24977 & 0.0210116 \\
\hline 7.5175 & 0.272418 & 0.0178561 & 0.264436 & 0.0309274 & 0.254165 & 0.0210417 \\
\hline 7.5275 & 0.268333 & 0.0203584 & 0.266152 & 0.0312646 & 0.254255 & 0.0278768 \\
\hline 17.5375 & 0.272023 & 0.0175437 & 0.271671 & 0.0361893 & 0.253983 & 0.0252949 \\
\hline 17.5475 & 0.272079 & 0.0150152 & 0.273762 & 0.035729 & 0.25725 & 0.029535 \\
\hline 17.5575 & 0.272492 & 0.0188677 & 0.278085 & 0.0398315 & 0.256698 & 0.0292488 \\
\hline 17.5675 & 0.273077 & 0.0201227 & 0.2807 & 0.0460029 & 0.261402 & 0.0349717 \\
\hline 17.5775 & 0.272952 & 0.0168184 & 0.285331 & 0.0507624 & 0.264214 & 0.0348256 \\
\hline 17.5875 & 0.275241 & 0.0199584 & 0.285144 & 0.0460808 & 0.267708 & 0.0374605 \\
\hline 17.5975 & 0.27586 & 0.0202369 & 0.287502 & 0.0538281 & 0.263582 & 0.0427778 \\
\hline 17.6075 & 0.273536 & 0.0178405 & 0.292794 & 0.0558113 & 0.26704 & 0.0381937 \\
\hline 17.6175 & 0.274735 & 0.0186372 & 0.292363 & 0.0569126 & 0.271835 & 2068 \\
\hline 17.6275 & 0.276783 & 0.0206846 & 0.297167 & 0.0583316 & 0.2682 & 0.0410723 \\
\hline 17.6375 & 0.274297 & 0.0206853 & 0.29752 & 0.0581216 & 0.273495 & 0.0447656 \\
\hline 17.64 & 0.279518 & 0.015 & 0.297 & 0.0616872 & 0.27 & 7677 \\
\hline 17.6575 & 0.276786 & 0.0188718 & 0.296209 & 0.0556286 & 0.27352 & 0.0507327 \\
\hline 17.6675 & 0.277788 & 0.0185444 & 0.296191 & 0.0573186 & 0.273624 & 5682 \\
\hline 17.6775 & 0.278992 & 0.0206119 & 0.294753 & 0.0559202 & 0.274011 & 0.0465408 \\
\hline 17.6875 & 0.278828 & 0.0222376 & 0.294553 & 0.0548603 & 0.274752 & 0.0434125 \\
\hline 17.6975 & 0.279179 & 0.0225066 & 28969 & & & 2861 \\
\hline 17.7075 & 0.276601 & 0.018628 & 0.288545 & 0.0540521 & 0.268448 & 0.0438246 \\
\hline 17.7175 & 0.27575 & 0.0226587 & 0.284338 & 0.0427906 & 0.269673 & 0.0437126 \\
\hline 17.7275 & .276248 & 0.0206199 & 0.282152 & 0.0432108 & 0.266944 & 1927 \\
\hline 17.7375 & 0.273389 & 0.0222955 & 0.282593 & 0.0426949 & 0.261681 & 0.0361481 \\
\hline 17.7475 & 0.272596 & 0.018775 & 0.277103 & 0.0368849 & 0.264539 & 0.0344771 \\
\hline 17.7575 & 0.273246 & 0.0173699 & 0.276696 & 0.0347175 & 0.259141 & 0.030444 \\
\hline 17.7675 & 0.270418 & 0.0189816 & 0.270198 & 0.0344871 & 0.258072 & 0.0289906 \\
\hline 17.7775 & 0.267637 & 0.0183061 & 0.267827 & 0.0299871 & 0.2 & 0.0278137 \\
\hline 17.7875 & 0.268044 & 0.0171841 & 0.264298 & 0.0267568 & 0.255849 & 0.0246546 \\
\hline 17.7975 & 0.26181 & 0.0184847 & 0.263022 & 0.0203076 & 0.256302 & 0.0229858 \\
\hline 17.8075 & 0.261985 & 0.0165641 & 0.264674 & 0.0285071 & 0.253083 & 0.0230757 \\
\hline 17.8175 & 0.260651 & 0.0184669 & 0.25828 & 0.0259826 & 0.252727 & 0.0228459 \\
\hline 17.8275 & 0.260046 & 0.0194004 & 0.257025 & 0.0258312 & 0.249333 & 0.0215132 \\
\hline 17.8375 & 0.256722 & 0.0172398 & 0.259436 & 0.0206705 & 0.249129 & 0.0222253 \\
\hline 17.8475 & 0.255869 & 0.0172363 & 0.260223 & 0.0216136 & 0.247901 & 0.0179839 \\
\hline 17.8575 & 0.257138 & 0.0153677 & 0.255018 & 0.0192317 & 0.248411 & 0.0188751 \\
\hline 17.8675 & 0.25638 & 0.0160291 & 0.258506 & 0.0201254 & 0.249883 & 0.0202048 \\
\hline 17.8775 & 0.25193 & 0.0173411 & 0.256093 & 0.0225977 & 0.248581 & 0.0191713 \\
\hline 17.8875 & 0.256194 & 0.0166316 & 0.258519 & 0.0209203 & 0.246777 & 0.0171828 \\
\hline 17.8975 & 0.253097 & 0.0157328 & 0.259496 & 0.0202734 & 0.247799 & 0.020372 \\
\hline
\end{tabular}




\begin{tabular}{|c|c|c|c|c|c|c|}
\hline 17.9075 & 0.25253 & 0.0140847 & 0.258042 & 0.0179188 & 0.253162 & 0.0200381 \\
\hline 7.9175 & 0.252842 & 0.0185177 & 0.256938 & 0.0207854 & 0.249834 & 0.0184587 \\
\hline 7.9275 & 0.251364 & 0.016855 & 0.25928 & 0.0185865 & 0.252 & 0.019629 \\
\hline 7.9375 & 0.25166 & 0.0165771 & 0.256499 & 0.0198784 & 0.254838 & 0.0194168 \\
\hline 17.9475 & 0.254467 & 0.0180761 & 0.257897 & 0.0220805 & 0.252736 & 0.0181266 \\
\hline 7.9575 & 0.254023 & 0.0179324 & 0.262401 & 0.0201736 & 0.253414 & 0.0173715 \\
\hline 7.9675 & 0.254194 & 0.019193 & 0.264948 & 0.0210397 & 0.256569 & 0.0159319 \\
\hline 17.9775 & 0.259564 & 0.0175618 & 0.264288 & 0.0201455 & 0.253597 & 0.0185122 \\
\hline 17.9875 & 0.256015 & 0.0161721 & 0.26276 & 0.0214153 & 0.25507 & 0.018051 \\
\hline 17.9975 & 0.258853 & 0.0189544 & 0.263395 & 0.0180765 & 0.258486 & 0.0175191 \\
\hline 18.0075 & 0.259444 & 0.0184899 & 0.263164 & 0.0206395 & 0.25937 & 0.0186358 \\
\hline 18.0175 & 0.257777 & 0.0184719 & 0.262875 & 0.0194685 & 0.259171 & 0.018588 \\
\hline 18.0275 & 0.26104 & 0.0183429 & 0.265709 & 0.0207507 & 0.256398 & 0.0160258 \\
\hline 18.0375 & 0.26081 & 0.0177438 & 0.262499 & 0.0218533 & 0.260004 & 0.0169288 \\
\hline 18.0475 & 0.259208 & 0.0158155 & 0.265906 & 0.0200015 & 0.259953 & 0.0166501 \\
\hline 18.0575 & 0.260294 & 0.0190759 & 0.261518 & 0.0177527 & 0.260744 & 0.0172673 \\
\hline 18.0675 & 0.264389 & 0.0179629 & 0.266726 & 0.0190402 & 0.262862 & 0.0179121 \\
\hline 18.0775 & 0.2628 & 0.0175158 & 0.263682 & 0.016653 & 0.260582 & 0.0165388 \\
\hline 18.0875 & 0.26572 & 0.0171709 & 0.270858 & 414 & 3618 & 5741 \\
\hline 18.0975 & 0.264778 & 0.0174317 & 0.262907 & 0.0178867 & 0.264926 & 0.0179217 \\
\hline 18.1075 & 0.26397 & 0.0175825 & 0.266523 & 0.0172733 & 0.265866 & 0.0196901 \\
\hline 18.1175 & 0.260577 & 0.0212816 & 0.269926 & 0.0176993 & 0.268522 & 0.0193392 \\
\hline 18.1275 & 0.267823 & 0.0161561 & 0.272616 & 0.016036 & 0.267088 & 0.0154521 \\
\hline 18.1375 & 0.262968 & 0.0184593 & 0.272753 & 0.0207217 & 0.267782 & 8024 \\
\hline 18.1475 & 0.267284 & 0.0166537 & 0.272183 & 0.020266 & 0.269197 & 0.0149537 \\
\hline 18.1575 & 0.26707 & 0.0168649 & 0.267661 & 0.019153 & 0.272532 & 0.0162431 \\
\hline 18.1675 & 0.26 & & & & & 326 \\
\hline 18.1775 & 0.26966 & 0.0180125 & 0.274891 & 0.0171567 & 0.276069 & 0.017438 \\
\hline 18.1875 & 0.273393 & 0.0204116 & 0.279252 & 0.0166218 & 0.279294 & 0.0173519 \\
\hline 18.1975 & 0.274191 & 0.0159811 & 0.279049 & 0.0176389 & 0.282126 & 0.01853 \\
\hline 18.2075 & 0.273858 & 0.0193191 & 0.281185 & 0.016848 & 0.281706 & 0.0203905 \\
\hline 18.2175 & 0.276794 & 0.0185127 & 0.286127 & 0.0191207 & 0.282959 & 0.018032 \\
\hline 18.2275 & 0.281261 & 0.0167372 & 0.286431 & 0.0196072 & 0.285655 & 0.0161878 \\
\hline 18.2375 & 0.278367 & 0.0191581 & 0.288082 & 0.0178089 & 0.288771 & 0.0188187 \\
\hline 18.2475 & 0.279184 & 0.0202427 & 0.287924 & 0.019786 & 0.287944 & 0.0174533 \\
\hline 18.2575 & 0.284049 & 0.0197917 & 0.294331 & 0.018232 & 0.291616 & 0.0153661 \\
\hline 18.2675 & 0.285574 & 0.0161547 & 0.292465 & 0.0202982 & 0.296033 & 0.0159315 \\
\hline 18.2775 & 0.28643 & 0.0177672 & 0.298199 & 0.0213313 & 0.300076 & 0.0178986 \\
\hline 18.2875 & 0.290679 & 0.020414 & 0.297666 & 0.0172721 & 0.303302 & 0.016682 \\
\hline 18.2975 & 0.292335 & 0.0172856 & 0.305812 & 0.0175594 & 0.307178 & 0.019155 \\
\hline 18.3075 & 0.294282 & 0.0186582 & 0.304537 & 0.0215103 & 0.304274 & 0.016933 \\
\hline 18.3175 & 0.296219 & 0.0168618 & 0.309043 & 0.0203873 & 0.310963 & 0.0183815 \\
\hline 18.3275 & 0.301632 & 0.0181762 & 0.313961 & 0.0207339 & 0.31477 & 0.0166 \\
\hline 18.3375 & 0.30305 & 0.018437 & 0.315731 & 0.0216027 & 0.321998 & 0.0174633 \\
\hline 18.3475 & 0.30395 & 0.0178127 & 0.320603 & 0.0206323 & 0.324127 & 0.017991 \\
\hline 18.3575 & 0.311339 & 0.0208725 & 0.32637 & 0.0209001 & 0.327939 & 0.018618 \\
\hline 18.3675 & 0.31543 & 0.0200214 & 0.32793 & 0.0214138 & 0.332941 & 0.019338 \\
\hline
\end{tabular}




\begin{tabular}{|c|c|c|c|c|c|c|}
\hline 18.3775 & 0.317584 & 0.0198961 & 0.338547 & 0.0198462 & 0.339766 & 0.020786 \\
\hline 8.3875 & 0.323606 & 0.0208423 & 0.342107 & 0.0206525 & 0.344928 & 0.0203171 \\
\hline 8.3975 & 0.324457 & 0.0202624 & 0.347257 & 0.0241031 & 0.350047 & 0.0199805 \\
\hline 3.4075 & .329932 & 0.0191228 & 0.350586 & 0.0200477 & 0.353144 & 0.0227172 \\
\hline 3.4175 & 0.335041 & 0.0182381 & 0.352348 & 0.0218484 & 0.361227 & 0.0204413 \\
\hline 8.4275 & 0.341768 & 0.0212092 & 0.358868 & 0.0235601 & 0.369659 & 0.0242238 \\
\hline 8.4375 & 0.344551 & 0.0201888 & 0.375433 & 0.021285 & 0.375056 & 0.0225037 \\
\hline 18.4475 & 0.353472 & 0.0201962 & 0.378643 & 0.0221174 & 0.382036 & 0.0210349 \\
\hline 18.4575 & 0.352665 & 0.0235797 & 0.387938 & 0.0237248 & 0.387188 & 0.0227757 \\
\hline 18.4675 & 0.36448 & 0.0211162 & 0.393936 & 0.0244788 & 0.399583 & 0.0237925 \\
\hline 18.4775 & 0.371339 & 0.0242087 & 0.403968 & 0.0250395 & 0.404377 & 0.0232655 \\
\hline 18.4875 & 0.380813 & 0.0223911 & 0.412081 & 0.0259589 & 0.416547 & 0.0267917 \\
\hline 18.4975 & 0.382661 & 0.0187711 & 0.42232 & 0.023856 & 0.425048 & 0.0250059 \\
\hline 18.5075 & 0.393436 & 0.0239046 & 0.428315 & 0.0254432 & 5774 & 0.023352 \\
\hline 18.5175 & 0.401305 & 0.0241008 & 0.437138 & 0.023947 & 0.443917 & 0.0251415 \\
\hline 18.5275 & 0.408573 & 0.0217917 & 0.446825 & 0.024836 & 0.4 & 0.0275704 \\
\hline 18.5375 & 0.420172 & 0.0242988 & 0.462099 & 0.0269386 & 0.468448 & 0.0278098 \\
\hline 18.5475 & 0.426702 & 0.0209218 & 0.475945 & 0.025241 & 0.474744 & 0.0284026 \\
\hline 18.5575 & 0.439406 & 0.0228175 & 6786 & 0.0272414 & 108 & 0.0282222 \\
\hline 18.5675 & 0.450863 & 0.0245704 & 0.498307 & 0.0253549 & 0.504093 & 0.0297659 \\
\hline 18.5775 & 0.456183 & 0.0237982 & 0.512475 & 0.0286374 & 0.515477 & 0.0277891 \\
\hline 18.5875 & 0.46 & 0.0270546 & 5107 & 3719 & 056 & 1426 \\
\hline 18.5975 & 0.485648 & 0.0250828 & 0.541323 & 0.0278211 & 0.540751 & 0.0312985 \\
\hline 18.6075 & 0.495529 & 0.0254152 & 0.551706 & 0.0311704 & 0.561636 & 0.0297666 \\
\hline 18.6175 & 0.509008 & 0.0286579 & 0.571604 & 0.0271876 & 0.578276 & 0.0334122 \\
\hline 18.6275 & 0.520218 & 0.0218933 & 0.583349 & 0.029721 & 0.586691 & 0.0308518 \\
\hline 18.6375 & 0.535559 & 0.023071 & 0.593224 & 0.0267812 & 0.606708 & 0.033522 \\
\hline 18.6475 & 0.546527 & 0.0280838 & 0.610332 & 0.0310534 & 0.616479 & 0.0335625 \\
\hline 18.6575 & 0.568054 & 0.0296972 & 0.632127 & 0.0314885 & 0.64139 & 0.0345746 \\
\hline 18.6675 & 0.57 & 0.0257379 & & 0.0321611 & & 0.033012 \\
\hline 18.6775 & 0.595537 & 0.0271547 & 0.668289 & 0.0361441 & 0.672351 & 0.0302004 \\
\hline 18.6875 & 0.609098 & 0.0304522 & 0.678002 & 0.0322935 & 0.687674 & 0.0316683 \\
\hline 18.6975 & 0.623725 & 0.0279422 & 0.696447 & 0.0309394 & 0.703614 & 0.0353478 \\
\hline 18.7075 & 0.638171 & 0.0337353 & 0.709609 & 0.0345598 & 0.720327 & 0.0360753 \\
\hline 18.7175 & 0.660012 & 0.0306748 & 0.732063 & 0.0358432 & 0.736872 & 0.0350506 \\
\hline 18.7275 & 0.672982 & 0.0304444 & 0.742103 & 0.0322736 & 0.753241 & 0.036365 \\
\hline 18.7375 & 0.688379 & 0.0301698 & 0.75841 & 0.0351024 & 0.774464 & 0.036437 \\
\hline 18.7475 & 0.704102 & 0.0328192 & 0.773305 & 0.0323087 & 723 & 0.0360957 \\
\hline 18.7575 & 0.726738 & 0.0298096 & 0.794145 & 0.0328589 & 0.799053 & 0.0357874 \\
\hline 18.7675 & 0.741847 & 0.0345029 & 0.809097 & 0.0355898 & 0.819161 & 0.03396 \\
\hline 18.7775 & 0.757964 & 0.0309693 & 0.819803 & 0.0381696 & 0.834761 & 0.0361389 \\
\hline 18.7875 & 0.774366 & 0.0337751 & 0.835929 & 0.0328475 & 0.84547 & 0.0358657 \\
\hline 18.7975 & 0.791914 & 0.0291513 & 0.85193 & 0.0380855 & 0.861046 & 0.0327489 \\
\hline 18.8075 & 0.804027 & 0.0320038 & 0.86134 & 0.0409057 & 0.875645 & 0.0417309 \\
\hline 18.8175 & 0.81997 & 0.0325123 & 0.874661 & 0.0362814 & 0.883149 & 0.035616 \\
\hline 18.8275 & 0.83435 & 0.0340404 & 0.885018 & 0.0347895 & 0.895407 & 0.0389376 \\
\hline 18.8375 & 0.847254 & 0.0328603 & 0.899876 & 0.0332706 & 0.909722 & 0.036917 \\
\hline
\end{tabular}




\begin{tabular}{|c|c|c|c|c|c|c|}
\hline 18.8475 & 0.862337 & 0.0320948 & 0.905111 & 0.0371115 & 0.915498 & 0.0345665 \\
\hline 18.8575 & 0.868985 & 0.0335666 & 0.912243 & 0.0357589 & 0.919283 & 0.0355757 \\
\hline 18.8675 & 0.882648 & 0.0349603 & 0.921195 & 0.0323046 & 0.926712 & 0.0324203 \\
\hline 18.8775 & 0.895289 & 0.0379314 & 0.934279 & 0.0350611 & 0.934186 & 0.0343779 \\
\hline 18.8875 & 0.905749 & 0.0367872 & 0.937306 & 0.0408167 & 0.947553 & 0.0321104 \\
\hline 18.8975 & 0.919232 & 0.0347055 & 0.946588 & 0.0351085 & 0.944817 & 0.0329004 \\
\hline 18.9075 & 0.924253 & 0.0358568 & 0.947426 & 0.0339745 & 0.946633 & 0.0340939 \\
\hline 18.9175 & 0.929925 & 0.0336909 & 0.949931 & 0.0308295 & 0.950912 & 0.0322898 \\
\hline 18.9275 & 0.941367 & 0.0329471 & 0.949888 & 0.032504 & 0.951428 & 0.0334766 \\
\hline 18.9375 & 0.942469 & 0.0338616 & 0.948018 & 0.0332003 & 0.954679 & 0.0324411 \\
\hline 18.9475 & 0.94801 & 0.0321909 & 0.946516 & 0.0317562 & 0.952361 & 0.0338124 \\
\hline 18.9575 & 0.94968 & 0.0313252 & 0.94312 & 0.035416 & 0.950974 & 0.0315183 \\
\hline 18.9675 & 0.952063 & 0.0318176 & 0.940686 & 0.037347 & 0.948333 & 0.031221 \\
\hline 18.9775 & 0.952144 & 0.0331186 & 0.931784 & 0.0355237 & 0.939278 & 0.032468 \\
\hline 18.9875 & 0.957574 & 0.0311003 & 0.932727 & 0.0377672 & 0.935959 & 0.0327862 \\
\hline 18.9975 & 0.949064 & 0.0362144 & 0.923995 & 0.0358172 & 0.92707 & 0.0311896 \\
\hline 19.0075 & 0.944863 & 0.0329669 & 0.919831 & 0.0385411 & 0.925973 & 0.0381388 \\
\hline 19.0175 & 0.942932 & 0.0371041 & 0.91002 & 0.0432724 & 0.909244 & 0.0335086 \\
\hline 19.0275 & 0.941305 & 0.0331101 & 0.905093 & 0.0345193 & 0.899275 & 0.03713 \\
\hline 19.0375 & 0.932564 & 0.0342091 & 0.898884 & 0.0384595 & 0.892104 & 0.0338332 \\
\hline 19.0475 & 0.930594 & 0.0373838 & 0.880134 & 0.0369389 & 0.879622 & 0.0377712 \\
\hline 19.0575 & 0.921759 & 0.0311966 & 0.867346 & 0.0364037 & 0.867273 & 0.0355267 \\
\hline 19.0675 & 0.907877 & 0.0322335 & 0.856797 & 0.0335239 & 0.856038 & 0.0331591 \\
\hline 19.0775 & 0.904354 & 0.0328562 & 0.843478 & 0.038625 & 0.837394 & 0.0339144 \\
\hline 19.0875 & 0.890122 & 0.0360877 & 0.824613 & 0.0368731 & 0.826925 & 0.0362075 \\
\hline 19.0975 & 0.874546 & 0.030224 & 0.806918 & 0.0368217 & 0.811041 & 0.0342638 \\
\hline 19.1075 & 0.86703 & 0.0354925 & 0.789601 & 0.0360009 & 0.794372 & 0.0332137 \\
\hline 19.1175 & 0.85443 & 0.0352335 & 0.775499 & 0.0358503 & 0.778059 & 0.030962 \\
\hline 19.1275 & 0.836238 & 0.0330711 & 0.763255 & 0.0397472 & 0.759302 & 0.0338154 \\
\hline 19.1375 & 0.8272 & 0.0375257 & 0.746332 & 0.0406385 & 0.752894 & 0.0363022 \\
\hline 19.1475 & 0.803607 & 0.0363529 & 0.724266 & 0.0330587 & 0.729429 & 0.0310926 \\
\hline 19.1575 & 0.794543 & 0.0319183 & 0.7144 & 0.0421435 & 0.714701 & 0.0350432 \\
\hline 19.1675 & 0.776672 & 0.0333931 & 0.694542 & 0.0290914 & 0.699382 & 0.0312481 \\
\hline 19.1775 & 0.765008 & 0.0319487 & 0.679668 & 0.0380647 & 0.687349 & 0.0295751 \\
\hline 19.1875 & 0.748319 & 0.0293689 & 0.663282 & 0.0341255 & 0.660235 & 0.0311398 \\
\hline 19.1975 & 0.729744 & 0.0330401 & 0.645623 & 0.0352189 & 0.642892 & 0.032066 \\
\hline 19.2075 & 0.715653 & 0.0315018 & 0.626683 & 0.0281225 & 0.626856 & 0.0311552 \\
\hline 19.2175 & 0.692569 & 0.0317647 & 0.608981 & 0.0301881 & 0.611007 & 0.0288269 \\
\hline 19.2275 & 0.679185 & 0.0299296 & 0.59205 & 0.0280159 & 0.594979 & 0.0290829 \\
\hline 19.2375 & 0.657527 & 0.0300427 & 0.581046 & 0.0295865 & 0.584797 & 0.0276759 \\
\hline 19.2475 & 0.640206 & 0.0309407 & 0.562063 & 0.0302897 & 0.564915 & 0.0306903 \\
\hline 19.2575 & 0.626307 & 0.0306608 & 0.548887 & 0.0297791 & 0.550237 & 0.026336 \\
\hline 19.2675 & 0.604382 & 0.0296758 & 0.530054 & 0.0281056 & 0.529508 & 0.0256862 \\
\hline 19.2775 & 0.592543 & 0.0282188 & 0.511307 & 0.0289015 & 0.514048 & 0.0282603 \\
\hline 19.2875 & 0.578072 & 0.024166 & 0.499184 & 0.0303214 & 0.505493 & 0.0286084 \\
\hline 19.2975 & 0.560907 & 0.0272482 & 0.489019 & 0.0282963 & 0.48538 & 0.0281426 \\
\hline 19.3075 & 0.538438 & 0.0260162 & 0.471776 & 0.0262476 & 0.473565 & 0.0245517 \\
\hline
\end{tabular}




\begin{tabular}{|c|c|c|c|c|c|c|}
\hline 19.3175 & 0.524776 & 0.0268587 & 0.459101 & 0.027717 & 0.46124 & 0.0237576 \\
\hline 19.3275 & 0.517941 & 0.0256303 & 0.448518 & 0.0286429 & 0.449701 & 0.0217731 \\
\hline 19.3375 & 0.501592 & 0.0256583 & 0.428277 & 0.02496 & 0.43661 & 0.0240582 \\
\hline 19.3475 & 0.486873 & 0.0260891 & 0.419576 & 0.0235144 & 0.422866 & 0.0223624 \\
\hline 19.3575 & 0.471363 & 0.0241216 & 0.407417 & 0.0244272 & 0.410387 & 0.0261088 \\
\hline 19.3675 & 0.457524 & 0.0231399 & 0.398759 & 0.0236621 & 0.398193 & 0.0196217 \\
\hline 19.3775 & 0.446745 & 0.0241334 & 0.385402 & 0.0238311 & 0.388615 & 0.0205791 \\
\hline 19.3875 & 0.433418 & 0.0235357 & 0.379797 & 0.0248996 & 0.379925 & 0.0253105 \\
\hline 19.3975 & 0.420438 & 0.0228985 & 0.364487 & 0.023989 & 0.370148 & 0.022503 \\
\hline 19.4075 & 0.406374 & 0.021046 & 0.359333 & 0.0216985 & 0.356049 & 0.022674 \\
\hline 19.4175 & 0.400399 & 0.0206125 & 0.348855 & 0.0195737 & 0.351511 & 0.0185876 \\
\hline 19.4275 & 0.385986 & 0.0203909 & 0.340866 & 0.0229449 & 0.343862 & 0.0206988 \\
\hline 19.4375 & 0.377226 & 0.0222745 & 0.333556 & 0.0203045 & 0.333394 & 0.0196465 \\
\hline 19.4475 & 0.366236 & 0.0199678 & 0.326132 & 0.0213912 & 0.325944 & 0.0194362 \\
\hline 19.4575 & 0.354345 & 0.0215396 & 0.316926 & 0.0218532 & 0.32229 & 0.0174591 \\
\hline 19.4675 & 0.349933 & 0.0202995 & 0.311764 & 0.0217604 & 0.312934 & 0.0202012 \\
\hline 19.4775 & 0.342522 & 0.0216696 & 0.303976 & 0.0181613 & 0.305201 & 0.0184758 \\
\hline 19.4875 & 0.334522 & 0.0215778 & 0.294945 & 0.018063 & 0.297794 & 0.0185148 \\
\hline 19.4975 & 0.32755 & 0.0175116 & 0.288358 & 0.018471 & 0.293052 & 0.0207075 \\
\hline 19.5075 & 0.320271 & 0.0183193 & 0.282079 & 0.0209026 & 0.284759 & 0.0184664 \\
\hline 19.5175 & 0.314313 & 0.0221443 & 0.275438 & 0.0206388 & 0.281578 & 0.016365 \\
\hline 19.5275 & 0.308454 & 0.0197268 & 0.26943 & 0.0196856 & 0.27645 & 0.0168276 \\
\hline 19.5375 & 0.301727 & 0.0204653 & 0.266514 & 0.0190602 & 0.273012 & 0.0175268 \\
\hline 19.5475 & 0.29482 & 0.0213855 & 0.262963 & 0.0181867 & 0.262176 & 0.0188625 \\
\hline 19.5575 & 0.284115 & 0.0200834 & 0.253669 & 0.0187718 & 0.258625 & 0.0172276 \\
\hline 19.5675 & 0.280483 & 0.0184968 & 0.252719 & 0.0185582 & 0.259607 & 0.0158132 \\
\hline 19.5775 & 0.27801 & 0.018749 & 0.248218 & 0.0200148 & 0.251392 & 0.0176492 \\
\hline 19.5875 & 0.274918 & 0.0191295 & 0.245892 & 0.0158811 & 0.246639 & 0.0179015 \\
\hline 19.5975 & 0.26666 & 0.0180222 & 0.235986 & 0.0184067 & 0.239329 & 0.016353 \\
\hline 19.6075 & 0.259435 & 0.0175437 & 0.232175 & 0.0174518 & 0.239818 & 0.0177022 \\
\hline 19.6175 & 0.25587 & 0.0187507 & 0.226715 & 0.0209486 & 0.230979 & 0.0155652 \\
\hline 19.6275 & 0.2538 & 0.0176923 & 0.223359 & 0.0175424 & 0.229139 & 0.0164706 \\
\hline 19.6375 & 0.249384 & 0.0176907 & 0.217733 & 0.0188704 & 0.226301 & 0.0158485 \\
\hline 19.6475 & 0.245456 & 0.017904 & 0.21666 & 0.0174279 & 0.220225 & 0.0178263 \\
\hline 19.6575 & 0.236585 & 0.0156496 & 0.215838 & 0.0174004 & 0.214537 & 0.0151276 \\
\hline 19.6675 & 0.235356 & 0.016743 & 0.21083 & 0.0169453 & 0.213535 & 0.013483 \\
\hline 19.6775 & 0.232027 & 0.0152417 & 0.203139 & 0.0150832 & 0.210947 & 0.0170592 \\
\hline 19.6875 & 0.225827 & 0.0165537 & 0.20088 & 0.0153669 & 0.208083 & 0.0134802 \\
\hline 19.6975 & 0.223737 & 0.0163743 & 0.199354 & 0.0154029 & 0.203467 & 0.0144075 \\
\hline 19.7075 & 0.221912 & 0.0164414 & 0.197524 & 0.0147745 & 0.203587 & 0.0144466 \\
\hline 19.7175 & 0.216525 & 0.0159137 & 0.193407 & 0.0165813 & 0.195709 & 0.0145531 \\
\hline 19.7275 & 0.210488 & 0.0178211 & 0.192283 & 0.0176808 & 0.193387 & 0.0166121 \\
\hline 19.7375 & 0.208535 & 0.0166064 & 0.188384 & 0.014652 & 0.192492 & 0.0138715 \\
\hline 19.7475 & 0.203446 & 0.0152211 & 0.189115 & 0.0151267 & 0.188954 & 0.0135774 \\
\hline 19.7575 & 0.201608 & 0.0151921 & 0.184131 & 0.015075 & 0.185262 & 0.0164582 \\
\hline 19.7675 & 0.199719 & 0.0157982 & 0.182569 & 0.0139762 & 0.18552 & 0.0151137 \\
\hline 19.7775 & 0.19572 & 0.0177744 & 0.183478 & 0.0146893 & 0.183458 & 0.0143645 \\
\hline
\end{tabular}




\begin{tabular}{|c|c|c|c|c|c|c|}
\hline 19.7875 & 0.191818 & 0.0156358 & 0.181591 & 0.0156954 & 0.179193 & 0.012729 \\
\hline 9.7975 & 0.189998 & 0.0139165 & 0.18197 & 0.0152223 & 0.182785 & 0.014771 \\
\hline .8075 & 0.187323 & 0.0151794 & 0.182621 & 0.0149825 & 0.181447 & 0.0139461 \\
\hline .8175 & 0.184155 & 0.0158608 & 0.182417 & 0.0154485 & 0.177664 & 0.0137121 \\
\hline .8275 & 0.183555 & 0.0153431 & 0.186847 & 0.014583 & 0.179449 & 0.0139403 \\
\hline 9.8375 & 0.180965 & 0.0140269 & 0.189429 & 0.0167989 & 0.183752 & 0.0144556 \\
\hline 9.8475 & 0.180618 & 0.0148848 & 0.193823 & 0.0158109 & 0.185224 & 0.0150025 \\
\hline 19.8575 & 0.181934 & 0.0145241 & 0.200594 & 0.0181556 & 0.186452 & 0.014336 \\
\hline 19.8675 & 0.18196 & 0.0147546 & 0.205841 & 0.0173435 & 0.19279 & 0.0125003 \\
\hline 9.8775 & 0.18215 & 0.0134437 & 0.216244 & 0.0187776 & 0.197839 & 0.014826 \\
\hline 19.8875 & 0.180451 & 0.0149408 & 0.228088 & 0.0198591 & 0.206689 & 0.0148665 \\
\hline 19.8975 & 0.189199 & 0.015301 & 0.237817 & 0.0181383 & 0.215718 & 0.0145132 \\
\hline 19.9075 & 0.188957 & 0.0163423 & 0.253541 & 0.020972 & 0.22189 & 0.0170258 \\
\hline 19.9175 & 0.195381 & 0.0160969 & 0.268447 & 0.0224744 & 0.232376 & 0.0165351 \\
\hline 19.9275 & 0.201365 & 0.0142936 & 0.284779 & 0.0247721 & 0.244391 & 0.0177372 \\
\hline 19.9375 & 0.207181 & 0.0162529 & 0.300085 & 0.0262182 & 0.258387 & 0.0190619 \\
\hline 19.9475 & 0.216736 & 0.0174832 & 0.320966 & 0.0285481 & 0.271477 & 0.0194325 \\
\hline 19.9575 & 0.228366 & 0.0164258 & 0.340276 & 0.0251458 & 0.287659 & 0.0190354 \\
\hline 19.9675 & 0.238289 & 0.0155957 & 0.362399 & 0.0284755 & 0.307134 & 0.020119 \\
\hline 19.9775 & 0.251984 & 0.0163113 & 0.387676 & 0.0282903 & 0.323293 & 0.021724 \\
\hline 19.9875 & 0.26534 & 0.0188538 & 0.410591 & 0.0248526 & 0.343705 & 0.0215427 \\
\hline 19.9975 & 0.28371 & 0.0188838 & 0.43 & 389 & 314 & 538 \\
\hline 20.0075 & 0.294586 & 0.0188272 & 0.456421 & 0.0291408 & 0.382292 & 0.0224829 \\
\hline 20.0175 & 0.319437 & 0.018088 & 0.484313 & 0.032105 & 0.402838 & 0.0264917 \\
\hline 20.0275 & 0.338537 & 0.0225497 & 0.513053 & 0.0322004 & 0.426015 & 0.0266635 \\
\hline 20.0375 & 0.355482 & 0.0224685 & 0.535818 & 0.0327591 & 0.452322 & 0.0288525 \\
\hline 20.0475 & 0.377965 & 0.0243084 & 0.55767 & 0.0351492 & 0.46908 & 0.0316081 \\
\hline 20.0575 & 0.400201 & 0.0220919 & 0.591507 & 0.032148 & 0.491921 & 0.0280167 \\
\hline 20.0675 & 0.421687 & 0.0238763 & 0.60986 & 0.0305142 & 0.512421 & 0.0327746 \\
\hline 20.0775 & 0.444281 & 0.0219224 & 263 & 0.0348755 & & 0.0317783 \\
\hline 20.0875 & 0.467722 & 0.0245059 & 0.650207 & 0.0311597 & 0.558993 & 0.0370442 \\
\hline 20.0975 & 0.494119 & 0.0249321 & 0.668532 & 0.0372727 & 0.570334 & 0.0372032 \\
\hline 20.1075 & 0.516443 & 0.0291244 & 0.681405 & 0.034093 & 0.590785 & 0.0381828 \\
\hline 20.1175 & 0.533544 & 0.0318346 & 0.70347 & 0.0368049 & 0.608048 & 0.0382929 \\
\hline 20.1275 & 0.558379 & 0.0305952 & 0.715435 & 0.0354656 & 0.621104 & 0.0384145 \\
\hline 20.1375 & 0.580439 & 0.0310786 & 0.71758 & 0.0395318 & 0.632297 & 0.040871 \\
\hline 20.1475 & 0.597018 & 0.0269504 & 0.717644 & 0.0328802 & 0.640968 & 0.0432554 \\
\hline 20.1575 & 0.618497 & 0.0291064 & 0.727816 & 0.0377658 & 0.650885 & 0.0398684 \\
\hline 20.1675 & 0.627718 & 0.0373341 & 0.724348 & 0.0378073 & 0.649187 & 0.039478 \\
\hline 20.1775 & 0.641904 & 0.0330361 & 0.722466 & 0.0384364 & 0.651132 & 0.0409538 \\
\hline 20.1875 & 0.659446 & 0.0268922 & 0.710774 & 0.0392193 & 0.647807 & 0.044272 \\
\hline 20.1975 & 0.666842 & 0.0348913 & 0.696203 & 0.0399185 & 0.639244 & 0.0409156 \\
\hline 20.2075 & 0.67368 & 0.0341852 & 0.673327 & 0.0421012 & 0.629655 & 0.0422976 \\
\hline 20.2175 & 0.673022 & 0.0319059 & 0.648753 & 0.0410327 & 0.620192 & 0.0388846 \\
\hline 20.2275 & 0.681074 & 0.0313422 & 0.630097 & 0.0410441 & 0.598098 & 0.0384577 \\
\hline 20.2375 & 0.673647 & 0.0291072 & 0.600643 & 0.0397667 & 0.585361 & 0.0384458 \\
\hline 20.2475 & 0.668863 & 0.0317004 & 0.573446 & 0.0426648 & 0.56259 & 0.0386043 \\
\hline
\end{tabular}




\begin{tabular}{|c|c|c|c|c|c|c|}
\hline 0.2575 & 0.652472 & 0.0328442 & 0.542058 & 0.0408265 & 0.535512 & 0.0353717 \\
\hline 0.2675 & 0.643658 & 0.032891 & 0.507422 & 0.04079 & 0.509813 & 0.0344534 \\
\hline .2775 & 0.623564 & 0.0313412 & 0.472346 & 0.0370164 & 0.486124 & 0.0315036 \\
\hline .2875 & 0.60136 & 0.0322646 & 0.438392 & 0.038913 & 0.455518 & 0.0303839 \\
\hline .2975 & 0.577415 & 0.0284584 & 0.415584 & 0.0367744 & 0.428945 & 0.0307256 \\
\hline 0.3075 & 0.550888 & 0.029599 & 0.374872 & 0.0336397 & 0.398588 & 0.0274788 \\
\hline .3175 & 0.525711 & 0.0311199 & 0.347304 & 0.0328179 & 0.371669 & 0.0272935 \\
\hline .3275 & 0.496239 & 0.0276417 & 0.317823 & 0.029644 & 0.337689 & 0.027176 \\
\hline 0.3375 & 0.466139 & 0.0273015 & 0.289516 & 0.0286613 & 0.311675 & 0.0236603 \\
\hline 0.3475 & 0.438778 & 0.0283153 & 0.266961 & 0.0239308 & 0.285018 & 0.0198861 \\
\hline 0.3575 & 0.407563 & 0.0260279 & 0.241822 & 0.0246211 & 0.266023 & 0.0214183 \\
\hline .3675 & 0.374275 & 0.024223 & 0.225545 & 0.0232472 & 0.243499 & 0.0191474 \\
\hline .3775 & 0.349797 & 0.022847 & 0.205211 & 0.0210194 & 0.223356 & 0.0180675 \\
\hline 0.3875 & 0.31696 & 0.0219988 & 0.190432 & 0.0221605 & 0.204198 & 0.0165257 \\
\hline 0.3975 & 0.290021 & 0.0196725 & 0.174163 & 0.0179884 & 0.190511 & 0.0154596 \\
\hline 0.4075 & 0.269029 & 0.0203919 & 0.166231 & 0.0170722 & 0.177627 & 0.0157573 \\
\hline 0.4175 & 0.243662 & 0.0194782 & 0.156724 & 0.0163025 & 0.164859 & 0.0144644 \\
\hline 0.4275 & 0.223648 & 0.0178391 & 0.145065 & 0.0158407 & 0.156285 & 0.0107867 \\
\hline 20.4375 & 0.204944 & 0.0157132 & 0.13572 & 0.0131071 & 0.147937 & 0.0145317 \\
\hline 20.4475 & 0.190375 & 0.0168543 & 0.133422 & 0.0153075 & 0.143347 & 0.0148275 \\
\hline 20.4575 & 0.179065 & 0.014269 & 0.131169 & 0.0117807 & 0.13402 & 0.0130246 \\
\hline 20.4675 & 0.167538 & 0.0162902 & 247 & 0.0135939 & 0.131407 & 0.0121706 \\
\hline 20.4775 & 0.155108 & 0.0149344 & 0.123376 & 0.0115259 & 0.124555 & 0.0113384 \\
\hline 20.4875 & 0.145586 & 0.0130904 & 0.12382 & 0.0137757 & 0.123274 & 0.0117624 \\
\hline 20.4975 & 0.138404 & 0.0135742 & 0.120288 & 0.0132546 & 0.1219 & 0.0105041 \\
\hline 20.5075 & 0.134469 & 0.0124334 & 0.11747 & 0.0135432 & 0.120956 & 0.012212 \\
\hline 20.5175 & 0.131606 & 0.0121796 & 0.117551 & 0.012753 & 0.117721 & 0.0129805 \\
\hline 20.5275 & 0.126221 & 0.0123359 & 0.117847 & 0.0118932 & 0.118663 & 0.0125364 \\
\hline 20.5375 & 0.12364 & 0.0121458 & 0.116618 & 0.0116717 & 0.117865 & 0.0126014 \\
\hline 20.5475 & 0.121612 & 0.0115305 & 0.115636 & 0.0119658 & 0.117917 & 0.0123839 \\
\hline 20.5575 & 0.119769 & 0.011008 & 0.117639 & 0.0107455 & 0.118712 & 0.0137347 \\
\hline 20.5675 & 0.118709 & 0.0124789 & 0.117085 & 0.0108681 & 0.115908 & 0.0101961 \\
\hline 20.5775 & 0.118079 & 0.0115739 & 0.114105 & 0.0108523 & 0.117957 & 0.0118236 \\
\hline 20.5875 & 0.118041 & 0.0110098 & 0.114849 & 0.0104763 & 0.1185 & 0.0101914 \\
\hline 20.5975 & 0.115967 & 0.011626 & 0.115836 & 0.0121939 & 0.116052 & 0.0115851 \\
\hline 20.6075 & 0.116969 & 0.0113237 & 0.113722 & 0.0116853 & 0.118192 & 0.0113784 \\
\hline 20.6175 & 0.115505 & 0.010976 & 0.114822 & 0.010374 & 0.120984 & 0.0120393 \\
\hline 20.6275 & 0.118359 & 0.0117918 & 0.115646 & 0.0117192 & 0.11864 & 0.011067 \\
\hline 20.6375 & 0.115595 & 0.0116338 & 0.117103 & 0.0130523 & 0.118178 & 0.0120945 \\
\hline 20.6475 & 0.116104 & 0.0130696 & 0.119069 & 0.0138731 & 0.120591 & 0.0143499 \\
\hline 20.6575 & 0.118011 & 0.0113157 & 0.120199 & 0.0128409 & 0.12236 & 0.0136016 \\
\hline 20.6675 & 0.117866 & 0.0119834 & 0.119111 & 0.0132071 & 0.120401 & 0.0123274 \\
\hline 20.6775 & 0.118209 & 0.0144115 & 0.121691 & 0.0117666 & 0.120512 & 0.0125852 \\
\hline 20.6875 & 0.118133 & 0.011512 & 0.121438 & 0.0124564 & 0.123757 & 0.0125533 \\
\hline 20.6975 & 0.11864 & 0.0109563 & 0.120472 & 0.0119712 & 0.124555 & 0.012512 \\
\hline 20.7075 & 0.118259 & 0.0127835 & 0.123508 & 0.0113906 & 0.124884 & 0.0132757 \\
\hline 20.7175 & 0.120684 & 0.0102706 & 0.123513 & 0.012811 & 0.123596 & 0.01220 \\
\hline
\end{tabular}




$\begin{array}{rrrrrrr}20.7275 & 0.118631 & 0.011741 & 0.122346 & 0.0118668 & 0.126703 & 0.0129907 \\ 20.7375 & 0.118144 & 0.010767 & 0.123104 & 0.013198 & 0.127316 & 0.0139152 \\ 20.7475 & 0.119716 & 0.0113733 & 0.12642 & 0.0141945 & 0.127875 & 0.0125254 \\ 20.7575 & 0.121788 & 0.0131854 & 0.126912 & 0.0122759 & 0.130735 & 0.0129991 \\ 20.7675 & 0.121323 & 0.0113461 & 0.127899 & 0.0144837 & 0.131744 & 0.0147225 \\ 20.7775 & 0.123991 & 0.0125657 & 0.130617 & 0.0120501 & 0.130555 & 0.0144173 \\ 20.7875 & 0.122118 & 0.0129542 & 0.127728 & 0.012098 & 0.132474 & 0.014708 \\ 20.7975 & 0.1258 & 0.0129092 & 0.1312 & 0.0137313 & 0.134877 & 0.0134656 \\ 20.8075 & 0.124928 & 0.0116019 & 0.133537 & 0.0119806 & 0.138352 & 0.0140852 \\ 20.8175 & 0.126647 & 0.0130767 & 0.133322 & 0.0132387 & 0.138561 & 0.0138619 \\ 20.8275 & 0.128221 & 0.0120344 & 0.133736 & 0.0127696 & 0.141101 & 0.0139103 \\ 20.8375 & 0.133423 & 0.0118589 & 0.135134 & 0.0132912 & 0.141917 & 0.0145221 \\ 20.8475 & 0.129537 & 0.00973234 & 0.138037 & 0.0141632 & 0.143592 & 0.016081 \\ 20.8575 & 0.131546 & 0.0116686 & 0.141896 & 0.0131597 & 0.145366 & 0.0153376 \\ 20.8675 & 0.13334 & 0.012376 & 0.141816 & 0.0115275 & 0.146359 & 0.0151564 \\ 20.8775 & 0.135749 & 0.0130146 & 0.143941 & 0.0145237 & 0.148408 & 0.0157669 \\ 20.8875 & 0.134915 & 0.0122776 & 0.1446 & 0.0131884 & 0.146793 & 0.0155792 \\ 20.8975 & 0.137328 & 0.0121952 & 0.148357 & 0.0136347 & 0.149933 & 0.0155658 \\ 20.9075 & 0.139437 & 0.0130133 & 0.148216 & 0.0115203 & 0.154151 & 0.014014 \\ 20.9175 & 0.142181 & 0.0137032 & 0.149508 & 0.0148422 & 0.154449 & 0.0146238 \\ 20.9275 & 0.143403 & 0.0143722 & 0.149071 & 0.014 & 0.156588 & 0.0159087 \\ 20.9375 & 0.142513 & 0.0148248 & 0.153573 & 0.0124852 & 0.153492 & 0.0158284 \\ 20.9475 & 0.146523 & 0.0138526 & 0.155321 & 0.0126501 & 0.158878 & 0.0152746 \\ 20.9575 & 0.145696 & 0.0127689 & 0.15843 & 0.013723 & 0.159346 & 0.0145742 \\ 20.9675 & 0.147725 & 0.0128134 & 0.154231 & 0.013994 & 0.163308 & 0.0176479 \\ 20.9775 & 0.153466 & 0.0140714 & 0.158379 & 0.0134817 & 0.16256 & 0.0164706 \\ 20.9875 & 0.15287 & 0.013607 & 0.160056 & 0.0133428 & 0.165764 & 0.0162785 \\ 20.9975 & 0.152387 & 0.0139763 & 0.164337 & 0.0153696 & 0.164902 & 0.0150312\end{array}$




\begin{tabular}{|c|c|c|c|c|c|c|}
\hline $\begin{array}{l}\mathrm{SB}>400 \mu \mathrm{m} \\
\text { mean }\end{array}$ & std & $\begin{array}{l}\text { SB X1 } \\
\text { mean }\end{array}$ & std & $\begin{array}{l}\text { SB SNF } \\
\text { mean }\end{array}$ & std & $\begin{array}{l}\text { SB SF } \\
\text { mean }\end{array}$ \\
\hline 0.301405 & 0.106735 & 0.308687 & 0.11143 & 0.207508 & 0.0201546 & 2.26705 \\
\hline 0.304656 & 0.110732 & 0.310137 & 0.108058 & 0.206511 & 0.0142846 & 2.22612 \\
\hline 0.303527 & 0.112638 & 0.315931 & 0.113678 & 0.210907 & 0.0169403 & 2.22689 \\
\hline 0.302083 & 0.112682 & 0.308801 & 0.110392 & 0.208779 & 0.015025 & 2.24404 \\
\hline 0.296841 & 0.101034 & 0.302719 & 0.114408 & 0.205839 & 0.01184 & 2.23019 \\
\hline 0.296671 & 0.103648 & 0.306009 & 0.102969 & 0.208633 & 0.0155606 & 2.24663 \\
\hline 0.298286 & 0.106989 & 0.30645 & 0.112916 & 0.207516 & 0.0176179 & 2.21478 \\
\hline 0.297871 & 0.103766 & 0.303618 & 0.109196 & 0.202652 & 0.0127888 & 2.21132 \\
\hline 0.296421 & 0.111086 & 0.304958 & 0.109611 & 0.205249 & 0.0114203 & 2.20511 \\
\hline 0.29582 & 0.107633 & 0.308482 & 0.111941 & 0.201042 & 0.016722 & 2.22559 \\
\hline 0.295062 & 0.103503 & 0.302535 & 0.107534 & 0.200532 & 0.014472 & 2.22557 \\
\hline 0.295744 & 0.105704 & 0.304841 & 0.103103 & 0.202207 & 0.0137443 & 2.2116 \\
\hline 0.293859 & 0.102985 & 0.311067 & 0.11518 & 0.206187 & 0.0165375 & 2.2145 \\
\hline 0.295007 & 0.105927 & 0.301094 & 0.109106 & 0.197679 & 0.0140839 & 2.20371 \\
\hline 0.292021 & 0.102103 & 0.303709 & 0.108588 & 0.20063 & 0.0131395 & 2.20423 \\
\hline 0.295662 & 0.109189 & 0.302503 & 0.118517 & 0.198765 & 0.0144961 & 2.19836 \\
\hline 0.295325 & 0.102074 & 0.306898 & 0.107371 & 0.202522 & 0.0167546 & 2.24487 \\
\hline 0.29476 & 0.100578 & 0.305782 & 0.114464 & 0.20357 & 0.0183039 & 2.21676 \\
\hline 0.299959 & 0.105998 & 0.30831 & 0.106408 & 0.208729 & 0.0180684 & 2.22763 \\
\hline 0.302645 & 0.106875 & 0.30877 & 0.113979 & 0.210373 & 0.0150397 & 2.23912 \\
\hline 0.303739 & 0.109545 & 0.315307 & 0.121606 & 0.216098 & 0.0143232 & 2.22939 \\
\hline 0.308595 & 0.109233 & 0.323118 & 0.11579 & 0.216713 & 0.016274 & 2.25873 \\
\hline 0.312258 & 0.105802 & 0.320944 & 0.114184 & 0.224228 & 0.0167299 & 2.23157 \\
\hline 0.322832 & 0.111033 & 0.327772 & 0.11891 & 0.224229 & 0.0158453 & 2.2213 \\
\hline 0.325782 & 0.11318 & 0.335759 & 0.120594 & 0.225353 & 0.0146191 & 2.2515 \\
\hline 0.328262 & 0.113772 & 0.332906 & 0.118561 & 0.227717 & 0.0187557 & 2.28104 \\
\hline 0.325031 & 0.112747 & 0.32266 & 0.120953 & 0.224952 & 0.0167007 & 2.24066 \\
\hline 0.324587 & 0.108909 & 0.323395 & 0.117069 & 0.222697 & 0.0128967 & 2.27249 \\
\hline 0.321836 & 0.111826 & 0.320343 & 0.12387 & 0.222966 & 0.0160793 & 2.2444 \\
\hline 0.313619 & 0.109053 & 0.321871 & 0.118168 & 0.21837 & 0.0165654 & 2.20182 \\
\hline 0.305886 & 0.105725 & 0.314741 & 0.119057 & 0.217483 & 0.0141103 & 2.23863 \\
\hline 0.303477 & 0.107752 & 0.308351 & 0.116626 & 0.211371 & 0.0145995 & 2.20453 \\
\hline 0.300926 & 0.105698 & 0.309562 & 0.117825 & 0.215614 & 0.0160385 & 2.20318 \\
\hline 0.298621 & 0.109518 & 0.307094 & 0.112969 & 0.217671 & 0.0153202 & 2.1886 \\
\hline 0.297101 & 0.102307 & 0.302818 & 0.110933 & 0.215103 & 0.0140671 & 2.17309 \\
\hline 0.299734 & 0.109481 & 0.307004 & 0.12029 & 0.218269 & 0.0145846 & 2.19934 \\
\hline 0.305512 & 0.109454 & 0.304061 & 0.1133 & 0.232976 & 0.0215142 & 2.18793 \\
\hline 0.313425 & 0.11121 & 0.31698 & 0.117024 & 0.254039 & 0.0216309 & 2.23924 \\
\hline 0.332374 & 0.114624 & 0.334086 & 0.124367 & 0.296554 & 0.0250523 & 2.2608 \\
\hline 0.36287 & 0.122626 & 0.363993 & 0.134331 & 0.359281 & 0.0327462 & 2.36981 \\
\hline 0.410251 & 0.136244 & 0.405212 & 0.162832 & 0.466959 & 0.0449386 & 2.4847 \\
\hline 0.47964 & 0.153458 & 0.458066 & 0.179872 & 0.606444 & 0.0580343 & 2.69445 \\
\hline 0.561202 & 0.176729 & 0.531656 & 0.202839 & 0.78837 & 0.0726182 & 2.96039 \\
\hline 0.667036 & 0.205507 & 0.625153 & 0.25539 & 1.0177 & 0.110837 & 3.24064 \\
\hline 0.794112 & 0.246879 & 0.726693 & 0.2906 & 1.275 & 0.143062 & 3.61264 \\
\hline
\end{tabular}




\begin{tabular}{|c|c|c|c|c|c|c|}
\hline 0.922619 & 0.280766 & 0.827076 & 0.338911 & 1.53985 & 0.179596 & 3.94844 \\
\hline 1.03754 & 0.32275 & 0.92193 & 0.38439 & 1.77575 & 0.20439 & 4.23805 \\
\hline 1.12945 & 0.351517 & 0.997562 & 0.404046 & 1.95232 & 0.211892 & 4.54869 \\
\hline 1.18454 & 0.358708 & 1.03541 & 0.433121 & 2.04788 & 0.2165 & 4.64071 \\
\hline 1.19075 & 0.36248 & 1.03113 & 0.425199 & 2.05295 & 0.230785 & 4.62252 \\
\hline 1.14733 & 0.355675 & 0.998692 & 0.40832 & 1.95366 & 0.216282 & 4.53226 \\
\hline 1.06799 & 0.321039 & 0.922986 & 0.371008 & 1.77308 & 0.203659 & 4.24779 \\
\hline 0.965371 & 0.3019 & 0.826326 & 0.338303 & 1.53331 & 0.166427 & 3.96532 \\
\hline 0.849417 & 0.262104 & 0.719981 & 0.291374 & 1.28793 & 0.131374 & 3.63702 \\
\hline 0.727459 & 0.22456 & 0.634273 & 0.25819 & 1.04649 & 0.106603 & 3.26762 \\
\hline 0.624678 & 0.193074 & 0.552862 & 0.225438 & 0.832537 & 0.0892648 & 2.98713 \\
\hline 0.548433 & 0.16968 & 0.495119 & 0.206384 & 0.653595 & 0.0658854 & 2.73518 \\
\hline 0.483809 & 0.157097 & 0.441898 & 0.172671 & 0.517751 & 0.0444023 & 2.54658 \\
\hline 0.445515 & 0.145822 & 0.41604 & 0.16779 & 0.427232 & 0.0406478 & 2.4497 \\
\hline 0.427175 & 0.139774 & 0.410751 & 0.170068 & 0.366832 & 0.0354811 & 2.36178 \\
\hline 0.44238 & 0.14431 & 0.429937 & 0.174395 & 0.348152 & 0.0341047 & 2.35305 \\
\hline 0.501028 & 0.158765 & 0.492124 & 0.205547 & 0.375542 & 0.033471 & 2.42599 \\
\hline 0.622488 & 0.197096 & 0.625702 & 0.273439 & 0.448361 & 0.0410147 & 2.65436 \\
\hline 0.843279 & 0.269944 & 0.83627 & 0.381326 & 0.602861 & 0.0603015 & 3.0602 \\
\hline 1.20196 & 0.390512 & 1.16679 & 0.544323 & 0.851281 & 0.08076 & 3.69562 \\
\hline 1.73956 & 0.575867 & 1.68261 & 0.824195 & 1.2178 & 0.128112 & 4.66518 \\
\hline 2.51414 & 0.837395 & 2.38746 & 1.19222 & 1.7422 & 0.190994 & 6.05742 \\
\hline 3.53011 & 1.17816 & 3.30963 & 1.67876 & 2.4423 & 0.259821 & 7.85826 \\
\hline 4.78911 & 1.61949 & 4.4363 & 2.26747 & 3.29573 & 0.350514 & 10.0376 \\
\hline 6.24112 & 2.10333 & 5.72337 & 2.93666 & 4.2536 & 0.476974 & 12.5634 \\
\hline 7.74644 & 2.62177 & 7.00565 & 3.58587 & 5.24586 & 0.590371 & 15.0729 \\
\hline 9.18462 & 3.12504 & 8.1862 & 4.20222 & 6.18516 & 0.676862 & 17.5132 \\
\hline 10.3506 & 3.51327 & 9.1074 & 4.65871 & 6.92793 & 0.755638 & 19.4821 \\
\hline 11.0906 & 3.79291 & 9.66204 & 4.982 & 7.35558 & 0.802061 & 20.7207 \\
\hline 11.2946 & 3.84876 & 9.73003 & 5.02615 & 7.4404 & 0.817311 & 20.9289 \\
\hline 10.9805 & 3.75103 & 9.28289 & 4.79974 & 7.17631 & 0.790804 & 20.2087 \\
\hline 10.1406 & 3.47394 & 8.52538 & 4.43293 & 6.58509 & 0.745883 & 18.7626 \\
\hline 8.94618 & 3.05488 & 7.44728 & 3.86628 & 5.79957 & 0.651236 & 16.6874 \\
\hline 7.54223 & 2.56541 & 6.20425 & 3.22901 & 4.82574 & 0.529498 & 14.2816 \\
\hline 6.10243 & 2.07587 & 4.98819 & 2.58356 & 3.88855 & 0.424243 & 11.831 \\
\hline 4.76854 & 1.62721 & 3.87888 & 1.98508 & 3.03865 & 0.338053 & 9.58169 \\
\hline 3.62642 & 1.22655 & 2.96262 & 1.51594 & 2.28861 & 0.252183 & 7.653 \\
\hline 2.7241 & 0.914173 & 2.22124 & 1.10317 & 1.7002 & 0.176317 & 6.12153 \\
\hline 2.05667 & 0.672769 & 1.69994 & 0.822942 & 1.28203 & 0.140309 & 5.01296 \\
\hline 1.55113 & 0.510153 & 1.29346 & 0.635097 & 0.964635 & 0.0995608 & 4.15967 \\
\hline 1.18906 & 0.393388 & 1.0076 & 0.491736 & 0.737116 & 0.0700289 & 3.53289 \\
\hline 0.936346 & 0.30393 & 0.804514 & 0.367258 & 0.571295 & 0.0515317 & 3.11701 \\
\hline 0.754374 & 0.244348 & 0.653943 & 0.287976 & 0.459817 & 0.0415526 & 2.79794 \\
\hline 0.62588 & 0.199882 & 0.545005 & 0.237343 & 0.382824 & 0.0296623 & 2.56781 \\
\hline 0.532416 & 0.172672 & 0.467788 & 0.18772 & 0.320754 & 0.0224117 & 2.4246 \\
\hline 0.462496 & 0.147902 & 0.416318 & 0.160499 & 0.279257 & 0.0236065 & 2.32891 \\
\hline 0.411787 & 0.131439 & 0.375455 & 0.15611 & 0.251075 & 0.0188884 & 2.22138 \\
\hline
\end{tabular}




\begin{tabular}{|c|c|c|c|c|c|c|}
\hline 0.376857 & 0.13422 & 0.365689 & 0.143013 & 0.235823 & 0.0184049 & 2.16409 \\
\hline 0.357537 & 0.125818 & 0.353624 & 0.135396 & 0.228827 & 0.0196343 & 2.12041 \\
\hline 0.349063 & 0.123017 & 0.351998 & 0.13518 & 0.230167 & 0.0193892 & 2.12755 \\
\hline 0.334676 & 0.11295 & 0.36345 & 0.143291 & 0.245096 & 0.0261421 & 2.12955 \\
\hline 0.332745 & 0.107975 & 0.367933 & 0.138046 & 0.257039 & 0.0282007 & 2.16679 \\
\hline 0.340259 & 0.122857 & 0.377293 & 0.149385 & 0.275938 & 0.0238608 & 2.17966 \\
\hline 0.345259 & 0.128193 & 0.380871 & 0.150545 & 0.291092 & 0.0252262 & 2.26163 \\
\hline 0.349321 & 0.131699 & 0.396303 & 0.150479 & 0.307689 & 0.0285898 & 2.30729 \\
\hline 0.357671 & 0.128954 & 0.400382 & 0.156765 & 0.3205 & 0.0305121 & 2.34532 \\
\hline 0.362179 & 0.120646 & 0.403593 & 0.161035 & 0.322581 & 0.0287649 & 2.37597 \\
\hline 0.365242 & 0.126768 & 0.394992 & 0.15717 & 0.324975 & 0.028041 & 2.40083 \\
\hline 0.361921 & 0.129666 & 0.390673 & 0.154935 & 0.315294 & 0.0264028 & 2.385 \\
\hline 0.353917 & 0.132698 & 0.387107 & 0.152566 & 0.299061 & 0.0291838 & 2.39445 \\
\hline 0.343491 & 0.127873 & 0.367996 & 0.147188 & 0.282108 & 0.0238274 & 2.37641 \\
\hline 0.335946 & 0.120959 & 0.353201 & 0.134942 & 0.264062 & 0.0193023 & 2.329 \\
\hline 0.32734 & 0.117511 & 0.338839 & 0.126049 & 0.245692 & 0.0189113 & 2.30572 \\
\hline 0.313841 & 0.114797 & 0.324438 & 0.12248 & 0.227351 & 0.0168124 & 2.25332 \\
\hline 0.304222 & 0.114343 & 0.322614 & 0.126107 & 0.212391 & 0.0160212 & 2.24438 \\
\hline 0.297066 & 0.107767 & 0.316523 & 0.118459 & 0.200822 & 0.0131792 & 2.20157 \\
\hline 0.294331 & 0.105938 & 0.308624 & 0.115244 & 0.193976 & 0.013225 & 2.14537 \\
\hline 0.289057 & 0.102034 & 0.302599 & 0.117071 & 0.185852 & 0.0131275 & 2.1195 \\
\hline 0.287102 & 0.102746 & 0.303824 & 0.115368 & 0.182476 & 0.0134558 & 2.08954 \\
\hline 0.286987 & 0.103101 & 0.299923 & 0.115882 & 0.182631 & 0.013154 & 2.07054 \\
\hline 0.290404 & 0.104406 & 0.310419 & 0.119066 & 0.18193 & 0.0139612 & 2.05216 \\
\hline 0.299512 & 0.108599 & 0.326413 & 0.126641 & 0.182409 & 0.0135025 & 2.03342 \\
\hline 0.312763 & 0.1109 & 0.35402 & 0.135697 & 0.190295 & 0.0139952 & 2.0309 \\
\hline 0.337107 & 0.112017 & 0.387438 & 0.151238 & 0.203176 & 0.0126854 & 2.02306 \\
\hline 0.374851 & 0.120356 & 0.438565 & 0.179398 & 0.22129 & 0.0123775 & 2.02361 \\
\hline 0.435706 & 0.139439 & 0.528339 & 0.216428 & 0.249639 & 0.0157115 & 2.06502 \\
\hline 0.525766 & 0.161611 & 0.658184 & 0.27863 & 0.297006 & 0.0205493 & 2.11373 \\
\hline 0.644769 & 0.187185 & 0.838328 & 0.352795 & 0.36308 & 0.0291593 & 2.17742 \\
\hline 0.80403 & 0.221655 & 1.06585 & 0.4499 & 0.448346 & 0.0386632 & 2.26574 \\
\hline 1.00484 & 0.279961 & 1.3422 & 0.586116 & 0.548835 & 0.0462272 & 2.38176 \\
\hline 1.24317 & 0.350487 & 1.65603 & 0.738172 & 0.669816 & 0.0576188 & 2.52048 \\
\hline 1.50418 & 0.422499 & 1.99616 & 0.884357 & 0.796993 & 0.0718903 & 2.65862 \\
\hline 1.76739 & 0.485598 & 2.33261 & 1.04278 & 0.914228 & 0.0824123 & 2.77446 \\
\hline 1.99975 & 0.548513 & 2.62883 & 1.19158 & 1.02309 & 0.0886223 & 2.87576 \\
\hline 2.18478 & 0.59709 & 2.85265 & 1.29126 & 1.10644 & 0.0992407 & 2.99498 \\
\hline 2.30193 & 0.62716 & 2.96735 & 1.33311 & 1.14736 & 0.106344 & 3.06978 \\
\hline 2.32455 & 0.635853 & 2.96864 & 1.33483 & 1.14424 & 0.107357 & 3.04752 \\
\hline 2.26139 & 0.621383 & 2.83253 & 1.2735 & 1.10496 & 0.101203 & 3.00556 \\
\hline 2.11518 & 0.576291 & 2.62941 & 1.1646 & 1.0296 & 0.096212 & 2.89699 \\
\hline 1.90413 & 0.518793 & 2.33956 & 1.03089 & 0.919819 & 0.0795879 & 2.77613 \\
\hline 1.66039 & 0.46054 & 2.00591 & 0.880313 & 0.796082 & 0.0685334 & 2.65473 \\
\hline 1.40368 & 0.390891 & 1.66569 & 0.717452 & 0.671587 & 0.0574162 & 2.48588 \\
\hline 1.16 & 0.320983 & 1.36255 & 0.573979 & 0.558863 & 0.0425947 & 2.3481 \\
\hline 0.95214 & 0.267476 & 1.10761 & 0.451712 & 0.459656 & 0.0328311 & 2.26231 \\
\hline
\end{tabular}




\begin{tabular}{|c|c|c|c|c|c|c|}
\hline 0.778745 & 0.223546 & 0.905387 & 0.364836 & 0.380952 & 0.0256029 & 2.16465 \\
\hline 0.651788 & 0.193568 & 0.746742 & 0.300044 & 0.325935 & 0.021689 & 2.08963 \\
\hline 0.559978 & 0.165415 & 0.647243 & 0.246455 & 0.291486 & 0.0193821 & 2.04419 \\
\hline 0.501353 & 0.146831 & 0.590655 & 0.222645 & 0.270325 & 0.0172872 & 2.01471 \\
\hline 0.469183 & 0.14056 & 0.546215 & 0.199974 & 0.257542 & 0.0175151 & 2.02301 \\
\hline 0.450278 & 0.136382 & 0.521495 & 0.191282 & 0.252011 & 0.015264 & 2.00817 \\
\hline 0.434556 & 0.134846 & 0.510498 & 0.186851 & 0.248095 & 0.0126222 & 1.99517 \\
\hline 0.426725 & 0.133323 & 0.500644 & 0.181503 & 0.240823 & 0.015278 & 2.00217 \\
\hline 0.420338 & 0.134424 & 0.479409 & 0.18048 & 0.237179 & 0.0147405 & 2.00935 \\
\hline 0.407154 & 0.13307 & 0.458802 & 0.171211 & 0.235576 & 0.0154482 & 2.02089 \\
\hline 0.391031 & 0.1285 & 0.433704 & 0.160275 & 0.22889 & 0.0146087 & 2.00782 \\
\hline 0.372923 & 0.124724 & 0.408099 & 0.148062 & 0.216873 & 0.0140069 & 1.99593 \\
\hline 0.353708 & 0.116045 & 0.384644 & 0.144042 & 0.208053 & 0.0115156 & 2.00099 \\
\hline 0.338744 & 0.112947 & 0.363698 & 0.133346 & 0.198747 & 0.0106627 & 2.00007 \\
\hline 0.327846 & 0.113506 & 0.344482 & 0.125015 & 0.195545 & 0.00993633 & 2.00998 \\
\hline 0.317603 & 0.107031 & 0.335581 & 0.124535 & 0.193926 & 0.0113217 & 2.04297 \\
\hline 0.311076 & 0.100894 & 0.329372 & 0.122134 & 0.194349 & 0.0120945 & 2.08797 \\
\hline 0.315145 & 0.102524 & 0.32409 & 0.121432 & 0.203467 & 0.0136228 & 2.15856 \\
\hline 0.326438 & 0.110596 & 0.334488 & 0.127329 & 0.215116 & 0.0150431 & 2.28644 \\
\hline 0.346635 & 0.118254 & 0.35587 & 0.137845 & 0.233306 & 0.0175646 & 2.48138 \\
\hline 0.375611 & 0.121282 & 0.378907 & 0.138514 & 0.262196 & 0.0194287 & 2.73297 \\
\hline 0.412239 & 0.126899 & 0.413405 & 0.145295 & 0.298956 & 0.0199477 & 3.09541 \\
\hline 0.463297 & 0.13967 & 0.458681 & 0.165515 & 0.346322 & 0.0236429 & 3.5966 \\
\hline 0.523 & 0.160167 & 0.510415 & 0.180859 & 0.407298 & 0.0318296 & 4.19223 \\
\hline 0.588843 & 0.17355 & 0.57374 & 0.202611 & 0.477706 & 0.0405266 & 4.94463 \\
\hline 0.66889 & 0.189289 & 0.640982 & 0.23069 & 0.549335 & 0.0477551 & 5.7714 \\
\hline 0.753473 & 0.212831 & 0.703966 & 0.251623 & 0.623658 & 0.0547991 & 6.63497 \\
\hline 0.831586 & 0.239769 & 0.771852 & 0.271892 & 0.698599 & 0.0604395 & 7.45958 \\
\hline 0.903442 & 0.263303 & 0.835163 & 0.295429 & 0.760895 & 0.0712701 & 8.21712 \\
\hline 0.957615 & 0.283234 & 0.87121 & 0.309238 & 0.806209 & 0.0735183 & 8.83745 \\
\hline 0.98755 & 0.299085 & 0.891144 & 0.310166 & 0.832373 & 0.072362 & 9.19713 \\
\hline 0.996762 & 0.298111 & 0.891669 & 0.310804 & 0.831719 & 0.074484 & 9.27493 \\
\hline 0.985044 & 0.289662 & 0.874711 & 0.307372 & 0.812981 & 0.0703834 & 9.07119 \\
\hline 0.958074 & 0.279757 & 0.838683 & 0.296215 & 0.778119 & 0.0643628 & 8.71355 \\
\hline 0.913959 & 0.264259 & 0.799337 & 0.28467 & 0.726501 & 0.0595233 & 8.22988 \\
\hline 0.863529 & 0.248888 & 0.771613 & 0.277635 & 0.675143 & 0.053512 & 7.68159 \\
\hline 0.824016 & 0.238193 & 0.750396 & 0.271294 & 0.624499 & 0.0427745 & 7.19325 \\
\hline 0.79843 & 0.227209 & 0.737163 & 0.267383 & 0.590004 & 0.0377481 & 6.79893 \\
\hline 0.791459 & 0.224583 & 0.73412 & 0.262622 & 0.567045 & 0.0403301 & 6.58103 \\
\hline 0.802884 & 0.22864 & 0.754314 & 0.273936 & 0.553876 & 0.036669 & 6.53038 \\
\hline 0.825578 & 0.232661 & 0.788877 & 0.300048 & 0.560058 & 0.0368268 & 6.61117 \\
\hline 0.857763 & 0.240478 & 0.827902 & 0.32288 & 0.575952 & 0.0448562 & 6.84031 \\
\hline 0.894948 & 0.25049 & 0.866698 & 0.331081 & 0.589046 & 0.0438771 & 7.06688 \\
\hline 0.925455 & 0.261797 & 0.885186 & 0.335698 & 0.600049 & 0.0436904 & 7.22244 \\
\hline 0.9419 & 0.265494 & 0.899677 & 0.344373 & 0.604876 & 0.0423049 & 7.29915 \\
\hline 0.945092 & 0.267158 & 0.901004 & 0.343095 & 0.601477 & 0.0414725 & 7.23966 \\
\hline 0.928815 & 0.265625 & 0.872978 & 0.32832 & 0.585795 & 0.0406707 & 7.03121 \\
\hline
\end{tabular}




\begin{tabular}{|c|c|c|c|c|c|c|}
\hline 0.896101 & 0.257663 & 0.831844 & 0.307529 & 0.557308 & 0.0401937 & 6.65747 \\
\hline 0.854469 & 0.245539 & 0.79433 & 0.292759 & 0.526256 & 0.0372796 & 6.21576 \\
\hline 0.800523 & 0.228658 & 0.754504 & 0.277459 & 0.496722 & 0.0299141 & 5.70833 \\
\hline 0.740724 & 0.206615 & 0.705575 & 0.252949 & 0.460272 & 0.0286796 & 5.15287 \\
\hline 0.691924 & 0.190264 & 0.661811 & 0.231549 & 0.424372 & 0.03007 & 4.65589 \\
\hline 0.650961 & 0.181141 & 0.622078 & 0.217124 & 0.398554 & 0.0286348 & 4.20989 \\
\hline 0.611639 & 0.171948 & 0.584813 & 0.204106 & 0.373926 & 0.027642 & 3.79727 \\
\hline 0.580134 & 0.161482 & 0.557383 & 0.191471 & 0.349281 & 0.0215223 & 3.47118 \\
\hline 0.558101 & 0.158715 & 0.53922 & 0.1804 & 0.330443 & 0.0205738 & 3.20802 \\
\hline 0.539122 & 0.154422 & 0.520488 & 0.173819 & 0.314624 & 0.0204336 & 2.97128 \\
\hline 0.525704 & 0.15302 & 0.506717 & 0.171121 & 0.302833 & 0.0194453 & 2.78465 \\
\hline 0.51423 & 0.150707 & 0.497199 & 0.165102 & 0.289576 & 0.0214027 & 2.63199 \\
\hline 0.499805 & 0.146984 & 0.48068 & 0.159596 & 0.276509 & 0.020021 & 2.50839 \\
\hline 0.485448 & 0.145644 & 0.470257 & 0.158623 & 0.264286 & 0.0187317 & 2.40091 \\
\hline 0.468684 & 0.143604 & 0.455598 & 0.15358 & 0.252195 & 0.016548 & 2.31534 \\
\hline 0.456246 & 0.145323 & 0.435287 & 0.15252 & 0.242166 & 0.0146855 & 2.23076 \\
\hline 0.440509 & 0.136602 & 0.425411 & 0.151627 & 0.231557 & 0.0163674 & 2.1613 \\
\hline 0.423929 & 0.131254 & 0.416105 & 0.144146 & 0.218503 & 0.0148076 & 2.1087 \\
\hline 0.409651 & 0.131745 & 0.401517 & 0.141564 & 0.210852 & 0.015712 & 2.04696 \\
\hline 0.397179 & 0.12779 & 0.389835 & 0.140898 & 0.20743 & 0.0138333 & 1.98956 \\
\hline 0.387311 & 0.129363 & 0.384254 & 0.141786 & 0.200883 & 0.0139005 & 1.95899 \\
\hline 0.378214 & 0.128334 & 0.381385 & 0.146725 & 0.193327 & 0.011364 & 1.93358 \\
\hline 0.372849 & 0.126622 & 0.379529 & 0.148268 & 0.191337 & 0.0114634 & 1.92274 \\
\hline 0.366381 & 0.124237 & 0.381737 & 0.144595 & 0.193668 & 0.0119929 & 1.9095 \\
\hline 0.367195 & 0.123314 & 0.385409 & 0.149215 & 0.196442 & 0.0108395 & 1.90467 \\
\hline 0.370775 & 0.12364 & 0.388345 & 0.146214 & 0.201656 & 0.0135851 & 1.91316 \\
\hline 0.375848 & 0.129573 & 0.388824 & 0.146106 & 0.207841 & 0.0145945 & 1.92712 \\
\hline 0.383307 & 0.133534 & 0.395524 & 0.152166 & 0.213895 & 0.0130022 & 1.91507 \\
\hline 0.390748 & 0.131999 & 0.406339 & 0.156672 & 0.227105 & 0.0142201 & 1.9441 \\
\hline 0.403299 & 0.135149 & 0.41645 & 0.159797 & 0.247886 & 0.0171306 & 1.97362 \\
\hline 0.422696 & 0.143067 & 0.43672 & 0.167502 & 0.281109 & 0.0179059 & 2.0227 \\
\hline 0.450195 & 0.151553 & 0.472575 & 0.184915 & 0.329265 & 0.0222421 & 2.0947 \\
\hline 0.49052 & 0.158741 & 0.518163 & 0.205931 & 0.396884 & 0.0304104 & 2.1926 \\
\hline 0.552139 & 0.176036 & 0.588332 & 0.240898 & 0.506759 & 0.0374853 & 2.35718 \\
\hline 0.640934 & 0.204763 & 0.680065 & 0.282539 & 0.667198 & 0.0469872 & 2.5971 \\
\hline 0.763619 & 0.243334 & 0.798719 & 0.342557 & 0.883594 & 0.0654907 & 2.94332 \\
\hline 0.924988 & 0.297715 & 0.95129 & 0.424463 & 1.17341 & 0.0785575 & 3.39756 \\
\hline 1.13679 & 0.362175 & 1.15995 & 0.523642 & 1.53697 & 0.103085 & 3.94453 \\
\hline 1.39566 & 0.446126 & 1.41575 & 0.647692 & 1.97639 & 0.146032 & 4.61785 \\
\hline 1.69158 & 0.543041 & 1.68099 & 0.786597 & 2.47683 & 0.195348 & 5.41398 \\
\hline 2.00852 & 0.654902 & 1.97759 & 0.934614 & 3.01369 & 0.238984 & 6.23053 \\
\hline 2.32835 & 0.770898 & 2.27474 & 1.0754 & 3.54086 & 0.274383 & 7.02476 \\
\hline 2.63742 & 0.872527 & 2.52657 & 1.1987 & 4.01424 & 0.30405 & 7.78565 \\
\hline 2.88107 & 0.953138 & 2.69282 & 1.29943 & 4.38106 & 0.32427 & 8.37833 \\
\hline 3.05486 & 1.00882 & 2.81864 & 1.37 & 4.62469 & 0.346757 & 8.76324 \\
\hline 3.13747 & 1.04176 & 2.85067 & 1.38107 & 4.72411 & 0.346413 & 8.9004 \\
\hline 3.12482 & 1.04399 & 2.78947 & 1.35222 & 4.64264 & 0.345403 & 8.80119 \\
\hline
\end{tabular}




\begin{tabular}{|c|c|c|c|c|c|c|}
\hline 2.99993 & 0.996599 & 2.64829 & 1.285 & 4.39459 & 0.331619 & 8.41864 \\
\hline 2.79284 & 0.92674 & 2.44289 & 1.17609 & 4.01472 & 0.305917 & 7.85982 \\
\hline 2.52044 & 0.840686 & 2.18433 & 1.04041 & 3.55078 & 0.277739 & 7.14655 \\
\hline 2.20614 & 0.735803 & 1.90165 & 0.909514 & 3.02688 & 0.22792 & 6.33781 \\
\hline 1.8852 & 0.620922 & 1.61043 & 0.762178 & 2.48763 & 0.183544 & 5.51245 \\
\hline 1.57673 & 0.516727 & 1.3502 & 0.623193 & 1.98078 & 0.141132 & 4.72954 \\
\hline 1.30275 & 0.424726 & 1.13464 & 0.517167 & 1.54734 & 0.106248 & 4.03254 \\
\hline 1.07298 & 0.349849 & 0.95197 & 0.429403 & 1.18417 & 0.0846665 & 3.44904 \\
\hline 0.890764 & 0.289402 & 0.805519 & 0.353893 & 0.890539 & 0.0580483 & 2.98488 \\
\hline 0.756088 & 0.241295 & 0.706255 & 0.302115 & 0.665786 & 0.0427609 & 2.62152 \\
\hline 0.663859 & 0.211459 & 0.646119 & 0.261338 & 0.508722 & 0.0344843 & 2.36989 \\
\hline 0.611149 & 0.195879 & 0.620026 & 0.2482 & 0.411423 & 0.0246101 & 2.21111 \\
\hline 0.58214 & 0.186062 & 0.609941 & 0.249825 & 0.353137 & 0.019643 & 2.10387 \\
\hline 0.583848 & 0.190078 & 0.623811 & 0.254461 & 0.323474 & 0.0204366 & 2.04502 \\
\hline 0.613044 & 0.202225 & 0.664488 & 0.271784 & 0.321013 & 0.0207822 & 2.03927 \\
\hline 0.662612 & 0.217418 & 0.730034 & 0.296375 & 0.340413 & 0.0201965 & 2.06438 \\
\hline 0.742486 & 0.240399 & 0.828442 & 0.328501 & 0.379745 & 0.0229455 & 2.11901 \\
\hline 0.855916 & 0.270246 & 0.978705 & 0.391818 & 0.433591 & 0.0278689 & 2.20537 \\
\hline 1.02479 & 0.318885 & 1.1936 & 0.477481 & 0.510566 & 0.0341381 & 2.28692 \\
\hline 1.28325 & 0.396967 & 1.51542 & 0.604967 & 0.617378 & 0.0435289 & 2.39367 \\
\hline 1.66037 & 0.515563 & 2.01323 & 0.822775 & 0.759494 & 0.0553272 & 2.55696 \\
\hline 2.22578 & 0.69241 & 2.75674 & 1.12624 & 0.933832 & 0.0647383 & 2.76393 \\
\hline 3.08262 & 0.962003 & 3.87345 & 1.56708 & 1.16927 & 0.0713231 & 3.01826 \\
\hline 4.34763 & 1.34644 & 5.48925 & 2.22595 & 1.49542 & 0.091603 & 3.32548 \\
\hline 6.14942 & 1.91107 & 7.77966 & 3.15677 & 1.91424 & 0.123485 & 3.6874 \\
\hline 8.65161 & 2.69878 & 10.9424 & 4.4446 & 2.45534 & 0.15895 & 4.1563 \\
\hline 12.0309 & 3.75266 & 15.1334 & 6.15421 & 3.13465 & 0.202013 & 4.74669 \\
\hline 16.4352 & 5.14042 & 20.4153 & 8.31057 & 4.00558 & 0.267314 & 5.46666 \\
\hline 21.8645 & 6.8651 & 26.7938 & 10.9232 & 5.05605 & 0.354042 & 6.33902 \\
\hline 28.2951 & 8.92127 & 34.1742 & 13.9735 & 6.27023 & 0.436557 & 7.33242 \\
\hline 35.5082 & 11.2121 & 42.182 & 17.2683 & 7.60805 & 0.53029 & 8.36714 \\
\hline 43.1398 & 13.6467 & 50.3936 & 20.674 & 8.98722 & 0.647369 & 9.44524 \\
\hline 50.7233 & 16.0645 & 58.2255 & 23.9004 & 10.3188 & 0.753848 & 10.5374 \\
\hline 57.6377 & 18.2401 & 64.94 & 26.68 & 11.5096 & 0.82849 & 11.5265 \\
\hline 63.2683 & 20.0769 & 70.0198 & 28.7817 & 12.4145 & 0.881681 & 12.2611 \\
\hline 67.035 & 21.3143 & 72.878 & 29.9643 & 12.951 & 0.926282 & 12.7053 \\
\hline 68.5714 & 21.8003 & 73.1562 & 30.1799 & 13.0726 & 0.923292 & 12.796 \\
\hline 67.7008 & 21.5563 & 70.8242 & 29.2608 & 12.7629 & 0.889428 & 12.491 \\
\hline 64.4782 & 20.5806 & 66.1622 & 27.354 & 12.0228 & 0.837275 & 11.8958 \\
\hline 59.2883 & 18.9255 & 59.7257 & 24.791 & 10.9309 & 0.75365 & 10.9824 \\
\hline 52.619 & 16.785 & 51.9951 & 21.645 & 9.61012 & 0.679382 & 9.84788 \\
\hline 45.0643 & 14.4082 & 43.6741 & 18.1927 & 8.15136 & 0.588674 & 8.58715 \\
\hline 37.2484 & 11.9419 & 35.4532 & 14.8295 & 6.69027 & 0.478474 & 7.39325 \\
\hline 29.7353 & 9.53724 & 27.8149 & 11.6563 & 5.30643 & 0.390249 & 6.3114 \\
\hline 22.9734 & 7.38673 & 21.1262 & 8.83956 & 4.07131 & 0.316362 & 5.25825 \\
\hline 17.1597 & 5.54772 & 15.4913 & 6.48717 & 3.04163 & 0.224359 & 4.3699 \\
\hline 12.408 & 4.01896 & 11.0078 & 4.59522 & 2.21145 & 0.152987 & 3.67314 \\
\hline
\end{tabular}




\begin{tabular}{|c|c|c|c|c|c|c|}
\hline 8.73362 & 2.82712 & 7.64904 & 3.19861 & 1.56974 & 0.105512 & 3.15665 \\
\hline 6.00113 & 1.95392 & 5.19477 & 2.17265 & 1.10361 & 0.0711724 & 2.77657 \\
\hline 4.05601 & 1.33511 & 3.49904 & 1.46057 & 0.786174 & 0.0516535 & 2.50174 \\
\hline 2.74265 & 0.89866 & 2.39628 & 0.989606 & 0.568602 & 0.0361629 & 2.3171 \\
\hline 1.90699 & 0.61873 & 1.70905 & 0.689266 & 0.429239 & 0.0292557 & 2.21368 \\
\hline 1.41458 & 0.461495 & 1.33102 & 0.541336 & 0.355055 & 0.0266109 & 2.16107 \\
\hline 1.16721 & 0.383117 & 1.17372 & 0.473446 & 0.322755 & 0.0253118 & 2.1421 \\
\hline 1.09628 & 0.363022 & 1.18866 & 0.466129 & 0.319099 & 0.0196485 & 2.14668 \\
\hline 1.1618 & 0.385689 & 1.34221 & 0.515734 & 0.343115 & 0.0199806 & 2.14391 \\
\hline 1.34931 & 0.434085 & 1.61642 & 0.621539 & 0.389441 & 0.0240918 & 2.1861 \\
\hline 1.65477 & 0.525491 & 2.02064 & 0.775616 & 0.453042 & 0.0315252 & 2.26176 \\
\hline 2.08567 & 0.663704 & 2.56754 & 0.997697 & 0.544783 & 0.0374096 & 2.3543 \\
\hline 2.64857 & 0.838763 & 3.258 & 1.27887 & 0.670624 & 0.0447566 & 2.47575 \\
\hline 3.34138 & 1.05183 & 4.09496 & 1.59059 & 0.822397 & 0.0544719 & 2.60957 \\
\hline 4.16462 & 1.31003 & 5.07442 & 1.96982 & 0.994912 & 0.0704468 & 2.75601 \\
\hline 5.09551 & 1.61109 & 6.14645 & 2.3853 & 1.18411 & 0.0822348 & 2.93166 \\
\hline 6.07656 & 1.91256 & 7.22699 & 2.81386 & 1.37734 & 0.0798762 & 3.09912 \\
\hline 7.0695 & 2.235 & 8.2801 & 3.25528 & 1.57448 & 0.0962844 & 3.24567 \\
\hline 8.00922 & 2.54796 & 9.22545 & 3.62095 & 1.7567 & 0.117444 & 3.40128 \\
\hline 8.78781 & 2.79048 & 9.94813 & 3.89783 & 1.89461 & 0.115539 & 3.51214 \\
\hline 9.35657 & 2.96643 & 10.4021 & 4.0892 & 1.99933 & 0.13196 & 3.57858 \\
\hline 9.66426 & 3.06547 & 10.5151 & 4.13564 & 2.05438 & 0.144878 & 3.64308 \\
\hline 9.65862 & 3.06268 & 10.3162 & 4.05995 & 2.02918 & 0.136225 & 3.64275 \\
\hline 9.35525 & 2.97216 & 9.81264 & 3.87932 & 1.94405 & 0.128979 & 3.56854 \\
\hline 8.76222 & 2.78988 & 9.00626 & 3.57199 & 1.8119 & 0.116335 & 3.46459 \\
\hline 7.9414 & 2.5297 & 8.01591 & 3.17443 & 1.64057 & 0.105987 & 3.32815 \\
\hline 6.984 & 2.24655 & 6.92155 & 2.74412 & 1.4437 & 0.0960417 & 3.1798 \\
\hline 5.93452 & 1.91495 & 5.77495 & 2.31308 & 1.2423 & 0.08165 & 3.00898 \\
\hline 4.88764 & 1.57689 & 4.66078 & 1.86153 & 1.04082 & 0.066141 & 2.83254 \\
\hline 3.90986 & 1.26575 & 3.65764 & 1.4562 & 0.847344 & 0.0537372 & 2.6894 \\
\hline 3.04431 & 0.99068 & 2.79242 & 1.10629 & 0.681468 & 0.0467017 & 2.56724 \\
\hline 2.30389 & 0.748646 & 2.0704 & 0.811067 & 0.549258 & 0.0356838 & 2.43993 \\
\hline 1.69667 & 0.550576 & 1.51129 & 0.591619 & 0.433533 & 0.0274243 & 2.36779 \\
\hline 1.2301 & 0.400448 & 1.09174 & 0.431047 & 0.34861 & 0.0240066 & 2.31411 \\
\hline 0.881441 & 0.291692 & 0.782046 & 0.303877 & 0.286755 & 0.0182613 & 2.23318 \\
\hline 0.635818 & 0.210948 & 0.567075 & 0.217378 & 0.239693 & 0.0136457 & 2.22069 \\
\hline 0.463413 & 0.156627 & 0.414133 & 0.160986 & 0.208033 & 0.0128152 & 2.22457 \\
\hline 0.34683 & 0.124578 & 0.31655 & 0.127162 & 0.188046 & 0.01153 & 2.17331 \\
\hline 0.273763 & 0.105551 & 0.25107 & 0.101317 & 0.175112 & 0.0111809 & 2.15809 \\
\hline 0.230708 & 0.0996417 & 0.213116 & 0.0888635 & 0.167986 & 0.00962738 & 2.17727 \\
\hline 0.200658 & 0.0877232 & 0.194014 & 0.0809568 & 0.163532 & 0.0117392 & 2.17757 \\
\hline 0.183091 & 0.0830077 & 0.178248 & 0.0715275 & 0.161385 & 0.0115909 & 2.18562 \\
\hline 0.174549 & 0.0790721 & 0.172702 & 0.0721986 & 0.162679 & 0.00980729 & 2.19674 \\
\hline 0.169548 & 0.0795156 & 0.169672 & 0.0747933 & 0.161311 & 0.0105517 & 2.21503 \\
\hline 0.168105 & 0.082991 & 0.169838 & 0.077344 & 0.162557 & 0.0118261 & 2.21892 \\
\hline 0.16788 & 0.0816286 & 0.169564 & 0.0793709 & 0.165594 & 0.0122762 & 2.23852 \\
\hline 0.167521 & 0.080238 & 0.169467 & 0.0757913 & 0.169452 & 0.0121086 & 2.26687 \\
\hline
\end{tabular}




\begin{tabular}{|c|c|c|c|c|c|c|}
\hline 0.169988 & 0.0814222 & 0.171192 & 0.0758179 & 0.170698 & 0.0118805 & 2.26679 \\
\hline 0.171894 & 0.0827488 & 0.173165 & 0.0787328 & 0.170996 & 0.012848 & 2.25903 \\
\hline 0.172134 & 0.0803376 & 0.174622 & 0.0787779 & 0.175085 & 0.0111951 & 2.28109 \\
\hline 0.174402 & 0.0858209 & 0.177833 & 0.0828147 & 0.182862 & 0.0130785 & 2.30106 \\
\hline 0.178086 & 0.0889257 & 0.181045 & 0.0860011 & 0.190733 & 0.0136419 & 2.33797 \\
\hline 0.183027 & 0.0877 & 0.183237 & 0.0858833 & 0.200782 & 0.0106478 & 2.35288 \\
\hline 0.187916 & 0.0895258 & 0.192121 & 0.0890881 & 0.214852 & 0.012575 & 2.40133 \\
\hline 0.19342 & 0.0912335 & 0.204621 & 0.0962997 & 0.23578 & 0.01354 & 2.43386 \\
\hline 0.205159 & 0.0962232 & 0.218496 & 0.109232 & 0.268041 & 0.0146619 & 2.48122 \\
\hline 0.223174 & 0.10638 & 0.239814 & 0.126027 & 0.31176 & 0.0164656 & 2.57761 \\
\hline 0.245069 & 0.114573 & 0.265511 & 0.14716 & 0.37361 & 0.0213875 & 2.71033 \\
\hline 0.274228 & 0.125345 & 0.302629 & 0.194088 & 0.454766 & 0.0301856 & 2.87886 \\
\hline 0.312301 & 0.136034 & 0.355038 & 0.26178 & 0.566086 & 0.0386928 & 3.09635 \\
\hline 0.359618 & 0.148843 & 0.418635 & 0.334223 & 0.711747 & 0.0440866 & 3.39576 \\
\hline 0.422197 & 0.167823 & 0.500131 & 0.432688 & 0.896421 & 0.0581731 & 3.70501 \\
\hline 0.500231 & 0.203418 & 0.595537 & 0.559972 & 1.11604 & 0.0714759 & 4.0963 \\
\hline 0.589236 & 0.237886 & 0.705957 & 0.701926 & 1.36784 & 0.0907241 & 4.59449 \\
\hline 0.686487 & 0.27628 & 0.827499 & 0.864483 & 1.64794 & 0.103825 & 5.11626 \\
\hline 0.79149 & 0.322369 & 0.949433 & 1.03914 & 1.94565 & 0.120789 & 5.6217 \\
\hline 0.899258 & 0.367648 & 1.07187 & 1.21251 & 2.23843 & 0.14007 & 6.17742 \\
\hline 0.99636 & 0.408352 & 1.18844 & 1.37194 & 2.51026 & 0.164536 & 6.6782 \\
\hline 1.09009 & 0.456524 & 1.28457 & 1.51914 & 2.74586 & 0.171833 & 7.08747 \\
\hline 1.16342 & 0.486208 & 1.34406 & 1.62555 & 2.91906 & 0.185284 & 7.40739 \\
\hline 1.20262 & 0.499002 & 1.37192 & 1.66773 & 3.00133 & 0.19812 & 7.59811 \\
\hline 1.21915 & 0.510764 & 1.3705 & 1.69441 & 3.01794 & 0.201733 & 7.64285 \\
\hline 1.20426 & 0.510788 & 1.32953 & 1.64848 & 2.9541 & 0.185573 & 7.58298 \\
\hline 1.15737 & 0.492658 & 1.25985 & 1.54246 & 2.79845 & 0.176453 & 7.3602 \\
\hline 1.09016 & 0.457953 & 1.17047 & 1.42166 & 2.57875 & 0.163032 & 6.97784 \\
\hline 1.00065 & 0.420151 & 1.04754 & 1.26036 & 2.29696 & 0.137901 & 6.57841 \\
\hline 0.895448 & 0.383226 & 0.929903 & 1.09324 & 2.00321 & 0.13354 & 6.11763 \\
\hline 0.791645 & 0.336693 & 0.806283 & 0.905831 & 1.70694 & 0.112303 & 5.62412 \\
\hline 0.684227 & 0.285367 & 0.691258 & 0.732615 & 1.41277 & 0.0849779 & 5.09845 \\
\hline 0.595236 & 0.254178 & 0.58615 & 0.587295 & 1.15989 & 0.071677 & 4.67631 \\
\hline 0.510952 & 0.218871 & 0.494717 & 0.462323 & 0.939926 & 0.058404 & 4.31514 \\
\hline 0.441644 & 0.194969 & 0.415741 & 0.335834 & 0.748609 & 0.0494313 & 4.04222 \\
\hline 0.382616 & 0.160148 & 0.359954 & 0.24821 & 0.59913 & 0.0441819 & 3.86587 \\
\hline 0.340402 & 0.145861 & 0.316739 & 0.200006 & 0.493103 & 0.036404 & 3.63708 \\
\hline 0.304897 & 0.136958 & 0.290419 & 0.163824 & 0.409486 & 0.0291076 & 3.57888 \\
\hline 0.281496 & 0.131042 & 0.274148 & 0.136459 & 0.349712 & 0.0242671 & 3.55219 \\
\hline 0.26749 & 0.124599 & 0.250626 & 0.120827 & 0.31363 & 0.0208224 & 3.50128 \\
\hline 0.252309 & 0.122608 & 0.245018 & 0.111573 & 0.289759 & 0.0239937 & 3.4261 \\
\hline 0.244981 & 0.110424 & 0.240974 & 0.11498 & 0.272726 & 0.0257093 & 3.38189 \\
\hline 0.237858 & 0.105731 & 0.24318 & 0.10871 & 0.263474 & 0.0218123 & 3.38224 \\
\hline 0.235971 & 0.104499 & 0.246535 & 0.105663 & 0.263743 & 0.0208935 & 3.35695 \\
\hline 0.235037 & 0.106046 & 0.243467 & 0.106908 & 0.265142 & 0.0172754 & 3.32365 \\
\hline 0.234865 & 0.107126 & 0.244987 & 0.110067 & 0.272151 & 0.0169821 & 3.34296 \\
\hline 0.238367 & 0.11036 & 0.246808 & 0.108375 & 0.27929 & 0.0149712 & 3.34193 \\
\hline
\end{tabular}




\begin{tabular}{|c|c|c|c|c|c|c|}
\hline 0.240259 & 0.104818 & 0.247076 & 0.105152 & 0.291366 & 0.0244187 & 3.30676 \\
\hline 0.248954 & 0.110105 & 0.252998 & 0.106551 & 0.307845 & 0.022544 & 3.34272 \\
\hline 0.25617 & 0.112281 & 0.261795 & 0.118868 & 0.329025 & 0.0222564 & 3.35771 \\
\hline 0.262403 & 0.108013 & 0.269398 & 0.121473 & 0.354547 & 0.028397 & 3.36441 \\
\hline 0.272997 & 0.112244 & 0.28064 & 0.128357 & 0.379885 & 0.0267315 & 3.40052 \\
\hline 0.282011 & 0.111523 & 0.296098 & 0.135897 & 0.41207 & 0.0326978 & 3.44747 \\
\hline 0.297815 & 0.117531 & 0.308611 & 0.134725 & 0.442399 & 0.0334053 & 3.48123 \\
\hline 0.311346 & 0.124142 & 0.323759 & 0.149728 & 0.475895 & 0.0366609 & 3.56186 \\
\hline 0.323219 & 0.122719 & 0.337564 & 0.157505 & 0.51244 & 0.0377843 & 3.60963 \\
\hline 0.339495 & 0.127568 & 0.351092 & 0.167931 & 0.546646 & 0.0410919 & 3.6571 \\
\hline 0.352798 & 0.130634 & 0.362274 & 0.179165 & 0.586065 & 0.0467371 & 3.75075 \\
\hline 0.36698 & 0.132827 & 0.38375 & 0.20165 & 0.627239 & 0.0489208 & 3.79071 \\
\hline 0.385423 & 0.139711 & 0.407054 & 0.225756 & 0.661063 & 0.0498674 & 3.89221 \\
\hline 0.402452 & 0.150204 & 0.426387 & 0.244071 & 0.707026 & 0.041594 & 3.95707 \\
\hline 0.423218 & 0.166105 & 0.446211 & 0.268689 & 0.751786 & 0.042591 & 4.03181 \\
\hline 0.438586 & 0.167121 & 0.460228 & 0.285813 & 0.796918 & 0.0475565 & 4.12815 \\
\hline 0.452841 & 0.169441 & 0.475708 & 0.304434 & 0.829703 & 0.0479264 & 4.19409 \\
\hline 0.469675 & 0.17697 & 0.494587 & 0.331554 & 0.868429 & 0.0487716 & 4.2507 \\
\hline 0.484564 & 0.186783 & 0.504033 & 0.346603 & 0.89328 & 0.0497712 & 4.28171 \\
\hline 0.489914 & 0.198035 & 0.512556 & 0.356103 & 0.890999 & 0.0503062 & 4.29754 \\
\hline 0.492921 & 0.199019 & 0.509767 & 0.354734 & 0.88971 & 0.0519601 & 4.26117 \\
\hline 0.488641 & 0.196208 & 0.497335 & 0.349415 & 0.864481 & 0.0516863 & 4.2419 \\
\hline 0.478854 & 0.192654 & 0.490542 & 0.352077 & 0.831193 & 0.0492199 & 4.20328 \\
\hline 0.46763 & 0.187017 & 0.478263 & 0.340979 & 0.782683 & 0.0475892 & 4.09476 \\
\hline 0.454034 & 0.18512 & 0.461823 & 0.338194 & 0.728379 & 0.0410541 & 3.98443 \\
\hline 0.435789 & 0.184334 & 0.437558 & 0.318276 & 0.667684 & 0.0294894 & 3.88418 \\
\hline 0.412561 & 0.181118 & 0.408823 & 0.301095 & 0.602421 & 0.0367164 & 3.7435 \\
\hline 0.390738 & 0.172612 & 0.389161 & 0.292023 & 0.54394 & 0.0307304 & 3.66942 \\
\hline 0.370197 & 0.175112 & 0.370002 & 0.285531 & 0.490371 & 0.0291405 & 3.57853 \\
\hline 0.35761 & 0.171075 & 0.352907 & 0.265869 & 0.44622 & 0.0227159 & 3.44511 \\
\hline 0.340876 & 0.163786 & 0.343501 & 0.26225 & 0.40526 & 0.019155 & 3.37574 \\
\hline 0.330036 & 0.165791 & 0.334522 & 0.257518 & 0.37417 & 0.0216121 & 3.3222 \\
\hline 0.32337 & 0.165859 & 0.327766 & 0.253398 & 0.349518 & 0.0197755 & 3.27709 \\
\hline 0.317323 & 0.158483 & 0.320727 & 0.249955 & 0.332081 & 0.0165487 & 3.28408 \\
\hline 0.311838 & 0.154534 & 0.31339 & 0.237044 & 0.321084 & 0.0172092 & 3.26918 \\
\hline 0.310278 & 0.163332 & 0.312009 & 0.239898 & 0.316791 & 0.0212081 & 3.27252 \\
\hline 0.310719 & 0.169674 & 0.314326 & 0.231058 & 0.314331 & 0.0206615 & 3.24132 \\
\hline 0.310593 & 0.157702 & 0.314794 & 0.216943 & 0.312989 & 0.0197039 & 3.31764 \\
\hline 0.311897 & 0.158354 & 0.317321 & 0.215891 & 0.31506 & 0.0196044 & 3.35709 \\
\hline 0.313252 & 0.15895 & 0.318855 & 0.215501 & 0.323614 & 0.0197624 & 3.33443 \\
\hline 0.311751 & 0.156982 & 0.319025 & 0.208902 & 0.328031 & 0.0181358 & 3.42681 \\
\hline 0.314062 & 0.151469 & 0.317346 & 0.194354 & 0.33339 & 0.0191147 & 3.44265 \\
\hline 0.313962 & 0.160242 & 0.320002 & 0.190173 & 0.335332 & 0.025755 & 3.4585 \\
\hline 0.314859 & 0.160504 & 0.321852 & 0.196601 & 0.346897 & 0.0249856 & 3.47428 \\
\hline 0.315677 & 0.161447 & 0.324388 & 0.192799 & 0.344496 & 0.0219798 & 3.52318 \\
\hline 0.318424 & 0.162637 & 0.316945 & 0.178781 & 0.345533 & 0.0212615 & 3.55758 \\
\hline 0.316296 & 0.154239 & 0.317279 & 0.168387 & 0.345567 & 0.023398 & 3.59928 \\
\hline
\end{tabular}




\begin{tabular}{|c|c|c|c|c|c|c|}
\hline 0.315275 & 0.160641 & 0.311428 & 0.169444 & 0.345719 & 0.0236214 & 3.62263 \\
\hline 0.31471 & 0.164486 & 0.321141 & 0.168793 & 0.350052 & 0.0236602 & 3.65921 \\
\hline 0.313589 & 0.171508 & 0.317219 & 0.157605 & 0.349658 & 0.0224828 & 3.66147 \\
\hline 0.312953 & 0.164269 & 0.317117 & 0.155625 & 0.354829 & 0.0249996 & 3.71966 \\
\hline 0.316308 & 0.165044 & 0.321917 & 0.153044 & 0.355674 & 0.0279649 & 3.75717 \\
\hline 0.316683 & 0.171077 & 0.321856 & 0.154492 & 0.355803 & 0.02133 & 3.84095 \\
\hline 0.321109 & 0.177026 & 0.329338 & 0.153386 & 0.361652 & 0.0249951 & 3.8931 \\
\hline 0.331188 & 0.180926 & 0.341486 & 0.154579 & 0.379238 & 0.0280349 & 3.976 \\
\hline 0.339271 & 0.185747 & 0.352842 & 0.157412 & 0.398063 & 0.0284992 & 4.13856 \\
\hline 0.353188 & 0.18455 & 0.380094 & 0.163256 & 0.420075 & 0.0325171 & 4.35679 \\
\hline 0.374775 & 0.207382 & 0.399441 & 0.174356 & 0.445194 & 0.0359809 & 4.51281 \\
\hline 0.395885 & 0.219785 & 0.437673 & 0.187232 & 0.483076 & 0.0374168 & 4.78107 \\
\hline 0.422202 & 0.228932 & 0.471153 & 0.213697 & 0.522551 & 0.0352422 & 5.04717 \\
\hline 0.455203 & 0.240125 & 0.514686 & 0.240125 & 0.572424 & 0.044832 & 5.41671 \\
\hline 0.493027 & 0.268256 & 0.559292 & 0.269614 & 0.626839 & 0.045095 & 5.72629 \\
\hline 0.530514 & 0.290128 & 0.608603 & 0.300128 & 0.674825 & 0.0532268 & 6.06146 \\
\hline 0.565788 & 0.305472 & 0.651811 & 0.330972 & 0.732995 & 0.0532861 & 6.39242 \\
\hline 0.606419 & 0.33823 & 0.693177 & 0.3459 & 0.780308 & 0.0621752 & 6.75486 \\
\hline 0.633408 & 0.350188 & 0.723231 & 0.36429 & 0.828316 & 0.0593006 & 6.97735 \\
\hline 0.664638 & 0.370007 & 0.746434 & 0.374711 & 0.857213 & 0.0678454 & 7.15261 \\
\hline 0.680792 & 0.383744 & 0.75398 & 0.387098 & 0.869193 & 0.0684601 & 7.25188 \\
\hline 0.686313 & 0.37746 & 0.754823 & 0.386219 & 0.88014 & 0.0647953 & 7.27763 \\
\hline 0.680583 & 0.376627 & 0.735609 & 0.369397 & 0.866388 & 0.0574937 & 7.24742 \\
\hline 0.667306 & 0.361316 & 0.706607 & 0.351078 & 0.837922 & 0.0627825 & 7.09914 \\
\hline 0.644228 & 0.342424 & 0.680182 & 0.331935 & 0.803116 & 0.0587948 & 6.87438 \\
\hline 0.616697 & 0.317117 & 0.647225 & 0.298023 & 0.748522 & 0.0515251 & 6.58341 \\
\hline 0.587826 & 0.290006 & 0.600032 & 0.267951 & 0.706264 & 0.0515851 & 6.24071 \\
\hline 0.556498 & 0.267116 & 0.548747 & 0.24216 & 0.654641 & 0.044061 & 6.00921 \\
\hline 0.51934 & 0.242098 & 0.525417 & 0.220791 & 0.610766 & 0.03857 & 5.71482 \\
\hline 0.493742 & 0.22507 & 0.482829 & 0.192299 & 0.562102 & 0.0318643 & 5.39292 \\
\hline 0.466809 & 0.210089 & 0.449678 & 0.180971 & 0.53023 & 0.036165 & 5.15741 \\
\hline 0.444819 & 0.192156 & 0.427808 & 0.175341 & 0.492702 & 0.0308751 & 4.9542 \\
\hline 0.422705 & 0.187368 & 0.408881 & 0.160208 & 0.464742 & 0.031061 & 4.78852 \\
\hline 0.408877 & 0.179234 & 0.394551 & 0.159007 & 0.441589 & 0.0246756 & 4.65071 \\
\hline 0.398611 & 0.173005 & 0.378389 & 0.15381 & 0.429736 & 0.0269686 & 4.56206 \\
\hline 0.388123 & 0.174433 & 0.370679 & 0.153299 & 0.415957 & 0.0247664 & 4.46882 \\
\hline 0.386261 & 0.185084 & 0.364527 & 0.148449 & 0.41271 & 0.0240632 & 4.44359 \\
\hline 0.380541 & 0.175437 & 0.35406 & 0.139173 & 0.394001 & 0.0265526 & 4.41204 \\
\hline 0.377432 & 0.179979 & 0.350381 & 0.137091 & 0.393207 & 0.0219366 & 4.3556 \\
\hline 0.378662 & 0.185499 & 0.358948 & 0.149811 & 0.39696 & 0.0213097 & 4.34829 \\
\hline 0.382698 & 0.189869 & 0.353149 & 0.147498 & 0.390084 & 0.0309783 & 4.26756 \\
\hline 0.380824 & 0.190971 & 0.35065 & 0.149049 & 0.390534 & 0.0227882 & 4.26594 \\
\hline 0.381824 & 0.194826 & 0.347938 & 0.14198 & 0.393936 & 0.0189791 & 4.27883 \\
\hline 0.385142 & 0.194993 & 0.353269 & 0.146322 & 0.39138 & 0.025652 & 4.273 \\
\hline 0.386538 & 0.209223 & 0.354931 & 0.144917 & 0.391771 & 0.0231682 & 4.26684 \\
\hline 0.384802 & 0.201336 & 0.357318 & 0.147613 & 0.391167 & 0.0206284 & 4.26931 \\
\hline 0.391977 & 0.213269 & 0.353341 & 0.141449 & 0.390935 & 0.0279325 & 4.26516 \\
\hline
\end{tabular}




\begin{tabular}{|c|c|c|c|c|c|c|}
\hline 0.392604 & 0.21617 & 0.359126 & 0.145813 & 0.393426 & 0.0226633 & 4.29264 \\
\hline 0.401588 & 0.216555 & 0.365582 & 0.155306 & 0.399975 & 0.0257843 & 4.35138 \\
\hline 0.402911 & 0.216038 & 0.374822 & 0.150157 & 0.403243 & 0.0234961 & 4.37902 \\
\hline 0.413748 & 0.239938 & 0.381162 & 0.160368 & 0.408233 & 0.0278467 & 4.42605 \\
\hline 0.420185 & 0.230683 & 0.392274 & 0.156559 & 0.418365 & 0.0266725 & 4.52613 \\
\hline 0.431619 & 0.232926 & 0.400222 & 0.160519 & 0.432384 & 0.0263109 & 4.60651 \\
\hline 0.446889 & 0.252634 & 0.4137 & 0.155322 & 0.447941 & 0.0285337 & 4.81191 \\
\hline 0.461943 & 0.246391 & 0.44535 & 0.186208 & 0.465081 & 0.0235906 & 4.98527 \\
\hline 0.48369 & 0.2733 & 0.459381 & 0.178791 & 0.49756 & 0.0340678 & 5.26262 \\
\hline 0.517501 & 0.293942 & 0.499573 & 0.207009 & 0.527918 & 0.0307861 & 5.65283 \\
\hline 0.555779 & 0.329688 & 0.548352 & 0.217352 & 0.572157 & 0.0383831 & 6.01328 \\
\hline 0.599919 & 0.364939 & 0.595772 & 0.243493 & 0.618863 & 0.0509835 & 6.61848 \\
\hline 0.650221 & 0.39285 & 0.654659 & 0.270917 & 0.682692 & 0.0615216 & 7.21887 \\
\hline 0.713699 & 0.440398 & 0.7282 & 0.302188 & 0.75715 & 0.0636241 & 7.97149 \\
\hline 0.795368 & 0.485834 & 0.814155 & 0.337937 & 0.835002 & 0.0752566 & 8.76868 \\
\hline 0.88661 & 0.577793 & 0.902858 & 0.391005 & 0.923046 & 0.0914935 & 9.67787 \\
\hline 0.963891 & 0.623976 & 0.994102 & 0.420639 & 1.01304 & 0.0936975 & 10.6656 \\
\hline 1.05298 & 0.685039 & 1.07445 & 0.459839 & 1.11107 & 0.114447 & 11.591 \\
\hline 1.14172 & 0.790428 & 1.15046 & 0.486367 & 1.18647 & 0.126837 & 12.4324 \\
\hline 1.22215 & 0.861892 & 1.21077 & 0.524454 & 1.26704 & 0.139877 & 13.2361 \\
\hline 1.28493 & 0.88249 & 1.28295 & 0.558781 & 1.33183 & 0.148101 & 13.8814 \\
\hline 1.32911 & 0.917458 & 1.30148 & 0.558322 & 1.36922 & 0.151423 & 14.3497 \\
\hline 1.34938 & 0.91895 & 1.31823 & 0.56966 & 1.37763 & 0.155605 & 14.611 \\
\hline 1.3628 & 0.935055 & 1.28378 & 0.539327 & 1.37145 & 0.150202 & 14.4928 \\
\hline 1.34646 & 0.9286 & 1.27153 & 0.539763 & 1.34302 & 0.147381 & 14.2743 \\
\hline 1.3171 & 0.914768 & 1.22619 & 0.51928 & 1.29643 & 0.134954 & 13.8714 \\
\hline 1.25255 & 0.847242 & 1.15901 & 0.506089 & 1.22615 & 0.127967 & 13.2905 \\
\hline 1.19001 & 0.799235 & 1.08741 & 0.468389 & 1.15124 & 0.114821 & 12.746 \\
\hline 1.12063 & 0.757776 & 1.01224 & 0.43391 & 1.05672 & 0.097336 & 11.9609 \\
\hline 1.04185 & 0.683442 & 0.94856 & 0.407582 & 0.98826 & 0.0975887 & 11.2457 \\
\hline 0.964493 & 0.637999 & 0.875779 & 0.365666 & 0.90813 & 0.0678399 & 10.5519 \\
\hline 0.89017 & 0.587768 & 0.819963 & 0.357007 & 0.835077 & 0.058176 & 9.91715 \\
\hline 0.832095 & 0.537958 & 0.764113 & 0.311116 & 0.767797 & 0.0587927 & 9.50348 \\
\hline 0.776452 & 0.50369 & 0.734694 & 0.320834 & 0.719934 & 0.0510806 & 9.09349 \\
\hline 0.738141 & 0.460837 & 0.686322 & 0.289804 & 0.681436 & 0.0511618 & 8.81822 \\
\hline 0.698272 & 0.451595 & 0.668567 & 0.286091 & 0.647365 & 0.0410718 & 8.61173 \\
\hline 0.679734 & 0.435315 & 0.648767 & 0.286943 & 0.619381 & 0.0313908 & 8.67573 \\
\hline 0.663615 & 0.418638 & 0.640278 & 0.280754 & 0.607864 & 0.0389783 & 8.7262 \\
\hline 0.652589 & 0.390355 & 0.642906 & 0.290999 & 0.601284 & 0.031957 & 8.85314 \\
\hline 0.648659 & 0.395084 & 0.636522 & 0.280235 & 0.59892 & 0.0432301 & 8.95609 \\
\hline 0.648111 & 0.3911 & 0.646084 & 0.294025 & 0.598874 & 0.0381895 & 9.33001 \\
\hline 0.647902 & 0.381913 & 0.65544 & 0.309892 & 0.617907 & 0.0446498 & 9.65088 \\
\hline 0.65728 & 0.390233 & 0.671291 & 0.31542 & 0.621808 & 0.0392404 & 9.99678 \\
\hline 0.671784 & 0.384913 & 0.690016 & 0.329579 & 0.629226 & 0.0400584 & 10.4347 \\
\hline 0.686804 & 0.389517 & 0.708301 & 0.33833 & 0.656287 & 0.0424043 & 10.9676 \\
\hline 0.701301 & 0.398407 & 0.732611 & 0.354816 & 0.674192 & 0.0461824 & 11.5251 \\
\hline 0.729193 & 0.430738 & 0.767835 & 0.379215 & 0.70532 & 0.0522659 & 12.2797 \\
\hline
\end{tabular}




\begin{tabular}{|c|c|c|c|c|c|c|}
\hline 0.757559 & 0.439579 & 0.807115 & 0.400727 & 0.743744 & 0.0500931 & 13.1265 \\
\hline 0.802489 & 0.474499 & 0.860383 & 0.436877 & 0.782653 & 0.0550128 & 14.3142 \\
\hline 0.859626 & 0.523175 & 0.945155 & 0.497787 & 0.845549 & 0.0537513 & 15.9475 \\
\hline 0.938113 & 0.58956 & 1.064 & 0.577313 & 0.932381 & 0.0685313 & 18.1253 \\
\hline 1.05005 & 0.67645 & 1.2361 & 0.694522 & 1.0681 & 0.0787304 & 21.594 \\
\hline 1.23421 & 0.822086 & 1.4809 & 0.837463 & 1.25639 & 0.0882433 & 26.5893 \\
\hline 1.47942 & 1.00538 & 1.88081 & 1.09562 & 1.54329 & 0.113704 & 33.982 \\
\hline 1.86401 & 1.29756 & 2.45248 & 1.49893 & 1.96464 & 0.129893 & 44.8513 \\
\hline 2.43797 & 1.76073 & 3.28351 & 2.02708 & 2.58857 & 0.187473 & 60.8162 \\
\hline 3.2704 & 2.3789 & 4.46787 & 2.81763 & 3.47236 & 0.233557 & 83.7091 \\
\hline 4.4564 & 3.32901 & 6.08874 & 3.8666 & 4.714 & 0.314095 & 115.53 \\
\hline 6.06848 & 4.58979 & 8.30852 & 5.33036 & 6.39116 & 0.452 & 159.485 \\
\hline 8.24099 & 6.25173 & 11.198 & 7.16914 & 8.67245 & 0.604677 & 217.491 \\
\hline 11.1307 & 8.51304 & 14.9134 & 9.63513 & 11.619 & 0.818885 & 291.832 \\
\hline 14.8034 & 11.4122 & 19.5287 & 12.6294 & 15.2631 & 1.04547 & 386.63 \\
\hline 19.3411 & 14.9342 & 25.0942 & 16.229 & 19.7363 & 1.39397 & 500.823 \\
\hline 24.7502 & 19.1835 & 31.5532 & 20.4737 & 24.9782 & 1.74931 & 635.35 \\
\hline 31.0366 & 24.0457 & 38.8303 & 25.3203 & 31.0223 & 2.2071 & 788.032 \\
\hline 38.0003 & 29.5226 & 46.5039 & 30.251 & 37.4553 & 2.62381 & 954.263 \\
\hline 45.5419 & 35.6312 & 54.4471 & 35.4681 & 44.3957 & 3.15239 & 1128.13 \\
\hline 53.2702 & 41.7139 & 62.3304 & 40.5179 & 51.2113 & 3.59605 & 1304.18 \\
\hline 60.6966 & 47.4528 & 69.446 & 45.2448 & 57.6616 & 4.04778 & 1467.9 \\
\hline 67.6057 & 52.9674 & 75.5149 & 48.9825 & 63.4149 & 4.5032 & 1613.48 \\
\hline 73.2673 & 57.4763 & 80.0848 & 52.115 & 67.8632 & 4.80237 & 1726.77 \\
\hline 77.5559 & 60.913 & 82.8787 & 54.0522 & 71.0353 & 5.14113 & 1806.12 \\
\hline 80.0751 & 63.0531 & 83.509 & 54.4057 & 72.3199 & 5.08934 & 1839.23 \\
\hline 80.555 & 63.4126 & 82.1536 & 53.4507 & 71.8206 & 5.13461 & 1827.86 \\
\hline 79.0952 & 62.4699 & 78.7382 & 51.316 & 69.6078 & 4.89791 & 1771.84 \\
\hline 75.6981 & 59.781 & 73.6831 & 47.9922 & 65.7734 & 4.65259 & 1673.66 \\
\hline 70.6429 & 55.9479 & 67.0878 & 43.7705 & 60.6819 & 4.2615 & 1543.41 \\
\hline 64.2524 & 50.9157 & 59.7524 & 38.8979 & 54.4858 & 3.82542 & 1387.29 \\
\hline 57.0271 & 45.173 & 51.78 & 33.5889 & 47.754 & 3.38237 & 1215.92 \\
\hline 49.3611 & 39.1963 & 43.8766 & 28.4466 & 40.8022 & 2.97603 & 1039.97 \\
\hline 41.7251 & 33.2639 & 36.1749 & 23.5578 & 34.0506 & 2.37639 & 868.368 \\
\hline 34.3891 & 27.3711 & 29.0978 & 18.8918 & 27.7359 & 1.99148 & 706.721 \\
\hline 27.6833 & 22.1416 & 22.9526 & 14.8644 & 22.1033 & 1.61426 & 562.352 \\
\hline 21.7749 & 17.3608 & 17.6548 & 11.4375 & 17.1603 & 1.23426 & 436.835 \\
\hline 16.7491 & 13.327 & 13.3174 & 8.59378 & 13.0718 & 0.907897 & 331.916 \\
\hline 12.6177 & 9.99465 & 9.85315 & 6.30104 & 9.74091 & 0.6897 & 247.052 \\
\hline 9.30655 & 7.29275 & 7.15185 & 4.54584 & 7.13622 & 0.501568 & 179.822 \\
\hline 6.79284 & 5.34542 & 5.14876 & 3.19753 & 5.18712 & 0.353035 & 128.947 \\
\hline 4.892 & 3.80318 & 3.67944 & 2.24968 & 3.69742 & 0.255662 & 91.16 \\
\hline 3.49335 & 2.6663 & 2.63366 & 1.59079 & 2.64536 & 0.192723 & 63.8324 \\
\hline 2.5091 & 1.86738 & 1.90874 & 1.09133 & 1.9161 & 0.142505 & 44.6391 \\
\hline 1.83294 & 1.31926 & 1.41439 & 0.760179 & 1.41507 & 0.0945908 & 31.4279 \\
\hline 1.36549 & 0.925359 & 1.10902 & 0.581204 & 1.09306 & 0.0697589 & 22.5637 \\
\hline 1.07621 & 0.677773 & 0.899481 & 0.435672 & 0.865589 & 0.0506758 & 16.8046 \\
\hline
\end{tabular}




\begin{tabular}{|c|c|c|c|c|c|c|}
\hline 0.878841 & 0.5082 & 0.762014 & 0.345078 & 0.733573 & 0.0459525 & 13.0441 \\
\hline 0.767686 & 0.444395 & 0.690678 & 0.297054 & 0.646375 & 0.0438198 & 10.6655 \\
\hline 0.683956 & 0.35747 & 0.64285 & 0.271698 & 0.593725 & 0.0352859 & 9.11927 \\
\hline 0.643586 & 0.318156 & 0.603266 & 0.245015 & 0.559156 & 0.033398 & 8.21384 \\
\hline 0.620308 & 0.309949 & 0.599583 & 0.238052 & 0.541745 & 0.0304902 & 7.5785 \\
\hline 0.609664 & 0.292473 & 0.598366 & 0.233071 & 0.538124 & 0.0350722 & 7.20473 \\
\hline 0.600326 & 0.272621 & 0.592209 & 0.23034 & 0.533723 & 0.030353 & 6.91542 \\
\hline 0.599244 & 0.278472 & 0.589347 & 0.226832 & 0.525171 & 0.0291192 & 6.79009 \\
\hline 0.602319 & 0.275621 & 0.59533 & 0.233519 & 0.53573 & 0.0360621 & 6.69508 \\
\hline 0.602591 & 0.271675 & 0.596114 & 0.234921 & 0.538841 & 0.0347248 & 6.72511 \\
\hline 0.606686 & 0.273269 & 0.60782 & 0.231734 & 0.545387 & 0.0258346 & 6.74992 \\
\hline 0.609439 & 0.271488 & 0.613884 & 0.229863 & 0.547117 & 0.0336726 & 6.74647 \\
\hline 0.617412 & 0.27538 & 0.619817 & 0.236401 & 0.554814 & 0.0319455 & 6.84831 \\
\hline 0.626037 & 0.291395 & 0.623023 & 0.235929 & 0.557007 & 0.0315981 & 6.93522 \\
\hline 0.636082 & 0.296274 & 0.647718 & 0.254377 & 0.577087 & 0.031506 & 7.09056 \\
\hline 0.647104 & 0.287591 & 0.662121 & 0.258567 & 0.585407 & 0.0300946 & 7.25994 \\
\hline 0.661483 & 0.31624 & 0.673271 & 0.258747 & 0.6 & 0.0432121 & 7.53899 \\
\hline 0.678038 & 0.314477 & 0.703584 & 0.290286 & 0.611641 & 0.0293485 & 7.82217 \\
\hline 0.699737 & 0.342799 & 0.708888 & 0.281203 & 0.643842 & 0.0385499 & 8.19344 \\
\hline 0.723182 & 0.35158 & 0.739595 & 0.301332 & 0.660965 & 0.0344486 & 8.64727 \\
\hline 0.746362 & 0.371501 & 0.759335 & 0.308835 & 0.691799 & 0.0388221 & 9.07916 \\
\hline 0.776847 & 0.382889 & 0.807327 & 0.340427 & 0.7235 & 0.0313108 & 9.77474 \\
\hline 0.817437 & 0.417727 & 0.863309 & 0.378356 & 0.771054 & 0.0427931 & 10.6034 \\
\hline 0.867649 & 0.448879 & 0.935946 & 0.415973 & 0.828402 & 0.0450555 & 11.7747 \\
\hline 0.931253 & 0.501845 & 1.0259 & 0.477447 & 0.904183 & 0.061527 & 13.2654 \\
\hline 1.01982 & 0.577655 & 1.15593 & 0.561399 & 0.997876 & 0.059365 & 15.208 \\
\hline 1.12967 & 0.648758 & 1.30126 & 0.644763 & 1.09916 & 0.0665797 & 17.9848 \\
\hline 1.27699 & 0.784801 & 1.49538 & 0.780185 & 1.27449 & 0.0666354 & 21.5895 \\
\hline 1.48187 & 0.953026 & 1.78043 & 0.971858 & 1.4719 & 0.0896469 & 26.2593 \\
\hline 1.73934 & 1.16616 & 2.12293 & 1.19102 & 1.75276 & 0.109787 & 32.6515 \\
\hline 2.08808 & 1.45708 & 2.57125 & 1.50111 & 2.09607 & 0.126755 & 40.6804 \\
\hline 2.50941 & 1.79365 & 3.14158 & 1.86912 & 2.52907 & 0.157004 & 50.8736 \\
\hline 3.05277 & 2.24562 & 3.81097 & 2.29614 & 3.05818 & 0.202038 & 63.4304 \\
\hline 3.70552 & 2.8019 & 4.62439 & 2.8827 & 3.67139 & 0.236072 & 78.7432 \\
\hline 4.47133 & 3.47472 & 5.55253 & 3.4974 & 4.44856 & 0.268326 & 96.3723 \\
\hline 5.37856 & 4.23152 & 6.59737 & 4.22566 & 5.24779 & 0.342678 & 116.146 \\
\hline 6.37888 & 5.08699 & 7.68635 & 4.96902 & 6.1887 & 0.396635 & 138.592 \\
\hline 7.445 & 6.01555 & 8.84942 & 5.67848 & 7.15376 & 0.471163 & 161.535 \\
\hline 8.54736 & 6.94541 & 10.0693 & 6.56095 & 8.11683 & 0.565127 & 184.928 \\
\hline 9.683 & 7.91838 & 11.119 & 7.31325 & 9.07257 & 0.623092 & 207.521 \\
\hline 10.7205 & 8.85495 & 12.077 & 7.87311 & 9.93753 & 0.65782 & 227.669 \\
\hline 11.6358 & 9.61458 & 12.8242 & 8.42015 & 10.6781 & 0.719834 & 244.904 \\
\hline 12.3754 & 10.2547 & 13.3564 & 8.82747 & 11.1687 & 0.759952 & 257.307 \\
\hline 12.8711 & 10.7366 & 13.5631 & 8.97929 & 11.5207 & 0.770559 & 264.628 \\
\hline 13.0963 & 10.9111 & 13.5184 & 8.88322 & 11.5797 & 0.799311 & 266.199 \\
\hline 13.0289 & 10.8987 & 13.165 & 8.68496 & 11.3738 & 0.734388 & 261.42 \\
\hline 12.7093 & 10.6197 & 12.5338 & 8.18672 & 10.9215 & 0.731507 & 251.189 \\
\hline
\end{tabular}




\begin{tabular}{|c|c|c|c|c|c|c|}
\hline 12.0965 & 10.0215 & 11.6903 & 7.70131 & 10.3063 & 0.699595 & 236.014 \\
\hline 11.2649 & 9.34914 & 10.6708 & 7.03879 & 9.4857 & 0.629963 & 216.574 \\
\hline 10.2699 & 8.43362 & 9.50327 & 6.22659 & 8.5342 & 0.546712 & 194.366 \\
\hline 9.18706 & 7.56656 & 8.32558 & 5.38793 & 7.52879 & 0.477244 & 170.507 \\
\hline 8.01935 & 6.59182 & 7.10035 & 4.59966 & 6.51319 & 0.409042 & 146.092 \\
\hline 6.86336 & 5.5997 & 5.97529 & 3.83106 & 5.52892 & 0.358424 & 122.518 \\
\hline 5.79503 & 4.68184 & 4.91113 & 3.09264 & 4.58497 & 0.272311 & 100.704 \\
\hline 4.75788 & 3.75465 & 3.9954 & 2.52666 & 3.75286 & 0.245235 & 80.7752 \\
\hline 3.87099 & 3.00234 & 3.18145 & 1.93281 & 3.00982 & 0.202301 & 63.5225 \\
\hline 3.09608 & 2.36819 & 2.52915 & 1.48653 & 2.3963 & 0.152914 & 48.8703 \\
\hline 2.44181 & 1.79892 & 1.99341 & 1.12943 & 1.89644 & 0.12256 & 37.103 \\
\hline 1.92546 & 1.35601 & 1.55696 & 0.85982 & 1.48221 & 0.0885858 & 27.4214 \\
\hline 1.51696 & 0.981349 & 1.24315 & 0.653621 & 1.17546 & 0.0735234 & 20.0897 \\
\hline 1.20261 & 0.741741 & 1.002 & 0.473882 & 0.931464 & 0.0536377 & 14.4503 \\
\hline 0.971024 & 0.548713 & 0.827871 & 0.376175 & 0.762029 & 0.0417242 & 10.2713 \\
\hline 0.799771 & 0.393679 & 0.715281 & 0.307863 & 0.635252 & 0.0400324 & 7.34005 \\
\hline 0.681305 & 0.297584 & 0.629414 & 0.24693 & 0.545645 & 0.0341183 & 5.28206 \\
\hline 0.596127 & 0.238528 & 0.568495 & 0.20306 & 0.485222 & 0.025143 & 3.80293 \\
\hline 0.541953 & 0.201672 & 0.535077 & 0.204513 & 0.443073 & 0.0277336 & 2.81 \\
\hline 0.50938 & 0.179208 & 0.518577 & 0.18074 & 0.420569 & 0.0203859 & 2.1554 \\
\hline 0.491504 & 0.157837 & 0.518244 & 0.186381 & 0.404813 & 0.0185172 & 1.70763 \\
\hline 0.483418 & 0.160588 & 0.515433 & 0.171158 & 0.395194 & 0.0210286 & 1.46974 \\
\hline 0.481568 & 0.154695 & 0.525314 & 0.182433 & 0.398716 & 0.0223021 & 1.3249 \\
\hline 0.487272 & 0.150253 & 0.544744 & 0.184241 & 0.397821 & 0.0220474 & 1.24488 \\
\hline 0.500155 & 0.15591 & 0.557056 & 0.17743 & 0.414337 & 0.0273 & 1.18049 \\
\hline 0.513356 & 0.158882 & 0.594784 & 0.195431 & 0.422096 & 0.0213309 & 1.19108 \\
\hline 0.532394 & 0.170551 & 0.625181 & 0.199896 & 0.444381 & 0.0251995 & 1.23638 \\
\hline 0.553567 & 0.171243 & 0.656972 & 0.207201 & 0.469645 & 0.0274419 & 1.28319 \\
\hline 0.574093 & 0.180113 & 0.685117 & 0.227717 & 0.493239 & 0.0286956 & 1.37207 \\
\hline 0.610507 & 0.194857 & 0.743652 & 0.231662 & 0.527581 & 0.0266672 & 1.45147 \\
\hline 0.63593 & 0.200173 & 0.789551 & 0.252361 & 0.564041 & 0.032905 & 1.60655 \\
\hline 0.669983 & 0.205652 & 0.842989 & 0.26567 & 0.602645 & 0.0351019 & 1.72592 \\
\hline 0.702424 & 0.221846 & 0.882957 & 0.272524 & 0.633732 & 0.0283386 & 1.88319 \\
\hline 0.735983 & 0.234681 & 0.936582 & 0.303103 & 0.676968 & 0.0335966 & 1.99967 \\
\hline 0.769873 & 0.242048 & 0.982816 & 0.300666 & 0.717428 & 0.0410803 & 2.13937 \\
\hline 0.794882 & 0.253389 & 1.01527 & 0.318238 & 0.743162 & 0.0379929 & 2.27426 \\
\hline 0.823104 & 0.260832 & 1.04295 & 0.332268 & 0.774849 & 0.0402283 & 2.38963 \\
\hline 0.836417 & 0.267056 & 1.05324 & 0.325496 & 0.795191 & 0.0419099 & 2.43594 \\
\hline 0.846948 & 0.276489 & 1.05159 & 0.334027 & 0.799751 & 0.0351225 & 2.49378 \\
\hline 0.853773 & 0.271568 & 1.0379 & 0.322286 & 0.800086 & 0.0371911 & 2.44812 \\
\hline 0.851708 & 0.272668 & 1.01903 & 0.312807 & 0.793677 & 0.0439895 & 2.4081 \\
\hline 0.832676 & 0.266549 & 0.982069 & 0.304112 & 0.779275 & 0.0408944 & 2.30001 \\
\hline 0.811268 & 0.255425 & 0.940319 & 0.288827 & 0.752212 & 0.040066 & 2.22659 \\
\hline 0.786885 & 0.249089 & 0.904935 & 0.274978 & 0.720175 & 0.0368815 & 2.09601 \\
\hline 0.754635 & 0.235061 & 0.841925 & 0.242702 & 0.694726 & 0.0473598 & 1.95961 \\
\hline 0.724393 & 0.223296 & 0.793338 & 0.241323 & 0.66264 & 0.0360349 & 1.82303 \\
\hline 0.686964 & 0.20665 & 0.733395 & 0.211078 & 0.619974 & 0.0305857 & 1.6727 \\
\hline
\end{tabular}




\begin{tabular}{|c|c|c|c|c|c|c|}
\hline 0.654313 & 0.20207 & 0.682971 & 0.207818 & 0.590568 & 0.0297961 & 1.51928 \\
\hline 0.623022 & 0.188669 & 0.643047 & 0.177283 & 0.555223 & 0.0285821 & 1.3937 \\
\hline 0.599066 & 0.179119 & 0.59866 & 0.170153 & 0.52272 & 0.026378 & 1.27106 \\
\hline 0.575317 & 0.173504 & 0.570832 & 0.155541 & 0.495409 & 0.0299286 & 1.15542 \\
\hline 0.553239 & 0.16258 & 0.53033 & 0.15133 & 0.480254 & 0.0242672 & 1.06154 \\
\hline 0.538518 & 0.157355 & 0.520843 & 0.153128 & 0.460998 & 0.0236612 & 0.983258 \\
\hline 0.527089 & 0.154056 & 0.498962 & 0.144355 & 0.447583 & 0.0269995 & 0.928208 \\
\hline 0.520447 & 0.156245 & 0.486599 & 0.139912 & 0.43303 & 0.0221468 & 0.884471 \\
\hline 0.515332 & 0.147008 & 0.49061 & 0.147956 & 0.438351 & 0.0233345 & 0.862443 \\
\hline 0.505241 & 0.144803 & 0.480578 & 0.149696 & 0.427301 & 0.0194773 & 0.819232 \\
\hline 0.50745 & 0.149012 & 0.479408 & 0.131527 & 0.420731 & 0.0217608 & 0.805138 \\
\hline 0.509032 & 0.140085 & 0.474126 & 0.136446 & 0.420145 & 0.0252402 & 0.798861 \\
\hline 0.500282 & 0.140751 & 0.476407 & 0.155449 & 0.416642 & 0.0261866 & 0.777904 \\
\hline 0.505159 & 0.146356 & 0.483248 & 0.144179 & 0.419566 & 0.0257975 & 0.780864 \\
\hline 0.502258 & 0.144942 & 0.480016 & 0.15145 & 0.417012 & 0.0263763 & 0.746201 \\
\hline 0.502454 & 0.145127 & 0.474658 & 0.148185 & 0.41686 & 0.0210794 & 0.786715 \\
\hline 0.505799 & 0.145503 & 0.480195 & 0.158852 & 0.418636 & 0.0237086 & 0.775397 \\
\hline 0.502984 & 0.149998 & 0.475304 & 0.158039 & 0.416594 & 0.0218048 & 0.774744 \\
\hline 0.505316 & 0.145318 & 0.47746 & 0.152936 & 0.42111 & 0.0220344 & 0.752733 \\
\hline 0.500574 & 0.148397 & 0.473522 & 0.163047 & 0.42287 & 0.0236016 & 0.769149 \\
\hline 0.502235 & 0.148356 & 0.472632 & 0.1592 & 0.41584 & 0.0254319 & 0.770274 \\
\hline 0.503877 & 0.151496 & 0.48538 & 0.161049 & 0.421668 & 0.027377 & 0.778586 \\
\hline 0.502169 & 0.158805 & 0.471373 & 0.157105 & 0.421964 & 0.0213102 & 0.784047 \\
\hline 0.497166 & 0.152018 & 0.477966 & 0.169525 & 0.425419 & 0.0274753 & 0.754121 \\
\hline 0.49608 & 0.153792 & 0.486465 & 0.164267 & 0.432035 & 0.0231981 & 0.765926 \\
\hline 0.501395 & 0.159073 & 0.478165 & 0.158756 & 0.434984 & 0.0281147 & 0.810545 \\
\hline 0.497631 & 0.156778 & 0.487297 & 0.175054 & 0.439155 & 0.0305352 & 0.803673 \\
\hline 0.494968 & 0.155257 & 0.484344 & 0.160604 & 0.44598 & 0.0222155 & 0.791888 \\
\hline 0.495265 & 0.160606 & 0.489376 & 0.168722 & 0.447495 & 0.0272548 & 0.837824 \\
\hline 0.499652 & 0.16346 & 0.49432 & 0.169731 & 0.464667 & 0.0228913 & 0.833367 \\
\hline 0.500511 & 0.170306 & 0.518027 & 0.183827 & 0.478272 & 0.0294152 & 0.850556 \\
\hline 0.508453 & 0.170903 & 0.522193 & 0.195703 & 0.489796 & 0.0291557 & 0.865901 \\
\hline 0.511193 & 0.167347 & 0.536164 & 0.188829 & 0.518621 & 0.0349549 & 0.875445 \\
\hline 0.517296 & 0.169061 & 0.558857 & 0.207107 & 0.547769 & 0.0316015 & 0.925844 \\
\hline 0.530127 & 0.175033 & 0.585625 & 0.23621 & 0.582763 & 0.0411671 & 0.921419 \\
\hline 0.54703 & 0.17123 & 0.629368 & 0.273681 & 0.619892 & 0.0510425 & 0.961756 \\
\hline 0.563735 & 0.179995 & 0.673551 & 0.320663 & 0.69493 & 0.062944 & 1.01267 \\
\hline 0.581371 & 0.171421 & 0.719057 & 0.390201 & 0.755942 & 0.0659191 & 1.05146 \\
\hline 0.6102 & 0.173642 & 0.787837 & 0.456206 & 0.838393 & 0.0899002 & 1.11835 \\
\hline 0.644504 & 0.187086 & 0.843685 & 0.562181 & 0.937492 & 0.112477 & 1.13847 \\
\hline 0.672321 & 0.185485 & 0.93391 & 0.676179 & 1.0405 & 0.130986 & 1.24425 \\
\hline 0.713857 & 0.19192 & 0.98891 & 0.754232 & 1.15632 & 0.158102 & 1.32558 \\
\hline 0.75406 & 0.202974 & 1.07429 & 0.876782 & 1.2703 & 0.189679 & 1.40113 \\
\hline 0.791868 & 0.209518 & 1.14437 & 0.995855 & 1.36722 & 0.195913 & 1.48866 \\
\hline 0.82986 & 0.221206 & 1.20988 & 1.07572 & 1.48174 & 0.224726 & 1.59662 \\
\hline 0.866823 & 0.226792 & 1.27163 & 1.1586 & 1.5697 & 0.249619 & 1.6247 \\
\hline 0.907993 & 0.235832 & 1.29489 & 1.18967 & 1.63898 & 0.272789 & 1.70562 \\
\hline
\end{tabular}




\begin{tabular}{|c|c|c|c|c|c|c|}
\hline 0.925095 & 0.231448 & 1.3385 & 1.27083 & 1.70586 & 0.275156 & 1.74369 \\
\hline 0.943573 & 0.234698 & 1.35964 & 1.27335 & 1.73531 & 0.283197 & 1.83523 \\
\hline 0.953571 & 0.243216 & 1.35041 & 1.28107 & 1.73821 & 0.277698 & 1.83934 \\
\hline 0.951411 & 0.246449 & 1.33018 & 1.24716 & 1.70573 & 0.279185 & 1.88117 \\
\hline 0.940527 & 0.235646 & 1.28768 & 1.17499 & 1.65989 & 0.25917 & 1.86901 \\
\hline 0.920105 & 0.233625 & 1.23 & 1.08049 & 1.59759 & 0.233377 & 1.86012 \\
\hline 0.897998 & 0.232015 & 1.16908 & 0.96387 & 1.48986 & 0.226893 & 1.85729 \\
\hline 0.863362 & 0.220245 & 1.10277 & 0.853312 & 1.41002 & 0.207932 & 1.82167 \\
\hline 0.830393 & 0.204629 & 1.02398 & 0.778528 & 1.30681 & 0.168492 & 1.73524 \\
\hline 0.79205 & 0.197211 & 0.948656 & 0.646707 & 1.20099 & 0.157349 & 1.7022 \\
\hline 0.759695 & 0.189227 & 0.895363 & 0.548919 & 1.08705 & 0.131958 & 1.65866 \\
\hline 0.721475 & 0.175516 & 0.83463 & 0.457803 & 0.994922 & 0.101924 & 1.61889 \\
\hline 0.69019 & 0.173422 & 0.776899 & 0.387428 & 0.901314 & 0.0853623 & 1.55272 \\
\hline 0.65756 & 0.156519 & 0.724853 & 0.309146 & 0.831519 & 0.0747444 & 1.47917 \\
\hline 0.636677 & 0.157522 & 0.696221 & 0.261873 & 0.757822 & 0.0561471 & 1.44727 \\
\hline 0.619008 & 0.147537 & 0.671819 & 0.223943 & 0.716913 & 0.0523917 & 1.37065 \\
\hline 0.600294 & 0.139631 & 0.647211 & 0.185599 & 0.665203 & 0.0408354 & 1.34839 \\
\hline 0.59627 & 0.141186 & 0.636295 & 0.179065 & 0.643273 & 0.0361491 & 1.32006 \\
\hline 0.594194 & 0.143364 & 0.622488 & 0.158603 & 0.617752 & 0.0344724 & 1.2898 \\
\hline 0.588746 & 0.144902 & 0.622061 & 0.153083 & 0.605531 & 0.0326515 & 1.25515 \\
\hline 0.583918 & 0.141998 & 0.615849 & 0.159019 & 0.59703 & 0.0367619 & 1.25327 \\
\hline 0.589162 & 0.139478 & 0.611876 & 0.159146 & 0.590977 & 0.0260758 & 1.19822 \\
\hline 0.590584 & 0.145825 & 0.619873 & 0.148018 & 0.592555 & 0.035672 & 1.18411 \\
\hline 0.593155 & 0.145262 & 0.624776 & 0.147673 & 0.583833 & 0.0331898 & 1.18067 \\
\hline 0.592503 & 0.139288 & 0.622067 & 0.150236 & 0.590767 & 0.0304155 & 1.15946 \\
\hline 0.598525 & 0.145133 & 0.618119 & 0.141224 & 0.57714 & 0.03187 & 1.14244 \\
\hline 0.596437 & 0.142808 & 0.611745 & 0.14296 & 0.575241 & 0.0331706 & 1.0795 \\
\hline 0.599735 & 0.149425 & 0.614227 & 0.14328 & 0.581716 & 0.0328236 & 1.08449 \\
\hline 0.596646 & 0.153989 & 0.612009 & 0.136268 & 0.571636 & 0.0325634 & 1.08347 \\
\hline 0.601338 & 0.147174 & 0.612689 & 0.141323 & 0.569969 & 0.0294356 & 1.03525 \\
\hline 0.600992 & 0.150961 & 0.607658 & 0.145244 & 0.567852 & 0.0279797 & 1.01083 \\
\hline 0.60227 & 0.158003 & 0.612127 & 0.137795 & 0.575147 & 0.029943 & 0.99381 \\
\hline 0.598574 & 0.161111 & 0.603396 & 0.140462 & 0.565799 & 0.0316103 & 0.976633 \\
\hline 0.605251 & 0.171778 & 0.608224 & 0.144292 & 0.557761 & 0.0287538 & 0.962553 \\
\hline 0.607657 & 0.169988 & 0.597816 & 0.138688 & 0.553078 & 0.0259733 & 0.92181 \\
\hline 0.611311 & 0.177197 & 0.595259 & 0.144106 & 0.551491 & 0.0246614 & 0.902105 \\
\hline 0.612783 & 0.186089 & 0.60153 & 0.133839 & 0.557257 & 0.0298573 & 0.883382 \\
\hline 0.613191 & 0.191183 & 0.601441 & 0.144678 & 0.560626 & 0.0262672 & 0.899177 \\
\hline 0.614702 & 0.197033 & 0.611387 & 0.153494 & 0.555628 & 0.0243012 & 0.882492 \\
\hline 0.628811 & 0.210316 & 0.620946 & 0.162644 & 0.560983 & 0.0252315 & 0.887771 \\
\hline 0.629209 & 0.214541 & 0.627961 & 0.172275 & 0.554395 & 0.0282828 & 0.867553 \\
\hline 0.638978 & 0.226714 & 0.63401 & 0.168599 & 0.563942 & 0.0249909 & 0.863166 \\
\hline 0.639852 & 0.232969 & 0.649326 & 0.176988 & 0.563831 & 0.022369 & 0.843882 \\
\hline 0.649867 & 0.238307 & 0.665706 & 0.19208 & 0.562176 & 0.0303506 & 0.84708 \\
\hline 0.661686 & 0.253207 & 0.693478 & 0.203198 & 0.578075 & 0.0305456 & 0.84762 \\
\hline 0.674415 & 0.256986 & 0.714067 & 0.2277 & 0.574686 & 0.0297061 & 0.854713 \\
\hline 0.68713 & 0.25615 & 0.74331 & 0.244872 & 0.59032 & 0.0277566 & 0.859415 \\
\hline
\end{tabular}




\begin{tabular}{|c|c|c|c|c|c|c|}
\hline 0.706568 & 0.272995 & 0.782631 & 0.271665 & 0.595018 & 0.0268647 & 0.874807 \\
\hline 0.727283 & 0.272798 & 0.833295 & 0.345211 & 0.607334 & 0.0246084 & 0.873661 \\
\hline 0.750001 & 0.278787 & 0.886086 & 0.370503 & 0.622549 & 0.0279782 & 0.855433 \\
\hline 0.777118 & 0.293908 & 0.950302 & 0.448881 & 0.637742 & 0.0395489 & 0.866277 \\
\hline 0.807746 & 0.297011 & 1.01579 & 0.519454 & 0.658244 & 0.0252317 & 0.87378 \\
\hline 0.839369 & 0.309382 & 1.08991 & 0.626478 & 0.68022 & 0.029073 & 0.868806 \\
\hline 0.88199 & 0.317834 & 1.15956 & 0.70768 & 0.690951 & 0.0309533 & 0.878285 \\
\hline 0.91668 & 0.334041 & 1.23475 & 0.762895 & 0.709761 & 0.0315073 & 0.905859 \\
\hline 0.939901 & 0.335025 & 1.31254 & 0.890275 & 0.73496 & 0.0322868 & 0.909234 \\
\hline 0.982874 & 0.351877 & 1.35839 & 0.910934 & 0.761322 & 0.0385169 & 0.908904 \\
\hline 1.01314 & 0.362276 & 1.42465 & 0.984 & 0.772592 & 0.0419272 & 0.904914 \\
\hline 1.04339 & 0.366819 & 1.45991 & 1.05471 & 0.782976 & 0.0385873 & 0.904824 \\
\hline 1.06288 & 0.371763 & 1.48819 & 1.06608 & 0.802038 & 0.0374528 & 0.92449 \\
\hline 1.08014 & 0.368349 & 1.51969 & 1.10649 & 0.801071 & 0.0399719 & 0.922648 \\
\hline 1.08111 & 0.37207 & 1.49514 & 1.10003 & 0.803609 & 0.0399919 & 0.945498 \\
\hline 1.08945 & 0.376494 & 1.47521 & 1.04079 & 0.803734 & 0.0356141 & 0.952078 \\
\hline 1.07659 & 0.378352 & 1.44248 & 1.01518 & 0.793625 & 0.0404826 & 0.954248 \\
\hline 1.06788 & 0.369609 & 1.38278 & 0.936056 & 0.788108 & 0.0312041 & 0.93508 \\
\hline 1.04039 & 0.364612 & 1.32366 & 0.870647 & 0.781233 & 0.0341036 & 0.95352 \\
\hline 1.01788 & 0.352489 & 1.26123 & 0.770662 & 0.762721 & 0.0318899 & 0.94314 \\
\hline 0.993219 & 0.358782 & 1.19771 & 0.680871 & 0.732957 & 0.0309219 & 0.943078 \\
\hline 0.960642 & 0.347574 & 1.13699 & 0.619275 & 0.72995 & 0.0335263 & 0.931594 \\
\hline 0.928921 & 0.330745 & 1.07128 & 0.536897 & 0.720252 & 0.0347131 & 0.933707 \\
\hline 0.906038 & 0.324718 & 1.0041 & 0.455542 & 0.701432 & 0.0303149 & 0.947441 \\
\hline 0.879349 & 0.312276 & 0.946665 & 0.377715 & 0.694321 & 0.0313268 & 0.939087 \\
\hline 0.852552 & 0.299585 & 0.906628 & 0.333928 & 0.677977 & 0.024378 & 0.939103 \\
\hline 0.83346 & 0.298688 & 0.879364 & 0.287337 & 0.678581 & 0.0314258 & 0.946684 \\
\hline 0.816465 & 0.279317 & 0.839181 & 0.258425 & 0.678326 & 0.0311266 & 0.958308 \\
\hline 0.8021 & 0.27486 & 0.8308 & 0.247787 & 0.679137 & 0.0326687 & 0.949013 \\
\hline 0.790369 & 0.271161 & 0.798891 & 0.214627 & 0.684063 & 0.0310545 & 0.967529 \\
\hline 0.785808 & 0.253674 & 0.799636 & 0.215137 & 0.698588 & 0.0346048 & 0.968512 \\
\hline 0.782468 & 0.249026 & 0.803527 & 0.203627 & 0.701736 & 0.0378198 & 0.988896 \\
\hline 0.782357 & 0.245154 & 0.799592 & 0.200081 & 0.713358 & 0.0342396 & 0.978078 \\
\hline 0.782736 & 0.234859 & 0.798061 & 0.207915 & 0.725642 & 0.0399213 & 0.98545 \\
\hline 0.78491 & 0.238507 & 0.806755 & 0.209662 & 0.742234 & 0.0389345 & 0.979516 \\
\hline 0.790954 & 0.225028 & 0.805382 & 0.207811 & 0.75511 & 0.043488 & 0.982734 \\
\hline 0.794552 & 0.217228 & 0.821216 & 0.222329 & 0.769763 & 0.0516984 & 1.00535 \\
\hline 0.793757 & 0.208021 & 0.827639 & 0.21754 & 0.790872 & 0.0557267 & 0.979685 \\
\hline 0.80547 & 0.207591 & 0.835826 & 0.231432 & 0.799021 & 0.0515314 & 1.01182 \\
\hline 0.802792 & 0.204428 & 0.836603 & 0.240888 & 0.812457 & 0.0576119 & 1.00007 \\
\hline 0.810155 & 0.202541 & 0.846587 & 0.244699 & 0.808818 & 0.0560944 & 1.02316 \\
\hline 0.810696 & 0.189141 & 0.848216 & 0.232051 & 0.812812 & 0.0490703 & 1.02922 \\
\hline 0.813314 & 0.191265 & 0.833401 & 0.224358 & 0.812772 & 0.0494173 & 1.01771 \\
\hline 0.805348 & 0.191173 & 0.840706 & 0.213107 & 0.810021 & 0.0473145 & 0.991536 \\
\hline 0.801176 & 0.185344 & 0.827206 & 0.21115 & 0.79448 & 0.0523918 & 1.00753 \\
\hline 0.799537 & 0.18084 & 0.817371 & 0.199197 & 0.780631 & 0.0554041 & 1.03086 \\
\hline 0.790571 & 0.177413 & 0.823865 & 0.197476 & 0.773339 & 0.043036 & 1.0057 \\
\hline
\end{tabular}




\begin{tabular}{|c|c|c|c|c|c|c|}
\hline 0.787066 & 0.176623 & 0.80075 & 0.1795 & 0.764456 & 0.0414102 & 1.01586 \\
\hline 0.787299 & 0.17441 & 0.78361 & 0.175781 & 0.739311 & 0.0459334 & 0.992877 \\
\hline 0.778116 & 0.181299 & 0.770144 & 0.163657 & 0.729037 & 0.0391567 & 1.0136 \\
\hline 0.768202 & 0.168878 & 0.767791 & 0.1518 & 0.70632 & 0.0414793 & 1.01422 \\
\hline 0.760028 & 0.170792 & 0.754837 & 0.147433 & 0.694102 & 0.0345143 & 1.00695 \\
\hline 0.753474 & 0.163426 & 0.753482 & 0.150691 & 0.683775 & 0.0370791 & 1.01983 \\
\hline 0.753413 & 0.167148 & 0.737833 & 0.134942 & 0.680469 & 0.0358745 & 1.01596 \\
\hline 0.745707 & 0.166809 & 0.733353 & 0.138718 & 0.661868 & 0.0277433 & 1.03796 \\
\hline 0.737515 & 0.160636 & 0.736682 & 0.146371 & 0.655722 & 0.028933 & 1.03816 \\
\hline 0.740705 & 0.165871 & 0.729038 & 0.130742 & 0.652122 & 0.0270626 & 1.05905 \\
\hline 0.736453 & 0.157696 & 0.726037 & 0.135859 & 0.65133 & 0.0322531 & 1.06039 \\
\hline 0.741648 & 0.163753 & 0.727716 & 0.149767 & 0.651284 & 0.033729 & 1.0498 \\
\hline 0.74165 & 0.160362 & 0.721752 & 0.132694 & 0.638651 & 0.0381901 & 1.07382 \\
\hline 0.734579 & 0.156418 & 0.718816 & 0.133852 & 0.641949 & 0.0268132 & 1.07844 \\
\hline 0.739291 & 0.164341 & 0.736635 & 0.139074 & 0.643364 & 0.033422 & 1.08473 \\
\hline 0.733672 & 0.159899 & 0.721273 & 0.133605 & 0.646594 & 0.0312129 & 1.06746 \\
\hline 0.736762 & 0.157763 & 0.734822 & 0.135727 & 0.646846 & 0.0248489 & 1.08007 \\
\hline 0.743033 & 0.165553 & 0.733244 & 0.14196 & 0.657419 & 0.0241766 & 1.08251 \\
\hline 0.744274 & 0.160399 & 0.729937 & 0.131416 & 0.665597 & 0.0289356 & 1.07995 \\
\hline 0.745942 & 0.156222 & 0.72924 & 0.143942 & 0.670428 & 0.0380079 & 1.06423 \\
\hline 0.744755 & 0.163653 & 0.730188 & 0.135284 & 0.662525 & 0.0263524 & 1.11017 \\
\hline 0.74484 & 0.162248 & 0.735545 & 0.139352 & 0.672463 & 0.0341496 & 1.08045 \\
\hline 0.748404 & 0.160023 & 0.747538 & 0.142018 & 0.677414 & 0.0294717 & 1.07959 \\
\hline 0.753862 & 0.16613 & 0.736735 & 0.13873 & 0.684543 & 0.0304196 & 1.07958 \\
\hline 0.756816 & 0.168012 & 0.737468 & 0.136263 & 0.684644 & 0.0371923 & 1.08184 \\
\hline 0.75423 & 0.159539 & 0.738011 & 0.141859 & 0.679519 & 0.0356973 & 1.07946 \\
\hline 0.755241 & 0.159391 & 0.727222 & 0.145068 & 0.686901 & 0.0294899 & 1.05841 \\
\hline 0.754135 & 0.159464 & 0.734629 & 0.133522 & 0.688302 & 0.0251231 & 1.06094 \\
\hline 0.760772 & 0.160657 & 0.724483 & 0.134077 & 0.68462 & 0.0293682 & 1.03126 \\
\hline 0.762356 & 0.159431 & 0.733996 & 0.145872 & 0.693395 & 0.030544 & 1.06455 \\
\hline 0.75614 & 0.160993 & 0.72602 & 0.139908 & 0.683153 & 0.0294326 & 1.05593 \\
\hline 0.759856 & 0.157897 & 0.730918 & 0.129962 & 0.682149 & 0.0402482 & 1.02381 \\
\hline 0.756343 & 0.154948 & 0.725434 & 0.137848 & 0.678047 & 0.0304814 & 1.02067 \\
\hline 0.763097 & 0.16387 & 0.726827 & 0.138415 & 0.685153 & 0.0262581 & 1.01268 \\
\hline 0.758965 & 0.161698 & 0.720961 & 0.138357 & 0.673093 & 0.0326689 & 1.0084 \\
\hline 0.755887 & 0.166861 & 0.716738 & 0.136979 & 0.674699 & 0.0296815 & 0.998405 \\
\hline 0.760615 & 0.168269 & 0.720183 & 0.134036 & 0.681583 & 0.0337241 & 0.98488 \\
\hline 0.759198 & 0.16533 & 0.714937 & 0.135709 & 0.676599 & 0.0311505 & 0.967675 \\
\hline 0.760204 & 0.176822 & 0.723766 & 0.137797 & 0.668297 & 0.0331291 & 0.961722 \\
\hline 0.756887 & 0.168471 & 0.722755 & 0.139067 & 0.664218 & 0.0251892 & 0.937761 \\
\hline 0.763155 & 0.179601 & 0.72116 & 0.143853 & 0.665139 & 0.0270228 & 0.960729 \\
\hline 0.763289 & 0.186167 & 0.716435 & 0.131352 & 0.667226 & 0.0275341 & 0.926402 \\
\hline 0.759794 & 0.18054 & 0.716087 & 0.132914 & 0.66951 & 0.0297782 & 0.928714 \\
\hline 0.765937 & 0.189908 & 0.718934 & 0.136615 & 0.665467 & 0.0286792 & 0.9465 \\
\hline 0.768404 & 0.198457 & 0.734607 & 0.134218 & 0.664581 & 0.0306002 & 0.902974 \\
\hline 0.772078 & 0.211112 & 0.733092 & 0.134761 & 0.667679 & 0.0344616 & 0.922519 \\
\hline 0.774462 & 0.211923 & 0.744234 & 0.139767 & 0.673655 & 0.0302672 & 0.919786 \\
\hline
\end{tabular}




\begin{tabular}{|c|c|c|c|c|c|c|}
\hline 0.780737 & 0.213248 & 0.744573 & 0.13331 & 0.668861 & 0.0344112 & 0.920665 \\
\hline 0.787512 & 0.216757 & 0.762462 & 0.148223 & 0.671129 & 0.0308896 & 0.912276 \\
\hline 0.788723 & 0.222864 & 0.768666 & 0.142526 & 0.676727 & 0.0357525 & 0.91997 \\
\hline 0.798958 & 0.232138 & 0.765552 & 0.14826 & 0.687021 & 0.0323587 & 0.908766 \\
\hline 0.800006 & 0.247532 & 0.77912 & 0.153408 & 0.685227 & 0.033981 & 0.900545 \\
\hline 0.809385 & 0.248477 & 0.786392 & 0.158904 & 0.689741 & 0.0310421 & 0.925554 \\
\hline 0.817836 & 0.243418 & 0.812968 & 0.176506 & 0.692693 & 0.0365423 & 0.913016 \\
\hline 0.825246 & 0.260459 & 0.816414 & 0.169249 & 0.69685 & 0.0327009 & 0.900185 \\
\hline 0.832524 & 0.277644 & 0.829411 & 0.173577 & 0.698374 & 0.0330119 & 0.930157 \\
\hline 0.841057 & 0.278986 & 0.84544 & 0.192835 & 0.709967 & 0.0331612 & 0.919799 \\
\hline 0.846843 & 0.285764 & 0.849123 & 0.202233 & 0.714872 & 0.0381504 & 0.94526 \\
\hline 0.850233 & 0.294306 & 0.860443 & 0.198454 & 0.707797 & 0.0321952 & 0.939599 \\
\hline 0.860585 & 0.296584 & 0.859141 & 0.198244 & 0.723566 & 0.0316049 & 0.94033 \\
\hline 0.862519 & 0.304279 & 0.870909 & 0.200732 & 0.712538 & 0.0369196 & 0.940332 \\
\hline 0.869362 & 0.314586 & 0.859401 & 0.194125 & 0.717841 & 0.0315881 & 0.974042 \\
\hline 0.869159 & 0.32677 & 0.868214 & 0.193462 & 0.723109 & 0.0333529 & 0.971233 \\
\hline 0.867009 & 0.334549 & 0.862064 & 0.193508 & 0.719374 & 0.0363329 & 0.993341 \\
\hline 0.876156 & 0.346812 & 0.857401 & 0.195509 & 0.719015 & 0.0312001 & 1.01303 \\
\hline 0.870451 & 0.353838 & 0.85567 & 0.189649 & 0.72424 & 0.0315715 & 1.01052 \\
\hline 0.873966 & 0.34107 & 0.855202 & 0.181887 & 0.719965 & 0.037232 & 1.02556 \\
\hline 0.871667 & 0.341692 & 0.838969 & 0.179369 & 0.72095 & 0.0298625 & 1.04035 \\
\hline 0.869681 & 0.356897 & 0.835841 & 0.176211 & 0.720726 & 0.0307792 & 1.04187 \\
\hline 0.872891 & 0.369571 & 0.82541 & 0.171192 & 0.719136 & 0.0295647 & 1.06414 \\
\hline 0.86521 & 0.365365 & 0.824565 & 0.164357 & 0.708515 & 0.0247041 & 1.06025 \\
\hline 0.870612 & 0.371174 & 0.825186 & 0.164061 & 0.718436 & 0.0340018 & 1.09754 \\
\hline 0.868095 & 0.383928 & 0.814585 & 0.168955 & 0.719436 & 0.0318268 & 1.09491 \\
\hline 0.864162 & 0.397228 & 0.814766 & 0.16256 & 0.71852 & 0.0319587 & 1.11349 \\
\hline 0.865262 & 0.401326 & 0.813967 & 0.17065 & 0.711138 & 0.0280039 & 1.09689 \\
\hline 0.862845 & 0.407215 & 0.803786 & 0.170208 & 0.71356 & 0.0307398 & 1.09555 \\
\hline 0.859015 & 0.392065 & 0.799957 & 0.158512 & 0.721985 & 0.0301524 & 1.10131 \\
\hline 0.862638 & 0.398222 & 0.797979 & 0.167115 & 0.709227 & 0.0328855 & 1.09032 \\
\hline 0.864578 & 0.41505 & 0.80069 & 0.172258 & 0.711845 & 0.0351863 & 1.08696 \\
\hline 0.862957 & 0.394258 & 0.809326 & 0.164399 & 0.712872 & 0.0280671 & 1.08142 \\
\hline 0.862408 & 0.400251 & 0.805045 & 0.177064 & 0.711479 & 0.034403 & 1.0571 \\
\hline 0.855013 & 0.391859 & 0.804127 & 0.173578 & 0.707507 & 0.0354975 & 1.06748 \\
\hline 0.867639 & 0.402475 & 0.80737 & 0.179742 & 0.711788 & 0.037729 & 1.04161 \\
\hline 0.86278 & 0.397333 & 0.810845 & 0.181879 & 0.707018 & 0.0366716 & 1.04078 \\
\hline 0.869126 & 0.388815 & 0.798011 & 0.190227 & 0.708905 & 0.0335539 & 1.03523 \\
\hline 0.869204 & 0.384562 & 0.808596 & 0.182214 & 0.708832 & 0.0334923 & 1.01794 \\
\hline 0.872501 & 0.38404 & 0.806971 & 0.182536 & 0.706142 & 0.032453 & 1.03191 \\
\hline 0.86881 & 0.384129 & 0.815294 & 0.199147 & 0.708845 & 0.0295526 & 1.04153 \\
\hline 0.870411 & 0.374438 & 0.812051 & 0.177266 & 0.712765 & 0.036666 & 1.03573 \\
\hline 0.869236 & 0.353725 & 0.817246 & 0.181388 & 0.707966 & 0.0288637 & 1.02032 \\
\hline 0.863776 & 0.359389 & 0.822739 & 0.181395 & 0.711305 & 0.0399992 & 1.01425 \\
\hline 0.873329 & 0.380688 & 0.822118 & 0.183483 & 0.713941 & 0.0253115 & 1.01145 \\
\hline 0.871456 & 0.338238 & 0.817531 & 0.187884 & 0.712935 & 0.0292912 & 1.01758 \\
\hline 0.875546 & 0.344339 & 0.836421 & 0.197195 & 0.717167 & 0.0290296 & 1.00617 \\
\hline
\end{tabular}




\begin{tabular}{|c|c|c|c|c|c|c|}
\hline 0.881671 & 0.344404 & 0.850197 & 0.198335 & 0.711094 & 0.0292947 & 1.02059 \\
\hline 0.884666 & 0.33856 & 0.842858 & 0.189097 & 0.720112 & 0.0278273 & 1.03144 \\
\hline 0.881333 & 0.319881 & 0.850495 & 0.193995 & 0.714288 & 0.0301379 & 1.03174 \\
\hline 0.889241 & 0.321998 & 0.865867 & 0.196985 & 0.717287 & 0.0292258 & 1.04993 \\
\hline 0.895502 & 0.305938 & 0.861771 & 0.197953 & 0.715115 & 0.0298177 & 1.07736 \\
\hline 0.89927 & 0.296484 & 0.887231 & 0.216329 & 0.718846 & 0.0311929 & 1.06634 \\
\hline 0.902204 & 0.292342 & 0.896749 & 0.226218 & 0.72494 & 0.0323231 & 1.06317 \\
\hline 0.914845 & 0.283984 & 0.905682 & 0.21551 & 0.731492 & 0.0347523 & 1.10891 \\
\hline 0.916518 & 0.280845 & 0.915942 & 0.218414 & 0.736248 & 0.0272548 & 1.11087 \\
\hline 0.930466 & 0.277134 & 0.937282 & 0.225291 & 0.724204 & 0.0249918 & 1.1318 \\
\hline 0.938483 & 0.277981 & 0.945953 & 0.229482 & 0.735137 & 0.0380082 & 1.13074 \\
\hline 0.951091 & 0.272465 & 0.966898 & 0.243463 & 0.734246 & 0.0341112 & 1.16036 \\
\hline 0.954276 & 0.272835 & 0.978242 & 0.25603 & 0.736558 & 0.034251 & 1.17078 \\
\hline 0.963132 & 0.273702 & 0.9873 & 0.260289 & 0.738809 & 0.0190936 & 1.19256 \\
\hline 0.970048 & 0.265991 & 0.994345 & 0.254019 & 0.745249 & 0.0345834 & 1.2179 \\
\hline 0.976456 & 0.272022 & 1.00204 & 0.26438 & 0.745194 & 0.0345801 & 1.2108 \\
\hline 0.986462 & 0.270268 & 1.01337 & 0.265655 & 0.739708 & 0.0424445 & 1.22091 \\
\hline 0.984229 & 0.263645 & 1.00433 & 0.254088 & 0.737445 & 0.0308986 & 1.21865 \\
\hline 0.98445 & 0.268314 & 1.01106 & 0.250686 & 0.747547 & 0.0225416 & 1.2348 \\
\hline 0.980431 & 0.255617 & 0.993926 & 0.255201 & 0.743327 & 0.0300627 & 1.19801 \\
\hline 0.985131 & 0.257513 & 0.98799 & 0.238547 & 0.743371 & 0.0358559 & 1.19837 \\
\hline 0.96948 & 0.233884 & 0.97499 & 0.233326 & 0.735841 & 0.0333795 & 1.18177 \\
\hline 0.967626 & 0.23356 & 0.972421 & 0.230024 & 0.735224 & 0.0282823 & 1.18666 \\
\hline 0.95467 & 0.220795 & 0.957722 & 0.211239 & 0.731105 & 0.0323611 & 1.17115 \\
\hline 0.941628 & 0.221636 & 0.936677 & 0.194408 & 0.730836 & 0.0302855 & 1.157 \\
\hline 0.934398 & 0.20917 & 0.925353 & 0.195784 & 0.730002 & 0.029562 & 1.14864 \\
\hline 0.922866 & 0.194906 & 0.925449 & 0.199726 & 0.728141 & 0.0279473 & 1.12701 \\
\hline 0.908961 & 0.186261 & 0.902058 & 0.180381 & 0.721787 & 0.0301479 & 1.10358 \\
\hline 0.901471 & 0.185027 & 0.893303 & 0.172566 & 0.715113 & 0.0259164 & 1.09992 \\
\hline 0.885296 & 0.173234 & 0.873234 & 0.160783 & 0.722565 & 0.0328643 & 1.08121 \\
\hline 0.874389 & 0.171872 & 0.871742 & 0.154547 & 0.721182 & 0.0230258 & 1.07556 \\
\hline 0.866203 & 0.158205 & 0.867935 & 0.149191 & 0.715955 & 0.0350557 & 1.08293 \\
\hline 0.865907 & 0.159324 & 0.845389 & 0.144241 & 0.71836 & 0.0286316 & 1.0713 \\
\hline 0.852961 & 0.155227 & 0.836105 & 0.142745 & 0.716552 & 0.0326043 & 1.05952 \\
\hline 0.849821 & 0.154259 & 0.847955 & 0.146992 & 0.718927 & 0.0367505 & 1.04883 \\
\hline 0.848631 & 0.138505 & 0.843494 & 0.149915 & 0.716731 & 0.0247875 & 1.06748 \\
\hline 0.844833 & 0.151012 & 0.847339 & 0.144127 & 0.717515 & 0.0251399 & 1.04175 \\
\hline 0.847508 & 0.14316 & 0.85587 & 0.14471 & 0.715825 & 0.0255594 & 1.06879 \\
\hline 0.844624 & 0.150505 & 0.848781 & 0.143998 & 0.711451 & 0.0302084 & 1.06407 \\
\hline 0.848075 & 0.148093 & 0.846289 & 0.132769 & 0.71878 & 0.0277688 & 1.06303 \\
\hline 0.849768 & 0.14573 & 0.838213 & 0.14058 & 0.704012 & 0.0244001 & 1.05056 \\
\hline 0.847721 & 0.141639 & 0.859614 & 0.144989 & 0.718114 & 0.0286931 & 1.07249 \\
\hline 0.846939 & 0.136152 & 0.859545 & 0.149222 & 0.724545 & 0.0323089 & 1.06292 \\
\hline 0.852884 & 0.14403 & 0.862369 & 0.148033 & 0.721147 & 0.0333808 & 1.05903 \\
\hline 0.861313 & 0.143625 & 0.866186 & 0.151309 & 0.724589 & 0.0316205 & 1.0814 \\
\hline 0.860409 & 0.144443 & 0.883919 & 0.145 & 0.725428 & 0.0279311 & 1.0648 \\
\hline 0.866778 & 0.14935 & 0.889876 & 0.154418 & 0.729172 & 0.0362368 & 1.08351 \\
\hline
\end{tabular}




\begin{tabular}{|c|c|c|c|c|c|c|}
\hline 0.875234 & 0.150549 & 0.89991 & 0.155976 & 0.738971 & 0.0308812 & 1.0906 \\
\hline 0.886333 & 0.148104 & 0.91542 & 0.164386 & 0.742791 & 0.0252031 & 1.0835 \\
\hline 0.892455 & 0.155309 & 0.935867 & 0.174193 & 0.748592 & 0.0331054 & 1.10682 \\
\hline 0.905478 & 0.153424 & 0.959217 & 0.179748 & 0.75713 & 0.027638 & 1.11421 \\
\hline 0.914646 & 0.160034 & 0.981274 & 0.197279 & 0.765058 & 0.0337724 & 1.10463 \\
\hline 0.929446 & 0.16042 & 1.00608 & 0.209141 & 0.790836 & 0.0323537 & 1.10225 \\
\hline 0.942507 & 0.157899 & 1.02648 & 0.217736 & 0.79595 & 0.0325747 & 1.10271 \\
\hline 0.954534 & 0.16419 & 1.06302 & 0.24548 & 0.810704 & 0.0325652 & 1.1451 \\
\hline 0.972053 & 0.16248 & 1.06999 & 0.270025 & 0.816246 & 0.0348637 & 1.107 \\
\hline 0.989118 & 0.1644 & 1.11878 & 0.279595 & 0.838417 & 0.0358857 & 1.14765 \\
\hline 1.0069 & 0.169563 & 1.13113 & 0.294805 & 0.859083 & 0.0416097 & 1.10211 \\
\hline 1.03372 & 0.173783 & 1.16502 & 0.314963 & 0.861833 & 0.0356785 & 1.13297 \\
\hline 1.0492 & 0.181498 & 1.18712 & 0.339725 & 0.891038 & 0.0338984 & 1.12717 \\
\hline 1.05802 & 0.176136 & 1.22379 & 0.351476 & 0.893713 & 0.0347871 & 1.11716 \\
\hline 1.07581 & 0.177149 & 1.20984 & 0.348049 & 0.907592 & 0.0361419 & 1.12244 \\
\hline 1.08843 & 0.182752 & 1.23555 & 0.354271 & 0.922391 & 0.046723 & 1.13889 \\
\hline 1.09157 & 0.174856 & 1.24759 & 0.38675 & 0.918394 & 0.0405443 & 1.1418 \\
\hline 1.09721 & 0.175236 & 1.2275 & 0.374737 & 0.929285 & 0.0468162 & 1.11709 \\
\hline 1.09506 & 0.181969 & 1.23754 & 0.356521 & 0.927541 & 0.0408065 & 1.12984 \\
\hline 1.09454 & 0.177795 & 1.21751 & 0.334865 & 0.909183 & 0.0382191 & 1.12159 \\
\hline 1.0844 & 0.179524 & 1.18083 & 0.326958 & 0.894833 & 0.0414884 & 1.104 \\
\hline 1.07272 & 0.177028 & 1.16741 & 0.318987 & 0.893038 & 0.0413067 & 1.10184 \\
\hline 1.05303 & 0.174554 & 1.13209 & 0.306025 & 0.878782 & 0.0415412 & 1.10011 \\
\hline 1.03218 & 0.1673 & 1.08531 & 0.277784 & 0.856344 & 0.0390053 & 1.10708 \\
\hline 1.011 & 0.163445 & 1.07011 & 0.242609 & 0.843562 & 0.0436084 & 1.09961 \\
\hline 0.991051 & 0.15965 & 1.03335 & 0.232806 & 0.826638 & 0.0363842 & 1.08177 \\
\hline 0.967845 & 0.155896 & 1.00258 & 0.208957 & 0.813702 & 0.0377381 & 1.09531 \\
\hline 0.94579 & 0.151208 & 0.96922 & 0.194402 & 0.796864 & 0.0320749 & 1.07391 \\
\hline 0.921858 & 0.15257 & 0.935213 & 0.166963 & 0.777263 & 0.0354037 & 1.07253 \\
\hline 0.910075 & 0.150264 & 0.922149 & 0.165633 & 0.768272 & 0.0317831 & 1.08449 \\
\hline 0.882569 & 0.145471 & 0.903451 & 0.144654 & 0.748851 & 0.0268741 & 1.04784 \\
\hline 0.873023 & 0.147143 & 0.883075 & 0.157079 & 0.746277 & 0.025915 & 1.05393 \\
\hline 0.859983 & 0.143144 & 0.863838 & 0.132681 & 0.732846 & 0.0304691 & 1.04825 \\
\hline 0.84959 & 0.143765 & 0.856003 & 0.140007 & 0.726635 & 0.0241683 & 1.06416 \\
\hline 0.835902 & 0.139839 & 0.848535 & 0.130808 & 0.719701 & 0.0253746 & 1.07086 \\
\hline 0.828523 & 0.150522 & 0.828674 & 0.125532 & 0.71485 & 0.0327013 & 1.04174 \\
\hline 0.823075 & 0.148187 & 0.819649 & 0.123414 & 0.714985 & 0.029095 & 1.0591 \\
\hline 0.814451 & 0.144346 & 0.824297 & 0.136975 & 0.701643 & 0.0202541 & 1.06166 \\
\hline 0.809947 & 0.140552 & 0.813111 & 0.122175 & 0.702861 & 0.0309218 & 1.06323 \\
\hline 0.81005 & 0.148343 & 0.817037 & 0.116201 & 0.698122 & 0.0304617 & 1.07278 \\
\hline 0.802895 & 0.139408 & 0.798763 & 0.118049 & 0.696139 & 0.0281016 & 1.06721 \\
\hline 0.796888 & 0.141691 & 0.790818 & 0.110693 & 0.701742 & 0.0266436 & 1.08643 \\
\hline 0.798885 & 0.141173 & 0.794178 & 0.119421 & 0.70629 & 0.0251203 & 1.06473 \\
\hline 0.795226 & 0.138513 & 0.797483 & 0.110429 & 0.702506 & 0.028947 & 1.07778 \\
\hline 0.786277 & 0.143003 & 0.786389 & 0.124538 & 0.704491 & 0.0210866 & 1.10001 \\
\hline 0.785829 & 0.137858 & 0.787815 & 0.106729 & 0.705542 & 0.0324214 & 1.09557 \\
\hline 0.784816 & 0.138392 & 0.782223 & 0.113574 & 0.699081 & 0.0218965 & 1.08669 \\
\hline
\end{tabular}




\begin{tabular}{|c|c|c|c|c|c|c|}
\hline 0.782335 & 0.135537 & 0.791952 & 0.113482 & 0.702396 & 0.0281725 & 1.11935 \\
\hline 0.780738 & 0.139624 & 0.778199 & 0.0996043 & 0.703765 & 0.0289203 & 1.10632 \\
\hline 0.784195 & 0.129034 & 0.776456 & 0.115113 & 0.702822 & 0.0302031 & 1.1155 \\
\hline 0.781762 & 0.132938 & 0.774346 & 0.105477 & 0.700633 & 0.0329531 & 1.11526 \\
\hline 0.777867 & 0.132114 & 0.777764 & 0.0961469 & 0.700899 & 0.0254439 & 1.1319 \\
\hline 0.779479 & 0.135422 & 0.77053 & 0.101629 & 0.705892 & 0.0341651 & 1.14594 \\
\hline 0.776384 & 0.136003 & 0.76479 & 0.0937145 & 0.694743 & 0.026982 & 1.14453 \\
\hline 0.774883 & 0.139881 & 0.771483 & 0.100086 & 0.70085 & 0.0216479 & 1.1443 \\
\hline 0.779591 & 0.136126 & 0.774001 & 0.0979894 & 0.696218 & 0.0311815 & 1.1416 \\
\hline 0.773122 & 0.133773 & 0.760395 & 0.0972726 & 0.702476 & 0.030837 & 1.13957 \\
\hline 0.77035 & 0.134715 & 0.774867 & 0.0964683 & 0.700712 & 0.0304849 & 1.13758 \\
\hline 0.776001 & 0.133456 & 0.767705 & 0.0922729 & 0.694378 & 0.029144 & 1.14056 \\
\hline 0.769798 & 0.132786 & 0.757497 & 0.0983181 & 0.700386 & 0.0314289 & 1.15091 \\
\hline 0.766521 & 0.132581 & 0.75302 & 0.0959541 & 0.690117 & 0.0238111 & 1.14328 \\
\hline 0.769658 & 0.126236 & 0.758861 & 0.0753228 & 0.696919 & 0.030694 & 1.14385 \\
\hline 0.768832 & 0.124259 & 0.765624 & 0.0813003 & 0.693261 & 0.032249 & 1.15457 \\
\hline 0.769928 & 0.13574 & 0.768717 & 0.102223 & 0.701802 & 0.0335086 & 1.1537 \\
\hline 0.768237 & 0.123124 & 0.761568 & 0.0875149 & 0.696155 & 0.0311945 & 1.15699 \\
\hline 0.767048 & 0.131247 & 0.759973 & 0.0858791 & 0.69073 & 0.0369788 & 1.15963 \\
\hline 0.767705 & 0.122501 & 0.757045 & 0.0809012 & 0.695479 & 0.0304133 & 1.14709 \\
\hline 0.769528 & 0.12683 & 0.756142 & 0.0913569 & 0.698649 & 0.033883 & 1.11514 \\
\hline 0.763725 & 0.125044 & 0.750202 & 0.0797283 & 0.694988 & 0.026924 & 1.12214 \\
\hline 0.769345 & 0.128465 & 0.758161 & 0.0985121 & 0.695845 & 0.0262428 & 1.12835 \\
\hline 0.772588 & 0.124354 & 0.758526 & 0.09003 & 0.697511 & 0.0248502 & 1.10186 \\
\hline 0.77117 & 0.124531 & 0.771722 & 0.0940057 & 0.692267 & 0.0246872 & 1.10476 \\
\hline 0.764921 & 0.118504 & 0.74656 & 0.0889708 & 0.692319 & 0.0272955 & 1.06819 \\
\hline 0.764416 & 0.122539 & 0.757397 & 0.0890688 & 0.70102 & 0.0240955 & 1.07193 \\
\hline 0.766073 & 0.128801 & 0.756824 & 0.0802817 & 0.696353 & 0.0285281 & 1.07148 \\
\hline 0.771405 & 0.12373 & 0.762742 & 0.0807595 & 0.697337 & 0.0315426 & 1.06125 \\
\hline 0.763513 & 0.117789 & 0.765809 & 0.0950346 & 0.687316 & 0.0253184 & 1.03774 \\
\hline 0.765494 & 0.120922 & 0.756739 & 0.0873904 & 0.695534 & 0.0320336 & 1.04277 \\
\hline 0.761055 & 0.116019 & 0.751974 & 0.0817807 & 0.699814 & 0.0288414 & 1.03306 \\
\hline 0.767305 & 0.120404 & 0.760163 & 0.0923333 & 0.698703 & 0.0286132 & 1.0367 \\
\hline 0.760388 & 0.118714 & 0.754556 & 0.0878169 & 0.68638 & 0.026779 & 1.02273 \\
\hline 0.769406 & 0.124932 & 0.756307 & 0.0833338 & 0.693927 & 0.0244874 & 1.02491 \\
\hline 0.768111 & 0.125424 & 0.748639 & 0.0886789 & 0.693029 & 0.0336311 & 1.01309 \\
\hline 0.770485 & 0.12557 & 0.749095 & 0.0794184 & 0.693304 & 0.0284076 & 1.00885 \\
\hline 0.765653 & 0.124238 & 0.760805 & 0.0824528 & 0.702695 & 0.0317771 & 1.03458 \\
\hline 0.768488 & 0.120157 & 0.765323 & 0.090687 & 0.700902 & 0.0308868 & 1.0037 \\
\hline 0.765366 & 0.120489 & 0.754201 & 0.0844634 & 0.69778 & 0.0265069 & 0.985522 \\
\hline 0.763988 & 0.119888 & 0.75886 & 0.0912683 & 0.702056 & 0.0311756 & 0.999847 \\
\hline 0.76921 & 0.119453 & 0.775262 & 0.0888096 & 0.693208 & 0.0263698 & 1.00357 \\
\hline 0.766435 & 0.119392 & 0.76248 & 0.0898088 & 0.697536 & 0.0263979 & 1.01301 \\
\hline 0.762043 & 0.119414 & 0.759262 & 0.0860805 & 0.698726 & 0.0361966 & 1.01291 \\
\hline 0.768604 & 0.122865 & 0.759837 & 0.0848015 & 0.694243 & 0.0311507 & 1.00419 \\
\hline 0.763939 & 0.121625 & 0.752601 & 0.0831129 & 0.694155 & 0.0261458 & 1.02123 \\
\hline 0.767643 & 0.122193 & 0.759122 & 0.082373 & 0.696458 & 0.0201736 & 0.999229 \\
\hline
\end{tabular}




\begin{tabular}{|c|c|c|c|c|c|c|}
\hline 0.764035 & 0.12167 & 0.755372 & 0.0833492 & 0.699124 & 0.0310885 & 0.98843 \\
\hline 0.764693 & 0.121556 & 0.758216 & 0.0689831 & 0.695741 & 0.0284452 & 1.00086 \\
\hline 0.762737 & 0.120104 & 0.748287 & 0.0779699 & 0.688628 & 0.0256525 & 1.00632 \\
\hline 0.766237 & 0.122116 & 0.744384 & 0.077764 & 0.686768 & 0.0340516 & 0.992557 \\
\hline 0.766241 & 0.123405 & 0.766669 & 0.0889948 & 0.691812 & 0.0237556 & 0.987002 \\
\hline 0.764802 & 0.112437 & 0.752488 & 0.0836813 & 0.695532 & 0.0253376 & 1.00611 \\
\hline 0.761268 & 0.118356 & 0.751991 & 0.0824023 & 0.702011 & 0.0270836 & 0.97565 \\
\hline 0.766147 & 0.112909 & 0.747929 & 0.0743534 & 0.690045 & 0.0271741 & 0.982012 \\
\hline 0.769213 & 0.117201 & 0.755709 & 0.0894492 & 0.692579 & 0.0257379 & 0.993897 \\
\hline 0.764902 & 0.117928 & 0.756003 & 0.0817325 & 0.689247 & 0.0250636 & 0.985412 \\
\hline 0.764386 & 0.122028 & 0.757052 & 0.0814349 & 0.691871 & 0.0311492 & 0.987554 \\
\hline 0.764359 & 0.117178 & 0.750756 & 0.090324 & 0.688852 & 0.033251 & 0.976179 \\
\hline 0.760221 & 0.114832 & 0.757372 & 0.0854812 & 0.694673 & 0.024009 & 0.982074 \\
\hline 0.762779 & 0.121693 & 0.755834 & 0.0838391 & 0.688665 & 0.0217652 & 0.982044 \\
\hline 0.761617 & 0.113516 & 0.744028 & 0.078823 & 0.687473 & 0.025644 & 0.982391 \\
\hline 0.76468 & 0.111531 & 0.748038 & 0.0762145 & 0.683085 & 0.0269587 & 0.989911 \\
\hline 0.763439 & 0.117404 & 0.740602 & 0.0796954 & 0.684688 & 0.0264757 & 0.962672 \\
\hline 0.761591 & 0.118621 & 0.740433 & 0.0670427 & 0.683765 & 0.0249843 & 0.988446 \\
\hline 0.759982 & 0.110574 & 0.752252 & 0.0733748 & 0.695193 & 0.0250323 & 0.989052 \\
\hline 0.758121 & 0.117012 & 0.757627 & 0.0880767 & 0.684336 & 0.0289141 & 0.966383 \\
\hline 0.76234 & 0.110086 & 0.754056 & 0.0850616 & 0.687841 & 0.0299674 & 0.988633 \\
\hline 0.762921 & 0.116973 & 0.749482 & 0.0825474 & 0.692437 & 0.0263481 & 0.98133 \\
\hline 0.756071 & 0.114604 & 0.751893 & 0.0842839 & 0.686756 & 0.0236223 & 0.9893 \\
\hline 0.763159 & 0.117152 & 0.747514 & 0.0821669 & 0.683664 & 0.0326996 & 0.991356 \\
\hline 0.763296 & 0.11485 & 0.745301 & 0.0790531 & 0.686959 & 0.0278028 & 0.988734 \\
\hline 0.760732 & 0.114178 & 0.751951 & 0.0716439 & 0.682268 & 0.0278231 & 0.979042 \\
\hline 0.761672 & 0.12094 & 0.755104 & 0.0800713 & 0.682919 & 0.0271465 & 1.00373 \\
\hline 0.766145 & 0.112738 & 0.743936 & 0.0860862 & 0.68318 & 0.0204316 & 1.01535 \\
\hline 0.762995 & 0.118339 & 0.753107 & 0.0835957 & 0.690232 & 0.0305405 & 0.987903 \\
\hline 0.76334 & 0.120219 & 0.738081 & 0.0796088 & 0.684955 & 0.0170511 & 0.995297 \\
\hline 0.762171 & 0.122038 & 0.752501 & 0.0801323 & 0.681625 & 0.0316106 & 0.990568 \\
\hline 0.76195 & 0.116635 & 0.746173 & 0.085734 & 0.679854 & 0.0230431 & 0.998897 \\
\hline 0.760236 & 0.118444 & 0.743515 & 0.0868761 & 0.68362 & 0.0308558 & 1.02251 \\
\hline 0.759197 & 0.115353 & 0.753235 & 0.0766919 & 0.684403 & 0.0231436 & 1.01524 \\
\hline 0.764101 & 0.11482 & 0.754956 & 0.0798846 & 0.680625 & 0.0222828 & 1.02048 \\
\hline 0.770075 & 0.116614 & 0.758077 & 0.0858088 & 0.68766 & 0.0231338 & 1.03945 \\
\hline 0.759688 & 0.113158 & 0.749745 & 0.0838562 & 0.679469 & 0.0285783 & 1.04168 \\
\hline 0.765064 & 0.11652 & 0.755127 & 0.0810219 & 0.678194 & 0.0273453 & 1.03671 \\
\hline 0.759492 & 0.115481 & 0.752118 & 0.0810168 & 0.682189 & 0.0219686 & 1.02313 \\
\hline 0.764325 & 0.112524 & 0.751212 & 0.0766755 & 0.68437 & 0.0269283 & 1.04384 \\
\hline 0.765361 & 0.11824 & 0.761375 & 0.0831553 & 0.683583 & 0.025813 & 1.05906 \\
\hline 0.760305 & 0.117079 & 0.755184 & 0.0828283 & 0.684124 & 0.0222621 & 1.05177 \\
\hline 0.764841 & 0.120365 & 0.759304 & 0.08703 & 0.681248 & 0.0287762 & 1.04885 \\
\hline 0.761101 & 0.111318 & 0.749479 & 0.0894545 & 0.68555 & 0.0259845 & 1.05046 \\
\hline 0.756174 & 0.113933 & 0.750219 & 0.0838986 & 0.679222 & 0.0234373 & 1.06252 \\
\hline 0.756285 & 0.122014 & 0.753951 & 0.0940546 & 0.686463 & 0.0274068 & 1.05948 \\
\hline 0.762576 & 0.1177 & 0.749282 & 0.0776435 & 0.679307 & 0.0233716 & 1.06082 \\
\hline
\end{tabular}




\begin{tabular}{|c|c|c|c|c|c|c|}
\hline 0.753671 & 0.114578 & 0.748458 & 0.0810592 & 0.675879 & 0.0299834 & 1.06929 \\
\hline 0.757302 & 0.122188 & 0.758154 & 0.0848329 & 0.672244 & 0.0274505 & 1.04552 \\
\hline 0.752928 & 0.113584 & 0.754149 & 0.0853794 & 0.683287 & 0.0341036 & 1.05346 \\
\hline 0.761819 & 0.10873 & 0.754 & 0.0823324 & 0.676053 & 0.0322457 & 1.04534 \\
\hline 0.75917 & 0.120204 & 0.74712 & 0.0847006 & 0.675015 & 0.0282661 & 1.03937 \\
\hline 0.756148 & 0.112108 & 0.751255 & 0.0881886 & 0.678801 & 0.0331338 & 1.02066 \\
\hline 0.756258 & 0.112677 & 0.75294 & 0.0758733 & 0.667439 & 0.0240804 & 1.02289 \\
\hline 0.75407 & 0.104295 & 0.751902 & 0.0829328 & 0.673907 & 0.0246692 & 1.02093 \\
\hline 0.75862 & 0.114214 & 0.746908 & 0.0830064 & 0.66796 & 0.0324558 & 1.00563 \\
\hline 0.752245 & 0.110667 & 0.751915 & 0.0885602 & 0.671241 & 0.0232935 & 1.01967 \\
\hline 0.749235 & 0.114917 & 0.75722 & 0.0938217 & 0.664238 & 0.0263118 & 0.997414 \\
\hline 0.747243 & 0.104927 & 0.750246 & 0.0890714 & 0.672513 & 0.0257943 & 0.990956 \\
\hline 0.747107 & 0.11383 & 0.745323 & 0.0870138 & 0.673945 & 0.0245345 & 0.989588 \\
\hline 0.744971 & 0.0988313 & 0.746282 & 0.0814619 & 0.670986 & 0.0240971 & 0.9851 \\
\hline 0.743424 & 0.105142 & 0.744798 & 0.0823383 & 0.667133 & 0.0215928 & 0.977756 \\
\hline 0.744313 & 0.106871 & 0.748338 & 0.0841383 & 0.669752 & 0.0245446 & 0.961601 \\
\hline 0.748951 & 0.107701 & 0.741782 & 0.0812553 & 0.663595 & 0.0212996 & 0.968869 \\
\hline 0.740813 & 0.105567 & 0.75237 & 0.0857508 & 0.658196 & 0.0221678 & 0.974919 \\
\hline 0.738473 & 0.097034 & 0.740037 & 0.0845546 & 0.664438 & 0.0275852 & 0.945913 \\
\hline 0.742802 & 0.10374 & 0.734139 & 0.0800211 & 0.662822 & 0.0273123 & 0.945732 \\
\hline 0.737944 & 0.105029 & 0.734217 & 0.0810967 & 0.656889 & 0.0245335 & 0.955708 \\
\hline 0.738118 & 0.104422 & 0.744698 & 0.0841562 & 0.657953 & 0.0224908 & 0.93932 \\
\hline 0.740429 & 0.105674 & 0.730524 & 0.0753256 & 0.649966 & 0.0274046 & 0.958892 \\
\hline 0.732888 & 0.091667 & 0.736745 & 0.0778362 & 0.656601 & 0.0269177 & 0.95587 \\
\hline 0.732939 & 0.100269 & 0.739094 & 0.0904415 & 0.65244 & 0.0261344 & 0.928793 \\
\hline 0.733852 & 0.0983867 & 0.731237 & 0.0805684 & 0.654708 & 0.0289357 & 0.949125 \\
\hline 0.735565 & 0.102449 & 0.735003 & 0.0897039 & 0.653351 & 0.0260107 & 0.959145 \\
\hline 0.730319 & 0.0933935 & 0.737207 & 0.0857049 & 0.644545 & 0.0261581 & 0.953538 \\
\hline 0.728333 & 0.095163 & 0.738429 & 0.077185 & 0.646603 & 0.0209794 & 0.941718 \\
\hline 0.730251 & 0.0941426 & 0.730174 & 0.0805476 & 0.647903 & 0.0234109 & 0.943891 \\
\hline 0.726526 & 0.0942901 & 0.724081 & 0.0788281 & 0.651014 & 0.0239913 & 0.956998 \\
\hline 0.729813 & 0.0861888 & 0.723354 & 0.0780007 & 0.648255 & 0.0227642 & 0.983028 \\
\hline 0.725771 & 0.0956369 & 0.730283 & 0.0797259 & 0.640983 & 0.0328041 & 0.948502 \\
\hline 0.724633 & 0.0964602 & 0.719206 & 0.0681826 & 0.647186 & 0.0210487 & 0.967639 \\
\hline 0.729111 & 0.0964957 & 0.729588 & 0.0796526 & 0.647385 & 0.028097 & 0.980438 \\
\hline 0.725879 & 0.0946523 & 0.724627 & 0.0813631 & 0.642634 & 0.0196635 & 0.989709 \\
\hline 0.725802 & 0.0934177 & 0.728265 & 0.0694427 & 0.646017 & 0.0261809 & 0.99585 \\
\hline 0.723934 & 0.0903887 & 0.727828 & 0.08045 & 0.646924 & 0.0174005 & 1.01001 \\
\hline 0.720627 & 0.0947472 & 0.734785 & 0.0753034 & 0.639929 & 0.0239284 & 1.02196 \\
\hline 0.718235 & 0.0900133 & 0.729746 & 0.075493 & 0.638999 & 0.0289283 & 1.03191 \\
\hline 0.722826 & 0.0949898 & 0.734033 & 0.0800045 & 0.634123 & 0.0276001 & 1.03928 \\
\hline 0.719157 & 0.0924085 & 0.719298 & 0.076855 & 0.638564 & 0.0240001 & 1.07124 \\
\hline 0.7126 & 0.0874779 & 0.724189 & 0.0747195 & 0.639499 & 0.0316636 & 1.06819 \\
\hline 0.715867 & 0.0881301 & 0.727065 & 0.0757341 & 0.638032 & 0.0188277 & 1.09198 \\
\hline 0.713509 & 0.0861054 & 0.724075 & 0.0673161 & 0.634491 & 0.0193622 & 1.10951 \\
\hline 0.718124 & 0.0916317 & 0.737494 & 0.0801671 & 0.634876 & 0.0223892 & 1.12602 \\
\hline 0.712495 & 0.0915223 & 0.727693 & 0.0745392 & 0.636828 & 0.0259215 & 1.12639 \\
\hline
\end{tabular}




\begin{tabular}{|c|c|c|c|c|c|c|}
\hline 0.709125 & 0.0807458 & 0.723219 & 0.0684382 & 0.64218 & 0.0256609 & 1.14928 \\
\hline 0.716288 & 0.0933179 & 0.715126 & 0.0739055 & 0.638747 & 0.0216749 & 1.13131 \\
\hline 0.705031 & 0.0898764 & 0.72433 & 0.0691531 & 0.629723 & 0.0176872 & 1.14087 \\
\hline 0.710268 & 0.0843942 & 0.718809 & 0.071447 & 0.629433 & 0.0242888 & 1.16072 \\
\hline 0.70247 & 0.0914398 & 0.717122 & 0.0733727 & 0.628465 & 0.0229791 & 1.16258 \\
\hline 0.705489 & 0.0865743 & 0.722596 & 0.0754474 & 0.630136 & 0.017723 & 1.16346 \\
\hline 0.703241 & 0.0856445 & 0.714446 & 0.067822 & 0.630273 & 0.0198326 & 1.14189 \\
\hline 0.704536 & 0.0869098 & 0.711265 & 0.065246 & 0.624361 & 0.0242521 & 1.14423 \\
\hline 0.701156 & 0.0824091 & 0.714533 & 0.0669621 & 0.62682 & 0.0226309 & 1.14109 \\
\hline 0.699176 & 0.086539 & 0.701786 & 0.0703251 & 0.625511 & 0.0253043 & 1.11414 \\
\hline 0.696128 & 0.0897877 & 0.709804 & 0.0660481 & 0.625334 & 0.0239826 & 1.1029 \\
\hline 0.695855 & 0.0845968 & 0.709313 & 0.0755712 & 0.628296 & 0.0184486 & 1.10315 \\
\hline 0.695042 & 0.0839696 & 0.704076 & 0.0708757 & 0.621802 & 0.0226274 & 1.10417 \\
\hline 0.694005 & 0.0795948 & 0.699742 & 0.0687401 & 0.624912 & 0.0268837 & 1.06636 \\
\hline 0.694608 & 0.0811549 & 0.705685 & 0.0714102 & 0.621073 & 0.0243621 & 1.08519 \\
\hline 0.689263 & 0.0787577 & 0.709119 & 0.0720991 & 0.621815 & 0.0241458 & 1.08389 \\
\hline 0.691101 & 0.0797468 & 0.705671 & 0.0691752 & 0.622531 & 0.01532 & 1.0895 \\
\hline 0.690596 & 0.0815115 & 0.712302 & 0.0763456 & 0.627519 & 0.0155805 & 1.08195 \\
\hline 0.695768 & 0.0833251 & 0.709547 & 0.0722268 & 0.625239 & 0.0206732 & 1.08232 \\
\hline 0.701039 & 0.0840494 & 0.722338 & 0.0664469 & 0.620262 & 0.0193316 & 1.08603 \\
\hline 0.696754 & 0.081599 & 0.719872 & 0.079288 & 0.627074 & 0.0285975 & 1.0939 \\
\hline 0.702974 & 0.077333 & 0.755779 & 0.0815532 & 0.631445 & 0.0243965 & 1.12916 \\
\hline 0.706042 & 0.0845142 & 0.755805 & 0.0958036 & 0.639595 & 0.0261488 & 1.13636 \\
\hline 0.711146 & 0.0737684 & 0.761948 & 0.0948172 & 0.642864 & 0.0178237 & 1.17701 \\
\hline 0.725941 & 0.0864155 & 0.781665 & 0.108151 & 0.658261 & 0.0248233 & 1.20445 \\
\hline 0.736519 & 0.0861328 & 0.794135 & 0.099376 & 0.665879 & 0.026188 & 1.24548 \\
\hline 0.745411 & 0.0874218 & 0.805401 & 0.123186 & 0.67681 & 0.0237303 & 1.33753 \\
\hline 0.75665 & 0.0864011 & 0.831074 & 0.138421 & 0.676028 & 0.0270242 & 1.42618 \\
\hline 0.770664 & 0.0847559 & 0.852404 & 0.136987 & 0.690814 & 0.0293292 & 1.52736 \\
\hline 0.785802 & 0.091725 & 0.868738 & 0.153976 & 0.687827 & 0.0255392 & 1.6628 \\
\hline 0.794789 & 0.0868154 & 0.876392 & 0.150301 & 0.711304 & 0.0291339 & 1.85093 \\
\hline 0.809033 & 0.0936003 & 0.905673 & 0.16318 & 0.714815 & 0.0288643 & 2.10893 \\
\hline 0.821524 & 0.0962739 & 0.906236 & 0.163825 & 0.72281 & 0.0359531 & 2.36145 \\
\hline 0.833114 & 0.0977866 & 0.925237 & 0.166909 & 0.733119 & 0.0309053 & 2.72176 \\
\hline 0.841423 & 0.094882 & 0.942438 & 0.189027 & 0.742868 & 0.0331614 & 3.2249 \\
\hline 0.855701 & 0.101364 & 0.939159 & 0.188828 & 0.751805 & 0.0350246 & 3.77616 \\
\hline 0.852448 & 0.10819 & 0.934928 & 0.175309 & 0.748469 & 0.0346737 & 4.35815 \\
\hline 0.853893 & 0.108141 & 0.947725 & 0.178176 & 0.752844 & 0.0293195 & 5.10259 \\
\hline 0.863192 & 0.117997 & 0.942869 & 0.184649 & 0.751573 & 0.0364931 & 6.00072 \\
\hline 0.859748 & 0.116102 & 0.932918 & 0.182092 & 0.751382 & 0.0270949 & 6.85881 \\
\hline 0.858686 & 0.11956 & 0.910807 & 0.174765 & 0.738866 & 0.0267356 & 7.97525 \\
\hline 0.854788 & 0.137988 & 0.902172 & 0.168839 & 0.744829 & 0.0317572 & 9.15766 \\
\hline 0.846922 & 0.138018 & 0.903372 & 0.183615 & 0.739332 & 0.0295949 & 10.4108 \\
\hline 0.843934 & 0.152021 & 0.887023 & 0.162968 & 0.735849 & 0.0320166 & 11.69 \\
\hline 0.837181 & 0.156381 & 0.885289 & 0.1597 & 0.729349 & 0.0245465 & 12.934 \\
\hline 0.829792 & 0.174652 & 0.865427 & 0.16748 & 0.732734 & 0.0297742 & 14.293 \\
\hline 0.822643 & 0.189773 & 0.855914 & 0.183598 & 0.724037 & 0.0249492 & 15.4947 \\
\hline
\end{tabular}




\begin{tabular}{|c|c|c|c|c|c|c|}
\hline 0.82213 & 0.213459 & 0.849209 & 0.182693 & 0.721315 & 0.0246897 & 16.6514 \\
\hline 0.807812 & 0.212919 & 0.831647 & 0.185818 & 0.716888 & 0.0289356 & 17.6194 \\
\hline 0.79973 & 0.217741 & 0.826746 & 0.182289 & 0.718804 & 0.0329662 & 18.5174 \\
\hline 0.796768 & 0.235548 & 0.832983 & 0.194977 & 0.702991 & 0.0373228 & 19.2301 \\
\hline 0.788764 & 0.246772 & 0.809462 & 0.177388 & 0.69945 & 0.0327313 & 19.7861 \\
\hline 0.78641 & 0.251301 & 0.79719 & 0.188334 & 0.698019 & 0.0288447 & 19.9108 \\
\hline 0.77764 & 0.24859 & 0.790752 & 0.200675 & 0.702785 & 0.0319859 & 19.9456 \\
\hline 0.775886 & 0.2551 & 0.784081 & 0.176859 & 0.696859 & 0.0253275 & 19.6697 \\
\hline 0.762681 & 0.260384 & 0.772915 & 0.171676 & 0.675152 & 0.0285855 & 19.0416 \\
\hline 0.761723 & 0.257471 & 0.754436 & 0.16982 & 0.679877 & 0.0286356 & 18.4268 \\
\hline 0.747648 & 0.234158 & 0.744211 & 0.147853 & 0.669471 & 0.0298475 & 17.4087 \\
\hline 0.742187 & 0.226214 & 0.733195 & 0.151784 & 0.653461 & 0.0300205 & 16.2454 \\
\hline 0.739167 & 0.223681 & 0.726888 & 0.131735 & 0.65221 & 0.022735 & 15.0644 \\
\hline 0.721727 & 0.200823 & 0.715625 & 0.134607 & 0.644678 & 0.0332739 & 13.7368 \\
\hline 0.716349 & 0.19133 & 0.70541 & 0.123093 & 0.637838 & 0.0303985 & 12.4629 \\
\hline 0.702991 & 0.170997 & 0.688001 & 0.105763 & 0.619389 & 0.0263769 & 11.0974 \\
\hline 0.695854 & 0.161212 & 0.68204 & 0.094568 & 0.62252 & 0.0239757 & 9.85888 \\
\hline 0.687179 & 0.153848 & 0.682779 & 0.0883349 & 0.610292 & 0.0240986 & 8.54868 \\
\hline 0.674016 & 0.125016 & 0.663897 & 0.076942 & 0.60255 & 0.0244727 & 7.42176 \\
\hline 0.66614 & 0.12472 & 0.664949 & 0.0751326 & 0.596611 & 0.0286461 & 6.40743 \\
\hline 0.657268 & 0.107296 & 0.661744 & 0.0723697 & 0.591498 & 0.0172249 & 5.41277 \\
\hline 0.650162 & 0.102387 & 0.652634 & 0.0636212 & 0.578307 & 0.0233367 & 4.60072 \\
\hline 0.649577 & 0.0878264 & 0.643516 & 0.0609519 & 0.582717 & 0.018595 & 3.87436 \\
\hline 0.640451 & 0.0908383 & 0.640515 & 0.0638706 & 0.569322 & 0.0255036 & 3.24846 \\
\hline 0.644707 & 0.0808857 & 0.632877 & 0.0615648 & 0.582413 & 0.0231746 & 2.75451 \\
\hline 0.636105 & 0.0769749 & 0.631078 & 0.0503339 & 0.568815 & 0.0223753 & 2.34713 \\
\hline 0.632742 & 0.0769389 & 0.626496 & 0.0551879 & 0.560817 & 0.0179258 & 1.97609 \\
\hline 0.630747 & 0.0720922 & 0.623867 & 0.0550159 & 0.559627 & 0.0180242 & 1.72792 \\
\hline 0.624549 & 0.0677836 & 0.632668 & 0.0592187 & 0.55616 & 0.0222186 & 1.51612 \\
\hline 0.619622 & 0.0716867 & 0.615777 & 0.052055 & 0.556851 & 0.0217917 & 1.35475 \\
\hline 0.622567 & 0.0656178 & 0.617149 & 0.0449052 & 0.54975 & 0.0238493 & 1.22993 \\
\hline 0.616569 & 0.0689415 & 0.602195 & 0.0517908 & 0.550632 & 0.024473 & 1.1115 \\
\hline 0.608994 & 0.066965 & 0.610354 & 0.0589274 & 0.541489 & 0.0210899 & 1.06625 \\
\hline 0.603474 & 0.0651898 & 0.596479 & 0.0509776 & 0.541647 & 0.0163035 & 0.978749 \\
\hline 0.606568 & 0.0657452 & 0.605792 & 0.0573135 & 0.540513 & 0.019801 & 0.98021 \\
\hline 0.603385 & 0.0644695 & 0.596228 & 0.0548478 & 0.54343 & 0.0170563 & 0.958205 \\
\hline 0.599805 & 0.0576817 & 0.596774 & 0.0490531 & 0.530204 & 0.0239378 & 0.936563 \\
\hline 0.595395 & 0.0623451 & 0.603644 & 0.0510916 & 0.535502 & 0.0214428 & 0.932248 \\
\hline 0.59659 & 0.0602541 & 0.588308 & 0.0542987 & 0.535178 & 0.02105 & 0.909832 \\
\hline 0.592978 & 0.0674178 & 0.594361 & 0.0587719 & 0.53717 & 0.0197314 & 0.915826 \\
\hline 0.591511 & 0.0640165 & 0.589111 & 0.0591209 & 0.52771 & 0.0208022 & 0.929077 \\
\hline 0.588636 & 0.0646262 & 0.586629 & 0.0481403 & 0.533067 & 0.0274892 & 0.943794 \\
\hline 0.58686 & 0.0630157 & 0.577892 & 0.0516572 & 0.535807 & 0.0162889 & 0.937044 \\
\hline 0.590983 & 0.0571722 & 0.583531 & 0.0546829 & 0.527536 & 0.0264317 & 0.960615 \\
\hline 0.589712 & 0.0597173 & 0.58375 & 0.0462009 & 0.535477 & 0.0263291 & 0.992792 \\
\hline 0.586592 & 0.0678093 & 0.591554 & 0.0502923 & 0.542041 & 0.0203571 & 1.01302 \\
\hline 0.590698 & 0.0639662 & 0.582961 & 0.0529576 & 0.531841 & 0.0237867 & 1.07792 \\
\hline
\end{tabular}




\begin{tabular}{|c|c|c|c|c|c|c|}
\hline 0.592683 & 0.0612317 & 0.596248 & 0.0549721 & 0.543153 & 0.0193075 & 1.10778 \\
\hline 0.590477 & 0.0680053 & 0.587777 & 0.0452224 & 0.539274 & 0.0197647 & 1.1901 \\
\hline 0.593479 & 0.0584934 & 0.593574 & 0.0509285 & 0.551105 & 0.018239 & 1.25518 \\
\hline 0.592304 & 0.0643692 & 0.600772 & 0.0571594 & 0.56053 & 0.0239728 & 1.36526 \\
\hline 0.598081 & 0.059553 & 0.596632 & 0.0559654 & 0.560607 & 0.0220581 & 1.47078 \\
\hline 0.601928 & 0.0641009 & 0.601026 & 0.0496656 & 0.569074 & 0.0225895 & 1.60108 \\
\hline 0.602184 & 0.0646847 & 0.608787 & 0.0562048 & 0.579576 & 0.0254877 & 1.7815 \\
\hline 0.609507 & 0.0669311 & 0.618276 & 0.0562014 & 0.58984 & 0.0250529 & 1.96471 \\
\hline 0.618491 & 0.0718874 & 0.617727 & 0.0614559 & 0.605095 & 0.0218077 & 2.16063 \\
\hline 0.624147 & 0.0765419 & 0.622461 & 0.0619909 & 0.609842 & 0.0200211 & 2.44111 \\
\hline 0.625542 & 0.0819821 & 0.631775 & 0.0553196 & 0.620486 & 0.0262991 & 2.70057 \\
\hline 0.633547 & 0.082684 & 0.637709 & 0.0569245 & 0.625527 & 0.0228424 & 2.99544 \\
\hline 0.641759 & 0.083028 & 0.633364 & 0.0691568 & 0.638452 & 0.0192358 & 3.32111 \\
\hline 0.65222 & 0.0890417 & 0.656142 & 0.0596698 & 0.645767 & 0.0227322 & 3.65218 \\
\hline 0.653596 & 0.0904265 & 0.648407 & 0.0681567 & 0.659877 & 0.0332556 & 4.02041 \\
\hline 0.657469 & 0.094662 & 0.655743 & 0.0745524 & 0.656985 & 0.0238707 & 4.39351 \\
\hline 0.660972 & 0.0997346 & 0.660074 & 0.0790826 & 0.665453 & 0.0271433 & 4.70981 \\
\hline 0.660312 & 0.100526 & 0.661361 & 0.0818789 & 0.671237 & 0.0274619 & 5.06449 \\
\hline 0.669126 & 0.111574 & 0.663163 & 0.0793838 & 0.672053 & 0.025753 & 5.4101 \\
\hline 0.673199 & 0.108731 & 0.652426 & 0.0834511 & 0.677183 & 0.0261565 & 5.67749 \\
\hline 0.671457 & 0.103205 & 0.661119 & 0.0777708 & 0.674386 & 0.0232831 & 5.92372 \\
\hline 0.668531 & 0.108727 & 0.65875 & 0.0799503 & 0.664594 & 0.02232 & 6.12288 \\
\hline 0.662604 & 0.113896 & 0.648183 & 0.0789853 & 0.659947 & 0.0222867 & 6.26816 \\
\hline 0.666674 & 0.110098 & 0.642011 & 0.078528 & 0.656922 & 0.0259684 & 6.32932 \\
\hline 0.65573 & 0.112374 & 0.647753 & 0.0807222 & 0.647124 & 0.0241592 & 6.35545 \\
\hline 0.651626 & 0.108083 & 0.632436 & 0.0897514 & 0.634078 & 0.0213865 & 6.31338 \\
\hline 0.649795 & 0.109583 & 0.619723 & 0.0753907 & 0.621902 & 0.0301133 & 6.14225 \\
\hline 0.642617 & 0.110172 & 0.61659 & 0.0792393 & 0.609553 & 0.0219439 & 5.9413 \\
\hline 0.632021 & 0.106577 & 0.604207 & 0.0713427 & 0.595348 & 0.022154 & 5.66514 \\
\hline 0.629588 & 0.102905 & 0.605137 & 0.0693262 & 0.596438 & 0.0214175 & 5.3918 \\
\hline 0.614654 & 0.0952755 & 0.603013 & 0.0629592 & 0.586642 & 0.021806 & 5.02486 \\
\hline 0.61262 & 0.0984407 & 0.586905 & 0.0708599 & 0.572361 & 0.0228293 & 4.69165 \\
\hline 0.598009 & 0.0824632 & 0.583625 & 0.0645817 & 0.551619 & 0.0230587 & 4.2813 \\
\hline 0.589241 & 0.0844279 & 0.568334 & 0.0524075 & 0.540237 & 0.0277448 & 3.91646 \\
\hline 0.586792 & 0.0794179 & 0.566475 & 0.0568623 & 0.539734 & 0.0184303 & 3.51161 \\
\hline 0.580226 & 0.0712346 & 0.566968 & 0.054817 & 0.527073 & 0.0252717 & 3.14936 \\
\hline 0.572911 & 0.070749 & 0.554559 & 0.046988 & 0.515377 & 0.0253253 & 2.82189 \\
\hline 0.5696 & 0.0700241 & 0.555491 & 0.0501238 & 0.510168 & 0.0231998 & 2.52594 \\
\hline 0.564208 & 0.0689214 & 0.555895 & 0.0488575 & 0.508161 & 0.017746 & 2.19163 \\
\hline 0.557713 & 0.0666264 & 0.540405 & 0.0482158 & 0.502149 & 0.0209656 & 1.9357 \\
\hline 0.559485 & 0.0628733 & 0.546107 & 0.0410864 & 0.501401 & 0.0192098 & 1.69319 \\
\hline 0.551893 & 0.0606349 & 0.54062 & 0.0464164 & 0.503664 & 0.0198472 & 1.49548 \\
\hline 0.55189 & 0.0575349 & 0.535916 & 0.0420626 & 0.491831 & 0.0176484 & 1.33914 \\
\hline 0.544848 & 0.0561976 & 0.534618 & 0.0389236 & 0.495323 & 0.0181018 & 1.19083 \\
\hline 0.54308 & 0.0576161 & 0.534459 & 0.0445016 & 0.488674 & 0.0176775 & 1.08747 \\
\hline 0.543865 & 0.0569805 & 0.529715 & 0.0501465 & 0.485496 & 0.0171217 & 0.976335 \\
\hline 0.54102 & 0.0625929 & 0.537392 & 0.0442005 & 0.484122 & 0.0205107 & 0.873897 \\
\hline
\end{tabular}




\begin{tabular}{|c|c|c|c|c|c|c|}
\hline 0.539434 & 0.0568732 & 0.529009 & 0.0453839 & 0.480207 & 0.0201236 & 0.824656 \\
\hline 534658 & 0.0531949 & 0.52544 & 0.0435471 & 0.48043 & 0.0164074 & 0.799983 \\
\hline .536803 & 0.0589563 & 0.533882 & 0.0485008 & 0.482525 & 0.020327 & 0.742294 \\
\hline .536521 & 0.0550757 & 0.530613 & 0.044285 & 0.482783 & 0.015954 & 0.724025 \\
\hline 0.535272 & 0.0556836 & 0.520676 & 0.0413471 & 0.484451 & 0.0205903 & 0.693485 \\
\hline 0.531487 & 0.0567198 & 0.526934 & 0.0401845 & 0.486831 & 0.0199457 & 0.683843 \\
\hline 0.532638 & 0.0615181 & 0.527039 & 0.0382143 & 0.483226 & 0.0227057 & 0.685132 \\
\hline 0.529727 & 0.0578876 & 0.531536 & 0.0483228 & 0.478115 & 0.0197927 & 0.669163 \\
\hline 0.534513 & 0.0602032 & 0.524828 & 0.0368536 & 0.478388 & 0.0172519 & 0.680042 \\
\hline 0.52751 & 0.0516728 & 0.531839 & 0.0424754 & 0.477432 & 0.0197527 & 0.664942 \\
\hline 0.528614 & 0.0615514 & 0.52083 & 0.0401548 & 0.475035 & 0.0204272 & 0.688814 \\
\hline 0.527405 & 0.0525312 & 0.519195 & 0.0432336 & 0.480238 & 0.0156208 & 0.670131 \\
\hline 0.528536 & 0.0609315 & 0.524907 & 0.0392914 & 0.479423 & 0.0221413 & 0.65997 \\
\hline 0.524328 & 0.0536597 & 0.520634 & 0.0462192 & 0.477552 & 0.0160699 & 0.672836 \\
\hline 0.52387 & 0.0578975 & 0.526279 & 0.0408813 & 0.480002 & 0.0212488 & 0.669566 \\
\hline 0.529498 & 0.0545294 & 0.530804 & 0.0511775 & 0.476006 & 0.0212077 & 0.684012 \\
\hline 0.521981 & 0.0499342 & 0.525998 & 0.0370907 & 0.477457 & 0.0184031 & 0.675455 \\
\hline 0.523435 & 0.050997 & 0.526204 & 0.0363077 & 0.472365 & 0.0219761 & 0.69468 \\
\hline 0.523397 & 0.0508484 & 0.515405 & 0.0392834 & 0.482979 & 0.0207571 & 0.704468 \\
\hline 0.524999 & 0.0553293 & 0.526213 & 0.035758 & 0.478818 & 0.0189188 & 0.689886 \\
\hline 0.526462 & 0.0517656 & 0.527389 & 0.0313657 & 0.485167 & 0.0178833 & 0.710064 \\
\hline 0.530225 & 0.0502455 & 0.52375 & 0753 & 0.4867 & 0.0206228 & 0.711096 \\
\hline 0.526831 & 0.0502449 & 0.527475 & 0.0412664 & 0.489769 & 0.0212398 & 0.734439 \\
\hline 0.525582 & 0.0480542 & 0.5244 & 0.047902 & 0.491853 & 0.0207333 & 0.742899 \\
\hline 0.53155 & 0.0558829 & 0.545304 & 0.0440849 & 0.503384 & 0.0209922 & 0.748141 \\
\hline 0.540956 & 0.0484457 & 0.541754 & 0.0407442 & 0.516451 & 0.0248607 & 0.772615 \\
\hline 0.542237 & 0.0521342 & 0.546754 & 0.0468355 & 0.522708 & 0.0212328 & 0.78676 \\
\hline 0.545324 & 0.0567485 & 0.563838 & 0.046337 & 0.544748 & 0.0242525 & 0.781597 \\
\hline 0.556734 & 0.0540277 & 0.578769 & 0.0473522 & 0.562022 & 0.0264694 & 0.866774 \\
\hline 0.563473 & 0.058913 & 0.594012 & 0.0426692 & 0.591712 & 0.0376976 & 0.860003 \\
\hline 0.575763 & 0.0570095 & 0.611478 & 0.0560389 & 0.620869 & 0.0302461 & 0.908624 \\
\hline 0.593398 & 0.060144 & 0.640988 & 0.05164 & 0.651031 & 0.0408739 & 0.967646 \\
\hline 0.613478 & 0.0582471 & 0.662945 & 0.0637403 & 0.695636 & 0.0394708 & 0.998702 \\
\hline 0.634389 & 0.0601333 & 0.678811 & 0.0625835 & 0.731793 & 0.0456525 & 1.05547 \\
\hline 0.660617 & 0.0701384 & 0.712819 & 0.0683656 & 0.778227 & 0.0527896 & 1.10576 \\
\hline 0.684173 & 0.073958 & 0.742222 & 0.0826584 & 0.832758 & 0.0693591 & 1.15654 \\
\hline 0.70957 & 0.0822358 & 0.773291 & 0.0873106 & 0.891669 & 0.077141 & 1.20823 \\
\hline 0.73912 & 0.0884808 & 0.815055 & 0.0955848 & 0.943429 & 0.0758064 & 1.25921 \\
\hline 0.770848 & 0.0926352 & 0.845891 & 0.0959571 & 1.00546 & 0.0907785 & 1.31178 \\
\hline 0.800235 & 0.109493 & 0.876272 & 0.107538 & 1.06921 & 0.0971614 & 1.35878 \\
\hline 0.834872 & 0.110093 & 0.917807 & 0.114419 & 1.12753 & 0.0962622 & 1.43269 \\
\hline 0.868695 & 0.128171 & 0.948869 & 0.116559 & 1.18491 & 0.114925 & 1.43442 \\
\hline 0.900844 & 0.134304 & 0.963829 & 0.130755 & 1.2321 & 0.128214 & 1.48362 \\
\hline 0.92591 & 0.142901 & 0.982133 & 0.126764 & 1.28585 & 0.13516 & 1.50973 \\
\hline 0.949545 & 0.158522 & 1.0074 & 0.12886 & 1.31245 & 0.136812 & 1.55548 \\
\hline 0.970892 & 0.153852 & 1.01818 & 0.122852 & 1.3499 & 0.130513 & 1.51722 \\
\hline 0.991141 & 0.162777 & 1.02751 & 0.139349 & 1.37036 & 0.1446 & 1.51346 \\
\hline
\end{tabular}




\begin{tabular}{|c|c|c|c|c|c|c|}
\hline 1.00062 & 0.171777 & 1.02621 & 0.14042 & 1.39989 & 0.151167 & 1.48044 \\
\hline 1.00387 & 0.168331 & 1.02436 & 0.135656 & 1.38908 & 0.138281 & 1.47822 \\
\hline 1.00621 & 0.172016 & 1.01065 & 0.134749 & 1.36943 & 0.155568 & 1.45569 \\
\hline 0.998687 & 0.162517 & 0.985875 & 0.132486 & 1.34267 & 0.135805 & 1.42903 \\
\hline 0.983295 & 0.159249 & 0.956454 & 0.100633 & 1.31143 & 0.137208 & 1.37162 \\
\hline 0.963084 & 0.157416 & 0.938573 & 0.120026 & 1.27714 & 0.138834 & 1.31633 \\
\hline 0.940319 & 0.151158 & 0.913562 & 0.104015 & 1.22756 & 0.112724 & 1.26981 \\
\hline 0.912079 & 0.150326 & 0.871157 & 0.0951285 & 1.16662 & 0.119221 & 1.18034 \\
\hline 0.892124 & 0.142609 & 0.830437 & 0.0823338 & 1.10752 & 0.105897 & 1.13848 \\
\hline 0.847103 & 0.122679 & 0.795401 & 0.0891119 & 1.04228 & 0.100368 & 1.09815 \\
\hline 0.820314 & 0.114111 & 0.756469 & 0.0782539 & 0.985139 & 0.0922952 & 1.00533 \\
\hline 0.784303 & 0.105334 & 0.725555 & 0.0764785 & 0.923947 & 0.0839511 & 0.955422 \\
\hline 0.747169 & 0.094049 & 0.680757 & 0.0601226 & 0.856598 & 0.0616258 & 0.886144 \\
\hline 0.712921 & 0.0910451 & 0.656952 & 0.0575085 & 0.790916 & 0.0614877 & 0.837097 \\
\hline 0.682763 & 0.0801482 & 0.630683 & 0.0478254 & 0.742868 & 0.0518916 & 0.802682 \\
\hline 0.649508 & 0.0724751 & 0.607303 & 0.0416708 & 0.684806 & 0.0372444 & 0.750993 \\
\hline 0.620573 & 0.068017 & 0.588788 & 0.0382569 & 0.641523 & 0.0341236 & 0.727158 \\
\hline 0.598293 & 0.058825 & 0.557099 & 0.0443416 & 0.612161 & 0.0336355 & 0.701454 \\
\hline 0.572917 & 0.0583033 & 0.552194 & 0.0414544 & 0.573761 & 0.0276664 & 0.6 \\
\hline 0.553647 & 0.0582918 & 0.532319 & 0.0347525 & 0.541807 & 0.025394 & 0.640288 \\
\hline 0.538455 & 0.0504241 & 0.513413 & 0.0364114 & 0.518881 & 0.0238533 & 0.628912 \\
\hline 0.521236 & 0.0492713 & 0.49 & 0.03 & 0.49459 & 0.0227296 & 0.617881 \\
\hline 0.50863 & 0.0479055 & 0.50123 & 0.0298411 & 0.483987 & 0.0221971 & 0.608833 \\
\hline 0.499281 & 0.0396545 & 0.490525 & 0.0276905 & 0.465255 & 0.0251402 & 0.592432 \\
\hline 0.492475 & 0.0459986 & 0.487603 & 0.0422787 & 0.453376 & 0.0237981 & 0.588683 \\
\hline 0.482146 & 0.0432001 & 0.485144 & 0.0337481 & 0.443459 & 0.0194136 & 0.572375 \\
\hline 0.48285 & 0.04189 & 0.476792 & 0.0304425 & 0.438623 & 0.0191527 & 0.571554 \\
\hline 0.472614 & 0.0418257 & 0.472174 & 0.0355851 & 0.431025 & 0.0178432 & 0.571727 \\
\hline 0.472873 & 0.0429698 & 0.459925 & 0.0281929 & 0.429924 & 0.0182529 & 0.569337 \\
\hline 0.46383 & 0.0417512 & 0.467407 & 0.0342654 & 0.427622 & 0.0178258 & 0.57234 \\
\hline 0.462131 & 0.0403191 & 0.468777 & 0.0345064 & 0.422352 & 0.0148654 & 0.569101 \\
\hline 0.464613 & 0.0432366 & 0.46577 & 0.0410903 & 0.419975 & 0.0151795 & 0.580774 \\
\hline 0.458039 & 0.0408694 & 0.45796 & 0.0372953 & 0.41822 & 0.0157281 & 0.557806 \\
\hline 0.45709 & 0.0402375 & 0.461558 & 0.0289495 & 0.414966 & 0.0199121 & 0.566791 \\
\hline 0.452737 & 0.0453727 & 0.456697 & 0.0318028 & 0.4168 & 0.0209632 & 0.569126 \\
\hline 0.458298 & 0.0379159 & 0.447552 & 0.0321349 & 0.412803 & 0.0176571 & 0.552421 \\
\hline 0.452141 & 0.0390733 & 0.461108 & 0.029552 & 0.409258 & 0.0178475 & 0.567158 \\
\hline 0.450348 & 0.040088 & 0.452107 & 0.032946 & 0.406598 & 0.0178461 & 0.556756 \\
\hline 0.448143 & 0.0405042 & 0.457895 & 0.0322302 & 0.404858 & 0.0141529 & 0.552035 \\
\hline 0.448184 & 0.0371829 & 0.45114 & 0.0318616 & 0.404667 & 0.0171891 & 0.575312 \\
\hline 0.449343 & 0.0403175 & 0.448096 & 0.0287022 & 0.404529 & 0.018679 & 0.551822 \\
\hline 0.444468 & 0.0399389 & 0.441572 & 0.0312753 & 0.401741 & 0.0224681 & 0.54929 \\
\hline 0.446561 & 0.0406305 & 0.446133 & 0.0227668 & 0.406682 & 0.0187688 & 0.556181 \\
\hline 0.443664 & 0.0368543 & 0.440352 & 0.0247691 & 0.407912 & 0.0125779 & 0.547841 \\
\hline 0.441493 & 0.0387783 & 0.447146 & 0.0319876 & 0.406576 & 0.0196698 & 0.560443 \\
\hline 0.444125 & 0.038787 & 0.453629 & 0.0347057 & 0.409913 & 0.0203471 & 0.54141 \\
\hline 0.446034 & 0.0386277 & 0.444677 & 0.0361365 & 0.40415 & 0.0184981 & 0.544383 \\
\hline
\end{tabular}




\begin{tabular}{|c|c|c|c|c|c|c|}
\hline 0.439256 & 0.0346763 & 0.44573 & 0.0335984 & 0.402906 & 0.0135638 & 0.549983 \\
\hline 0.439418 & 0.0397398 & 0.443079 & 0.0292813 & 0.4 & 0.0131973 & 0.539933 \\
\hline 0.442186 & 0.0383895 & 0.448479 & 0.0311479 & 0.406094 & 0.022215 & 0.534689 \\
\hline 0.442147 & 0.0398316 & 0.445609 & 0.0277698 & 0.40164 & 0.0197579 & 0.530854 \\
\hline 0.443759 & 0.0374421 & 0.454575 & 0.027824 & 0.40047 & 0.0160269 & 0.534535 \\
\hline 0.443707 & 0.0363641 & 0.455173 & 0.0356867 & 0.407038 & 0.0206433 & 0.518729 \\
\hline 0.445572 & 0.0356468 & 0.452974 & 0.0361686 & 0.398538 & 0.0143205 & 0.527501 \\
\hline 0.446513 & 0.0358761 & 0.450527 & 0.0331936 & 0.402223 & 0.0198894 & 0.527125 \\
\hline 0.442629 & 0.037845 & 0.453409 & 0.0358462 & 0.399002 & 0.0191991 & 0.528463 \\
\hline 0.44405 & 0.0372259 & 0.462468 & 0.0353554 & 0.403671 & 0.0209461 & 0.518777 \\
\hline 0.447743 & 0.0367591 & 0.463156 & 0.0321281 & 0.410298 & 0.0157509 & 0.533135 \\
\hline 0.44916 & 0.0376382 & 0.462059 & 0.029978 & 0.409772 & 0.0209279 & 0.528831 \\
\hline 0.446033 & 0.0377157 & 0.468595 & 0.0350907 & 0.415834 & 0.0199825 & 0.521193 \\
\hline 0.44691 & 0.038594 & 0.466147 & 0.0282314 & 0.415295 & 0.0238987 & 0.516082 \\
\hline 0.448814 & 0.0361185 & 0.4618 & 0.036094 & 0.413495 & 0.015086 & 0.510819 \\
\hline 0.447325 & 0.0360089 & 0.461151 & 0.0347651 & 0.420836 & 0.0170124 & 0.51446 \\
\hline 0.445778 & 0.0352165 & 0.461147 & 0.0355579 & 0.416495 & 0.016722 & 0.532657 \\
\hline 0.446018 & 0.0339277 & 0.460744 & 0.0322535 & 0.42427 & 0.0172214 & 0.508531 \\
\hline 0.447087 & 0.0360519 & 0.462905 & 0.03063 & 0.426901 & 0.0165026 & 0.527196 \\
\hline 0.447993 & 0.0376247 & 0.460953 & 0.0290026 & 0.426163 & 0.0176957 & 0.510385 \\
\hline 0.448411 & 0.0372974 & 0.451233 & 0.0292941 & 0.428292 & 0.0200139 & 0.527835 \\
\hline 0.447676 & 0.0382552 & 0.458857 & 0.0266351 & 0.42715 & 0.0226283 & 0.534738 \\
\hline 0.444679 & 0.0356717 & 0.447216 & 0.0308956 & 0.43179 & 0.0155308 & 0.531475 \\
\hline 0.443684 & 0.031904 & 0.445017 & 0.0297537 & 0.436677 & 0.0180693 & 0.522591 \\
\hline 0.448001 & 0.0357534 & 0.449031 & 0.0299489 & 0.431352 & 0.0179968 & 0.537332 \\
\hline 0.444882 & 0.0372949 & 0.440165 & 0.0319653 & 0.430728 & 0.0239315 & 0.52641 \\
\hline 0.444823 & 0.040879 & 0.452021 & 0.0352505 & 0.441814 & 0.0186116 & 0.527584 \\
\hline 0.443141 & 0.042262 & 0.45058 & 0.0320641 & 0.438549 & 0.0219439 & 0.535206 \\
\hline 0.440914 & 0.0371465 & 0.433055 & 0.0245267 & 0.444919 & 0.0172201 & 0.535427 \\
\hline 0.438151 & 0.0377812 & 0.43455 & 0.0259569 & 0.440354 & 0.0158394 & 0.524137 \\
\hline 0.440871 & 0.0388147 & 0.438909 & 0.0271416 & 0.4431 & 0.0201687 & 0.530618 \\
\hline 0.434921 & 0.0420688 & 0.430862 & 0.0316588 & 0.445726 & 0.0195846 & 0.526469 \\
\hline 0.438576 & 0.0414251 & 0.44081 & 0.0301192 & 0.442375 & 0.0188333 & 0.538802 \\
\hline 0.437331 & 0.0381148 & 0.429213 & 0.0298737 & 0.444257 & 0.0256246 & 0.532415 \\
\hline 0.435469 & 0.0439342 & 0.433507 & 0.0322583 & 0.442887 & 0.0172371 & 0.524664 \\
\hline 0.432138 & 0.0379136 & 0.434804 & 0.0343242 & 0.440547 & 0.0173015 & 0.532001 \\
\hline 0.432125 & 0.0386383 & 0.420173 & 0.028211 & 0.442825 & 0.0208971 & 0.530249 \\
\hline 0.42998 & 0.0400728 & 0.426084 & 0.0288286 & 0.435989 & 0.0211324 & 0.520471 \\
\hline 0.428452 & 0.0376399 & 0.416297 & 0.0204009 & 0.431914 & 0.018096 & 0.526004 \\
\hline 0.423692 & 0.0376557 & 0.414131 & 0.0353871 & 0.422874 & 0.0190701 & 0.50621 \\
\hline 0.419401 & 0.0360338 & 0.415197 & 0.0322332 & 0.420474 & 0.0182175 & 0.534534 \\
\hline 0.42109 & 0.0338802 & 0.417213 & 0.0252988 & 0.417662 & 0.0122951 & 0.526074 \\
\hline 0.417031 & 0.035633 & 0.41481 & 0.0205661 & 0.408779 & 0.0126309 & 0.517439 \\
\hline 0.417944 & 0.0381403 & 0.411228 & 0.0279494 & 0.411051 & 0.0201222 & 0.503301 \\
\hline 0.411195 & 0.0343786 & 0.403551 & 0.0332775 & 0.400942 & 0.0147351 & 0.49242 \\
\hline 0.408374 & 0.0359658 & 0.406789 & 0.0263897 & 0.391774 & 0.0160697 & 0.509823 \\
\hline 0.401694 & 0.0335558 & 0.398097 & 0.0282741 & 0.38851 & 0.0149234 & 0.484627 \\
\hline
\end{tabular}




\begin{tabular}{|c|c|c|c|c|c|c|}
\hline 0.401475 & 0.0337123 & 0.39936 & 0.0294571 & 0.384151 & 0.0193837 & 0.498425 \\
\hline 0.400069 & 0.0358333 & 0.400008 & 0.0241821 & 0.384807 & 0.0148081 & 0.495916 \\
\hline 0.395006 & 0.0350554 & 0.396981 & 0.0327569 & 0.379624 & 0.0187437 & .473071 \\
\hline 0.399485 & 0.0310641 & 0.391072 & 0.0299095 & 0.368199 & 0.0184375 & 0.484358 \\
\hline 0.38842 & 0.0310711 & 0.395391 & 0.0279784 & 0.370146 & 0.0178655 & 0.470872 \\
\hline 0.388665 & 0.0316776 & 0.388369 & 0.0191231 & 0.364004 & 0.0158059 & 0.469748 \\
\hline 0.389497 & 0.0286036 & 0.386457 & 0.0302409 & 0.363875 & 0.0142398 & 0.482686 \\
\hline 0.388637 & 0.0319692 & 0.390196 & 0.0221356 & 0.363519 & 0.020292 & 0.470264 \\
\hline 0.385268 & 0.0318821 & 0.387957 & 0.0295484 & 0.360907 & 0.0182947 & 0.478936 \\
\hline 0.384809 & 0.0297 & 0.389876 & 0.0279417 & 0.357785 & 0.0167071 & 0.474933 \\
\hline 0.380645 & 0.0312553 & 0.384699 & 0.0298785 & 0.356204 & 0.0143909 & 0.483068 \\
\hline 0.377726 & 0.0288532 & 0.383516 & 0.0260367 & 0.354213 & 0.0180597 & 0.455878 \\
\hline 0.375381 & 0.0330427 & 0.373106 & 0.0217247 & 0.351504 & 0.0156729 & 0.459289 \\
\hline 0.38131 & 0.0334375 & 0.378723 & 0.0235752 & 0.34851 & 0.0192152 & 0.463278 \\
\hline 0.371878 & 0.0318066 & 0.37175 & 0.0162644 & 0.353525 & 0.014827 & 0.459746 \\
\hline 0.371836 & 0.027471 & 0.375303 & 0.0246921 & 0.348863 & 0.0153023 & 0.4461 \\
\hline 0.37107 & 0.0318352 & 0.374814 & 0.0283775 & 0.348893 & 0.0168292 & 0.463354 \\
\hline 0.369067 & 0.028572 & 0.370103 & 0.0262242 & 0.349793 & 0.0135764 & 0.445783 \\
\hline 0.365407 & 0.0302918 & 0.366164 & 0.02 & 0.3 & 0.01 & 48113 \\
\hline 0.363814 & 0.0306469 & 0.364866 & 0.0296898 & 0.342594 & 0.0140024 & 0.451382 \\
\hline 0.362403 & 0.0285831 & 0.363019 & 0.0209717 & 0.333897 & 0.0144009 & 0.454423 \\
\hline 0.36446 & 0.0304 & 0.364666 & 044 & 0.336504 & 7038 & 5679 \\
\hline 0.36193 & 0.0292807 & 0.360095 & 0.0247132 & 0.336044 & 0.0138537 & 0.438064 \\
\hline 0.360441 & 0.0317864 & 0.364268 & 0.0260682 & 0.33645 & 0.0185271 & 0.455674 \\
\hline 0.354516 & 0.0280529 & 0.360905 & 0.0246477 & 0.331843 & 0.0147678 & 0.455143 \\
\hline 0.353518 & 0.0291776 & 0.353309 & 0.0300634 & 0.334024 & 0.0177825 & 0.450816 \\
\hline 0.355187 & 0.0280374 & 0.359639 & 0.0214211 & 0.32672 & 0.0149284 & 0.44354 \\
\hline 0.357599 & 0.0277567 & 0.355569 & 0.019179 & 0.327954 & 0.0176358 & 0.440393 \\
\hline 0.350255 & 0.0278123 & 0.354969 & 0.0188604 & 0.329143 & 0.0169686 & 0.425687 \\
\hline 0.350793 & 0.0282517 & 0.357817 & 0.0214076 & 0.324859 & 0.011571 & 0.428787 \\
\hline 0.349555 & 0.0265078 & 0.348366 & 0.0232475 & 0.327199 & 0.0135231 & 0.429706 \\
\hline 0.34921 & 0.0277458 & 0.357513 & 0.0218038 & 0.327696 & 0.0158422 & 0.437475 \\
\hline 0.347849 & 0.0239974 & 0.350646 & 0.0254279 & 0.326269 & 0.013864 & 0.438539 \\
\hline 0.350157 & 0.0279 & 0.348517 & 0.0260842 & 0.323946 & 0.0170505 & 0.433899 \\
\hline 0.346265 & 0.0268037 & 0.353033 & 0.0259264 & 0.324282 & 0.0129055 & 0.437607 \\
\hline 0.348417 & 0.0273073 & 0.354302 & 0.0266583 & 0.327375 & 0.0169762 & 0.443514 \\
\hline 0.347572 & 0.0312467 & 0.350417 & 0.0201617 & 0.322353 & 0.0154777 & 0.422961 \\
\hline 0.345508 & 0.0290026 & 0.347677 & 0.0182801 & 0.325434 & 0.018709 & 0.435805 \\
\hline 0.346703 & 0.0274389 & 0.346413 & 0.0213956 & 0.323016 & 0.0161187 & 0.431045 \\
\hline 0.345465 & 0.0271659 & 0.353314 & 0.0258247 & 0.32384 & 0.0161862 & 0.43657 \\
\hline 0.346967 & 0.0290584 & 0.350003 & 0.0203981 & 0.324543 & 0.0165774 & 0.429246 \\
\hline 0.347251 & 0.0254999 & 0.355366 & 0.0237927 & 0.328706 & 0.0156096 & 0.42496 \\
\hline 0.349208 & 0.0275332 & 0.359108 & 0.0233109 & 0.336022 & 0.0169089 & 0.441032 \\
\hline 0.347396 & 0.0292956 & 0.353636 & 0.0262258 & 0.334969 & 0.016741 & 0.439477 \\
\hline 0.350816 & 0.0277999 & 0.361711 & 0.023256 & 0.337345 & 0.0149603 & 0.432867 \\
\hline 0.35098 & 0.0271625 & 0.364187 & 0.0292382 & 0.341803 & 0.015345 & 0.440617 \\
\hline 0.355974 & 0.0277914 & 0.372445 & 0.0283031 & 0.347526 & 0.0186873 & 0.460382 \\
\hline
\end{tabular}




\begin{tabular}{|c|c|c|c|c|c|c|}
\hline 0.358986 & 0.0287695 & 0.371106 & 0.0279312 & 0.350389 & 0.0186788 & 0.460806 \\
\hline 0.369146 & 0.0278576 & 0.381592 & 0.028381 & 0.360717 & 0.0155083 & 0.470588 \\
\hline 0.375136 & 0.0286341 & 0.396778 & 0.0320446 & 0.368679 & 0.0170051 & 0.47361 \\
\hline 380469 & 0.0276221 & 0.406476 & 0.0309305 & 0.377006 & 0.0158336 & 0.481107 \\
\hline 0.386173 & 0.029775 & 0.405125 & 0.0329599 & 0.398271 & 0.0162245 & 0.50555 \\
\hline 0.391899 & 0.0310076 & 0.41799 & 0.0365811 & 0.407015 & 0.0170719 & 0.509076 \\
\hline 0.406601 & 0.0354212 & 0.424168 & 0.04066 & 0.421487 & 0.0157775 & 0.513595 \\
\hline 0.413971 & 0.0353219 & 0.445936 & 0.0431036 & 0.44217 & 0.0198121 & 0.544598 \\
\hline 0.424543 & 0.0420321 & 0.457763 & 0.0484866 & 0.459342 & 0.0261641 & 0.557364 \\
\hline 0.435385 & 0.0418606 & 0.468915 & 0.048262 & 0.472039 & 0.0189555 & 0.559018 \\
\hline 0.453747 & 0.0494724 & 0.479725 & 0.0518964 & 0.492576 & 0.0189174 & 0.593498 \\
\hline 0.464705 & 0.0491427 & 0.494206 & 0.0631042 & 0.517385 & 0.0223507 & 0.607107 \\
\hline 0.476586 & 0.0535808 & 0.514799 & 0.0580688 & 0.532266 & 0.0151618 & 0.611955 \\
\hline 0.491543 & 0.0594796 & 0.526565 & 0.0629657 & 0.553971 & 0.0222865 & 0.635754 \\
\hline 0.511806 & 0.0617126 & 0.540793 & 0.0758684 & 0.574643 & 0.0214618 & 0.6613 \\
\hline 0.521856 & 0.0658591 & 0.554153 & 0.0665572 & 0.5917 & 0.0237729 & 0.672975 \\
\hline 0.534537 & 0.0703352 & 0.563491 & 0.0778713 & 0.613373 & 0.0285312 & 0.701737 \\
\hline 0.544831 & 0.0762353 & 0.576489 & 0.0681481 & 0.631762 & 0.0318014 & 0.703903 \\
\hline 0.555765 & 0.0762026 & 0.588484 & 0.0701704 & 0.641743 & 0.0259183 & 0.706512 \\
\hline 0.567474 & 0.0777174 & 0.589085 & 0.0683531 & 0.656873 & 0.0313818 & 0.724015 \\
\hline 0.577443 & 0.0817859 & 0.588654 & 0.0671515 & 0.675727 & 0.0327963 & 0.723274 \\
\hline 0.586264 & 0.0809115 & 0.586271 & 0.0760226 & 0.67239 & 0.026418 & 0.726437 \\
\hline 0.58761 & 0.0794569 & 0.58316 & 0.0690281 & 0.684147 & 0.0319127 & 0.713357 \\
\hline 0.585656 & 0.0809705 & 0.590378 & 0.0717178 & 0.689146 & 0.0360915 & 0.725761 \\
\hline 0.58912 & 0.0809518 & 0.581359 & 0.077765 & 0.679632 & 0.0295235 & 0.698522 \\
\hline 0.586983 & 0.0827331 & 0.573979 & 0.0608476 & 0.673907 & 0.0236784 & 0.727899 \\
\hline 0.583656 & 0.0755819 & 0.565541 & 0.0702577 & 0.663436 & 0.0301339 & 0.702997 \\
\hline 0.568777 & 0.0738286 & 0.548886 & 0.0577082 & 0.652603 & 0.0362848 & 0.707964 \\
\hline 0.563306 & 0.0712285 & 0.537688 & 0.0533707 & 0.63547 & 0.0258986 & 0.671476 \\
\hline 0.549657 & 0.0676246 & 0.522067 & 0.0471196 & 0.615043 & 0.0280215 & 0.654165 \\
\hline 0.537543 & 0.056655 & 0.500567 & 0.0460074 & 0.603712 & 0.0296525 & 0.624571 \\
\hline 0.522302 & 0.0575515 & 0.494213 & 0.0412052 & 0.579377 & 0.0272789 & 0.620182 \\
\hline 0.507777 & 0.0566867 & 0.473201 & 0.0422399 & 0.557557 & 0.0312242 & 0.594417 \\
\hline 0.489715 & 0.0533996 & 0.457416 & 0.0314412 & 0.540994 & 0.0258737 & 0.569904 \\
\hline 0.471478 & 0.0501328 & 0.443713 & 0.0365546 & 0.514235 & 0.0273159 & 0.555993 \\
\hline 0.457149 & 0.0444854 & 0.425245 & 0.0299615 & 0.482806 & 0.0193363 & 0.531561 \\
\hline 0.439923 & 0.0430616 & 0.409666 & 0.0355294 & 0.460869 & 0.0205565 & 0.505132 \\
\hline 0.424962 & 0.0394851 & 0.399808 & 0.0295306 & 0.447807 & 0.0238067 & 0.492208 \\
\hline 0.406392 & 0.0355145 & 0.382475 & 0.0234908 & 0.419298 & 0.020143 & 0.470888 \\
\hline 0.393275 & 0.0313148 & 0.371953 & 0.0152138 & 0.399249 & 0.0172037 & 0.462291 \\
\hline 0.381502 & 0.0309591 & 0.361065 & 0.0246954 & 0.391015 & 0.0215668 & 0.440067 \\
\hline 0.371555 & 0.0285956 & 0.352008 & 0.0252994 & 0.371178 & 0.0187227 & 0.430989 \\
\hline 0.361755 & 0.0283096 & 0.34189 & 0.0189318 & 0.353336 & 0.0228171 & 0.424402 \\
\hline 0.355181 & 0.0286522 & 0.34211 & 0.0303332 & 0.350046 & 0.0143282 & 0.41805 \\
\hline 0.342257 & 0.0286755 & 0.334003 & 0.0195048 & 0.335526 & 0.0170213 & 0.402572 \\
\hline 0.338714 & 0.0233063 & 0.32845 & 0.0251455 & 0.330156 & 0.0175537 & 0.407153 \\
\hline 0.329227 & 0.028924 & 0.321969 & 0.019798 & 0.315474 & 0.0154344 & 0.409577 \\
\hline
\end{tabular}




\begin{tabular}{|c|c|c|c|c|c|c|}
\hline 0.328207 & 0.0268143 & 0.323996 & 0.0252299 & 0.316726 & 0.0168537 & 0.406828 \\
\hline 0.324393 & 0.0257523 & 0.316517 & 0.023792 & 0.311022 & 0.0168535 & 0.387461 \\
\hline 0.315901 & 0.0268508 & 0.315926 & 0.025141 & 0.309344 & 0.0210127 & 0.390184 \\
\hline 0.315175 & 0.0241217 & 0.316907 & 0.0195148 & 0.304958 & 0.0104925 & 0.388948 \\
\hline 0.313489 & 0.0231732 & 0.310027 & 0.0230787 & 0.303111 & 0.0113705 & 0.383744 \\
\hline 0.311286 & 0.0261465 & 0.312031 & 0.0243748 & 0.302774 & 0.0129783 & 0.3908 \\
\hline 0.313456 & 0.0254801 & 0.304384 & 0.0175278 & 0.295838 & 0.0147579 & 0.391398 \\
\hline 0.309595 & 0.0259047 & 0.313116 & 0.0224096 & 0.297754 & 0.0159275 & 0.391301 \\
\hline 0.307199 & 0.0259803 & 0.310749 & 0.0222453 & 0.296615 & 0.0182349 & 0.376526 \\
\hline 0.307574 & 0.0258205 & 0.311588 & 0.02109 & 0.290512 & 0.0163451 & 0.382768 \\
\hline 0.308207 & 0.0252783 & 0.300284 & 0.0205372 & 0.292673 & 0.0120241 & 0.375931 \\
\hline 0.303097 & 0.0252834 & 0.303458 & 0.0238919 & 0.289224 & 0.0145434 & 0.367125 \\
\hline 0.303892 & 0.0269113 & 0.298525 & 0.0225769 & 0.288329 & 0.0171424 & 0.370066 \\
\hline 0.298995 & 0.0240548 & 0.296751 & 0.0222386 & 0.282395 & 0.0142138 & 0.370324 \\
\hline 0.30351 & 0.0230952 & 0.299338 & 0.0200882 & 0.282617 & 0.016611 & 0.366802 \\
\hline 0.300423 & 0.0257255 & 0.302361 & 0.0204555 & 0.283508 & 0.0173629 & 0.363711 \\
\hline 0.299076 & 0.0229595 & 0.30109 & 0.0184389 & 0.285317 & 0.0177184 & 0.365954 \\
\hline 0.296749 & 0.0250719 & 0.296815 & 0.0206117 & 0.273842 & 0.0138342 & 0.37024 \\
\hline 0.297308 & 0.0232252 & 0.292637 & 0.0173581 & 0.274783 & 0.013893 & 0.364688 \\
\hline 0.295097 & 0.0222812 & 0.296769 & 0.0223972 & 0.277553 & 0.0155967 & 0.373243 \\
\hline 0.2923 & 0.024874 & 0.289373 & 0.0186873 & 0.276121 & 0.0155281 & 0.357431 \\
\hline 0.289166 & 0.0245271 & 0.292838 & 0.018996 & 0.267467 & 0.0131609 & 0.368785 \\
\hline 0.290942 & 0.023155 & 0.293197 & 0.0224255 & 0.265498 & 0.0135769 & 0.359529 \\
\hline 0.288731 & 0.023577 & 0.29208 & 0.0194603 & 0.265898 & 0.0141002 & 0.358981 \\
\hline 0.286412 & 0.0229862 & 0.29044 & 0.0166819 & 0.268474 & 0.0141549 & 0.344414 \\
\hline 0.287516 & 0.0213225 & 0.288178 & 0.0188223 & 0.268206 & 0.0165416 & 0.355978 \\
\hline 0.284331 & 0.0239364 & 0.290739 & 0.0184721 & 0.26431 & 0.0148252 & 0.357948 \\
\hline 0.283557 & 0.0232409 & 0.283141 & 0.0232214 & 0.263489 & 0.0110044 & 0.353782 \\
\hline 0.282989 & 0.0211928 & 0.28579 & 0.0194251 & 0.265134 & 0.0169254 & 0.355003 \\
\hline 0.284751 & 0.0255233 & 0.280449 & 0.0177733 & 0.258599 & 0.0136566 & 0.353974 \\
\hline 0.279692 & 0.0227718 & 0.280293 & 0.0210475 & 0.255546 & 0.0153423 & 0.351172 \\
\hline 0.27815 & 0.0221017 & 0.279489 & 0.0203061 & 0.257566 & 0.0116739 & 0.358061 \\
\hline 0.278423 & 0.0207999 & 0.286867 & 0.0199123 & 0.258793 & 0.01149 & 0.357117 \\
\hline 0.27994 & 0.0226689 & 0.28008 & 0.0183879 & 0.260148 & 0.0121742 & 0.353041 \\
\hline 0.280626 & 0.0253013 & 0.277779 & 0.018558 & 0.25552 & 0.0111247 & 0.353493 \\
\hline 0.277566 & 0.0198453 & 0.281663 & 0.0215032 & 0.259922 & 0.0150564 & 0.360668 \\
\hline 0.281966 & 0.0236646 & 0.280999 & 0.024556 & 0.260046 & 0.013033 & 0.345433 \\
\hline 0.274559 & 0.020775 & 0.277772 & 0.0209642 & 0.255709 & 0.0122067 & 0.346094 \\
\hline 0.278442 & 0.0223581 & 0.282026 & 0.0153962 & 0.258439 & 0.010954 & 0.334144 \\
\hline 0.273416 & 0.0208756 & 0.283658 & 0.0242186 & 0.256848 & 0.00922117 & 0.353049 \\
\hline 0.276851 & 0.0207978 & 0.279639 & 0.0177952 & 0.259559 & 0.0158335 & 0.34509 \\
\hline 0.274383 & 0.018626 & 0.275144 & 0.0229916 & 0.26185 & 0.0157154 & 0.352896 \\
\hline 0.275114 & 0.0211584 & 0.275533 & 0.0148286 & 0.255736 & 0.0124974 & 0.361903 \\
\hline 0.275897 & 0.0244391 & 0.275803 & 0.02021 & 0.256361 & 0.0142218 & 0.35531 \\
\hline 0.276158 & 0.0212103 & 0.276887 & 0.0214737 & 0.256012 & 0.0152786 & 0.350449 \\
\hline 0.273623 & 0.0209088 & 0.272893 & 0.0187178 & 0.260718 & 0.013051 & 0.344431 \\
\hline 0.271224 & 0.0223356 & 0.280602 & 0.0179683 & 0.260765 & 0.0117901 & 0.343951 \\
\hline
\end{tabular}




\begin{tabular}{|c|c|c|c|c|c|c|}
\hline 0.27233 & 0.0210216 & 0.27412 & 0.0209203 & 0.257982 & 0.00934941 & 0.331298 \\
\hline 0.271748 & 0.0194464 & 0.271916 & 0.0162068 & 0.258131 & 0.0127139 & 0.338477 \\
\hline 0.272403 & 0.024004 & 0.277775 & 0.0215939 & 0.255906 & 0.0144028 & .346172 \\
\hline 0.272544 & 0.0195157 & 0.276407 & 0.0205147 & 0.261936 & 0.0170737 & .350509 \\
\hline 0.269835 & 0.020706 & 0.277048 & 0.0122776 & 0.263135 & 0.0103681 & 0.3455 \\
\hline 0.271481 & 0.0222822 & 0.271532 & 0.022029 & 0.261213 & 0.0139539 & 347309 \\
\hline 0.270782 & 0.0207057 & 0.275464 & 0.0156341 & 0.255851 & 0.0109707 & 0.336806 \\
\hline 0.270452 & 0.0230775 & 0.272351 & 0.0195711 & 0.260729 & 0.0136486 & 0.344535 \\
\hline 0.269078 & 0.0179427 & 0.27476 & 0.021697 & 0.259164 & 0.0139141 & 0.339272 \\
\hline 0.270142 & 0.0214056 & 0.269812 & 0.0193894 & 0.260187 & 0.0145563 & 0.340807 \\
\hline 0.272882 & 0.0205479 & 0.271753 & 0.0205855 & 0.261323 & 0.0123258 & 0.341503 \\
\hline 0.270145 & 0.0238305 & 0.269683 & 0.0212365 & 0.260684 & 0.0108759 & 0.336682 \\
\hline 0.270476 & 0.019679 & 0.265316 & 0.0188411 & 0.261477 & 0.0175706 & 0.330529 \\
\hline 0.270793 & 0.0213706 & 0.273912 & 0.0199248 & 0.258304 & 0.0148587 & 0.35035 \\
\hline 0.269583 & 0.0174348 & 0.27077 & 0.0159474 & 0.257504 & 0.013459 & 0.333739 \\
\hline 0.267251 & 0.0206949 & 0.278392 & 0.0162054 & 0.259649 & 0.017781 & 0.337163 \\
\hline 0.269835 & 0.0190401 & 0.27059 & 0.0173784 & 0.259099 & 0.0109478 & 0.346297 \\
\hline 0.267768 & 0.0217936 & 0.268835 & 0.0189252 & 0.260861 & 0.0170058 & 0.326312 \\
\hline 0.268459 & 0.02 & 0.27 & 226 & 0.255313 & 512 & 0.345453 \\
\hline 0.26701 & 0.0206291 & 0.261665 & 0.015721 & 0.251063 & 0.0169964 & 0.34336 \\
\hline 0.266304 & 0.0204485 & 0.261776 & 0.0168088 & 0.25469 & 0.0114121 & 0.337609 \\
\hline 0.26 & 0.01 & 6068 & 0.0 & 0.260021 & 912 & 0.338492 \\
\hline 0.263384 & 0.0240523 & 0.260388 & 0.0141556 & 0.249275 & 0.0120857 & 0.33932 \\
\hline 0.265551 & 0.0222953 & 0.26793 & 0.0224945 & 0.254666 & 0.01 & .345342 \\
\hline 0.263288 & 0.0200868 & 0.261265 & 0.0265667 & 0.252723 & 0.0157176 & 0.336355 \\
\hline 0.263719 & 0.0212301 & 0.257024 & 0.0204219 & 0.251131 & 0.0117838 & 0.33458 \\
\hline 0.263091 & 0.0215113 & 0.265298 & 0.0155137 & 0.24986 & 298 & 0.334462 \\
\hline 0.261512 & 0.0179379 & 0.262517 & 0.0213234 & 0.245047 & 0.0135779 & 0.328406 \\
\hline 0.26107 & 0.020931 & 0.261576 & 0.0195948 & 0.244202 & 0.0161001 & 0.338948 \\
\hline 0.259995 & 0.0161011 & 0.265807 & 0.023043 & 0.24406 & 0.0139055 & 0.325044 \\
\hline 0.257515 & 0.0216574 & 0.266043 & 0.0183182 & 0.247227 & 0.0147327 & 0.330919 \\
\hline 0.259625 & 0.0222267 & 0.26003 & 0.0197557 & 0.24266 & 0.0135949 & 0.330978 \\
\hline 0.258292 & 0.0214667 & 0.251723 & 0.0175915 & 0.24453 & 0.0139724 & 0.335887 \\
\hline 0.257895 & 0.0199427 & 0.259618 & 0.0207703 & 0.24406 & 0.0134211 & 0.337256 \\
\hline 0.255663 & 0.0196079 & 0.256709 & 0.0185247 & 0.242874 & 0.0177739 & 0.335113 \\
\hline 0.259214 & 0.0196657 & 0.251136 & 0.0159116 & 0.241289 & 0.0109185 & 0.327719 \\
\hline 0.253874 & 0.0184075 & 0.259195 & 0.0183691 & 0.235618 & 0.0149047 & 0.331958 \\
\hline 0.256245 & 0.0220393 & 0.254895 & 0.0173708 & 0.239193 & 0.0161947 & 0.335915 \\
\hline 0.255998 & 0.0246763 & 0.257002 & 0.0136024 & 0.241215 & 0.00991097 & 0.330656 \\
\hline 0.251528 & 0.0208915 & 0.259581 & 0.0184446 & 0.242797 & 0.00986263 & 0.334659 \\
\hline 0.254536 & 0.0211256 & 0.256159 & 0.0163115 & 0.23685 & 0.014024 & 0.323421 \\
\hline 0.250648 & 0.0193535 & 0.249937 & 0.0185203 & 0.239852 & 0.0116542 & 0.325123 \\
\hline 0.253006 & 0.0194885 & 0.252428 & 0.0191627 & 0.237274 & 0.0156142 & 0.309492 \\
\hline 0.25205 & 0.0194539 & 0.249043 & 0.018624 & 0.233996 & 0.0129199 & 0.321638 \\
\hline 0.250688 & 0.0187332 & 0.249677 & 0.018005 & 0.233935 & 0.0160161 & 0.326289 \\
\hline 0.248529 & 0.0212056 & 0.252301 & 0.0171399 & 0.229808 & 0.0118027 & 0.319769 \\
\hline 0.250306 & 0.0182643 & 0.24949 & 0.018858 & 0.23256 & 0.0108665 & 0.305782 \\
\hline
\end{tabular}




\begin{tabular}{|c|c|c|c|c|c|c|}
\hline 0.251463 & 0.0182002 & 0.253578 & 0.02015 & 0.232992 & 0.0119223 & 0.313841 \\
\hline 0.248709 & 0.0207648 & 0.254303 & 0.0184494 & 0.237569 & 0.0125443 & 0.302673 \\
\hline .246496 & 0.0227633 & 0.251872 & 0.0175173 & 0.23309 & 0.0122934 & .311881 \\
\hline .249053 & 0.0206274 & 0.250921 & 0.0129434 & 0.230535 & 0.0165905 & 0.309531 \\
\hline 0.249388 & 0.0198743 & 0.256284 & 0.0200453 & 0.227447 & 0.0114636 & 0.318276 \\
\hline 0.246153 & 0.0202295 & 0.25093 & 0.0167111 & 0.230494 & 0.0135953 & 0.309221 \\
\hline 0.247263 & 0.0161742 & 0.249256 & 0.014967 & 0.232009 & 0.0105185 & 0.31135 \\
\hline 0.248388 & 0.0185832 & 0.249104 & 0.0189622 & 0.231216 & 0.0144359 & 0.320389 \\
\hline 0.248886 & 0.0192135 & 0.248567 & 0.0196055 & 0.227687 & 0.0130605 & 0.316859 \\
\hline 0.243966 & 0.0201 & 0.24928 & 0.0152275 & 0.22827 & 0.0163027 & 0.304095 \\
\hline 0.245984 & 0.0198557 & 0.244103 & 0.0212808 & 0.230039 & 0.0143527 & 0.316771 \\
\hline 0.246619 & 0.0203059 & 0.249729 & 0.0148449 & 0.226066 & 0.0130365 & 0.305995 \\
\hline 0.243885 & 0.0203236 & 0.249222 & 0.0162527 & 0.225548 & 0.0143107 & 0.316063 \\
\hline 0.244142 & 0.0191914 & 0.239958 & 0.018313 & 0.228722 & 0.00963702 & 0.309687 \\
\hline 0.242839 & 0.0194459 & 0.242053 & 0.0174349 & 0.230675 & 0.0145003 & 0.315254 \\
\hline 0.2441 & 0.0204005 & 0.245141 & 0.0218096 & 0.228096 & 0.0149033 & 0.306365 \\
\hline 0.240792 & 0.0215489 & 0.24736 & 0.0181951 & 0.225006 & 0.0113689 & 0.31909 \\
\hline 0.243978 & 0.0183609 & 0.240479 & 0.0160237 & 0.223869 & 0.0124561 & 0.310816 \\
\hline 0.242092 & 896 & 0.239783 & 0.0150053 & 0.221842 & 0.01 & 0.31096 \\
\hline 0.240548 & 0.0190705 & 0.239509 & 0.0157455 & 0.220865 & 0.010363 & 0.307723 \\
\hline 0.244732 & 0.0167889 & 0.242724 & 0.0145197 & 0.228192 & 0.0129917 & 0.323991 \\
\hline 0.239913 & 0.02 & 0.246638 & 0.017768 & 0.220105 & 0.0117048 & 0.312889 \\
\hline 0.24167 & 0.0188752 & 0.23946 & 0.0170648 & 0.226677 & 0.0085934 & 0.313757 \\
\hline 0.241576 & 0.0193533 & 0.241325 & 0.0183522 & 0.223923 & 0.013 & 958 \\
\hline 0.24233 & 0.0196278 & 0.239015 & 0.0162932 & 0.222874 & 0.0140699 & 0.320946 \\
\hline 0.241414 & 0.0170615 & 0.240801 & 0.0139794 & 0.223047 & 0.0128332 & 0.318251 \\
\hline 0.242051 & 0.01 & 0.24 & 0.0191691 & 0.227514 & 0.0111752 & 0.311489 \\
\hline 0.238251 & 0.0179771 & 0.241441 & 0.0167696 & 0.222959 & 0.0137755 & 0.318998 \\
\hline 0.242766 & 0.0195603 & 0.245962 & 0.0166851 & 0.229751 & 0.0128231 & 0.309029 \\
\hline 0.23877 & 0.0168384 & 0.240097 & 0.0165497 & 0.226562 & 0.0176553 & 0.313237 \\
\hline 0.236963 & 0.0190499 & 0.246465 & 0.0170518 & 0.228166 & 0.0127141 & 0.316989 \\
\hline 0.240669 & 0.0226951 & 0.243331 & 0.0190475 & 0.226343 & 0.0126649 & 0.309623 \\
\hline 0.241753 & 0.0201936 & 0.238283 & 0.0163493 & 0.225768 & 0.0106195 & 0.325161 \\
\hline 0.241546 & 0.019194 & 0.244287 & 0.0173442 & 0.229309 & 0.0104908 & 0.319047 \\
\hline 0.2399 & 0.0191493 & 0.243007 & 0.016279 & 0.224167 & 0.0134748 & 0.306999 \\
\hline 0.242895 & 0.0209726 & 0.247024 & 0.0166146 & 0.229621 & 0.0131704 & 0.323818 \\
\hline 0.242089 & 0.0226664 & 0.246012 & 0.0152845 & 0.233942 & 0.0154876 & 0.312928 \\
\hline 0.241252 & 0.0240997 & 0.252852 & 0.0215979 & 0.234685 & 0.0126689 & 0.324029 \\
\hline 0.242222 & 0.0213705 & 0.250318 & 0.0175329 & 0.231926 & 0.012808 & 0.323517 \\
\hline 0.247175 & 0.0224903 & 0.25203 & 0.0161387 & 0.236712 & 0.0114929 & 0.329103 \\
\hline 0.245804 & 0.0227961 & 0.250576 & 0.0147274 & 0.235166 & 0.0158787 & 0.324428 \\
\hline 0.244314 & 0.0223154 & 0.254492 & 0.0196384 & 0.239352 & 0.0137516 & 0.336575 \\
\hline 0.246509 & 0.0268868 & 0.25379 & 0.0175235 & 0.237318 & 0.014859 & 0.333048 \\
\hline 0.247693 & 0.0251447 & 0.255917 & 0.0205671 & 0.241722 & 0.0154185 & 0.334611 \\
\hline 0.247657 & 0.0276929 & 0.254831 & 0.0215646 & 0.240685 & 0.0140956 & 0.350017 \\
\hline 0.244981 & 0.0270855 & 0.249607 & 0.0143519 & 0.241218 & 0.0148036 & 0.335902 \\
\hline 0.251571 & 0.0234811 & 0.256378 & 0.0187581 & 0.242351 & 0.0106048 & 0.325902 \\
\hline
\end{tabular}




\begin{tabular}{|c|c|c|c|c|c|c|}
\hline 0.250008 & 0.0259477 & 0.255064 & 0.0199358 & 0.241341 & 0.0129963 & 0.326483 \\
\hline 0.249892 & 0.0231455 & 0.253806 & 0.0248881 & 0.241948 & 0.0130385 & 0.330562 \\
\hline 0.252571 & 0.0271395 & 0.258383 & 0.0172118 & 0.242545 & 0.014866 & 0.336719 \\
\hline 0.249921 & 0.0238468 & 0.252601 & 0.0164026 & 0.248246 & 0.0101076 & 0.334478 \\
\hline 0.250331 & 0.0230793 & 0.253388 & 0.0131723 & 0.246107 & 0.0131547 & 0.338109 \\
\hline 0.252099 & 0.0255735 & 0.256048 & 0.0193946 & 0.250777 & 0.0128171 & 0.319648 \\
\hline 0.252742 & 0.0231047 & 0.258311 & 0.0141232 & 0.247431 & 0.0131083 & 0.34208 \\
\hline 0.255258 & 0.0268187 & 0.260147 & 0.0201097 & 0.253413 & 0.0105149 & 0.333944 \\
\hline 0.256351 & 0.0241326 & 0.263836 & 0.0191044 & 0.250032 & 0.0135433 & 0.326671 \\
\hline 0.255943 & 0.0236602 & 0.259487 & 0.0158847 & 0.252223 & 0.0129857 & 0.332019 \\
\hline 0.25743 & 0.0245884 & 0.260871 & 0.0186991 & 0.252298 & 0.0143453 & 0.343343 \\
\hline 0.257249 & 0.0188523 & 0.262819 & 0.0202098 & 0.249627 & 0.0137455 & 0.329618 \\
\hline 0.255477 & 0.0198979 & 0.270161 & 0.0183526 & 0.25412 & 0.0140129 & 0.341155 \\
\hline 0.258344 & 0.0222658 & 0.260238 & 0.0192801 & 0.257667 & 0.016199 & 0.336944 \\
\hline 0.261828 & 0.0220842 & 0.268572 & 0.0220231 & 0.25681 & 0.0158564 & 0.339102 \\
\hline 0.26102 & 0.0225969 & 0.266673 & 0.0191169 & 0.261477 & 0.0124781 & 0.34365 \\
\hline 0.260155 & 0.0207476 & 0.267656 & 0.0208556 & 0.257788 & 0.014514 & 0.3456 \\
\hline 0.266038 & 0.0221664 & 0.265785 & 0.0213336 & 0.261365 & 0.0158476 & 0.346393 \\
\hline 0.267019 & 0.0202618 & 0.266245 & 0.0210613 & 0.268302 & 0.0147805 & 0.341154 \\
\hline 0.267779 & 0.0209159 & 0.274791 & 0.0252935 & 0.263527 & 0.018795 & 0.334057 \\
\hline 0.267781 & 0.0214809 & 0.271457 & 0.0202732 & 0.268973 & 0.0157109 & 0.354107 \\
\hline 0.26875 & 0.0235524 & 0.275352 & 0.0191334 & 0.266185 & 0.0132741 & 0.336843 \\
\hline 0.270464 & 0.0223235 & 0.26787 & 0.0245271 & 0.26971 & 0.0147169 & 0.35018 \\
\hline 0.271061 & 0.0223944 & 0.267733 & 0.0196004 & 0.266688 & 0.0146543 & 0.344728 \\
\hline 0.271638 & 0.0236215 & 0.268184 & 0.0234804 & 0.267068 & 0.0120918 & 0.351041 \\
\hline 0.269486 & 0.0227742 & 0.26969 & 0.017459 & 0.265482 & 0.0125264 & 0.340079 \\
\hline 0.27286 & 0.0259272 & 0.272632 & 0.0183165 & 0.260571 & 0.0130128 & 0.331753 \\
\hline 0.272538 & 0.0209632 & 0.268195 & 0.0205682 & 0.26332 & 0.0141657 & 0.337056 \\
\hline 0.269521 & 0.022658 & 0.26556 & 0.0218682 & 0.262707 & 0.0159349 & 0.330528 \\
\hline 0.265391 & 0.0218144 & 0.267707 & 0.0214511 & 0.258521 & 0.0112296 & 0.327129 \\
\hline 0.267954 & 0.0224864 & 0.262487 & 0.018865 & 0.256265 & 0.0150678 & 0.316782 \\
\hline 0.266939 & 0.0226194 & 0.259656 & 0.0222046 & 0.254925 & 0.0134471 & 0.333422 \\
\hline 0.268981 & 0.017304 & 0.259591 & 0.0166557 & 0.252805 & 0.0116476 & 0.328058 \\
\hline 0.262768 & 0.0193443 & 0.257709 & 0.0265198 & 0.25351 & 0.0135655 & 0.319768 \\
\hline 0.259154 & 0.0229892 & 0.257516 & 0.0196323 & 0.246149 & 0.0113043 & 0.327053 \\
\hline 0.260949 & 0.0222363 & 0.255739 & 0.0195419 & 0.251416 & 0.0133039 & 0.316702 \\
\hline 0.25776 & 0.0205417 & 0.257054 & 0.0167733 & 0.247845 & 0.012272 & 0.311872 \\
\hline 0.254859 & 0.021891 & 0.255497 & 0.0199886 & 0.239172 & 0.0115766 & 0.298045 \\
\hline 0.253505 & 0.0203552 & 0.255124 & 0.0195213 & 0.241168 & 0.0106515 & 0.309436 \\
\hline 0.254621 & 0.0194292 & 0.254615 & 0.0173472 & 0.240706 & 0.0126877 & 0.307795 \\
\hline 0.25249 & 0.0216788 & 0.251867 & 0.0225492 & 0.243037 & 0.0104659 & 0.314546 \\
\hline 0.252046 & 0.0193311 & 0.25302 & 0.0170564 & 0.238423 & 0.0141793 & 0.303471 \\
\hline 0.252147 & 0.018876 & 0.247917 & 0.0168012 & 0.233707 & 0.0164485 & 0.308355 \\
\hline 0.248933 & 0.0185569 & 0.250714 & 0.0199517 & 0.236393 & 0.0139696 & 0.303482 \\
\hline 0.253845 & 0.0159292 & 0.249856 & 0.0166009 & 0.233377 & 0.0136561 & 0.292654 \\
\hline 0.251139 & 0.0161398 & 0.252335 & 0.0123594 & 0.237451 & 0.0116811 & 0.311322 \\
\hline 0.249853 & 0.0179097 & 0.2513 & 0.0165841 & 0.237987 & 0.0126542 & 0.307467 \\
\hline
\end{tabular}




\begin{tabular}{|c|c|c|c|c|c|c|}
\hline 0.249025 & 0.0164395 & 0.249536 & 0.0167244 & 0.235949 & 0.0145444 & 9223 \\
\hline .252548 & 0.0198259 & 0.254759 & 0.0205105 & 0.237248 & 0.012908 & 0.309331 \\
\hline .252831 & 0.018713 & 0.257525 & 0.0154323 & 0.236615 & 0.00991837 & .298056 \\
\hline .255291 & 0.0195176 & 0.256507 & 0.020142 & 0.241656 & 0.0139047 & 0.291896 \\
\hline .252353 & 0.0165294 & 0.257268 & 0.0178659 & 0.239308 & 0.0163823 & 0.303195 \\
\hline 0.251937 & 0.0191557 & 0.255553 & 0.0180463 & 0.244697 & 0.0146415 & 0.310598 \\
\hline 0.251873 & 0.0207293 & 0.256795 & 0.0177784 & 0.240976 & 0.0134199 & 0.300678 \\
\hline 0.252263 & 0.0208261 & 0.25925 & 0.0189469 & 0.247129 & 0.0129592 & 0.311644 \\
\hline 0.258079 & 0.0166328 & 0.258986 & 0.0219466 & 0.245406 & 0.0119765 & 0.305946 \\
\hline 0.254085 & 0.0185682 & 0.256075 & 0.0148886 & 0.247387 & 0.0175978 & 0.303451 \\
\hline 0.253126 & 0.0185076 & 0.256075 & 0.0202035 & 0.242464 & 0.0150191 & 0.309253 \\
\hline 0.254903 & 0.0225748 & 0.258635 & 0.0192246 & 0.245912 & 0.0149196 & 0.307295 \\
\hline 0.256273 & 0.017863 & 0.256115 & 0.0153183 & 0.246749 & 0.0142435 & 0.313538 \\
\hline 0.258393 & 0.0190977 & 0.260923 & 0.0188947 & 0.247973 & 0.010754 & 0.318148 \\
\hline 0.261111 & 0.018439 & 0.265182 & 0.0205123 & 0.246762 & 0.0115915 & 0.31732 \\
\hline 0.257666 & 0.0196063 & 0.260926 & 0.0221442 & 0.247753 & 0.0151198 & 0.312263 \\
\hline 0.264595 & 0.0217965 & 0.261877 & 0.011037 & 0.251967 & 0.011825 & 0.312608 \\
\hline 0.260472 & 0.0244301 & 0.260859 & 0.0208183 & 0.253035 & 0.012847 & 0.303665 \\
\hline 0.26167 & 0.02 & 0.25995 & 0.01 & 773 & 0.0157579 & 30616 \\
\hline 0.264825 & 0.0204195 & 0.262575 & 0.0191238 & 0.251524 & 0.0105994 & 0.303651 \\
\hline 0.263051 & 0.0200883 & 0.266499 & 0.0168837 & 0.257151 & 0.0158074 & 0.309745 \\
\hline 0.264069 & 0.02 & 0.266911 & 426 & 49 & 0.01 & .31594 \\
\hline 0.26511 & 0.0202823 & 0.264232 & 0.0196532 & 0.255878 & 0.0129313 & 0.31094 \\
\hline 0.267197 & 0.0187056 & 0.268831 & 0.0151321 & 0.257619 & 0.0141202 & 0.31066 \\
\hline 0.270144 & 0.0206941 & 0.274288 & 0.0172473 & 0.253901 & 0.0157259 & 0.316517 \\
\hline 0.268773 & 0.0216954 & 0.271761 & 0.0153745 & 0.259944 & 0.0136107 & 0.310459 \\
\hline 0.268571 & 0.0198878 & 0.27472 & 0.0171145 & 0.263356 & 0.0130558 & 0.315395 \\
\hline 0.273234 & 0.0188722 & 0.276043 & 0.0217503 & 0.261851 & 0.012495 & 0.300027 \\
\hline 0.274231 & 0.0182641 & 0.275298 & 0.0194158 & 0.265964 & 0.0165959 & 0.325597 \\
\hline 0.276139 & 0.0200633 & 0.27659 & 0.0215965 & 0.262218 & 0.0148951 & 0.318926 \\
\hline 0.278938 & 0.0213483 & 0.279104 & 0.0213919 & 0.266599 & 0.0131159 & 0.319211 \\
\hline 0.279483 & 0.0181982 & 0.27651 & 0.0134142 & 0.269312 & 0.0108876 & 0.331506 \\
\hline 0.279414 & 0.0182487 & 0.285527 & 0.0182795 & 0.269264 & 0.0158174 & 0.318617 \\
\hline 0.281158 & 0.018505 & 0.287448 & 0.0195663 & 0.273643 & 0.0114633 & 0.329573 \\
\hline 0.285469 & 0.0186154 & 0.288939 & 0.0200272 & 0.278145 & 0.0145442 & 0.329574 \\
\hline 0.288672 & 0.0185489 & 0.290449 & 0.0193032 & 0.278011 & 0.0139262 & 0.327839 \\
\hline 0.29118 & 0.0221315 & 0.293782 & 0.0192664 & 0.284767 & 0.0118785 & 0.333681 \\
\hline 0.292188 & 0.0226427 & 0.295712 & 0.0223447 & 0.282282 & 0.011905 & 0.325146 \\
\hline 0.294505 & 0.0203038 & 0.300121 & 0.0206755 & 0.285985 & 0.0137477 & 0.342654 \\
\hline 0.293566 & 0.0209393 & 0.303414 & 0.0194929 & 0.281008 & 0.0175788 & 0.338996 \\
\hline 0.297534 & 0.0166629 & 0.304867 & 0.0205022 & 0.292217 & 0.0148348 & 0.346029 \\
\hline 0.300455 & 0.0213895 & 0.307159 & 0.0182492 & 0.303584 & 0.0158216 & 0.346311 \\
\hline 0.304243 & 0.0189451 & 0.311678 & 0.0165537 & 0.2971 & 0.0128455 & 0.351917 \\
\hline 0.305326 & 0.0197182 & 0.31077 & 0.0210675 & 0.302513 & 0.0149744 & 0.345724 \\
\hline 0.309887 & 0.0216058 & 0.312151 & 0.0202877 & 0.307319 & 0.018011 & 0.344396 \\
\hline 0.312048 & 0.0218274 & 0.32455 & 0.0162842 & 0.311035 & 0.0104974 & 0.355931 \\
\hline 0.315995 & 0.0196804 & 0.324464 & 0.0193158 & 0.313614 & 0.0129968 & 0.365586 \\
\hline
\end{tabular}




\begin{tabular}{|c|c|c|c|c|c|c|}
\hline 0.322401 & 0.0189547 & 0.328647 & 0.019343 & 0.319728 & 0.0146505 & 0.3 \\
\hline 0.322496 & 0.0212055 & 0.334587 & 0.017437 & 0.327856 & 0.0139217 & 0.3666 \\
\hline 0.330632 & 0.0196879 & 0.341166 & 0.0193546 & 0.326911 & 0.0145971 & 0.368865 \\
\hline 0.329745 & 0.0198719 & .334387 & 0.0219895 & 0.334797 & 0.0148965 & 0.3727 \\
\hline 0.341503 & 0.0236771 & .344126 & 0.0169604 & 0.341305 & 0.0153693 & 0.36944 \\
\hline 0.344811 & 0.0230978 & 0.351596 & 0.0253277 & 0.343831 & 0.0155535 & 0.37076 \\
\hline 0.347607 & 0.0206893 & 0.362356 & 0.0202448 & 0.359843 & 0.0161063 & 0.392019 \\
\hline 0.351906 & 0.0216859 & 0.368949 & 0.021168 & 0.358847 & 0.0241575 & 0.389321 \\
\hline 0.356593 & 0.0238197 & 0.376103 & 0.0249576 & 0.374329 & 0.0184786 & 0.391758 \\
\hline 0.367299 & 0.0212957 & 0.373629 & 0.0168554 & 0.378843 & 0.016309 & 0.382292 \\
\hline 0.371058 & 0.0217055 & 0.3821 & 0.0190488 & 0.382375 & 0.016032 & 0.390263 \\
\hline 0.382511 & 0.0202653 & 0.393662 & 0.0270993 & 0.391795 & 0.0131222 & 0.405344 \\
\hline 0.38704 & 0.0237636 & 0.399597 & 0.018787 & 0.401832 & 0.0208458 & 0.415503 \\
\hline 0.394257 & 0.024591 & 0.412057 & 0.0225585 & 0.417209 & 0.0177888 & 0.440509 \\
\hline 0.401963 & 0.0243175 & 0.425042 & 0.0268172 & 0.427746 & 0.0202967 & 0.433404 \\
\hline 0.41468 & 0.0239572 & 0.425175 & 0.0218068 & 0.434242 & 0.0217048 & 0.430772 \\
\hline 0.420292 & 0.0224679 & 0.431904 & 0.0230492 & 0.453694 & 0.018051 & 0.437694 \\
\hline 0.43303 & 0.0270287 & 0.451444 & 0.0257424 & 0.458096 & 0.018933 & 0.448171 \\
\hline 0.444213 & 0.0226441 & 0.45954 & 0.0195778 & 0.47171 & 0.020557 & 0.448803 \\
\hline 0.451826 & 0.0258365 & 0.465541 & 0.0195421 & 0.48069 & 0.0235713 & 0.46984 \\
\hline 0.465081 & 0.0246095 & 0.486913 & 0.0247774 & 0.503254 & 0.0207355 & 0.478013 \\
\hline 0.474963 & 0.0222392 & 0.496331 & 0.0241229 & 0.509344 & 0.0199169 & 0.491279 \\
\hline 0.487332 & 0.025502 & 0.505245 & 0.0237538 & 0.528877 & 0.018907 & 0.500347 \\
\hline 0.499277 & 0.0260887 & 0.524045 & 0.0227386 & 0.543634 & 0.0200675 & 0.510165 \\
\hline 0.513245 & 0.02 & & 0.02 & 0.562452 & 0.0 & \\
\hline 0.525005 & 0.0251323 & 0.558073 & 0.0225456 & 0.570826 & 0.0165235 & 0.514434 \\
\hline 0.540221 & 0.0265003 & 0.558459 & 0.024943 & 0.591267 & 0.0208055 & 0.551466 \\
\hline 0.556755 & 0.0262625 & 0.591097 & 0.0265439 & 0.605661 & 0.0253969 & 0.53799 \\
\hline 0.570366 & 0.028522 & 0.594685 & 0.0303151 & 0.622564 & 0.0239715 & 0.541721 \\
\hline 0.585289 & 0.0279042 & 0.617288 & 0.0175908 & 0.643001 & 0.0265125 & 0.579883 \\
\hline 0.59922 & 0.0301049 & 0.639387 & 0.0212707 & 0.654574 & 0.0264548 & 0.58198 \\
\hline 0.620139 & 0.0291919 & 0.644425 & 0.0286708 & 0.672716 & 0.0202292 & 0.598639 \\
\hline 0.632026 & 0.030376 & 0.665703 & 0.0228046 & 0.692206 & 0.0166971 & 0.596296 \\
\hline 0.647774 & 0.0312636 & 0.6803 & 0.0306381 & 0.707317 & 0.0206571 & 0.620647 \\
\hline 0.661859 & 0.0262497 & 0.695472 & 0.0302066 & 0.721745 & 0.0240791 & 0.637122 \\
\hline 0.678294 & 0.0315979 & 0.701715 & 0.0266521 & 0.74038 & 0.0260881 & 0.648112 \\
\hline 0.695027 & 0.0272985 & 0.728148 & 0.0363027 & 0.752107 & 0.0212028 & 0.651535 \\
\hline 0.714537 & 0.0302504 & 0.746214 & 0.0210226 & 0.782806 & 0.0221272 & 0.666351 \\
\hline 0.72687 & 0.0358867 & 0.76443 & 0.0286046 & 0.788835 & 0.0300879 & 0.679241 \\
\hline 0.74464 & 0.0329808 & 0.775273 & 0.0301175 & 0.817467 & 0.0256139 & 0.689324 \\
\hline 0.765413 & 0.0329589 & 0.793387 & 0.0265463 & 0.827224 & 0.0255009 & 0.695261 \\
\hline 0.776516 & 0.034469 & 0.804558 & 0.0303222 & 0.841719 & 0.0309188 & 0.724238 \\
\hline 0.789633 & 0.0335298 & 0.81668 & 0.0244764 & 0.855422 & 0.0270313 & 0.723409 \\
\hline 0.806584 & 0.0328269 & 0.838846 & 0.0303673 & 0.863762 & 0.0292946 & 0.74356 \\
\hline 0.822753 & 0.0342329 & 0.843953 & 0.0305898 & 0.879883 & 0.0303741 & 0.739605 \\
\hline 0.841144 & 0.0310525 & 0.859676 & 0.027155 & 0.894282 & 0.021986 & 0.742947 \\
\hline 0.851784 & 0.0315948 & 0.874343 & 0.0254948 & 0.911158 & 0.0306164 & 0.766735 \\
\hline
\end{tabular}




\begin{tabular}{|c|c|c|c|c|c|c|}
\hline 0.865463 & 0.0325401 & 0.894189 & 0.0379775 & 0.917468 & 0.024621 & 0.774827 \\
\hline 0.875264 & 0.0350821 & 0.897684 & 0.0394417 & 0.927549 & 0.0216385 & 0.773016 \\
\hline 0.89137 & 0.034832 & 0.90525 & 0.0284221 & 0.935135 & 0.0267759 & 0.795449 \\
\hline 0.89786 & 0.0334404 & 0.921731 & 0.0401188 & 0.930089 & 0.0282339 & 0.795953 \\
\hline 0.907989 & 0.0281971 & 0.92676 & 0.0311428 & 0.951941 & 0.0291146 & 0.824272 \\
\hline 0.919662 & 0.0361628 & 0.926131 & 0.0401681 & 0.961426 & 0.027854 & 0.826801 \\
\hline 0.924247 & 0.0364583 & 0.940907 & 0.0338074 & 0.959757 & 0.029191 & 0.829475 \\
\hline 0.929783 & 0.0344865 & 0.939835 & 0.0305851 & 0.967559 & 0.0199547 & 0.832529 \\
\hline 0.933497 & 0.0316707 & 0.948476 & 0.0369252 & 0.963502 & 0.0236022 & 0.85217 \\
\hline 0.941957 & 0.0347977 & 0.952949 & 0.036814 & 0.96873 & 0.0244649 & 0.845742 \\
\hline 0.947601 & 0.0346026 & 0.956301 & 0.0311772 & 0.967145 & 0.0250172 & 0.850407 \\
\hline 0.947307 & 0.0298364 & 0.948333 & 0.0406847 & 0.953022 & 0.0319778 & 0.868806 \\
\hline 0.954921 & 0.0316146 & 0.965795 & 0.0272638 & 0.9638 & 0.0262456 & 0.881659 \\
\hline 0.953854 & 0.0355495 & 0.956302 & 0.0336316 & 0.955216 & 0.0288643 & 0.873993 \\
\hline 0.954713 & 0.0321229 & 0.953957 & 0.0332246 & 0.949406 & 0.0272166 & 0.878795 \\
\hline 0.946021 & 0.033541 & 0.950893 & 0.0328395 & 0.951729 & 0.0271549 & 0.889599 \\
\hline 0.938327 & 0.0360917 & 0.941494 & 0.0276489 & 0.934739 & 0.0272295 & 0.884116 \\
\hline 0.938705 & 0.0347615 & 0.933506 & 0.0318147 & 0.934796 & 0.0283444 & 0.891177 \\
\hline 0.938308 & 0.0346398 & 0.930504 & 0.0297254 & 0.922923 & 0.0307706 & 0.900298 \\
\hline 0.927156 & 0.0363764 & 0.92206 & 0.033863 & 0.908957 & 0.0273958 & 0.905223 \\
\hline 0.923768 & 0.0316868 & 0.910546 & 0.0351635 & 0.895107 & 0.0279129 & 0.90233 \\
\hline 0.920656 & 0.0375168 & 0.898423 & 0.0341396 & 0.876041 & 0.0271332 & 0.91527 \\
\hline 0.901714 & 0.0351604 & 0.886075 & 0.0313758 & 0.864285 & 0.027928 & 0.892902 \\
\hline 0.896334 & 0.0349893 & 0.885484 & 0.0323233 & 0.860319 & 0.0302198 & 0.907426 \\
\hline 0.883857 & 0.0333975 & 0.867651 & 0.0339184 & 0.844861 & 0.0236658 & 0.902206 \\
\hline 0.876555 & 0.0327102 & 0.850575 & 0.0301315 & 0.820688 & 0.0277322 & 0.891177 \\
\hline 0.862799 & 0.0347948 & 0.844478 & 0.0387539 & 0.802419 & 0.021734 & 0.894094 \\
\hline 0.84386 & 0.0332224 & 0.825184 & 0.0297723 & 0.793972 & 0.0267491 & 0.910327 \\
\hline 0.836079 & 0.0388406 & 0.810516 & 0.0285787 & 0.771204 & 0.0260231 & 0.879467 \\
\hline 0.823555 & 0.0307893 & 0.803445 & 0.0251958 & 0.755328 & 0.0261474 & 0.867001 \\
\hline 0.806464 & 0.0340701 & 0.776573 & 0.0299291 & 0.740027 & 0.0235432 & 0.871759 \\
\hline 0.785427 & 0.03798 & 0.773373 & 0.0290228 & 0.719495 & 0.024949 & 0.861737 \\
\hline 0.768556 & 0.0328597 & 0.743431 & 0.0304555 & 0.706509 & 0.0259197 & 0.850744 \\
\hline 0.759876 & 0.0326164 & 0.728435 & 0.024645 & 0.682654 & 0.0218836 & 0.819658 \\
\hline 0.741223 & 0.0319877 & 0.706083 & 0.0290139 & 0.659962 & 0.017507 & 0.836369 \\
\hline 0.725023 & 0.0325695 & 0.694365 & 0.0251753 & 0.649345 & 0.0223821 & 0.813577 \\
\hline 0.704589 & 0.0296819 & 0.673586 & 0.0253742 & 0.629123 & 0.0238431 & 0.808563 \\
\hline 0.688292 & 0.0312848 & 0.655165 & 0.0225838 & 0.610157 & 0.0277509 & 0.788899 \\
\hline 0.667866 & 0.0293814 & 0.642017 & 0.0277181 & 0.59974 & 0.0242178 & 0.76045 \\
\hline 0.658667 & 0.0350867 & 0.631687 & 0.0200558 & 0.578914 & 0.0219269 & 0.740352 \\
\hline 0.639084 & 0.030987 & 0.597062 & 0.0249929 & 0.56302 & 0.0222141 & 0.705057 \\
\hline 0.619575 & 0.0282167 & 0.579906 & 0.0297153 & 0.541777 & 0.0228326 & 0.697487 \\
\hline 0.606965 & 0.0319889 & 0.574755 & 0.0213591 & 0.527519 & 0.0189671 & 0.66689 \\
\hline 0.585553 & 0.0327679 & 0.556904 & 0.0259151 & 0.507079 & 0.0241988 & 0.648434 \\
\hline 0.573779 & 0.0265435 & 0.540366 & 0.0276016 & 0.496136 & 0.0177872 & 0.62875 \\
\hline 0.554929 & 0.0291507 & 0.526021 & 0.0266893 & 0.472435 & 0.0196936 & 0.622544 \\
\hline 0.539613 & 0.0297695 & 0.514344 & 0.0279486 & 0.468209 & 0.0232798 & 0.588399 \\
\hline
\end{tabular}




\begin{tabular}{|c|c|c|c|c|c|c|}
\hline 0.519337 & 0.0296895 & 0.494356 & 0.0270112 & 0.454205 & 0.0187359 & 0.574432 \\
\hline 0.510709 & 0.0270657 & 0.48496 & 0.0227396 & 0.437126 & 0.0194244 & 0.558717 \\
\hline 498991 & 0.028809 & 0.476734 & 0.020807 & 0.424165 & 0.0167827 & 0.53179 \\
\hline 478735 & 0.0296366 & 0.453007 & 0.0285272 & 0.411657 & 0.0183361 & 0.52463 \\
\hline 0.469461 & 0.0247595 & 0.440991 & 0.0234478 & 0.395801 & 0.0148318 & 0.495064 \\
\hline 0.454628 & 0.0283711 & 0.43437 & 0.0201006 & 0.392233 & 0.0123143 & 0.484339 \\
\hline 0.440259 & 0.026572 & 0.422172 & 0.017471 & 0.378769 & 0.0170953 & 0.475085 \\
\hline 0.428599 & 0.0268449 & 0.409621 & 0.0267353 & 0.365347 & 0.0161878 & 0.447431 \\
\hline 0.414444 & 0.0209805 & 0.394251 & 0.0199 & 0.357783 & 0.0168658 & 0.442534 \\
\hline 0.408827 & 0.0265715 & 0.389489 & 0.0199475 & 0.345334 & 0.0157748 & 0.434324 \\
\hline 0.39428 & 0.0260292 & 0.380105 & 0.0238093 & 0.338486 & 0.016373 & 0.412674 \\
\hline 0.385188 & 0.0224988 & 0.369911 & 0.0209516 & 0.329135 & 0.0144613 & 0.407943 \\
\hline 0.374183 & 0.0242303 & 0.357356 & 0.0226074 & 0.324084 & 0.0183182 & 0.413909 \\
\hline 0.366746 & 0.023162 & 0.349036 & 0.0165927 & 0.316217 & 0.016353 & 0.398871 \\
\hline 0.35454 & 0.0224336 & 0.341579 & 0.0234899 & 0.308416 & 0.0133923 & 0.371687 \\
\hline 0.351319 & 0.0200522 & 0.333537 & 0.0181249 & 0.297677 & 0.0134822 & 0.37356 \\
\hline 0.340472 & 0.0207968 & 0.327593 & 0.0194635 & 0.298884 & 0.0150595 & 0.369481 \\
\hline 0.333976 & 0.0235745 & 0.314819 & 0.0198322 & 0.284394 & 0.0132749 & 0.350979 \\
\hline 0.325558 & 0.0195544 & 0.316047 & 0.0 & 0.2 & 0.015 & 8251 \\
\hline 0.314044 & 0.0193497 & 0.298715 & 0.0162566 & 0.272769 & 0.0142717 & 0.35808 \\
\hline 0.312812 & 0.0218994 & 0.290498 & 0.0191574 & 0.267813 & 0.0147484 & 0.340242 \\
\hline 0.305617 & 0.0215518 & $0.2 \varepsilon$ & 742 & 549 & 0.011 & 812 \\
\hline 0.293526 & 0.0209484 & 0.283053 & 0.0145006 & 0.263085 & 0.01183 & 0.328661 \\
\hline 0.292658 & 0.0215426 & 0.279885 & 0.014526 & 0.257159 & 0.012118 & 0.331536 \\
\hline 0.287913 & 0.0161515 & 0.273956 & 0.0223633 & 0.254094 & 0.0144669 & 0.312722 \\
\hline 0.280134 & 0.0215614 & 0.270118 & 0.0234124 & 0.248718 & 0.0125288 & 0.307532 \\
\hline 0.274834 & 0.0207211 & 0.263855 & 0.0149604 & 0.243831 & 0.0148843 & 0.320321 \\
\hline 0.269115 & 0.018943 & 0.261307 & 0.0167382 & 0.24132 & 0.0102792 & 0.301661 \\
\hline 0.263996 & 0.0212707 & 0.25119 & 0.0174653 & 0.240327 & 0.0117913 & 0.30079 \\
\hline 0.259318 & 0.0198921 & 0.247881 & 5279 & 0.226652 & 0.0080209 & 0.308737 \\
\hline 0.253119 & 0.0199672 & 0.24268 & 0.0171696 & 0.228584 & 0.0139423 & 0.291798 \\
\hline 0.247208 & 0.0201169 & 0.239642 & 0.0169694 & 0.223156 & 0.0135666 & 0.298016 \\
\hline 0.241851 & 0.0203895 & 0.23095 & 0.018758 & 0.220553 & 0.0108394 & 0.290064 \\
\hline 0.24113 & 0.0189432 & 0.229886 & 0.0124592 & 0.21909 & 0.0145042 & 0.294277 \\
\hline 0.233949 & 0.018586 & 0.229556 & 0.0165456 & 0.215285 & 0.0140299 & 0.281975 \\
\hline 0.231749 & 0.0197656 & 0.220429 & 0.0214143 & 0.20883 & 0.011679 & 0.274241 \\
\hline 0.227022 & 0.0170633 & 0.217865 & 0.0140195 & 0.211607 & 0.014491 & 0.282741 \\
\hline 0.222098 & 0.017143 & 0.218872 & 0.0157955 & 0.203375 & 0.0118938 & 0.284983 \\
\hline 0.219065 & 0.015641 & 0.211248 & 0.0172965 & 0.20253 & 0.0122299 & 0.270667 \\
\hline 0.21135 & 0.0161552 & 0.212339 & 0.0122309 & 0.200249 & 0.0132644 & 0.289946 \\
\hline 0.210463 & 0.0152144 & 0.207178 & 0.0151919 & 0.196024 & 0.0137027 & 0.289967 \\
\hline 0.206925 & 0.0182444 & 0.201294 & 0.0145458 & 0.190694 & 0.0128149 & 0.281494 \\
\hline 0.203669 & 0.0170069 & 0.198961 & 0.0184978 & 0.187399 & 0.0106001 & 0.294852 \\
\hline 0.201121 & 0.0170341 & 0.197373 & 0.0135829 & 0.183807 & 0.0106938 & 0.277735 \\
\hline 0.197029 & 0.016028 & 0.196577 & 0.017063 & 0.184258 & 0.0107909 & 0.290293 \\
\hline 0.195624 & 0.0145396 & 0.190536 & 0.0153441 & 0.182349 & 0.00899204 & 0.28485 \\
\hline 0.19469 & 0.0160783 & 0.188772 & 0.0137414 & 0.176579 & 0.0108333 & 0.288522 \\
\hline
\end{tabular}




\begin{tabular}{|c|c|c|c|c|c|c|}
\hline 0.190229 & 0.0164782 & 0.186398 & 0.0137703 & 0.180187 & 0.0119627 & 0.289413 \\
\hline 0.189797 & 0.0170903 & 0.185369 & 0.0176812 & 0.178047 & 0.0129813 & 0.282079 \\
\hline 0.185415 & 0.0151045 & 0.18535 & 0.0176004 & 0.176437 & 0.0107589 & 0.299605 \\
\hline 0.188423 & 0.0163761 & 0.189512 & 0.0115211 & 0.175167 & 0.00855991 & 0.296005 \\
\hline 0.184042 & 0.0148513 & 0.194854 & 0.0164561 & 0.171708 & 0.0128839 & 0.296044 \\
\hline 0.187148 & 0.0170777 & 0.192042 & 0.0159669 & 0.173535 & 0.0101539 & 0.300791 \\
\hline 0.184448 & 0.0154713 & 0.200598 & 0.0155887 & 0.17028 & 0.0109145 & 0.304163 \\
\hline 0.18333 & 0.0155769 & 0.198472 & 0.0159787 & 0.174616 & 0.0143748 & 0.310073 \\
\hline 0.190341 & 0.0192335 & 0.199134 & 0.019688 & 0.174707 & 0.011485 & 0.320992 \\
\hline 0.192043 & 0.016992 & 0.211422 & 0.0137863 & 0.181251 & 0.0124467 & 0.330293 \\
\hline 0.198356 & 0.0219237 & 0.215727 & 0.0204123 & 0.180838 & 0.0101474 & 0.339803 \\
\hline 0.199474 & 0.0179701 & 0.226831 & 0.0256388 & 0.189136 & 0.00945993 & 0.353321 \\
\hline 0.207473 & 0.0203754 & 0.236468 & 0.0214887 & 0.192334 & 0.0100439 & 0.379948 \\
\hline 0.213718 & 0.0219681 & 0.246516 & 0.0231266 & 0.203912 & 0.0145602 & 0.385529 \\
\hline 0.223958 & 0.0218934 & 0.255307 & 0.0270988 & 0.206801 & 0.009867 & 0.404385 \\
\hline 0.235642 & 0.0229086 & 0.27826 & 0.0214218 & 0.218899 & 0.0132573 & 0.433588 \\
\hline 0.241368 & 0.0233329 & 0.289123 & 0.0219221 & 0.225116 & 0.0153914 & 0.462164 \\
\hline 0.256712 & 0.0268666 & 0.311595 & 0.0286566 & 0.240791 & 0.0152889 & 0.498664 \\
\hline 0.272946 & 0.03357 & 0.333205 & 0.036909 & 0.249073 & 0.0134413 & 0.526158 \\
\hline 0.29342 & 0.0339954 & 0.349694 & 0.0325025 & 0.263 & 0.01866 & 0.55789 \\
\hline 0.307754 & 0.0343822 & 0.370307 & 0.0373491 & 0.277202 & 0.0175174 & 0.600128 \\
\hline 0.324393 & 0.0304907 & 0.401829 & 0.0397664 & 0.295493 & 0.0121085 & 0.64042 \\
\hline 0.347023 & 0.0387142 & 0.419372 & 0.0440708 & 0.307301 & 0.0184157 & 0.708125 \\
\hline 0.366519 & 0.048655 & 0.445215 & 0.0440767 & 0.326556 & 0.0141577 & 0.738874 \\
\hline 0.392654 & 0.0497997 & 0.461542 & 0.0524005 & 0.336524 & 0.021419 & 0.776923 \\
\hline 0.413602 & 0.0475914 & 0.485675 & 0.0568858 & 0.357804 & 0.0157467 & 0.842352 \\
\hline 0.431078 & 0.0502186 & 0.512192 & 0.0564348 & 0.376902 & 0.0195132 & 0.872137 \\
\hline 0.459438 & 0.0617185 & 0.530558 & 0.0588019 & 0.392693 & 0.018354 & 0.914993 \\
\hline 0.482968 & 0.0613779 & 0.552928 & 0.0620198 & 0.41471 & 0.0209146 & 0.956749 \\
\hline 0.501597 & 0.0617201 & 0.57377 & 0.0709712 & 0.425071 & 0.0187971 & 1.01615 \\
\hline 0.523293 & 0.0605718 & 0.600875 & 0.061017 & 0.43768 & 0.024792 & 1.07953 \\
\hline 0.549587 & 0.0715152 & 0.625932 & 0.0704187 & 0.456084 & 0.0206363 & 1.09864 \\
\hline 0.564872 & 0.0730411 & 0.637022 & 0.0740886 & 0.471954 & 0.0240183 & 1.13166 \\
\hline 0.591932 & 0.0761344 & 0.645604 & 0.0652037 & 0.482551 & 0.0231195 & 1.19049 \\
\hline 0.60553 & 0.0753933 & 0.671366 & 0.0737202 & 0.494653 & 0.017657 & 1.20697 \\
\hline 0.624263 & 0.0785491 & 0.679182 & 0.0843268 & 0.499058 & 0.0191195 & 1.25253 \\
\hline 0.638719 & 0.0874475 & 0.693704 & 0.0762112 & 0.519804 & 0.0180128 & 1.2656 \\
\hline 0.645857 & 0.0849936 & 0.693299 & 0.0817989 & 0.523865 & 0.0205641 & 1.31298 \\
\hline 0.660579 & 0.0855712 & 0.689641 & 0.0715775 & 0.530098 & 0.0210934 & 1.28799 \\
\hline 0.673719 & 0.0870461 & 0.687451 & 0.0746154 & 0.528843 & 0.0209327 & 1.29374 \\
\hline 0.678218 & 0.0864738 & 0.694993 & 0.0917725 & 0.531904 & 0.0172651 & 1.32219 \\
\hline 0.678603 & 0.0901956 & 0.677514 & 0.0641346 & 0.526589 & 0.02202 & 1.27718 \\
\hline 0.680099 & 0.0849619 & 0.671481 & 0.076633 & 0.525173 & 0.0181738 & 1.28363 \\
\hline 0.676225 & 0.0915346 & 0.670008 & 0.0746842 & 0.515504 & 0.0255059 & 1.26805 \\
\hline 0.659845 & 0.0845131 & 0.635146 & 0.0639704 & 0.499653 & 0.0235768 & 1.23331 \\
\hline 0.654834 & 0.0900173 & 0.613488 & 0.0685828 & 0.491009 & 0.0232158 & 1.18864 \\
\hline 0.640969 & 0.0906144 & 0.587578 & 0.0653042 & 0.479914 & 0.0171441 & 1.16398 \\
\hline
\end{tabular}




\begin{tabular}{|c|c|c|c|c|c|c|}
\hline 0.623423 & 0.0974956 & 0.56221 & 0.0566241 & 0.466791 & 0.0232728 & 1.09986 \\
\hline 0.595721 & 0.0760391 & 0.539551 & 0.0656734 & 0.444417 & 0.0267321 & 1.04062 \\
\hline 0.570754 & 0.0770756 & 0.512363 & 0.0589665 & 0.41812 & 0.0209443 & 1.00589 \\
\hline 0.543605 & 0.0800625 & 0.472536 & 0.0539325 & 0.397403 & 0.0160808 & 0.937743 \\
\hline 0.517655 & 0.0691244 & 0.436814 & 0.0518452 & 0.379754 & 0.0161136 & 0.892747 \\
\hline 0.486319 & 0.0684888 & 0.405946 & 0.0485934 & 0.352435 & 0.0168641 & 0.807136 \\
\hline 0.460364 & 0.0551941 & 0.380926 & 0.0433355 & 0.328061 & 0.0151431 & 0.753108 \\
\hline 0.427574 & 0.0541027 & 0.350903 & 0.0371334 & 0.311098 & 0.0145673 & 0.699886 \\
\hline 0.395753 & 0.0525673 & 0.313946 & 0.0325805 & 0.282099 & 0.0167015 & 0.651188 \\
\hline 0.365623 & 0.0494382 & 0.294427 & 0.0368234 & 0.265727 & 0.0148407 & 0.581909 \\
\hline 0.334039 & 0.038628 & 0.268049 & 0.0349519 & 0.239611 & 0.0115253 & 0.524715 \\
\hline 0.310458 & 0.0396597 & 0.24503 & 0.0271148 & 0.223408 & 0.0146582 & 0.460552 \\
\hline 0.283082 & 0.0369391 & 0.229076 & 0.0276106 & 0.211032 & 0.013924 & 0.431263 \\
\hline 0.257979 & 0.0328493 & 0.204773 & 0.0259694 & 0.191978 & 0.0102878 & 0.398806 \\
\hline 0.239092 & 0.032314 & 0.187881 & 0.0159819 & 0.180399 & 0.0117804 & 0.354746 \\
\hline 0.21877 & 0.02823 & 0.180295 & 0.0254973 & 0.170258 & 0.0112147 & 0.31477 \\
\hline 0.199855 & 0.023227 & 0.167797 & 0.0160554 & 0.158761 & 0.0107385 & 0.296285 \\
\hline 0.183308 & 0.0229314 & 0.157847 & 0.0181694 & 0.148132 & 0.008828 & 0.266233 \\
\hline 0.174477 & 0.0230966 & 0.144992 & 0.0148585 & 0.139917 & 0.01 & 0.251682 \\
\hline 0.163038 & 0.0192025 & 0.139349 & 0.0153584 & 0.137897 & 0.00750698 & 0.240008 \\
\hline 0.151692 & 0.0204451 & 0.134025 & 0.0140838 & 0.129973 & 0.0097802 & 0.226888 \\
\hline 0.145093 & 0.0163941 & 0.127988 & 0.0124497 & 0.125196 & 0.00 & 0.213403 \\
\hline 0.139195 & 0.0189691 & 0.126993 & 0.0118024 & 0.119386 & 0.00911401 & 0.201708 \\
\hline 0.13065 & 0.0140711 & 0.12502 & 0.0139651 & 0.120359 & 0.0113471 & 0.189462 \\
\hline 0.128649 & 0.0159062 & 0.122477 & 0.0115524 & 0.121071 & 0.00768478 & 0.191041 \\
\hline 0.124572 & 0.0139756 & 0.116421 & 0.0107779 & 0.116245 & 0.00964147 & 0.181853 \\
\hline 0.122325 & 0.0153868 & 0.113045 & 0.0117456 & 0.11227 & 0.00950028 & 0.1787 \\
\hline 0.119906 & 0.013354 & 0.119499 & 0.0134584 & 0.112786 & 0.00899454 & 0.169678 \\
\hline 0.1173 & 0.013272 & 0.117738 & 0.0124982 & 0.11224 & 0.00985398 & 0.179615 \\
\hline 0.116113 & 0.0143102 & 0.114644 & 0.0104714 & 0.111676 & 0.0112589 & 0.166976 \\
\hline 0.115252 & 0.0127595 & 0.11949 & 0.0123551 & 0.112859 & 0.00898895 & 0.162673 \\
\hline 0.11507 & 0.0137879 & 0.115628 & 0.0123007 & 0.111511 & 0.00853906 & 0.162909 \\
\hline 0.116144 & 0.0134696 & 0.111161 & 0.0115936 & 0.109335 & 0.0105326 & 0.161114 \\
\hline 0.112622 & 0.0122366 & 0.114557 & 0.012062 & 0.11073 & 0.00761779 & 0.156315 \\
\hline 0.115475 & 0.011998 & 0.11537 & 0.0143539 & 0.110709 & 0.00820042 & 0.149447 \\
\hline 0.113941 & 0.0118714 & 0.113759 & 0.0126852 & 0.111451 & 0.00908741 & 0.159289 \\
\hline 0.11375 & 0.0128454 & 0.115878 & 0.0161959 & 0.112363 & 0.0106027 & 0.161113 \\
\hline 0.116157 & 0.0129152 & 0.117864 & 0.0095703 & 0.112533 & 0.0097241 & 0.151005 \\
\hline 0.114423 & 0.0119411 & 0.117962 & 0.0130394 & 0.115973 & 0.00742504 & 0.155367 \\
\hline 0.116063 & 0.0122881 & 0.118699 & 0.0147603 & 0.111351 & 0.00801164 & 0.151179 \\
\hline 0.117356 & 0.0126546 & 0.116455 & 0.013001 & 0.112465 & 0.00626227 & 0.15099 \\
\hline 0.118171 & 0.0122658 & 0.117199 & 0.00999868 & 0.115852 & 0.00920016 & 0.148608 \\
\hline 0.118309 & 0.013035 & 0.118403 & 0.0126529 & 0.116381 & 0.00747057 & 0.153271 \\
\hline 0.11512 & 0.0129173 & 0.116911 & 0.0116672 & 0.114499 & 0.00825664 & 0.148804 \\
\hline 0.119325 & 0.0126728 & 0.115186 & 0.0130972 & 0.116902 & 0.00834933 & 0.155221 \\
\hline 0.119245 & 0.0136971 & 0.116236 & 0.0130681 & 0.116792 & 0.0107288 & 0.149554 \\
\hline 0.116671 & 0.0112578 & 0.121118 & 0.0126381 & 0.11669 & 0.00833264 & 0.15104 \\
\hline
\end{tabular}




$\begin{array}{rrrrrrr}0.119664 & 0.0142356 & 0.119406 & 0.0114989 & 0.118334 & 0.00799637 & 0.142269 \\ 0.119763 & 0.0110782 & 0.121059 & 0.0129529 & 0.120062 & 0.00855003 & 0.147912 \\ 0.119522 & 0.0119959 & 0.12556 & 0.0107001 & 0.119952 & 0.00805147 & 0.150607 \\ 0.123118 & 0.0121898 & 0.12268 & 0.0149585 & 0.120696 & 0.00893863 & 0.150098 \\ 0.123268 & 0.0124314 & 0.125319 & 0.0117904 & 0.121825 & 0.00992392 & 0.145599 \\ 0.12686 & 0.0140353 & 0.131911 & 0.013575 & 0.125968 & 0.0104449 & 0.149271 \\ 0.125704 & 0.0126282 & 0.128954 & 0.011646 & 0.127894 & 0.0111466 & 0.146207 \\ 0.123556 & 0.0131579 & 0.133899 & 0.0150805 & 0.127585 & 0.00892522 & 0.154732 \\ 0.128843 & 0.0139357 & 0.134126 & 0.0119092 & 0.126093 & 0.0106256 & 0.154176 \\ 0.129797 & 0.0129211 & 0.131537 & 0.0109294 & 0.129879 & 0.0106193 & 0.150355 \\ 0.130465 & 0.0115365 & 0.134457 & 0.0119196 & 0.13128 & 0.00853928 & 0.15331 \\ 0.131077 & 0.0130072 & 0.134958 & 0.0152801 & 0.133015 & 0.00898211 & 0.15843 \\ 0.134745 & 0.0135511 & 0.134534 & 0.011851 & 0.135017 & 0.0108925 & 0.154184 \\ 0.131281 & 0.0119629 & 0.135285 & 0.0145822 & 0.137144 & 0.0117821 & 0.153067 \\ 0.134657 & 0.0145226 & 0.140407 & 0.0121281 & 0.140074 & 0.00954814 & 0.152042 \\ 0.136694 & 0.0124812 & 0.137638 & 0.0101792 & 0.138872 & 0.00956613 & 0.155261 \\ 0.136471 & 0.0129786 & 0.14438 & 0.0143844 & 0.141546 & 0.00976785 & 0.158635 \\ 0.138883 & 0.0117745 & 0.144458 & 0.0119797 & 0.13917 & 0.00978675 & 0.159652 \\ 0.142152 & 0.0136392 & 0.142396 & 0.0120494 & 0.144723 & 0.00937127 & 0.15631 \\ 0.141137 & 0.0132362 & 0.144791 & 0.0128454 & 0.143478 & 0.00857167 & 0.160901 \\ 0.142066 & 0.0125946 & 0.149694 & 0.0151822 & 0.148972 & 0.00836343 & 0.156739 \\ 0.145587 & 0.0129048 & 0.15198 & 0.0140668 & 0.147402 & 0.0101437 & 0.160881 \\ 0.146445 & 0.0136061 & 0.15065 & 0.0122329 & 0.149141 & 0.0121304 & 0.171541 \\ 0.147491 & 0.0127335 & 0.153143 & 0.0120528 & 0.15354 & 0.0120383 & 0.161166 \\ 0.149338 & 0.0138244 & 0.157278 & 0.0132148 & 0.154635 & 0.0114896 & 0.163658 \\ 0.151166 & 0.0141718 & 0.155228 & 0.0141783 & 0.154523 & 0.00981945 & 0.160297 \\ 0.152406 & 0.0141078 & 0.160557 & 0.0119961 & 0.158626 & 0.0119239 & 0.165805 \\ 0.15632 & 0.0144533 & 0.156118 & 0.0134256 & 0.160641 & 0.00787009 & 0.172904\end{array}$




\begin{tabular}{|c|c|c|c|c|c|c|}
\hline 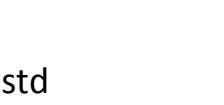 & $\begin{array}{l}\text { SB Ru } \\
\text { mean }\end{array}$ & std & $\begin{array}{l}\text { SB Rc } \\
\text { mean }\end{array}$ & std & $\begin{array}{l}\text { SB Rb } \\
\text { mean }\end{array}$ & std \\
\hline 0.160022 & 0.414779 & 0.0396361 & 0.246999 & 0.0355575 & 0.219046 & 0.0237054 \\
\hline 0.16577 & 0.41935 & 0.0391818 & 0.251183 & 0.0463229 & 0.225464 & 0.0283012 \\
\hline 0.206033 & 0.419251 & 0.0398095 & 0.245345 & 0.0365502 & 0.221423 & 0.0219308 \\
\hline 0.171586 & 0.413871 & 0.0383521 & 0.240785 & 0.0426016 & 0.224599 & 0.0212836 \\
\hline 0.184532 & 0.410389 & 0.0365561 & 0.24347 & 0.0388571 & 0.220209 & 0.0197176 \\
\hline 0.148431 & 0.412056 & 0.0378681 & 0.243633 & 0.0405405 & 0.22251 & 0.0259831 \\
\hline 0.193611 & 0.40759 & 0.0379245 & 0.237773 & 0.0406711 & 0.221521 & 0.0184433 \\
\hline 0.168888 & 0.401381 & 0.0338912 & 0.240675 & 0.038309 & 0.225026 & 0.0203709 \\
\hline 0.176389 & 0.406426 & 0.0372156 & 0.238894 & 0.0358067 & 0.218111 & 0.0201055 \\
\hline 0.172527 & 0.397683 & 0.0326627 & 0.239532 & 0.0384017 & 0.220209 & 0.02481 \\
\hline 0.190425 & 0.405531 & 0.0430814 & 0.233267 & 0.0429858 & 0.219208 & 0.0241948 \\
\hline 0.161995 & 0.403367 & 0.0388538 & 0.23921 & 0.0430773 & 0.213844 & 0.0229495 \\
\hline 0.162602 & 0.395222 & 0.0368999 & 0.236391 & 0.0400993 & 0.216479 & 0.0237501 \\
\hline 0.186156 & 0.398615 & 0.0313438 & 0.236967 & 0.0424654 & 0.217257 & 0.019222 \\
\hline 0.186654 & 0.402177 & 0.0366532 & 0.23957 & 0.0386176 & 0.21929 & 0.0234442 \\
\hline 0.194399 & 0.408318 & 0.0342755 & 0.241328 & 0.0416089 & 0.216931 & 0.0225813 \\
\hline 0.187099 & 0.419174 & 0.0373018 & 0.237714 & 0.0439192 & 0.219767 & 0.0292699 \\
\hline 0.16485 & 0.430185 & 0.0332631 & 0.245069 & 0.0426619 & 0.223269 & 0.0236013 \\
\hline 0.179337 & 0.447275 & 0.036634 & 0.243699 & 0.0383746 & 0.228212 & 0.0186804 \\
\hline 0.159489 & 0.480381 & 0.0358655 & 0.245426 & 0.0437852 & 0.237251 & 0.0235309 \\
\hline 0.165406 & 0.50984 & 0.0422821 & 0.247269 & 0.04035 & 0.243977 & 0.025029 \\
\hline 0.197574 & 0.537378 & 0.0449791 & 0.258171 & 0.0456306 & 0.249737 & 0.0264067 \\
\hline 0.185353 & 0.561891 & 0.0495212 & 0.262633 & 0.0449508 & 0.255356 & 0.0313032 \\
\hline 0.17414 & 0.589192 & 0.0498859 & 0.263091 & 0.0436256 & 0.266288 & 0.0261491 \\
\hline 0.163043 & 0.602898 & 0.0550131 & 0.268014 & 0.0404423 & 0.269325 & 0.0296341 \\
\hline 0.162079 & 0.605333 & 0.0491874 & 0.270719 & 0.0487615 & 0.275542 & 0.0308929 \\
\hline 0.15601 & 0.599334 & 0.046535 & 0.266128 & 0.0443956 & 0.274284 & 0.0255819 \\
\hline 0.174494 & 0.574484 & 0.0504109 & 0.263813 & 0.0455 & 0.266597 & 0.0253243 \\
\hline 0.164734 & 0.54204 & 0.0469827 & 0.262365 & 0.0438652 & 0.260685 & 0.0319286 \\
\hline 0.182823 & 0.506494 & 0.0355557 & 0.258535 & 0.0445171 & 0.258395 & 0.0253376 \\
\hline 0.179726 & 0.479336 & 0.0412564 & 0.251627 & 0.0416743 & 0.247983 & 0.0326018 \\
\hline 0.169899 & 0.450696 & 0.0360121 & 0.248331 & 0.0431184 & 0.246854 & 0.0280415 \\
\hline 0.17532 & 0.428913 & 0.0299521 & 0.246648 & 0.0446053 & 5079 & 0.0270135 \\
\hline 0.185129 & 0.422042 & 0.03451 & 0.243389 & 0.0452872 & 0.241654 & 0.0269365 \\
\hline 0.189456 & 0.408039 & 0.0293579 & 0.24687 & 0.0444906 & 0.249726 & 0.0271943 \\
\hline 0.18209 & 0.407933 & 0.0322783 & 0.245801 & 0.0443404 & 0.259872 & 0.0301359 \\
\hline 0.195429 & 0.414621 & 0.0396938 & 0.250566 & 0.0412233 & 0.277791 & 0.0341378 \\
\hline 0.169443 & 0.438689 & 0.0397684 & 0.257073 & 0.0460656 & 0.301789 & 0.0353424 \\
\hline 0.179955 & 0.476361 & 0.0406175 & 0.275119 & 0.0471082 & 0.352458 & 0.0434241 \\
\hline 0.202413 & 0.522055 & 0.0507679 & 0.292417 & 0.0489617 & 0.4366 & 0.0493406 \\
\hline 0.203411 & 0.599969 & 0.0748351 & 0.330233 & 0.0570488 & 0.561215 & 0.0732286 \\
\hline 0.24186 & 0.698031 & 0.0917319 & 0.374297 & 0.0717831 & 0.742332 & 0.0866274 \\
\hline 0.303656 & 0.836977 & 0.121366 & 0.433196 & 0.0907014 & 0.98571 & 0.134976 \\
\hline 0.31926 & 0.990951 & 0.141433 & 0.502639 & 0.110434 & 1.25159 & 0.182546 \\
\hline 0.394536 & 1.14341 & 0.178969 & 0.587764 & 0.140717 & 1.56761 & 0.222819 \\
\hline
\end{tabular}




\begin{tabular}{|c|c|c|c|c|c|c|}
\hline 0.420632 & 1.28568 & 0.226073 & 0.667085 & 0.157056 & 1.86813 & 0.256165 \\
\hline 0.520988 & 1.40252 & 0.249954 & 0.742437 & 0.175464 & 2.14262 & 0.289038 \\
\hline 0.554873 & 1.48735 & 0.274139 & 0.79659 & 0.191581 & 2.34299 & 0.328902 \\
\hline 0.602498 & 1.50518 & 0.280148 & 0.822916 & 0.205557 & 2.45027 & 0.350094 \\
\hline 0.604933 & 1.46969 & 0.275106 & 0.823344 & 0.201074 & 2.43444 & 0.342894 \\
\hline 0.573879 & 1.38364 & 0.24771 & 0.796944 & 0.196706 & 2.29733 & 0.319785 \\
\hline 0.525416 & 1.25249 & 0.205545 & 0.739779 & 0.183993 & 2.07287 & 0.280669 \\
\hline 0.462485 & 1.12347 & 0.17336 & 0.678028 & 0.160496 & 1.8072 & 0.247623 \\
\hline 0.391206 & 0.99204 & 0.14253 & 0.605555 & 0.142253 & 1.52015 & 0.197365 \\
\hline 0.336138 & 0.870748 & 0.117453 & 0.527486 & 0.113274 & 1.22294 & 0.149434 \\
\hline 0.306971 & 0.782109 & 0.0910478 & 0.466042 & 0.0956164 & 0.980306 & 0.132817 \\
\hline 0.286734 & 0.72466 & 0.078848 & 0.419074 & 0.0818846 & 0.783347 & 0.108273 \\
\hline 0.238991 & 0.709886 & 0.0653528 & 0.390516 & 0.0716093 & 0.640875 & 0.0836078 \\
\hline 0.194521 & 0.738804 & 0.0686709 & 0.377867 & 0.0725376 & 0.559247 & 0.0646695 \\
\hline 0.190783 & 0.853126 & 0.0828101 & 0.382455 & 0.070806 & 0.521708 & 0.0562581 \\
\hline 0.217264 & 1.10376 & 0.129987 & 0.414189 & 0.0756218 & 0.554832 & 0.0652626 \\
\hline 0.203042 & 1.55542 & 0.209171 & 0.500352 & 0.10054 & 0.677424 & 0.086533 \\
\hline 0.253781 & 2.34215 & 0.345329 & 0.66796 & 0.135826 & 0.923079 & 0.119677 \\
\hline 0.317715 & 3.64376 & 0.572464 & 0.937576 & 0.199493 & 1.34633 & 0.184922 \\
\hline 0.383592 & 5.62537 & 0.926661 & 1.37171 & 0.313344 & 2.04834 & 0.280934 \\
\hline 0.542937 & 8.44253 & 1.40865 & 2.01437 & 0.450914 & 3.06845 & 0.447785 \\
\hline 0.750873 & 12.2101 & 2.04905 & 2.92299 & 0.661356 & 4.50316 & 0.660096 \\
\hline 1.06537 & 16.8529 & 2.87702 & 4.131 & 0.950293 & 6.36087 & 0.935287 \\
\hline 1.38095 & 22.1276 & 3.79694 & 5.59828 & 1.30941 & 8.61156 & 1.2528 \\
\hline 1.78915 & 27.6748 & 4.74677 & 7.25256 & 1.70152 & 11.1332 & 1.63472 \\
\hline 2.17842 & 32.803 & 5.67446 & 8.93467 & 2.13338 & 13.7215 & 2.0616 \\
\hline 2.53349 & 36.8195 & 6.43405 & 10.4855 & 2.51131 & 15.9906 & 2.38812 \\
\hline 2.90862 & 39.2923 & 6.87822 & 11.7283 & 2.79938 & 17.711 & 2.6499 \\
\hline 3.05502 & 39.784 & 6.93486 & 12.4307 & 2.97403 & 18.6586 & 2.72887 \\
\hline 3.02898 & 38.2154 & 6.61746 & 12.5689 & 3.00386 & 18.7991 & 2.77821 \\
\hline 2.94936 & 34.884 & 6.02732 & 12.1025 & 2.89787 & 17.9197 & 2.65871 \\
\hline 2.73256 & 30.31 & 5.26192 & 11.0648 & 2.64949 & 16.2397 & 2.39585 \\
\hline 2.43959 & 25.0975 & 4.37385 & 9.63293 & 2.29325 & 14.0038 & 2.06507 \\
\hline 2.08148 & 19.9156 & 3.4448 & 8.01363 & 1.91122 & 11.6049 & 1.70604 \\
\hline 1.69195 & 15.2515 & 2.60462 & 6.41742 & 1.51561 & 9.18915 & 1.3531 \\
\hline 1.3427 & 11.3054 & 1.90058 & 4.96812 & 1.18166 & 7.02319 & 1.03108 \\
\hline 1.01744 & 8.21728 & 1.34788 & 3.73584 & 0.872079 & 5.20804 & 0.758673 \\
\hline 0.81116 & 5.97628 & 0.958682 & 2.79073 & 0.639089 & 3.78015 & 0.571447 \\
\hline 0.640996 & 4.35746 & 0.684798 & 2.07037 & 0.456461 & 2.73882 & 0.392251 \\
\hline 0.500362 & 3.18835 & 0.486381 & 1.54169 & 0.334216 & 1.99315 & 0.292056 \\
\hline 0.396618 & 2.35431 & 0.334799 & 1.15663 & 0.247063 & 1.44732 & 0.204247 \\
\hline 0.304363 & 1.71533 & 0.229184 & 0.891641 & 0.187674 & 1.06649 & 0.151268 \\
\hline 0.270885 & 1.2448 & 0.163976 & 0.711917 & 0.145544 & 0.810432 & 0.110011 \\
\hline 0.238037 & 0.899874 & 0.113025 & 0.575207 & 0.109165 & 0.616842 & 0.0809196 \\
\hline 0.227721 & 0.67836 & 0.0847103 & 0.462546 & 0.0813608 & 0.480845 & 0.0573667 \\
\hline 0.200637 & 0.553018 & 0.0745453 & 0.394225 & 0.0726806 & 0.374114 & 0.048181 \\
\hline 0.202568 & 0.519776 & 0.0460902 & 0.335109 & 0.0618823 & 0.301669 & 0.0419586 \\
\hline
\end{tabular}




\begin{tabular}{|c|c|c|c|c|c|c|}
\hline 0.201751 & 0.564538 & 0.0558998 & 0.308778 & 0.059213 & 0.251713 & 314285 \\
\hline 0.178575 & .652739 & 0.0792542 & 0.296745 & 0.0584095 & 0.221957 & 0.0219746 \\
\hline 0.154082 & 0.748911 & 0.0692138 & 0.290096 & 0.0541415 & 0.196997 & 0.0230328 \\
\hline 0.160857 & .836583 & 0.0823285 & 0.289686 & 0.0512471 & 0.188548 & 0.0191509 \\
\hline 0.172921 & 0.909331 & 0.0913932 & 0.284501 & 0.0517361 & 0.18424 & 0.0232631 \\
\hline 0.190444 & 0.946955 & 0.082618 & 0.284743 & 0.0553594 & 0.188152 & 0.0245834 \\
\hline 0.182423 & 0.948749 & 0.0801244 & 0.284315 & 0.0601538 & 0.188173 & 0.0251011 \\
\hline 0.174235 & 0.92243 & 0.0838813 & 0.302104 & 0.0555074 & 0.195288 & 0.0244242 \\
\hline 0.19396 & 0.882207 & 0.0803486 & 0.295961 & 0.0512778 & 0.200075 & 0.0224616 \\
\hline 0.189818 & 0.82019 & 0.0688446 & 0.298639 & 0.0538295 & 0.202626 & 0.021926 \\
\hline 0.19456 & 0.750233 & 0.0724076 & 0.29363 & 0.0594645 & 0.201318 & 0.0204065 \\
\hline 0.179661 & 0.686684 & 0.0668843 & 0.286089 & 0.0564176 & 0.20144 & 0.0263339 \\
\hline 0.188216 & 0.630613 & 0.0597287 & 0.276777 & 0.0602158 & 0.197753 & 0.0275165 \\
\hline 0.165452 & 0.583034 & 0.0517372 & 0.274425 & 0.054987 & 0.190334 & 0.0316435 \\
\hline 0.172059 & 0.539362 & 0.0503024 & 0.262882 & 0.0483742 & 0.186336 & 0.0300205 \\
\hline 0.168784 & 0.506829 & 0.0425248 & 0.253696 & 0.0545172 & 0.178588 & 0.0281629 \\
\hline 0.167617 & 0.493019 & 0.0464127 & 0.247937 & 0.0506259 & 0.176021 & 0.0261969 \\
\hline 0.163091 & 0.480785 & 0.0498256 & 0.238044 & 0.0445792 & 0.169505 & 0.0240613 \\
\hline 0.1701 & 0.468171 & 0.0500392 & 0.238755 & 0.0444248 & 0.168879 & 0.0265156 \\
\hline 0.155226 & 0.460166 & 0.0442455 & 0.235559 & 0.0426678 & 0.166745 & 0.0286308 \\
\hline 0.16378 & 0.463103 & 0.044424 & 0.229565 & 0.0416682 & 0.160602 & 0.0215446 \\
\hline 0.157392 & 0.463384 & 0.0421288 & 0.229104 & 0.0427841 & 0.161322 & 2841 \\
\hline 0.167363 & 0.465225 & 0.0441868 & 0.228669 & 0.0433415 & 0.159296 & 0.0187902 \\
\hline 0.159707 & 0.472918 & 0.0429315 & 0.230049 & 0.0445107 & 0.155979 & 0.0190128 \\
\hline 0.176953 & 0.4846 & 0.0576437 & 0.234786 & 0.040864 & 0.155041 & 0.0186474 \\
\hline 0.176211 & 0.500134 & 0.0579359 & 0.237706 & 0.043393 & 0.155096 & 0.0159533 \\
\hline 0.162876 & 0.548624 & 0.0417728 & 0.24953 & 0.0446532 & 0.156999 & 0.0186099 \\
\hline 0.150336 & 0.621829 & 0.0578389 & 0.263707 & 0.0492235 & 0.15732 & 0.018254 \\
\hline 0.154159 & 0.733151 & 0.0755131 & 0.289869 & 0.053361 & 0.159332 & 0.0190356 \\
\hline 0.165447 & 0.901361 & 0.0945223 & 0.328578 & 0.0633153 & 0.172497 & 4035 \\
\hline 0.167305 & 1.10062 & 0.120343 & 0.384146 & 0.0750933 & 0.189605 & 0.0225377 \\
\hline 0.173985 & 1.36621 & 0.167179 & 0.455441 & 0.0899772 & 0.208135 & 0.0249998 \\
\hline 0.210932 & 1.65545 & 0.215623 & 0.538877 & 0.104358 & 0.227542 & 0.0282125 \\
\hline 0.232875 & 1.95597 & 0.253286 & 0.6445 & 0.131263 & 0.247282 & 0.0275734 \\
\hline 0.267814 & 2.23596 & 0.286937 & 0.753832 & 0.160839 & 0.274658 & 0.0316162 \\
\hline 0.315838 & 2.49923 & 0.34824 & 0.853796 & 0.186434 & 0.302387 & 0.0380221 \\
\hline 0.356849 & 2.66634 & 0.360185 & 0.946176 & 0.20335 & 0.319498 & 0.0414578 \\
\hline 0.369412 & 2.73463 & 0.357234 & 1.01835 & 0.222931 & 0.339123 & 0.0430333 \\
\hline 0.379433 & 2.69635 & 0.355114 & 1.05405 & 0.231485 & 0.348079 & 0.0434706 \\
\hline 0.390821 & 2.56921 & 0.342125 & 1.05275 & 0.22621 & 0.348633 & 0.0438207 \\
\hline 0.378106 & 2.34373 & 0.327446 & 1.02065 & 0.227367 & 0.339182 & 0.043324 \\
\hline 0.346855 & 2.0774 & 0.266492 & 0.954176 & 0.204884 & 0.317827 & 0.0394036 \\
\hline 0.316508 & 1.79716 & 0.211083 & 0.863824 & 0.179553 & 0.293644 & 0.0393016 \\
\hline 0.288181 & 1.50629 & 0.183903 & 0.759076 & 0.163811 & 0.27412 & 0.0367155 \\
\hline 0.249607 & 1.24398 & 0.148201 & 0.649748 & 0.132611 & 0.251693 & 0.0296308 \\
\hline 0.231684 & 1.0316 & 0.10605 & 0.553196 & 0.105403 & 0.220747 & 0.0287586 \\
\hline 0.211538 & 0.869455 & 0.0817224 & 0.477328 & 0.094372 & 0.201049 & 0.0234934 \\
\hline
\end{tabular}




\begin{tabular}{|c|c|c|c|c|c|c|}
\hline 0.208586 & 0.748016 & 0.0815606 & 0.410089 & 0.081813 & 0.187498 & 0.0201103 \\
\hline 0.195352 & 0.657011 & 0.0618082 & 0.357823 & 0.072386 & 0.175309 & 0.0197475 \\
\hline 0.178548 & 0.600478 & 0.0627628 & 0.327543 & 0.0628895 & 0.168888 & 0.022516 \\
\hline 0.168031 & 0.566817 & 0.0577855 & 0.304567 & 0.0550219 & 0.164991 & 0.0229972 \\
\hline 0.154599 & 0.552668 & 0.0524753 & 0.286818 & 0.0539314 & 0.161584 & 0.0209612 \\
\hline 0.161134 & 0.537422 & 0.0458312 & 0.281349 & 0.0519544 & 0.158096 & 0.0186029 \\
\hline 0.157125 & 0.518776 & 0.0529291 & 0.280024 & 0.0534809 & 0.159332 & 0.0186319 \\
\hline 0.15934 & 0.510149 & 0.0544202 & 0.279765 & 0.0532175 & 0.159608 & 0.0193065 \\
\hline 0.165537 & 0.491147 & 0.0503137 & 0.273552 & 0.0519362 & 0.15552 & 0.0160795 \\
\hline 0.171677 & 0.481904 & 0.0469151 & 0.264783 & 0.0452813 & 0.15792 & 0.0165566 \\
\hline 0.153937 & 0.46683 & 0.0459422 & 0.260609 & 0.0455169 & 0.157954 & 0.0214131 \\
\hline 0.147496 & 0.457897 & 0.0440893 & 0.25744 & 0.0481641 & 0.155008 & 0.0190706 \\
\hline 0.145908 & 0.442727 & 0.0399623 & 0.254546 & 0.0464386 & 0.155099 & 0.0166795 \\
\hline 0.164254 & 0.439813 & 0.0444369 & 0.252066 & 0.0465109 & 0.154524 & 0.0178563 \\
\hline 0.176856 & 0.452786 & 0.0434794 & 0.249115 & 0.0459794 & 0.157444 & 0.0157116 \\
\hline 0.17255 & 0.476643 & 0.0449971 & 0.251344 & 0.0486539 & 0.160992 & 0.0130008 \\
\hline 0.18349 & 0.511202 & 0.0545458 & 0.259534 & 0.0484861 & 0.163179 & 0.015409 \\
\hline 0.202083 & 0.557362 & 0.061634 & 0.26881 & 0.050898 & 0.174221 & 0.0177616 \\
\hline 0.263991 & 0.648317 & 0.0732325 & 0.279578 & 0.0525047 & 0.19107 & 0.0188579 \\
\hline 0.345202 & 0.765752 & 0.0776672 & 0.302499 & 0.0521145 & 0.205246 & 0.0223158 \\
\hline 0.435045 & 0.900999 & 0.0979088 & 0.335623 & 0.0578388 & 0.225712 & 0.0246639 \\
\hline 0.610406 & 1.07931 & 0.128565 & 0.37266 & 0.0666694 & 0.262959 & 0.0312059 \\
\hline 0.859032 & 1.29066 & 0.164403 & 0.409914 & 0.0785477 & 0.306288 & 0.0339434 \\
\hline 1.16661 & 1.54737 & 0.198104 & 0.454475 & 0.0885999 & 0.35881 & 0.0408006 \\
\hline 1.50178 & 1.79685 & 0.252396 & 0.512448 & 0.0979466 & 0.423893 & 0.0511654 \\
\hline 1.89809 & 2.0345 & 0.293679 & 0.573939 & 0.109909 & 0.487563 & 0.0609923 \\
\hline 2.36437 & 2.28126 & 0.334839 & 0.63857 & 0.126426 & 0.548578 & 0.0711326 \\
\hline 2.77441 & 2.45143 & 0.351943 & 0.69737 & 0.138002 & 0.612241 & 0.0782725 \\
\hline 3.15346 & 2.57729 & 0.385454 & 0.739495 & 0.144443 & 0.67392 & 0.0726579 \\
\hline 3.46552 & 2.60156 & 0.387041 & 0.775923 & 0.150823 & 0.713847 & 0.0828939 \\
\hline 3.63319 & 2.57066 & 0.371819 & 0.79309 & 0.152345 & 0.734847 & 0.0924278 \\
\hline 3.65226 & 2.47152 & 0.355611 & 0.792396 & 0.149758 & 0.737372 & 0.0841013 \\
\hline 3.47961 & 2.32149 & 0.303208 & 0.779075 & 0.147059 & 0.722181 & 0.0835424 \\
\hline 3.18836 & 2.18482 & 0.259645 & 0.752909 & 0.139801 & 0.68891 & 0.0888289 \\
\hline 2.87428 & 2.06505 & 0.236225 & 0.720224 & 0.13796 & 0.64479 & 0.0874173 \\
\hline 2.55533 & 1.97998 & 0.194747 & 0.693174 & 0.139398 & 0.608436 & 0.078461 \\
\hline 2.20692 & 1.96384 & 0.175529 & 0.679532 & 0.133124 & 0.584037 & 0.0773579 \\
\hline 1.83612 & 1.99468 & 0.164553 & 0.666186 & 0.128026 & 0.56717 & 0.0813974 \\
\hline 1.59583 & 2.07006 & 0.174311 & 0.671707 & 0.132292 & 0.56766 & 0.0839056 \\
\hline 1.4796 & 2.17723 & 0.194131 & 0.693818 & 0.135742 & 0.584783 & 0.0934254 \\
\hline 1.44991 & 2.28556 & 0.195554 & 0.726679 & 0.146817 & 0.606949 & 0.104334 \\
\hline 1.56628 & 2.36676 & 0.215895 & 0.767673 & 0.159611 & 0.6368 & 0.112589 \\
\hline 1.68866 & 2.40594 & 0.209415 & 0.791789 & 0.162727 & 0.670722 & 0.124168 \\
\hline 1.73552 & 2.41933 & 0.208862 & 0.818673 & 0.171734 & 0.690981 & 0.126026 \\
\hline 1.81636 & 2.36217 & 0.211076 & 0.839346 & 0.179995 & 0.693454 & 0.126251 \\
\hline 1.8523 & 2.24518 & 0.203679 & 0.832831 & 0.174747 & 0.686935 & 0.128935 \\
\hline 1.8061 & 2.10134 & 0.186873 & 0.816741 & 0.170884 & 0.669041 & 0.121772 \\
\hline
\end{tabular}




\begin{tabular}{|c|c|c|c|c|c|c|}
\hline 1.70688 & 1.93437 & 0.161422 & 0.788875 & 0.167475 & 0.63417 & 0.112344 \\
\hline 1.55182 & 1.78388 & 0.140806 & 0.744311 & 0.152752 & 0.596299 & 0.103505 \\
\hline 1.37442 & 1.63516 & 0.136441 & 0.696915 & 0.142706 & 0.552556 & 0.087516 \\
\hline 1.21717 & 1.51364 & 0.117082 & 0.653203 & 0.130915 & 0.504783 & 0.0736265 \\
\hline 1.0319 & 1.41792 & 0.130858 & 0.606419 & 0.117693 & 0.4587 & 0.0662973 \\
\hline 0.871395 & 1.35424 & 0.154494 & 0.571357 & 0.111086 & 0.417985 & 0.0577794 \\
\hline 0.738234 & 1.32246 & 0.153872 & 0.539422 & 0.0964943 & 0.389124 & 0.0499428 \\
\hline 0.606248 & 1.29738 & 0.176582 & 0.512652 & 0.0904162 & 0.360146 & 0.0419667 \\
\hline 0.520106 & 1.30093 & 0.205855 & 0.49515 & 0.0863253 & 0.336816 & 0.0367855 \\
\hline 0.432181 & 1.29498 & 0.212551 & 0.478322 & 0.0790497 & 0.318 & 0.0313562 \\
\hline 0.330893 & 1.28183 & 0.226583 & 0.466585 & 0.0802231 & 0.302196 & 0.0281696 \\
\hline 0.294268 & 1.24224 & 0.236544 & 0.452354 & 0.0802325 & 0.285984 & 0.0275213 \\
\hline 0.235627 & 1.20135 & 0.23554 & 0.4412 & 0.0760733 & 0.267445 & 0.0318281 \\
\hline 0.216414 & 1.1731 & 0.220129 & 0.431418 & 0.0755095 & 0.255995 & 0.0331037 \\
\hline 0.227346 & 1.11667 & 0.211236 & 0.416802 & 0.0741999 & 0.244472 & 0.02852 \\
\hline 0.195806 & 1.0483 & 0.187765 & 0.402647 & 0.0708396 & 0.231759 & 0.0282533 \\
\hline 0.179996 & 0.980288 & 0.177256 & 0.387008 & 0.0743008 & 0.220505 & 0.0256265 \\
\hline 0.179312 & 0.918492 & 0.157258 & 0.374476 & 0.0730607 & 0.212796 & 0.0249635 \\
\hline 0.177999 & 0.863146 & 0.134505 & 0.363614 & 0.0696689 & 0.201451 & 0.0216684 \\
\hline 0.165103 & 0.808613 & 0.109214 & 0.357273 & 0.069115 & 0.191564 & 0.0226112 \\
\hline 0.161259 & 0.763783 & 0.0965207 & 0.351541 & 0.0707793 & 0.185493 & 0.0237966 \\
\hline 0.165491 & 0.724996 & 0.085409 & 0.346203 & 0.0713323 & 0.184153 & 0.0229235 \\
\hline 0.160775 & 0.696142 & 0.073047 & 0.33978 & 0.0681469 & 0.186644 & 0.022013 \\
\hline 0.155469 & 0.688602 & 0.057198 & 0.335277 & 0.0656545 & 0.183802 & 0.0239503 \\
\hline 0.15816 & 0.67567 & 0.0657209 & 0.344636 & 0.0734224 & 0.182462 & 0.0222661 \\
\hline 0.167998 & 0.668129 & 0.0633623 & 0.348353 & 0.0751263 & 0.188272 & 0.0213031 \\
\hline 0.147215 & 0.678123 & 0.0638557 & 0.35004 & 0.072992 & 0.194529 & 0.0223557 \\
\hline 0.133963 & 0.695654 & 0.0665904 & 0.362858 & 0.0772024 & 0.204572 & 0.0221784 \\
\hline 0.159267 & 0.707233 & 0.0592568 & 0.37102 & 0.0766269 & 0.219833 & 0.0266239 \\
\hline 0.169077 & 0.728111 & 0.0620194 & 0.383261 & 0.0779693 & 0.240046 & 0.0305457 \\
\hline 0.184832 & 0.757625 & 0.0730086 & 0.398179 & 0.0797105 & 0.276417 & 0.0374056 \\
\hline 0.184231 & 0.808231 & 0.0911439 & 0.42905 & 0.0867582 & 0.334225 & 0.0454802 \\
\hline 0.187061 & 0.888503 & 0.104915 & 0.472992 & 0.104659 & 0.414789 & 0.0582545 \\
\hline 0.203635 & 0.989288 & 0.147048 & 0.535061 & 0.126221 & 0.533476 & 0.0754359 \\
\hline 0.232789 & 1.12715 & 0.197747 & 0.622159 & 0.158303 & 0.707991 & 0.103437 \\
\hline 0.273296 & 1.30614 & 0.250731 & 0.738947 & 0.206158 & 0.952198 & 0.144358 \\
\hline 0.333704 & 1.54525 & 0.327318 & 0.892795 & 0.271183 & 1.26327 & 0.189557 \\
\hline 0.421772 & 1.80915 & 0.428082 & 1.10512 & 0.366338 & 1.64555 & 0.251925 \\
\hline 0.493954 & 2.07187 & 0.525099 & 1.34861 & 0.4697 & 2.09529 & 0.323979 \\
\hline 0.615027 & 2.34832 & 0.6212 & 1.60521 & 0.581613 & 2.60486 & 0.400865 \\
\hline 0.726601 & 2.60798 & 0.708442 & 1.8853 & 0.693239 & 3.14987 & 0.488037 \\
\hline 0.858675 & 2.80599 & 0.783777 & 2.17807 & 0.820622 & 3.67608 & 0.576976 \\
\hline 0.98145 & 2.96101 & 0.834631 & 2.42995 & 0.921284 & 4.11252 & 0.63827 \\
\hline 1.04404 & 3.01803 & 0.867073 & 2.61347 & 1.01394 & 4.43672 & 0.697568 \\
\hline 1.05571 & 2.99844 & 0.868046 & 2.73104 & 1.07224 & 4.64042 & 0.727157 \\
\hline 1.05319 & 2.87266 & 0.813532 & 2.77487 & 1.09063 & 4.66712 & 0.726697 \\
\hline 1.0447 & 2.66509 & 0.730663 & 2.73677 & 1.065 & 4.52328 & 0.714358 \\
\hline
\end{tabular}




\begin{tabular}{|c|c|c|c|c|c|c|}
\hline 1.02438 & 2.41506 & 0.641119 & 2.59692 & 0.998794 & 4.22464 & 0.655836 \\
\hline 0.960508 & 2.13711 & 0.538906 & 2.38771 & 0.914769 & 3.81116 & 0.598718 \\
\hline 0.834119 & 1.86687 & 0.450141 & 2.14531 & 0.811562 & 3.30921 & 0.522871 \\
\hline 0.725458 & 1.61422 & 0.35758 & 1.86289 & 0.679517 & 2.77818 & 0.43674 \\
\hline 0.664389 & 1.39522 & 0.281396 & 1.57529 & 0.548794 & 2.25347 & 0.343531 \\
\hline 0.53966 & 1.22077 & 0.190838 & 1.3253 & 0.437114 & 1.77442 & 0.279358 \\
\hline 0.404033 & 1.091 & 0.14244 & 1.10463 & 0.338937 & 1.35896 & 0.215263 \\
\hline 0.322197 & 1.02984 & 0.130927 & 0.916149 & 0.262771 & 1.02998 & 0.154315 \\
\hline 0.26498 & 0.999771 & 0.104177 & 0.777405 & 0.203518 & 0.766878 & 0.117494 \\
\hline 0.200738 & 1.02287 & 0.113112 & 0.680333 & 0.161343 & 0.569959 & 0.0865421 \\
\hline 0.191812 & 1.0728 & 0.10621 & 0.622553 & 0.14084 & 0.440766 & 0.0661403 \\
\hline 0.196447 & 1.16356 & 0.123774 & 0.585168 & 0.123361 & 0.353883 & 0.0472697 \\
\hline 0.185007 & 1.32398 & 0.152098 & 0.577926 & 0.117987 & 0.302526 & 0.0379307 \\
\hline 0.158504 & 1.52816 & 0.164754 & 0.600636 & 0.125148 & 0.285166 & 0.0366403 \\
\hline 0.16518 & 1.83986 & 0.200462 & 0.641628 & 0.14006 & 0.285903 & 0.0339081 \\
\hline 0.165343 & 2.28161 & 0.241063 & 0.711369 & 0.150221 & 0.304102 & 0.032995 \\
\hline 0.176781 & 2.97141 & 0.312235 & 0.815179 & 0.171888 & 0.343121 & 0.0412645 \\
\hline 0.166894 & 4.05505 & 0.440543 & 0.966786 & 0.203758 & 0.394629 & 0.0522589 \\
\hline 0.171295 & 5.74313 & 0.628074 & 1.19566 & 0.251982 & 0.46533 & 0.0567554 \\
\hline 0.204379 & 8.38141 & 0.917134 & 1.54095 & 0.334885 & 0.55987 & 0.0655621 \\
\hline 0.233865 & 12.4056 & 1.33707 & 2.05498 & 0.459993 & 0.678172 & 0.0839173 \\
\hline 0.268688 & 18.4923 & 1.97796 & 2.83072 & 0.620686 & 0.804052 & 0.103555 \\
\hline 0.276409 & 27.298 & 2.95891 & 4.02139 & 0.881526 & 0.972153 & 0.126149 \\
\hline 0.306787 & 39.7666 & 4.34894 & 5.73171 & 1.26589 & 1.19256 & 0.155451 \\
\hline 0.387316 & 56.6119 & 6.22428 & 8.16561 & 1.81362 & 1.46168 & 0.188494 \\
\hline 0.475231 & 78.3704 & 8.6131 & 11.5597 & 2.59571 & 1.81243 & 0.23374 \\
\hline 0.575863 & 105.103 & 11.6619 & 16.0411 & 3.62427 & 2.23488 & 0.281961 \\
\hline 0.706292 & 136.663 & 15.0444 & 21.7353 & 4.9341 & 2.73948 & 0.354119 \\
\hline 0.873879 & 171.932 & 18.8688 & 28.7174 & 6.52448 & 3.34365 & 0.43364 \\
\hline 1.06766 & 208.599 & 22.9286 & 36.7636 & 8.36496 & 4.02534 & 0.514927 \\
\hline 1.28853 & 244.311 & 26.762 & 45.6023 & 10.41 & 4.75021 & 0.61474 \\
\hline 1.48078 & 276.027 & 30.1598 & 54.7337 & 12.4443 & 5.4706 & 0.708985 \\
\hline 1.7154 & 300.771 & 33.0485 & 63.4784 & 14.4096 & 6.17501 & 0.794177 \\
\hline 1.91459 & 315.942 & 34.5749 & 71.1436 & 16.1931 & 6.76637 & 0.889364 \\
\hline 2.121 & 319.66 & 34.904 & 76.9843 & 17.549 & 7.16651 & 0.958337 \\
\hline 2.21995 & 311.934 & 34.1207 & 80.3603 & 18.2882 & 7.36714 & 0.981657 \\
\hline 2.1869 & 293.258 & 31.9574 & 80.8958 & 18.4044 & 7.31548 & 0.968255 \\
\hline 2.13807 & 265.994 & 29.1223 & 78.6998 & 17.9533 & 7.00791 & 0.933576 \\
\hline 1.98354 & 232.175 & 25.4698 & 73.871 & 16.7763 & 6.48629 & 0.866342 \\
\hline 1.81087 & 195.567 & 21.5322 & 66.8446 & 15.1272 & 5.83097 & 0.764528 \\
\hline 1.63395 & 158.707 & 17.3056 & 58.3529 & 13.2221 & 5.07112 & 0.660088 \\
\hline 1.38569 & 124.291 & 13.6039 & 49.1925 & 11.1685 & 4.26023 & 0.555368 \\
\hline 1.14481 & 94.0059 & 10.2653 & 40.0522 & 9.11193 & 3.46254 & 0.44974 \\
\hline 0.939361 & 68.5629 & 7.55858 & 31.5075 & 7.16621 & 2.72086 & 0.35341 \\
\hline 0.7321 & 48.3875 & 5.21479 & 23.9645 & 5.44275 & 2.07515 & 0.27 \\
\hline 0.585496 & 33.1661 & 3.61575 & 17.6288 & 3.98897 & 1.53274 & 0.197927 \\
\hline 0.476776 & 22.1363 & 2.4503 & 12.5612 & 2.85286 & 1.12479 & 0.144973 \\
\hline
\end{tabular}




\begin{tabular}{|c|c|c|c|c|c|c|}
\hline 0.36452 & 14.5889 & 1.59693 & 8.68895 & 1.97818 & 0.815594 & 0.108366 \\
\hline 0.295287 & 9.6333 & 1.05062 & 5.8865 & 1.33105 & 0.586025 & 0.0806417 \\
\hline 0.251891 & 6.59916 & 0.701319 & 3.93434 & 0.895154 & 0.436818 & 0.0551451 \\
\hline 0.233237 & 4.90536 & 0.518745 & 2.65832 & 0.606203 & 0.339204 & 0.0381174 \\
\hline 0.202658 & 4.15864 & 0.459353 & 1.86528 & 0.421588 & 0.283039 & 0.0331798 \\
\hline 0.195601 & 4.12452 & 0.436774 & 1.42217 & 0.31767 & 0.248812 & 0.0323331 \\
\hline 0.195085 & 4.66599 & 0.507012 & 1.2266 & 0.274567 & 0.235329 & 0.030931 \\
\hline 0.179603 & 5.76921 & 0.640019 & 1.21364 & 0.270153 & 0.240601 & 0.0302998 \\
\hline 0.184354 & 7.4146 & 0.846357 & 1.37688 & 0.301387 & 0.257355 & 0.0273158 \\
\hline 0.222827 & 9.58861 & 1.06609 & 1.66927 & 0.36385 & 0.291364 & 0.0324125 \\
\hline 0.234451 & 12.3926 & 1.38606 & 2.09677 & 0.443474 & 0.332689 & 0.0378496 \\
\hline 0.222675 & 15.8511 & 1.76991 & 2.68469 & 0.572698 & 0.387243 & 0.045733 \\
\hline 0.225669 & 19.8973 & 2.1997 & 3.43709 & 0.743404 & 0.460696 & 0.0552112 \\
\hline 0.23536 & 24.4516 & 2.76818 & 4.35069 & 0.931736 & 0.544223 & 0.0668222 \\
\hline 0.257291 & 29.0733 & 3.26394 & 5.39723 & 1.15401 & 0.650716 & 0.0824423 \\
\hline 0.273345 & 33.6665 & 3.76085 & 6.56132 & 1.4188 & 0.763744 & 0.0952968 \\
\hline 0.314878 & 37.8128 & 4.22402 & 7.77408 & 1.68735 & 0.86667 & 0.11285 \\
\hline 0.350744 & 41.1351 & 4.61502 & 8.93062 & 1.92661 & 0.964188 & 0.123027 \\
\hline 0.380592 & 43.2526 & 4.77553 & 9.97394 & 2.15403 & 1.05924 & 0.127202 \\
\hline 0.414932 & 43.9461 & 4.91366 & 10.7978 & 2.33133 & 1.1283 & 0.136751 \\
\hline 0.408291 & 43.3091 & 4.86113 & 11.3379 & 2.41757 & 1.16492 & 0.141304 \\
\hline 0.402337 & 41.1487 & 4.51635 & 11.5492 & 2.46503 & 1.18096 & 0.143656 \\
\hline 0.422955 & 37.8252 & 4.18775 & 11.3711 & 2.45167 & 1.15592 & 0.143971 \\
\hline 0.402062 & 33.6124 & 3.73391 & 10.8373 & 2.33584 & 1.09509 & 0.13961 \\
\hline 0.378027 & 28.9356 & 3.17754 & 9.98093 & 2.14808 & 1.01996 & 0.121146 \\
\hline 0.391215 & 23.9887 & 2.67446 & 8.88796 & 1.90285 & 0.916281 & 0.108973 \\
\hline 0.35582 & 19.2363 & 2.12334 & 7.68011 & 1.63662 & 0.8016 & 0.106865 \\
\hline 0.302892 & 14.9006 & 1.62061 & 6.42992 & 1.38391 & 0.694175 & 0.0911108 \\
\hline 0.269537 & 11.2031 & 1.24825 & 5.20791 & 1.11443 & 0.585694 & 0.0683918 \\
\hline 0.25632 & 8.16731 & 0.897816 & 4.08978 & 0.876545 & 0.494386 & 0.055018 \\
\hline 0.234812 & 5.75848 & 0.622969 & 3.11307 & 0.66669 & 0.412923 & 0.0468616 \\
\hline 0.215638 & 3.96282 & 0.425562 & 2.29899 & 0.490785 & 0.338997 & 0.0406233 \\
\hline 0.20956 & 2.65207 & 0.28083 & 1.64461 & 0.339924 & 0.285035 & 0.03493 \\
\hline 0.193767 & 1.75287 & 0.176816 & 1.15582 & 0.236008 & 0.246511 & 0.0301776 \\
\hline 0.18463 & 1.15255 & 0.11926 & 0.804753 & 0.167934 & 0.216011 & 0.0277268 \\
\hline 0.196863 & 0.75222 & 0.0616321 & 0.551646 & 0.114126 & 0.192769 & 0.0266002 \\
\hline 0.169753 & 0.506909 & 0.0481993 & 0.382109 & 0.0792216 & 0.180502 & 0.0253503 \\
\hline 0.167208 & 0.362724 & 0.0337335 & 0.269737 & 0.0553222 & 0.173193 & 0.0240138 \\
\hline 0.183412 & 0.281363 & 0.0238308 & 0.197681 & 0.0390885 & 0.167262 & 0.0221116 \\
\hline 0.18248 & 0.234124 & 0.0166445 & 0.15415 & 0.0289387 & 0.16438 & 0.0211213 \\
\hline 0.182121 & 0.20701 & 0.0192809 & 0.129995 & 0.0261451 & 0.166708 & 0.023508 \\
\hline 0.185889 & 0.197873 & 0.0172684 & 0.113788 & 0.0228414 & 0.170361 & 0.023664 \\
\hline 0.211282 & 0.191767 & 0.0154614 & 0.103413 & 0.0202582 & 0.169486 & 0.0227189 \\
\hline 0.200126 & 0.186963 & 0.0156058 & 0.0996979 & 0.0218506 & 0.168319 & 0.0253497 \\
\hline 0.199387 & 0.190523 & 0.0201605 & 0.0997846 & 0.0224863 & 0.16863 & 0.0231887 \\
\hline 0.209777 & 0.188627 & 0.0161383 & 0.0988547 & 0.0219518 & 0.171468 & 0.0245505 \\
\hline 0.194355 & 0.190322 & 0.0141799 & 0.0994506 & 0.0228482 & 0.173218 & 0.0282257 \\
\hline
\end{tabular}




\begin{tabular}{|c|c|c|c|c|c|c|}
\hline 0.198765 & 0.192102 & 0.0171661 & 0.0985683 & 0.0242527 & 0.177917 & 0.0261348 \\
\hline 0.204147 & 0.199308 & 0.0200549 & 0.0970648 & 0.0234554 & 0.183889 & 0.028003 \\
\hline .195397 & 0.199326 & 0.0204313 & 0.0992521 & 0.0214846 & 0.188298 & 0.0297735 \\
\hline .193201 & 0.205697 & 0.0238199 & 0.1025 & 0.0193513 & 0.194302 & 0.0296095 \\
\hline 209717 & 0.208873 & 0.0252635 & 0.103163 & 0.02199 & 0.203488 & 0.0335941 \\
\hline 0.206325 & 0.212258 & 0.0230074 & 0.103118 & 0.0237582 & 0.216905 & 0.0395995 \\
\hline 0.218796 & 0.218726 & 0.0204418 & 0.107149 & 0.0239229 & 0.237668 & 0.045287 \\
\hline 0.218917 & 0.229298 & 0.0258348 & 0.112688 & 0.0242674 & 0.271404 & 0.0595414 \\
\hline 0.234838 & 0.242397 & 0.0297456 & 0.118982 & 0.0269296 & 0.318512 & 0.0814513 \\
\hline 0.259214 & 0.261053 & 0.0334557 & 0.131209 & 0.0304205 & 0.387853 & 0.113254 \\
\hline 0.261253 & 0.284598 & 0.0426304 & 0.143373 & 0.0326001 & 0.485415 & 0.157354 \\
\hline 0.252833 & 0.315305 & 0.0495054 & 0.158145 & 0.0356143 & 0.620356 & 0.217699 \\
\hline 0.260103 & 0.353489 & 0.070448 & 0.183308 & 0.0423039 & 0.804346 & 0.300584 \\
\hline 0.290524 & 0.400307 & 0.0768463 & 0.209256 & 0.0494222 & 1.0354 & 0.413638 \\
\hline 0.332115 & 0.445493 & 0.0915985 & 0.242893 & 0.0599416 & 1.32885 & 0.544966 \\
\hline 0.374999 & 0.497081 & 0.115894 & 0.29083 & 0.0735528 & 1.68536 & 0.711647 \\
\hline 0.4306 & 0.553543 & 0.138522 & 0.334566 & 0.085441 & 2.09264 & 0.908915 \\
\hline .486546 & 0.607212 & 0.160115 & 0.383068 & 0.100335 & 2.53779 & 1.11493 \\
\hline 0.585383 & 0.654231 & 0.175781 & 0.436075 & 0.11 & 986 & 1.35028 \\
\hline 0.651267 & 0.700043 & 0.198575 & 0.489876 & 0.131949 & 3.45608 & 1.58117 \\
\hline 0.674209 & 0.733227 & 0.220587 & 0.537192 & 0.15018 & 3.85858 & 1.78246 \\
\hline 0.710393 & 0.741809 & 0.223575 & 0.5788 & 0.15 & 671 & 1.96204 \\
\hline 0.785517 & 0.735195 & 0.21643 & 0.602719 & 0.164185 & 4.43583 & 2.05594 \\
\hline 0.832314 & 0.721508 & 0.208029 & 0.615999 & 0.163966 & 4.5412 & 2.1197 \\
\hline 0.797494 & 0.691028 & 0.210182 & 0.617558 & 0.163707 & 4.50802 & 2.12386 \\
\hline 0.801526 & 0.650417 & 0.188036 & 0.600692 & 0.162793 & 4.33348 & 2.02995 \\
\hline 0.767366 & 0.59752 & 0.151934 & 0.568573 & 0.155573 & 4.05291 & 1.89721 \\
\hline 0.712771 & 0.548644 & 0.14319 & 0.531557 & 0.144132 & 3.70079 & 1.72652 \\
\hline 0.684434 & 0.489929 & 0.120367 & 0.486213 & 0.132595 & 3.25931 & 1.52366 \\
\hline 0.601243 & 0.435851 & 0.0950606 & 0.433719 & 0.111881 & 2.79441 & 1.2984 \\
\hline 0.559631 & 0.392 & 0.0759368 & 0.379602 & 0.0941799 & 2.33878 & 1.06292 \\
\hline 0.511091 & 0.358017 & 0.0599978 & 0.327227 & 0.0825611 & 1.9031 & 0.836695 \\
\hline 0.491934 & 0.327066 & 0.050012 & 0.278693 & 0.0688717 & 1.52923 & 0.663687 \\
\hline 0.484041 & 0.300183 & 0.044633 & 0.241794 & 0.0561971 & 1.20018 & 0.486578 \\
\hline 0.494985 & 0.287084 & 0.0325455 & 0.211209 & 0.0436469 & 0.92906 & 0.363366 \\
\hline 0.488875 & 0.277279 & 0.0230789 & 0.185504 & 0.0367456 & 0.719742 & 0.260875 \\
\hline 0.434322 & 0.273386 & 0.0256938 & 0.168382 & 0.0363774 & 0.574505 & 0.180483 \\
\hline 0.433709 & 0.267594 & 0.0245257 & 0.152881 & 0.0319647 & 0.46586 & 0.132756 \\
\hline 0.462747 & 0.26476 & 0.0251188 & 0.146346 & 0.0301913 & 0.394361 & 0.0901936 \\
\hline 0.454565 & 0.266132 & 0.0237251 & 0.140275 & 0.0281151 & 0.347223 & 0.0711231 \\
\hline 0.459256 & 0.264302 & 0.0227372 & 0.134861 & 0.0268965 & 0.308523 & 0.0570991 \\
\hline 0.469886 & 0.265198 & 0.0203939 & 0.134541 & 0.0289634 & 0.286747 & 0.0523803 \\
\hline 0.503883 & 0.26629 & 0.0192212 & 0.130591 & 0.0286315 & 0.276006 & 0.0436421 \\
\hline 0.467901 & 0.270475 & 0.0209615 & 0.135186 & 0.0276782 & 0.269635 & 0.0372518 \\
\hline 0.444926 & 0.272652 & 0.0223048 & 0.135444 & 0.0272935 & 0.266712 & 0.0328994 \\
\hline 0.480923 & 0.280089 & 0.0214074 & 0.133073 & 0.0294522 & 0.269315 & 0.0322772 \\
\hline 0.512567 & 0.281561 & 0.0202344 & 0.136929 & 0.0282287 & 0.276721 & 0.034798 \\
\hline
\end{tabular}




\begin{tabular}{|c|c|c|c|c|c|c|}
\hline 0.479486 & 0.285391 & 0.024223 & 0.141224 & 0.0306677 & 0.281628 & 0.0364699 \\
\hline 0.480046 & 0.292868 & 0.0222008 & 0.142641 & 0.0302105 & 0.296273 & 0.0425639 \\
\hline 0.448061 & 0.305886 & 0.0241197 & 0.147336 & 0.03441 & 0.317892 & 0.043517 \\
\hline .435445 & 0.310749 & 0.02553 & 0.153447 & 0.0357153 & 0.334809 & 0.045883 \\
\hline 0.440175 & 0.320321 & 0.0295099 & 0.163906 & 0.0352219 & 0.355043 & 0.0526614 \\
\hline 0.426905 & 0.331099 & 0.0237998 & 0.168019 & 0.0371316 & 0.384026 & 0.0593045 \\
\hline 0.424421 & 0.342707 & 0.0290757 & 0.174767 & 0.0410644 & 0.424184 & 0.0644552 \\
\hline 0.438124 & 0.361073 & 0.0314123 & 0.181982 & 0.0403164 & 0.467323 & 0.0768737 \\
\hline 0.411337 & 0.367519 & 0.0350936 & 0.193686 & 0.0476184 & 0.503158 & 0.0980425 \\
\hline 0.400695 & 0.381707 & 0.0388362 & 0.200576 & 0.0453254 & 0.553966 & 0.10603 \\
\hline 0.404137 & 0.399431 & 0.0391835 & 0.211658 & 0.051959 & 0.610267 & 0.128802 \\
\hline 0.379301 & 0.412799 & 0.048803 & 0.218419 & 0.0543121 & 0.679604 & 0.157047 \\
\hline 0.380649 & 0.429057 & 0.0531026 & 0.23048 & 0.0556503 & 0.740739 & 0.176684 \\
\hline 0.37955 & 0.448407 & 0.0580534 & 0.238617 & 0.0569567 & 0.808468 & 0.213261 \\
\hline 0.379863 & 0.460932 & 0.0627101 & 0.248458 & 0.0553872 & 0.888143 & 0.249111 \\
\hline 0.392551 & 0.473176 & 0.0689127 & 0.256474 & 0.0540786 & 0.959492 & 0.272503 \\
\hline 0.367919 & 0.483464 & 0.06043 & 0.268824 & 0.0535183 & 1.02434 & 0.303942 \\
\hline 0.394116 & 0.489441 & 0.0649455 & 0.269968 & 0.0531424 & 1.05911 & 0.317291 \\
\hline 0.384328 & 0.488788 & 0.067 & 0.27 & 0.0584762 & .08693 & 0.320969 \\
\hline 0.413025 & 0.492238 & 0.0731597 & 0.279351 & 0.0577313 & 1.09184 & 0.328278 \\
\hline 0.374756 & 0.490807 & 0.0672521 & 0.278822 & 0.0585405 & 1.07889 & 0.321111 \\
\hline 0.363063 & 0.483555 & 0.07 & 683 & 0.0533982 & 578 & 1555 \\
\hline 0.336077 & 0.475834 & 0.0714451 & 0.276643 & 0.057282 & 0.992051 & 0.284055 \\
\hline 0.358512 & 0.463601 & 0.0687355 & 0.269569 & 0.0539627 & 0.935675 & 0.264271 \\
\hline 0.353552 & 0.452255 & 0.0597076 & 0.259222 & 0.0483981 & 0.861229 & 0.231908 \\
\hline 0.362549 & 0.441546 & 0.0619708 & 0.250953 & 0.0436082 & 0.787663 & 0.208747 \\
\hline 0.355375 & 0.427858 & 0.0573399 & 0.23777 & 0.0462286 & 0.710921 & 0.173381 \\
\hline 0.306924 & 0.417899 & 0.059459 & 0.231577 & 0.0424421 & 0.644127 & 0.150495 \\
\hline 0.291642 & 0.411414 & 0.0600052 & 0.223293 & 0.0349385 & 0.586939 & 0.120758 \\
\hline 0.307576 & 0.399316 & 0.0543517 & 0.21192 & 0.0361263 & 0.532873 & 0.0980453 \\
\hline 0.310326 & 0.392742 & 0.0513102 & 0.205719 & 0.0373933 & 0.486785 & 0.0966524 \\
\hline 0.295856 & 0.385896 & 0.0493079 & 0.202022 & 0.0336453 & 0.451033 & 0.0837427 \\
\hline 0.291507 & 0.38234 & 0.0484484 & 0.198845 & 0.0340082 & 0.426003 & 0.0807738 \\
\hline 0.281027 & 0.376008 & 0.0526076 & 0.196344 & 0.0307973 & 0.40632 & 0.0821446 \\
\hline 0.275311 & 0.377478 & 0.0499922 & 0.195863 & 0.0325632 & 0.395132 & 0.0824815 \\
\hline 0.271227 & 0.376876 & 0.0471855 & 0.194074 & 0.0312663 & 0.382976 & 0.0781067 \\
\hline 0.275428 & 0.367235 & 0.0454157 & 0.190635 & 0.0306002 & 0.378464 & 0.0737101 \\
\hline 0.282193 & 0.364271 & 0.0456539 & 0.19569 & 0.0314604 & 0.380566 & 0.0756953 \\
\hline 0.274227 & 0.3582 & 0.0446466 & 0.194642 & 0.0307029 & 0.379737 & 0.0687589 \\
\hline 0.29159 & 0.35379 & 0.0419382 & 0.193704 & 0.0344392 & 0.377141 & 0.0701277 \\
\hline 0.269596 & 0.35258 & 0.0390377 & 0.200869 & 0.0369015 & 0.378963 & 0.0772261 \\
\hline 0.286023 & 0.347298 & 0.0441226 & 0.194122 & 0.0340023 & 0.374017 & 0.0741249 \\
\hline 0.283282 & 0.34595 & 0.0359601 & 0.198759 & 0.0336537 & 0.372275 & 0.0729559 \\
\hline 0.290009 & 0.34843 & 0.0333665 & 0.19851 & 0.0332483 & 0.377857 & 0.0700233 \\
\hline 0.317937 & 0.34726 & 0.0364751 & 0.192075 & 0.0364655 & 0.371953 & 0.0645912 \\
\hline 0.285453 & 0.341279 & 0.0360724 & 0.191477 & 0.0343068 & 0.362607 & 0.0690339 \\
\hline 0.28932 & 0.340011 & 0.0370476 & 0.195132 & 0.032419 & 0.367726 & 0.0596109 \\
\hline
\end{tabular}




\begin{tabular}{|c|c|c|c|c|c|c|}
\hline 0.283624 & 0.338374 & 0.0347176 & 0.194649 & 0.0315497 & 0.370765 & 0.0578565 \\
\hline 0.293823 & 0.339693 & 0.0385408 & 0.19054 & 0.0328529 & 0.366004 & 0.056336 \\
\hline 0.271934 & 0.338343 & 0.0312017 & 0.189129 & 0.0312662 & 0.364576 & 0.0541807 \\
\hline 0.29637 & 0.343967 & 0.0281184 & 0.189004 & 0.0365332 & 0.362606 & 0.0486983 \\
\hline 0.318947 & 0.349372 & 0.0315589 & 0.191492 & 0.0330416 & 0.361262 & 0.0508518 \\
\hline 0.296444 & 0.365015 & 0.0387823 & 0.195344 & 0.0294612 & 0.365807 & 0.0456158 \\
\hline 0.3435 & 0.375971 & 0.0431871 & 0.201712 & 0.0330712 & 0.365161 & 0.0409401 \\
\hline 0.329053 & 0.39779 & 0.0512116 & 0.207746 & 0.035293 & 0.379882 & 0.0469186 \\
\hline 0.397206 & 0.42352 & 0.0608825 & 0.217739 & 0.0442171 & 0.39297 & 0.0538143 \\
\hline 0.448652 & 0.453331 & 0.0727065 & 0.225836 & 0.0421401 & 0.422651 & 0.0514414 \\
\hline 0.428543 & 0.488401 & 0.0968175 & 0.246945 & 0.0519135 & 0.437036 & 0.0565618 \\
\hline 0.534778 & 0.53496 & 0.122317 & 0.267123 & 0.0618256 & 0.46998 & 0.0637013 \\
\hline 0.591345 & 0.571774 & 0.141024 & 0.292266 & 0.07088 & 0.510222 & 0.074709 \\
\hline 0.703508 & 0.608121 & 0.163888 & 0.322651 & 0.101284 & 0.54978 & 0.0862812 \\
\hline 0.794583 & 0.644719 & 0.185703 & 0.348094 & 0.110957 & 0.595116 & 0.0997288 \\
\hline 0.893829 & 0.673271 & 0.210287 & 0.384455 & 0.131852 & 0.637067 & 0.118732 \\
\hline 1.04419 & 0.692244 & 0.219829 & 0.423048 & 0.151405 & 0.678052 & 0.129087 \\
\hline 1.1206 & 0.712531 & 0.219171 & 0.442555 & 0.169149 & 0.710746 & 0.133774 \\
\hline 1.18026 & 0.725947 & 0.212831 & 0.459251 & 0.173803 & 0.740785 & 0.147253 \\
\hline 1.2682 & 0.723694 & 0.218333 & 0.480028 & 0.184704 & 0.752311 & 0.156063 \\
\hline 1.28299 & 0.702966 & 0.203847 & 0.486495 & 0.184246 & 0.769001 & 0.156362 \\
\hline 1.3078 & 0.681779 & 0.188145 & 0.492925 & 0.189847 & 0.765853 & 0.140092 \\
\hline 1.3373 & 0.654712 & 0.173303 & 0.480469 & 0.188491 & 0.744358 & 0.153085 \\
\hline 1.21149 & 0.62065 & 0.142377 & 0.461392 & 0.17055 & 0.715924 & 0.135296 \\
\hline 1.12294 & 0.596443 & 0.124155 & 0.453711 & 0.16779 & 0.691281 & 0.128685 \\
\hline 1.06681 & 0.568649 & 0.110463 & 0.417285 & 0.138445 & 0.652741 & 0.121729 \\
\hline 0.907683 & 0.53244 & 0.0886932 & 0.394842 & 0.128029 & 0.599265 & 0.10161 \\
\hline 0.831469 & 0.506824 & 0.0768048 & 0.366112 & 0.117147 & 0.567747 & 0.0833955 \\
\hline 0.692919 & 0.487438 & 0.0611439 & 0.3403 & 0.100132 & 0.537114 & 0.0810465 \\
\hline 0.625329 & 0.465248 & 0.048636 & 0.312692 & 0.0796104 & 0.497884 & 0.0721645 \\
\hline 0.548307 & 0.4484 & 0.043142 & 0.293246 & 0.0706107 & 0.4696 & 0.0634993 \\
\hline 0.460787 & 0.442317 & 0.0391011 & 0.278959 & 0.0658632 & 0.451119 & 0.0549394 \\
\hline 0.470242 & 0.436441 & 0.0373051 & 0.261205 & 0.0584194 & 0.430741 & 0.0601912 \\
\hline 0.396925 & 0.435844 & 0.0366166 & 0.251172 & 0.0560811 & 0.419792 & 0.0548619 \\
\hline 0.380844 & 0.429451 & 0.0346282 & 0.244436 & 0.0510566 & 0.413373 & 0.047838 \\
\hline 0.393427 & 0.429707 & 0.0349235 & 0.243172 & 0.0575648 & 0.405737 & 0.0469284 \\
\hline 0.378897 & 0.432711 & 0.0346092 & 0.232356 & 0.0479726 & 0.401545 & 0.0513337 \\
\hline 0.414616 & 0.440352 & 0.0416407 & 0.234629 & 0.0518663 & 0.401063 & 0.0463017 \\
\hline 0.381466 & 0.445277 & 0.0368202 & 0.232933 & 0.0567834 & 0.399191 & 0.044605 \\
\hline 0.330583 & 0.45152 & 0.0468856 & 0.231184 & 0.0551096 & 0.397759 & 0.0457713 \\
\hline 0.32476 & 0.456506 & 0.0457967 & 0.233435 & 0.0500724 & 0.390367 & 0.0445316 \\
\hline 0.353219 & 0.464406 & 0.0477797 & 0.235431 & 0.0587181 & 0.404338 & 0.0466189 \\
\hline 0.403963 & 0.464667 & 0.0454788 & 0.234016 & 0.0541465 & 0.396389 & 0.0473597 \\
\hline 0.340748 & 0.469363 & 0.0489783 & 0.238847 & 0.0561782 & 0.402124 & 0.0568162 \\
\hline 0.390254 & 0.480917 & 0.0526268 & 0.23891 & 0.0577274 & 0.412607 & 0.0532457 \\
\hline 0.361019 & 0.491975 & 0.0477667 & 0.239514 & 0.0542661 & 0.415621 & 0.0602198 \\
\hline 0.366686 & 0.500803 & 0.0508172 & 0.241133 & 0.0577705 & 0.416954 & 0.0590726 \\
\hline
\end{tabular}




\begin{tabular}{|c|c|c|c|c|c|c|}
\hline 0.334409 & 0.512036 & 0.057327 & 0.245288 & 0.0584729 & 0.415861 & 0.0583236 \\
\hline 0.334572 & 0.52361 & 0.0586562 & 0.253826 & 0.0572301 & 0.433366 & 0.0761684 \\
\hline 0.354397 & 0.534575 & 0.0611116 & 0.251648 & 0.0564633 & 0.440863 & 0.0859735 \\
\hline .404465 & 0.552252 & 0.05731 & 0.265902 & 0.0541382 & 0.448147 & 0.092416 \\
\hline 0.353417 & 0.571522 & 0.0627667 & 0.265077 & 0.0561717 & 0.462679 & 0.113081 \\
\hline 0.378221 & 0.592655 & 0.0619286 & 0.277657 & 0.057807 & 0.483372 & 0.123212 \\
\hline 0.385356 & 0.618338 & 0.0630686 & 0.286193 & 0.0621336 & 0.492613 & 0.136886 \\
\hline 0.423764 & 0.648172 & 0.0600547 & 0.300416 & 0.0563486 & 0.523379 & 0.153678 \\
\hline 0.466223 & 0.6902 & 0.0672015 & 0.316725 & 0.0555505 & 0.554373 & 0.163932 \\
\hline 0.462513 & 0.735999 & 0.0793012 & 0.340048 & 0.0582307 & 0.586894 & 0.172219 \\
\hline 0.492188 & 0.797919 & 0.0749817 & 0.364728 & 0.0570977 & 0.630933 & 0.176971 \\
\hline 0.539728 & 0.854376 & 0.083675 & 0.39714 & 0.0698721 & 0.684925 & 0.189104 \\
\hline 0.593269 & 0.921618 & 0.0919713 & 0.442137 & 0.074501 & 0.750045 & 0.177215 \\
\hline 0.681703 & 0.9799 & 0.102314 & 0.494166 & 0.0845099 & 0.822689 & 0.186468 \\
\hline 0.711132 & 1.04959 & 0.100289 & 0.547035 & 0.0953475 & 0.915648 & 0.185285 \\
\hline 0.806525 & 1.11515 & 0.111439 & 0.601289 & 0.116908 & 0.98759 & 0.196725 \\
\hline 0.935542 & 1.1589 & 0.121583 & 0.66275 & 0.13189 & 1.06946 & 0.19213 \\
\hline 1.03953 & 1.20217 & 0.126275 & 0.722212 & 0.13706 & 1.15412 & 0.208856 \\
\hline 1.10596 & 1.22799 & 0.115905 & 0.771166 & 0.1417 & 1.21542 & 0.185757 \\
\hline 1.19798 & 1.24342 & 0.125696 & 0.82157 & 0.156401 & 1.27468 & 0.181972 \\
\hline 1.25335 & 1.23796 & 0.131843 & 0.860598 & 0.170507 & 1.32341 & 0.194486 \\
\hline 1.26674 & 1.21076 & 0.129443 & & 0.172768 & 1.35676 & 0.180684 \\
\hline 1.39346 & 1.17744 & 0.128508 & 0.877073 & 0.174673 & 1.35358 & 0.170946 \\
\hline 1.28953 & 1.13822 & 0.121773 & 0.874015 & 0.169088 & 1.33169 & 0.175599 \\
\hline 1.2724 & 1.08245 & 0.107369 & 0.848094 & 0.159512 & 1.28133 & 0.170977 \\
\hline 1.18677 & 1.01939 & 0.0923853 & 0.816556 & 0.149724 & 1.2281 & 0.145281 \\
\hline 1.14523 & 0.959515 & 0.0843269 & 0.768536 & 0.139898 & 1.15411 & 0.145809 \\
\hline 1.05906 & 0.908932 & 0.0851578 & 0.721166 & 0.134635 & 1.0934 & 0.132757 \\
\hline 1.04951 & 0.848055 & 0.0716519 & 0.673565 & 0.11972 & 0.999141 & 0.116658 \\
\hline 0.914169 & 0.79847 & 0.0640948 & 0.607834 & 0.106081 & 0.933809 & 0.120068 \\
\hline 0.862519 & 0.756643 & 0.058822 & 0.557092 & 0.0911928 & 0.856746 & 0.0939447 \\
\hline 0.833132 & 0.722452 & 0.056677 & 0.513532 & 0.0893557 & 0.791585 & 0.0954774 \\
\hline 0.766016 & 0.692784 & 0.0554346 & 0.468541 & 0.0813546 & 0.735071 & 0.0811952 \\
\hline 0.697188 & 0.669286 & 0.0595939 & 0.445982 & 0.0788157 & 0.702996 & 0.0703068 \\
\hline 0.720437 & 0.644298 & 0.0543259 & 0.407812 & 0.0688536 & 0.654694 & 0.0655283 \\
\hline 0.680983 & 0.627374 & 0.0498818 & 0.394423 & 0.0693227 & 0.638622 & 0.0612402 \\
\hline 0.699999 & 0.614612 & 0.0522305 & 0.373747 & 0.0640568 & 0.61315 & 0.0634182 \\
\hline 0.65339 & 0.602556 & 0.0523941 & 0.360642 & 0.064085 & 0.606194 & 0.0584741 \\
\hline 0.676062 & 0.600437 & 0.0520977 & 0.357729 & 0.0717773 & 0.606317 & 0.0499105 \\
\hline 0.705047 & 0.599846 & 0.0501272 & 0.348788 & 0.065604 & 0.602476 & 0.0502971 \\
\hline 0.745155 & 0.588492 & 0.0527778 & 0.34706 & 0.0654982 & 0.602196 & 0.0581046 \\
\hline 0.771886 & 0.586565 & 0.0471047 & 0.346056 & 0.0611277 & 0.614942 & 0.0462526 \\
\hline 0.7218 & 0.585814 & 0.0557068 & 0.347696 & 0.0703055 & 0.632585 & 0.0546557 \\
\hline 0.860991 & 0.587189 & 0.0597443 & 0.349467 & 0.0689336 & 0.625461 & 0.0593916 \\
\hline 0.798548 & 0.597569 & 0.0606842 & 0.349246 & 0.0687127 & 0.659119 & 0.061241 \\
\hline 0.870843 & 0.607401 & 0.0625854 & 0.361075 & 0.0754383 & 0.670905 & 0.0648628 \\
\hline 0.861564 & 0.626962 & 0.0681339 & 0.365762 & 0.0732358 & 0.706396 & 0.058183 \\
\hline
\end{tabular}




\begin{tabular}{|c|c|c|c|c|c|c|}
\hline 1.07279 & 0.657798 & 0.078679 & 0.368591 & 0.073338 & 0.748654 & 0.0642073 \\
\hline 1.1039 & 0.697703 & 0.0905234 & 0.385495 & 0.0864582 & 0.802743 & 0.0766527 \\
\hline 1.2166 & 0.756659 & 0.101686 & 0.408111 & 0.0888236 & 0.871327 & 0.0713357 \\
\hline 1.3809 & 0.866712 & 0.141175 & 0.437551 & 0.0976833 & 0.985489 & 0.100265 \\
\hline 1.66068 & 1.02118 & 0.186568 & 0.474868 & 0.118689 & 1.14465 & 0.099102 \\
\hline 2.05264 & 1.25242 & 0.251674 & 0.536511 & 0.139913 & 1.40254 & 0.132939 \\
\hline 2.53178 & 1.58237 & 0.360383 & 0.64647 & 0.186417 & 1.75713 & 0.160997 \\
\hline 3.53636 & 2.03715 & 0.492013 & 0.795657 & 0.229813 & 2.29496 & 0.214554 \\
\hline 4.46693 & 2.66385 & 0.697632 & 1.00131 & 0.337886 & 3.08297 & 0.280945 \\
\hline 6.3201 & 3.50103 & 0.957707 & 1.30326 & 0.436778 & 4.15416 & 0.363372 \\
\hline 8.80965 & 4.57599 & 1.29247 & 1.73502 & 0.619932 & 5.66729 & 0.504527 \\
\hline 12.1371 & 5.93178 & 1.7426 & 2.30481 & 0.84928 & 7.68128 & 0.665466 \\
\hline 16.3524 & 7.55976 & 2.22774 & 3.0801 & 1.15952 & 10.3583 & 0.921693 \\
\hline 21.7905 & 9.47848 & 2.81905 & 4.07925 & 1.56075 & 13.7564 & 1.28922 \\
\hline 28.8871 & 11.6463 & 3.47515 & 5.2999 & 2.05533 & 17.9028 & 1.58111 \\
\hline 36.9856 & 13.9727 & 4.21035 & 6.81219 & 2.64751 & 22.7908 & 2.07478 \\
\hline 47.2077 & 16.4544 & 4.95215 & 8.53117 & 3.32292 & 28.4736 & 2.62301 \\
\hline 59.1444 & 18.8764 & 5.77182 & 10.5238 & 4.13052 & 34.8362 & 3.23421 \\
\hline 71.3179 & 21.1195 & 6.48406 & 12.6163 & 4.96955 & 41.4284 & 3.76185 \\
\hline 83.9831 & 23.0558 & 7.064 & 14.8104 & 5.78641 & 48.3172 & 4.49601 \\
\hline 97.4547 & 24.5471 & 7.51067 & 16.9769 & 6.66282 & 54.8546 & 5.16745 \\
\hline 110.626 & 25.4989 & 7.75338 & 19.0446 & 7.53979 & 60.7473 & 5.60481 \\
\hline 120.904 & 25.8543 & 7.91146 & 20.8463 & 8.20873 & 65.7786 & 6.08034 \\
\hline 130.133 & 25.5682 & 7.81083 & 22.1464 & 8.72621 & 69.165 & 6.51586 \\
\hline 136.315 & 24.5991 & 7.49717 & 22.9565 & 9.09012 & 71.0412 & 6.65515 \\
\hline 138.065 & 23.1701 & 7.03853 & 23.3481 & 9.23555 & 71.1657 & 6.75469 \\
\hline 138.966 & 21.2308 & 6.40434 & 23.0778 & 9.09575 & 69.5385 & 6.66104 \\
\hline 134.992 & 19.0353 & 5.73417 & 22.2221 & 8.78167 & 66.1697 & 6.33425 \\
\hline 126.999 & 16.6273 & 4.9526 & 20.8848 & 8.20776 & 61.3628 & 5.88699 \\
\hline 116.651 & 14.2027 & 4.21297 & 19.1821 & 7.53238 & 55.5451 & 5.30132 \\
\hline 105.279 & 11.8697 & 3.50348 & 17.1643 & 6.73402 & 49.1532 & 4.79761 \\
\hline 93.0746 & 9.72116 & 2.83011 & 14.9821 & 5.89349 & 42.3293 & 4.09695 \\
\hline 80.1195 & 7.78789 & 2.24175 & 12.7585 & 4.91494 & 35.5958 & 3.54082 \\
\hline 67.1034 & 6.13921 & 1.72404 & 10.6271 & 4.12104 & 29.159 & 2.85697 \\
\hline 55.4222 & 4.79814 & 1.30303 & 8.67006 & 3.34135 & 23.3846 & 2.34296 \\
\hline 44.3065 & 3.72744 & 0.952541 & 6.88162 & 2.61987 & 18.3032 & 1.80481 \\
\hline 34.868 & 2.90462 & 0.690578 & 5.3681 & 2.00318 & 14.0027 & 1.36465 \\
\hline 26.2886 & 2.27251 & 0.488293 & 4.139 & 1.52361 & 10.4951 & 1.04319 \\
\hline 20.0783 & 1.81366 & 0.338936 & 3.11617 & 1.14233 & 7.71 & 0.761811 \\
\hline 14.6221 & 1.48735 & 0.239591 & 2.32424 & 0.793567 & 5.58116 & 0.536254 \\
\hline 10.5904 & 1.27544 & 0.173582 & 1.73918 & 0.570606 & 4.0226 & 0.408542 \\
\hline 7.66741 & 1.12414 & 0.13498 & 1.30439 & 0.401079 & 2.89151 & 0.293672 \\
\hline 5.49714 & 1.03835 & 0.108238 & 1.00042 & 0.279016 & 2.09028 & 0.193954 \\
\hline 3.94747 & 0.976321 & 0.0901172 & 0.781852 & 0.200022 & 1.54 & 0.149505 \\
\hline 2.80515 & 0.937873 & 0.083513 & 0.63751 & 0.157571 & 1.16088 & 0.114471 \\
\hline 2.17489 & 0.916148 & 0.0765444 & 0.539808 & 0.114717 & 0.927858 & 0.0851394 \\
\hline 1.649 & 0.904194 & 0.0569866 & 0.477916 & 0.0948945 & 0.78089 & 0.076989 \\
\hline
\end{tabular}




\begin{tabular}{|c|c|c|c|c|c|c|}
\hline 1.29427 & 0.894505 & 0.0642197 & 0.441993 & 0.0711823 & 0.694851 & 0.0628987 \\
\hline 1.00349 & 0.897566 & 0.0621366 & 0.418793 & 0.0710184 & 0.620651 & 0.0593832 \\
\hline 0.895768 & 0.900136 & 0.0602234 & 0.402385 & 0.0677591 & 0.591155 & 0.0583691 \\
\hline 0.87032 & 0.900759 & 0.0661016 & 0.392083 & 0.0629876 & 0.568316 & 0.0613838 \\
\hline 0.799799 & 0.903414 & 0.0649131 & 0.400862 & 0.0656671 & 0.554124 & 0.0594834 \\
\hline 0.678094 & 0.901297 & 0.0616461 & 0.391317 & 0.0631244 & 0.554327 & 0.0584392 \\
\hline 0.73876 & 0.895774 & 0.0636004 & 0.39223 & 0.0586558 & 0.558583 & 0.0616487 \\
\hline 0.639922 & 0.904017 & 0.0560937 & 0.395822 & 0.0622388 & 0.556957 & 0.0537569 \\
\hline 0.660904 & 0.901494 & 0.0541111 & 0.397833 & 0.0560699 & 0.565074 & 0.0651751 \\
\hline 0.655779 & 0.902366 & 0.0586835 & 0.399483 & 0.0538525 & 0.556466 & 0.0638433 \\
\hline 0.688612 & 0.900071 & 0.0569755 & 0.395695 & 0.0524721 & 0.565074 & 0.0561913 \\
\hline 0.650905 & 0.909005 & 0.0545434 & 0.402432 & 0.0516957 & 0.564914 & 0.0497883 \\
\hline 0.68014 & 0.913881 & 0.0647529 & 0.406857 & 0.0597635 & 0.582316 & 0.0595777 \\
\hline 0.658876 & 0.919766 & 0.0629884 & 0.408013 & 0.0484858 & 0.587877 & 0.0623491 \\
\hline 0.712528 & 0.925793 & 0.0574715 & 0.413483 & 0.0513401 & 0.594169 & 0.0585457 \\
\hline 0.683407 & 0.930172 & 0.0558305 & 0.413092 & 0.053007 & 0.608881 & 0.0565344 \\
\hline 0.662523 & 0.931478 & 0.0548824 & 0.422527 & 0.0581017 & 0.622013 & 0.0665531 \\
\hline 0.678849 & 0.94037 & 0.0568321 & 0.425647 & 0.0575544 & 0.636475 & 0.054245 \\
\hline 0.72128 & 0.95215 & 0.0566033 & 0.431182 & 0.0624174 & 0.654052 & 0.0611873 \\
\hline 0.6796 & 0.963955 & 0.0585311 & 0.432918 & 0.061006 & 0.674666 & 0.0536096 \\
\hline 0.692431 & 0.979753 & 0.0621035 & 0.452505 & 0.0629322 & 0.701624 & 0.0616244 \\
\hline 0.699198 & 1.00602 & 0.0662468 & 0.464769 & 0.0674404 & 0.741003 & 0.0590997 \\
\hline 0.821349 & 1.04252 & 0.070192 & 0.478107 & 0.0627917 & 0.780586 & 0.0652913 \\
\hline 0.883137 & 1.09222 & 0.084643 & 0.494707 & 0.0787425 & 0.847045 & 0.0583353 \\
\hline 0.925422 & 1.14833 & 0.0974181 & 0.519052 & 0.0897336 & 0.93253 & 0.066181 \\
\hline 1.02588 & 1.22942 & 0.127416 & 0.543535 & 0.0961227 & 1.0329 & 0.0780081 \\
\hline 1.24048 & 1.33472 & 0.163615 & 0.583569 & 0.110882 & 1.17995 & 0.1021 \\
\hline 1.49935 & 1.46551 & 0.194428 & 0.635088 & 0.132187 & 1.36194 & 0.100436 \\
\hline 1.71006 & 1.63386 & 0.255027 & 0.715766 & 0.160044 & 1.61191 & 0.136446 \\
\hline 2.01291 & 1.83938 & 0.309244 & 0.809912 & 0.182438 & 1.91788 & 0.148003 \\
\hline 2.60598 & 2.08404 & 0.364174 & 0.915296 & 0.235449 & 2.31792 & 0.185028 \\
\hline 3.19752 & 2.36493 & 0.451597 & 1.0694 & 0.306281 & 2.83008 & 0.22104 \\
\hline 3.93239 & 2.66594 & 0.545963 & 1.26351 & 0.375878 & 3.40534 & 0.276986 \\
\hline 4.91553 & 3.02033 & 0.644584 & 1.47093 & 0.464496 & 4.12168 & 0.318378 \\
\hline 5.78639 & 3.34411 & 0.764117 & 1.72691 & 0.55219 & 4.94186 & 0.416006 \\
\hline 7.06943 & 3.70127 & 0.84405 & 2.00945 & 0.663663 & 5.81256 & 0.467385 \\
\hline 8.31663 & 4.03312 & 0.954305 & 2.3183 & 0.787632 & 6.7619 & 0.558242 \\
\hline 9.74184 & 4.31326 & 1.02293 & 2.63361 & 0.910561 & 7.76362 & 0.645602 \\
\hline 11.0604 & 4.52578 & 1.10188 & 2.9633 & 1.03108 & 8.71861 & 0.699105 \\
\hline 12.7199 & 4.71424 & 1.14075 & 3.254 & 1.18213 & 9.57618 & 0.802721 \\
\hline 13.4995 & 4.80004 & 1.15543 & 3.53592 & 1.29794 & 10.3446 & 0.842044 \\
\hline 15.0982 & 4.77845 & 1.16559 & 3.76439 & 1.34158 & 10.9441 & 0.910437 \\
\hline 15.7835 & 4.6669 & 1.1298 & 3.90017 & 1.40019 & 11.3124 & 0.968173 \\
\hline 16.0888 & 4.46015 & 1.05861 & 4.01099 & 1.43491 & 11.4354 & 0.954964 \\
\hline 16.4338 & 4.1955 & 0.981635 & 4.0052 & 1.46994 & 11.3345 & 0.998023 \\
\hline 16.1014 & 3.89265 & 0.894877 & 3.90518 & 1.42075 & 10.9486 & 0.936274 \\
\hline 15.4019 & 3.56098 & 0.802957 & 3.77826 & 1.36024 & 10.3709 & 0.919485 \\
\hline
\end{tabular}




\begin{tabular}{|c|c|c|c|c|c|c|}
\hline 14.6984 & 3.20116 & 0.699196 & 3.53551 & 1.27609 & 9.61624 & 0.807514 \\
\hline 13.4855 & 2.84631 & 0.584551 & 3.2669 & 1.15037 & 8.69913 & 0.759821 \\
\hline 12.2525 & 2.51239 & 0.492747 & 2.97313 & 1.03343 & 7.71246 & 0.707605 \\
\hline 10.6962 & 2.19546 & 0.397253 & 2.63698 & 0.906695 & 6.73259 & 0.573564 \\
\hline 9.43463 & 1.92101 & 0.312251 & 2.29946 & 0.771784 & 5.73654 & 0.507073 \\
\hline 7.91218 & 1.67941 & 0.239867 & 1.98439 & 0.657942 & 4.7837 & 0.422057 \\
\hline 6.50159 & 1.49125 & 0.180481 & 1.6825 & 0.515246 & 3.9269 & 0.358698 \\
\hline 5.34666 & 1.34136 & 0.142546 & 1.42162 & 0.420169 & 3.17792 & 0.271479 \\
\hline 4.11664 & 1.21589 & 0.113558 & 1.20454 & 0.339327 & 2.54614 & 0.2147 \\
\hline 3.33296 & 1.12884 & 0.0819461 & 0.995894 & 0.25411 & 2.00722 & 0.171975 \\
\hline 2.52625 & 1.06885 & 0.0730541 & 0.855646 & 0.201135 & 1.59158 & 0.142571 \\
\hline 1.95669 & 1.02131 & 0.0728794 & 0.712081 & 0.145533 & 1.2642 & 0.110309 \\
\hline 1.4565 & 0.997908 & 0.0645688 & 0.629883 & 0.111501 & 1.00531 & 0.0844182 \\
\hline 1.11662 & 0.982817 & 0.0511274 & 0.563451 & 0.091212 & 0.821789 & 0.068581 \\
\hline 0.824799 & 0.987425 & 0.0510307 & 0.509201 & 0.0789942 & 0.677769 & 0.0486258 \\
\hline 0.624661 & 0.998873 & 0.0539311 & 0.474253 & 0.0586258 & 0.590268 & 0.0528097 \\
\hline 0.485432 & 1.0114 & 0.0639671 & 0.448888 & 0.052324 & 0.514516 & 0.0419877 \\
\hline 0.360759 & 1.03813 & 0.0641015 & 0.439285 & 0.0619266 & 0.470005 & 0.0337875 \\
\hline 0.295291 & 1.06783 & 0.0669633 & 0.435349 & 0.0497591 & 0.440696 & 0.0389016 \\
\hline 0.231438 & 1.09326 & 0.0741798 & 0.436853 & 0.0579241 & 0.424193 & 0.0387495 \\
\hline 0.219043 & 1.12441 & 0.0787399 & 0.431503 & 0.0536226 & 0.415522 & 0.0332388 \\
\hline 0.178611 & 1.15247 & 0.0737908 & 0.446256 & 0.0580101 & 0.408429 & 0.032897 \\
\hline 0.179137 & 1.18329 & 0.0852458 & 0.45702 & 0.0619345 & 0.401088 & 0.0321218 \\
\hline 0.181837 & 1.19932 & 0.0893441 & 0.465619 & 0.0653782 & 0.394602 & 0.037129 \\
\hline 0.163251 & 1.21654 & 0.0886371 & 0.472622 & 0.0658093 & 0.404341 & 0.0328114 \\
\hline 0.154999 & 1.22481 & 0.089394 & 0.479139 & 0.0757418 & 0.408361 & 0.0323227 \\
\hline 0.17943 & 1.2238 & 0.090029 & 0.490562 & 0.0705054 & 0.418966 & 0.03505 \\
\hline 0.177925 & 1.22328 & 0.0906855 & 0.498674 & 0.0774615 & 0.42114 & 0.0343264 \\
\hline 0.210087 & 1.20405 & 0.0879674 & 0.515604 & 0.0752039 & 0.435291 & 0.0375256 \\
\hline 0.21379 & 1.18783 & 0.0734355 & 0.51103 & 0.0859726 & 0.447773 & 0.0351021 \\
\hline 0.246172 & 1.18295 & 0.0763366 & 0.518058 & 0.0775389 & 0.460986 & 0.0427404 \\
\hline 0.259319 & 1.16188 & 0.0732436 & 0.513151 & 0.0778294 & 0.473506 & 0.0346694 \\
\hline 0.286466 & 1.13895 & 0.066399 & 0.520118 & 0.064821 & 0.481906 & 0.0419616 \\
\hline 0.300574 & 1.11047 & 0.0648459 & 0.517772 & 0.0721642 & 0.498333 & 0.0409041 \\
\hline 0.32333 & 1.08857 & 0.0608382 & 0.515713 & 0.0769016 & 0.507576 & 0.0449691 \\
\hline 0.336652 & 1.06631 & 0.0536377 & 0.510321 & 0.0686841 & 0.511766 & 0.0368668 \\
\hline 0.386705 & 1.03951 & 0.0562582 & 0.512242 & 0.0708935 & 0.529377 & 0.0327129 \\
\hline 0.406718 & 1.02039 & 0.0625783 & 0.503202 & 0.0722087 & 0.530136 & 0.0475164 \\
\hline 0.419256 & 1.01097 & 0.0535226 & 0.500363 & 0.0633363 & 0.536228 & 0.0396697 \\
\hline 0.371615 & 0.988862 & 0.0550076 & 0.494616 & 0.0617128 & 0.536842 & 0.0517814 \\
\hline 0.375552 & 0.970465 & 0.0592776 & 0.48856 & 0.0641572 & 0.541704 & 0.0426934 \\
\hline 0.380761 & 0.96191 & 0.0579486 & 0.483249 & 0.0679359 & 0.541783 & 0.0446009 \\
\hline 0.366281 & 0.959218 & 0.0624579 & 0.468943 & 0.0617193 & 0.529335 & 0.049887 \\
\hline 0.310866 & 0.958338 & 0.0625162 & 0.467741 & 0.0528721 & 0.526645 & 0.0553571 \\
\hline 0.293787 & 0.949807 & 0.0628559 & 0.466197 & 0.0646975 & 0.51284 & 0.0495861 \\
\hline 0.265387 & 0.947241 & 0.0619349 & 0.454895 & 0.0667171 & 0.518314 & 0.0635861 \\
\hline 0.263664 & 0.949563 & 0.0597253 & 0.455649 & 0.0590531 & 0.510994 & 0.0600625 \\
\hline
\end{tabular}




\begin{tabular}{|c|c|c|c|c|c|c|}
\hline 0.239323 & 0.949586 & 0.0601531 & 0.446184 & 0.0529012 & 0.501009 & 0.0676795 \\
\hline 0.20151 & 0.952886 & 0.0523668 & 0.438833 & 0.0605439 & 0.494602 & 0.0704392 \\
\hline 0.182293 & 0.958173 & 0.0491987 & 0.448904 & 0.0682066 & 0.483002 & 0.0837324 \\
\hline 0.177616 & 0.96749 & 0.0616882 & 0.452047 & 0.0673244 & 0.490085 & 0.0813358 \\
\hline 0.154393 & 0.969837 & 0.0636359 & 0.448615 & 0.0609147 & 0.487985 & 0.0845174 \\
\hline 0.154072 & 0.97998 & 0.0649936 & 0.448209 & 0.0579059 & 0.482122 & 0.0796312 \\
\hline 0.114836 & 0.974893 & 0.0555938 & 0.447372 & 0.0600655 & 0.481405 & 0.0875627 \\
\hline 0.119183 & 0.977131 & 0.0562559 & 0.460188 & 0.0577207 & 0.48212 & 0.088649 \\
\hline 0.124637 & 0.970829 & 0.0569205 & 0.455004 & 0.0604347 & 0.490327 & 0.08562 \\
\hline 0.0990258 & 0.973648 & 0.0530231 & 0.46204 & 0.057163 & 0.481442 & 0.0833675 \\
\hline 0.105388 & 0.973507 & 0.0510112 & 0.459646 & 0.0633873 & 0.478934 & 0.0706467 \\
\hline 0.107512 & 0.955779 & 0.0536249 & 0.459404 & 0.0709501 & 0.486386 & 0.0715792 \\
\hline 0.114997 & 0.950234 & 0.0594816 & 0.465048 & 0.0635551 & 0.492191 & 0.0722369 \\
\hline 0.119884 & 0.946964 & 0.0632066 & 0.464595 & 0.0576193 & 0.488654 & 0.0706703 \\
\hline 0.107877 & 0.945337 & 0.0545257 & 0.458018 & 0.0587977 & 0.480985 & 0.0767619 \\
\hline 0.112351 & 0.947586 & 0.0517848 & 0.463312 & 0.0647178 & 0.489826 & 0.0618618 \\
\hline 0.106332 & 0.942309 & 0.0543078 & .466665 & 0.0677396 & 0.489605 & 9927 \\
\hline 0.11404 & 0.943077 & 0.0473838 & 0.4647 & 0.0706412 & 0.48942 & 0.0530158 \\
\hline 0.105791 & 0.946353 & 0.0541303 & 0.465523 & 0.0623014 & 0.489369 & 0.0664286 \\
\hline 0.116616 & 0.928581 & 7196 & 494 & 478 & 0.485123 & 0.04 \\
\hline 0.107601 & 0.926796 & 0.0625468 & 0.459797 & 0.0618275 & 0.482991 & 0.0516537 \\
\hline 0.104479 & 0.928612 & 0.0510837 & 0.460955 & 0.0600459 & 0.48634 & 0.0484477 \\
\hline 0.110972 & 0.917202 & 0.052172 & 0.458044 & 0.0594642 & 0.477237 & 0.0427297 \\
\hline 0.0856878 & 0.910616 & 0.0553309 & 0.457272 & 0.0659642 & 0.48239 & 0.0440347 \\
\hline 0.0966248 & 0.913599 & 0.0599032 & 0.461546 & 0.066204 & 0.489949 & 0.043745 \\
\hline 0.109693 & 0.903571 & 0.0536792 & 0.453005 & 0.058198 & 0.494954 & 0.0375638 \\
\hline 0.105158 & 0.891733 & 0.0574151 & 0.444533 & 0.0576705 & 0.48172 & 0.0390412 \\
\hline 0.111329 & 0.887036 & 0.0518489 & 0.441326 & 0.0599543 & 0.490259 & 0.0428678 \\
\hline 0.103886 & 0.884104 & 0.0459089 & 0.451184 & 0.0635569 & 0.483441 & 0.0366457 \\
\hline 0.107036 & 0.891436 & 0.0570807 & 0.444151 & 0.0642872 & 0.494367 & 0.0386191 \\
\hline 0.0975881 & 0.89357 & 0.0508015 & 0.450207 & 0.0605744 & 0.484012 & 0.0363178 \\
\hline 0.0956286 & 0.893497 & 0.0529496 & 0.442195 & 0.0577774 & 0.496061 & 0.0394199 \\
\hline 0.114313 & 0.889824 & 0.0552349 & 0.445829 & 0.0605803 & 0.492909 & 0.0344918 \\
\hline 0.140166 & 0.891016 & 0.0523136 & 0.447144 & 0.0488557 & 0.489592 & 0.0379214 \\
\hline 0.121139 & 0.899487 & 0.0506571 & 0.45543 & 0.0543712 & 0.490277 & 0.0364788 \\
\hline 0.100607 & 0.904651 & 0.0577001 & 0.446332 & 0.0534447 & 0.493947 & 0.040404 \\
\hline 0.140177 & 0.902841 & 0.0433651 & 0.45942 & 0.0464325 & 0.507118 & 0.0405272 \\
\hline 0.139595 & 0.903527 & 0.0520583 & 0.452295 & 0.0469341 & 0.509601 & 0.0269612 \\
\hline 0.130291 & 0.908797 & 0.0487619 & 0.457698 & 0.0573051 & 0.517932 & 0.0372214 \\
\hline 0.141934 & 0.923197 & 0.0436261 & 0.466695 & 0.0467316 & 0.529321 & 0.0449686 \\
\hline 0.168186 & 0.92241 & 0.0556714 & 0.465329 & 0.0478154 & 0.536827 & 0.0488036 \\
\hline 0.161584 & 0.925891 & 0.0477818 & 0.474809 & 0.0530765 & 0.546774 & 0.0400659 \\
\hline 0.183394 & 0.935068 & 0.0483851 & 0.475908 & 0.0536258 & 0.554946 & 0.0431185 \\
\hline 0.185237 & 0.942821 & 0.0468763 & 0.475854 & 0.0470964 & 0.576167 & 0.0407528 \\
\hline 0.211069 & 0.93519 & 0.0479648 & 0.483879 & 0.0546683 & 0.58023 & 0.040752 \\
\hline 0.212701 & 0.938114 & 0.0465401 & 0.489424 & 0.054454 & 0.579291 & 0.0462271 \\
\hline 0.211772 & 0.942098 & 0.0480756 & 0.493763 & 0.0599562 & 0.588157 & 0.0479928 \\
\hline
\end{tabular}




\begin{tabular}{|c|c|c|c|c|c|c|}
\hline 0.21523 & 0.944767 & 0.0452953 & 0.501153 & 0.0558547 & 0.59655 & 477 \\
\hline 0.267783 & 0.951486 & 0.0429054 & 0.493337 & 0.059477 & 0.606089 & 0.0512384 \\
\hline 0.217568 & 0.944481 & 0.0395433 & 0.494171 & 0.0573739 & 0.606887 & 0.0473102 \\
\hline 0.263352 & 0.953397 & 0.0535906 & 0.495938 & 0.0601603 & 0.599401 & 0.0465667 \\
\hline 0.241577 & .955098 & 0.0523374 & 0.496272 & 0.0606185 & 0.599404 & 0.0428862 \\
\hline 0.240196 & 0.950016 & 0.0439409 & 0.499254 & 0.0640947 & 0.600043 & 0.044774 \\
\hline 0.267886 & 0.949683 & 0.0435456 & 0.49497 & 0.0612241 & 0.592889 & 0.0445035 \\
\hline 0.248756 & 0.949833 & 0.0516228 & 0.488585 & 0.0661872 & 0.590607 & 0.0488613 \\
\hline 0.232097 & 0.944505 & 0.0519528 & 0.485656 & 0.0554956 & 0.576749 & 0.0370544 \\
\hline 0.227129 & 0.948604 & 0.0514881 & 0.482907 & 0.0573227 & 0.581052 & 0.0300366 \\
\hline 0.231706 & 0.951507 & 0.0494571 & 0.478094 & 0.0502083 & 0.563997 & 0.0406183 \\
\hline 0.226277 & 0.956868 & 0.0479285 & 0.483469 & 0.0547166 & 0.564382 & 0.0426423 \\
\hline 0.227101 & 0.965098 & 0.0479768 & 0.477143 & 0.0430273 & 0.563623 & 0.0360813 \\
\hline 0.198007 & 0.971804 & 9043 & 0.482603 & 0.057 & 0.556934 & 0.0355999 \\
\hline 0.195566 & 0.975183 & 0.0477251 & 0.475274 & 0.058077 & 0.552871 & 0.0316167 \\
\hline 0.174351 & 0.976961 & 0.0472091 & 0.477585 & 0.0549095 & 0.548699 & 0.0385979 \\
\hline 0.184482 & 0.990757 & 0.0517089 & 0.474406 & 0.0541867 & 0.553451 & 0.0410961 \\
\hline 0.177815 & 0.997215 & 0.0470506 & 0.482161 & 0.0569496 & 0.557962 & 0.0464594 \\
\hline 0.172905 & 1.00435 & 0.0533798 & 0.475933 & 0.050881 & 0.55086 & 0.0422359 \\
\hline 0.177777 & 1.02075 & 0.049827 & 0.487059 & 0.0539117 & 0.553285 & 0.0366171 \\
\hline 0.157701 & 1.02977 & 0.0560179 & 0.482865 & 0.050365 & 0.556171 & 0.0463488 \\
\hline 0.153105 & 1.02999 & 0.0580947 & 0.480144 & 0.0524789 & 0.555309 & 0.0401321 \\
\hline 0.149825 & 1.04374 & 0.0604038 & 0.486208 & 0.0510979 & 0.569942 & 0.0328322 \\
\hline 0.149388 & 1.0555 & 0.0604657 & 0.490277 & 0.056 & 0.558293 & 0.0452767 \\
\hline 0.100961 & 1.06701 & 0.04 & 849 & 48 & 966 & 881 \\
\hline 0.139985 & 1.07781 & 0.0631206 & 0.492121 & 0.0500057 & 0.575338 & 0.0484218 \\
\hline 0.125408 & 1.08914 & 0.0645734 & 0.502941 & 0.0560674 & 0.573061 & 0.0462462 \\
\hline 0.115112 & 1.08802 & 0.0582639 & 0.506949 & 0.0615067 & 0.585705 & 0.0463827 \\
\hline 0.136573 & 1.10534 & 0.0625755 & 0.507747 & 0.0545955 & 0.583235 & 0.0474614 \\
\hline 0.116914 & 1.1255 & 0.0659912 & 0.508599 & 0.0519391 & 0.585858 & 0.0443639 \\
\hline 0.11968 & 1.13141 & 0.0731253 & 0.508392 & 0.056983 & 0.597228 & 0.04645 \\
\hline 0.0953681 & 1.15162 & 0.0750047 & 0.521514 & 0.0588211 & 0.589419 & 0.0525044 \\
\hline 0.0993864 & 1.15232 & 0.0746848 & 0.518647 & 0.0597634 & 0.606957 & 0.0450545 \\
\hline 0.113893 & 1.1551 & 0.080002 & 0.519956 & 0.0590579 & 0.60267 & 0.0430324 \\
\hline 0.102234 & 1.16983 & 0.0894533 & 0.528839 & 0.0651164 & 0.613281 & 0.0546689 \\
\hline 0.100125 & 1.1893 & 0.0852721 & 0.53174 & 0.0630769 & 0.614482 & 0.0416395 \\
\hline 0.104779 & 1.20651 & 0.0823271 & 0.541744 & 0.0714383 & 0.623381 & 0.0502625 \\
\hline 0.113182 & 1.22277 & 0.0951548 & 0.544519 & 0.0574875 & 0.611587 & 0.0518808 \\
\hline 0.120008 & 1.22776 & 0.0909103 & 0.549907 & 0.0615972 & 0.638781 & 0.0475032 \\
\hline 0.101536 & 1.23816 & 0.0878654 & 0.557596 & 0.0746422 & 0.629826 & 0.0489991 \\
\hline 0.106209 & 1.25158 & 0.0949434 & 0.554834 & 0.0691966 & 0.642675 & 0.0435933 \\
\hline 0.101772 & 1.26659 & 0.0989945 & 0.563054 & 0.0640514 & 0.634114 & 0.0480808 \\
\hline 0.111063 & 1.28313 & 0.0966303 & 0.579214 & 0.0812915 & 0.65394 & 0.0486887 \\
\hline 0.106265 & 1.29427 & 0.0978902 & 0.565969 & 0.064758 & 0.659959 & 0.0510101 \\
\hline 0.112765 & 1.31188 & 0.0906657 & 0.58448 & 0.0767114 & 0.668679 & 0.0457487 \\
\hline 0.109546 & 1.33123 & 0.0944289 & 0.585387 & 0.0714786 & 0.683328 & 0.0597014 \\
\hline 0.0959754 & 1.35646 & 0.0914292 & 0.600997 & 0.0757611 & 0.696263 & 0.0587796 \\
\hline
\end{tabular}




\begin{tabular}{|c|c|c|c|c|c|c|}
\hline 0.0986272 & 1.37365 & 0.109913 & 0.605784 & 0.0797692 & 0.716389 & 0.0434225 \\
\hline 0.111244 & 1.3956 & 0.108055 & 0.616379 & 0.0752908 & 0.733399 & 0.0556829 \\
\hline 0.109576 & 1.40873 & 0.0998991 & 0.630181 & 0.0825142 & 0.762234 & 0.0530671 \\
\hline 0.124514 & 1.42707 & 0.0931185 & 0.637595 & 0.0775188 & 0.778369 & 0.0474859 \\
\hline 0.132466 & 1.4497 & 0.105576 & 0.648717 & 0.0797071 & 0.814543 & 0.0453475 \\
\hline 0.107465 & 1.46131 & 0.104742 & 0.66565 & 0.0855513 & 0.83898 & 0.0536224 \\
\hline 0.121345 & 1.48402 & 0.0865545 & 0.672157 & 0.0800394 & 0.869035 & 0.0500474 \\
\hline 0.121525 & 1.50007 & 0.0974198 & 0.685074 & 0.075741 & 0.902035 & 0.0468661 \\
\hline 0.0974662 & 1.51212 & 0.0981638 & 0.699254 & 0.0932091 & 0.931838 & 0.0694084 \\
\hline 0.0997277 & 1.50593 & 0.0932069 & 0.717821 & 0.088717 & 0.951119 & 0.0699575 \\
\hline 0.106369 & 1.51957 & 0.0903157 & 0.736347 & 0.0786742 & 0.975344 & 0.062439 \\
\hline 0.125371 & 1.53356 & 0.082873 & 0.738861 & 0.0776036 & 0.978991 & 0.0607615 \\
\hline 0.107215 & 1.52073 & 0.0870462 & 0.745118 & 0.0820754 & 1.00295 & 0.0673997 \\
\hline 0.102887 & 1.51985 & 0.087795 & 0.745417 & 0.0790102 & 0.997341 & 0.058588 \\
\hline 0.116002 & 1.51104 & 0.078524 & 0.754793 & 0.0895043 & 0.99607 & 0.0716464 \\
\hline 0.119942 & 1.4991 & 0.0797169 & 0.745091 & 0.087418 & 0.981326 & 0.0670539 \\
\hline 0.110054 & 1.50127 & 0.0802391 & 0.746429 & 0.0861003 & 0.977253 & 0.0702543 \\
\hline 0.121189 & 1.48776 & 0.0764272 & 0.752824 & 0.088283 & 0.946736 & 0.0594436 \\
\hline 0.127786 & 1.49568 & 0.0691331 & 0.737617 & 0.0884952 & 0.93141 & 0.0547967 \\
\hline 0.10163 & 1.47986 & 0.0685322 & 0.736386 & 0.0845716 & 0.910098 & 0.066991 \\
\hline 0.131457 & 1.46264 & 0.0782369 & 0.725901 & 0.078551 & 0.87532 & 0.06174 \\
\hline 0.129522 & 1.46254 & 0.077536 & 0.727076 & 0.0765138 & 0.856714 & 0.0592957 \\
\hline 0.116715 & 1.46593 & 0.0791023 & 0.707497 & 0.0745259 & 0.828916 & 0.0607172 \\
\hline 0.119186 & 1.45548 & 0.0806012 & 0.707354 & 0.0748536 & 0.810036 & 0.0602629 \\
\hline 0.119706 & 1.45712 & 0.0780898 & 0.698242 & 0.0738303 & 0.805187 & 0.0619163 \\
\hline 0.127488 & 1.45464 & 0.0839931 & 0.692975 & 0.0834936 & 0.788724 & 0.0522901 \\
\hline 0.12951 & 1.44791 & 0.0791638 & 0.676699 & 0.0740966 & 0.769893 & 0.0577744 \\
\hline 0.111333 & 1.45126 & 0.0806122 & 0.687694 & 0.0766131 & 0.759177 & 0.0496366 \\
\hline 0.115274 & 1.4638 & 0.0789038 & 0.682268 & 0.06378 & 0.749593 & 0.057582 \\
\hline 0.130557 & 1.45484 & 0.0757527 & 0.67995 & 0.0740189 & 0.744677 & 0.0576745 \\
\hline 0.130787 & 1.46027 & 0.0947016 & 0.681228 & 0.0748137 & 0.730918 & 0.0543545 \\
\hline 0.115063 & 1.45879 & 0.0848792 & 0.673913 & 0.0688411 & 0.728116 & 0.0540607 \\
\hline 0.129937 & 1.45405 & 0.0709876 & 0.678709 & 0.0565981 & 0.741078 & 0.0573711 \\
\hline 0.133075 & 1.46412 & 0.0946729 & 0.686596 & 0.0662865 & 0.735098 & 0.0564154 \\
\hline 0.122555 & 1.46872 & 0.0932926 & 0.687669 & 0.0627953 & 0.741219 & 0.0485724 \\
\hline 0.140359 & 1.4625 & 0.0834404 & 0.682345 & 0.0592416 & 0.733568 & 0.057408 \\
\hline 0.110717 & 1.46182 & 0.0977664 & 0.676928 & 0.0611871 & 0.726604 & 0.050198 \\
\hline 0.103571 & 1.47381 & 0.0893253 & 0.692047 & 0.0664222 & 0.724592 & 0.0580498 \\
\hline 0.129624 & 1.45664 & 0.0811133 & 0.685647 & 0.0565706 & 0.732702 & 0.0477554 \\
\hline 0.144031 & 1.4662 & 0.0858918 & 0.688029 & 0.0534326 & 0.731253 & 0.0488882 \\
\hline 0.112192 & 1.45196 & 0.0859922 & 0.684497 & 0.0489543 & 0.7269 & 0.0529937 \\
\hline 0.12518 & 1.45174 & 0.0820036 & 0.691247 & 0.0594911 & 0.738753 & 0.0366997 \\
\hline 0.130511 & 1.46747 & 0.0992565 & 0.680406 & 0.0654076 & 0.73133 & 0.0530785 \\
\hline 0.118723 & 1.45933 & 0.0762015 & 0.678487 & 0.0612776 & 0.729637 & 0.0426805 \\
\hline 0.11636 & 1.46283 & 0.0781547 & 0.67693 & 0.0555937 & 0.723904 & 0.0498202 \\
\hline 0.131096 & 1.44634 & 0.082724 & 0.681393 & 0.0522278 & 0.723105 & 0.0517714 \\
\hline 0.143549 & 1.44201 & 0.0858348 & 0.687733 & 0.0526539 & 0.714817 & 0.0467929 \\
\hline
\end{tabular}




\begin{tabular}{|c|c|c|c|c|c|c|}
\hline 0.136276 & 1.45242 & 0.0873006 & 0.689451 & 0.0521975 & 0.731521 & 185 \\
\hline 0.126007 & 1.44528 & 0.084596 & 0.690703 & 0.0574705 & 0.715024 & 0.0420244 \\
\hline 0.130741 & 1.43918 & 0.0824084 & 0.687913 & 0.0530957 & 0.72331 & 0.044195 \\
\hline 0.139856 & 1.43417 & 0.0926384 & 0.677398 & 0.0538715 & 0.715612 & 0.0427187 \\
\hline 0.126269 & 1.43607 & 0.0948336 & 0.674555 & 0.0594002 & 0.706387 & 0.0529919 \\
\hline 0.149448 & 1.43897 & 0.0835318 & 0.687769 & 0.0597779 & 0.69702 & 0.0447088 \\
\hline 0.148618 & 1.43942 & 0.0840978 & 0.682086 & 0.0590175 & 0.707963 & 0.0405342 \\
\hline 0.176727 & 1.44247 & 0.0878757 & 0.680799 & 0.049923 & 0.704263 & 0.0417453 \\
\hline 0.157729 & 1.43168 & 0.0868608 & 0.673746 & 0.0647753 & 0.705942 & 0.0396809 \\
\hline 0.140273 & 1.42993 & 0.0837346 & 0.67168 & 0.0566203 & 0.716826 & 0.0362717 \\
\hline 0.145458 & 1.43225 & 0.0846835 & 0.680466 & 0.0568194 & 0.708044 & 0.0383841 \\
\hline 0.157558 & 1.42493 & 0.0844184 & 0.675313 & 0.058188 & 0.702459 & 0.0487179 \\
\hline 0.149854 & 1.43644 & 0.0897241 & 0.680962 & 0.0565477 & 0.697253 & 0.0385034 \\
\hline 0.177615 & 1.42284 & 0.0805134 & 0.674271 & 0.0538832 & 0.715939 & 0.0350569 \\
\hline 0.161862 & 1.428 & 0.0791623 & 0.676304 & 0.0578812 & 0.706813 & 0.0392494 \\
\hline 0.148426 & 1.4341 & 0.0826446 & 0.680857 & 0.0633094 & 0.706298 & 0.0384029 \\
\hline 0.178968 & 1.42731 & 0.0904582 & 0.675371 & 0.049972 & 0.723015 & 0.0455593 \\
\hline 0.175119 & 1.42123 & 0.0813506 & 0.671137 & 0.0564534 & 0.720855 & 0.0403153 \\
\hline 0.169396 & 1.42402 & 0.0761734 & 0.679774 & 0.0531446 & 0.722385 & 0.0356037 \\
\hline 0.178347 & 1.43277 & 0.080 & 0.666996 & 0.0553267 & 0.724746 & 0.0381882 \\
\hline 0.156199 & 1.43029 & 0.0776472 & 0.674625 & 0.0606538 & 0.722913 & 0.0371799 \\
\hline 0.149483 & 1.43258 & 0.0740146 & 0.679693 & 0.0592796 & 0.726121 & 0.0412048 \\
\hline 0.153729 & 1.42644 & 0.0760987 & 0.683367 & 0.064441 & 0.745147 & 0.0393016 \\
\hline 0.148997 & 1.42871 & 0.0804492 & 0.678533 & 0.0530732 & 0.738521 & 0.0434697 \\
\hline 0.147587 & 1.43095 & 0.0898775 & 0.683492 & 0.0607816 & 0.749332 & 9964 \\
\hline 0.153141 & 1.42735 & 0.0733113 & 0.672703 & 0.0527252 & 0.74742 & 0.0384829 \\
\hline 0.13044 & 1.43407 & 0.0822363 & 0.666256 & 0.0661105 & 0.758117 & 0.0426639 \\
\hline 0.1327 & 1.43051 & 0.0703865 & 0.678221 & 0.0566225 & 0.763687 & 0.0439412 \\
\hline 0.143774 & 1.42891 & 0.0738019 & 0.68928 & 0.0569503 & 0.75394 & 0.0444991 \\
\hline 0.160439 & 1.42771 & 0.0750869 & 0.683008 & 0.0570962 & 0.755614 & 0.0322368 \\
\hline 0.140228 & 1.42443 & 0.0743853 & 0.6862 & 0.0564705 & 0.742444 & 0.0479825 \\
\hline 0.128434 & 1.421 & 0.064614 & 0.674476 & 0.0592512 & 0.75518 & 0.0360921 \\
\hline 0.132323 & 1.41954 & 0.0602367 & 0.677305 & 0.0547401 & 0.753817 & 0.0426651 \\
\hline 0.139347 & 1.42117 & 0.0639833 & 0.679967 & 0.0559991 & 0.748533 & 0.0390675 \\
\hline 0.151528 & 1.43177 & 0.0715197 & 0.682718 & 0.0623105 & 0.745884 & 0.037312 \\
\hline 0.137552 & 1.42573 & 0.0761374 & 0.683741 & 0.0482168 & 0.752362 & 0.0338552 \\
\hline 0.148017 & 1.42565 & 0.0755555 & 0.675258 & 0.0495927 & 0.739711 & 0.0445456 \\
\hline 0.126374 & 1.42398 & 0.0627133 & 0.677397 & 0.0512878 & 0.74545 & 0.0421443 \\
\hline 0.131907 & 1.42435 & 0.0747298 & 0.684295 & 0.0660261 & 0.737237 & 0.0358137 \\
\hline 0.124387 & 1.4239 & 0.0723194 & 0.682904 & 0.047258 & 0.736862 & 0.0375312 \\
\hline 0.113901 & 1.43056 & 0.0707082 & 0.68522 & 0.0495224 & 0.728491 & 0.0494707 \\
\hline 0.106511 & 1.43785 & 0.0728798 & 0.681095 & 0.0590283 & 0.735471 & 0.0364231 \\
\hline 0.100003 & 1.43852 & 0.0726106 & 0.691886 & 0.0540961 & 0.744929 & 0.0349661 \\
\hline 0.113532 & 1.43773 & 0.0664133 & 0.689904 & 0.0525229 & 0.738242 & 0.0434046 \\
\hline 0.115479 & 1.44136 & 0.0591987 & 0.688596 & 0.0486318 & 0.745958 & 0.0407946 \\
\hline 0.0973816 & 1.43503 & 0.066186 & 0.688698 & 0.0573378 & 0.74937 & 0.0488894 \\
\hline 0.116596 & 1.44782 & 0.0673478 & 0.693072 & 0.0527958 & 0.742546 & 0.0436018 \\
\hline
\end{tabular}




\begin{tabular}{|c|c|c|c|c|c|c|}
\hline 0.12733 & 1.46201 & 0.064085 & 0.701945 & 0.0493321 & 0.752577 & 0.0503197 \\
\hline 0.103567 & 1.45823 & 0.073576 & 0.70256 & 0.0553229 & 0.759288 & 0.0411441 \\
\hline 0.114731 & .46066 & 0.0518151 & 0.699135 & 0.0527816 & 0.76338 & 0.0416992 \\
\hline 0.0864103 & 1.46554 & 0.0577179 & 0.706642 & 0.0553796 & 0.755107 & 0.0366157 \\
\hline 0.102652 & 1.47524 & 0.0619933 & 0.70869 & 0.0532072 & 0.771793 & 0.0483243 \\
\hline 0.110633 & 1.49206 & 0.0765503 & 0.710195 & 0.0557015 & 0.777158 & 0.0490705 \\
\hline 0.108219 & 1.50115 & 0.0740523 & 0.724573 & 0.0514681 & 0.790668 & 0.0249848 \\
\hline 0.0929882 & 1.49985 & 0.0736347 & 0.721568 & 0.0577958 & 0.789826 & 0.0467696 \\
\hline 0.107978 & 1.50052 & 0.0613065 & 0.709895 & 0.0503488 & 0.795873 & 0.042744 \\
\hline 0.0921147 & 1.5048 & 0.0565175 & 0.725835 & 0.059349 & 0.797704 & 0.0501656 \\
\hline 0.109468 & 1.5107 & 0.0673994 & 0.728825 & 0.0576603 & 0.793442 & 0.0486732 \\
\hline 0.0997048 & 1.5126 & 0.0724425 & 0.728089 & 0.0557355 & 0.815747 & 0.0361851 \\
\hline 0.101014 & 1.51108 & 0.0677963 & 0.72819 & 0.0564205 & 0.810566 & 0.0509148 \\
\hline 0.0949478 & 1.52205 & 0.0771082 & 0.724842 & 0.0529003 & 0.807529 & 0.0470599 \\
\hline 0.124819 & 1.52687 & 0.081972 & 0.734444 & 0.056922 & 0.814768 & 0.0392135 \\
\hline 0.114417 & 1.52307 & 0.0782438 & 0.729813 & 0.0568382 & 0.813457 & 0.042182 \\
\hline 0.105116 & 1.53602 & 0.0827066 & 0.747336 & 0.0519527 & 0.814479 & 0.0383847 \\
\hline 0.13312 & 1.53975 & 0.0826963 & 0.748136 & 0.0631964 & 0.810587 & 0.0491775 \\
\hline 0.105803 & 1.54167 & 0.0769634 & 0.739059 & 0.0575539 & 0.812804 & 0.0396101 \\
\hline 0.141253 & 1.54945 & 0.081095 & 0.746509 & 0.0661577 & 0.812312 & 0.0389265 \\
\hline 0.119535 & 1.54151 & 0.0802948 & 0.744183 & 0.0691603 & 0.804545 & 0.0519286 \\
\hline 0.126789 & 1.5529 & 0.0767267 & 0.739053 & 0.0626825 & 0.801772 & 0.042699 \\
\hline 0.127347 & 1.55556 & 0.0695443 & 0.755672 & 0.0631265 & 0.812699 & 0.047273 \\
\hline 0.133267 & 1.55205 & 0.0736161 & 0.743988 & 0.0629309 & 0.81481 & 0.0440766 \\
\hline 0.118152 & 1.54619 & 0.07579 & 0.754696 & 0.0581053 & 0.803433 & 0.0438268 \\
\hline 0.138595 & 1.55032 & 0.0696044 & 0.754305 & 0.0597397 & 0.801877 & 0.0475628 \\
\hline 0.133465 & 1.55694 & 0.0637263 & 0.750389 & 0.0640989 & 0.797165 & 0.0493592 \\
\hline 0.128714 & 1.55885 & 0.0688473 & 0.752321 & 0.063207 & 0.793631 & 0.045193 \\
\hline 0.126074 & 1.55541 & 0.0702426 & 0.749311 & 0.0587598 & 0.801961 & 0.0436202 \\
\hline 0.125741 & 1.55698 & 0.064 & 446 & 0.0554944 & 0.799126 & 0.055086 \\
\hline 0.140655 & 1.55154 & 0.0623909 & 0.752119 & 0.0655757 & 0.801564 & 0.0424261 \\
\hline 0.127346 & 1.56089 & 0.0733978 & 0.749856 & 0.0666222 & 0.800035 & 0.0363693 \\
\hline 0.129572 & 1.56661 & 0.0684013 & 0.759447 & 0.0644025 & 0.801081 & 0.0442136 \\
\hline 0.119106 & 1.56384 & 0.0745823 & 0.759052 & 0.0639786 & 0.791772 & 0.0613219 \\
\hline 0.124878 & 1.56047 & 0.0789593 & 0.76074 & 0.0654077 & 0.796291 & 0.0487655 \\
\hline 0.120441 & 1.56229 & 0.0577207 & 0.756411 & 0.0605393 & 0.790899 & 0.0492933 \\
\hline 0.120574 & 1.56481 & 0.0613023 & 0.75915 & 0.0538275 & 0.800428 & 0.0615938 \\
\hline 0.110327 & 1.57068 & 0.0702538 & 0.761205 & 0.0597949 & 0.800733 & 0.0340748 \\
\hline 0.125751 & 1.57142 & 0.0695562 & 0.763558 & 0.0704736 & 0.806813 & 0.0513196 \\
\hline 0.116677 & 1.57608 & 0.0592985 & 0.757293 & 0.0562213 & 0.805177 & 0.0534771 \\
\hline 0.0997382 & 1.58083 & 0.0669258 & 0.763829 & 0.0583867 & 0.802575 & 0.057773 \\
\hline 0.112855 & 1.5721 & 0.0707836 & 0.766819 & 0.062463 & 0.797343 & 0.0462066 \\
\hline 0.0964331 & 1.57163 & 0.0705061 & 0.768036 & 0.0559786 & 0.807705 & 0.0461591 \\
\hline 0.120216 & 1.57596 & 0.0726533 & 0.75997 & 0.0598332 & 0.798517 & 0.0474374 \\
\hline 0.0954949 & 1.57909 & 0.0770238 & 0.781376 & 0.0550849 & 0.803479 & 0.0445884 \\
\hline 0.11373 & 1.57918 & 0.0786322 & 0.778394 & 0.0620065 & 0.808577 & 0.0468623 \\
\hline 0.0996956 & 1.56716 & 0.0721334 & 0.769866 & 0.0529928 & 0.798053 & 0.043234 \\
\hline
\end{tabular}




\begin{tabular}{|c|c|c|c|c|c|c|}
\hline 0.104135 & 1.56763 & 0.064515 & 0.779272 & 0.0519322 & 0.801878 & 0.0461123 \\
\hline 0.122797 & 1.56109 & 0.0689022 & 0.780185 & 0.0637278 & 0.807569 & 0.0451369 \\
\hline 0.130519 & 1.57561 & 0.0771475 & 0.788644 & 0.0664932 & 0.806562 & 0.0490311 \\
\hline 0.12435 & .57767 & 0.0680973 & 0.777092 & 0.0500322 & 0.803807 & 0.0412602 \\
\hline 0.110387 & 1.57506 & 0.0582271 & 0.786307 & 0.048273 & 0.7974 & 0.0400097 \\
\hline 0.107734 & 1.57339 & 0.0658025 & 0.794014 & 0.0580038 & 0.794778 & 0.0465919 \\
\hline 0.120612 & 1.57794 & 0.0636144 & 0.79036 & 0.0565468 & 0.802918 & 0.0450892 \\
\hline 0.136106 & 1.57751 & 0.0662865 & 0.79721 & 0.0677277 & 0.806416 & 0.0410566 \\
\hline 0.11363 & 1.58694 & 0.0732274 & 0.801145 & 0.053955 & 0.802711 & 0.0465478 \\
\hline 0.123941 & 1.57748 & 0.0717567 & 0.809603 & 0.0646253 & 0.807226 & 0.0501288 \\
\hline 0.142552 & 1.58299 & 0.0706363 & 0.805598 & 0.0612991 & 0.806218 & 0.0409289 \\
\hline 0.118101 & 1.58174 & 0.0585242 & 0.818462 & 0.0719463 & 0.805016 & 0.0496787 \\
\hline 0.15518 & 1.58502 & 0.0587863 & 0.818702 & 0.0603227 & 0.807084 & 0.0386712 \\
\hline 0.160735 & 1.58493 & 0.071372 & 0.830341 & 0.0655625 & 0.808609 & 0.038869 \\
\hline 0.147355 & 1.59009 & 0.0717941 & 0.824689 & 0.069106 & 0.80424 & 0.0401795 \\
\hline 0.136205 & 1.58796 & 0.0698703 & 0.830984 & 0.070482 & 0.808446 & 0.0491096 \\
\hline 0.127383 & 1.57521 & 0.0783181 & 0.824256 & 0.071608 & 0.80423 & 0.039639 \\
\hline 0.140695 & 1.56836 & 0.0732019 & 0.822285 & 0.0707647 & 0.799663 & 0.0424809 \\
\hline 0.154621 & 1.56857 & 0.0690545 & 0.828928 & 0.0536488 & 345 & 0.0494668 \\
\hline 0.145037 & 1.56341 & 0.068504 & 0.831632 & 0.075699 & 0.800727 & 0.0432205 \\
\hline 0.139299 & 1.5549 & 0.0649954 & 0.821443 & 0.065727 & 0.803284 & 0.0463589 \\
\hline 0.156506 & 1.57361 & 0.076402 & 0.829545 & 0.0597551 & 0.802543 & 9754 \\
\hline 0.118228 & 1.56426 & 0.0695018 & 0.814159 & 0.0749875 & 0.805624 & 0.0472321 \\
\hline 0.166047 & 1.56258 & 0.0655978 & 0.807905 & 0.0682965 & 0.790529 & 0.0390317 \\
\hline 0.129375 & 1.55306 & 0.0681627 & 0.805571 & 0.0529738 & 0.794593 & 0.0492651 \\
\hline 0.128036 & 1.55747 & 0.0533595 & 0.802881 & 0.0661912 & 0.798747 & 0.0382495 \\
\hline 0.153714 & 1.55044 & 0.0567079 & 0.78638 & 0.0572224 & 0.803587 & 0.0590674 \\
\hline 0.136165 & 1.55177 & 0.0594334 & 0.788948 & 0.0588802 & 0.792952 & 0.0479382 \\
\hline 0.130034 & 1.5511 & 0.0594069 & 0.799351 & 0.0520464 & 0.803411 & 0.0463048 \\
\hline 0.120909 & 1.54476 & 0.0647086 & 0.780732 & 0.0497616 & 0.797103 & 0.0429976 \\
\hline 0.121381 & 1.54006 & 0.0723961 & 0.796192 & 0.0523929 & 0.805882 & 0.0503047 \\
\hline 0.122273 & 1.54432 & 0.0712991 & 0.781112 & 0.0511034 & 0.800564 & 0.0374798 \\
\hline 0.116813 & 1.53103 & 0.0677252 & 0.781111 & 0.0421346 & 0.795236 & 0.0468855 \\
\hline 0.121112 & 1.53726 & 0.0651253 & 0.767379 & 0.0506091 & 0.790283 & 0.0436326 \\
\hline 0.107763 & 1.53675 & 0.0654749 & 0.767135 & 0.0460797 & 0.791099 & 0.0462539 \\
\hline 0.0930421 & 1.53696 & 0.0667607 & 0.763834 & 0.0415229 & 0.79101 & 0.037477 \\
\hline 0.128549 & 1.53587 & 0.0634537 & 0.779912 & 0.0491864 & 0.78892 & 0.0345875 \\
\hline 0.108321 & 1.53982 & 0.0550708 & 0.781744 & 0.0422293 & 0.776443 & 0.0395642 \\
\hline 0.115789 & 1.53355 & 0.0654397 & 0.764008 & 0.0463258 & 0.799896 & 0.0520658 \\
\hline 0.133302 & 1.52896 & 0.0613919 & 0.777059 & 0.0389091 & 0.797168 & 0.045362 \\
\hline 0.109531 & 1.5335 & 0.0576506 & 0.768997 & 0.0425294 & 0.789252 & 0.0458159 \\
\hline 0.101351 & 1.53498 & 0.0557406 & 0.777057 & 0.0447304 & 0.779545 & 0.0417192 \\
\hline 0.12186 & 1.53502 & 0.0617743 & 0.770315 & 0.0480907 & 0.798378 & 0.0417272 \\
\hline 0.127702 & 1.53488 & 0.0604936 & 0.771572 & 0.0489533 & 0.791945 & 0.0410373 \\
\hline 0.12629 & 1.53853 & 0.0555329 & 0.765965 & 0.0484983 & 0.780523 & 0.0374345 \\
\hline 0.121473 & 1.53337 & 0.0666688 & 0.766403 & 0.0452696 & 0.793591 & 0.0415964 \\
\hline 0.117611 & 1.53945 & 0.0588993 & 0.771906 & 0.0488304 & 0.783305 & 0.0399279 \\
\hline
\end{tabular}




\begin{tabular}{|c|c|c|c|c|c|c|}
\hline 0.134219 & 1.53108 & 0.0667765 & 0.763935 & 0.0523155 & 0.785684 & 0.0445043 \\
\hline 0.134347 & 1.52848 & 0.0701449 & 0.769154 & 0.0435145 & 0.80099 & 0.0461264 \\
\hline 0.125737 & .54123 & 0.0624605 & 0.772706 & 0.0464552 & 0.793613 & 0.0459674 \\
\hline 0.0937242 & 1.5386 & 0.0581081 & 0.778956 & 0.0550613 & 0.811638 & 0.0466851 \\
\hline 0.107025 & 1.53422 & 0.0558564 & 0.778468 & 0.0635104 & 0.802683 & 0.0372577 \\
\hline 0.102598 & 1.53267 & 0.0518984 & 0.777767 & 0.0522141 & 0.811126 & 0.0515974 \\
\hline 0.124164 & 1.532 & 0.0609812 & 0.775592 & 0.0492324 & 0.813369 & 0.0538791 \\
\hline 0.113545 & 1.53864 & 0.0635382 & 0.792342 & 0.0571504 & 0.822516 & 0.050985 \\
\hline 0.0962854 & 1.52811 & 0.0605505 & 0.780608 & 0.0580237 & 0.823679 & 0.0382978 \\
\hline 0.0916729 & 1.5312 & 0.0654952 & 0.782932 & 0.0491395 & 0.820683 & 0.0472139 \\
\hline 0.122175 & 1.52481 & 0.0703936 & 0.782882 & 0.0525794 & 0.834329 & 0.0486966 \\
\hline 0.119316 & 1.53179 & 0.0608043 & 0.787682 & 0.0552238 & 0.838448 & 0.0552162 \\
\hline 0.108993 & 1.53094 & 0.0643771 & 0.791934 & 0.0524261 & 0.836264 & 0.0475845 \\
\hline 0.122923 & 1.53292 & 0.0561144 & 0.793335 & 0.0630411 & 0.83189 & 0.0424029 \\
\hline 0.126043 & 1.53059 & 0.0593528 & 0.791985 & 0.0656933 & 0.840648 & 0.0534698 \\
\hline 0.117316 & 1.52673 & 0.0678479 & 0.797435 & 0.0629854 & 0.841905 & 0.0488257 \\
\hline 0.0966087 & 1.5197 & 0.0673275 & 0.798116 & 0.0680535 & 0.846002 & 0.0545543 \\
\hline 0.111149 & 1.51803 & 0.0700699 & 0.790168 & 0.0661879 & 0.824411 & 0.0635993 \\
\hline 0.117772 & 1.50372 & 0.0607611 & 0.793897 & 0.060715 & 0.849549 & 0.0600047 \\
\hline 0.117138 & 1.50433 & 0.0510343 & 0.796367 & 0.0632465 & 0.836271 & 0.0531544 \\
\hline 0.102703 & 1.50482 & 0.0547119 & 0.782362 & 0.0533161 & 0.829873 & 0.0651703 \\
\hline 0.119977 & 1.5075 & 0.061281 & 0.777732 & 0.0595509 & 0.824838 & 0.0545876 \\
\hline 0.105531 & 1.50015 & 0.0587242 & 0.776193 & 0.0529735 & 0.824902 & 0.0551116 \\
\hline 0.126841 & 1.49402 & 0.0558405 & 0.773407 & 0.0636627 & 0.805467 & 0.056041 \\
\hline 0.116562 & 1.49149 & 0.0517557 & 0.770556 & 0.0578345 & 0.797621 & 0.0499634 \\
\hline 0.111971 & 1.49072 & 0.0596235 & 0.767164 & 0.0475061 & 0.801479 & 0.0592235 \\
\hline 0.109037 & 1.48207 & 0.0596705 & 0.758278 & 0.0498877 & 0.794264 & 0.0599751 \\
\hline 0.104749 & 1.49471 & 0.0559577 & 0.763949 & 0.0481993 & 0.779729 & 0.0542444 \\
\hline 0.109255 & 1.48922 & 0.0521746 & 0.757874 & 0.0414251 & 0.77721 & 0.0485145 \\
\hline 0.11438 & 1.48055 & 0.047977 & 0.753478 & 0.0453979 & 0.781762 & 0.0607356 \\
\hline 0.0945218 & 1.47757 & 0.0621726 & 0.748391 & 0.0484535 & 0.771867 & 0.0477799 \\
\hline 0.10174 & 1.4862 & 0.062023 & 0.75371 & 0.0499029 & 0.761806 & 0.0504145 \\
\hline 0.107972 & 1.47955 & 0.0602048 & 0.744635 & 0.0568326 & 0.764948 & 0.0422283 \\
\hline 0.123813 & 1.46933 & 0.0607988 & 0.752556 & 0.0496103 & 0.756094 & 0.0409968 \\
\hline 0.0952014 & 1.46772 & 0.0541214 & 0.756894 & 0.0556572 & 0.763287 & 0.0471034 \\
\hline 0.135145 & 1.46862 & 0.0547373 & 0.74769 & 0.0519924 & 0.753339 & 0.0411459 \\
\hline 0.103407 & 1.46153 & 0.0588223 & 0.746542 & 0.054731 & 0.753225 & 0.0422874 \\
\hline 0.107641 & 1.47377 & 0.0507262 & 0.739047 & 0.0503094 & 0.760197 & 0.0576463 \\
\hline 0.118156 & 1.48108 & 0.0490915 & 0.736207 & 0.051418 & 0.760412 & 0.0445954 \\
\hline 0.0897647 & 1.46726 & 0.0548435 & 0.744451 & 0.0524598 & 0.760693 & 0.0371283 \\
\hline 0.109571 & 1.44943 & 0.0502261 & 0.741 & 0.0580138 & 0.741443 & 0.0387979 \\
\hline 0.117554 & 1.45583 & 0.0599534 & 0.740248 & 0.0566227 & 0.755355 & 0.0435005 \\
\hline 0.104986 & 1.46923 & 0.059348 & 0.734245 & 0.0505046 & 0.745192 & 0.0413415 \\
\hline 0.119706 & 1.46739 & 0.0600514 & 0.738171 & 0.0551344 & 0.747067 & 0.0438906 \\
\hline 0.100902 & 1.46567 & 0.0590657 & 0.739293 & 0.0547999 & 0.746533 & 0.0405718 \\
\hline 0.116636 & 1.45815 & 0.0585188 & 0.742295 & 0.0559217 & 0.746096 & 0.0447626 \\
\hline 0.116317 & 1.44938 & 0.0560432 & 0.738646 & 0.0473141 & 0.752568 & 0.0391208 \\
\hline
\end{tabular}




\begin{tabular}{|c|c|c|c|c|c|c|}
\hline 0.114914 & 1.45371 & 0.0545629 & 0.732818 & 0.0504899 & 0.745195 & 0.0398837 \\
\hline 0.130613 & 1.45288 & 0.0580172 & 0.737833 & 0.0430191 & 0.745103 & 0.042018 \\
\hline 0.120375 & 1.4571 & 0.0584201 & 0.731133 & 0.0615085 & 0.740647 & 0.037223 \\
\hline 0.117448 & 1.45596 & 0.0553873 & 0.737333 & 0.0544944 & 0.736876 & 0.0447853 \\
\hline 0.120181 & 1.4581 & 0.0629995 & 0.73952 & 0.0488411 & 0.741673 & 0.0396074 \\
\hline 0.13542 & 1.4483 & 0.0562951 & 0.733849 & 0.0502776 & 0.741609 & 0.0463985 \\
\hline 0.123395 & 1.4526 & 0.0526361 & 0.723043 & 0.0559598 & 0.741791 & 0.0439341 \\
\hline 0.109578 & 1.45505 & 0.0588559 & 0.722679 & 0.0531836 & 0.754304 & 0.0444128 \\
\hline 0.116429 & 1.45333 & 0.0628687 & 0.732335 & 0.0530789 & 0.738958 & 0.0388653 \\
\hline 0.127539 & 1.45306 & 0.0606069 & 0.726365 & 0.0531308 & 0.745253 & 0.0372756 \\
\hline 0.141349 & 1.44904 & 0.0611978 & 0.735287 & 0.0441106 & 0.748884 & 0.0383395 \\
\hline 0.110686 & 1.45304 & 0.0702767 & 0.726486 & 0.0423018 & 0.727948 & 0.0312685 \\
\hline 0.119289 & 1.44245 & 0.0610482 & 0.716734 & 0.0449344 & 0.738494 & 0.0375602 \\
\hline 0.12643 & 1.43848 & 0.0584353 & 0.731278 & 0.0486147 & 0.731843 & 0.0337077 \\
\hline 0.133294 & 1.44612 & 0.0598928 & 0.72637 & 0.0554235 & 0.735571 & 0.038458 \\
\hline 0.120555 & 1.44431 & 0.0626223 & 0.722173 & 0.051607 & 0.726496 & 0.0358794 \\
\hline 0.116849 & 1.44583 & 0.0622106 & 0.733304 & 0.048825 & 0.727046 & 0.0330033 \\
\hline 0.127674 & 1.45108 & 0.0608255 & 0.727789 & 0.0406132 & 0.722478 & 0.0422693 \\
\hline 0.1346 & 1.44883 & 0.0625487 & 0.725192 & 0.0427397 & 0.726108 & 0.0300383 \\
\hline 0.118696 & 1.44524 & 0.0556799 & 0.727931 & 0.0503624 & 0.715039 & 0.0359449 \\
\hline 0.119205 & 1.44488 & 0.0528755 & 0.722378 & 0.0402644 & 0.715462 & 0.0388813 \\
\hline 0.127663 & 1.44211 & 0.0530483 & 0.725321 & 0.0457366 & 0.730783 & 0.0328245 \\
\hline 0.113933 & 1.43794 & 0.0603954 & 0.71587 & 0.0477017 & 0.722759 & 0.0314694 \\
\hline 0.133134 & 1.44337 & 0.0675583 & 0.72142 & 0.0503917 & 0.722292 & 0.032909 \\
\hline 0.123874 & 1.44552 & 0.0547399 & 0.717727 & 0.0502287 & 0.727508 & 0.0411293 \\
\hline 0.117315 & 1.44608 & 0.0547304 & 0.724234 & 0.0438371 & 0.726391 & 0.0338682 \\
\hline 0.114393 & 1.44548 & 0.054883 & 0.715634 & 0.0552907 & 0.715543 & 0.0355487 \\
\hline 0.112568 & 1.44961 & 0.0563291 & 0.719113 & 0.0427315 & 0.718196 & 0.0401164 \\
\hline 0.109852 & 1.45263 & 0.0577055 & 0.716042 & 0.0480262 & 0.722044 & 0.0371636 \\
\hline 0.10579 & 1.44504 & 0.055743 & 0.714085 & 0.0525491 & 0.719988 & 0.027324 \\
\hline 0.106494 & 1.44864 & 0.057704 & 0.724451 & 0.0407932 & 0.723548 & 0.0384499 \\
\hline 0.106722 & 1.44982 & 0.0552133 & 0.720981 & 0.0472071 & 0.712051 & 0.0356444 \\
\hline 0.112546 & 1.44288 & 0.0518074 & 0.715406 & 0.0451563 & 0.718495 & 0.0349925 \\
\hline 0.101032 & 1.44106 & 0.0511899 & 0.725979 & 0.0548072 & 0.716048 & 0.0369409 \\
\hline 0.100331 & 1.44964 & 0.0512486 & 0.713557 & 0.0386812 & 0.729548 & 0.0321596 \\
\hline 0.108138 & 1.44274 & 0.0471421 & 0.715368 & 0.0395173 & 0.722097 & 0.0320038 \\
\hline 0.112448 & 1.43545 & 0.0531765 & 0.716685 & 0.0491949 & 0.726867 & 0.0388908 \\
\hline 0.10246 & 1.44043 & 0.0523568 & 0.708658 & 0.0399995 & 0.725204 & 0.0323259 \\
\hline 0.090305 & 1.44907 & 0.0527732 & 0.711863 & 0.0516793 & 0.716204 & 0.0326056 \\
\hline 0.106735 & 1.4467 & 0.0535375 & 0.712235 & 0.0487612 & 0.717183 & 0.0333776 \\
\hline 0.118077 & 1.44343 & 0.0608723 & 0.717225 & 0.0433395 & 0.72088 & 0.041923 \\
\hline 0.096478 & 1.45058 & 0.0523891 & 0.713871 & 0.0460109 & 0.712876 & 0.042581 \\
\hline 0.0832042 & 1.44363 & 0.0508161 & 0.711503 & 0.0473238 & 0.722856 & 0.0366014 \\
\hline 0.117023 & 1.43842 & 0.0532124 & 0.701758 & 0.0463577 & 0.711417 & 0.0455133 \\
\hline 0.0926013 & 1.44248 & 0.0579004 & 0.714115 & 0.0435705 & 0.717366 & 0.0452398 \\
\hline 0.100939 & 1.44724 & 0.0596303 & 0.713043 & 0.0452317 & 0.72077 & 0.0391757 \\
\hline 0.120275 & 1.44093 & 0.0566159 & 0.711543 & 0.0464278 & 0.708272 & 0.0392626 \\
\hline
\end{tabular}




\begin{tabular}{|c|c|c|c|c|c|c|}
\hline 0.0939606 & 1.44359 & 0.0616014 & 0.708812 & 0.0369639 & 0.721427 & 0.03 \\
\hline 0.0842214 & 1.45007 & 0.062405 & 0.70272 & 0.0426486 & 0.708915 & 0.0300002 \\
\hline 0.0999885 & 1.43869 & 0.0611387 & 0.715645 & 0.0480288 & 0.703048 & 0.0322299 \\
\hline 0.110293 & 1.4322 & 0.0607602 & 0.707316 & 0.0478565 & 0.70996 & 0.0336835 \\
\hline 0.0776421 & 1.43642 & 0.056165 & 0.712483 & 0.0442152 & 0.712322 & 0.0482027 \\
\hline 0.0915889 & 1.44318 & 0.0667222 & 0.712262 & 0.0404747 & 0.706357 & 0.0360474 \\
\hline 0.086063 & 1.4446 & 0.0705593 & 0.713571 & 0.0413902 & 0.709612 & 0.0293688 \\
\hline 0.101992 & 1.43383 & 0.0660199 & 0.703638 & 0.0456606 & 0.714095 & 0.0390504 \\
\hline 0.0746534 & 1.43732 & 0.0646394 & 0.70297 & 0.0426774 & 0.713744 & 0.04198 \\
\hline 0.0855542 & 1.44026 & 0.0710845 & 0.712357 & 0.0462822 & 0.720351 & 0.0346563 \\
\hline 0.108161 & 1.44099 & 0.0664617 & 0.706174 & 0.0451315 & 0.707926 & 0.0366404 \\
\hline 0.101956 & 1.44192 & 0.0636711 & 0.70777 & 0.0499448 & 0.708499 & 0.0393154 \\
\hline 0.0910303 & 1.43313 & 0.0686477 & 0.711913 & 0.0403623 & 0.708991 & 0.0369952 \\
\hline 0.0939386 & 1.43238 & 0.0681786 & 0.706604 & 0.039825 & 0.704686 & 0.0365297 \\
\hline 0.0945612 & 1.43492 & 0.0659388 & 0.709312 & 0.0498011 & 0.704779 & 0.0271222 \\
\hline 0.0961785 & 1.43405 & 0.0602211 & 0.704673 & 0.0329015 & 0.711034 & 0.047597 \\
\hline 0.0952835 & 1.43015 & 0.0538639 & 0.709054 & 0.0407793 & 0.715312 & 0.0391871 \\
\hline 0.0975665 & 1.42829 & 0.0679791 & 0.699821 & 0.034378 & 0.707416 & 0.0311132 \\
\hline 0.0833344 & 1.42968 & 0.0701344 & 0.706547 & 0.0406763 & 0.704723 & 0.0428949 \\
\hline 0.0986814 & 1.43243 & 0.0627319 & 0.700816 & 0.0352045 & 0.704986 & 0.0397759 \\
\hline 0.102459 & 1.42978 & 0.063488 & 0.701931 & 0.042045 & 0.709106 & 0.0370273 \\
\hline 0.102086 & 1.43046 & 0.0650735 & 0.698764 & 0.0430619 & 0.708581 & 0.0397009 \\
\hline 0.0907508 & 1.42747 & 0.0607949 & 0.70484 & 0.0438915 & 0.705243 & 0.0355643 \\
\hline 0.084617 & 1.42464 & 0.0593368 & 0.694686 & 0.0365648 & 0.701459 & 0.0341421 \\
\hline 0.0800393 & 1.42835 & 0.055 & 0.71293 & 0.03 & 0.702776 & 0.0411242 \\
\hline 0.0893979 & 1.43225 & 0.0626138 & 0.701373 & 0.0441493 & 0.715643 & 0.0378789 \\
\hline 0.0848255 & 1.43469 & 0.0676252 & 0.708654 & 0.034705 & 0.705705 & 0.0321479 \\
\hline 0.10123 & 1.42808 & 0.0608416 & 0.713249 & 0.0427223 & 0.70646 & 0.0341822 \\
\hline 0.0834103 & 1.43078 & 0.0573655 & 0.70319 & 0.0415474 & 0.713435 & 0.0447263 \\
\hline 0.08442 & 1.43152 & 0.0618994 & 0.707951 & 0.0414091 & 0.706478 & 0.0435314 \\
\hline 0.092184 & 1.43013 & 0.0700764 & 0.698384 & 0.0468413 & 0.711652 & 0.0388516 \\
\hline 0.0847422 & 1.42726 & 0.0681325 & 0.703888 & 0.0408153 & 0.706329 & 0.0365042 \\
\hline 0.10528 & 1.42626 & 0.0660946 & 0.716766 & 0.045921 & 0.710823 & 0.0448864 \\
\hline 0.088319 & 1.43071 & 0.066968 & 0.698231 & 0.0444724 & 0.697274 & 0.040783 \\
\hline 0.0879489 & 1.42432 & 0.0626167 & 0.70237 & 0.040989 & 0.719305 & 0.0316244 \\
\hline 0.0747808 & 1.42808 & 0.0545267 & 0.708323 & 0.044114 & 0.703831 & 0.0297215 \\
\hline 0.107153 & 1.43291 & 0.0533226 & 0.701672 & 0.0456043 & 0.710519 & 0.0349561 \\
\hline 0.0922435 & 1.43258 & 0.0547848 & 0.706459 & 0.0466093 & 0.70611 & 0.0350053 \\
\hline 0.0946685 & 1.43331 & 0.0627637 & 0.70808 & 0.0395232 & 0.709816 & 0.0433641 \\
\hline 0.0831862 & 1.42854 & 0.0692448 & 0.694842 & 0.0477242 & 0.717435 & 0.0387902 \\
\hline 0.0808928 & 1.42404 & 0.067044 & 0.708871 & 0.0436945 & 0.706019 & 0.0503785 \\
\hline 0.0936033 & 1.42579 & 0.0651897 & 0.710633 & 0.0454222 & 0.714552 & 0.0438861 \\
\hline 0.0909978 & 1.4285 & 0.0662643 & 0.701262 & 0.0507984 & 0.702076 & 0.0301835 \\
\hline 0.0899109 & 1.4258 & 0.0579193 & 0.710524 & 0.0468911 & 0.70807 & 0.0410478 \\
\hline 0.0869359 & 1.4187 & 0.0516015 & 0.704519 & 0.0323442 & 0.705759 & 0.0343498 \\
\hline 0.102791 & 1.41786 & 0.0571067 & 0.704325 & 0.038557 & 0.69322 & 0.0435681 \\
\hline 0.103897 & 1.4193 & 0.0615703 & 0.6882 & 0.039008 & 0.70776 & 0.0305519 \\
\hline
\end{tabular}




\begin{tabular}{|c|c|c|c|c|c|c|}
\hline 0.111141 & 1.41779 & 0.0572319 & 0.702602 & 0.0407582 & 0.707954 & 0.0361382 \\
\hline 0.0930845 & 1.42381 & 0.0564567 & 0.710208 & 0.0353545 & 0.69587 & 0.0412692 \\
\hline 0.100337 & 1.42428 & 0.058445 & 0.711408 & 0.0352387 & 0.696859 & 0.041147 \\
\hline 0.0970268 & 1.42523 & 0.0537068 & 0.708877 & 0.0526105 & 0.697988 & 0.0345394 \\
\hline 0.0934533 & 1.42188 & 0.05259 & 0.688264 & 0.0354081 & 0.690446 & 0.0323362 \\
\hline 0.102269 & 1.41116 & 0.0492129 & 0.700696 & 0.0445768 & 0.691817 & 0.0348633 \\
\hline 0.103097 & 1.41164 & 0.0474799 & 0.704712 & 0.0420848 & 0.698726 & 0.0396029 \\
\hline 0.0917362 & 1.41244 & 0.0506337 & 0.704338 & 0.0347312 & 0.698793 & 0.0304982 \\
\hline 0.0870884 & 1.41048 & 0.047564 & 0.710483 & 0.0362231 & 0.694688 & 0.0396707 \\
\hline 0.0914956 & 1.40736 & 0.0410239 & 0.701478 & 0.0327707 & 0.701924 & 0.0396269 \\
\hline 0.0828858 & 1.40345 & 0.0459529 & 0.705594 & 0.0414773 & 0.689736 & 0.0389484 \\
\hline 0.0814288 & 1.40104 & 0.0569702 & 0.698025 & 0.0385372 & 0.68755 & 0.0389658 \\
\hline 0.0839852 & 1.40193 & 0.0569253 & 0.696273 & 0.0389343 & 0.677546 & 0.0379909 \\
\hline 0.0635735 & 1.40638 & 0.0517255 & 0.699004 & 0.0446172 & 0.68621 & 0.0382456 \\
\hline 0.0941396 & 1.40429 & 0.0524744 & 0.697212 & 0.0332009 & 0.689365 & 0.0387463 \\
\hline 0.0809527 & 1.39767 & 0.0545313 & 0.686953 & 0.0435552 & 0.683576 & 0.0422947 \\
\hline 0.066298 & 1.39922 & 0.0539372 & 0.68505 & 0.0411085 & 0.6898 & 0.0387223 \\
\hline 0.085098 & 1.40097 & 0.0496372 & 0.689762 & 0.0285716 & 0.683834 & 0.0367775 \\
\hline 0.0920013 & 1.39478 & 0.0478433 & 0.690435 & 0.03 & 0.681249 & 0.0302107 \\
\hline 0.0750664 & 1.39054 & 0.0516981 & 0.682171 & 0.0425431 & 0.688506 & 0.0430949 \\
\hline 0.075285 & 1.38561 & 0.0553938 & 0.676238 & 0.0315671 & 0.67548 & 0.036499 \\
\hline 0.0833774 & 1.37985 & 0.0547451 & 0.689547 & 0.0 & 0.680883 & 0.0356976 \\
\hline 0.101601 & 1.37685 & 0.0480913 & 0.686722 & 0.0427786 & 0.672187 & 0.0353369 \\
\hline 0.0944045 & 1.377 & 0.0458378 & 0.683823 & 0.036541 & 0.676625 & 0.0382414 \\
\hline 0.0918071 & 1.3787 & 0.047658 & 0.684354 & 0.0384494 & 0.682983 & 0.0410309 \\
\hline 0.0989941 & 1.3807 & 0.0481636 & 0.69172 & 0.0399527 & 0.667443 & 0.0365098 \\
\hline 0.0720013 & 1.3806 & 0.0509647 & 0.686749 & 0.0380663 & 0.678116 & 0.0380251 \\
\hline 0.0953796 & 1.37743 & 0.0477988 & 0.674093 & 0.0368449 & 0.666854 & 0.0437418 \\
\hline 0.0773762 & 1.3812 & 0.0433423 & 0.684769 & 0.0424707 & 0.669187 & 0.033523 \\
\hline 0.0856515 & 1.37822 & 0.049098 & 0.693087 & 1588 & 0.672567 & 0.0362368 \\
\hline 0.0839852 & 1.36886 & 0.0499625 & 0.674342 & 0.0376929 & 0.669445 & 0.0304569 \\
\hline 0.102915 & 1.36196 & 0.048627 & 0.687362 & 0.038992 & 0.670334 & 0.0345338 \\
\hline 0.0890668 & 1.3511 & 0.0485462 & 0.678928 & 0.0396002 & 0.671008 & 0.0357837 \\
\hline 0.100101 & 1.35325 & 0.0461845 & 0.683736 & 0.0288632 & 0.675785 & 0.0338475 \\
\hline 0.100848 & 1.35538 & 0.0447136 & 0.680473 & 0.0370245 & 0.676351 & 0.0387953 \\
\hline 0.0958809 & 1.34795 & 0.0417582 & 0.679801 & 0.0389532 & 0.663388 & 0.0336504 \\
\hline 0.0869473 & 1.34859 & 0.0397262 & 0.674091 & 0.0345715 & 0.668891 & 0.03388 \\
\hline 0.0900176 & 1.35188 & 0.0453627 & 0.683908 & 0.0347493 & 0.665998 & 0.0431302 \\
\hline 0.0965016 & 1.34831 & 0.0438496 & 0.677492 & 0.0345978 & 0.667345 & 0.0403247 \\
\hline 0.111044 & 1.34279 & 0.0431634 & 0.678682 & 0.0417489 & 0.659188 & 0.0260266 \\
\hline 0.102288 & 1.34259 & 0.0501032 & 0.668356 & 0.0322185 & 0.649725 & 0.0395195 \\
\hline 0.115255 & 1.34077 & 0.0511664 & 0.667002 & 0.0371394 & 0.664454 & 0.0306287 \\
\hline 0.111153 & 1.33221 & 0.0490672 & 0.676557 & 0.0310664 & 0.654895 & 0.0343323 \\
\hline 0.115234 & 1.32616 & 0.0439368 & 0.670007 & 0.043694 & 0.658756 & 0.0397719 \\
\hline 0.116095 & 1.3326 & 0.0443122 & 0.675206 & 0.0325685 & 0.648842 & 0.0350391 \\
\hline 0.117553 & 1.32808 & 0.0458225 & 0.666336 & 0.0370152 & 0.660341 & 0.0389992 \\
\hline 0.110593 & 1.32214 & 0.0471637 & 0.662234 & 0.0393488 & 0.655538 & 0.033655 \\
\hline
\end{tabular}




\begin{tabular}{|c|c|c|c|c|c|c|}
\hline 0.121764 & 1.32455 & 0.0474255 & 0.662765 & 0.0386788 & 0.651129 & 5824 \\
\hline 0.13066 & 1.31344 & 0.0465202 & 0.662807 & 0.0352626 & 0.658852 & 0.0350145 \\
\hline 0.124806 & 1.31236 & 0.0548358 & 0.660699 & 0.0387252 & 0.648503 & 0.0451047 \\
\hline 0.116169 & 1.31701 & 0.0440938 & 0.664908 & 0.0303001 & 0.645317 & 0.0342461 \\
\hline 0.132494 & 1.30741 & 0.0370866 & 0.657695 & 0.034679 & 0.646222 & 0.0332672 \\
\hline 0.12832 & 1.29996 & 0.0426088 & 0.659454 & 0.0404995 & 0.65101 & 0.0331829 \\
\hline 0.100938 & 1.29832 & 0.0400102 & 0.64918 & 0.0397684 & 0.655495 & 0.0382063 \\
\hline 0.114661 & 1.29391 & 0.0422849 & 0.650616 & 0.0367764 & 0.640721 & 0.0362784 \\
\hline 0.142009 & 1.29404 & 0.0455336 & 0.657215 & 0.0303267 & 0.639142 & 0.0329983 \\
\hline 0.102742 & 1.29112 & 0.0514845 & 0.656295 & 0.0369011 & 0.640073 & 0.0234456 \\
\hline 0.102448 & 1.28606 & 0.0429055 & 0.648343 & 0.0374234 & 0.640201 & 0.0316082 \\
\hline 0.112877 & 1.2886 & 0.0368601 & 0.645493 & 0.0417361 & 0.628811 & 0.0371566 \\
\hline 0.120204 & 1.29091 & 0.0439749 & 0.649132 & 0.0356741 & 0.637475 & 0.0361977 \\
\hline 0.0937429 & 1.29 & 0.0448191 & 0.653296 & 0.0358702 & 0.639892 & 0.0324678 \\
\hline 0.132092 & 1.28949 & 0.0463644 & 0.645241 & 0.0392723 & 0.632864 & 0.0388697 \\
\hline 0.105841 & 1.28256 & 0.0475854 & 0.645778 & 0.0430778 & 0.633163 & 0.0311302 \\
\hline 0.109382 & 1.27832 & 0.0485041 & 0.643876 & 0.0305927 & 0.623358 & 0.0297944 \\
\hline 0.0986863 & 1.27303 & 0.0477047 & 0.649457 & 0.0434509 & 0.641232 & 0.0300353 \\
\hline 0.0848537 & 1.26334 & 0.0446719 & 0.630073 & 0.0262246 & 0.623468 & 0.0278704 \\
\hline 0.107542 & 1.26619 & 0.0495323 & 0.634629 & 0.0387835 & 0.620549 & 0.0265695 \\
\hline 0.10933 & 1.27555 & 0.0463816 & 0.632629 & 0.0366656 & 0.628093 & 0.034993 \\
\hline 0.0767579 & 1.27203 & 0.0474896 & 0.636568 & 0.0336887 & 0.623285 & 0.0316096 \\
\hline 0.123485 & 1.26655 & 0.0452769 & 0.634687 & 0.0343284 & 0.630536 & 0.0317789 \\
\hline 0.101539 & 1.26389 & 0.0453247 & 0.638589 & 0.0289661 & 0.621599 & 0.0355444 \\
\hline 0.120757 & 1.26292 & 345 & 129 & 0.03 & 0.626974 & 0.02 \\
\hline 0.121374 & 1.26179 & 0.0491146 & 0.634949 & 0.0406403 & 0.628314 & 0.0285436 \\
\hline 0.139132 & 1.26725 & 0.0462614 & 0.63457 & 0.0361106 & 0.621101 & 0.0290496 \\
\hline 0.14786 & 1.26181 & 0.0423614 & 0.632954 & 0.0388922 & 0.632231 & 0.0355163 \\
\hline 0.171164 & 1.25182 & 0.0397695 & 0.63022 & 0.038676 & 0.631681 & 0.0373888 \\
\hline 0.193526 & 1.2578 & 0.0410193 & 0.627513 & 0.0356612 & 0.646315 & 0.0312616 \\
\hline 0.247582 & 1.25569 & 0.0404354 & 0.637804 & 0.0337322 & 0.63748 & 0.0302564 \\
\hline 0.263696 & 1.2572 & 0.0432561 & 0.635656 & 0.0329598 & 0.634947 & 0.0400308 \\
\hline 0.301069 & 1.26035 & 0.0485941 & 0.632457 & 0.0323479 & 0.636765 & 0.0330026 \\
\hline 0.367921 & 1.25187 & 0.0477282 & 0.620578 & 0.0443002 & 0.642379 & 0.0297983 \\
\hline 0.457773 & 1.24381 & 0.0462035 & 0.62931 & 0.036889 & 0.644444 & 0.0301814 \\
\hline 0.544134 & 1.23931 & 0.0447766 & 0.626925 & 0.0383305 & 0.645415 & 0.0239017 \\
\hline 0.61111 & 1.23966 & 0.0479479 & 0.637331 & 0.0379938 & 0.643675 & 0.0293549 \\
\hline 0.775703 & 1.23323 & 0.0394006 & 0.633383 & 0.037907 & 0.647648 & 0.0247844 \\
\hline 0.905482 & 1.22799 & 0.0444086 & 0.631661 & 0.0344462 & 0.649411 & 0.0333066 \\
\hline 1.03042 & 1.22743 & 0.0482201 & 0.623174 & 0.0399218 & 0.642735 & 0.0403567 \\
\hline 1.25979 & 1.22959 & 0.043825 & 0.630183 & 0.0394075 & 0.644019 & 0.0336604 \\
\hline 1.45157 & 1.22922 & 0.049796 & 0.621751 & 0.0327154 & 0.654031 & 0.0309568 \\
\hline 1.64597 & 1.22865 & 0.042481 & 0.623846 & 0.0332633 & 0.648715 & 0.0330369 \\
\hline 1.8613 & 1.22818 & 0.0372315 & 0.623411 & 0.0290942 & 0.64805 & 0.0314094 \\
\hline 2.05954 & 1.22641 & 0.0446238 & 0.622175 & 0.0322717 & 0.650809 & 0.0302424 \\
\hline 2.30303 & 1.22634 & 0.0430132 & 0.61582 & 0.0371494 & 0.647098 & 0.0301503 \\
\hline 2.52664 & 1.22403 & 0.0382491 & 0.618534 & 0.0414334 & 0.646057 & 0.037371 \\
\hline
\end{tabular}




\begin{tabular}{|c|c|c|c|c|c|c|}
\hline 2.64708 & 1.21732 & 0.0387648 & 0.619539 & 0.0381463 & 0.647917 & 0.0427563 \\
\hline 2.85552 & 1.21144 & 0.0426931 & 0.615855 & 0.0314918 & 0.648256 & 0.0295209 \\
\hline 2.9436 & 1.21871 & 0.0484709 & 0.615472 & 0.0280206 & 0.65567 & 0.0374932 \\
\hline 3.13415 & 1.21172 & 0.0442949 & 0.618943 & 0.0351935 & 0.652338 & 0.0358214 \\
\hline 3.18473 & .20188 & 0.038266 & 0.615694 & 0.0382387 & 0.645687 & 0.0320862 \\
\hline 3.27805 & 1.20438 & 0.0406414 & 0.621583 & 0.0295541 & 0.645814 & 0.0329423 \\
\hline 3.1794 & 1.1977 & 0.0452878 & 0.621093 & 0.0400778 & 0.63877 & 0.0351166 \\
\hline 3.2049 & 1.19896 & 0.0412365 & 0.612823 & 0.031728 & 0.638136 & 0.0407829 \\
\hline 3.15581 & 1.2016 & 0.0433144 & 0.614414 & 0.0375489 & 0.649966 & 0.0302774 \\
\hline 2.92544 & 1.19239 & 0.0420737 & 0.606068 & 0.0338892 & 0.640013 & 0.0346894 \\
\hline 2.81208 & 1.18456 & 0.0387743 & 0.615071 & 0.0338894 & 0.62722 & 0.0347962 \\
\hline 2.62761 & 1.18989 & 0.0387616 & 0.60626 & 0.0335584 & 0.636043 & 0.0233067 \\
\hline 2.42302 & 1.18603 & 0.0414131 & 0.605911 & 0.0358204 & 0.635739 & 0.0424043 \\
\hline 2.20952 & 1.18289 & 0.0399852 & 0.608238 & 0.0375094 & 0.627804 & 0.0275227 \\
\hline 2.06231 & 1.18746 & 0.0394933 & 0.604042 & 0.0369318 & 0.608782 & 0.0269517 \\
\hline 1.70888 & 1.18411 & 0.0347292 & 0.600434 & 0.0302122 & 0.613611 & 0.0269963 \\
\hline 1.55105 & 1.18499 & 0.0350125 & 0.600592 & 0.0424811 & 0.610043 & 0.0363631 \\
\hline 1.32821 & 1.18179 & 0.0376538 & 0.598649 & 0.0296819 & 0.60587 & 0.0358204 \\
\hline 1.13807 & 1.18205 & 0.0403792 & 0.586968 & 0.0266326 & 0.596864 & 0.0221463 \\
\hline 0.983309 & 1.17823 & 0.0416415 & 0.6007 & 0.0302499 & 0.596658 & 0.0335061 \\
\hline 0.815623 & 1.17295 & 0.0428417 & 0.601306 & 0.0373753 & 0.588801 & 0.0290933 \\
\hline 0.737745 & 1.16431 & 0.0408649 & 0.585992 & 0.0377624 & 1407 & 4479 \\
\hline 0.568641 & 1.15245 & 0.0398811 & 0.597308 & 0.0292843 & 0.589551 & 0.0369605 \\
\hline 0.458828 & 1.14778 & 0.0367321 & 0.59298 & 0.0343232 & 0.589792 & 0.0229101 \\
\hline 0.3762 & 1.14771 & 0.0402464 & 0.59546 & 0.0287628 & 0.594297 & 0.0333934 \\
\hline 0.295295 & 1.14143 & 0.0390267 & 0.586606 & 0.0308155 & 0.577949 & 0.026002 \\
\hline 0.251799 & 1.14904 & 0.0454263 & 0.588334 & 0.0332392 & 0.584366 & 0.037819 \\
\hline 0.218094 & 1.14721 & 0.047414 & 0.58236 & 0.0276027 & 0.573505 & 0.0282392 \\
\hline 0.169754 & 1.13532 & 0.0437373 & 0.575531 & 0.0245452 & 0.573374 & 0.0279674 \\
\hline 0.130181 & 1.13318 & 0.0474821 & 0.579485 & 0.0274258 & 0.567476 & 0.0300739 \\
\hline 0.144582 & 1.13011 & 0.0371114 & 0.584674 & 0.037816 & 0.568268 & 0.03263 \\
\hline 0.109767 & 1.12587 & 0.0425994 & 0.57808 & 0.0386217 & 0.569458 & 0.0260791 \\
\hline 0.0917397 & 1.12134 & 0.041478 & 0.57527 & 0.0334841 & 0.567356 & 0.0242645 \\
\hline 0.0849886 & 1.12323 & 0.0388567 & 0.569083 & 0.0271563 & 0.567197 & 0.0278374 \\
\hline 0.0962389 & 1.11955 & 0.0342347 & 0.568567 & 0.0321331 & 0.568997 & 0.0255178 \\
\hline 0.0976248 & 1.10813 & 0.0374481 & 0.571145 & 0.0418494 & 0.560981 & 0.0322487 \\
\hline 0.0667772 & 1.10662 & 0.0398175 & 0.568055 & 0.0306499 & 0.564754 & 0.0271755 \\
\hline 0.0793177 & 1.10932 & 0.0379554 & 0.565448 & 0.0302939 & 0.564424 & 0.0267492 \\
\hline 0.0932561 & 1.11169 & 0.0327562 & 0.562594 & 0.0307871 & 0.551263 & 0.0351726 \\
\hline 0.0847462 & 1.11206 & 0.0368962 & 0.562348 & 0.0268158 & 0.562397 & 0.0304819 \\
\hline 0.0808763 & 1.10958 & 0.0355342 & 0.560285 & 0.027625 & 0.562745 & 0.0268183 \\
\hline 0.0972219 & 1.10407 & 0.033239 & 0.561679 & 0.0358705 & 0.567264 & 0.0272288 \\
\hline 0.0730407 & 1.10054 & 0.0385399 & 0.560585 & 0.0209071 & 0.561813 & 0.0243766 \\
\hline 0.0943243 & 1.09297 & 0.0427998 & 0.561157 & 0.0311355 & 0.561021 & 0.0254645 \\
\hline 0.0894272 & 1.09562 & 0.0425609 & 0.554678 & 0.035197 & 0.554965 & 0.0245457 \\
\hline 0.0748405 & 1.09804 & 0.0437915 & 0.563027 & 0.0318454 & 0.56858 & 0.0321969 \\
\hline 0.0810419 & 1.09429 & 0.0409617 & 0.555545 & 0.0252018 & 0.56722 & 0.0212145 \\
\hline
\end{tabular}




\begin{tabular}{|c|c|c|c|c|c|c|}
\hline 0.0886521 & 1.09041 & 0.035411 & 0.561254 & 0.0273942 & 0.581944 & 0.0278186 \\
\hline 0.1251 & 1.09362 & 0.0340454 & 0.559634 & 0.0290692 & 0.59107 & 0.0289373 \\
\hline 0.119815 & 1.09859 & 0.0456682 & 0.565122 & 0.0245927 & 0.593245 & 0.0372034 \\
\hline 0.142889 & 1.0939 & 0.0464831 & 0.575789 & 0.0344069 & 0.608365 & 0.0313305 \\
\hline 0.189147 & 1.08507 & 0.0384128 & 0.57068 & 0.0285438 & 0.618517 & 0.0361685 \\
\hline 0.18405 & 1.08552 & 0.0321804 & 0.566301 & 0.0375976 & 0.619547 & 0.0320458 \\
\hline 0.17315 & 1.09018 & 0.0410703 & 0.57592 & 0.0319609 & 0.650298 & 0.0309435 \\
\hline 0.254773 & 1.09175 & 0.0460985 & 0.57747 & 0.0296528 & 0.66052 & 0.0365374 \\
\hline 0.254246 & 1.09243 & 0.0424833 & 0.568147 & 0.023575 & 0.663748 & 0.0330116 \\
\hline 0.301366 & 1.09582 & 0.0425474 & 0.578589 & 0.0310268 & 0.682468 & 0.0331501 \\
\hline 0.354547 & 1.09666 & 0.0413641 & 0.583165 & 0.032244 & 0.690537 & 0.0363073 \\
\hline 0.40947 & 1.09994 & 0.0409002 & 0.589926 & 0.029986 & 0.703173 & 0.0393528 \\
\hline 0.425118 & 1.0998 & 0.0472461 & 0.591497 & 0.0315321 & 0.71835 & 0.0286891 \\
\hline 0.497074 & 1.09428 & 0.0410081 & 0.595081 & 0.0376407 & 0.728671 & 0.0283762 \\
\hline 0.508554 & 1.0834 & 0.0400868 & 0.599664 & 0.0279214 & 0.727396 & 0.0305934 \\
\hline 0.589033 & 1.08354 & 0.0466132 & 0.592441 & 0.0291183 & 0.738715 & 0.0352586 \\
\hline 0.6463 & 1.08885 & 0.047766 & 0.601189 & 0.0317899 & 0.73387 & 0.027196 \\
\hline 0.679752 & 1.08136 & 0.0445354 & 0.603122 & 0.0302052 & 0.748683 & 0.0306015 \\
\hline 0.739567 & 1.0754 & 0.0397121 & 0.60104 & 0.0291126 & 0.738722 & 0.0426365 \\
\hline 0.784587 & 1.06367 & 0.0407103 & 0.603086 & 0.033343 & 0.741363 & 0.0389861 \\
\hline 0.822 & 1.07028 & 0.0426148 & 0.601036 & 0.0321403 & 0.72634 & 0.0395776 \\
\hline 0.859581 & 1.06924 & 0.0398985 & 0.585429 & 3096 & 0.713327 & 0.036719 \\
\hline 0.856775 & 1.06153 & 0.0385777 & 0.58271 & 0.0328563 & 0.698326 & 0.0322881 \\
\hline 0.891109 & 1.05494 & 0.0348802 & 0.5849 & 0.0283292 & 0.689774 & 0.0380274 \\
\hline 0.896958 & 1.04964 & 0.0384269 & 0.576596 & 0.0333576 & 0.67875 & 0.0413512 \\
\hline 0.884145 & 1.04544 & 0.0346073 & 0.572757 & 0.0335762 & 0.669127 & 0.0297553 \\
\hline 0.828954 & 1.04268 & 0.0327783 & 0.568982 & 0.0310892 & 0.656739 & 0.023745 \\
\hline 0.827294 & 1.0379 & 0.033478 & 0.563003 & 0.0281782 & 0.632083 & 0.0335872 \\
\hline 0.785922 & 1.03699 & 0.0308571 & 0.563215 & 0.0261628 & 0.622463 & 0.0260879 \\
\hline 0.775906 & 1.03101 & 0.0275941 & 0.552125 & 0.0273525 & 0.612715 & 0.0342167 \\
\hline 0.691692 & 1.02365 & 0.0334749 & 0.545786 & 0.0328932 & 0.602831 & 0.0282029 \\
\hline 0.630661 & 1.02108 & 0.0382108 & 0.541412 & 0.0260563 & 0.58583 & 0.024058 \\
\hline 0.565208 & 1.02505 & 0.0424249 & 0.542036 & 0.0267737 & 0.574448 & 0.0330356 \\
\hline 0.546689 & 1.021 & 0.0369895 & 0.539039 & 0.0306272 & 0.572475 & 0.0365318 \\
\hline 0.4416 & 1.01809 & 0.0320007 & 0.540047 & 0.0322354 & 0.553166 & 0.0274394 \\
\hline 0.425172 & 1.02446 & 0.0334486 & 0.533162 & 0.0270018 & 0.545719 & 0.0341688 \\
\hline 0.368043 & 1.02003 & 0.0371281 & 0.519094 & 0.0285073 & 0.534192 & 0.0309347 \\
\hline 0.324495 & 1.01101 & 0.032105 & 0.529307 & 0.0320441 & 0.531175 & 0.0273708 \\
\hline 0.262367 & 1.00743 & 0.0314257 & 0.528213 & 0.0305746 & 0.532945 & 0.0237351 \\
\hline 0.204157 & 1.00376 & 0.0343616 & 0.523665 & 0.0287829 & 0.519223 & 0.0287663 \\
\hline 0.192404 & 1.00383 & 0.0391575 & 0.522634 & 0.029289 & 0.521777 & 0.0276957 \\
\hline 0.198821 & 1.00416 & 0.0410451 & 0.517844 & 0.0293813 & 0.518049 & 0.0297293 \\
\hline 0.146839 & 0.999259 & 0.0382439 & 0.519167 & 0.0285114 & 0.525199 & 0.029378 \\
\hline 0.129758 & 1.00184 & 0.0307479 & 0.524983 & 0.0261598 & 0.521158 & 0.0336887 \\
\hline 0.119906 & 1.00225 & 0.0322748 & 0.522673 & 0.0248386 & 0.512147 & 0.0333258 \\
\hline 0.0950385 & 1.00283 & 0.0331524 & 0.513186 & 0.0331692 & 0.522159 & 0.0293272 \\
\hline 0.0936402 & 0.997656 & 0.030806 & 0.511912 & 0.0275873 & 0.50957 & 0.0336619 \\
\hline
\end{tabular}




\begin{tabular}{|c|c|c|c|c|c|c|}
\hline 0.0777605 & 0.995641 & 0.0248131 & 0.507538 & 0.0314702 & 0.510631 & 0.0306905 \\
\hline 0.0868432 & 1.00018 & 0.0274932 & 0.506082 & 0.0331467 & 0.517521 & 0.0280041 \\
\hline 0.0673065 & 1.00103 & 0.0285663 & 0.513611 & 0.0284657 & 0.518715 & 0.0227337 \\
\hline 0.0839607 & 0.994646 & 0.0337611 & 0.508998 & 0.0303382 & 0.513087 & 0.0225682 \\
\hline 0.0576869 & 0.989575 & 0.0268119 & 0.510376 & 0.0254544 & 0.500917 & 0.0311938 \\
\hline 0.0557535 & 0.994026 & 0.0292375 & 0.508439 & 0.0308078 & 0.511113 & 0.0306995 \\
\hline 0.0669254 & 0.986697 & 0.0312665 & 0.511828 & 0.0257597 & 0.509965 & 0.0238229 \\
\hline 0.0534328 & 0.985704 & 0.0378245 & 0.502898 & 0.0306536 & 0.500635 & 0.0256 \\
\hline 0.0574546 & 0.986157 & 0.0369664 & 0.498732 & 0.0275059 & 0.507395 & 0.0352704 \\
\hline 0.06157 & 0.985242 & 0.0355686 & 0.511228 & 0.0283829 & 0.499903 & 0.0237093 \\
\hline 0.0618464 & 0.986021 & 0.0314934 & 0.500227 & 0.0240717 & 0.498078 & 0.0220636 \\
\hline 0.0669563 & 0.984 & 0.0292699 & 0.502894 & 0.0269589 & 0.50103 & 0.0341259 \\
\hline 0.0550048 & 0.983025 & 0.0302248 & 0.504987 & 0.0344568 & 0.492419 & 0.0321105 \\
\hline 0.0623739 & 0.97959 & 0.0342939 & 0.49372 & 0.0258949 & 0.498776 & 0.020413 \\
\hline 0.0594516 & 0.989415 & 0.0348635 & 0.501125 & 0.0299033 & 0.500619 & 0.0264417 \\
\hline 0.0656972 & 0.985847 & 0.0339773 & 0.504234 & 0.026278 & 0.501326 & 0.0238557 \\
\hline 0.0807908 & 0.97992 & 0.0308538 & 0.507019 & 0.0251673 & 0.49496 & 0.0247089 \\
\hline 0.0745334 & 0.985873 & 0.0341175 & 0.495599 & 0.0321173 & 0.498029 & 0.0255838 \\
\hline 0.0605111 & 0.985581 & 0.028 & 0.49852 & 0.026 & 0.502293 & 0.0262693 \\
\hline 0.0736016 & 0.985735 & 0.0296775 & 0.500766 & 0.0283596 & 0.502263 & 0.0273452 \\
\hline 0.0610092 & 0.995959 & 0.0314054 & 0.498589 & 0.0316555 & 0.509547 & 0.0288777 \\
\hline 0.0596503 & 1.00212 & 0.02 & 0.506157 & 281 & 0.49874 & 0.0289097 \\
\hline 0.0690135 & 1.01507 & 0.0357827 & 0.506052 & 0.0277745 & 0.496133 & 0.0292669 \\
\hline 0.0754769 & 1.03533 & 0.0288714 & 0.51268 & 0.0285148 & 0.499395 & 0.0275495 \\
\hline 0.0585721 & 1.03935 & 0.0350804 & 0.509427 & 0.0247627 & 0.510475 & 0.0297807 \\
\hline 0.0789442 & 1.05267 & 0.036399 & 0.517518 & 0.0266684 & 0.508793 & 0.0252758 \\
\hline 0.0791659 & 1.0735 & 0.0386439 & 0.513086 & 0.0252351 & 0.513902 & 0.0204485 \\
\hline 0.0659972 & 1.09941 & 0.0370176 & 0.523538 & 0.0265218 & 0.521085 & 0.0347628 \\
\hline 0.0815162 & 1.11994 & 0.040708 & 0.537775 & 0.0251959 & 0.528297 & 0.0227271 \\
\hline 0.0758593 & 1.15043 & 0.0463005 & 344 & 0.025859 & 0.526328 & 0.0237673 \\
\hline 0.0755625 & 1.177 & 0.0367798 & 0.560166 & 0.0243233 & 0.547126 & 0.0307846 \\
\hline 0.0812186 & 1.21044 & 0.0388332 & 0.574344 & 0.029582 & 0.547777 & 0.0300401 \\
\hline 0.0747864 & 1.2479 & 0.0394834 & 0.587802 & 0.0308331 & 0.569014 & 0.0342412 \\
\hline 0.108779 & 1.27617 & 0.0433167 & 0.602877 & 0.0322254 & 0.580434 & 0.0305591 \\
\hline 0.100156 & 1.30305 & 0.0483299 & 0.619771 & 0.0355966 & 0.59682 & 0.0337179 \\
\hline 0.112132 & 1.33558 & 0.0497461 & 0.64098 & 0.0359484 & 0.601878 & 0.0323842 \\
\hline 0.117632 & 1.35695 & 0.0557929 & 0.665854 & 0.0340825 & 0.624447 & 0.0399849 \\
\hline 0.132728 & 1.38296 & 0.0552849 & 0.693647 & 0.0444446 & 0.635083 & 0.0280732 \\
\hline 0.12888 & 1.40543 & 0.0590002 & 0.714338 & 0.0350941 & 0.660092 & 0.036398 \\
\hline 0.153683 & 1.42928 & 0.0590506 & 0.746032 & 0.0444739 & 0.668935 & 0.038271 \\
\hline 0.12878 & 1.43847 & 0.062565 & 0.756033 & 0.04026 & 0.678959 & 0.0380035 \\
\hline 0.131847 & 1.44415 & 0.0612564 & 0.786891 & 0.0496505 & 0.702156 & 0.0384283 \\
\hline 0.132497 & 1.4448 & 0.0663833 & 0.805832 & 0.0480736 & 0.710537 & 0.0456257 \\
\hline 0.161418 & 1.43298 & 0.0709873 & 0.817973 & 0.0330012 & 0.726272 & 0.0357718 \\
\hline 0.13577 & 1.42346 & 0.0647483 & 0.823334 & 0.0420481 & 0.731921 & 0.0483982 \\
\hline 0.149659 & 1.40159 & 0.0575754 & 0.844574 & 0.0444265 & 0.734734 & 0.0389093 \\
\hline 0.141784 & 1.38089 & 0.0555056 & 0.849158 & 0.0538167 & 0.727876 & 0.0345122 \\
\hline
\end{tabular}




\begin{tabular}{|c|c|c|c|c|c|c|}
\hline 0.131101 & 1.35566 & 0.0553103 & 0.84465 & 0.0457149 & 0.730547 & \\
\hline 0.122191 & 1.31954 & 0.053833 & 0.847461 & 0.0470761 & 0.726661 & 0.0342682 \\
\hline 0.137941 & 1.28145 & 0.0551468 & 0.839858 & 0.0438848 & 0.709411 & 0.0393938 \\
\hline 0.134816 & 1.2427 & 0.0524113 & 0.823188 & 0.0488769 & 0.705479 & 0.0375913 \\
\hline 0.154089 & 1.2091 & 0.0463008 & 0.814578 & 0.0520033 & 0.69312 & 0.0385905 \\
\hline 0.129251 & 1.17891 & 0.0434291 & 0.792843 & 0.040854 & 0.686248 & 0.0389527 \\
\hline 0.105734 & 1.14885 & 0.0347058 & 0.765612 & 0.0420046 & 0.657828 & 0.0355056 \\
\hline 0.114535 & 1.09424 & 0.0401009 & 0.747202 & 0.0418846 & 0.642468 & 0.0334102 \\
\hline 0.110393 & 1.05661 & 0.0381485 & 0.71959 & 0.0422306 & 0.616448 & 0.035555 \\
\hline 0.0856758 & 1.03515 & 0.0331474 & 0.699745 & 0.0351849 & 0.613105 & 0.0355483 \\
\hline 0.0961957 & 1.01895 & 0.0269604 & 0.666268 & 0.0278828 & 0.583526 & 0.0373329 \\
\hline 0.0945416 & 0.989303 & 0.0314493 & 0.64715 & 0.0343187 & 0.567516 & 0.0276147 \\
\hline 0.0664684 & 0.956434 & 0.0331016 & 0.620265 & 0.0390466 & 0.551923 & 0.0357574 \\
\hline 0.0845601 & 0.946384 & 0.0300704 & 0.590936 & 0.0301783 & 0.539808 & 0.0241573 \\
\hline 0.0646871 & 0.925975 & 0.0281772 & 0.564204 & 0.0355494 & 0.517163 & 0.0287582 \\
\hline 0.0654316 & 0.917137 & 0.0311034 & 0.549923 & 0.0262974 & 0.512381 & 0.0291403 \\
\hline 0.0630177 & 0.902863 & 0.0322811 & 0.534135 & 0.0263536 & 0.502427 & 0.0242057 \\
\hline 0.0629234 & 0.890641 & 0.0286527 & 0.524515 & 0.0213768 & 0.492158 & 0.028645 \\
\hline 0.0609184 & 0.878122 & 0.0287167 & 0.507819 & 0.0274654 & 0.472716 & 0.0226257 \\
\hline 0.0532949 & 0.876492 & 0.0272135 & 0.495379 & 0.0233792 & 0.470441 & 0.0268364 \\
\hline 0.0556345 & 0.877079 & 0.0250134 & 0.483165 & 0.027791 & 0.46301 & 0.0244757 \\
\hline 0.0487454 & 0.870332 & 0.0280173 & 0.476948 & 0.0272756 & 0.45858 & 0.0216473 \\
\hline 0.0627396 & 0.86488 & 0.0306825 & 0.469131 & 0.0289171 & 0.45979 & 0.0249595 \\
\hline 0.0479176 & 0.860683 & 0.0325882 & 0.466403 & 0.0233735 & 0.450767 & 0.0273768 \\
\hline 0.0588351 & 0.86 & 0.03 & 0.45 & 257 & 924 & 8231 \\
\hline 0.056295 & 0.855877 & 0.0265924 & 0.455665 & 0.0277762 & 0.445027 & 0.0213628 \\
\hline 0.0500858 & 0.840883 & 0.0266218 & 0.449565 & 0.0263953 & 0.440817 & 0.0241374 \\
\hline 0.0563991 & 0.846881 & 0.0298911 & 0.452392 & 0.019406 & 0.441068 & 0.0250988 \\
\hline 0.047789 & 0.844805 & 0.0302588 & 0.447492 & 0.0231088 & 0.429166 & 0.0272465 \\
\hline 0.0508902 & 0.839044 & 0.0318025 & 0.452697 & 0.0291124 & 0.442328 & 0.021821 \\
\hline 0.0492149 & 0.835386 & 0.0287696 & 0.433888 & 0.0250138 & 0.438667 & 0.0266548 \\
\hline 0.0560203 & 0.835678 & 0.0243192 & 0.442506 & 0.0224293 & 0.438416 & 0.0188464 \\
\hline 0.0681233 & 0.827435 & 0.0244899 & 0.437472 & 0.0200283 & 0.438193 & 0.0257513 \\
\hline 0.0635961 & 0.824146 & 0.02689 & 0.436375 & 0.0278457 & 0.429288 & 0.0218017 \\
\hline 0.0624461 & 0.825567 & 0.0238017 & 0.440658 & 0.0259922 & 0.431649 & 0.0283204 \\
\hline 0.0521204 & 0.826646 & 0.0273617 & 0.434044 & 0.0240818 & 0.429854 & 0.0227922 \\
\hline 0.0637521 & 0.824646 & 0.0277782 & 0.431192 & 0.0224394 & 0.426282 & 0.0257832 \\
\hline 0.054732 & 0.816918 & 0.0281824 & 0.427055 & 0.0208279 & 0.420004 & 0.0249756 \\
\hline 0.060663 & 0.811372 & 0.0306241 & 0.434 & 0.0171725 & 0.419686 & 0.0278194 \\
\hline 0.0543202 & 0.812777 & 0.026341 & 0.429447 & 0.0190491 & 0.422033 & 0.0250487 \\
\hline 0.0481495 & 0.812797 & 0.0286626 & 0.426129 & 0.0193778 & 0.42269 & 0.0242191 \\
\hline 0.0475975 & 0.81037 & 0.03043 & 0.428854 & 0.0244361 & 0.419975 & 0.0230774 \\
\hline 0.0571003 & 0.807278 & 0.0250027 & 0.429965 & 0.0326343 & 0.417403 & 0.0208025 \\
\hline 0.0519781 & 0.807162 & 0.0301895 & 0.429104 & 0.022067 & 0.42036 & 0.0229904 \\
\hline 0.045744 & 0.805998 & 0.0297676 & 0.425639 & 0.0192912 & 0.427794 & 0.0191488 \\
\hline 0.0381278 & 0.800611 & 0.0291384 & 0.41818 & 0.0231802 & 0.418376 & 0.0252245 \\
\hline 0.0472112 & 0.798446 & 0.0272907 & 0.425943 & 0.0228411 & 0.411109 & 0.018867 \\
\hline
\end{tabular}




\begin{tabular}{|c|c|c|c|c|c|c|}
\hline 0.0585236 & 0.799559 & 0.0252802 & 0.416174 & 0.0276892 & 0.420487 & 289776 \\
\hline 0.0525688 & 0.79406 & 0.0223841 & 0.425549 & 0.020934 & 0.40255 & 0.0242846 \\
\hline 0.0520464 & 0.793785 & 0.0223561 & 0.420998 & 0.0209242 & 0.412752 & 0.0213645 \\
\hline .0466211 & 0.793083 & 0.0242945 & 0.414535 & 0.0235736 & 0.401182 & 0.0186313 \\
\hline 0.051882 & 0.790297 & 0.0271956 & 0.418547 & 0.0229766 & 0.4127 & 0.0246903 \\
\hline 0.0426328 & 0.784639 & 0.0286496 & 0.414075 & 0.0255316 & 0.406895 & 0.025058 \\
\hline 0.0501727 & 0.781718 & 0.0250923 & 0.417973 & 0.0197513 & 0.406731 & 0.0220611 \\
\hline 0.0460051 & 0.782267 & 0.0252504 & 0.408558 & 0.0266879 & 0.411543 & 0.0211635 \\
\hline 0.0479068 & 0.778265 & 0.0304413 & 0.415969 & 0.0227603 & 0.41771 & 0.0268133 \\
\hline 0.0478654 & 0.773204 & 0.0204881 & 0.404638 & 0.0195327 & 0.412348 & 0.0216291 \\
\hline 0.0497992 & 0.775517 & 0.0212796 & 0.409613 & 0.0256083 & 0.41648 & 0.0264609 \\
\hline 0.0444827 & 0.774037 & 0.0271788 & 0.412966 & 0.0229141 & 0.411026 & 0.0217219 \\
\hline 0.0466107 & 0.771369 & 0.0311748 & 0.41488 & 0.0218931 & 0.410093 & 0.0203103 \\
\hline 0.0517436 & 0.769335 & 0.0242462 & 0.403637 & 0.0194502 & 0.408929 & 0.0266093 \\
\hline 0.0426326 & 0.766253 & 0.0288659 & 0.409942 & 0.027525 & 0.417264 & 0.0234312 \\
\hline 0.0481029 & 0.765391 & 0.0350609 & 0.412239 & 0.0270171 & 0.422269 & 1257 \\
\hline 0.0551079 & 0.763987 & 0.0276808 & 0.406511 & 0.0274668 & 0.416145 & 0.021439 \\
\hline 0.0546802 & 0.767928 & 0.0229508 & 0.409568 & 0.0197776 & 0.419414 & 0.0270722 \\
\hline 0.0496825 & 0.766906 & 323 & 0.405215 & 0.02 & 0.4 & 0.02 \\
\hline 0.0495973 & 0.763863 & 0.0249923 & 0.414932 & 0.0203115 & 0.425453 & 0.0247 \\
\hline 0.0380337 & 0.767488 & 0.0214457 & 0.409172 & 0.0205701 & 0.425839 & 0.022281 \\
\hline 0.0493943 & 0.759622 & 0.02 & 5054 & 5398 & 353 & 939 \\
\hline 0.0500854 & 0.761974 & 0.0172957 & 0.40667 & 0.0225893 & 0.428895 & 0.0289793 \\
\hline 0.0501358 & 0.754699 & 0.0208951 & 0.414075 & 0.0214057 & 0.438935 & 0.0268533 \\
\hline 0.0429537 & 0.759601 & 0.0225604 & 0.413302 & 0.0261318 & 0.436167 & 0.0294979 \\
\hline 0.0396657 & 0.760155 & 0.024064 & 0.410504 & 0.0204087 & 0.446774 & 0.0231608 \\
\hline 0.0425759 & 0.751605 & 0.0280242 & 0.414179 & 0.0160888 & 0.453986 & 0.0263629 \\
\hline 0.0558062 & 0.755217 & 0.0308293 & 0.408594 & 0.0222962 & 267 & 0.0269307 \\
\hline 0.0440352 & 0.754813 & 0.0269256 & 0.410436 & 0.0238468 & 0.461518 & 0.0274097 \\
\hline 0.0434042 & 365 & & 557 & 5391 & 331 & 708 \\
\hline 0.049587 & 0.745734 & 0.0243574 & 0.415566 & 0.0243846 & 0.4 & 0.0248163 \\
\hline 0.0508302 & 0.736656 & 0.0246841 & 0.416518 & 0.0245228 & 0.456758 & 0.0298259 \\
\hline 0.0526076 & 0.737246 & 0.0307926 & 0.415878 & 0.0244511 & 323 & 0.0236086 \\
\hline 0.0447252 & 0.739708 & 0.0289651 & 0.413791 & 0.0252899 & 0.4 & 0.0313497 \\
\hline 0.0468624 & 0.740267 & 0.0280587 & 0.401214 & 0.026158 & 0.458756 & 0.0275176 \\
\hline 0.0477446 & 0.732437 & 0.02851 & 0.409297 & 0.0145159 & 0.447736 & 0.0221352 \\
\hline 0.0396583 & 0.724488 & 0.0290959 & 0.407344 & 0.0223179 & 0.443875 & 0.0281479 \\
\hline 0.0321133 & 0.730549 & 0.0267629 & 0.400575 & 0.020534 & 0.442666 & 0.0247246 \\
\hline 0.0497316 & 0.730013 & 0.0244461 & 0.400748 & 0.0292758 & 0.43308 & 0.0321437 \\
\hline 0.0510215 & 0.732742 & 0.0278276 & 0.399314 & 0.0166532 & 0.427346 & 0.0309577 \\
\hline 0.0465795 & 0.722781 & 0.0267416 & 0.401081 & 0.0253415 & 0.422558 & 0.0242924 \\
\hline 0.0423502 & 0.719619 & 0.0229066 & 0.394278 & 0.0220089 & 0.425 & 0.0285972 \\
\hline 0.0539785 & 0.715668 & 0.0262214 & 0.395829 & 0.0262688 & 0.417226 & 0.0255041 \\
\hline 0.0423958 & 0.715107 & 0.0293698 & 0.385969 & 0.0224635 & 0.406818 & 0.0257446 \\
\hline 0.0670519 & 0.712548 & 0.0249735 & 0.389074 & 0.0249674 & 0.399683 & 0.0215209 \\
\hline 0.0432747 & 0.710616 & 0.0169691 & 0.382327 & 0.0206576 & 0.397186 & 0.0237525 \\
\hline 0.0572399 & 0.704753 & 0.0292499 & 0.385885 & 0.0192884 & 0.386924 & 0.0256159 \\
\hline
\end{tabular}




\begin{tabular}{|c|c|c|c|c|c|c|}
\hline 0.0600781 & 0.695981 & 0.0271685 & 0.373345 & 0.0162088 & 0.380427 & 0.019958 \\
\hline 0.037758 & 0.692845 & 0.0232895 & 0.373387 & 0.0203095 & 0.380548 & 0.0292195 \\
\hline 0.0530108 & 0.692121 & 0.0213035 & 0.38155 & 0.0269735 & 0.378579 & 0.0252618 \\
\hline 0.0467394 & .689781 & 0.0283921 & 0.380379 & 0.0170963 & 0.377401 & 0.0236705 \\
\hline 0.0448662 & 0.686648 & 0.0281109 & 0.369529 & 0.0189686 & 0.373727 & 0.0264051 \\
\hline 0.0473737 & 0.685629 & 0.0263065 & 0.366321 & 0.0247872 & 0.372295 & 0.0235615 \\
\hline 0.0586769 & 0.68352 & 0.0256219 & 0.362965 & 0.0190437 & 0.358029 & 0.0195621 \\
\hline 0.0486992 & 0.683685 & 0.0257512 & 0.3662 & 0.0210297 & 0.363197 & 0.0240371 \\
\hline 0.038133 & 0.67838 & 0.0250555 & 0.364216 & 0.0172361 & 0.366463 & 0.0209879 \\
\hline 0.0452548 & 0.681411 & 0.0227036 & 0.35796 & 0.0130407 & 0.357891 & 0.0218827 \\
\hline 0.0451016 & 0.673937 & 0.0215311 & 0.358705 & 0.0144574 & 0.355772 & 0.0238566 \\
\hline 0.0474529 & 0.672542 & 0.0217196 & 0.368693 & 0.0185423 & 0.35376 & 0.0225251 \\
\hline 0.0407141 & 0.671245 & 0.0196844 & 0.361625 & 0.0158067 & 0.347441 & 0.0205858 \\
\hline 0.0428905 & 0.676854 & 0.0226516 & 0.353863 & 0.0207267 & 0.356192 & 0.0189762 \\
\hline 0.0455852 & 0.667599 & 0.0264728 & 0.355378 & 0.0213734 & 0.354444 & 0.0202569 \\
\hline 0.0376696 & 0.67301 & 0.0273741 & 0.354422 & 0.0216122 & 18959 & 0.0257788 \\
\hline 0.039481 & 0.670157 & 0.0237428 & 0.353786 & 0.0177418 & 0.355766 & 0.0197548 \\
\hline 0.0439456 & 0.661641 & 0.0238591 & 0.354316 & 0.0183332 & 0.346011 & 0.0207166 \\
\hline 0.0411927 & 0.658701 & 929 & 008 & 0.0159219 & 0062 & 0.022082 \\
\hline 0.0462417 & 0.659992 & 0.0261251 & 0.350929 & 0.0148095 & 0.34856 & 0.0193733 \\
\hline 0.056527 & 0.660849 & 0.0198343 & 0.346138 & 0.0190564 & 0.342703 & 0.0240187 \\
\hline 0.0340806 & 0.661062 & 0.02 & 145 & 411 & 008 & 5929 \\
\hline 0.0497663 & 0.654721 & 0.0212547 & 0.343284 & 0.0154633 & 0.340916 & 0.0220572 \\
\hline 0.0391072 & 0.659094 & 0.0197492 & 0.344713 & 0.0206748 & 0.340908 & 0.0219941 \\
\hline 0.043448 & 0.656345 & 0.0222248 & 0.346898 & 0.019487 & 0.344208 & 0.0264802 \\
\hline 0.0349256 & 0.653124 & 0.0212379 & 0.339777 & 0.0200909 & 0.331576 & 0.0190112 \\
\hline 0.0381083 & 0.644772 & 0.0285652 & 0.342723 & 0.0209833 & 0.332668 & 0.0184463 \\
\hline 0.0444462 & 0.644196 & 0.0209477 & 0.337164 & 0.0187544 & 0.340051 & 0.0232909 \\
\hline 0.0375173 & 0.646986 & 0.0236757 & 0.342121 & 0.017516 & 0.334679 & 0.0175857 \\
\hline 0.0353466 & 0.65278 & 0.0252559 & 0.340733 & 0.014122 & 0.336133 & 0.0234568 \\
\hline 0.0513267 & 0.641094 & 0.0217831 & 0.337174 & 0.0232763 & 0.337137 & 0.026862 \\
\hline 0.0379722 & 0.638855 & 0.022666 & 0.340955 & 0.0275116 & 0.337196 & 0.0195013 \\
\hline 0.0502899 & 0.640201 & 0.0218142 & 0.335805 & 0.0219982 & 0.331789 & 0.019015 \\
\hline 0.0441164 & 0.640256 & 0.0227464 & 0.340434 & 0.0214446 & 0.336536 & 0.0207594 \\
\hline 0.0445084 & 0.647253 & 0.0237528 & 0.336574 & 0.0231635 & 0.334589 & 0.023015 \\
\hline 0.0513135 & 0.642715 & 0.0270494 & 0.340623 & 0.0174381 & 0.329531 & 0.0229888 \\
\hline 0.0394538 & 0.648174 & 0.0197681 & 0.340641 & 0.0197299 & 0.339213 & 0.0219334 \\
\hline 0.0494087 & 0.64538 & 0.0219327 & 0.340812 & 0.0171191 & 0.342088 & 0.0203416 \\
\hline 0.0422088 & 0.645536 & 0.0253367 & 0.341921 & 0.0182839 & 0.338069 & 0.0176739 \\
\hline 0.052899 & 0.644112 & 0.0232682 & 0.340677 & 0.0230709 & 0.345479 & 0.0214831 \\
\hline 0.0404605 & 0.645435 & 0.0190802 & 0.338562 & 0.019938 & 0.346402 & 0.0247856 \\
\hline 0.0424381 & 0.650987 & 0.0256138 & 0.336275 & 0.0186467 & 0.353182 & 0.0245818 \\
\hline 0.0431262 & 0.660208 & 0.0284975 & 0.338627 & 0.0171146 & 0.364503 & 0.0247813 \\
\hline 0.0418752 & 0.6576 & 0.0265624 & 0.343672 & 0.0197344 & 0.369209 & 0.0322257 \\
\hline 0.0469988 & 0.660765 & 0.0217493 & 0.347242 & 0.0193345 & 0.38537 & 0.030603 \\
\hline 0.0366562 & 0.663251 & 0.0188219 & 0.346609 & 0.0232601 & 0.391893 & 0.0348606 \\
\hline 0.0437251 & 0.676303 & 0.02516 & 0.348854 & 0.0214186 & 0.397409 & 0.0281381 \\
\hline
\end{tabular}




\begin{tabular}{|c|c|c|c|c|c|c|}
\hline 0.0449088 & 0.677483 & 0.0243285 & 0.356654 & 0.0156115 & 0.415237 & 0.0356679 \\
\hline 0.0499905 & 0.688757 & 0.0197285 & 0.365711 & 0.0181508 & 0.43848 & 0.0448374 \\
\hline 0.0349152 & 0.699272 & 0.0170322 & 0.368957 & 0.0216061 & 0.457067 & 0.0418102 \\
\hline .0564789 & .712325 & 0.0270482 & .370526 & 0.0196264 & 0.483391 & 0.0599672 \\
\hline 0.0486349 & 0.727796 & 0.0296314 & 0.376651 & 0.0206413 & 0.49885 & 0.0571215 \\
\hline 0.0485971 & 0.722466 & 0.0254026 & 0.395938 & 0.0251583 & 0.524004 & 0.0601528 \\
\hline 0.0472588 & 0.73706 & 0.0231146 & 0.404614 & 0.0207165 & 0.557225 & 0.0687583 \\
\hline 0.0524757 & 0.747916 & 0.0205102 & 0.411753 & 0.0239632 & 0.579563 & 0.0807572 \\
\hline 0.0559703 & 0.762385 & 0.0256252 & 0.420148 & 0.0240425 & 0.606909 & 0.0877522 \\
\hline 0.042621 & 0.77201 & 0.0260746 & 0.431654 & 0.0195671 & 0.635732 & 0.0952725 \\
\hline 0.0549267 & 0.780269 & 0.0215486 & 0.44473 & 0.0211101 & 0.667017 & 0.0998635 \\
\hline 0.0469485 & 0.791484 & 0.0318056 & 0.456858 & 0.0216308 & 0.705199 & 0.0944304 \\
\hline 0.0442162 & 0.797776 & 0.0327868 & 0.480029 & 0.0221039 & 0.727811 & 0.127768 \\
\hline 0.0508555 & 0.809909 & 0.027085 & 0.482453 & 0.0316144 & 0.749183 & 0.127622 \\
\hline 0.0626759 & 0.813176 & 0.0290726 & 0.502075 & 0.0257446 & 0.769638 & 0.124448 \\
\hline 0.0550165 & 0.811581 & 0.0308201 & 0.508342 & 0.0248248 & 0.7 & 0.135094 \\
\hline 0.0532766 & 0.812008 & 0.029293 & 0.509957 & 0.0285781 & 0.814163 & 0.141253 \\
\hline 0.0613202 & 0.815067 & 0.0228122 & 0.511584 & 0.0254675 & 0.83 & 0.134367 \\
\hline 0.0508464 & 0.81 & 0.0259702 & 0.53078 & 0.0321322 & 0.8 & 0.146021 \\
\hline 0.0707803 & 0.798804 & 0.026084 & 0.531086 & 0.0311215 & 0.833574 & 0.14123 \\
\hline 0.0537283 & 0.794804 & 0.02633 & 0.538802 & 0.0394089 & 0.834374 & 0.135727 \\
\hline 0.0651188 & 0.7 & 0.022 & 0.540488 & 5965 & 286 & 35173 \\
\hline 0.057123 & 0.775008 & 0.0303802 & 0.538413 & 0.028645 & 0.807028 & 0.135549 \\
\hline 0.0607703 & 0.767637 & 0.0223655 & 0.532334 & 0.0369477 & 0.771113 & 0.126266 \\
\hline 0.071286 & 0.755825 & 0.0180923 & 0.526797 & 0.0240686 & 0.769833 & 0.113973 \\
\hline 0.0576698 & 0.747913 & 0.0217213 & 0.525714 & 0.0295341 & 0.737492 & 0.123207 \\
\hline 0.0606999 & 0.727245 & 0.02121 & 0.51483 & 0.0302727 & 0.71335 & 0.113353 \\
\hline 0.0642087 & 0.705739 & 0.02423 & 0.503305 & 0.0284523 & 0.67 & 0.0974304 \\
\hline 0.0527581 & 0.692136 & 0.0253577 & 0.503775 & 0.0293333 & 0.6 & 0.085624 \\
\hline 0.0557089 & 0.68 & 0.02 & 0.481711 & 0.0285453 & & 132 \\
\hline 0.0589636 & 0.673245 & 0.0193362 & 0.468009 & 0.0268178 & 0.5 & 0.0741484 \\
\hline 0.0530875 & 0.658519 & 0.0220925 & 0.451696 & 0.0294765 & 0.542476 & 0.0673739 \\
\hline 0.0583146 & 0.647089 & 0.0217116 & 0.43574 & 0.0230254 & 0.513141 & 0.0635922 \\
\hline 0.0620079 & 0.637638 & 0.014804 & 0.421981 & 0.0232822 & 0.48993 & 0.0476258 \\
\hline 0.0533696 & 0.624485 & 0.0217441 & 0.413334 & 0.0178441 & 0.462913 & 0.0519121 \\
\hline 0.0540233 & 0.616813 & 0.0214898 & 0.397397 & 0.0243879 & 0.435431 & 0.0379729 \\
\hline 0.04195 & 0.602195 & 0.0186244 & 0.389743 & 0.0181314 & 0.405328 & 0.0302479 \\
\hline 0.0392748 & 0.593813 & 0.0187413 & 0.36742 & 0.0169124 & 0.388454 & 0.0347714 \\
\hline 0.0410138 & 0.588403 & 0.0214799 & 0.357884 & 0.0222937 & 0.37357 & 0.0356044 \\
\hline 0.0402857 & 0.590264 & 0.0232297 & 0.353341 & 0.0178316 & 0.365094 & 0.0264946 \\
\hline 0.0382588 & 0.587836 & 0.0290248 & 0.340797 & 0.0173109 & 0.351892 & 0.024761 \\
\hline 0.0411116 & 0.574135 & 0.0223212 & 0.338911 & 0.0167027 & 0.333162 & 0.0200853 \\
\hline 0.046213 & 0.570394 & 0.0179717 & 0.331315 & 0.0208092 & 0.323021 & 0.0213067 \\
\hline 0.040953 & 0.571483 & 0.0293355 & 0.326713 & 0.0211033 & 0.320284 & 0.0267759 \\
\hline 0.0341542 & 0.572715 & 0.0252861 & 0.319795 & 0.0187405 & 0.320408 & 0.0260058 \\
\hline 0.036261 & 0.561584 & 0.0204817 & 0.32 & 0.022593 & 0.305116 & 0.0220761 \\
\hline 0.0418148 & 0.557058 & 0.0253809 & 0.309518 & 0.0194989 & 0.304268 & 0.0227584 \\
\hline
\end{tabular}




\begin{tabular}{|c|c|c|c|c|c|c|}
\hline 0.0448648 & 0.557769 & 0.02605 & 0.310902 & 0.0141982 & 0.300295 & 0.0147141 \\
\hline 0.0419151 & .555902 & 0.0165157 & 0.312904 & 0.0156141 & 0.302312 & 0.019235 \\
\hline 0.0404016 & 0.558905 & 0.0244151 & .304847 & 0.0182399 & 0.299267 & 0.0240413 \\
\hline 0399947 & .547085 & 0.0223886 & 0.30958 & 0.0160625 & 0.300842 & 0.0214179 \\
\hline 0.0352383 & 0.556616 & .0167739 & 0.297802 & 0.0157153 & 0.298879 & 0.0151275 \\
\hline 0.0444747 & 0.549672 & 0.02116 & 0.304247 & 0.019214 & 0.294814 & 0.0203473 \\
\hline 0.0384344 & 0.543581 & 0.0204405 & 0.308084 & 0.0161646 & 0.290172 & 0.0241629 \\
\hline 0.0355205 & 0.538018 & 0.0247455 & 0.299853 & 0.021649 & 0.295095 & 0.0187486 \\
\hline 0.0395421 & 0.536289 & 0.0218367 & 0.291377 & 0.015218 & 0.29595 & 0.0178175 \\
\hline 0.0357673 & 0.544097 & 0.0216103 & 0.297038 & 0.0173939 & 0.29236 & 0.0204468 \\
\hline 0.0469588 & 0.534984 & 0.0180879 & 0.298412 & 0.0198015 & 0.293354 & 0.0185378 \\
\hline 0.0432845 & 0.536754 & 0.0230375 & 0.296714 & 0.0172905 & 0.28703 & 0.0205477 \\
\hline 0.0486171 & 0.53656 & 0.021383 & 0.288338 & 0.0196591 & 0.289979 & 0.0186529 \\
\hline 0.0386798 & 0.528944 & 0.0218459 & 0.288332 & 0.0134976 & 0.285249 & 0.0205026 \\
\hline 0.0431221 & 0.528747 & 0.029477 & 0.293629 & 0.0176751 & 0.288418 & 0.020304 \\
\hline 0.0459433 & 0.531676 & 0.0214465 & 0.287515 & 0.0185891 & 0.282906 & 0.0230515 \\
\hline 0.0368743 & 0.520433 & 0.0183491 & 0.285997 & 0.0187387 & 0.286876 & 0.0192381 \\
\hline 0.0380321 & 0.532344 & 0.0212389 & 0.290984 & 0.0180403 & 0.279697 & 0.016542 \\
\hline 0.0365383 & 0.520787 & 0.0217893 & 0.287692 & 0.018 & 0.2831 & 0.01 \\
\hline 0.0383428 & 0.525189 & 0.0202209 & 0.283285 & 0.0140184 & 0.274744 & 0.0178223 \\
\hline 0.0294809 & 0.515541 & 0.022715 & 0.286854 & 0.0208952 & 0.278566 & 0.022182 \\
\hline 0.0419079 & 0.52 & 0.020392 & 0.2843 & 45 & 283437 & 458 \\
\hline 0.039431 & 0.511983 & 0.0204906 & 0.282969 & 0.0131457 & 0.273953 & 0.0168841 \\
\hline 0.0420719 & 0.504531 & 0.0225559 & 0.284537 & 0.0233943 & 0.276833 & 0.0155869 \\
\hline 0.039911 & 0.507283 & 0.0259529 & 0.278227 & 0.0144627 & 0.276987 & 0.0205825 \\
\hline 0.0354551 & 0.514708 & 0.0198623 & 0.277837 & 0.0131031 & 0.273524 & 0.0190459 \\
\hline 0.0381465 & 0.50871 & 0.0225048 & 0.276856 & 0.0177332 & 0.275546 & 0.0209097 \\
\hline 0.0341122 & 0.50433 & 0.0229726 & 0.273921 & 0.015517 & 0.275223 & 0.0201422 \\
\hline 0.0440397 & 0.503455 & 0.0233934 & 0.272743 & 0.0180769 & 0.270936 & 0.0189398 \\
\hline 0.0350447 & 0.511 & 0.021 & 0.276356 & 0.016 & 0.275417 & 982 \\
\hline 0.0404944 & 0.51034 & 0.0213928 & 0.26727 & 0.0154132 & 0.274247 & 0.0178481 \\
\hline 0.0443656 & 0.508658 & 0.0222969 & 0.268205 & 0.0164777 & 0.269555 & 0.0213876 \\
\hline 0.0359424 & 0.49691 & 0.0216432 & 0.27318 & 0.0144613 & 0.271647 & 0.0146854 \\
\hline 0.0281955 & 0.497804 & 0.0224531 & 0.276225 & 0.017228 & 0.274439 & 0.0190167 \\
\hline 0.0338622 & 0.498626 & 0.0215172 & 0.271548 & 0.0144541 & 0.270423 & 0.0207952 \\
\hline 0.0365744 & 0.49779 & 0.0249147 & 0.269326 & 0.0147475 & 0.269207 & 0.0177413 \\
\hline 0.0206119 & 0.500024 & 0.0242046 & 0.268599 & 0.0166966 & 0.268105 & 0.0179647 \\
\hline 0.0312019 & 0.496932 & 0.0169653 & 0.268487 & 0.0147575 & 0.270618 & 0.0163692 \\
\hline 0.0438715 & 0.49261 & 0.0198098 & 0.270028 & 0.0181813 & 0.26529 & 0.0201737 \\
\hline 0.0394494 & 0.489863 & 0.0222472 & 0.268349 & 0.0157069 & 0.277597 & 0.016808 \\
\hline 0.0377763 & 0.48683 & 0.0270252 & 0.264748 & 0.0176709 & 0.268625 & 0.0183295 \\
\hline 0.0346687 & 0.490114 & 0.0227698 & 0.271276 & 0.0163439 & 0.273049 & 0.0209648 \\
\hline 0.0391935 & 0.49612 & 0.0183822 & 0.26672 & 0.0144621 & 0.269919 & 0.0184228 \\
\hline 0.0496102 & 0.489784 & 0.020022 & 0.272499 & 0.016983 & 0.272763 & 0.0192906 \\
\hline 0.0356873 & 0.485948 & 0.0243208 & 0.272982 & 0.0196362 & 0.269692 & 0.0173109 \\
\hline 0.0415323 & 0.492964 & 0.0245783 & 0.26959 & 0.017414 & 0.280325 & 0.0263023 \\
\hline 0.0360291 & 0.477085 & 0.0221343 & 0.258469 & 0.0194164 & 0.286673 & 0.0207159 \\
\hline
\end{tabular}




\begin{tabular}{|c|c|c|c|c|c|c|}
\hline 0.0399994 & 0.482793 & 0.0179749 & 0.257018 & 0.0145516 & 0.271959 & 0.0167625 \\
\hline 0.0382244 & 0.477815 & 0.0207709 & 0.267558 & 0.0194075 & 0.271413 & 0.0231129 \\
\hline 0.0318982 & 0.476233 & 0.0261687 & 0.261895 & 0.0116931 & 0.278919 & 0.017669 \\
\hline 0372994 & .481425 & 0.020569 & .266175 & 0.0131232 & 0.276942 & 0.01909 \\
\hline 0.0379482 & 0.480211 & 0.0203949 & 0.261854 & 0.0174585 & 0.281627 & 0.0171868 \\
\hline 0.0403691 & 0.481538 & 0.0190014 & 0.270081 & 0.0163304 & 0.279327 & 0.0200482 \\
\hline 0.0336139 & 0.479563 & 0.019813 & 0.263765 & 0.0168103 & 0.277725 & 0.018891 \\
\hline 0.034002 & 0.472193 & 0.0174757 & 0.270792 & 0.0182967 & 0.281172 & 0.0182884 \\
\hline 0.0325003 & 0.475423 & 0.0262877 & 0.258205 & 0.0173405 & 0.278743 & 0.018992 \\
\hline 0.0324401 & 0.474708 & 0.0201931 & 0.263103 & 0.0163969 & 0.278186 & 0.0176481 \\
\hline 0.0349006 & 0.473325 & 0.0231739 & 0.264415 & 0.0136093 & 0.279324 & 0.0183133 \\
\hline 0.0392216 & 0.474827 & 0.0157827 & 0.257946 & 0.0139462 & 0.278761 & 0.0227503 \\
\hline 0.0318716 & 0.468484 & 0.0219865 & 0.260655 & 0.0176079 & 0.277522 & 0.0221045 \\
\hline 0.0453572 & 0.473354 & 0.0239037 & 0.265357 & 0.0122671 & 0.276146 & 0.0186435 \\
\hline 0.0344242 & 0.470872 & 0.0170311 & 0.261012 & 0.0150994 & 0.280223 & 0.0189235 \\
\hline 0.0345738 & 0.467145 & 0.0240564 & 0.258438 & 0.0153863 & 0.283218 & 0.0155841 \\
\hline 0.0366106 & 0.471403 & 0.0227685 & 0.265287 & 0.0173219 & 0.268833 & 0.0186138 \\
\hline 0.0290035 & 0.465356 & 0.0167021 & 0.262326 & 0.0155179 & 0.270531 & 0.0218325 \\
\hline 0.0355494 & 0.454191 & 0.0190121 & 0.2 & 0.01 & 52899 & 0.0183797 \\
\hline 0.0434815 & 0.461317 & 0.0176881 & 0.257661 & 0.0183184 & 0.265688 & 0.0177869 \\
\hline 0.0363845 & 0.459885 & 0.0181255 & 5961 & 0.0156811 & 0.260898 & 0.0145303 \\
\hline 0.0347338 & 0.4 & 304 & 03 & 0.01 & 649 & 393 \\
\hline 0.0389378 & 0.462774 & 0.0240838 & 0.257313 & 0.0132644 & 0.270725 & 0.0220085 \\
\hline 0.0327146 & 0.46286 & 0.0170108 & 0.258223 & 0.0161087 & 0.259827 & 0.0187211 \\
\hline 0.0360397 & 0.459155 & 0.0191234 & 0.256421 & 0.018986 & 0.261021 & 0.016711 \\
\hline 0.0408513 & 0.454208 & 0.0150299 & 0.253605 & 0.015782 & 0.261881 & 0.0169508 \\
\hline 0.0491888 & 0.447552 & 0.0199944 & 0.255981 & 0.0154621 & 0.260825 & 0.0207438 \\
\hline 0.0349903 & 0.452745 & 0.0218852 & 0.25401 & 0.0158756 & 0.259459 & 0.0245501 \\
\hline 0.0390549 & 0.450426 & 0.0218848 & 0.254488 & 0.0101616 & 0.256774 & 0.0175321 \\
\hline 0.0346003 & 0.447074 & 0.0151857 & 077 & 771 & 0.262632 & 0.018387 \\
\hline 0.0436333 & 0.451604 & 0.0154663 & 0.249205 & 0.016532 & 0.254295 & 0.01697 \\
\hline 0.0324173 & 0.447869 & 0.0221176 & 0.25264 & 0.0147766 & 0.249357 & 0.0153469 \\
\hline 0.036047 & 0.445657 & 0.0168379 & 0.248768 & 0.0157841 & 0.253934 & 0.019646 \\
\hline 0.0439519 & 0.443887 & 0.0197189 & 0.248142 & 0.0175681 & 0.249508 & 0.0138252 \\
\hline 0.0363799 & 0.449644 & 0.0183612 & 0.254785 & 0.0149886 & 0.247946 & 0.0158805 \\
\hline 0.0338196 & 0.445566 & 0.0208859 & 0.247596 & 0.017711 & 0.251211 & 0.0206203 \\
\hline 0.0314052 & 0.439542 & 0.0166092 & 0.246462 & 0.00994866 & 0.242652 & 0.0182082 \\
\hline 0.0438828 & 0.446119 & 0.0208477 & 0.246378 & 0.0129158 & 0.245739 & 0.0169437 \\
\hline 0.0342599 & 0.436403 & 0.0172917 & 0.246179 & 0.0164997 & 0.243385 & 0.0167754 \\
\hline 0.0342557 & 0.442726 & 0.0220037 & 0.251691 & 0.0192403 & 0.245278 & 0.0159876 \\
\hline 0.0425152 & 0.439183 & 0.0200439 & 0.245721 & 0.0145341 & 0.246308 & 0.0155484 \\
\hline 0.0285654 & 0.440211 & 0.0135956 & 0.245767 & 0.0146846 & 0.243722 & 0.0182558 \\
\hline 0.0355198 & 0.435953 & 0.0146302 & 0.248512 & 0.0177029 & 0.251354 & 0.017443 \\
\hline 0.0351915 & 0.441405 & 0.0158365 & 0.250501 & 0.0149875 & 0.245208 & 0.0198635 \\
\hline 0.0270796 & 0.436094 & 0.0189741 & 0.248537 & 0.0143796 & 0.241318 & 0.0195417 \\
\hline 0.0455328 & 0.435273 & 0.0168509 & 0.24368 & 0.014441 & 0.246712 & 0.0164668 \\
\hline 0.0389961 & 0.432771 & 0.0174915 & 0.248786 & 0.0129048 & 0.239188 & 0.0220268 \\
\hline
\end{tabular}




\begin{tabular}{|c|c|c|c|c|c|c|}
\hline 0.0416297 & 0.433166 & 0.0179146 & 0.240254 & 0.0131614 & 0.243194 & 0.0157493 \\
\hline 0.0373225 & 0.430728 & 0.0175184 & 0.245782 & 0.0152361 & 0.246277 & 0.0167773 \\
\hline 0.0274715 & 0.429069 & 0.0195657 & 0.245068 & 0.0135705 & 0.252021 & 0.019115 \\
\hline 0.0326819 & .437419 & 0.0193088 & 0.243448 & 0.0146671 & 0.242717 & 0.0201787 \\
\hline 0.038475 & 0.428302 & 0.0200783 & 0.239429 & 0.0156483 & 0.240162 & 0.0170537 \\
\hline 0.033838 & 0.427432 & 0.0179746 & 0.241428 & 0.0140709 & 0.239273 & 0.0124491 \\
\hline 0.0386222 & 0.433987 & 0.0115112 & 0.236101 & 0.0137512 & 0.238551 & 0.0168564 \\
\hline 0.0472658 & 0.429551 & 0.0174449 & 0.24119 & 0.0128769 & 0.240909 & 0.0177575 \\
\hline 0.0298682 & 0.427399 & 0.0179721 & 0.244653 & 0.0193901 & 0.240026 & 0.0162068 \\
\hline 0.0369498 & 0.424716 & 0.0166305 & 0.23566 & 0.017091 & 0.237969 & 0.0179128 \\
\hline 0.0423897 & 0.423655 & 0.0172403 & 0.241492 & 0.0142742 & 0.241434 & 0.0184477 \\
\hline 0.0346627 & 0.422788 & 0.0260153 & 0.24092 & 0.0160347 & 0.24213 & 0.0186156 \\
\hline 0.0434039 & 0.426262 & 0.0194732 & 0.240367 & 0.0172558 & 0.2373 & 0.0147779 \\
\hline 0.0316453 & 0.422864 & 0.0206504 & 0.235397 & 0.0145531 & 0.243111 & 0.020314 \\
\hline 0.0313298 & 0.416766 & 0.013355 & 0.244711 & 0.0148191 & 0.241291 & 0.0168781 \\
\hline 0.0397436 & 0.418768 & 0.0179042 & 0.233287 & 0.0140818 & 0.236018 & 0.0164308 \\
\hline 0.0348478 & 0.424208 & 0.0124565 & 0.238637 & 0.0213324 & 0.235243 & 0.0178082 \\
\hline 0.0322181 & 0.42154 & 0.0181256 & 0.241361 & 0.0224114 & 0.24453 & 0.01 \\
\hline 0.03 & 0.419797 & 0.0171931 & 081 & 0.0185817 & 114 & 502 \\
\hline 0.0338597 & 0.414411 & 0.0122228 & 0.236947 & 0.0177449 & 0.235501 & 0.0179089 \\
\hline 0.0427373 & 0.416822 & 0.0142669 & 0.241412 & 0.0149486 & 0.234452 & 0.0194229 \\
\hline 0.033264 & 0.422664 & 0.01 & 442 & 0.0155749 & 748 & 739 \\
\hline 0.0344603 & 0.416331 & 0.0194614 & 0.238081 & 0.0168544 & 0.235255 & 0.0169395 \\
\hline 0.0394936 & 0.419402 & 0.0189437 & 0.236341 & 0.0160139 & 0.23881 & 0.0175817 \\
\hline 0.0385123 & 0.417415 & 0.0191749 & 0.239817 & 0.014286 & 0.237645 & 0.0179959 \\
\hline 0.0359626 & 0.412125 & 0.0152211 & 0.233679 & 0.0115277 & 0.235661 & 0.0160781 \\
\hline 0.0313752 & 0.419755 & 0.0160282 & 0.235001 & 0.0131759 & 0.243434 & 0.0232771 \\
\hline 0.0297175 & 0.421599 & 0.0189616 & 0.237909 & 0.0197348 & 0.231824 & 0.0174158 \\
\hline 0.0315153 & 0.42267 & 0.0223296 & 0.243036 & 0.0159872 & 0.236164 & 0.0181435 \\
\hline 0.0352495 & 0.416733 & 0.0175163 & & 0.01 & & 893 \\
\hline 0.0433127 & 0.416327 & 0.0177315 & 0.235454 & 0.0114623 & 0.239167 & 0.017945 \\
\hline 0.0354092 & 0.417398 & 0.0193892 & 0.236777 & 0.0132609 & 0.241086 & 0.0180503 \\
\hline 0.0270859 & 0.423137 & 0.0140097 & 0.238967 & 0.0107688 & 0.244887 & 0.0196256 \\
\hline 0.0367421 & 0.416384 & 0.0139708 & 0.24475 & 0.0156334 & 0.241105 & 0.0188978 \\
\hline 0.0346756 & 0.416706 & 0.0203707 & 0.244787 & 0.0149402 & 0.237215 & 0.0142422 \\
\hline 0.0387486 & 0.416952 & 0.0187537 & 0.246291 & 0.0174945 & 0.234962 & 0.0170258 \\
\hline 0.0411288 & 0.41525 & 0.0159114 & 0.246176 & 0.015149 & 0.249713 & 0.0182447 \\
\hline 0.0344498 & 0.417493 & 0.019311 & 0.242911 & 0.0125316 & 0.243127 & 0.017051 \\
\hline 0.0362179 & 0.419527 & 0.0166981 & 0.239863 & 0.0157595 & 0.246516 & 0.0171495 \\
\hline 0.0295133 & 0.419275 & 0.0196602 & 0.245013 & 0.014245 & 0.244413 & 0.0193676 \\
\hline 0.0287029 & 0.424448 & 0.0123149 & 0.246666 & 0.0175636 & 0.244709 & 0.0172198 \\
\hline 0.0397375 & 0.42318 & 0.0136604 & 0.245794 & 0.0205397 & 0.245031 & 0.0147768 \\
\hline 0.0376683 & 0.419833 & 0.0158042 & 0.249554 & 0.0115385 & 0.246643 & 0.0212603 \\
\hline 0.0296935 & 0.421532 & 0.0172049 & 0.24718 & 0.0139153 & 0.248771 & 0.0168964 \\
\hline 0.0346275 & 0.419851 & 0.0146768 & 0.246465 & 0.0177814 & 0.256126 & 0.0207353 \\
\hline 0.0389252 & 0.415456 & 0.0159521 & 0.249726 & 0.0147597 & 0.2492 & 0.0189501 \\
\hline 0.0345616 & 0.412278 & 0.0170378 & 0.245706 & 0.0142602 & 0.250508 & 0.0201534 \\
\hline
\end{tabular}




\begin{tabular}{|c|c|c|c|c|c|c|}
\hline 0.0366429 & 0.41553 & 0.0165187 & 0.254095 & 0.0149798 & 0.25509 & 0.0203739 \\
\hline 0.0414786 & 0.417036 & 0.0159911 & 0.259145 & 0.0165377 & 0.255337 & 0.0160383 \\
\hline 0.0346457 & 0.416898 & 0.0154961 & 0.255001 & 0.0188547 & 0.259704 & 0.0186095 \\
\hline 0461404 & 0.41838 & 0.0175184 & 0.251447 & 0.0159876 & 0.263762 & 0.0222539 \\
\hline 0.0321862 & 0.418841 & 0.0143975 & 0.251598 & 0.0133169 & 0.262454 & 0.018963 \\
\hline 0.0300424 & 0.420267 & 0.0155532 & 0.25341 & 0.0153976 & 0.263948 & 0.0195644 \\
\hline 0.041793 & 0.415252 & 0.018078 & 0.256249 & 0.0140649 & 0.273994 & 0.0187149 \\
\hline 0.0370777 & 0.419197 & 0.013021 & 0.252839 & 0.0173876 & 0.272726 & 0.0216376 \\
\hline 0.0330266 & 0.420535 & 0.0144428 & 0.251188 & 0.0146294 & 0.269874 & 0.021699 \\
\hline 0.0262478 & 0.420393 & 0.0162391 & 0.259402 & 0.0156704 & 0.27934 & 0.0248237 \\
\hline 0.0424888 & 0.416072 & 0.0163563 & 0.256676 & 0.0153439 & 0.280738 & 0.0217083 \\
\hline 0.0354099 & 0.412331 & 0.0147233 & 0.256174 & 0.0138372 & 0.292204 & 0.0247353 \\
\hline 0.0389657 & 0.417238 & 0.0179344 & 0.255878 & 0.0155225 & 0.292495 & 0.0252661 \\
\hline 0.0341831 & 0.414896 & 0.0164641 & 0.256793 & 0.0134732 & 0.29322 & 0.0294909 \\
\hline 0.0330551 & 0.423781 & 0.0186681 & 0.259334 & 0.0166146 & 0.297913 & 0.0235727 \\
\hline 0.0357706 & 0.421632 & 0.0180672 & 0.259344 & 0.0144451 & 0.302368 & 0.0240532 \\
\hline 0.0361021 & 0.416106 & 0.0152429 & 0.270644 & 0.0177375 & 0.310848 & 0.0282386 \\
\hline 0.0381735 & 0.416957 & 0.0185382 & 0.25617 & 0.0151141 & 0.314342 & 0.0188869 \\
\hline 0.0334017 & 0.415034 & 0.0144833 & 327 & 0.0 & 89 & 0.0300223 \\
\hline 0.0283496 & 0.412605 & 0.0129988 & 0.263001 & 0.0174547 & 0.313103 & 0.0337054 \\
\hline 0.0457688 & 0.412453 & 0.0175376 & 0.260936 & 0.0129992 & 0.310941 & 0.0307936 \\
\hline 0.0398106 & 0.41 & 347 & 526 & 0.01 & 64 & 738 \\
\hline 0.0449681 & 0.413886 & 0.012134 & 0.262811 & 0.0178237 & 0.314604 & 0.0288104 \\
\hline 0.0428704 & 0.408409 & 0.0125936 & 0.263617 & 0.0167188 & 0.317571 & 0.0326987 \\
\hline 0.0393857 & 0.408273 & 0.0145721 & 0.264979 & 0.015477 & 0.310722 & 0.0256027 \\
\hline 0.037476 & 0.407172 & 0.0151952 & 0.262813 & 0.0173756 & 0.312554 & 0.0355438 \\
\hline 0.0358508 & 0.407262 & 0.0197233 & 0.260321 & 0.0167814 & 0.303266 & 0.0256318 \\
\hline 0.0364007 & 0.403747 & 0.013149 & 0.261773 & 0.0141852 & 0.300111 & 0.0252843 \\
\hline 0.035492 & 0.401483 & 0.0151225 & 0.256079 & 0.0163401 & 0.299576 & 0.0242542 \\
\hline 0.0369888 & 0.402863 & 0.0170897 & 0.260762 & 0.0170128 & 0.296168 & 0.0252723 \\
\hline 0.0307645 & 0.402401 & 0.0194179 & 0.262478 & 0.0172493 & 0.292766 & 0.0199751 \\
\hline 0.0395873 & 0.401665 & 0.016253 & 0.257007 & 0.016432 & 0.290819 & 0.0316359 \\
\hline 0.0420003 & 0.398453 & 0.0154712 & 0.254034 & 0.0123125 & 0.282391 & 0.0223119 \\
\hline 0.0277698 & 0.390333 & 0.0138211 & 0.249715 & 0.0152955 & 0.276785 & 0.0249936 \\
\hline 0.0307725 & 0.393466 & 0.0128197 & 0.247372 & 0.0185381 & 0.267664 & 0.0220174 \\
\hline 0.0319938 & 0.394308 & 0.0151051 & 0.246247 & 0.0150065 & 0.268985 & 0.024277 \\
\hline 0.0330772 & 0.392272 & 0.013335 & 0.25404 & 0.0174201 & 0.271682 & 0.0189224 \\
\hline 0.0359896 & 0.391373 & 0.0133689 & 0.252699 & 0.0171021 & 0.265604 & 0.0164757 \\
\hline 0.0361928 & 0.39216 & 0.0103582 & 0.252019 & 0.0145797 & 0.262246 & 0.0178199 \\
\hline 0.0377474 & 0.390431 & 0.0130987 & 0.252723 & 0.0146483 & 0.258747 & 0.0162847 \\
\hline 0.0381546 & 0.391202 & 0.0163059 & 0.253397 & 0.0161954 & 0.24736 & 0.0168355 \\
\hline 0.0341811 & 0.389374 & 0.0160497 & 0.247749 & 0.0125935 & 0.254858 & 0.0187629 \\
\hline 0.0287212 & 0.391476 & 0.0175517 & 0.246892 & 0.017383 & 0.260245 & 0.0245282 \\
\hline 0.0346388 & 0.399185 & 0.0107967 & 0.242999 & 0.0155415 & 0.260838 & 0.0206307 \\
\hline 0.035162 & 0.3874 & 0.012002 & 0.242197 & 0.0150966 & 0.261973 & 0.0176447 \\
\hline 0.0261867 & 0.388429 & 0.0134618 & 0.247891 & 0.0140523 & 0.257529 & 0.0181638 \\
\hline 0.0361893 & 0.388309 & 0.0121326 & 0.249143 & 0.014907 & 0.256182 & 0.0230698 \\
\hline
\end{tabular}




\begin{tabular}{|c|c|c|c|c|c|c|}
\hline 0.0347512 & 0.389598 & 0.015028 & 0.252357 & 0.0164369 & 0.260373 & 0.0218091 \\
\hline 0.041671 & .390695 & 0.0147185 & 0.247623 & 0.011059 & 0.26149 & 0.0169612 \\
\hline 0.0371317 & 0.3924 & 0.0135137 & 0.251607 & 0.0173483 & 0.259441 & 0.0190671 \\
\hline 0.0292654 & .390996 & 0.0123391 & 0.248925 & 0.017209 & 0.261915 & 0.0213596 \\
\hline 0.0324212 & 389741 & 0.010756 & 0.24903 & 0.0162515 & 0.258016 & 0.0179402 \\
\hline 0.0485707 & 0.393148 & 0.017555 & 0.253955 & 0.0161479 & 0.256512 & 0.0181962 \\
\hline 0.0329451 & 0.389643 & 0.0181227 & 0.256247 & 0.0165983 & 0.26519 & 0.0220422 \\
\hline 0.0340001 & 0.387549 & 0.0150368 & 0.250628 & 0.0143536 & 0.264771 & 0.0193533 \\
\hline 0.0406995 & 0.390098 & 0.0165614 & 0.250244 & 0.0182757 & 0.263623 & 0.0168542 \\
\hline 0.0419439 & 0.391317 & 0.018234 & 0.248506 & 0.0141684 & 0.261164 & 0.0173376 \\
\hline 0.0356507 & 0.391253 & 0.0114351 & 0.253695 & 0.0113784 & 0.269301 & 0.0195349 \\
\hline 0.0348849 & 0.395612 & 0.0155457 & 0.260104 & 0.0125173 & 0.265301 & 0.0161759 \\
\hline 0.0323531 & 0.395965 & 0.0148675 & 0.25632 & 0.017904 & 0.268364 & 0.0194802 \\
\hline 0.0367166 & 0.393924 & 0.0142721 & 0.255489 & 0.0125374 & 0.264683 & 0.0251788 \\
\hline 0.0286205 & 0.393323 & 0.0117365 & 0.255014 & 0.0192305 & 0.263516 & 0.0165632 \\
\hline 0.0337605 & 0.392257 & 0.0158998 & 0.259916 & 0.0133176 & 0.272508 & 0.0221598 \\
\hline 0.0411414 & 0.39805 & 0.0184206 & 0.258963 & 0.0139807 & 0.263056 & 0.0160776 \\
\hline 0.0319093 & 0.399477 & 0.0138977 & 0.260356 & 0.0131017 & 0.258639 & 0.0179728 \\
\hline 0.033852 & 0.393985 & 0.0136109 & 24 & 0.0124227 & 0.27 & 4964 \\
\hline 0.0327059 & 0.396113 & 0.015441 & 0.261164 & 0.0117176 & 0.267759 & 0.0212574 \\
\hline 0.0329393 & 0.401479 & 0.0173921 & 0.269363 & 0.0171357 & 0.268982 & 0.0200847 \\
\hline 0.028204 & 40246 & 0.0141212 & 59 & 0.015 & 92 & 3944 \\
\hline 0.0355349 & 0.396777 & 0.015397 & 0.263926 & 0.0154669 & 0.265845 & 0.0194711 \\
\hline 0.0235923 & 0.402671 & 0.0185724 & 0.270886 & 0.0173917 & 0.279744 & 0.0202696 \\
\hline 0.0388001 & 0.403811 & 0.0143848 & 0.266135 & 0.0136081 & 0.272299 & 0.0193847 \\
\hline 0.0342074 & 0.406873 & 0.0140398 & 0.265204 & 0.0160464 & 0.277598 & 0.0168999 \\
\hline 0.0308666 & 0.404387 & 0.020345 & 0.270961 & 0.0178563 & 0.277412 & 0.0195002 \\
\hline 0.0312072 & 0.408715 & 0.014211 & 0.272486 & 0.0138924 & 0.281272 & 0.0180937 \\
\hline 0.0445887 & 0.413484 & 0.0173323 & 0.276873 & 0.019943 & 0.2747 & 0.0175223 \\
\hline 0.0389423 & 0.416411 & 0.0137442 & 0.279181 & 0.0159222 & 921 & 0.0177627 \\
\hline 0.0444246 & 0.416918 & 0.0143402 & 0.275219 & 0.0161122 & 0.285841 & 0.0196293 \\
\hline 0.0240386 & 0.417583 & 0.0146087 & 0.275876 & 0.0181036 & 0.293176 & 0.0176219 \\
\hline 0.0439062 & 0.417305 & 0.0178338 & 0.276442 & 0.0188613 & 0.285674 & 0.0202713 \\
\hline 0.0346897 & 0.421159 & 0.0193026 & 0.285536 & 0.0172213 & 0.2912 & 0.0205852 \\
\hline 0.0382366 & 0.425309 & 0.0146197 & 0.287387 & 0.0118997 & 0.287358 & 0.0178015 \\
\hline 0.0326898 & 0.423145 & 0.0145366 & 0.2891 & 0.0118113 & 0.297056 & 0.0214003 \\
\hline 0.040071 & 0.425518 & 0.0136378 & 0.283936 & 0.0146948 & 0.297397 & 0.0222132 \\
\hline 0.0345933 & 0.426004 & 0.0164048 & 0.29168 & 0.0185203 & 0.304643 & 0.0208435 \\
\hline 0.0363562 & 0.430601 & 0.0176897 & 0.292157 & 0.0160708 & 0.305203 & 0.0239154 \\
\hline 0.0369077 & 0.437366 & 0.0141739 & 0.298291 & 0.01646 & 0.309319 & 0.0194727 \\
\hline 0.0381477 & 0.440396 & 0.0167175 & 0.294288 & 0.017727 & 0.312765 & 0.0201476 \\
\hline 0.0364585 & 0.444573 & 0.0189691 & 0.311851 & 0.0152371 & 0.307971 & 0.020459 \\
\hline 0.0425807 & 0.448332 & 0.0176173 & 0.308453 & 0.0153966 & 0.319724 & 0.0196662 \\
\hline 0.0344847 & 0.454416 & 0.0155608 & 0.31468 & 0.0188084 & 0.323874 & 0.0245532 \\
\hline 0.0385776 & 0.460929 & 0.0153041 & 0.312455 & 0.0243675 & 0.334231 & 0.0262079 \\
\hline 0.0369052 & 0.465225 & 0.0143314 & 0.314506 & 0.0172435 & 0.345327 & 0.0255198 \\
\hline 0.0352727 & 0.470955 & 0.0174713 & 0.322637 & 0.0153639 & 0.335443 & 0.0224756 \\
\hline
\end{tabular}




\begin{tabular}{|c|c|c|c|c|c|c|}
\hline 0.0396855 & 0.471109 & 0.0161128 & 0.324539 & 0.017481 & 0.341925 & 0.0202469 \\
\hline 0.0459344 & 0.479158 & 0.014111 & 0.331857 & 0.0193889 & 0.35012 & 0.0193457 \\
\hline 0.0383598 & 0.484454 & 0.0179686 & 0.337397 & 0.0167927 & 0.353456 & 0.0228626 \\
\hline 0.0421719 & .489257 & 0.0167941 & 0.339259 & 0.0190265 & 0.358608 & 0.0180711 \\
\hline 0.03751 & 0.498705 & 0.0172208 & 0.34538 & 0.0253614 & 0.365806 & 0.0195915 \\
\hline 0.044368 & 0.508893 & 0.013581 & 0.344521 & 0.0163891 & 0.365603 & 0.0242904 \\
\hline 0.048557 & 0.518629 & 0.0157543 & 0.350572 & 0.0179242 & 0.381997 & 0.0281026 \\
\hline 0.0374921 & 0.523263 & 0.0166316 & 0.36561 & 0.019196 & 0.387923 & 0.0301186 \\
\hline 0.0505527 & 0.530342 & 0.02019 & 0.36597 & 0.0196331 & 0.392311 & 0.0193866 \\
\hline 0.0388099 & 0.53919 & 0.0176756 & 0.37659 & 0.0179669 & 0.404557 & 0.0203034 \\
\hline 0.0390637 & 0.547247 & 0.015956 & 0.381241 & 0.0181212 & 0.418569 & 0.0282249 \\
\hline 0.0397629 & 0.55789 & 0.0186256 & 0.390789 & 0.0169445 & 0.426584 & 0.0230505 \\
\hline 0.047739 & 0.572542 & 0.0191619 & 0.403404 & 0.0227726 & 0.427528 & 0.0238725 \\
\hline 0.0408826 & 0.577282 & 0.0178193 & 0.410035 & 0.0180107 & 0.440122 & 0.0221292 \\
\hline 0.0419429 & 0.593078 & 0.0190404 & 0.41881 & 0.0208276 & 0.448514 & 0.0267911 \\
\hline 0.0407602 & 0.610323 & 0.0187357 & 0.425859 & 0.0187285 & 0.452033 & 0.0267201 \\
\hline 0.0445503 & 0.615465 & 0.0210464 & 0.432829 & 0.0187172 & 0.467269 & 0.0266842 \\
\hline 0.0446157 & 0.625962 & 0.0230385 & 0.45063 & 0.025 & 0.478266 & 0.0250656 \\
\hline 0.0531105 & 834 & 0.0229899 & 0.45 & 0.02 & 0.493231 & 0.032 \\
\hline 0.0435292 & 0.651341 & 0.0167815 & 0.47026 & 0.0188701 & 0.508898 & 0.0240535 \\
\hline 0.0518816 & 0.666004 & 0.0187523 & 0.480667 & 0.0224866 & 0.51834 & 0.0264792 \\
\hline 0.0616761 & 43 & 0.0208961 & 17 & 0.02 & 0.524782 & 185 \\
\hline 0.0486968 & 0.700541 & 0.0212243 & 0.507863 & 0.0233678 & 0.54801 & 0.0339522 \\
\hline 0.0501058 & 0.712904 & 0.0269648 & 0.513147 & 0.0274151 & 0.555662 & 0.027912 \\
\hline 0.0517564 & 0.728546 & 0.0193262 & 0.524424 & 0.0281981 & 0.580081 & 0.0313744 \\
\hline 0.0407645 & 0.746362 & 0.0202056 & 0.554655 & 0.0266867 & 0.572858 & 0.0269076 \\
\hline 0.0551209 & 0.755085 & 0.0203742 & 0.565292 & 0.0229142 & 0.59868 & 0.031674 \\
\hline 0.0560542 & 0.766122 & 0.0234326 & 0.578651 & 0.0280626 & 0.626534 & 0.0304952 \\
\hline 0.0490676 & 0.782113 & 0.0263525 & 0.586925 & 0.0252837 & 0.633774 & 0.0308626 \\
\hline 0.0548789 & & 0.0269 & & 0.02 & 446 & 0.0422688 \\
\hline 0.0492161 & 0.809389 & 0.0256855 & 0.625527 & 0.0259288 & 0.662398 & 0.0279641 \\
\hline 0.036757 & 0.824901 & 0.0195607 & 0.641372 & 0.0267596 & 0.677676 & 0.0308556 \\
\hline 0.0535237 & 0.843292 & 0.0231534 & 0.657285 & 0.0266259 & 0.70034 & 0.0338806 \\
\hline 0.0514744 & 0.854862 & 0.0192724 & 0.685383 & 0.0271961 & 0.71128 & 0.0281962 \\
\hline 0.0613098 & 0.867851 & 0.0202639 & 0.686731 & 0.0233361 & 0.736713 & 0.0351319 \\
\hline 0.0630335 & 0.876502 & 0.0221038 & 0.722028 & 0.0242996 & 0.74652 & 0.03479 \\
\hline 0.0620185 & 0.886373 & 0.0230033 & 0.719338 & 0.0326287 & 0.753571 & 0.0376029 \\
\hline 0.0631364 & 0.904726 & 0.0207525 & 0.730561 & 0.0274392 & 0.773043 & 0.0377463 \\
\hline 0.0530376 & 0.913157 & 0.0227166 & 0.759976 & 0.0297125 & 0.792554 & 0.0333439 \\
\hline 0.046779 & 0.923297 & 0.0223315 & 0.773144 & 0.0290197 & 0.805731 & 0.0382373 \\
\hline 0.0524637 & 0.925443 & 0.0226282 & 0.792968 & 0.0268663 & 0.815342 & 0.0325052 \\
\hline 0.0609754 & 0.938672 & 0.0213403 & 0.802554 & 0.0301176 & 0.835288 & 0.032294 \\
\hline 0.0679108 & 0.951694 & 0.0267806 & 0.812878 & 0.0196902 & 0.850343 & 0.0390061 \\
\hline 0.0567089 & 0.956644 & 0.0216653 & 0.831944 & 0.026491 & 0.85567 & 0.0381103 \\
\hline 0.0742667 & 0.95806 & 0.0169288 & 0.848887 & 0.0353537 & 0.866599 & 0.0321775 \\
\hline 0.0638075 & 0.968851 & 0.0198667 & 0.855637 & 0.0295533 & 0.876796 & 0.0401276 \\
\hline 0.0689664 & 0.974993 & 0.015953 & 0.869656 & 0.0272496 & 0.892621 & 0.0447493 \\
\hline
\end{tabular}




\begin{tabular}{|c|c|c|c|c|c|c|}
\hline 0.0628893 & 0.975755 & 0.0169561 & 0.884752 & 0.0265174 & 0.914257 & 0.0451445 \\
\hline 0.0597283 & .972147 & 0.0200548 & 0.89988 & 0.0259811 & 0.902435 & 0.0364524 \\
\hline 0.0629695 & 0.971667 & 0.0202804 & 0.907697 & 0.0243486 & 0.929067 & 0.032946 \\
\hline .0496599 & .968315 & 0.0199656 & 0.910855 & 0.0286247 & 0.92174 & 0.0400309 \\
\hline .0580825 & .971818 & 0.0207719 & 0.926247 & 0.025498 & 0.917314 & 0.0327214 \\
\hline 0.05814 & 0.968725 & 0.0246105 & 0.936096 & 0.0296694 & 0.943165 & 0.0464089 \\
\hline 0.0648555 & 0.966367 & 0.0266052 & 0.94382 & 0.031541 & 0.949431 & 0.0353945 \\
\hline 0.0588387 & 0.967878 & 0.0218142 & 0.938864 & 0.0305535 & 0.943408 & 0.035188 \\
\hline 0.0548317 & 0.957383 & 0.020135 & 0.949195 & 0.0301632 & 0.939789 & 0.0363271 \\
\hline 0.0802022 & 0.948779 & 0.0239552 & 0.94917 & 0.0289116 & 0.947006 & 0.0325766 \\
\hline 0.0472783 & 0.940516 & 0.021491 & 0.956894 & 0.0325299 & 0.944213 & 0.0316117 \\
\hline 0.0576431 & 0.933683 & 0.021626 & 0.953866 & 0.0338575 & 0.947977 & 0.0411948 \\
\hline 0.083169 & 0.933143 & 0.0235243 & 0.953535 & 0.0304989 & 0.939525 & 0.0333223 \\
\hline 0.0585421 & 0.924452 & 0.0234001 & 0.949436 & 0.0261882 & 0.932703 & 0.040192 \\
\hline 0.0601066 & 0.912761 & 0.0270636 & 0.934665 & 0.0348068 & 0.933639 & 0.0412737 \\
\hline 0.0680318 & 0.900055 & 0.0240331 & 0.943739 & 0.0320082 & 0.925112 & 0.0404035 \\
\hline 0.0599696 & 0.890455 & 0.0195784 & 0.946967 & 0.0333088 & 0.921067 & 0.0401594 \\
\hline 0.0536944 & 0.873049 & 0.0199216 & 0.939268 & 0.032976 & 0.902638 & 0.0387432 \\
\hline 0.055433 & 0.86 & 0.017567 & 0.931257 & 0.0282739 & 0.90 & 0.0456721 \\
\hline 0.0571688 & 0.848362 & 0.0239047 & 0.923999 & 0.0286709 & 0.894608 & 0.0461311 \\
\hline 0.0595534 & 0.83491 & 0.0209001 & 0.907968 & 0.0263509 & 0.887993 & 0.0435281 \\
\hline 0.0662731 & 0.82 & 0.0237178 & 0.89 & 0.0342898 & 331 & 749 \\
\hline 0.0639529 & 0.810684 & 0.0238247 & 0.893278 & 0.0308382 & 0.859483 & 0.0418677 \\
\hline 0.0431788 & 0.791541 & 0.0258565 & 0.881777 & 0.0358725 & 0.841776 & 0.0264098 \\
\hline 0.0567606 & 0.776719 & 0.0272842 & 0.863987 & 0.0299888 & 0.832202 & 0.024421 \\
\hline 0.0502742 & 0.766121 & 0.0198046 & 0.85004 & 0.0250093 & 0.819982 & 0.0293084 \\
\hline 0.0721674 & 0.751121 & 0.0158127 & 0.835149 & 0.0321872 & 0.805622 & 0.0327737 \\
\hline 0.0606053 & 0.735851 & 0.0195358 & 0.819828 & 0.0202368 & 0.793141 & 0.0303243 \\
\hline 0.063327 & 0.71986 & 0.0222635 & 0.810727 & 0.026333 & 0.764662 & 0.0284328 \\
\hline 0.0693294 & 0.702482 & 0.0182763 & 0.790815 & 0.0353506 & 0.757591 & 0.0373221 \\
\hline 0.0673099 & 0.684332 & 0.0178206 & 0.782704 & 0.0292132 & 0.736426 & 0.0277104 \\
\hline 0.0724869 & 0.667626 & 0.0212963 & 0.758544 & 0.0269977 & 0.724528 & 0.0324112 \\
\hline 0.0678885 & 0.653423 & 0.0233952 & 0.752536 & 0.0285852 & 0.712149 & 0.0401421 \\
\hline 0.0582169 & 0.638065 & 0.0227133 & 0.731379 & 0.0268082 & 0.698303 & 0.0306327 \\
\hline 0.0553115 & 0.618278 & 0.0201146 & 0.71607 & 0.0259126 & 0.679432 & 0.0295201 \\
\hline 0.0765005 & 0.604878 & 0.0166912 & 0.696106 & 0.0215413 & 0.656409 & 0.0327598 \\
\hline 0.0658757 & 0.592379 & 0.0188488 & 0.688606 & 0.0261793 & 0.63387 & 0.0232882 \\
\hline 0.0723515 & 0.580488 & 0.0229229 & 0.663628 & 0.0308037 & 0.619202 & 0.0290145 \\
\hline 0.0521904 & 0.566796 & 0.0231919 & 0.646129 & 0.0264734 & 0.611352 & 0.0320327 \\
\hline 0.0681785 & 0.554433 & 0.0219174 & 0.626391 & 0.0254521 & 0.587574 & 0.030118 \\
\hline 0.0587321 & 0.539603 & 0.018659 & 0.620975 & 0.0296443 & 0.577475 & 0.0331435 \\
\hline 0.0546033 & 0.529373 & 0.0166572 & 0.592926 & 0.0175485 & 0.569084 & 0.0233754 \\
\hline 0.0653275 & 0.523387 & 0.0156105 & 0.573961 & 0.0204951 & 0.542699 & 0.0236894 \\
\hline 0.0458256 & 0.505531 & 0.0170759 & 0.557676 & 0.0238973 & 0.530406 & 0.0288133 \\
\hline 0.0483486 & 0.490913 & 0.0175536 & 0.547483 & 0.0229567 & 0.51951 & 0.0267812 \\
\hline 0.055513 & 0.484832 & 0.0169598 & 0.5286 & 0.0216894 & 0.499925 & 0.0232159 \\
\hline 0.0463638 & 0.475742 & 0.018815 & 0.515107 & 0.024656 & 0.488914 & 0.0221391 \\
\hline
\end{tabular}




\begin{tabular}{|c|c|c|c|c|c|c|}
\hline 0.0546257 & 0.464394 & 0.0184341 & 0.502353 & 0.0195272 & 0.469276 & 0.0287758 \\
\hline 0.0592901 & 0.453569 & 0.0160815 & 0.482675 & 0.0256678 & 0.468766 & 0.0280096 \\
\hline 0.0387106 & 0.444288 & 0.0126846 & .477908 & 0.0195175 & 0.448609 & 0.0252795 \\
\hline 0533253 & 0.432365 & 0.0145221 & .464131 & 0.0231387 & 0.436 & 0.02719 \\
\hline 0.0458121 & 0.41953 & 0.0148983 & 0.449414 & 0.0209002 & 0.428887 & 0.0215529 \\
\hline 0.0583217 & 0.414076 & 0.0124657 & 0.438765 & 0.0293498 & 0.413865 & 0.0225472 \\
\hline 0.0542186 & 0.405982 & 0.0133523 & 0.420779 & 0.0210994 & 0.402422 & 0.0333723 \\
\hline 0.0453495 & 0.396132 & 0.0159635 & 0.411186 & 0.0164323 & 0.402276 & 0.0246522 \\
\hline 0.041335 & 0.39163 & 0.0134076 & 0.404861 & 0.0179353 & 0.38989 & 0.0240087 \\
\hline 0.0517301 & 0.386767 & 0.0115846 & 0.398061 & 0.0188258 & 0.380071 & 0.0179707 \\
\hline 0.0539654 & 0.378732 & 0.0101309 & 0.382772 & 0.0190948 & 0.365584 & 0.0237639 \\
\hline 0.0370652 & 0.375071 & 0.0103774 & 0.374549 & 0.0231174 & 0.349952 & 0.0198644 \\
\hline 0.0388154 & 0.370044 & 0.0106426 & 0.355247 & 0.0193805 & 0.345211 & 0.0192353 \\
\hline 0.0407964 & 0.360159 & 0.0105497 & 0.357702 & 0.0163879 & 0.33516 & 0.0214502 \\
\hline 0.0365464 & 0.35249 & 0.0149426 & 0.341413 & 0.0154753 & 0.33309 & 0.0175944 \\
\hline 0.0333821 & 0.348444 & 0.0117632 & 0.337006 & 0.0130727 & 0.323752 & 0.0155544 \\
\hline 0.0411668 & 0.344128 & 0.0109243 & 0.330269 & 0.0148596 & 0.315594 & 0.0276043 \\
\hline 0.0395034 & 0.338532 & 0.0115371 & 0.319989 & 0.0161723 & 0.316969 & 0.0231928 \\
\hline 0.0411823 & 0.33 & 0.015158 & 44 & 0.0134432 & & 5106 \\
\hline 0.0335574 & 0.330677 & 0.0102832 & 0.313477 & 0.0184671 & 0.28999 & 0.0176235 \\
\hline 0.0460177 & 0.324907 & 0.0112658 & 0.294548 & 0.018357 & 0.292934 & 0.022905 \\
\hline 0.036022 & 0.31 & 0.0162722 & 95 & 0.015 & 57 & 452 \\
\hline 0.03925 & 0.309706 & 0.0137571 & 0.286219 & 0.0162968 & 0.276215 & 0.0140843 \\
\hline 0.0322716 & 0.308114 & 0.0107267 & 0.288027 & 0.0104409 & 0.276982 & 0.0229223 \\
\hline 0.0346414 & 0.305957 & 0.0101654 & 0.272664 & 0.0132386 & 0.268775 & 0.0200901 \\
\hline 0.0411858 & 0.301387 & 0.0131925 & 0.270745 & 0.0129937 & 0.263251 & 0.0215282 \\
\hline 0.0306591 & 0.295343 & 0.0142065 & 0.260436 & 0.0178948 & 0.260384 & 0.0204678 \\
\hline 0.0268385 & 0.294584 & 0.0101172 & 0.259895 & 0.012901 & 0.258867 & 0.019892 \\
\hline 0.0381088 & 0.290623 & 0.00818192 & 0.260199 & 0.0172787 & 0.246676 & 0.0210143 \\
\hline 0.0348602 & 0.284869 & 0.0114583 & & 0.0171978 & 24311 & 0.0172687 \\
\hline 0.0361705 & 0.283231 & 0.0116043 & 0.24 & 0.0167303 & 0.239289 & 0.0185594 \\
\hline 0.0378473 & 0.276476 & 0.0127805 & 0.239952 & 0.0140873 & 0.237602 & 0.0154251 \\
\hline 0.0347298 & 0.272525 & 0.0139374 & 0.232611 & 0.0140705 & 0.227968 & 0.0157703 \\
\hline 0.0306058 & 0.269772 & 0.0107791 & 0.231221 & 0.0105265 & 0.229418 & 0.0125239 \\
\hline 0.0382161 & 0.267669 & 0.0107551 & 0.225468 & 0.0136278 & 0.221845 & 0.0180972 \\
\hline 0.0271581 & 0.265296 & 0.0109784 & 0.223545 & 0.0145706 & 0.221429 & 0.0155388 \\
\hline 0.0356827 & 0.261597 & 0.010477 & 0.216211 & 0.0155012 & 0.216135 & 0.0153627 \\
\hline 0.0326509 & 0.261201 & 0.0132117 & 0.213982 & 0.014128 & 0.212964 & 0.0146097 \\
\hline 0.0322201 & 0.259833 & 0.010473 & 0.210096 & 0.010217 & 0.207638 & 0.0196462 \\
\hline 0.0311154 & 0.255473 & 0.00976098 & 0.210227 & 0.0112513 & 0.209606 & 0.0124068 \\
\hline 0.0378099 & 0.25492 & 0.0101239 & 0.204755 & 0.015626 & 0.200679 & 0.0162127 \\
\hline 0.0338507 & 0.251372 & 0.010012 & 0.199423 & 0.0131597 & 0.197333 & 0.0179001 \\
\hline 0.0339479 & 0.247581 & 0.0109653 & 0.199042 & 0.012334 & 0.192417 & 0.0152791 \\
\hline 0.0354896 & 0.248974 & 0.0116044 & 0.193134 & 0.0126111 & 0.188635 & 0.0184498 \\
\hline 0.0383387 & 0.249254 & 0.0119215 & 0.190233 & 0.0131945 & 0.196382 & 0.0132816 \\
\hline 0.0396512 & 0.250715 & 0.00938004 & 0.189643 & 0.0131971 & 0.18804 & 0.0118942 \\
\hline 0.0381752 & 0.250464 & 0.00967716 & 0.190023 & 0.0123089 & 0.187883 & 0.0167628 \\
\hline
\end{tabular}




\begin{tabular}{|c|c|c|c|c|c|c|}
\hline 0.0316589 & .25298 & 0.010526 & 0.189343 & 0.0148985 & 0.183809 & 0.0128855 \\
\hline 0.0339491 & 0.256699 & 0.0100508 & 0.184591 & 0.0102186 & 0.188164 & 0.0149639 \\
\hline 0.0327655 & 0.261576 & 0.00850483 & 0.184643 & 0.0106205 & 0.188796 & 0.0164008 \\
\hline 0320728 & 0.26698 & 0.00916157 & .183584 & 0.0124738 & 0.182353 & 0.01404 \\
\hline 0.0301792 & 0.273797 & 0.0110121 & 0.182528 & 0.0145553 & 0.19107 & 0.015013 \\
\hline 0.0384695 & 0.280203 & 0.0120425 & 0.183721 & 0.0160807 & 0.189401 & 0.017355 \\
\hline 0.0415115 & 0.284996 & 0.0124784 & 0.186081 & 0.0148133 & 0.192529 & 0.0148511 \\
\hline 0.0368676 & 0.295719 & 0.012366 & 0.195317 & 0.0119537 & 0.198702 & 0.0194626 \\
\hline 0.0339903 & 0.310272 & 0.0116014 & 0.196748 & 0.013842 & 0.204635 & 0.0133582 \\
\hline 0.0411568 & 0.323902 & 0.0114976 & 0.197633 & 0.0142698 & 0.210276 & 0.0168589 \\
\hline 0.0458891 & 0.336717 & 0.0109787 & 0.207135 & 0.0141042 & 0.215411 & 0.0121909 \\
\hline 0.0326953 & 0.350819 & 0.0111387 & 0.212722 & 0.0132352 & 0.230429 & 0.0143525 \\
\hline 0.0401049 & 0.366509 & 0.0119812 & 0.226473 & 0.013906 & 0.233228 & 0.0184979 \\
\hline 0.0515572 & 0.387037 & 0.0144152 & 0.234862 & 0.013526 & 0.245426 & 0.0221248 \\
\hline 0.0394696 & 0.405807 & 0.0137833 & 0.246761 & 0.0155626 & 0.259552 & 0.0165705 \\
\hline 0.0523867 & 0.425861 & 0.0134653 & 0.256068 & 0.0172037 & 0.272059 & 0.0208442 \\
\hline 0.0336263 & 0.446545 & 0.0101592 & 0.27182 & 0.0144898 & 0.285752 & 0.0233213 \\
\hline 0.0560612 & 0.465416 & 0.010617 & 0.292741 & 0.0162033 & 0.30561 & 0.0156332 \\
\hline 0.0490591 & 0.48622 & 0.0121822 & 932 & 0.01 & 42 & 0.02 \\
\hline 0.0603253 & 0.505935 & 0.0140123 & 0.322045 & 0.0118688 & 0.343841 & 0.0185248 \\
\hline 0.0574051 & 0.529698 & 0.0151862 & 0.339704 & 0.0171032 & 0.349795 & 0.0172956 \\
\hline 0.0539122 & 0.54 & 0.0168355 & 546 & 0.01 & 575 & 5811 \\
\hline 0.0656639 & 0.564156 & 0.0168158 & 0.388838 & 0.0201768 & 0.395477 & 0.0228594 \\
\hline 0.0732415 & 0.586118 & 0.0158174 & 0.410568 & 0.0207992 & 0.411866 & 0.0273884 \\
\hline 0.0602273 & 0.604491 & 0.014134 & 0.436697 & 0.02 & 0.437621 & 0.0261478 \\
\hline 0.0542664 & 0.622502 & 0.0176964 & 0.455886 & 0.0234878 & 0.458697 & 0.0260232 \\
\hline 0.0782791 & 0.635551 & 0.023812 & 0.472058 & 0.0259701 & 0.491165 & 0.028345 \\
\hline 0.0755266 & 0.64784 & 0.0172335 & 0.501802 & 0.0267867 & 0.494893 & 0.0238509 \\
\hline 0.067301 & 0.665585 & 0.0212245 & 0.519384 & 0.0192007 & 0.518857 & 0.025514 \\
\hline 0.0741438 & 0.677589 & 0.0197984 & 0.541693 & 0.0197367 & 0.537361 & 0.0279279 \\
\hline 0.0749942 & 0.680898 & 0.0201059 & 0.558471 & 0.025 & 0.557485 & 0.0241026 \\
\hline 0.0879594 & 0.683094 & 0.0202966 & 0.581921 & 0.0234809 & 0.571465 & 0.0235049 \\
\hline 0.0925311 & 0.687328 & 0.0189221 & 0.601203 & 0.0246205 & 0.589893 & 0.0349122 \\
\hline 0.0967296 & 0.692113 & 0.020981 & 0.609388 & 0.0256675 & 0.592655 & 0.030601 \\
\hline 0.079853 & 0.686562 & 0.0251264 & 0.634672 & 0.0303463 & 0.608836 & 0.0273712 \\
\hline 0.0849879 & 0.672171 & 0.021696 & 0.639239 & 0.0322624 & 0.616039 & 0.0262889 \\
\hline 0.102612 & 0.659537 & 0.0173055 & 0.652833 & 0.0354838 & 0.610406 & 0.026554 \\
\hline 0.0854993 & 0.646843 & 0.0139434 & 0.659701 & 0.0274104 & 0.616983 & 0.0347793 \\
\hline 0.101276 & 0.633347 & 0.015193 & 0.65317 & 0.0321107 & 0.619344 & 0.0287841 \\
\hline 0.0929962 & 0.61498 & 0.0195891 & 0.662145 & 0.0325084 & 0.616628 & 0.0272638 \\
\hline 0.073405 & 0.592149 & 0.0170789 & 0.664338 & 0.0288474 & 0.604736 & 0.0268609 \\
\hline 0.106723 & 0.568012 & 0.015572 & 0.664166 & 0.0253508 & 0.594829 & 0.0286354 \\
\hline 0.120978 & 0.542192 & 0.0141073 & 0.644667 & 0.0324011 & 0.58672 & 0.0336446 \\
\hline 0.0922195 & 0.513 & 0.0153968 & 0.64113 & 0.0249493 & 0.57005 & 0.0281693 \\
\hline 0.099384 & 0.482993 & 0.0120556 & 0.609956 & 0.0319298 & 0.556207 & 0.0306544 \\
\hline 0.0939418 & 0.45482 & 0.0122267 & 0.598443 & 0.0240237 & 0.541707 & 0.0280303 \\
\hline 0.0997095 & 0.427684 & 0.0144778 & 0.579055 & 0.0200652 & 0.517206 & 0.028462 \\
\hline
\end{tabular}




\begin{tabular}{|c|c|c|c|c|c|c|}
\hline 0.0885118 & 0.4036 & 0.0131102 & 0.550964 & 0.030309 & 0.491369 & 5071 \\
\hline 0.0784901 & 0.377438 & 0.0136907 & 0.525346 & 0.0272267 & 0.453994 & 0.0262647 \\
\hline 0.0737988 & 0.349235 & 0.0144286 & .502879 & 0.0218817 & 0.429826 & 0.0278824 \\
\hline 0786694 & .327918 & 0.0130222 & 0.47282 & 0.020437 & 0.405457 & 0.0268368 \\
\hline 0.0832691 & 0.303406 & 0.0102286 & 0.440703 & 0.0251306 & 0.373194 & 0.0203622 \\
\hline 0.0612264 & 0.281539 & 0.0117688 & 0.408029 & 0.0162226 & 0.345709 & 0.0169579 \\
\hline 0.0578752 & 0.269357 & 0.012716 & 0.378165 & 0.0183529 & 0.320792 & 0.0185395 \\
\hline 0.0674407 & 0.25369 & 0.0113593 & 0.349063 & 0.0138694 & 0.294327 & 0.0192807 \\
\hline 0.051268 & 0.235403 & 0.010396 & 0.323796 & 0.0187361 & 0.266897 & 0.0131805 \\
\hline 0.0470863 & 0.224187 & 0.0100283 & 0.296916 & 0.0151954 & 0.25239 & 0.01772 \\
\hline 0.0540734 & 0.213877 & 0.00893492 & 0.272312 & 0.0159684 & 0.22935 & 0.0150071 \\
\hline 0.0415996 & 0.205153 & 0.0107251 & 0.254477 & 0.0193958 & 0.215886 & 0.0176982 \\
\hline 0.047576 & 0.200263 & 0.00783811 & 0.229368 & 0.0126385 & 0.192531 & 0.0182062 \\
\hline 0.0477401 & 0.194241 & 0.00692413 & 0.205016 & 0.0136874 & 0.179318 & 0.015408 \\
\hline 0.0300142 & 0.188731 & 0.00861725 & 0.193949 & 0.0137532 & 0.167808 & 0.0148396 \\
\hline 0.0365469 & 0.184174 & 0.00867567 & 0.178955 & 0.0138361 & 0.1548 & 9584 \\
\hline 0.028323 & 0.182258 & 0.00820797 & 0.169769 & 0.0140171 & 0.153115 & 0.0160662 \\
\hline 0.035907 & 0.179604 & 0.00773793 & 0.155363 & 0.0104546 & 0.140002 & 1528 \\
\hline 0.0323528 & 0.176644 & 0.007 & 262 & 037 & 13 & 473 \\
\hline 0.0299665 & 0.175718 & 0.00876643 & 0.140681 & 0.0130642 & 0.129518 & 0.0141955 \\
\hline 0.0270815 & 0.17281 & 0.00836613 & 0.137292 & 0.0136263 & 0.126734 & 0.0115328 \\
\hline 0.031839 & 646 & 0.00 & 839 & 0.00 & & 359 \\
\hline 0.023076 & 0.172019 & 0.00778423 & 0.124343 & 0.0100539 & 0.123188 & 0.0125388 \\
\hline 0.0252878 & 0.170635 & 0.00790476 & 0.12606 & 0.0121374 & 0.120441 & 0.0132091 \\
\hline 0.0364125 & 0.168426 & 0.00921928 & 0.11696 & 0.00789095 & 0.115536 & 0.0120762 \\
\hline 0.0260675 & 0.168203 & 0.0104183 & 0.117971 & 0.0100183 & 0.114628 & 0.0125982 \\
\hline 0.0322828 & 0.168011 & 0.00751165 & 0.118163 & 0.0104184 & 0.116286 & 0.0124621 \\
\hline 0.0224529 & 0.169347 & 0.00895898 & 0.118144 & 0.0114755 & 0.112013 & 0.0111058 \\
\hline 0.0256496 & 0.170642 & 0.0095648 & 0.116209 & 0.00815836 & 0.112089 & 0.0118751 \\
\hline 0.0286439 & 0.169194 & 0.010 & 0.113818 & 0.010363 & 0.115911 & 2722 \\
\hline 0.0210231 & 0.167986 & 0.00741552 & 0.112355 & 0.0107474 & 0.112942 & 0.0133818 \\
\hline 0.0259321 & 0.16953 & 0.00855666 & 0.116629 & 0.0101421 & 0.1117 & 0.010972 \\
\hline 0.0207879 & 0.172645 & 0.00872184 & 0.112854 & 0.0108656 & 0.117062 & 0.0110926 \\
\hline 0.0220968 & 0.171524 & 0.00807526 & 0.114237 & 0.00634563 & 0.119274 & 0.0122442 \\
\hline 0.0207714 & 0.168712 & 0.00744397 & 0.115609 & 0.0107075 & 0.118575 & 0.0144215 \\
\hline 0.0251081 & 0.169957 & 0.00732525 & 0.117696 & 0.0122816 & 0.117445 & 0.0119207 \\
\hline 0.0198871 & 0.171598 & 0.0108847 & 0.117639 & 0.00963507 & 0.1132 & 0.0147284 \\
\hline 0.0304519 & 0.173181 & 0.0113088 & 0.116846 & 0.0108413 & 0.112352 & 0.00919493 \\
\hline 0.0254495 & 0.173956 & 0.00972527 & 0.114391 & 0.0111164 & 0.115713 & 0.01146 \\
\hline 0.0226373 & 0.174948 & 0.00819171 & 0.11273 & 0.0119975 & 0.11606 & 0.0115266 \\
\hline 0.0216702 & 0.176199 & 0.00781007 & 0.118237 & 0.011209 & 0.116497 & 0.0108618 \\
\hline 0.0261412 & 0.175196 & 0.00759933 & 0.120065 & 0.0122582 & 0.118386 & 0.0103616 \\
\hline 0.0235591 & 0.177419 & 0.0072629 & 0.119782 & 0.0105779 & 0.117551 & 0.0112159 \\
\hline 0.0252912 & 0.179955 & 0.00914775 & 0.118832 & 0.0106206 & 0.118355 & 0.0104166 \\
\hline 0.0252539 & 0.178117 & 0.0101948 & 0.120608 & 0.0122131 & 0.119774 & 0.0106866 \\
\hline 0.0286642 & 0.174614 & 0.00852516 & 0.120549 & 0.0124759 & 0.11982 & 0.0125921 \\
\hline 0.0198433 & 0.173785 & 0.00748054 & 0.122196 & 0.0106185 & 0.125282 & 0.0118697 \\
\hline
\end{tabular}




$\begin{array}{rrrrrrr}0.0267957 & 0.176744 & 0.00869528 & 0.121443 & 0.0094337 & 0.126649 & 0.0143971 \\ 0.0205817 & 0.180199 & 0.0102275 & 0.119844 & 0.0101525 & 0.123433 & 0.0138334 \\ 0.0237775 & 0.179986 & 0.0126081 & 0.124366 & 0.0126281 & 0.12195 & 0.0120719 \\ 0.0275517 & 0.180411 & 0.00982481 & 0.123528 & 0.0089807 & 0.128604 & 0.0139932 \\ 0.0238738 & 0.182535 & 0.0102076 & 0.126564 & 0.0126246 & 0.129054 & 0.0117067 \\ 0.0248718 & 0.185136 & 0.00951424 & 0.12547 & 0.00966249 & 0.130736 & 0.0132092 \\ 0.0230128 & 0.187087 & 0.00862778 & 0.130993 & 0.00813954 & 0.126265 & 0.00929173 \\ 0.0272171 & 0.18818 & 0.010425 & 0.128428 & 0.00887737 & 0.133585 & 0.0131707 \\ 0.0195466 & 0.189007 & 0.0108048 & 0.127718 & 0.0109008 & 0.132981 & 0.015578 \\ 0.023979 & 0.18987 & 0.00858879 & 0.130624 & 0.00973808 & 0.136269 & 0.0120752 \\ 0.0260836 & 0.190732 & 0.00810961 & 0.132461 & 0.0118196 & 0.136967 & 0.0143256 \\ 0.0305166 & 0.189754 & 0.0110841 & 0.132284 & 0.0112092 & 0.136613 & 0.0116072 \\ 0.0246101 & 0.193048 & 0.0104957 & 0.133125 & 0.011096 & 0.138814 & 0.0158401 \\ 0.0211813 & 0.196322 & 0.0114637 & 0.139623 & 0.0115045 & 0.137149 & 0.013881 \\ 0.0290172 & 0.195233 & 0.0102278 & 0.140044 & 0.0108475 & 0.133634 & 0.0120355 \\ 0.0222481 & 0.193596 & 0.00885864 & 0.143092 & 0.0131492 & 0.140449 & 0.0143364 \\ 0.02394 & 0.197949 & 0.00890883 & 0.139486 & 0.00843923 & 0.143957 & 0.00897351 \\ 0.0311431 & 0.197629 & 0.00898524 & 0.143378 & 0.0100056 & 0.142836 & 0.0169299 \\ 0.0232894 & 0.194099 & 0.00905429 & 0.144162 & 0.0110193 & 0.147553 & 0.0125955 \\ 0.0252591 & 0.197444 & 0.0107786 & 0.145446 & 0.0118549 & 0.150248 & 0.0106334 \\ 0.0261131 & 0.199907 & 0.00967535 & 0.146529 & 0.014243 & 0.154051 & 0.0135478 \\ 0.0288547 & 0.20141 & 0.00861808 & 0.152456 & 0.0106938 & 0.150096 & 0.0140619 \\ 0.0235059 & 0.201863 & 0.00996916 & 0.149859 & 0.0151307 & 0.151975 & 0.0146699 \\ 0.0255689 & 0.20092 & 0.00988273 & 0.153107 & 0.010649 & 0.156639 & 0.0171358 \\ 0.0271253 & 0.204356 & 0.00790213 & 0.153167 & 0.00707604 & 0.157039 & 0.0139374 \\ 0.026893 & 0.201933 & 0.00898684 & 0.153682 & 0.0104289 & 0.156597 & 0.0107228 \\ 0.0321093 & 0.203049 & 0.00975137 & 0.159159 & 0.0151188 & 0.156721 & 0.0151804 \\ 0.0241523 & 0.208082 & 0.0093383 & 0.158896 & 0.0106804 & 0.161528 & 0.0131388\end{array}$




\begin{tabular}{|c|c|c|c|c|c|c|}
\hline $\begin{array}{l}\text { SB Gy } \\
\text { mean }\end{array}$ & std & $\begin{array}{l}\text { SB X1 } \\
\text { mean }\end{array}$ & std & $\begin{array}{l}\text { SB SNF } \\
\text { mean }\end{array}$ & std & $\begin{array}{l}\text { SB SF } \\
\text { mean }\end{array}$ \\
\hline 0.372716 & 0.0213241 & 0.308687 & 0.11143 & 0.207508 & 0.0201546 & 2.26705 \\
\hline 0.380266 & 0.0261759 & 0.310137 & 0.108058 & 0.206511 & 0.0142846 & 2.22612 \\
\hline 0.381494 & 0.0303519 & 0.315931 & 0.113678 & 0.210907 & 0.0169403 & 2.22689 \\
\hline 0.383343 & 0.029217 & 0.308801 & 0.110392 & 0.208779 & 0.015025 & 2.24404 \\
\hline 0.372164 & 0.0223948 & 0.302719 & 0.114408 & 0.205839 & 0.01184 & 2.23019 \\
\hline 0.380273 & 0.0258454 & 0.306009 & 0.102969 & 0.208633 & 0.0155606 & 2.24663 \\
\hline 0.37502 & 0.0201682 & 0.30645 & 0.112916 & 0.207516 & 0.0176179 & 2.21478 \\
\hline 0.384129 & 0.0277388 & 0.303618 & 0.109196 & 0.202652 & 0.0127888 & 2.21132 \\
\hline 0.376635 & 0.0294942 & 0.304958 & 0.109611 & 0.205249 & 0.0114203 & 2.20511 \\
\hline 0.383255 & 0.033965 & 0.308482 & 0.111941 & 0.201042 & 0.016722 & 2.22559 \\
\hline 0.372901 & 0.0254062 & 0.302535 & 0.107534 & 0.200532 & 0.014472 & 2.22557 \\
\hline 0.371613 & 0.0267283 & 0.304841 & 0.103103 & 0.202207 & 0.0137443 & 2.2116 \\
\hline 0.375027 & 0.0257698 & 0.311067 & 0.11518 & 0.206187 & 0.0165375 & 2.2145 \\
\hline 0.376517 & 0.0267551 & 0.301094 & 0.109106 & 0.197679 & 0.0140839 & 2.20371 \\
\hline 0.372764 & 0.023906 & 0.303709 & 0.108588 & 0.20063 & 0.0131395 & 2.20423 \\
\hline 0.373007 & 0.0307179 & 0.302503 & 0.118517 & 0.198765 & 0.0144961 & 2.19836 \\
\hline 0.371699 & 0.0209773 & 0.306898 & 0.107371 & 0.202522 & 0.0167546 & 2.24487 \\
\hline 0.380479 & 0.031546 & 0.305782 & 0.114464 & 0.20357 & 0.0183039 & 2.21676 \\
\hline 0.384404 & 0.0293727 & 0.30831 & 0.106408 & 0.208729 & 0.0180684 & 2.22763 \\
\hline 0.389884 & 0.0297627 & 0.30877 & 0.113979 & 0.210373 & 0.0150397 & 2.23912 \\
\hline 0.391656 & 0.0274233 & 0.315307 & 0.121606 & 0.216098 & 0.0143232 & 2.22939 \\
\hline 0.399688 & 0.0238505 & 0.323118 & 0.11579 & 0.216713 & 0.016274 & 2.25873 \\
\hline 0.410974 & 0.0250151 & 0.320944 & 0.114184 & 0.224228 & 0.0167299 & 2.23157 \\
\hline 0.408896 & 0.0235667 & 0.327772 & 0.11891 & 0.224229 & 0.0158453 & 2.2213 \\
\hline 0.421748 & 0.0236502 & 0.335759 & 0.120594 & 0.225353 & 0.0146191 & 2.2515 \\
\hline 0.4241 & 0.0249547 & 0.332906 & 0.118561 & 0.227717 & 0.0187557 & 2.28104 \\
\hline 0.416492 & 0.0285785 & 0.32266 & 0.120953 & 0.224952 & 0.0167007 & 2.24066 \\
\hline 0.415808 & 0.0241955 & 0.323395 & 0.117069 & 0.222697 & 0.0128967 & 2.27249 \\
\hline 0.416166 & 0.0305974 & 0.320343 & 0.12387 & 0.222966 & 0.0160793 & 2.2444 \\
\hline 0.407442 & 0.023805 & 0.321871 & 0.118168 & 0.21837 & 0.0165654 & 2.20182 \\
\hline 0.398192 & 0.0233872 & 0.314741 & 0.119057 & 0.217483 & 0.0141103 & 2.23863 \\
\hline 0.394454 & 0.0223466 & 0.308351 & 0.116626 & 0.211371 & 0.0145995 & 2.20453 \\
\hline 0.387544 & 0.0273999 & 0.309562 & 0.117825 & 0.215614 & 0.0160385 & 2.20318 \\
\hline 0.383359 & 0.0315601 & 0.307094 & 0.112969 & 0.217671 & 0.0153202 & 2.1886 \\
\hline 0.370008 & 0.0225174 & 0.302818 & 0.110933 & 0.215103 & 0.0140671 & 2.17309 \\
\hline 0.370649 & 0.0260253 & 0.307004 & 0.12029 & 0.218269 & 0.0145846 & 2.19934 \\
\hline 0.371458 & 0.0252193 & 0.304061 & 0.1133 & 0.232976 & 0.0215142 & 2.18793 \\
\hline 0.374266 & 0.0231795 & 0.31698 & 0.117024 & 0.254039 & 0.0216309 & 2.23924 \\
\hline 0.378974 & 0.0269045 & 0.334086 & 0.124367 & 0.296554 & 0.0250523 & 2.2608 \\
\hline 0.380536 & 0.0242832 & 0.363993 & 0.134331 & 0.359281 & 0.0327462 & 2.36981 \\
\hline 0.390441 & 0.0236091 & 0.405212 & 0.162832 & 0.466959 & 0.0449386 & 2.4847 \\
\hline 0.399948 & 0.0243715 & 0.458066 & 0.179872 & 0.606444 & 0.0580343 & 2.69445 \\
\hline 0.412937 & 0.0172393 & 0.531656 & 0.202839 & 0.78837 & 0.0726182 & 2.96039 \\
\hline 0.43221 & 0.0303715 & 0.625153 & 0.25539 & 1.0177 & 0.110837 & 3.24064 \\
\hline 0.450375 & 0.025193 & 0.726693 & 0.2906 & 1.275 & 0.143062 & 3.61264 \\
\hline
\end{tabular}




\begin{tabular}{|c|c|c|c|c|c|c|}
\hline 0.472457 & 0.0277405 & 0.827076 & 0.338911 & 1.53985 & 0.179596 & 3.94844 \\
\hline 0.498 & 0.0292648 & 0.92193 & 0.38439 & 1.77575 & 0.20439 & 4.23805 \\
\hline 0.512533 & 0.0328701 & 0.997562 & 0.404046 & 1.95232 & 0.211892 & 4.54869 \\
\hline 0.515413 & 0.0293187 & 1.03541 & 0.433121 & 2.04788 & 0.2165 & 4.64071 \\
\hline 0.530284 & 0.0355724 & 1.03113 & 0.425199 & 2.05295 & 0.230785 & 4.62252 \\
\hline 0.520182 & 0.03097 & 0.998692 & 0.40832 & 1.95366 & 0.216282 & 4.53226 \\
\hline 0.511319 & 0.0291712 & 0.922986 & 0.371008 & 1.77308 & 0.203659 & 4.24779 \\
\hline 0.495357 & 0.0286304 & 0.826326 & 0.338303 & 1.53331 & 0.166427 & 3.96532 \\
\hline 0.476559 & 0.0297605 & 0.719981 & 0.291374 & 1.28793 & 0.131374 & 3.63702 \\
\hline 0.455196 & 0.0236857 & 0.634273 & 0.25819 & 1.04649 & 0.106603 & 3.26762 \\
\hline 0.431886 & 0.0298639 & 0.552862 & 0.225438 & 0.832537 & 0.0892648 & 2.98713 \\
\hline 0.425728 & 0.0258066 & 0.495119 & 0.206384 & 0.653595 & 0.0658854 & 2.73518 \\
\hline 0.405181 & 0.0233254 & 0.441898 & 0.172671 & 0.517751 & 0.0444023 & 2.54658 \\
\hline 0.393977 & 0.0290388 & 0.41604 & 0.16779 & 0.427232 & 0.0406478 & 2.4497 \\
\hline 0.395433 & 0.0249935 & 0.410751 & 0.170068 & 0.366832 & 0.0354811 & 2.36178 \\
\hline 0.397644 & 0.0212563 & 0.429937 & 0.174395 & 0.348152 & 0.0341047 & 2.35305 \\
\hline 0.396738 & 0.0225985 & 0.492124 & 0.205547 & 0.375542 & 0.033471 & 2.42599 \\
\hline 0.41182 & 0.0234825 & 0.625702 & 0.273439 & 0.448361 & 0.0410147 & 2.65436 \\
\hline 0.426622 & 0.023352 & 0.83627 & 0.381326 & 0.602861 & 0.0603015 & 3.0602 \\
\hline 0.473306 & 0.0280582 & 1.16679 & 0.544323 & 0.851281 & 0.08076 & 3.69562 \\
\hline 0.546224 & 0.0327075 & 1.68261 & 0.824195 & 1.2178 & 0.128112 & 4.66518 \\
\hline 0.632605 & 0.0390847 & 2.38746 & 1.19222 & 1.7422 & 0.190994 & 6.05742 \\
\hline 0.751081 & 0.0510928 & 3.30963 & 1.67876 & 2.4423 & 0.259821 & 7.85826 \\
\hline 0.903525 & 0.0600441 & 4.4363 & 2.26747 & 3.29573 & 0.350514 & 10.0376 \\
\hline 1.10555 & 0.0808396 & 5.72337 & 2.93666 & 4.2536 & 0.476974 & 12.5634 \\
\hline 1.30088 & 0.105435 & 7.00565 & 3.58587 & 5.24586 & 0.590371 & 15.0729 \\
\hline 1.51293 & 0.128142 & 8.1862 & 4.20222 & 6.18516 & 0.676862 & 17.5132 \\
\hline 1.69474 & 0.124461 & 9.1074 & 4.65871 & 6.92793 & 0.755638 & 19.4821 \\
\hline 1.82472 & 0.14738 & 9.66204 & 4.982 & 7.35558 & 0.802061 & 20.7207 \\
\hline 1.89005 & 0.139062 & 9.73003 & 5.02615 & 7.4404 & 0.817311 & 20.9289 \\
\hline 1.89167 & 0.147937 & 9.28289 & 4.79974 & 7.17631 & 0.790804 & 20.2087 \\
\hline 1.82637 & 0.1431 & 8.52538 & 4.43293 & 6.58509 & 0.745883 & 18.7626 \\
\hline 1.68839 & 0.129044 & 7.44728 & 3.86628 & 5.79957 & 0.651236 & 16.6874 \\
\hline 1.54518 & 0.101766 & 6.20425 & 3.22901 & 4.82574 & 0.529498 & 14.2816 \\
\hline 1.34241 & 0.0978442 & 4.98819 & 2.58356 & 3.88855 & 0.424243 & 11.831 \\
\hline 1.17637 & 0.0739933 & 3.87888 & 1.98508 & 3.03865 & 0.338053 & 9.58169 \\
\hline 1.048 & 0.0608868 & 2.96262 & 1.51594 & 2.28861 & 0.252183 & 7.653 \\
\hline 0.929764 & 0.0550786 & 2.22124 & 1.10317 & 1.7002 & 0.176317 & 6.12153 \\
\hline 0.844002 & 0.0460938 & 1.69994 & 0.822942 & 1.28203 & 0.140309 & 5.01296 \\
\hline 0.798807 & 0.042529 & 1.29346 & 0.635097 & 0.964635 & 0.0995608 & 4.15967 \\
\hline 0.759484 & 0.0497701 & 1.0076 & 0.491736 & 0.737116 & 0.0700289 & 3.53289 \\
\hline 0.739432 & 0.0408895 & 0.804514 & 0.367258 & 0.571295 & 0.0515317 & 3.11701 \\
\hline 0.722478 & 0.0376147 & 0.653943 & 0.287976 & 0.459817 & 0.0415526 & 2.79794 \\
\hline 0.711702 & 0.036852 & 0.545005 & 0.237343 & 0.382824 & 0.0296623 & 2.56781 \\
\hline 0.713068 & 0.0344655 & 0.467788 & 0.18772 & 0.320754 & 0.0224117 & 2.4246 \\
\hline 0.715768 & 0.0485585 & 0.416318 & 0.160499 & 0.279257 & 0.0236065 & 2.32891 \\
\hline 0.707745 & 0.0404225 & 0.375455 & 0.15611 & 0.251075 & 0.0188884 & 2.22138 \\
\hline
\end{tabular}




\begin{tabular}{|c|c|c|c|c|c|c|}
\hline 0.70294 & 0.0483059 & 0.365689 & 0.143013 & 0.235823 & 0.0184049 & 2.16409 \\
\hline 0.69537 & 0.0524448 & 0.353624 & 0.135396 & 0.228827 & 0.0196343 & 2.12041 \\
\hline 0.658041 & 0.0426863 & 0.351998 & 0.13518 & 0.230167 & 0.0193892 & 2.12755 \\
\hline 0.619547 & 0.054873 & 0.36345 & 0.143291 & 0.245096 & 0.0261421 & 2.12955 \\
\hline 0.596756 & 0.0603676 & 0.367933 & 0.138046 & 0.257039 & 0.0282007 & 2.16679 \\
\hline 0.571603 & 0.0478929 & 0.377293 & 0.149385 & 0.275938 & 0.0238608 & 2.17966 \\
\hline 0.554683 & 0.0407247 & 0.380871 & 0.150545 & 0.291092 & 0.0252262 & 2.26163 \\
\hline 0.560185 & 0.0433066 & 0.396303 & 0.150479 & 0.307689 & 0.0285898 & 2.30729 \\
\hline 0.56399 & 0.0399822 & 0.400382 & 0.156765 & 0.3205 & 0.0305121 & 2.34532 \\
\hline 0.570893 & 0.0320076 & 0.403593 & 0.161035 & 0.322581 & 0.0287649 & 2.37597 \\
\hline 0.593637 & 0.0300065 & 0.394992 & 0.15717 & 0.324975 & 0.028041 & 2.40083 \\
\hline 0.617765 & 0.0384793 & 0.390673 & 0.154935 & 0.315294 & 0.0264028 & 2.385 \\
\hline 0.638362 & 0.0392277 & 0.387107 & 0.152566 & 0.299061 & 0.0291838 & 2.39445 \\
\hline 0.654538 & 0.0403441 & 0.367996 & 0.147188 & 0.282108 & 0.0238274 & 2.37641 \\
\hline 0.663806 & 0.0299902 & 0.353201 & 0.134942 & 0.264062 & 0.0193023 & 2.329 \\
\hline 0.672772 & 0.0273933 & 0.338839 & 0.126049 & 0.245692 & 0.0189113 & 2.30572 \\
\hline 0.673502 & 0.0333002 & 0.324438 & 0.12248 & 0.227351 & 0.0168124 & 2.25332 \\
\hline 0.682997 & 0.0307671 & 0.322614 & 0.126107 & 0.212391 & 0.0160212 & 2.24438 \\
\hline 0.700228 & 0.0397011 & 0.316523 & 0.118459 & 0.200822 & 0.0131792 & 2.20157 \\
\hline 0.705575 & 0.0356558 & 0.308624 & 0.115244 & 0.193976 & 0.013225 & 2.14537 \\
\hline 0.735114 & 0.0378579 & 0.302599 & 0.117071 & 0.185852 & 0.0131275 & 2.1195 \\
\hline 0.767705 & 0.0382508 & 0.303824 & 0.115368 & 0.182476 & 0.0134558 & 2.08954 \\
\hline 0.84213 & 0.0382054 & 0.299923 & 0.115882 & 0.182631 & 0.013154 & 2.07054 \\
\hline 0.963136 & 0.0428276 & 0.310419 & 0.119066 & 0.18193 & 0.0139612 & 2.05216 \\
\hline 1.15721 & 0.054753 & 0.326413 & 0.126641 & 0.182409 & 0.0135025 & 2.03342 \\
\hline 1.49855 & 0.068892 & 0.35402 & 0.135697 & 0.190295 & 0.0139952 & 2.0309 \\
\hline 2.05752 & 0.0840323 & 0.387438 & 0.151238 & 0.203176 & 0.0126854 & 2.02306 \\
\hline 2.92723 & 0.119234 & 0.438565 & 0.179398 & 0.22129 & 0.0123775 & 2.02361 \\
\hline 4.27618 & 0.156162 & 0.528339 & 0.216428 & 0.249639 & 0.0157115 & 2.06502 \\
\hline 6.28791 & 0.231019 & 0.658184 & 0.27863 & 0.297006 & 0.0205493 & 2.11373 \\
\hline 9.13014 & 0.310094 & 0.838328 & 0.352795 & 0.36308 & 0.0291593 & 2.17742 \\
\hline 12.9534 & 0.451718 & 1.06585 & 0.4499 & 0.448346 & 0.0386632 & 2.26574 \\
\hline 17.8921 & 0.650147 & 1.3422 & 0.586116 & 0.548835 & 0.0462272 & 2.38176 \\
\hline 23.8371 & 0.826144 & 1.65603 & 0.738172 & 0.669816 & 0.0576188 & 2.52048 \\
\hline 30.6409 & 1.0168 & 1.99616 & 0.884357 & 0.796993 & 0.0718903 & 2.65862 \\
\hline 37.7573 & 1.32311 & 2.33261 & 1.04278 & 0.914228 & 0.0824123 & 2.77446 \\
\hline 44.5817 & 1.5909 & 2.62883 & 1.19158 & 1.02309 & 0.0886223 & 2.87576 \\
\hline 50.4373 & 1.81811 & 2.85265 & 1.29126 & 1.10644 & 0.0992407 & 2.99498 \\
\hline 54.5841 & 1.91153 & 2.96735 & 1.33311 & 1.14736 & 0.106344 & 3.06978 \\
\hline 56.4233 & 1.97923 & 2.96864 & 1.33483 & 1.14424 & 0.107357 & 3.04752 \\
\hline 55.7988 & 1.94306 & 2.83253 & 1.2735 & 1.10496 & 0.101203 & 3.00556 \\
\hline 52.7842 & 1.81533 & 2.62941 & 1.1646 & 1.0296 & 0.096212 & 2.89699 \\
\hline 47.7514 & 1.65581 & 2.33956 & 1.03089 & 0.919819 & 0.0795879 & 2.77613 \\
\hline 41.3805 & 1.43442 & 2.00591 & 0.880313 & 0.796082 & 0.0685334 & 2.65473 \\
\hline 34.315 & 1.16852 & 1.66569 & 0.717452 & 0.671587 & 0.0574162 & 2.48588 \\
\hline 27.3487 & 0.939717 & 1.36255 & 0.573979 & 0.558863 & 0.0425947 & 2.3481 \\
\hline 21.0934 & 0.739198 & 1.10761 & 0.451712 & 0.459656 & 0.0328311 & 2.26231 \\
\hline
\end{tabular}




\begin{tabular}{|c|c|c|c|c|c|c|}
\hline 15.7949 & 0.558569 & 0.905387 & 0.364836 & 0.380952 & 0.0256029 & 2.16465 \\
\hline 11.6894 & 0.411496 & 0.746742 & 0.300044 & 0.325935 & 0.021689 & 2.08963 \\
\hline 8.75696 & 0.310487 & 0.647243 & 0.246455 & 0.291486 & 0.0193821 & 2.04419 \\
\hline 6.78892 & 0.229867 & 0.590655 & 0.222645 & 0.270325 & 0.0172872 & 2.01471 \\
\hline 5.58353 & 0.192327 & 0.546215 & 0.199974 & 0.257542 & 0.0175151 & 2.02301 \\
\hline 4.90036 & 0.176586 & 0.521495 & 0.191282 & 0.252011 & 0.015264 & 2.00817 \\
\hline 4.54975 & 0.171108 & 0.510498 & 0.186851 & 0.248095 & 0.0126222 & 1.99517 \\
\hline 4.34428 & 0.160519 & 0.500644 & 0.181503 & 0.240823 & 0.015278 & 2.00217 \\
\hline 4.16705 & 0.143103 & 0.479409 & 0.18048 & 0.237179 & 0.0147405 & 2.00935 \\
\hline 3.91412 & 0.138431 & 0.458802 & 0.171211 & 0.235576 & 0.0154482 & 2.02089 \\
\hline 3.59086 & 0.140698 & 0.433704 & 0.160275 & 0.22889 & 0.0146087 & 2.00782 \\
\hline 3.1958 & 0.111948 & 0.408099 & 0.148062 & 0.216873 & 0.0140069 & 1.99593 \\
\hline 2.75212 & 0.0897652 & 0.384644 & 0.144042 & 0.208053 & 0.0115156 & 2.00099 \\
\hline 2.2936 & 0.0769859 & 0.363698 & 0.133346 & 0.198747 & 0.0106627 & 2.00007 \\
\hline 1.88007 & 0.071502 & 0.344482 & 0.125015 & 0.195545 & 0.00993633 & 2.00998 \\
\hline 1.49248 & 0.0614355 & 0.335581 & 0.124535 & 0.193926 & 0.0113217 & 2.04297 \\
\hline 1.1772 & 0.0450162 & 0.329372 & 0.122134 & 0.194349 & 0.0120945 & 2.08797 \\
\hline 0.941556 & 0.0349625 & 0.32409 & 0.121432 & 0.203467 & 0.0136228 & 2.15856 \\
\hline 0.769069 & 0.0357297 & 0.334488 & 0.127329 & 0.215116 & 0.0150431 & 2.28644 \\
\hline 0.655884 & 0.0295008 & 0.35587 & 0.137845 & 0.233306 & 0.0175646 & 2.48138 \\
\hline 0.601404 & 0.0281233 & 0.378907 & 0.138514 & 0.262196 & 0.0194287 & 2.73297 \\
\hline 0.595001 & 0.0322782 & 0.413405 & 0.145295 & 0.298956 & 0.0199477 & 3.09541 \\
\hline 0.621957 & 0.0407109 & 0.458681 & 0.165515 & 0.346322 & 0.0236429 & 3.5966 \\
\hline 0.687865 & 0.0452526 & 0.510415 & 0.180859 & 0.407298 & 0.0318296 & 4.19223 \\
\hline 0.786704 & 0.0632332 & 0.57374 & 0.202611 & 0.477706 & 0.0405266 & 4.94463 \\
\hline 0.910689 & 0.0808785 & 0.640982 & 0.23069 & 0.549335 & 0.0477551 & 5.7714 \\
\hline 1.05246 & 0.0953228 & 0.703966 & 0.251623 & 0.623658 & 0.0547991 & 6.63497 \\
\hline 1.19908 & 0.124151 & 0.771852 & 0.271892 & 0.698599 & 0.0604395 & 7.45958 \\
\hline 1.33325 & 0.14497 & 0.835163 & 0.295429 & 0.760895 & 0.0712701 & 8.21712 \\
\hline 1.44697 & 0.15292 & 0.87121 & 0.309238 & 0.806209 & 0.0735183 & 8.83745 \\
\hline 1.53845 & 0.17251 & 0.891144 & 0.310166 & 0.832373 & 0.072362 & 9.19713 \\
\hline 1.58605 & 0.187399 & 0.891669 & 0.310804 & 0.831719 & 0.074484 & 9.27493 \\
\hline 1.57439 & 0.183575 & 0.874711 & 0.307372 & 0.812981 & 0.0703834 & 9.07119 \\
\hline 1.51674 & 0.181831 & 0.838683 & 0.296215 & 0.778119 & 0.0643628 & 8.71355 \\
\hline 1.43153 & 0.152791 & 0.799337 & 0.28467 & 0.726501 & 0.0595233 & 8.22988 \\
\hline 1.32344 & 0.140409 & 0.771613 & 0.277635 & 0.675143 & 0.053512 & 7.68159 \\
\hline 1.1962 & 0.131814 & 0.750396 & 0.271294 & 0.624499 & 0.0427745 & 7.19325 \\
\hline 1.0775 & 0.110467 & 0.737163 & 0.267383 & 0.590004 & 0.0377481 & 6.79893 \\
\hline 0.976032 & 0.0815855 & 0.73412 & 0.262622 & 0.567045 & 0.0403301 & 6.58103 \\
\hline 0.886271 & 0.0589615 & 0.754314 & 0.273936 & 0.553876 & 0.036669 & 6.53038 \\
\hline 0.834327 & 0.053636 & 0.788877 & 0.300048 & 0.560058 & 0.0368268 & 6.61117 \\
\hline 0.804654 & 0.0393803 & 0.827902 & 0.32288 & 0.575952 & 0.0448562 & 6.84031 \\
\hline 0.783499 & 0.0354921 & 0.866698 & 0.331081 & 0.589046 & 0.0438771 & 7.06688 \\
\hline 0.778389 & 0.0426232 & 0.885186 & 0.335698 & 0.600049 & 0.0436904 & 7.22244 \\
\hline 0.779036 & 0.0401601 & 0.899677 & 0.344373 & 0.604876 & 0.0423049 & 7.29915 \\
\hline 0.780779 & 0.0297967 & 0.901004 & 0.343095 & 0.601477 & 0.0414725 & 7.23966 \\
\hline 0.776833 & 0.0336248 & 0.872978 & 0.32832 & 0.585795 & 0.0406707 & 7.03121 \\
\hline
\end{tabular}




\begin{tabular}{|c|c|c|c|c|c|c|}
\hline 0.77079 & 0.0328023 & 0.831844 & 0.307529 & 0.557308 & 0.0401937 & 6.65747 \\
\hline 0.755934 & 0.0271153 & 0.79433 & 0.292759 & 0.526256 & 0.0372796 & 6.21576 \\
\hline 0.738013 & 0.0314278 & 0.754504 & 0.277459 & 0.496722 & 0.0299141 & 5.70833 \\
\hline 0.718921 & 0.0326773 & 0.705575 & 0.252949 & 0.460272 & 0.0286796 & 5.15287 \\
\hline 0.703013 & 0.0336959 & 0.661811 & 0.231549 & 0.424372 & 0.03007 & 4.65589 \\
\hline 0.697636 & 0.0343313 & 0.622078 & 0.217124 & 0.398554 & 0.0286348 & 4.20989 \\
\hline 0.69787 & 0.0314584 & 0.584813 & 0.204106 & 0.373926 & 0.027642 & 3.79727 \\
\hline 0.688885 & 0.030187 & 0.557383 & 0.191471 & 0.349281 & 0.0215223 & 3.47118 \\
\hline 0.679076 & 0.0364028 & 0.53922 & 0.1804 & 0.330443 & 0.0205738 & 3.20802 \\
\hline 0.678533 & 0.0334932 & 0.520488 & 0.173819 & 0.314624 & 0.0204336 & 2.97128 \\
\hline 0.678414 & 0.030876 & 0.506717 & 0.171121 & 0.302833 & 0.0194453 & 2.78465 \\
\hline 0.670694 & 0.0337615 & 0.497199 & 0.165102 & 0.289576 & 0.0214027 & 2.63199 \\
\hline 0.661744 & 0.0384873 & 0.48068 & 0.159596 & 0.276509 & 0.020021 & 2.50839 \\
\hline 0.650916 & 0.0377832 & 0.470257 & 0.158623 & 0.264286 & 0.0187317 & 2.40091 \\
\hline 0.638708 & 0.0352241 & 0.455598 & 0.15358 & 0.252195 & 0.016548 & 2.31534 \\
\hline 0.626833 & 0.0333965 & 0.435287 & 0.15252 & 0.242166 & 0.0146855 & 2.23076 \\
\hline 0.602146 & 0.0349021 & 0.425411 & 0.151627 & 0.231557 & 0.0163674 & 2.1613 \\
\hline 0.577522 & 0.0303192 & 0.416105 & 0.144146 & 0.218503 & 0.0148076 & 2.1087 \\
\hline 0.559569 & 0.0285785 & 0.401517 & 0.141564 & 0.210852 & 0.015712 & 2.04696 \\
\hline 0.536042 & 0.0271029 & 0.389835 & 0.140898 & 0.20743 & 0.0138333 & 1.98956 \\
\hline 0.51879 & 0.0226814 & 0.384254 & 0.141786 & 0.200883 & 0.0139005 & 1.95899 \\
\hline 0.505612 & 0.0245369 & 0.381385 & 0.146725 & 0.193327 & 0.011364 & 1.93358 \\
\hline 0.498301 & 0.0284991 & 0.379529 & 0.148268 & 0.191337 & 0.0114634 & 1.92274 \\
\hline 0.495228 & 0.0276086 & 0.381737 & 0.144595 & 0.193668 & 0.0119929 & 1.9095 \\
\hline 0.490933 & 0.0229089 & 0.385409 & 0.149215 & 0.196442 & 0.0108395 & 1.90467 \\
\hline 0.489591 & 0.0224953 & 0.388345 & 0.146214 & 0.201656 & 0.0135851 & 1.91316 \\
\hline 0.487884 & 0.0231697 & 0.388824 & 0.146106 & 0.207841 & 0.0145945 & 1.92712 \\
\hline 0.487348 & 0.0263137 & 0.395524 & 0.152166 & 0.213895 & 0.0130022 & 1.91507 \\
\hline 0.493624 & 0.0259613 & 0.406339 & 0.156672 & 0.227105 & 0.0142201 & 1.9441 \\
\hline 0.505962 & 0.0293031 & 0.41645 & 0.159797 & 0.247886 & 0.0171306 & 1.97362 \\
\hline 0.524971 & 0.0298974 & 0.43672 & 0.167502 & 0.281109 & 0.0179059 & 2.0227 \\
\hline 0.526903 & 0.0262914 & 0.472575 & 0.184915 & 0.329265 & 0.0222421 & 2.0947 \\
\hline 0.533581 & 0.0270116 & 0.518163 & 0.205931 & 0.396884 & 0.0304104 & 2.1926 \\
\hline 0.546232 & 0.0264137 & 0.588332 & 0.240898 & 0.506759 & 0.0374853 & 2.35718 \\
\hline 0.564335 & 0.0262866 & 0.680065 & 0.282539 & 0.667198 & 0.0469872 & 2.5971 \\
\hline 0.579431 & 0.0323719 & 0.798719 & 0.342557 & 0.883594 & 0.0654907 & 2.94332 \\
\hline 0.593735 & 0.0338645 & 0.95129 & 0.424463 & 1.17341 & 0.0785575 & 3.39756 \\
\hline 0.607313 & 0.0266893 & 1.15995 & 0.523642 & 1.53697 & 0.103085 & 3.94453 \\
\hline 0.636288 & 0.029684 & 1.41575 & 0.647692 & 1.97639 & 0.146032 & 4.61785 \\
\hline 0.665248 & 0.0321439 & 1.68099 & 0.786597 & 2.47683 & 0.195348 & 5.41398 \\
\hline 0.694318 & 0.0242754 & 1.97759 & 0.934614 & 3.01369 & 0.238984 & 6.23053 \\
\hline 0.72288 & 0.0294067 & 2.27474 & 1.0754 & 3.54086 & 0.274383 & 7.02476 \\
\hline 0.766697 & 0.0365183 & 2.52657 & 1.1987 & 4.01424 & 0.30405 & 7.78565 \\
\hline 0.801274 & 0.0439138 & 2.69282 & 1.29943 & 4.38106 & 0.32427 & 8.37833 \\
\hline 0.818638 & 0.049581 & 2.81864 & 1.37 & 4.62469 & 0.346757 & 8.76324 \\
\hline 0.831094 & 0.0442562 & 2.85067 & 1.38107 & 4.72411 & 0.346413 & 8.9004 \\
\hline 0.832441 & 0.0470938 & 2.78947 & 1.35222 & 4.64264 & 0.345403 & 8.80119 \\
\hline
\end{tabular}




\begin{tabular}{|c|c|c|c|c|c|c|}
\hline 0.829957 & 0.0516645 & 2.64829 & 1.285 & 4.39459 & 0.331619 & 8.41864 \\
\hline 0.821808 & 0.0501677 & 2.44289 & 1.17609 & 4.01472 & 0.305917 & 7.85982 \\
\hline 0.799577 & 0.0422057 & 2.18433 & 1.04041 & 3.55078 & 0.277739 & 7.14655 \\
\hline 0.768415 & 0.0347857 & 1.90165 & 0.909514 & 3.02688 & 0.22792 & 6.33781 \\
\hline 0.747946 & 0.0336694 & 1.61043 & 0.762178 & 2.48763 & 0.183544 & 5.51245 \\
\hline 0.726036 & 0.0316346 & 1.3502 & 0.623193 & 1.98078 & 0.141132 & 4.72954 \\
\hline 0.70718 & 0.0323375 & 1.13464 & 0.517167 & 1.54734 & 0.106248 & 4.03254 \\
\hline 0.694931 & 0.0337009 & 0.95197 & 0.429403 & 1.18417 & 0.0846665 & 3.44904 \\
\hline 0.684584 & 0.0325681 & 0.805519 & 0.353893 & 0.890539 & 0.0580483 & 2.98488 \\
\hline 0.690149 & 0.0376781 & 0.706255 & 0.302115 & 0.665786 & 0.0427609 & 2.62152 \\
\hline 0.702845 & 0.0354651 & 0.646119 & 0.261338 & 0.508722 & 0.0344843 & 2.36989 \\
\hline 0.723058 & 0.0328066 & 0.620026 & 0.2482 & 0.411423 & 0.0246101 & 2.21111 \\
\hline 0.762223 & 0.0336426 & 0.609941 & 0.249825 & 0.353137 & 0.019643 & 2.10387 \\
\hline 0.807597 & 0.0337152 & 0.623811 & 0.254461 & 0.323474 & 0.0204366 & 2.04502 \\
\hline 0.865142 & 0.0291717 & 0.664488 & 0.271784 & 0.321013 & 0.0207822 & 2.03927 \\
\hline 0.950853 & 0.0315156 & 0.730034 & 0.296375 & 0.340413 & 0.0201965 & 2.06438 \\
\hline 1.05422 & 0.0436613 & 0.828442 & 0.328501 & 0.379745 & 0.0229455 & 2.11901 \\
\hline 1.20483 & 0.0552122 & 0.978705 & 0.391818 & 0.433591 & 0.0278689 & 2.20537 \\
\hline 1.43591 & 0.0651619 & 1.1936 & 0.477481 & 0.510566 & 0.0341381 & 2.28692 \\
\hline 1.77145 & 0.0719451 & 1.51542 & 0.604967 & 0.617378 & 0.0435289 & 2.39367 \\
\hline 2.27874 & 0.0847984 & 2.01323 & 0.822775 & 0.759494 & 0.0553272 & 2.55696 \\
\hline 3.07655 & 0.114858 & 2.75674 & 1.12624 & 0.933832 & 0.0647383 & 2.76393 \\
\hline 4.30867 & 0.167359 & 3.87345 & 1.56708 & 1.16927 & 0.0713231 & 3.01826 \\
\hline 6.18951 & 0.225492 & 5.48925 & 2.22595 & 1.49542 & 0.091603 & 3.32548 \\
\hline 8.98532 & 0.318582 & 7.77966 & 3.15677 & 1.91424 & 0.123485 & 3.6874 \\
\hline 13.0242 & 0.468534 & 10.9424 & 4.4446 & 2.45534 & 0.15895 & 4.1563 \\
\hline 18.686 & 0.651179 & 15.1334 & 6.15421 & 3.13465 & 0.202013 & 4.74669 \\
\hline 26.2776 & 0.945931 & 20.4153 & 8.31057 & 4.00558 & 0.267314 & 5.46666 \\
\hline 36.0684 & 1.27473 & 26.7938 & 10.9232 & 5.05605 & 0.354042 & 6.33902 \\
\hline 48.0681 & 1.66116 & 34.1742 & 13.9735 & 6.27023 & 0.436557 & 7.33242 \\
\hline 62.1404 & 2.1598 & 42.182 & 17.2683 & 7.60805 & 0.53029 & 8.36714 \\
\hline 77.8349 & 2.68989 & 50.3936 & 20.674 & 8.98722 & 0.647369 & 9.44524 \\
\hline 94.196 & 3.26809 & 58.2255 & 23.9004 & 10.3188 & 0.753848 & 10.5374 \\
\hline 110.173 & 3.79421 & 64.94 & 26.68 & 11.5096 & 0.82849 & 11.5265 \\
\hline 124.549 & 4.37613 & 70.0198 & 28.7817 & 12.4145 & 0.881681 & 12.2611 \\
\hline 135.845 & 4.77371 & 72.878 & 29.9643 & 12.951 & 0.926282 & 12.7053 \\
\hline 143.108 & 5.02775 & 73.1562 & 30.1799 & 13.0726 & 0.923292 & 12.796 \\
\hline 145.455 & 5.11556 & 70.8242 & 29.2608 & 12.7629 & 0.889428 & 12.491 \\
\hline 142.602 & 5.03641 & 66.1622 & 27.354 & 12.0228 & 0.837275 & 11.8958 \\
\hline 134.904 & 4.82674 & 59.7257 & 24.791 & 10.9309 & 0.75365 & 10.9824 \\
\hline 123.152 & 4.30528 & 51.9951 & 21.645 & 9.61012 & 0.679382 & 9.84788 \\
\hline 108.47 & 3.72035 & 43.6741 & 18.1927 & 8.15136 & 0.588674 & 8.58715 \\
\hline 92.1945 & 3.15397 & 35.4532 & 14.8295 & 6.69027 & 0.478474 & 7.39325 \\
\hline 75.703 & 2.5538 & 27.8149 & 11.6563 & 5.30643 & 0.390249 & 6.3114 \\
\hline 59.9368 & 2.0304 & 21.1262 & 8.83956 & 4.07131 & 0.316362 & 5.25825 \\
\hline 45.8596 & 1.58508 & 15.4913 & 6.48717 & 3.04163 & 0.224359 & 4.3699 \\
\hline 34.0088 & 1.18261 & 11.0078 & 4.59522 & 2.21145 & 0.152987 & 3.67314 \\
\hline
\end{tabular}




\begin{tabular}{|c|c|c|c|c|c|c|}
\hline 24.4163 & 0.869565 & 7.64904 & 3.19861 & 1.56974 & 0.105512 & 3.15665 \\
\hline 17.0133 & 0.608204 & 5.19477 & 2.17265 & 1.10361 & 0.0711724 & 2.77657 \\
\hline 11.5521 & 0.379906 & 3.49904 & 1.46057 & 0.786174 & 0.0516535 & 2.50174 \\
\hline 7.70807 & 0.23998 & 2.39628 & 0.989606 & 0.568602 & 0.0361629 & 2.3171 \\
\hline 5.14452 & 0.177302 & 1.70905 & 0.689266 & 0.429239 & 0.0292557 & 2.21368 \\
\hline 3.52825 & 0.126587 & 1.33102 & 0.541336 & 0.355055 & 0.0266109 & 2.16107 \\
\hline 2.58904 & 0.0830851 & 1.17372 & 0.473446 & 0.322755 & 0.0253118 & 2.1421 \\
\hline 2.14404 & 0.0747842 & 1.18866 & 0.466129 & 0.319099 & 0.0196485 & 2.14668 \\
\hline 2.05985 & 0.0754324 & 1.34221 & 0.515734 & 0.343115 & 0.0199806 & 2.14391 \\
\hline 2.25991 & 0.0801706 & 1.61642 & 0.621539 & 0.389441 & 0.0240918 & 2.1861 \\
\hline 2.70744 & 0.0867646 & 2.02064 & 0.775616 & 0.453042 & 0.0315252 & 2.26176 \\
\hline 3.40804 & 0.112464 & 2.56754 & 0.997697 & 0.544783 & 0.0374096 & 2.3543 \\
\hline 4.36658 & 0.156567 & 3.258 & 1.27887 & 0.670624 & 0.0447566 & 2.47575 \\
\hline 5.63177 & 0.195426 & 4.09496 & 1.59059 & 0.822397 & 0.0544719 & 2.60957 \\
\hline 7.19252 & 0.251477 & 5.07442 & 1.96982 & 0.994912 & 0.0704468 & 2.75601 \\
\hline 9.01366 & 0.323583 & 6.14645 & 2.3853 & 1.18411 & 0.0822348 & 2.93166 \\
\hline 11.0824 & 0.409603 & 7.22699 & 2.81386 & 1.37734 & 0.0798762 & 3.09912 \\
\hline 13.2466 & 0.471928 & 8.2801 & 3.25528 & 1.57448 & 0.0962844 & 3.24567 \\
\hline 15.3667 & 0.538853 & 9.22545 & 3.62095 & 1.7567 & 0.117444 & 3.40128 \\
\hline 17.3661 & 0.619849 & 9.94813 & 3.89783 & 1.89461 & 0.115539 & 3.51214 \\
\hline 19.0481 & 0.674242 & 10.4021 & 4.0892 & 1.99933 & 0.13196 & 3.57858 \\
\hline 20.208 & 0.726503 & 10.5151 & 4.13564 & 2.05438 & 0.144878 & 3.64308 \\
\hline 20.7467 & 0.714494 & 10.3162 & 4.05995 & 2.02918 & 0.136225 & 3.64275 \\
\hline 20.6332 & 0.686782 & 9.81264 & 3.87932 & 1.94405 & 0.128979 & 3.56854 \\
\hline 19.8924 & 0.670902 & 9.00626 & 3.57199 & 1.8119 & 0.116335 & 3.46459 \\
\hline 18.5386 & 0.62738 & 8.01591 & 3.17443 & 1.64057 & 0.105987 & 3.32815 \\
\hline 16.723 & 0.583533 & 6.92155 & 2.74412 & 1.4437 & 0.0960417 & 3.1798 \\
\hline 14.5656 & 0.501739 & 5.77495 & 2.31308 & 1.2423 & 0.08165 & 3.00898 \\
\hline 12.2713 & 0.412997 & 4.66078 & 1.86153 & 1.04082 & 0.066141 & 2.83254 \\
\hline 10.0426 & 0.349927 & 3.65764 & 1.4562 & 0.847344 & 0.0537372 & 2.6894 \\
\hline 7.93488 & 0.266115 & 2.79242 & 1.10629 & 0.681468 & 0.0467017 & 2.56724 \\
\hline 6.06653 & 0.220508 & 2.0704 & 0.811067 & 0.549258 & 0.0356838 & 2.43993 \\
\hline 4.51484 & 0.180521 & 1.51129 & 0.591619 & 0.433533 & 0.0274243 & 2.36779 \\
\hline 3.27056 & 0.126859 & 1.09174 & 0.431047 & 0.34861 & 0.0240066 & 2.31411 \\
\hline 2.29831 & 0.0883192 & 0.782046 & 0.303877 & 0.286755 & 0.0182613 & 2.23318 \\
\hline 1.57484 & 0.0710781 & 0.567075 & 0.217378 & 0.239693 & 0.0136457 & 2.22069 \\
\hline 1.05527 & 0.0494537 & 0.414133 & 0.160986 & 0.208033 & 0.0128152 & 2.22457 \\
\hline 0.703673 & 0.0314026 & 0.31655 & 0.127162 & 0.188046 & 0.01153 & 2.17331 \\
\hline 0.476854 & 0.0253823 & 0.25107 & 0.101317 & 0.175112 & 0.0111809 & 2.15809 \\
\hline 0.329256 & 0.0185118 & 0.213116 & 0.0888635 & 0.167986 & 0.00962738 & 2.17727 \\
\hline 0.231128 & 0.0146133 & 0.194014 & 0.0809568 & 0.163532 & 0.0117392 & 2.17757 \\
\hline 0.172857 & 0.0115835 & 0.178248 & 0.0715275 & 0.161385 & 0.0115909 & 2.18562 \\
\hline 0.143106 & 0.00800486 & 0.172702 & 0.0721986 & 0.162679 & 0.00980729 & 2.19674 \\
\hline 0.126101 & 0.0101835 & 0.169672 & 0.0747933 & 0.161311 & 0.0105517 & 2.21503 \\
\hline 0.112878 & 0.0121306 & 0.169838 & 0.077344 & 0.162557 & 0.0118261 & 2.21892 \\
\hline 0.10546 & 0.012742 & 0.169564 & 0.0793709 & 0.165594 & 0.0122762 & 2.23852 \\
\hline 0.0980207 & 0.00988317 & 0.169467 & 0.0757913 & 0.169452 & 0.0121086 & 2.26687 \\
\hline
\end{tabular}




\begin{tabular}{|c|c|c|c|c|c|c|}
\hline 0.0971087 & 0.00770268 & 0.171192 & 0.0758179 & 0.170698 & 0.0118805 & 2.26679 \\
\hline 0.0952662 & 0.00924388 & 0.173165 & 0.0787328 & 0.170996 & 0.012848 & 2.25903 \\
\hline 0.0924062 & 0.0105983 & 0.174622 & 0.0787779 & 0.175085 & 0.0111951 & 2.28109 \\
\hline 0.0917546 & 0.00793704 & 0.177833 & 0.0828147 & 0.182862 & 0.0130785 & 2.30106 \\
\hline 0.0921165 & 0.00549585 & 0.181045 & 0.0860011 & 0.190733 & 0.0136419 & 2.33797 \\
\hline 0.0898673 & 0.00722141 & 0.183237 & 0.0858833 & 0.200782 & 0.0106478 & 2.35288 \\
\hline 0.0878878 & 0.0070874 & 0.192121 & 0.0890881 & 0.214852 & 0.012575 & 2.40133 \\
\hline 0.0897843 & 0.00506456 & 0.204621 & 0.0962997 & 0.23578 & 0.01354 & 2.43386 \\
\hline 0.0898014 & 0.0074409 & 0.218496 & 0.109232 & 0.268041 & 0.0146619 & 2.48122 \\
\hline 0.0892425 & 0.00756517 & 0.239814 & 0.126027 & 0.31176 & 0.0164656 & 2.57761 \\
\hline 0.0902533 & 0.00888054 & 0.265511 & 0.14716 & 0.37361 & 0.0213875 & 2.71033 \\
\hline 0.0947633 & 0.00872779 & 0.302629 & 0.194088 & 0.454766 & 0.0301856 & 2.87886 \\
\hline 0.100709 & 0.0110056 & 0.355038 & 0.26178 & 0.566086 & 0.0386928 & 3.09635 \\
\hline 0.108737 & 0.0141541 & 0.418635 & 0.334223 & 0.711747 & 0.0440866 & 3.39576 \\
\hline 0.11304 & 0.0171639 & 0.500131 & 0.432688 & 0.896421 & 0.0581731 & 3.70501 \\
\hline 0.121754 & 0.020825 & 0.595537 & 0.559972 & 1.11604 & 0.0714759 & 4.0963 \\
\hline 0.137492 & 0.0262649 & 0.705957 & 0.701926 & 1.36784 & 0.0907241 & 4.59449 \\
\hline 0.150986 & 0.0340488 & 0.827499 & 0.864483 & 1.64794 & 0.103825 & 5.11626 \\
\hline 0.163956 & 0.0409616 & 0.949433 & 1.03914 & 1.94565 & 0.120789 & 5.6217 \\
\hline 0.181431 & 0.0517584 & 1.07187 & 1.21251 & 2.23843 & 0.14007 & 6.17742 \\
\hline 0.199876 & 0.0584596 & 1.18844 & 1.37194 & 2.51026 & 0.164536 & 6.6782 \\
\hline 0.213217 & 0.0687115 & 1.28457 & 1.51914 & 2.74586 & 0.171833 & 7.08747 \\
\hline 0.225729 & 0.0734272 & 1.34406 & 1.62555 & 2.91906 & 0.185284 & 7.40739 \\
\hline 0.237254 & 0.0788782 & 1.37192 & 1.66773 & 3.00133 & 0.19812 & 7.59811 \\
\hline 0.245219 & 0.089259 & 1.3705 & 1.69441 & 3.01794 & 0.201733 & 7.64285 \\
\hline 0.251795 & 0.0957245 & 1.32953 & 1.64848 & 2.9541 & 0.185573 & 7.58298 \\
\hline 0.255958 & 0.0866469 & 1.25985 & 1.54246 & 2.79845 & 0.176453 & 7.3602 \\
\hline 0.247662 & 0.0827964 & 1.17047 & 1.42166 & 2.57875 & 0.163032 & 6.97784 \\
\hline 0.23464 & 0.0813667 & 1.04754 & 1.26036 & 2.29696 & 0.137901 & 6.57841 \\
\hline 0.229318 & 0.0808938 & 0.929903 & 1.09324 & 2.00321 & 0.13354 & 6.11763 \\
\hline 0.220743 & 0.0665006 & 0.806283 & 0.905831 & 1.70694 & 0.112303 & 5.62412 \\
\hline 0.207048 & 0.0522694 & 0.691258 & 0.732615 & 1.41277 & 0.0849779 & 5.09845 \\
\hline 0.194389 & 0.0370583 & 0.58615 & 0.587295 & 1.15989 & 0.071677 & 4.67631 \\
\hline 0.181991 & 0.0376021 & 0.494717 & 0.462323 & 0.939926 & 0.058404 & 4.31514 \\
\hline 0.170625 & 0.0297795 & 0.415741 & 0.335834 & 0.748609 & 0.0494313 & 4.04222 \\
\hline 0.160793 & 0.026302 & 0.359954 & 0.24821 & 0.59913 & 0.0441819 & 3.86587 \\
\hline 0.153482 & 0.0204461 & 0.316739 & 0.200006 & 0.493103 & 0.036404 & 3.63708 \\
\hline 0.149322 & 0.0161264 & 0.290419 & 0.163824 & 0.409486 & 0.0291076 & 3.57888 \\
\hline 0.139231 & 0.0132671 & 0.274148 & 0.136459 & 0.349712 & 0.0242671 & 3.55219 \\
\hline 0.137264 & 0.0105368 & 0.250626 & 0.120827 & 0.31363 & 0.0208224 & 3.50128 \\
\hline 0.132414 & 0.0118896 & 0.245018 & 0.111573 & 0.289759 & 0.0239937 & 3.4261 \\
\hline 0.130831 & 0.0140143 & 0.240974 & 0.11498 & 0.272726 & 0.0257093 & 3.38189 \\
\hline 0.131222 & 0.0108052 & 0.24318 & 0.10871 & 0.263474 & 0.0218123 & 3.38224 \\
\hline 0.125637 & 0.00946786 & 0.246535 & 0.105663 & 0.263743 & 0.0208935 & 3.35695 \\
\hline 0.124988 & 0.0118189 & 0.243467 & 0.106908 & 0.265142 & 0.0172754 & 3.32365 \\
\hline 0.123967 & 0.0127261 & 0.244987 & 0.110067 & 0.272151 & 0.0169821 & 3.34296 \\
\hline 0.12186 & 0.0104646 & 0.246808 & 0.108375 & 0.27929 & 0.0149712 & 3.34193 \\
\hline
\end{tabular}




\begin{tabular}{|c|c|c|c|c|c|c|}
\hline 0.123058 & 0.0108462 & 0.247076 & 0.105152 & 0.291366 & 0.0244187 & 3.30676 \\
\hline 0.12637 & 0.0121867 & 0.252998 & 0.106551 & 0.307845 & 0.022544 & 3.34272 \\
\hline 0.125766 & 0.0112009 & 0.261795 & 0.118868 & 0.329025 & 0.0222564 & 3.35771 \\
\hline 0.128551 & 0.0140629 & 0.269398 & 0.121473 & 0.354547 & 0.028397 & 3.36441 \\
\hline 0.130737 & 0.0112497 & 0.28064 & 0.128357 & 0.379885 & 0.0267315 & 3.40052 \\
\hline 0.133933 & 0.0111301 & 0.296098 & 0.135897 & 0.41207 & 0.0326978 & 3.44747 \\
\hline 0.130692 & 0.0129497 & 0.308611 & 0.134725 & 0.442399 & 0.0334053 & 3.48123 \\
\hline 0.133694 & 0.0118204 & 0.323759 & 0.149728 & 0.475895 & 0.0366609 & 3.56186 \\
\hline 0.137587 & 0.0115689 & 0.337564 & 0.157505 & 0.51244 & 0.0377843 & 3.60963 \\
\hline 0.138529 & 0.0133954 & 0.351092 & 0.167931 & 0.546646 & 0.0410919 & 3.6571 \\
\hline 0.142767 & 0.0145518 & 0.362274 & 0.179165 & 0.586065 & 0.0467371 & 3.75075 \\
\hline 0.14467 & 0.0132928 & 0.38375 & 0.20165 & 0.627239 & 0.0489208 & 3.79071 \\
\hline 0.142328 & 0.0114496 & 0.407054 & 0.225756 & 0.661063 & 0.0498674 & 3.89221 \\
\hline 0.143778 & 0.013685 & 0.426387 & 0.244071 & 0.707026 & 0.041594 & 3.95707 \\
\hline 0.144424 & 0.0127217 & 0.446211 & 0.268689 & 0.751786 & 0.042591 & 4.03181 \\
\hline 0.152002 & 0.0135893 & 0.460228 & 0.285813 & 0.796918 & 0.0475565 & 4.12815 \\
\hline 0.150444 & 0.0141152 & 0.475708 & 0.304434 & 0.829703 & 0.0479264 & 4.19409 \\
\hline 0.152471 & 0.0163489 & 0.494587 & 0.331554 & 0.868429 & 0.0487716 & 4.2507 \\
\hline 0.155092 & 0.017643 & 0.504033 & 0.346603 & 0.89328 & 0.0497712 & 4.28171 \\
\hline 0.159695 & 0.021127 & 0.512556 & 0.356103 & 0.890999 & 0.0503062 & 4.29754 \\
\hline 0.162607 & 0.0204253 & 0.509767 & 0.354734 & 0.88971 & 0.0519601 & 4.26117 \\
\hline 0.163466 & 0.0185693 & 0.497335 & 0.349415 & 0.864481 & 0.0516863 & 4.2419 \\
\hline 0.165249 & 0.0185024 & 0.490542 & 0.352077 & 0.831193 & 0.0492199 & 4.20328 \\
\hline 0.165146 & 0.0179473 & 0.478263 & 0.340979 & 0.782683 & 0.0475892 & 4.09476 \\
\hline 0.162119 & 0.0196238 & 0.461823 & 0.338194 & 0.728379 & 0.0410541 & 3.98443 \\
\hline 0.162968 & 0.0163457 & 0.437558 & 0.318276 & 0.667684 & 0.0294894 & 3.88418 \\
\hline 0.156878 & 0.0146794 & 0.408823 & 0.301095 & 0.602421 & 0.0367164 & 3.7435 \\
\hline 0.15257 & 0.0152804 & 0.389161 & 0.292023 & 0.54394 & 0.0307304 & 3.66942 \\
\hline 0.148613 & 0.0139917 & 0.370002 & 0.285531 & 0.490371 & 0.0291405 & 3.57853 \\
\hline 0.149875 & 0.0147743 & 0.352907 & 0.265869 & 0.44622 & 0.0227159 & 3.44511 \\
\hline 0.150155 & 0.0141225 & 0.343501 & 0.26225 & 0.40526 & 0.019155 & 3.37574 \\
\hline 0.145958 & 0.0110783 & 0.334522 & 0.257518 & 0.37417 & 0.0216121 & 3.3222 \\
\hline 0.142247 & 0.0106191 & 0.327766 & 0.253398 & 0.349518 & 0.0197755 & 3.27709 \\
\hline 0.143534 & 0.0128977 & 0.320727 & 0.249955 & 0.332081 & 0.0165487 & 3.28408 \\
\hline 0.147571 & 0.0145961 & 0.31339 & 0.237044 & 0.321084 & 0.0172092 & 3.26918 \\
\hline 0.145757 & 0.0149061 & 0.312009 & 0.239898 & 0.316791 & 0.0212081 & 3.27252 \\
\hline 0.143223 & 0.0119269 & 0.314326 & 0.231058 & 0.314331 & 0.0206615 & 3.24132 \\
\hline 0.14271 & 0.0109012 & 0.314794 & 0.216943 & 0.312989 & 0.0197039 & 3.31764 \\
\hline 0.148662 & 0.0121301 & 0.317321 & 0.215891 & 0.31506 & 0.0196044 & 3.35709 \\
\hline 0.146175 & 0.0142393 & 0.318855 & 0.215501 & 0.323614 & 0.0197624 & 3.33443 \\
\hline 0.141352 & 0.0123073 & 0.319025 & 0.208902 & 0.328031 & 0.0181358 & 3.42681 \\
\hline 0.145824 & 0.00935231 & 0.317346 & 0.194354 & 0.33339 & 0.0191147 & 3.44265 \\
\hline 0.145983 & 0.012859 & 0.320002 & 0.190173 & 0.335332 & 0.025755 & 3.4585 \\
\hline 0.148751 & 0.0145125 & 0.321852 & 0.196601 & 0.346897 & 0.0249856 & 3.47428 \\
\hline 0.145033 & 0.0135608 & 0.324388 & 0.192799 & 0.344496 & 0.0219798 & 3.52318 \\
\hline 0.145449 & 0.0118917 & 0.316945 & 0.178781 & 0.345533 & 0.0212615 & 3.55758 \\
\hline 0.147925 & 0.0135539 & 0.317279 & 0.168387 & 0.345567 & 0.023398 & 3.59928 \\
\hline
\end{tabular}




\begin{tabular}{|c|c|c|c|c|c|c|}
\hline 0.149046 & 0.0151232 & 0.311428 & 0.169444 & 0.345719 & 0.0236214 & 3.62263 \\
\hline 0.145052 & 0.0120739 & 0.321141 & 0.168793 & 0.350052 & 0.0236602 & 3.65921 \\
\hline 0.148941 & 0.0173923 & 0.317219 & 0.157605 & 0.349658 & 0.0224828 & 3.66147 \\
\hline 0.148862 & 0.0163482 & 0.317117 & 0.155625 & 0.354829 & 0.0249996 & 3.71966 \\
\hline 0.150637 & 0.0123972 & 0.321917 & 0.153044 & 0.355674 & 0.0279649 & 3.75717 \\
\hline 0.151626 & 0.0160471 & 0.321856 & 0.154492 & 0.355803 & 0.02133 & 3.84095 \\
\hline 0.154435 & 0.0144171 & 0.329338 & 0.153386 & 0.361652 & 0.0249951 & 3.8931 \\
\hline 0.152992 & 0.0148324 & 0.341486 & 0.154579 & 0.379238 & 0.0280349 & 3.976 \\
\hline 0.154502 & 0.01426 & 0.352842 & 0.157412 & 0.398063 & 0.0284992 & 4.13856 \\
\hline 0.15881 & 0.0130098 & 0.380094 & 0.163256 & 0.420075 & 0.0325171 & 4.35679 \\
\hline 0.161974 & 0.015563 & 0.399441 & 0.174356 & 0.445194 & 0.0359809 & 4.51281 \\
\hline 0.167055 & 0.0147217 & 0.437673 & 0.187232 & 0.483076 & 0.0374168 & 4.78107 \\
\hline 0.171432 & 0.0124857 & 0.471153 & 0.213697 & 0.522551 & 0.0352422 & 5.04717 \\
\hline 0.176481 & 0.0151044 & 0.514686 & 0.240125 & 0.572424 & 0.044832 & 5.41671 \\
\hline 0.184784 & 0.0134373 & 0.559292 & 0.269614 & 0.626839 & 0.045095 & 5.72629 \\
\hline 0.192962 & 0.0148093 & 0.608603 & 0.300128 & 0.674825 & 0.0532268 & 6.06146 \\
\hline 0.197919 & 0.0163869 & 0.651811 & 0.330972 & 0.732995 & 0.0532861 & 6.39242 \\
\hline 0.201462 & 0.0122642 & 0.693177 & 0.3459 & 0.780308 & 0.0621752 & 6.75486 \\
\hline 0.209317 & 0.0193193 & 0.723231 & 0.36429 & 0.828316 & 0.0593006 & 6.97735 \\
\hline 0.213598 & 0.0175184 & 0.746434 & 0.374711 & 0.857213 & 0.0678454 & 7.15261 \\
\hline 0.218603 & 0.0203201 & 0.75398 & 0.387098 & 0.869193 & 0.0684601 & 7.25188 \\
\hline 0.228703 & 0.0170431 & 0.754823 & 0.386219 & 0.88014 & 0.0647953 & 7.27763 \\
\hline 0.230293 & 0.0188532 & 0.735609 & 0.369397 & 0.866388 & 0.0574937 & 7.24742 \\
\hline 0.229489 & 0.0173821 & 0.706607 & 0.351078 & 0.837922 & 0.0627825 & 7.09914 \\
\hline 0.233927 & 0.0188456 & 0.680182 & 0.331935 & 0.803116 & 0.0587948 & 6.87438 \\
\hline 0.228963 & 0.0150076 & 0.647225 & 0.298023 & 0.748522 & 0.0515251 & 6.58341 \\
\hline 0.230005 & 0.0216778 & 0.600032 & 0.267951 & 0.706264 & 0.0515851 & 6.24071 \\
\hline 0.232828 & 0.021338 & 0.548747 & 0.24216 & 0.654641 & 0.044061 & 6.00921 \\
\hline 0.229174 & 0.0175924 & 0.525417 & 0.220791 & 0.610766 & 0.03857 & 5.71482 \\
\hline 0.221265 & 0.0160429 & 0.482829 & 0.192299 & 0.562102 & 0.0318643 & 5.39292 \\
\hline 0.219507 & 0.0195085 & 0.449678 & 0.180971 & 0.53023 & 0.036165 & 5.15741 \\
\hline 0.228013 & 0.0179282 & 0.427808 & 0.175341 & 0.492702 & 0.0308751 & 4.9542 \\
\hline 0.222046 & 0.0166245 & 0.408881 & 0.160208 & 0.464742 & 0.031061 & 4.78852 \\
\hline 0.221653 & 0.0144172 & 0.394551 & 0.159007 & 0.441589 & 0.0246756 & 4.65071 \\
\hline 0.220926 & 0.0182145 & 0.378389 & 0.15381 & 0.429736 & 0.0269686 & 4.56206 \\
\hline 0.221841 & 0.0163525 & 0.370679 & 0.153299 & 0.415957 & 0.0247664 & 4.46882 \\
\hline 0.21921 & 0.0186856 & 0.364527 & 0.148449 & 0.41271 & 0.0240632 & 4.44359 \\
\hline 0.220505 & 0.0152808 & 0.35406 & 0.139173 & 0.394001 & 0.0265526 & 4.41204 \\
\hline 0.215325 & 0.0176884 & 0.350381 & 0.137091 & 0.393207 & 0.0219366 & 4.3556 \\
\hline 0.216778 & 0.0143858 & 0.358948 & 0.149811 & 0.39696 & 0.0213097 & 4.34829 \\
\hline 0.214662 & 0.0181113 & 0.353149 & 0.147498 & 0.390084 & 0.0309783 & 4.26756 \\
\hline 0.215885 & 0.0139064 & 0.35065 & 0.149049 & 0.390534 & 0.0227882 & 4.26594 \\
\hline 0.211902 & 0.0200428 & 0.347938 & 0.14198 & 0.393936 & 0.0189791 & 4.27883 \\
\hline 0.214447 & 0.0199218 & 0.353269 & 0.146322 & 0.39138 & 0.025652 & 4.273 \\
\hline 0.212056 & 0.0163734 & 0.354931 & 0.144917 & 0.391771 & 0.0231682 & 4.26684 \\
\hline 0.206556 & 0.0202983 & 0.357318 & 0.147613 & 0.391167 & 0.0206284 & 4.26931 \\
\hline 0.207812 & 0.0174127 & 0.353341 & 0.141449 & 0.390935 & 0.0279325 & 4.26516 \\
\hline
\end{tabular}




\begin{tabular}{|c|c|c|c|c|c|c|}
\hline 0.216472 & 0.0152578 & 0.359126 & 0.145813 & 0.393426 & 0.0226633 & 4.29264 \\
\hline 0.211488 & 0.0161351 & 0.365582 & 0.155306 & 0.399975 & 0.0257843 & 4.35138 \\
\hline 0.213214 & 0.0156818 & 0.374822 & 0.150157 & 0.403243 & 0.0234961 & 4.37902 \\
\hline 0.211663 & 0.0179413 & 0.381162 & 0.160368 & 0.408233 & 0.0278467 & 4.42605 \\
\hline 0.214777 & 0.0150893 & 0.392274 & 0.156559 & 0.418365 & 0.0266725 & 4.52613 \\
\hline 0.221949 & 0.0150355 & 0.400222 & 0.160519 & 0.432384 & 0.0263109 & 4.60651 \\
\hline 0.22571 & 0.0182687 & 0.4137 & 0.155322 & 0.447941 & 0.0285337 & 4.81191 \\
\hline 0.224718 & 0.0205449 & 0.44535 & 0.186208 & 0.465081 & 0.0235906 & 4.98527 \\
\hline 0.227831 & 0.0202068 & 0.459381 & 0.178791 & 0.49756 & 0.0340678 & 5.26262 \\
\hline 0.239124 & 0.017129 & 0.499573 & 0.207009 & 0.527918 & 0.0307861 & 5.65283 \\
\hline 0.238537 & 0.0217245 & 0.548352 & 0.217352 & 0.572157 & 0.0383831 & 6.01328 \\
\hline 0.251113 & 0.0176954 & 0.595772 & 0.243493 & 0.618863 & 0.0509835 & 6.61848 \\
\hline 0.256171 & 0.0214945 & 0.654659 & 0.270917 & 0.682692 & 0.0615216 & 7.21887 \\
\hline 0.271015 & 0.0184257 & 0.7282 & 0.302188 & 0.75715 & 0.0636241 & 7.97149 \\
\hline 0.275798 & 0.0222717 & 0.814155 & 0.337937 & 0.835002 & 0.0752566 & 8.76868 \\
\hline 0.295402 & 0.0223111 & 0.902858 & 0.391005 & 0.923046 & 0.0914935 & 9.67787 \\
\hline 0.315952 & 0.0268241 & 0.994102 & 0.420639 & 1.01304 & 0.0936975 & 10.6656 \\
\hline 0.327325 & 0.0238547 & 1.07445 & 0.459839 & 1.11107 & 0.114447 & 11.591 \\
\hline 0.342119 & 0.0193821 & 1.15046 & 0.486367 & 1.18647 & 0.126837 & 12.4324 \\
\hline 0.369209 & 0.028592 & 1.21077 & 0.524454 & 1.26704 & 0.139877 & 13.2361 \\
\hline 0.373247 & 0.0239276 & 1.28295 & 0.558781 & 1.33183 & 0.148101 & 13.8814 \\
\hline 0.392341 & 0.0215759 & 1.30148 & 0.558322 & 1.36922 & 0.151423 & 14.3497 \\
\hline 0.410861 & 0.0253978 & 1.31823 & 0.56966 & 1.37763 & 0.155605 & 14.611 \\
\hline 0.42209 & 0.0323473 & 1.28378 & 0.539327 & 1.37145 & 0.150202 & 14.4928 \\
\hline 0.438091 & 0.0238554 & 1.27153 & 0.539763 & 1.34302 & 0.147381 & 14.2743 \\
\hline 0.449086 & 0.0348606 & 1.22619 & 0.51928 & 1.29643 & 0.134954 & 13.8714 \\
\hline 0.457838 & 0.0342788 & 1.15901 & 0.506089 & 1.22615 & 0.127967 & 13.2905 \\
\hline 0.464444 & 0.0262884 & 1.08741 & 0.468389 & 1.15124 & 0.114821 & 12.746 \\
\hline 0.473033 & 0.0295967 & 1.01224 & 0.43391 & 1.05672 & 0.097336 & 11.9609 \\
\hline 0.469797 & 0.026727 & 0.94856 & 0.407582 & 0.98826 & 0.0975887 & 11.2457 \\
\hline 0.464054 & 0.0281947 & 0.875779 & 0.365666 & 0.90813 & 0.0678399 & 10.5519 \\
\hline 0.46515 & 0.0270696 & 0.819963 & 0.357007 & 0.835077 & 0.058176 & 9.91715 \\
\hline 0.4688 & 0.02286 & 0.764113 & 0.311116 & 0.767797 & 0.0587927 & 9.50348 \\
\hline 0.456843 & 0.0251147 & 0.734694 & 0.320834 & 0.719934 & 0.0510806 & 9.09349 \\
\hline 0.442797 & 0.0195384 & 0.686322 & 0.289804 & 0.681436 & 0.0511618 & 8.81822 \\
\hline 0.422219 & 0.0277837 & 0.668567 & 0.286091 & 0.647365 & 0.0410718 & 8.61173 \\
\hline 0.416284 & 0.0312156 & 0.648767 & 0.286943 & 0.619381 & 0.0313908 & 8.67573 \\
\hline 0.397437 & 0.0305841 & 0.640278 & 0.280754 & 0.607864 & 0.0389783 & 8.7262 \\
\hline 0.385937 & 0.0216929 & 0.642906 & 0.290999 & 0.601284 & 0.031957 & 8.85314 \\
\hline 0.356066 & 0.0284117 & 0.636522 & 0.280235 & 0.59892 & 0.0432301 & 8.95609 \\
\hline 0.345045 & 0.0246834 & 0.646084 & 0.294025 & 0.598874 & 0.0381895 & 9.33001 \\
\hline 0.326019 & 0.0180854 & 0.65544 & 0.309892 & 0.617907 & 0.0446498 & 9.65088 \\
\hline 0.31662 & 0.0246355 & 0.671291 & 0.31542 & 0.621808 & 0.0392404 & 9.99678 \\
\hline 0.299395 & 0.0223628 & 0.690016 & 0.329579 & 0.629226 & 0.0400584 & 10.4347 \\
\hline 0.289159 & 0.0217964 & 0.708301 & 0.33833 & 0.656287 & 0.0424043 & 10.9676 \\
\hline 0.283025 & 0.0236775 & 0.732611 & 0.354816 & 0.674192 & 0.0461824 & 11.5251 \\
\hline 0.275868 & 0.019359 & 0.767835 & 0.379215 & 0.70532 & 0.0522659 & 12.2797 \\
\hline
\end{tabular}




\begin{tabular}{|c|c|c|c|c|c|c|}
\hline 0.272521 & 0.0171116 & 0.807115 & 0.400727 & 0.743744 & 0.0500931 & 13.1265 \\
\hline 0.269378 & 0.0227351 & 0.860383 & 0.436877 & 0.782653 & 0.0550128 & 14.3142 \\
\hline 0.272195 & 0.0167459 & 0.945155 & 0.497787 & 0.845549 & 0.0537513 & 15.9475 \\
\hline 0.274476 & 0.0157399 & 1.064 & 0.577313 & 0.932381 & 0.0685313 & 18.1253 \\
\hline 0.272904 & 0.0190724 & 1.2361 & 0.694522 & 1.0681 & 0.0787304 & 21.594 \\
\hline 0.271792 & 0.0181558 & 1.4809 & 0.837463 & 1.25639 & 0.0882433 & 26.5893 \\
\hline 0.284012 & 0.018211 & 1.88081 & 1.09562 & 1.54329 & 0.113704 & 33.982 \\
\hline 0.291995 & 0.0205171 & 2.45248 & 1.49893 & 1.96464 & 0.129893 & 44.8513 \\
\hline 0.302678 & 0.0243775 & 3.28351 & 2.02708 & 2.58857 & 0.187473 & 60.8162 \\
\hline 0.335683 & 0.0260234 & 4.46787 & 2.81763 & 3.47236 & 0.233557 & 83.7091 \\
\hline 0.355731 & 0.0235856 & 6.08874 & 3.8666 & 4.714 & 0.314095 & 115.53 \\
\hline 0.405823 & 0.0274803 & 8.30852 & 5.33036 & 6.39116 & 0.452 & 159.485 \\
\hline 0.451694 & 0.0346639 & 11.198 & 7.16914 & 8.67245 & 0.604677 & 217.491 \\
\hline 0.536835 & 0.0434415 & 14.9134 & 9.63513 & 11.619 & 0.818885 & 291.832 \\
\hline 0.629114 & 0.0379553 & 19.5287 & 12.6294 & 15.2631 & 1.04547 & 386.63 \\
\hline 0.765389 & 0.0469514 & 25.0942 & 16.229 & 19.7363 & 1.39397 & 500.823 \\
\hline 0.925909 & 0.07662 & 31.5532 & 20.4737 & 24.9782 & 1.74931 & 635.35 \\
\hline 1.1057 & 0.0844052 & 38.8303 & 25.3203 & 31.0223 & 2.2071 & 788.032 \\
\hline 1.33524 & 0.09238 & 46.5039 & 30.251 & 37.4553 & 2.62381 & 954.263 \\
\hline 1.57232 & 0.127661 & 54.4471 & 35.4681 & 44.3957 & 3.15239 & 1128.13 \\
\hline 1.85819 & 0.132348 & 62.3304 & 40.5179 & 51.2113 & 3.59605 & 1304.18 \\
\hline 2.15061 & 0.165043 & 69.446 & 45.2448 & 57.6616 & 4.04778 & 1467.9 \\
\hline 2.42904 & 0.183177 & 75.5149 & 48.9825 & 63.4149 & 4.5032 & 1613.48 \\
\hline 2.68121 & 0.201296 & 80.0848 & 52.115 & 67.8632 & 4.80237 & 1726.77 \\
\hline 2.9117 & 0.225226 & 82.8787 & 54.0522 & 71.0353 & 5.14113 & 1806.12 \\
\hline 3.08435 & 0.213983 & 83.509 & 54.4057 & 72.3199 & 5.08934 & 1839.23 \\
\hline 3.19734 & 0.231403 & 82.1536 & 53.4507 & 71.8206 & 5.13461 & 1827.86 \\
\hline 3.26731 & 0.233598 & 78.7382 & 51.316 & 69.6078 & 4.89791 & 1771.84 \\
\hline 3.24591 & 0.232052 & 73.6831 & 47.9922 & 65.7734 & 4.65259 & 1673.66 \\
\hline 3.14064 & 0.26008 & 67.0878 & 43.7705 & 60.6819 & 4.2615 & 1543.41 \\
\hline 2.99782 & 0.196674 & 59.7524 & 38.8979 & 54.4858 & 3.82542 & 1387.29 \\
\hline 2.74969 & 0.201956 & 51.78 & 33.5889 & 47.754 & 3.38237 & 1215.92 \\
\hline 2.5149 & 0.190806 & 43.8766 & 28.4466 & 40.8022 & 2.97603 & 1039.97 \\
\hline 2.23372 & 0.160146 & 36.1749 & 23.5578 & 34.0506 & 2.37639 & 868.368 \\
\hline 1.94913 & 0.146123 & 29.0978 & 18.8918 & 27.7359 & 1.99148 & 706.721 \\
\hline 1.68283 & 0.126273 & 22.9526 & 14.8644 & 22.1033 & 1.61426 & 562.352 \\
\hline 1.44562 & 0.106121 & 17.6548 & 11.4375 & 17.1603 & 1.23426 & 436.835 \\
\hline 1.20703 & 0.0797906 & 13.3174 & 8.59378 & 13.0718 & 0.907897 & 331.916 \\
\hline 1.02663 & 0.0812693 & 9.85315 & 6.30104 & 9.74091 & 0.6897 & 247.052 \\
\hline 0.846431 & 0.04934 & 7.15185 & 4.54584 & 7.13622 & 0.501568 & 179.822 \\
\hline 0.729146 & 0.0418396 & 5.14876 & 3.19753 & 5.18712 & 0.353035 & 128.947 \\
\hline 0.631396 & 0.0375157 & 3.67944 & 2.24968 & 3.69742 & 0.255662 & 91.16 \\
\hline 0.560745 & 0.0394308 & 2.63366 & 1.59079 & 2.64536 & 0.192723 & 63.8324 \\
\hline 0.498128 & 0.0401024 & 1.90874 & 1.09133 & 1.9161 & 0.142505 & 44.6391 \\
\hline 0.454944 & 0.0310433 & 1.41439 & 0.760179 & 1.41507 & 0.0945908 & 31.4279 \\
\hline 0.42886 & 0.0288671 & 1.10902 & 0.581204 & 1.09306 & 0.0697589 & 22.5637 \\
\hline 0.409371 & 0.0235671 & 0.899481 & 0.435672 & 0.865589 & 0.0506758 & 16.8046 \\
\hline
\end{tabular}




\begin{tabular}{|c|c|c|c|c|c|c|}
\hline 0.402467 & 0.0335621 & 0.762014 & 0.345078 & 0.733573 & 0.0459525 & 13.0441 \\
\hline 0.394832 & 0.0266795 & 0.690678 & 0.297054 & 0.646375 & 0.0438198 & 10.6655 \\
\hline 0.382728 & 0.0240049 & 0.64285 & 0.271698 & 0.593725 & 0.0352859 & 9.11927 \\
\hline 0.386637 & 0.0252377 & 0.603266 & 0.245015 & 0.559156 & 0.033398 & 8.21384 \\
\hline 0.391509 & 0.0312597 & 0.599583 & 0.238052 & 0.541745 & 0.0304902 & 7.5785 \\
\hline 0.391369 & 0.0255311 & 0.598366 & 0.233071 & 0.538124 & 0.0350722 & 7.20473 \\
\hline 0.390883 & 0.0278155 & 0.592209 & 0.23034 & 0.533723 & 0.030353 & 6.91542 \\
\hline 0.392718 & 0.0245059 & 0.589347 & 0.226832 & 0.525171 & 0.0291192 & 6.79009 \\
\hline 0.400349 & 0.0263058 & 0.59533 & 0.233519 & 0.53573 & 0.0360621 & 6.69508 \\
\hline 0.402413 & 0.0265483 & 0.596114 & 0.234921 & 0.538841 & 0.0347248 & 6.72511 \\
\hline 0.4025 & 0.0308385 & 0.60782 & 0.231734 & 0.545387 & 0.0258346 & 6.74992 \\
\hline 0.398191 & 0.0234157 & 0.613884 & 0.229863 & 0.547117 & 0.0336726 & 6.74647 \\
\hline 0.406334 & 0.0325259 & 0.619817 & 0.236401 & 0.554814 & 0.0319455 & 6.84831 \\
\hline 0.406229 & 0.0267422 & 0.623023 & 0.235929 & 0.557007 & 0.0315981 & 6.93522 \\
\hline 0.417607 & 0.0333537 & 0.647718 & 0.254377 & 0.577087 & 0.031506 & 7.09056 \\
\hline 0.399099 & 0.0274648 & 0.662121 & 0.258567 & 0.585407 & 0.0300946 & 7.25994 \\
\hline 0.419612 & 0.0267127 & 0.673271 & 0.258747 & 0.6 & 0.0432121 & 7.53899 \\
\hline 0.410395 & 0.0211471 & 0.703584 & 0.290286 & 0.611641 & 0.0293485 & 7.82217 \\
\hline 0.418447 & 0.0267329 & 0.708888 & 0.281203 & 0.643842 & 0.0385499 & 8.19344 \\
\hline 0.412196 & 0.0303997 & 0.739595 & 0.301332 & 0.660965 & 0.0344486 & 8.64727 \\
\hline 0.41821 & 0.0250826 & 0.759335 & 0.308835 & 0.691799 & 0.0388221 & 9.07916 \\
\hline 0.418667 & 0.0217823 & 0.807327 & 0.340427 & 0.7235 & 0.0313108 & 9.77474 \\
\hline 0.427949 & 0.0257889 & 0.863309 & 0.378356 & 0.771054 & 0.0427931 & 10.6034 \\
\hline 0.434326 & 0.0250777 & 0.935946 & 0.415973 & 0.828402 & 0.0450555 & 11.7747 \\
\hline 0.426772 & 0.0268691 & 1.0259 & 0.477447 & 0.904183 & 0.061527 & 13.2654 \\
\hline 0.429214 & 0.0234067 & 1.15593 & 0.561399 & 0.997876 & 0.059365 & 15.208 \\
\hline 0.439701 & 0.0380626 & 1.30126 & 0.644763 & 1.09916 & 0.0665797 & 17.9848 \\
\hline 0.442922 & 0.0244442 & 1.49538 & 0.780185 & 1.27449 & 0.0666354 & 21.5895 \\
\hline 0.44718 & 0.0247349 & 1.78043 & 0.971858 & 1.4719 & 0.0896469 & 26.2593 \\
\hline 0.446651 & 0.0297062 & 2.12293 & 1.19102 & 1.75276 & 0.109787 & 32.6515 \\
\hline 0.459183 & 0.031136 & 2.57125 & 1.50111 & 2.09607 & 0.126755 & 40.6804 \\
\hline 0.467402 & 0.0211286 & 3.14158 & 1.86912 & 2.52907 & 0.157004 & 50.8736 \\
\hline 0.485948 & 0.0247192 & 3.81097 & 2.29614 & 3.05818 & 0.202038 & 63.4304 \\
\hline 0.511451 & 0.0284367 & 4.62439 & 2.8827 & 3.67139 & 0.236072 & 78.7432 \\
\hline 0.524214 & 0.0358143 & 5.55253 & 3.4974 & 4.44856 & 0.268326 & 96.3723 \\
\hline 0.558387 & 0.0329121 & 6.59737 & 4.22566 & 5.24779 & 0.342678 & 116.146 \\
\hline 0.574362 & 0.0316822 & 7.68635 & 4.96902 & 6.1887 & 0.396635 & 138.592 \\
\hline 0.602703 & 0.0379904 & 8.84942 & 5.67848 & 7.15376 & 0.471163 & 161.535 \\
\hline 0.642011 & 0.0426871 & 10.0693 & 6.56095 & 8.11683 & 0.565127 & 184.928 \\
\hline 0.692209 & 0.0379365 & 11.119 & 7.31325 & 9.07257 & 0.623092 & 207.521 \\
\hline 0.723016 & 0.044246 & 12.077 & 7.87311 & 9.93753 & 0.65782 & 227.669 \\
\hline 0.77547 & 0.0421708 & 12.8242 & 8.42015 & 10.6781 & 0.719834 & 244.904 \\
\hline 0.801192 & 0.0512674 & 13.3564 & 8.82747 & 11.1687 & 0.759952 & 257.307 \\
\hline 0.829224 & 0.0459896 & 13.5631 & 8.97929 & 11.5207 & 0.770559 & 264.628 \\
\hline 0.859626 & 0.0533253 & 13.5184 & 8.88322 & 11.5797 & 0.799311 & 266.199 \\
\hline 0.851537 & 0.0530327 & 13.165 & 8.68496 & 11.3738 & 0.734388 & 261.42 \\
\hline 0.867506 & 0.0575767 & 12.5338 & 8.18672 & 10.9215 & 0.731507 & 251.189 \\
\hline
\end{tabular}




\begin{tabular}{|c|c|c|c|c|c|c|}
\hline 0.862619 & 0.0542283 & 11.6903 & 7.70131 & 10.3063 & 0.699595 & 236.014 \\
\hline 0.834795 & 0.0449096 & 10.6708 & 7.03879 & 9.4857 & 0.629963 & 216.574 \\
\hline 0.814521 & 0.0525104 & 9.50327 & 6.22659 & 8.5342 & 0.546712 & 194.366 \\
\hline 0.770355 & 0.0444869 & 8.32558 & 5.38793 & 7.52879 & 0.477244 & 170.507 \\
\hline 0.752082 & 0.0476568 & 7.10035 & 4.59966 & 6.51319 & 0.409042 & 146.092 \\
\hline 0.693114 & 0.035964 & 5.97529 & 3.83106 & 5.52892 & 0.358424 & 122.518 \\
\hline 0.667821 & 0.0468528 & 4.91113 & 3.09264 & 4.58497 & 0.272311 & 100.704 \\
\hline 0.616414 & 0.0259887 & 3.9954 & 2.52666 & 3.75286 & 0.245235 & 80.7752 \\
\hline 0.583336 & 0.0351381 & 3.18145 & 1.93281 & 3.00982 & 0.202301 & 63.5225 \\
\hline 0.544108 & 0.0259237 & 2.52915 & 1.48653 & 2.3963 & 0.152914 & 48.8703 \\
\hline 0.513457 & 0.0320141 & 1.99341 & 1.12943 & 1.89644 & 0.12256 & 37.103 \\
\hline 0.496591 & 0.0348952 & 1.55696 & 0.85982 & 1.48221 & 0.0885858 & 27.4214 \\
\hline 0.469278 & 0.0243022 & 1.24315 & 0.653621 & 1.17546 & 0.0735234 & 20.0897 \\
\hline 0.459781 & 0.0229088 & 1.002 & 0.473882 & 0.931464 & 0.0536377 & 14.4503 \\
\hline 0.447451 & 0.0263791 & 0.827871 & 0.376175 & 0.762029 & 0.0417242 & 10.2713 \\
\hline 0.442388 & 0.0270518 & 0.715281 & 0.307863 & 0.635252 & 0.0400324 & 7.34005 \\
\hline 0.448034 & 0.0286166 & 0.629414 & 0.24693 & 0.545645 & 0.0341183 & 5.28206 \\
\hline 0.444267 & 0.0303522 & 0.568495 & 0.20306 & 0.485222 & 0.025143 & 3.80293 \\
\hline 0.448898 & 0.0246633 & 0.535077 & 0.204513 & 0.443073 & 0.0277336 & 2.81 \\
\hline 0.461794 & 0.0303523 & 0.518577 & 0.18074 & 0.420569 & 0.0203859 & 2.1554 \\
\hline 0.466309 & 0.0255527 & 0.518244 & 0.186381 & 0.404813 & 0.0185172 & 1.70763 \\
\hline 0.48029 & 0.0315092 & 0.515433 & 0.171158 & 0.395194 & 0.0210286 & 1.46974 \\
\hline 0.485308 & 0.0319769 & 0.525314 & 0.182433 & 0.398716 & 0.0223021 & 1.3249 \\
\hline 0.510351 & 0.0366843 & 0.544744 & 0.184241 & 0.397821 & 0.0220474 & 1.24488 \\
\hline 0.536309 & 0.0334227 & 0.557056 & 0.17743 & 0.414337 & 0.0273 & 1.18049 \\
\hline 0.544772 & 0.0306367 & 0.594784 & 0.195431 & 0.422096 & 0.0213309 & 1.19108 \\
\hline 0.563274 & 0.0281968 & 0.625181 & 0.199896 & 0.444381 & 0.0251995 & 1.23638 \\
\hline 0.591913 & 0.0354163 & 0.656972 & 0.207201 & 0.469645 & 0.0274419 & 1.28319 \\
\hline 0.603271 & 0.0338848 & 0.685117 & 0.227717 & 0.493239 & 0.0286956 & 1.37207 \\
\hline 0.612546 & 0.0457993 & 0.743652 & 0.231662 & 0.527581 & 0.0266672 & 1.45147 \\
\hline 0.620041 & 0.034554 & 0.789551 & 0.252361 & 0.564041 & 0.032905 & 1.60655 \\
\hline 0.624411 & 0.0382925 & 0.842989 & 0.26567 & 0.602645 & 0.0351019 & 1.72592 \\
\hline 0.637539 & 0.0355504 & 0.882957 & 0.272524 & 0.633732 & 0.0283386 & 1.88319 \\
\hline 0.630633 & 0.0300967 & 0.936582 & 0.303103 & 0.676968 & 0.0335966 & 1.99967 \\
\hline 0.622165 & 0.0399371 & 0.982816 & 0.300666 & 0.717428 & 0.0410803 & 2.13937 \\
\hline 0.620751 & 0.0396978 & 1.01527 & 0.318238 & 0.743162 & 0.0379929 & 2.27426 \\
\hline 0.612024 & 0.0277623 & 1.04295 & 0.332268 & 0.774849 & 0.0402283 & 2.38963 \\
\hline 0.595935 & 0.0398092 & 1.05324 & 0.325496 & 0.795191 & 0.0419099 & 2.43594 \\
\hline 0.579034 & 0.0376433 & 1.05159 & 0.334027 & 0.799751 & 0.0351225 & 2.49378 \\
\hline 0.564589 & 0.0324687 & 1.0379 & 0.322286 & 0.800086 & 0.0371911 & 2.44812 \\
\hline 0.550919 & 0.0409481 & 1.01903 & 0.312807 & 0.793677 & 0.0439895 & 2.4081 \\
\hline 0.549309 & 0.0304739 & 0.982069 & 0.304112 & 0.779275 & 0.0408944 & 2.30001 \\
\hline 0.527049 & 0.0320083 & 0.940319 & 0.288827 & 0.752212 & 0.040066 & 2.22659 \\
\hline 0.516527 & 0.0246431 & 0.904935 & 0.274978 & 0.720175 & 0.0368815 & 2.09601 \\
\hline 0.506816 & 0.034279 & 0.841925 & 0.242702 & 0.694726 & 0.0473598 & 1.95961 \\
\hline 0.514774 & 0.030142 & 0.793338 & 0.241323 & 0.66264 & 0.0360349 & 1.82303 \\
\hline 0.500613 & 0.0264534 & 0.733395 & 0.211078 & 0.619974 & 0.0305857 & 1.6727 \\
\hline
\end{tabular}




\begin{tabular}{|c|c|c|c|c|c|c|}
\hline 0.494271 & 0.0367363 & 0.682971 & 0.207818 & 0.590568 & 0.0297961 & 1.51928 \\
\hline 0.483743 & 0.0288246 & 0.643047 & 0.177283 & 0.555223 & 0.0285821 & 1.3937 \\
\hline 0.489656 & 0.0326344 & 0.59866 & 0.170153 & 0.52272 & 0.026378 & 1.27106 \\
\hline 0.488864 & 0.0301915 & 0.570832 & 0.155541 & 0.495409 & 0.0299286 & 1.15542 \\
\hline 0.487944 & 0.02942 & 0.53033 & 0.15133 & 0.480254 & 0.0242672 & 1.06154 \\
\hline 0.49832 & 0.0318196 & 0.520843 & 0.153128 & 0.460998 & 0.0236612 & 0.983258 \\
\hline 0.482086 & 0.0341074 & 0.498962 & 0.144355 & 0.447583 & 0.0269995 & 0.928208 \\
\hline 0.488836 & 0.0341462 & 0.486599 & 0.139912 & 0.43303 & 0.0221468 & 0.884471 \\
\hline 0.492362 & 0.0274356 & 0.49061 & 0.147956 & 0.438351 & 0.0233345 & 0.862443 \\
\hline 0.496203 & 0.0244791 & 0.480578 & 0.149696 & 0.427301 & 0.0194773 & 0.819232 \\
\hline 0.512617 & 0.0280552 & 0.479408 & 0.131527 & 0.420731 & 0.0217608 & 0.805138 \\
\hline 0.519124 & 0.0321731 & 0.474126 & 0.136446 & 0.420145 & 0.0252402 & 0.798861 \\
\hline 0.516768 & 0.0332242 & 0.476407 & 0.155449 & 0.416642 & 0.0261866 & 0.777904 \\
\hline 0.527857 & 0.0257864 & 0.483248 & 0.144179 & 0.419566 & 0.0257975 & 0.780864 \\
\hline 0.52979 & 0.0347802 & 0.480016 & 0.15145 & 0.417012 & 0.0263763 & 0.746201 \\
\hline 0.536778 & 0.0296195 & 0.474658 & 0.148185 & 0.41686 & 0.0210794 & 0.786715 \\
\hline 0.540895 & 0.0306319 & 0.480195 & 0.158852 & 0.418636 & 0.0237086 & 0.775397 \\
\hline 0.531995 & 0.0315901 & 0.475304 & 0.158039 & 0.416594 & 0.0218048 & 0.774744 \\
\hline 0.535237 & 0.0252574 & 0.47746 & 0.152936 & 0.42111 & 0.0220344 & 0.752733 \\
\hline 0.536276 & 0.0237866 & 0.473522 & 0.163047 & 0.42287 & 0.0236016 & 0.769149 \\
\hline 0.531516 & 0.0303314 & 0.472632 & 0.1592 & 0.41584 & 0.0254319 & 0.770274 \\
\hline 0.526396 & 0.0300142 & 0.48538 & 0.161049 & 0.421668 & 0.027377 & 0.778586 \\
\hline 0.52519 & 0.031379 & 0.471373 & 0.157105 & 0.421964 & 0.0213102 & 0.784047 \\
\hline 0.514254 & 0.0379234 & 0.477966 & 0.169525 & 0.425419 & 0.0274753 & 0.754121 \\
\hline 0.519105 & 0.0299345 & 0.486465 & 0.164267 & 0.432035 & 0.0231981 & 0.765926 \\
\hline 0.508814 & 0.0327403 & 0.478165 & 0.158756 & 0.434984 & 0.0281147 & 0.810545 \\
\hline 0.508996 & 0.0302509 & 0.487297 & 0.175054 & 0.439155 & 0.0305352 & 0.803673 \\
\hline 0.511892 & 0.0273709 & 0.484344 & 0.160604 & 0.44598 & 0.0222155 & 0.791888 \\
\hline 0.506185 & 0.0282694 & 0.489376 & 0.168722 & 0.447495 & 0.0272548 & 0.837824 \\
\hline 0.497407 & 0.0327149 & 0.49432 & 0.169731 & 0.464667 & 0.0228913 & 0.833367 \\
\hline 0.500387 & 0.035269 & 0.518027 & 0.183827 & 0.478272 & 0.0294152 & 0.850556 \\
\hline 0.499985 & 0.0297676 & 0.522193 & 0.195703 & 0.489796 & 0.0291557 & 0.865901 \\
\hline 0.500343 & 0.0290652 & 0.536164 & 0.188829 & 0.518621 & 0.0349549 & 0.875445 \\
\hline 0.498261 & 0.031389 & 0.558857 & 0.207107 & 0.547769 & 0.0316015 & 0.925844 \\
\hline 0.497765 & 0.0350265 & 0.585625 & 0.23621 & 0.582763 & 0.0411671 & 0.921419 \\
\hline 0.502759 & 0.0253394 & 0.629368 & 0.273681 & 0.619892 & 0.0510425 & 0.961756 \\
\hline 0.508809 & 0.0309231 & 0.673551 & 0.320663 & 0.69493 & 0.062944 & 1.01267 \\
\hline 0.507504 & 0.03 & 0.719057 & 0.390201 & 0.755942 & 0.0659191 & 1.05146 \\
\hline 0.517793 & 0.0317664 & 0.787837 & 0.456206 & 0.838393 & 0.0899002 & 1.11835 \\
\hline 0.498252 & 0.031584 & 0.843685 & 0.562181 & 0.937492 & 0.112477 & 1.13847 \\
\hline 0.506567 & 0.0314694 & 0.93391 & 0.676179 & 1.0405 & 0.130986 & 1.24425 \\
\hline 0.508302 & 0.0300906 & 0.98891 & 0.754232 & 1.15632 & 0.158102 & 1.32558 \\
\hline 0.515181 & 0.0313064 & 1.07429 & 0.876782 & 1.2703 & 0.189679 & 1.40113 \\
\hline 0.529988 & 0.032009 & 1.14437 & 0.995855 & 1.36722 & 0.195913 & 1.48866 \\
\hline 0.526081 & 0.0344199 & 1.20988 & 1.07572 & 1.48174 & 0.224726 & 1.59662 \\
\hline 0.52763 & 0.0307745 & 1.27163 & 1.1586 & 1.5697 & 0.249619 & 1.6247 \\
\hline 0.536364 & 0.0339432 & 1.29489 & 1.18967 & 1.63898 & 0.272789 & 1.70562 \\
\hline
\end{tabular}




\begin{tabular}{|c|c|c|c|c|c|c|}
\hline 0.531632 & 0.029309 & 1.3385 & 1.27083 & 1.70586 & 0.275156 & 1.74369 \\
\hline 0.535063 & 0.0317246 & 1.35964 & 1.27335 & 1.73531 & 0.283197 & 1.83523 \\
\hline 0.536896 & 0.0292475 & 1.35041 & 1.28107 & 1.73821 & 0.277698 & 1.83934 \\
\hline 0.552434 & 0.0300414 & 1.33018 & 1.24716 & 1.70573 & 0.279185 & 1.88117 \\
\hline 0.537546 & 0.0317409 & 1.28768 & 1.17499 & 1.65989 & 0.25917 & 1.86901 \\
\hline 0.545144 & 0.0341375 & 1.23 & 1.08049 & 1.59759 & 0.233377 & 1.86012 \\
\hline 0.542429 & 0.033526 & 1.16908 & 0.96387 & 1.48986 & 0.226893 & 1.85729 \\
\hline 0.54085 & 0.0383404 & 1.10277 & 0.853312 & 1.41002 & 0.207932 & 1.82167 \\
\hline 0.536669 & 0.0328047 & 1.02398 & 0.778528 & 1.30681 & 0.168492 & 1.73524 \\
\hline 0.551657 & 0.0363924 & 0.948656 & 0.646707 & 1.20099 & 0.157349 & 1.7022 \\
\hline 0.54173 & 0.0286275 & 0.895363 & 0.548919 & 1.08705 & 0.131958 & 1.65866 \\
\hline 0.538144 & 0.0308595 & 0.83463 & 0.457803 & 0.994922 & 0.101924 & 1.61889 \\
\hline 0.541285 & 0.0329498 & 0.776899 & 0.387428 & 0.901314 & 0.0853623 & 1.55272 \\
\hline 0.532176 & 0.0326602 & 0.724853 & 0.309146 & 0.831519 & 0.0747444 & 1.47917 \\
\hline 0.540369 & 0.0314973 & 0.696221 & 0.261873 & 0.757822 & 0.0561471 & 1.44727 \\
\hline 0.536917 & 0.0341104 & 0.671819 & 0.223943 & 0.716913 & 0.0523917 & 1.37065 \\
\hline 0.534042 & 0.0275518 & 0.647211 & 0.185599 & 0.665203 & 0.0408354 & 1.34839 \\
\hline 0.535944 & 0.0321645 & 0.636295 & 0.179065 & 0.643273 & 0.0361491 & 1.32006 \\
\hline 0.537269 & 0.0380972 & 0.622488 & 0.158603 & 0.617752 & 0.0344724 & 1.2898 \\
\hline 0.541942 & 0.0354047 & 0.622061 & 0.153083 & 0.605531 & 0.0326515 & 1.25515 \\
\hline 0.544956 & 0.0357864 & 0.615849 & 0.159019 & 0.59703 & 0.0367619 & 1.25327 \\
\hline 0.553937 & 0.0246584 & 0.611876 & 0.159146 & 0.590977 & 0.0260758 & 1.19822 \\
\hline 0.545372 & 0.0356501 & 0.619873 & 0.148018 & 0.592555 & 0.035672 & 1.18411 \\
\hline 0.544355 & 0.0357503 & 0.624776 & 0.147673 & 0.583833 & 0.0331898 & 1.18067 \\
\hline 0.560736 & 0.0349845 & 0.622067 & 0.150236 & 0.590767 & 0.0304155 & 1.15946 \\
\hline 0.549312 & 0.0366083 & 0.618119 & 0.141224 & 0.57714 & 0.03187 & 1.14244 \\
\hline 0.558819 & 0.0351244 & 0.611745 & 0.14296 & 0.575241 & 0.0331706 & 1.0795 \\
\hline 0.574146 & 0.0316236 & 0.614227 & 0.14328 & 0.581716 & 0.0328236 & 1.08449 \\
\hline 0.580255 & 0.0338467 & 0.612009 & 0.136268 & 0.571636 & 0.0325634 & 1.08347 \\
\hline 0.572242 & 0.0336161 & 0.612689 & 0.141323 & 0.569969 & 0.0294356 & 1.03525 \\
\hline 0.582454 & 0.0395294 & 0.607658 & 0.145244 & 0.567852 & 0.0279797 & 1.01083 \\
\hline 0.578188 & 0.0377369 & 0.612127 & 0.137795 & 0.575147 & 0.029943 & 0.99381 \\
\hline 0.5952 & 0.0361818 & 0.603396 & 0.140462 & 0.565799 & 0.0316103 & 0.976633 \\
\hline 0.601039 & 0.0394392 & 0.608224 & 0.144292 & 0.557761 & 0.0287538 & 0.962553 \\
\hline 0.594709 & 0.0404258 & 0.597816 & 0.138688 & 0.553078 & 0.0259733 & 0.92181 \\
\hline 0.609546 & 0.0347107 & 0.595259 & 0.144106 & 0.551491 & 0.0246614 & 0.902105 \\
\hline 0.613087 & 0.0374328 & 0.60153 & 0.133839 & 0.557257 & 0.0298573 & 0.883382 \\
\hline 0.615333 & 0.0324741 & 0.601441 & 0.144678 & 0.560626 & 0.0262672 & 0.899177 \\
\hline 0.620346 & 0.0405127 & 0.611387 & 0.153494 & 0.555628 & 0.0243012 & 0.882492 \\
\hline 0.622352 & 0.0408533 & 0.620946 & 0.162644 & 0.560983 & 0.0252315 & 0.887771 \\
\hline 0.628185 & 0.0437528 & 0.627961 & 0.172275 & 0.554395 & 0.0282828 & 0.867553 \\
\hline 0.632571 & 0.0307099 & 0.63401 & 0.168599 & 0.563942 & 0.0249909 & 0.863166 \\
\hline 0.645231 & 0.0401332 & 0.649326 & 0.176988 & 0.563831 & 0.022369 & 0.843882 \\
\hline 0.645953 & 0.0386255 & 0.665706 & 0.19208 & 0.562176 & 0.0303506 & 0.84708 \\
\hline 0.661167 & 0.0287804 & 0.693478 & 0.203198 & 0.578075 & 0.0305456 & 0.84762 \\
\hline 0.663739 & 0.0340969 & 0.714067 & 0.2277 & 0.574686 & 0.0297061 & 0.854713 \\
\hline 0.658416 & 0.0341712 & 0.74331 & 0.244872 & 0.59032 & 0.0277566 & 0.859415 \\
\hline
\end{tabular}




\begin{tabular}{|c|c|c|c|c|c|c|}
\hline 0.670306 & 0.0361895 & 0.782631 & 0.271665 & 0.595018 & 0.0268647 & 0.874807 \\
\hline 0.674703 & 0.0536382 & 0.833295 & 0.345211 & 0.607334 & 0.0246084 & 0.873661 \\
\hline 0.70241 & 0.0475 & 0.886086 & 0.370503 & 0.622549 & 0.0279782 & 0.855433 \\
\hline 0.709017 & 0.0359953 & 0.950302 & 0.448881 & 0.637742 & 0.0395489 & 0.866277 \\
\hline 0.727361 & 0.0410253 & 1.01579 & 0.519454 & 0.658244 & 0.0252317 & 0.87378 \\
\hline 0.737523 & 0.04234 & 1.08991 & 0.626478 & 0.68022 & 0.029073 & 0.868806 \\
\hline 0.757445 & 0.0409128 & 1.15956 & 0.70768 & 0.690951 & 0.0309533 & 0.878285 \\
\hline 0.78509 & 0.0448646 & 1.23475 & 0.762895 & 0.709761 & 0.0315073 & 0.905859 \\
\hline 0.791242 & 0.0440612 & 1.31254 & 0.890275 & 0.73496 & 0.0322868 & 0.909234 \\
\hline 0.823496 & 0.0372528 & 1.35839 & 0.910934 & 0.761322 & 0.0385169 & 0.908904 \\
\hline 0.838171 & 0.0347606 & 1.42465 & 0.984 & 0.772592 & 0.0419272 & 0.904914 \\
\hline 0.873773 & 0.0504667 & 1.45991 & 1.05471 & 0.782976 & 0.0385873 & 0.904824 \\
\hline 0.898378 & 0.0516233 & 1.48819 & 1.06608 & 0.802038 & 0.0374528 & 0.92449 \\
\hline 0.9108 & 0.0362479 & 1.51969 & 1.10649 & 0.801071 & 0.0399719 & 0.922648 \\
\hline 0.922713 & 0.0385786 & 1.49514 & 1.10003 & 0.803609 & 0.0399919 & 0.945498 \\
\hline 0.931869 & 0.0478539 & 1.47521 & 1.04079 & 0.803734 & 0.0356141 & 0.952078 \\
\hline 0.940912 & 0.0421535 & 1.44248 & 1.01518 & 0.793625 & 0.0404826 & 0.954248 \\
\hline 0.935832 & 0.0477847 & 1.38278 & 0.936056 & 0.788108 & 0.0312041 & 0.93508 \\
\hline 0.943566 & 0.0378 & 1.32366 & 0.870647 & 0.781233 & 0.0341036 & 0.95352 \\
\hline 0.913593 & 0.0441624 & 1.26123 & 0.770662 & 0.762721 & 0.0318899 & 0.94314 \\
\hline 0.909733 & 0.0398984 & 1.19771 & 0.680871 & 0.732957 & 0.0309219 & 0.943078 \\
\hline 0.8905 & 0.0394057 & 1.13699 & 0.619275 & 0.72995 & 0.0335263 & 0.931594 \\
\hline 0.891554 & 0.046985 & 1.07128 & 0.536897 & 0.720252 & 0.0347131 & 0.933707 \\
\hline 0.862648 & 0.0398647 & 1.0041 & 0.455542 & 0.701432 & 0.0303149 & 0.947441 \\
\hline 0.8515 & 0.0427416 & 0.946665 & 0.377715 & 0.694321 & 0.0313268 & 0.939087 \\
\hline 0.829702 & 0.0408523 & 0.906628 & 0.333928 & 0.677977 & 0.024378 & 0.939103 \\
\hline 0.815555 & 0.0521006 & 0.879364 & 0.287337 & 0.678581 & 0.0314258 & 0.946684 \\
\hline 0.808203 & 0.0413252 & 0.839181 & 0.258425 & 0.678326 & 0.0311266 & 0.958308 \\
\hline 0.793894 & 0.0433545 & 0.8308 & 0.247787 & 0.679137 & 0.0326687 & 0.949013 \\
\hline 0.776222 & 0.0411668 & 0.798891 & 0.214627 & 0.684063 & 0.0310545 & 0.967529 \\
\hline 0.781374 & 0.0404124 & 0.799636 & 0.215137 & 0.698588 & 0.0346048 & 0.968512 \\
\hline 0.761058 & 0.0446235 & 0.803527 & 0.203627 & 0.701736 & 0.0378198 & 0.988896 \\
\hline 0.778096 & 0.0411386 & 0.799592 & 0.200081 & 0.713358 & 0.0342396 & 0.978078 \\
\hline 0.781627 & 0.0482708 & 0.798061 & 0.207915 & 0.725642 & 0.0399213 & 0.98545 \\
\hline 0.770627 & 0.0375871 & 0.806755 & 0.209662 & 0.742234 & 0.0389345 & 0.979516 \\
\hline 0.765513 & 0.042357 & 0.805382 & 0.207811 & 0.75511 & 0.043488 & 0.982734 \\
\hline 0.762918 & 0.0385652 & 0.821216 & 0.222329 & 0.769763 & 0.0516984 & 1.00535 \\
\hline 0.779373 & 0.0404215 & 0.827639 & 0.21754 & 0.790872 & 0.0557267 & 0.979685 \\
\hline 0.774678 & 0.032388 & 0.835826 & 0.231432 & 0.799021 & 0.0515314 & 1.01182 \\
\hline 0.766351 & 0.0465723 & 0.836603 & 0.240888 & 0.812457 & 0.0576119 & 1.00007 \\
\hline 0.78033 & 0.0351847 & 0.846587 & 0.244699 & 0.808818 & 0.0560944 & 1.02316 \\
\hline 0.780188 & 0.0421092 & 0.848216 & 0.232051 & 0.812812 & 0.0490703 & 1.02922 \\
\hline 0.791442 & 0.0535511 & 0.833401 & 0.224358 & 0.812772 & 0.0494173 & 1.01771 \\
\hline 0.785988 & 0.0328184 & 0.840706 & 0.213107 & 0.810021 & 0.0473145 & 0.991536 \\
\hline 0.78693 & 0.0429107 & 0.827206 & 0.21115 & 0.79448 & 0.0523918 & 1.00753 \\
\hline 0.785935 & 0.0389105 & 0.817371 & 0.199197 & 0.780631 & 0.0554041 & 1.03086 \\
\hline 0.793247 & 0.0388883 & 0.823865 & 0.197476 & 0.773339 & 0.043036 & 1.0057 \\
\hline
\end{tabular}




\begin{tabular}{|c|c|c|c|c|c|c|}
\hline 0.787463 & 0.0359528 & 0.80075 & 0.1795 & 0.764456 & 0.0414102 & 1.01586 \\
\hline 0.784345 & 0.0370571 & 0.78361 & 0.175781 & 0.739311 & 0.0459334 & 0.992877 \\
\hline 0.783924 & 0.047076 & 0.770144 & 0.163657 & 0.729037 & 0.0391567 & 1.0136 \\
\hline 0.790671 & 0.0467898 & 0.767791 & 0.1518 & 0.70632 & 0.0414793 & 1.01422 \\
\hline 0.780129 & 0.0458087 & 0.754837 & 0.147433 & 0.694102 & 0.0345143 & 1.00695 \\
\hline 0.785858 & 0.0442374 & 0.753482 & 0.150691 & 0.683775 & 0.0370791 & 1.01983 \\
\hline 0.786615 & 0.0338482 & 0.737833 & 0.134942 & 0.680469 & 0.0358745 & 1.01596 \\
\hline 0.78751 & 0.0358372 & 0.733353 & 0.138718 & 0.661868 & 0.0277433 & 1.03796 \\
\hline 0.783053 & 0.0321582 & 0.736682 & 0.146371 & 0.655722 & 0.028933 & 1.03816 \\
\hline 0.799136 & 0.0349048 & 0.729038 & 0.130742 & 0.652122 & 0.0270626 & 1.05905 \\
\hline 0.785444 & 0.0363052 & 0.726037 & 0.135859 & 0.65133 & 0.0322531 & 1.06039 \\
\hline 0.787049 & 0.0422475 & 0.727716 & 0.149767 & 0.651284 & 0.033729 & 1.0498 \\
\hline 0.800235 & 0.0369471 & 0.721752 & 0.132694 & 0.638651 & 0.0381901 & 1.07382 \\
\hline 0.781372 & 0.0387392 & 0.718816 & 0.133852 & 0.641949 & 0.0268132 & 1.07844 \\
\hline 0.780209 & 0.0353501 & 0.736635 & 0.139074 & 0.643364 & 0.033422 & 1.08473 \\
\hline 0.775177 & 0.0364375 & 0.721273 & 0.133605 & 0.646594 & 0.0312129 & 1.06746 \\
\hline 0.785519 & 0.0388733 & 0.734822 & 0.135727 & 0.646846 & 0.0248489 & 1.08007 \\
\hline 0.788284 & 0.0400767 & 0.733244 & 0.14196 & 0.657419 & 0.0241766 & 1.08251 \\
\hline 0.790441 & 0.0421395 & 0.729937 & 0.131416 & 0.665597 & 0.0289356 & 1.07995 \\
\hline 0.795409 & 0.0249923 & 0.72924 & 0.143942 & 0.670428 & 0.0380079 & 1.06423 \\
\hline 0.788191 & 0.0381458 & 0.730188 & 0.135284 & 0.662525 & 0.0263524 & 1.11017 \\
\hline 0.780685 & 0.0390506 & 0.735545 & 0.139352 & 0.672463 & 0.0341496 & 1.08045 \\
\hline 0.788784 & 0.0357649 & 0.747538 & 0.142018 & 0.677414 & 0.0294717 & 1.07959 \\
\hline 0.781709 & 0.0338451 & 0.736735 & 0.13873 & 0.684543 & 0.0304196 & 1.07958 \\
\hline 0.783956 & 0.0382216 & 0.737468 & 0.136263 & 0.684644 & 0.0371923 & 1.08184 \\
\hline 0.786749 & 0.0400844 & 0.738011 & 0.141859 & 0.679519 & 0.0356973 & 1.07946 \\
\hline 0.789212 & 0.0456323 & 0.727222 & 0.145068 & 0.686901 & 0.0294899 & 1.05841 \\
\hline 0.791909 & 0.0374232 & 0.734629 & 0.133522 & 0.688302 & 0.0251231 & 1.06094 \\
\hline 0.792617 & 0.0435274 & 0.724483 & 0.134077 & 0.68462 & 0.0293682 & 1.03126 \\
\hline 0.777178 & 0.0378316 & 0.733996 & 0.145872 & 0.693395 & 0.030544 & 1.06455 \\
\hline 0.790279 & 0.0342244 & 0.72602 & 0.139908 & 0.683153 & 0.0294326 & 1.05593 \\
\hline 0.775678 & 0.0384353 & 0.730918 & 0.129962 & 0.682149 & 0.0402482 & 1.02381 \\
\hline 0.779416 & 0.044976 & 0.725434 & 0.137848 & 0.678047 & 0.0304814 & 1.02067 \\
\hline 0.778754 & 0.0357754 & 0.726827 & 0.138415 & 0.685153 & 0.0262581 & 1.01268 \\
\hline 0.78316 & 0.0332347 & 0.720961 & 0.138357 & 0.673093 & 0.0326689 & 1.0084 \\
\hline 0.78055 & 0.0292006 & 0.716738 & 0.136979 & 0.674699 & 0.0296815 & 0.998405 \\
\hline 0.778849 & 0.0452984 & 0.720183 & 0.134036 & 0.681583 & 0.0337241 & 0.98488 \\
\hline 0.787727 & 0.0401288 & 0.714937 & 0.135709 & 0.676599 & 0.0311505 & 0.967675 \\
\hline 0.77356 & 0.0366987 & 0.723766 & 0.137797 & 0.668297 & 0.0331291 & 0.961722 \\
\hline 0.776739 & 0.0406859 & 0.722755 & 0.139067 & 0.664218 & 0.0251892 & 0.937761 \\
\hline 0.777949 & 0.0417168 & 0.72116 & 0.143853 & 0.665139 & 0.0270228 & 0.960729 \\
\hline 0.774702 & 0.0334462 & 0.716435 & 0.131352 & 0.667226 & 0.0275341 & 0.926402 \\
\hline 0.789741 & 0.0340262 & 0.716087 & 0.132914 & 0.66951 & 0.0297782 & 0.928714 \\
\hline 0.785639 & 0.0387791 & 0.718934 & 0.136615 & 0.665467 & 0.0286792 & 0.9465 \\
\hline 0.778517 & 0.0460602 & 0.734607 & 0.134218 & 0.664581 & 0.0306002 & 0.902974 \\
\hline 0.808252 & 0.0466859 & 0.733092 & 0.134761 & 0.667679 & 0.0344616 & 0.922519 \\
\hline 0.800784 & 0.0478725 & 0.744234 & 0.139767 & 0.673655 & 0.0302672 & 0.919786 \\
\hline
\end{tabular}




\begin{tabular}{|c|c|c|c|c|c|c|}
\hline 0.783904 & 0.0436964 & 0.744573 & 0.13331 & 0.668861 & 0.0344112 & 0.920665 \\
\hline 0.794798 & 0.0393305 & 0.762462 & 0.148223 & 0.671129 & 0.0308896 & 0.912276 \\
\hline 0.809032 & 0.0449165 & 0.768666 & 0.142526 & 0.676727 & 0.0357525 & 0.91997 \\
\hline 0.802052 & 0.0517099 & 0.765552 & 0.14826 & 0.687021 & 0.0323587 & 0.908766 \\
\hline 0.811904 & 0.042012 & 0.77912 & 0.153408 & 0.685227 & 0.033981 & 0.900545 \\
\hline 0.812943 & 0.0401844 & 0.786392 & 0.158904 & 0.689741 & 0.0310421 & 0.925554 \\
\hline 0.812696 & 0.0410657 & 0.812968 & 0.176506 & 0.692693 & 0.0365423 & 0.913016 \\
\hline 0.833888 & 0.0494377 & 0.816414 & 0.169249 & 0.69685 & 0.0327009 & 0.900185 \\
\hline 0.836063 & 0.0459244 & 0.829411 & 0.173577 & 0.698374 & 0.0330119 & 0.930157 \\
\hline 0.835401 & 0.0384831 & 0.84544 & 0.192835 & 0.709967 & 0.0331612 & 0.919799 \\
\hline 0.838328 & 0.0404735 & 0.849123 & 0.202233 & 0.714872 & 0.0381504 & 0.94526 \\
\hline 0.847189 & 0.0421177 & 0.860443 & 0.198454 & 0.707797 & 0.0321952 & 0.939599 \\
\hline 0.851799 & 0.0486422 & 0.859141 & 0.198244 & 0.723566 & 0.0316049 & 0.94033 \\
\hline 0.857238 & 0.0393893 & 0.870909 & 0.200732 & 0.712538 & 0.0369196 & 0.940332 \\
\hline 0.852991 & 0.0350676 & 0.859401 & 0.194125 & 0.717841 & 0.0315881 & 0.974042 \\
\hline 0.850693 & 0.047416 & 0.868214 & 0.193462 & 0.723109 & 0.0333529 & 0.971233 \\
\hline 0.865483 & 0.044718 & 0.862064 & 0.193508 & 0.719374 & 0.0363329 & 0.993341 \\
\hline 0.858223 & 0.0389013 & 0.857401 & 0.195509 & 0.719015 & 0.0312001 & 1.01303 \\
\hline 0.8593 & 0.0420967 & 0.85567 & 0.189649 & 0.72424 & 0.0315715 & 1.01052 \\
\hline 0.853605 & 0.0439302 & 0.855202 & 0.181887 & 0.719965 & 0.037232 & 1.02556 \\
\hline 0.867295 & 0.038949 & 0.838969 & 0.179369 & 0.72095 & 0.0298625 & 1.04035 \\
\hline 0.865055 & 0.0396619 & 0.835841 & 0.176211 & 0.720726 & 0.0307792 & 1.04187 \\
\hline 0.861049 & 0.0372425 & 0.82541 & 0.171192 & 0.719136 & 0.0295647 & 1.06414 \\
\hline 0.867804 & 0.0433166 & 0.824565 & 0.164357 & 0.708515 & 0.0247041 & 1.06025 \\
\hline 0.862898 & 0.0494286 & 0.825186 & 0.164061 & 0.718436 & 0.0340018 & 1.09754 \\
\hline 0.854558 & 0.0502689 & 0.814585 & 0.168955 & 0.719436 & 0.0318268 & 1.09491 \\
\hline 0.855722 & 0.0438907 & 0.814766 & 0.16256 & 0.71852 & 0.0319587 & 1.11349 \\
\hline 0.839339 & 0.042 & 0.813967 & 0.17065 & 0.711138 & 0.0280039 & 1.09689 \\
\hline 0.856157 & 0.0472226 & 0.803786 & 0.170208 & 0.71356 & 0.0307398 & 1.09555 \\
\hline 0.855652 & 0.0457681 & 0.799957 & 0.158512 & 0.721985 & 0.0301524 & 1.10131 \\
\hline 0.849431 & 0.0429653 & 0.797979 & 0.167115 & 0.709227 & 0.0328855 & 1.09032 \\
\hline 0.850104 & 0.0419436 & 0.80069 & 0.172258 & 0.711845 & 0.0351863 & 1.08696 \\
\hline 0.842301 & 0.0420128 & 0.809326 & 0.164399 & 0.712872 & 0.0280671 & 1.08142 \\
\hline 0.846107 & 0.0490515 & 0.805045 & 0.177064 & 0.711479 & 0.034403 & 1.0571 \\
\hline 0.84826 & 0.0352851 & 0.804127 & 0.173578 & 0.707507 & 0.0354975 & 1.06748 \\
\hline 0.852145 & 0.0398839 & 0.80737 & 0.179742 & 0.711788 & 0.037729 & 1.04161 \\
\hline 0.854201 & 0.0492003 & 0.810845 & 0.181879 & 0.707018 & 0.0366716 & 1.04078 \\
\hline 0.844532 & 0.044169 & 0.798011 & 0.190227 & 0.708905 & 0.0335539 & 1.03523 \\
\hline 0.853946 & 0.0476224 & 0.808596 & 0.182214 & 0.708832 & 0.0334923 & 1.01794 \\
\hline 0.849007 & 0.0331306 & 0.806971 & 0.182536 & 0.706142 & 0.032453 & 1.03191 \\
\hline 0.849937 & 0.0399491 & 0.815294 & 0.199147 & 0.708845 & 0.0295526 & 1.04153 \\
\hline 0.867199 & 0.0457264 & 0.812051 & 0.177266 & 0.712765 & 0.036666 & 1.03573 \\
\hline 0.853516 & 0.0452766 & 0.817246 & 0.181388 & 0.707966 & 0.0288637 & 1.02032 \\
\hline 0.845464 & 0.0363502 & 0.822739 & 0.181395 & 0.711305 & 0.0399992 & 1.01425 \\
\hline 0.853684 & 0.0398429 & 0.822118 & 0.183483 & 0.713941 & 0.0253115 & 1.01145 \\
\hline 0.84905 & 0.0426153 & 0.817531 & 0.187884 & 0.712935 & 0.0292912 & 1.01758 \\
\hline 0.846502 & 0.0362238 & 0.836421 & 0.197195 & 0.717167 & 0.0290296 & 1.00617 \\
\hline
\end{tabular}




\begin{tabular}{|c|c|c|c|c|c|c|}
\hline 0.86067 & 0.0529792 & 0.850197 & 0.198335 & 0.711094 & 0.0292947 & 1.02059 \\
\hline 0.853421 & 0.0345412 & 0.842858 & 0.189097 & 0.720112 & 0.0278273 & 1.03144 \\
\hline 0.847347 & 0.0358793 & 0.850495 & 0.193995 & 0.714288 & 0.0301379 & 1.03174 \\
\hline 0.858604 & 0.0415698 & 0.865867 & 0.196985 & 0.717287 & 0.0292258 & 1.04993 \\
\hline 0.872815 & 0.0411475 & 0.861771 & 0.197953 & 0.715115 & 0.0298177 & 1.07736 \\
\hline 0.853107 & 0.0423984 & 0.887231 & 0.216329 & 0.718846 & 0.0311929 & 1.06634 \\
\hline 0.847318 & 0.0404679 & 0.896749 & 0.226218 & 0.72494 & 0.0323231 & 1.06317 \\
\hline 0.858323 & 0.0458025 & 0.905682 & 0.21551 & 0.731492 & 0.0347523 & 1.10891 \\
\hline 0.876023 & 0.0543616 & 0.915942 & 0.218414 & 0.736248 & 0.0272548 & 1.11087 \\
\hline 0.868542 & 0.0553168 & 0.937282 & 0.225291 & 0.724204 & 0.0249918 & 1.1318 \\
\hline 0.870829 & 0.0405221 & 0.945953 & 0.229482 & 0.735137 & 0.0380082 & 1.13074 \\
\hline 0.857045 & 0.0462058 & 0.966898 & 0.243463 & 0.734246 & 0.0341112 & 1.16036 \\
\hline 0.868709 & 0.0441931 & 0.978242 & 0.25603 & 0.736558 & 0.034251 & 1.17078 \\
\hline 0.871892 & 0.0408822 & 0.9873 & 0.260289 & 0.738809 & 0.0190936 & 1.19256 \\
\hline 0.866368 & 0.0429572 & 0.994345 & 0.254019 & 0.745249 & 0.0345834 & 1.2179 \\
\hline 0.869632 & 0.0444799 & 1.00204 & 0.26438 & 0.745194 & 0.0345801 & 1.2108 \\
\hline 0.865805 & 0.049376 & 1.01337 & 0.265655 & 0.739708 & 0.0424445 & 1.22091 \\
\hline 0.853362 & 0.042648 & 1.00433 & 0.254088 & 0.737445 & 0.0308986 & 1.21865 \\
\hline 0.871804 & 0.0440495 & 1.01106 & 0.250686 & 0.747547 & 0.0225416 & 1.2348 \\
\hline 0.863925 & 0.0504499 & 0.993926 & 0.255201 & 0.743327 & 0.0300627 & 1.19801 \\
\hline 0.865308 & 0.039761 & 0.98799 & 0.238547 & 0.743371 & 0.0358559 & 1.19837 \\
\hline 0.871377 & 0.042618 & 0.97499 & 0.233326 & 0.735841 & 0.0333795 & 1.18177 \\
\hline 0.849912 & 0.044299 & 0.972421 & 0.230024 & 0.735224 & 0.0282823 & 1.18666 \\
\hline 0.858324 & 0.0338172 & 0.957722 & 0.211239 & 0.731105 & 0.0323611 & 1.17115 \\
\hline 0.855068 & 0.0335291 & 0.936677 & 0.194408 & 0.730836 & 0.0302855 & 1.157 \\
\hline 0.864126 & 0.046805 & 0.925353 & 0.195784 & 0.730002 & 0.029562 & 1.14864 \\
\hline 0.861802 & 0.0479044 & 0.925449 & 0.199726 & 0.728141 & 0.0279473 & 1.12701 \\
\hline 0.86135 & 0.0392057 & 0.902058 & 0.180381 & 0.721787 & 0.0301479 & 1.10358 \\
\hline 0.858117 & 0.0383222 & 0.893303 & 0.172566 & 0.715113 & 0.0259164 & 1.09992 \\
\hline 0.853455 & 0.0517437 & 0.873234 & 0.160783 & 0.722565 & 0.0328643 & 1.08121 \\
\hline 0.856188 & 0.0446416 & 0.871742 & 0.154547 & 0.721182 & 0.0230258 & 1.07556 \\
\hline 0.85703 & 0.0426268 & 0.867935 & 0.149191 & 0.715955 & 0.0350557 & 1.08293 \\
\hline 0.858527 & 0.0408017 & 0.845389 & 0.144241 & 0.71836 & 0.0286316 & 1.0713 \\
\hline 0.853067 & 0.0357398 & 0.836105 & 0.142745 & 0.716552 & 0.0326043 & 1.05952 \\
\hline 0.854128 & 0.0495628 & 0.847955 & 0.146992 & 0.718927 & 0.0367505 & 1.04883 \\
\hline 0.843801 & 0.043209 & 0.843494 & 0.149915 & 0.716731 & 0.0247875 & 1.06748 \\
\hline 0.853948 & 0.0464292 & 0.847339 & 0.144127 & 0.717515 & 0.0251399 & 1.04175 \\
\hline 0.843348 & 0.0419318 & 0.85587 & 0.14471 & 0.715825 & 0.0255594 & 1.06879 \\
\hline 0.844394 & 0.0415323 & 0.848781 & 0.143998 & 0.711451 & 0.0302084 & 1.06407 \\
\hline 0.845652 & 0.0423525 & 0.846289 & 0.132769 & 0.71878 & 0.0277688 & 1.06303 \\
\hline 0.850051 & 0.0486715 & 0.838213 & 0.14058 & 0.704012 & 0.0244001 & 1.05056 \\
\hline 0.843894 & 0.0417067 & 0.859614 & 0.144989 & 0.718114 & 0.0286931 & 1.07249 \\
\hline 0.844557 & 0.0447975 & 0.859545 & 0.149222 & 0.724545 & 0.0323089 & 1.06292 \\
\hline 0.842529 & 0.0496598 & 0.862369 & 0.148033 & 0.721147 & 0.0333808 & 1.05903 \\
\hline 0.838313 & 0.0419638 & 0.866186 & 0.151309 & 0.724589 & 0.0316205 & 1.0814 \\
\hline 0.848315 & 0.0417945 & 0.883919 & 0.145 & 0.725428 & 0.0279311 & 1.0648 \\
\hline 0.838973 & 0.046321 & 0.889876 & 0.154418 & 0.729172 & 0.0362368 & 1.08351 \\
\hline
\end{tabular}




\begin{tabular}{|c|c|c|c|c|c|c|}
\hline 0.838761 & 0.0405873 & 0.89991 & 0.155976 & 0.738971 & 0.0308812 & 1.0906 \\
\hline 0.83377 & 0.0407967 & 0.91542 & 0.164386 & 0.742791 & 0.0252031 & 1.0835 \\
\hline 0.831158 & 0.0371386 & 0.935867 & 0.174193 & 0.748592 & 0.0331054 & 1.10682 \\
\hline 0.843229 & 0.0345951 & 0.959217 & 0.179748 & 0.75713 & 0.027638 & 1.11421 \\
\hline 0.834201 & 0.0473336 & 0.981274 & 0.197279 & 0.765058 & 0.0337724 & 1.10463 \\
\hline 0.843699 & 0.0420765 & 1.00608 & 0.209141 & 0.790836 & 0.0323537 & 1.10225 \\
\hline 0.840037 & 0.0391193 & 1.02648 & 0.217736 & 0.79595 & 0.0325747 & 1.10271 \\
\hline 0.842435 & 0.0368118 & 1.06302 & 0.24548 & 0.810704 & 0.0325652 & 1.1451 \\
\hline 0.850208 & 0.0395694 & 1.06999 & 0.270025 & 0.816246 & 0.0348637 & 1.107 \\
\hline 0.85989 & 0.0454629 & 1.11878 & 0.279595 & 0.838417 & 0.0358857 & 1.14765 \\
\hline 0.861339 & 0.0386847 & 1.13113 & 0.294805 & 0.859083 & 0.0416097 & 1.10211 \\
\hline 0.865213 & 0.0440706 & 1.16502 & 0.314963 & 0.861833 & 0.0356785 & 1.13297 \\
\hline 0.853685 & 0.0369935 & 1.18712 & 0.339725 & 0.891038 & 0.0338984 & 1.12717 \\
\hline 0.881142 & 0.0492657 & 1.22379 & 0.351476 & 0.893713 & 0.0347871 & 1.11716 \\
\hline 0.879144 & 0.0625007 & 1.20984 & 0.348049 & 0.907592 & 0.0361419 & 1.12244 \\
\hline 0.885408 & 0.0420382 & 1.23555 & 0.354271 & 0.922391 & 0.046723 & 1.13889 \\
\hline 0.880438 & 0.0462595 & 1.24759 & 0.38675 & 0.918394 & 0.0405443 & 1.1418 \\
\hline 0.891436 & 0.0437047 & 1.2275 & 0.374737 & 0.929285 & 0.0468162 & 1.11709 \\
\hline 0.903551 & 0.0396681 & 1.23754 & 0.356521 & 0.927541 & 0.0408065 & 1.12984 \\
\hline 0.893358 & 0.048967 & 1.21751 & 0.334865 & 0.909183 & 0.0382191 & 1.12159 \\
\hline 0.886757 & 0.0368487 & 1.18083 & 0.326958 & 0.894833 & 0.0414884 & 1.104 \\
\hline 0.892582 & 0.0481696 & 1.16741 & 0.318987 & 0.893038 & 0.0413067 & 1.10184 \\
\hline 0.891804 & 0.0444942 & 1.13209 & 0.306025 & 0.878782 & 0.0415412 & 1.10011 \\
\hline 0.888349 & 0.0309159 & 1.08531 & 0.277784 & 0.856344 & 0.0390053 & 1.10708 \\
\hline 0.894446 & 0.0410535 & 1.07011 & 0.242609 & 0.843562 & 0.0436084 & 1.09961 \\
\hline 0.878732 & 0.0393413 & 1.03335 & 0.232806 & 0.826638 & 0.0363842 & 1.08177 \\
\hline 0.882313 & 0.0483119 & 1.00258 & 0.208957 & 0.813702 & 0.0377381 & 1.09531 \\
\hline 0.866698 & 0.0351767 & 0.96922 & 0.194402 & 0.796864 & 0.0320749 & 1.07391 \\
\hline 0.869799 & 0.0465492 & 0.935213 & 0.166963 & 0.777263 & 0.0354037 & 1.07253 \\
\hline 0.867358 & 0.0395226 & 0.922149 & 0.165633 & 0.768272 & 0.0317831 & 1.08449 \\
\hline 0.859306 & 0.0373821 & 0.903451 & 0.144654 & 0.748851 & 0.0268741 & 1.04784 \\
\hline 0.842867 & 0.0369896 & 0.883075 & 0.157079 & 0.746277 & 0.025915 & 1.05393 \\
\hline 0.856837 & 0.0509439 & 0.863838 & 0.132681 & 0.732846 & 0.0304691 & 1.04825 \\
\hline 0.837738 & 0.0371347 & 0.856003 & 0.140007 & 0.726635 & 0.0241683 & 1.06416 \\
\hline 0.83811 & 0.0351286 & 0.848535 & 0.130808 & 0.719701 & 0.0253746 & 1.07086 \\
\hline 0.838832 & 0.0374277 & 0.828674 & 0.125532 & 0.71485 & 0.0327013 & 1.04174 \\
\hline 0.848751 & 0.0459633 & 0.819649 & 0.123414 & 0.714985 & 0.029095 & 1.0591 \\
\hline 0.846058 & 0.0388449 & 0.824297 & 0.136975 & 0.701643 & 0.0202541 & 1.06166 \\
\hline 0.844326 & 0.0351475 & 0.813111 & 0.122175 & 0.702861 & 0.0309218 & 1.06323 \\
\hline 0.839198 & 0.0451275 & 0.817037 & 0.116201 & 0.698122 & 0.0304617 & 1.07278 \\
\hline 0.839707 & 0.0402739 & 0.798763 & 0.118049 & 0.696139 & 0.0281016 & 1.06721 \\
\hline 0.840237 & 0.0499812 & 0.790818 & 0.110693 & 0.701742 & 0.0266436 & 1.08643 \\
\hline 0.851748 & 0.0436735 & 0.794178 & 0.119421 & 0.70629 & 0.0251203 & 1.06473 \\
\hline 0.849307 & 0.039688 & 0.797483 & 0.110429 & 0.702506 & 0.028947 & 1.07778 \\
\hline 0.845985 & 0.0446901 & 0.786389 & 0.124538 & 0.704491 & 0.0210866 & 1.10001 \\
\hline 0.849418 & 0.0425667 & 0.787815 & 0.106729 & 0.705542 & 0.0324214 & 1.09557 \\
\hline 0.846145 & 0.0390052 & 0.782223 & 0.113574 & 0.699081 & 0.0218965 & 1.08669 \\
\hline
\end{tabular}




\begin{tabular}{|c|c|c|c|c|c|c|}
\hline 0.848405 & 0.0486996 & 0.791952 & 0.113482 & 0.702396 & 0.0281725 & 1.11935 \\
\hline 0.847176 & 0.0405875 & 0.778199 & 0.0996043 & 0.703765 & 0.0289203 & 1.10632 \\
\hline 0.843671 & 0.0335678 & 0.776456 & 0.115113 & 0.702822 & 0.0302031 & 1.1155 \\
\hline 0.847196 & 0.0346854 & 0.774346 & 0.105477 & 0.700633 & 0.0329531 & 1.11526 \\
\hline 0.847905 & 0.0429883 & 0.777764 & 0.0961469 & 0.700899 & 0.0254439 & 1.1319 \\
\hline 0.846509 & 0.036298 & 0.77053 & 0.101629 & 0.705892 & 0.0341651 & 1.14594 \\
\hline 0.858672 & 0.0421144 & 0.76479 & 0.0937145 & 0.694743 & 0.026982 & 1.14453 \\
\hline 0.857593 & 0.0378118 & 0.771483 & 0.100086 & 0.70085 & 0.0216479 & 1.1443 \\
\hline 0.853528 & 0.033279 & 0.774001 & 0.0979894 & 0.696218 & 0.0311815 & 1.1416 \\
\hline 0.869 & 0.0469867 & 0.760395 & 0.0972726 & 0.702476 & 0.030837 & 1.13957 \\
\hline 0.860515 & 0.0455446 & 0.774867 & 0.0964683 & 0.700712 & 0.0304849 & 1.13758 \\
\hline 0.85465 & 0.0447772 & 0.767705 & 0.0922729 & 0.694378 & 0.029144 & 1.14056 \\
\hline 0.860897 & 0.0417809 & 0.757497 & 0.0983181 & 0.700386 & 0.0314289 & 1.15091 \\
\hline 0.855308 & 0.0460532 & 0.75302 & 0.0959541 & 0.690117 & 0.0238111 & 1.14328 \\
\hline 0.86099 & 0.0476998 & 0.758861 & 0.0753228 & 0.696919 & 0.030694 & 1.14385 \\
\hline 0.862828 & 0.0399831 & 0.765624 & 0.0813003 & 0.693261 & 0.032249 & 1.15457 \\
\hline 0.873284 & 0.048336 & 0.768717 & 0.102223 & 0.701802 & 0.0335086 & 1.1537 \\
\hline 0.864503 & 0.0461755 & 0.761568 & 0.0875149 & 0.696155 & 0.0311945 & 1.15699 \\
\hline 0.866981 & 0.0332354 & 0.759973 & 0.0858791 & 0.69073 & 0.0369788 & 1.15963 \\
\hline 0.8529 & 0.0410277 & 0.757045 & 0.0809012 & 0.695479 & 0.0304133 & 1.14709 \\
\hline 0.870564 & 0.0374355 & 0.756142 & 0.0913569 & 0.698649 & 0.033883 & 1.11514 \\
\hline 0.856012 & 0.0463113 & 0.750202 & 0.0797283 & 0.694988 & 0.026924 & 1.12214 \\
\hline 0.867813 & 0.0355794 & 0.758161 & 0.0985121 & 0.695845 & 0.0262428 & 1.12835 \\
\hline 0.874754 & 0.0388627 & 0.758526 & 0.09003 & 0.697511 & 0.0248502 & 1.10186 \\
\hline 0.861233 & 0.039427 & 0.771722 & 0.0940057 & 0.692267 & 0.0246872 & 1.10476 \\
\hline 0.854731 & 0.046543 & 0.74656 & 0.0889708 & 0.692319 & 0.0272955 & 1.06819 \\
\hline 0.85619 & 0.045326 & 0.757397 & 0.0890688 & 0.70102 & 0.0240955 & 1.07193 \\
\hline 0.867438 & 0.0408561 & 0.756824 & 0.0802817 & 0.696353 & 0.0285281 & 1.07148 \\
\hline 0.864015 & 0.0438959 & 0.762742 & 0.0807595 & 0.697337 & 0.0315426 & 1.06125 \\
\hline 0.849034 & 0.0427056 & 0.765809 & 0.0950346 & 0.687316 & 0.0253184 & 1.03774 \\
\hline 0.86661 & 0.0383892 & 0.756739 & 0.0873904 & 0.695534 & 0.0320336 & 1.04277 \\
\hline 0.859966 & 0.0380701 & 0.751974 & 0.0817807 & 0.699814 & 0.0288414 & 1.03306 \\
\hline 0.865129 & 0.0434513 & 0.760163 & 0.0923333 & 0.698703 & 0.0286132 & 1.0367 \\
\hline 0.862237 & 0.0432781 & 0.754556 & 0.0878169 & 0.68638 & 0.026779 & 1.02273 \\
\hline 0.844717 & 0.0438368 & 0.756307 & 0.0833338 & 0.693927 & 0.0244874 & 1.02491 \\
\hline 0.842625 & 0.0456076 & 0.748639 & 0.0886789 & 0.693029 & 0.0336311 & 1.01309 \\
\hline 0.857397 & 0.0460697 & 0.749095 & 0.0794184 & 0.693304 & 0.0284076 & 1.00885 \\
\hline 0.851467 & 0.0365942 & 0.760805 & 0.0824528 & 0.702695 & 0.0317771 & 1.03458 \\
\hline 0.863979 & 0.0406875 & 0.765323 & 0.090687 & 0.700902 & 0.0308868 & 1.0037 \\
\hline 0.851104 & 0.0410449 & 0.754201 & 0.0844634 & 0.69778 & 0.0265069 & 0.985522 \\
\hline 0.838598 & 0.0380401 & 0.75886 & 0.0912683 & 0.702056 & 0.0311756 & 0.999847 \\
\hline 0.854898 & 0.0439535 & 0.775262 & 0.0888096 & 0.693208 & 0.0263698 & 1.00357 \\
\hline 0.838285 & 0.0403687 & 0.76248 & 0.0898088 & 0.697536 & 0.0263979 & 1.01301 \\
\hline 0.841855 & 0.0450822 & 0.759262 & 0.0860805 & 0.698726 & 0.0361966 & 1.01291 \\
\hline 0.84438 & 0.0369434 & 0.759837 & 0.0848015 & 0.694243 & 0.0311507 & 1.00419 \\
\hline 0.849254 & 0.0342205 & 0.752601 & 0.0831129 & 0.694155 & 0.0261458 & 1.02123 \\
\hline 0.848752 & 0.0458902 & 0.759122 & 0.082373 & 0.696458 & 0.0201736 & 0.999229 \\
\hline
\end{tabular}




\begin{tabular}{|c|c|c|c|c|c|c|}
\hline 0.850698 & 0.039333 & 0.755372 & 0.0833492 & 0.699124 & 0.0310885 & 0.98843 \\
\hline 0.840479 & 0.0396731 & 0.758216 & 0.0689831 & 0.695741 & 0.0284452 & 1.00086 \\
\hline 0.843469 & 0.0359548 & 0.748287 & 0.0779699 & 0.688628 & 0.0256525 & 1.00632 \\
\hline 0.846546 & 0.039098 & 0.744384 & 0.077764 & 0.686768 & 0.0340516 & 0.992557 \\
\hline 0.831275 & 0.0428439 & 0.766669 & 0.0889948 & 0.691812 & 0.0237556 & 0.987002 \\
\hline 0.833879 & 0.0401753 & 0.752488 & 0.0836813 & 0.695532 & 0.0253376 & 1.00611 \\
\hline 0.842216 & 0.048601 & 0.751991 & 0.0824023 & 0.702011 & 0.0270836 & 0.97565 \\
\hline 0.834308 & 0.0489264 & 0.747929 & 0.0743534 & 0.690045 & 0.0271741 & 0.982012 \\
\hline 0.848004 & 0.0345427 & 0.755709 & 0.0894492 & 0.692579 & 0.0257379 & 0.993897 \\
\hline 0.832748 & 0.0374267 & 0.756003 & 0.0817325 & 0.689247 & 0.0250636 & 0.985412 \\
\hline 0.834295 & 0.0359662 & 0.757052 & 0.0814349 & 0.691871 & 0.0311492 & 0.987554 \\
\hline 0.8285 & 0.0434347 & 0.750756 & 0.090324 & 0.688852 & 0.033251 & 0.976179 \\
\hline 0.83892 & 0.0444121 & 0.757372 & 0.0854812 & 0.694673 & 0.024009 & 0.982074 \\
\hline 0.834213 & 0.0368092 & 0.755834 & 0.0838391 & 0.688665 & 0.0217652 & 0.982044 \\
\hline 0.848231 & 0.0481967 & 0.744028 & 0.078823 & 0.687473 & 0.025644 & 0.982391 \\
\hline 0.840758 & 0.0333417 & 0.748038 & 0.0762145 & 0.683085 & 0.0269587 & 0.989911 \\
\hline 0.836375 & 0.0401974 & 0.740602 & 0.0796954 & 0.684688 & 0.0264757 & 0.962672 \\
\hline 0.837 & 0.0491307 & 0.740433 & 0.0670427 & 0.683765 & 0.0249843 & 0.988446 \\
\hline 0.831435 & 0.0455257 & 0.752252 & 0.0733748 & 193 & 0.0250323 & 0.989052 \\
\hline 0.823336 & 0.0392367 & 0.757627 & 0.0880767 & 0.684336 & 0.0289141 & 0.966383 \\
\hline 0.844217 & 0.0428871 & 0.754056 & 0.0850616 & 0.687841 & 0.0299674 & 0.988633 \\
\hline 0.834626 & 0.0339781 & 0.749482 & 0.0825474 & 437 & 0.0263481 & 0.98133 \\
\hline 0.820977 & 0.0456489 & 0.751893 & 0.0842839 & 0.686756 & 0.0236223 & 0.9893 \\
\hline 0.832424 & 0.0494099 & 0.747514 & 0.0821669 & 0.683664 & 0.0326996 & 0.991356 \\
\hline 0.835103 & 0.0405389 & 0.745301 & 0.0790531 & 0.686959 & 0.0278028 & 0.988734 \\
\hline 0.841842 & 0.0490687 & 0.751951 & 0.0716439 & 0.682268 & 0.0278231 & 0.979042 \\
\hline 0.834478 & 0.0321205 & 0.755104 & 0.0800713 & 0.682919 & 0.0271465 & 1.00373 \\
\hline 0.831536 & 0.0409308 & 0.743936 & 0.0860862 & 0.68318 & 0.0204316 & 1.01535 \\
\hline 0.829607 & 0.0469951 & 0.753107 & 0.0835957 & 0.690232 & 0.0305405 & 0.987903 \\
\hline 0.839029 & 0.0457984 & 0.738081 & 0.0796088 & 0.684955 & 0.0170511 & 0.995297 \\
\hline 0.833083 & 0.0386086 & 0.752501 & 0.0801323 & 0.681625 & 0.0316106 & 0.990568 \\
\hline 0.828297 & 0.0341597 & 0.746173 & 0.085734 & 0.679854 & 0.0230431 & 0.998897 \\
\hline 0.833078 & 0.0361229 & 0.743515 & 0.0868761 & 0.68362 & 0.0308558 & 1.02251 \\
\hline 0.8285 & 0.0342609 & 0.753235 & 0.0766919 & 0.684403 & 0.0231436 & 1.01524 \\
\hline 0.842399 & 0.0380671 & 0.754956 & 0.0798846 & 0.680625 & 0.0222828 & 1.02048 \\
\hline 0.839311 & 0.0342687 & 0.758077 & 0.0858088 & 0.68766 & 0.0231338 & 1.03945 \\
\hline 0.843657 & 0.0384446 & 0.749745 & 0.0838562 & 0.679469 & 0.0285783 & 1.04168 \\
\hline 0.833385 & 0.0336941 & 0.755127 & 0.0810219 & 0.678194 & 0.0273453 & 1.03671 \\
\hline 0.82016 & 0.0308444 & 0.752118 & 0.0810168 & 0.682189 & 0.0219686 & 1.02313 \\
\hline 0.841726 & 0.046134 & 0.751212 & 0.0766755 & 0.68437 & 0.0269283 & 1.04384 \\
\hline 0.842455 & 0.0413516 & 0.761375 & 0.0831553 & 0.683583 & 0.025813 & 1.05906 \\
\hline 0.827631 & 0.0335236 & 0.755184 & 0.0828283 & 0.684124 & 0.0222621 & 1.05177 \\
\hline 0.824714 & 0.0391878 & 0.759304 & 0.08703 & 0.681248 & 0.0287762 & 1.04885 \\
\hline 0.830326 & 0.0373922 & 0.749479 & 0.0894545 & 0.68555 & 0.0259845 & 1.05046 \\
\hline 0.828435 & 0.0404531 & 0.750219 & 0.0838986 & 0.679222 & 0.0234373 & 1.06252 \\
\hline 0.82206 & 0.0451394 & 0.753951 & 0.0940546 & 0.686463 & 0.0274068 & 1.05948 \\
\hline 0.825563 & 0.0407062 & 0.749282 & 0.0776435 & 0.679307 & 0.0233716 & 1.06082 \\
\hline
\end{tabular}




\begin{tabular}{|c|c|c|c|c|c|c|}
\hline 0.825658 & 0.0386597 & 0.748458 & 0.0810592 & 0.675879 & 0.0299834 & 1.06929 \\
\hline 0.824935 & 0.0356302 & 0.758154 & 0.0848329 & 0.672244 & 0.0274505 & 1.04552 \\
\hline 0.817907 & 0.0395812 & 0.754149 & 0.0853794 & 0.683287 & 0.0341036 & 1.05346 \\
\hline 0.805257 & 0.0335708 & 0.754 & 0.0823324 & 0.676053 & 0.0322457 & 1.04534 \\
\hline 0.816671 & 0.0295689 & 0.74712 & 0.0847006 & 0.675015 & 0.0282661 & 1.03937 \\
\hline 0.815968 & 0.042772 & 0.751255 & 0.0881886 & 0.678801 & 0.0331338 & 1.02066 \\
\hline 0.809455 & 0.0346701 & 0.75294 & 0.0758733 & 0.667439 & 0.0240804 & 1.02289 \\
\hline 0.812395 & 0.037794 & 0.751902 & 0.0829328 & 0.673907 & 0.0246692 & 1.02093 \\
\hline 0.809081 & 0.039483 & 0.746908 & 0.0830064 & 0.66796 & 0.0324558 & 1.00563 \\
\hline 0.816266 & 0.0410126 & 0.751915 & 0.0885602 & 0.671241 & 0.0232935 & 1.01967 \\
\hline 0.806826 & 0.0447412 & 0.75722 & 0.0938217 & 0.664238 & 0.0263118 & 0.997414 \\
\hline 0.80421 & 0.0419473 & 0.750246 & 0.0890714 & 0.672513 & 0.0257943 & 0.990956 \\
\hline 0.802999 & 0.0340117 & 0.745323 & 0.0870138 & 0.673945 & 0.0245345 & 0.989588 \\
\hline 0.796246 & 0.0412301 & 0.746282 & 0.0814619 & 0.670986 & 0.0240971 & 0.9851 \\
\hline 0.80549 & 0.0365467 & 0.744798 & 0.0823383 & 0.667133 & 0.0215928 & 0.977756 \\
\hline 0.803771 & 0.0461712 & 0.748338 & 0.0841383 & 0.669752 & 0.0245446 & 0.961601 \\
\hline 0.800426 & 0.0332329 & 0.741782 & 0.0812553 & 0.663595 & 0.0212996 & 0.968869 \\
\hline 0.797057 & 0.0308849 & 0.75237 & 0.0857508 & 0.658196 & 0.0221678 & 0.974919 \\
\hline 0.79206 & 0.0370994 & 0.740037 & 0.0845546 & 0.664438 & 0.0275852 & 0.945913 \\
\hline 0.785618 & 0.0376571 & 0.734139 & 0.0800211 & 0.662822 & 0.0273123 & 0.945732 \\
\hline 0.785065 & 0.0338744 & 0.734217 & 0.0810967 & 0.656889 & 0.0245335 & 0.955708 \\
\hline 0.795263 & 0.0414314 & 0.744698 & 0.0841562 & 0.657953 & 0.0224908 & 0.93932 \\
\hline 0.793077 & 0.0377466 & 0.730524 & 0.0753256 & 0.649966 & 0.0274046 & 0.958892 \\
\hline 0.794192 & 0.0421001 & 0.736745 & 0.0778362 & 0.656601 & 0.0269177 & 0.95587 \\
\hline 0.783169 & 0.028723 & 0.739094 & 0.0904415 & 0.65244 & 0.0261344 & 0.928793 \\
\hline 0.78685 & 0.0301117 & 0.731237 & 0.0805684 & 0.654708 & 0.0289357 & 0.949125 \\
\hline 0.778919 & 0.045914 & 0.735003 & 0.0897039 & 0.653351 & 0.0260107 & 0.959145 \\
\hline 0.787884 & 0.030787 & 0.737207 & 0.0857049 & 0.644545 & 0.0261581 & 0.953538 \\
\hline 0.773412 & 0.0426539 & 0.738429 & 0.077185 & 0.646603 & 0.0209794 & 0.941718 \\
\hline 0.785745 & 0.0506185 & 0.730174 & 0.0805476 & 0.647903 & 0.0234109 & 0.943891 \\
\hline 0.781002 & 0.0505028 & 0.724081 & 0.0788281 & 0.651014 & 0.0239913 & 0.956998 \\
\hline 0.757036 & 0.0415234 & 0.723354 & 0.0780007 & 0.648255 & 0.0227642 & 0.983028 \\
\hline 0.773511 & 0.0469891 & 0.730283 & 0.0797259 & 0.640983 & 0.0328041 & 0.948502 \\
\hline 0.770256 & 0.032475 & 0.719206 & 0.0681826 & 0.647186 & 0.0210487 & 0.967639 \\
\hline 0.767002 & 0.0327726 & 0.729588 & 0.0796526 & 0.647385 & 0.028097 & 0.980438 \\
\hline 0.768627 & 0.0425142 & 0.724627 & 0.0813631 & 0.642634 & 0.0196635 & 0.989709 \\
\hline 0.764625 & 0.0396065 & 0.728265 & 0.0694427 & 0.646017 & 0.0261809 & 0.99585 \\
\hline 0.762762 & 0.0376237 & 0.727828 & 0.08045 & 0.646924 & 0.0174005 & 1.01001 \\
\hline 0.763434 & 0.0479703 & 0.734785 & 0.0753034 & 0.639929 & 0.0239284 & 1.02196 \\
\hline 0.766899 & 0.0389631 & 0.729746 & 0.075493 & 0.638999 & 0.0289283 & 1.03191 \\
\hline 0.753749 & 0.0400127 & 0.734033 & 0.0800045 & 0.634123 & 0.0276001 & 1.03928 \\
\hline 0.751424 & 0.0437233 & 0.719298 & 0.076855 & 0.638564 & 0.0240001 & 1.07124 \\
\hline 0.759219 & 0.0417551 & 0.724189 & 0.0747195 & 0.639499 & 0.0316636 & 1.06819 \\
\hline 0.754418 & 0.0328176 & 0.727065 & 0.0757341 & 0.638032 & 0.0188277 & 1.09198 \\
\hline 0.758648 & 0.0366057 & 0.724075 & 0.0673161 & 0.634491 & 0.0193622 & 1.10951 \\
\hline 0.747859 & 0.03344 & 0.737494 & 0.0801671 & 0.634876 & 0.0223892 & 1.12602 \\
\hline 0.743691 & 0.0358288 & 0.727693 & 0.0745392 & 0.636828 & 0.0259215 & 1.12639 \\
\hline
\end{tabular}




\begin{tabular}{|c|c|c|c|c|c|c|}
\hline 0.747686 & 0.0348459 & 0.723219 & 0.0684382 & 0.64218 & 0.0256609 & 1.14928 \\
\hline 0.750112 & 0.0327794 & 0.715126 & 0.0739055 & 0.638747 & 0.0216749 & 1.13131 \\
\hline 0.744809 & 0.0394794 & 0.72433 & 0.0691531 & 0.629723 & 0.0176872 & 1.14087 \\
\hline 0.746438 & 0.0346799 & 0.718809 & 0.071447 & 0.629433 & 0.0242888 & 1.16072 \\
\hline 0.732498 & 0.0431975 & 0.717122 & 0.0733727 & 0.628465 & 0.0229791 & 1.16258 \\
\hline 0.736583 & 0.0394093 & 0.722596 & 0.0754474 & 0.630136 & 0.017723 & 1.16346 \\
\hline 0.734938 & 0.0380376 & 0.714446 & 0.067822 & 0.630273 & 0.0198326 & 1.14189 \\
\hline 0.733256 & 0.0360894 & 0.711265 & 0.065246 & 0.624361 & 0.0242521 & 1.14423 \\
\hline 0.733258 & 0.0379431 & 0.714533 & 0.0669621 & 0.62682 & 0.0226309 & 1.14109 \\
\hline 0.730751 & 0.0328783 & 0.701786 & 0.0703251 & 0.625511 & 0.0253043 & 1.11414 \\
\hline 0.739689 & 0.0355194 & 0.709804 & 0.0660481 & 0.625334 & 0.0239826 & 1.1029 \\
\hline 0.747122 & 0.0420051 & 0.709313 & 0.0755712 & 0.628296 & 0.0184486 & 1.10315 \\
\hline 0.738511 & 0.0301019 & 0.704076 & 0.0708757 & 0.621802 & 0.0226274 & 1.10417 \\
\hline 0.735766 & 0.0424107 & 0.699742 & 0.0687401 & 0.624912 & 0.0268837 & 1.06636 \\
\hline 0.719198 & 0.0322756 & 0.705685 & 0.0714102 & 0.621073 & 0.0243621 & 1.08519 \\
\hline 0.728386 & 0.0339125 & 0.709119 & 0.0720991 & 0.621815 & 0.0241458 & 1.08389 \\
\hline 0.723433 & 0.037033 & 0.705671 & 0.0691752 & 0.622531 & 0.01532 & 1.0895 \\
\hline 0.729049 & 0.0341901 & 0.712302 & 0.0763456 & 0.627519 & 0.0155805 & 1.08195 \\
\hline 0.714672 & 0.0294171 & 0.709547 & 0.0722268 & 0.625239 & 0.0206732 & 1.08232 \\
\hline 0.73132 & 0.0391078 & 0.722338 & 0.0664469 & 0.620262 & 0.0193316 & 1.08603 \\
\hline 0.730561 & 0.0337336 & 0.719872 & 0.079288 & 0.627074 & 0.0285975 & 1.0939 \\
\hline 0.721762 & 0.0359656 & 0.755779 & 0.0815532 & 0.631445 & 0.0243965 & 1.12916 \\
\hline 0.710792 & 0.037101 & 0.755805 & 0.0958036 & 0.639595 & 0.0261488 & 1.13636 \\
\hline 0.718703 & 0.0346457 & 0.761948 & 0.0948172 & 0.642864 & 0.0178237 & 1.17701 \\
\hline 0.729363 & 0.0377169 & 0.781665 & 0.108151 & 0.658261 & 0.0248233 & 1.20445 \\
\hline 0.710576 & 0.0406256 & 0.794135 & 0.099376 & 0.665879 & 0.026188 & 1.24548 \\
\hline 0.724276 & 0.0346723 & 0.805401 & 0.123186 & 0.67681 & 0.0237303 & 1.33753 \\
\hline 0.725914 & 0.0364443 & 0.831074 & 0.138421 & 0.676028 & 0.0270242 & 1.42618 \\
\hline 0.726804 & 0.0311733 & 0.852404 & 0.136987 & 0.690814 & 0.0293292 & 1.52736 \\
\hline 0.73818 & 0.0334408 & 0.868738 & 0.153976 & 0.687827 & 0.0255392 & 1.6628 \\
\hline 0.735685 & 0.0449901 & 0.876392 & 0.150301 & 0.711304 & 0.0291339 & 1.85093 \\
\hline 0.739028 & 0.0442798 & 0.905673 & 0.16318 & 0.714815 & 0.0288643 & 2.10893 \\
\hline 0.750783 & 0.0415977 & 0.906236 & 0.163825 & 0.72281 & 0.0359531 & 2.36145 \\
\hline 0.739918 & 0.0343087 & 0.925237 & 0.166909 & 0.733119 & 0.0309053 & 2.72176 \\
\hline 0.743519 & 0.0387593 & 0.942438 & 0.189027 & 0.742868 & 0.0331614 & 3.2249 \\
\hline 0.744245 & 0.0405671 & 0.939159 & 0.188828 & 0.751805 & 0.0350246 & 3.77616 \\
\hline 0.745565 & 0.0296239 & 0.934928 & 0.175309 & 0.748469 & 0.0346737 & 4.35815 \\
\hline 0.742393 & 0.0383868 & 0.947725 & 0.178176 & 0.752844 & 0.0293195 & 5.10259 \\
\hline 0.750577 & 0.0340317 & 0.942869 & 0.184649 & 0.751573 & 0.0364931 & 6.00072 \\
\hline 0.753851 & 0.0384571 & 0.932918 & 0.182092 & 0.751382 & 0.0270949 & 6.85881 \\
\hline 0.738608 & 0.0320571 & 0.910807 & 0.174765 & 0.738866 & 0.0267356 & 7.97525 \\
\hline 0.743537 & 0.0382348 & 0.902172 & 0.168839 & 0.744829 & 0.0317572 & 9.15766 \\
\hline 0.734513 & 0.039927 & 0.903372 & 0.183615 & 0.739332 & 0.0295949 & 10.4108 \\
\hline 0.737462 & 0.0331416 & 0.887023 & 0.162968 & 0.735849 & 0.0320166 & 11.69 \\
\hline 0.732495 & 0.0369019 & 0.885289 & 0.1597 & 0.729349 & 0.0245465 & 12.934 \\
\hline 0.732133 & 0.0382046 & 0.865427 & 0.16748 & 0.732734 & 0.0297742 & 14.293 \\
\hline 0.712374 & 0.0290937 & 0.855914 & 0.183598 & 0.724037 & 0.0249492 & 15.4947 \\
\hline
\end{tabular}




\begin{tabular}{|c|c|c|c|c|c|c|}
\hline 0.724592 & 0.0406875 & 0.849209 & 0.182693 & 0.721315 & 0.0246897 & 16.6514 \\
\hline 0.713781 & 0.0276943 & 0.831647 & 0.185818 & 0.716888 & 0.0289356 & 17.6194 \\
\hline 0.699677 & 0.0386789 & 0.826746 & 0.182289 & 0.718804 & 0.0329662 & 18.5174 \\
\hline 0.703071 & 0.0356178 & 0.832983 & 0.194977 & 0.702991 & 0.0373228 & 19.2301 \\
\hline 0.704591 & 0.0387725 & 0.809462 & 0.177388 & 0.69945 & 0.0327313 & 19.7861 \\
\hline 0.690454 & 0.0260051 & 0.79719 & 0.188334 & 0.698019 & 0.0288447 & 19.9108 \\
\hline 0.682903 & 0.0394224 & 0.790752 & 0.200675 & 0.702785 & 0.0319859 & 19.9456 \\
\hline 0.688745 & 0.0310815 & 0.784081 & 0.176859 & 0.696859 & 0.0253275 & 19.6697 \\
\hline 0.680676 & 0.0356054 & 0.772915 & 0.171676 & 0.675152 & 0.0285855 & 19.0416 \\
\hline 0.67357 & 0.0396407 & 0.754436 & 0.16982 & 0.679877 & 0.0286356 & 18.4268 \\
\hline 0.679237 & 0.0360753 & 0.744211 & 0.147853 & 0.669471 & 0.0298475 & 17.4087 \\
\hline 0.667754 & 0.042197 & 0.733195 & 0.151784 & 0.653461 & 0.0300205 & 16.2454 \\
\hline 0.665785 & 0.0337319 & 0.726888 & 0.131735 & 0.65221 & 0.022735 & 15.0644 \\
\hline 0.663807 & 0.0331811 & 0.715625 & 0.134607 & 0.644678 & 0.0332739 & 13.7368 \\
\hline 0.667833 & 0.0343418 & 0.70541 & 0.123093 & 0.637838 & 0.0303985 & 12.4629 \\
\hline 0.670583 & 0.0285583 & 0.688001 & 0.105763 & 0.619389 & 0.0263769 & 11.0974 \\
\hline 0.66528 & 0.0287997 & 0.68204 & 0.094568 & 0.62252 & 0.0239757 & 9.85888 \\
\hline 0.650799 & 0.0415258 & 0.682779 & 0.0883349 & 0.610292 & 0.0240986 & 8.54868 \\
\hline 0.664775 & 0.0356457 & 0.663897 & 0.076942 & 0.6 & 0.02 & 7.42176 \\
\hline 0.661938 & 0.0261695 & 0.664949 & 0.0751326 & 0.596611 & 0.0286461 & 6.40743 \\
\hline 0.664496 & 0.0409443 & 0.661744 & 0.0723697 & 0.591498 & 0.0172249 & 5.41277 \\
\hline 0.663474 & 0.0364521 & 0.652634 & 0.0636212 & 0.578307 & 0.0233367 & 4.60072 \\
\hline 0.654036 & 0.0366044 & 0.643516 & 0.0609519 & 0.582717 & 0.018595 & 3.87436 \\
\hline 0.654275 & 0.0357727 & 0.640515 & 0.0638706 & 0.569322 & 0.0255036 & 3.24846 \\
\hline 0.64794 & 0.0331241 & 0.632877 & 0.0615648 & 0.582413 & 0.0231746 & 2.75451 \\
\hline 0.648437 & 0.0330404 & 0.631078 & 0.0503339 & 0.568815 & 0.0223753 & 2.34713 \\
\hline 0.64517 & 0.0295818 & 0.626496 & 0.0551879 & 0.560817 & 0.0179258 & 1.97609 \\
\hline 0.639766 & 0.0319626 & 0.623867 & 0.0550159 & 0.559627 & 0.0180242 & 1.72792 \\
\hline 0.644024 & 0.0388813 & 0.632668 & 0.0592187 & 0.55616 & 0.0222186 & 1.51612 \\
\hline 0.648865 & 0.029835 & 0.615777 & 0.052055 & 0.556851 & 0.0217917 & 1.35475 \\
\hline 0.639032 & 0.0378634 & 0.617149 & 0.0449052 & 0.54975 & 0.0238493 & 1.22993 \\
\hline 0.642343 & 0.0358957 & 0.602195 & 0.0517908 & 0.550632 & 0.024473 & 1.1115 \\
\hline 0.645051 & 0.0320855 & 0.610354 & 0.0589274 & 0.541489 & 0.0210899 & 1.06625 \\
\hline 0.640542 & 0.0328498 & 0.596479 & 0.0509776 & 0.541647 & 0.0163035 & 0.978749 \\
\hline 0.633393 & 0.0313291 & 0.605792 & 0.0573135 & 0.540513 & 0.019801 & 0.98021 \\
\hline 0.62859 & 0.034828 & 0.596228 & 0.0548478 & 0.54343 & 0.0170563 & 0.958205 \\
\hline 0.635022 & 0.0365342 & 0.596774 & 0.0490531 & 0.530204 & 0.0239378 & 0.936563 \\
\hline 0.625513 & 0.034567 & 0.603644 & 0.0510916 & 0.535502 & 0.0214428 & 0.932248 \\
\hline 0.633564 & 0.0424712 & 0.588308 & 0.0542987 & 0.535178 & 0.02105 & 0.909832 \\
\hline 0.628083 & 0.038744 & 0.594361 & 0.0587719 & 0.53717 & 0.0197314 & 0.915826 \\
\hline 0.624813 & 0.0324154 & 0.589111 & 0.0591209 & 0.52771 & 0.0208022 & 0.929077 \\
\hline 0.619539 & 0.0343992 & 0.586629 & 0.0481403 & 0.533067 & 0.0274892 & 0.943794 \\
\hline 0.606679 & 0.0299114 & 0.577892 & 0.0516572 & 0.535807 & 0.0162889 & 0.937044 \\
\hline 0.614601 & 0.0374819 & 0.583531 & 0.0546829 & 0.527536 & 0.0264317 & 0.960615 \\
\hline 0.625925 & 0.0317952 & 0.58375 & 0.0462009 & 0.535477 & 0.0263291 & 0.992792 \\
\hline 0.622233 & 0.0292699 & 0.591554 & 0.0502923 & 0.542041 & 0.0203571 & 1.01302 \\
\hline 0.621663 & 0.029896 & 0.582961 & 0.0529576 & 0.531841 & 0.0237867 & 1.07792 \\
\hline
\end{tabular}




\begin{tabular}{|c|c|c|c|c|c|c|}
\hline 0.620508 & 0.039744 & 0.596248 & 0.0549721 & 0.543153 & 0.0193075 & 1.10778 \\
\hline 0.614682 & 0.0334725 & 0.587777 & 0.0452224 & 0.539274 & 0.0197647 & 1.1901 \\
\hline 0.609719 & 0.025528 & 0.593574 & 0.0509285 & 0.551105 & 0.018239 & 1.25518 \\
\hline 0.613734 & 0.0296645 & 0.600772 & 0.0571594 & 0.56053 & 0.0239728 & 1.36526 \\
\hline 0.59804 & 0.0348238 & 0.596632 & 0.0559654 & 0.560607 & 0.0220581 & 1.47078 \\
\hline 0.618982 & 0.0294414 & 0.601026 & 0.0496656 & 0.569074 & 0.0225895 & 1.60108 \\
\hline 0.602109 & 0.0292139 & 0.608787 & 0.0562048 & 0.579576 & 0.0254877 & 1.7815 \\
\hline 0.607041 & 0.0342343 & 0.618276 & 0.0562014 & 0.58984 & 0.0250529 & 1.96471 \\
\hline 0.60642 & 0.0335709 & 0.617727 & 0.0614559 & 0.605095 & 0.0218077 & 2.16063 \\
\hline 0.606957 & 0.0298983 & 0.622461 & 0.0619909 & 0.609842 & 0.0200211 & 2.44111 \\
\hline 0.613392 & 0.0307011 & 0.631775 & 0.0553196 & 0.620486 & 0.0262991 & 2.70057 \\
\hline 0.60149 & 0.0306966 & 0.637709 & 0.0569245 & 0.625527 & 0.0228424 & 2.99544 \\
\hline 0.603475 & 0.0393442 & 0.633364 & 0.0691568 & 0.638452 & 0.0192358 & 3.32111 \\
\hline 0.611593 & 0.0296038 & 0.656142 & 0.0596698 & 0.645767 & 0.0227322 & 3.65218 \\
\hline 0.61281 & 0.0297517 & 0.648407 & 0.0681567 & 0.659877 & 0.0332556 & 4.02041 \\
\hline 0.614398 & 0.0310258 & 0.655743 & 0.0745524 & 0.656985 & 0.0238707 & 4.39351 \\
\hline 0.593953 & 0.0301143 & 0.660074 & 0.0790826 & 0.665453 & 0.0271433 & 4.70981 \\
\hline 0.609395 & 0.0327554 & 0.661361 & 0.0818789 & 0.671237 & 0.0274619 & 5.06449 \\
\hline 0.608117 & 0.0324191 & 0.663163 & 0.079 & 0.672053 & 0.025753 & 5.4101 \\
\hline 0.61434 & 0.0283861 & 0.652426 & 0.0834511 & 0.677183 & 0.0261565 & 5.67749 \\
\hline 0.605251 & 0.039904 & 0.661119 & 0.0777708 & 0.674386 & 0.0232831 & 5.92372 \\
\hline 0.609052 & 0.0264609 & 0.65875 & 0.07 & 0.664594 & 0.02232 & 6.12288 \\
\hline 0.609565 & 0.0261956 & 0.648183 & 0.0789853 & 0.659947 & 0.0222867 & 6.26816 \\
\hline 0.59914 & 0.0351034 & 0.642011 & 0.078528 & 0.656922 & 0.0259684 & 6.32932 \\
\hline 0.600031 & 0.0340094 & 0.647753 & 0.0807222 & 0.647124 & 0.0241592 & 6.35545 \\
\hline 0.597082 & 0.0289319 & 0.632436 & 0.0897514 & 0.634078 & 0.0213865 & 6.31338 \\
\hline 0.59975 & 0.0371901 & 0.619723 & 0.0753907 & 0.621902 & 0.0301133 & 6.14225 \\
\hline 0.59224 & 0.0309263 & 0.61659 & 0.0792393 & 0.609553 & 0.0219439 & 5.9413 \\
\hline 0.597002 & 0.0304517 & 0.604207 & 0.0713427 & 0.595348 & 0.022154 & 5.66514 \\
\hline 0.608273 & 0.0305447 & 0.605137 & 0.0693262 & 0.596438 & 0.0214175 & 5.3918 \\
\hline 0.60161 & 0.028748 & 0.603013 & 0.0629592 & 0.586642 & 0.021806 & 5.02486 \\
\hline 0.588488 & 0.0331903 & 0.586905 & 0.0708599 & 0.572361 & 0.0228293 & 4.69165 \\
\hline 0.597193 & 0.0282843 & 0.583625 & 0.0645817 & 0.551619 & 0.0230587 & 4.2813 \\
\hline 0.594522 & 0.0427784 & 0.568334 & 0.0524075 & 0.540237 & 0.0277448 & 3.91646 \\
\hline 0.593506 & 0.0324615 & 0.566475 & 0.0568623 & 0.539734 & 0.0184303 & 3.51161 \\
\hline 0.592461 & 0.0319687 & 0.566968 & 0.054817 & 0.527073 & 0.0252717 & 3.14936 \\
\hline 0.58575 & 0.0314932 & 0.554559 & 0.046988 & 0.515377 & 0.0253253 & 2.82189 \\
\hline 0.587034 & 0.0360401 & 0.555491 & 0.0501238 & 0.510168 & 0.0231998 & 2.52594 \\
\hline 0.58191 & 0.0306646 & 0.555895 & 0.0488575 & 0.508161 & 0.017746 & 2.19163 \\
\hline 0.5894 & 0.029097 & 0.540405 & 0.0482158 & 0.502149 & 0.0209656 & 1.9357 \\
\hline 0.584979 & 0.0356675 & 0.546107 & 0.0410864 & 0.501401 & 0.0192098 & 1.69319 \\
\hline 0.578005 & 0.0240229 & 0.54062 & 0.0464164 & 0.503664 & 0.0198472 & 1.49548 \\
\hline 0.587613 & 0.0299813 & 0.535916 & 0.0420626 & 0.491831 & 0.0176484 & 1.33914 \\
\hline 0.574736 & 0.0330834 & 0.534618 & 0.0389236 & 0.495323 & 0.0181018 & 1.19083 \\
\hline 0.580404 & 0.0252783 & 0.534459 & 0.0445016 & 0.488674 & 0.0176775 & 1.08747 \\
\hline 0.579819 & 0.0291071 & 0.529715 & 0.0501465 & 0.485496 & 0.0171217 & 0.976335 \\
\hline 0.581624 & 0.0315587 & 0.537392 & 0.0442005 & 0.484122 & 0.0205107 & 0.873897 \\
\hline
\end{tabular}




\begin{tabular}{|c|c|c|c|c|c|c|}
\hline 0.589563 & 0.0253373 & 0.529009 & 0.0453839 & 0.480207 & 0.0201236 & 0.824656 \\
\hline 0.584381 & 0.0320799 & 0.52544 & 0.0435471 & 0.48043 & 0.0164074 & 0.799983 \\
\hline 0.569007 & 0.0275484 & 0.533882 & 0.0485008 & 0.482525 & 0.020327 & 0.742294 \\
\hline 0.573777 & 0.0308751 & 0.530613 & 0.044285 & 0.482783 & 0.015954 & 0.724025 \\
\hline 0.569933 & 0.0381553 & 0.520676 & 0.0413471 & 0.484451 & 0.0205903 & 0.693485 \\
\hline 0.571889 & 0.027839 & 0.526934 & 0.0401845 & 0.486831 & 0.0199457 & 0.683843 \\
\hline 0.569986 & 0.0246392 & 0.527039 & 0.0382143 & 0.483226 & 0.0227057 & 0.685132 \\
\hline 0.57006 & 0.0299981 & 0.531536 & 0.0483228 & 0.478115 & 0.0197927 & 0.669163 \\
\hline 0.566935 & 0.0291506 & 0.524828 & 0.0368536 & 0.478388 & 0.0172519 & 0.680042 \\
\hline 0.571812 & 0.0373532 & 0.531839 & 0.0424754 & 0.477432 & 0.0197527 & 0.664942 \\
\hline 0.56695 & 0.0341429 & 0.52083 & 0.0401548 & 0.475035 & 0.0204272 & 0.688814 \\
\hline 0.574429 & 0.037602 & 0.519195 & 0.0432336 & 0.480238 & 0.0156208 & 0.670131 \\
\hline 0.568419 & 0.0308518 & 0.524907 & 0.0392914 & 0.479423 & 0.0221413 & 0.65997 \\
\hline 0.565168 & 0.0362642 & 0.520634 & 0.0462192 & 0.477552 & 0.0160699 & 0.672836 \\
\hline 0.572088 & 0.0308017 & 0.526279 & 0.0408813 & 0.480002 & 0.0212488 & 0.669566 \\
\hline 0.572505 & 0.0247762 & 0.530804 & 0.0511775 & 0.476006 & 0.0212077 & 0.684012 \\
\hline 0.575143 & 0.036004 & 0.525998 & 0.0370907 & 0.477457 & 0.0184031 & 0.675455 \\
\hline 0.568843 & 0.0284158 & 0.526204 & 0.0363077 & 0.472365 & 0.0219761 & 0.69468 \\
\hline 0.570164 & 0.0264156 & 0.515405 & 0.0392834 & 0.482979 & 0.0207571 & 0.704468 \\
\hline 0.570641 & 0.0311307 & 0.526213 & 0.035758 & 0.478818 & 0.0189188 & 0.689886 \\
\hline 0.567125 & 0.0322773 & 0.527389 & 0.0313657 & 0.485167 & 0.0178833 & 0.710064 \\
\hline 0.579777 & 0.0284526 & 0.52375 & 0.0410753 & 0.4867 & 0.0206228 & 0.711096 \\
\hline 0.584805 & 0.0296736 & 0.527475 & 0.0412664 & 0.489769 & 0.0212398 & 0.734439 \\
\hline 0.593259 & 0.0269557 & 0.5244 & 0.047902 & 0.491853 & 0.0207333 & 0.742899 \\
\hline 0.596197 & 0.0293207 & 0.545304 & 0.0440849 & 0.503384 & 0.0209922 & 0.748141 \\
\hline 0.609112 & 0.0428777 & 0.541754 & 0.0407442 & 0.516451 & 0.0248607 & 0.772615 \\
\hline 0.620558 & 0.0300785 & 0.546754 & 0.0468355 & 0.522708 & 0.0212328 & 0.78676 \\
\hline 0.642015 & 0.0398168 & 0.563838 & 0.046337 & 0.544748 & 0.0242525 & 0.781597 \\
\hline 0.667338 & 0.0307963 & 0.578769 & 0.0473522 & 0.562022 & 0.0264694 & 0.866774 \\
\hline 0.689825 & 0.0375692 & 0.594012 & 0.0426692 & 0.591712 & 0.0376976 & 0.860003 \\
\hline 0.754784 & 0.0469969 & 0.611478 & 0.0560389 & 0.620869 & 0.0302461 & 0.908624 \\
\hline 0.794788 & 0.0574424 & 0.640988 & 0.05164 & 0.651031 & 0.0408739 & 0.967646 \\
\hline 0.845547 & 0.0570102 & 0.662945 & 0.0637403 & 0.695636 & 0.0394708 & 0.998702 \\
\hline 0.931309 & 0.0678012 & 0.678811 & 0.0625835 & 0.731793 & 0.0456525 & 1.05547 \\
\hline 1.02711 & 0.0738474 & 0.712819 & 0.0683656 & 0.778227 & 0.0527896 & 1.10576 \\
\hline 1.11775 & 0.0870677 & 0.742222 & 0.0826584 & 0.832758 & 0.0693591 & 1.15654 \\
\hline 1.24148 & 0.0885916 & 0.773291 & 0.0873106 & 0.891669 & 0.077141 & 1.20823 \\
\hline 1.37005 & 0.104781 & 0.815055 & 0.0955848 & 0.943429 & 0.0758064 & 1.25921 \\
\hline 1.51808 & 0.118082 & 0.845891 & 0.0959571 & 1.00546 & 0.0907785 & 1.31178 \\
\hline 1.69648 & 0.139804 & 0.876272 & 0.107538 & 1.06921 & 0.0971614 & 1.35878 \\
\hline 1.87414 & 0.158274 & 0.917807 & 0.114419 & 1.12753 & 0.0962622 & 1.43269 \\
\hline 2.03117 & 0.179235 & 0.948869 & 0.116559 & 1.18491 & 0.114925 & 1.43442 \\
\hline 2.22872 & 0.196784 & 0.963829 & 0.130755 & 1.2321 & 0.128214 & 1.48362 \\
\hline 2.42686 & 0.212458 & 0.982133 & 0.126764 & 1.28585 & 0.13516 & 1.50973 \\
\hline 2.56792 & 0.242895 & 1.0074 & 0.12886 & 1.31245 & 0.136812 & 1.55548 \\
\hline 2.76836 & 0.262607 & 1.01818 & 0.122852 & 1.3499 & 0.130513 & 1.51722 \\
\hline 2.95114 & 0.283023 & 1.02751 & 0.139349 & 1.37036 & 0.1446 & 1.51346 \\
\hline
\end{tabular}




\begin{tabular}{|c|c|c|c|c|c|c|}
\hline 3.08097 & 0.273188 & 1.02621 & 0.14042 & 1.39989 & 0.151167 & 1.48044 \\
\hline 3.20692 & 0.285975 & 1.02436 & 0.135656 & 1.38908 & 0.138281 & 1.47822 \\
\hline 3.33998 & 0.305627 & 1.01065 & 0.134749 & 1.36943 & 0.155568 & 1.45569 \\
\hline 3.3747 & 0.31093 & 0.985875 & 0.132486 & 1.34267 & 0.135805 & 1.42903 \\
\hline 3.44085 & 0.331503 & 0.956454 & 0.100633 & 1.31143 & 0.137208 & 1.37162 \\
\hline 3.43042 & 0.31145 & 0.938573 & 0.120026 & 1.27714 & 0.138834 & 1.31633 \\
\hline 3.36867 & 0.331777 & 0.913562 & 0.104015 & 1.22756 & 0.112724 & 1.26981 \\
\hline 3.32149 & 0.322542 & 0.871157 & 0.0951285 & 1.16662 & 0.119221 & 1.18034 \\
\hline 3.21567 & 0.294367 & 0.830437 & 0.0823338 & 1.10752 & 0.105897 & 1.13848 \\
\hline 3.08892 & 0.266367 & 0.795401 & 0.0891119 & 1.04228 & 0.100368 & 1.09815 \\
\hline 2.94177 & 0.269756 & 0.756469 & 0.0782539 & 0.985139 & 0.0922952 & 1.00533 \\
\hline 2.78912 & 0.267491 & 0.725555 & 0.0764785 & 0.923947 & 0.0839511 & 0.955422 \\
\hline 2.58594 & 0.226465 & 0.680757 & 0.0601226 & 0.856598 & 0.0616258 & 0.886144 \\
\hline 2.39791 & 0.220079 & 0.656952 & 0.0575085 & 0.790916 & 0.0614877 & 0.837097 \\
\hline 2.23152 & 0.199131 & 0.630683 & 0.0478254 & 0.742868 & 0.0518916 & 0.802682 \\
\hline 2.01156 & 0.173751 & 0.607303 & 0.0416708 & 0.684806 & 0.0372444 & 0.750993 \\
\hline 1.83504 & 0.160404 & 0.588788 & 0.0382569 & 0.641523 & 0.0341236 & 0.727158 \\
\hline 1.6425 & 0.159776 & 0.557099 & 0.0443416 & 0.612161 & 0.0336355 & 0.701454 \\
\hline 1.47125 & 0.115277 & 0.552194 & 0.0414544 & 0.573761 & 0.0276664 & 0.669257 \\
\hline 1.30639 & 0.0863896 & 0.532319 & 0.0347525 & 0.541807 & 0.025394 & 0.640288 \\
\hline 1.18545 & 0.0879692 & 0.513413 & 0.0364114 & 0.518881 & 0.0238533 & 0.628912 \\
\hline 1.07001 & 0.0754804 & 0.496009 & 0.0329285 & 0.49459 & 0.0227296 & 0.617881 \\
\hline 0.96032 & 0.0553705 & 0.50123 & 0.0298411 & 0.483987 & 0.0221971 & 0.608833 \\
\hline 0.866156 & 0.0558392 & 0.490525 & 0.0276905 & 0.465255 & 0.0251402 & 0.592432 \\
\hline 0.791763 & 0.0448238 & 0.487603 & 0.0422787 & 0.453376 & 0.0237981 & 0.588683 \\
\hline 0.708699 & 0.036652 & 0.485144 & 0.0337481 & 0.443459 & 0.0194136 & 0.572375 \\
\hline 0.665438 & 0.0376188 & 0.476792 & 0.0304425 & 0.438623 & 0.0191527 & 0.571554 \\
\hline 0.629585 & 0.0417429 & 0.472174 & 0.0355851 & 0.431025 & 0.0178432 & 0.571727 \\
\hline 0.596897 & 0.0328971 & 0.459925 & 0.0281929 & 0.429924 & 0.0182529 & 0.569337 \\
\hline 0.573365 & 0.0277303 & 0.467407 & 0.0342654 & 0.427622 & 0.0178258 & 0.57234 \\
\hline 0.537057 & 0.0353449 & 0.468777 & 0.0345064 & 0.422352 & 0.0148654 & 0.569101 \\
\hline 0.525711 & 0.0301985 & 0.46577 & 0.0410903 & 0.419975 & 0.0151795 & 0.580774 \\
\hline 0.509527 & 0.0298111 & 0.45796 & 0.0372953 & 0.41822 & 0.0157281 & 0.557806 \\
\hline 0.506275 & 0.0291974 & 0.461558 & 0.0289495 & 0.414966 & 0.0199121 & 0.566791 \\
\hline 0.496285 & 0.035569 & 0.456697 & 0.0318028 & 0.4168 & 0.0209632 & 0.569126 \\
\hline 0.48673 & 0.026952 & 0.447552 & 0.0321349 & 0.412803 & 0.0176571 & 0.552421 \\
\hline 0.491151 & 0.0220831 & 0.461108 & 0.029552 & 0.409258 & 0.0178475 & 0.567158 \\
\hline 0.495678 & 0.0324598 & 0.452107 & 0.032946 & 0.406598 & 0.0178461 & 0.556756 \\
\hline 0.480039 & 0.0227731 & 0.457895 & 0.0322302 & 0.404858 & 0.0141529 & 0.552035 \\
\hline 0.477471 & 0.0241979 & 0.45114 & 0.0318616 & 0.404667 & 0.0171891 & 0.575312 \\
\hline 0.478648 & 0.0287182 & 0.448096 & 0.0287022 & 0.404529 & 0.018679 & 0.551822 \\
\hline 0.47481 & 0.0312918 & 0.441572 & 0.0312753 & 0.401741 & 0.0224681 & 0.54929 \\
\hline 0.474828 & 0.0255929 & 0.446133 & 0.0227668 & 0.406682 & 0.0187688 & 0.556181 \\
\hline 0.471292 & 0.026936 & 0.440352 & 0.0247691 & 0.407912 & 0.0125779 & 0.547841 \\
\hline 0.461825 & 0.0343717 & 0.447146 & 0.0319876 & 0.406576 & 0.0196698 & 0.560443 \\
\hline 0.466353 & 0.0267699 & 0.453629 & 0.0347057 & 0.409913 & 0.0203471 & 0.54141 \\
\hline 0.460746 & 0.0286394 & 0.444677 & 0.0361365 & 0.40415 & 0.0184981 & 0.544383 \\
\hline
\end{tabular}




\begin{tabular}{|c|c|c|c|c|c|c|}
\hline 0.464268 & 0.0283781 & 0.44573 & 0.0335984 & 0.402906 & 0.0135638 & 0.549983 \\
\hline 0.467135 & 0.026792 & 0.443079 & 0.0292813 & 0.4 & 0.0131973 & 0.539933 \\
\hline 0.457713 & 0.0275041 & 0.448479 & 0.0311479 & 0.406094 & 0.022215 & .534689 \\
\hline 0.46725 & 0.0250168 & 0.445609 & 0.0277698 & 0.40164 & 0.0197579 & 530854 \\
\hline 0.459912 & 0.0247551 & 0.454575 & 0.027824 & 0.40047 & 0.0160269 & 0.534535 \\
\hline 0.449109 & 0.0261545 & 0.455173 & 0.0356867 & 0.407038 & 0.0206433 & .518729 \\
\hline 0.459394 & 0.0288714 & 0.452974 & 0.0361686 & 0.398538 & 0.0143205 & 0.527501 \\
\hline 0.461332 & 0.0276065 & 0.450527 & 0.0331936 & 0.402223 & 0.0198894 & 0.527125 \\
\hline 0.454717 & 0.0230333 & 0.453409 & 0.0358462 & 0.399002 & 0.0191991 & 0.528463 \\
\hline 0.460029 & 0.0298299 & 0.462468 & 0.0353554 & 0.403671 & 0.0209461 & 0.518777 \\
\hline 0.455058 & 0.0258198 & 0.463156 & 0.0321281 & 0.410298 & 0.0157509 & 0.533135 \\
\hline 0.455738 & 0.0324506 & 0.462059 & 0.029978 & 0.409772 & 0.0209279 & 0.528831 \\
\hline 0.458927 & 0.024397 & 0.468595 & 0.0350907 & 0.415834 & 0.0199825 & 0.521193 \\
\hline 0.46657 & 0.0273751 & 0.466147 & 0.0282314 & 0.415295 & 0.0238987 & 0.516082 \\
\hline 0.460982 & 0.0245355 & 0.4618 & 0.036094 & 0.413495 & 0.015086 & 0.510819 \\
\hline 0.45933 & 0.0300135 & 0.461151 & 0.0347651 & 0.420836 & 0.0170124 & 0.51446 \\
\hline 0.457775 & 0.0274367 & 0.461147 & 0.0355579 & 0.416495 & 0.016722 & 0.532657 \\
\hline 0.455296 & 0.0235804 & 0.460744 & 0.0322535 & 0.42427 & 0.0172214 & 0.508531 \\
\hline 0.454438 & 0.0274683 & 0.46 & 0.03063 & 0.4 & 0.0165026 & 7196 \\
\hline 0.454822 & 0.0306505 & 0.460953 & 0.0290026 & 0.426163 & 0.0176957 & 0.510385 \\
\hline 0.447171 & 0.0223377 & 0.451233 & 0.0292941 & 0.428292 & 0.0200139 & 0.527835 \\
\hline 0.450297 & 0.02 & 0.4 & 351 & 715 & 0.0226283 & 1738 \\
\hline 0.447978 & 0.0234843 & 0.447216 & 0.0308956 & 0.43179 & 0.0155308 & 0.531475 \\
\hline 0.44849 & 0.0191251 & 0.445017 & 0.0297537 & 0.436677 & 0.0180693 & 0.522591 \\
\hline 0.439888 & 0.0284899 & 0.449031 & 0.0299489 & 0.431352 & 0.0179968 & 0.537332 \\
\hline 0.444822 & 0.0291303 & 0.440165 & 0.0319653 & 0.430728 & 0.0239315 & 0.52641 \\
\hline 0.444286 & 0.0232663 & 0.452021 & 0.0352505 & 0.441814 & 0.0186116 & 0.527584 \\
\hline 0.448896 & 0.025368 & 0.45058 & 0.0320641 & 0.438549 & 0.0219439 & 0.535206 \\
\hline 0.446733 & 0.0262391 & 0.433055 & 0.0245267 & 0.444919 & 0.0172201 & 0.535427 \\
\hline 0.448952 & 0.0316397 & 0.43455 & 0.0259569 & 0.440354 & 0.0158394 & 0.524137 \\
\hline 0.438856 & 0.0256272 & 0.438909 & 0.0271416 & 0.4431 & 0.0201687 & 0.530618 \\
\hline 0.430536 & 0.0234954 & 0.430862 & 0.0316588 & 0.445726 & 0.0195846 & 0.526469 \\
\hline 0.433829 & 0.0220303 & 0.44081 & 0.0301192 & 0.442375 & 0.0188333 & 0.538802 \\
\hline 0.446647 & 0.0299802 & 0.429213 & 0.0298737 & 0.444257 & 0.0256246 & 0.532415 \\
\hline 0.428597 & 0.0298484 & 0.433507 & 0.0322583 & 0.442887 & 0.0172371 & 0.524664 \\
\hline 0.43277 & 0.0308257 & 0.434804 & 0.0343242 & 0.440547 & 0.0173015 & 0.532001 \\
\hline 0.431558 & 0.0233478 & 0.420173 & 0.028211 & 0.442825 & 0.0208971 & 0.530249 \\
\hline 0.429473 & 0.0268805 & 0.426084 & 0.0288286 & 0.435989 & 0.0211324 & 0.520471 \\
\hline 0.430543 & 0.0262861 & 0.416297 & 0.0204009 & 0.431914 & 0.018096 & 0.526004 \\
\hline 0.429612 & 0.0299126 & 0.414131 & 0.0353871 & 0.422874 & 0.0190701 & 0.50621 \\
\hline 0.432457 & 0.0221065 & 0.415197 & 0.0322332 & 0.420474 & 0.0182175 & 0.534534 \\
\hline 0.42918 & 0.0247558 & 0.417213 & 0.0252988 & 0.417662 & 0.0122951 & 0.526074 \\
\hline 0.433287 & 0.0211033 & 0.41481 & 0.0205661 & 0.408779 & 0.0126309 & 0.517439 \\
\hline 0.422265 & 0.0291305 & 0.411228 & 0.0279494 & 0.411051 & 0.0201222 & 0.503301 \\
\hline 0.426941 & 0.0249578 & 0.403551 & 0.0332775 & 0.400942 & 0.0147351 & 0.49242 \\
\hline 0.421887 & 0.0206214 & 0.406789 & 0.0263897 & 0.391774 & 0.0160697 & 0.509823 \\
\hline 0.426264 & 0.0257914 & 0.398097 & 0.0282741 & 0.38851 & 0.0149234 & 0.484627 \\
\hline
\end{tabular}




\begin{tabular}{|c|c|c|c|c|c|c|}
\hline 0.41676 & 0.0268354 & 0.39936 & 0.0294571 & 0.384151 & 0.0193837 & 0.498425 \\
\hline 0.421209 & 0.020349 & 0.400008 & 0.0241821 & 0.384807 & 0.0148081 & 0.495916 \\
\hline 0.417547 & 0.0273405 & 0.396981 & 0.0327569 & 0.379624 & 0.0187437 & 0.473071 \\
\hline 0.421017 & 0.0258252 & 0.391072 & 0.0299095 & 0.368199 & 0.0184375 & 0.484358 \\
\hline 0.416177 & 0.0242499 & 0.395391 & 0.0279784 & 0.370146 & 0.0178655 & 0.470872 \\
\hline 0.411402 & 0.032982 & 0.388369 & 0.0191231 & 0.364004 & 0.0158059 & 0.469748 \\
\hline 0.412767 & 0.0320025 & 0.386457 & 0.0302409 & 0.363875 & 0.0142398 & 0.482686 \\
\hline 0.414777 & 0.0273483 & 0.390196 & 0.0221356 & 0.363519 & 0.020292 & 0.470264 \\
\hline 0.411709 & 0.0206306 & 0.387957 & 0.0295484 & 0.360907 & 0.0182947 & 0.478936 \\
\hline 0.415038 & 0.027779 & 0.389876 & 0.0279417 & 0.357785 & 0.0167071 & 0.474933 \\
\hline 0.403879 & 0.0324922 & 0.384699 & 0.0298785 & 0.356204 & 0.0143909 & 0.483068 \\
\hline 0.405351 & 0.0269945 & 0.383516 & 0.0260367 & 0.354213 & 0.0180597 & 0.455878 \\
\hline 0.406875 & 0.0245078 & 0.373106 & 0.0217247 & 0.351504 & 0.0156729 & 0.459289 \\
\hline 0.407713 & 0.021255 & 0.378723 & 0.0235752 & 0.34851 & 0.0192152 & 0.463278 \\
\hline 0.400086 & 0.0241934 & 0.37175 & 0.0162644 & 0.353525 & 0.014827 & 0.459746 \\
\hline 0.399691 & 0.0237797 & 0.375303 & 0.0246921 & 0.348863 & 0.0153023 & 0.4461 \\
\hline 0.411241 & 0.0279025 & 0.374814 & 0.0283775 & 0.348893 & 0.0168292 & 0.463354 \\
\hline 0.400062 & 0.0235072 & 0.370103 & 0.0262242 & 0.349793 & 0.0135764 & 0.445783 \\
\hline 0.399545 & 0.0298336 & 0.366164 & 0.02 & 0.3 & 0.01 & 48113 \\
\hline 0.402075 & 0.0193604 & 0.364866 & 0.0296898 & 0.342594 & 0.0140024 & 0.451382 \\
\hline 0.395129 & 0.0294175 & 0.363019 & 0.0209717 & 0.333897 & 0.0144009 & 0.454423 \\
\hline 0.39 & 0.023 & 0.364666 & 044 & 0.33 & 038 & 5679 \\
\hline 0.388081 & 0.0201332 & 0.360095 & 0.0247132 & 0.336044 & 0.0138537 & 0.438064 \\
\hline 0.383301 & 0.0234168 & 0.364268 & 0.0260682 & 0.33645 & 0.0185271 & 0.455674 \\
\hline 0.384514 & 0.0259593 & 0.360905 & 0.0246477 & 0.331843 & 0.0147678 & 0.455143 \\
\hline 0.383478 & 0.0243364 & 0.353309 & 0.0300634 & 0.334024 & 0.0177825 & 0.450816 \\
\hline 0.390956 & 0.0201808 & 0.359639 & 0.0214211 & 0.32672 & 0.0149284 & 0.44354 \\
\hline 0.38649 & 0.028489 & 0.355569 & 0.019179 & 0.327954 & 0.0176358 & 0.440393 \\
\hline 0.383669 & 0.0228458 & 0.354969 & 0.0188604 & 0.329143 & 0.0169686 & 0.425687 \\
\hline 0.386343 & 0.024239 & 0.357817 & 0.0214076 & 0.324859 & 0.011571 & 0.428787 \\
\hline 0.380068 & 0.0222764 & 0.348366 & 0.023 & 0.327199 & 0.013 & 0.429706 \\
\hline 0.377566 & 0.0268287 & 0.357513 & 0.0218038 & 0.327696 & 0.0158422 & 0.437475 \\
\hline 0.380191 & 0.0248201 & 0.350646 & 0.0254279 & 0.326269 & 0.013864 & 0.438539 \\
\hline 0.378618 & 0.0205123 & 0.348517 & 0.0260842 & 0.323946 & 0.0170505 & 0.433899 \\
\hline 0.381004 & 0.0180105 & 0.353033 & 0.0259264 & 0.324282 & 0.0129055 & 0.437607 \\
\hline 0.378779 & 0.020012 & 0.354302 & 0.0266583 & 0.327375 & 0.0169762 & 0.443514 \\
\hline 0.36375 & 0.0186736 & 0.350417 & 0.0201617 & 0.322353 & 0.0154777 & 0.422961 \\
\hline 0.374134 & 0.0216623 & 0.347677 & 0.0182801 & 0.325434 & 0.018709 & 0.435805 \\
\hline 0.370352 & 0.0260606 & 0.346413 & 0.0213956 & 0.323016 & 0.0161187 & 0.431045 \\
\hline 0.371657 & 0.026431 & 0.353314 & 0.0258247 & 0.32384 & 0.0161862 & 0.43657 \\
\hline 0.361418 & 0.023488 & 0.350003 & 0.0203981 & 0.324543 & 0.0165774 & 0.429246 \\
\hline 0.36631 & 0.0250857 & 0.355366 & 0.0237927 & 0.328706 & 0.0156096 & 0.42496 \\
\hline 0.368611 & 0.022338 & 0.359108 & 0.0233109 & 0.336022 & 0.0169089 & 0.441032 \\
\hline 0.372983 & 0.0247618 & 0.353636 & 0.0262258 & 0.334969 & 0.016741 & 0.439477 \\
\hline 0.373695 & 0.0203167 & 0.361711 & 0.023256 & 0.337345 & 0.0149603 & 0.432867 \\
\hline 0.372565 & 0.0238763 & 0.364187 & 0.0292382 & 0.341803 & 0.015345 & 0.440617 \\
\hline 0.367507 & 0.0252263 & 0.372445 & 0.0283031 & 0.347526 & 0.0186873 & 0.460382 \\
\hline
\end{tabular}




\begin{tabular}{|c|c|c|c|c|c|c|}
\hline 0.373101 & 0.0273198 & 0.371106 & 0.0279312 & 0.350389 & 0.0186788 & 0.460806 \\
\hline 0.372747 & 0.0227219 & 0.381592 & 0.028381 & 0.360717 & 0.0155083 & 0.470588 \\
\hline 0.374305 & 0.0237585 & 0.396778 & 0.0320446 & 0.368679 & 0.0170051 & 0.47361 \\
\hline .385034 & 0.028005 & 0.406476 & 0.0309305 & 0.377006 & 0.0158336 & 0.481107 \\
\hline 0.382458 & 0.026142 & 0.405125 & 0.0329599 & 0.398271 & 0.0162245 & 0.50555 \\
\hline 0.387572 & 0.022275 & 0.41799 & 0.0365811 & 0.407015 & 0.0170719 & 0.509076 \\
\hline 0.390933 & 0.0248179 & 0.424168 & 0.04066 & 0.421487 & 0.0157775 & 0.513595 \\
\hline 0.393666 & 0.0231348 & 0.445936 & 0.0431036 & 0.44217 & 0.0198121 & 0.544598 \\
\hline 0.403038 & 0.0248605 & 0.457763 & 0.0484866 & 0.459342 & 0.0261641 & 0.557364 \\
\hline 0.412109 & 0.0293871 & 0.468915 & 0.048262 & 0.472039 & 0.0189555 & 0.559018 \\
\hline 0.422185 & 0.0243386 & 0.479725 & 0.0518964 & 0.492576 & 0.0189174 & 0.593498 \\
\hline 0.438379 & 0.0236905 & 0.494206 & 0.0631042 & 0.517385 & 0.0223507 & 0.607107 \\
\hline 0.44605 & 0.0311423 & 0.514799 & 0.0580688 & 0.532266 & 0.0151618 & 0.611955 \\
\hline 0.457509 & 0.030931 & 0.526565 & 0.0629657 & 0.553971 & 0.0222865 & 0.635754 \\
\hline 0.482695 & 0.0248145 & 0.540793 & 0.0758684 & 0.574643 & 0.0214618 & 0.6613 \\
\hline 0.492618 & 0.0331723 & 0.554153 & 0.0665572 & 0.5917 & 0.0237729 & 0.672975 \\
\hline 0.517868 & 0.0280956 & 0.563491 & 0.0778713 & 0.613373 & 0.0285312 & 0.701737 \\
\hline 0.550447 & 0.0301159 & 0.576489 & 0.0681481 & 0.631762 & 0.0318014 & 0.703903 \\
\hline 0.567068 & 0.0416313 & 0.588484 & 0.0701704 & 0.641743 & 0.0259183 & 0.706512 \\
\hline 0.599097 & 0.0285495 & 0.589085 & 0.0683531 & 0.656873 & 0.0313818 & 0.724015 \\
\hline 0.634362 & 0.0391401 & 0.588654 & 0.0671515 & 0.675727 & 0.0327963 & 0.723274 \\
\hline 0.667313 & 0.0428992 & 0.586271 & 0.0760226 & 0.67239 & 0.026418 & 0.726437 \\
\hline 0.691694 & 0.0380604 & 0.58316 & 0.0690281 & 0.684147 & 0.0319127 & 0.713357 \\
\hline 0.739457 & 0.0502417 & 0.590378 & 0.0717178 & 0.689146 & 0.0360915 & 0.725761 \\
\hline 0.758465 & 0.0459265 & 0.581359 & 0.077765 & 0.679632 & 0.0295235 & 0.698522 \\
\hline 0.787311 & 0.0473008 & 0.573979 & 0.0608476 & 0.673907 & 0.0236784 & 0.727899 \\
\hline 0.814967 & 0.0406562 & 0.565541 & 0.0702577 & 0.663436 & 0.0301339 & 0.702997 \\
\hline 0.846948 & 0.0578279 & 0.548886 & 0.0577082 & 0.652603 & 0.0362848 & 0.707964 \\
\hline 0.865456 & 0.0513862 & 0.537688 & 0.0533707 & 0.63547 & 0.0258986 & 0.671476 \\
\hline 0.879251 & 0.0628924 & 0.522067 & 0.0471196 & 0.615043 & 0.0280215 & 0.654165 \\
\hline 0.894939 & 0.0611724 & 0.500567 & 0.0460074 & 0.603712 & 0.0296525 & 0.624571 \\
\hline 0.903477 & 0.0581665 & 0.494213 & 0.0412052 & 0.579377 & 0.0272789 & 0.620182 \\
\hline 0.896992 & 0.0692615 & 0.473201 & 0.0422399 & 0.557557 & 0.0312242 & 0.594417 \\
\hline 0.893701 & 0.0633693 & 0.457416 & 0.0314412 & 0.540994 & 0.0258737 & 0.569904 \\
\hline 0.878145 & 0.0576213 & 0.443713 & 0.0365546 & 0.514235 & 0.0273159 & 0.555993 \\
\hline 0.860963 & 0.0551546 & 0.425245 & 0.0299615 & 0.482806 & 0.0193363 & 0.531561 \\
\hline 0.842802 & 0.0663398 & 0.409666 & 0.0355294 & 0.460869 & 0.0205565 & 0.505132 \\
\hline 0.811444 & 0.0547667 & 0.399808 & 0.0295306 & 0.447807 & 0.0238067 & 0.492208 \\
\hline 0.78513 & 0.0595718 & 0.382475 & 0.0234908 & 0.419298 & 0.020143 & 0.470888 \\
\hline 0.750425 & 0.0570436 & 0.371953 & 0.0152138 & 0.399249 & 0.0172037 & 0.462291 \\
\hline 0.713574 & 0.0514614 & 0.361065 & 0.0246954 & 0.391015 & 0.0215668 & 0.440067 \\
\hline 0.683845 & 0.0378127 & 0.352008 & 0.0252994 & 0.371178 & 0.0187227 & 0.430989 \\
\hline 0.634551 & 0.0507087 & 0.34189 & 0.0189318 & 0.353336 & 0.0228171 & 0.424402 \\
\hline 0.601395 & 0.039391 & 0.34211 & 0.0303332 & 0.350046 & 0.0143282 & 0.41805 \\
\hline 0.585071 & 0.0389024 & 0.334003 & 0.0195048 & 0.335526 & 0.0170213 & 0.402572 \\
\hline 0.54545 & 0.0333785 & 0.32845 & 0.0251455 & 0.330156 & 0.0175537 & 0.407153 \\
\hline 0.518376 & 0.0328267 & 0.321969 & 0.019798 & 0.315474 & 0.0154344 & 0.409577 \\
\hline
\end{tabular}




\begin{tabular}{|c|c|c|c|c|c|c|}
\hline 0.495429 & 0.0273562 & 0.323996 & 0.0252299 & 0.316726 & 0.0168537 & 0.406828 \\
\hline 0.470301 & 0.0297434 & 0.316517 & 0.023792 & 0.311022 & 0.0168535 & 0.387461 \\
\hline 0.444803 & 0.0247156 & 0.315926 & 0.025141 & 0.309344 & 0.0210127 & .390184 \\
\hline .433332 & 0.036724 & 0.316907 & 0.0195148 & 0.304958 & 0.0104925 & 0.388948 \\
\hline .420973 & 0.0250667 & 0.310027 & 0.0230787 & 0.303111 & 0.0113705 & 0.383744 \\
\hline 0.407953 & 0.034263 & 0.312031 & 0.0243748 & 0.302774 & 0.0129783 & 0.3908 \\
\hline 0.406882 & 0.0233342 & 0.304384 & 0.0175278 & 0.295838 & 0.0147579 & 0.391398 \\
\hline 0.399343 & 0.0295255 & 0.313116 & 0.0224096 & 0.297754 & 0.0159275 & 0.391301 \\
\hline 0.390484 & 0.0243817 & 0.310749 & 0.0222453 & 0.296615 & 0.0182349 & 0.376526 \\
\hline 0.379143 & 0.0262167 & 0.311588 & 0.02109 & 0.290512 & 0.0163451 & 0.382768 \\
\hline 0.383608 & 0.0248558 & 0.300284 & 0.0205372 & 0.292673 & 0.0120241 & 0.375931 \\
\hline 0.379453 & 0.0244322 & 0.303458 & 0.0238919 & 0.289224 & 0.0145434 & 0.367125 \\
\hline 0.370782 & 0.0258836 & 0.298525 & 0.0225769 & 0.288329 & 0.0171424 & 0.370066 \\
\hline 0.372391 & 0.026099 & 0.296751 & 0.0222386 & 0.282395 & 0.0142138 & 0.370324 \\
\hline 0.364336 & 0.0270242 & 0.299338 & 0.0200882 & 0.282617 & 0.016611 & 0.366802 \\
\hline 0.351734 & 0.024795 & 0.302361 & 0.0204555 & 0.283508 & 0.0173629 & 0.363711 \\
\hline 0.352457 & 0.0249939 & 0.30109 & 0.0184389 & 0.285317 & 0.0177184 & 0.365954 \\
\hline 0.353244 & 0.022364 & 0.296815 & 0.0206117 & 0.273842 & 0.0138342 & 0.37024 \\
\hline 0.341526 & 0.02 & 0.25 & 0.01 & 0.27 & 0.013893 & .364688 \\
\hline 0.341346 & 0.0234739 & 0.296769 & 0.0223972 & 0.277553 & 0.0155967 & 0.373243 \\
\hline 0.344861 & 0.0213764 & 0.289373 & 0.0186873 & 0.276121 & 0.0155281 & 0.357431 \\
\hline 0.329023 & 0.02 & 838 & 0.0 & 167 & 1609 & 0.368785 \\
\hline 0.327122 & 0.0235436 & 0.293197 & 0.0224255 & 0.265498 & 0.0135769 & 0.359529 \\
\hline 0.313202 & 0.0197215 & 0.29208 & 0.0194603 & 0.265898 & 0.0141002 & 0.358981 \\
\hline 0.317661 & 0.0191942 & 0.29044 & 0.0166819 & 0.268474 & 0.0141549 & 0.344414 \\
\hline 0.312922 & 0.0261367 & 0.288178 & 0.0188223 & 0.268206 & 0.0165416 & 0.355978 \\
\hline 0.306693 & 0.0220535 & 0.290739 & 0.0184721 & 0.26431 & 0.0148252 & 0.357948 \\
\hline 0.306369 & 0.0243737 & 0.283141 & 0.0232214 & 0.263489 & 0.0110044 & 0.353782 \\
\hline 0.304682 & 0.0215716 & 0.28579 & 0.0194251 & 0.265134 & 0.0169254 & 0.355003 \\
\hline 0.295589 & 0.0186517 & 0.280449 & 0.0177733 & 0.258599 & 0.0136566 & 0.353974 \\
\hline 0.287162 & 0.0258775 & 0.280293 & 0.0210475 & 0.255546 & 0.0153423 & 0.351172 \\
\hline 0.296714 & 0.0209079 & 0.279489 & 0.0203061 & 0.257566 & 0.0116739 & 0.358061 \\
\hline 0.294479 & 0.0192243 & 0.286867 & 0.0199123 & 0.258793 & 0.01149 & 0.357117 \\
\hline 0.288366 & 0.0208972 & 0.28008 & 0.0183879 & 0.260148 & 0.0121742 & 0.353041 \\
\hline 0.292331 & 0.0235089 & 0.277779 & 0.018558 & 0.25552 & 0.0111247 & 0.353493 \\
\hline 0.293592 & 0.021106 & 0.281663 & 0.0215032 & 0.259922 & 0.0150564 & 0.360668 \\
\hline 0.286132 & 0.0147861 & 0.280999 & 0.024556 & 0.260046 & 0.013033 & 0.345433 \\
\hline 0.287881 & 0.0229284 & 0.277772 & 0.0209642 & 0.255709 & 0.0122067 & 0.346094 \\
\hline 0.283464 & 0.0210654 & 0.282026 & 0.0153962 & 0.258439 & 0.010954 & 0.334144 \\
\hline 0.28629 & 0.0254145 & 0.283658 & 0.0242186 & 0.256848 & 0.00922117 & 0.353049 \\
\hline 0.2804 & 0.0190923 & 0.279639 & 0.0177952 & 0.259559 & 0.0158335 & 0.34509 \\
\hline 0.28157 & 0.0230214 & 0.275144 & 0.0229916 & 0.26185 & 0.0157154 & 0.352896 \\
\hline 0.283389 & 0.020536 & 0.275533 & 0.0148286 & 0.255736 & 0.0124974 & 0.361903 \\
\hline 0.286848 & 0.0219463 & 0.275803 & 0.02021 & 0.256361 & 0.0142218 & 0.35531 \\
\hline 0.276703 & 0.0203844 & 0.276887 & 0.0214737 & 0.256012 & 0.0152786 & 0.350449 \\
\hline 0.279708 & 0.0231304 & 0.272893 & 0.0187178 & 0.260718 & 0.013051 & 0.344431 \\
\hline 0.285129 & 0.0159699 & 0.280602 & 0.0179683 & 0.260765 & 0.0117901 & 0.343951 \\
\hline
\end{tabular}




\begin{tabular}{|c|c|c|c|c|c|c|}
\hline 0.277747 & 0.014617 & 0.27412 & 0.0209203 & 0.257982 & 0.00934941 & 0.331298 \\
\hline 0.277063 & 0.0238026 & 0.271916 & 0.0162068 & 0.258131 & 0.0127139 & 0.338477 \\
\hline 0.274781 & 0.0159088 & 0.277775 & 0.0215939 & 0.255906 & 0.0144028 & 346172 \\
\hline 0.280104 & 0.0215283 & 0.276407 & 0.0205147 & 0.261936 & 0.0170737 & 350509 \\
\hline 0.276817 & 0.0146052 & 0.277048 & 0.0122776 & 0.263135 & 0.0103681 & 0.3455 \\
\hline 0.271715 & 0.0211279 & 0.271532 & 0.022029 & 0.261213 & 0.0139539 & 347309 \\
\hline 0.272281 & 0.0191874 & 0.275464 & 0.0156341 & 0.255851 & 0.0109707 & 0.336806 \\
\hline 0.264315 & 0.0190926 & 0.272351 & 0.0195711 & 0.260729 & 0.0136486 & 0.344535 \\
\hline 0.27503 & 0.0219475 & 0.27476 & 0.021697 & 0.259164 & 0.0139141 & 0.339272 \\
\hline 0.272855 & 0.024648 & 0.269812 & 0.0193894 & 0.260187 & 0.0145563 & 0.340807 \\
\hline 0.26698 & 0.0200084 & 0.271753 & 0.0205855 & 0.261323 & 0.0123258 & 0.341503 \\
\hline 0.268665 & 0.0191836 & 0.269683 & 0.0212365 & 0.260684 & 0.0108759 & 0.336682 \\
\hline 0.274406 & 0.025276 & 0.265316 & 0.0188411 & 0.261477 & 0.0175706 & 0.330529 \\
\hline 0.264842 & 0.0164092 & 0.273912 & 0.0199248 & 0.258304 & 0.0148587 & 0.35035 \\
\hline 0.266152 & 0.0147587 & 0.27077 & 0.0159474 & 0.257504 & 0.013459 & 0.333739 \\
\hline 0.265782 & 0.019789 & 0.278392 & 0.0162054 & 0.259649 & 0.017781 & 0.337163 \\
\hline 0.269076 & 0.0192794 & 0.27059 & 0.0173784 & 0.259099 & 0.0109478 & 0.346297 \\
\hline 0.265818 & 0.0196514 & 0.268835 & 0.0189252 & 0.260861 & 0.0170058 & 0.326312 \\
\hline 0.265564 & 0.0175797 & 0.272724 & 0.0154226 & 0.255313 & 0.0137512 & 0.345453 \\
\hline 0.266491 & 0.0193402 & 0.261665 & 0.015721 & 0.251063 & 0.0169964 & 0.34336 \\
\hline 0.263352 & 0.019464 & 0.261776 & 0.0168088 & 0.25469 & 0.0114121 & 0.337609 \\
\hline 0.265606 & 0.0195495 & 0.26068 & 0.0186471 & 0.260021 & 0.0111912 & 0.338492 \\
\hline 0.262767 & 0.0185545 & 0.260388 & 0.0141556 & 0.249275 & 0.0120857 & 0.33932 \\
\hline 0.266319 & 0.017548 & 0.26793 & 0.0224945 & 0.25 & 0.0121221 & 0.345342 \\
\hline 0.264803 & 0.0175329 & 0.261265 & 0.0265667 & 0.252723 & 0.0157176 & 0.336355 \\
\hline 0.263926 & 0.021087 & 0.257024 & 0.0204219 & 0.251131 & 0.0117838 & 0.33458 \\
\hline 0.259475 & 0.0190185 & 0.265298 & 0.0155137 & 86 & 0.0123298 & 0.334462 \\
\hline 0.262233 & 0.0176373 & 0.262517 & 0.0213234 & 0.245047 & 0.0135779 & 0.328406 \\
\hline 0.264868 & 0.0187316 & 0.261576 & 0.0195948 & 0.244202 & 0.0161001 & 0.338948 \\
\hline 0.260026 & 0.0191576 & 0.265807 & 0.023043 & 0.24406 & 0.0139055 & 0.325044 \\
\hline 0.259806 & 0.017218 & 0.266043 & 0.0183182 & 0.247227 & 0.0147327 & 0.330919 \\
\hline 0.259026 & 0.0191524 & 0.26003 & 0.0197557 & 0.24266 & 0.0135949 & 0.330978 \\
\hline 0.256145 & 0.0225233 & 0.251723 & 0.0175915 & 0.24453 & 0.0139724 & 0.335887 \\
\hline 0.255501 & 0.0185535 & 0.259618 & 0.0207703 & 0.24406 & 0.0134211 & 0.337256 \\
\hline 0.257449 & 0.0178059 & 0.256709 & 0.0185247 & 0.242874 & 0.0177739 & 0.335113 \\
\hline 0.25981 & 0.0170009 & 0.251136 & 0.0159116 & 0.241289 & 0.0109185 & 0.327719 \\
\hline 0.256918 & 0.0205417 & 0.259195 & 0.0183691 & 0.235618 & 0.0149047 & 0.331958 \\
\hline 0.253793 & 0.0196498 & 0.254895 & 0.0173708 & 0.239193 & 0.0161947 & 0.335915 \\
\hline 0.257019 & 0.019751 & 0.257002 & 0.0136024 & 0.241215 & 0.00991097 & 0.330656 \\
\hline 0.252662 & 0.0188534 & 0.259581 & 0.0184446 & 0.242797 & 0.00986263 & 0.334659 \\
\hline 0.254022 & 0.0193541 & 0.256159 & 0.0163115 & 0.23685 & 0.014024 & 0.323421 \\
\hline 0.258615 & 0.0182826 & 0.249937 & 0.0185203 & 0.239852 & 0.0116542 & 0.325123 \\
\hline 0.248169 & 0.0179587 & 0.252428 & 0.0191627 & 0.237274 & 0.0156142 & 0.309492 \\
\hline 0.248547 & 0.0173315 & 0.249043 & 0.018624 & 0.233996 & 0.0129199 & 0.321638 \\
\hline 0.252594 & 0.0214461 & 0.249677 & 0.018005 & 0.233935 & 0.0160161 & 0.326289 \\
\hline 0.257026 & 0.0181573 & 0.252301 & 0.0171399 & 0.229808 & 0.0118027 & 0.319769 \\
\hline 0.246938 & 0.01977 & 0.24949 & 0.018858 & 0.23256 & 0.0108665 & 0.305782 \\
\hline
\end{tabular}




\begin{tabular}{|c|c|c|c|c|c|c|}
\hline 0.247233 & 0.0189572 & 0.253578 & 0.02015 & 0.232992 & 0.0119223 & 0.313841 \\
\hline 0.251283 & 0.0188273 & 0.254303 & 0.0184494 & 0.237569 & 0.0125443 & 0.302673 \\
\hline 0.253949 & 0.0204754 & 0.251872 & 0.0175173 & 0.23309 & 0.0122934 & 0.311881 \\
\hline 0.251024 & 0.0180801 & 0.250921 & 0.0129434 & 0.230535 & 0.0165905 & 0.309531 \\
\hline 0.250638 & 0.0206616 & 0.256284 & 0.0200453 & 0.227447 & 0.0114636 & 0.318276 \\
\hline 0.24842 & 0.0177291 & 0.25093 & 0.0167111 & 0.230494 & 0.0135953 & 0.309221 \\
\hline 0.248078 & 0.0201888 & 0.249256 & 0.014967 & 0.232009 & 0.0105185 & 0.31135 \\
\hline 0.245072 & 0.021382 & 0.249104 & 0.0189622 & 0.231216 & 0.0144359 & 0.320389 \\
\hline 0.246583 & 0.0188218 & 0.248567 & 0.0196055 & 0.227687 & 0.0130605 & 0.316859 \\
\hline 0.250689 & 0.0138213 & 0.24928 & 0.0152275 & 0.22827 & 0.0163027 & 0.304095 \\
\hline 0.253925 & 0.0182461 & 0.244103 & 0.0212808 & 0.230039 & 0.0143527 & 0.316771 \\
\hline 0.245077 & 0.0174209 & 0.249729 & 0.0148449 & 0.226066 & 0.0130365 & 0.305995 \\
\hline 0.246931 & 0.0213814 & 0.249222 & 0.0162527 & 0.225548 & 0.0143107 & 0.316063 \\
\hline 0.249779 & 0.0198636 & 0.239958 & 0.018313 & 0.228722 & 0.00963702 & 0.309687 \\
\hline 0.255666 & 0.0205387 & 0.242053 & 0.0174349 & 0.230675 & 0.0145003 & 0.315254 \\
\hline 0.250918 & 0.0175241 & 0.245141 & 0.0218096 & 0.228096 & 0.0149033 & 0.306365 \\
\hline 0.245217 & 0.0187661 & 0.24736 & 0.0181951 & 0.225006 & 0.0113689 & 0.31909 \\
\hline 0.244498 & 0.0197807 & 0.240479 & 0.0160237 & 0.223869 & 0.0124561 & 0.310816 \\
\hline 0.245013 & 0.0212035 & 0.239783 & 0.0150053 & 0.221842 & 0.0146421 & 0.31096 \\
\hline 0.245348 & 0.0216577 & 0.239509 & 0.0157455 & 0.220865 & 0.010363 & 0.307723 \\
\hline 0.241634 & 0.017676 & 0.242724 & 0.0145197 & 0.228192 & 0.0129917 & 0.323991 \\
\hline 0.246731 & 0.0208495 & 0.246638 & 0.017768 & 0.220105 & 0.0117048 & 0.312889 \\
\hline 0.247196 & 0.0213043 & 0.23946 & 0.0170648 & 0.226677 & 0.0085934 & 0.313757 \\
\hline 0.244297 & 0.0217774 & 0.241325 & 0.0183522 & 0.223923 & 0.0130422 & 0.323958 \\
\hline 0.243253 & 0.0209588 & 0.239015 & 0.0162932 & 0.222874 & 0.0140699 & 0.320946 \\
\hline 0.24023 & 0.0197353 & 0.240801 & 0.0139794 & 0.223047 & 0.0128332 & 0.318251 \\
\hline 0.246558 & 0.0163443 & 0.24389 & 0.0191691 & 0.227514 & 0.0111752 & 0.311489 \\
\hline 0.244721 & 0.0193173 & 0.241441 & 0.0167696 & 0.222959 & 0.0137755 & 0.318998 \\
\hline 0.242825 & 0.0164349 & 0.245962 & 0.0166851 & 0.229751 & 0.0128231 & 0.309029 \\
\hline 0.241185 & 0.0196896 & 0.240097 & 0.0165497 & 0.226562 & 0.0176553 & 0.313237 \\
\hline 0.242918 & 0.0176806 & 0.246465 & 0.0170518 & 0.228166 & 0.0127141 & 0.316989 \\
\hline 0.243438 & 0.0130118 & 0.243331 & 0.0190475 & 0.226343 & 0.0126649 & 0.309623 \\
\hline 0.241478 & 0.0184988 & 0.238283 & 0.0163493 & 0.225768 & 0.0106195 & 0.325161 \\
\hline 0.241188 & 0.0179706 & 0.244287 & 0.0173442 & 0.229309 & 0.0104908 & 0.319047 \\
\hline 0.24426 & 0.0193528 & 0.243007 & 0.016279 & 0.224167 & 0.0134748 & 0.306999 \\
\hline 0.245041 & 0.0206107 & 0.247024 & 0.0166146 & 0.229621 & 0.0131704 & 0.323818 \\
\hline 0.238916 & 0.0192538 & 0.246012 & 0.0152845 & 0.233942 & 0.0154876 & 0.312928 \\
\hline 0.238169 & 0.0168474 & 0.252852 & 0.0215979 & 0.234685 & 0.0126689 & 0.324029 \\
\hline 0.245847 & 0.0213944 & 0.250318 & 0.0175329 & 0.231926 & 0.012808 & 0.323517 \\
\hline 0.244371 & 0.019353 & 0.25203 & 0.0161387 & 0.236712 & 0.0114929 & 0.329103 \\
\hline 0.245136 & 0.0221091 & 0.250576 & 0.0147274 & 0.235166 & 0.0158787 & 0.324428 \\
\hline 0.243365 & 0.0190316 & 0.254492 & 0.0196384 & 0.239352 & 0.0137516 & 0.336575 \\
\hline 0.243906 & 0.0151155 & 0.25379 & 0.0175235 & 0.237318 & 0.014859 & 0.333048 \\
\hline 0.236897 & 0.0164737 & 0.255917 & 0.0205671 & 0.241722 & 0.0154185 & 0.334611 \\
\hline 0.239821 & 0.0166612 & 0.254831 & 0.0215646 & 0.240685 & 0.0140956 & 0.350017 \\
\hline 0.241067 & 0.0201339 & 0.249607 & 0.0143519 & 0.241218 & 0.0148036 & 0.335902 \\
\hline 0.236623 & 0.0163735 & 0.256378 & 0.0187581 & 0.242351 & 0.0106048 & 0.325902 \\
\hline
\end{tabular}




\begin{tabular}{|c|c|c|c|c|c|c|}
\hline 0.238258 & 0.0174438 & 0.255064 & 0.0199358 & 0.241341 & 0.0129963 & 0.326483 \\
\hline 0.236096 & 0.0174141 & 0.253806 & 0.0248881 & 0.241948 & 0.0130385 & 0.330562 \\
\hline .243527 & 0.0202658 & 0.258383 & 0.0172118 & 0.242545 & 0.014866 & 0.336719 \\
\hline 0.235115 & 0.0248477 & 0.252601 & 0.0164026 & 0.248246 & 0.0101076 & 0.334478 \\
\hline 0.240025 & 0.0201476 & 0.253388 & 0.0131723 & 0.246107 & 0.0131547 & 0.338109 \\
\hline 0.238301 & 0.0167876 & 0.256048 & 0.0193946 & 0.250777 & 0.0128171 & 0.319648 \\
\hline 0.239849 & 0.01664 & 0.258311 & 0.0141232 & 0.247431 & 0.0131083 & 0.34208 \\
\hline 0.240216 & 0.0204838 & 0.260147 & 0.0201097 & 0.253413 & 0.0105149 & 0.333944 \\
\hline 0.246191 & 0.0185599 & 0.263836 & 0.0191044 & 0.250032 & 0.0135433 & 0.326671 \\
\hline 0.247733 & 0.0157628 & 0.259487 & 0.0158847 & 0.252223 & 0.0129857 & 0.332019 \\
\hline 0.242771 & 0.0205872 & 0.260871 & 0.0186991 & 0.252298 & 0.0143453 & 0.343343 \\
\hline 0.251116 & 0.0176693 & 0.262819 & 0.0202098 & 0.249627 & 0.0137455 & 0.329618 \\
\hline 0.242566 & 0.0211194 & 0.270161 & 0.0183526 & 0.25412 & 0.0140129 & 0.341155 \\
\hline 0.241041 & 0.0153999 & 0.260238 & 0.0192801 & 0.257667 & 0.016199 & 0.336944 \\
\hline 0.245332 & 0.0193527 & 0.268572 & 0.0220231 & 0.25681 & 0.0158564 & 0.339102 \\
\hline 0.249698 & 0.0209161 & 0.266673 & 0.0191169 & 0.261477 & 0.0124781 & 0.34365 \\
\hline 0.246361 & 0.0187447 & 0.267656 & 0.0208556 & 0.257788 & 0.014514 & 0.3456 \\
\hline 0.255628 & 0.0170824 & 0.265785 & 0.0213336 & 0.261365 & 0.0158476 & 0.346393 \\
\hline 0.248338 & 0.0215798 & 0.266245 & 0.0210613 & 0.268302 & 0.0147805 & 0.341154 \\
\hline 0.250683 & 0.0190933 & 0.274791 & 0.0252935 & 0.263527 & 0.018795 & 0.334057 \\
\hline 0.251378 & 0.017501 & 0.271457 & 0.0202732 & 0.268973 & 0.0157109 & 0.354107 \\
\hline 0.250708 & 0.0181034 & 0.275352 & 0.0191334 & 0.266185 & 0.0132741 & 0.336843 \\
\hline 0.263537 & 0.0201218 & 0.26787 & 0.0245271 & 0.26971 & 0.0147169 & 0.35018 \\
\hline 0.258382 & 0.0224865 & 0.267733 & 0.0196004 & 0.266688 & 0.0146543 & 0.344728 \\
\hline 0.258724 & 0.0173847 & 0.268184 & 0.0234804 & 0.267068 & 0.0120918 & 0.351041 \\
\hline 0.253556 & 0.0144528 & 0.26969 & 0.017459 & 0.265482 & 0.0125264 & 0.340079 \\
\hline 0.256851 & 0.0160574 & 0.272632 & 0.0183165 & 0.260571 & 0.0130128 & 0.331753 \\
\hline 0.261341 & 0.0136159 & 0.268195 & 0.0205682 & 0.26332 & 0.0141657 & 0.337056 \\
\hline 0.263329 & 0.0173735 & 0.26556 & 0.0218682 & 0.262707 & 0.0159349 & 0.330528 \\
\hline 0.259604 & 0.0187586 & 0.267707 & 0.0214511 & 0.258521 & 0.0112296 & 0.327129 \\
\hline 0.265189 & 0.0166286 & 0.262487 & 0.018865 & 0.256265 & 0.0150678 & 0.316782 \\
\hline 0.266129 & 0.0209993 & 0.259656 & 0.0222046 & 0.254925 & 0.0134471 & 0.333422 \\
\hline 0.256983 & 0.0212161 & 0.259591 & 0.0166557 & 0.252805 & 0.0116476 & 0.328058 \\
\hline 0.263538 & 0.0221196 & 0.257709 & 0.0265198 & 0.25351 & 0.0135655 & 0.319768 \\
\hline 0.261732 & 0.0191258 & 0.257516 & 0.0196323 & 0.246149 & 0.0113043 & 0.327053 \\
\hline 0.263179 & 0.0173206 & 0.255739 & 0.0195419 & 0.251416 & 0.0133039 & 0.316702 \\
\hline 0.25676 & 0.0197114 & 0.257054 & 0.0167733 & 0.247845 & 0.012272 & 0.311872 \\
\hline 0.261455 & 0.0213859 & 0.255497 & 0.0199886 & 0.239172 & 0.0115766 & 0.298045 \\
\hline 0.267623 & 0.0200966 & 0.255124 & 0.0195213 & 0.241168 & 0.0106515 & 0.309436 \\
\hline 0.262211 & 0.0183186 & 0.254615 & 0.0173472 & 0.240706 & 0.0126877 & 0.307795 \\
\hline 0.259937 & 0.0206268 & 0.251867 & 0.0225492 & 0.243037 & 0.0104659 & 0.314546 \\
\hline 0.260841 & 0.0194416 & 0.25302 & 0.0170564 & 0.238423 & 0.0141793 & 0.303471 \\
\hline 0.256609 & 0.022605 & 0.247917 & 0.0168012 & 0.233707 & 0.0164485 & 0.308355 \\
\hline 0.255887 & 0.0183815 & 0.250714 & 0.0199517 & 0.236393 & 0.0139696 & 0.303482 \\
\hline 0.256306 & 0.0210167 & 0.249856 & 0.0166009 & 0.233377 & 0.0136561 & 0.292654 \\
\hline 0.264449 & 0.0219444 & 0.252335 & 0.0123594 & 0.237451 & 0.0116811 & 0.311322 \\
\hline 0.257322 & 0.0206406 & 0.2513 & 0.0165841 & 0.237987 & 0.0126542 & 0.307467 \\
\hline
\end{tabular}




\begin{tabular}{|c|c|c|c|c|c|c|}
\hline 0.253595 & 0.0208055 & 0.249536 & 0.0167244 & 0.235949 & 0.0145444 & 0.299223 \\
\hline 0.255076 & 0.017184 & 0.254759 & 0.0205105 & 0.237248 & 0.012908 & 0.309331 \\
\hline 0.253853 & 0.0218653 & 0.257525 & 0.0154323 & 0.236615 & 0.00991837 & 0.298056 \\
\hline 0.256118 & 0.0196864 & 0.256507 & 0.020142 & 0.241656 & 0.0139047 & 0.291896 \\
\hline 0.256559 & 0.0199679 & 0.257268 & 0.0178659 & 0.239308 & 0.0163823 & 0.303195 \\
\hline 0.253577 & 0.0193584 & 0.255553 & 0.0180463 & 0.244697 & 0.0146415 & 0.310598 \\
\hline 0.259815 & 0.0197162 & 0.256795 & 0.0177784 & 0.240976 & 0.0134199 & 0.300678 \\
\hline 0.258973 & 0.0180138 & 0.25925 & 0.0189469 & 0.247129 & 0.0129592 & 0.311644 \\
\hline 0.255449 & 0.0201288 & 0.258986 & 0.0219466 & 0.245406 & 0.0119765 & 0.305946 \\
\hline 0.254879 & 0.0157095 & 0.256075 & 0.0148886 & 0.247387 & 0.0175978 & 0.303451 \\
\hline 0.261572 & 0.0169001 & 0.256075 & 0.0202035 & 0.242464 & 0.0150191 & 0.309253 \\
\hline 0.253651 & 0.0135474 & 0.258635 & 0.0192246 & 0.245912 & 0.0149196 & 0.307295 \\
\hline 0.25704 & 0.0152767 & 0.256115 & 0.0153183 & 0.246749 & 0.0142435 & 0.313538 \\
\hline 0.256059 & 0.0157284 & 0.260923 & 0.0188947 & 0.247973 & 0.010754 & 0.318148 \\
\hline 0.25869 & 0.0208011 & 0.265182 & 0.0205123 & 0.246762 & 0.0115915 & 0.31732 \\
\hline 0.257611 & 0.0234462 & 0.260926 & 0.0221442 & 0.247753 & 0.0151198 & 0.312263 \\
\hline 0.259557 & 0.0152981 & 0.261877 & 0.011037 & 0.251967 & 0.011825 & 0.312608 \\
\hline 0.265486 & 0.0175017 & 0.260859 & 0.0208183 & 0.253035 & 0.012847 & 0.303665 \\
\hline 0.259496 & 0.0186388 & 0.25995 & 0.0165057 & 0.252773 & 0.0157579 & 0.30616 \\
\hline 0.268564 & 0.0170582 & 0.262575 & 0.0191238 & 0.251524 & 0.0105994 & 0.303651 \\
\hline 0.264503 & 0.01785 & 0.266499 & 0.0168837 & 0.257151 & 0.0158074 & 0.309745 \\
\hline 0.269809 & 0.0210246 & 0.266911 & 0.0175426 & 0.24949 & 0.0143336 & 0.31594 \\
\hline 0.27401 & 0.0193395 & 0.264232 & 0.0196532 & 0.255878 & 0.0129313 & 0.31094 \\
\hline 0.268753 & 0.0165677 & 0.268831 & 0.0151321 & 0.257619 & 0.0141202 & 0.31066 \\
\hline 0.264448 & 0.0184198 & 0.274288 & 0.0172473 & 0.253901 & 0.0157259 & 0.316517 \\
\hline 0.27084 & 0.0181668 & 0.271761 & 0.0153745 & 0.259944 & 0.0136107 & 0.310459 \\
\hline 0.26634 & 0.0201062 & 0.27472 & 0.0171145 & 0.263356 & 0.0130558 & 0.315395 \\
\hline 0.269314 & 0.0249999 & 0.276043 & 0.0217503 & 0.261851 & 0.012495 & 0.300027 \\
\hline 0.27931 & 0.0208432 & 0.275298 & 0.0194158 & 0.265964 & 0.0165959 & 0.325597 \\
\hline 0.274173 & 0.0178583 & 0.27659 & 0.0215965 & 0.262218 & 0.0148951 & 0.318926 \\
\hline 0.275497 & 0.0168754 & 0.279104 & 0.0213919 & 0.266599 & 0.0131159 & 0.319211 \\
\hline 0.274046 & 0.0199992 & 0.27651 & 0.0134142 & 0.269312 & 0.0108876 & 0.331506 \\
\hline 0.274229 & 0.0150713 & 0.285527 & 0.0182795 & 0.269264 & 0.0158174 & 0.318617 \\
\hline 0.282176 & 0.0217481 & 0.287448 & 0.0195663 & 0.273643 & 0.0114633 & 0.329573 \\
\hline 0.277188 & 0.0182436 & 0.288939 & 0.0200272 & 0.278145 & 0.0145442 & 0.329574 \\
\hline 0.283395 & 0.029438 & 0.290449 & 0.0193032 & 0.278011 & 0.0139262 & 0.327839 \\
\hline 0.293447 & 0.02147 & 0.293782 & 0.0192664 & 0.284767 & 0.0118785 & 0.333681 \\
\hline 0.291957 & 0.0242524 & 0.295712 & 0.0223447 & 0.282282 & 0.011905 & 0.325146 \\
\hline 0.288238 & 0.0201036 & 0.300121 & 0.0206755 & 0.285985 & 0.0137477 & 0.342654 \\
\hline 0.297085 & 0.0198579 & 0.303414 & 0.0194929 & 0.281008 & 0.0175788 & 0.338996 \\
\hline 0.292414 & 0.0222423 & 0.304867 & 0.0205022 & 0.292217 & 0.0148348 & 0.346029 \\
\hline 0.298343 & 0.0215823 & 0.307159 & 0.0182492 & 0.303584 & 0.0158216 & 0.346311 \\
\hline 0.302435 & 0.0193748 & 0.311678 & 0.0165537 & 0.2971 & 0.0128455 & 0.351917 \\
\hline 0.305318 & 0.0186204 & 0.31077 & 0.0210675 & 0.302513 & 0.0149744 & 0.345724 \\
\hline 0.307052 & 0.0172961 & 0.312151 & 0.0202877 & 0.307319 & 0.018011 & 0.344396 \\
\hline 0.315578 & 0.0246177 & 0.32455 & 0.0162842 & 0.311035 & 0.0104974 & 0.355931 \\
\hline 0.309535 & 0.0160914 & 0.324464 & 0.0193158 & 0.313614 & 0.0129968 & 0.365586 \\
\hline
\end{tabular}




\begin{tabular}{|c|c|c|c|c|c|c|}
\hline 0.3195 & 0.0205146 & 0.328647 & 0.019343 & 0.319728 & 0.0146505 & 0.344061 \\
\hline 0.327723 & 0.0227885 & 0.334587 & 0.017437 & 0.327856 & 0.0139217 & 0.3666 \\
\hline 0.325154 & 0.0228474 & 0.341166 & 0.0193546 & 0.326911 & 0.0145971 & 0.368865 \\
\hline 0.337107 & 0.02517 & 0.334387 & 0.0219895 & 0.334797 & 0.0148965 & 0.3727 \\
\hline 0.340481 & 0.0240806 & 0.344126 & 0.0169604 & 0.341305 & 0.0153693 & 0.36944 \\
\hline 0.344691 & 0.0226591 & 0.351596 & 0.0253277 & 0.343831 & 0.0155535 & 0.37076 \\
\hline 0.345723 & 0.0215362 & 0.362356 & 0.0202448 & 0.359843 & 0.0161063 & 0.392019 \\
\hline 0.351097 & 0.0225799 & 0.368949 & 0.021168 & 0.358847 & 0.0241575 & 0.389321 \\
\hline 0.359662 & 0.0228517 & 0.376103 & 0.0249576 & 0.374329 & 0.0184786 & 0.391758 \\
\hline 0.373022 & 0.0228105 & 0.373629 & 0.0168554 & 0.378843 & 0.016309 & 0.382292 \\
\hline 0.382661 & 0.0192277 & 0.3821 & 0.0190488 & 0.382375 & 0.016032 & 0.390263 \\
\hline 0.384454 & 0.0237667 & 0.393662 & 0.0270993 & 0.391795 & 0.0131222 & 0.405344 \\
\hline 0.401172 & 0.0299731 & 0.399597 & 0.018787 & 0.401832 & 0.0208458 & 0.415503 \\
\hline 0.409669 & 0.0193708 & 0.412057 & 0.0225585 & 0.417209 & 0.0177888 & 0.440509 \\
\hline 0.427833 & 0.0221403 & 0.425042 & 0.0268172 & 0.427746 & 0.0202967 & 0.433404 \\
\hline 0.423884 & 0.0196037 & 0.425175 & 0.0218068 & 0.434242 & 0.0217048 & 0.430772 \\
\hline 0.431286 & 0.0274319 & 0.431904 & 0.0230492 & 0.453694 & 0.018051 & 0.437694 \\
\hline 0.43932 & 0.0374163 & 0.451444 & 0.0257424 & 0.458096 & 0.018933 & 0.448171 \\
\hline 0.460301 & 0.0220143 & 0.45954 & 0.0195778 & 0.47171 & 0.020557 & 0.448803 \\
\hline 0.467498 & 0.0226374 & 0.465541 & 0.0195421 & 0.48069 & 0.0235713 & 0.46984 \\
\hline 0.485763 & 0.0245541 & 0.486913 & 0.0247774 & 0.503254 & 0.0207355 & 0.478013 \\
\hline 0.501215 & 0.0206865 & 0.496331 & 0.0241 & 0.50 & 0.0199169 & 0.491279 \\
\hline 0.517999 & 0.022964 & 0.505245 & 0.0237538 & 0.528877 & 0.018907 & 0.500347 \\
\hline 0.525829 & 0.0304329 & 0.524045 & 0.0227386 & 0.543634 & 0.0200675 & 0.510165 \\
\hline 0.539617 & 0.0311932 & 0.542483 & 0.0245725 & 0.562452 & 0.0222842 & 0.50458 \\
\hline 0.554711 & 0.0293446 & 0.558073 & 0.0225456 & 0.570826 & 0.0165235 & 0.514434 \\
\hline 0.563485 & 0.0282601 & 0.558459 & 0.024943 & 0.591267 & 0.0208055 & 0.551466 \\
\hline 0.584863 & 0.0374057 & 0.591097 & 0.0265439 & 0.605661 & 0.0253969 & 0.53799 \\
\hline 0.597974 & 0.0312914 & 0.594685 & 0.0303151 & 0.622564 & 0.0239715 & 0.541721 \\
\hline 0.621668 & 0.0234541 & 0.617288 & 0.0175908 & 0.643001 & 0.0265125 & 0.579883 \\
\hline 0.630266 & 0.0262221 & 0.639387 & 0.0212707 & 0.654574 & 0.0264548 & 0.58198 \\
\hline 0.651383 & 0.0262129 & 0.644425 & 0.0286708 & 0.672716 & 0.0202292 & 0.598639 \\
\hline 0.664579 & 0.0329937 & 0.665703 & 0.0228046 & 0.692206 & 0.0166971 & 0.596296 \\
\hline 0.679424 & 0.0293892 & 0.6803 & 0.0306381 & 0.707317 & 0.0206571 & 0.620647 \\
\hline 0.702803 & 0.037263 & 0.695472 & 0.0302066 & 0.721745 & 0.0240791 & 0.637122 \\
\hline 0.717543 & 0.0302581 & 0.701715 & 0.0266521 & 0.74038 & 0.0260881 & 0.648112 \\
\hline 0.738762 & 0.0269172 & 0.728148 & 0.0363027 & 0.752107 & 0.0212028 & 0.651535 \\
\hline 0.750317 & 0.0294285 & 0.746214 & 0.0210226 & 0.782806 & 0.0221272 & 0.666351 \\
\hline 0.76257 & 0.0321664 & 0.76443 & 0.0286046 & 0.788835 & 0.0300879 & 0.679241 \\
\hline 0.785038 & 0.0280653 & 0.775273 & 0.0301175 & 0.817467 & 0.0256139 & 0.689324 \\
\hline 0.792314 & 0.0317504 & 0.793387 & 0.0265463 & 0.827224 & 0.0255009 & 0.695261 \\
\hline 0.809248 & 0.0408336 & 0.804558 & 0.0303222 & 0.841719 & 0.0309188 & 0.724238 \\
\hline 0.821245 & 0.0360119 & 0.81668 & 0.0244764 & 0.855422 & 0.0270313 & 0.723409 \\
\hline 0.838519 & 0.0383004 & 0.838846 & 0.0303673 & 0.863762 & 0.0292946 & 0.74356 \\
\hline 0.850703 & 0.0372058 & 0.843953 & 0.0305898 & 0.879883 & 0.0303741 & 0.739605 \\
\hline 0.876087 & 0.0366784 & 0.859676 & 0.027155 & 0.894282 & 0.021986 & 0.742947 \\
\hline 0.881935 & 0.0355927 & 0.874343 & 0.0254948 & 0.911158 & 0.0306164 & 0.766735 \\
\hline
\end{tabular}




\begin{tabular}{|c|c|c|c|c|c|c|}
\hline 0.901103 & 0.0318896 & 0.894189 & 0.0379775 & 0.917468 & 0.024621 & 0.774827 \\
\hline 0.89541 & 0.0478576 & 0.897684 & 0.0394417 & 0.927549 & 0.0216385 & 0.773016 \\
\hline 0.916155 & 0.0409685 & 0.90525 & 0.0284221 & 0.935135 & 0.0267759 & 0.795449 \\
\hline 0.914801 & 0.0343062 & 0.921731 & 0.0401188 & 0.930089 & 0.0282339 & 0.795953 \\
\hline 0.927805 & 0.0376201 & 0.92676 & 0.0311428 & 0.951941 & 0.0291146 & 0.824272 \\
\hline 0.933855 & 0.0371549 & 0.926131 & 0.0401681 & 0.961426 & 0.027854 & 0.826801 \\
\hline 0.944003 & 0.0326804 & 0.940907 & 0.0338074 & 0.959757 & 0.029191 & 0.829475 \\
\hline 0.940753 & 0.0308843 & 0.939835 & 0.0305851 & 0.967559 & 0.0199547 & 0.832529 \\
\hline 0.946405 & 0.0362133 & 0.948476 & 0.0369252 & 0.963502 & 0.0236022 & 0.85217 \\
\hline 0.95059 & 0.0333961 & 0.952949 & 0.036814 & 0.96873 & 0.0244649 & 0.845742 \\
\hline 0.946407 & 0.0356351 & 0.956301 & 0.0311772 & 0.967145 & 0.0250172 & 0.850407 \\
\hline 0.951156 & 0.0344957 & 0.948333 & 0.0406847 & 0.953022 & 0.0319778 & 0.868806 \\
\hline 0.949571 & 0.0386788 & 0.965795 & 0.0272638 & 0.9638 & 0.0262456 & 0.881659 \\
\hline 0.96223 & 0.0307557 & 0.956302 & 0.0336316 & 0.955216 & 0.0288643 & 0.873993 \\
\hline 0.934966 & 0.0353849 & 0.953957 & 0.0332246 & 0.949406 & 0.0272166 & 0.878795 \\
\hline 0.938129 & 0.0375357 & 0.950893 & 0.0328395 & 0.951729 & 0.0271549 & 0.889599 \\
\hline 0.929999 & 0.0445537 & 0.941494 & 0.0276489 & 0.934739 & 0.0272295 & 0.884116 \\
\hline 0.93228 & 0.0345616 & 0.933506 & 0.0318147 & 0.934796 & 0.0283444 & 0.891177 \\
\hline 0.922845 & 0.0465651 & 0.930504 & 0.0297254 & 0.922923 & 0.0307706 & 0.900298 \\
\hline 0.904975 & 0.0380633 & 0.92206 & 0.033863 & 0.908957 & 0.0273958 & 0.905223 \\
\hline 0.910688 & 0.0389107 & 0.910546 & 0.0351635 & 0.895107 & 0.0279129 & 0.90233 \\
\hline 0.883186 & 0.0409587 & 0.898423 & 0.0341396 & 0.876041 & 0.0271332 & 0.91527 \\
\hline 0.867318 & 0.0472885 & 0.886075 & 0.0313758 & 0.864285 & 0.027928 & 0.892902 \\
\hline 0.877362 & 0.0377859 & 0.885484 & 0.0323233 & 0.860319 & 0.0302198 & 0.907426 \\
\hline 0.849909 & 0.0402789 & 0.867651 & 0.0339184 & 0.844861 & 0.0236658 & 0.902206 \\
\hline 0.840253 & 0.0356852 & 0.850575 & 0.0301315 & 0.820688 & 0.0277322 & 0.891177 \\
\hline 0.841596 & 0.0378234 & 0.844478 & 0.0387539 & 0.802419 & 0.021734 & 0.894094 \\
\hline 0.816361 & 0.0312058 & 0.825184 & 0.0297723 & 0.793972 & 0.0267491 & 0.910327 \\
\hline 0.795635 & 0.0394129 & 0.810516 & 0.0285787 & 0.771204 & 0.0260231 & 0.879467 \\
\hline 0.787729 & 0.0297737 & 0.803445 & 0.0251958 & 0.755328 & 0.0261474 & 0.867001 \\
\hline 0.761763 & 0.035504 & 0.776573 & 0.0299291 & 0.740027 & 0.0235432 & 0.871759 \\
\hline 0.740243 & 0.0424939 & 0.773373 & 0.0290228 & 0.719495 & 0.024949 & 0.861737 \\
\hline 0.727485 & 0.0338681 & 0.743431 & 0.0304555 & 0.706509 & 0.0259197 & 0.850744 \\
\hline 0.714827 & 0.0376142 & 0.728435 & 0.024645 & 0.682654 & 0.0218836 & 0.819658 \\
\hline 0.692815 & 0.0308686 & 0.706083 & 0.0290139 & 0.659962 & 0.017507 & 0.836369 \\
\hline 0.679532 & 0.0350642 & 0.694365 & 0.0251753 & 0.649345 & 0.0223821 & 0.813577 \\
\hline 0.665615 & 0.0308921 & 0.673586 & 0.0253742 & 0.629123 & 0.0238431 & 0.808563 \\
\hline 0.652341 & 0.0393036 & 0.655165 & 0.0225838 & 0.610157 & 0.0277509 & 0.788899 \\
\hline 0.623003 & 0.0272862 & 0.642017 & 0.0277181 & 0.59974 & 0.0242178 & 0.76045 \\
\hline 0.606887 & 0.0294067 & 0.631687 & 0.0200558 & 0.578914 & 0.0219269 & 0.740352 \\
\hline 0.595097 & 0.0231564 & 0.597062 & 0.0249929 & 0.56302 & 0.0222141 & 0.705057 \\
\hline 0.584111 & 0.0269978 & 0.579906 & 0.0297153 & 0.541777 & 0.0228326 & 0.697487 \\
\hline 0.55871 & 0.0229015 & 0.574755 & 0.0213591 & 0.527519 & 0.0189671 & 0.66689 \\
\hline 0.552983 & 0.026084 & 0.556904 & 0.0259151 & 0.507079 & 0.0241988 & 0.648434 \\
\hline 0.527301 & 0.03269 & 0.540366 & 0.0276016 & 0.496136 & 0.0177872 & 0.62875 \\
\hline 0.511796 & 0.0218702 & 0.526021 & 0.0266893 & 0.472435 & 0.0196936 & 0.622544 \\
\hline 0.499199 & 0.024619 & 0.514344 & 0.0279486 & 0.468209 & 0.0232798 & 0.588399 \\
\hline
\end{tabular}




\begin{tabular}{|c|c|c|c|c|c|c|}
\hline 0.483743 & 0.025518 & 0.494356 & 0.0270112 & 0.454205 & 0.0187359 & 0.574432 \\
\hline 0.4725 & 0.027152 & 0.48496 & 0.0227396 & 0.437126 & 0.0194244 & 0.558717 \\
\hline 0.45968 & 0.0233471 & 0.476734 & 0.020807 & 0.424165 & 0.0167827 & 0.53179 \\
\hline 0.440072 & 0.0213094 & 0.453007 & 0.0285272 & 0.411657 & 0.0183361 & 0.52463 \\
\hline 0.429148 & 0.0217406 & 0.440991 & 0.0234478 & 0.395801 & 0.0148318 & 0.495064 \\
\hline 0.424296 & 0.0284059 & 0.43437 & 0.0201006 & 0.392233 & 0.0123143 & 0.484339 \\
\hline 0.41029 & 0.025059 & 0.422172 & 0.017471 & 0.378769 & 0.0170953 & 0.475085 \\
\hline 0.398369 & 0.0234502 & 0.409621 & 0.0267353 & 0.365347 & 0.0161878 & 0.447431 \\
\hline 0.38978 & 0.025594 & 0.394251 & 0.0199 & 0.357783 & 0.0168658 & 0.442534 \\
\hline 0.382655 & 0.021233 & 0.389489 & 0.0199475 & 0.345334 & 0.0157748 & 0.434324 \\
\hline 0.365665 & 0.0218366 & 0.380105 & 0.0238093 & 0.338486 & 0.016373 & 0.412674 \\
\hline 0.352491 & 0.0212516 & 0.369911 & 0.0209516 & 0.329135 & 0.0144613 & 0.407943 \\
\hline 0.349241 & 0.0195367 & 0.357356 & 0.0226074 & 0.324084 & 0.0183182 & 0.413909 \\
\hline 0.343536 & 0.0227338 & 0.349036 & 0.0165927 & 0.316217 & 0.016353 & 0.398871 \\
\hline 0.331035 & 0.0167502 & 0.341579 & 0.0234899 & 0.308416 & 0.0133923 & 0.371687 \\
\hline 0.324078 & 0.0231953 & 0.333537 & 0.0181249 & 0.297677 & 0.0134822 & 0.37356 \\
\hline 0.317946 & 0.017791 & 0.327593 & 0.0194635 & 0.298884 & 0.0150595 & 0.369481 \\
\hline 0.310056 & 0.0192998 & 0.314819 & 0.0198322 & 0.284394 & 0.0132749 & 0.350979 \\
\hline 0.301954 & 0.0207472 & 0.316047 & 0.0199201 & 0.284192 & 0.0159167 & 0.358251 \\
\hline 0.303676 & 0.0238232 & 0.298715 & 0.0162566 & 0.272769 & 0.0142717 & 0.35808 \\
\hline 0.294041 & 0.0178128 & 0.290498 & 0.0191574 & 0.267813 & 0.0147484 & 0.340242 \\
\hline 0.286996 & 0.019739 & 0.284665 & 0.01742 & 0.267649 & 0.0116553 & 0.340812 \\
\hline 0.279818 & 0.0207907 & 0.283053 & 0.0145006 & 0.263085 & 0.01183 & 0.328661 \\
\hline 0.281401 & 0.0227153 & 0.279885 & 0.014526 & 0.257159 & 0.012118 & 0.331536 \\
\hline 0.270529 & 0.0246204 & 0.273956 & 0.0223633 & 0.254094 & 0.0144669 & 0.312722 \\
\hline 0.256943 & 0.0173768 & 0.270118 & 0.0234124 & 0.248718 & 0.0125288 & 0.307532 \\
\hline 0.258185 & 0.0232346 & 0.263855 & 0.0149604 & 0.243831 & 0.0148843 & 0.320321 \\
\hline 0.256905 & 0.0208102 & 0.261307 & 0.0167382 & 0.24132 & 0.0102792 & 0.301661 \\
\hline 0.25036 & 0.021373 & 0.25119 & 0.0174653 & 0.240327 & 0.0117913 & 0.30079 \\
\hline 0.248073 & 0.018938 & 0.247881 & 0.015279 & 0.226652 & 0.0080209 & 0.308737 \\
\hline 0.239229 & 0.0160789 & 0.24268 & 0.0171696 & 0.228584 & 0.0139423 & 0.291798 \\
\hline 0.238353 & 0.0185316 & 0.239642 & 0.0169694 & 0.223156 & 0.0135666 & 0.298016 \\
\hline 0.231503 & 0.0213584 & 0.23095 & 0.018758 & 0.220553 & 0.0108394 & 0.290064 \\
\hline 0.22582 & 0.0197257 & 0.229886 & 0.0124592 & 0.21909 & 0.0145042 & 0.294277 \\
\hline 0.226466 & 0.0180866 & 0.229556 & 0.0165456 & 0.215285 & 0.0140299 & 0.281975 \\
\hline 0.21989 & 0.0179634 & 0.220429 & 0.0214143 & 0.20883 & 0.011679 & 0.274241 \\
\hline 0.214064 & 0.013042 & 0.217865 & 0.0140195 & 0.211607 & 0.014491 & 0.282741 \\
\hline 0.216541 & 0.0221379 & 0.218872 & 0.0157955 & 0.203375 & 0.0118938 & 0.284983 \\
\hline 0.211227 & 0.0181608 & 0.211248 & 0.0172965 & 0.20253 & 0.0122299 & 0.270667 \\
\hline 0.204811 & 0.0179078 & 0.212339 & 0.0122309 & 0.200249 & 0.0132644 & 0.289946 \\
\hline 0.201039 & 0.0151144 & 0.207178 & 0.0151919 & 0.196024 & 0.0137027 & 0.289967 \\
\hline 0.202518 & 0.0159277 & 0.201294 & 0.0145458 & 0.190694 & 0.0128149 & 0.281494 \\
\hline 0.194279 & 0.0164434 & 0.198961 & 0.0184978 & 0.187399 & 0.0106001 & 0.294852 \\
\hline 0.197835 & 0.0154831 & 0.197373 & 0.0135829 & 0.183807 & 0.0106938 & 0.277735 \\
\hline 0.195647 & 0.0125549 & 0.196577 & 0.017063 & 0.184258 & 0.0107909 & 0.290293 \\
\hline 0.185034 & 0.0187747 & 0.190536 & 0.0153441 & 0.182349 & 0.00899204 & 0.28485 \\
\hline 0.182264 & 0.0164126 & 0.188772 & 0.0137414 & 0.176579 & 0.0108333 & 0.288522 \\
\hline
\end{tabular}




\begin{tabular}{|c|c|c|c|c|c|c|}
\hline 0.184546 & 0.0162756 & 0.186398 & 0.0137703 & 0.180187 & 0.0119627 & 89413 \\
\hline 0.176161 & 0.0133583 & 0.185369 & 0.0176812 & 0.178047 & 0.0129813 & 0.282079 \\
\hline .178668 & 0.0176569 & 0.18535 & 0.0176004 & 0.176437 & 0.0107589 & .299605 \\
\hline .178399 & 0.0156962 & 0.189512 & 0.0115211 & 0.175167 & 0.00855991 & 0.296005 \\
\hline 0.175592 & 0.0150016 & 0.194854 & 0.0164561 & 0.171708 & 0.0128839 & 0.296044 \\
\hline 0.171834 & 0.0170267 & 0.192042 & 0.0159669 & 0.173535 & 0.0101539 & 0.300791 \\
\hline 0.172957 & 0.0152562 & 0.200598 & 0.0155887 & 0.17028 & 0.0109145 & 0.304163 \\
\hline 0.16968 & 0.0177297 & 0.198472 & 0.0159787 & 0.174616 & 0.0143748 & 0.310073 \\
\hline 0.170593 & 0.0152035 & 0.199134 & 0.019688 & 0.174707 & 0.011485 & 0.320992 \\
\hline 0.176077 & 0.0158216 & 0.211422 & 0.0137863 & 0.181251 & 0.0124467 & 0.330293 \\
\hline 0.168323 & 0.0166852 & 0.215727 & 0.0204123 & 0.180838 & 0.0101474 & 0.339803 \\
\hline 0.172568 & 0.0117677 & 0.226831 & 0.0256388 & 0.189136 & 0.00945993 & 0.353321 \\
\hline 0.173641 & 0.0113944 & 0.236468 & 0.0214887 & 0.192334 & 0.0100439 & 0.379948 \\
\hline 0.18346 & 0.0130802 & 0.246516 & 0.0231266 & 0.203912 & 0.0145602 & 0.385529 \\
\hline 0.185565 & 0.0207585 & 0.255307 & 0.0270988 & 0.206801 & 0.009867 & 0.404385 \\
\hline 0.185791 & 0.014251 & 0.27826 & 0.0214218 & 0.218899 & 0.0132573 & 3588 \\
\hline 0.193425 & 0.0125441 & 0.289123 & 0.0219221 & 0.225116 & 0.0153914 & 0.462164 \\
\hline 0.201144 & 0.0149717 & 0.311595 & 0.0286566 & 0.240791 & 0.0152889 & 0.498664 \\
\hline 0.207621 & 0.016598 & 0.333205 & 0.0 & .249073 & 0.0134413 & 158 \\
\hline 0.22018 & 0.0165287 & 0.349694 & 0.0325025 & 0.263 & 0.01866 & 0.55789 \\
\hline 0.232894 & 0.0163789 & 0.370307 & 0.0373491 & 0.277202 & 0.0175174 & 0.600128 \\
\hline 0.252947 & 0.0216025 & 0.401829 & 0.03 & 0.295493 & 0.0121085 & 042 \\
\hline 0.260909 & 0.0170744 & 0.419372 & 0.0440708 & 0.307301 & 0.0184157 & 0.708125 \\
\hline 0.283519 & 0.0149221 & 0.445215 & 0.0440767 & 0.326556 & 0.0141577 & 0.738874 \\
\hline 0.296743 & 0.0211051 & 0.461542 & 0.0524005 & 0.336524 & 0.021419 & 0.776923 \\
\hline 0.318113 & 0.0213555 & 0.485675 & 0.0568858 & 0.357804 & 0.0157467 & 0.842352 \\
\hline 0.339535 & 0.0215182 & 0.512192 & 0.0564348 & 0.376902 & 0.0195132 & 0.872137 \\
\hline 0.364699 & 0.0174984 & 0.530558 & 0.0588019 & 0.392693 & 0.018354 & 0.914993 \\
\hline 0.385029 & 0.0224131 & 0.552928 & 0.0620198 & 0.41471 & 0.0209146 & 0.956749 \\
\hline 0.413086 & 0.02279 & 0.57377 & 0.0709712 & 0.425071 & 0.0187971 & 1.01615 \\
\hline 0.430027 & 0.0232263 & 0.600875 & 0.061017 & 0.43768 & 0.024792 & 1.07953 \\
\hline 0.459782 & 0.0274964 & 0.625932 & 0.0704187 & 0.456084 & 0.0206363 & 1.09864 \\
\hline 0.487285 & 0.0283681 & 0.637022 & 0.0740886 & 0.471954 & 0.0240183 & 1.13166 \\
\hline 0.51823 & 0.0222783 & 0.645604 & 0.0652037 & 0.482551 & 0.0231195 & 1.19049 \\
\hline 0.524127 & 0.021622 & 0.671366 & 0.0737202 & 0.494653 & 0.017657 & 1.20697 \\
\hline 0.549737 & 0.0294006 & 0.679182 & 0.0843268 & 0.499058 & 0.0191195 & 1.25253 \\
\hline 0.58469 & 0.0302703 & 0.693704 & 0.0762112 & 0.519804 & 0.0180128 & 1.2656 \\
\hline 0.601346 & 0.034337 & 0.693299 & 0.0817989 & 0.523865 & 0.0205641 & 1.31298 \\
\hline 0.614706 & 0.0314508 & 0.689641 & 0.0715775 & 0.530098 & 0.0210934 & 1.28799 \\
\hline 0.638468 & 0.035929 & 0.687451 & 0.0746154 & 0.528843 & 0.0209327 & 1.29374 \\
\hline 0.655274 & 0.0293698 & 0.694993 & 0.0917725 & 0.531904 & 0.0172651 & 1.32219 \\
\hline 0.675482 & 0.0419135 & 0.677514 & 0.0641346 & 0.526589 & 0.02202 & 1.27718 \\
\hline 0.688909 & 0.0401016 & 0.671481 & 0.076633 & 0.525173 & 0.0181738 & 1.28363 \\
\hline 0.682992 & 0.0326616 & 0.670008 & 0.0746842 & 0.515504 & 0.0255059 & 1.26805 \\
\hline 0.69623 & 0.0283086 & 0.635146 & 0.0639704 & 0.499653 & 0.0235768 & 1.23331 \\
\hline 0.697505 & 0.0363935 & 0.613488 & 0.0685828 & 0.491009 & 0.0232158 & 1.18864 \\
\hline 0.696156 & 0.033383 & 0.587578 & 0.0653042 & 0.479914 & 0.0171441 & 1.16398 \\
\hline
\end{tabular}




\begin{tabular}{|c|c|c|c|c|c|c|}
\hline 0.705591 & 0.0330066 & 0.56221 & 0.0566241 & 0.466791 & 2728 & \\
\hline 0.684975 & 0.0376951 & 0.539551 & 0.0656734 & 0.444417 & 0.0267321 & 1.04062 \\
\hline 679611 & 0.0293509 & 0.512363 & 0.0589665 & 0.41812 & 0.0209443 & 1.00589 \\
\hline 0.666364 & 0.0345181 & 0.472536 & 0.0539325 & 0.397403 & 0.0160808 & 0.937743 \\
\hline 0.649087 & 0.030015 & 0.436814 & 0.0518452 & 0.379754 & 0.0161136 & 0.892747 \\
\hline 0.622488 & 0.038419 & 0.405946 & 0.0485934 & 0.352435 & 0.0168641 & 0.807136 \\
\hline 0.597601 & 0.0355082 & 0.380926 & 0.0433355 & 0.328061 & 0.0151431 & 0.753108 \\
\hline 0.570857 & 0.0287481 & 0.350903 & 0.0371334 & 0.311098 & 0.0145673 & 0.699886 \\
\hline 0.543768 & 0.0275989 & 0.313946 & 0.0325805 & 0.282099 & 0.0167015 & 0.651188 \\
\hline 0.517087 & 0.0298766 & 0.294427 & 0.0368234 & 0.265727 & 0.0148407 & 0.581909 \\
\hline 0.485464 & 0.0242198 & 0.268049 & 0.0349519 & 0.239611 & 0.0115253 & 0.524715 \\
\hline 0.453349 & 0.0260756 & 0.24503 & 0.0271148 & 0.223408 & 0.0146582 & 0.460552 \\
\hline 0.410406 & 0.0244614 & 0.229076 & 0.0276106 & 0.211032 & 0.013924 & 0.431263 \\
\hline 0.38467 & 0.02 & 0.204773 & 0.0259694 & 0.19 & 0.0102878 & 0.398806 \\
\hline 0.352581 & 0.0220554 & 0.187881 & 0.0159819 & 0.180399 & 0.0117804 & 0.354746 \\
\hline 0.324813 & 0.026543 & 0.180295 & 0.0254973 & 0.170258 & 0.0112147 & 0.31477 \\
\hline 0.297898 & 0.0189259 & 0.167797 & 0.0160554 & 0.158761 & 0.0107385 & 0.296285 \\
\hline 0.274267 & 0.0207864 & 0.157847 & 0.0181694 & 0.148132 & 0.008828 & 0.266233 \\
\hline 0.248251 & 0.0183081 & 0.144992 & 0.0148585 & 0.139917 & 0.0100364 & 0.251682 \\
\hline 0.227485 & 563 & 0.139349 & 0.0153584 & 0.137897 & 0.00750698 & 0.240008 \\
\hline 0.208268 & 0.0166217 & 0.134025 & 0.0140838 & 0.129973 & 0.0097802 & 0.226888 \\
\hline 0.193768 & 0.016054 & 0.127988 & 0.0124497 & 0.125196 & 0.00928703 & 0.213403 \\
\hline 0.176482 & 0.01787 & 0.126993 & 0.0118024 & 0.119386 & 0.00911401 & 0.201708 \\
\hline 0.16936 & 0.015722 & 0.12502 & 0.0139651 & 0.120359 & 0.0113471 & 0.189462 \\
\hline 0.1562 & 0.01 & 0.122477 & 524 & 71 & 0.00 & 0.1 \\
\hline 0.143865 & 0.0142472 & 0.116421 & 0.0107779 & 0.116245 & 0.00964147 & 0.181853 \\
\hline 0.141479 & 0.0129628 & 0.113045 & 0.0117456 & 0.11227 & 0.00950028 & 0.1787 \\
\hline 0.136995 & 0.0128425 & 0.119499 & 0.0134584 & 0.112786 & 0.00899454 & 0.169678 \\
\hline 0.128589 & 0.010756 & 0.117738 & 0.0124982 & 0.11224 & 0.00985398 & 0.179615 \\
\hline 0.127038 & 0.0154334 & 0.114644 & 0.0104714 & 0.111676 & 0.0112589 & 0.166976 \\
\hline 0.121689 & 0.0136291 & 0.11949 & 0.0123551 & 0.112859 & 0.00898895 & 0.162673 \\
\hline 0.11865 & 0.0121283 & 0.115628 & 0.0123007 & 0.111511 & 0.00853906 & 0.162909 \\
\hline 0.117497 & 0.0121142 & 0.111161 & 0.0115936 & 0.109335 & 0.0105326 & 0.161114 \\
\hline 0.119976 & 0.0131515 & 0.114557 & 0.012062 & 0.11073 & 0.00761779 & 0.156315 \\
\hline 0.116757 & 0.0103795 & 0.11537 & 0.0143539 & 0.110709 & 0.00820042 & 0.149447 \\
\hline 0.11976 & 0.0133118 & 0.113759 & 0.0126852 & 0.111451 & 0.00908741 & 0.159289 \\
\hline 0.116324 & 0.012109 & 0.115878 & 0.0161959 & 0.112363 & 0.0106027 & 0.161113 \\
\hline 0.11407 & 0.0134326 & 0.117864 & 0.0095703 & 0.112533 & 0.0097241 & 0.151005 \\
\hline 0.118929 & 0.013987 & 0.117962 & 0.0130394 & 0.115973 & 0.00742504 & 0.155367 \\
\hline 0.112528 & 0.0142674 & 0.118699 & 0.0147603 & 0.111351 & 0.00801164 & 0.151179 \\
\hline 0.115467 & 0.0116095 & 0.116455 & 0.013001 & 0.112465 & 0.00626227 & 0.15099 \\
\hline 0.115997 & 0.0127482 & 0.117199 & 0.00999868 & 0.115852 & 0.00920016 & 0.148608 \\
\hline 0.117921 & 0.0142658 & 0.118403 & 0.0126529 & 0.116381 & 0.00747057 & 0.153271 \\
\hline 0.116065 & 0.0112226 & 0.116911 & 0.0116672 & 0.114499 & 0.00825664 & 0.148804 \\
\hline 0.11707 & 0.0112436 & 0.115186 & 0.0130972 & 0.116902 & 0.00834933 & 0.155221 \\
\hline 0.121072 & 0.0121146 & 0.116236 & 0.0130681 & 0.116792 & 0.0107288 & 0.149554 \\
\hline 0.116314 & 0.0135722 & 0.121118 & 0.0126381 & 0.11669 & 0.00833264 & 0.15104 \\
\hline
\end{tabular}




$\begin{array}{rrrrrrr}0.119483 & 0.0134712 & 0.119406 & 0.0114989 & 0.118334 & 0.00799637 & 0.142269 \\ 0.127106 & 0.0139928 & 0.121059 & 0.0129529 & 0.120062 & 0.00855003 & 0.147912 \\ 0.119163 & 0.0141353 & 0.12556 & 0.0107001 & 0.119952 & 0.00805147 & 0.150607 \\ 0.125174 & 0.0118564 & 0.12268 & 0.0149585 & 0.120696 & 0.00893863 & 0.150098 \\ 0.124137 & 0.0147327 & 0.125319 & 0.0117904 & 0.121825 & 0.00992392 & 0.145599 \\ 0.126827 & 0.0142199 & 0.131911 & 0.013575 & 0.125968 & 0.0104449 & 0.149271 \\ 0.126994 & 0.0133714 & 0.128954 & 0.011646 & 0.127894 & 0.0111466 & 0.146207 \\ 0.129578 & 0.0153655 & 0.133899 & 0.0150805 & 0.127585 & 0.00892522 & 0.154732 \\ 0.131812 & 0.0117325 & 0.134126 & 0.0119092 & 0.126093 & 0.0106256 & 0.154176 \\ 0.132591 & 0.0149199 & 0.131537 & 0.0109294 & 0.129879 & 0.0106193 & 0.150355 \\ 0.128748 & 0.0141268 & 0.134457 & 0.0119196 & 0.13128 & 0.00853928 & 0.15331 \\ 0.133345 & 0.0133648 & 0.134958 & 0.0152801 & 0.133015 & 0.00898211 & 0.15843 \\ 0.136582 & 0.0133202 & 0.134534 & 0.011851 & 0.135017 & 0.0108925 & 0.154184 \\ 0.138386 & 0.0169455 & 0.135285 & 0.0145822 & 0.137144 & 0.0117821 & 0.153067 \\ 0.140049 & 0.0116715 & 0.140407 & 0.0121281 & 0.140074 & 0.00954814 & 0.152042 \\ 0.138681 & 0.0105405 & 0.137638 & 0.0101792 & 0.138872 & 0.00956613 & 0.155261 \\ 0.138787 & 0.0148804 & 0.14438 & 0.0143844 & 0.141546 & 0.00976785 & 0.158635 \\ 0.145747 & 0.0142234 & 0.144458 & 0.0119797 & 0.13917 & 0.00978675 & 0.159652 \\ 0.147458 & 0.014226 & 0.142396 & 0.0120494 & 0.144723 & 0.00937127 & 0.15631 \\ 0.144941 & 0.0126385 & 0.144791 & 0.0128454 & 0.143478 & 0.00857167 & 0.160901 \\ 0.145555 & 0.0158219 & 0.149694 & 0.0151822 & 0.148972 & 0.00836343 & 0.156739 \\ 0.151266 & 0.0173448 & 0.15198 & 0.0140668 & 0.147402 & 0.0101437 & 0.160881 \\ 0.15358 & 0.0149336 & 0.15065 & 0.0122329 & 0.149141 & 0.0121304 & 0.171541 \\ 0.155817 & 0.0162382 & 0.153143 & 0.0120528 & 0.15354 & 0.0120383 & 0.161166 \\ 0.155209 & 0.0138266 & 0.157278 & 0.0132148 & 0.154635 & 0.0114896 & 0.163658 \\ 0.157689 & 0.0142665 & 0.155228 & 0.0141783 & 0.154523 & 0.00981945 & 0.160297 \\ 0.155235 & 0.0143532 & 0.160557 & 0.0119961 & 0.158626 & 0.0119239 & 0.165805 \\ 0.158778 & 0.0152725 & 0.156118 & 0.0134256 & 0.160641 & 0.00787009 & 0.172904\end{array}$




\begin{tabular}{|c|c|c|c|c|c|c|}
\hline 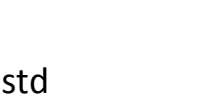 & $\begin{array}{l}\text { SB Ru } \\
\text { mean }\end{array}$ & std & $\begin{array}{l}\text { SB Rc } \\
\text { mean }\end{array}$ & std & $\begin{array}{l}\text { SB Rb } \\
\text { mean }\end{array}$ & std \\
\hline 0.160022 & 0.414779 & 0.0396361 & 0.246999 & 0.0355575 & 0.219046 & 0.0237054 \\
\hline 0.16577 & 0.41935 & 0.0391818 & 0.251183 & 0.0463229 & 0.225464 & 0.0283012 \\
\hline 0.206033 & 0.419251 & 0.0398095 & 0.245345 & 0.0365502 & 0.221423 & 0.0219308 \\
\hline 0.171586 & 0.413871 & 0.0383521 & 0.240785 & 0.0426016 & 0.224599 & 0.0212836 \\
\hline 0.184532 & 0.410389 & 0.0365561 & 0.24347 & 0.0388571 & 0.220209 & 0.0197176 \\
\hline 0.148431 & 0.412056 & 0.0378681 & 0.243633 & 0.0405405 & 0.22251 & 0.0259831 \\
\hline 0.193611 & 0.40759 & 0.0379245 & 0.237773 & 0.0406711 & 0.221521 & 0.0184433 \\
\hline 0.168888 & 0.401381 & 0.0338912 & 0.240675 & 0.038309 & 0.225026 & 0.0203709 \\
\hline 0.176389 & 0.406426 & 0.0372156 & 0.238894 & 0.0358067 & 0.218111 & 0.0201055 \\
\hline 0.172527 & 0.397683 & 0.0326627 & 0.239532 & 0.0384017 & 0.220209 & 0.02481 \\
\hline 0.190425 & 0.405531 & 0.0430814 & 0.233267 & 0.0429858 & 0.219208 & 0.0241948 \\
\hline 0.161995 & 0.403367 & 0.0388538 & 0.23921 & 0.0430773 & 0.213844 & 0.0229495 \\
\hline 0.162602 & 0.395222 & 0.0368999 & 0.236391 & 0.0400993 & 0.216479 & 0.0237501 \\
\hline 0.186156 & 0.398615 & 0.0313438 & 0.236967 & 0.0424654 & 0.217257 & 0.019222 \\
\hline 0.186654 & 0.402177 & 0.0366532 & 0.23957 & 0.0386176 & 0.21929 & 0.0234442 \\
\hline 0.194399 & 0.408318 & 0.0342755 & 0.241328 & 0.0416089 & 0.216931 & 0.0225813 \\
\hline 0.187099 & 0.419174 & 0.0373018 & 0.237714 & 0.0439192 & 0.219767 & 0.0292699 \\
\hline 0.16485 & 0.430185 & 0.0332631 & 0.245069 & 0.0426619 & 0.223269 & 0.0236013 \\
\hline 0.179337 & 0.447275 & 0.036634 & 0.243699 & 0.0383746 & 0.228212 & 0.0186804 \\
\hline 0.159489 & 0.480381 & 0.0358655 & 0.245426 & 0.0437852 & 0.237251 & 0.0235309 \\
\hline 0.165406 & 0.50984 & 0.0422821 & 0.247269 & 0.04035 & 0.243977 & 0.025029 \\
\hline 0.197574 & 0.537378 & 0.0449791 & 0.258171 & 0.0456306 & 0.249737 & 0.0264067 \\
\hline 0.185353 & 0.561891 & 0.0495212 & 0.262633 & 0.0449508 & 0.255356 & 0.0313032 \\
\hline 0.17414 & 0.589192 & 0.0498859 & 0.263091 & 0.0436256 & 0.266288 & 0.0261491 \\
\hline 0.163043 & 0.602898 & 0.0550131 & 0.268014 & 0.0404423 & 0.269325 & 0.0296341 \\
\hline 0.162079 & 0.605333 & 0.0491874 & 0.270719 & 0.0487615 & 0.275542 & 0.0308929 \\
\hline 0.15601 & 0.599334 & 0.046535 & 0.266128 & 0.0443956 & 0.274284 & 0.0255819 \\
\hline 0.174494 & 0.574484 & 0.0504109 & 0.263813 & 0.0455 & 0.266597 & 0.0253243 \\
\hline 0.164734 & 0.54204 & 0.0469827 & 0.262365 & 0.0438652 & 0.260685 & 0.0319286 \\
\hline 0.182823 & 0.506494 & 0.0355557 & 0.258535 & 0.0445171 & 0.258395 & 0.0253376 \\
\hline 0.179726 & 0.479336 & 0.0412564 & 0.251627 & 0.0416743 & 0.247983 & 0.0326018 \\
\hline 0.169899 & 0.450696 & 0.0360121 & 0.248331 & 0.0431184 & 0.246854 & 0.0280415 \\
\hline 0.17532 & 0.428913 & 0.0299521 & 0.246648 & 0.0446053 & 5079 & 0.0270135 \\
\hline 0.185129 & 0.422042 & 0.03451 & 0.243389 & 0.0452872 & 0.241654 & 0.0269365 \\
\hline 0.189456 & 0.408039 & 0.0293579 & 0.24687 & 0.0444906 & 0.249726 & 0.0271943 \\
\hline 0.18209 & 0.407933 & 0.0322783 & 0.245801 & 0.0443404 & 0.259872 & 0.0301359 \\
\hline 0.195429 & 0.414621 & 0.0396938 & 0.250566 & 0.0412233 & 0.277791 & 0.0341378 \\
\hline 0.169443 & 0.438689 & 0.0397684 & 0.257073 & 0.0460656 & 0.301789 & 0.0353424 \\
\hline 0.179955 & 0.476361 & 0.0406175 & 0.275119 & 0.0471082 & 0.352458 & 0.0434241 \\
\hline 0.202413 & 0.522055 & 0.0507679 & 0.292417 & 0.0489617 & 0.4366 & 0.0493406 \\
\hline 0.203411 & 0.599969 & 0.0748351 & 0.330233 & 0.0570488 & 0.561215 & 0.0732286 \\
\hline 0.24186 & 0.698031 & 0.0917319 & 0.374297 & 0.0717831 & 0.742332 & 0.0866274 \\
\hline 0.303656 & 0.836977 & 0.121366 & 0.433196 & 0.0907014 & 0.98571 & 0.134976 \\
\hline 0.31926 & 0.990951 & 0.141433 & 0.502639 & 0.110434 & 1.25159 & 0.182546 \\
\hline 0.394536 & 1.14341 & 0.178969 & 0.587764 & 0.140717 & 1.56761 & 0.222819 \\
\hline
\end{tabular}




\begin{tabular}{|c|c|c|c|c|c|c|}
\hline 0.420632 & 1.28568 & 0.226073 & 0.667085 & 0.157056 & 1.86813 & 0.256165 \\
\hline 0.520988 & 1.40252 & 0.249954 & 0.742437 & 0.175464 & 2.14262 & 0.289038 \\
\hline 0.554873 & 1.48735 & 0.274139 & 0.79659 & 0.191581 & 2.34299 & 0.328902 \\
\hline 0.602498 & 1.50518 & 0.280148 & 0.822916 & 0.205557 & 2.45027 & 0.350094 \\
\hline 0.604933 & 1.46969 & 0.275106 & 0.823344 & 0.201074 & 2.43444 & 0.342894 \\
\hline 0.573879 & 1.38364 & 0.24771 & 0.796944 & 0.196706 & 2.29733 & 0.319785 \\
\hline 0.525416 & 1.25249 & 0.205545 & 0.739779 & 0.183993 & 2.07287 & 0.280669 \\
\hline 0.462485 & 1.12347 & 0.17336 & 0.678028 & 0.160496 & 1.8072 & 0.247623 \\
\hline 0.391206 & 0.99204 & 0.14253 & 0.605555 & 0.142253 & 1.52015 & 0.197365 \\
\hline 0.336138 & 0.870748 & 0.117453 & 0.527486 & 0.113274 & 1.22294 & 0.149434 \\
\hline 0.306971 & 0.782109 & 0.0910478 & 0.466042 & 0.0956164 & 0.980306 & 0.132817 \\
\hline 0.286734 & 0.72466 & 0.078848 & 0.419074 & 0.0818846 & 0.783347 & 0.108273 \\
\hline 0.238991 & 0.709886 & 0.0653528 & 0.390516 & 0.0716093 & 0.640875 & 0.0836078 \\
\hline 0.194521 & 0.738804 & 0.0686709 & 0.377867 & 0.0725376 & 0.559247 & 0.0646695 \\
\hline 0.190783 & 0.853126 & 0.0828101 & 0.382455 & 0.070806 & 0.521708 & 0.0562581 \\
\hline 0.217264 & 1.10376 & 0.129987 & 0.414189 & 0.0756218 & 0.554832 & 0.0652626 \\
\hline 0.203042 & 1.55542 & 0.209171 & 0.500352 & 0.10054 & 0.677424 & 0.086533 \\
\hline 0.253781 & 2.34215 & 0.345329 & 0.66796 & 0.135826 & 0.923079 & 0.119677 \\
\hline 0.317715 & 3.64376 & 0.572464 & 0.937576 & 0.199493 & 1.34633 & 0.184922 \\
\hline 0.383592 & 5.62537 & 0.926661 & 1.37171 & 0.313344 & 2.04834 & 0.280934 \\
\hline 0.542937 & 8.44253 & 1.40865 & 2.01437 & 0.450914 & 3.06845 & 0.447785 \\
\hline 0.750873 & 12.2101 & 2.04905 & 2.92299 & 0.661356 & 4.50316 & 0.660096 \\
\hline 1.06537 & 16.8529 & 2.87702 & 4.131 & 0.950293 & 6.36087 & 0.935287 \\
\hline 1.38095 & 22.1276 & 3.79694 & 5.59828 & 1.30941 & 8.61156 & 1.2528 \\
\hline 1.78915 & 27.6748 & 4.74677 & 7.25256 & 1.70152 & 11.1332 & 1.63472 \\
\hline 2.17842 & 32.803 & 5.67446 & 8.93467 & 2.13338 & 13.7215 & 2.0616 \\
\hline 2.53349 & 36.8195 & 6.43405 & 10.4855 & 2.51131 & 15.9906 & 2.38812 \\
\hline 2.90862 & 39.2923 & 6.87822 & 11.7283 & 2.79938 & 17.711 & 2.6499 \\
\hline 3.05502 & 39.784 & 6.93486 & 12.4307 & 2.97403 & 18.6586 & 2.72887 \\
\hline 3.02898 & 38.2154 & 6.61746 & 12.5689 & 3.00386 & 18.7991 & 2.77821 \\
\hline 2.94936 & 34.884 & 6.02732 & 12.1025 & 2.89787 & 17.9197 & 2.65871 \\
\hline 2.73256 & 30.31 & 5.26192 & 11.0648 & 2.64949 & 16.2397 & 2.39585 \\
\hline 2.43959 & 25.0975 & 4.37385 & 9.63293 & 2.29325 & 14.0038 & 2.06507 \\
\hline 2.08148 & 19.9156 & 3.4448 & 8.01363 & 1.91122 & 11.6049 & 1.70604 \\
\hline 1.69195 & 15.2515 & 2.60462 & 6.41742 & 1.51561 & 9.18915 & 1.3531 \\
\hline 1.3427 & 11.3054 & 1.90058 & 4.96812 & 1.18166 & 7.02319 & 1.03108 \\
\hline 1.01744 & 8.21728 & 1.34788 & 3.73584 & 0.872079 & 5.20804 & 0.758673 \\
\hline 0.81116 & 5.97628 & 0.958682 & 2.79073 & 0.639089 & 3.78015 & 0.571447 \\
\hline 0.640996 & 4.35746 & 0.684798 & 2.07037 & 0.456461 & 2.73882 & 0.392251 \\
\hline 0.500362 & 3.18835 & 0.486381 & 1.54169 & 0.334216 & 1.99315 & 0.292056 \\
\hline 0.396618 & 2.35431 & 0.334799 & 1.15663 & 0.247063 & 1.44732 & 0.204247 \\
\hline 0.304363 & 1.71533 & 0.229184 & 0.891641 & 0.187674 & 1.06649 & 0.151268 \\
\hline 0.270885 & 1.2448 & 0.163976 & 0.711917 & 0.145544 & 0.810432 & 0.110011 \\
\hline 0.238037 & 0.899874 & 0.113025 & 0.575207 & 0.109165 & 0.616842 & 0.0809196 \\
\hline 0.227721 & 0.67836 & 0.0847103 & 0.462546 & 0.0813608 & 0.480845 & 0.0573667 \\
\hline 0.200637 & 0.553018 & 0.0745453 & 0.394225 & 0.0726806 & 0.374114 & 0.048181 \\
\hline 0.202568 & 0.519776 & 0.0460902 & 0.335109 & 0.0618823 & 0.301669 & 0.0419586 \\
\hline
\end{tabular}




\begin{tabular}{|c|c|c|c|c|c|c|}
\hline 0.201751 & 0.564538 & 0.0558998 & 0.308778 & 0.059213 & 0.251713 & 314285 \\
\hline 0.178575 & .652739 & 0.0792542 & 0.296745 & 0.0584095 & 0.221957 & 0.0219746 \\
\hline 0.154082 & 0.748911 & 0.0692138 & 0.290096 & 0.0541415 & 0.196997 & 0.0230328 \\
\hline 0.160857 & .836583 & 0.0823285 & 0.289686 & 0.0512471 & 0.188548 & 0.0191509 \\
\hline 0.172921 & 0.909331 & 0.0913932 & 0.284501 & 0.0517361 & 0.18424 & 0.0232631 \\
\hline 0.190444 & 0.946955 & 0.082618 & 0.284743 & 0.0553594 & 0.188152 & 0.0245834 \\
\hline 0.182423 & 0.948749 & 0.0801244 & 0.284315 & 0.0601538 & 0.188173 & 0.0251011 \\
\hline 0.174235 & 0.92243 & 0.0838813 & 0.302104 & 0.0555074 & 0.195288 & 0.0244242 \\
\hline 0.19396 & 0.882207 & 0.0803486 & 0.295961 & 0.0512778 & 0.200075 & 0.0224616 \\
\hline 0.189818 & 0.82019 & 0.0688446 & 0.298639 & 0.0538295 & 0.202626 & 0.021926 \\
\hline 0.19456 & 0.750233 & 0.0724076 & 0.29363 & 0.0594645 & 0.201318 & 0.0204065 \\
\hline 0.179661 & 0.686684 & 0.0668843 & 0.286089 & 0.0564176 & 0.20144 & 0.0263339 \\
\hline 0.188216 & 0.630613 & 0.0597287 & 0.276777 & 0.0602158 & 0.197753 & 0.0275165 \\
\hline 0.165452 & 0.583034 & 0.0517372 & 0.274425 & 0.054987 & 0.190334 & 0.0316435 \\
\hline 0.172059 & 0.539362 & 0.0503024 & 0.262882 & 0.0483742 & 0.186336 & 0.0300205 \\
\hline 0.168784 & 0.506829 & 0.0425248 & 0.253696 & 0.0545172 & 0.178588 & 0.0281629 \\
\hline 0.167617 & 0.493019 & 0.0464127 & 0.247937 & 0.0506259 & 0.176021 & 0.0261969 \\
\hline 0.163091 & 0.480785 & 0.0498256 & 0.238044 & 0.0445792 & 0.169505 & 0.0240613 \\
\hline 0.1701 & 0.468171 & 0.0500392 & 0.238755 & 0.0444248 & 0.168879 & 0.0265156 \\
\hline 0.155226 & 0.460166 & 0.0442455 & 0.235559 & 0.0426678 & 0.166745 & 0.0286308 \\
\hline 0.16378 & 0.463103 & 0.044424 & 0.229565 & 0.0416682 & 0.160602 & 0.0215446 \\
\hline 0.157392 & 0.463384 & 0.0421288 & 0.229104 & 0.0427841 & 0.161322 & 2841 \\
\hline 0.167363 & 0.465225 & 0.0441868 & 0.228669 & 0.0433415 & 0.159296 & 0.0187902 \\
\hline 0.159707 & 0.472918 & 0.0429315 & 0.230049 & 0.0445107 & 0.155979 & 0.0190128 \\
\hline 0.176953 & 0.4846 & 0.0576437 & 0.234786 & 0.040864 & 0.155041 & 0.0186474 \\
\hline 0.176211 & 0.500134 & 0.0579359 & 0.237706 & 0.043393 & 0.155096 & 0.0159533 \\
\hline 0.162876 & 0.548624 & 0.0417728 & 0.24953 & 0.0446532 & 0.156999 & 0.0186099 \\
\hline 0.150336 & 0.621829 & 0.0578389 & 0.263707 & 0.0492235 & 0.15732 & 0.018254 \\
\hline 0.154159 & 0.733151 & 0.0755131 & 0.289869 & 0.053361 & 0.159332 & 0.0190356 \\
\hline 0.165447 & 0.901361 & 0.0945223 & 0.328578 & 0.0633153 & 0.172497 & 4035 \\
\hline 0.167305 & 1.10062 & 0.120343 & 0.384146 & 0.0750933 & 0.189605 & 0.0225377 \\
\hline 0.173985 & 1.36621 & 0.167179 & 0.455441 & 0.0899772 & 0.208135 & 0.0249998 \\
\hline 0.210932 & 1.65545 & 0.215623 & 0.538877 & 0.104358 & 0.227542 & 0.0282125 \\
\hline 0.232875 & 1.95597 & 0.253286 & 0.6445 & 0.131263 & 0.247282 & 0.0275734 \\
\hline 0.267814 & 2.23596 & 0.286937 & 0.753832 & 0.160839 & 0.274658 & 0.0316162 \\
\hline 0.315838 & 2.49923 & 0.34824 & 0.853796 & 0.186434 & 0.302387 & 0.0380221 \\
\hline 0.356849 & 2.66634 & 0.360185 & 0.946176 & 0.20335 & 0.319498 & 0.0414578 \\
\hline 0.369412 & 2.73463 & 0.357234 & 1.01835 & 0.222931 & 0.339123 & 0.0430333 \\
\hline 0.379433 & 2.69635 & 0.355114 & 1.05405 & 0.231485 & 0.348079 & 0.0434706 \\
\hline 0.390821 & 2.56921 & 0.342125 & 1.05275 & 0.22621 & 0.348633 & 0.0438207 \\
\hline 0.378106 & 2.34373 & 0.327446 & 1.02065 & 0.227367 & 0.339182 & 0.043324 \\
\hline 0.346855 & 2.0774 & 0.266492 & 0.954176 & 0.204884 & 0.317827 & 0.0394036 \\
\hline 0.316508 & 1.79716 & 0.211083 & 0.863824 & 0.179553 & 0.293644 & 0.0393016 \\
\hline 0.288181 & 1.50629 & 0.183903 & 0.759076 & 0.163811 & 0.27412 & 0.0367155 \\
\hline 0.249607 & 1.24398 & 0.148201 & 0.649748 & 0.132611 & 0.251693 & 0.0296308 \\
\hline 0.231684 & 1.0316 & 0.10605 & 0.553196 & 0.105403 & 0.220747 & 0.0287586 \\
\hline 0.211538 & 0.869455 & 0.0817224 & 0.477328 & 0.094372 & 0.201049 & 0.0234934 \\
\hline
\end{tabular}




\begin{tabular}{|c|c|c|c|c|c|c|}
\hline 0.208586 & 0.748016 & 0.0815606 & 0.410089 & 0.081813 & 0.187498 & 0.0201103 \\
\hline 0.195352 & 0.657011 & 0.0618082 & 0.357823 & 0.072386 & 0.175309 & 0.0197475 \\
\hline 0.178548 & 0.600478 & 0.0627628 & 0.327543 & 0.0628895 & 0.168888 & 0.022516 \\
\hline 0.168031 & 0.566817 & 0.0577855 & 0.304567 & 0.0550219 & 0.164991 & 0.0229972 \\
\hline 0.154599 & 0.552668 & 0.0524753 & 0.286818 & 0.0539314 & 0.161584 & 0.0209612 \\
\hline 0.161134 & 0.537422 & 0.0458312 & 0.281349 & 0.0519544 & 0.158096 & 0.0186029 \\
\hline 0.157125 & 0.518776 & 0.0529291 & 0.280024 & 0.0534809 & 0.159332 & 0.0186319 \\
\hline 0.15934 & 0.510149 & 0.0544202 & 0.279765 & 0.0532175 & 0.159608 & 0.0193065 \\
\hline 0.165537 & 0.491147 & 0.0503137 & 0.273552 & 0.0519362 & 0.15552 & 0.0160795 \\
\hline 0.171677 & 0.481904 & 0.0469151 & 0.264783 & 0.0452813 & 0.15792 & 0.0165566 \\
\hline 0.153937 & 0.46683 & 0.0459422 & 0.260609 & 0.0455169 & 0.157954 & 0.0214131 \\
\hline 0.147496 & 0.457897 & 0.0440893 & 0.25744 & 0.0481641 & 0.155008 & 0.0190706 \\
\hline 0.145908 & 0.442727 & 0.0399623 & 0.254546 & 0.0464386 & 0.155099 & 0.0166795 \\
\hline 0.164254 & 0.439813 & 0.0444369 & 0.252066 & 0.0465109 & 0.154524 & 0.0178563 \\
\hline 0.176856 & 0.452786 & 0.0434794 & 0.249115 & 0.0459794 & 0.157444 & 0.0157116 \\
\hline 0.17255 & 0.476643 & 0.0449971 & 0.251344 & 0.0486539 & 0.160992 & 0.0130008 \\
\hline 0.18349 & 0.511202 & 0.0545458 & 0.259534 & 0.0484861 & 0.163179 & 0.015409 \\
\hline 0.202083 & 0.557362 & 0.061634 & 0.26881 & 0.050898 & 0.174221 & 0.0177616 \\
\hline 0.263991 & 0.648317 & 0.0732325 & 0.279578 & 0.0525047 & 0.19107 & 0.0188579 \\
\hline 0.345202 & 0.765752 & 0.0776672 & 0.302499 & 0.0521145 & 0.205246 & 0.0223158 \\
\hline 0.435045 & 0.900999 & 0.0979088 & 0.335623 & 0.0578388 & 0.225712 & 0.0246639 \\
\hline 0.610406 & 1.07931 & 0.128565 & 0.37266 & 0.0666694 & 0.262959 & 0.0312059 \\
\hline 0.859032 & 1.29066 & 0.164403 & 0.409914 & 0.0785477 & 0.306288 & 0.0339434 \\
\hline 1.16661 & 1.54737 & 0.198104 & 0.454475 & 0.0885999 & 0.35881 & 0.0408006 \\
\hline 1.50178 & 1.79685 & 0.252396 & 0.512448 & 0.0979466 & 0.423893 & 0.0511654 \\
\hline 1.89809 & 2.0345 & 0.293679 & 0.573939 & 0.109909 & 0.487563 & 0.0609923 \\
\hline 2.36437 & 2.28126 & 0.334839 & 0.63857 & 0.126426 & 0.548578 & 0.0711326 \\
\hline 2.77441 & 2.45143 & 0.351943 & 0.69737 & 0.138002 & 0.612241 & 0.0782725 \\
\hline 3.15346 & 2.57729 & 0.385454 & 0.739495 & 0.144443 & 0.67392 & 0.0726579 \\
\hline 3.46552 & 2.60156 & 0.387041 & 0.775923 & 0.150823 & 0.713847 & 0.0828939 \\
\hline 3.63319 & 2.57066 & 0.371819 & 0.79309 & 0.152345 & 0.734847 & 0.0924278 \\
\hline 3.65226 & 2.47152 & 0.355611 & 0.792396 & 0.149758 & 0.737372 & 0.0841013 \\
\hline 3.47961 & 2.32149 & 0.303208 & 0.779075 & 0.147059 & 0.722181 & 0.0835424 \\
\hline 3.18836 & 2.18482 & 0.259645 & 0.752909 & 0.139801 & 0.68891 & 0.0888289 \\
\hline 2.87428 & 2.06505 & 0.236225 & 0.720224 & 0.13796 & 0.64479 & 0.0874173 \\
\hline 2.55533 & 1.97998 & 0.194747 & 0.693174 & 0.139398 & 0.608436 & 0.078461 \\
\hline 2.20692 & 1.96384 & 0.175529 & 0.679532 & 0.133124 & 0.584037 & 0.0773579 \\
\hline 1.83612 & 1.99468 & 0.164553 & 0.666186 & 0.128026 & 0.56717 & 0.0813974 \\
\hline 1.59583 & 2.07006 & 0.174311 & 0.671707 & 0.132292 & 0.56766 & 0.0839056 \\
\hline 1.4796 & 2.17723 & 0.194131 & 0.693818 & 0.135742 & 0.584783 & 0.0934254 \\
\hline 1.44991 & 2.28556 & 0.195554 & 0.726679 & 0.146817 & 0.606949 & 0.104334 \\
\hline 1.56628 & 2.36676 & 0.215895 & 0.767673 & 0.159611 & 0.6368 & 0.112589 \\
\hline 1.68866 & 2.40594 & 0.209415 & 0.791789 & 0.162727 & 0.670722 & 0.124168 \\
\hline 1.73552 & 2.41933 & 0.208862 & 0.818673 & 0.171734 & 0.690981 & 0.126026 \\
\hline 1.81636 & 2.36217 & 0.211076 & 0.839346 & 0.179995 & 0.693454 & 0.126251 \\
\hline 1.8523 & 2.24518 & 0.203679 & 0.832831 & 0.174747 & 0.686935 & 0.128935 \\
\hline 1.8061 & 2.10134 & 0.186873 & 0.816741 & 0.170884 & 0.669041 & 0.121772 \\
\hline
\end{tabular}




\begin{tabular}{|c|c|c|c|c|c|c|}
\hline 1.70688 & 1.93437 & 0.161422 & 0.788875 & 0.167475 & 0.63417 & 0.112344 \\
\hline 1.55182 & 1.78388 & 0.140806 & 0.744311 & 0.152752 & 0.596299 & 0.103505 \\
\hline 1.37442 & 1.63516 & 0.136441 & 0.696915 & 0.142706 & 0.552556 & 0.087516 \\
\hline 1.21717 & 1.51364 & 0.117082 & 0.653203 & 0.130915 & 0.504783 & 0.0736265 \\
\hline 1.0319 & 1.41792 & 0.130858 & 0.606419 & 0.117693 & 0.4587 & 0.0662973 \\
\hline 0.871395 & 1.35424 & 0.154494 & 0.571357 & 0.111086 & 0.417985 & 0.0577794 \\
\hline 0.738234 & 1.32246 & 0.153872 & 0.539422 & 0.0964943 & 0.389124 & 0.0499428 \\
\hline 0.606248 & 1.29738 & 0.176582 & 0.512652 & 0.0904162 & 0.360146 & 0.0419667 \\
\hline 0.520106 & 1.30093 & 0.205855 & 0.49515 & 0.0863253 & 0.336816 & 0.0367855 \\
\hline 0.432181 & 1.29498 & 0.212551 & 0.478322 & 0.0790497 & 0.318 & 0.0313562 \\
\hline 0.330893 & 1.28183 & 0.226583 & 0.466585 & 0.0802231 & 0.302196 & 0.0281696 \\
\hline 0.294268 & 1.24224 & 0.236544 & 0.452354 & 0.0802325 & 0.285984 & 0.0275213 \\
\hline 0.235627 & 1.20135 & 0.23554 & 0.4412 & 0.0760733 & 0.267445 & 0.0318281 \\
\hline 0.216414 & 1.1731 & 0.220129 & 0.431418 & 0.0755095 & 0.255995 & 0.0331037 \\
\hline 0.227346 & 1.11667 & 0.211236 & 0.416802 & 0.0741999 & 0.244472 & 0.02852 \\
\hline 0.195806 & 1.0483 & 0.187765 & 0.402647 & 0.0708396 & 0.231759 & 0.0282533 \\
\hline 0.179996 & 0.980288 & 0.177256 & 0.387008 & 0.0743008 & 0.220505 & 0.0256265 \\
\hline 0.179312 & 0.918492 & 0.157258 & 0.374476 & 0.0730607 & 0.212796 & 0.0249635 \\
\hline 0.177999 & 0.863146 & 0.134505 & 0.363614 & 0.0696689 & 0.201451 & 0.0216684 \\
\hline 0.165103 & 0.808613 & 0.109214 & 0.357273 & 0.069115 & 0.191564 & 0.0226112 \\
\hline 0.161259 & 0.763783 & 0.0965207 & 0.351541 & 0.0707793 & 0.185493 & 0.0237966 \\
\hline 0.165491 & 0.724996 & 0.085409 & 0.346203 & 0.0713323 & 0.184153 & 0.0229235 \\
\hline 0.160775 & 0.696142 & 0.073047 & 0.33978 & 0.0681469 & 0.186644 & 0.022013 \\
\hline 0.155469 & 0.688602 & 0.057198 & 0.335277 & 0.0656545 & 0.183802 & 0.0239503 \\
\hline 0.15816 & 0.67567 & 0.0657209 & 0.344636 & 0.0734224 & 0.182462 & 0.0222661 \\
\hline 0.167998 & 0.668129 & 0.0633623 & 0.348353 & 0.0751263 & 0.188272 & 0.0213031 \\
\hline 0.147215 & 0.678123 & 0.0638557 & 0.35004 & 0.072992 & 0.194529 & 0.0223557 \\
\hline 0.133963 & 0.695654 & 0.0665904 & 0.362858 & 0.0772024 & 0.204572 & 0.0221784 \\
\hline 0.159267 & 0.707233 & 0.0592568 & 0.37102 & 0.0766269 & 0.219833 & 0.0266239 \\
\hline 0.169077 & 0.728111 & 0.0620194 & 0.383261 & 0.0779693 & 0.240046 & 0.0305457 \\
\hline 0.184832 & 0.757625 & 0.0730086 & 0.398179 & 0.0797105 & 0.276417 & 0.0374056 \\
\hline 0.184231 & 0.808231 & 0.0911439 & 0.42905 & 0.0867582 & 0.334225 & 0.0454802 \\
\hline 0.187061 & 0.888503 & 0.104915 & 0.472992 & 0.104659 & 0.414789 & 0.0582545 \\
\hline 0.203635 & 0.989288 & 0.147048 & 0.535061 & 0.126221 & 0.533476 & 0.0754359 \\
\hline 0.232789 & 1.12715 & 0.197747 & 0.622159 & 0.158303 & 0.707991 & 0.103437 \\
\hline 0.273296 & 1.30614 & 0.250731 & 0.738947 & 0.206158 & 0.952198 & 0.144358 \\
\hline 0.333704 & 1.54525 & 0.327318 & 0.892795 & 0.271183 & 1.26327 & 0.189557 \\
\hline 0.421772 & 1.80915 & 0.428082 & 1.10512 & 0.366338 & 1.64555 & 0.251925 \\
\hline 0.493954 & 2.07187 & 0.525099 & 1.34861 & 0.4697 & 2.09529 & 0.323979 \\
\hline 0.615027 & 2.34832 & 0.6212 & 1.60521 & 0.581613 & 2.60486 & 0.400865 \\
\hline 0.726601 & 2.60798 & 0.708442 & 1.8853 & 0.693239 & 3.14987 & 0.488037 \\
\hline 0.858675 & 2.80599 & 0.783777 & 2.17807 & 0.820622 & 3.67608 & 0.576976 \\
\hline 0.98145 & 2.96101 & 0.834631 & 2.42995 & 0.921284 & 4.11252 & 0.63827 \\
\hline 1.04404 & 3.01803 & 0.867073 & 2.61347 & 1.01394 & 4.43672 & 0.697568 \\
\hline 1.05571 & 2.99844 & 0.868046 & 2.73104 & 1.07224 & 4.64042 & 0.727157 \\
\hline 1.05319 & 2.87266 & 0.813532 & 2.77487 & 1.09063 & 4.66712 & 0.726697 \\
\hline 1.0447 & 2.66509 & 0.730663 & 2.73677 & 1.065 & 4.52328 & 0.714358 \\
\hline
\end{tabular}




\begin{tabular}{|c|c|c|c|c|c|c|}
\hline 1.02438 & 2.41506 & 0.641119 & 2.59692 & 0.998794 & 4.22464 & 0.655836 \\
\hline 0.960508 & 2.13711 & 0.538906 & 2.38771 & 0.914769 & 3.81116 & 0.598718 \\
\hline 0.834119 & 1.86687 & 0.450141 & 2.14531 & 0.811562 & 3.30921 & 0.522871 \\
\hline 0.725458 & 1.61422 & 0.35758 & 1.86289 & 0.679517 & 2.77818 & 0.43674 \\
\hline 0.664389 & 1.39522 & 0.281396 & 1.57529 & 0.548794 & 2.25347 & 0.343531 \\
\hline 0.53966 & 1.22077 & 0.190838 & 1.3253 & 0.437114 & 1.77442 & 0.279358 \\
\hline 0.404033 & 1.091 & 0.14244 & 1.10463 & 0.338937 & 1.35896 & 0.215263 \\
\hline 0.322197 & 1.02984 & 0.130927 & 0.916149 & 0.262771 & 1.02998 & 0.154315 \\
\hline 0.26498 & 0.999771 & 0.104177 & 0.777405 & 0.203518 & 0.766878 & 0.117494 \\
\hline 0.200738 & 1.02287 & 0.113112 & 0.680333 & 0.161343 & 0.569959 & 0.0865421 \\
\hline 0.191812 & 1.0728 & 0.10621 & 0.622553 & 0.14084 & 0.440766 & 0.0661403 \\
\hline 0.196447 & 1.16356 & 0.123774 & 0.585168 & 0.123361 & 0.353883 & 0.0472697 \\
\hline 0.185007 & 1.32398 & 0.152098 & 0.577926 & 0.117987 & 0.302526 & 0.0379307 \\
\hline 0.158504 & 1.52816 & 0.164754 & 0.600636 & 0.125148 & 0.285166 & 0.0366403 \\
\hline 0.16518 & 1.83986 & 0.200462 & 0.641628 & 0.14006 & 0.285903 & 0.0339081 \\
\hline 0.165343 & 2.28161 & 0.241063 & 0.711369 & 0.150221 & 0.304102 & 0.032995 \\
\hline 0.176781 & 2.97141 & 0.312235 & 0.815179 & 0.171888 & 0.343121 & 0.0412645 \\
\hline 0.166894 & 4.05505 & 0.440543 & 0.966786 & 0.203758 & 0.394629 & 0.0522589 \\
\hline 0.171295 & 5.74313 & 0.628074 & 1.19566 & 0.251982 & 0.46533 & 0.0567554 \\
\hline 0.204379 & 8.38141 & 0.917134 & 1.54095 & 0.334885 & 0.55987 & 0.0655621 \\
\hline 0.233865 & 12.4056 & 1.33707 & 2.05498 & 0.459993 & 0.678172 & 0.0839173 \\
\hline 0.268688 & 18.4923 & 1.97796 & 2.83072 & 0.620686 & 0.804052 & 0.103555 \\
\hline 0.276409 & 27.298 & 2.95891 & 4.02139 & 0.881526 & 0.972153 & 0.126149 \\
\hline 0.306787 & 39.7666 & 4.34894 & 5.73171 & 1.26589 & 1.19256 & 0.155451 \\
\hline 0.387316 & 56.6119 & 6.22428 & 8.16561 & 1.81362 & 1.46168 & 0.188494 \\
\hline 0.475231 & 78.3704 & 8.6131 & 11.5597 & 2.59571 & 1.81243 & 0.23374 \\
\hline 0.575863 & 105.103 & 11.6619 & 16.0411 & 3.62427 & 2.23488 & 0.281961 \\
\hline 0.706292 & 136.663 & 15.0444 & 21.7353 & 4.9341 & 2.73948 & 0.354119 \\
\hline 0.873879 & 171.932 & 18.8688 & 28.7174 & 6.52448 & 3.34365 & 0.43364 \\
\hline 1.06766 & 208.599 & 22.9286 & 36.7636 & 8.36496 & 4.02534 & 0.514927 \\
\hline 1.28853 & 244.311 & 26.762 & 45.6023 & 10.41 & 4.75021 & 0.61474 \\
\hline 1.48078 & 276.027 & 30.1598 & 54.7337 & 12.4443 & 5.4706 & 0.708985 \\
\hline 1.7154 & 300.771 & 33.0485 & 63.4784 & 14.4096 & 6.17501 & 0.794177 \\
\hline 1.91459 & 315.942 & 34.5749 & 71.1436 & 16.1931 & 6.76637 & 0.889364 \\
\hline 2.121 & 319.66 & 34.904 & 76.9843 & 17.549 & 7.16651 & 0.958337 \\
\hline 2.21995 & 311.934 & 34.1207 & 80.3603 & 18.2882 & 7.36714 & 0.981657 \\
\hline 2.1869 & 293.258 & 31.9574 & 80.8958 & 18.4044 & 7.31548 & 0.968255 \\
\hline 2.13807 & 265.994 & 29.1223 & 78.6998 & 17.9533 & 7.00791 & 0.933576 \\
\hline 1.98354 & 232.175 & 25.4698 & 73.871 & 16.7763 & 6.48629 & 0.866342 \\
\hline 1.81087 & 195.567 & 21.5322 & 66.8446 & 15.1272 & 5.83097 & 0.764528 \\
\hline 1.63395 & 158.707 & 17.3056 & 58.3529 & 13.2221 & 5.07112 & 0.660088 \\
\hline 1.38569 & 124.291 & 13.6039 & 49.1925 & 11.1685 & 4.26023 & 0.555368 \\
\hline 1.14481 & 94.0059 & 10.2653 & 40.0522 & 9.11193 & 3.46254 & 0.44974 \\
\hline 0.939361 & 68.5629 & 7.55858 & 31.5075 & 7.16621 & 2.72086 & 0.35341 \\
\hline 0.7321 & 48.3875 & 5.21479 & 23.9645 & 5.44275 & 2.07515 & 0.27 \\
\hline 0.585496 & 33.1661 & 3.61575 & 17.6288 & 3.98897 & 1.53274 & 0.197927 \\
\hline 0.476776 & 22.1363 & 2.4503 & 12.5612 & 2.85286 & 1.12479 & 0.144973 \\
\hline
\end{tabular}




\begin{tabular}{|c|c|c|c|c|c|c|}
\hline 0.36452 & 14.5889 & 1.59693 & 8.68895 & 1.97818 & 0.815594 & 0.108366 \\
\hline 0.295287 & 9.6333 & 1.05062 & 5.8865 & 1.33105 & 0.586025 & 0.0806417 \\
\hline 0.251891 & 6.59916 & 0.701319 & 3.93434 & 0.895154 & 0.436818 & 0.0551451 \\
\hline 0.233237 & 4.90536 & 0.518745 & 2.65832 & 0.606203 & 0.339204 & 0.0381174 \\
\hline 0.202658 & 4.15864 & 0.459353 & 1.86528 & 0.421588 & 0.283039 & 0.0331798 \\
\hline 0.195601 & 4.12452 & 0.436774 & 1.42217 & 0.31767 & 0.248812 & 0.0323331 \\
\hline 0.195085 & 4.66599 & 0.507012 & 1.2266 & 0.274567 & 0.235329 & 0.030931 \\
\hline 0.179603 & 5.76921 & 0.640019 & 1.21364 & 0.270153 & 0.240601 & 0.0302998 \\
\hline 0.184354 & 7.4146 & 0.846357 & 1.37688 & 0.301387 & 0.257355 & 0.0273158 \\
\hline 0.222827 & 9.58861 & 1.06609 & 1.66927 & 0.36385 & 0.291364 & 0.0324125 \\
\hline 0.234451 & 12.3926 & 1.38606 & 2.09677 & 0.443474 & 0.332689 & 0.0378496 \\
\hline 0.222675 & 15.8511 & 1.76991 & 2.68469 & 0.572698 & 0.387243 & 0.045733 \\
\hline 0.225669 & 19.8973 & 2.1997 & 3.43709 & 0.743404 & 0.460696 & 0.0552112 \\
\hline 0.23536 & 24.4516 & 2.76818 & 4.35069 & 0.931736 & 0.544223 & 0.0668222 \\
\hline 0.257291 & 29.0733 & 3.26394 & 5.39723 & 1.15401 & 0.650716 & 0.0824423 \\
\hline 0.273345 & 33.6665 & 3.76085 & 6.56132 & 1.4188 & 0.763744 & 0.0952968 \\
\hline 0.314878 & 37.8128 & 4.22402 & 7.77408 & 1.68735 & 0.86667 & 0.11285 \\
\hline 0.350744 & 41.1351 & 4.61502 & 8.93062 & 1.92661 & 0.964188 & 0.123027 \\
\hline 0.380592 & 43.2526 & 4.77553 & 9.97394 & 2.15403 & 1.05924 & 0.127202 \\
\hline 0.414932 & 43.9461 & 4.91366 & 10.7978 & 2.33133 & 1.1283 & 0.136751 \\
\hline 0.408291 & 43.3091 & 4.86113 & 11.3379 & 2.41757 & 1.16492 & 0.141304 \\
\hline 0.402337 & 41.1487 & 4.51635 & 11.5492 & 2.46503 & 1.18096 & 0.143656 \\
\hline 0.422955 & 37.8252 & 4.18775 & 11.3711 & 2.45167 & 1.15592 & 0.143971 \\
\hline 0.402062 & 33.6124 & 3.73391 & 10.8373 & 2.33584 & 1.09509 & 0.13961 \\
\hline 0.378027 & 28.9356 & 3.17754 & 9.98093 & 2.14808 & 1.01996 & 0.121146 \\
\hline 0.391215 & 23.9887 & 2.67446 & 8.88796 & 1.90285 & 0.916281 & 0.108973 \\
\hline 0.35582 & 19.2363 & 2.12334 & 7.68011 & 1.63662 & 0.8016 & 0.106865 \\
\hline 0.302892 & 14.9006 & 1.62061 & 6.42992 & 1.38391 & 0.694175 & 0.0911108 \\
\hline 0.269537 & 11.2031 & 1.24825 & 5.20791 & 1.11443 & 0.585694 & 0.0683918 \\
\hline 0.25632 & 8.16731 & 0.897816 & 4.08978 & 0.876545 & 0.494386 & 0.055018 \\
\hline 0.234812 & 5.75848 & 0.622969 & 3.11307 & 0.66669 & 0.412923 & 0.0468616 \\
\hline 0.215638 & 3.96282 & 0.425562 & 2.29899 & 0.490785 & 0.338997 & 0.0406233 \\
\hline 0.20956 & 2.65207 & 0.28083 & 1.64461 & 0.339924 & 0.285035 & 0.03493 \\
\hline 0.193767 & 1.75287 & 0.176816 & 1.15582 & 0.236008 & 0.246511 & 0.0301776 \\
\hline 0.18463 & 1.15255 & 0.11926 & 0.804753 & 0.167934 & 0.216011 & 0.0277268 \\
\hline 0.196863 & 0.75222 & 0.0616321 & 0.551646 & 0.114126 & 0.192769 & 0.0266002 \\
\hline 0.169753 & 0.506909 & 0.0481993 & 0.382109 & 0.0792216 & 0.180502 & 0.0253503 \\
\hline 0.167208 & 0.362724 & 0.0337335 & 0.269737 & 0.0553222 & 0.173193 & 0.0240138 \\
\hline 0.183412 & 0.281363 & 0.0238308 & 0.197681 & 0.0390885 & 0.167262 & 0.0221116 \\
\hline 0.18248 & 0.234124 & 0.0166445 & 0.15415 & 0.0289387 & 0.16438 & 0.0211213 \\
\hline 0.182121 & 0.20701 & 0.0192809 & 0.129995 & 0.0261451 & 0.166708 & 0.023508 \\
\hline 0.185889 & 0.197873 & 0.0172684 & 0.113788 & 0.0228414 & 0.170361 & 0.023664 \\
\hline 0.211282 & 0.191767 & 0.0154614 & 0.103413 & 0.0202582 & 0.169486 & 0.0227189 \\
\hline 0.200126 & 0.186963 & 0.0156058 & 0.0996979 & 0.0218506 & 0.168319 & 0.0253497 \\
\hline 0.199387 & 0.190523 & 0.0201605 & 0.0997846 & 0.0224863 & 0.16863 & 0.0231887 \\
\hline 0.209777 & 0.188627 & 0.0161383 & 0.0988547 & 0.0219518 & 0.171468 & 0.0245505 \\
\hline 0.194355 & 0.190322 & 0.0141799 & 0.0994506 & 0.0228482 & 0.173218 & 0.0282257 \\
\hline
\end{tabular}




\begin{tabular}{|c|c|c|c|c|c|c|}
\hline 0.198765 & 0.192102 & 0.0171661 & 0.0985683 & 0.0242527 & 0.177917 & 0.0261348 \\
\hline 0.204147 & 0.199308 & 0.0200549 & 0.0970648 & 0.0234554 & 0.183889 & 0.028003 \\
\hline .195397 & 0.199326 & 0.0204313 & 0.0992521 & 0.0214846 & 0.188298 & 0.0297735 \\
\hline .193201 & 0.205697 & 0.0238199 & 0.1025 & 0.0193513 & 0.194302 & 0.0296095 \\
\hline 209717 & 0.208873 & 0.0252635 & 0.103163 & 0.02199 & 0.203488 & 0.0335941 \\
\hline 0.206325 & 0.212258 & 0.0230074 & 0.103118 & 0.0237582 & 0.216905 & 0.0395995 \\
\hline 0.218796 & 0.218726 & 0.0204418 & 0.107149 & 0.0239229 & 0.237668 & 0.045287 \\
\hline 0.218917 & 0.229298 & 0.0258348 & 0.112688 & 0.0242674 & 0.271404 & 0.0595414 \\
\hline 0.234838 & 0.242397 & 0.0297456 & 0.118982 & 0.0269296 & 0.318512 & 0.0814513 \\
\hline 0.259214 & 0.261053 & 0.0334557 & 0.131209 & 0.0304205 & 0.387853 & 0.113254 \\
\hline 0.261253 & 0.284598 & 0.0426304 & 0.143373 & 0.0326001 & 0.485415 & 0.157354 \\
\hline 0.252833 & 0.315305 & 0.0495054 & 0.158145 & 0.0356143 & 0.620356 & 0.217699 \\
\hline 0.260103 & 0.353489 & 0.070448 & 0.183308 & 0.0423039 & 0.804346 & 0.300584 \\
\hline 0.290524 & 0.400307 & 0.0768463 & 0.209256 & 0.0494222 & 1.0354 & 0.413638 \\
\hline 0.332115 & 0.445493 & 0.0915985 & 0.242893 & 0.0599416 & 1.32885 & 0.544966 \\
\hline 0.374999 & 0.497081 & 0.115894 & 0.29083 & 0.0735528 & 1.68536 & 0.711647 \\
\hline 0.4306 & 0.553543 & 0.138522 & 0.334566 & 0.085441 & 2.09264 & 0.908915 \\
\hline .486546 & 0.607212 & 0.160115 & 0.383068 & 0.100335 & 2.53779 & 1.11493 \\
\hline 0.585383 & 0.654231 & 0.175781 & 0.436075 & 0.11 & 986 & 1.35028 \\
\hline 0.651267 & 0.700043 & 0.198575 & 0.489876 & 0.131949 & 3.45608 & 1.58117 \\
\hline 0.674209 & 0.733227 & 0.220587 & 0.537192 & 0.15018 & 3.85858 & 1.78246 \\
\hline 0.710393 & 0.741809 & 0.223575 & 0.5788 & 0.15 & 671 & 1.96204 \\
\hline 0.785517 & 0.735195 & 0.21643 & 0.602719 & 0.164185 & 4.43583 & 2.05594 \\
\hline 0.832314 & 0.721508 & 0.208029 & 0.615999 & 0.163966 & 4.5412 & 2.1197 \\
\hline 0.797494 & 0.691028 & 0.210182 & 0.617558 & 0.163707 & 4.50802 & 2.12386 \\
\hline 0.801526 & 0.650417 & 0.188036 & 0.600692 & 0.162793 & 4.33348 & 2.02995 \\
\hline 0.767366 & 0.59752 & 0.151934 & 0.568573 & 0.155573 & 4.05291 & 1.89721 \\
\hline 0.712771 & 0.548644 & 0.14319 & 0.531557 & 0.144132 & 3.70079 & 1.72652 \\
\hline 0.684434 & 0.489929 & 0.120367 & 0.486213 & 0.132595 & 3.25931 & 1.52366 \\
\hline 0.601243 & 0.435851 & 0.0950606 & 0.433719 & 0.111881 & 2.79441 & 1.2984 \\
\hline 0.559631 & 0.392 & 0.0759368 & 0.379602 & 0.0941799 & 2.33878 & 1.06292 \\
\hline 0.511091 & 0.358017 & 0.0599978 & 0.327227 & 0.0825611 & 1.9031 & 0.836695 \\
\hline 0.491934 & 0.327066 & 0.050012 & 0.278693 & 0.0688717 & 1.52923 & 0.663687 \\
\hline 0.484041 & 0.300183 & 0.044633 & 0.241794 & 0.0561971 & 1.20018 & 0.486578 \\
\hline 0.494985 & 0.287084 & 0.0325455 & 0.211209 & 0.0436469 & 0.92906 & 0.363366 \\
\hline 0.488875 & 0.277279 & 0.0230789 & 0.185504 & 0.0367456 & 0.719742 & 0.260875 \\
\hline 0.434322 & 0.273386 & 0.0256938 & 0.168382 & 0.0363774 & 0.574505 & 0.180483 \\
\hline 0.433709 & 0.267594 & 0.0245257 & 0.152881 & 0.0319647 & 0.46586 & 0.132756 \\
\hline 0.462747 & 0.26476 & 0.0251188 & 0.146346 & 0.0301913 & 0.394361 & 0.0901936 \\
\hline 0.454565 & 0.266132 & 0.0237251 & 0.140275 & 0.0281151 & 0.347223 & 0.0711231 \\
\hline 0.459256 & 0.264302 & 0.0227372 & 0.134861 & 0.0268965 & 0.308523 & 0.0570991 \\
\hline 0.469886 & 0.265198 & 0.0203939 & 0.134541 & 0.0289634 & 0.286747 & 0.0523803 \\
\hline 0.503883 & 0.26629 & 0.0192212 & 0.130591 & 0.0286315 & 0.276006 & 0.0436421 \\
\hline 0.467901 & 0.270475 & 0.0209615 & 0.135186 & 0.0276782 & 0.269635 & 0.0372518 \\
\hline 0.444926 & 0.272652 & 0.0223048 & 0.135444 & 0.0272935 & 0.266712 & 0.0328994 \\
\hline 0.480923 & 0.280089 & 0.0214074 & 0.133073 & 0.0294522 & 0.269315 & 0.0322772 \\
\hline 0.512567 & 0.281561 & 0.0202344 & 0.136929 & 0.0282287 & 0.276721 & 0.034798 \\
\hline
\end{tabular}




\begin{tabular}{|c|c|c|c|c|c|c|}
\hline 0.479486 & 0.285391 & 0.024223 & 0.141224 & 0.0306677 & 0.281628 & 0.0364699 \\
\hline 0.480046 & 0.292868 & 0.0222008 & 0.142641 & 0.0302105 & 0.296273 & 0.0425639 \\
\hline 0.448061 & 0.305886 & 0.0241197 & 0.147336 & 0.03441 & 0.317892 & 0.043517 \\
\hline .435445 & 0.310749 & 0.02553 & 0.153447 & 0.0357153 & 0.334809 & 0.045883 \\
\hline 0.440175 & 0.320321 & 0.0295099 & 0.163906 & 0.0352219 & 0.355043 & 0.0526614 \\
\hline 0.426905 & 0.331099 & 0.0237998 & 0.168019 & 0.0371316 & 0.384026 & 0.0593045 \\
\hline 0.424421 & 0.342707 & 0.0290757 & 0.174767 & 0.0410644 & 0.424184 & 0.0644552 \\
\hline 0.438124 & 0.361073 & 0.0314123 & 0.181982 & 0.0403164 & 0.467323 & 0.0768737 \\
\hline 0.411337 & 0.367519 & 0.0350936 & 0.193686 & 0.0476184 & 0.503158 & 0.0980425 \\
\hline 0.400695 & 0.381707 & 0.0388362 & 0.200576 & 0.0453254 & 0.553966 & 0.10603 \\
\hline 0.404137 & 0.399431 & 0.0391835 & 0.211658 & 0.051959 & 0.610267 & 0.128802 \\
\hline 0.379301 & 0.412799 & 0.048803 & 0.218419 & 0.0543121 & 0.679604 & 0.157047 \\
\hline 0.380649 & 0.429057 & 0.0531026 & 0.23048 & 0.0556503 & 0.740739 & 0.176684 \\
\hline 0.37955 & 0.448407 & 0.0580534 & 0.238617 & 0.0569567 & 0.808468 & 0.213261 \\
\hline 0.379863 & 0.460932 & 0.0627101 & 0.248458 & 0.0553872 & 0.888143 & 0.249111 \\
\hline 0.392551 & 0.473176 & 0.0689127 & 0.256474 & 0.0540786 & 0.959492 & 0.272503 \\
\hline 0.367919 & 0.483464 & 0.06043 & 0.268824 & 0.0535183 & 1.02434 & 0.303942 \\
\hline 0.394116 & 0.489441 & 0.0649455 & 0.269968 & 0.0531424 & 1.05911 & 0.317291 \\
\hline 0.384328 & 0.488788 & 0.067 & 0.27 & 0.0584762 & .08693 & 0.320969 \\
\hline 0.413025 & 0.492238 & 0.0731597 & 0.279351 & 0.0577313 & 1.09184 & 0.328278 \\
\hline 0.374756 & 0.490807 & 0.0672521 & 0.278822 & 0.0585405 & 1.07889 & 0.321111 \\
\hline 0.363063 & 0.483555 & 0.07 & 683 & 0.0533982 & 578 & 1555 \\
\hline 0.336077 & 0.475834 & 0.0714451 & 0.276643 & 0.057282 & 0.992051 & 0.284055 \\
\hline 0.358512 & 0.463601 & 0.0687355 & 0.269569 & 0.0539627 & 0.935675 & 0.264271 \\
\hline 0.353552 & 0.452255 & 0.0597076 & 0.259222 & 0.0483981 & 0.861229 & 0.231908 \\
\hline 0.362549 & 0.441546 & 0.0619708 & 0.250953 & 0.0436082 & 0.787663 & 0.208747 \\
\hline 0.355375 & 0.427858 & 0.0573399 & 0.23777 & 0.0462286 & 0.710921 & 0.173381 \\
\hline 0.306924 & 0.417899 & 0.059459 & 0.231577 & 0.0424421 & 0.644127 & 0.150495 \\
\hline 0.291642 & 0.411414 & 0.0600052 & 0.223293 & 0.0349385 & 0.586939 & 0.120758 \\
\hline 0.307576 & 0.399316 & 0.0543517 & 0.21192 & 0.0361263 & 0.532873 & 0.0980453 \\
\hline 0.310326 & 0.392742 & 0.0513102 & 0.205719 & 0.0373933 & 0.486785 & 0.0966524 \\
\hline 0.295856 & 0.385896 & 0.0493079 & 0.202022 & 0.0336453 & 0.451033 & 0.0837427 \\
\hline 0.291507 & 0.38234 & 0.0484484 & 0.198845 & 0.0340082 & 0.426003 & 0.0807738 \\
\hline 0.281027 & 0.376008 & 0.0526076 & 0.196344 & 0.0307973 & 0.40632 & 0.0821446 \\
\hline 0.275311 & 0.377478 & 0.0499922 & 0.195863 & 0.0325632 & 0.395132 & 0.0824815 \\
\hline 0.271227 & 0.376876 & 0.0471855 & 0.194074 & 0.0312663 & 0.382976 & 0.0781067 \\
\hline 0.275428 & 0.367235 & 0.0454157 & 0.190635 & 0.0306002 & 0.378464 & 0.0737101 \\
\hline 0.282193 & 0.364271 & 0.0456539 & 0.19569 & 0.0314604 & 0.380566 & 0.0756953 \\
\hline 0.274227 & 0.3582 & 0.0446466 & 0.194642 & 0.0307029 & 0.379737 & 0.0687589 \\
\hline 0.29159 & 0.35379 & 0.0419382 & 0.193704 & 0.0344392 & 0.377141 & 0.0701277 \\
\hline 0.269596 & 0.35258 & 0.0390377 & 0.200869 & 0.0369015 & 0.378963 & 0.0772261 \\
\hline 0.286023 & 0.347298 & 0.0441226 & 0.194122 & 0.0340023 & 0.374017 & 0.0741249 \\
\hline 0.283282 & 0.34595 & 0.0359601 & 0.198759 & 0.0336537 & 0.372275 & 0.0729559 \\
\hline 0.290009 & 0.34843 & 0.0333665 & 0.19851 & 0.0332483 & 0.377857 & 0.0700233 \\
\hline 0.317937 & 0.34726 & 0.0364751 & 0.192075 & 0.0364655 & 0.371953 & 0.0645912 \\
\hline 0.285453 & 0.341279 & 0.0360724 & 0.191477 & 0.0343068 & 0.362607 & 0.0690339 \\
\hline 0.28932 & 0.340011 & 0.0370476 & 0.195132 & 0.032419 & 0.367726 & 0.0596109 \\
\hline
\end{tabular}




\begin{tabular}{|c|c|c|c|c|c|c|}
\hline 0.283624 & 0.338374 & 0.0347176 & 0.194649 & 0.0315497 & 0.370765 & 0.0578565 \\
\hline 0.293823 & 0.339693 & 0.0385408 & 0.19054 & 0.0328529 & 0.366004 & 0.056336 \\
\hline 0.271934 & 0.338343 & 0.0312017 & 0.189129 & 0.0312662 & 0.364576 & 0.0541807 \\
\hline 0.29637 & 0.343967 & 0.0281184 & 0.189004 & 0.0365332 & 0.362606 & 0.0486983 \\
\hline 0.318947 & 0.349372 & 0.0315589 & 0.191492 & 0.0330416 & 0.361262 & 0.0508518 \\
\hline 0.296444 & 0.365015 & 0.0387823 & 0.195344 & 0.0294612 & 0.365807 & 0.0456158 \\
\hline 0.3435 & 0.375971 & 0.0431871 & 0.201712 & 0.0330712 & 0.365161 & 0.0409401 \\
\hline 0.329053 & 0.39779 & 0.0512116 & 0.207746 & 0.035293 & 0.379882 & 0.0469186 \\
\hline 0.397206 & 0.42352 & 0.0608825 & 0.217739 & 0.0442171 & 0.39297 & 0.0538143 \\
\hline 0.448652 & 0.453331 & 0.0727065 & 0.225836 & 0.0421401 & 0.422651 & 0.0514414 \\
\hline 0.428543 & 0.488401 & 0.0968175 & 0.246945 & 0.0519135 & 0.437036 & 0.0565618 \\
\hline 0.534778 & 0.53496 & 0.122317 & 0.267123 & 0.0618256 & 0.46998 & 0.0637013 \\
\hline 0.591345 & 0.571774 & 0.141024 & 0.292266 & 0.07088 & 0.510222 & 0.074709 \\
\hline 0.703508 & 0.608121 & 0.163888 & 0.322651 & 0.101284 & 0.54978 & 0.0862812 \\
\hline 0.794583 & 0.644719 & 0.185703 & 0.348094 & 0.110957 & 0.595116 & 0.0997288 \\
\hline 0.893829 & 0.673271 & 0.210287 & 0.384455 & 0.131852 & 0.637067 & 0.118732 \\
\hline 1.04419 & 0.692244 & 0.219829 & 0.423048 & 0.151405 & 0.678052 & 0.129087 \\
\hline 1.1206 & 0.712531 & 0.219171 & 0.442555 & 0.169149 & 0.710746 & 0.133774 \\
\hline 1.18026 & 0.725947 & 0.212831 & 0.459251 & 0.173803 & 0.740785 & 0.147253 \\
\hline 1.2682 & 0.723694 & 0.218333 & 0.480028 & 0.184704 & 0.752311 & 0.156063 \\
\hline 1.28299 & 0.702966 & 0.203847 & 0.486495 & 0.184246 & 0.769001 & 0.156362 \\
\hline 1.3078 & 0.681779 & 0.188145 & 0.492925 & 0.189847 & 0.765853 & 0.140092 \\
\hline 1.3373 & 0.654712 & 0.173303 & 0.480469 & 0.188491 & 0.744358 & 0.153085 \\
\hline 1.21149 & 0.62065 & 0.142377 & 0.461392 & 0.17055 & 0.715924 & 0.135296 \\
\hline 1.12294 & 0.596443 & 0.124155 & 0.453711 & 0.16779 & 0.691281 & 0.128685 \\
\hline 1.06681 & 0.568649 & 0.110463 & 0.417285 & 0.138445 & 0.652741 & 0.121729 \\
\hline 0.907683 & 0.53244 & 0.0886932 & 0.394842 & 0.128029 & 0.599265 & 0.10161 \\
\hline 0.831469 & 0.506824 & 0.0768048 & 0.366112 & 0.117147 & 0.567747 & 0.0833955 \\
\hline 0.692919 & 0.487438 & 0.0611439 & 0.3403 & 0.100132 & 0.537114 & 0.0810465 \\
\hline 0.625329 & 0.465248 & 0.048636 & 0.312692 & 0.0796104 & 0.497884 & 0.0721645 \\
\hline 0.548307 & 0.4484 & 0.043142 & 0.293246 & 0.0706107 & 0.4696 & 0.0634993 \\
\hline 0.460787 & 0.442317 & 0.0391011 & 0.278959 & 0.0658632 & 0.451119 & 0.0549394 \\
\hline 0.470242 & 0.436441 & 0.0373051 & 0.261205 & 0.0584194 & 0.430741 & 0.0601912 \\
\hline 0.396925 & 0.435844 & 0.0366166 & 0.251172 & 0.0560811 & 0.419792 & 0.0548619 \\
\hline 0.380844 & 0.429451 & 0.0346282 & 0.244436 & 0.0510566 & 0.413373 & 0.047838 \\
\hline 0.393427 & 0.429707 & 0.0349235 & 0.243172 & 0.0575648 & 0.405737 & 0.0469284 \\
\hline 0.378897 & 0.432711 & 0.0346092 & 0.232356 & 0.0479726 & 0.401545 & 0.0513337 \\
\hline 0.414616 & 0.440352 & 0.0416407 & 0.234629 & 0.0518663 & 0.401063 & 0.0463017 \\
\hline 0.381466 & 0.445277 & 0.0368202 & 0.232933 & 0.0567834 & 0.399191 & 0.044605 \\
\hline 0.330583 & 0.45152 & 0.0468856 & 0.231184 & 0.0551096 & 0.397759 & 0.0457713 \\
\hline 0.32476 & 0.456506 & 0.0457967 & 0.233435 & 0.0500724 & 0.390367 & 0.0445316 \\
\hline 0.353219 & 0.464406 & 0.0477797 & 0.235431 & 0.0587181 & 0.404338 & 0.0466189 \\
\hline 0.403963 & 0.464667 & 0.0454788 & 0.234016 & 0.0541465 & 0.396389 & 0.0473597 \\
\hline 0.340748 & 0.469363 & 0.0489783 & 0.238847 & 0.0561782 & 0.402124 & 0.0568162 \\
\hline 0.390254 & 0.480917 & 0.0526268 & 0.23891 & 0.0577274 & 0.412607 & 0.0532457 \\
\hline 0.361019 & 0.491975 & 0.0477667 & 0.239514 & 0.0542661 & 0.415621 & 0.0602198 \\
\hline 0.366686 & 0.500803 & 0.0508172 & 0.241133 & 0.0577705 & 0.416954 & 0.0590726 \\
\hline
\end{tabular}




\begin{tabular}{|c|c|c|c|c|c|c|}
\hline 0.334409 & 0.512036 & 0.057327 & 0.245288 & 0.0584729 & 0.415861 & 0.0583236 \\
\hline 0.334572 & 0.52361 & 0.0586562 & 0.253826 & 0.0572301 & 0.433366 & 0.0761684 \\
\hline 0.354397 & 0.534575 & 0.0611116 & 0.251648 & 0.0564633 & 0.440863 & 0.0859735 \\
\hline .404465 & 0.552252 & 0.05731 & 0.265902 & 0.0541382 & 0.448147 & 0.092416 \\
\hline 0.353417 & 0.571522 & 0.0627667 & 0.265077 & 0.0561717 & 0.462679 & 0.113081 \\
\hline 0.378221 & 0.592655 & 0.0619286 & 0.277657 & 0.057807 & 0.483372 & 0.123212 \\
\hline 0.385356 & 0.618338 & 0.0630686 & 0.286193 & 0.0621336 & 0.492613 & 0.136886 \\
\hline 0.423764 & 0.648172 & 0.0600547 & 0.300416 & 0.0563486 & 0.523379 & 0.153678 \\
\hline 0.466223 & 0.6902 & 0.0672015 & 0.316725 & 0.0555505 & 0.554373 & 0.163932 \\
\hline 0.462513 & 0.735999 & 0.0793012 & 0.340048 & 0.0582307 & 0.586894 & 0.172219 \\
\hline 0.492188 & 0.797919 & 0.0749817 & 0.364728 & 0.0570977 & 0.630933 & 0.176971 \\
\hline 0.539728 & 0.854376 & 0.083675 & 0.39714 & 0.0698721 & 0.684925 & 0.189104 \\
\hline 0.593269 & 0.921618 & 0.0919713 & 0.442137 & 0.074501 & 0.750045 & 0.177215 \\
\hline 0.681703 & 0.9799 & 0.102314 & 0.494166 & 0.0845099 & 0.822689 & 0.186468 \\
\hline 0.711132 & 1.04959 & 0.100289 & 0.547035 & 0.0953475 & 0.915648 & 0.185285 \\
\hline 0.806525 & 1.11515 & 0.111439 & 0.601289 & 0.116908 & 0.98759 & 0.196725 \\
\hline 0.935542 & 1.1589 & 0.121583 & 0.66275 & 0.13189 & 1.06946 & 0.19213 \\
\hline 1.03953 & 1.20217 & 0.126275 & 0.722212 & 0.13706 & 1.15412 & 0.208856 \\
\hline 1.10596 & 1.22799 & 0.115905 & 0.771166 & 0.1417 & 1.21542 & 0.185757 \\
\hline 1.19798 & 1.24342 & 0.125696 & 0.82157 & 0.156401 & 1.27468 & 0.181972 \\
\hline 1.25335 & 1.23796 & 0.131843 & 0.860598 & 0.170507 & 1.32341 & 0.194486 \\
\hline 1.26674 & 1.21076 & 0.129443 & & 0.172768 & 1.35676 & 0.180684 \\
\hline 1.39346 & 1.17744 & 0.128508 & 0.877073 & 0.174673 & 1.35358 & 0.170946 \\
\hline 1.28953 & 1.13822 & 0.121773 & 0.874015 & 0.169088 & 1.33169 & 0.175599 \\
\hline 1.2724 & 1.08245 & 0.107369 & 0.848094 & 0.159512 & 1.28133 & 0.170977 \\
\hline 1.18677 & 1.01939 & 0.0923853 & 0.816556 & 0.149724 & 1.2281 & 0.145281 \\
\hline 1.14523 & 0.959515 & 0.0843269 & 0.768536 & 0.139898 & 1.15411 & 0.145809 \\
\hline 1.05906 & 0.908932 & 0.0851578 & 0.721166 & 0.134635 & 1.0934 & 0.132757 \\
\hline 1.04951 & 0.848055 & 0.0716519 & 0.673565 & 0.11972 & 0.999141 & 0.116658 \\
\hline 0.914169 & 0.79847 & 0.0640948 & 0.607834 & 0.106081 & 0.933809 & 0.120068 \\
\hline 0.862519 & 0.756643 & 0.058822 & 0.557092 & 0.0911928 & 0.856746 & 0.0939447 \\
\hline 0.833132 & 0.722452 & 0.056677 & 0.513532 & 0.0893557 & 0.791585 & 0.0954774 \\
\hline 0.766016 & 0.692784 & 0.0554346 & 0.468541 & 0.0813546 & 0.735071 & 0.0811952 \\
\hline 0.697188 & 0.669286 & 0.0595939 & 0.445982 & 0.0788157 & 0.702996 & 0.0703068 \\
\hline 0.720437 & 0.644298 & 0.0543259 & 0.407812 & 0.0688536 & 0.654694 & 0.0655283 \\
\hline 0.680983 & 0.627374 & 0.0498818 & 0.394423 & 0.0693227 & 0.638622 & 0.0612402 \\
\hline 0.699999 & 0.614612 & 0.0522305 & 0.373747 & 0.0640568 & 0.61315 & 0.0634182 \\
\hline 0.65339 & 0.602556 & 0.0523941 & 0.360642 & 0.064085 & 0.606194 & 0.0584741 \\
\hline 0.676062 & 0.600437 & 0.0520977 & 0.357729 & 0.0717773 & 0.606317 & 0.0499105 \\
\hline 0.705047 & 0.599846 & 0.0501272 & 0.348788 & 0.065604 & 0.602476 & 0.0502971 \\
\hline 0.745155 & 0.588492 & 0.0527778 & 0.34706 & 0.0654982 & 0.602196 & 0.0581046 \\
\hline 0.771886 & 0.586565 & 0.0471047 & 0.346056 & 0.0611277 & 0.614942 & 0.0462526 \\
\hline 0.7218 & 0.585814 & 0.0557068 & 0.347696 & 0.0703055 & 0.632585 & 0.0546557 \\
\hline 0.860991 & 0.587189 & 0.0597443 & 0.349467 & 0.0689336 & 0.625461 & 0.0593916 \\
\hline 0.798548 & 0.597569 & 0.0606842 & 0.349246 & 0.0687127 & 0.659119 & 0.061241 \\
\hline 0.870843 & 0.607401 & 0.0625854 & 0.361075 & 0.0754383 & 0.670905 & 0.0648628 \\
\hline 0.861564 & 0.626962 & 0.0681339 & 0.365762 & 0.0732358 & 0.706396 & 0.058183 \\
\hline
\end{tabular}




\begin{tabular}{|c|c|c|c|c|c|c|}
\hline 1.07279 & 0.657798 & 0.078679 & 0.368591 & 0.073338 & 0.748654 & 0.0642073 \\
\hline 1.1039 & 0.697703 & 0.0905234 & 0.385495 & 0.0864582 & 0.802743 & 0.0766527 \\
\hline 1.2166 & 0.756659 & 0.101686 & 0.408111 & 0.0888236 & 0.871327 & 0.0713357 \\
\hline 1.3809 & 0.866712 & 0.141175 & 0.437551 & 0.0976833 & 0.985489 & 0.100265 \\
\hline 1.66068 & 1.02118 & 0.186568 & 0.474868 & 0.118689 & 1.14465 & 0.099102 \\
\hline 2.05264 & 1.25242 & 0.251674 & 0.536511 & 0.139913 & 1.40254 & 0.132939 \\
\hline 2.53178 & 1.58237 & 0.360383 & 0.64647 & 0.186417 & 1.75713 & 0.160997 \\
\hline 3.53636 & 2.03715 & 0.492013 & 0.795657 & 0.229813 & 2.29496 & 0.214554 \\
\hline 4.46693 & 2.66385 & 0.697632 & 1.00131 & 0.337886 & 3.08297 & 0.280945 \\
\hline 6.3201 & 3.50103 & 0.957707 & 1.30326 & 0.436778 & 4.15416 & 0.363372 \\
\hline 8.80965 & 4.57599 & 1.29247 & 1.73502 & 0.619932 & 5.66729 & 0.504527 \\
\hline 12.1371 & 5.93178 & 1.7426 & 2.30481 & 0.84928 & 7.68128 & 0.665466 \\
\hline 16.3524 & 7.55976 & 2.22774 & 3.0801 & 1.15952 & 10.3583 & 0.921693 \\
\hline 21.7905 & 9.47848 & 2.81905 & 4.07925 & 1.56075 & 13.7564 & 1.28922 \\
\hline 28.8871 & 11.6463 & 3.47515 & 5.2999 & 2.05533 & 17.9028 & 1.58111 \\
\hline 36.9856 & 13.9727 & 4.21035 & 6.81219 & 2.64751 & 22.7908 & 2.07478 \\
\hline 47.2077 & 16.4544 & 4.95215 & 8.53117 & 3.32292 & 28.4736 & 2.62301 \\
\hline 59.1444 & 18.8764 & 5.77182 & 10.5238 & 4.13052 & 34.8362 & 3.23421 \\
\hline 71.3179 & 21.1195 & 6.48406 & 12.6163 & 4.96955 & 41.4284 & 3.76185 \\
\hline 83.9831 & 23.0558 & 7.064 & 14.8104 & 5.78641 & 48.3172 & 4.49601 \\
\hline 97.4547 & 24.5471 & 7.51067 & 16.9769 & 6.66282 & 54.8546 & 5.16745 \\
\hline 110.626 & 25.4989 & 7.75338 & 19.0446 & 7.53979 & 60.7473 & 5.60481 \\
\hline 120.904 & 25.8543 & 7.91146 & 20.8463 & 8.20873 & 65.7786 & 6.08034 \\
\hline 130.133 & 25.5682 & 7.81083 & 22.1464 & 8.72621 & 69.165 & 6.51586 \\
\hline 136.315 & 24.5991 & 7.49717 & 22.9565 & 9.09012 & 71.0412 & 6.65515 \\
\hline 138.065 & 23.1701 & 7.03853 & 23.3481 & 9.23555 & 71.1657 & 6.75469 \\
\hline 138.966 & 21.2308 & 6.40434 & 23.0778 & 9.09575 & 69.5385 & 6.66104 \\
\hline 134.992 & 19.0353 & 5.73417 & 22.2221 & 8.78167 & 66.1697 & 6.33425 \\
\hline 126.999 & 16.6273 & 4.9526 & 20.8848 & 8.20776 & 61.3628 & 5.88699 \\
\hline 116.651 & 14.2027 & 4.21297 & 19.1821 & 7.53238 & 55.5451 & 5.30132 \\
\hline 105.279 & 11.8697 & 3.50348 & 17.1643 & 6.73402 & 49.1532 & 4.79761 \\
\hline 93.0746 & 9.72116 & 2.83011 & 14.9821 & 5.89349 & 42.3293 & 4.09695 \\
\hline 80.1195 & 7.78789 & 2.24175 & 12.7585 & 4.91494 & 35.5958 & 3.54082 \\
\hline 67.1034 & 6.13921 & 1.72404 & 10.6271 & 4.12104 & 29.159 & 2.85697 \\
\hline 55.4222 & 4.79814 & 1.30303 & 8.67006 & 3.34135 & 23.3846 & 2.34296 \\
\hline 44.3065 & 3.72744 & 0.952541 & 6.88162 & 2.61987 & 18.3032 & 1.80481 \\
\hline 34.868 & 2.90462 & 0.690578 & 5.3681 & 2.00318 & 14.0027 & 1.36465 \\
\hline 26.2886 & 2.27251 & 0.488293 & 4.139 & 1.52361 & 10.4951 & 1.04319 \\
\hline 20.0783 & 1.81366 & 0.338936 & 3.11617 & 1.14233 & 7.71 & 0.761811 \\
\hline 14.6221 & 1.48735 & 0.239591 & 2.32424 & 0.793567 & 5.58116 & 0.536254 \\
\hline 10.5904 & 1.27544 & 0.173582 & 1.73918 & 0.570606 & 4.0226 & 0.408542 \\
\hline 7.66741 & 1.12414 & 0.13498 & 1.30439 & 0.401079 & 2.89151 & 0.293672 \\
\hline 5.49714 & 1.03835 & 0.108238 & 1.00042 & 0.279016 & 2.09028 & 0.193954 \\
\hline 3.94747 & 0.976321 & 0.0901172 & 0.781852 & 0.200022 & 1.54 & 0.149505 \\
\hline 2.80515 & 0.937873 & 0.083513 & 0.63751 & 0.157571 & 1.16088 & 0.114471 \\
\hline 2.17489 & 0.916148 & 0.0765444 & 0.539808 & 0.114717 & 0.927858 & 0.0851394 \\
\hline 1.649 & 0.904194 & 0.0569866 & 0.477916 & 0.0948945 & 0.78089 & 0.076989 \\
\hline
\end{tabular}




\begin{tabular}{|c|c|c|c|c|c|c|}
\hline 1.29427 & 0.894505 & 0.0642197 & 0.441993 & 0.0711823 & 0.694851 & 0.0628987 \\
\hline 1.00349 & 0.897566 & 0.0621366 & 0.418793 & 0.0710184 & 0.620651 & 0.0593832 \\
\hline 0.895768 & 0.900136 & 0.0602234 & 0.402385 & 0.0677591 & 0.591155 & 0.0583691 \\
\hline 0.87032 & 0.900759 & 0.0661016 & 0.392083 & 0.0629876 & 0.568316 & 0.0613838 \\
\hline 0.799799 & 0.903414 & 0.0649131 & 0.400862 & 0.0656671 & 0.554124 & 0.0594834 \\
\hline 0.678094 & 0.901297 & 0.0616461 & 0.391317 & 0.0631244 & 0.554327 & 0.0584392 \\
\hline 0.73876 & 0.895774 & 0.0636004 & 0.39223 & 0.0586558 & 0.558583 & 0.0616487 \\
\hline 0.639922 & 0.904017 & 0.0560937 & 0.395822 & 0.0622388 & 0.556957 & 0.0537569 \\
\hline 0.660904 & 0.901494 & 0.0541111 & 0.397833 & 0.0560699 & 0.565074 & 0.0651751 \\
\hline 0.655779 & 0.902366 & 0.0586835 & 0.399483 & 0.0538525 & 0.556466 & 0.0638433 \\
\hline 0.688612 & 0.900071 & 0.0569755 & 0.395695 & 0.0524721 & 0.565074 & 0.0561913 \\
\hline 0.650905 & 0.909005 & 0.0545434 & 0.402432 & 0.0516957 & 0.564914 & 0.0497883 \\
\hline 0.68014 & 0.913881 & 0.0647529 & 0.406857 & 0.0597635 & 0.582316 & 0.0595777 \\
\hline 0.658876 & 0.919766 & 0.0629884 & 0.408013 & 0.0484858 & 0.587877 & 0.0623491 \\
\hline 0.712528 & 0.925793 & 0.0574715 & 0.413483 & 0.0513401 & 0.594169 & 0.0585457 \\
\hline 0.683407 & 0.930172 & 0.0558305 & 0.413092 & 0.053007 & 0.608881 & 0.0565344 \\
\hline 0.662523 & 0.931478 & 0.0548824 & 0.422527 & 0.0581017 & 0.622013 & 0.0665531 \\
\hline 0.678849 & 0.94037 & 0.0568321 & 0.425647 & 0.0575544 & 0.636475 & 0.054245 \\
\hline 0.72128 & 0.95215 & 0.0566033 & 0.431182 & 0.0624174 & 0.654052 & 0.0611873 \\
\hline 0.6796 & 0.963955 & 0.0585311 & 0.432918 & 0.061006 & 0.674666 & 0.0536096 \\
\hline 0.692431 & 0.979753 & 0.0621035 & 0.452505 & 0.0629322 & 0.701624 & 0.0616244 \\
\hline 0.699198 & 1.00602 & 0.0662468 & 0.464769 & 0.0674404 & 0.741003 & 0.0590997 \\
\hline 0.821349 & 1.04252 & 0.070192 & 0.478107 & 0.0627917 & 0.780586 & 0.0652913 \\
\hline 0.883137 & 1.09222 & 0.084643 & 0.494707 & 0.0787425 & 0.847045 & 0.0583353 \\
\hline 0.925422 & 1.14833 & 0.0974181 & 0.519052 & 0.0897336 & 0.93253 & 0.066181 \\
\hline 1.02588 & 1.22942 & 0.127416 & 0.543535 & 0.0961227 & 1.0329 & 0.0780081 \\
\hline 1.24048 & 1.33472 & 0.163615 & 0.583569 & 0.110882 & 1.17995 & 0.1021 \\
\hline 1.49935 & 1.46551 & 0.194428 & 0.635088 & 0.132187 & 1.36194 & 0.100436 \\
\hline 1.71006 & 1.63386 & 0.255027 & 0.715766 & 0.160044 & 1.61191 & 0.136446 \\
\hline 2.01291 & 1.83938 & 0.309244 & 0.809912 & 0.182438 & 1.91788 & 0.148003 \\
\hline 2.60598 & 2.08404 & 0.364174 & 0.915296 & 0.235449 & 2.31792 & 0.185028 \\
\hline 3.19752 & 2.36493 & 0.451597 & 1.0694 & 0.306281 & 2.83008 & 0.22104 \\
\hline 3.93239 & 2.66594 & 0.545963 & 1.26351 & 0.375878 & 3.40534 & 0.276986 \\
\hline 4.91553 & 3.02033 & 0.644584 & 1.47093 & 0.464496 & 4.12168 & 0.318378 \\
\hline 5.78639 & 3.34411 & 0.764117 & 1.72691 & 0.55219 & 4.94186 & 0.416006 \\
\hline 7.06943 & 3.70127 & 0.84405 & 2.00945 & 0.663663 & 5.81256 & 0.467385 \\
\hline 8.31663 & 4.03312 & 0.954305 & 2.3183 & 0.787632 & 6.7619 & 0.558242 \\
\hline 9.74184 & 4.31326 & 1.02293 & 2.63361 & 0.910561 & 7.76362 & 0.645602 \\
\hline 11.0604 & 4.52578 & 1.10188 & 2.9633 & 1.03108 & 8.71861 & 0.699105 \\
\hline 12.7199 & 4.71424 & 1.14075 & 3.254 & 1.18213 & 9.57618 & 0.802721 \\
\hline 13.4995 & 4.80004 & 1.15543 & 3.53592 & 1.29794 & 10.3446 & 0.842044 \\
\hline 15.0982 & 4.77845 & 1.16559 & 3.76439 & 1.34158 & 10.9441 & 0.910437 \\
\hline 15.7835 & 4.6669 & 1.1298 & 3.90017 & 1.40019 & 11.3124 & 0.968173 \\
\hline 16.0888 & 4.46015 & 1.05861 & 4.01099 & 1.43491 & 11.4354 & 0.954964 \\
\hline 16.4338 & 4.1955 & 0.981635 & 4.0052 & 1.46994 & 11.3345 & 0.998023 \\
\hline 16.1014 & 3.89265 & 0.894877 & 3.90518 & 1.42075 & 10.9486 & 0.936274 \\
\hline 15.4019 & 3.56098 & 0.802957 & 3.77826 & 1.36024 & 10.3709 & 0.919485 \\
\hline
\end{tabular}




\begin{tabular}{|c|c|c|c|c|c|c|}
\hline 14.6984 & 3.20116 & 0.699196 & 3.53551 & 1.27609 & 9.61624 & 0.807514 \\
\hline 13.4855 & 2.84631 & 0.584551 & 3.2669 & 1.15037 & 8.69913 & 0.759821 \\
\hline 12.2525 & 2.51239 & 0.492747 & 2.97313 & 1.03343 & 7.71246 & 0.707605 \\
\hline 10.6962 & 2.19546 & 0.397253 & 2.63698 & 0.906695 & 6.73259 & 0.573564 \\
\hline 9.43463 & 1.92101 & 0.312251 & 2.29946 & 0.771784 & 5.73654 & 0.507073 \\
\hline 7.91218 & 1.67941 & 0.239867 & 1.98439 & 0.657942 & 4.7837 & 0.422057 \\
\hline 6.50159 & 1.49125 & 0.180481 & 1.6825 & 0.515246 & 3.9269 & 0.358698 \\
\hline 5.34666 & 1.34136 & 0.142546 & 1.42162 & 0.420169 & 3.17792 & 0.271479 \\
\hline 4.11664 & 1.21589 & 0.113558 & 1.20454 & 0.339327 & 2.54614 & 0.2147 \\
\hline 3.33296 & 1.12884 & 0.0819461 & 0.995894 & 0.25411 & 2.00722 & 0.171975 \\
\hline 2.52625 & 1.06885 & 0.0730541 & 0.855646 & 0.201135 & 1.59158 & 0.142571 \\
\hline 1.95669 & 1.02131 & 0.0728794 & 0.712081 & 0.145533 & 1.2642 & 0.110309 \\
\hline 1.4565 & 0.997908 & 0.0645688 & 0.629883 & 0.111501 & 1.00531 & 0.0844182 \\
\hline 1.11662 & 0.982817 & 0.0511274 & 0.563451 & 0.091212 & 0.821789 & 0.068581 \\
\hline 0.824799 & 0.987425 & 0.0510307 & 0.509201 & 0.0789942 & 0.677769 & 0.0486258 \\
\hline 0.624661 & 0.998873 & 0.0539311 & 0.474253 & 0.0586258 & 0.590268 & 0.0528097 \\
\hline 0.485432 & 1.0114 & 0.0639671 & 0.448888 & 0.052324 & 0.514516 & 0.0419877 \\
\hline 0.360759 & 1.03813 & 0.0641015 & 0.439285 & 0.0619266 & 0.470005 & 0.0337875 \\
\hline 0.295291 & 1.06783 & 0.0669633 & 0.435349 & 0.0497591 & 0.440696 & 0.0389016 \\
\hline 0.231438 & 1.09326 & 0.0741798 & 0.436853 & 0.0579241 & 0.424193 & 0.0387495 \\
\hline 0.219043 & 1.12441 & 0.0787399 & 0.431503 & 0.0536226 & 0.415522 & 0.0332388 \\
\hline 0.178611 & 1.15247 & 0.0737908 & 0.446256 & 0.0580101 & 0.408429 & 0.032897 \\
\hline 0.179137 & 1.18329 & 0.0852458 & 0.45702 & 0.0619345 & 0.401088 & 0.0321218 \\
\hline 0.181837 & 1.19932 & 0.0893441 & 0.465619 & 0.0653782 & 0.394602 & 0.037129 \\
\hline 0.163251 & 1.21654 & 0.0886371 & 0.472622 & 0.0658093 & 0.404341 & 0.0328114 \\
\hline 0.154999 & 1.22481 & 0.089394 & 0.479139 & 0.0757418 & 0.408361 & 0.0323227 \\
\hline 0.17943 & 1.2238 & 0.090029 & 0.490562 & 0.0705054 & 0.418966 & 0.03505 \\
\hline 0.177925 & 1.22328 & 0.0906855 & 0.498674 & 0.0774615 & 0.42114 & 0.0343264 \\
\hline 0.210087 & 1.20405 & 0.0879674 & 0.515604 & 0.0752039 & 0.435291 & 0.0375256 \\
\hline 0.21379 & 1.18783 & 0.0734355 & 0.51103 & 0.0859726 & 0.447773 & 0.0351021 \\
\hline 0.246172 & 1.18295 & 0.0763366 & 0.518058 & 0.0775389 & 0.460986 & 0.0427404 \\
\hline 0.259319 & 1.16188 & 0.0732436 & 0.513151 & 0.0778294 & 0.473506 & 0.0346694 \\
\hline 0.286466 & 1.13895 & 0.066399 & 0.520118 & 0.064821 & 0.481906 & 0.0419616 \\
\hline 0.300574 & 1.11047 & 0.0648459 & 0.517772 & 0.0721642 & 0.498333 & 0.0409041 \\
\hline 0.32333 & 1.08857 & 0.0608382 & 0.515713 & 0.0769016 & 0.507576 & 0.0449691 \\
\hline 0.336652 & 1.06631 & 0.0536377 & 0.510321 & 0.0686841 & 0.511766 & 0.0368668 \\
\hline 0.386705 & 1.03951 & 0.0562582 & 0.512242 & 0.0708935 & 0.529377 & 0.0327129 \\
\hline 0.406718 & 1.02039 & 0.0625783 & 0.503202 & 0.0722087 & 0.530136 & 0.0475164 \\
\hline 0.419256 & 1.01097 & 0.0535226 & 0.500363 & 0.0633363 & 0.536228 & 0.0396697 \\
\hline 0.371615 & 0.988862 & 0.0550076 & 0.494616 & 0.0617128 & 0.536842 & 0.0517814 \\
\hline 0.375552 & 0.970465 & 0.0592776 & 0.48856 & 0.0641572 & 0.541704 & 0.0426934 \\
\hline 0.380761 & 0.96191 & 0.0579486 & 0.483249 & 0.0679359 & 0.541783 & 0.0446009 \\
\hline 0.366281 & 0.959218 & 0.0624579 & 0.468943 & 0.0617193 & 0.529335 & 0.049887 \\
\hline 0.310866 & 0.958338 & 0.0625162 & 0.467741 & 0.0528721 & 0.526645 & 0.0553571 \\
\hline 0.293787 & 0.949807 & 0.0628559 & 0.466197 & 0.0646975 & 0.51284 & 0.0495861 \\
\hline 0.265387 & 0.947241 & 0.0619349 & 0.454895 & 0.0667171 & 0.518314 & 0.0635861 \\
\hline 0.263664 & 0.949563 & 0.0597253 & 0.455649 & 0.0590531 & 0.510994 & 0.0600625 \\
\hline
\end{tabular}




\begin{tabular}{|c|c|c|c|c|c|c|}
\hline 0.239323 & 0.949586 & 0.0601531 & 0.446184 & 0.0529012 & 0.501009 & 0.0676795 \\
\hline 0.20151 & 0.952886 & 0.0523668 & 0.438833 & 0.0605439 & 0.494602 & 0.0704392 \\
\hline 0.182293 & 0.958173 & 0.0491987 & 0.448904 & 0.0682066 & 0.483002 & 0.0837324 \\
\hline 0.177616 & 0.96749 & 0.0616882 & 0.452047 & 0.0673244 & 0.490085 & 0.0813358 \\
\hline 0.154393 & 0.969837 & 0.0636359 & 0.448615 & 0.0609147 & 0.487985 & 0.0845174 \\
\hline 0.154072 & 0.97998 & 0.0649936 & 0.448209 & 0.0579059 & 0.482122 & 0.0796312 \\
\hline 0.114836 & 0.974893 & 0.0555938 & 0.447372 & 0.0600655 & 0.481405 & 0.0875627 \\
\hline 0.119183 & 0.977131 & 0.0562559 & 0.460188 & 0.0577207 & 0.48212 & 0.088649 \\
\hline 0.124637 & 0.970829 & 0.0569205 & 0.455004 & 0.0604347 & 0.490327 & 0.08562 \\
\hline 0.0990258 & 0.973648 & 0.0530231 & 0.46204 & 0.057163 & 0.481442 & 0.0833675 \\
\hline 0.105388 & 0.973507 & 0.0510112 & 0.459646 & 0.0633873 & 0.478934 & 0.0706467 \\
\hline 0.107512 & 0.955779 & 0.0536249 & 0.459404 & 0.0709501 & 0.486386 & 0.0715792 \\
\hline 0.114997 & 0.950234 & 0.0594816 & 0.465048 & 0.0635551 & 0.492191 & 0.0722369 \\
\hline 0.119884 & 0.946964 & 0.0632066 & 0.464595 & 0.0576193 & 0.488654 & 0.0706703 \\
\hline 0.107877 & 0.945337 & 0.0545257 & 0.458018 & 0.0587977 & 0.480985 & 0.0767619 \\
\hline 0.112351 & 0.947586 & 0.0517848 & 0.463312 & 0.0647178 & 0.489826 & 0.0618618 \\
\hline 0.106332 & 0.942309 & 0.0543078 & .466665 & 0.0677396 & 0.489605 & 9927 \\
\hline 0.11404 & 0.943077 & 0.0473838 & 0.4647 & 0.0706412 & 0.48942 & 0.0530158 \\
\hline 0.105791 & 0.946353 & 0.0541303 & 0.465523 & 0.0623014 & 0.489369 & 0.0664286 \\
\hline 0.116616 & 0.928581 & 7196 & 494 & 478 & 0.485123 & 0.04 \\
\hline 0.107601 & 0.926796 & 0.0625468 & 0.459797 & 0.0618275 & 0.482991 & 0.0516537 \\
\hline 0.104479 & 0.928612 & 0.0510837 & 0.460955 & 0.0600459 & 0.48634 & 0.0484477 \\
\hline 0.110972 & 0.917202 & 0.052172 & 0.458044 & 0.0594642 & 0.477237 & 0.0427297 \\
\hline 0.0856878 & 0.910616 & 0.0553309 & 0.457272 & 0.0659642 & 0.48239 & 0.0440347 \\
\hline 0.0966248 & 0.913599 & 0.0599032 & 0.461546 & 0.066204 & 0.489949 & 0.043745 \\
\hline 0.109693 & 0.903571 & 0.0536792 & 0.453005 & 0.058198 & 0.494954 & 0.0375638 \\
\hline 0.105158 & 0.891733 & 0.0574151 & 0.444533 & 0.0576705 & 0.48172 & 0.0390412 \\
\hline 0.111329 & 0.887036 & 0.0518489 & 0.441326 & 0.0599543 & 0.490259 & 0.0428678 \\
\hline 0.103886 & 0.884104 & 0.0459089 & 0.451184 & 0.0635569 & 0.483441 & 0.0366457 \\
\hline 0.107036 & 0.891436 & 0.0570807 & 0.444151 & 0.0642872 & 0.494367 & 0.0386191 \\
\hline 0.0975881 & 0.89357 & 0.0508015 & 0.450207 & 0.0605744 & 0.484012 & 0.0363178 \\
\hline 0.0956286 & 0.893497 & 0.0529496 & 0.442195 & 0.0577774 & 0.496061 & 0.0394199 \\
\hline 0.114313 & 0.889824 & 0.0552349 & 0.445829 & 0.0605803 & 0.492909 & 0.0344918 \\
\hline 0.140166 & 0.891016 & 0.0523136 & 0.447144 & 0.0488557 & 0.489592 & 0.0379214 \\
\hline 0.121139 & 0.899487 & 0.0506571 & 0.45543 & 0.0543712 & 0.490277 & 0.0364788 \\
\hline 0.100607 & 0.904651 & 0.0577001 & 0.446332 & 0.0534447 & 0.493947 & 0.040404 \\
\hline 0.140177 & 0.902841 & 0.0433651 & 0.45942 & 0.0464325 & 0.507118 & 0.0405272 \\
\hline 0.139595 & 0.903527 & 0.0520583 & 0.452295 & 0.0469341 & 0.509601 & 0.0269612 \\
\hline 0.130291 & 0.908797 & 0.0487619 & 0.457698 & 0.0573051 & 0.517932 & 0.0372214 \\
\hline 0.141934 & 0.923197 & 0.0436261 & 0.466695 & 0.0467316 & 0.529321 & 0.0449686 \\
\hline 0.168186 & 0.92241 & 0.0556714 & 0.465329 & 0.0478154 & 0.536827 & 0.0488036 \\
\hline 0.161584 & 0.925891 & 0.0477818 & 0.474809 & 0.0530765 & 0.546774 & 0.0400659 \\
\hline 0.183394 & 0.935068 & 0.0483851 & 0.475908 & 0.0536258 & 0.554946 & 0.0431185 \\
\hline 0.185237 & 0.942821 & 0.0468763 & 0.475854 & 0.0470964 & 0.576167 & 0.0407528 \\
\hline 0.211069 & 0.93519 & 0.0479648 & 0.483879 & 0.0546683 & 0.58023 & 0.040752 \\
\hline 0.212701 & 0.938114 & 0.0465401 & 0.489424 & 0.054454 & 0.579291 & 0.0462271 \\
\hline 0.211772 & 0.942098 & 0.0480756 & 0.493763 & 0.0599562 & 0.588157 & 0.0479928 \\
\hline
\end{tabular}




\begin{tabular}{|c|c|c|c|c|c|c|}
\hline 0.21523 & 0.944767 & 0.0452953 & 0.501153 & 0.0558547 & 0.59655 & 477 \\
\hline 0.267783 & 0.951486 & 0.0429054 & 0.493337 & 0.059477 & 0.606089 & 0.0512384 \\
\hline 0.217568 & 0.944481 & 0.0395433 & 0.494171 & 0.0573739 & 0.606887 & 0.0473102 \\
\hline 0.263352 & 0.953397 & 0.0535906 & 0.495938 & 0.0601603 & 0.599401 & 0.0465667 \\
\hline 0.241577 & .955098 & 0.0523374 & 0.496272 & 0.0606185 & 0.599404 & 0.0428862 \\
\hline 0.240196 & 0.950016 & 0.0439409 & 0.499254 & 0.0640947 & 0.600043 & 0.044774 \\
\hline 0.267886 & 0.949683 & 0.0435456 & 0.49497 & 0.0612241 & 0.592889 & 0.0445035 \\
\hline 0.248756 & 0.949833 & 0.0516228 & 0.488585 & 0.0661872 & 0.590607 & 0.0488613 \\
\hline 0.232097 & 0.944505 & 0.0519528 & 0.485656 & 0.0554956 & 0.576749 & 0.0370544 \\
\hline 0.227129 & 0.948604 & 0.0514881 & 0.482907 & 0.0573227 & 0.581052 & 0.0300366 \\
\hline 0.231706 & 0.951507 & 0.0494571 & 0.478094 & 0.0502083 & 0.563997 & 0.0406183 \\
\hline 0.226277 & 0.956868 & 0.0479285 & 0.483469 & 0.0547166 & 0.564382 & 0.0426423 \\
\hline 0.227101 & 0.965098 & 0.0479768 & 0.477143 & 0.0430273 & 0.563623 & 0.0360813 \\
\hline 0.198007 & 0.971804 & 9043 & 0.482603 & 0.057 & 0.556934 & 0.0355999 \\
\hline 0.195566 & 0.975183 & 0.0477251 & 0.475274 & 0.058077 & 0.552871 & 0.0316167 \\
\hline 0.174351 & 0.976961 & 0.0472091 & 0.477585 & 0.0549095 & 0.548699 & 0.0385979 \\
\hline 0.184482 & 0.990757 & 0.0517089 & 0.474406 & 0.0541867 & 0.553451 & 0.0410961 \\
\hline 0.177815 & 0.997215 & 0.0470506 & 0.482161 & 0.0569496 & 0.557962 & 0.0464594 \\
\hline 0.172905 & 1.00435 & 0.0533798 & 0.475933 & 0.050881 & 0.55086 & 0.0422359 \\
\hline 0.177777 & 1.02075 & 0.049827 & 0.487059 & 0.0539117 & 0.553285 & 0.0366171 \\
\hline 0.157701 & 1.02977 & 0.0560179 & 0.482865 & 0.050365 & 0.556171 & 0.0463488 \\
\hline 0.153105 & 1.02999 & 0.0580947 & 0.480144 & 0.0524789 & 0.555309 & 0.0401321 \\
\hline 0.149825 & 1.04374 & 0.0604038 & 0.486208 & 0.0510979 & 0.569942 & 0.0328322 \\
\hline 0.149388 & 1.0555 & 0.0604657 & 0.490277 & 0.056 & 0.558293 & 0.0452767 \\
\hline 0.100961 & 1.06701 & 0.04 & 849 & 48 & 966 & 881 \\
\hline 0.139985 & 1.07781 & 0.0631206 & 0.492121 & 0.0500057 & 0.575338 & 0.0484218 \\
\hline 0.125408 & 1.08914 & 0.0645734 & 0.502941 & 0.0560674 & 0.573061 & 0.0462462 \\
\hline 0.115112 & 1.08802 & 0.0582639 & 0.506949 & 0.0615067 & 0.585705 & 0.0463827 \\
\hline 0.136573 & 1.10534 & 0.0625755 & 0.507747 & 0.0545955 & 0.583235 & 0.0474614 \\
\hline 0.116914 & 1.1255 & 0.0659912 & 0.508599 & 0.0519391 & 0.585858 & 0.0443639 \\
\hline 0.11968 & 1.13141 & 0.0731253 & 0.508392 & 0.056983 & 0.597228 & 0.04645 \\
\hline 0.0953681 & 1.15162 & 0.0750047 & 0.521514 & 0.0588211 & 0.589419 & 0.0525044 \\
\hline 0.0993864 & 1.15232 & 0.0746848 & 0.518647 & 0.0597634 & 0.606957 & 0.0450545 \\
\hline 0.113893 & 1.1551 & 0.080002 & 0.519956 & 0.0590579 & 0.60267 & 0.0430324 \\
\hline 0.102234 & 1.16983 & 0.0894533 & 0.528839 & 0.0651164 & 0.613281 & 0.0546689 \\
\hline 0.100125 & 1.1893 & 0.0852721 & 0.53174 & 0.0630769 & 0.614482 & 0.0416395 \\
\hline 0.104779 & 1.20651 & 0.0823271 & 0.541744 & 0.0714383 & 0.623381 & 0.0502625 \\
\hline 0.113182 & 1.22277 & 0.0951548 & 0.544519 & 0.0574875 & 0.611587 & 0.0518808 \\
\hline 0.120008 & 1.22776 & 0.0909103 & 0.549907 & 0.0615972 & 0.638781 & 0.0475032 \\
\hline 0.101536 & 1.23816 & 0.0878654 & 0.557596 & 0.0746422 & 0.629826 & 0.0489991 \\
\hline 0.106209 & 1.25158 & 0.0949434 & 0.554834 & 0.0691966 & 0.642675 & 0.0435933 \\
\hline 0.101772 & 1.26659 & 0.0989945 & 0.563054 & 0.0640514 & 0.634114 & 0.0480808 \\
\hline 0.111063 & 1.28313 & 0.0966303 & 0.579214 & 0.0812915 & 0.65394 & 0.0486887 \\
\hline 0.106265 & 1.29427 & 0.0978902 & 0.565969 & 0.064758 & 0.659959 & 0.0510101 \\
\hline 0.112765 & 1.31188 & 0.0906657 & 0.58448 & 0.0767114 & 0.668679 & 0.0457487 \\
\hline 0.109546 & 1.33123 & 0.0944289 & 0.585387 & 0.0714786 & 0.683328 & 0.0597014 \\
\hline 0.0959754 & 1.35646 & 0.0914292 & 0.600997 & 0.0757611 & 0.696263 & 0.0587796 \\
\hline
\end{tabular}




\begin{tabular}{|c|c|c|c|c|c|c|}
\hline 0.0986272 & 1.37365 & 0.109913 & 0.605784 & 0.0797692 & 0.716389 & 0.0434225 \\
\hline 0.111244 & 1.3956 & 0.108055 & 0.616379 & 0.0752908 & 0.733399 & 0.0556829 \\
\hline 0.109576 & 1.40873 & 0.0998991 & 0.630181 & 0.0825142 & 0.762234 & 0.0530671 \\
\hline 0.124514 & 1.42707 & 0.0931185 & 0.637595 & 0.0775188 & 0.778369 & 0.0474859 \\
\hline 0.132466 & 1.4497 & 0.105576 & 0.648717 & 0.0797071 & 0.814543 & 0.0453475 \\
\hline 0.107465 & 1.46131 & 0.104742 & 0.66565 & 0.0855513 & 0.83898 & 0.0536224 \\
\hline 0.121345 & 1.48402 & 0.0865545 & 0.672157 & 0.0800394 & 0.869035 & 0.0500474 \\
\hline 0.121525 & 1.50007 & 0.0974198 & 0.685074 & 0.075741 & 0.902035 & 0.0468661 \\
\hline 0.0974662 & 1.51212 & 0.0981638 & 0.699254 & 0.0932091 & 0.931838 & 0.0694084 \\
\hline 0.0997277 & 1.50593 & 0.0932069 & 0.717821 & 0.088717 & 0.951119 & 0.0699575 \\
\hline 0.106369 & 1.51957 & 0.0903157 & 0.736347 & 0.0786742 & 0.975344 & 0.062439 \\
\hline 0.125371 & 1.53356 & 0.082873 & 0.738861 & 0.0776036 & 0.978991 & 0.0607615 \\
\hline 0.107215 & 1.52073 & 0.0870462 & 0.745118 & 0.0820754 & 1.00295 & 0.0673997 \\
\hline 0.102887 & 1.51985 & 0.087795 & 0.745417 & 0.0790102 & 0.997341 & 0.058588 \\
\hline 0.116002 & 1.51104 & 0.078524 & 0.754793 & 0.0895043 & 0.99607 & 0.0716464 \\
\hline 0.119942 & 1.4991 & 0.0797169 & 0.745091 & 0.087418 & 0.981326 & 0.0670539 \\
\hline 0.110054 & 1.50127 & 0.0802391 & 0.746429 & 0.0861003 & 0.977253 & 0.0702543 \\
\hline 0.121189 & 1.48776 & 0.0764272 & 0.752824 & 0.088283 & 0.946736 & 0.0594436 \\
\hline 0.127786 & 1.49568 & 0.0691331 & 0.737617 & 0.0884952 & 0.93141 & 0.0547967 \\
\hline 0.10163 & 1.47986 & 0.0685322 & 0.736386 & 0.0845716 & 0.910098 & 0.066991 \\
\hline 0.131457 & 1.46264 & 0.0782369 & 0.725901 & 0.078551 & 0.87532 & 0.06174 \\
\hline 0.129522 & 1.46254 & 0.077536 & 0.727076 & 0.0765138 & 0.856714 & 0.0592957 \\
\hline 0.116715 & 1.46593 & 0.0791023 & 0.707497 & 0.0745259 & 0.828916 & 0.0607172 \\
\hline 0.119186 & 1.45548 & 0.0806012 & 0.707354 & 0.0748536 & 0.810036 & 0.0602629 \\
\hline 0.119706 & 1.45712 & 0.0780898 & 0.698242 & 0.0738303 & 0.805187 & 0.0619163 \\
\hline 0.127488 & 1.45464 & 0.0839931 & 0.692975 & 0.0834936 & 0.788724 & 0.0522901 \\
\hline 0.12951 & 1.44791 & 0.0791638 & 0.676699 & 0.0740966 & 0.769893 & 0.0577744 \\
\hline 0.111333 & 1.45126 & 0.0806122 & 0.687694 & 0.0766131 & 0.759177 & 0.0496366 \\
\hline 0.115274 & 1.4638 & 0.0789038 & 0.682268 & 0.06378 & 0.749593 & 0.057582 \\
\hline 0.130557 & 1.45484 & 0.0757527 & 0.67995 & 0.0740189 & 0.744677 & 0.0576745 \\
\hline 0.130787 & 1.46027 & 0.0947016 & 0.681228 & 0.0748137 & 0.730918 & 0.0543545 \\
\hline 0.115063 & 1.45879 & 0.0848792 & 0.673913 & 0.0688411 & 0.728116 & 0.0540607 \\
\hline 0.129937 & 1.45405 & 0.0709876 & 0.678709 & 0.0565981 & 0.741078 & 0.0573711 \\
\hline 0.133075 & 1.46412 & 0.0946729 & 0.686596 & 0.0662865 & 0.735098 & 0.0564154 \\
\hline 0.122555 & 1.46872 & 0.0932926 & 0.687669 & 0.0627953 & 0.741219 & 0.0485724 \\
\hline 0.140359 & 1.4625 & 0.0834404 & 0.682345 & 0.0592416 & 0.733568 & 0.057408 \\
\hline 0.110717 & 1.46182 & 0.0977664 & 0.676928 & 0.0611871 & 0.726604 & 0.050198 \\
\hline 0.103571 & 1.47381 & 0.0893253 & 0.692047 & 0.0664222 & 0.724592 & 0.0580498 \\
\hline 0.129624 & 1.45664 & 0.0811133 & 0.685647 & 0.0565706 & 0.732702 & 0.0477554 \\
\hline 0.144031 & 1.4662 & 0.0858918 & 0.688029 & 0.0534326 & 0.731253 & 0.0488882 \\
\hline 0.112192 & 1.45196 & 0.0859922 & 0.684497 & 0.0489543 & 0.7269 & 0.0529937 \\
\hline 0.12518 & 1.45174 & 0.0820036 & 0.691247 & 0.0594911 & 0.738753 & 0.0366997 \\
\hline 0.130511 & 1.46747 & 0.0992565 & 0.680406 & 0.0654076 & 0.73133 & 0.0530785 \\
\hline 0.118723 & 1.45933 & 0.0762015 & 0.678487 & 0.0612776 & 0.729637 & 0.0426805 \\
\hline 0.11636 & 1.46283 & 0.0781547 & 0.67693 & 0.0555937 & 0.723904 & 0.0498202 \\
\hline 0.131096 & 1.44634 & 0.082724 & 0.681393 & 0.0522278 & 0.723105 & 0.0517714 \\
\hline 0.143549 & 1.44201 & 0.0858348 & 0.687733 & 0.0526539 & 0.714817 & 0.0467929 \\
\hline
\end{tabular}




\begin{tabular}{|c|c|c|c|c|c|c|}
\hline 0.136276 & 1.45242 & 0.0873006 & 0.689451 & 0.0521975 & 0.731521 & 185 \\
\hline 0.126007 & 1.44528 & 0.084596 & 0.690703 & 0.0574705 & 0.715024 & 0.0420244 \\
\hline 0.130741 & 1.43918 & 0.0824084 & 0.687913 & 0.0530957 & 0.72331 & 0.044195 \\
\hline 0.139856 & 1.43417 & 0.0926384 & 0.677398 & 0.0538715 & 0.715612 & 0.0427187 \\
\hline 0.126269 & 1.43607 & 0.0948336 & 0.674555 & 0.0594002 & 0.706387 & 0.0529919 \\
\hline 0.149448 & 1.43897 & 0.0835318 & 0.687769 & 0.0597779 & 0.69702 & 0.0447088 \\
\hline 0.148618 & 1.43942 & 0.0840978 & 0.682086 & 0.0590175 & 0.707963 & 0.0405342 \\
\hline 0.176727 & 1.44247 & 0.0878757 & 0.680799 & 0.049923 & 0.704263 & 0.0417453 \\
\hline 0.157729 & 1.43168 & 0.0868608 & 0.673746 & 0.0647753 & 0.705942 & 0.0396809 \\
\hline 0.140273 & 1.42993 & 0.0837346 & 0.67168 & 0.0566203 & 0.716826 & 0.0362717 \\
\hline 0.145458 & 1.43225 & 0.0846835 & 0.680466 & 0.0568194 & 0.708044 & 0.0383841 \\
\hline 0.157558 & 1.42493 & 0.0844184 & 0.675313 & 0.058188 & 0.702459 & 0.0487179 \\
\hline 0.149854 & 1.43644 & 0.0897241 & 0.680962 & 0.0565477 & 0.697253 & 0.0385034 \\
\hline 0.177615 & 1.42284 & 0.0805134 & 0.674271 & 0.0538832 & 0.715939 & 0.0350569 \\
\hline 0.161862 & 1.428 & 0.0791623 & 0.676304 & 0.0578812 & 0.706813 & 0.0392494 \\
\hline 0.148426 & 1.4341 & 0.0826446 & 0.680857 & 0.0633094 & 0.706298 & 0.0384029 \\
\hline 0.178968 & 1.42731 & 0.0904582 & 0.675371 & 0.049972 & 0.723015 & 0.0455593 \\
\hline 0.175119 & 1.42123 & 0.0813506 & 0.671137 & 0.0564534 & 0.720855 & 0.0403153 \\
\hline 0.169396 & 1.42402 & 0.0761734 & 0.679774 & 0.0531446 & 0.722385 & 0.0356037 \\
\hline 0.178347 & 1.43277 & 0.080 & 0.666996 & 0.0553267 & 0.724746 & 0.0381882 \\
\hline 0.156199 & 1.43029 & 0.0776472 & 0.674625 & 0.0606538 & 0.722913 & 0.0371799 \\
\hline 0.149483 & 1.43258 & 0.0740146 & 0.679693 & 0.0592796 & 0.726121 & 0.0412048 \\
\hline 0.153729 & 1.42644 & 0.0760987 & 0.683367 & 0.064441 & 0.745147 & 0.0393016 \\
\hline 0.148997 & 1.42871 & 0.0804492 & 0.678533 & 0.0530732 & 0.738521 & 0.0434697 \\
\hline 0.147587 & 1.43095 & 0.0898775 & 0.683492 & 0.0607816 & 0.749332 & 9964 \\
\hline 0.153141 & 1.42735 & 0.0733113 & 0.672703 & 0.0527252 & 0.74742 & 0.0384829 \\
\hline 0.13044 & 1.43407 & 0.0822363 & 0.666256 & 0.0661105 & 0.758117 & 0.0426639 \\
\hline 0.1327 & 1.43051 & 0.0703865 & 0.678221 & 0.0566225 & 0.763687 & 0.0439412 \\
\hline 0.143774 & 1.42891 & 0.0738019 & 0.68928 & 0.0569503 & 0.75394 & 0.0444991 \\
\hline 0.160439 & 1.42771 & 0.0750869 & 0.683008 & 0.0570962 & 0.755614 & 0.0322368 \\
\hline 0.140228 & 1.42443 & 0.0743853 & 0.6862 & 0.0564705 & 0.742444 & 0.0479825 \\
\hline 0.128434 & 1.421 & 0.064614 & 0.674476 & 0.0592512 & 0.75518 & 0.0360921 \\
\hline 0.132323 & 1.41954 & 0.0602367 & 0.677305 & 0.0547401 & 0.753817 & 0.0426651 \\
\hline 0.139347 & 1.42117 & 0.0639833 & 0.679967 & 0.0559991 & 0.748533 & 0.0390675 \\
\hline 0.151528 & 1.43177 & 0.0715197 & 0.682718 & 0.0623105 & 0.745884 & 0.037312 \\
\hline 0.137552 & 1.42573 & 0.0761374 & 0.683741 & 0.0482168 & 0.752362 & 0.0338552 \\
\hline 0.148017 & 1.42565 & 0.0755555 & 0.675258 & 0.0495927 & 0.739711 & 0.0445456 \\
\hline 0.126374 & 1.42398 & 0.0627133 & 0.677397 & 0.0512878 & 0.74545 & 0.0421443 \\
\hline 0.131907 & 1.42435 & 0.0747298 & 0.684295 & 0.0660261 & 0.737237 & 0.0358137 \\
\hline 0.124387 & 1.4239 & 0.0723194 & 0.682904 & 0.047258 & 0.736862 & 0.0375312 \\
\hline 0.113901 & 1.43056 & 0.0707082 & 0.68522 & 0.0495224 & 0.728491 & 0.0494707 \\
\hline 0.106511 & 1.43785 & 0.0728798 & 0.681095 & 0.0590283 & 0.735471 & 0.0364231 \\
\hline 0.100003 & 1.43852 & 0.0726106 & 0.691886 & 0.0540961 & 0.744929 & 0.0349661 \\
\hline 0.113532 & 1.43773 & 0.0664133 & 0.689904 & 0.0525229 & 0.738242 & 0.0434046 \\
\hline 0.115479 & 1.44136 & 0.0591987 & 0.688596 & 0.0486318 & 0.745958 & 0.0407946 \\
\hline 0.0973816 & 1.43503 & 0.066186 & 0.688698 & 0.0573378 & 0.74937 & 0.0488894 \\
\hline 0.116596 & 1.44782 & 0.0673478 & 0.693072 & 0.0527958 & 0.742546 & 0.0436018 \\
\hline
\end{tabular}




\begin{tabular}{|c|c|c|c|c|c|c|}
\hline 0.12733 & 1.46201 & 0.064085 & 0.701945 & 0.0493321 & 0.752577 & 0.0503197 \\
\hline 0.103567 & 1.45823 & 0.073576 & 0.70256 & 0.0553229 & 0.759288 & 0.0411441 \\
\hline 0.114731 & .46066 & 0.0518151 & 0.699135 & 0.0527816 & 0.76338 & 0.0416992 \\
\hline 0.0864103 & 1.46554 & 0.0577179 & 0.706642 & 0.0553796 & 0.755107 & 0.0366157 \\
\hline 0.102652 & 1.47524 & 0.0619933 & 0.70869 & 0.0532072 & 0.771793 & 0.0483243 \\
\hline 0.110633 & 1.49206 & 0.0765503 & 0.710195 & 0.0557015 & 0.777158 & 0.0490705 \\
\hline 0.108219 & 1.50115 & 0.0740523 & 0.724573 & 0.0514681 & 0.790668 & 0.0249848 \\
\hline 0.0929882 & 1.49985 & 0.0736347 & 0.721568 & 0.0577958 & 0.789826 & 0.0467696 \\
\hline 0.107978 & 1.50052 & 0.0613065 & 0.709895 & 0.0503488 & 0.795873 & 0.042744 \\
\hline 0.0921147 & 1.5048 & 0.0565175 & 0.725835 & 0.059349 & 0.797704 & 0.0501656 \\
\hline 0.109468 & 1.5107 & 0.0673994 & 0.728825 & 0.0576603 & 0.793442 & 0.0486732 \\
\hline 0.0997048 & 1.5126 & 0.0724425 & 0.728089 & 0.0557355 & 0.815747 & 0.0361851 \\
\hline 0.101014 & 1.51108 & 0.0677963 & 0.72819 & 0.0564205 & 0.810566 & 0.0509148 \\
\hline 0.0949478 & 1.52205 & 0.0771082 & 0.724842 & 0.0529003 & 0.807529 & 0.0470599 \\
\hline 0.124819 & 1.52687 & 0.081972 & 0.734444 & 0.056922 & 0.814768 & 0.0392135 \\
\hline 0.114417 & 1.52307 & 0.0782438 & 0.729813 & 0.0568382 & 0.813457 & 0.042182 \\
\hline 0.105116 & 1.53602 & 0.0827066 & 0.747336 & 0.0519527 & 0.814479 & 0.0383847 \\
\hline 0.13312 & 1.53975 & 0.0826963 & 0.748136 & 0.0631964 & 0.810587 & 0.0491775 \\
\hline 0.105803 & 1.54167 & 0.0769634 & 0.739059 & 0.0575539 & 0.812804 & 0.0396101 \\
\hline 0.141253 & 1.54945 & 0.081095 & 0.746509 & 0.0661577 & 0.812312 & 0.0389265 \\
\hline 0.119535 & 1.54151 & 0.0802948 & 0.744183 & 0.0691603 & 0.804545 & 0.0519286 \\
\hline 0.126789 & 1.5529 & 0.0767267 & 0.739053 & 0.0626825 & 0.801772 & 0.042699 \\
\hline 0.127347 & 1.55556 & 0.0695443 & 0.755672 & 0.0631265 & 0.812699 & 0.047273 \\
\hline 0.133267 & 1.55205 & 0.0736161 & 0.743988 & 0.0629309 & 0.81481 & 0.0440766 \\
\hline 0.118152 & 1.54619 & 0.07579 & 0.754696 & 0.0581053 & 0.803433 & 0.0438268 \\
\hline 0.138595 & 1.55032 & 0.0696044 & 0.754305 & 0.0597397 & 0.801877 & 0.0475628 \\
\hline 0.133465 & 1.55694 & 0.0637263 & 0.750389 & 0.0640989 & 0.797165 & 0.0493592 \\
\hline 0.128714 & 1.55885 & 0.0688473 & 0.752321 & 0.063207 & 0.793631 & 0.045193 \\
\hline 0.126074 & 1.55541 & 0.0702426 & 0.749311 & 0.0587598 & 0.801961 & 0.0436202 \\
\hline 0.125741 & 1.55698 & 0.064 & 446 & 0.0554944 & 0.799126 & 0.055086 \\
\hline 0.140655 & 1.55154 & 0.0623909 & 0.752119 & 0.0655757 & 0.801564 & 0.0424261 \\
\hline 0.127346 & 1.56089 & 0.0733978 & 0.749856 & 0.0666222 & 0.800035 & 0.0363693 \\
\hline 0.129572 & 1.56661 & 0.0684013 & 0.759447 & 0.0644025 & 0.801081 & 0.0442136 \\
\hline 0.119106 & 1.56384 & 0.0745823 & 0.759052 & 0.0639786 & 0.791772 & 0.0613219 \\
\hline 0.124878 & 1.56047 & 0.0789593 & 0.76074 & 0.0654077 & 0.796291 & 0.0487655 \\
\hline 0.120441 & 1.56229 & 0.0577207 & 0.756411 & 0.0605393 & 0.790899 & 0.0492933 \\
\hline 0.120574 & 1.56481 & 0.0613023 & 0.75915 & 0.0538275 & 0.800428 & 0.0615938 \\
\hline 0.110327 & 1.57068 & 0.0702538 & 0.761205 & 0.0597949 & 0.800733 & 0.0340748 \\
\hline 0.125751 & 1.57142 & 0.0695562 & 0.763558 & 0.0704736 & 0.806813 & 0.0513196 \\
\hline 0.116677 & 1.57608 & 0.0592985 & 0.757293 & 0.0562213 & 0.805177 & 0.0534771 \\
\hline 0.0997382 & 1.58083 & 0.0669258 & 0.763829 & 0.0583867 & 0.802575 & 0.057773 \\
\hline 0.112855 & 1.5721 & 0.0707836 & 0.766819 & 0.062463 & 0.797343 & 0.0462066 \\
\hline 0.0964331 & 1.57163 & 0.0705061 & 0.768036 & 0.0559786 & 0.807705 & 0.0461591 \\
\hline 0.120216 & 1.57596 & 0.0726533 & 0.75997 & 0.0598332 & 0.798517 & 0.0474374 \\
\hline 0.0954949 & 1.57909 & 0.0770238 & 0.781376 & 0.0550849 & 0.803479 & 0.0445884 \\
\hline 0.11373 & 1.57918 & 0.0786322 & 0.778394 & 0.0620065 & 0.808577 & 0.0468623 \\
\hline 0.0996956 & 1.56716 & 0.0721334 & 0.769866 & 0.0529928 & 0.798053 & 0.043234 \\
\hline
\end{tabular}




\begin{tabular}{|c|c|c|c|c|c|c|}
\hline 0.104135 & 1.56763 & 0.064515 & 0.779272 & 0.0519322 & 0.801878 & 0.0461123 \\
\hline 0.122797 & 1.56109 & 0.0689022 & 0.780185 & 0.0637278 & 0.807569 & 0.0451369 \\
\hline 0.130519 & 1.57561 & 0.0771475 & 0.788644 & 0.0664932 & 0.806562 & 0.0490311 \\
\hline 0.12435 & .57767 & 0.0680973 & 0.777092 & 0.0500322 & 0.803807 & 0.0412602 \\
\hline 0.110387 & 1.57506 & 0.0582271 & 0.786307 & 0.048273 & 0.7974 & 0.0400097 \\
\hline 0.107734 & 1.57339 & 0.0658025 & 0.794014 & 0.0580038 & 0.794778 & 0.0465919 \\
\hline 0.120612 & 1.57794 & 0.0636144 & 0.79036 & 0.0565468 & 0.802918 & 0.0450892 \\
\hline 0.136106 & 1.57751 & 0.0662865 & 0.79721 & 0.0677277 & 0.806416 & 0.0410566 \\
\hline 0.11363 & 1.58694 & 0.0732274 & 0.801145 & 0.053955 & 0.802711 & 0.0465478 \\
\hline 0.123941 & 1.57748 & 0.0717567 & 0.809603 & 0.0646253 & 0.807226 & 0.0501288 \\
\hline 0.142552 & 1.58299 & 0.0706363 & 0.805598 & 0.0612991 & 0.806218 & 0.0409289 \\
\hline 0.118101 & 1.58174 & 0.0585242 & 0.818462 & 0.0719463 & 0.805016 & 0.0496787 \\
\hline 0.15518 & 1.58502 & 0.0587863 & 0.818702 & 0.0603227 & 0.807084 & 0.0386712 \\
\hline 0.160735 & 1.58493 & 0.071372 & 0.830341 & 0.0655625 & 0.808609 & 0.038869 \\
\hline 0.147355 & 1.59009 & 0.0717941 & 0.824689 & 0.069106 & 0.80424 & 0.0401795 \\
\hline 0.136205 & 1.58796 & 0.0698703 & 0.830984 & 0.070482 & 0.808446 & 0.0491096 \\
\hline 0.127383 & 1.57521 & 0.0783181 & 0.824256 & 0.071608 & 0.80423 & 0.039639 \\
\hline 0.140695 & 1.56836 & 0.0732019 & 0.822285 & 0.0707647 & 0.799663 & 0.0424809 \\
\hline 0.154621 & 1.56857 & 0.0690545 & 0.828928 & 0.0536488 & 345 & 0.0494668 \\
\hline 0.145037 & 1.56341 & 0.068504 & 0.831632 & 0.075699 & 0.800727 & 0.0432205 \\
\hline 0.139299 & 1.5549 & 0.0649954 & 0.821443 & 0.065727 & 0.803284 & 0.0463589 \\
\hline 0.156506 & 1.57361 & 0.076402 & 0.829545 & 0.0597551 & 0.802543 & 9754 \\
\hline 0.118228 & 1.56426 & 0.0695018 & 0.814159 & 0.0749875 & 0.805624 & 0.0472321 \\
\hline 0.166047 & 1.56258 & 0.0655978 & 0.807905 & 0.0682965 & 0.790529 & 0.0390317 \\
\hline 0.129375 & 1.55306 & 0.0681627 & 0.805571 & 0.0529738 & 0.794593 & 0.0492651 \\
\hline 0.128036 & 1.55747 & 0.0533595 & 0.802881 & 0.0661912 & 0.798747 & 0.0382495 \\
\hline 0.153714 & 1.55044 & 0.0567079 & 0.78638 & 0.0572224 & 0.803587 & 0.0590674 \\
\hline 0.136165 & 1.55177 & 0.0594334 & 0.788948 & 0.0588802 & 0.792952 & 0.0479382 \\
\hline 0.130034 & 1.5511 & 0.0594069 & 0.799351 & 0.0520464 & 0.803411 & 0.0463048 \\
\hline 0.120909 & 1.54476 & 0.0647086 & 0.780732 & 0.0497616 & 0.797103 & 0.0429976 \\
\hline 0.121381 & 1.54006 & 0.0723961 & 0.796192 & 0.0523929 & 0.805882 & 0.0503047 \\
\hline 0.122273 & 1.54432 & 0.0712991 & 0.781112 & 0.0511034 & 0.800564 & 0.0374798 \\
\hline 0.116813 & 1.53103 & 0.0677252 & 0.781111 & 0.0421346 & 0.795236 & 0.0468855 \\
\hline 0.121112 & 1.53726 & 0.0651253 & 0.767379 & 0.0506091 & 0.790283 & 0.0436326 \\
\hline 0.107763 & 1.53675 & 0.0654749 & 0.767135 & 0.0460797 & 0.791099 & 0.0462539 \\
\hline 0.0930421 & 1.53696 & 0.0667607 & 0.763834 & 0.0415229 & 0.79101 & 0.037477 \\
\hline 0.128549 & 1.53587 & 0.0634537 & 0.779912 & 0.0491864 & 0.78892 & 0.0345875 \\
\hline 0.108321 & 1.53982 & 0.0550708 & 0.781744 & 0.0422293 & 0.776443 & 0.0395642 \\
\hline 0.115789 & 1.53355 & 0.0654397 & 0.764008 & 0.0463258 & 0.799896 & 0.0520658 \\
\hline 0.133302 & 1.52896 & 0.0613919 & 0.777059 & 0.0389091 & 0.797168 & 0.045362 \\
\hline 0.109531 & 1.5335 & 0.0576506 & 0.768997 & 0.0425294 & 0.789252 & 0.0458159 \\
\hline 0.101351 & 1.53498 & 0.0557406 & 0.777057 & 0.0447304 & 0.779545 & 0.0417192 \\
\hline 0.12186 & 1.53502 & 0.0617743 & 0.770315 & 0.0480907 & 0.798378 & 0.0417272 \\
\hline 0.127702 & 1.53488 & 0.0604936 & 0.771572 & 0.0489533 & 0.791945 & 0.0410373 \\
\hline 0.12629 & 1.53853 & 0.0555329 & 0.765965 & 0.0484983 & 0.780523 & 0.0374345 \\
\hline 0.121473 & 1.53337 & 0.0666688 & 0.766403 & 0.0452696 & 0.793591 & 0.0415964 \\
\hline 0.117611 & 1.53945 & 0.0588993 & 0.771906 & 0.0488304 & 0.783305 & 0.0399279 \\
\hline
\end{tabular}




\begin{tabular}{|c|c|c|c|c|c|c|}
\hline 0.134219 & 1.53108 & 0.0667765 & 0.763935 & 0.0523155 & 0.785684 & 0.0445043 \\
\hline 0.134347 & 1.52848 & 0.0701449 & 0.769154 & 0.0435145 & 0.80099 & 0.0461264 \\
\hline 0.125737 & .54123 & 0.0624605 & 0.772706 & 0.0464552 & 0.793613 & 0.0459674 \\
\hline 0.0937242 & 1.5386 & 0.0581081 & 0.778956 & 0.0550613 & 0.811638 & 0.0466851 \\
\hline 0.107025 & 1.53422 & 0.0558564 & 0.778468 & 0.0635104 & 0.802683 & 0.0372577 \\
\hline 0.102598 & 1.53267 & 0.0518984 & 0.777767 & 0.0522141 & 0.811126 & 0.0515974 \\
\hline 0.124164 & 1.532 & 0.0609812 & 0.775592 & 0.0492324 & 0.813369 & 0.0538791 \\
\hline 0.113545 & 1.53864 & 0.0635382 & 0.792342 & 0.0571504 & 0.822516 & 0.050985 \\
\hline 0.0962854 & 1.52811 & 0.0605505 & 0.780608 & 0.0580237 & 0.823679 & 0.0382978 \\
\hline 0.0916729 & 1.5312 & 0.0654952 & 0.782932 & 0.0491395 & 0.820683 & 0.0472139 \\
\hline 0.122175 & 1.52481 & 0.0703936 & 0.782882 & 0.0525794 & 0.834329 & 0.0486966 \\
\hline 0.119316 & 1.53179 & 0.0608043 & 0.787682 & 0.0552238 & 0.838448 & 0.0552162 \\
\hline 0.108993 & 1.53094 & 0.0643771 & 0.791934 & 0.0524261 & 0.836264 & 0.0475845 \\
\hline 0.122923 & 1.53292 & 0.0561144 & 0.793335 & 0.0630411 & 0.83189 & 0.0424029 \\
\hline 0.126043 & 1.53059 & 0.0593528 & 0.791985 & 0.0656933 & 0.840648 & 0.0534698 \\
\hline 0.117316 & 1.52673 & 0.0678479 & 0.797435 & 0.0629854 & 0.841905 & 0.0488257 \\
\hline 0.0966087 & 1.5197 & 0.0673275 & 0.798116 & 0.0680535 & 0.846002 & 0.0545543 \\
\hline 0.111149 & 1.51803 & 0.0700699 & 0.790168 & 0.0661879 & 0.824411 & 0.0635993 \\
\hline 0.117772 & 1.50372 & 0.0607611 & 0.793897 & 0.060715 & 0.849549 & 0.0600047 \\
\hline 0.117138 & 1.50433 & 0.0510343 & 0.796367 & 0.0632465 & 0.836271 & 0.0531544 \\
\hline 0.102703 & 1.50482 & 0.0547119 & 0.782362 & 0.0533161 & 0.829873 & 0.0651703 \\
\hline 0.119977 & 1.5075 & 0.061281 & 0.777732 & 0.0595509 & 0.824838 & 0.0545876 \\
\hline 0.105531 & 1.50015 & 0.0587242 & 0.776193 & 0.0529735 & 0.824902 & 0.0551116 \\
\hline 0.126841 & 1.49402 & 0.0558405 & 0.773407 & 0.0636627 & 0.805467 & 0.056041 \\
\hline 0.116562 & 1.49149 & 0.0517557 & 0.770556 & 0.0578345 & 0.797621 & 0.0499634 \\
\hline 0.111971 & 1.49072 & 0.0596235 & 0.767164 & 0.0475061 & 0.801479 & 0.0592235 \\
\hline 0.109037 & 1.48207 & 0.0596705 & 0.758278 & 0.0498877 & 0.794264 & 0.0599751 \\
\hline 0.104749 & 1.49471 & 0.0559577 & 0.763949 & 0.0481993 & 0.779729 & 0.0542444 \\
\hline 0.109255 & 1.48922 & 0.0521746 & 0.757874 & 0.0414251 & 0.77721 & 0.0485145 \\
\hline 0.11438 & 1.48055 & 0.047977 & 0.753478 & 0.0453979 & 0.781762 & 0.0607356 \\
\hline 0.0945218 & 1.47757 & 0.0621726 & 0.748391 & 0.0484535 & 0.771867 & 0.0477799 \\
\hline 0.10174 & 1.4862 & 0.062023 & 0.75371 & 0.0499029 & 0.761806 & 0.0504145 \\
\hline 0.107972 & 1.47955 & 0.0602048 & 0.744635 & 0.0568326 & 0.764948 & 0.0422283 \\
\hline 0.123813 & 1.46933 & 0.0607988 & 0.752556 & 0.0496103 & 0.756094 & 0.0409968 \\
\hline 0.0952014 & 1.46772 & 0.0541214 & 0.756894 & 0.0556572 & 0.763287 & 0.0471034 \\
\hline 0.135145 & 1.46862 & 0.0547373 & 0.74769 & 0.0519924 & 0.753339 & 0.0411459 \\
\hline 0.103407 & 1.46153 & 0.0588223 & 0.746542 & 0.054731 & 0.753225 & 0.0422874 \\
\hline 0.107641 & 1.47377 & 0.0507262 & 0.739047 & 0.0503094 & 0.760197 & 0.0576463 \\
\hline 0.118156 & 1.48108 & 0.0490915 & 0.736207 & 0.051418 & 0.760412 & 0.0445954 \\
\hline 0.0897647 & 1.46726 & 0.0548435 & 0.744451 & 0.0524598 & 0.760693 & 0.0371283 \\
\hline 0.109571 & 1.44943 & 0.0502261 & 0.741 & 0.0580138 & 0.741443 & 0.0387979 \\
\hline 0.117554 & 1.45583 & 0.0599534 & 0.740248 & 0.0566227 & 0.755355 & 0.0435005 \\
\hline 0.104986 & 1.46923 & 0.059348 & 0.734245 & 0.0505046 & 0.745192 & 0.0413415 \\
\hline 0.119706 & 1.46739 & 0.0600514 & 0.738171 & 0.0551344 & 0.747067 & 0.0438906 \\
\hline 0.100902 & 1.46567 & 0.0590657 & 0.739293 & 0.0547999 & 0.746533 & 0.0405718 \\
\hline 0.116636 & 1.45815 & 0.0585188 & 0.742295 & 0.0559217 & 0.746096 & 0.0447626 \\
\hline 0.116317 & 1.44938 & 0.0560432 & 0.738646 & 0.0473141 & 0.752568 & 0.0391208 \\
\hline
\end{tabular}




\begin{tabular}{|c|c|c|c|c|c|c|}
\hline 0.114914 & 1.45371 & 0.0545629 & 0.732818 & 0.0504899 & 0.745195 & 0.0398837 \\
\hline 0.130613 & 1.45288 & 0.0580172 & 0.737833 & 0.0430191 & 0.745103 & 0.042018 \\
\hline 0.120375 & 1.4571 & 0.0584201 & 0.731133 & 0.0615085 & 0.740647 & 0.037223 \\
\hline 0.117448 & 1.45596 & 0.0553873 & 0.737333 & 0.0544944 & 0.736876 & 0.0447853 \\
\hline 0.120181 & 1.4581 & 0.0629995 & 0.73952 & 0.0488411 & 0.741673 & 0.0396074 \\
\hline 0.13542 & 1.4483 & 0.0562951 & 0.733849 & 0.0502776 & 0.741609 & 0.0463985 \\
\hline 0.123395 & 1.4526 & 0.0526361 & 0.723043 & 0.0559598 & 0.741791 & 0.0439341 \\
\hline 0.109578 & 1.45505 & 0.0588559 & 0.722679 & 0.0531836 & 0.754304 & 0.0444128 \\
\hline 0.116429 & 1.45333 & 0.0628687 & 0.732335 & 0.0530789 & 0.738958 & 0.0388653 \\
\hline 0.127539 & 1.45306 & 0.0606069 & 0.726365 & 0.0531308 & 0.745253 & 0.0372756 \\
\hline 0.141349 & 1.44904 & 0.0611978 & 0.735287 & 0.0441106 & 0.748884 & 0.0383395 \\
\hline 0.110686 & 1.45304 & 0.0702767 & 0.726486 & 0.0423018 & 0.727948 & 0.0312685 \\
\hline 0.119289 & 1.44245 & 0.0610482 & 0.716734 & 0.0449344 & 0.738494 & 0.0375602 \\
\hline 0.12643 & 1.43848 & 0.0584353 & 0.731278 & 0.0486147 & 0.731843 & 0.0337077 \\
\hline 0.133294 & 1.44612 & 0.0598928 & 0.72637 & 0.0554235 & 0.735571 & 0.038458 \\
\hline 0.120555 & 1.44431 & 0.0626223 & 0.722173 & 0.051607 & 0.726496 & 0.0358794 \\
\hline 0.116849 & 1.44583 & 0.0622106 & 0.733304 & 0.048825 & 0.727046 & 0.0330033 \\
\hline 0.127674 & 1.45108 & 0.0608255 & 0.727789 & 0.0406132 & 0.722478 & 0.0422693 \\
\hline 0.1346 & 1.44883 & 0.0625487 & 0.725192 & 0.0427397 & 0.726108 & 0.0300383 \\
\hline 0.118696 & 1.44524 & 0.0556799 & 0.727931 & 0.0503624 & 0.715039 & 0.0359449 \\
\hline 0.119205 & 1.44488 & 0.0528755 & 0.722378 & 0.0402644 & 0.715462 & 0.0388813 \\
\hline 0.127663 & 1.44211 & 0.0530483 & 0.725321 & 0.0457366 & 0.730783 & 0.0328245 \\
\hline 0.113933 & 1.43794 & 0.0603954 & 0.71587 & 0.0477017 & 0.722759 & 0.0314694 \\
\hline 0.133134 & 1.44337 & 0.0675583 & 0.72142 & 0.0503917 & 0.722292 & 0.032909 \\
\hline 0.123874 & 1.44552 & 0.0547399 & 0.717727 & 0.0502287 & 0.727508 & 0.0411293 \\
\hline 0.117315 & 1.44608 & 0.0547304 & 0.724234 & 0.0438371 & 0.726391 & 0.0338682 \\
\hline 0.114393 & 1.44548 & 0.054883 & 0.715634 & 0.0552907 & 0.715543 & 0.0355487 \\
\hline 0.112568 & 1.44961 & 0.0563291 & 0.719113 & 0.0427315 & 0.718196 & 0.0401164 \\
\hline 0.109852 & 1.45263 & 0.0577055 & 0.716042 & 0.0480262 & 0.722044 & 0.0371636 \\
\hline 0.10579 & 1.44504 & 0.055743 & 0.714085 & 0.0525491 & 0.719988 & 0.027324 \\
\hline 0.106494 & 1.44864 & 0.057704 & 0.724451 & 0.0407932 & 0.723548 & 0.0384499 \\
\hline 0.106722 & 1.44982 & 0.0552133 & 0.720981 & 0.0472071 & 0.712051 & 0.0356444 \\
\hline 0.112546 & 1.44288 & 0.0518074 & 0.715406 & 0.0451563 & 0.718495 & 0.0349925 \\
\hline 0.101032 & 1.44106 & 0.0511899 & 0.725979 & 0.0548072 & 0.716048 & 0.0369409 \\
\hline 0.100331 & 1.44964 & 0.0512486 & 0.713557 & 0.0386812 & 0.729548 & 0.0321596 \\
\hline 0.108138 & 1.44274 & 0.0471421 & 0.715368 & 0.0395173 & 0.722097 & 0.0320038 \\
\hline 0.112448 & 1.43545 & 0.0531765 & 0.716685 & 0.0491949 & 0.726867 & 0.0388908 \\
\hline 0.10246 & 1.44043 & 0.0523568 & 0.708658 & 0.0399995 & 0.725204 & 0.0323259 \\
\hline 0.090305 & 1.44907 & 0.0527732 & 0.711863 & 0.0516793 & 0.716204 & 0.0326056 \\
\hline 0.106735 & 1.4467 & 0.0535375 & 0.712235 & 0.0487612 & 0.717183 & 0.0333776 \\
\hline 0.118077 & 1.44343 & 0.0608723 & 0.717225 & 0.0433395 & 0.72088 & 0.041923 \\
\hline 0.096478 & 1.45058 & 0.0523891 & 0.713871 & 0.0460109 & 0.712876 & 0.042581 \\
\hline 0.0832042 & 1.44363 & 0.0508161 & 0.711503 & 0.0473238 & 0.722856 & 0.0366014 \\
\hline 0.117023 & 1.43842 & 0.0532124 & 0.701758 & 0.0463577 & 0.711417 & 0.0455133 \\
\hline 0.0926013 & 1.44248 & 0.0579004 & 0.714115 & 0.0435705 & 0.717366 & 0.0452398 \\
\hline 0.100939 & 1.44724 & 0.0596303 & 0.713043 & 0.0452317 & 0.72077 & 0.0391757 \\
\hline 0.120275 & 1.44093 & 0.0566159 & 0.711543 & 0.0464278 & 0.708272 & 0.0392626 \\
\hline
\end{tabular}




\begin{tabular}{|c|c|c|c|c|c|c|}
\hline 0.0939606 & 1.44359 & 0.0616014 & 0.708812 & 0.0369639 & 0.721427 & 0.03 \\
\hline 0.0842214 & 1.45007 & 0.062405 & 0.70272 & 0.0426486 & 0.708915 & 0.0300002 \\
\hline 0.0999885 & 1.43869 & 0.0611387 & 0.715645 & 0.0480288 & 0.703048 & 0.0322299 \\
\hline 0.110293 & 1.4322 & 0.0607602 & 0.707316 & 0.0478565 & 0.70996 & 0.0336835 \\
\hline 0.0776421 & 1.43642 & 0.056165 & 0.712483 & 0.0442152 & 0.712322 & 0.0482027 \\
\hline 0.0915889 & 1.44318 & 0.0667222 & 0.712262 & 0.0404747 & 0.706357 & 0.0360474 \\
\hline 0.086063 & 1.4446 & 0.0705593 & 0.713571 & 0.0413902 & 0.709612 & 0.0293688 \\
\hline 0.101992 & 1.43383 & 0.0660199 & 0.703638 & 0.0456606 & 0.714095 & 0.0390504 \\
\hline 0.0746534 & 1.43732 & 0.0646394 & 0.70297 & 0.0426774 & 0.713744 & 0.04198 \\
\hline 0.0855542 & 1.44026 & 0.0710845 & 0.712357 & 0.0462822 & 0.720351 & 0.0346563 \\
\hline 0.108161 & 1.44099 & 0.0664617 & 0.706174 & 0.0451315 & 0.707926 & 0.0366404 \\
\hline 0.101956 & 1.44192 & 0.0636711 & 0.70777 & 0.0499448 & 0.708499 & 0.0393154 \\
\hline 0.0910303 & 1.43313 & 0.0686477 & 0.711913 & 0.0403623 & 0.708991 & 0.0369952 \\
\hline 0.0939386 & 1.43238 & 0.0681786 & 0.706604 & 0.039825 & 0.704686 & 0.0365297 \\
\hline 0.0945612 & 1.43492 & 0.0659388 & 0.709312 & 0.0498011 & 0.704779 & 0.0271222 \\
\hline 0.0961785 & 1.43405 & 0.0602211 & 0.704673 & 0.0329015 & 0.711034 & 0.047597 \\
\hline 0.0952835 & 1.43015 & 0.0538639 & 0.709054 & 0.0407793 & 0.715312 & 0.0391871 \\
\hline 0.0975665 & 1.42829 & 0.0679791 & 0.699821 & 0.034378 & 0.707416 & 0.0311132 \\
\hline 0.0833344 & 1.42968 & 0.0701344 & 0.706547 & 0.0406763 & 0.704723 & 0.0428949 \\
\hline 0.0986814 & 1.43243 & 0.0627319 & 0.700816 & 0.0352045 & 0.704986 & 0.0397759 \\
\hline 0.102459 & 1.42978 & 0.063488 & 0.701931 & 0.042045 & 0.709106 & 0.0370273 \\
\hline 0.102086 & 1.43046 & 0.0650735 & 0.698764 & 0.0430619 & 0.708581 & 0.0397009 \\
\hline 0.0907508 & 1.42747 & 0.0607949 & 0.70484 & 0.0438915 & 0.705243 & 0.0355643 \\
\hline 0.084617 & 1.42464 & 0.0593368 & 0.694686 & 0.0365648 & 0.701459 & 0.0341421 \\
\hline 0.0800393 & 1.42835 & 0.055 & 0.71293 & 0.03 & 0.702776 & 0.0411242 \\
\hline 0.0893979 & 1.43225 & 0.0626138 & 0.701373 & 0.0441493 & 0.715643 & 0.0378789 \\
\hline 0.0848255 & 1.43469 & 0.0676252 & 0.708654 & 0.034705 & 0.705705 & 0.0321479 \\
\hline 0.10123 & 1.42808 & 0.0608416 & 0.713249 & 0.0427223 & 0.70646 & 0.0341822 \\
\hline 0.0834103 & 1.43078 & 0.0573655 & 0.70319 & 0.0415474 & 0.713435 & 0.0447263 \\
\hline 0.08442 & 1.43152 & 0.0618994 & 0.707951 & 0.0414091 & 0.706478 & 0.0435314 \\
\hline 0.092184 & 1.43013 & 0.0700764 & 0.698384 & 0.0468413 & 0.711652 & 0.0388516 \\
\hline 0.0847422 & 1.42726 & 0.0681325 & 0.703888 & 0.0408153 & 0.706329 & 0.0365042 \\
\hline 0.10528 & 1.42626 & 0.0660946 & 0.716766 & 0.045921 & 0.710823 & 0.0448864 \\
\hline 0.088319 & 1.43071 & 0.066968 & 0.698231 & 0.0444724 & 0.697274 & 0.040783 \\
\hline 0.0879489 & 1.42432 & 0.0626167 & 0.70237 & 0.040989 & 0.719305 & 0.0316244 \\
\hline 0.0747808 & 1.42808 & 0.0545267 & 0.708323 & 0.044114 & 0.703831 & 0.0297215 \\
\hline 0.107153 & 1.43291 & 0.0533226 & 0.701672 & 0.0456043 & 0.710519 & 0.0349561 \\
\hline 0.0922435 & 1.43258 & 0.0547848 & 0.706459 & 0.0466093 & 0.70611 & 0.0350053 \\
\hline 0.0946685 & 1.43331 & 0.0627637 & 0.70808 & 0.0395232 & 0.709816 & 0.0433641 \\
\hline 0.0831862 & 1.42854 & 0.0692448 & 0.694842 & 0.0477242 & 0.717435 & 0.0387902 \\
\hline 0.0808928 & 1.42404 & 0.067044 & 0.708871 & 0.0436945 & 0.706019 & 0.0503785 \\
\hline 0.0936033 & 1.42579 & 0.0651897 & 0.710633 & 0.0454222 & 0.714552 & 0.0438861 \\
\hline 0.0909978 & 1.4285 & 0.0662643 & 0.701262 & 0.0507984 & 0.702076 & 0.0301835 \\
\hline 0.0899109 & 1.4258 & 0.0579193 & 0.710524 & 0.0468911 & 0.70807 & 0.0410478 \\
\hline 0.0869359 & 1.4187 & 0.0516015 & 0.704519 & 0.0323442 & 0.705759 & 0.0343498 \\
\hline 0.102791 & 1.41786 & 0.0571067 & 0.704325 & 0.038557 & 0.69322 & 0.0435681 \\
\hline 0.103897 & 1.4193 & 0.0615703 & 0.6882 & 0.039008 & 0.70776 & 0.0305519 \\
\hline
\end{tabular}




\begin{tabular}{|c|c|c|c|c|c|c|}
\hline 0.111141 & 1.41779 & 0.0572319 & 0.702602 & 0.0407582 & 0.707954 & 0.0361382 \\
\hline 0.0930845 & 1.42381 & 0.0564567 & 0.710208 & 0.0353545 & 0.69587 & 0.0412692 \\
\hline 0.100337 & 1.42428 & 0.058445 & 0.711408 & 0.0352387 & 0.696859 & 0.041147 \\
\hline 0.0970268 & 1.42523 & 0.0537068 & 0.708877 & 0.0526105 & 0.697988 & 0.0345394 \\
\hline 0.0934533 & 1.42188 & 0.05259 & 0.688264 & 0.0354081 & 0.690446 & 0.0323362 \\
\hline 0.102269 & 1.41116 & 0.0492129 & 0.700696 & 0.0445768 & 0.691817 & 0.0348633 \\
\hline 0.103097 & 1.41164 & 0.0474799 & 0.704712 & 0.0420848 & 0.698726 & 0.0396029 \\
\hline 0.0917362 & 1.41244 & 0.0506337 & 0.704338 & 0.0347312 & 0.698793 & 0.0304982 \\
\hline 0.0870884 & 1.41048 & 0.047564 & 0.710483 & 0.0362231 & 0.694688 & 0.0396707 \\
\hline 0.0914956 & 1.40736 & 0.0410239 & 0.701478 & 0.0327707 & 0.701924 & 0.0396269 \\
\hline 0.0828858 & 1.40345 & 0.0459529 & 0.705594 & 0.0414773 & 0.689736 & 0.0389484 \\
\hline 0.0814288 & 1.40104 & 0.0569702 & 0.698025 & 0.0385372 & 0.68755 & 0.0389658 \\
\hline 0.0839852 & 1.40193 & 0.0569253 & 0.696273 & 0.0389343 & 0.677546 & 0.0379909 \\
\hline 0.0635735 & 1.40638 & 0.0517255 & 0.699004 & 0.0446172 & 0.68621 & 0.0382456 \\
\hline 0.0941396 & 1.40429 & 0.0524744 & 0.697212 & 0.0332009 & 0.689365 & 0.0387463 \\
\hline 0.0809527 & 1.39767 & 0.0545313 & 0.686953 & 0.0435552 & 0.683576 & 0.0422947 \\
\hline 0.066298 & 1.39922 & 0.0539372 & 0.68505 & 0.0411085 & 0.6898 & 0.0387223 \\
\hline 0.085098 & 1.40097 & 0.0496372 & 0.689762 & 0.0285716 & 0.683834 & 0.0367775 \\
\hline 0.0920013 & 1.39478 & 0.0478433 & 0.690435 & 0.03 & 0.681249 & 0.0302107 \\
\hline 0.0750664 & 1.39054 & 0.0516981 & 0.682171 & 0.0425431 & 0.688506 & 0.0430949 \\
\hline 0.075285 & 1.38561 & 0.0553938 & 0.676238 & 0.0315671 & 0.67548 & 0.036499 \\
\hline 0.0833774 & 1.37985 & 0.0547451 & 0.689547 & 0.0 & 0.680883 & 0.0356976 \\
\hline 0.101601 & 1.37685 & 0.0480913 & 0.686722 & 0.0427786 & 0.672187 & 0.0353369 \\
\hline 0.0944045 & 1.377 & 0.0458378 & 0.683823 & 0.036541 & 0.676625 & 0.0382414 \\
\hline 0.0918071 & 1.3787 & 0.047658 & 0.684354 & 0.0384494 & 0.682983 & 0.0410309 \\
\hline 0.0989941 & 1.3807 & 0.0481636 & 0.69172 & 0.0399527 & 0.667443 & 0.0365098 \\
\hline 0.0720013 & 1.3806 & 0.0509647 & 0.686749 & 0.0380663 & 0.678116 & 0.0380251 \\
\hline 0.0953796 & 1.37743 & 0.0477988 & 0.674093 & 0.0368449 & 0.666854 & 0.0437418 \\
\hline 0.0773762 & 1.3812 & 0.0433423 & 0.684769 & 0.0424707 & 0.669187 & 0.033523 \\
\hline 0.0856515 & 1.37822 & 0.049098 & 0.693087 & 1588 & 0.672567 & 0.0362368 \\
\hline 0.0839852 & 1.36886 & 0.0499625 & 0.674342 & 0.0376929 & 0.669445 & 0.0304569 \\
\hline 0.102915 & 1.36196 & 0.048627 & 0.687362 & 0.038992 & 0.670334 & 0.0345338 \\
\hline 0.0890668 & 1.3511 & 0.0485462 & 0.678928 & 0.0396002 & 0.671008 & 0.0357837 \\
\hline 0.100101 & 1.35325 & 0.0461845 & 0.683736 & 0.0288632 & 0.675785 & 0.0338475 \\
\hline 0.100848 & 1.35538 & 0.0447136 & 0.680473 & 0.0370245 & 0.676351 & 0.0387953 \\
\hline 0.0958809 & 1.34795 & 0.0417582 & 0.679801 & 0.0389532 & 0.663388 & 0.0336504 \\
\hline 0.0869473 & 1.34859 & 0.0397262 & 0.674091 & 0.0345715 & 0.668891 & 0.03388 \\
\hline 0.0900176 & 1.35188 & 0.0453627 & 0.683908 & 0.0347493 & 0.665998 & 0.0431302 \\
\hline 0.0965016 & 1.34831 & 0.0438496 & 0.677492 & 0.0345978 & 0.667345 & 0.0403247 \\
\hline 0.111044 & 1.34279 & 0.0431634 & 0.678682 & 0.0417489 & 0.659188 & 0.0260266 \\
\hline 0.102288 & 1.34259 & 0.0501032 & 0.668356 & 0.0322185 & 0.649725 & 0.0395195 \\
\hline 0.115255 & 1.34077 & 0.0511664 & 0.667002 & 0.0371394 & 0.664454 & 0.0306287 \\
\hline 0.111153 & 1.33221 & 0.0490672 & 0.676557 & 0.0310664 & 0.654895 & 0.0343323 \\
\hline 0.115234 & 1.32616 & 0.0439368 & 0.670007 & 0.043694 & 0.658756 & 0.0397719 \\
\hline 0.116095 & 1.3326 & 0.0443122 & 0.675206 & 0.0325685 & 0.648842 & 0.0350391 \\
\hline 0.117553 & 1.32808 & 0.0458225 & 0.666336 & 0.0370152 & 0.660341 & 0.0389992 \\
\hline 0.110593 & 1.32214 & 0.0471637 & 0.662234 & 0.0393488 & 0.655538 & 0.033655 \\
\hline
\end{tabular}




\begin{tabular}{|c|c|c|c|c|c|c|}
\hline 0.121764 & 1.32455 & 0.0474255 & 0.662765 & 0.0386788 & 0.651129 & 5824 \\
\hline 0.13066 & 1.31344 & 0.0465202 & 0.662807 & 0.0352626 & 0.658852 & 0.0350145 \\
\hline 0.124806 & 1.31236 & 0.0548358 & 0.660699 & 0.0387252 & 0.648503 & 0.0451047 \\
\hline 0.116169 & 1.31701 & 0.0440938 & 0.664908 & 0.0303001 & 0.645317 & 0.0342461 \\
\hline 0.132494 & 1.30741 & 0.0370866 & 0.657695 & 0.034679 & 0.646222 & 0.0332672 \\
\hline 0.12832 & 1.29996 & 0.0426088 & 0.659454 & 0.0404995 & 0.65101 & 0.0331829 \\
\hline 0.100938 & 1.29832 & 0.0400102 & 0.64918 & 0.0397684 & 0.655495 & 0.0382063 \\
\hline 0.114661 & 1.29391 & 0.0422849 & 0.650616 & 0.0367764 & 0.640721 & 0.0362784 \\
\hline 0.142009 & 1.29404 & 0.0455336 & 0.657215 & 0.0303267 & 0.639142 & 0.0329983 \\
\hline 0.102742 & 1.29112 & 0.0514845 & 0.656295 & 0.0369011 & 0.640073 & 0.0234456 \\
\hline 0.102448 & 1.28606 & 0.0429055 & 0.648343 & 0.0374234 & 0.640201 & 0.0316082 \\
\hline 0.112877 & 1.2886 & 0.0368601 & 0.645493 & 0.0417361 & 0.628811 & 0.0371566 \\
\hline 0.120204 & 1.29091 & 0.0439749 & 0.649132 & 0.0356741 & 0.637475 & 0.0361977 \\
\hline 0.0937429 & 1.29 & 0.0448191 & 0.653296 & 0.0358702 & 0.639892 & 0.0324678 \\
\hline 0.132092 & 1.28949 & 0.0463644 & 0.645241 & 0.0392723 & 0.632864 & 0.0388697 \\
\hline 0.105841 & 1.28256 & 0.0475854 & 0.645778 & 0.0430778 & 0.633163 & 0.0311302 \\
\hline 0.109382 & 1.27832 & 0.0485041 & 0.643876 & 0.0305927 & 0.623358 & 0.0297944 \\
\hline 0.0986863 & 1.27303 & 0.0477047 & 0.649457 & 0.0434509 & 0.641232 & 0.0300353 \\
\hline 0.0848537 & 1.26334 & 0.0446719 & 0.630073 & 0.0262246 & 0.623468 & 0.0278704 \\
\hline 0.107542 & 1.26619 & 0.0495323 & 0.634629 & 0.0387835 & 0.620549 & 0.0265695 \\
\hline 0.10933 & 1.27555 & 0.0463816 & 0.632629 & 0.0366656 & 0.628093 & 0.034993 \\
\hline 0.0767579 & 1.27203 & 0.0474896 & 0.636568 & 0.0336887 & 0.623285 & 0.0316096 \\
\hline 0.123485 & 1.26655 & 0.0452769 & 0.634687 & 0.0343284 & 0.630536 & 0.0317789 \\
\hline 0.101539 & 1.26389 & 0.0453247 & 0.638589 & 0.0289661 & 0.621599 & 0.0355444 \\
\hline 0.120757 & 1.26292 & 345 & 129 & 0.03 & 0.626974 & 0.02 \\
\hline 0.121374 & 1.26179 & 0.0491146 & 0.634949 & 0.0406403 & 0.628314 & 0.0285436 \\
\hline 0.139132 & 1.26725 & 0.0462614 & 0.63457 & 0.0361106 & 0.621101 & 0.0290496 \\
\hline 0.14786 & 1.26181 & 0.0423614 & 0.632954 & 0.0388922 & 0.632231 & 0.0355163 \\
\hline 0.171164 & 1.25182 & 0.0397695 & 0.63022 & 0.038676 & 0.631681 & 0.0373888 \\
\hline 0.193526 & 1.2578 & 0.0410193 & 0.627513 & 0.0356612 & 0.646315 & 0.0312616 \\
\hline 0.247582 & 1.25569 & 0.0404354 & 0.637804 & 0.0337322 & 0.63748 & 0.0302564 \\
\hline 0.263696 & 1.2572 & 0.0432561 & 0.635656 & 0.0329598 & 0.634947 & 0.0400308 \\
\hline 0.301069 & 1.26035 & 0.0485941 & 0.632457 & 0.0323479 & 0.636765 & 0.0330026 \\
\hline 0.367921 & 1.25187 & 0.0477282 & 0.620578 & 0.0443002 & 0.642379 & 0.0297983 \\
\hline 0.457773 & 1.24381 & 0.0462035 & 0.62931 & 0.036889 & 0.644444 & 0.0301814 \\
\hline 0.544134 & 1.23931 & 0.0447766 & 0.626925 & 0.0383305 & 0.645415 & 0.0239017 \\
\hline 0.61111 & 1.23966 & 0.0479479 & 0.637331 & 0.0379938 & 0.643675 & 0.0293549 \\
\hline 0.775703 & 1.23323 & 0.0394006 & 0.633383 & 0.037907 & 0.647648 & 0.0247844 \\
\hline 0.905482 & 1.22799 & 0.0444086 & 0.631661 & 0.0344462 & 0.649411 & 0.0333066 \\
\hline 1.03042 & 1.22743 & 0.0482201 & 0.623174 & 0.0399218 & 0.642735 & 0.0403567 \\
\hline 1.25979 & 1.22959 & 0.043825 & 0.630183 & 0.0394075 & 0.644019 & 0.0336604 \\
\hline 1.45157 & 1.22922 & 0.049796 & 0.621751 & 0.0327154 & 0.654031 & 0.0309568 \\
\hline 1.64597 & 1.22865 & 0.042481 & 0.623846 & 0.0332633 & 0.648715 & 0.0330369 \\
\hline 1.8613 & 1.22818 & 0.0372315 & 0.623411 & 0.0290942 & 0.64805 & 0.0314094 \\
\hline 2.05954 & 1.22641 & 0.0446238 & 0.622175 & 0.0322717 & 0.650809 & 0.0302424 \\
\hline 2.30303 & 1.22634 & 0.0430132 & 0.61582 & 0.0371494 & 0.647098 & 0.0301503 \\
\hline 2.52664 & 1.22403 & 0.0382491 & 0.618534 & 0.0414334 & 0.646057 & 0.037371 \\
\hline
\end{tabular}




\begin{tabular}{|c|c|c|c|c|c|c|}
\hline 2.64708 & 1.21732 & 0.0387648 & 0.619539 & 0.0381463 & 0.647917 & 0.0427563 \\
\hline 2.85552 & 1.21144 & 0.0426931 & 0.615855 & 0.0314918 & 0.648256 & 0.0295209 \\
\hline 2.9436 & 1.21871 & 0.0484709 & 0.615472 & 0.0280206 & 0.65567 & 0.0374932 \\
\hline 3.13415 & 1.21172 & 0.0442949 & 0.618943 & 0.0351935 & 0.652338 & 0.0358214 \\
\hline 3.18473 & .20188 & 0.038266 & 0.615694 & 0.0382387 & 0.645687 & 0.0320862 \\
\hline 3.27805 & 1.20438 & 0.0406414 & 0.621583 & 0.0295541 & 0.645814 & 0.0329423 \\
\hline 3.1794 & 1.1977 & 0.0452878 & 0.621093 & 0.0400778 & 0.63877 & 0.0351166 \\
\hline 3.2049 & 1.19896 & 0.0412365 & 0.612823 & 0.031728 & 0.638136 & 0.0407829 \\
\hline 3.15581 & 1.2016 & 0.0433144 & 0.614414 & 0.0375489 & 0.649966 & 0.0302774 \\
\hline 2.92544 & 1.19239 & 0.0420737 & 0.606068 & 0.0338892 & 0.640013 & 0.0346894 \\
\hline 2.81208 & 1.18456 & 0.0387743 & 0.615071 & 0.0338894 & 0.62722 & 0.0347962 \\
\hline 2.62761 & 1.18989 & 0.0387616 & 0.60626 & 0.0335584 & 0.636043 & 0.0233067 \\
\hline 2.42302 & 1.18603 & 0.0414131 & 0.605911 & 0.0358204 & 0.635739 & 0.0424043 \\
\hline 2.20952 & 1.18289 & 0.0399852 & 0.608238 & 0.0375094 & 0.627804 & 0.0275227 \\
\hline 2.06231 & 1.18746 & 0.0394933 & 0.604042 & 0.0369318 & 0.608782 & 0.0269517 \\
\hline 1.70888 & 1.18411 & 0.0347292 & 0.600434 & 0.0302122 & 0.613611 & 0.0269963 \\
\hline 1.55105 & 1.18499 & 0.0350125 & 0.600592 & 0.0424811 & 0.610043 & 0.0363631 \\
\hline 1.32821 & 1.18179 & 0.0376538 & 0.598649 & 0.0296819 & 0.60587 & 0.0358204 \\
\hline 1.13807 & 1.18205 & 0.0403792 & 0.586968 & 0.0266326 & 0.596864 & 0.0221463 \\
\hline 0.983309 & 1.17823 & 0.0416415 & 0.6007 & 0.0302499 & 0.596658 & 0.0335061 \\
\hline 0.815623 & 1.17295 & 0.0428417 & 0.601306 & 0.0373753 & 0.588801 & 0.0290933 \\
\hline 0.737745 & 1.16431 & 0.0408649 & 0.585992 & 0.0377624 & 1407 & 4479 \\
\hline 0.568641 & 1.15245 & 0.0398811 & 0.597308 & 0.0292843 & 0.589551 & 0.0369605 \\
\hline 0.458828 & 1.14778 & 0.0367321 & 0.59298 & 0.0343232 & 0.589792 & 0.0229101 \\
\hline 0.3762 & 1.14771 & 0.0402464 & 0.59546 & 0.0287628 & 0.594297 & 0.0333934 \\
\hline 0.295295 & 1.14143 & 0.0390267 & 0.586606 & 0.0308155 & 0.577949 & 0.026002 \\
\hline 0.251799 & 1.14904 & 0.0454263 & 0.588334 & 0.0332392 & 0.584366 & 0.037819 \\
\hline 0.218094 & 1.14721 & 0.047414 & 0.58236 & 0.0276027 & 0.573505 & 0.0282392 \\
\hline 0.169754 & 1.13532 & 0.0437373 & 0.575531 & 0.0245452 & 0.573374 & 0.0279674 \\
\hline 0.130181 & 1.13318 & 0.0474821 & 0.579485 & 0.0274258 & 0.567476 & 0.0300739 \\
\hline 0.144582 & 1.13011 & 0.0371114 & 0.584674 & 0.037816 & 0.568268 & 0.03263 \\
\hline 0.109767 & 1.12587 & 0.0425994 & 0.57808 & 0.0386217 & 0.569458 & 0.0260791 \\
\hline 0.0917397 & 1.12134 & 0.041478 & 0.57527 & 0.0334841 & 0.567356 & 0.0242645 \\
\hline 0.0849886 & 1.12323 & 0.0388567 & 0.569083 & 0.0271563 & 0.567197 & 0.0278374 \\
\hline 0.0962389 & 1.11955 & 0.0342347 & 0.568567 & 0.0321331 & 0.568997 & 0.0255178 \\
\hline 0.0976248 & 1.10813 & 0.0374481 & 0.571145 & 0.0418494 & 0.560981 & 0.0322487 \\
\hline 0.0667772 & 1.10662 & 0.0398175 & 0.568055 & 0.0306499 & 0.564754 & 0.0271755 \\
\hline 0.0793177 & 1.10932 & 0.0379554 & 0.565448 & 0.0302939 & 0.564424 & 0.0267492 \\
\hline 0.0932561 & 1.11169 & 0.0327562 & 0.562594 & 0.0307871 & 0.551263 & 0.0351726 \\
\hline 0.0847462 & 1.11206 & 0.0368962 & 0.562348 & 0.0268158 & 0.562397 & 0.0304819 \\
\hline 0.0808763 & 1.10958 & 0.0355342 & 0.560285 & 0.027625 & 0.562745 & 0.0268183 \\
\hline 0.0972219 & 1.10407 & 0.033239 & 0.561679 & 0.0358705 & 0.567264 & 0.0272288 \\
\hline 0.0730407 & 1.10054 & 0.0385399 & 0.560585 & 0.0209071 & 0.561813 & 0.0243766 \\
\hline 0.0943243 & 1.09297 & 0.0427998 & 0.561157 & 0.0311355 & 0.561021 & 0.0254645 \\
\hline 0.0894272 & 1.09562 & 0.0425609 & 0.554678 & 0.035197 & 0.554965 & 0.0245457 \\
\hline 0.0748405 & 1.09804 & 0.0437915 & 0.563027 & 0.0318454 & 0.56858 & 0.0321969 \\
\hline 0.0810419 & 1.09429 & 0.0409617 & 0.555545 & 0.0252018 & 0.56722 & 0.0212145 \\
\hline
\end{tabular}




\begin{tabular}{|c|c|c|c|c|c|c|}
\hline 0.0886521 & 1.09041 & 0.035411 & 0.561254 & 0.0273942 & 0.581944 & 0.0278186 \\
\hline 0.1251 & 1.09362 & 0.0340454 & 0.559634 & 0.0290692 & 0.59107 & 0.0289373 \\
\hline 0.119815 & 1.09859 & 0.0456682 & 0.565122 & 0.0245927 & 0.593245 & 0.0372034 \\
\hline 0.142889 & 1.0939 & 0.0464831 & 0.575789 & 0.0344069 & 0.608365 & 0.0313305 \\
\hline 0.189147 & 1.08507 & 0.0384128 & 0.57068 & 0.0285438 & 0.618517 & 0.0361685 \\
\hline 0.18405 & 1.08552 & 0.0321804 & 0.566301 & 0.0375976 & 0.619547 & 0.0320458 \\
\hline 0.17315 & 1.09018 & 0.0410703 & 0.57592 & 0.0319609 & 0.650298 & 0.0309435 \\
\hline 0.254773 & 1.09175 & 0.0460985 & 0.57747 & 0.0296528 & 0.66052 & 0.0365374 \\
\hline 0.254246 & 1.09243 & 0.0424833 & 0.568147 & 0.023575 & 0.663748 & 0.0330116 \\
\hline 0.301366 & 1.09582 & 0.0425474 & 0.578589 & 0.0310268 & 0.682468 & 0.0331501 \\
\hline 0.354547 & 1.09666 & 0.0413641 & 0.583165 & 0.032244 & 0.690537 & 0.0363073 \\
\hline 0.40947 & 1.09994 & 0.0409002 & 0.589926 & 0.029986 & 0.703173 & 0.0393528 \\
\hline 0.425118 & 1.0998 & 0.0472461 & 0.591497 & 0.0315321 & 0.71835 & 0.0286891 \\
\hline 0.497074 & 1.09428 & 0.0410081 & 0.595081 & 0.0376407 & 0.728671 & 0.0283762 \\
\hline 0.508554 & 1.0834 & 0.0400868 & 0.599664 & 0.0279214 & 0.727396 & 0.0305934 \\
\hline 0.589033 & 1.08354 & 0.0466132 & 0.592441 & 0.0291183 & 0.738715 & 0.0352586 \\
\hline 0.6463 & 1.08885 & 0.047766 & 0.601189 & 0.0317899 & 0.73387 & 0.027196 \\
\hline 0.679752 & 1.08136 & 0.0445354 & 0.603122 & 0.0302052 & 0.748683 & 0.0306015 \\
\hline 0.739567 & 1.0754 & 0.0397121 & 0.60104 & 0.0291126 & 0.738722 & 0.0426365 \\
\hline 0.784587 & 1.06367 & 0.0407103 & 0.603086 & 0.033343 & 0.741363 & 0.0389861 \\
\hline 0.822 & 1.07028 & 0.0426148 & 0.601036 & 0.0321403 & 0.72634 & 0.0395776 \\
\hline 0.859581 & 1.06924 & 0.0398985 & 0.585429 & 3096 & 0.713327 & 0.036719 \\
\hline 0.856775 & 1.06153 & 0.0385777 & 0.58271 & 0.0328563 & 0.698326 & 0.0322881 \\
\hline 0.891109 & 1.05494 & 0.0348802 & 0.5849 & 0.0283292 & 0.689774 & 0.0380274 \\
\hline 0.896958 & 1.04964 & 0.0384269 & 0.576596 & 0.0333576 & 0.67875 & 0.0413512 \\
\hline 0.884145 & 1.04544 & 0.0346073 & 0.572757 & 0.0335762 & 0.669127 & 0.0297553 \\
\hline 0.828954 & 1.04268 & 0.0327783 & 0.568982 & 0.0310892 & 0.656739 & 0.023745 \\
\hline 0.827294 & 1.0379 & 0.033478 & 0.563003 & 0.0281782 & 0.632083 & 0.0335872 \\
\hline 0.785922 & 1.03699 & 0.0308571 & 0.563215 & 0.0261628 & 0.622463 & 0.0260879 \\
\hline 0.775906 & 1.03101 & 0.0275941 & 0.552125 & 0.0273525 & 0.612715 & 0.0342167 \\
\hline 0.691692 & 1.02365 & 0.0334749 & 0.545786 & 0.0328932 & 0.602831 & 0.0282029 \\
\hline 0.630661 & 1.02108 & 0.0382108 & 0.541412 & 0.0260563 & 0.58583 & 0.024058 \\
\hline 0.565208 & 1.02505 & 0.0424249 & 0.542036 & 0.0267737 & 0.574448 & 0.0330356 \\
\hline 0.546689 & 1.021 & 0.0369895 & 0.539039 & 0.0306272 & 0.572475 & 0.0365318 \\
\hline 0.4416 & 1.01809 & 0.0320007 & 0.540047 & 0.0322354 & 0.553166 & 0.0274394 \\
\hline 0.425172 & 1.02446 & 0.0334486 & 0.533162 & 0.0270018 & 0.545719 & 0.0341688 \\
\hline 0.368043 & 1.02003 & 0.0371281 & 0.519094 & 0.0285073 & 0.534192 & 0.0309347 \\
\hline 0.324495 & 1.01101 & 0.032105 & 0.529307 & 0.0320441 & 0.531175 & 0.0273708 \\
\hline 0.262367 & 1.00743 & 0.0314257 & 0.528213 & 0.0305746 & 0.532945 & 0.0237351 \\
\hline 0.204157 & 1.00376 & 0.0343616 & 0.523665 & 0.0287829 & 0.519223 & 0.0287663 \\
\hline 0.192404 & 1.00383 & 0.0391575 & 0.522634 & 0.029289 & 0.521777 & 0.0276957 \\
\hline 0.198821 & 1.00416 & 0.0410451 & 0.517844 & 0.0293813 & 0.518049 & 0.0297293 \\
\hline 0.146839 & 0.999259 & 0.0382439 & 0.519167 & 0.0285114 & 0.525199 & 0.029378 \\
\hline 0.129758 & 1.00184 & 0.0307479 & 0.524983 & 0.0261598 & 0.521158 & 0.0336887 \\
\hline 0.119906 & 1.00225 & 0.0322748 & 0.522673 & 0.0248386 & 0.512147 & 0.0333258 \\
\hline 0.0950385 & 1.00283 & 0.0331524 & 0.513186 & 0.0331692 & 0.522159 & 0.0293272 \\
\hline 0.0936402 & 0.997656 & 0.030806 & 0.511912 & 0.0275873 & 0.50957 & 0.0336619 \\
\hline
\end{tabular}




\begin{tabular}{|c|c|c|c|c|c|c|}
\hline 0.0777605 & 0.995641 & 0.0248131 & 0.507538 & 0.0314702 & 0.510631 & 0.0306905 \\
\hline 0.0868432 & 1.00018 & 0.0274932 & 0.506082 & 0.0331467 & 0.517521 & 0.0280041 \\
\hline 0.0673065 & 1.00103 & 0.0285663 & 0.513611 & 0.0284657 & 0.518715 & 0.0227337 \\
\hline 0.0839607 & 0.994646 & 0.0337611 & 0.508998 & 0.0303382 & 0.513087 & 0.0225682 \\
\hline 0.0576869 & 0.989575 & 0.0268119 & 0.510376 & 0.0254544 & 0.500917 & 0.0311938 \\
\hline 0.0557535 & 0.994026 & 0.0292375 & 0.508439 & 0.0308078 & 0.511113 & 0.0306995 \\
\hline 0.0669254 & 0.986697 & 0.0312665 & 0.511828 & 0.0257597 & 0.509965 & 0.0238229 \\
\hline 0.0534328 & 0.985704 & 0.0378245 & 0.502898 & 0.0306536 & 0.500635 & 0.0256 \\
\hline 0.0574546 & 0.986157 & 0.0369664 & 0.498732 & 0.0275059 & 0.507395 & 0.0352704 \\
\hline 0.06157 & 0.985242 & 0.0355686 & 0.511228 & 0.0283829 & 0.499903 & 0.0237093 \\
\hline 0.0618464 & 0.986021 & 0.0314934 & 0.500227 & 0.0240717 & 0.498078 & 0.0220636 \\
\hline 0.0669563 & 0.984 & 0.0292699 & 0.502894 & 0.0269589 & 0.50103 & 0.0341259 \\
\hline 0.0550048 & 0.983025 & 0.0302248 & 0.504987 & 0.0344568 & 0.492419 & 0.0321105 \\
\hline 0.0623739 & 0.97959 & 0.0342939 & 0.49372 & 0.0258949 & 0.498776 & 0.020413 \\
\hline 0.0594516 & 0.989415 & 0.0348635 & 0.501125 & 0.0299033 & 0.500619 & 0.0264417 \\
\hline 0.0656972 & 0.985847 & 0.0339773 & 0.504234 & 0.026278 & 0.501326 & 0.0238557 \\
\hline 0.0807908 & 0.97992 & 0.0308538 & 0.507019 & 0.0251673 & 0.49496 & 0.0247089 \\
\hline 0.0745334 & 0.985873 & 0.0341175 & 0.495599 & 0.0321173 & 0.498029 & 0.0255838 \\
\hline 0.0605111 & 0.985581 & 0.028 & 0.49852 & 0.026 & 0.502293 & 0.0262693 \\
\hline 0.0736016 & 0.985735 & 0.0296775 & 0.500766 & 0.0283596 & 0.502263 & 0.0273452 \\
\hline 0.0610092 & 0.995959 & 0.0314054 & 0.498589 & 0.0316555 & 0.509547 & 0.0288777 \\
\hline 0.0596503 & 1.00212 & 0.02 & 0.506157 & 281 & 0.49874 & 0.0289097 \\
\hline 0.0690135 & 1.01507 & 0.0357827 & 0.506052 & 0.0277745 & 0.496133 & 0.0292669 \\
\hline 0.0754769 & 1.03533 & 0.0288714 & 0.51268 & 0.0285148 & 0.499395 & 0.0275495 \\
\hline 0.0585721 & 1.03935 & 0.0350804 & 0.509427 & 0.0247627 & 0.510475 & 0.0297807 \\
\hline 0.0789442 & 1.05267 & 0.036399 & 0.517518 & 0.0266684 & 0.508793 & 0.0252758 \\
\hline 0.0791659 & 1.0735 & 0.0386439 & 0.513086 & 0.0252351 & 0.513902 & 0.0204485 \\
\hline 0.0659972 & 1.09941 & 0.0370176 & 0.523538 & 0.0265218 & 0.521085 & 0.0347628 \\
\hline 0.0815162 & 1.11994 & 0.040708 & 0.537775 & 0.0251959 & 0.528297 & 0.0227271 \\
\hline 0.0758593 & 1.15043 & 0.0463005 & 344 & 0.025859 & 0.526328 & 0.0237673 \\
\hline 0.0755625 & 1.177 & 0.0367798 & 0.560166 & 0.0243233 & 0.547126 & 0.0307846 \\
\hline 0.0812186 & 1.21044 & 0.0388332 & 0.574344 & 0.029582 & 0.547777 & 0.0300401 \\
\hline 0.0747864 & 1.2479 & 0.0394834 & 0.587802 & 0.0308331 & 0.569014 & 0.0342412 \\
\hline 0.108779 & 1.27617 & 0.0433167 & 0.602877 & 0.0322254 & 0.580434 & 0.0305591 \\
\hline 0.100156 & 1.30305 & 0.0483299 & 0.619771 & 0.0355966 & 0.59682 & 0.0337179 \\
\hline 0.112132 & 1.33558 & 0.0497461 & 0.64098 & 0.0359484 & 0.601878 & 0.0323842 \\
\hline 0.117632 & 1.35695 & 0.0557929 & 0.665854 & 0.0340825 & 0.624447 & 0.0399849 \\
\hline 0.132728 & 1.38296 & 0.0552849 & 0.693647 & 0.0444446 & 0.635083 & 0.0280732 \\
\hline 0.12888 & 1.40543 & 0.0590002 & 0.714338 & 0.0350941 & 0.660092 & 0.036398 \\
\hline 0.153683 & 1.42928 & 0.0590506 & 0.746032 & 0.0444739 & 0.668935 & 0.038271 \\
\hline 0.12878 & 1.43847 & 0.062565 & 0.756033 & 0.04026 & 0.678959 & 0.0380035 \\
\hline 0.131847 & 1.44415 & 0.0612564 & 0.786891 & 0.0496505 & 0.702156 & 0.0384283 \\
\hline 0.132497 & 1.4448 & 0.0663833 & 0.805832 & 0.0480736 & 0.710537 & 0.0456257 \\
\hline 0.161418 & 1.43298 & 0.0709873 & 0.817973 & 0.0330012 & 0.726272 & 0.0357718 \\
\hline 0.13577 & 1.42346 & 0.0647483 & 0.823334 & 0.0420481 & 0.731921 & 0.0483982 \\
\hline 0.149659 & 1.40159 & 0.0575754 & 0.844574 & 0.0444265 & 0.734734 & 0.0389093 \\
\hline 0.141784 & 1.38089 & 0.0555056 & 0.849158 & 0.0538167 & 0.727876 & 0.0345122 \\
\hline
\end{tabular}




\begin{tabular}{|c|c|c|c|c|c|c|}
\hline 0.131101 & 1.35566 & 0.0553103 & 0.84465 & 0.0457149 & 0.730547 & \\
\hline 0.122191 & 1.31954 & 0.053833 & 0.847461 & 0.0470761 & 0.726661 & 0.0342682 \\
\hline 0.137941 & 1.28145 & 0.0551468 & 0.839858 & 0.0438848 & 0.709411 & 0.0393938 \\
\hline 0.134816 & 1.2427 & 0.0524113 & 0.823188 & 0.0488769 & 0.705479 & 0.0375913 \\
\hline 0.154089 & 1.2091 & 0.0463008 & 0.814578 & 0.0520033 & 0.69312 & 0.0385905 \\
\hline 0.129251 & 1.17891 & 0.0434291 & 0.792843 & 0.040854 & 0.686248 & 0.0389527 \\
\hline 0.105734 & 1.14885 & 0.0347058 & 0.765612 & 0.0420046 & 0.657828 & 0.0355056 \\
\hline 0.114535 & 1.09424 & 0.0401009 & 0.747202 & 0.0418846 & 0.642468 & 0.0334102 \\
\hline 0.110393 & 1.05661 & 0.0381485 & 0.71959 & 0.0422306 & 0.616448 & 0.035555 \\
\hline 0.0856758 & 1.03515 & 0.0331474 & 0.699745 & 0.0351849 & 0.613105 & 0.0355483 \\
\hline 0.0961957 & 1.01895 & 0.0269604 & 0.666268 & 0.0278828 & 0.583526 & 0.0373329 \\
\hline 0.0945416 & 0.989303 & 0.0314493 & 0.64715 & 0.0343187 & 0.567516 & 0.0276147 \\
\hline 0.0664684 & 0.956434 & 0.0331016 & 0.620265 & 0.0390466 & 0.551923 & 0.0357574 \\
\hline 0.0845601 & 0.946384 & 0.0300704 & 0.590936 & 0.0301783 & 0.539808 & 0.0241573 \\
\hline 0.0646871 & 0.925975 & 0.0281772 & 0.564204 & 0.0355494 & 0.517163 & 0.0287582 \\
\hline 0.0654316 & 0.917137 & 0.0311034 & 0.549923 & 0.0262974 & 0.512381 & 0.0291403 \\
\hline 0.0630177 & 0.902863 & 0.0322811 & 0.534135 & 0.0263536 & 0.502427 & 0.0242057 \\
\hline 0.0629234 & 0.890641 & 0.0286527 & 0.524515 & 0.0213768 & 0.492158 & 0.028645 \\
\hline 0.0609184 & 0.878122 & 0.0287167 & 0.507819 & 0.0274654 & 0.472716 & 0.0226257 \\
\hline 0.0532949 & 0.876492 & 0.0272135 & 0.495379 & 0.0233792 & 0.470441 & 0.0268364 \\
\hline 0.0556345 & 0.877079 & 0.0250134 & 0.483165 & 0.027791 & 0.46301 & 0.0244757 \\
\hline 0.0487454 & 0.870332 & 0.0280173 & 0.476948 & 0.0272756 & 0.45858 & 0.0216473 \\
\hline 0.0627396 & 0.86488 & 0.0306825 & 0.469131 & 0.0289171 & 0.45979 & 0.0249595 \\
\hline 0.0479176 & 0.860683 & 0.0325882 & 0.466403 & 0.0233735 & 0.450767 & 0.0273768 \\
\hline 0.0588351 & 0.86 & 0.03 & 0.45 & 257 & 924 & 8231 \\
\hline 0.056295 & 0.855877 & 0.0265924 & 0.455665 & 0.0277762 & 0.445027 & 0.0213628 \\
\hline 0.0500858 & 0.840883 & 0.0266218 & 0.449565 & 0.0263953 & 0.440817 & 0.0241374 \\
\hline 0.0563991 & 0.846881 & 0.0298911 & 0.452392 & 0.019406 & 0.441068 & 0.0250988 \\
\hline 0.047789 & 0.844805 & 0.0302588 & 0.447492 & 0.0231088 & 0.429166 & 0.0272465 \\
\hline 0.0508902 & 0.839044 & 0.0318025 & 0.452697 & 0.0291124 & 0.442328 & 0.021821 \\
\hline 0.0492149 & 0.835386 & 0.0287696 & 0.433888 & 0.0250138 & 0.438667 & 0.0266548 \\
\hline 0.0560203 & 0.835678 & 0.0243192 & 0.442506 & 0.0224293 & 0.438416 & 0.0188464 \\
\hline 0.0681233 & 0.827435 & 0.0244899 & 0.437472 & 0.0200283 & 0.438193 & 0.0257513 \\
\hline 0.0635961 & 0.824146 & 0.02689 & 0.436375 & 0.0278457 & 0.429288 & 0.0218017 \\
\hline 0.0624461 & 0.825567 & 0.0238017 & 0.440658 & 0.0259922 & 0.431649 & 0.0283204 \\
\hline 0.0521204 & 0.826646 & 0.0273617 & 0.434044 & 0.0240818 & 0.429854 & 0.0227922 \\
\hline 0.0637521 & 0.824646 & 0.0277782 & 0.431192 & 0.0224394 & 0.426282 & 0.0257832 \\
\hline 0.054732 & 0.816918 & 0.0281824 & 0.427055 & 0.0208279 & 0.420004 & 0.0249756 \\
\hline 0.060663 & 0.811372 & 0.0306241 & 0.434 & 0.0171725 & 0.419686 & 0.0278194 \\
\hline 0.0543202 & 0.812777 & 0.026341 & 0.429447 & 0.0190491 & 0.422033 & 0.0250487 \\
\hline 0.0481495 & 0.812797 & 0.0286626 & 0.426129 & 0.0193778 & 0.42269 & 0.0242191 \\
\hline 0.0475975 & 0.81037 & 0.03043 & 0.428854 & 0.0244361 & 0.419975 & 0.0230774 \\
\hline 0.0571003 & 0.807278 & 0.0250027 & 0.429965 & 0.0326343 & 0.417403 & 0.0208025 \\
\hline 0.0519781 & 0.807162 & 0.0301895 & 0.429104 & 0.022067 & 0.42036 & 0.0229904 \\
\hline 0.045744 & 0.805998 & 0.0297676 & 0.425639 & 0.0192912 & 0.427794 & 0.0191488 \\
\hline 0.0381278 & 0.800611 & 0.0291384 & 0.41818 & 0.0231802 & 0.418376 & 0.0252245 \\
\hline 0.0472112 & 0.798446 & 0.0272907 & 0.425943 & 0.0228411 & 0.411109 & 0.018867 \\
\hline
\end{tabular}




\begin{tabular}{|c|c|c|c|c|c|c|}
\hline 0.0585236 & 0.799559 & 0.0252802 & 0.416174 & 0.0276892 & 0.420487 & 289776 \\
\hline 0.0525688 & 0.79406 & 0.0223841 & 0.425549 & 0.020934 & 0.40255 & 0.0242846 \\
\hline 0.0520464 & 0.793785 & 0.0223561 & 0.420998 & 0.0209242 & 0.412752 & 0.0213645 \\
\hline .0466211 & 0.793083 & 0.0242945 & 0.414535 & 0.0235736 & 0.401182 & 0.0186313 \\
\hline 0.051882 & 0.790297 & 0.0271956 & 0.418547 & 0.0229766 & 0.4127 & 0.0246903 \\
\hline 0.0426328 & 0.784639 & 0.0286496 & 0.414075 & 0.0255316 & 0.406895 & 0.025058 \\
\hline 0.0501727 & 0.781718 & 0.0250923 & 0.417973 & 0.0197513 & 0.406731 & 0.0220611 \\
\hline 0.0460051 & 0.782267 & 0.0252504 & 0.408558 & 0.0266879 & 0.411543 & 0.0211635 \\
\hline 0.0479068 & 0.778265 & 0.0304413 & 0.415969 & 0.0227603 & 0.41771 & 0.0268133 \\
\hline 0.0478654 & 0.773204 & 0.0204881 & 0.404638 & 0.0195327 & 0.412348 & 0.0216291 \\
\hline 0.0497992 & 0.775517 & 0.0212796 & 0.409613 & 0.0256083 & 0.41648 & 0.0264609 \\
\hline 0.0444827 & 0.774037 & 0.0271788 & 0.412966 & 0.0229141 & 0.411026 & 0.0217219 \\
\hline 0.0466107 & 0.771369 & 0.0311748 & 0.41488 & 0.0218931 & 0.410093 & 0.0203103 \\
\hline 0.0517436 & 0.769335 & 0.0242462 & 0.403637 & 0.0194502 & 0.408929 & 0.0266093 \\
\hline 0.0426326 & 0.766253 & 0.0288659 & 0.409942 & 0.027525 & 0.417264 & 0.0234312 \\
\hline 0.0481029 & 0.765391 & 0.0350609 & 0.412239 & 0.0270171 & 0.422269 & 1257 \\
\hline 0.0551079 & 0.763987 & 0.0276808 & 0.406511 & 0.0274668 & 0.416145 & 0.021439 \\
\hline 0.0546802 & 0.767928 & 0.0229508 & 0.409568 & 0.0197776 & 0.419414 & 0.0270722 \\
\hline 0.0496825 & 0.766906 & 323 & 0.405215 & 0.02 & 0.4 & 0.02 \\
\hline 0.0495973 & 0.763863 & 0.0249923 & 0.414932 & 0.0203115 & 0.425453 & 0.0247 \\
\hline 0.0380337 & 0.767488 & 0.0214457 & 0.409172 & 0.0205701 & 0.425839 & 0.022281 \\
\hline 0.0493943 & 0.759622 & 0.02 & 5054 & 5398 & 353 & 939 \\
\hline 0.0500854 & 0.761974 & 0.0172957 & 0.40667 & 0.0225893 & 0.428895 & 0.0289793 \\
\hline 0.0501358 & 0.754699 & 0.0208951 & 0.414075 & 0.0214057 & 0.438935 & 0.0268533 \\
\hline 0.0429537 & 0.759601 & 0.0225604 & 0.413302 & 0.0261318 & 0.436167 & 0.0294979 \\
\hline 0.0396657 & 0.760155 & 0.024064 & 0.410504 & 0.0204087 & 0.446774 & 0.0231608 \\
\hline 0.0425759 & 0.751605 & 0.0280242 & 0.414179 & 0.0160888 & 0.453986 & 0.0263629 \\
\hline 0.0558062 & 0.755217 & 0.0308293 & 0.408594 & 0.0222962 & 267 & 0.0269307 \\
\hline 0.0440352 & 0.754813 & 0.0269256 & 0.410436 & 0.0238468 & 0.461518 & 0.0274097 \\
\hline 0.0434042 & 365 & & 557 & 5391 & 331 & 708 \\
\hline 0.049587 & 0.745734 & 0.0243574 & 0.415566 & 0.0243846 & 0.4 & 0.0248163 \\
\hline 0.0508302 & 0.736656 & 0.0246841 & 0.416518 & 0.0245228 & 0.456758 & 0.0298259 \\
\hline 0.0526076 & 0.737246 & 0.0307926 & 0.415878 & 0.0244511 & 323 & 0.0236086 \\
\hline 0.0447252 & 0.739708 & 0.0289651 & 0.413791 & 0.0252899 & 0.4 & 0.0313497 \\
\hline 0.0468624 & 0.740267 & 0.0280587 & 0.401214 & 0.026158 & 0.458756 & 0.0275176 \\
\hline 0.0477446 & 0.732437 & 0.02851 & 0.409297 & 0.0145159 & 0.447736 & 0.0221352 \\
\hline 0.0396583 & 0.724488 & 0.0290959 & 0.407344 & 0.0223179 & 0.443875 & 0.0281479 \\
\hline 0.0321133 & 0.730549 & 0.0267629 & 0.400575 & 0.020534 & 0.442666 & 0.0247246 \\
\hline 0.0497316 & 0.730013 & 0.0244461 & 0.400748 & 0.0292758 & 0.43308 & 0.0321437 \\
\hline 0.0510215 & 0.732742 & 0.0278276 & 0.399314 & 0.0166532 & 0.427346 & 0.0309577 \\
\hline 0.0465795 & 0.722781 & 0.0267416 & 0.401081 & 0.0253415 & 0.422558 & 0.0242924 \\
\hline 0.0423502 & 0.719619 & 0.0229066 & 0.394278 & 0.0220089 & 0.425 & 0.0285972 \\
\hline 0.0539785 & 0.715668 & 0.0262214 & 0.395829 & 0.0262688 & 0.417226 & 0.0255041 \\
\hline 0.0423958 & 0.715107 & 0.0293698 & 0.385969 & 0.0224635 & 0.406818 & 0.0257446 \\
\hline 0.0670519 & 0.712548 & 0.0249735 & 0.389074 & 0.0249674 & 0.399683 & 0.0215209 \\
\hline 0.0432747 & 0.710616 & 0.0169691 & 0.382327 & 0.0206576 & 0.397186 & 0.0237525 \\
\hline 0.0572399 & 0.704753 & 0.0292499 & 0.385885 & 0.0192884 & 0.386924 & 0.0256159 \\
\hline
\end{tabular}




\begin{tabular}{|c|c|c|c|c|c|c|}
\hline 0.0600781 & 0.695981 & 0.0271685 & 0.373345 & 0.0162088 & 0.380427 & 0.019958 \\
\hline 0.037758 & 0.692845 & 0.0232895 & 0.373387 & 0.0203095 & 0.380548 & 0.0292195 \\
\hline 0.0530108 & 0.692121 & 0.0213035 & 0.38155 & 0.0269735 & 0.378579 & 0.0252618 \\
\hline 0.0467394 & .689781 & 0.0283921 & 0.380379 & 0.0170963 & 0.377401 & 0.0236705 \\
\hline 0.0448662 & 0.686648 & 0.0281109 & 0.369529 & 0.0189686 & 0.373727 & 0.0264051 \\
\hline 0.0473737 & 0.685629 & 0.0263065 & 0.366321 & 0.0247872 & 0.372295 & 0.0235615 \\
\hline 0.0586769 & 0.68352 & 0.0256219 & 0.362965 & 0.0190437 & 0.358029 & 0.0195621 \\
\hline 0.0486992 & 0.683685 & 0.0257512 & 0.3662 & 0.0210297 & 0.363197 & 0.0240371 \\
\hline 0.038133 & 0.67838 & 0.0250555 & 0.364216 & 0.0172361 & 0.366463 & 0.0209879 \\
\hline 0.0452548 & 0.681411 & 0.0227036 & 0.35796 & 0.0130407 & 0.357891 & 0.0218827 \\
\hline 0.0451016 & 0.673937 & 0.0215311 & 0.358705 & 0.0144574 & 0.355772 & 0.0238566 \\
\hline 0.0474529 & 0.672542 & 0.0217196 & 0.368693 & 0.0185423 & 0.35376 & 0.0225251 \\
\hline 0.0407141 & 0.671245 & 0.0196844 & 0.361625 & 0.0158067 & 0.347441 & 0.0205858 \\
\hline 0.0428905 & 0.676854 & 0.0226516 & 0.353863 & 0.0207267 & 0.356192 & 0.0189762 \\
\hline 0.0455852 & 0.667599 & 0.0264728 & 0.355378 & 0.0213734 & 0.354444 & 0.0202569 \\
\hline 0.0376696 & 0.67301 & 0.0273741 & 0.354422 & 0.0216122 & 18959 & 0.0257788 \\
\hline 0.039481 & 0.670157 & 0.0237428 & 0.353786 & 0.0177418 & 0.355766 & 0.0197548 \\
\hline 0.0439456 & 0.661641 & 0.0238591 & 0.354316 & 0.0183332 & 0.346011 & 0.0207166 \\
\hline 0.0411927 & 0.658701 & 929 & 008 & 0.0159219 & 0062 & 0.022082 \\
\hline 0.0462417 & 0.659992 & 0.0261251 & 0.350929 & 0.0148095 & 0.34856 & 0.0193733 \\
\hline 0.056527 & 0.660849 & 0.0198343 & 0.346138 & 0.0190564 & 0.342703 & 0.0240187 \\
\hline 0.0340806 & 0.661062 & 0.02 & 145 & 411 & 008 & 5929 \\
\hline 0.0497663 & 0.654721 & 0.0212547 & 0.343284 & 0.0154633 & 0.340916 & 0.0220572 \\
\hline 0.0391072 & 0.659094 & 0.0197492 & 0.344713 & 0.0206748 & 0.340908 & 0.0219941 \\
\hline 0.043448 & 0.656345 & 0.0222248 & 0.346898 & 0.019487 & 0.344208 & 0.0264802 \\
\hline 0.0349256 & 0.653124 & 0.0212379 & 0.339777 & 0.0200909 & 0.331576 & 0.0190112 \\
\hline 0.0381083 & 0.644772 & 0.0285652 & 0.342723 & 0.0209833 & 0.332668 & 0.0184463 \\
\hline 0.0444462 & 0.644196 & 0.0209477 & 0.337164 & 0.0187544 & 0.340051 & 0.0232909 \\
\hline 0.0375173 & 0.646986 & 0.0236757 & 0.342121 & 0.017516 & 0.334679 & 0.0175857 \\
\hline 0.0353466 & 0.65278 & 0.0252559 & 0.340733 & 0.014122 & 0.336133 & 0.0234568 \\
\hline 0.0513267 & 0.641094 & 0.0217831 & 0.337174 & 0.0232763 & 0.337137 & 0.026862 \\
\hline 0.0379722 & 0.638855 & 0.022666 & 0.340955 & 0.0275116 & 0.337196 & 0.0195013 \\
\hline 0.0502899 & 0.640201 & 0.0218142 & 0.335805 & 0.0219982 & 0.331789 & 0.019015 \\
\hline 0.0441164 & 0.640256 & 0.0227464 & 0.340434 & 0.0214446 & 0.336536 & 0.0207594 \\
\hline 0.0445084 & 0.647253 & 0.0237528 & 0.336574 & 0.0231635 & 0.334589 & 0.023015 \\
\hline 0.0513135 & 0.642715 & 0.0270494 & 0.340623 & 0.0174381 & 0.329531 & 0.0229888 \\
\hline 0.0394538 & 0.648174 & 0.0197681 & 0.340641 & 0.0197299 & 0.339213 & 0.0219334 \\
\hline 0.0494087 & 0.64538 & 0.0219327 & 0.340812 & 0.0171191 & 0.342088 & 0.0203416 \\
\hline 0.0422088 & 0.645536 & 0.0253367 & 0.341921 & 0.0182839 & 0.338069 & 0.0176739 \\
\hline 0.052899 & 0.644112 & 0.0232682 & 0.340677 & 0.0230709 & 0.345479 & 0.0214831 \\
\hline 0.0404605 & 0.645435 & 0.0190802 & 0.338562 & 0.019938 & 0.346402 & 0.0247856 \\
\hline 0.0424381 & 0.650987 & 0.0256138 & 0.336275 & 0.0186467 & 0.353182 & 0.0245818 \\
\hline 0.0431262 & 0.660208 & 0.0284975 & 0.338627 & 0.0171146 & 0.364503 & 0.0247813 \\
\hline 0.0418752 & 0.6576 & 0.0265624 & 0.343672 & 0.0197344 & 0.369209 & 0.0322257 \\
\hline 0.0469988 & 0.660765 & 0.0217493 & 0.347242 & 0.0193345 & 0.38537 & 0.030603 \\
\hline 0.0366562 & 0.663251 & 0.0188219 & 0.346609 & 0.0232601 & 0.391893 & 0.0348606 \\
\hline 0.0437251 & 0.676303 & 0.02516 & 0.348854 & 0.0214186 & 0.397409 & 0.0281381 \\
\hline
\end{tabular}




\begin{tabular}{|c|c|c|c|c|c|c|}
\hline 0.0449088 & 0.677483 & 0.0243285 & 0.356654 & 0.0156115 & 0.415237 & 0.0356679 \\
\hline 0.0499905 & 0.688757 & 0.0197285 & 0.365711 & 0.0181508 & 0.43848 & 0.0448374 \\
\hline 0.0349152 & 0.699272 & 0.0170322 & 0.368957 & 0.0216061 & 0.457067 & 0.0418102 \\
\hline .0564789 & .712325 & 0.0270482 & .370526 & 0.0196264 & 0.483391 & 0.0599672 \\
\hline 0.0486349 & 0.727796 & 0.0296314 & 0.376651 & 0.0206413 & 0.49885 & 0.0571215 \\
\hline 0.0485971 & 0.722466 & 0.0254026 & 0.395938 & 0.0251583 & 0.524004 & 0.0601528 \\
\hline 0.0472588 & 0.73706 & 0.0231146 & 0.404614 & 0.0207165 & 0.557225 & 0.0687583 \\
\hline 0.0524757 & 0.747916 & 0.0205102 & 0.411753 & 0.0239632 & 0.579563 & 0.0807572 \\
\hline 0.0559703 & 0.762385 & 0.0256252 & 0.420148 & 0.0240425 & 0.606909 & 0.0877522 \\
\hline 0.042621 & 0.77201 & 0.0260746 & 0.431654 & 0.0195671 & 0.635732 & 0.0952725 \\
\hline 0.0549267 & 0.780269 & 0.0215486 & 0.44473 & 0.0211101 & 0.667017 & 0.0998635 \\
\hline 0.0469485 & 0.791484 & 0.0318056 & 0.456858 & 0.0216308 & 0.705199 & 0.0944304 \\
\hline 0.0442162 & 0.797776 & 0.0327868 & 0.480029 & 0.0221039 & 0.727811 & 0.127768 \\
\hline 0.0508555 & 0.809909 & 0.027085 & 0.482453 & 0.0316144 & 0.749183 & 0.127622 \\
\hline 0.0626759 & 0.813176 & 0.0290726 & 0.502075 & 0.0257446 & 0.769638 & 0.124448 \\
\hline 0.0550165 & 0.811581 & 0.0308201 & 0.508342 & 0.0248248 & 0.7 & 0.135094 \\
\hline 0.0532766 & 0.812008 & 0.029293 & 0.509957 & 0.0285781 & 0.814163 & 0.141253 \\
\hline 0.0613202 & 0.815067 & 0.0228122 & 0.511584 & 0.0254675 & 0.83 & 0.134367 \\
\hline 0.0508464 & 0.81 & 0.0259702 & 0.53078 & 0.0321322 & 0.8 & 0.146021 \\
\hline 0.0707803 & 0.798804 & 0.026084 & 0.531086 & 0.0311215 & 0.833574 & 0.14123 \\
\hline 0.0537283 & 0.794804 & 0.02633 & 0.538802 & 0.0394089 & 0.834374 & 0.135727 \\
\hline 0.0651188 & 0.7 & 0.022 & 0.540488 & 5965 & 286 & 35173 \\
\hline 0.057123 & 0.775008 & 0.0303802 & 0.538413 & 0.028645 & 0.807028 & 0.135549 \\
\hline 0.0607703 & 0.767637 & 0.0223655 & 0.532334 & 0.0369477 & 0.771113 & 0.126266 \\
\hline 0.071286 & 0.755825 & 0.0180923 & 0.526797 & 0.0240686 & 0.769833 & 0.113973 \\
\hline 0.0576698 & 0.747913 & 0.0217213 & 0.525714 & 0.0295341 & 0.737492 & 0.123207 \\
\hline 0.0606999 & 0.727245 & 0.02121 & 0.51483 & 0.0302727 & 0.71335 & 0.113353 \\
\hline 0.0642087 & 0.705739 & 0.02423 & 0.503305 & 0.0284523 & 0.67 & 0.0974304 \\
\hline 0.0527581 & 0.692136 & 0.0253577 & 0.503775 & 0.0293333 & 0.6 & 0.085624 \\
\hline 0.0557089 & 0.68 & 0.02 & 0.481711 & 0.0285453 & & 132 \\
\hline 0.0589636 & 0.673245 & 0.0193362 & 0.468009 & 0.0268178 & 0.5 & 0.0741484 \\
\hline 0.0530875 & 0.658519 & 0.0220925 & 0.451696 & 0.0294765 & 0.542476 & 0.0673739 \\
\hline 0.0583146 & 0.647089 & 0.0217116 & 0.43574 & 0.0230254 & 0.513141 & 0.0635922 \\
\hline 0.0620079 & 0.637638 & 0.014804 & 0.421981 & 0.0232822 & 0.48993 & 0.0476258 \\
\hline 0.0533696 & 0.624485 & 0.0217441 & 0.413334 & 0.0178441 & 0.462913 & 0.0519121 \\
\hline 0.0540233 & 0.616813 & 0.0214898 & 0.397397 & 0.0243879 & 0.435431 & 0.0379729 \\
\hline 0.04195 & 0.602195 & 0.0186244 & 0.389743 & 0.0181314 & 0.405328 & 0.0302479 \\
\hline 0.0392748 & 0.593813 & 0.0187413 & 0.36742 & 0.0169124 & 0.388454 & 0.0347714 \\
\hline 0.0410138 & 0.588403 & 0.0214799 & 0.357884 & 0.0222937 & 0.37357 & 0.0356044 \\
\hline 0.0402857 & 0.590264 & 0.0232297 & 0.353341 & 0.0178316 & 0.365094 & 0.0264946 \\
\hline 0.0382588 & 0.587836 & 0.0290248 & 0.340797 & 0.0173109 & 0.351892 & 0.024761 \\
\hline 0.0411116 & 0.574135 & 0.0223212 & 0.338911 & 0.0167027 & 0.333162 & 0.0200853 \\
\hline 0.046213 & 0.570394 & 0.0179717 & 0.331315 & 0.0208092 & 0.323021 & 0.0213067 \\
\hline 0.040953 & 0.571483 & 0.0293355 & 0.326713 & 0.0211033 & 0.320284 & 0.0267759 \\
\hline 0.0341542 & 0.572715 & 0.0252861 & 0.319795 & 0.0187405 & 0.320408 & 0.0260058 \\
\hline 0.036261 & 0.561584 & 0.0204817 & 0.32 & 0.022593 & 0.305116 & 0.0220761 \\
\hline 0.0418148 & 0.557058 & 0.0253809 & 0.309518 & 0.0194989 & 0.304268 & 0.0227584 \\
\hline
\end{tabular}




\begin{tabular}{|c|c|c|c|c|c|c|}
\hline 0.0448648 & 0.557769 & 0.02605 & 0.310902 & 0.0141982 & 0.300295 & 0.0147141 \\
\hline 0.0419151 & .555902 & 0.0165157 & 0.312904 & 0.0156141 & 0.302312 & 0.019235 \\
\hline 0.0404016 & 0.558905 & 0.0244151 & .304847 & 0.0182399 & 0.299267 & 0.0240413 \\
\hline 0399947 & .547085 & 0.0223886 & 0.30958 & 0.0160625 & 0.300842 & 0.0214179 \\
\hline 0.0352383 & 0.556616 & .0167739 & 0.297802 & 0.0157153 & 0.298879 & 0.0151275 \\
\hline 0.0444747 & 0.549672 & 0.02116 & 0.304247 & 0.019214 & 0.294814 & 0.0203473 \\
\hline 0.0384344 & 0.543581 & 0.0204405 & 0.308084 & 0.0161646 & 0.290172 & 0.0241629 \\
\hline 0.0355205 & 0.538018 & 0.0247455 & 0.299853 & 0.021649 & 0.295095 & 0.0187486 \\
\hline 0.0395421 & 0.536289 & 0.0218367 & 0.291377 & 0.015218 & 0.29595 & 0.0178175 \\
\hline 0.0357673 & 0.544097 & 0.0216103 & 0.297038 & 0.0173939 & 0.29236 & 0.0204468 \\
\hline 0.0469588 & 0.534984 & 0.0180879 & 0.298412 & 0.0198015 & 0.293354 & 0.0185378 \\
\hline 0.0432845 & 0.536754 & 0.0230375 & 0.296714 & 0.0172905 & 0.28703 & 0.0205477 \\
\hline 0.0486171 & 0.53656 & 0.021383 & 0.288338 & 0.0196591 & 0.289979 & 0.0186529 \\
\hline 0.0386798 & 0.528944 & 0.0218459 & 0.288332 & 0.0134976 & 0.285249 & 0.0205026 \\
\hline 0.0431221 & 0.528747 & 0.029477 & 0.293629 & 0.0176751 & 0.288418 & 0.020304 \\
\hline 0.0459433 & 0.531676 & 0.0214465 & 0.287515 & 0.0185891 & 0.282906 & 0.0230515 \\
\hline 0.0368743 & 0.520433 & 0.0183491 & 0.285997 & 0.0187387 & 0.286876 & 0.0192381 \\
\hline 0.0380321 & 0.532344 & 0.0212389 & 0.290984 & 0.0180403 & 0.279697 & 0.016542 \\
\hline 0.0365383 & 0.520787 & 0.0217893 & 0.287692 & 0.018 & 0.2831 & 0.01 \\
\hline 0.0383428 & 0.525189 & 0.0202209 & 0.283285 & 0.0140184 & 0.274744 & 0.0178223 \\
\hline 0.0294809 & 0.515541 & 0.022715 & 0.286854 & 0.0208952 & 0.278566 & 0.022182 \\
\hline 0.0419079 & 0.52 & 0.020392 & 0.2843 & 45 & 283437 & 458 \\
\hline 0.039431 & 0.511983 & 0.0204906 & 0.282969 & 0.0131457 & 0.273953 & 0.0168841 \\
\hline 0.0420719 & 0.504531 & 0.0225559 & 0.284537 & 0.0233943 & 0.276833 & 0.0155869 \\
\hline 0.039911 & 0.507283 & 0.0259529 & 0.278227 & 0.0144627 & 0.276987 & 0.0205825 \\
\hline 0.0354551 & 0.514708 & 0.0198623 & 0.277837 & 0.0131031 & 0.273524 & 0.0190459 \\
\hline 0.0381465 & 0.50871 & 0.0225048 & 0.276856 & 0.0177332 & 0.275546 & 0.0209097 \\
\hline 0.0341122 & 0.50433 & 0.0229726 & 0.273921 & 0.015517 & 0.275223 & 0.0201422 \\
\hline 0.0440397 & 0.503455 & 0.0233934 & 0.272743 & 0.0180769 & 0.270936 & 0.0189398 \\
\hline 0.0350447 & 0.511 & 0.021 & 0.276356 & 0.016 & 0.275417 & 982 \\
\hline 0.0404944 & 0.51034 & 0.0213928 & 0.26727 & 0.0154132 & 0.274247 & 0.0178481 \\
\hline 0.0443656 & 0.508658 & 0.0222969 & 0.268205 & 0.0164777 & 0.269555 & 0.0213876 \\
\hline 0.0359424 & 0.49691 & 0.0216432 & 0.27318 & 0.0144613 & 0.271647 & 0.0146854 \\
\hline 0.0281955 & 0.497804 & 0.0224531 & 0.276225 & 0.017228 & 0.274439 & 0.0190167 \\
\hline 0.0338622 & 0.498626 & 0.0215172 & 0.271548 & 0.0144541 & 0.270423 & 0.0207952 \\
\hline 0.0365744 & 0.49779 & 0.0249147 & 0.269326 & 0.0147475 & 0.269207 & 0.0177413 \\
\hline 0.0206119 & 0.500024 & 0.0242046 & 0.268599 & 0.0166966 & 0.268105 & 0.0179647 \\
\hline 0.0312019 & 0.496932 & 0.0169653 & 0.268487 & 0.0147575 & 0.270618 & 0.0163692 \\
\hline 0.0438715 & 0.49261 & 0.0198098 & 0.270028 & 0.0181813 & 0.26529 & 0.0201737 \\
\hline 0.0394494 & 0.489863 & 0.0222472 & 0.268349 & 0.0157069 & 0.277597 & 0.016808 \\
\hline 0.0377763 & 0.48683 & 0.0270252 & 0.264748 & 0.0176709 & 0.268625 & 0.0183295 \\
\hline 0.0346687 & 0.490114 & 0.0227698 & 0.271276 & 0.0163439 & 0.273049 & 0.0209648 \\
\hline 0.0391935 & 0.49612 & 0.0183822 & 0.26672 & 0.0144621 & 0.269919 & 0.0184228 \\
\hline 0.0496102 & 0.489784 & 0.020022 & 0.272499 & 0.016983 & 0.272763 & 0.0192906 \\
\hline 0.0356873 & 0.485948 & 0.0243208 & 0.272982 & 0.0196362 & 0.269692 & 0.0173109 \\
\hline 0.0415323 & 0.492964 & 0.0245783 & 0.26959 & 0.017414 & 0.280325 & 0.0263023 \\
\hline 0.0360291 & 0.477085 & 0.0221343 & 0.258469 & 0.0194164 & 0.286673 & 0.0207159 \\
\hline
\end{tabular}




\begin{tabular}{|c|c|c|c|c|c|c|}
\hline 0.0399994 & 0.482793 & 0.0179749 & 0.257018 & 0.0145516 & 0.271959 & 0.0167625 \\
\hline 0.0382244 & 0.477815 & 0.0207709 & 0.267558 & 0.0194075 & 0.271413 & 0.0231129 \\
\hline 0.0318982 & 0.476233 & 0.0261687 & 0.261895 & 0.0116931 & 0.278919 & 0.017669 \\
\hline 0372994 & .481425 & 0.020569 & .266175 & 0.0131232 & 0.276942 & 0.01909 \\
\hline 0.0379482 & 0.480211 & 0.0203949 & 0.261854 & 0.0174585 & 0.281627 & 0.0171868 \\
\hline 0.0403691 & 0.481538 & 0.0190014 & 0.270081 & 0.0163304 & 0.279327 & 0.0200482 \\
\hline 0.0336139 & 0.479563 & 0.019813 & 0.263765 & 0.0168103 & 0.277725 & 0.018891 \\
\hline 0.034002 & 0.472193 & 0.0174757 & 0.270792 & 0.0182967 & 0.281172 & 0.0182884 \\
\hline 0.0325003 & 0.475423 & 0.0262877 & 0.258205 & 0.0173405 & 0.278743 & 0.018992 \\
\hline 0.0324401 & 0.474708 & 0.0201931 & 0.263103 & 0.0163969 & 0.278186 & 0.0176481 \\
\hline 0.0349006 & 0.473325 & 0.0231739 & 0.264415 & 0.0136093 & 0.279324 & 0.0183133 \\
\hline 0.0392216 & 0.474827 & 0.0157827 & 0.257946 & 0.0139462 & 0.278761 & 0.0227503 \\
\hline 0.0318716 & 0.468484 & 0.0219865 & 0.260655 & 0.0176079 & 0.277522 & 0.0221045 \\
\hline 0.0453572 & 0.473354 & 0.0239037 & 0.265357 & 0.0122671 & 0.276146 & 0.0186435 \\
\hline 0.0344242 & 0.470872 & 0.0170311 & 0.261012 & 0.0150994 & 0.280223 & 0.0189235 \\
\hline 0.0345738 & 0.467145 & 0.0240564 & 0.258438 & 0.0153863 & 0.283218 & 0.0155841 \\
\hline 0.0366106 & 0.471403 & 0.0227685 & 0.265287 & 0.0173219 & 0.268833 & 0.0186138 \\
\hline 0.0290035 & 0.465356 & 0.0167021 & 0.262326 & 0.0155179 & 0.270531 & 0.0218325 \\
\hline 0.0355494 & 0.454191 & 0.0190121 & 0.2 & 0.01 & 52899 & 0.0183797 \\
\hline 0.0434815 & 0.461317 & 0.0176881 & 0.257661 & 0.0183184 & 0.265688 & 0.0177869 \\
\hline 0.0363845 & 0.459885 & 0.0181255 & 5961 & 0.0156811 & 0.260898 & 0.0145303 \\
\hline 0.0347338 & 0.4 & 304 & 03 & 0.01 & 649 & 393 \\
\hline 0.0389378 & 0.462774 & 0.0240838 & 0.257313 & 0.0132644 & 0.270725 & 0.0220085 \\
\hline 0.0327146 & 0.46286 & 0.0170108 & 0.258223 & 0.0161087 & 0.259827 & 0.0187211 \\
\hline 0.0360397 & 0.459155 & 0.0191234 & 0.256421 & 0.018986 & 0.261021 & 0.016711 \\
\hline 0.0408513 & 0.454208 & 0.0150299 & 0.253605 & 0.015782 & 0.261881 & 0.0169508 \\
\hline 0.0491888 & 0.447552 & 0.0199944 & 0.255981 & 0.0154621 & 0.260825 & 0.0207438 \\
\hline 0.0349903 & 0.452745 & 0.0218852 & 0.25401 & 0.0158756 & 0.259459 & 0.0245501 \\
\hline 0.0390549 & 0.450426 & 0.0218848 & 0.254488 & 0.0101616 & 0.256774 & 0.0175321 \\
\hline 0.0346003 & 0.447074 & 0.0151857 & 077 & 771 & 0.262632 & 0.018387 \\
\hline 0.0436333 & 0.451604 & 0.0154663 & 0.249205 & 0.016532 & 0.254295 & 0.01697 \\
\hline 0.0324173 & 0.447869 & 0.0221176 & 0.25264 & 0.0147766 & 0.249357 & 0.0153469 \\
\hline 0.036047 & 0.445657 & 0.0168379 & 0.248768 & 0.0157841 & 0.253934 & 0.019646 \\
\hline 0.0439519 & 0.443887 & 0.0197189 & 0.248142 & 0.0175681 & 0.249508 & 0.0138252 \\
\hline 0.0363799 & 0.449644 & 0.0183612 & 0.254785 & 0.0149886 & 0.247946 & 0.0158805 \\
\hline 0.0338196 & 0.445566 & 0.0208859 & 0.247596 & 0.017711 & 0.251211 & 0.0206203 \\
\hline 0.0314052 & 0.439542 & 0.0166092 & 0.246462 & 0.00994866 & 0.242652 & 0.0182082 \\
\hline 0.0438828 & 0.446119 & 0.0208477 & 0.246378 & 0.0129158 & 0.245739 & 0.0169437 \\
\hline 0.0342599 & 0.436403 & 0.0172917 & 0.246179 & 0.0164997 & 0.243385 & 0.0167754 \\
\hline 0.0342557 & 0.442726 & 0.0220037 & 0.251691 & 0.0192403 & 0.245278 & 0.0159876 \\
\hline 0.0425152 & 0.439183 & 0.0200439 & 0.245721 & 0.0145341 & 0.246308 & 0.0155484 \\
\hline 0.0285654 & 0.440211 & 0.0135956 & 0.245767 & 0.0146846 & 0.243722 & 0.0182558 \\
\hline 0.0355198 & 0.435953 & 0.0146302 & 0.248512 & 0.0177029 & 0.251354 & 0.017443 \\
\hline 0.0351915 & 0.441405 & 0.0158365 & 0.250501 & 0.0149875 & 0.245208 & 0.0198635 \\
\hline 0.0270796 & 0.436094 & 0.0189741 & 0.248537 & 0.0143796 & 0.241318 & 0.0195417 \\
\hline 0.0455328 & 0.435273 & 0.0168509 & 0.24368 & 0.014441 & 0.246712 & 0.0164668 \\
\hline 0.0389961 & 0.432771 & 0.0174915 & 0.248786 & 0.0129048 & 0.239188 & 0.0220268 \\
\hline
\end{tabular}




\begin{tabular}{|c|c|c|c|c|c|c|}
\hline 0.0416297 & 0.433166 & 0.0179146 & 0.240254 & 0.0131614 & 0.243194 & 0.0157493 \\
\hline 0.0373225 & 0.430728 & 0.0175184 & 0.245782 & 0.0152361 & 0.246277 & 0.0167773 \\
\hline 0.0274715 & 0.429069 & 0.0195657 & 0.245068 & 0.0135705 & 0.252021 & 0.019115 \\
\hline 0.0326819 & .437419 & 0.0193088 & 0.243448 & 0.0146671 & 0.242717 & 0.0201787 \\
\hline 0.038475 & 0.428302 & 0.0200783 & 0.239429 & 0.0156483 & 0.240162 & 0.0170537 \\
\hline 0.033838 & 0.427432 & 0.0179746 & 0.241428 & 0.0140709 & 0.239273 & 0.0124491 \\
\hline 0.0386222 & 0.433987 & 0.0115112 & 0.236101 & 0.0137512 & 0.238551 & 0.0168564 \\
\hline 0.0472658 & 0.429551 & 0.0174449 & 0.24119 & 0.0128769 & 0.240909 & 0.0177575 \\
\hline 0.0298682 & 0.427399 & 0.0179721 & 0.244653 & 0.0193901 & 0.240026 & 0.0162068 \\
\hline 0.0369498 & 0.424716 & 0.0166305 & 0.23566 & 0.017091 & 0.237969 & 0.0179128 \\
\hline 0.0423897 & 0.423655 & 0.0172403 & 0.241492 & 0.0142742 & 0.241434 & 0.0184477 \\
\hline 0.0346627 & 0.422788 & 0.0260153 & 0.24092 & 0.0160347 & 0.24213 & 0.0186156 \\
\hline 0.0434039 & 0.426262 & 0.0194732 & 0.240367 & 0.0172558 & 0.2373 & 0.0147779 \\
\hline 0.0316453 & 0.422864 & 0.0206504 & 0.235397 & 0.0145531 & 0.243111 & 0.020314 \\
\hline 0.0313298 & 0.416766 & 0.013355 & 0.244711 & 0.0148191 & 0.241291 & 0.0168781 \\
\hline 0.0397436 & 0.418768 & 0.0179042 & 0.233287 & 0.0140818 & 0.236018 & 0.0164308 \\
\hline 0.0348478 & 0.424208 & 0.0124565 & 0.238637 & 0.0213324 & 0.235243 & 0.0178082 \\
\hline 0.0322181 & 0.42154 & 0.0181256 & 0.241361 & 0.0224114 & 0.24453 & 0.01 \\
\hline 0.03 & 0.419797 & 0.0171931 & 081 & 0.0185817 & 114 & 502 \\
\hline 0.0338597 & 0.414411 & 0.0122228 & 0.236947 & 0.0177449 & 0.235501 & 0.0179089 \\
\hline 0.0427373 & 0.416822 & 0.0142669 & 0.241412 & 0.0149486 & 0.234452 & 0.0194229 \\
\hline 0.033264 & 0.422664 & 0.01 & 442 & 0.0155749 & 748 & 739 \\
\hline 0.0344603 & 0.416331 & 0.0194614 & 0.238081 & 0.0168544 & 0.235255 & 0.0169395 \\
\hline 0.0394936 & 0.419402 & 0.0189437 & 0.236341 & 0.0160139 & 0.23881 & 0.0175817 \\
\hline 0.0385123 & 0.417415 & 0.0191749 & 0.239817 & 0.014286 & 0.237645 & 0.0179959 \\
\hline 0.0359626 & 0.412125 & 0.0152211 & 0.233679 & 0.0115277 & 0.235661 & 0.0160781 \\
\hline 0.0313752 & 0.419755 & 0.0160282 & 0.235001 & 0.0131759 & 0.243434 & 0.0232771 \\
\hline 0.0297175 & 0.421599 & 0.0189616 & 0.237909 & 0.0197348 & 0.231824 & 0.0174158 \\
\hline 0.0315153 & 0.42267 & 0.0223296 & 0.243036 & 0.0159872 & 0.236164 & 0.0181435 \\
\hline 0.0352495 & 0.416733 & 0.0175163 & & 0.01 & & 893 \\
\hline 0.0433127 & 0.416327 & 0.0177315 & 0.235454 & 0.0114623 & 0.239167 & 0.017945 \\
\hline 0.0354092 & 0.417398 & 0.0193892 & 0.236777 & 0.0132609 & 0.241086 & 0.0180503 \\
\hline 0.0270859 & 0.423137 & 0.0140097 & 0.238967 & 0.0107688 & 0.244887 & 0.0196256 \\
\hline 0.0367421 & 0.416384 & 0.0139708 & 0.24475 & 0.0156334 & 0.241105 & 0.0188978 \\
\hline 0.0346756 & 0.416706 & 0.0203707 & 0.244787 & 0.0149402 & 0.237215 & 0.0142422 \\
\hline 0.0387486 & 0.416952 & 0.0187537 & 0.246291 & 0.0174945 & 0.234962 & 0.0170258 \\
\hline 0.0411288 & 0.41525 & 0.0159114 & 0.246176 & 0.015149 & 0.249713 & 0.0182447 \\
\hline 0.0344498 & 0.417493 & 0.019311 & 0.242911 & 0.0125316 & 0.243127 & 0.017051 \\
\hline 0.0362179 & 0.419527 & 0.0166981 & 0.239863 & 0.0157595 & 0.246516 & 0.0171495 \\
\hline 0.0295133 & 0.419275 & 0.0196602 & 0.245013 & 0.014245 & 0.244413 & 0.0193676 \\
\hline 0.0287029 & 0.424448 & 0.0123149 & 0.246666 & 0.0175636 & 0.244709 & 0.0172198 \\
\hline 0.0397375 & 0.42318 & 0.0136604 & 0.245794 & 0.0205397 & 0.245031 & 0.0147768 \\
\hline 0.0376683 & 0.419833 & 0.0158042 & 0.249554 & 0.0115385 & 0.246643 & 0.0212603 \\
\hline 0.0296935 & 0.421532 & 0.0172049 & 0.24718 & 0.0139153 & 0.248771 & 0.0168964 \\
\hline 0.0346275 & 0.419851 & 0.0146768 & 0.246465 & 0.0177814 & 0.256126 & 0.0207353 \\
\hline 0.0389252 & 0.415456 & 0.0159521 & 0.249726 & 0.0147597 & 0.2492 & 0.0189501 \\
\hline 0.0345616 & 0.412278 & 0.0170378 & 0.245706 & 0.0142602 & 0.250508 & 0.0201534 \\
\hline
\end{tabular}




\begin{tabular}{|c|c|c|c|c|c|c|}
\hline 0.0366429 & 0.41553 & 0.0165187 & 0.254095 & 0.0149798 & 0.25509 & 0.0203739 \\
\hline 0.0414786 & 0.417036 & 0.0159911 & 0.259145 & 0.0165377 & 0.255337 & 0.0160383 \\
\hline 0.0346457 & 0.416898 & 0.0154961 & 0.255001 & 0.0188547 & 0.259704 & 0.0186095 \\
\hline 0461404 & 0.41838 & 0.0175184 & 0.251447 & 0.0159876 & 0.263762 & 0.0222539 \\
\hline 0.0321862 & 0.418841 & 0.0143975 & 0.251598 & 0.0133169 & 0.262454 & 0.018963 \\
\hline 0.0300424 & 0.420267 & 0.0155532 & 0.25341 & 0.0153976 & 0.263948 & 0.0195644 \\
\hline 0.041793 & 0.415252 & 0.018078 & 0.256249 & 0.0140649 & 0.273994 & 0.0187149 \\
\hline 0.0370777 & 0.419197 & 0.013021 & 0.252839 & 0.0173876 & 0.272726 & 0.0216376 \\
\hline 0.0330266 & 0.420535 & 0.0144428 & 0.251188 & 0.0146294 & 0.269874 & 0.021699 \\
\hline 0.0262478 & 0.420393 & 0.0162391 & 0.259402 & 0.0156704 & 0.27934 & 0.0248237 \\
\hline 0.0424888 & 0.416072 & 0.0163563 & 0.256676 & 0.0153439 & 0.280738 & 0.0217083 \\
\hline 0.0354099 & 0.412331 & 0.0147233 & 0.256174 & 0.0138372 & 0.292204 & 0.0247353 \\
\hline 0.0389657 & 0.417238 & 0.0179344 & 0.255878 & 0.0155225 & 0.292495 & 0.0252661 \\
\hline 0.0341831 & 0.414896 & 0.0164641 & 0.256793 & 0.0134732 & 0.29322 & 0.0294909 \\
\hline 0.0330551 & 0.423781 & 0.0186681 & 0.259334 & 0.0166146 & 0.297913 & 0.0235727 \\
\hline 0.0357706 & 0.421632 & 0.0180672 & 0.259344 & 0.0144451 & 0.302368 & 0.0240532 \\
\hline 0.0361021 & 0.416106 & 0.0152429 & 0.270644 & 0.0177375 & 0.310848 & 0.0282386 \\
\hline 0.0381735 & 0.416957 & 0.0185382 & 0.25617 & 0.0151141 & 0.314342 & 0.0188869 \\
\hline 0.0334017 & 0.415034 & 0.0144833 & 327 & 0.0 & 89 & 0.0300223 \\
\hline 0.0283496 & 0.412605 & 0.0129988 & 0.263001 & 0.0174547 & 0.313103 & 0.0337054 \\
\hline 0.0457688 & 0.412453 & 0.0175376 & 0.260936 & 0.0129992 & 0.310941 & 0.0307936 \\
\hline 0.0398106 & 0.41 & 347 & 526 & 0.01 & 64 & 738 \\
\hline 0.0449681 & 0.413886 & 0.012134 & 0.262811 & 0.0178237 & 0.314604 & 0.0288104 \\
\hline 0.0428704 & 0.408409 & 0.0125936 & 0.263617 & 0.0167188 & 0.317571 & 0.0326987 \\
\hline 0.0393857 & 0.408273 & 0.0145721 & 0.264979 & 0.015477 & 0.310722 & 0.0256027 \\
\hline 0.037476 & 0.407172 & 0.0151952 & 0.262813 & 0.0173756 & 0.312554 & 0.0355438 \\
\hline 0.0358508 & 0.407262 & 0.0197233 & 0.260321 & 0.0167814 & 0.303266 & 0.0256318 \\
\hline 0.0364007 & 0.403747 & 0.013149 & 0.261773 & 0.0141852 & 0.300111 & 0.0252843 \\
\hline 0.035492 & 0.401483 & 0.0151225 & 0.256079 & 0.0163401 & 0.299576 & 0.0242542 \\
\hline 0.0369888 & 0.402863 & 0.0170897 & 0.260762 & 0.0170128 & 0.296168 & 0.0252723 \\
\hline 0.0307645 & 0.402401 & 0.0194179 & 0.262478 & 0.0172493 & 0.292766 & 0.0199751 \\
\hline 0.0395873 & 0.401665 & 0.016253 & 0.257007 & 0.016432 & 0.290819 & 0.0316359 \\
\hline 0.0420003 & 0.398453 & 0.0154712 & 0.254034 & 0.0123125 & 0.282391 & 0.0223119 \\
\hline 0.0277698 & 0.390333 & 0.0138211 & 0.249715 & 0.0152955 & 0.276785 & 0.0249936 \\
\hline 0.0307725 & 0.393466 & 0.0128197 & 0.247372 & 0.0185381 & 0.267664 & 0.0220174 \\
\hline 0.0319938 & 0.394308 & 0.0151051 & 0.246247 & 0.0150065 & 0.268985 & 0.024277 \\
\hline 0.0330772 & 0.392272 & 0.013335 & 0.25404 & 0.0174201 & 0.271682 & 0.0189224 \\
\hline 0.0359896 & 0.391373 & 0.0133689 & 0.252699 & 0.0171021 & 0.265604 & 0.0164757 \\
\hline 0.0361928 & 0.39216 & 0.0103582 & 0.252019 & 0.0145797 & 0.262246 & 0.0178199 \\
\hline 0.0377474 & 0.390431 & 0.0130987 & 0.252723 & 0.0146483 & 0.258747 & 0.0162847 \\
\hline 0.0381546 & 0.391202 & 0.0163059 & 0.253397 & 0.0161954 & 0.24736 & 0.0168355 \\
\hline 0.0341811 & 0.389374 & 0.0160497 & 0.247749 & 0.0125935 & 0.254858 & 0.0187629 \\
\hline 0.0287212 & 0.391476 & 0.0175517 & 0.246892 & 0.017383 & 0.260245 & 0.0245282 \\
\hline 0.0346388 & 0.399185 & 0.0107967 & 0.242999 & 0.0155415 & 0.260838 & 0.0206307 \\
\hline 0.035162 & 0.3874 & 0.012002 & 0.242197 & 0.0150966 & 0.261973 & 0.0176447 \\
\hline 0.0261867 & 0.388429 & 0.0134618 & 0.247891 & 0.0140523 & 0.257529 & 0.0181638 \\
\hline 0.0361893 & 0.388309 & 0.0121326 & 0.249143 & 0.014907 & 0.256182 & 0.0230698 \\
\hline
\end{tabular}




\begin{tabular}{|c|c|c|c|c|c|c|}
\hline 0.0347512 & 0.389598 & 0.015028 & 0.252357 & 0.0164369 & 0.260373 & 0.0218091 \\
\hline 0.041671 & .390695 & 0.0147185 & 0.247623 & 0.011059 & 0.26149 & 0.0169612 \\
\hline 0.0371317 & 0.3924 & 0.0135137 & 0.251607 & 0.0173483 & 0.259441 & 0.0190671 \\
\hline 0.0292654 & .390996 & 0.0123391 & 0.248925 & 0.017209 & 0.261915 & 0.0213596 \\
\hline 0.0324212 & 389741 & 0.010756 & 0.24903 & 0.0162515 & 0.258016 & 0.0179402 \\
\hline 0.0485707 & 0.393148 & 0.017555 & 0.253955 & 0.0161479 & 0.256512 & 0.0181962 \\
\hline 0.0329451 & 0.389643 & 0.0181227 & 0.256247 & 0.0165983 & 0.26519 & 0.0220422 \\
\hline 0.0340001 & 0.387549 & 0.0150368 & 0.250628 & 0.0143536 & 0.264771 & 0.0193533 \\
\hline 0.0406995 & 0.390098 & 0.0165614 & 0.250244 & 0.0182757 & 0.263623 & 0.0168542 \\
\hline 0.0419439 & 0.391317 & 0.018234 & 0.248506 & 0.0141684 & 0.261164 & 0.0173376 \\
\hline 0.0356507 & 0.391253 & 0.0114351 & 0.253695 & 0.0113784 & 0.269301 & 0.0195349 \\
\hline 0.0348849 & 0.395612 & 0.0155457 & 0.260104 & 0.0125173 & 0.265301 & 0.0161759 \\
\hline 0.0323531 & 0.395965 & 0.0148675 & 0.25632 & 0.017904 & 0.268364 & 0.0194802 \\
\hline 0.0367166 & 0.393924 & 0.0142721 & 0.255489 & 0.0125374 & 0.264683 & 0.0251788 \\
\hline 0.0286205 & 0.393323 & 0.0117365 & 0.255014 & 0.0192305 & 0.263516 & 0.0165632 \\
\hline 0.0337605 & 0.392257 & 0.0158998 & 0.259916 & 0.0133176 & 0.272508 & 0.0221598 \\
\hline 0.0411414 & 0.39805 & 0.0184206 & 0.258963 & 0.0139807 & 0.263056 & 0.0160776 \\
\hline 0.0319093 & 0.399477 & 0.0138977 & 0.260356 & 0.0131017 & 0.258639 & 0.0179728 \\
\hline 0.033852 & 0.393985 & 0.0136109 & 24 & 0.0124227 & 0.27 & 4964 \\
\hline 0.0327059 & 0.396113 & 0.015441 & 0.261164 & 0.0117176 & 0.267759 & 0.0212574 \\
\hline 0.0329393 & 0.401479 & 0.0173921 & 0.269363 & 0.0171357 & 0.268982 & 0.0200847 \\
\hline 0.028204 & 40246 & 0.0141212 & 59 & 0.015 & 92 & 3944 \\
\hline 0.0355349 & 0.396777 & 0.015397 & 0.263926 & 0.0154669 & 0.265845 & 0.0194711 \\
\hline 0.0235923 & 0.402671 & 0.0185724 & 0.270886 & 0.0173917 & 0.279744 & 0.0202696 \\
\hline 0.0388001 & 0.403811 & 0.0143848 & 0.266135 & 0.0136081 & 0.272299 & 0.0193847 \\
\hline 0.0342074 & 0.406873 & 0.0140398 & 0.265204 & 0.0160464 & 0.277598 & 0.0168999 \\
\hline 0.0308666 & 0.404387 & 0.020345 & 0.270961 & 0.0178563 & 0.277412 & 0.0195002 \\
\hline 0.0312072 & 0.408715 & 0.014211 & 0.272486 & 0.0138924 & 0.281272 & 0.0180937 \\
\hline 0.0445887 & 0.413484 & 0.0173323 & 0.276873 & 0.019943 & 0.2747 & 0.0175223 \\
\hline 0.0389423 & 0.416411 & 0.0137442 & 0.279181 & 0.0159222 & 921 & 0.0177627 \\
\hline 0.0444246 & 0.416918 & 0.0143402 & 0.275219 & 0.0161122 & 0.285841 & 0.0196293 \\
\hline 0.0240386 & 0.417583 & 0.0146087 & 0.275876 & 0.0181036 & 0.293176 & 0.0176219 \\
\hline 0.0439062 & 0.417305 & 0.0178338 & 0.276442 & 0.0188613 & 0.285674 & 0.0202713 \\
\hline 0.0346897 & 0.421159 & 0.0193026 & 0.285536 & 0.0172213 & 0.2912 & 0.0205852 \\
\hline 0.0382366 & 0.425309 & 0.0146197 & 0.287387 & 0.0118997 & 0.287358 & 0.0178015 \\
\hline 0.0326898 & 0.423145 & 0.0145366 & 0.2891 & 0.0118113 & 0.297056 & 0.0214003 \\
\hline 0.040071 & 0.425518 & 0.0136378 & 0.283936 & 0.0146948 & 0.297397 & 0.0222132 \\
\hline 0.0345933 & 0.426004 & 0.0164048 & 0.29168 & 0.0185203 & 0.304643 & 0.0208435 \\
\hline 0.0363562 & 0.430601 & 0.0176897 & 0.292157 & 0.0160708 & 0.305203 & 0.0239154 \\
\hline 0.0369077 & 0.437366 & 0.0141739 & 0.298291 & 0.01646 & 0.309319 & 0.0194727 \\
\hline 0.0381477 & 0.440396 & 0.0167175 & 0.294288 & 0.017727 & 0.312765 & 0.0201476 \\
\hline 0.0364585 & 0.444573 & 0.0189691 & 0.311851 & 0.0152371 & 0.307971 & 0.020459 \\
\hline 0.0425807 & 0.448332 & 0.0176173 & 0.308453 & 0.0153966 & 0.319724 & 0.0196662 \\
\hline 0.0344847 & 0.454416 & 0.0155608 & 0.31468 & 0.0188084 & 0.323874 & 0.0245532 \\
\hline 0.0385776 & 0.460929 & 0.0153041 & 0.312455 & 0.0243675 & 0.334231 & 0.0262079 \\
\hline 0.0369052 & 0.465225 & 0.0143314 & 0.314506 & 0.0172435 & 0.345327 & 0.0255198 \\
\hline 0.0352727 & 0.470955 & 0.0174713 & 0.322637 & 0.0153639 & 0.335443 & 0.0224756 \\
\hline
\end{tabular}




\begin{tabular}{|c|c|c|c|c|c|c|}
\hline 0.0396855 & 0.471109 & 0.0161128 & 0.324539 & 0.017481 & 0.341925 & 0.0202469 \\
\hline 0.0459344 & 0.479158 & 0.014111 & 0.331857 & 0.0193889 & 0.35012 & 0.0193457 \\
\hline 0.0383598 & 0.484454 & 0.0179686 & 0.337397 & 0.0167927 & 0.353456 & 0.0228626 \\
\hline 0.0421719 & .489257 & 0.0167941 & 0.339259 & 0.0190265 & 0.358608 & 0.0180711 \\
\hline 0.03751 & 0.498705 & 0.0172208 & 0.34538 & 0.0253614 & 0.365806 & 0.0195915 \\
\hline 0.044368 & 0.508893 & 0.013581 & 0.344521 & 0.0163891 & 0.365603 & 0.0242904 \\
\hline 0.048557 & 0.518629 & 0.0157543 & 0.350572 & 0.0179242 & 0.381997 & 0.0281026 \\
\hline 0.0374921 & 0.523263 & 0.0166316 & 0.36561 & 0.019196 & 0.387923 & 0.0301186 \\
\hline 0.0505527 & 0.530342 & 0.02019 & 0.36597 & 0.0196331 & 0.392311 & 0.0193866 \\
\hline 0.0388099 & 0.53919 & 0.0176756 & 0.37659 & 0.0179669 & 0.404557 & 0.0203034 \\
\hline 0.0390637 & 0.547247 & 0.015956 & 0.381241 & 0.0181212 & 0.418569 & 0.0282249 \\
\hline 0.0397629 & 0.55789 & 0.0186256 & 0.390789 & 0.0169445 & 0.426584 & 0.0230505 \\
\hline 0.047739 & 0.572542 & 0.0191619 & 0.403404 & 0.0227726 & 0.427528 & 0.0238725 \\
\hline 0.0408826 & 0.577282 & 0.0178193 & 0.410035 & 0.0180107 & 0.440122 & 0.0221292 \\
\hline 0.0419429 & 0.593078 & 0.0190404 & 0.41881 & 0.0208276 & 0.448514 & 0.0267911 \\
\hline 0.0407602 & 0.610323 & 0.0187357 & 0.425859 & 0.0187285 & 0.452033 & 0.0267201 \\
\hline 0.0445503 & 0.615465 & 0.0210464 & 0.432829 & 0.0187172 & 0.467269 & 0.0266842 \\
\hline 0.0446157 & 0.625962 & 0.0230385 & 0.45063 & 0.025 & 0.478266 & 0.0250656 \\
\hline 0.0531105 & 834 & 0.0229899 & 0.45 & 0.02 & 0.493231 & 0.032 \\
\hline 0.0435292 & 0.651341 & 0.0167815 & 0.47026 & 0.0188701 & 0.508898 & 0.0240535 \\
\hline 0.0518816 & 0.666004 & 0.0187523 & 0.480667 & 0.0224866 & 0.51834 & 0.0264792 \\
\hline 0.0616761 & 43 & 0.0208961 & 17 & 0.02 & 0.524782 & 185 \\
\hline 0.0486968 & 0.700541 & 0.0212243 & 0.507863 & 0.0233678 & 0.54801 & 0.0339522 \\
\hline 0.0501058 & 0.712904 & 0.0269648 & 0.513147 & 0.0274151 & 0.555662 & 0.027912 \\
\hline 0.0517564 & 0.728546 & 0.0193262 & 0.524424 & 0.0281981 & 0.580081 & 0.0313744 \\
\hline 0.0407645 & 0.746362 & 0.0202056 & 0.554655 & 0.0266867 & 0.572858 & 0.0269076 \\
\hline 0.0551209 & 0.755085 & 0.0203742 & 0.565292 & 0.0229142 & 0.59868 & 0.031674 \\
\hline 0.0560542 & 0.766122 & 0.0234326 & 0.578651 & 0.0280626 & 0.626534 & 0.0304952 \\
\hline 0.0490676 & 0.782113 & 0.0263525 & 0.586925 & 0.0252837 & 0.633774 & 0.0308626 \\
\hline 0.0548789 & & 0.0269 & & 0.02 & 446 & 0.0422688 \\
\hline 0.0492161 & 0.809389 & 0.0256855 & 0.625527 & 0.0259288 & 0.662398 & 0.0279641 \\
\hline 0.036757 & 0.824901 & 0.0195607 & 0.641372 & 0.0267596 & 0.677676 & 0.0308556 \\
\hline 0.0535237 & 0.843292 & 0.0231534 & 0.657285 & 0.0266259 & 0.70034 & 0.0338806 \\
\hline 0.0514744 & 0.854862 & 0.0192724 & 0.685383 & 0.0271961 & 0.71128 & 0.0281962 \\
\hline 0.0613098 & 0.867851 & 0.0202639 & 0.686731 & 0.0233361 & 0.736713 & 0.0351319 \\
\hline 0.0630335 & 0.876502 & 0.0221038 & 0.722028 & 0.0242996 & 0.74652 & 0.03479 \\
\hline 0.0620185 & 0.886373 & 0.0230033 & 0.719338 & 0.0326287 & 0.753571 & 0.0376029 \\
\hline 0.0631364 & 0.904726 & 0.0207525 & 0.730561 & 0.0274392 & 0.773043 & 0.0377463 \\
\hline 0.0530376 & 0.913157 & 0.0227166 & 0.759976 & 0.0297125 & 0.792554 & 0.0333439 \\
\hline 0.046779 & 0.923297 & 0.0223315 & 0.773144 & 0.0290197 & 0.805731 & 0.0382373 \\
\hline 0.0524637 & 0.925443 & 0.0226282 & 0.792968 & 0.0268663 & 0.815342 & 0.0325052 \\
\hline 0.0609754 & 0.938672 & 0.0213403 & 0.802554 & 0.0301176 & 0.835288 & 0.032294 \\
\hline 0.0679108 & 0.951694 & 0.0267806 & 0.812878 & 0.0196902 & 0.850343 & 0.0390061 \\
\hline 0.0567089 & 0.956644 & 0.0216653 & 0.831944 & 0.026491 & 0.85567 & 0.0381103 \\
\hline 0.0742667 & 0.95806 & 0.0169288 & 0.848887 & 0.0353537 & 0.866599 & 0.0321775 \\
\hline 0.0638075 & 0.968851 & 0.0198667 & 0.855637 & 0.0295533 & 0.876796 & 0.0401276 \\
\hline 0.0689664 & 0.974993 & 0.015953 & 0.869656 & 0.0272496 & 0.892621 & 0.0447493 \\
\hline
\end{tabular}




\begin{tabular}{|c|c|c|c|c|c|c|}
\hline 0.0628893 & 0.975755 & 0.0169561 & 0.884752 & 0.0265174 & 0.914257 & 0.0451445 \\
\hline 0.0597283 & .972147 & 0.0200548 & 0.89988 & 0.0259811 & 0.902435 & 0.0364524 \\
\hline 0.0629695 & 0.971667 & 0.0202804 & 0.907697 & 0.0243486 & 0.929067 & 0.032946 \\
\hline .0496599 & .968315 & 0.0199656 & 0.910855 & 0.0286247 & 0.92174 & 0.0400309 \\
\hline .0580825 & .971818 & 0.0207719 & 0.926247 & 0.025498 & 0.917314 & 0.0327214 \\
\hline 0.05814 & 0.968725 & 0.0246105 & 0.936096 & 0.0296694 & 0.943165 & 0.0464089 \\
\hline 0.0648555 & 0.966367 & 0.0266052 & 0.94382 & 0.031541 & 0.949431 & 0.0353945 \\
\hline 0.0588387 & 0.967878 & 0.0218142 & 0.938864 & 0.0305535 & 0.943408 & 0.035188 \\
\hline 0.0548317 & 0.957383 & 0.020135 & 0.949195 & 0.0301632 & 0.939789 & 0.0363271 \\
\hline 0.0802022 & 0.948779 & 0.0239552 & 0.94917 & 0.0289116 & 0.947006 & 0.0325766 \\
\hline 0.0472783 & 0.940516 & 0.021491 & 0.956894 & 0.0325299 & 0.944213 & 0.0316117 \\
\hline 0.0576431 & 0.933683 & 0.021626 & 0.953866 & 0.0338575 & 0.947977 & 0.0411948 \\
\hline 0.083169 & 0.933143 & 0.0235243 & 0.953535 & 0.0304989 & 0.939525 & 0.0333223 \\
\hline 0.0585421 & 0.924452 & 0.0234001 & 0.949436 & 0.0261882 & 0.932703 & 0.040192 \\
\hline 0.0601066 & 0.912761 & 0.0270636 & 0.934665 & 0.0348068 & 0.933639 & 0.0412737 \\
\hline 0.0680318 & 0.900055 & 0.0240331 & 0.943739 & 0.0320082 & 0.925112 & 0.0404035 \\
\hline 0.0599696 & 0.890455 & 0.0195784 & 0.946967 & 0.0333088 & 0.921067 & 0.0401594 \\
\hline 0.0536944 & 0.873049 & 0.0199216 & 0.939268 & 0.032976 & 0.902638 & 0.0387432 \\
\hline 0.055433 & 0.86 & 0.017567 & 0.931257 & 0.0282739 & 0.90 & 0.0456721 \\
\hline 0.0571688 & 0.848362 & 0.0239047 & 0.923999 & 0.0286709 & 0.894608 & 0.0461311 \\
\hline 0.0595534 & 0.83491 & 0.0209001 & 0.907968 & 0.0263509 & 0.887993 & 0.0435281 \\
\hline 0.0662731 & 0.82 & 0.0237178 & 0.89 & 0.0342898 & 331 & 749 \\
\hline 0.0639529 & 0.810684 & 0.0238247 & 0.893278 & 0.0308382 & 0.859483 & 0.0418677 \\
\hline 0.0431788 & 0.791541 & 0.0258565 & 0.881777 & 0.0358725 & 0.841776 & 0.0264098 \\
\hline 0.0567606 & 0.776719 & 0.0272842 & 0.863987 & 0.0299888 & 0.832202 & 0.024421 \\
\hline 0.0502742 & 0.766121 & 0.0198046 & 0.85004 & 0.0250093 & 0.819982 & 0.0293084 \\
\hline 0.0721674 & 0.751121 & 0.0158127 & 0.835149 & 0.0321872 & 0.805622 & 0.0327737 \\
\hline 0.0606053 & 0.735851 & 0.0195358 & 0.819828 & 0.0202368 & 0.793141 & 0.0303243 \\
\hline 0.063327 & 0.71986 & 0.0222635 & 0.810727 & 0.026333 & 0.764662 & 0.0284328 \\
\hline 0.0693294 & 0.702482 & 0.0182763 & 0.790815 & 0.0353506 & 0.757591 & 0.0373221 \\
\hline 0.0673099 & 0.684332 & 0.0178206 & 0.782704 & 0.0292132 & 0.736426 & 0.0277104 \\
\hline 0.0724869 & 0.667626 & 0.0212963 & 0.758544 & 0.0269977 & 0.724528 & 0.0324112 \\
\hline 0.0678885 & 0.653423 & 0.0233952 & 0.752536 & 0.0285852 & 0.712149 & 0.0401421 \\
\hline 0.0582169 & 0.638065 & 0.0227133 & 0.731379 & 0.0268082 & 0.698303 & 0.0306327 \\
\hline 0.0553115 & 0.618278 & 0.0201146 & 0.71607 & 0.0259126 & 0.679432 & 0.0295201 \\
\hline 0.0765005 & 0.604878 & 0.0166912 & 0.696106 & 0.0215413 & 0.656409 & 0.0327598 \\
\hline 0.0658757 & 0.592379 & 0.0188488 & 0.688606 & 0.0261793 & 0.63387 & 0.0232882 \\
\hline 0.0723515 & 0.580488 & 0.0229229 & 0.663628 & 0.0308037 & 0.619202 & 0.0290145 \\
\hline 0.0521904 & 0.566796 & 0.0231919 & 0.646129 & 0.0264734 & 0.611352 & 0.0320327 \\
\hline 0.0681785 & 0.554433 & 0.0219174 & 0.626391 & 0.0254521 & 0.587574 & 0.030118 \\
\hline 0.0587321 & 0.539603 & 0.018659 & 0.620975 & 0.0296443 & 0.577475 & 0.0331435 \\
\hline 0.0546033 & 0.529373 & 0.0166572 & 0.592926 & 0.0175485 & 0.569084 & 0.0233754 \\
\hline 0.0653275 & 0.523387 & 0.0156105 & 0.573961 & 0.0204951 & 0.542699 & 0.0236894 \\
\hline 0.0458256 & 0.505531 & 0.0170759 & 0.557676 & 0.0238973 & 0.530406 & 0.0288133 \\
\hline 0.0483486 & 0.490913 & 0.0175536 & 0.547483 & 0.0229567 & 0.51951 & 0.0267812 \\
\hline 0.055513 & 0.484832 & 0.0169598 & 0.5286 & 0.0216894 & 0.499925 & 0.0232159 \\
\hline 0.0463638 & 0.475742 & 0.018815 & 0.515107 & 0.024656 & 0.488914 & 0.0221391 \\
\hline
\end{tabular}




\begin{tabular}{|c|c|c|c|c|c|c|}
\hline 0.0546257 & 0.464394 & 0.0184341 & 0.502353 & 0.0195272 & 0.469276 & 0.0287758 \\
\hline 0.0592901 & 0.453569 & 0.0160815 & 0.482675 & 0.0256678 & 0.468766 & 0.0280096 \\
\hline 0.0387106 & 0.444288 & 0.0126846 & .477908 & 0.0195175 & 0.448609 & 0.0252795 \\
\hline 0533253 & 0.432365 & 0.0145221 & .464131 & 0.0231387 & 0.436 & 0.02719 \\
\hline 0.0458121 & 0.41953 & 0.0148983 & 0.449414 & 0.0209002 & 0.428887 & 0.0215529 \\
\hline 0.0583217 & 0.414076 & 0.0124657 & 0.438765 & 0.0293498 & 0.413865 & 0.0225472 \\
\hline 0.0542186 & 0.405982 & 0.0133523 & 0.420779 & 0.0210994 & 0.402422 & 0.0333723 \\
\hline 0.0453495 & 0.396132 & 0.0159635 & 0.411186 & 0.0164323 & 0.402276 & 0.0246522 \\
\hline 0.041335 & 0.39163 & 0.0134076 & 0.404861 & 0.0179353 & 0.38989 & 0.0240087 \\
\hline 0.0517301 & 0.386767 & 0.0115846 & 0.398061 & 0.0188258 & 0.380071 & 0.0179707 \\
\hline 0.0539654 & 0.378732 & 0.0101309 & 0.382772 & 0.0190948 & 0.365584 & 0.0237639 \\
\hline 0.0370652 & 0.375071 & 0.0103774 & 0.374549 & 0.0231174 & 0.349952 & 0.0198644 \\
\hline 0.0388154 & 0.370044 & 0.0106426 & 0.355247 & 0.0193805 & 0.345211 & 0.0192353 \\
\hline 0.0407964 & 0.360159 & 0.0105497 & 0.357702 & 0.0163879 & 0.33516 & 0.0214502 \\
\hline 0.0365464 & 0.35249 & 0.0149426 & 0.341413 & 0.0154753 & 0.33309 & 0.0175944 \\
\hline 0.0333821 & 0.348444 & 0.0117632 & 0.337006 & 0.0130727 & 0.323752 & 0.0155544 \\
\hline 0.0411668 & 0.344128 & 0.0109243 & 0.330269 & 0.0148596 & 0.315594 & 0.0276043 \\
\hline 0.0395034 & 0.338532 & 0.0115371 & 0.319989 & 0.0161723 & 0.316969 & 0.0231928 \\
\hline 0.0411823 & 0.33 & 0.015158 & 44 & 0.0134432 & & 5106 \\
\hline 0.0335574 & 0.330677 & 0.0102832 & 0.313477 & 0.0184671 & 0.28999 & 0.0176235 \\
\hline 0.0460177 & 0.324907 & 0.0112658 & 0.294548 & 0.018357 & 0.292934 & 0.022905 \\
\hline 0.036022 & 0.31 & 0.0162722 & 95 & 0.015 & 57 & 452 \\
\hline 0.03925 & 0.309706 & 0.0137571 & 0.286219 & 0.0162968 & 0.276215 & 0.0140843 \\
\hline 0.0322716 & 0.308114 & 0.0107267 & 0.288027 & 0.0104409 & 0.276982 & 0.0229223 \\
\hline 0.0346414 & 0.305957 & 0.0101654 & 0.272664 & 0.0132386 & 0.268775 & 0.0200901 \\
\hline 0.0411858 & 0.301387 & 0.0131925 & 0.270745 & 0.0129937 & 0.263251 & 0.0215282 \\
\hline 0.0306591 & 0.295343 & 0.0142065 & 0.260436 & 0.0178948 & 0.260384 & 0.0204678 \\
\hline 0.0268385 & 0.294584 & 0.0101172 & 0.259895 & 0.012901 & 0.258867 & 0.019892 \\
\hline 0.0381088 & 0.290623 & 0.00818192 & 0.260199 & 0.0172787 & 0.246676 & 0.0210143 \\
\hline 0.0348602 & 0.284869 & 0.0114583 & & 0.0171978 & 24311 & 0.0172687 \\
\hline 0.0361705 & 0.283231 & 0.0116043 & 0.24 & 0.0167303 & 0.239289 & 0.0185594 \\
\hline 0.0378473 & 0.276476 & 0.0127805 & 0.239952 & 0.0140873 & 0.237602 & 0.0154251 \\
\hline 0.0347298 & 0.272525 & 0.0139374 & 0.232611 & 0.0140705 & 0.227968 & 0.0157703 \\
\hline 0.0306058 & 0.269772 & 0.0107791 & 0.231221 & 0.0105265 & 0.229418 & 0.0125239 \\
\hline 0.0382161 & 0.267669 & 0.0107551 & 0.225468 & 0.0136278 & 0.221845 & 0.0180972 \\
\hline 0.0271581 & 0.265296 & 0.0109784 & 0.223545 & 0.0145706 & 0.221429 & 0.0155388 \\
\hline 0.0356827 & 0.261597 & 0.010477 & 0.216211 & 0.0155012 & 0.216135 & 0.0153627 \\
\hline 0.0326509 & 0.261201 & 0.0132117 & 0.213982 & 0.014128 & 0.212964 & 0.0146097 \\
\hline 0.0322201 & 0.259833 & 0.010473 & 0.210096 & 0.010217 & 0.207638 & 0.0196462 \\
\hline 0.0311154 & 0.255473 & 0.00976098 & 0.210227 & 0.0112513 & 0.209606 & 0.0124068 \\
\hline 0.0378099 & 0.25492 & 0.0101239 & 0.204755 & 0.015626 & 0.200679 & 0.0162127 \\
\hline 0.0338507 & 0.251372 & 0.010012 & 0.199423 & 0.0131597 & 0.197333 & 0.0179001 \\
\hline 0.0339479 & 0.247581 & 0.0109653 & 0.199042 & 0.012334 & 0.192417 & 0.0152791 \\
\hline 0.0354896 & 0.248974 & 0.0116044 & 0.193134 & 0.0126111 & 0.188635 & 0.0184498 \\
\hline 0.0383387 & 0.249254 & 0.0119215 & 0.190233 & 0.0131945 & 0.196382 & 0.0132816 \\
\hline 0.0396512 & 0.250715 & 0.00938004 & 0.189643 & 0.0131971 & 0.18804 & 0.0118942 \\
\hline 0.0381752 & 0.250464 & 0.00967716 & 0.190023 & 0.0123089 & 0.187883 & 0.0167628 \\
\hline
\end{tabular}




\begin{tabular}{|c|c|c|c|c|c|c|}
\hline 0.0316589 & .25298 & 0.010526 & 0.189343 & 0.0148985 & 0.183809 & 0.0128855 \\
\hline 0.0339491 & 0.256699 & 0.0100508 & 0.184591 & 0.0102186 & 0.188164 & 0.0149639 \\
\hline 0.0327655 & 0.261576 & 0.00850483 & 0.184643 & 0.0106205 & 0.188796 & 0.0164008 \\
\hline 0320728 & 0.26698 & 0.00916157 & .183584 & 0.0124738 & 0.182353 & 0.01404 \\
\hline 0.0301792 & 0.273797 & 0.0110121 & 0.182528 & 0.0145553 & 0.19107 & 0.015013 \\
\hline 0.0384695 & 0.280203 & 0.0120425 & 0.183721 & 0.0160807 & 0.189401 & 0.017355 \\
\hline 0.0415115 & 0.284996 & 0.0124784 & 0.186081 & 0.0148133 & 0.192529 & 0.0148511 \\
\hline 0.0368676 & 0.295719 & 0.012366 & 0.195317 & 0.0119537 & 0.198702 & 0.0194626 \\
\hline 0.0339903 & 0.310272 & 0.0116014 & 0.196748 & 0.013842 & 0.204635 & 0.0133582 \\
\hline 0.0411568 & 0.323902 & 0.0114976 & 0.197633 & 0.0142698 & 0.210276 & 0.0168589 \\
\hline 0.0458891 & 0.336717 & 0.0109787 & 0.207135 & 0.0141042 & 0.215411 & 0.0121909 \\
\hline 0.0326953 & 0.350819 & 0.0111387 & 0.212722 & 0.0132352 & 0.230429 & 0.0143525 \\
\hline 0.0401049 & 0.366509 & 0.0119812 & 0.226473 & 0.013906 & 0.233228 & 0.0184979 \\
\hline 0.0515572 & 0.387037 & 0.0144152 & 0.234862 & 0.013526 & 0.245426 & 0.0221248 \\
\hline 0.0394696 & 0.405807 & 0.0137833 & 0.246761 & 0.0155626 & 0.259552 & 0.0165705 \\
\hline 0.0523867 & 0.425861 & 0.0134653 & 0.256068 & 0.0172037 & 0.272059 & 0.0208442 \\
\hline 0.0336263 & 0.446545 & 0.0101592 & 0.27182 & 0.0144898 & 0.285752 & 0.0233213 \\
\hline 0.0560612 & 0.465416 & 0.010617 & 0.292741 & 0.0162033 & 0.30561 & 0.0156332 \\
\hline 0.0490591 & 0.48622 & 0.0121822 & 932 & 0.01 & 42 & 0.02 \\
\hline 0.0603253 & 0.505935 & 0.0140123 & 0.322045 & 0.0118688 & 0.343841 & 0.0185248 \\
\hline 0.0574051 & 0.529698 & 0.0151862 & 0.339704 & 0.0171032 & 0.349795 & 0.0172956 \\
\hline 0.0539122 & 0.54 & 0.0168355 & 546 & 0.01 & 575 & 5811 \\
\hline 0.0656639 & 0.564156 & 0.0168158 & 0.388838 & 0.0201768 & 0.395477 & 0.0228594 \\
\hline 0.0732415 & 0.586118 & 0.0158174 & 0.410568 & 0.0207992 & 0.411866 & 0.0273884 \\
\hline 0.0602273 & 0.604491 & 0.014134 & 0.436697 & 0.02 & 0.437621 & 0.0261478 \\
\hline 0.0542664 & 0.622502 & 0.0176964 & 0.455886 & 0.0234878 & 0.458697 & 0.0260232 \\
\hline 0.0782791 & 0.635551 & 0.023812 & 0.472058 & 0.0259701 & 0.491165 & 0.028345 \\
\hline 0.0755266 & 0.64784 & 0.0172335 & 0.501802 & 0.0267867 & 0.494893 & 0.0238509 \\
\hline 0.067301 & 0.665585 & 0.0212245 & 0.519384 & 0.0192007 & 0.518857 & 0.025514 \\
\hline 0.0741438 & 0.677589 & 0.0197984 & 0.541693 & 0.0197367 & 0.537361 & 0.0279279 \\
\hline 0.0749942 & 0.680898 & 0.0201059 & 0.558471 & 0.025 & 0.557485 & 0.0241026 \\
\hline 0.0879594 & 0.683094 & 0.0202966 & 0.581921 & 0.0234809 & 0.571465 & 0.0235049 \\
\hline 0.0925311 & 0.687328 & 0.0189221 & 0.601203 & 0.0246205 & 0.589893 & 0.0349122 \\
\hline 0.0967296 & 0.692113 & 0.020981 & 0.609388 & 0.0256675 & 0.592655 & 0.030601 \\
\hline 0.079853 & 0.686562 & 0.0251264 & 0.634672 & 0.0303463 & 0.608836 & 0.0273712 \\
\hline 0.0849879 & 0.672171 & 0.021696 & 0.639239 & 0.0322624 & 0.616039 & 0.0262889 \\
\hline 0.102612 & 0.659537 & 0.0173055 & 0.652833 & 0.0354838 & 0.610406 & 0.026554 \\
\hline 0.0854993 & 0.646843 & 0.0139434 & 0.659701 & 0.0274104 & 0.616983 & 0.0347793 \\
\hline 0.101276 & 0.633347 & 0.015193 & 0.65317 & 0.0321107 & 0.619344 & 0.0287841 \\
\hline 0.0929962 & 0.61498 & 0.0195891 & 0.662145 & 0.0325084 & 0.616628 & 0.0272638 \\
\hline 0.073405 & 0.592149 & 0.0170789 & 0.664338 & 0.0288474 & 0.604736 & 0.0268609 \\
\hline 0.106723 & 0.568012 & 0.015572 & 0.664166 & 0.0253508 & 0.594829 & 0.0286354 \\
\hline 0.120978 & 0.542192 & 0.0141073 & 0.644667 & 0.0324011 & 0.58672 & 0.0336446 \\
\hline 0.0922195 & 0.513 & 0.0153968 & 0.64113 & 0.0249493 & 0.57005 & 0.0281693 \\
\hline 0.099384 & 0.482993 & 0.0120556 & 0.609956 & 0.0319298 & 0.556207 & 0.0306544 \\
\hline 0.0939418 & 0.45482 & 0.0122267 & 0.598443 & 0.0240237 & 0.541707 & 0.0280303 \\
\hline 0.0997095 & 0.427684 & 0.0144778 & 0.579055 & 0.0200652 & 0.517206 & 0.028462 \\
\hline
\end{tabular}




\begin{tabular}{|c|c|c|c|c|c|c|}
\hline 0.0885118 & 0.4036 & 0.0131102 & 0.550964 & 0.030309 & 0.491369 & 5071 \\
\hline 0.0784901 & 0.377438 & 0.0136907 & 0.525346 & 0.0272267 & 0.453994 & 0.0262647 \\
\hline 0.0737988 & 0.349235 & 0.0144286 & .502879 & 0.0218817 & 0.429826 & 0.0278824 \\
\hline 0786694 & .327918 & 0.0130222 & 0.47282 & 0.020437 & 0.405457 & 0.0268368 \\
\hline 0.0832691 & 0.303406 & 0.0102286 & 0.440703 & 0.0251306 & 0.373194 & 0.0203622 \\
\hline 0.0612264 & 0.281539 & 0.0117688 & 0.408029 & 0.0162226 & 0.345709 & 0.0169579 \\
\hline 0.0578752 & 0.269357 & 0.012716 & 0.378165 & 0.0183529 & 0.320792 & 0.0185395 \\
\hline 0.0674407 & 0.25369 & 0.0113593 & 0.349063 & 0.0138694 & 0.294327 & 0.0192807 \\
\hline 0.051268 & 0.235403 & 0.010396 & 0.323796 & 0.0187361 & 0.266897 & 0.0131805 \\
\hline 0.0470863 & 0.224187 & 0.0100283 & 0.296916 & 0.0151954 & 0.25239 & 0.01772 \\
\hline 0.0540734 & 0.213877 & 0.00893492 & 0.272312 & 0.0159684 & 0.22935 & 0.0150071 \\
\hline 0.0415996 & 0.205153 & 0.0107251 & 0.254477 & 0.0193958 & 0.215886 & 0.0176982 \\
\hline 0.047576 & 0.200263 & 0.00783811 & 0.229368 & 0.0126385 & 0.192531 & 0.0182062 \\
\hline 0.0477401 & 0.194241 & 0.00692413 & 0.205016 & 0.0136874 & 0.179318 & 0.015408 \\
\hline 0.0300142 & 0.188731 & 0.00861725 & 0.193949 & 0.0137532 & 0.167808 & 0.0148396 \\
\hline 0.0365469 & 0.184174 & 0.00867567 & 0.178955 & 0.0138361 & 0.1548 & 9584 \\
\hline 0.028323 & 0.182258 & 0.00820797 & 0.169769 & 0.0140171 & 0.153115 & 0.0160662 \\
\hline 0.035907 & 0.179604 & 0.00773793 & 0.155363 & 0.0104546 & 0.140002 & 1528 \\
\hline 0.0323528 & 0.176644 & 0.007 & 262 & 037 & 13 & 473 \\
\hline 0.0299665 & 0.175718 & 0.00876643 & 0.140681 & 0.0130642 & 0.129518 & 0.0141955 \\
\hline 0.0270815 & 0.17281 & 0.00836613 & 0.137292 & 0.0136263 & 0.126734 & 0.0115328 \\
\hline 0.031839 & 646 & 0.00 & 839 & 0.00 & & 359 \\
\hline 0.023076 & 0.172019 & 0.00778423 & 0.124343 & 0.0100539 & 0.123188 & 0.0125388 \\
\hline 0.0252878 & 0.170635 & 0.00790476 & 0.12606 & 0.0121374 & 0.120441 & 0.0132091 \\
\hline 0.0364125 & 0.168426 & 0.00921928 & 0.11696 & 0.00789095 & 0.115536 & 0.0120762 \\
\hline 0.0260675 & 0.168203 & 0.0104183 & 0.117971 & 0.0100183 & 0.114628 & 0.0125982 \\
\hline 0.0322828 & 0.168011 & 0.00751165 & 0.118163 & 0.0104184 & 0.116286 & 0.0124621 \\
\hline 0.0224529 & 0.169347 & 0.00895898 & 0.118144 & 0.0114755 & 0.112013 & 0.0111058 \\
\hline 0.0256496 & 0.170642 & 0.0095648 & 0.116209 & 0.00815836 & 0.112089 & 0.0118751 \\
\hline 0.0286439 & 0.169194 & 0.010 & 0.113818 & 0.010363 & 0.115911 & 2722 \\
\hline 0.0210231 & 0.167986 & 0.00741552 & 0.112355 & 0.0107474 & 0.112942 & 0.0133818 \\
\hline 0.0259321 & 0.16953 & 0.00855666 & 0.116629 & 0.0101421 & 0.1117 & 0.010972 \\
\hline 0.0207879 & 0.172645 & 0.00872184 & 0.112854 & 0.0108656 & 0.117062 & 0.0110926 \\
\hline 0.0220968 & 0.171524 & 0.00807526 & 0.114237 & 0.00634563 & 0.119274 & 0.0122442 \\
\hline 0.0207714 & 0.168712 & 0.00744397 & 0.115609 & 0.0107075 & 0.118575 & 0.0144215 \\
\hline 0.0251081 & 0.169957 & 0.00732525 & 0.117696 & 0.0122816 & 0.117445 & 0.0119207 \\
\hline 0.0198871 & 0.171598 & 0.0108847 & 0.117639 & 0.00963507 & 0.1132 & 0.0147284 \\
\hline 0.0304519 & 0.173181 & 0.0113088 & 0.116846 & 0.0108413 & 0.112352 & 0.00919493 \\
\hline 0.0254495 & 0.173956 & 0.00972527 & 0.114391 & 0.0111164 & 0.115713 & 0.01146 \\
\hline 0.0226373 & 0.174948 & 0.00819171 & 0.11273 & 0.0119975 & 0.11606 & 0.0115266 \\
\hline 0.0216702 & 0.176199 & 0.00781007 & 0.118237 & 0.011209 & 0.116497 & 0.0108618 \\
\hline 0.0261412 & 0.175196 & 0.00759933 & 0.120065 & 0.0122582 & 0.118386 & 0.0103616 \\
\hline 0.0235591 & 0.177419 & 0.0072629 & 0.119782 & 0.0105779 & 0.117551 & 0.0112159 \\
\hline 0.0252912 & 0.179955 & 0.00914775 & 0.118832 & 0.0106206 & 0.118355 & 0.0104166 \\
\hline 0.0252539 & 0.178117 & 0.0101948 & 0.120608 & 0.0122131 & 0.119774 & 0.0106866 \\
\hline 0.0286642 & 0.174614 & 0.00852516 & 0.120549 & 0.0124759 & 0.11982 & 0.0125921 \\
\hline 0.0198433 & 0.173785 & 0.00748054 & 0.122196 & 0.0106185 & 0.125282 & 0.0118697 \\
\hline
\end{tabular}




$\begin{array}{rrrrrrr}0.0267957 & 0.176744 & 0.00869528 & 0.121443 & 0.0094337 & 0.126649 & 0.0143971 \\ 0.0205817 & 0.180199 & 0.0102275 & 0.119844 & 0.0101525 & 0.123433 & 0.0138334 \\ 0.0237775 & 0.179986 & 0.0126081 & 0.124366 & 0.0126281 & 0.12195 & 0.0120719 \\ 0.0275517 & 0.180411 & 0.00982481 & 0.123528 & 0.0089807 & 0.128604 & 0.0139932 \\ 0.0238738 & 0.182535 & 0.0102076 & 0.126564 & 0.0126246 & 0.129054 & 0.0117067 \\ 0.0248718 & 0.185136 & 0.00951424 & 0.12547 & 0.00966249 & 0.130736 & 0.0132092 \\ 0.0230128 & 0.187087 & 0.00862778 & 0.130993 & 0.00813954 & 0.126265 & 0.00929173 \\ 0.0272171 & 0.18818 & 0.010425 & 0.128428 & 0.00887737 & 0.133585 & 0.0131707 \\ 0.0195466 & 0.189007 & 0.0108048 & 0.127718 & 0.0109008 & 0.132981 & 0.015578 \\ 0.023979 & 0.18987 & 0.00858879 & 0.130624 & 0.00973808 & 0.136269 & 0.0120752 \\ 0.0260836 & 0.190732 & 0.00810961 & 0.132461 & 0.0118196 & 0.136967 & 0.0143256 \\ 0.0305166 & 0.189754 & 0.0110841 & 0.132284 & 0.0112092 & 0.136613 & 0.0116072 \\ 0.0246101 & 0.193048 & 0.0104957 & 0.133125 & 0.011096 & 0.138814 & 0.0158401 \\ 0.0211813 & 0.196322 & 0.0114637 & 0.139623 & 0.0115045 & 0.137149 & 0.013881 \\ 0.0290172 & 0.195233 & 0.0102278 & 0.140044 & 0.0108475 & 0.133634 & 0.0120355 \\ 0.0222481 & 0.193596 & 0.00885864 & 0.143092 & 0.0131492 & 0.140449 & 0.0143364 \\ 0.02394 & 0.197949 & 0.00890883 & 0.139486 & 0.00843923 & 0.143957 & 0.00897351 \\ 0.0311431 & 0.197629 & 0.00898524 & 0.143378 & 0.0100056 & 0.142836 & 0.0169299 \\ 0.0232894 & 0.194099 & 0.00905429 & 0.144162 & 0.0110193 & 0.147553 & 0.0125955 \\ 0.0252591 & 0.197444 & 0.0107786 & 0.145446 & 0.0118549 & 0.150248 & 0.0106334 \\ 0.0261131 & 0.199907 & 0.00967535 & 0.146529 & 0.014243 & 0.154051 & 0.0135478 \\ 0.0288547 & 0.20141 & 0.00861808 & 0.152456 & 0.0106938 & 0.150096 & 0.0140619 \\ 0.0235059 & 0.201863 & 0.00996916 & 0.149859 & 0.0151307 & 0.151975 & 0.0146699 \\ 0.0255689 & 0.20092 & 0.00988273 & 0.153107 & 0.010649 & 0.156639 & 0.0171358 \\ 0.0271253 & 0.204356 & 0.00790213 & 0.153167 & 0.00707604 & 0.157039 & 0.0139374 \\ 0.026893 & 0.201933 & 0.00898684 & 0.153682 & 0.0104289 & 0.156597 & 0.0107228 \\ 0.0321093 & 0.203049 & 0.00975137 & 0.159159 & 0.0151188 & 0.156721 & 0.0151804 \\ 0.0241523 & 0.208082 & 0.0093383 & 0.158896 & 0.0106804 & 0.161528 & 0.0131388\end{array}$




\begin{tabular}{|c|c|c|c|c|c|c|}
\hline $\begin{array}{l}\text { SB Gy } \\
\text { mean }\end{array}$ & std & $\begin{array}{l}\text { SB X1 } \\
\text { mean }\end{array}$ & std & $\begin{array}{l}\text { SB SNF } \\
\text { mean }\end{array}$ & std & $\begin{array}{l}\text { SB SF } \\
\text { mean }\end{array}$ \\
\hline 0.372716 & 0.0213241 & 0.308687 & 0.11143 & 0.207508 & 0.0201546 & 2.26705 \\
\hline 0.380266 & 0.0261759 & 0.310137 & 0.108058 & 0.206511 & 0.0142846 & 2.22612 \\
\hline 0.381494 & 0.0303519 & 0.315931 & 0.113678 & 0.210907 & 0.0169403 & 2.22689 \\
\hline 0.383343 & 0.029217 & 0.308801 & 0.110392 & 0.208779 & 0.015025 & 2.24404 \\
\hline 0.372164 & 0.0223948 & 0.302719 & 0.114408 & 0.205839 & 0.01184 & 2.23019 \\
\hline 0.380273 & 0.0258454 & 0.306009 & 0.102969 & 0.208633 & 0.0155606 & 2.24663 \\
\hline 0.37502 & 0.0201682 & 0.30645 & 0.112916 & 0.207516 & 0.0176179 & 2.21478 \\
\hline 0.384129 & 0.0277388 & 0.303618 & 0.109196 & 0.202652 & 0.0127888 & 2.21132 \\
\hline 0.376635 & 0.0294942 & 0.304958 & 0.109611 & 0.205249 & 0.0114203 & 2.20511 \\
\hline 0.383255 & 0.033965 & 0.308482 & 0.111941 & 0.201042 & 0.016722 & 2.22559 \\
\hline 0.372901 & 0.0254062 & 0.302535 & 0.107534 & 0.200532 & 0.014472 & 2.22557 \\
\hline 0.371613 & 0.0267283 & 0.304841 & 0.103103 & 0.202207 & 0.0137443 & 2.2116 \\
\hline 0.375027 & 0.0257698 & 0.311067 & 0.11518 & 0.206187 & 0.0165375 & 2.2145 \\
\hline 0.376517 & 0.0267551 & 0.301094 & 0.109106 & 0.197679 & 0.0140839 & 2.20371 \\
\hline 0.372764 & 0.023906 & 0.303709 & 0.108588 & 0.20063 & 0.0131395 & 2.20423 \\
\hline 0.373007 & 0.0307179 & 0.302503 & 0.118517 & 0.198765 & 0.0144961 & 2.19836 \\
\hline 0.371699 & 0.0209773 & 0.306898 & 0.107371 & 0.202522 & 0.0167546 & 2.24487 \\
\hline 0.380479 & 0.031546 & 0.305782 & 0.114464 & 0.20357 & 0.0183039 & 2.21676 \\
\hline 0.384404 & 0.0293727 & 0.30831 & 0.106408 & 0.208729 & 0.0180684 & 2.22763 \\
\hline 0.389884 & 0.0297627 & 0.30877 & 0.113979 & 0.210373 & 0.0150397 & 2.23912 \\
\hline 0.391656 & 0.0274233 & 0.315307 & 0.121606 & 0.216098 & 0.0143232 & 2.22939 \\
\hline 0.399688 & 0.0238505 & 0.323118 & 0.11579 & 0.216713 & 0.016274 & 2.25873 \\
\hline 0.410974 & 0.0250151 & 0.320944 & 0.114184 & 0.224228 & 0.0167299 & 2.23157 \\
\hline 0.408896 & 0.0235667 & 0.327772 & 0.11891 & 0.224229 & 0.0158453 & 2.2213 \\
\hline 0.421748 & 0.0236502 & 0.335759 & 0.120594 & 0.225353 & 0.0146191 & 2.2515 \\
\hline 0.4241 & 0.0249547 & 0.332906 & 0.118561 & 0.227717 & 0.0187557 & 2.28104 \\
\hline 0.416492 & 0.0285785 & 0.32266 & 0.120953 & 0.224952 & 0.0167007 & 2.24066 \\
\hline 0.415808 & 0.0241955 & 0.323395 & 0.117069 & 0.222697 & 0.0128967 & 2.27249 \\
\hline 0.416166 & 0.0305974 & 0.320343 & 0.12387 & 0.222966 & 0.0160793 & 2.2444 \\
\hline 0.407442 & 0.023805 & 0.321871 & 0.118168 & 0.21837 & 0.0165654 & 2.20182 \\
\hline 0.398192 & 0.0233872 & 0.314741 & 0.119057 & 0.217483 & 0.0141103 & 2.23863 \\
\hline 0.394454 & 0.0223466 & 0.308351 & 0.116626 & 0.211371 & 0.0145995 & 2.20453 \\
\hline 0.387544 & 0.0273999 & 0.309562 & 0.117825 & 0.215614 & 0.0160385 & 2.20318 \\
\hline 0.383359 & 0.0315601 & 0.307094 & 0.112969 & 0.217671 & 0.0153202 & 2.1886 \\
\hline 0.370008 & 0.0225174 & 0.302818 & 0.110933 & 0.215103 & 0.0140671 & 2.17309 \\
\hline 0.370649 & 0.0260253 & 0.307004 & 0.12029 & 0.218269 & 0.0145846 & 2.19934 \\
\hline 0.371458 & 0.0252193 & 0.304061 & 0.1133 & 0.232976 & 0.0215142 & 2.18793 \\
\hline 0.374266 & 0.0231795 & 0.31698 & 0.117024 & 0.254039 & 0.0216309 & 2.23924 \\
\hline 0.378974 & 0.0269045 & 0.334086 & 0.124367 & 0.296554 & 0.0250523 & 2.2608 \\
\hline 0.380536 & 0.0242832 & 0.363993 & 0.134331 & 0.359281 & 0.0327462 & 2.36981 \\
\hline 0.390441 & 0.0236091 & 0.405212 & 0.162832 & 0.466959 & 0.0449386 & 2.4847 \\
\hline 0.399948 & 0.0243715 & 0.458066 & 0.179872 & 0.606444 & 0.0580343 & 2.69445 \\
\hline 0.412937 & 0.0172393 & 0.531656 & 0.202839 & 0.78837 & 0.0726182 & 2.96039 \\
\hline 0.43221 & 0.0303715 & 0.625153 & 0.25539 & 1.0177 & 0.110837 & 3.24064 \\
\hline 0.450375 & 0.025193 & 0.726693 & 0.2906 & 1.275 & 0.143062 & 3.61264 \\
\hline
\end{tabular}




\begin{tabular}{|c|c|c|c|c|c|c|}
\hline 0.472457 & 0.0277405 & 0.827076 & 0.338911 & 1.53985 & 0.179596 & 3.94844 \\
\hline 0.498 & 0.0292648 & 0.92193 & 0.38439 & 1.77575 & 0.20439 & 4.23805 \\
\hline 0.512533 & 0.0328701 & 0.997562 & 0.404046 & 1.95232 & 0.211892 & 4.54869 \\
\hline 0.515413 & 0.0293187 & 1.03541 & 0.433121 & 2.04788 & 0.2165 & 4.64071 \\
\hline 0.530284 & 0.0355724 & 1.03113 & 0.425199 & 2.05295 & 0.230785 & 4.62252 \\
\hline 0.520182 & 0.03097 & 0.998692 & 0.40832 & 1.95366 & 0.216282 & 4.53226 \\
\hline 0.511319 & 0.0291712 & 0.922986 & 0.371008 & 1.77308 & 0.203659 & 4.24779 \\
\hline 0.495357 & 0.0286304 & 0.826326 & 0.338303 & 1.53331 & 0.166427 & 3.96532 \\
\hline 0.476559 & 0.0297605 & 0.719981 & 0.291374 & 1.28793 & 0.131374 & 3.63702 \\
\hline 0.455196 & 0.0236857 & 0.634273 & 0.25819 & 1.04649 & 0.106603 & 3.26762 \\
\hline 0.431886 & 0.0298639 & 0.552862 & 0.225438 & 0.832537 & 0.0892648 & 2.98713 \\
\hline 0.425728 & 0.0258066 & 0.495119 & 0.206384 & 0.653595 & 0.0658854 & 2.73518 \\
\hline 0.405181 & 0.0233254 & 0.441898 & 0.172671 & 0.517751 & 0.0444023 & 2.54658 \\
\hline 0.393977 & 0.0290388 & 0.41604 & 0.16779 & 0.427232 & 0.0406478 & 2.4497 \\
\hline 0.395433 & 0.0249935 & 0.410751 & 0.170068 & 0.366832 & 0.0354811 & 2.36178 \\
\hline 0.397644 & 0.0212563 & 0.429937 & 0.174395 & 0.348152 & 0.0341047 & 2.35305 \\
\hline 0.396738 & 0.0225985 & 0.492124 & 0.205547 & 0.375542 & 0.033471 & 2.42599 \\
\hline 0.41182 & 0.0234825 & 0.625702 & 0.273439 & 0.448361 & 0.0410147 & 2.65436 \\
\hline 0.426622 & 0.023352 & 0.83627 & 0.381326 & 0.602861 & 0.0603015 & 3.0602 \\
\hline 0.473306 & 0.0280582 & 1.16679 & 0.544323 & 0.851281 & 0.08076 & 3.69562 \\
\hline 0.546224 & 0.0327075 & 1.68261 & 0.824195 & 1.2178 & 0.128112 & 4.66518 \\
\hline 0.632605 & 0.0390847 & 2.38746 & 1.19222 & 1.7422 & 0.190994 & 6.05742 \\
\hline 0.751081 & 0.0510928 & 3.30963 & 1.67876 & 2.4423 & 0.259821 & 7.85826 \\
\hline 0.903525 & 0.0600441 & 4.4363 & 2.26747 & 3.29573 & 0.350514 & 10.0376 \\
\hline 1.10555 & 0.0808396 & 5.72337 & 2.93666 & 4.2536 & 0.476974 & 12.5634 \\
\hline 1.30088 & 0.105435 & 7.00565 & 3.58587 & 5.24586 & 0.590371 & 15.0729 \\
\hline 1.51293 & 0.128142 & 8.1862 & 4.20222 & 6.18516 & 0.676862 & 17.5132 \\
\hline 1.69474 & 0.124461 & 9.1074 & 4.65871 & 6.92793 & 0.755638 & 19.4821 \\
\hline 1.82472 & 0.14738 & 9.66204 & 4.982 & 7.35558 & 0.802061 & 20.7207 \\
\hline 1.89005 & 0.139062 & 9.73003 & 5.02615 & 7.4404 & 0.817311 & 20.9289 \\
\hline 1.89167 & 0.147937 & 9.28289 & 4.79974 & 7.17631 & 0.790804 & 20.2087 \\
\hline 1.82637 & 0.1431 & 8.52538 & 4.43293 & 6.58509 & 0.745883 & 18.7626 \\
\hline 1.68839 & 0.129044 & 7.44728 & 3.86628 & 5.79957 & 0.651236 & 16.6874 \\
\hline 1.54518 & 0.101766 & 6.20425 & 3.22901 & 4.82574 & 0.529498 & 14.2816 \\
\hline 1.34241 & 0.0978442 & 4.98819 & 2.58356 & 3.88855 & 0.424243 & 11.831 \\
\hline 1.17637 & 0.0739933 & 3.87888 & 1.98508 & 3.03865 & 0.338053 & 9.58169 \\
\hline 1.048 & 0.0608868 & 2.96262 & 1.51594 & 2.28861 & 0.252183 & 7.653 \\
\hline 0.929764 & 0.0550786 & 2.22124 & 1.10317 & 1.7002 & 0.176317 & 6.12153 \\
\hline 0.844002 & 0.0460938 & 1.69994 & 0.822942 & 1.28203 & 0.140309 & 5.01296 \\
\hline 0.798807 & 0.042529 & 1.29346 & 0.635097 & 0.964635 & 0.0995608 & 4.15967 \\
\hline 0.759484 & 0.0497701 & 1.0076 & 0.491736 & 0.737116 & 0.0700289 & 3.53289 \\
\hline 0.739432 & 0.0408895 & 0.804514 & 0.367258 & 0.571295 & 0.0515317 & 3.11701 \\
\hline 0.722478 & 0.0376147 & 0.653943 & 0.287976 & 0.459817 & 0.0415526 & 2.79794 \\
\hline 0.711702 & 0.036852 & 0.545005 & 0.237343 & 0.382824 & 0.0296623 & 2.56781 \\
\hline 0.713068 & 0.0344655 & 0.467788 & 0.18772 & 0.320754 & 0.0224117 & 2.4246 \\
\hline 0.715768 & 0.0485585 & 0.416318 & 0.160499 & 0.279257 & 0.0236065 & 2.32891 \\
\hline 0.707745 & 0.0404225 & 0.375455 & 0.15611 & 0.251075 & 0.0188884 & 2.22138 \\
\hline
\end{tabular}




\begin{tabular}{|c|c|c|c|c|c|c|}
\hline 0.70294 & 0.0483059 & 0.365689 & 0.143013 & 0.235823 & 0.0184049 & 2.16409 \\
\hline 0.69537 & 0.0524448 & 0.353624 & 0.135396 & 0.228827 & 0.0196343 & 2.12041 \\
\hline 0.658041 & 0.0426863 & 0.351998 & 0.13518 & 0.230167 & 0.0193892 & 2.12755 \\
\hline 0.619547 & 0.054873 & 0.36345 & 0.143291 & 0.245096 & 0.0261421 & 2.12955 \\
\hline 0.596756 & 0.0603676 & 0.367933 & 0.138046 & 0.257039 & 0.0282007 & 2.16679 \\
\hline 0.571603 & 0.0478929 & 0.377293 & 0.149385 & 0.275938 & 0.0238608 & 2.17966 \\
\hline 0.554683 & 0.0407247 & 0.380871 & 0.150545 & 0.291092 & 0.0252262 & 2.26163 \\
\hline 0.560185 & 0.0433066 & 0.396303 & 0.150479 & 0.307689 & 0.0285898 & 2.30729 \\
\hline 0.56399 & 0.0399822 & 0.400382 & 0.156765 & 0.3205 & 0.0305121 & 2.34532 \\
\hline 0.570893 & 0.0320076 & 0.403593 & 0.161035 & 0.322581 & 0.0287649 & 2.37597 \\
\hline 0.593637 & 0.0300065 & 0.394992 & 0.15717 & 0.324975 & 0.028041 & 2.40083 \\
\hline 0.617765 & 0.0384793 & 0.390673 & 0.154935 & 0.315294 & 0.0264028 & 2.385 \\
\hline 0.638362 & 0.0392277 & 0.387107 & 0.152566 & 0.299061 & 0.0291838 & 2.39445 \\
\hline 0.654538 & 0.0403441 & 0.367996 & 0.147188 & 0.282108 & 0.0238274 & 2.37641 \\
\hline 0.663806 & 0.0299902 & 0.353201 & 0.134942 & 0.264062 & 0.0193023 & 2.329 \\
\hline 0.672772 & 0.0273933 & 0.338839 & 0.126049 & 0.245692 & 0.0189113 & 2.30572 \\
\hline 0.673502 & 0.0333002 & 0.324438 & 0.12248 & 0.227351 & 0.0168124 & 2.25332 \\
\hline 0.682997 & 0.0307671 & 0.322614 & 0.126107 & 0.212391 & 0.0160212 & 2.24438 \\
\hline 0.700228 & 0.0397011 & 0.316523 & 0.118459 & 0.200822 & 0.0131792 & 2.20157 \\
\hline 0.705575 & 0.0356558 & 0.308624 & 0.115244 & 0.193976 & 0.013225 & 2.14537 \\
\hline 0.735114 & 0.0378579 & 0.302599 & 0.117071 & 0.185852 & 0.0131275 & 2.1195 \\
\hline 0.767705 & 0.0382508 & 0.303824 & 0.115368 & 0.182476 & 0.0134558 & 2.08954 \\
\hline 0.84213 & 0.0382054 & 0.299923 & 0.115882 & 0.182631 & 0.013154 & 2.07054 \\
\hline 0.963136 & 0.0428276 & 0.310419 & 0.119066 & 0.18193 & 0.0139612 & 2.05216 \\
\hline 1.15721 & 0.054753 & 0.326413 & 0.126641 & 0.182409 & 0.0135025 & 2.03342 \\
\hline 1.49855 & 0.068892 & 0.35402 & 0.135697 & 0.190295 & 0.0139952 & 2.0309 \\
\hline 2.05752 & 0.0840323 & 0.387438 & 0.151238 & 0.203176 & 0.0126854 & 2.02306 \\
\hline 2.92723 & 0.119234 & 0.438565 & 0.179398 & 0.22129 & 0.0123775 & 2.02361 \\
\hline 4.27618 & 0.156162 & 0.528339 & 0.216428 & 0.249639 & 0.0157115 & 2.06502 \\
\hline 6.28791 & 0.231019 & 0.658184 & 0.27863 & 0.297006 & 0.0205493 & 2.11373 \\
\hline 9.13014 & 0.310094 & 0.838328 & 0.352795 & 0.36308 & 0.0291593 & 2.17742 \\
\hline 12.9534 & 0.451718 & 1.06585 & 0.4499 & 0.448346 & 0.0386632 & 2.26574 \\
\hline 17.8921 & 0.650147 & 1.3422 & 0.586116 & 0.548835 & 0.0462272 & 2.38176 \\
\hline 23.8371 & 0.826144 & 1.65603 & 0.738172 & 0.669816 & 0.0576188 & 2.52048 \\
\hline 30.6409 & 1.0168 & 1.99616 & 0.884357 & 0.796993 & 0.0718903 & 2.65862 \\
\hline 37.7573 & 1.32311 & 2.33261 & 1.04278 & 0.914228 & 0.0824123 & 2.77446 \\
\hline 44.5817 & 1.5909 & 2.62883 & 1.19158 & 1.02309 & 0.0886223 & 2.87576 \\
\hline 50.4373 & 1.81811 & 2.85265 & 1.29126 & 1.10644 & 0.0992407 & 2.99498 \\
\hline 54.5841 & 1.91153 & 2.96735 & 1.33311 & 1.14736 & 0.106344 & 3.06978 \\
\hline 56.4233 & 1.97923 & 2.96864 & 1.33483 & 1.14424 & 0.107357 & 3.04752 \\
\hline 55.7988 & 1.94306 & 2.83253 & 1.2735 & 1.10496 & 0.101203 & 3.00556 \\
\hline 52.7842 & 1.81533 & 2.62941 & 1.1646 & 1.0296 & 0.096212 & 2.89699 \\
\hline 47.7514 & 1.65581 & 2.33956 & 1.03089 & 0.919819 & 0.0795879 & 2.77613 \\
\hline 41.3805 & 1.43442 & 2.00591 & 0.880313 & 0.796082 & 0.0685334 & 2.65473 \\
\hline 34.315 & 1.16852 & 1.66569 & 0.717452 & 0.671587 & 0.0574162 & 2.48588 \\
\hline 27.3487 & 0.939717 & 1.36255 & 0.573979 & 0.558863 & 0.0425947 & 2.3481 \\
\hline 21.0934 & 0.739198 & 1.10761 & 0.451712 & 0.459656 & 0.0328311 & 2.26231 \\
\hline
\end{tabular}




\begin{tabular}{|c|c|c|c|c|c|c|}
\hline 15.7949 & 0.558569 & 0.905387 & 0.364836 & 0.380952 & 0.0256029 & 2.16465 \\
\hline 11.6894 & 0.411496 & 0.746742 & 0.300044 & 0.325935 & 0.021689 & 2.08963 \\
\hline 8.75696 & 0.310487 & 0.647243 & 0.246455 & 0.291486 & 0.0193821 & 2.04419 \\
\hline 6.78892 & 0.229867 & 0.590655 & 0.222645 & 0.270325 & 0.0172872 & 2.01471 \\
\hline 5.58353 & 0.192327 & 0.546215 & 0.199974 & 0.257542 & 0.0175151 & 2.02301 \\
\hline 4.90036 & 0.176586 & 0.521495 & 0.191282 & 0.252011 & 0.015264 & 2.00817 \\
\hline 4.54975 & 0.171108 & 0.510498 & 0.186851 & 0.248095 & 0.0126222 & 1.99517 \\
\hline 4.34428 & 0.160519 & 0.500644 & 0.181503 & 0.240823 & 0.015278 & 2.00217 \\
\hline 4.16705 & 0.143103 & 0.479409 & 0.18048 & 0.237179 & 0.0147405 & 2.00935 \\
\hline 3.91412 & 0.138431 & 0.458802 & 0.171211 & 0.235576 & 0.0154482 & 2.02089 \\
\hline 3.59086 & 0.140698 & 0.433704 & 0.160275 & 0.22889 & 0.0146087 & 2.00782 \\
\hline 3.1958 & 0.111948 & 0.408099 & 0.148062 & 0.216873 & 0.0140069 & 1.99593 \\
\hline 2.75212 & 0.0897652 & 0.384644 & 0.144042 & 0.208053 & 0.0115156 & 2.00099 \\
\hline 2.2936 & 0.0769859 & 0.363698 & 0.133346 & 0.198747 & 0.0106627 & 2.00007 \\
\hline 1.88007 & 0.071502 & 0.344482 & 0.125015 & 0.195545 & 0.00993633 & 2.00998 \\
\hline 1.49248 & 0.0614355 & 0.335581 & 0.124535 & 0.193926 & 0.0113217 & 2.04297 \\
\hline 1.1772 & 0.0450162 & 0.329372 & 0.122134 & 0.194349 & 0.0120945 & 2.08797 \\
\hline 0.941556 & 0.0349625 & 0.32409 & 0.121432 & 0.203467 & 0.0136228 & 2.15856 \\
\hline 0.769069 & 0.0357297 & 0.334488 & 0.127329 & 0.215116 & 0.0150431 & 2.28644 \\
\hline 0.655884 & 0.0295008 & 0.35587 & 0.137845 & 0.233306 & 0.0175646 & 2.48138 \\
\hline 0.601404 & 0.0281233 & 0.378907 & 0.138514 & 0.262196 & 0.0194287 & 2.73297 \\
\hline 0.595001 & 0.0322782 & 0.413405 & 0.145295 & 0.298956 & 0.0199477 & 3.09541 \\
\hline 0.621957 & 0.0407109 & 0.458681 & 0.165515 & 0.346322 & 0.0236429 & 3.5966 \\
\hline 0.687865 & 0.0452526 & 0.510415 & 0.180859 & 0.407298 & 0.0318296 & 4.19223 \\
\hline 0.786704 & 0.0632332 & 0.57374 & 0.202611 & 0.477706 & 0.0405266 & 4.94463 \\
\hline 0.910689 & 0.0808785 & 0.640982 & 0.23069 & 0.549335 & 0.0477551 & 5.7714 \\
\hline 1.05246 & 0.0953228 & 0.703966 & 0.251623 & 0.623658 & 0.0547991 & 6.63497 \\
\hline 1.19908 & 0.124151 & 0.771852 & 0.271892 & 0.698599 & 0.0604395 & 7.45958 \\
\hline 1.33325 & 0.14497 & 0.835163 & 0.295429 & 0.760895 & 0.0712701 & 8.21712 \\
\hline 1.44697 & 0.15292 & 0.87121 & 0.309238 & 0.806209 & 0.0735183 & 8.83745 \\
\hline 1.53845 & 0.17251 & 0.891144 & 0.310166 & 0.832373 & 0.072362 & 9.19713 \\
\hline 1.58605 & 0.187399 & 0.891669 & 0.310804 & 0.831719 & 0.074484 & 9.27493 \\
\hline 1.57439 & 0.183575 & 0.874711 & 0.307372 & 0.812981 & 0.0703834 & 9.07119 \\
\hline 1.51674 & 0.181831 & 0.838683 & 0.296215 & 0.778119 & 0.0643628 & 8.71355 \\
\hline 1.43153 & 0.152791 & 0.799337 & 0.28467 & 0.726501 & 0.0595233 & 8.22988 \\
\hline 1.32344 & 0.140409 & 0.771613 & 0.277635 & 0.675143 & 0.053512 & 7.68159 \\
\hline 1.1962 & 0.131814 & 0.750396 & 0.271294 & 0.624499 & 0.0427745 & 7.19325 \\
\hline 1.0775 & 0.110467 & 0.737163 & 0.267383 & 0.590004 & 0.0377481 & 6.79893 \\
\hline 0.976032 & 0.0815855 & 0.73412 & 0.262622 & 0.567045 & 0.0403301 & 6.58103 \\
\hline 0.886271 & 0.0589615 & 0.754314 & 0.273936 & 0.553876 & 0.036669 & 6.53038 \\
\hline 0.834327 & 0.053636 & 0.788877 & 0.300048 & 0.560058 & 0.0368268 & 6.61117 \\
\hline 0.804654 & 0.0393803 & 0.827902 & 0.32288 & 0.575952 & 0.0448562 & 6.84031 \\
\hline 0.783499 & 0.0354921 & 0.866698 & 0.331081 & 0.589046 & 0.0438771 & 7.06688 \\
\hline 0.778389 & 0.0426232 & 0.885186 & 0.335698 & 0.600049 & 0.0436904 & 7.22244 \\
\hline 0.779036 & 0.0401601 & 0.899677 & 0.344373 & 0.604876 & 0.0423049 & 7.29915 \\
\hline 0.780779 & 0.0297967 & 0.901004 & 0.343095 & 0.601477 & 0.0414725 & 7.23966 \\
\hline 0.776833 & 0.0336248 & 0.872978 & 0.32832 & 0.585795 & 0.0406707 & 7.03121 \\
\hline
\end{tabular}




\begin{tabular}{|c|c|c|c|c|c|c|}
\hline 0.77079 & 0.0328023 & 0.831844 & 0.307529 & 0.557308 & 0.0401937 & 6.65747 \\
\hline 0.755934 & 0.0271153 & 0.79433 & 0.292759 & 0.526256 & 0.0372796 & 6.21576 \\
\hline 0.738013 & 0.0314278 & 0.754504 & 0.277459 & 0.496722 & 0.0299141 & 5.70833 \\
\hline 0.718921 & 0.0326773 & 0.705575 & 0.252949 & 0.460272 & 0.0286796 & 5.15287 \\
\hline 0.703013 & 0.0336959 & 0.661811 & 0.231549 & 0.424372 & 0.03007 & 4.65589 \\
\hline 0.697636 & 0.0343313 & 0.622078 & 0.217124 & 0.398554 & 0.0286348 & 4.20989 \\
\hline 0.69787 & 0.0314584 & 0.584813 & 0.204106 & 0.373926 & 0.027642 & 3.79727 \\
\hline 0.688885 & 0.030187 & 0.557383 & 0.191471 & 0.349281 & 0.0215223 & 3.47118 \\
\hline 0.679076 & 0.0364028 & 0.53922 & 0.1804 & 0.330443 & 0.0205738 & 3.20802 \\
\hline 0.678533 & 0.0334932 & 0.520488 & 0.173819 & 0.314624 & 0.0204336 & 2.97128 \\
\hline 0.678414 & 0.030876 & 0.506717 & 0.171121 & 0.302833 & 0.0194453 & 2.78465 \\
\hline 0.670694 & 0.0337615 & 0.497199 & 0.165102 & 0.289576 & 0.0214027 & 2.63199 \\
\hline 0.661744 & 0.0384873 & 0.48068 & 0.159596 & 0.276509 & 0.020021 & 2.50839 \\
\hline 0.650916 & 0.0377832 & 0.470257 & 0.158623 & 0.264286 & 0.0187317 & 2.40091 \\
\hline 0.638708 & 0.0352241 & 0.455598 & 0.15358 & 0.252195 & 0.016548 & 2.31534 \\
\hline 0.626833 & 0.0333965 & 0.435287 & 0.15252 & 0.242166 & 0.0146855 & 2.23076 \\
\hline 0.602146 & 0.0349021 & 0.425411 & 0.151627 & 0.231557 & 0.0163674 & 2.1613 \\
\hline 0.577522 & 0.0303192 & 0.416105 & 0.144146 & 0.218503 & 0.0148076 & 2.1087 \\
\hline 0.559569 & 0.0285785 & 0.401517 & 0.141564 & 0.210852 & 0.015712 & 2.04696 \\
\hline 0.536042 & 0.0271029 & 0.389835 & 0.140898 & 0.20743 & 0.0138333 & 1.98956 \\
\hline 0.51879 & 0.0226814 & 0.384254 & 0.141786 & 0.200883 & 0.0139005 & 1.95899 \\
\hline 0.505612 & 0.0245369 & 0.381385 & 0.146725 & 0.193327 & 0.011364 & 1.93358 \\
\hline 0.498301 & 0.0284991 & 0.379529 & 0.148268 & 0.191337 & 0.0114634 & 1.92274 \\
\hline 0.495228 & 0.0276086 & 0.381737 & 0.144595 & 0.193668 & 0.0119929 & 1.9095 \\
\hline 0.490933 & 0.0229089 & 0.385409 & 0.149215 & 0.196442 & 0.0108395 & 1.90467 \\
\hline 0.489591 & 0.0224953 & 0.388345 & 0.146214 & 0.201656 & 0.0135851 & 1.91316 \\
\hline 0.487884 & 0.0231697 & 0.388824 & 0.146106 & 0.207841 & 0.0145945 & 1.92712 \\
\hline 0.487348 & 0.0263137 & 0.395524 & 0.152166 & 0.213895 & 0.0130022 & 1.91507 \\
\hline 0.493624 & 0.0259613 & 0.406339 & 0.156672 & 0.227105 & 0.0142201 & 1.9441 \\
\hline 0.505962 & 0.0293031 & 0.41645 & 0.159797 & 0.247886 & 0.0171306 & 1.97362 \\
\hline 0.524971 & 0.0298974 & 0.43672 & 0.167502 & 0.281109 & 0.0179059 & 2.0227 \\
\hline 0.526903 & 0.0262914 & 0.472575 & 0.184915 & 0.329265 & 0.0222421 & 2.0947 \\
\hline 0.533581 & 0.0270116 & 0.518163 & 0.205931 & 0.396884 & 0.0304104 & 2.1926 \\
\hline 0.546232 & 0.0264137 & 0.588332 & 0.240898 & 0.506759 & 0.0374853 & 2.35718 \\
\hline 0.564335 & 0.0262866 & 0.680065 & 0.282539 & 0.667198 & 0.0469872 & 2.5971 \\
\hline 0.579431 & 0.0323719 & 0.798719 & 0.342557 & 0.883594 & 0.0654907 & 2.94332 \\
\hline 0.593735 & 0.0338645 & 0.95129 & 0.424463 & 1.17341 & 0.0785575 & 3.39756 \\
\hline 0.607313 & 0.0266893 & 1.15995 & 0.523642 & 1.53697 & 0.103085 & 3.94453 \\
\hline 0.636288 & 0.029684 & 1.41575 & 0.647692 & 1.97639 & 0.146032 & 4.61785 \\
\hline 0.665248 & 0.0321439 & 1.68099 & 0.786597 & 2.47683 & 0.195348 & 5.41398 \\
\hline 0.694318 & 0.0242754 & 1.97759 & 0.934614 & 3.01369 & 0.238984 & 6.23053 \\
\hline 0.72288 & 0.0294067 & 2.27474 & 1.0754 & 3.54086 & 0.274383 & 7.02476 \\
\hline 0.766697 & 0.0365183 & 2.52657 & 1.1987 & 4.01424 & 0.30405 & 7.78565 \\
\hline 0.801274 & 0.0439138 & 2.69282 & 1.29943 & 4.38106 & 0.32427 & 8.37833 \\
\hline 0.818638 & 0.049581 & 2.81864 & 1.37 & 4.62469 & 0.346757 & 8.76324 \\
\hline 0.831094 & 0.0442562 & 2.85067 & 1.38107 & 4.72411 & 0.346413 & 8.9004 \\
\hline 0.832441 & 0.0470938 & 2.78947 & 1.35222 & 4.64264 & 0.345403 & 8.80119 \\
\hline
\end{tabular}




\begin{tabular}{|c|c|c|c|c|c|c|}
\hline 0.829957 & 0.0516645 & 2.64829 & 1.285 & 4.39459 & 0.331619 & 8.41864 \\
\hline 0.821808 & 0.0501677 & 2.44289 & 1.17609 & 4.01472 & 0.305917 & 7.85982 \\
\hline 0.799577 & 0.0422057 & 2.18433 & 1.04041 & 3.55078 & 0.277739 & 7.14655 \\
\hline 0.768415 & 0.0347857 & 1.90165 & 0.909514 & 3.02688 & 0.22792 & 6.33781 \\
\hline 0.747946 & 0.0336694 & 1.61043 & 0.762178 & 2.48763 & 0.183544 & 5.51245 \\
\hline 0.726036 & 0.0316346 & 1.3502 & 0.623193 & 1.98078 & 0.141132 & 4.72954 \\
\hline 0.70718 & 0.0323375 & 1.13464 & 0.517167 & 1.54734 & 0.106248 & 4.03254 \\
\hline 0.694931 & 0.0337009 & 0.95197 & 0.429403 & 1.18417 & 0.0846665 & 3.44904 \\
\hline 0.684584 & 0.0325681 & 0.805519 & 0.353893 & 0.890539 & 0.0580483 & 2.98488 \\
\hline 0.690149 & 0.0376781 & 0.706255 & 0.302115 & 0.665786 & 0.0427609 & 2.62152 \\
\hline 0.702845 & 0.0354651 & 0.646119 & 0.261338 & 0.508722 & 0.0344843 & 2.36989 \\
\hline 0.723058 & 0.0328066 & 0.620026 & 0.2482 & 0.411423 & 0.0246101 & 2.21111 \\
\hline 0.762223 & 0.0336426 & 0.609941 & 0.249825 & 0.353137 & 0.019643 & 2.10387 \\
\hline 0.807597 & 0.0337152 & 0.623811 & 0.254461 & 0.323474 & 0.0204366 & 2.04502 \\
\hline 0.865142 & 0.0291717 & 0.664488 & 0.271784 & 0.321013 & 0.0207822 & 2.03927 \\
\hline 0.950853 & 0.0315156 & 0.730034 & 0.296375 & 0.340413 & 0.0201965 & 2.06438 \\
\hline 1.05422 & 0.0436613 & 0.828442 & 0.328501 & 0.379745 & 0.0229455 & 2.11901 \\
\hline 1.20483 & 0.0552122 & 0.978705 & 0.391818 & 0.433591 & 0.0278689 & 2.20537 \\
\hline 1.43591 & 0.0651619 & 1.1936 & 0.477481 & 0.510566 & 0.0341381 & 2.28692 \\
\hline 1.77145 & 0.0719451 & 1.51542 & 0.604967 & 0.617378 & 0.0435289 & 2.39367 \\
\hline 2.27874 & 0.0847984 & 2.01323 & 0.822775 & 0.759494 & 0.0553272 & 2.55696 \\
\hline 3.07655 & 0.114858 & 2.75674 & 1.12624 & 0.933832 & 0.0647383 & 2.76393 \\
\hline 4.30867 & 0.167359 & 3.87345 & 1.56708 & 1.16927 & 0.0713231 & 3.01826 \\
\hline 6.18951 & 0.225492 & 5.48925 & 2.22595 & 1.49542 & 0.091603 & 3.32548 \\
\hline 8.98532 & 0.318582 & 7.77966 & 3.15677 & 1.91424 & 0.123485 & 3.6874 \\
\hline 13.0242 & 0.468534 & 10.9424 & 4.4446 & 2.45534 & 0.15895 & 4.1563 \\
\hline 18.686 & 0.651179 & 15.1334 & 6.15421 & 3.13465 & 0.202013 & 4.74669 \\
\hline 26.2776 & 0.945931 & 20.4153 & 8.31057 & 4.00558 & 0.267314 & 5.46666 \\
\hline 36.0684 & 1.27473 & 26.7938 & 10.9232 & 5.05605 & 0.354042 & 6.33902 \\
\hline 48.0681 & 1.66116 & 34.1742 & 13.9735 & 6.27023 & 0.436557 & 7.33242 \\
\hline 62.1404 & 2.1598 & 42.182 & 17.2683 & 7.60805 & 0.53029 & 8.36714 \\
\hline 77.8349 & 2.68989 & 50.3936 & 20.674 & 8.98722 & 0.647369 & 9.44524 \\
\hline 94.196 & 3.26809 & 58.2255 & 23.9004 & 10.3188 & 0.753848 & 10.5374 \\
\hline 110.173 & 3.79421 & 64.94 & 26.68 & 11.5096 & 0.82849 & 11.5265 \\
\hline 124.549 & 4.37613 & 70.0198 & 28.7817 & 12.4145 & 0.881681 & 12.2611 \\
\hline 135.845 & 4.77371 & 72.878 & 29.9643 & 12.951 & 0.926282 & 12.7053 \\
\hline 143.108 & 5.02775 & 73.1562 & 30.1799 & 13.0726 & 0.923292 & 12.796 \\
\hline 145.455 & 5.11556 & 70.8242 & 29.2608 & 12.7629 & 0.889428 & 12.491 \\
\hline 142.602 & 5.03641 & 66.1622 & 27.354 & 12.0228 & 0.837275 & 11.8958 \\
\hline 134.904 & 4.82674 & 59.7257 & 24.791 & 10.9309 & 0.75365 & 10.9824 \\
\hline 123.152 & 4.30528 & 51.9951 & 21.645 & 9.61012 & 0.679382 & 9.84788 \\
\hline 108.47 & 3.72035 & 43.6741 & 18.1927 & 8.15136 & 0.588674 & 8.58715 \\
\hline 92.1945 & 3.15397 & 35.4532 & 14.8295 & 6.69027 & 0.478474 & 7.39325 \\
\hline 75.703 & 2.5538 & 27.8149 & 11.6563 & 5.30643 & 0.390249 & 6.3114 \\
\hline 59.9368 & 2.0304 & 21.1262 & 8.83956 & 4.07131 & 0.316362 & 5.25825 \\
\hline 45.8596 & 1.58508 & 15.4913 & 6.48717 & 3.04163 & 0.224359 & 4.3699 \\
\hline 34.0088 & 1.18261 & 11.0078 & 4.59522 & 2.21145 & 0.152987 & 3.67314 \\
\hline
\end{tabular}




\begin{tabular}{|c|c|c|c|c|c|c|}
\hline 24.4163 & 0.869565 & 7.64904 & 3.19861 & 1.56974 & 0.105512 & 3.15665 \\
\hline 17.0133 & 0.608204 & 5.19477 & 2.17265 & 1.10361 & 0.0711724 & 2.77657 \\
\hline 11.5521 & 0.379906 & 3.49904 & 1.46057 & 0.786174 & 0.0516535 & 2.50174 \\
\hline 7.70807 & 0.23998 & 2.39628 & 0.989606 & 0.568602 & 0.0361629 & 2.3171 \\
\hline 5.14452 & 0.177302 & 1.70905 & 0.689266 & 0.429239 & 0.0292557 & 2.21368 \\
\hline 3.52825 & 0.126587 & 1.33102 & 0.541336 & 0.355055 & 0.0266109 & 2.16107 \\
\hline 2.58904 & 0.0830851 & 1.17372 & 0.473446 & 0.322755 & 0.0253118 & 2.1421 \\
\hline 2.14404 & 0.0747842 & 1.18866 & 0.466129 & 0.319099 & 0.0196485 & 2.14668 \\
\hline 2.05985 & 0.0754324 & 1.34221 & 0.515734 & 0.343115 & 0.0199806 & 2.14391 \\
\hline 2.25991 & 0.0801706 & 1.61642 & 0.621539 & 0.389441 & 0.0240918 & 2.1861 \\
\hline 2.70744 & 0.0867646 & 2.02064 & 0.775616 & 0.453042 & 0.0315252 & 2.26176 \\
\hline 3.40804 & 0.112464 & 2.56754 & 0.997697 & 0.544783 & 0.0374096 & 2.3543 \\
\hline 4.36658 & 0.156567 & 3.258 & 1.27887 & 0.670624 & 0.0447566 & 2.47575 \\
\hline 5.63177 & 0.195426 & 4.09496 & 1.59059 & 0.822397 & 0.0544719 & 2.60957 \\
\hline 7.19252 & 0.251477 & 5.07442 & 1.96982 & 0.994912 & 0.0704468 & 2.75601 \\
\hline 9.01366 & 0.323583 & 6.14645 & 2.3853 & 1.18411 & 0.0822348 & 2.93166 \\
\hline 11.0824 & 0.409603 & 7.22699 & 2.81386 & 1.37734 & 0.0798762 & 3.09912 \\
\hline 13.2466 & 0.471928 & 8.2801 & 3.25528 & 1.57448 & 0.0962844 & 3.24567 \\
\hline 15.3667 & 0.538853 & 9.22545 & 3.62095 & 1.7567 & 0.117444 & 3.40128 \\
\hline 17.3661 & 0.619849 & 9.94813 & 3.89783 & 1.89461 & 0.115539 & 3.51214 \\
\hline 19.0481 & 0.674242 & 10.4021 & 4.0892 & 1.99933 & 0.13196 & 3.57858 \\
\hline 20.208 & 0.726503 & 10.5151 & 4.13564 & 2.05438 & 0.144878 & 3.64308 \\
\hline 20.7467 & 0.714494 & 10.3162 & 4.05995 & 2.02918 & 0.136225 & 3.64275 \\
\hline 20.6332 & 0.686782 & 9.81264 & 3.87932 & 1.94405 & 0.128979 & 3.56854 \\
\hline 19.8924 & 0.670902 & 9.00626 & 3.57199 & 1.8119 & 0.116335 & 3.46459 \\
\hline 18.5386 & 0.62738 & 8.01591 & 3.17443 & 1.64057 & 0.105987 & 3.32815 \\
\hline 16.723 & 0.583533 & 6.92155 & 2.74412 & 1.4437 & 0.0960417 & 3.1798 \\
\hline 14.5656 & 0.501739 & 5.77495 & 2.31308 & 1.2423 & 0.08165 & 3.00898 \\
\hline 12.2713 & 0.412997 & 4.66078 & 1.86153 & 1.04082 & 0.066141 & 2.83254 \\
\hline 10.0426 & 0.349927 & 3.65764 & 1.4562 & 0.847344 & 0.0537372 & 2.6894 \\
\hline 7.93488 & 0.266115 & 2.79242 & 1.10629 & 0.681468 & 0.0467017 & 2.56724 \\
\hline 6.06653 & 0.220508 & 2.0704 & 0.811067 & 0.549258 & 0.0356838 & 2.43993 \\
\hline 4.51484 & 0.180521 & 1.51129 & 0.591619 & 0.433533 & 0.0274243 & 2.36779 \\
\hline 3.27056 & 0.126859 & 1.09174 & 0.431047 & 0.34861 & 0.0240066 & 2.31411 \\
\hline 2.29831 & 0.0883192 & 0.782046 & 0.303877 & 0.286755 & 0.0182613 & 2.23318 \\
\hline 1.57484 & 0.0710781 & 0.567075 & 0.217378 & 0.239693 & 0.0136457 & 2.22069 \\
\hline 1.05527 & 0.0494537 & 0.414133 & 0.160986 & 0.208033 & 0.0128152 & 2.22457 \\
\hline 0.703673 & 0.0314026 & 0.31655 & 0.127162 & 0.188046 & 0.01153 & 2.17331 \\
\hline 0.476854 & 0.0253823 & 0.25107 & 0.101317 & 0.175112 & 0.0111809 & 2.15809 \\
\hline 0.329256 & 0.0185118 & 0.213116 & 0.0888635 & 0.167986 & 0.00962738 & 2.17727 \\
\hline 0.231128 & 0.0146133 & 0.194014 & 0.0809568 & 0.163532 & 0.0117392 & 2.17757 \\
\hline 0.172857 & 0.0115835 & 0.178248 & 0.0715275 & 0.161385 & 0.0115909 & 2.18562 \\
\hline 0.143106 & 0.00800486 & 0.172702 & 0.0721986 & 0.162679 & 0.00980729 & 2.19674 \\
\hline 0.126101 & 0.0101835 & 0.169672 & 0.0747933 & 0.161311 & 0.0105517 & 2.21503 \\
\hline 0.112878 & 0.0121306 & 0.169838 & 0.077344 & 0.162557 & 0.0118261 & 2.21892 \\
\hline 0.10546 & 0.012742 & 0.169564 & 0.0793709 & 0.165594 & 0.0122762 & 2.23852 \\
\hline 0.0980207 & 0.00988317 & 0.169467 & 0.0757913 & 0.169452 & 0.0121086 & 2.26687 \\
\hline
\end{tabular}




\begin{tabular}{|c|c|c|c|c|c|c|}
\hline 0.0971087 & 0.00770268 & 0.171192 & 0.0758179 & 0.170698 & 0.0118805 & 2.26679 \\
\hline 0.0952662 & 0.00924388 & 0.173165 & 0.0787328 & 0.170996 & 0.012848 & 2.25903 \\
\hline 0.0924062 & 0.0105983 & 0.174622 & 0.0787779 & 0.175085 & 0.0111951 & 2.28109 \\
\hline 0.0917546 & 0.00793704 & 0.177833 & 0.0828147 & 0.182862 & 0.0130785 & 2.30106 \\
\hline 0.0921165 & 0.00549585 & 0.181045 & 0.0860011 & 0.190733 & 0.0136419 & 2.33797 \\
\hline 0.0898673 & 0.00722141 & 0.183237 & 0.0858833 & 0.200782 & 0.0106478 & 2.35288 \\
\hline 0.0878878 & 0.0070874 & 0.192121 & 0.0890881 & 0.214852 & 0.012575 & 2.40133 \\
\hline 0.0897843 & 0.00506456 & 0.204621 & 0.0962997 & 0.23578 & 0.01354 & 2.43386 \\
\hline 0.0898014 & 0.0074409 & 0.218496 & 0.109232 & 0.268041 & 0.0146619 & 2.48122 \\
\hline 0.0892425 & 0.00756517 & 0.239814 & 0.126027 & 0.31176 & 0.0164656 & 2.57761 \\
\hline 0.0902533 & 0.00888054 & 0.265511 & 0.14716 & 0.37361 & 0.0213875 & 2.71033 \\
\hline 0.0947633 & 0.00872779 & 0.302629 & 0.194088 & 0.454766 & 0.0301856 & 2.87886 \\
\hline 0.100709 & 0.0110056 & 0.355038 & 0.26178 & 0.566086 & 0.0386928 & 3.09635 \\
\hline 0.108737 & 0.0141541 & 0.418635 & 0.334223 & 0.711747 & 0.0440866 & 3.39576 \\
\hline 0.11304 & 0.0171639 & 0.500131 & 0.432688 & 0.896421 & 0.0581731 & 3.70501 \\
\hline 0.121754 & 0.020825 & 0.595537 & 0.559972 & 1.11604 & 0.0714759 & 4.0963 \\
\hline 0.137492 & 0.0262649 & 0.705957 & 0.701926 & 1.36784 & 0.0907241 & 4.59449 \\
\hline 0.150986 & 0.0340488 & 0.827499 & 0.864483 & 1.64794 & 0.103825 & 5.11626 \\
\hline 0.163956 & 0.0409616 & 0.949433 & 1.03914 & 1.94565 & 0.120789 & 5.6217 \\
\hline 0.181431 & 0.0517584 & 1.07187 & 1.21251 & 2.23843 & 0.14007 & 6.17742 \\
\hline 0.199876 & 0.0584596 & 1.18844 & 1.37194 & 2.51026 & 0.164536 & 6.6782 \\
\hline 0.213217 & 0.0687115 & 1.28457 & 1.51914 & 2.74586 & 0.171833 & 7.08747 \\
\hline 0.225729 & 0.0734272 & 1.34406 & 1.62555 & 2.91906 & 0.185284 & 7.40739 \\
\hline 0.237254 & 0.0788782 & 1.37192 & 1.66773 & 3.00133 & 0.19812 & 7.59811 \\
\hline 0.245219 & 0.089259 & 1.3705 & 1.69441 & 3.01794 & 0.201733 & 7.64285 \\
\hline 0.251795 & 0.0957245 & 1.32953 & 1.64848 & 2.9541 & 0.185573 & 7.58298 \\
\hline 0.255958 & 0.0866469 & 1.25985 & 1.54246 & 2.79845 & 0.176453 & 7.3602 \\
\hline 0.247662 & 0.0827964 & 1.17047 & 1.42166 & 2.57875 & 0.163032 & 6.97784 \\
\hline 0.23464 & 0.0813667 & 1.04754 & 1.26036 & 2.29696 & 0.137901 & 6.57841 \\
\hline 0.229318 & 0.0808938 & 0.929903 & 1.09324 & 2.00321 & 0.13354 & 6.11763 \\
\hline 0.220743 & 0.0665006 & 0.806283 & 0.905831 & 1.70694 & 0.112303 & 5.62412 \\
\hline 0.207048 & 0.0522694 & 0.691258 & 0.732615 & 1.41277 & 0.0849779 & 5.09845 \\
\hline 0.194389 & 0.0370583 & 0.58615 & 0.587295 & 1.15989 & 0.071677 & 4.67631 \\
\hline 0.181991 & 0.0376021 & 0.494717 & 0.462323 & 0.939926 & 0.058404 & 4.31514 \\
\hline 0.170625 & 0.0297795 & 0.415741 & 0.335834 & 0.748609 & 0.0494313 & 4.04222 \\
\hline 0.160793 & 0.026302 & 0.359954 & 0.24821 & 0.59913 & 0.0441819 & 3.86587 \\
\hline 0.153482 & 0.0204461 & 0.316739 & 0.200006 & 0.493103 & 0.036404 & 3.63708 \\
\hline 0.149322 & 0.0161264 & 0.290419 & 0.163824 & 0.409486 & 0.0291076 & 3.57888 \\
\hline 0.139231 & 0.0132671 & 0.274148 & 0.136459 & 0.349712 & 0.0242671 & 3.55219 \\
\hline 0.137264 & 0.0105368 & 0.250626 & 0.120827 & 0.31363 & 0.0208224 & 3.50128 \\
\hline 0.132414 & 0.0118896 & 0.245018 & 0.111573 & 0.289759 & 0.0239937 & 3.4261 \\
\hline 0.130831 & 0.0140143 & 0.240974 & 0.11498 & 0.272726 & 0.0257093 & 3.38189 \\
\hline 0.131222 & 0.0108052 & 0.24318 & 0.10871 & 0.263474 & 0.0218123 & 3.38224 \\
\hline 0.125637 & 0.00946786 & 0.246535 & 0.105663 & 0.263743 & 0.0208935 & 3.35695 \\
\hline 0.124988 & 0.0118189 & 0.243467 & 0.106908 & 0.265142 & 0.0172754 & 3.32365 \\
\hline 0.123967 & 0.0127261 & 0.244987 & 0.110067 & 0.272151 & 0.0169821 & 3.34296 \\
\hline 0.12186 & 0.0104646 & 0.246808 & 0.108375 & 0.27929 & 0.0149712 & 3.34193 \\
\hline
\end{tabular}




\begin{tabular}{|c|c|c|c|c|c|c|}
\hline 0.123058 & 0.0108462 & 0.247076 & 0.105152 & 0.291366 & 0.0244187 & 3.30676 \\
\hline 0.12637 & 0.0121867 & 0.252998 & 0.106551 & 0.307845 & 0.022544 & 3.34272 \\
\hline 0.125766 & 0.0112009 & 0.261795 & 0.118868 & 0.329025 & 0.0222564 & 3.35771 \\
\hline 0.128551 & 0.0140629 & 0.269398 & 0.121473 & 0.354547 & 0.028397 & 3.36441 \\
\hline 0.130737 & 0.0112497 & 0.28064 & 0.128357 & 0.379885 & 0.0267315 & 3.40052 \\
\hline 0.133933 & 0.0111301 & 0.296098 & 0.135897 & 0.41207 & 0.0326978 & 3.44747 \\
\hline 0.130692 & 0.0129497 & 0.308611 & 0.134725 & 0.442399 & 0.0334053 & 3.48123 \\
\hline 0.133694 & 0.0118204 & 0.323759 & 0.149728 & 0.475895 & 0.0366609 & 3.56186 \\
\hline 0.137587 & 0.0115689 & 0.337564 & 0.157505 & 0.51244 & 0.0377843 & 3.60963 \\
\hline 0.138529 & 0.0133954 & 0.351092 & 0.167931 & 0.546646 & 0.0410919 & 3.6571 \\
\hline 0.142767 & 0.0145518 & 0.362274 & 0.179165 & 0.586065 & 0.0467371 & 3.75075 \\
\hline 0.14467 & 0.0132928 & 0.38375 & 0.20165 & 0.627239 & 0.0489208 & 3.79071 \\
\hline 0.142328 & 0.0114496 & 0.407054 & 0.225756 & 0.661063 & 0.0498674 & 3.89221 \\
\hline 0.143778 & 0.013685 & 0.426387 & 0.244071 & 0.707026 & 0.041594 & 3.95707 \\
\hline 0.144424 & 0.0127217 & 0.446211 & 0.268689 & 0.751786 & 0.042591 & 4.03181 \\
\hline 0.152002 & 0.0135893 & 0.460228 & 0.285813 & 0.796918 & 0.0475565 & 4.12815 \\
\hline 0.150444 & 0.0141152 & 0.475708 & 0.304434 & 0.829703 & 0.0479264 & 4.19409 \\
\hline 0.152471 & 0.0163489 & 0.494587 & 0.331554 & 0.868429 & 0.0487716 & 4.2507 \\
\hline 0.155092 & 0.017643 & 0.504033 & 0.346603 & 0.89328 & 0.0497712 & 4.28171 \\
\hline 0.159695 & 0.021127 & 0.512556 & 0.356103 & 0.890999 & 0.0503062 & 4.29754 \\
\hline 0.162607 & 0.0204253 & 0.509767 & 0.354734 & 0.88971 & 0.0519601 & 4.26117 \\
\hline 0.163466 & 0.0185693 & 0.497335 & 0.349415 & 0.864481 & 0.0516863 & 4.2419 \\
\hline 0.165249 & 0.0185024 & 0.490542 & 0.352077 & 0.831193 & 0.0492199 & 4.20328 \\
\hline 0.165146 & 0.0179473 & 0.478263 & 0.340979 & 0.782683 & 0.0475892 & 4.09476 \\
\hline 0.162119 & 0.0196238 & 0.461823 & 0.338194 & 0.728379 & 0.0410541 & 3.98443 \\
\hline 0.162968 & 0.0163457 & 0.437558 & 0.318276 & 0.667684 & 0.0294894 & 3.88418 \\
\hline 0.156878 & 0.0146794 & 0.408823 & 0.301095 & 0.602421 & 0.0367164 & 3.7435 \\
\hline 0.15257 & 0.0152804 & 0.389161 & 0.292023 & 0.54394 & 0.0307304 & 3.66942 \\
\hline 0.148613 & 0.0139917 & 0.370002 & 0.285531 & 0.490371 & 0.0291405 & 3.57853 \\
\hline 0.149875 & 0.0147743 & 0.352907 & 0.265869 & 0.44622 & 0.0227159 & 3.44511 \\
\hline 0.150155 & 0.0141225 & 0.343501 & 0.26225 & 0.40526 & 0.019155 & 3.37574 \\
\hline 0.145958 & 0.0110783 & 0.334522 & 0.257518 & 0.37417 & 0.0216121 & 3.3222 \\
\hline 0.142247 & 0.0106191 & 0.327766 & 0.253398 & 0.349518 & 0.0197755 & 3.27709 \\
\hline 0.143534 & 0.0128977 & 0.320727 & 0.249955 & 0.332081 & 0.0165487 & 3.28408 \\
\hline 0.147571 & 0.0145961 & 0.31339 & 0.237044 & 0.321084 & 0.0172092 & 3.26918 \\
\hline 0.145757 & 0.0149061 & 0.312009 & 0.239898 & 0.316791 & 0.0212081 & 3.27252 \\
\hline 0.143223 & 0.0119269 & 0.314326 & 0.231058 & 0.314331 & 0.0206615 & 3.24132 \\
\hline 0.14271 & 0.0109012 & 0.314794 & 0.216943 & 0.312989 & 0.0197039 & 3.31764 \\
\hline 0.148662 & 0.0121301 & 0.317321 & 0.215891 & 0.31506 & 0.0196044 & 3.35709 \\
\hline 0.146175 & 0.0142393 & 0.318855 & 0.215501 & 0.323614 & 0.0197624 & 3.33443 \\
\hline 0.141352 & 0.0123073 & 0.319025 & 0.208902 & 0.328031 & 0.0181358 & 3.42681 \\
\hline 0.145824 & 0.00935231 & 0.317346 & 0.194354 & 0.33339 & 0.0191147 & 3.44265 \\
\hline 0.145983 & 0.012859 & 0.320002 & 0.190173 & 0.335332 & 0.025755 & 3.4585 \\
\hline 0.148751 & 0.0145125 & 0.321852 & 0.196601 & 0.346897 & 0.0249856 & 3.47428 \\
\hline 0.145033 & 0.0135608 & 0.324388 & 0.192799 & 0.344496 & 0.0219798 & 3.52318 \\
\hline 0.145449 & 0.0118917 & 0.316945 & 0.178781 & 0.345533 & 0.0212615 & 3.55758 \\
\hline 0.147925 & 0.0135539 & 0.317279 & 0.168387 & 0.345567 & 0.023398 & 3.59928 \\
\hline
\end{tabular}




\begin{tabular}{|c|c|c|c|c|c|c|}
\hline 0.149046 & 0.0151232 & 0.311428 & 0.169444 & 0.345719 & 0.0236214 & 3.62263 \\
\hline 0.145052 & 0.0120739 & 0.321141 & 0.168793 & 0.350052 & 0.0236602 & 3.65921 \\
\hline 0.148941 & 0.0173923 & 0.317219 & 0.157605 & 0.349658 & 0.0224828 & 3.66147 \\
\hline 0.148862 & 0.0163482 & 0.317117 & 0.155625 & 0.354829 & 0.0249996 & 3.71966 \\
\hline 0.150637 & 0.0123972 & 0.321917 & 0.153044 & 0.355674 & 0.0279649 & 3.75717 \\
\hline 0.151626 & 0.0160471 & 0.321856 & 0.154492 & 0.355803 & 0.02133 & 3.84095 \\
\hline 0.154435 & 0.0144171 & 0.329338 & 0.153386 & 0.361652 & 0.0249951 & 3.8931 \\
\hline 0.152992 & 0.0148324 & 0.341486 & 0.154579 & 0.379238 & 0.0280349 & 3.976 \\
\hline 0.154502 & 0.01426 & 0.352842 & 0.157412 & 0.398063 & 0.0284992 & 4.13856 \\
\hline 0.15881 & 0.0130098 & 0.380094 & 0.163256 & 0.420075 & 0.0325171 & 4.35679 \\
\hline 0.161974 & 0.015563 & 0.399441 & 0.174356 & 0.445194 & 0.0359809 & 4.51281 \\
\hline 0.167055 & 0.0147217 & 0.437673 & 0.187232 & 0.483076 & 0.0374168 & 4.78107 \\
\hline 0.171432 & 0.0124857 & 0.471153 & 0.213697 & 0.522551 & 0.0352422 & 5.04717 \\
\hline 0.176481 & 0.0151044 & 0.514686 & 0.240125 & 0.572424 & 0.044832 & 5.41671 \\
\hline 0.184784 & 0.0134373 & 0.559292 & 0.269614 & 0.626839 & 0.045095 & 5.72629 \\
\hline 0.192962 & 0.0148093 & 0.608603 & 0.300128 & 0.674825 & 0.0532268 & 6.06146 \\
\hline 0.197919 & 0.0163869 & 0.651811 & 0.330972 & 0.732995 & 0.0532861 & 6.39242 \\
\hline 0.201462 & 0.0122642 & 0.693177 & 0.3459 & 0.780308 & 0.0621752 & 6.75486 \\
\hline 0.209317 & 0.0193193 & 0.723231 & 0.36429 & 0.828316 & 0.0593006 & 6.97735 \\
\hline 0.213598 & 0.0175184 & 0.746434 & 0.374711 & 0.857213 & 0.0678454 & 7.15261 \\
\hline 0.218603 & 0.0203201 & 0.75398 & 0.387098 & 0.869193 & 0.0684601 & 7.25188 \\
\hline 0.228703 & 0.0170431 & 0.754823 & 0.386219 & 0.88014 & 0.0647953 & 7.27763 \\
\hline 0.230293 & 0.0188532 & 0.735609 & 0.369397 & 0.866388 & 0.0574937 & 7.24742 \\
\hline 0.229489 & 0.0173821 & 0.706607 & 0.351078 & 0.837922 & 0.0627825 & 7.09914 \\
\hline 0.233927 & 0.0188456 & 0.680182 & 0.331935 & 0.803116 & 0.0587948 & 6.87438 \\
\hline 0.228963 & 0.0150076 & 0.647225 & 0.298023 & 0.748522 & 0.0515251 & 6.58341 \\
\hline 0.230005 & 0.0216778 & 0.600032 & 0.267951 & 0.706264 & 0.0515851 & 6.24071 \\
\hline 0.232828 & 0.021338 & 0.548747 & 0.24216 & 0.654641 & 0.044061 & 6.00921 \\
\hline 0.229174 & 0.0175924 & 0.525417 & 0.220791 & 0.610766 & 0.03857 & 5.71482 \\
\hline 0.221265 & 0.0160429 & 0.482829 & 0.192299 & 0.562102 & 0.0318643 & 5.39292 \\
\hline 0.219507 & 0.0195085 & 0.449678 & 0.180971 & 0.53023 & 0.036165 & 5.15741 \\
\hline 0.228013 & 0.0179282 & 0.427808 & 0.175341 & 0.492702 & 0.0308751 & 4.9542 \\
\hline 0.222046 & 0.0166245 & 0.408881 & 0.160208 & 0.464742 & 0.031061 & 4.78852 \\
\hline 0.221653 & 0.0144172 & 0.394551 & 0.159007 & 0.441589 & 0.0246756 & 4.65071 \\
\hline 0.220926 & 0.0182145 & 0.378389 & 0.15381 & 0.429736 & 0.0269686 & 4.56206 \\
\hline 0.221841 & 0.0163525 & 0.370679 & 0.153299 & 0.415957 & 0.0247664 & 4.46882 \\
\hline 0.21921 & 0.0186856 & 0.364527 & 0.148449 & 0.41271 & 0.0240632 & 4.44359 \\
\hline 0.220505 & 0.0152808 & 0.35406 & 0.139173 & 0.394001 & 0.0265526 & 4.41204 \\
\hline 0.215325 & 0.0176884 & 0.350381 & 0.137091 & 0.393207 & 0.0219366 & 4.3556 \\
\hline 0.216778 & 0.0143858 & 0.358948 & 0.149811 & 0.39696 & 0.0213097 & 4.34829 \\
\hline 0.214662 & 0.0181113 & 0.353149 & 0.147498 & 0.390084 & 0.0309783 & 4.26756 \\
\hline 0.215885 & 0.0139064 & 0.35065 & 0.149049 & 0.390534 & 0.0227882 & 4.26594 \\
\hline 0.211902 & 0.0200428 & 0.347938 & 0.14198 & 0.393936 & 0.0189791 & 4.27883 \\
\hline 0.214447 & 0.0199218 & 0.353269 & 0.146322 & 0.39138 & 0.025652 & 4.273 \\
\hline 0.212056 & 0.0163734 & 0.354931 & 0.144917 & 0.391771 & 0.0231682 & 4.26684 \\
\hline 0.206556 & 0.0202983 & 0.357318 & 0.147613 & 0.391167 & 0.0206284 & 4.26931 \\
\hline 0.207812 & 0.0174127 & 0.353341 & 0.141449 & 0.390935 & 0.0279325 & 4.26516 \\
\hline
\end{tabular}




\begin{tabular}{|c|c|c|c|c|c|c|}
\hline 0.216472 & 0.0152578 & 0.359126 & 0.145813 & 0.393426 & 0.0226633 & 4.29264 \\
\hline 0.211488 & 0.0161351 & 0.365582 & 0.155306 & 0.399975 & 0.0257843 & 4.35138 \\
\hline 0.213214 & 0.0156818 & 0.374822 & 0.150157 & 0.403243 & 0.0234961 & 4.37902 \\
\hline 0.211663 & 0.0179413 & 0.381162 & 0.160368 & 0.408233 & 0.0278467 & 4.42605 \\
\hline 0.214777 & 0.0150893 & 0.392274 & 0.156559 & 0.418365 & 0.0266725 & 4.52613 \\
\hline 0.221949 & 0.0150355 & 0.400222 & 0.160519 & 0.432384 & 0.0263109 & 4.60651 \\
\hline 0.22571 & 0.0182687 & 0.4137 & 0.155322 & 0.447941 & 0.0285337 & 4.81191 \\
\hline 0.224718 & 0.0205449 & 0.44535 & 0.186208 & 0.465081 & 0.0235906 & 4.98527 \\
\hline 0.227831 & 0.0202068 & 0.459381 & 0.178791 & 0.49756 & 0.0340678 & 5.26262 \\
\hline 0.239124 & 0.017129 & 0.499573 & 0.207009 & 0.527918 & 0.0307861 & 5.65283 \\
\hline 0.238537 & 0.0217245 & 0.548352 & 0.217352 & 0.572157 & 0.0383831 & 6.01328 \\
\hline 0.251113 & 0.0176954 & 0.595772 & 0.243493 & 0.618863 & 0.0509835 & 6.61848 \\
\hline 0.256171 & 0.0214945 & 0.654659 & 0.270917 & 0.682692 & 0.0615216 & 7.21887 \\
\hline 0.271015 & 0.0184257 & 0.7282 & 0.302188 & 0.75715 & 0.0636241 & 7.97149 \\
\hline 0.275798 & 0.0222717 & 0.814155 & 0.337937 & 0.835002 & 0.0752566 & 8.76868 \\
\hline 0.295402 & 0.0223111 & 0.902858 & 0.391005 & 0.923046 & 0.0914935 & 9.67787 \\
\hline 0.315952 & 0.0268241 & 0.994102 & 0.420639 & 1.01304 & 0.0936975 & 10.6656 \\
\hline 0.327325 & 0.0238547 & 1.07445 & 0.459839 & 1.11107 & 0.114447 & 11.591 \\
\hline 0.342119 & 0.0193821 & 1.15046 & 0.486367 & 1.18647 & 0.126837 & 12.4324 \\
\hline 0.369209 & 0.028592 & 1.21077 & 0.524454 & 1.26704 & 0.139877 & 13.2361 \\
\hline 0.373247 & 0.0239276 & 1.28295 & 0.558781 & 1.33183 & 0.148101 & 13.8814 \\
\hline 0.392341 & 0.0215759 & 1.30148 & 0.558322 & 1.36922 & 0.151423 & 14.3497 \\
\hline 0.410861 & 0.0253978 & 1.31823 & 0.56966 & 1.37763 & 0.155605 & 14.611 \\
\hline 0.42209 & 0.0323473 & 1.28378 & 0.539327 & 1.37145 & 0.150202 & 14.4928 \\
\hline 0.438091 & 0.0238554 & 1.27153 & 0.539763 & 1.34302 & 0.147381 & 14.2743 \\
\hline 0.449086 & 0.0348606 & 1.22619 & 0.51928 & 1.29643 & 0.134954 & 13.8714 \\
\hline 0.457838 & 0.0342788 & 1.15901 & 0.506089 & 1.22615 & 0.127967 & 13.2905 \\
\hline 0.464444 & 0.0262884 & 1.08741 & 0.468389 & 1.15124 & 0.114821 & 12.746 \\
\hline 0.473033 & 0.0295967 & 1.01224 & 0.43391 & 1.05672 & 0.097336 & 11.9609 \\
\hline 0.469797 & 0.026727 & 0.94856 & 0.407582 & 0.98826 & 0.0975887 & 11.2457 \\
\hline 0.464054 & 0.0281947 & 0.875779 & 0.365666 & 0.90813 & 0.0678399 & 10.5519 \\
\hline 0.46515 & 0.0270696 & 0.819963 & 0.357007 & 0.835077 & 0.058176 & 9.91715 \\
\hline 0.4688 & 0.02286 & 0.764113 & 0.311116 & 0.767797 & 0.0587927 & 9.50348 \\
\hline 0.456843 & 0.0251147 & 0.734694 & 0.320834 & 0.719934 & 0.0510806 & 9.09349 \\
\hline 0.442797 & 0.0195384 & 0.686322 & 0.289804 & 0.681436 & 0.0511618 & 8.81822 \\
\hline 0.422219 & 0.0277837 & 0.668567 & 0.286091 & 0.647365 & 0.0410718 & 8.61173 \\
\hline 0.416284 & 0.0312156 & 0.648767 & 0.286943 & 0.619381 & 0.0313908 & 8.67573 \\
\hline 0.397437 & 0.0305841 & 0.640278 & 0.280754 & 0.607864 & 0.0389783 & 8.7262 \\
\hline 0.385937 & 0.0216929 & 0.642906 & 0.290999 & 0.601284 & 0.031957 & 8.85314 \\
\hline 0.356066 & 0.0284117 & 0.636522 & 0.280235 & 0.59892 & 0.0432301 & 8.95609 \\
\hline 0.345045 & 0.0246834 & 0.646084 & 0.294025 & 0.598874 & 0.0381895 & 9.33001 \\
\hline 0.326019 & 0.0180854 & 0.65544 & 0.309892 & 0.617907 & 0.0446498 & 9.65088 \\
\hline 0.31662 & 0.0246355 & 0.671291 & 0.31542 & 0.621808 & 0.0392404 & 9.99678 \\
\hline 0.299395 & 0.0223628 & 0.690016 & 0.329579 & 0.629226 & 0.0400584 & 10.4347 \\
\hline 0.289159 & 0.0217964 & 0.708301 & 0.33833 & 0.656287 & 0.0424043 & 10.9676 \\
\hline 0.283025 & 0.0236775 & 0.732611 & 0.354816 & 0.674192 & 0.0461824 & 11.5251 \\
\hline 0.275868 & 0.019359 & 0.767835 & 0.379215 & 0.70532 & 0.0522659 & 12.2797 \\
\hline
\end{tabular}




\begin{tabular}{|c|c|c|c|c|c|c|}
\hline 0.272521 & 0.0171116 & 0.807115 & 0.400727 & 0.743744 & 0.0500931 & 13.1265 \\
\hline 0.269378 & 0.0227351 & 0.860383 & 0.436877 & 0.782653 & 0.0550128 & 14.3142 \\
\hline 0.272195 & 0.0167459 & 0.945155 & 0.497787 & 0.845549 & 0.0537513 & 15.9475 \\
\hline 0.274476 & 0.0157399 & 1.064 & 0.577313 & 0.932381 & 0.0685313 & 18.1253 \\
\hline 0.272904 & 0.0190724 & 1.2361 & 0.694522 & 1.0681 & 0.0787304 & 21.594 \\
\hline 0.271792 & 0.0181558 & 1.4809 & 0.837463 & 1.25639 & 0.0882433 & 26.5893 \\
\hline 0.284012 & 0.018211 & 1.88081 & 1.09562 & 1.54329 & 0.113704 & 33.982 \\
\hline 0.291995 & 0.0205171 & 2.45248 & 1.49893 & 1.96464 & 0.129893 & 44.8513 \\
\hline 0.302678 & 0.0243775 & 3.28351 & 2.02708 & 2.58857 & 0.187473 & 60.8162 \\
\hline 0.335683 & 0.0260234 & 4.46787 & 2.81763 & 3.47236 & 0.233557 & 83.7091 \\
\hline 0.355731 & 0.0235856 & 6.08874 & 3.8666 & 4.714 & 0.314095 & 115.53 \\
\hline 0.405823 & 0.0274803 & 8.30852 & 5.33036 & 6.39116 & 0.452 & 159.485 \\
\hline 0.451694 & 0.0346639 & 11.198 & 7.16914 & 8.67245 & 0.604677 & 217.491 \\
\hline 0.536835 & 0.0434415 & 14.9134 & 9.63513 & 11.619 & 0.818885 & 291.832 \\
\hline 0.629114 & 0.0379553 & 19.5287 & 12.6294 & 15.2631 & 1.04547 & 386.63 \\
\hline 0.765389 & 0.0469514 & 25.0942 & 16.229 & 19.7363 & 1.39397 & 500.823 \\
\hline 0.925909 & 0.07662 & 31.5532 & 20.4737 & 24.9782 & 1.74931 & 635.35 \\
\hline 1.1057 & 0.0844052 & 38.8303 & 25.3203 & 31.0223 & 2.2071 & 788.032 \\
\hline 1.33524 & 0.09238 & 46.5039 & 30.251 & 37.4553 & 2.62381 & 954.263 \\
\hline 1.57232 & 0.127661 & 54.4471 & 35.4681 & 44.3957 & 3.15239 & 1128.13 \\
\hline 1.85819 & 0.132348 & 62.3304 & 40.5179 & 51.2113 & 3.59605 & 1304.18 \\
\hline 2.15061 & 0.165043 & 69.446 & 45.2448 & 57.6616 & 4.04778 & 1467.9 \\
\hline 2.42904 & 0.183177 & 75.5149 & 48.9825 & 63.4149 & 4.5032 & 1613.48 \\
\hline 2.68121 & 0.201296 & 80.0848 & 52.115 & 67.8632 & 4.80237 & 1726.77 \\
\hline 2.9117 & 0.225226 & 82.8787 & 54.0522 & 71.0353 & 5.14113 & 1806.12 \\
\hline 3.08435 & 0.213983 & 83.509 & 54.4057 & 72.3199 & 5.08934 & 1839.23 \\
\hline 3.19734 & 0.231403 & 82.1536 & 53.4507 & 71.8206 & 5.13461 & 1827.86 \\
\hline 3.26731 & 0.233598 & 78.7382 & 51.316 & 69.6078 & 4.89791 & 1771.84 \\
\hline 3.24591 & 0.232052 & 73.6831 & 47.9922 & 65.7734 & 4.65259 & 1673.66 \\
\hline 3.14064 & 0.26008 & 67.0878 & 43.7705 & 60.6819 & 4.2615 & 1543.41 \\
\hline 2.99782 & 0.196674 & 59.7524 & 38.8979 & 54.4858 & 3.82542 & 1387.29 \\
\hline 2.74969 & 0.201956 & 51.78 & 33.5889 & 47.754 & 3.38237 & 1215.92 \\
\hline 2.5149 & 0.190806 & 43.8766 & 28.4466 & 40.8022 & 2.97603 & 1039.97 \\
\hline 2.23372 & 0.160146 & 36.1749 & 23.5578 & 34.0506 & 2.37639 & 868.368 \\
\hline 1.94913 & 0.146123 & 29.0978 & 18.8918 & 27.7359 & 1.99148 & 706.721 \\
\hline 1.68283 & 0.126273 & 22.9526 & 14.8644 & 22.1033 & 1.61426 & 562.352 \\
\hline 1.44562 & 0.106121 & 17.6548 & 11.4375 & 17.1603 & 1.23426 & 436.835 \\
\hline 1.20703 & 0.0797906 & 13.3174 & 8.59378 & 13.0718 & 0.907897 & 331.916 \\
\hline 1.02663 & 0.0812693 & 9.85315 & 6.30104 & 9.74091 & 0.6897 & 247.052 \\
\hline 0.846431 & 0.04934 & 7.15185 & 4.54584 & 7.13622 & 0.501568 & 179.822 \\
\hline 0.729146 & 0.0418396 & 5.14876 & 3.19753 & 5.18712 & 0.353035 & 128.947 \\
\hline 0.631396 & 0.0375157 & 3.67944 & 2.24968 & 3.69742 & 0.255662 & 91.16 \\
\hline 0.560745 & 0.0394308 & 2.63366 & 1.59079 & 2.64536 & 0.192723 & 63.8324 \\
\hline 0.498128 & 0.0401024 & 1.90874 & 1.09133 & 1.9161 & 0.142505 & 44.6391 \\
\hline 0.454944 & 0.0310433 & 1.41439 & 0.760179 & 1.41507 & 0.0945908 & 31.4279 \\
\hline 0.42886 & 0.0288671 & 1.10902 & 0.581204 & 1.09306 & 0.0697589 & 22.5637 \\
\hline 0.409371 & 0.0235671 & 0.899481 & 0.435672 & 0.865589 & 0.0506758 & 16.8046 \\
\hline
\end{tabular}




\begin{tabular}{|c|c|c|c|c|c|c|}
\hline 0.402467 & 0.0335621 & 0.762014 & 0.345078 & 0.733573 & 0.0459525 & 13.0441 \\
\hline 0.394832 & 0.0266795 & 0.690678 & 0.297054 & 0.646375 & 0.0438198 & 10.6655 \\
\hline 0.382728 & 0.0240049 & 0.64285 & 0.271698 & 0.593725 & 0.0352859 & 9.11927 \\
\hline 0.386637 & 0.0252377 & 0.603266 & 0.245015 & 0.559156 & 0.033398 & 8.21384 \\
\hline 0.391509 & 0.0312597 & 0.599583 & 0.238052 & 0.541745 & 0.0304902 & 7.5785 \\
\hline 0.391369 & 0.0255311 & 0.598366 & 0.233071 & 0.538124 & 0.0350722 & 7.20473 \\
\hline 0.390883 & 0.0278155 & 0.592209 & 0.23034 & 0.533723 & 0.030353 & 6.91542 \\
\hline 0.392718 & 0.0245059 & 0.589347 & 0.226832 & 0.525171 & 0.0291192 & 6.79009 \\
\hline 0.400349 & 0.0263058 & 0.59533 & 0.233519 & 0.53573 & 0.0360621 & 6.69508 \\
\hline 0.402413 & 0.0265483 & 0.596114 & 0.234921 & 0.538841 & 0.0347248 & 6.72511 \\
\hline 0.4025 & 0.0308385 & 0.60782 & 0.231734 & 0.545387 & 0.0258346 & 6.74992 \\
\hline 0.398191 & 0.0234157 & 0.613884 & 0.229863 & 0.547117 & 0.0336726 & 6.74647 \\
\hline 0.406334 & 0.0325259 & 0.619817 & 0.236401 & 0.554814 & 0.0319455 & 6.84831 \\
\hline 0.406229 & 0.0267422 & 0.623023 & 0.235929 & 0.557007 & 0.0315981 & 6.93522 \\
\hline 0.417607 & 0.0333537 & 0.647718 & 0.254377 & 0.577087 & 0.031506 & 7.09056 \\
\hline 0.399099 & 0.0274648 & 0.662121 & 0.258567 & 0.585407 & 0.0300946 & 7.25994 \\
\hline 0.419612 & 0.0267127 & 0.673271 & 0.258747 & 0.6 & 0.0432121 & 7.53899 \\
\hline 0.410395 & 0.0211471 & 0.703584 & 0.290286 & 0.611641 & 0.0293485 & 7.82217 \\
\hline 0.418447 & 0.0267329 & 0.708888 & 0.281203 & 0.643842 & 0.0385499 & 8.19344 \\
\hline 0.412196 & 0.0303997 & 0.739595 & 0.301332 & 0.660965 & 0.0344486 & 8.64727 \\
\hline 0.41821 & 0.0250826 & 0.759335 & 0.308835 & 0.691799 & 0.0388221 & 9.07916 \\
\hline 0.418667 & 0.0217823 & 0.807327 & 0.340427 & 0.7235 & 0.0313108 & 9.77474 \\
\hline 0.427949 & 0.0257889 & 0.863309 & 0.378356 & 0.771054 & 0.0427931 & 10.6034 \\
\hline 0.434326 & 0.0250777 & 0.935946 & 0.415973 & 0.828402 & 0.0450555 & 11.7747 \\
\hline 0.426772 & 0.0268691 & 1.0259 & 0.477447 & 0.904183 & 0.061527 & 13.2654 \\
\hline 0.429214 & 0.0234067 & 1.15593 & 0.561399 & 0.997876 & 0.059365 & 15.208 \\
\hline 0.439701 & 0.0380626 & 1.30126 & 0.644763 & 1.09916 & 0.0665797 & 17.9848 \\
\hline 0.442922 & 0.0244442 & 1.49538 & 0.780185 & 1.27449 & 0.0666354 & 21.5895 \\
\hline 0.44718 & 0.0247349 & 1.78043 & 0.971858 & 1.4719 & 0.0896469 & 26.2593 \\
\hline 0.446651 & 0.0297062 & 2.12293 & 1.19102 & 1.75276 & 0.109787 & 32.6515 \\
\hline 0.459183 & 0.031136 & 2.57125 & 1.50111 & 2.09607 & 0.126755 & 40.6804 \\
\hline 0.467402 & 0.0211286 & 3.14158 & 1.86912 & 2.52907 & 0.157004 & 50.8736 \\
\hline 0.485948 & 0.0247192 & 3.81097 & 2.29614 & 3.05818 & 0.202038 & 63.4304 \\
\hline 0.511451 & 0.0284367 & 4.62439 & 2.8827 & 3.67139 & 0.236072 & 78.7432 \\
\hline 0.524214 & 0.0358143 & 5.55253 & 3.4974 & 4.44856 & 0.268326 & 96.3723 \\
\hline 0.558387 & 0.0329121 & 6.59737 & 4.22566 & 5.24779 & 0.342678 & 116.146 \\
\hline 0.574362 & 0.0316822 & 7.68635 & 4.96902 & 6.1887 & 0.396635 & 138.592 \\
\hline 0.602703 & 0.0379904 & 8.84942 & 5.67848 & 7.15376 & 0.471163 & 161.535 \\
\hline 0.642011 & 0.0426871 & 10.0693 & 6.56095 & 8.11683 & 0.565127 & 184.928 \\
\hline 0.692209 & 0.0379365 & 11.119 & 7.31325 & 9.07257 & 0.623092 & 207.521 \\
\hline 0.723016 & 0.044246 & 12.077 & 7.87311 & 9.93753 & 0.65782 & 227.669 \\
\hline 0.77547 & 0.0421708 & 12.8242 & 8.42015 & 10.6781 & 0.719834 & 244.904 \\
\hline 0.801192 & 0.0512674 & 13.3564 & 8.82747 & 11.1687 & 0.759952 & 257.307 \\
\hline 0.829224 & 0.0459896 & 13.5631 & 8.97929 & 11.5207 & 0.770559 & 264.628 \\
\hline 0.859626 & 0.0533253 & 13.5184 & 8.88322 & 11.5797 & 0.799311 & 266.199 \\
\hline 0.851537 & 0.0530327 & 13.165 & 8.68496 & 11.3738 & 0.734388 & 261.42 \\
\hline 0.867506 & 0.0575767 & 12.5338 & 8.18672 & 10.9215 & 0.731507 & 251.189 \\
\hline
\end{tabular}




\begin{tabular}{|c|c|c|c|c|c|c|}
\hline 0.862619 & 0.0542283 & 11.6903 & 7.70131 & 10.3063 & 0.699595 & 236.014 \\
\hline 0.834795 & 0.0449096 & 10.6708 & 7.03879 & 9.4857 & 0.629963 & 216.574 \\
\hline 0.814521 & 0.0525104 & 9.50327 & 6.22659 & 8.5342 & 0.546712 & 194.366 \\
\hline 0.770355 & 0.0444869 & 8.32558 & 5.38793 & 7.52879 & 0.477244 & 170.507 \\
\hline 0.752082 & 0.0476568 & 7.10035 & 4.59966 & 6.51319 & 0.409042 & 146.092 \\
\hline 0.693114 & 0.035964 & 5.97529 & 3.83106 & 5.52892 & 0.358424 & 122.518 \\
\hline 0.667821 & 0.0468528 & 4.91113 & 3.09264 & 4.58497 & 0.272311 & 100.704 \\
\hline 0.616414 & 0.0259887 & 3.9954 & 2.52666 & 3.75286 & 0.245235 & 80.7752 \\
\hline 0.583336 & 0.0351381 & 3.18145 & 1.93281 & 3.00982 & 0.202301 & 63.5225 \\
\hline 0.544108 & 0.0259237 & 2.52915 & 1.48653 & 2.3963 & 0.152914 & 48.8703 \\
\hline 0.513457 & 0.0320141 & 1.99341 & 1.12943 & 1.89644 & 0.12256 & 37.103 \\
\hline 0.496591 & 0.0348952 & 1.55696 & 0.85982 & 1.48221 & 0.0885858 & 27.4214 \\
\hline 0.469278 & 0.0243022 & 1.24315 & 0.653621 & 1.17546 & 0.0735234 & 20.0897 \\
\hline 0.459781 & 0.0229088 & 1.002 & 0.473882 & 0.931464 & 0.0536377 & 14.4503 \\
\hline 0.447451 & 0.0263791 & 0.827871 & 0.376175 & 0.762029 & 0.0417242 & 10.2713 \\
\hline 0.442388 & 0.0270518 & 0.715281 & 0.307863 & 0.635252 & 0.0400324 & 7.34005 \\
\hline 0.448034 & 0.0286166 & 0.629414 & 0.24693 & 0.545645 & 0.0341183 & 5.28206 \\
\hline 0.444267 & 0.0303522 & 0.568495 & 0.20306 & 0.485222 & 0.025143 & 3.80293 \\
\hline 0.448898 & 0.0246633 & 0.535077 & 0.204513 & 0.443073 & 0.0277336 & 2.81 \\
\hline 0.461794 & 0.0303523 & 0.518577 & 0.18074 & 0.420569 & 0.0203859 & 2.1554 \\
\hline 0.466309 & 0.0255527 & 0.518244 & 0.186381 & 0.404813 & 0.0185172 & 1.70763 \\
\hline 0.48029 & 0.0315092 & 0.515433 & 0.171158 & 0.395194 & 0.0210286 & 1.46974 \\
\hline 0.485308 & 0.0319769 & 0.525314 & 0.182433 & 0.398716 & 0.0223021 & 1.3249 \\
\hline 0.510351 & 0.0366843 & 0.544744 & 0.184241 & 0.397821 & 0.0220474 & 1.24488 \\
\hline 0.536309 & 0.0334227 & 0.557056 & 0.17743 & 0.414337 & 0.0273 & 1.18049 \\
\hline 0.544772 & 0.0306367 & 0.594784 & 0.195431 & 0.422096 & 0.0213309 & 1.19108 \\
\hline 0.563274 & 0.0281968 & 0.625181 & 0.199896 & 0.444381 & 0.0251995 & 1.23638 \\
\hline 0.591913 & 0.0354163 & 0.656972 & 0.207201 & 0.469645 & 0.0274419 & 1.28319 \\
\hline 0.603271 & 0.0338848 & 0.685117 & 0.227717 & 0.493239 & 0.0286956 & 1.37207 \\
\hline 0.612546 & 0.0457993 & 0.743652 & 0.231662 & 0.527581 & 0.0266672 & 1.45147 \\
\hline 0.620041 & 0.034554 & 0.789551 & 0.252361 & 0.564041 & 0.032905 & 1.60655 \\
\hline 0.624411 & 0.0382925 & 0.842989 & 0.26567 & 0.602645 & 0.0351019 & 1.72592 \\
\hline 0.637539 & 0.0355504 & 0.882957 & 0.272524 & 0.633732 & 0.0283386 & 1.88319 \\
\hline 0.630633 & 0.0300967 & 0.936582 & 0.303103 & 0.676968 & 0.0335966 & 1.99967 \\
\hline 0.622165 & 0.0399371 & 0.982816 & 0.300666 & 0.717428 & 0.0410803 & 2.13937 \\
\hline 0.620751 & 0.0396978 & 1.01527 & 0.318238 & 0.743162 & 0.0379929 & 2.27426 \\
\hline 0.612024 & 0.0277623 & 1.04295 & 0.332268 & 0.774849 & 0.0402283 & 2.38963 \\
\hline 0.595935 & 0.0398092 & 1.05324 & 0.325496 & 0.795191 & 0.0419099 & 2.43594 \\
\hline 0.579034 & 0.0376433 & 1.05159 & 0.334027 & 0.799751 & 0.0351225 & 2.49378 \\
\hline 0.564589 & 0.0324687 & 1.0379 & 0.322286 & 0.800086 & 0.0371911 & 2.44812 \\
\hline 0.550919 & 0.0409481 & 1.01903 & 0.312807 & 0.793677 & 0.0439895 & 2.4081 \\
\hline 0.549309 & 0.0304739 & 0.982069 & 0.304112 & 0.779275 & 0.0408944 & 2.30001 \\
\hline 0.527049 & 0.0320083 & 0.940319 & 0.288827 & 0.752212 & 0.040066 & 2.22659 \\
\hline 0.516527 & 0.0246431 & 0.904935 & 0.274978 & 0.720175 & 0.0368815 & 2.09601 \\
\hline 0.506816 & 0.034279 & 0.841925 & 0.242702 & 0.694726 & 0.0473598 & 1.95961 \\
\hline 0.514774 & 0.030142 & 0.793338 & 0.241323 & 0.66264 & 0.0360349 & 1.82303 \\
\hline 0.500613 & 0.0264534 & 0.733395 & 0.211078 & 0.619974 & 0.0305857 & 1.6727 \\
\hline
\end{tabular}




\begin{tabular}{|c|c|c|c|c|c|c|}
\hline 0.494271 & 0.0367363 & 0.682971 & 0.207818 & 0.590568 & 0.0297961 & 1.51928 \\
\hline 0.483743 & 0.0288246 & 0.643047 & 0.177283 & 0.555223 & 0.0285821 & 1.3937 \\
\hline 0.489656 & 0.0326344 & 0.59866 & 0.170153 & 0.52272 & 0.026378 & 1.27106 \\
\hline 0.488864 & 0.0301915 & 0.570832 & 0.155541 & 0.495409 & 0.0299286 & 1.15542 \\
\hline 0.487944 & 0.02942 & 0.53033 & 0.15133 & 0.480254 & 0.0242672 & 1.06154 \\
\hline 0.49832 & 0.0318196 & 0.520843 & 0.153128 & 0.460998 & 0.0236612 & 0.983258 \\
\hline 0.482086 & 0.0341074 & 0.498962 & 0.144355 & 0.447583 & 0.0269995 & 0.928208 \\
\hline 0.488836 & 0.0341462 & 0.486599 & 0.139912 & 0.43303 & 0.0221468 & 0.884471 \\
\hline 0.492362 & 0.0274356 & 0.49061 & 0.147956 & 0.438351 & 0.0233345 & 0.862443 \\
\hline 0.496203 & 0.0244791 & 0.480578 & 0.149696 & 0.427301 & 0.0194773 & 0.819232 \\
\hline 0.512617 & 0.0280552 & 0.479408 & 0.131527 & 0.420731 & 0.0217608 & 0.805138 \\
\hline 0.519124 & 0.0321731 & 0.474126 & 0.136446 & 0.420145 & 0.0252402 & 0.798861 \\
\hline 0.516768 & 0.0332242 & 0.476407 & 0.155449 & 0.416642 & 0.0261866 & 0.777904 \\
\hline 0.527857 & 0.0257864 & 0.483248 & 0.144179 & 0.419566 & 0.0257975 & 0.780864 \\
\hline 0.52979 & 0.0347802 & 0.480016 & 0.15145 & 0.417012 & 0.0263763 & 0.746201 \\
\hline 0.536778 & 0.0296195 & 0.474658 & 0.148185 & 0.41686 & 0.0210794 & 0.786715 \\
\hline 0.540895 & 0.0306319 & 0.480195 & 0.158852 & 0.418636 & 0.0237086 & 0.775397 \\
\hline 0.531995 & 0.0315901 & 0.475304 & 0.158039 & 0.416594 & 0.0218048 & 0.774744 \\
\hline 0.535237 & 0.0252574 & 0.47746 & 0.152936 & 0.42111 & 0.0220344 & 0.752733 \\
\hline 0.536276 & 0.0237866 & 0.473522 & 0.163047 & 0.42287 & 0.0236016 & 0.769149 \\
\hline 0.531516 & 0.0303314 & 0.472632 & 0.1592 & 0.41584 & 0.0254319 & 0.770274 \\
\hline 0.526396 & 0.0300142 & 0.48538 & 0.161049 & 0.421668 & 0.027377 & 0.778586 \\
\hline 0.52519 & 0.031379 & 0.471373 & 0.157105 & 0.421964 & 0.0213102 & 0.784047 \\
\hline 0.514254 & 0.0379234 & 0.477966 & 0.169525 & 0.425419 & 0.0274753 & 0.754121 \\
\hline 0.519105 & 0.0299345 & 0.486465 & 0.164267 & 0.432035 & 0.0231981 & 0.765926 \\
\hline 0.508814 & 0.0327403 & 0.478165 & 0.158756 & 0.434984 & 0.0281147 & 0.810545 \\
\hline 0.508996 & 0.0302509 & 0.487297 & 0.175054 & 0.439155 & 0.0305352 & 0.803673 \\
\hline 0.511892 & 0.0273709 & 0.484344 & 0.160604 & 0.44598 & 0.0222155 & 0.791888 \\
\hline 0.506185 & 0.0282694 & 0.489376 & 0.168722 & 0.447495 & 0.0272548 & 0.837824 \\
\hline 0.497407 & 0.0327149 & 0.49432 & 0.169731 & 0.464667 & 0.0228913 & 0.833367 \\
\hline 0.500387 & 0.035269 & 0.518027 & 0.183827 & 0.478272 & 0.0294152 & 0.850556 \\
\hline 0.499985 & 0.0297676 & 0.522193 & 0.195703 & 0.489796 & 0.0291557 & 0.865901 \\
\hline 0.500343 & 0.0290652 & 0.536164 & 0.188829 & 0.518621 & 0.0349549 & 0.875445 \\
\hline 0.498261 & 0.031389 & 0.558857 & 0.207107 & 0.547769 & 0.0316015 & 0.925844 \\
\hline 0.497765 & 0.0350265 & 0.585625 & 0.23621 & 0.582763 & 0.0411671 & 0.921419 \\
\hline 0.502759 & 0.0253394 & 0.629368 & 0.273681 & 0.619892 & 0.0510425 & 0.961756 \\
\hline 0.508809 & 0.0309231 & 0.673551 & 0.320663 & 0.69493 & 0.062944 & 1.01267 \\
\hline 0.507504 & 0.03 & 0.719057 & 0.390201 & 0.755942 & 0.0659191 & 1.05146 \\
\hline 0.517793 & 0.0317664 & 0.787837 & 0.456206 & 0.838393 & 0.0899002 & 1.11835 \\
\hline 0.498252 & 0.031584 & 0.843685 & 0.562181 & 0.937492 & 0.112477 & 1.13847 \\
\hline 0.506567 & 0.0314694 & 0.93391 & 0.676179 & 1.0405 & 0.130986 & 1.24425 \\
\hline 0.508302 & 0.0300906 & 0.98891 & 0.754232 & 1.15632 & 0.158102 & 1.32558 \\
\hline 0.515181 & 0.0313064 & 1.07429 & 0.876782 & 1.2703 & 0.189679 & 1.40113 \\
\hline 0.529988 & 0.032009 & 1.14437 & 0.995855 & 1.36722 & 0.195913 & 1.48866 \\
\hline 0.526081 & 0.0344199 & 1.20988 & 1.07572 & 1.48174 & 0.224726 & 1.59662 \\
\hline 0.52763 & 0.0307745 & 1.27163 & 1.1586 & 1.5697 & 0.249619 & 1.6247 \\
\hline 0.536364 & 0.0339432 & 1.29489 & 1.18967 & 1.63898 & 0.272789 & 1.70562 \\
\hline
\end{tabular}




\begin{tabular}{|c|c|c|c|c|c|c|}
\hline 0.531632 & 0.029309 & 1.3385 & 1.27083 & 1.70586 & 0.275156 & 1.74369 \\
\hline 0.535063 & 0.0317246 & 1.35964 & 1.27335 & 1.73531 & 0.283197 & 1.83523 \\
\hline 0.536896 & 0.0292475 & 1.35041 & 1.28107 & 1.73821 & 0.277698 & 1.83934 \\
\hline 0.552434 & 0.0300414 & 1.33018 & 1.24716 & 1.70573 & 0.279185 & 1.88117 \\
\hline 0.537546 & 0.0317409 & 1.28768 & 1.17499 & 1.65989 & 0.25917 & 1.86901 \\
\hline 0.545144 & 0.0341375 & 1.23 & 1.08049 & 1.59759 & 0.233377 & 1.86012 \\
\hline 0.542429 & 0.033526 & 1.16908 & 0.96387 & 1.48986 & 0.226893 & 1.85729 \\
\hline 0.54085 & 0.0383404 & 1.10277 & 0.853312 & 1.41002 & 0.207932 & 1.82167 \\
\hline 0.536669 & 0.0328047 & 1.02398 & 0.778528 & 1.30681 & 0.168492 & 1.73524 \\
\hline 0.551657 & 0.0363924 & 0.948656 & 0.646707 & 1.20099 & 0.157349 & 1.7022 \\
\hline 0.54173 & 0.0286275 & 0.895363 & 0.548919 & 1.08705 & 0.131958 & 1.65866 \\
\hline 0.538144 & 0.0308595 & 0.83463 & 0.457803 & 0.994922 & 0.101924 & 1.61889 \\
\hline 0.541285 & 0.0329498 & 0.776899 & 0.387428 & 0.901314 & 0.0853623 & 1.55272 \\
\hline 0.532176 & 0.0326602 & 0.724853 & 0.309146 & 0.831519 & 0.0747444 & 1.47917 \\
\hline 0.540369 & 0.0314973 & 0.696221 & 0.261873 & 0.757822 & 0.0561471 & 1.44727 \\
\hline 0.536917 & 0.0341104 & 0.671819 & 0.223943 & 0.716913 & 0.0523917 & 1.37065 \\
\hline 0.534042 & 0.0275518 & 0.647211 & 0.185599 & 0.665203 & 0.0408354 & 1.34839 \\
\hline 0.535944 & 0.0321645 & 0.636295 & 0.179065 & 0.643273 & 0.0361491 & 1.32006 \\
\hline 0.537269 & 0.0380972 & 0.622488 & 0.158603 & 0.617752 & 0.0344724 & 1.2898 \\
\hline 0.541942 & 0.0354047 & 0.622061 & 0.153083 & 0.605531 & 0.0326515 & 1.25515 \\
\hline 0.544956 & 0.0357864 & 0.615849 & 0.159019 & 0.59703 & 0.0367619 & 1.25327 \\
\hline 0.553937 & 0.0246584 & 0.611876 & 0.159146 & 0.590977 & 0.0260758 & 1.19822 \\
\hline 0.545372 & 0.0356501 & 0.619873 & 0.148018 & 0.592555 & 0.035672 & 1.18411 \\
\hline 0.544355 & 0.0357503 & 0.624776 & 0.147673 & 0.583833 & 0.0331898 & 1.18067 \\
\hline 0.560736 & 0.0349845 & 0.622067 & 0.150236 & 0.590767 & 0.0304155 & 1.15946 \\
\hline 0.549312 & 0.0366083 & 0.618119 & 0.141224 & 0.57714 & 0.03187 & 1.14244 \\
\hline 0.558819 & 0.0351244 & 0.611745 & 0.14296 & 0.575241 & 0.0331706 & 1.0795 \\
\hline 0.574146 & 0.0316236 & 0.614227 & 0.14328 & 0.581716 & 0.0328236 & 1.08449 \\
\hline 0.580255 & 0.0338467 & 0.612009 & 0.136268 & 0.571636 & 0.0325634 & 1.08347 \\
\hline 0.572242 & 0.0336161 & 0.612689 & 0.141323 & 0.569969 & 0.0294356 & 1.03525 \\
\hline 0.582454 & 0.0395294 & 0.607658 & 0.145244 & 0.567852 & 0.0279797 & 1.01083 \\
\hline 0.578188 & 0.0377369 & 0.612127 & 0.137795 & 0.575147 & 0.029943 & 0.99381 \\
\hline 0.5952 & 0.0361818 & 0.603396 & 0.140462 & 0.565799 & 0.0316103 & 0.976633 \\
\hline 0.601039 & 0.0394392 & 0.608224 & 0.144292 & 0.557761 & 0.0287538 & 0.962553 \\
\hline 0.594709 & 0.0404258 & 0.597816 & 0.138688 & 0.553078 & 0.0259733 & 0.92181 \\
\hline 0.609546 & 0.0347107 & 0.595259 & 0.144106 & 0.551491 & 0.0246614 & 0.902105 \\
\hline 0.613087 & 0.0374328 & 0.60153 & 0.133839 & 0.557257 & 0.0298573 & 0.883382 \\
\hline 0.615333 & 0.0324741 & 0.601441 & 0.144678 & 0.560626 & 0.0262672 & 0.899177 \\
\hline 0.620346 & 0.0405127 & 0.611387 & 0.153494 & 0.555628 & 0.0243012 & 0.882492 \\
\hline 0.622352 & 0.0408533 & 0.620946 & 0.162644 & 0.560983 & 0.0252315 & 0.887771 \\
\hline 0.628185 & 0.0437528 & 0.627961 & 0.172275 & 0.554395 & 0.0282828 & 0.867553 \\
\hline 0.632571 & 0.0307099 & 0.63401 & 0.168599 & 0.563942 & 0.0249909 & 0.863166 \\
\hline 0.645231 & 0.0401332 & 0.649326 & 0.176988 & 0.563831 & 0.022369 & 0.843882 \\
\hline 0.645953 & 0.0386255 & 0.665706 & 0.19208 & 0.562176 & 0.0303506 & 0.84708 \\
\hline 0.661167 & 0.0287804 & 0.693478 & 0.203198 & 0.578075 & 0.0305456 & 0.84762 \\
\hline 0.663739 & 0.0340969 & 0.714067 & 0.2277 & 0.574686 & 0.0297061 & 0.854713 \\
\hline 0.658416 & 0.0341712 & 0.74331 & 0.244872 & 0.59032 & 0.0277566 & 0.859415 \\
\hline
\end{tabular}




\begin{tabular}{|c|c|c|c|c|c|c|}
\hline 0.670306 & 0.0361895 & 0.782631 & 0.271665 & 0.595018 & 0.0268647 & 0.874807 \\
\hline 0.674703 & 0.0536382 & 0.833295 & 0.345211 & 0.607334 & 0.0246084 & 0.873661 \\
\hline 0.70241 & 0.0475 & 0.886086 & 0.370503 & 0.622549 & 0.0279782 & 0.855433 \\
\hline 0.709017 & 0.0359953 & 0.950302 & 0.448881 & 0.637742 & 0.0395489 & 0.866277 \\
\hline 0.727361 & 0.0410253 & 1.01579 & 0.519454 & 0.658244 & 0.0252317 & 0.87378 \\
\hline 0.737523 & 0.04234 & 1.08991 & 0.626478 & 0.68022 & 0.029073 & 0.868806 \\
\hline 0.757445 & 0.0409128 & 1.15956 & 0.70768 & 0.690951 & 0.0309533 & 0.878285 \\
\hline 0.78509 & 0.0448646 & 1.23475 & 0.762895 & 0.709761 & 0.0315073 & 0.905859 \\
\hline 0.791242 & 0.0440612 & 1.31254 & 0.890275 & 0.73496 & 0.0322868 & 0.909234 \\
\hline 0.823496 & 0.0372528 & 1.35839 & 0.910934 & 0.761322 & 0.0385169 & 0.908904 \\
\hline 0.838171 & 0.0347606 & 1.42465 & 0.984 & 0.772592 & 0.0419272 & 0.904914 \\
\hline 0.873773 & 0.0504667 & 1.45991 & 1.05471 & 0.782976 & 0.0385873 & 0.904824 \\
\hline 0.898378 & 0.0516233 & 1.48819 & 1.06608 & 0.802038 & 0.0374528 & 0.92449 \\
\hline 0.9108 & 0.0362479 & 1.51969 & 1.10649 & 0.801071 & 0.0399719 & 0.922648 \\
\hline 0.922713 & 0.0385786 & 1.49514 & 1.10003 & 0.803609 & 0.0399919 & 0.945498 \\
\hline 0.931869 & 0.0478539 & 1.47521 & 1.04079 & 0.803734 & 0.0356141 & 0.952078 \\
\hline 0.940912 & 0.0421535 & 1.44248 & 1.01518 & 0.793625 & 0.0404826 & 0.954248 \\
\hline 0.935832 & 0.0477847 & 1.38278 & 0.936056 & 0.788108 & 0.0312041 & 0.93508 \\
\hline 0.943566 & 0.0378 & 1.32366 & 0.870647 & 0.781233 & 0.0341036 & 0.95352 \\
\hline 0.913593 & 0.0441624 & 1.26123 & 0.770662 & 0.762721 & 0.0318899 & 0.94314 \\
\hline 0.909733 & 0.0398984 & 1.19771 & 0.680871 & 0.732957 & 0.0309219 & 0.943078 \\
\hline 0.8905 & 0.0394057 & 1.13699 & 0.619275 & 0.72995 & 0.0335263 & 0.931594 \\
\hline 0.891554 & 0.046985 & 1.07128 & 0.536897 & 0.720252 & 0.0347131 & 0.933707 \\
\hline 0.862648 & 0.0398647 & 1.0041 & 0.455542 & 0.701432 & 0.0303149 & 0.947441 \\
\hline 0.8515 & 0.0427416 & 0.946665 & 0.377715 & 0.694321 & 0.0313268 & 0.939087 \\
\hline 0.829702 & 0.0408523 & 0.906628 & 0.333928 & 0.677977 & 0.024378 & 0.939103 \\
\hline 0.815555 & 0.0521006 & 0.879364 & 0.287337 & 0.678581 & 0.0314258 & 0.946684 \\
\hline 0.808203 & 0.0413252 & 0.839181 & 0.258425 & 0.678326 & 0.0311266 & 0.958308 \\
\hline 0.793894 & 0.0433545 & 0.8308 & 0.247787 & 0.679137 & 0.0326687 & 0.949013 \\
\hline 0.776222 & 0.0411668 & 0.798891 & 0.214627 & 0.684063 & 0.0310545 & 0.967529 \\
\hline 0.781374 & 0.0404124 & 0.799636 & 0.215137 & 0.698588 & 0.0346048 & 0.968512 \\
\hline 0.761058 & 0.0446235 & 0.803527 & 0.203627 & 0.701736 & 0.0378198 & 0.988896 \\
\hline 0.778096 & 0.0411386 & 0.799592 & 0.200081 & 0.713358 & 0.0342396 & 0.978078 \\
\hline 0.781627 & 0.0482708 & 0.798061 & 0.207915 & 0.725642 & 0.0399213 & 0.98545 \\
\hline 0.770627 & 0.0375871 & 0.806755 & 0.209662 & 0.742234 & 0.0389345 & 0.979516 \\
\hline 0.765513 & 0.042357 & 0.805382 & 0.207811 & 0.75511 & 0.043488 & 0.982734 \\
\hline 0.762918 & 0.0385652 & 0.821216 & 0.222329 & 0.769763 & 0.0516984 & 1.00535 \\
\hline 0.779373 & 0.0404215 & 0.827639 & 0.21754 & 0.790872 & 0.0557267 & 0.979685 \\
\hline 0.774678 & 0.032388 & 0.835826 & 0.231432 & 0.799021 & 0.0515314 & 1.01182 \\
\hline 0.766351 & 0.0465723 & 0.836603 & 0.240888 & 0.812457 & 0.0576119 & 1.00007 \\
\hline 0.78033 & 0.0351847 & 0.846587 & 0.244699 & 0.808818 & 0.0560944 & 1.02316 \\
\hline 0.780188 & 0.0421092 & 0.848216 & 0.232051 & 0.812812 & 0.0490703 & 1.02922 \\
\hline 0.791442 & 0.0535511 & 0.833401 & 0.224358 & 0.812772 & 0.0494173 & 1.01771 \\
\hline 0.785988 & 0.0328184 & 0.840706 & 0.213107 & 0.810021 & 0.0473145 & 0.991536 \\
\hline 0.78693 & 0.0429107 & 0.827206 & 0.21115 & 0.79448 & 0.0523918 & 1.00753 \\
\hline 0.785935 & 0.0389105 & 0.817371 & 0.199197 & 0.780631 & 0.0554041 & 1.03086 \\
\hline 0.793247 & 0.0388883 & 0.823865 & 0.197476 & 0.773339 & 0.043036 & 1.0057 \\
\hline
\end{tabular}




\begin{tabular}{|c|c|c|c|c|c|c|}
\hline 0.787463 & 0.0359528 & 0.80075 & 0.1795 & 0.764456 & 0.0414102 & 1.01586 \\
\hline 0.784345 & 0.0370571 & 0.78361 & 0.175781 & 0.739311 & 0.0459334 & 0.992877 \\
\hline 0.783924 & 0.047076 & 0.770144 & 0.163657 & 0.729037 & 0.0391567 & 1.0136 \\
\hline 0.790671 & 0.0467898 & 0.767791 & 0.1518 & 0.70632 & 0.0414793 & 1.01422 \\
\hline 0.780129 & 0.0458087 & 0.754837 & 0.147433 & 0.694102 & 0.0345143 & 1.00695 \\
\hline 0.785858 & 0.0442374 & 0.753482 & 0.150691 & 0.683775 & 0.0370791 & 1.01983 \\
\hline 0.786615 & 0.0338482 & 0.737833 & 0.134942 & 0.680469 & 0.0358745 & 1.01596 \\
\hline 0.78751 & 0.0358372 & 0.733353 & 0.138718 & 0.661868 & 0.0277433 & 1.03796 \\
\hline 0.783053 & 0.0321582 & 0.736682 & 0.146371 & 0.655722 & 0.028933 & 1.03816 \\
\hline 0.799136 & 0.0349048 & 0.729038 & 0.130742 & 0.652122 & 0.0270626 & 1.05905 \\
\hline 0.785444 & 0.0363052 & 0.726037 & 0.135859 & 0.65133 & 0.0322531 & 1.06039 \\
\hline 0.787049 & 0.0422475 & 0.727716 & 0.149767 & 0.651284 & 0.033729 & 1.0498 \\
\hline 0.800235 & 0.0369471 & 0.721752 & 0.132694 & 0.638651 & 0.0381901 & 1.07382 \\
\hline 0.781372 & 0.0387392 & 0.718816 & 0.133852 & 0.641949 & 0.0268132 & 1.07844 \\
\hline 0.780209 & 0.0353501 & 0.736635 & 0.139074 & 0.643364 & 0.033422 & 1.08473 \\
\hline 0.775177 & 0.0364375 & 0.721273 & 0.133605 & 0.646594 & 0.0312129 & 1.06746 \\
\hline 0.785519 & 0.0388733 & 0.734822 & 0.135727 & 0.646846 & 0.0248489 & 1.08007 \\
\hline 0.788284 & 0.0400767 & 0.733244 & 0.14196 & 0.657419 & 0.0241766 & 1.08251 \\
\hline 0.790441 & 0.0421395 & 0.729937 & 0.131416 & 0.665597 & 0.0289356 & 1.07995 \\
\hline 0.795409 & 0.0249923 & 0.72924 & 0.143942 & 0.670428 & 0.0380079 & 1.06423 \\
\hline 0.788191 & 0.0381458 & 0.730188 & 0.135284 & 0.662525 & 0.0263524 & 1.11017 \\
\hline 0.780685 & 0.0390506 & 0.735545 & 0.139352 & 0.672463 & 0.0341496 & 1.08045 \\
\hline 0.788784 & 0.0357649 & 0.747538 & 0.142018 & 0.677414 & 0.0294717 & 1.07959 \\
\hline 0.781709 & 0.0338451 & 0.736735 & 0.13873 & 0.684543 & 0.0304196 & 1.07958 \\
\hline 0.783956 & 0.0382216 & 0.737468 & 0.136263 & 0.684644 & 0.0371923 & 1.08184 \\
\hline 0.786749 & 0.0400844 & 0.738011 & 0.141859 & 0.679519 & 0.0356973 & 1.07946 \\
\hline 0.789212 & 0.0456323 & 0.727222 & 0.145068 & 0.686901 & 0.0294899 & 1.05841 \\
\hline 0.791909 & 0.0374232 & 0.734629 & 0.133522 & 0.688302 & 0.0251231 & 1.06094 \\
\hline 0.792617 & 0.0435274 & 0.724483 & 0.134077 & 0.68462 & 0.0293682 & 1.03126 \\
\hline 0.777178 & 0.0378316 & 0.733996 & 0.145872 & 0.693395 & 0.030544 & 1.06455 \\
\hline 0.790279 & 0.0342244 & 0.72602 & 0.139908 & 0.683153 & 0.0294326 & 1.05593 \\
\hline 0.775678 & 0.0384353 & 0.730918 & 0.129962 & 0.682149 & 0.0402482 & 1.02381 \\
\hline 0.779416 & 0.044976 & 0.725434 & 0.137848 & 0.678047 & 0.0304814 & 1.02067 \\
\hline 0.778754 & 0.0357754 & 0.726827 & 0.138415 & 0.685153 & 0.0262581 & 1.01268 \\
\hline 0.78316 & 0.0332347 & 0.720961 & 0.138357 & 0.673093 & 0.0326689 & 1.0084 \\
\hline 0.78055 & 0.0292006 & 0.716738 & 0.136979 & 0.674699 & 0.0296815 & 0.998405 \\
\hline 0.778849 & 0.0452984 & 0.720183 & 0.134036 & 0.681583 & 0.0337241 & 0.98488 \\
\hline 0.787727 & 0.0401288 & 0.714937 & 0.135709 & 0.676599 & 0.0311505 & 0.967675 \\
\hline 0.77356 & 0.0366987 & 0.723766 & 0.137797 & 0.668297 & 0.0331291 & 0.961722 \\
\hline 0.776739 & 0.0406859 & 0.722755 & 0.139067 & 0.664218 & 0.0251892 & 0.937761 \\
\hline 0.777949 & 0.0417168 & 0.72116 & 0.143853 & 0.665139 & 0.0270228 & 0.960729 \\
\hline 0.774702 & 0.0334462 & 0.716435 & 0.131352 & 0.667226 & 0.0275341 & 0.926402 \\
\hline 0.789741 & 0.0340262 & 0.716087 & 0.132914 & 0.66951 & 0.0297782 & 0.928714 \\
\hline 0.785639 & 0.0387791 & 0.718934 & 0.136615 & 0.665467 & 0.0286792 & 0.9465 \\
\hline 0.778517 & 0.0460602 & 0.734607 & 0.134218 & 0.664581 & 0.0306002 & 0.902974 \\
\hline 0.808252 & 0.0466859 & 0.733092 & 0.134761 & 0.667679 & 0.0344616 & 0.922519 \\
\hline 0.800784 & 0.0478725 & 0.744234 & 0.139767 & 0.673655 & 0.0302672 & 0.919786 \\
\hline
\end{tabular}




\begin{tabular}{|c|c|c|c|c|c|c|}
\hline 0.783904 & 0.0436964 & 0.744573 & 0.13331 & 0.668861 & 0.0344112 & 0.920665 \\
\hline 0.794798 & 0.0393305 & 0.762462 & 0.148223 & 0.671129 & 0.0308896 & 0.912276 \\
\hline 0.809032 & 0.0449165 & 0.768666 & 0.142526 & 0.676727 & 0.0357525 & 0.91997 \\
\hline 0.802052 & 0.0517099 & 0.765552 & 0.14826 & 0.687021 & 0.0323587 & 0.908766 \\
\hline 0.811904 & 0.042012 & 0.77912 & 0.153408 & 0.685227 & 0.033981 & 0.900545 \\
\hline 0.812943 & 0.0401844 & 0.786392 & 0.158904 & 0.689741 & 0.0310421 & 0.925554 \\
\hline 0.812696 & 0.0410657 & 0.812968 & 0.176506 & 0.692693 & 0.0365423 & 0.913016 \\
\hline 0.833888 & 0.0494377 & 0.816414 & 0.169249 & 0.69685 & 0.0327009 & 0.900185 \\
\hline 0.836063 & 0.0459244 & 0.829411 & 0.173577 & 0.698374 & 0.0330119 & 0.930157 \\
\hline 0.835401 & 0.0384831 & 0.84544 & 0.192835 & 0.709967 & 0.0331612 & 0.919799 \\
\hline 0.838328 & 0.0404735 & 0.849123 & 0.202233 & 0.714872 & 0.0381504 & 0.94526 \\
\hline 0.847189 & 0.0421177 & 0.860443 & 0.198454 & 0.707797 & 0.0321952 & 0.939599 \\
\hline 0.851799 & 0.0486422 & 0.859141 & 0.198244 & 0.723566 & 0.0316049 & 0.94033 \\
\hline 0.857238 & 0.0393893 & 0.870909 & 0.200732 & 0.712538 & 0.0369196 & 0.940332 \\
\hline 0.852991 & 0.0350676 & 0.859401 & 0.194125 & 0.717841 & 0.0315881 & 0.974042 \\
\hline 0.850693 & 0.047416 & 0.868214 & 0.193462 & 0.723109 & 0.0333529 & 0.971233 \\
\hline 0.865483 & 0.044718 & 0.862064 & 0.193508 & 0.719374 & 0.0363329 & 0.993341 \\
\hline 0.858223 & 0.0389013 & 0.857401 & 0.195509 & 0.719015 & 0.0312001 & 1.01303 \\
\hline 0.8593 & 0.0420967 & 0.85567 & 0.189649 & 0.72424 & 0.0315715 & 1.01052 \\
\hline 0.853605 & 0.0439302 & 0.855202 & 0.181887 & 0.719965 & 0.037232 & 1.02556 \\
\hline 0.867295 & 0.038949 & 0.838969 & 0.179369 & 0.72095 & 0.0298625 & 1.04035 \\
\hline 0.865055 & 0.0396619 & 0.835841 & 0.176211 & 0.720726 & 0.0307792 & 1.04187 \\
\hline 0.861049 & 0.0372425 & 0.82541 & 0.171192 & 0.719136 & 0.0295647 & 1.06414 \\
\hline 0.867804 & 0.0433166 & 0.824565 & 0.164357 & 0.708515 & 0.0247041 & 1.06025 \\
\hline 0.862898 & 0.0494286 & 0.825186 & 0.164061 & 0.718436 & 0.0340018 & 1.09754 \\
\hline 0.854558 & 0.0502689 & 0.814585 & 0.168955 & 0.719436 & 0.0318268 & 1.09491 \\
\hline 0.855722 & 0.0438907 & 0.814766 & 0.16256 & 0.71852 & 0.0319587 & 1.11349 \\
\hline 0.839339 & 0.042 & 0.813967 & 0.17065 & 0.711138 & 0.0280039 & 1.09689 \\
\hline 0.856157 & 0.0472226 & 0.803786 & 0.170208 & 0.71356 & 0.0307398 & 1.09555 \\
\hline 0.855652 & 0.0457681 & 0.799957 & 0.158512 & 0.721985 & 0.0301524 & 1.10131 \\
\hline 0.849431 & 0.0429653 & 0.797979 & 0.167115 & 0.709227 & 0.0328855 & 1.09032 \\
\hline 0.850104 & 0.0419436 & 0.80069 & 0.172258 & 0.711845 & 0.0351863 & 1.08696 \\
\hline 0.842301 & 0.0420128 & 0.809326 & 0.164399 & 0.712872 & 0.0280671 & 1.08142 \\
\hline 0.846107 & 0.0490515 & 0.805045 & 0.177064 & 0.711479 & 0.034403 & 1.0571 \\
\hline 0.84826 & 0.0352851 & 0.804127 & 0.173578 & 0.707507 & 0.0354975 & 1.06748 \\
\hline 0.852145 & 0.0398839 & 0.80737 & 0.179742 & 0.711788 & 0.037729 & 1.04161 \\
\hline 0.854201 & 0.0492003 & 0.810845 & 0.181879 & 0.707018 & 0.0366716 & 1.04078 \\
\hline 0.844532 & 0.044169 & 0.798011 & 0.190227 & 0.708905 & 0.0335539 & 1.03523 \\
\hline 0.853946 & 0.0476224 & 0.808596 & 0.182214 & 0.708832 & 0.0334923 & 1.01794 \\
\hline 0.849007 & 0.0331306 & 0.806971 & 0.182536 & 0.706142 & 0.032453 & 1.03191 \\
\hline 0.849937 & 0.0399491 & 0.815294 & 0.199147 & 0.708845 & 0.0295526 & 1.04153 \\
\hline 0.867199 & 0.0457264 & 0.812051 & 0.177266 & 0.712765 & 0.036666 & 1.03573 \\
\hline 0.853516 & 0.0452766 & 0.817246 & 0.181388 & 0.707966 & 0.0288637 & 1.02032 \\
\hline 0.845464 & 0.0363502 & 0.822739 & 0.181395 & 0.711305 & 0.0399992 & 1.01425 \\
\hline 0.853684 & 0.0398429 & 0.822118 & 0.183483 & 0.713941 & 0.0253115 & 1.01145 \\
\hline 0.84905 & 0.0426153 & 0.817531 & 0.187884 & 0.712935 & 0.0292912 & 1.01758 \\
\hline 0.846502 & 0.0362238 & 0.836421 & 0.197195 & 0.717167 & 0.0290296 & 1.00617 \\
\hline
\end{tabular}




\begin{tabular}{|c|c|c|c|c|c|c|}
\hline 0.86067 & 0.0529792 & 0.850197 & 0.198335 & 0.711094 & 0.0292947 & 1.02059 \\
\hline 0.853421 & 0.0345412 & 0.842858 & 0.189097 & 0.720112 & 0.0278273 & 1.03144 \\
\hline 0.847347 & 0.0358793 & 0.850495 & 0.193995 & 0.714288 & 0.0301379 & 1.03174 \\
\hline 0.858604 & 0.0415698 & 0.865867 & 0.196985 & 0.717287 & 0.0292258 & 1.04993 \\
\hline 0.872815 & 0.0411475 & 0.861771 & 0.197953 & 0.715115 & 0.0298177 & 1.07736 \\
\hline 0.853107 & 0.0423984 & 0.887231 & 0.216329 & 0.718846 & 0.0311929 & 1.06634 \\
\hline 0.847318 & 0.0404679 & 0.896749 & 0.226218 & 0.72494 & 0.0323231 & 1.06317 \\
\hline 0.858323 & 0.0458025 & 0.905682 & 0.21551 & 0.731492 & 0.0347523 & 1.10891 \\
\hline 0.876023 & 0.0543616 & 0.915942 & 0.218414 & 0.736248 & 0.0272548 & 1.11087 \\
\hline 0.868542 & 0.0553168 & 0.937282 & 0.225291 & 0.724204 & 0.0249918 & 1.1318 \\
\hline 0.870829 & 0.0405221 & 0.945953 & 0.229482 & 0.735137 & 0.0380082 & 1.13074 \\
\hline 0.857045 & 0.0462058 & 0.966898 & 0.243463 & 0.734246 & 0.0341112 & 1.16036 \\
\hline 0.868709 & 0.0441931 & 0.978242 & 0.25603 & 0.736558 & 0.034251 & 1.17078 \\
\hline 0.871892 & 0.0408822 & 0.9873 & 0.260289 & 0.738809 & 0.0190936 & 1.19256 \\
\hline 0.866368 & 0.0429572 & 0.994345 & 0.254019 & 0.745249 & 0.0345834 & 1.2179 \\
\hline 0.869632 & 0.0444799 & 1.00204 & 0.26438 & 0.745194 & 0.0345801 & 1.2108 \\
\hline 0.865805 & 0.049376 & 1.01337 & 0.265655 & 0.739708 & 0.0424445 & 1.22091 \\
\hline 0.853362 & 0.042648 & 1.00433 & 0.254088 & 0.737445 & 0.0308986 & 1.21865 \\
\hline 0.871804 & 0.0440495 & 1.01106 & 0.250686 & 0.747547 & 0.0225416 & 1.2348 \\
\hline 0.863925 & 0.0504499 & 0.993926 & 0.255201 & 0.743327 & 0.0300627 & 1.19801 \\
\hline 0.865308 & 0.039761 & 0.98799 & 0.238547 & 0.743371 & 0.0358559 & 1.19837 \\
\hline 0.871377 & 0.042618 & 0.97499 & 0.233326 & 0.735841 & 0.0333795 & 1.18177 \\
\hline 0.849912 & 0.044299 & 0.972421 & 0.230024 & 0.735224 & 0.0282823 & 1.18666 \\
\hline 0.858324 & 0.0338172 & 0.957722 & 0.211239 & 0.731105 & 0.0323611 & 1.17115 \\
\hline 0.855068 & 0.0335291 & 0.936677 & 0.194408 & 0.730836 & 0.0302855 & 1.157 \\
\hline 0.864126 & 0.046805 & 0.925353 & 0.195784 & 0.730002 & 0.029562 & 1.14864 \\
\hline 0.861802 & 0.0479044 & 0.925449 & 0.199726 & 0.728141 & 0.0279473 & 1.12701 \\
\hline 0.86135 & 0.0392057 & 0.902058 & 0.180381 & 0.721787 & 0.0301479 & 1.10358 \\
\hline 0.858117 & 0.0383222 & 0.893303 & 0.172566 & 0.715113 & 0.0259164 & 1.09992 \\
\hline 0.853455 & 0.0517437 & 0.873234 & 0.160783 & 0.722565 & 0.0328643 & 1.08121 \\
\hline 0.856188 & 0.0446416 & 0.871742 & 0.154547 & 0.721182 & 0.0230258 & 1.07556 \\
\hline 0.85703 & 0.0426268 & 0.867935 & 0.149191 & 0.715955 & 0.0350557 & 1.08293 \\
\hline 0.858527 & 0.0408017 & 0.845389 & 0.144241 & 0.71836 & 0.0286316 & 1.0713 \\
\hline 0.853067 & 0.0357398 & 0.836105 & 0.142745 & 0.716552 & 0.0326043 & 1.05952 \\
\hline 0.854128 & 0.0495628 & 0.847955 & 0.146992 & 0.718927 & 0.0367505 & 1.04883 \\
\hline 0.843801 & 0.043209 & 0.843494 & 0.149915 & 0.716731 & 0.0247875 & 1.06748 \\
\hline 0.853948 & 0.0464292 & 0.847339 & 0.144127 & 0.717515 & 0.0251399 & 1.04175 \\
\hline 0.843348 & 0.0419318 & 0.85587 & 0.14471 & 0.715825 & 0.0255594 & 1.06879 \\
\hline 0.844394 & 0.0415323 & 0.848781 & 0.143998 & 0.711451 & 0.0302084 & 1.06407 \\
\hline 0.845652 & 0.0423525 & 0.846289 & 0.132769 & 0.71878 & 0.0277688 & 1.06303 \\
\hline 0.850051 & 0.0486715 & 0.838213 & 0.14058 & 0.704012 & 0.0244001 & 1.05056 \\
\hline 0.843894 & 0.0417067 & 0.859614 & 0.144989 & 0.718114 & 0.0286931 & 1.07249 \\
\hline 0.844557 & 0.0447975 & 0.859545 & 0.149222 & 0.724545 & 0.0323089 & 1.06292 \\
\hline 0.842529 & 0.0496598 & 0.862369 & 0.148033 & 0.721147 & 0.0333808 & 1.05903 \\
\hline 0.838313 & 0.0419638 & 0.866186 & 0.151309 & 0.724589 & 0.0316205 & 1.0814 \\
\hline 0.848315 & 0.0417945 & 0.883919 & 0.145 & 0.725428 & 0.0279311 & 1.0648 \\
\hline 0.838973 & 0.046321 & 0.889876 & 0.154418 & 0.729172 & 0.0362368 & 1.08351 \\
\hline
\end{tabular}




\begin{tabular}{|c|c|c|c|c|c|c|}
\hline 0.838761 & 0.0405873 & 0.89991 & 0.155976 & 0.738971 & 0.0308812 & 1.0906 \\
\hline 0.83377 & 0.0407967 & 0.91542 & 0.164386 & 0.742791 & 0.0252031 & 1.0835 \\
\hline 0.831158 & 0.0371386 & 0.935867 & 0.174193 & 0.748592 & 0.0331054 & 1.10682 \\
\hline 0.843229 & 0.0345951 & 0.959217 & 0.179748 & 0.75713 & 0.027638 & 1.11421 \\
\hline 0.834201 & 0.0473336 & 0.981274 & 0.197279 & 0.765058 & 0.0337724 & 1.10463 \\
\hline 0.843699 & 0.0420765 & 1.00608 & 0.209141 & 0.790836 & 0.0323537 & 1.10225 \\
\hline 0.840037 & 0.0391193 & 1.02648 & 0.217736 & 0.79595 & 0.0325747 & 1.10271 \\
\hline 0.842435 & 0.0368118 & 1.06302 & 0.24548 & 0.810704 & 0.0325652 & 1.1451 \\
\hline 0.850208 & 0.0395694 & 1.06999 & 0.270025 & 0.816246 & 0.0348637 & 1.107 \\
\hline 0.85989 & 0.0454629 & 1.11878 & 0.279595 & 0.838417 & 0.0358857 & 1.14765 \\
\hline 0.861339 & 0.0386847 & 1.13113 & 0.294805 & 0.859083 & 0.0416097 & 1.10211 \\
\hline 0.865213 & 0.0440706 & 1.16502 & 0.314963 & 0.861833 & 0.0356785 & 1.13297 \\
\hline 0.853685 & 0.0369935 & 1.18712 & 0.339725 & 0.891038 & 0.0338984 & 1.12717 \\
\hline 0.881142 & 0.0492657 & 1.22379 & 0.351476 & 0.893713 & 0.0347871 & 1.11716 \\
\hline 0.879144 & 0.0625007 & 1.20984 & 0.348049 & 0.907592 & 0.0361419 & 1.12244 \\
\hline 0.885408 & 0.0420382 & 1.23555 & 0.354271 & 0.922391 & 0.046723 & 1.13889 \\
\hline 0.880438 & 0.0462595 & 1.24759 & 0.38675 & 0.918394 & 0.0405443 & 1.1418 \\
\hline 0.891436 & 0.0437047 & 1.2275 & 0.374737 & 0.929285 & 0.0468162 & 1.11709 \\
\hline 0.903551 & 0.0396681 & 1.23754 & 0.356521 & 0.927541 & 0.0408065 & 1.12984 \\
\hline 0.893358 & 0.048967 & 1.21751 & 0.334865 & 0.909183 & 0.0382191 & 1.12159 \\
\hline 0.886757 & 0.0368487 & 1.18083 & 0.326958 & 0.894833 & 0.0414884 & 1.104 \\
\hline 0.892582 & 0.0481696 & 1.16741 & 0.318987 & 0.893038 & 0.0413067 & 1.10184 \\
\hline 0.891804 & 0.0444942 & 1.13209 & 0.306025 & 0.878782 & 0.0415412 & 1.10011 \\
\hline 0.888349 & 0.0309159 & 1.08531 & 0.277784 & 0.856344 & 0.0390053 & 1.10708 \\
\hline 0.894446 & 0.0410535 & 1.07011 & 0.242609 & 0.843562 & 0.0436084 & 1.09961 \\
\hline 0.878732 & 0.0393413 & 1.03335 & 0.232806 & 0.826638 & 0.0363842 & 1.08177 \\
\hline 0.882313 & 0.0483119 & 1.00258 & 0.208957 & 0.813702 & 0.0377381 & 1.09531 \\
\hline 0.866698 & 0.0351767 & 0.96922 & 0.194402 & 0.796864 & 0.0320749 & 1.07391 \\
\hline 0.869799 & 0.0465492 & 0.935213 & 0.166963 & 0.777263 & 0.0354037 & 1.07253 \\
\hline 0.867358 & 0.0395226 & 0.922149 & 0.165633 & 0.768272 & 0.0317831 & 1.08449 \\
\hline 0.859306 & 0.0373821 & 0.903451 & 0.144654 & 0.748851 & 0.0268741 & 1.04784 \\
\hline 0.842867 & 0.0369896 & 0.883075 & 0.157079 & 0.746277 & 0.025915 & 1.05393 \\
\hline 0.856837 & 0.0509439 & 0.863838 & 0.132681 & 0.732846 & 0.0304691 & 1.04825 \\
\hline 0.837738 & 0.0371347 & 0.856003 & 0.140007 & 0.726635 & 0.0241683 & 1.06416 \\
\hline 0.83811 & 0.0351286 & 0.848535 & 0.130808 & 0.719701 & 0.0253746 & 1.07086 \\
\hline 0.838832 & 0.0374277 & 0.828674 & 0.125532 & 0.71485 & 0.0327013 & 1.04174 \\
\hline 0.848751 & 0.0459633 & 0.819649 & 0.123414 & 0.714985 & 0.029095 & 1.0591 \\
\hline 0.846058 & 0.0388449 & 0.824297 & 0.136975 & 0.701643 & 0.0202541 & 1.06166 \\
\hline 0.844326 & 0.0351475 & 0.813111 & 0.122175 & 0.702861 & 0.0309218 & 1.06323 \\
\hline 0.839198 & 0.0451275 & 0.817037 & 0.116201 & 0.698122 & 0.0304617 & 1.07278 \\
\hline 0.839707 & 0.0402739 & 0.798763 & 0.118049 & 0.696139 & 0.0281016 & 1.06721 \\
\hline 0.840237 & 0.0499812 & 0.790818 & 0.110693 & 0.701742 & 0.0266436 & 1.08643 \\
\hline 0.851748 & 0.0436735 & 0.794178 & 0.119421 & 0.70629 & 0.0251203 & 1.06473 \\
\hline 0.849307 & 0.039688 & 0.797483 & 0.110429 & 0.702506 & 0.028947 & 1.07778 \\
\hline 0.845985 & 0.0446901 & 0.786389 & 0.124538 & 0.704491 & 0.0210866 & 1.10001 \\
\hline 0.849418 & 0.0425667 & 0.787815 & 0.106729 & 0.705542 & 0.0324214 & 1.09557 \\
\hline 0.846145 & 0.0390052 & 0.782223 & 0.113574 & 0.699081 & 0.0218965 & 1.08669 \\
\hline
\end{tabular}




\begin{tabular}{|c|c|c|c|c|c|c|}
\hline 0.848405 & 0.0486996 & 0.791952 & 0.113482 & 0.702396 & 0.0281725 & 1.11935 \\
\hline 0.847176 & 0.0405875 & 0.778199 & 0.0996043 & 0.703765 & 0.0289203 & 1.10632 \\
\hline 0.843671 & 0.0335678 & 0.776456 & 0.115113 & 0.702822 & 0.0302031 & 1.1155 \\
\hline 0.847196 & 0.0346854 & 0.774346 & 0.105477 & 0.700633 & 0.0329531 & 1.11526 \\
\hline 0.847905 & 0.0429883 & 0.777764 & 0.0961469 & 0.700899 & 0.0254439 & 1.1319 \\
\hline 0.846509 & 0.036298 & 0.77053 & 0.101629 & 0.705892 & 0.0341651 & 1.14594 \\
\hline 0.858672 & 0.0421144 & 0.76479 & 0.0937145 & 0.694743 & 0.026982 & 1.14453 \\
\hline 0.857593 & 0.0378118 & 0.771483 & 0.100086 & 0.70085 & 0.0216479 & 1.1443 \\
\hline 0.853528 & 0.033279 & 0.774001 & 0.0979894 & 0.696218 & 0.0311815 & 1.1416 \\
\hline 0.869 & 0.0469867 & 0.760395 & 0.0972726 & 0.702476 & 0.030837 & 1.13957 \\
\hline 0.860515 & 0.0455446 & 0.774867 & 0.0964683 & 0.700712 & 0.0304849 & 1.13758 \\
\hline 0.85465 & 0.0447772 & 0.767705 & 0.0922729 & 0.694378 & 0.029144 & 1.14056 \\
\hline 0.860897 & 0.0417809 & 0.757497 & 0.0983181 & 0.700386 & 0.0314289 & 1.15091 \\
\hline 0.855308 & 0.0460532 & 0.75302 & 0.0959541 & 0.690117 & 0.0238111 & 1.14328 \\
\hline 0.86099 & 0.0476998 & 0.758861 & 0.0753228 & 0.696919 & 0.030694 & 1.14385 \\
\hline 0.862828 & 0.0399831 & 0.765624 & 0.0813003 & 0.693261 & 0.032249 & 1.15457 \\
\hline 0.873284 & 0.048336 & 0.768717 & 0.102223 & 0.701802 & 0.0335086 & 1.1537 \\
\hline 0.864503 & 0.0461755 & 0.761568 & 0.0875149 & 0.696155 & 0.0311945 & 1.15699 \\
\hline 0.866981 & 0.0332354 & 0.759973 & 0.0858791 & 0.69073 & 0.0369788 & 1.15963 \\
\hline 0.8529 & 0.0410277 & 0.757045 & 0.0809012 & 0.695479 & 0.0304133 & 1.14709 \\
\hline 0.870564 & 0.0374355 & 0.756142 & 0.0913569 & 0.698649 & 0.033883 & 1.11514 \\
\hline 0.856012 & 0.0463113 & 0.750202 & 0.0797283 & 0.694988 & 0.026924 & 1.12214 \\
\hline 0.867813 & 0.0355794 & 0.758161 & 0.0985121 & 0.695845 & 0.0262428 & 1.12835 \\
\hline 0.874754 & 0.0388627 & 0.758526 & 0.09003 & 0.697511 & 0.0248502 & 1.10186 \\
\hline 0.861233 & 0.039427 & 0.771722 & 0.0940057 & 0.692267 & 0.0246872 & 1.10476 \\
\hline 0.854731 & 0.046543 & 0.74656 & 0.0889708 & 0.692319 & 0.0272955 & 1.06819 \\
\hline 0.85619 & 0.045326 & 0.757397 & 0.0890688 & 0.70102 & 0.0240955 & 1.07193 \\
\hline 0.867438 & 0.0408561 & 0.756824 & 0.0802817 & 0.696353 & 0.0285281 & 1.07148 \\
\hline 0.864015 & 0.0438959 & 0.762742 & 0.0807595 & 0.697337 & 0.0315426 & 1.06125 \\
\hline 0.849034 & 0.0427056 & 0.765809 & 0.0950346 & 0.687316 & 0.0253184 & 1.03774 \\
\hline 0.86661 & 0.0383892 & 0.756739 & 0.0873904 & 0.695534 & 0.0320336 & 1.04277 \\
\hline 0.859966 & 0.0380701 & 0.751974 & 0.0817807 & 0.699814 & 0.0288414 & 1.03306 \\
\hline 0.865129 & 0.0434513 & 0.760163 & 0.0923333 & 0.698703 & 0.0286132 & 1.0367 \\
\hline 0.862237 & 0.0432781 & 0.754556 & 0.0878169 & 0.68638 & 0.026779 & 1.02273 \\
\hline 0.844717 & 0.0438368 & 0.756307 & 0.0833338 & 0.693927 & 0.0244874 & 1.02491 \\
\hline 0.842625 & 0.0456076 & 0.748639 & 0.0886789 & 0.693029 & 0.0336311 & 1.01309 \\
\hline 0.857397 & 0.0460697 & 0.749095 & 0.0794184 & 0.693304 & 0.0284076 & 1.00885 \\
\hline 0.851467 & 0.0365942 & 0.760805 & 0.0824528 & 0.702695 & 0.0317771 & 1.03458 \\
\hline 0.863979 & 0.0406875 & 0.765323 & 0.090687 & 0.700902 & 0.0308868 & 1.0037 \\
\hline 0.851104 & 0.0410449 & 0.754201 & 0.0844634 & 0.69778 & 0.0265069 & 0.985522 \\
\hline 0.838598 & 0.0380401 & 0.75886 & 0.0912683 & 0.702056 & 0.0311756 & 0.999847 \\
\hline 0.854898 & 0.0439535 & 0.775262 & 0.0888096 & 0.693208 & 0.0263698 & 1.00357 \\
\hline 0.838285 & 0.0403687 & 0.76248 & 0.0898088 & 0.697536 & 0.0263979 & 1.01301 \\
\hline 0.841855 & 0.0450822 & 0.759262 & 0.0860805 & 0.698726 & 0.0361966 & 1.01291 \\
\hline 0.84438 & 0.0369434 & 0.759837 & 0.0848015 & 0.694243 & 0.0311507 & 1.00419 \\
\hline 0.849254 & 0.0342205 & 0.752601 & 0.0831129 & 0.694155 & 0.0261458 & 1.02123 \\
\hline 0.848752 & 0.0458902 & 0.759122 & 0.082373 & 0.696458 & 0.0201736 & 0.999229 \\
\hline
\end{tabular}




\begin{tabular}{|c|c|c|c|c|c|c|}
\hline 0.850698 & 0.039333 & 0.755372 & 0.0833492 & 0.699124 & 0.0310885 & 0.98843 \\
\hline 0.840479 & 0.0396731 & 0.758216 & 0.0689831 & 0.695741 & 0.0284452 & 1.00086 \\
\hline 0.843469 & 0.0359548 & 0.748287 & 0.0779699 & 0.688628 & 0.0256525 & 1.00632 \\
\hline 0.846546 & 0.039098 & 0.744384 & 0.077764 & 0.686768 & 0.0340516 & 0.992557 \\
\hline 0.831275 & 0.0428439 & 0.766669 & 0.0889948 & 0.691812 & 0.0237556 & 0.987002 \\
\hline 0.833879 & 0.0401753 & 0.752488 & 0.0836813 & 0.695532 & 0.0253376 & 1.00611 \\
\hline 0.842216 & 0.048601 & 0.751991 & 0.0824023 & 0.702011 & 0.0270836 & 0.97565 \\
\hline 0.834308 & 0.0489264 & 0.747929 & 0.0743534 & 0.690045 & 0.0271741 & 0.982012 \\
\hline 0.848004 & 0.0345427 & 0.755709 & 0.0894492 & 0.692579 & 0.0257379 & 0.993897 \\
\hline 0.832748 & 0.0374267 & 0.756003 & 0.0817325 & 0.689247 & 0.0250636 & 0.985412 \\
\hline 0.834295 & 0.0359662 & 0.757052 & 0.0814349 & 0.691871 & 0.0311492 & 0.987554 \\
\hline 0.8285 & 0.0434347 & 0.750756 & 0.090324 & 0.688852 & 0.033251 & 0.976179 \\
\hline 0.83892 & 0.0444121 & 0.757372 & 0.0854812 & 0.694673 & 0.024009 & 0.982074 \\
\hline 0.834213 & 0.0368092 & 0.755834 & 0.0838391 & 0.688665 & 0.0217652 & 0.982044 \\
\hline 0.848231 & 0.0481967 & 0.744028 & 0.078823 & 0.687473 & 0.025644 & 0.982391 \\
\hline 0.840758 & 0.0333417 & 0.748038 & 0.0762145 & 0.683085 & 0.0269587 & 0.989911 \\
\hline 0.836375 & 0.0401974 & 0.740602 & 0.0796954 & 0.684688 & 0.0264757 & 0.962672 \\
\hline 0.837 & 0.0491307 & 0.740433 & 0.0670427 & 0.683765 & 0.0249843 & 0.988446 \\
\hline 0.831435 & 0.0455257 & 0.752252 & 0.0733748 & 193 & 0.0250323 & 0.989052 \\
\hline 0.823336 & 0.0392367 & 0.757627 & 0.0880767 & 0.684336 & 0.0289141 & 0.966383 \\
\hline 0.844217 & 0.0428871 & 0.754056 & 0.0850616 & 0.687841 & 0.0299674 & 0.988633 \\
\hline 0.834626 & 0.0339781 & 0.749482 & 0.0825474 & 437 & 0.0263481 & 0.98133 \\
\hline 0.820977 & 0.0456489 & 0.751893 & 0.0842839 & 0.686756 & 0.0236223 & 0.9893 \\
\hline 0.832424 & 0.0494099 & 0.747514 & 0.0821669 & 0.683664 & 0.0326996 & 0.991356 \\
\hline 0.835103 & 0.0405389 & 0.745301 & 0.0790531 & 0.686959 & 0.0278028 & 0.988734 \\
\hline 0.841842 & 0.0490687 & 0.751951 & 0.0716439 & 0.682268 & 0.0278231 & 0.979042 \\
\hline 0.834478 & 0.0321205 & 0.755104 & 0.0800713 & 0.682919 & 0.0271465 & 1.00373 \\
\hline 0.831536 & 0.0409308 & 0.743936 & 0.0860862 & 0.68318 & 0.0204316 & 1.01535 \\
\hline 0.829607 & 0.0469951 & 0.753107 & 0.0835957 & 0.690232 & 0.0305405 & 0.987903 \\
\hline 0.839029 & 0.0457984 & 0.738081 & 0.0796088 & 0.684955 & 0.0170511 & 0.995297 \\
\hline 0.833083 & 0.0386086 & 0.752501 & 0.0801323 & 0.681625 & 0.0316106 & 0.990568 \\
\hline 0.828297 & 0.0341597 & 0.746173 & 0.085734 & 0.679854 & 0.0230431 & 0.998897 \\
\hline 0.833078 & 0.0361229 & 0.743515 & 0.0868761 & 0.68362 & 0.0308558 & 1.02251 \\
\hline 0.8285 & 0.0342609 & 0.753235 & 0.0766919 & 0.684403 & 0.0231436 & 1.01524 \\
\hline 0.842399 & 0.0380671 & 0.754956 & 0.0798846 & 0.680625 & 0.0222828 & 1.02048 \\
\hline 0.839311 & 0.0342687 & 0.758077 & 0.0858088 & 0.68766 & 0.0231338 & 1.03945 \\
\hline 0.843657 & 0.0384446 & 0.749745 & 0.0838562 & 0.679469 & 0.0285783 & 1.04168 \\
\hline 0.833385 & 0.0336941 & 0.755127 & 0.0810219 & 0.678194 & 0.0273453 & 1.03671 \\
\hline 0.82016 & 0.0308444 & 0.752118 & 0.0810168 & 0.682189 & 0.0219686 & 1.02313 \\
\hline 0.841726 & 0.046134 & 0.751212 & 0.0766755 & 0.68437 & 0.0269283 & 1.04384 \\
\hline 0.842455 & 0.0413516 & 0.761375 & 0.0831553 & 0.683583 & 0.025813 & 1.05906 \\
\hline 0.827631 & 0.0335236 & 0.755184 & 0.0828283 & 0.684124 & 0.0222621 & 1.05177 \\
\hline 0.824714 & 0.0391878 & 0.759304 & 0.08703 & 0.681248 & 0.0287762 & 1.04885 \\
\hline 0.830326 & 0.0373922 & 0.749479 & 0.0894545 & 0.68555 & 0.0259845 & 1.05046 \\
\hline 0.828435 & 0.0404531 & 0.750219 & 0.0838986 & 0.679222 & 0.0234373 & 1.06252 \\
\hline 0.82206 & 0.0451394 & 0.753951 & 0.0940546 & 0.686463 & 0.0274068 & 1.05948 \\
\hline 0.825563 & 0.0407062 & 0.749282 & 0.0776435 & 0.679307 & 0.0233716 & 1.06082 \\
\hline
\end{tabular}




\begin{tabular}{|c|c|c|c|c|c|c|}
\hline 0.825658 & 0.0386597 & 0.748458 & 0.0810592 & 0.675879 & 0.0299834 & 1.06929 \\
\hline 0.824935 & 0.0356302 & 0.758154 & 0.0848329 & 0.672244 & 0.0274505 & 1.04552 \\
\hline 0.817907 & 0.0395812 & 0.754149 & 0.0853794 & 0.683287 & 0.0341036 & 1.05346 \\
\hline 0.805257 & 0.0335708 & 0.754 & 0.0823324 & 0.676053 & 0.0322457 & 1.04534 \\
\hline 0.816671 & 0.0295689 & 0.74712 & 0.0847006 & 0.675015 & 0.0282661 & 1.03937 \\
\hline 0.815968 & 0.042772 & 0.751255 & 0.0881886 & 0.678801 & 0.0331338 & 1.02066 \\
\hline 0.809455 & 0.0346701 & 0.75294 & 0.0758733 & 0.667439 & 0.0240804 & 1.02289 \\
\hline 0.812395 & 0.037794 & 0.751902 & 0.0829328 & 0.673907 & 0.0246692 & 1.02093 \\
\hline 0.809081 & 0.039483 & 0.746908 & 0.0830064 & 0.66796 & 0.0324558 & 1.00563 \\
\hline 0.816266 & 0.0410126 & 0.751915 & 0.0885602 & 0.671241 & 0.0232935 & 1.01967 \\
\hline 0.806826 & 0.0447412 & 0.75722 & 0.0938217 & 0.664238 & 0.0263118 & 0.997414 \\
\hline 0.80421 & 0.0419473 & 0.750246 & 0.0890714 & 0.672513 & 0.0257943 & 0.990956 \\
\hline 0.802999 & 0.0340117 & 0.745323 & 0.0870138 & 0.673945 & 0.0245345 & 0.989588 \\
\hline 0.796246 & 0.0412301 & 0.746282 & 0.0814619 & 0.670986 & 0.0240971 & 0.9851 \\
\hline 0.80549 & 0.0365467 & 0.744798 & 0.0823383 & 0.667133 & 0.0215928 & 0.977756 \\
\hline 0.803771 & 0.0461712 & 0.748338 & 0.0841383 & 0.669752 & 0.0245446 & 0.961601 \\
\hline 0.800426 & 0.0332329 & 0.741782 & 0.0812553 & 0.663595 & 0.0212996 & 0.968869 \\
\hline 0.797057 & 0.0308849 & 0.75237 & 0.0857508 & 0.658196 & 0.0221678 & 0.974919 \\
\hline 0.79206 & 0.0370994 & 0.740037 & 0.0845546 & 0.664438 & 0.0275852 & 0.945913 \\
\hline 0.785618 & 0.0376571 & 0.734139 & 0.0800211 & 0.662822 & 0.0273123 & 0.945732 \\
\hline 0.785065 & 0.0338744 & 0.734217 & 0.0810967 & 0.656889 & 0.0245335 & 0.955708 \\
\hline 0.795263 & 0.0414314 & 0.744698 & 0.0841562 & 0.657953 & 0.0224908 & 0.93932 \\
\hline 0.793077 & 0.0377466 & 0.730524 & 0.0753256 & 0.649966 & 0.0274046 & 0.958892 \\
\hline 0.794192 & 0.0421001 & 0.736745 & 0.0778362 & 0.656601 & 0.0269177 & 0.95587 \\
\hline 0.783169 & 0.028723 & 0.739094 & 0.0904415 & 0.65244 & 0.0261344 & 0.928793 \\
\hline 0.78685 & 0.0301117 & 0.731237 & 0.0805684 & 0.654708 & 0.0289357 & 0.949125 \\
\hline 0.778919 & 0.045914 & 0.735003 & 0.0897039 & 0.653351 & 0.0260107 & 0.959145 \\
\hline 0.787884 & 0.030787 & 0.737207 & 0.0857049 & 0.644545 & 0.0261581 & 0.953538 \\
\hline 0.773412 & 0.0426539 & 0.738429 & 0.077185 & 0.646603 & 0.0209794 & 0.941718 \\
\hline 0.785745 & 0.0506185 & 0.730174 & 0.0805476 & 0.647903 & 0.0234109 & 0.943891 \\
\hline 0.781002 & 0.0505028 & 0.724081 & 0.0788281 & 0.651014 & 0.0239913 & 0.956998 \\
\hline 0.757036 & 0.0415234 & 0.723354 & 0.0780007 & 0.648255 & 0.0227642 & 0.983028 \\
\hline 0.773511 & 0.0469891 & 0.730283 & 0.0797259 & 0.640983 & 0.0328041 & 0.948502 \\
\hline 0.770256 & 0.032475 & 0.719206 & 0.0681826 & 0.647186 & 0.0210487 & 0.967639 \\
\hline 0.767002 & 0.0327726 & 0.729588 & 0.0796526 & 0.647385 & 0.028097 & 0.980438 \\
\hline 0.768627 & 0.0425142 & 0.724627 & 0.0813631 & 0.642634 & 0.0196635 & 0.989709 \\
\hline 0.764625 & 0.0396065 & 0.728265 & 0.0694427 & 0.646017 & 0.0261809 & 0.99585 \\
\hline 0.762762 & 0.0376237 & 0.727828 & 0.08045 & 0.646924 & 0.0174005 & 1.01001 \\
\hline 0.763434 & 0.0479703 & 0.734785 & 0.0753034 & 0.639929 & 0.0239284 & 1.02196 \\
\hline 0.766899 & 0.0389631 & 0.729746 & 0.075493 & 0.638999 & 0.0289283 & 1.03191 \\
\hline 0.753749 & 0.0400127 & 0.734033 & 0.0800045 & 0.634123 & 0.0276001 & 1.03928 \\
\hline 0.751424 & 0.0437233 & 0.719298 & 0.076855 & 0.638564 & 0.0240001 & 1.07124 \\
\hline 0.759219 & 0.0417551 & 0.724189 & 0.0747195 & 0.639499 & 0.0316636 & 1.06819 \\
\hline 0.754418 & 0.0328176 & 0.727065 & 0.0757341 & 0.638032 & 0.0188277 & 1.09198 \\
\hline 0.758648 & 0.0366057 & 0.724075 & 0.0673161 & 0.634491 & 0.0193622 & 1.10951 \\
\hline 0.747859 & 0.03344 & 0.737494 & 0.0801671 & 0.634876 & 0.0223892 & 1.12602 \\
\hline 0.743691 & 0.0358288 & 0.727693 & 0.0745392 & 0.636828 & 0.0259215 & 1.12639 \\
\hline
\end{tabular}




\begin{tabular}{|c|c|c|c|c|c|c|}
\hline 0.747686 & 0.0348459 & 0.723219 & 0.0684382 & 0.64218 & 0.0256609 & 1.14928 \\
\hline 0.750112 & 0.0327794 & 0.715126 & 0.0739055 & 0.638747 & 0.0216749 & 1.13131 \\
\hline 0.744809 & 0.0394794 & 0.72433 & 0.0691531 & 0.629723 & 0.0176872 & 1.14087 \\
\hline 0.746438 & 0.0346799 & 0.718809 & 0.071447 & 0.629433 & 0.0242888 & 1.16072 \\
\hline 0.732498 & 0.0431975 & 0.717122 & 0.0733727 & 0.628465 & 0.0229791 & 1.16258 \\
\hline 0.736583 & 0.0394093 & 0.722596 & 0.0754474 & 0.630136 & 0.017723 & 1.16346 \\
\hline 0.734938 & 0.0380376 & 0.714446 & 0.067822 & 0.630273 & 0.0198326 & 1.14189 \\
\hline 0.733256 & 0.0360894 & 0.711265 & 0.065246 & 0.624361 & 0.0242521 & 1.14423 \\
\hline 0.733258 & 0.0379431 & 0.714533 & 0.0669621 & 0.62682 & 0.0226309 & 1.14109 \\
\hline 0.730751 & 0.0328783 & 0.701786 & 0.0703251 & 0.625511 & 0.0253043 & 1.11414 \\
\hline 0.739689 & 0.0355194 & 0.709804 & 0.0660481 & 0.625334 & 0.0239826 & 1.1029 \\
\hline 0.747122 & 0.0420051 & 0.709313 & 0.0755712 & 0.628296 & 0.0184486 & 1.10315 \\
\hline 0.738511 & 0.0301019 & 0.704076 & 0.0708757 & 0.621802 & 0.0226274 & 1.10417 \\
\hline 0.735766 & 0.0424107 & 0.699742 & 0.0687401 & 0.624912 & 0.0268837 & 1.06636 \\
\hline 0.719198 & 0.0322756 & 0.705685 & 0.0714102 & 0.621073 & 0.0243621 & 1.08519 \\
\hline 0.728386 & 0.0339125 & 0.709119 & 0.0720991 & 0.621815 & 0.0241458 & 1.08389 \\
\hline 0.723433 & 0.037033 & 0.705671 & 0.0691752 & 0.622531 & 0.01532 & 1.0895 \\
\hline 0.729049 & 0.0341901 & 0.712302 & 0.0763456 & 0.627519 & 0.0155805 & 1.08195 \\
\hline 0.714672 & 0.0294171 & 0.709547 & 0.0722268 & 0.625239 & 0.0206732 & 1.08232 \\
\hline 0.73132 & 0.0391078 & 0.722338 & 0.0664469 & 0.620262 & 0.0193316 & 1.08603 \\
\hline 0.730561 & 0.0337336 & 0.719872 & 0.079288 & 0.627074 & 0.0285975 & 1.0939 \\
\hline 0.721762 & 0.0359656 & 0.755779 & 0.0815532 & 0.631445 & 0.0243965 & 1.12916 \\
\hline 0.710792 & 0.037101 & 0.755805 & 0.0958036 & 0.639595 & 0.0261488 & 1.13636 \\
\hline 0.718703 & 0.0346457 & 0.761948 & 0.0948172 & 0.642864 & 0.0178237 & 1.17701 \\
\hline 0.729363 & 0.0377169 & 0.781665 & 0.108151 & 0.658261 & 0.0248233 & 1.20445 \\
\hline 0.710576 & 0.0406256 & 0.794135 & 0.099376 & 0.665879 & 0.026188 & 1.24548 \\
\hline 0.724276 & 0.0346723 & 0.805401 & 0.123186 & 0.67681 & 0.0237303 & 1.33753 \\
\hline 0.725914 & 0.0364443 & 0.831074 & 0.138421 & 0.676028 & 0.0270242 & 1.42618 \\
\hline 0.726804 & 0.0311733 & 0.852404 & 0.136987 & 0.690814 & 0.0293292 & 1.52736 \\
\hline 0.73818 & 0.0334408 & 0.868738 & 0.153976 & 0.687827 & 0.0255392 & 1.6628 \\
\hline 0.735685 & 0.0449901 & 0.876392 & 0.150301 & 0.711304 & 0.0291339 & 1.85093 \\
\hline 0.739028 & 0.0442798 & 0.905673 & 0.16318 & 0.714815 & 0.0288643 & 2.10893 \\
\hline 0.750783 & 0.0415977 & 0.906236 & 0.163825 & 0.72281 & 0.0359531 & 2.36145 \\
\hline 0.739918 & 0.0343087 & 0.925237 & 0.166909 & 0.733119 & 0.0309053 & 2.72176 \\
\hline 0.743519 & 0.0387593 & 0.942438 & 0.189027 & 0.742868 & 0.0331614 & 3.2249 \\
\hline 0.744245 & 0.0405671 & 0.939159 & 0.188828 & 0.751805 & 0.0350246 & 3.77616 \\
\hline 0.745565 & 0.0296239 & 0.934928 & 0.175309 & 0.748469 & 0.0346737 & 4.35815 \\
\hline 0.742393 & 0.0383868 & 0.947725 & 0.178176 & 0.752844 & 0.0293195 & 5.10259 \\
\hline 0.750577 & 0.0340317 & 0.942869 & 0.184649 & 0.751573 & 0.0364931 & 6.00072 \\
\hline 0.753851 & 0.0384571 & 0.932918 & 0.182092 & 0.751382 & 0.0270949 & 6.85881 \\
\hline 0.738608 & 0.0320571 & 0.910807 & 0.174765 & 0.738866 & 0.0267356 & 7.97525 \\
\hline 0.743537 & 0.0382348 & 0.902172 & 0.168839 & 0.744829 & 0.0317572 & 9.15766 \\
\hline 0.734513 & 0.039927 & 0.903372 & 0.183615 & 0.739332 & 0.0295949 & 10.4108 \\
\hline 0.737462 & 0.0331416 & 0.887023 & 0.162968 & 0.735849 & 0.0320166 & 11.69 \\
\hline 0.732495 & 0.0369019 & 0.885289 & 0.1597 & 0.729349 & 0.0245465 & 12.934 \\
\hline 0.732133 & 0.0382046 & 0.865427 & 0.16748 & 0.732734 & 0.0297742 & 14.293 \\
\hline 0.712374 & 0.0290937 & 0.855914 & 0.183598 & 0.724037 & 0.0249492 & 15.4947 \\
\hline
\end{tabular}




\begin{tabular}{|c|c|c|c|c|c|c|}
\hline 0.724592 & 0.0406875 & 0.849209 & 0.182693 & 0.721315 & 0.0246897 & 16.6514 \\
\hline 0.713781 & 0.0276943 & 0.831647 & 0.185818 & 0.716888 & 0.0289356 & 17.6194 \\
\hline 0.699677 & 0.0386789 & 0.826746 & 0.182289 & 0.718804 & 0.0329662 & 18.5174 \\
\hline 0.703071 & 0.0356178 & 0.832983 & 0.194977 & 0.702991 & 0.0373228 & 19.2301 \\
\hline 0.704591 & 0.0387725 & 0.809462 & 0.177388 & 0.69945 & 0.0327313 & 19.7861 \\
\hline 0.690454 & 0.0260051 & 0.79719 & 0.188334 & 0.698019 & 0.0288447 & 19.9108 \\
\hline 0.682903 & 0.0394224 & 0.790752 & 0.200675 & 0.702785 & 0.0319859 & 19.9456 \\
\hline 0.688745 & 0.0310815 & 0.784081 & 0.176859 & 0.696859 & 0.0253275 & 19.6697 \\
\hline 0.680676 & 0.0356054 & 0.772915 & 0.171676 & 0.675152 & 0.0285855 & 19.0416 \\
\hline 0.67357 & 0.0396407 & 0.754436 & 0.16982 & 0.679877 & 0.0286356 & 18.4268 \\
\hline 0.679237 & 0.0360753 & 0.744211 & 0.147853 & 0.669471 & 0.0298475 & 17.4087 \\
\hline 0.667754 & 0.042197 & 0.733195 & 0.151784 & 0.653461 & 0.0300205 & 16.2454 \\
\hline 0.665785 & 0.0337319 & 0.726888 & 0.131735 & 0.65221 & 0.022735 & 15.0644 \\
\hline 0.663807 & 0.0331811 & 0.715625 & 0.134607 & 0.644678 & 0.0332739 & 13.7368 \\
\hline 0.667833 & 0.0343418 & 0.70541 & 0.123093 & 0.637838 & 0.0303985 & 12.4629 \\
\hline 0.670583 & 0.0285583 & 0.688001 & 0.105763 & 0.619389 & 0.0263769 & 11.0974 \\
\hline 0.66528 & 0.0287997 & 0.68204 & 0.094568 & 0.62252 & 0.0239757 & 9.85888 \\
\hline 0.650799 & 0.0415258 & 0.682779 & 0.0883349 & 0.610292 & 0.0240986 & 8.54868 \\
\hline 0.664775 & 0.0356457 & 0.663897 & 0.076942 & 0.6 & 0.02 & 7.42176 \\
\hline 0.661938 & 0.0261695 & 0.664949 & 0.0751326 & 0.596611 & 0.0286461 & 6.40743 \\
\hline 0.664496 & 0.0409443 & 0.661744 & 0.0723697 & 0.591498 & 0.0172249 & 5.41277 \\
\hline 0.663474 & 0.0364521 & 0.652634 & 0.0636212 & 0.578307 & 0.0233367 & 4.60072 \\
\hline 0.654036 & 0.0366044 & 0.643516 & 0.0609519 & 0.582717 & 0.018595 & 3.87436 \\
\hline 0.654275 & 0.0357727 & 0.640515 & 0.0638706 & 0.569322 & 0.0255036 & 3.24846 \\
\hline 0.64794 & 0.0331241 & 0.632877 & 0.0615648 & 0.582413 & 0.0231746 & 2.75451 \\
\hline 0.648437 & 0.0330404 & 0.631078 & 0.0503339 & 0.568815 & 0.0223753 & 2.34713 \\
\hline 0.64517 & 0.0295818 & 0.626496 & 0.0551879 & 0.560817 & 0.0179258 & 1.97609 \\
\hline 0.639766 & 0.0319626 & 0.623867 & 0.0550159 & 0.559627 & 0.0180242 & 1.72792 \\
\hline 0.644024 & 0.0388813 & 0.632668 & 0.0592187 & 0.55616 & 0.0222186 & 1.51612 \\
\hline 0.648865 & 0.029835 & 0.615777 & 0.052055 & 0.556851 & 0.0217917 & 1.35475 \\
\hline 0.639032 & 0.0378634 & 0.617149 & 0.0449052 & 0.54975 & 0.0238493 & 1.22993 \\
\hline 0.642343 & 0.0358957 & 0.602195 & 0.0517908 & 0.550632 & 0.024473 & 1.1115 \\
\hline 0.645051 & 0.0320855 & 0.610354 & 0.0589274 & 0.541489 & 0.0210899 & 1.06625 \\
\hline 0.640542 & 0.0328498 & 0.596479 & 0.0509776 & 0.541647 & 0.0163035 & 0.978749 \\
\hline 0.633393 & 0.0313291 & 0.605792 & 0.0573135 & 0.540513 & 0.019801 & 0.98021 \\
\hline 0.62859 & 0.034828 & 0.596228 & 0.0548478 & 0.54343 & 0.0170563 & 0.958205 \\
\hline 0.635022 & 0.0365342 & 0.596774 & 0.0490531 & 0.530204 & 0.0239378 & 0.936563 \\
\hline 0.625513 & 0.034567 & 0.603644 & 0.0510916 & 0.535502 & 0.0214428 & 0.932248 \\
\hline 0.633564 & 0.0424712 & 0.588308 & 0.0542987 & 0.535178 & 0.02105 & 0.909832 \\
\hline 0.628083 & 0.038744 & 0.594361 & 0.0587719 & 0.53717 & 0.0197314 & 0.915826 \\
\hline 0.624813 & 0.0324154 & 0.589111 & 0.0591209 & 0.52771 & 0.0208022 & 0.929077 \\
\hline 0.619539 & 0.0343992 & 0.586629 & 0.0481403 & 0.533067 & 0.0274892 & 0.943794 \\
\hline 0.606679 & 0.0299114 & 0.577892 & 0.0516572 & 0.535807 & 0.0162889 & 0.937044 \\
\hline 0.614601 & 0.0374819 & 0.583531 & 0.0546829 & 0.527536 & 0.0264317 & 0.960615 \\
\hline 0.625925 & 0.0317952 & 0.58375 & 0.0462009 & 0.535477 & 0.0263291 & 0.992792 \\
\hline 0.622233 & 0.0292699 & 0.591554 & 0.0502923 & 0.542041 & 0.0203571 & 1.01302 \\
\hline 0.621663 & 0.029896 & 0.582961 & 0.0529576 & 0.531841 & 0.0237867 & 1.07792 \\
\hline
\end{tabular}




\begin{tabular}{|c|c|c|c|c|c|c|}
\hline 0.620508 & 0.039744 & 0.596248 & 0.0549721 & 0.543153 & 0.0193075 & 1.10778 \\
\hline 0.614682 & 0.0334725 & 0.587777 & 0.0452224 & 0.539274 & 0.0197647 & 1.1901 \\
\hline 0.609719 & 0.025528 & 0.593574 & 0.0509285 & 0.551105 & 0.018239 & 1.25518 \\
\hline 0.613734 & 0.0296645 & 0.600772 & 0.0571594 & 0.56053 & 0.0239728 & 1.36526 \\
\hline 0.59804 & 0.0348238 & 0.596632 & 0.0559654 & 0.560607 & 0.0220581 & 1.47078 \\
\hline 0.618982 & 0.0294414 & 0.601026 & 0.0496656 & 0.569074 & 0.0225895 & 1.60108 \\
\hline 0.602109 & 0.0292139 & 0.608787 & 0.0562048 & 0.579576 & 0.0254877 & 1.7815 \\
\hline 0.607041 & 0.0342343 & 0.618276 & 0.0562014 & 0.58984 & 0.0250529 & 1.96471 \\
\hline 0.60642 & 0.0335709 & 0.617727 & 0.0614559 & 0.605095 & 0.0218077 & 2.16063 \\
\hline 0.606957 & 0.0298983 & 0.622461 & 0.0619909 & 0.609842 & 0.0200211 & 2.44111 \\
\hline 0.613392 & 0.0307011 & 0.631775 & 0.0553196 & 0.620486 & 0.0262991 & 2.70057 \\
\hline 0.60149 & 0.0306966 & 0.637709 & 0.0569245 & 0.625527 & 0.0228424 & 2.99544 \\
\hline 0.603475 & 0.0393442 & 0.633364 & 0.0691568 & 0.638452 & 0.0192358 & 3.32111 \\
\hline 0.611593 & 0.0296038 & 0.656142 & 0.0596698 & 0.645767 & 0.0227322 & 3.65218 \\
\hline 0.61281 & 0.0297517 & 0.648407 & 0.0681567 & 0.659877 & 0.0332556 & 4.02041 \\
\hline 0.614398 & 0.0310258 & 0.655743 & 0.0745524 & 0.656985 & 0.0238707 & 4.39351 \\
\hline 0.593953 & 0.0301143 & 0.660074 & 0.0790826 & 0.665453 & 0.0271433 & 4.70981 \\
\hline 0.609395 & 0.0327554 & 0.661361 & 0.0818789 & 0.671237 & 0.0274619 & 5.06449 \\
\hline 0.608117 & 0.0324191 & 0.663163 & 0.079 & 0.672053 & 0.025753 & 5.4101 \\
\hline 0.61434 & 0.0283861 & 0.652426 & 0.0834511 & 0.677183 & 0.0261565 & 5.67749 \\
\hline 0.605251 & 0.039904 & 0.661119 & 0.0777708 & 0.674386 & 0.0232831 & 5.92372 \\
\hline 0.609052 & 0.0264609 & 0.65875 & 0.07 & 0.664594 & 0.02232 & 6.12288 \\
\hline 0.609565 & 0.0261956 & 0.648183 & 0.0789853 & 0.659947 & 0.0222867 & 6.26816 \\
\hline 0.59914 & 0.0351034 & 0.642011 & 0.078528 & 0.656922 & 0.0259684 & 6.32932 \\
\hline 0.600031 & 0.0340094 & 0.647753 & 0.0807222 & 0.647124 & 0.0241592 & 6.35545 \\
\hline 0.597082 & 0.0289319 & 0.632436 & 0.0897514 & 0.634078 & 0.0213865 & 6.31338 \\
\hline 0.59975 & 0.0371901 & 0.619723 & 0.0753907 & 0.621902 & 0.0301133 & 6.14225 \\
\hline 0.59224 & 0.0309263 & 0.61659 & 0.0792393 & 0.609553 & 0.0219439 & 5.9413 \\
\hline 0.597002 & 0.0304517 & 0.604207 & 0.0713427 & 0.595348 & 0.022154 & 5.66514 \\
\hline 0.608273 & 0.0305447 & 0.605137 & 0.0693262 & 0.596438 & 0.0214175 & 5.3918 \\
\hline 0.60161 & 0.028748 & 0.603013 & 0.0629592 & 0.586642 & 0.021806 & 5.02486 \\
\hline 0.588488 & 0.0331903 & 0.586905 & 0.0708599 & 0.572361 & 0.0228293 & 4.69165 \\
\hline 0.597193 & 0.0282843 & 0.583625 & 0.0645817 & 0.551619 & 0.0230587 & 4.2813 \\
\hline 0.594522 & 0.0427784 & 0.568334 & 0.0524075 & 0.540237 & 0.0277448 & 3.91646 \\
\hline 0.593506 & 0.0324615 & 0.566475 & 0.0568623 & 0.539734 & 0.0184303 & 3.51161 \\
\hline 0.592461 & 0.0319687 & 0.566968 & 0.054817 & 0.527073 & 0.0252717 & 3.14936 \\
\hline 0.58575 & 0.0314932 & 0.554559 & 0.046988 & 0.515377 & 0.0253253 & 2.82189 \\
\hline 0.587034 & 0.0360401 & 0.555491 & 0.0501238 & 0.510168 & 0.0231998 & 2.52594 \\
\hline 0.58191 & 0.0306646 & 0.555895 & 0.0488575 & 0.508161 & 0.017746 & 2.19163 \\
\hline 0.5894 & 0.029097 & 0.540405 & 0.0482158 & 0.502149 & 0.0209656 & 1.9357 \\
\hline 0.584979 & 0.0356675 & 0.546107 & 0.0410864 & 0.501401 & 0.0192098 & 1.69319 \\
\hline 0.578005 & 0.0240229 & 0.54062 & 0.0464164 & 0.503664 & 0.0198472 & 1.49548 \\
\hline 0.587613 & 0.0299813 & 0.535916 & 0.0420626 & 0.491831 & 0.0176484 & 1.33914 \\
\hline 0.574736 & 0.0330834 & 0.534618 & 0.0389236 & 0.495323 & 0.0181018 & 1.19083 \\
\hline 0.580404 & 0.0252783 & 0.534459 & 0.0445016 & 0.488674 & 0.0176775 & 1.08747 \\
\hline 0.579819 & 0.0291071 & 0.529715 & 0.0501465 & 0.485496 & 0.0171217 & 0.976335 \\
\hline 0.581624 & 0.0315587 & 0.537392 & 0.0442005 & 0.484122 & 0.0205107 & 0.873897 \\
\hline
\end{tabular}




\begin{tabular}{|c|c|c|c|c|c|c|}
\hline 0.589563 & 0.0253373 & 0.529009 & 0.0453839 & 0.480207 & 0.0201236 & 0.824656 \\
\hline 0.584381 & 0.0320799 & 0.52544 & 0.0435471 & 0.48043 & 0.0164074 & 0.799983 \\
\hline 0.569007 & 0.0275484 & 0.533882 & 0.0485008 & 0.482525 & 0.020327 & 0.742294 \\
\hline 0.573777 & 0.0308751 & 0.530613 & 0.044285 & 0.482783 & 0.015954 & 0.724025 \\
\hline 0.569933 & 0.0381553 & 0.520676 & 0.0413471 & 0.484451 & 0.0205903 & 0.693485 \\
\hline 0.571889 & 0.027839 & 0.526934 & 0.0401845 & 0.486831 & 0.0199457 & 0.683843 \\
\hline 0.569986 & 0.0246392 & 0.527039 & 0.0382143 & 0.483226 & 0.0227057 & 0.685132 \\
\hline 0.57006 & 0.0299981 & 0.531536 & 0.0483228 & 0.478115 & 0.0197927 & 0.669163 \\
\hline 0.566935 & 0.0291506 & 0.524828 & 0.0368536 & 0.478388 & 0.0172519 & 0.680042 \\
\hline 0.571812 & 0.0373532 & 0.531839 & 0.0424754 & 0.477432 & 0.0197527 & 0.664942 \\
\hline 0.56695 & 0.0341429 & 0.52083 & 0.0401548 & 0.475035 & 0.0204272 & 0.688814 \\
\hline 0.574429 & 0.037602 & 0.519195 & 0.0432336 & 0.480238 & 0.0156208 & 0.670131 \\
\hline 0.568419 & 0.0308518 & 0.524907 & 0.0392914 & 0.479423 & 0.0221413 & 0.65997 \\
\hline 0.565168 & 0.0362642 & 0.520634 & 0.0462192 & 0.477552 & 0.0160699 & 0.672836 \\
\hline 0.572088 & 0.0308017 & 0.526279 & 0.0408813 & 0.480002 & 0.0212488 & 0.669566 \\
\hline 0.572505 & 0.0247762 & 0.530804 & 0.0511775 & 0.476006 & 0.0212077 & 0.684012 \\
\hline 0.575143 & 0.036004 & 0.525998 & 0.0370907 & 0.477457 & 0.0184031 & 0.675455 \\
\hline 0.568843 & 0.0284158 & 0.526204 & 0.0363077 & 0.472365 & 0.0219761 & 0.69468 \\
\hline 0.570164 & 0.0264156 & 0.515405 & 0.0392834 & 0.482979 & 0.0207571 & 0.704468 \\
\hline 0.570641 & 0.0311307 & 0.526213 & 0.035758 & 0.478818 & 0.0189188 & 0.689886 \\
\hline 0.567125 & 0.0322773 & 0.527389 & 0.0313657 & 0.485167 & 0.0178833 & 0.710064 \\
\hline 0.579777 & 0.0284526 & 0.52375 & 0.0410753 & 0.4867 & 0.0206228 & 0.711096 \\
\hline 0.584805 & 0.0296736 & 0.527475 & 0.0412664 & 0.489769 & 0.0212398 & 0.734439 \\
\hline 0.593259 & 0.0269557 & 0.5244 & 0.047902 & 0.491853 & 0.0207333 & 0.742899 \\
\hline 0.596197 & 0.0293207 & 0.545304 & 0.0440849 & 0.503384 & 0.0209922 & 0.748141 \\
\hline 0.609112 & 0.0428777 & 0.541754 & 0.0407442 & 0.516451 & 0.0248607 & 0.772615 \\
\hline 0.620558 & 0.0300785 & 0.546754 & 0.0468355 & 0.522708 & 0.0212328 & 0.78676 \\
\hline 0.642015 & 0.0398168 & 0.563838 & 0.046337 & 0.544748 & 0.0242525 & 0.781597 \\
\hline 0.667338 & 0.0307963 & 0.578769 & 0.0473522 & 0.562022 & 0.0264694 & 0.866774 \\
\hline 0.689825 & 0.0375692 & 0.594012 & 0.0426692 & 0.591712 & 0.0376976 & 0.860003 \\
\hline 0.754784 & 0.0469969 & 0.611478 & 0.0560389 & 0.620869 & 0.0302461 & 0.908624 \\
\hline 0.794788 & 0.0574424 & 0.640988 & 0.05164 & 0.651031 & 0.0408739 & 0.967646 \\
\hline 0.845547 & 0.0570102 & 0.662945 & 0.0637403 & 0.695636 & 0.0394708 & 0.998702 \\
\hline 0.931309 & 0.0678012 & 0.678811 & 0.0625835 & 0.731793 & 0.0456525 & 1.05547 \\
\hline 1.02711 & 0.0738474 & 0.712819 & 0.0683656 & 0.778227 & 0.0527896 & 1.10576 \\
\hline 1.11775 & 0.0870677 & 0.742222 & 0.0826584 & 0.832758 & 0.0693591 & 1.15654 \\
\hline 1.24148 & 0.0885916 & 0.773291 & 0.0873106 & 0.891669 & 0.077141 & 1.20823 \\
\hline 1.37005 & 0.104781 & 0.815055 & 0.0955848 & 0.943429 & 0.0758064 & 1.25921 \\
\hline 1.51808 & 0.118082 & 0.845891 & 0.0959571 & 1.00546 & 0.0907785 & 1.31178 \\
\hline 1.69648 & 0.139804 & 0.876272 & 0.107538 & 1.06921 & 0.0971614 & 1.35878 \\
\hline 1.87414 & 0.158274 & 0.917807 & 0.114419 & 1.12753 & 0.0962622 & 1.43269 \\
\hline 2.03117 & 0.179235 & 0.948869 & 0.116559 & 1.18491 & 0.114925 & 1.43442 \\
\hline 2.22872 & 0.196784 & 0.963829 & 0.130755 & 1.2321 & 0.128214 & 1.48362 \\
\hline 2.42686 & 0.212458 & 0.982133 & 0.126764 & 1.28585 & 0.13516 & 1.50973 \\
\hline 2.56792 & 0.242895 & 1.0074 & 0.12886 & 1.31245 & 0.136812 & 1.55548 \\
\hline 2.76836 & 0.262607 & 1.01818 & 0.122852 & 1.3499 & 0.130513 & 1.51722 \\
\hline 2.95114 & 0.283023 & 1.02751 & 0.139349 & 1.37036 & 0.1446 & 1.51346 \\
\hline
\end{tabular}




\begin{tabular}{|c|c|c|c|c|c|c|}
\hline 3.08097 & 0.273188 & 1.02621 & 0.14042 & 1.39989 & 0.151167 & 1.48044 \\
\hline 3.20692 & 0.285975 & 1.02436 & 0.135656 & 1.38908 & 0.138281 & 1.47822 \\
\hline 3.33998 & 0.305627 & 1.01065 & 0.134749 & 1.36943 & 0.155568 & 1.45569 \\
\hline 3.3747 & 0.31093 & 0.985875 & 0.132486 & 1.34267 & 0.135805 & 1.42903 \\
\hline 3.44085 & 0.331503 & 0.956454 & 0.100633 & 1.31143 & 0.137208 & 1.37162 \\
\hline 3.43042 & 0.31145 & 0.938573 & 0.120026 & 1.27714 & 0.138834 & 1.31633 \\
\hline 3.36867 & 0.331777 & 0.913562 & 0.104015 & 1.22756 & 0.112724 & 1.26981 \\
\hline 3.32149 & 0.322542 & 0.871157 & 0.0951285 & 1.16662 & 0.119221 & 1.18034 \\
\hline 3.21567 & 0.294367 & 0.830437 & 0.0823338 & 1.10752 & 0.105897 & 1.13848 \\
\hline 3.08892 & 0.266367 & 0.795401 & 0.0891119 & 1.04228 & 0.100368 & 1.09815 \\
\hline 2.94177 & 0.269756 & 0.756469 & 0.0782539 & 0.985139 & 0.0922952 & 1.00533 \\
\hline 2.78912 & 0.267491 & 0.725555 & 0.0764785 & 0.923947 & 0.0839511 & 0.955422 \\
\hline 2.58594 & 0.226465 & 0.680757 & 0.0601226 & 0.856598 & 0.0616258 & 0.886144 \\
\hline 2.39791 & 0.220079 & 0.656952 & 0.0575085 & 0.790916 & 0.0614877 & 0.837097 \\
\hline 2.23152 & 0.199131 & 0.630683 & 0.0478254 & 0.742868 & 0.0518916 & 0.802682 \\
\hline 2.01156 & 0.173751 & 0.607303 & 0.0416708 & 0.684806 & 0.0372444 & 0.750993 \\
\hline 1.83504 & 0.160404 & 0.588788 & 0.0382569 & 0.641523 & 0.0341236 & 0.727158 \\
\hline 1.6425 & 0.159776 & 0.557099 & 0.0443416 & 0.612161 & 0.0336355 & 0.701454 \\
\hline 1.47125 & 0.115277 & 0.552194 & 0.0414544 & 0.573761 & 0.0276664 & 0.669257 \\
\hline 1.30639 & 0.0863896 & 0.532319 & 0.0347525 & 0.541807 & 0.025394 & 0.640288 \\
\hline 1.18545 & 0.0879692 & 0.513413 & 0.0364114 & 0.518881 & 0.0238533 & 0.628912 \\
\hline 1.07001 & 0.0754804 & 0.496009 & 0.0329285 & 0.49459 & 0.0227296 & 0.617881 \\
\hline 0.96032 & 0.0553705 & 0.50123 & 0.0298411 & 0.483987 & 0.0221971 & 0.608833 \\
\hline 0.866156 & 0.0558392 & 0.490525 & 0.0276905 & 0.465255 & 0.0251402 & 0.592432 \\
\hline 0.791763 & 0.0448238 & 0.487603 & 0.0422787 & 0.453376 & 0.0237981 & 0.588683 \\
\hline 0.708699 & 0.036652 & 0.485144 & 0.0337481 & 0.443459 & 0.0194136 & 0.572375 \\
\hline 0.665438 & 0.0376188 & 0.476792 & 0.0304425 & 0.438623 & 0.0191527 & 0.571554 \\
\hline 0.629585 & 0.0417429 & 0.472174 & 0.0355851 & 0.431025 & 0.0178432 & 0.571727 \\
\hline 0.596897 & 0.0328971 & 0.459925 & 0.0281929 & 0.429924 & 0.0182529 & 0.569337 \\
\hline 0.573365 & 0.0277303 & 0.467407 & 0.0342654 & 0.427622 & 0.0178258 & 0.57234 \\
\hline 0.537057 & 0.0353449 & 0.468777 & 0.0345064 & 0.422352 & 0.0148654 & 0.569101 \\
\hline 0.525711 & 0.0301985 & 0.46577 & 0.0410903 & 0.419975 & 0.0151795 & 0.580774 \\
\hline 0.509527 & 0.0298111 & 0.45796 & 0.0372953 & 0.41822 & 0.0157281 & 0.557806 \\
\hline 0.506275 & 0.0291974 & 0.461558 & 0.0289495 & 0.414966 & 0.0199121 & 0.566791 \\
\hline 0.496285 & 0.035569 & 0.456697 & 0.0318028 & 0.4168 & 0.0209632 & 0.569126 \\
\hline 0.48673 & 0.026952 & 0.447552 & 0.0321349 & 0.412803 & 0.0176571 & 0.552421 \\
\hline 0.491151 & 0.0220831 & 0.461108 & 0.029552 & 0.409258 & 0.0178475 & 0.567158 \\
\hline 0.495678 & 0.0324598 & 0.452107 & 0.032946 & 0.406598 & 0.0178461 & 0.556756 \\
\hline 0.480039 & 0.0227731 & 0.457895 & 0.0322302 & 0.404858 & 0.0141529 & 0.552035 \\
\hline 0.477471 & 0.0241979 & 0.45114 & 0.0318616 & 0.404667 & 0.0171891 & 0.575312 \\
\hline 0.478648 & 0.0287182 & 0.448096 & 0.0287022 & 0.404529 & 0.018679 & 0.551822 \\
\hline 0.47481 & 0.0312918 & 0.441572 & 0.0312753 & 0.401741 & 0.0224681 & 0.54929 \\
\hline 0.474828 & 0.0255929 & 0.446133 & 0.0227668 & 0.406682 & 0.0187688 & 0.556181 \\
\hline 0.471292 & 0.026936 & 0.440352 & 0.0247691 & 0.407912 & 0.0125779 & 0.547841 \\
\hline 0.461825 & 0.0343717 & 0.447146 & 0.0319876 & 0.406576 & 0.0196698 & 0.560443 \\
\hline 0.466353 & 0.0267699 & 0.453629 & 0.0347057 & 0.409913 & 0.0203471 & 0.54141 \\
\hline 0.460746 & 0.0286394 & 0.444677 & 0.0361365 & 0.40415 & 0.0184981 & 0.544383 \\
\hline
\end{tabular}




\begin{tabular}{|c|c|c|c|c|c|c|}
\hline 0.464268 & 0.0283781 & 0.44573 & 0.0335984 & 0.402906 & 0.0135638 & 0.549983 \\
\hline 0.467135 & 0.026792 & 0.443079 & 0.0292813 & 0.4 & 0.0131973 & 0.539933 \\
\hline 0.457713 & 0.0275041 & 0.448479 & 0.0311479 & 0.406094 & 0.022215 & .534689 \\
\hline 0.46725 & 0.0250168 & 0.445609 & 0.0277698 & 0.40164 & 0.0197579 & 530854 \\
\hline 0.459912 & 0.0247551 & 0.454575 & 0.027824 & 0.40047 & 0.0160269 & 0.534535 \\
\hline 0.449109 & 0.0261545 & 0.455173 & 0.0356867 & 0.407038 & 0.0206433 & .518729 \\
\hline 0.459394 & 0.0288714 & 0.452974 & 0.0361686 & 0.398538 & 0.0143205 & 0.527501 \\
\hline 0.461332 & 0.0276065 & 0.450527 & 0.0331936 & 0.402223 & 0.0198894 & 0.527125 \\
\hline 0.454717 & 0.0230333 & 0.453409 & 0.0358462 & 0.399002 & 0.0191991 & 0.528463 \\
\hline 0.460029 & 0.0298299 & 0.462468 & 0.0353554 & 0.403671 & 0.0209461 & 0.518777 \\
\hline 0.455058 & 0.0258198 & 0.463156 & 0.0321281 & 0.410298 & 0.0157509 & 0.533135 \\
\hline 0.455738 & 0.0324506 & 0.462059 & 0.029978 & 0.409772 & 0.0209279 & 0.528831 \\
\hline 0.458927 & 0.024397 & 0.468595 & 0.0350907 & 0.415834 & 0.0199825 & 0.521193 \\
\hline 0.46657 & 0.0273751 & 0.466147 & 0.0282314 & 0.415295 & 0.0238987 & 0.516082 \\
\hline 0.460982 & 0.0245355 & 0.4618 & 0.036094 & 0.413495 & 0.015086 & 0.510819 \\
\hline 0.45933 & 0.0300135 & 0.461151 & 0.0347651 & 0.420836 & 0.0170124 & 0.51446 \\
\hline 0.457775 & 0.0274367 & 0.461147 & 0.0355579 & 0.416495 & 0.016722 & 0.532657 \\
\hline 0.455296 & 0.0235804 & 0.460744 & 0.0322535 & 0.42427 & 0.0172214 & 0.508531 \\
\hline 0.454438 & 0.0274683 & 0.46 & 0.03063 & 0.4 & 0.0165026 & 7196 \\
\hline 0.454822 & 0.0306505 & 0.460953 & 0.0290026 & 0.426163 & 0.0176957 & 0.510385 \\
\hline 0.447171 & 0.0223377 & 0.451233 & 0.0292941 & 0.428292 & 0.0200139 & 0.527835 \\
\hline 0.450297 & 0.02 & 0.4 & 351 & 715 & 0.0226283 & 1738 \\
\hline 0.447978 & 0.0234843 & 0.447216 & 0.0308956 & 0.43179 & 0.0155308 & 0.531475 \\
\hline 0.44849 & 0.0191251 & 0.445017 & 0.0297537 & 0.436677 & 0.0180693 & 0.522591 \\
\hline 0.439888 & 0.0284899 & 0.449031 & 0.0299489 & 0.431352 & 0.0179968 & 0.537332 \\
\hline 0.444822 & 0.0291303 & 0.440165 & 0.0319653 & 0.430728 & 0.0239315 & 0.52641 \\
\hline 0.444286 & 0.0232663 & 0.452021 & 0.0352505 & 0.441814 & 0.0186116 & 0.527584 \\
\hline 0.448896 & 0.025368 & 0.45058 & 0.0320641 & 0.438549 & 0.0219439 & 0.535206 \\
\hline 0.446733 & 0.0262391 & 0.433055 & 0.0245267 & 0.444919 & 0.0172201 & 0.535427 \\
\hline 0.448952 & 0.0316397 & 0.43455 & 0.0259569 & 0.440354 & 0.0158394 & 0.524137 \\
\hline 0.438856 & 0.0256272 & 0.438909 & 0.0271416 & 0.4431 & 0.0201687 & 0.530618 \\
\hline 0.430536 & 0.0234954 & 0.430862 & 0.0316588 & 0.445726 & 0.0195846 & 0.526469 \\
\hline 0.433829 & 0.0220303 & 0.44081 & 0.0301192 & 0.442375 & 0.0188333 & 0.538802 \\
\hline 0.446647 & 0.0299802 & 0.429213 & 0.0298737 & 0.444257 & 0.0256246 & 0.532415 \\
\hline 0.428597 & 0.0298484 & 0.433507 & 0.0322583 & 0.442887 & 0.0172371 & 0.524664 \\
\hline 0.43277 & 0.0308257 & 0.434804 & 0.0343242 & 0.440547 & 0.0173015 & 0.532001 \\
\hline 0.431558 & 0.0233478 & 0.420173 & 0.028211 & 0.442825 & 0.0208971 & 0.530249 \\
\hline 0.429473 & 0.0268805 & 0.426084 & 0.0288286 & 0.435989 & 0.0211324 & 0.520471 \\
\hline 0.430543 & 0.0262861 & 0.416297 & 0.0204009 & 0.431914 & 0.018096 & 0.526004 \\
\hline 0.429612 & 0.0299126 & 0.414131 & 0.0353871 & 0.422874 & 0.0190701 & 0.50621 \\
\hline 0.432457 & 0.0221065 & 0.415197 & 0.0322332 & 0.420474 & 0.0182175 & 0.534534 \\
\hline 0.42918 & 0.0247558 & 0.417213 & 0.0252988 & 0.417662 & 0.0122951 & 0.526074 \\
\hline 0.433287 & 0.0211033 & 0.41481 & 0.0205661 & 0.408779 & 0.0126309 & 0.517439 \\
\hline 0.422265 & 0.0291305 & 0.411228 & 0.0279494 & 0.411051 & 0.0201222 & 0.503301 \\
\hline 0.426941 & 0.0249578 & 0.403551 & 0.0332775 & 0.400942 & 0.0147351 & 0.49242 \\
\hline 0.421887 & 0.0206214 & 0.406789 & 0.0263897 & 0.391774 & 0.0160697 & 0.509823 \\
\hline 0.426264 & 0.0257914 & 0.398097 & 0.0282741 & 0.38851 & 0.0149234 & 0.484627 \\
\hline
\end{tabular}




\begin{tabular}{|c|c|c|c|c|c|c|}
\hline 0.41676 & 0.0268354 & 0.39936 & 0.0294571 & 0.384151 & 0.0193837 & 0.498425 \\
\hline 0.421209 & 0.020349 & 0.400008 & 0.0241821 & 0.384807 & 0.0148081 & 0.495916 \\
\hline 0.417547 & 0.0273405 & 0.396981 & 0.0327569 & 0.379624 & 0.0187437 & 0.473071 \\
\hline 0.421017 & 0.0258252 & 0.391072 & 0.0299095 & 0.368199 & 0.0184375 & 0.484358 \\
\hline 0.416177 & 0.0242499 & 0.395391 & 0.0279784 & 0.370146 & 0.0178655 & 0.470872 \\
\hline 0.411402 & 0.032982 & 0.388369 & 0.0191231 & 0.364004 & 0.0158059 & 0.469748 \\
\hline 0.412767 & 0.0320025 & 0.386457 & 0.0302409 & 0.363875 & 0.0142398 & 0.482686 \\
\hline 0.414777 & 0.0273483 & 0.390196 & 0.0221356 & 0.363519 & 0.020292 & 0.470264 \\
\hline 0.411709 & 0.0206306 & 0.387957 & 0.0295484 & 0.360907 & 0.0182947 & 0.478936 \\
\hline 0.415038 & 0.027779 & 0.389876 & 0.0279417 & 0.357785 & 0.0167071 & 0.474933 \\
\hline 0.403879 & 0.0324922 & 0.384699 & 0.0298785 & 0.356204 & 0.0143909 & 0.483068 \\
\hline 0.405351 & 0.0269945 & 0.383516 & 0.0260367 & 0.354213 & 0.0180597 & 0.455878 \\
\hline 0.406875 & 0.0245078 & 0.373106 & 0.0217247 & 0.351504 & 0.0156729 & 0.459289 \\
\hline 0.407713 & 0.021255 & 0.378723 & 0.0235752 & 0.34851 & 0.0192152 & 0.463278 \\
\hline 0.400086 & 0.0241934 & 0.37175 & 0.0162644 & 0.353525 & 0.014827 & 0.459746 \\
\hline 0.399691 & 0.0237797 & 0.375303 & 0.0246921 & 0.348863 & 0.0153023 & 0.4461 \\
\hline 0.411241 & 0.0279025 & 0.374814 & 0.0283775 & 0.348893 & 0.0168292 & 0.463354 \\
\hline 0.400062 & 0.0235072 & 0.370103 & 0.0262242 & 0.349793 & 0.0135764 & 0.445783 \\
\hline 0.399545 & 0.0298336 & 0.366164 & 0.02 & 0.3 & 0.01 & 48113 \\
\hline 0.402075 & 0.0193604 & 0.364866 & 0.0296898 & 0.342594 & 0.0140024 & 0.451382 \\
\hline 0.395129 & 0.0294175 & 0.363019 & 0.0209717 & 0.333897 & 0.0144009 & 0.454423 \\
\hline 0.39 & 0.023 & 0.364666 & 044 & 0.33 & 038 & 5679 \\
\hline 0.388081 & 0.0201332 & 0.360095 & 0.0247132 & 0.336044 & 0.0138537 & 0.438064 \\
\hline 0.383301 & 0.0234168 & 0.364268 & 0.0260682 & 0.33645 & 0.0185271 & 0.455674 \\
\hline 0.384514 & 0.0259593 & 0.360905 & 0.0246477 & 0.331843 & 0.0147678 & 0.455143 \\
\hline 0.383478 & 0.0243364 & 0.353309 & 0.0300634 & 0.334024 & 0.0177825 & 0.450816 \\
\hline 0.390956 & 0.0201808 & 0.359639 & 0.0214211 & 0.32672 & 0.0149284 & 0.44354 \\
\hline 0.38649 & 0.028489 & 0.355569 & 0.019179 & 0.327954 & 0.0176358 & 0.440393 \\
\hline 0.383669 & 0.0228458 & 0.354969 & 0.0188604 & 0.329143 & 0.0169686 & 0.425687 \\
\hline 0.386343 & 0.024239 & 0.357817 & 0.0214076 & 0.324859 & 0.011571 & 0.428787 \\
\hline 0.380068 & 0.0222764 & 0.348366 & 0.023 & 0.327199 & 0.013 & 0.429706 \\
\hline 0.377566 & 0.0268287 & 0.357513 & 0.0218038 & 0.327696 & 0.0158422 & 0.437475 \\
\hline 0.380191 & 0.0248201 & 0.350646 & 0.0254279 & 0.326269 & 0.013864 & 0.438539 \\
\hline 0.378618 & 0.0205123 & 0.348517 & 0.0260842 & 0.323946 & 0.0170505 & 0.433899 \\
\hline 0.381004 & 0.0180105 & 0.353033 & 0.0259264 & 0.324282 & 0.0129055 & 0.437607 \\
\hline 0.378779 & 0.020012 & 0.354302 & 0.0266583 & 0.327375 & 0.0169762 & 0.443514 \\
\hline 0.36375 & 0.0186736 & 0.350417 & 0.0201617 & 0.322353 & 0.0154777 & 0.422961 \\
\hline 0.374134 & 0.0216623 & 0.347677 & 0.0182801 & 0.325434 & 0.018709 & 0.435805 \\
\hline 0.370352 & 0.0260606 & 0.346413 & 0.0213956 & 0.323016 & 0.0161187 & 0.431045 \\
\hline 0.371657 & 0.026431 & 0.353314 & 0.0258247 & 0.32384 & 0.0161862 & 0.43657 \\
\hline 0.361418 & 0.023488 & 0.350003 & 0.0203981 & 0.324543 & 0.0165774 & 0.429246 \\
\hline 0.36631 & 0.0250857 & 0.355366 & 0.0237927 & 0.328706 & 0.0156096 & 0.42496 \\
\hline 0.368611 & 0.022338 & 0.359108 & 0.0233109 & 0.336022 & 0.0169089 & 0.441032 \\
\hline 0.372983 & 0.0247618 & 0.353636 & 0.0262258 & 0.334969 & 0.016741 & 0.439477 \\
\hline 0.373695 & 0.0203167 & 0.361711 & 0.023256 & 0.337345 & 0.0149603 & 0.432867 \\
\hline 0.372565 & 0.0238763 & 0.364187 & 0.0292382 & 0.341803 & 0.015345 & 0.440617 \\
\hline 0.367507 & 0.0252263 & 0.372445 & 0.0283031 & 0.347526 & 0.0186873 & 0.460382 \\
\hline
\end{tabular}




\begin{tabular}{|c|c|c|c|c|c|c|}
\hline 0.373101 & 0.0273198 & 0.371106 & 0.0279312 & 0.350389 & 0.0186788 & 0.460806 \\
\hline 0.372747 & 0.0227219 & 0.381592 & 0.028381 & 0.360717 & 0.0155083 & 0.470588 \\
\hline 0.374305 & 0.0237585 & 0.396778 & 0.0320446 & 0.368679 & 0.0170051 & 0.47361 \\
\hline .385034 & 0.028005 & 0.406476 & 0.0309305 & 0.377006 & 0.0158336 & 0.481107 \\
\hline 0.382458 & 0.026142 & 0.405125 & 0.0329599 & 0.398271 & 0.0162245 & 0.50555 \\
\hline 0.387572 & 0.022275 & 0.41799 & 0.0365811 & 0.407015 & 0.0170719 & 0.509076 \\
\hline 0.390933 & 0.0248179 & 0.424168 & 0.04066 & 0.421487 & 0.0157775 & 0.513595 \\
\hline 0.393666 & 0.0231348 & 0.445936 & 0.0431036 & 0.44217 & 0.0198121 & 0.544598 \\
\hline 0.403038 & 0.0248605 & 0.457763 & 0.0484866 & 0.459342 & 0.0261641 & 0.557364 \\
\hline 0.412109 & 0.0293871 & 0.468915 & 0.048262 & 0.472039 & 0.0189555 & 0.559018 \\
\hline 0.422185 & 0.0243386 & 0.479725 & 0.0518964 & 0.492576 & 0.0189174 & 0.593498 \\
\hline 0.438379 & 0.0236905 & 0.494206 & 0.0631042 & 0.517385 & 0.0223507 & 0.607107 \\
\hline 0.44605 & 0.0311423 & 0.514799 & 0.0580688 & 0.532266 & 0.0151618 & 0.611955 \\
\hline 0.457509 & 0.030931 & 0.526565 & 0.0629657 & 0.553971 & 0.0222865 & 0.635754 \\
\hline 0.482695 & 0.0248145 & 0.540793 & 0.0758684 & 0.574643 & 0.0214618 & 0.6613 \\
\hline 0.492618 & 0.0331723 & 0.554153 & 0.0665572 & 0.5917 & 0.0237729 & 0.672975 \\
\hline 0.517868 & 0.0280956 & 0.563491 & 0.0778713 & 0.613373 & 0.0285312 & 0.701737 \\
\hline 0.550447 & 0.0301159 & 0.576489 & 0.0681481 & 0.631762 & 0.0318014 & 0.703903 \\
\hline 0.567068 & 0.0416313 & 0.588484 & 0.0701704 & 0.641743 & 0.0259183 & 0.706512 \\
\hline 0.599097 & 0.0285495 & 0.589085 & 0.0683531 & 0.656873 & 0.0313818 & 0.724015 \\
\hline 0.634362 & 0.0391401 & 0.588654 & 0.0671515 & 0.675727 & 0.0327963 & 0.723274 \\
\hline 0.667313 & 0.0428992 & 0.586271 & 0.0760226 & 0.67239 & 0.026418 & 0.726437 \\
\hline 0.691694 & 0.0380604 & 0.58316 & 0.0690281 & 0.684147 & 0.0319127 & 0.713357 \\
\hline 0.739457 & 0.0502417 & 0.590378 & 0.0717178 & 0.689146 & 0.0360915 & 0.725761 \\
\hline 0.758465 & 0.0459265 & 0.581359 & 0.077765 & 0.679632 & 0.0295235 & 0.698522 \\
\hline 0.787311 & 0.0473008 & 0.573979 & 0.0608476 & 0.673907 & 0.0236784 & 0.727899 \\
\hline 0.814967 & 0.0406562 & 0.565541 & 0.0702577 & 0.663436 & 0.0301339 & 0.702997 \\
\hline 0.846948 & 0.0578279 & 0.548886 & 0.0577082 & 0.652603 & 0.0362848 & 0.707964 \\
\hline 0.865456 & 0.0513862 & 0.537688 & 0.0533707 & 0.63547 & 0.0258986 & 0.671476 \\
\hline 0.879251 & 0.0628924 & 0.522067 & 0.0471196 & 0.615043 & 0.0280215 & 0.654165 \\
\hline 0.894939 & 0.0611724 & 0.500567 & 0.0460074 & 0.603712 & 0.0296525 & 0.624571 \\
\hline 0.903477 & 0.0581665 & 0.494213 & 0.0412052 & 0.579377 & 0.0272789 & 0.620182 \\
\hline 0.896992 & 0.0692615 & 0.473201 & 0.0422399 & 0.557557 & 0.0312242 & 0.594417 \\
\hline 0.893701 & 0.0633693 & 0.457416 & 0.0314412 & 0.540994 & 0.0258737 & 0.569904 \\
\hline 0.878145 & 0.0576213 & 0.443713 & 0.0365546 & 0.514235 & 0.0273159 & 0.555993 \\
\hline 0.860963 & 0.0551546 & 0.425245 & 0.0299615 & 0.482806 & 0.0193363 & 0.531561 \\
\hline 0.842802 & 0.0663398 & 0.409666 & 0.0355294 & 0.460869 & 0.0205565 & 0.505132 \\
\hline 0.811444 & 0.0547667 & 0.399808 & 0.0295306 & 0.447807 & 0.0238067 & 0.492208 \\
\hline 0.78513 & 0.0595718 & 0.382475 & 0.0234908 & 0.419298 & 0.020143 & 0.470888 \\
\hline 0.750425 & 0.0570436 & 0.371953 & 0.0152138 & 0.399249 & 0.0172037 & 0.462291 \\
\hline 0.713574 & 0.0514614 & 0.361065 & 0.0246954 & 0.391015 & 0.0215668 & 0.440067 \\
\hline 0.683845 & 0.0378127 & 0.352008 & 0.0252994 & 0.371178 & 0.0187227 & 0.430989 \\
\hline 0.634551 & 0.0507087 & 0.34189 & 0.0189318 & 0.353336 & 0.0228171 & 0.424402 \\
\hline 0.601395 & 0.039391 & 0.34211 & 0.0303332 & 0.350046 & 0.0143282 & 0.41805 \\
\hline 0.585071 & 0.0389024 & 0.334003 & 0.0195048 & 0.335526 & 0.0170213 & 0.402572 \\
\hline 0.54545 & 0.0333785 & 0.32845 & 0.0251455 & 0.330156 & 0.0175537 & 0.407153 \\
\hline 0.518376 & 0.0328267 & 0.321969 & 0.019798 & 0.315474 & 0.0154344 & 0.409577 \\
\hline
\end{tabular}




\begin{tabular}{|c|c|c|c|c|c|c|}
\hline 0.495429 & 0.0273562 & 0.323996 & 0.0252299 & 0.316726 & 0.0168537 & 0.406828 \\
\hline 0.470301 & 0.0297434 & 0.316517 & 0.023792 & 0.311022 & 0.0168535 & 0.387461 \\
\hline 0.444803 & 0.0247156 & 0.315926 & 0.025141 & 0.309344 & 0.0210127 & .390184 \\
\hline .433332 & 0.036724 & 0.316907 & 0.0195148 & 0.304958 & 0.0104925 & 0.388948 \\
\hline .420973 & 0.0250667 & 0.310027 & 0.0230787 & 0.303111 & 0.0113705 & 0.383744 \\
\hline 0.407953 & 0.034263 & 0.312031 & 0.0243748 & 0.302774 & 0.0129783 & 0.3908 \\
\hline 0.406882 & 0.0233342 & 0.304384 & 0.0175278 & 0.295838 & 0.0147579 & 0.391398 \\
\hline 0.399343 & 0.0295255 & 0.313116 & 0.0224096 & 0.297754 & 0.0159275 & 0.391301 \\
\hline 0.390484 & 0.0243817 & 0.310749 & 0.0222453 & 0.296615 & 0.0182349 & 0.376526 \\
\hline 0.379143 & 0.0262167 & 0.311588 & 0.02109 & 0.290512 & 0.0163451 & 0.382768 \\
\hline 0.383608 & 0.0248558 & 0.300284 & 0.0205372 & 0.292673 & 0.0120241 & 0.375931 \\
\hline 0.379453 & 0.0244322 & 0.303458 & 0.0238919 & 0.289224 & 0.0145434 & 0.367125 \\
\hline 0.370782 & 0.0258836 & 0.298525 & 0.0225769 & 0.288329 & 0.0171424 & 0.370066 \\
\hline 0.372391 & 0.026099 & 0.296751 & 0.0222386 & 0.282395 & 0.0142138 & 0.370324 \\
\hline 0.364336 & 0.0270242 & 0.299338 & 0.0200882 & 0.282617 & 0.016611 & 0.366802 \\
\hline 0.351734 & 0.024795 & 0.302361 & 0.0204555 & 0.283508 & 0.0173629 & 0.363711 \\
\hline 0.352457 & 0.0249939 & 0.30109 & 0.0184389 & 0.285317 & 0.0177184 & 0.365954 \\
\hline 0.353244 & 0.022364 & 0.296815 & 0.0206117 & 0.273842 & 0.0138342 & 0.37024 \\
\hline 0.341526 & 0.02 & 0.25 & 0.01 & 0.27 & 0.013893 & .364688 \\
\hline 0.341346 & 0.0234739 & 0.296769 & 0.0223972 & 0.277553 & 0.0155967 & 0.373243 \\
\hline 0.344861 & 0.0213764 & 0.289373 & 0.0186873 & 0.276121 & 0.0155281 & 0.357431 \\
\hline 0.329023 & 0.02 & 838 & 0.0 & 167 & 1609 & 0.368785 \\
\hline 0.327122 & 0.0235436 & 0.293197 & 0.0224255 & 0.265498 & 0.0135769 & 0.359529 \\
\hline 0.313202 & 0.0197215 & 0.29208 & 0.0194603 & 0.265898 & 0.0141002 & 0.358981 \\
\hline 0.317661 & 0.0191942 & 0.29044 & 0.0166819 & 0.268474 & 0.0141549 & 0.344414 \\
\hline 0.312922 & 0.0261367 & 0.288178 & 0.0188223 & 0.268206 & 0.0165416 & 0.355978 \\
\hline 0.306693 & 0.0220535 & 0.290739 & 0.0184721 & 0.26431 & 0.0148252 & 0.357948 \\
\hline 0.306369 & 0.0243737 & 0.283141 & 0.0232214 & 0.263489 & 0.0110044 & 0.353782 \\
\hline 0.304682 & 0.0215716 & 0.28579 & 0.0194251 & 0.265134 & 0.0169254 & 0.355003 \\
\hline 0.295589 & 0.0186517 & 0.280449 & 0.0177733 & 0.258599 & 0.0136566 & 0.353974 \\
\hline 0.287162 & 0.0258775 & 0.280293 & 0.0210475 & 0.255546 & 0.0153423 & 0.351172 \\
\hline 0.296714 & 0.0209079 & 0.279489 & 0.0203061 & 0.257566 & 0.0116739 & 0.358061 \\
\hline 0.294479 & 0.0192243 & 0.286867 & 0.0199123 & 0.258793 & 0.01149 & 0.357117 \\
\hline 0.288366 & 0.0208972 & 0.28008 & 0.0183879 & 0.260148 & 0.0121742 & 0.353041 \\
\hline 0.292331 & 0.0235089 & 0.277779 & 0.018558 & 0.25552 & 0.0111247 & 0.353493 \\
\hline 0.293592 & 0.021106 & 0.281663 & 0.0215032 & 0.259922 & 0.0150564 & 0.360668 \\
\hline 0.286132 & 0.0147861 & 0.280999 & 0.024556 & 0.260046 & 0.013033 & 0.345433 \\
\hline 0.287881 & 0.0229284 & 0.277772 & 0.0209642 & 0.255709 & 0.0122067 & 0.346094 \\
\hline 0.283464 & 0.0210654 & 0.282026 & 0.0153962 & 0.258439 & 0.010954 & 0.334144 \\
\hline 0.28629 & 0.0254145 & 0.283658 & 0.0242186 & 0.256848 & 0.00922117 & 0.353049 \\
\hline 0.2804 & 0.0190923 & 0.279639 & 0.0177952 & 0.259559 & 0.0158335 & 0.34509 \\
\hline 0.28157 & 0.0230214 & 0.275144 & 0.0229916 & 0.26185 & 0.0157154 & 0.352896 \\
\hline 0.283389 & 0.020536 & 0.275533 & 0.0148286 & 0.255736 & 0.0124974 & 0.361903 \\
\hline 0.286848 & 0.0219463 & 0.275803 & 0.02021 & 0.256361 & 0.0142218 & 0.35531 \\
\hline 0.276703 & 0.0203844 & 0.276887 & 0.0214737 & 0.256012 & 0.0152786 & 0.350449 \\
\hline 0.279708 & 0.0231304 & 0.272893 & 0.0187178 & 0.260718 & 0.013051 & 0.344431 \\
\hline 0.285129 & 0.0159699 & 0.280602 & 0.0179683 & 0.260765 & 0.0117901 & 0.343951 \\
\hline
\end{tabular}




\begin{tabular}{|c|c|c|c|c|c|c|}
\hline 0.277747 & 0.014617 & 0.27412 & 0.0209203 & 0.257982 & 0.00934941 & 0.331298 \\
\hline 0.277063 & 0.0238026 & 0.271916 & 0.0162068 & 0.258131 & 0.0127139 & 0.338477 \\
\hline 0.274781 & 0.0159088 & 0.277775 & 0.0215939 & 0.255906 & 0.0144028 & 346172 \\
\hline 0.280104 & 0.0215283 & 0.276407 & 0.0205147 & 0.261936 & 0.0170737 & 350509 \\
\hline 0.276817 & 0.0146052 & 0.277048 & 0.0122776 & 0.263135 & 0.0103681 & 0.3455 \\
\hline 0.271715 & 0.0211279 & 0.271532 & 0.022029 & 0.261213 & 0.0139539 & 347309 \\
\hline 0.272281 & 0.0191874 & 0.275464 & 0.0156341 & 0.255851 & 0.0109707 & 0.336806 \\
\hline 0.264315 & 0.0190926 & 0.272351 & 0.0195711 & 0.260729 & 0.0136486 & 0.344535 \\
\hline 0.27503 & 0.0219475 & 0.27476 & 0.021697 & 0.259164 & 0.0139141 & 0.339272 \\
\hline 0.272855 & 0.024648 & 0.269812 & 0.0193894 & 0.260187 & 0.0145563 & 0.340807 \\
\hline 0.26698 & 0.0200084 & 0.271753 & 0.0205855 & 0.261323 & 0.0123258 & 0.341503 \\
\hline 0.268665 & 0.0191836 & 0.269683 & 0.0212365 & 0.260684 & 0.0108759 & 0.336682 \\
\hline 0.274406 & 0.025276 & 0.265316 & 0.0188411 & 0.261477 & 0.0175706 & 0.330529 \\
\hline 0.264842 & 0.0164092 & 0.273912 & 0.0199248 & 0.258304 & 0.0148587 & 0.35035 \\
\hline 0.266152 & 0.0147587 & 0.27077 & 0.0159474 & 0.257504 & 0.013459 & 0.333739 \\
\hline 0.265782 & 0.019789 & 0.278392 & 0.0162054 & 0.259649 & 0.017781 & 0.337163 \\
\hline 0.269076 & 0.0192794 & 0.27059 & 0.0173784 & 0.259099 & 0.0109478 & 0.346297 \\
\hline 0.265818 & 0.0196514 & 0.268835 & 0.0189252 & 0.260861 & 0.0170058 & 0.326312 \\
\hline 0.265564 & 0.0175797 & 0.272724 & 0.0154226 & 0.255313 & 0.0137512 & 0.345453 \\
\hline 0.266491 & 0.0193402 & 0.261665 & 0.015721 & 0.251063 & 0.0169964 & 0.34336 \\
\hline 0.263352 & 0.019464 & 0.261776 & 0.0168088 & 0.25469 & 0.0114121 & 0.337609 \\
\hline 0.265606 & 0.0195495 & 0.26068 & 0.0186471 & 0.260021 & 0.0111912 & 0.338492 \\
\hline 0.262767 & 0.0185545 & 0.260388 & 0.0141556 & 0.249275 & 0.0120857 & 0.33932 \\
\hline 0.266319 & 0.017548 & 0.26793 & 0.0224945 & 0.25 & 0.0121221 & 0.345342 \\
\hline 0.264803 & 0.0175329 & 0.261265 & 0.0265667 & 0.252723 & 0.0157176 & 0.336355 \\
\hline 0.263926 & 0.021087 & 0.257024 & 0.0204219 & 0.251131 & 0.0117838 & 0.33458 \\
\hline 0.259475 & 0.0190185 & 0.265298 & 0.0155137 & 86 & 0.0123298 & 0.334462 \\
\hline 0.262233 & 0.0176373 & 0.262517 & 0.0213234 & 0.245047 & 0.0135779 & 0.328406 \\
\hline 0.264868 & 0.0187316 & 0.261576 & 0.0195948 & 0.244202 & 0.0161001 & 0.338948 \\
\hline 0.260026 & 0.0191576 & 0.265807 & 0.023043 & 0.24406 & 0.0139055 & 0.325044 \\
\hline 0.259806 & 0.017218 & 0.266043 & 0.0183182 & 0.247227 & 0.0147327 & 0.330919 \\
\hline 0.259026 & 0.0191524 & 0.26003 & 0.0197557 & 0.24266 & 0.0135949 & 0.330978 \\
\hline 0.256145 & 0.0225233 & 0.251723 & 0.0175915 & 0.24453 & 0.0139724 & 0.335887 \\
\hline 0.255501 & 0.0185535 & 0.259618 & 0.0207703 & 0.24406 & 0.0134211 & 0.337256 \\
\hline 0.257449 & 0.0178059 & 0.256709 & 0.0185247 & 0.242874 & 0.0177739 & 0.335113 \\
\hline 0.25981 & 0.0170009 & 0.251136 & 0.0159116 & 0.241289 & 0.0109185 & 0.327719 \\
\hline 0.256918 & 0.0205417 & 0.259195 & 0.0183691 & 0.235618 & 0.0149047 & 0.331958 \\
\hline 0.253793 & 0.0196498 & 0.254895 & 0.0173708 & 0.239193 & 0.0161947 & 0.335915 \\
\hline 0.257019 & 0.019751 & 0.257002 & 0.0136024 & 0.241215 & 0.00991097 & 0.330656 \\
\hline 0.252662 & 0.0188534 & 0.259581 & 0.0184446 & 0.242797 & 0.00986263 & 0.334659 \\
\hline 0.254022 & 0.0193541 & 0.256159 & 0.0163115 & 0.23685 & 0.014024 & 0.323421 \\
\hline 0.258615 & 0.0182826 & 0.249937 & 0.0185203 & 0.239852 & 0.0116542 & 0.325123 \\
\hline 0.248169 & 0.0179587 & 0.252428 & 0.0191627 & 0.237274 & 0.0156142 & 0.309492 \\
\hline 0.248547 & 0.0173315 & 0.249043 & 0.018624 & 0.233996 & 0.0129199 & 0.321638 \\
\hline 0.252594 & 0.0214461 & 0.249677 & 0.018005 & 0.233935 & 0.0160161 & 0.326289 \\
\hline 0.257026 & 0.0181573 & 0.252301 & 0.0171399 & 0.229808 & 0.0118027 & 0.319769 \\
\hline 0.246938 & 0.01977 & 0.24949 & 0.018858 & 0.23256 & 0.0108665 & 0.305782 \\
\hline
\end{tabular}




\begin{tabular}{|c|c|c|c|c|c|c|}
\hline 0.247233 & 0.0189572 & 0.253578 & 0.02015 & 0.232992 & 0.0119223 & 0.313841 \\
\hline 0.251283 & 0.0188273 & 0.254303 & 0.0184494 & 0.237569 & 0.0125443 & 0.302673 \\
\hline 0.253949 & 0.0204754 & 0.251872 & 0.0175173 & 0.23309 & 0.0122934 & 0.311881 \\
\hline 0.251024 & 0.0180801 & 0.250921 & 0.0129434 & 0.230535 & 0.0165905 & 0.309531 \\
\hline 0.250638 & 0.0206616 & 0.256284 & 0.0200453 & 0.227447 & 0.0114636 & 0.318276 \\
\hline 0.24842 & 0.0177291 & 0.25093 & 0.0167111 & 0.230494 & 0.0135953 & 0.309221 \\
\hline 0.248078 & 0.0201888 & 0.249256 & 0.014967 & 0.232009 & 0.0105185 & 0.31135 \\
\hline 0.245072 & 0.021382 & 0.249104 & 0.0189622 & 0.231216 & 0.0144359 & 0.320389 \\
\hline 0.246583 & 0.0188218 & 0.248567 & 0.0196055 & 0.227687 & 0.0130605 & 0.316859 \\
\hline 0.250689 & 0.0138213 & 0.24928 & 0.0152275 & 0.22827 & 0.0163027 & 0.304095 \\
\hline 0.253925 & 0.0182461 & 0.244103 & 0.0212808 & 0.230039 & 0.0143527 & 0.316771 \\
\hline 0.245077 & 0.0174209 & 0.249729 & 0.0148449 & 0.226066 & 0.0130365 & 0.305995 \\
\hline 0.246931 & 0.0213814 & 0.249222 & 0.0162527 & 0.225548 & 0.0143107 & 0.316063 \\
\hline 0.249779 & 0.0198636 & 0.239958 & 0.018313 & 0.228722 & 0.00963702 & 0.309687 \\
\hline 0.255666 & 0.0205387 & 0.242053 & 0.0174349 & 0.230675 & 0.0145003 & 0.315254 \\
\hline 0.250918 & 0.0175241 & 0.245141 & 0.0218096 & 0.228096 & 0.0149033 & 0.306365 \\
\hline 0.245217 & 0.0187661 & 0.24736 & 0.0181951 & 0.225006 & 0.0113689 & 0.31909 \\
\hline 0.244498 & 0.0197807 & 0.240479 & 0.0160237 & 0.223869 & 0.0124561 & 0.310816 \\
\hline 0.245013 & 0.0212035 & 0.239783 & 0.0150053 & 0.221842 & 0.0146421 & 0.31096 \\
\hline 0.245348 & 0.0216577 & 0.239509 & 0.0157455 & 0.220865 & 0.010363 & 0.307723 \\
\hline 0.241634 & 0.017676 & 0.242724 & 0.0145197 & 0.228192 & 0.0129917 & 0.323991 \\
\hline 0.246731 & 0.0208495 & 0.246638 & 0.017768 & 0.220105 & 0.0117048 & 0.312889 \\
\hline 0.247196 & 0.0213043 & 0.23946 & 0.0170648 & 0.226677 & 0.0085934 & 0.313757 \\
\hline 0.244297 & 0.0217774 & 0.241325 & 0.0183522 & 0.223923 & 0.0130422 & 0.323958 \\
\hline 0.243253 & 0.0209588 & 0.239015 & 0.0162932 & 0.222874 & 0.0140699 & 0.320946 \\
\hline 0.24023 & 0.0197353 & 0.240801 & 0.0139794 & 0.223047 & 0.0128332 & 0.318251 \\
\hline 0.246558 & 0.0163443 & 0.24389 & 0.0191691 & 0.227514 & 0.0111752 & 0.311489 \\
\hline 0.244721 & 0.0193173 & 0.241441 & 0.0167696 & 0.222959 & 0.0137755 & 0.318998 \\
\hline 0.242825 & 0.0164349 & 0.245962 & 0.0166851 & 0.229751 & 0.0128231 & 0.309029 \\
\hline 0.241185 & 0.0196896 & 0.240097 & 0.0165497 & 0.226562 & 0.0176553 & 0.313237 \\
\hline 0.242918 & 0.0176806 & 0.246465 & 0.0170518 & 0.228166 & 0.0127141 & 0.316989 \\
\hline 0.243438 & 0.0130118 & 0.243331 & 0.0190475 & 0.226343 & 0.0126649 & 0.309623 \\
\hline 0.241478 & 0.0184988 & 0.238283 & 0.0163493 & 0.225768 & 0.0106195 & 0.325161 \\
\hline 0.241188 & 0.0179706 & 0.244287 & 0.0173442 & 0.229309 & 0.0104908 & 0.319047 \\
\hline 0.24426 & 0.0193528 & 0.243007 & 0.016279 & 0.224167 & 0.0134748 & 0.306999 \\
\hline 0.245041 & 0.0206107 & 0.247024 & 0.0166146 & 0.229621 & 0.0131704 & 0.323818 \\
\hline 0.238916 & 0.0192538 & 0.246012 & 0.0152845 & 0.233942 & 0.0154876 & 0.312928 \\
\hline 0.238169 & 0.0168474 & 0.252852 & 0.0215979 & 0.234685 & 0.0126689 & 0.324029 \\
\hline 0.245847 & 0.0213944 & 0.250318 & 0.0175329 & 0.231926 & 0.012808 & 0.323517 \\
\hline 0.244371 & 0.019353 & 0.25203 & 0.0161387 & 0.236712 & 0.0114929 & 0.329103 \\
\hline 0.245136 & 0.0221091 & 0.250576 & 0.0147274 & 0.235166 & 0.0158787 & 0.324428 \\
\hline 0.243365 & 0.0190316 & 0.254492 & 0.0196384 & 0.239352 & 0.0137516 & 0.336575 \\
\hline 0.243906 & 0.0151155 & 0.25379 & 0.0175235 & 0.237318 & 0.014859 & 0.333048 \\
\hline 0.236897 & 0.0164737 & 0.255917 & 0.0205671 & 0.241722 & 0.0154185 & 0.334611 \\
\hline 0.239821 & 0.0166612 & 0.254831 & 0.0215646 & 0.240685 & 0.0140956 & 0.350017 \\
\hline 0.241067 & 0.0201339 & 0.249607 & 0.0143519 & 0.241218 & 0.0148036 & 0.335902 \\
\hline 0.236623 & 0.0163735 & 0.256378 & 0.0187581 & 0.242351 & 0.0106048 & 0.325902 \\
\hline
\end{tabular}




\begin{tabular}{|c|c|c|c|c|c|c|}
\hline 0.238258 & 0.0174438 & 0.255064 & 0.0199358 & 0.241341 & 0.0129963 & 0.326483 \\
\hline 0.236096 & 0.0174141 & 0.253806 & 0.0248881 & 0.241948 & 0.0130385 & 0.330562 \\
\hline .243527 & 0.0202658 & 0.258383 & 0.0172118 & 0.242545 & 0.014866 & 0.336719 \\
\hline 0.235115 & 0.0248477 & 0.252601 & 0.0164026 & 0.248246 & 0.0101076 & 0.334478 \\
\hline 0.240025 & 0.0201476 & 0.253388 & 0.0131723 & 0.246107 & 0.0131547 & 0.338109 \\
\hline 0.238301 & 0.0167876 & 0.256048 & 0.0193946 & 0.250777 & 0.0128171 & 0.319648 \\
\hline 0.239849 & 0.01664 & 0.258311 & 0.0141232 & 0.247431 & 0.0131083 & 0.34208 \\
\hline 0.240216 & 0.0204838 & 0.260147 & 0.0201097 & 0.253413 & 0.0105149 & 0.333944 \\
\hline 0.246191 & 0.0185599 & 0.263836 & 0.0191044 & 0.250032 & 0.0135433 & 0.326671 \\
\hline 0.247733 & 0.0157628 & 0.259487 & 0.0158847 & 0.252223 & 0.0129857 & 0.332019 \\
\hline 0.242771 & 0.0205872 & 0.260871 & 0.0186991 & 0.252298 & 0.0143453 & 0.343343 \\
\hline 0.251116 & 0.0176693 & 0.262819 & 0.0202098 & 0.249627 & 0.0137455 & 0.329618 \\
\hline 0.242566 & 0.0211194 & 0.270161 & 0.0183526 & 0.25412 & 0.0140129 & 0.341155 \\
\hline 0.241041 & 0.0153999 & 0.260238 & 0.0192801 & 0.257667 & 0.016199 & 0.336944 \\
\hline 0.245332 & 0.0193527 & 0.268572 & 0.0220231 & 0.25681 & 0.0158564 & 0.339102 \\
\hline 0.249698 & 0.0209161 & 0.266673 & 0.0191169 & 0.261477 & 0.0124781 & 0.34365 \\
\hline 0.246361 & 0.0187447 & 0.267656 & 0.0208556 & 0.257788 & 0.014514 & 0.3456 \\
\hline 0.255628 & 0.0170824 & 0.265785 & 0.0213336 & 0.261365 & 0.0158476 & 0.346393 \\
\hline 0.248338 & 0.0215798 & 0.266245 & 0.0210613 & 0.268302 & 0.0147805 & 0.341154 \\
\hline 0.250683 & 0.0190933 & 0.274791 & 0.0252935 & 0.263527 & 0.018795 & 0.334057 \\
\hline 0.251378 & 0.017501 & 0.271457 & 0.0202732 & 0.268973 & 0.0157109 & 0.354107 \\
\hline 0.250708 & 0.0181034 & 0.275352 & 0.0191334 & 0.266185 & 0.0132741 & 0.336843 \\
\hline 0.263537 & 0.0201218 & 0.26787 & 0.0245271 & 0.26971 & 0.0147169 & 0.35018 \\
\hline 0.258382 & 0.0224865 & 0.267733 & 0.0196004 & 0.266688 & 0.0146543 & 0.344728 \\
\hline 0.258724 & 0.0173847 & 0.268184 & 0.0234804 & 0.267068 & 0.0120918 & 0.351041 \\
\hline 0.253556 & 0.0144528 & 0.26969 & 0.017459 & 0.265482 & 0.0125264 & 0.340079 \\
\hline 0.256851 & 0.0160574 & 0.272632 & 0.0183165 & 0.260571 & 0.0130128 & 0.331753 \\
\hline 0.261341 & 0.0136159 & 0.268195 & 0.0205682 & 0.26332 & 0.0141657 & 0.337056 \\
\hline 0.263329 & 0.0173735 & 0.26556 & 0.0218682 & 0.262707 & 0.0159349 & 0.330528 \\
\hline 0.259604 & 0.0187586 & 0.267707 & 0.0214511 & 0.258521 & 0.0112296 & 0.327129 \\
\hline 0.265189 & 0.0166286 & 0.262487 & 0.018865 & 0.256265 & 0.0150678 & 0.316782 \\
\hline 0.266129 & 0.0209993 & 0.259656 & 0.0222046 & 0.254925 & 0.0134471 & 0.333422 \\
\hline 0.256983 & 0.0212161 & 0.259591 & 0.0166557 & 0.252805 & 0.0116476 & 0.328058 \\
\hline 0.263538 & 0.0221196 & 0.257709 & 0.0265198 & 0.25351 & 0.0135655 & 0.319768 \\
\hline 0.261732 & 0.0191258 & 0.257516 & 0.0196323 & 0.246149 & 0.0113043 & 0.327053 \\
\hline 0.263179 & 0.0173206 & 0.255739 & 0.0195419 & 0.251416 & 0.0133039 & 0.316702 \\
\hline 0.25676 & 0.0197114 & 0.257054 & 0.0167733 & 0.247845 & 0.012272 & 0.311872 \\
\hline 0.261455 & 0.0213859 & 0.255497 & 0.0199886 & 0.239172 & 0.0115766 & 0.298045 \\
\hline 0.267623 & 0.0200966 & 0.255124 & 0.0195213 & 0.241168 & 0.0106515 & 0.309436 \\
\hline 0.262211 & 0.0183186 & 0.254615 & 0.0173472 & 0.240706 & 0.0126877 & 0.307795 \\
\hline 0.259937 & 0.0206268 & 0.251867 & 0.0225492 & 0.243037 & 0.0104659 & 0.314546 \\
\hline 0.260841 & 0.0194416 & 0.25302 & 0.0170564 & 0.238423 & 0.0141793 & 0.303471 \\
\hline 0.256609 & 0.022605 & 0.247917 & 0.0168012 & 0.233707 & 0.0164485 & 0.308355 \\
\hline 0.255887 & 0.0183815 & 0.250714 & 0.0199517 & 0.236393 & 0.0139696 & 0.303482 \\
\hline 0.256306 & 0.0210167 & 0.249856 & 0.0166009 & 0.233377 & 0.0136561 & 0.292654 \\
\hline 0.264449 & 0.0219444 & 0.252335 & 0.0123594 & 0.237451 & 0.0116811 & 0.311322 \\
\hline 0.257322 & 0.0206406 & 0.2513 & 0.0165841 & 0.237987 & 0.0126542 & 0.307467 \\
\hline
\end{tabular}




\begin{tabular}{|c|c|c|c|c|c|c|}
\hline 0.253595 & 0.0208055 & 0.249536 & 0.0167244 & 0.235949 & 0.0145444 & 0.299223 \\
\hline 0.255076 & 0.017184 & 0.254759 & 0.0205105 & 0.237248 & 0.012908 & 0.309331 \\
\hline 0.253853 & 0.0218653 & 0.257525 & 0.0154323 & 0.236615 & 0.00991837 & 0.298056 \\
\hline 0.256118 & 0.0196864 & 0.256507 & 0.020142 & 0.241656 & 0.0139047 & 0.291896 \\
\hline 0.256559 & 0.0199679 & 0.257268 & 0.0178659 & 0.239308 & 0.0163823 & 0.303195 \\
\hline 0.253577 & 0.0193584 & 0.255553 & 0.0180463 & 0.244697 & 0.0146415 & 0.310598 \\
\hline 0.259815 & 0.0197162 & 0.256795 & 0.0177784 & 0.240976 & 0.0134199 & 0.300678 \\
\hline 0.258973 & 0.0180138 & 0.25925 & 0.0189469 & 0.247129 & 0.0129592 & 0.311644 \\
\hline 0.255449 & 0.0201288 & 0.258986 & 0.0219466 & 0.245406 & 0.0119765 & 0.305946 \\
\hline 0.254879 & 0.0157095 & 0.256075 & 0.0148886 & 0.247387 & 0.0175978 & 0.303451 \\
\hline 0.261572 & 0.0169001 & 0.256075 & 0.0202035 & 0.242464 & 0.0150191 & 0.309253 \\
\hline 0.253651 & 0.0135474 & 0.258635 & 0.0192246 & 0.245912 & 0.0149196 & 0.307295 \\
\hline 0.25704 & 0.0152767 & 0.256115 & 0.0153183 & 0.246749 & 0.0142435 & 0.313538 \\
\hline 0.256059 & 0.0157284 & 0.260923 & 0.0188947 & 0.247973 & 0.010754 & 0.318148 \\
\hline 0.25869 & 0.0208011 & 0.265182 & 0.0205123 & 0.246762 & 0.0115915 & 0.31732 \\
\hline 0.257611 & 0.0234462 & 0.260926 & 0.0221442 & 0.247753 & 0.0151198 & 0.312263 \\
\hline 0.259557 & 0.0152981 & 0.261877 & 0.011037 & 0.251967 & 0.011825 & 0.312608 \\
\hline 0.265486 & 0.0175017 & 0.260859 & 0.0208183 & 0.253035 & 0.012847 & 0.303665 \\
\hline 0.259496 & 0.0186388 & 0.25995 & 0.0165057 & 0.252773 & 0.0157579 & 0.30616 \\
\hline 0.268564 & 0.0170582 & 0.262575 & 0.0191238 & 0.251524 & 0.0105994 & 0.303651 \\
\hline 0.264503 & 0.01785 & 0.266499 & 0.0168837 & 0.257151 & 0.0158074 & 0.309745 \\
\hline 0.269809 & 0.0210246 & 0.266911 & 0.0175426 & 0.24949 & 0.0143336 & 0.31594 \\
\hline 0.27401 & 0.0193395 & 0.264232 & 0.0196532 & 0.255878 & 0.0129313 & 0.31094 \\
\hline 0.268753 & 0.0165677 & 0.268831 & 0.0151321 & 0.257619 & 0.0141202 & 0.31066 \\
\hline 0.264448 & 0.0184198 & 0.274288 & 0.0172473 & 0.253901 & 0.0157259 & 0.316517 \\
\hline 0.27084 & 0.0181668 & 0.271761 & 0.0153745 & 0.259944 & 0.0136107 & 0.310459 \\
\hline 0.26634 & 0.0201062 & 0.27472 & 0.0171145 & 0.263356 & 0.0130558 & 0.315395 \\
\hline 0.269314 & 0.0249999 & 0.276043 & 0.0217503 & 0.261851 & 0.012495 & 0.300027 \\
\hline 0.27931 & 0.0208432 & 0.275298 & 0.0194158 & 0.265964 & 0.0165959 & 0.325597 \\
\hline 0.274173 & 0.0178583 & 0.27659 & 0.0215965 & 0.262218 & 0.0148951 & 0.318926 \\
\hline 0.275497 & 0.0168754 & 0.279104 & 0.0213919 & 0.266599 & 0.0131159 & 0.319211 \\
\hline 0.274046 & 0.0199992 & 0.27651 & 0.0134142 & 0.269312 & 0.0108876 & 0.331506 \\
\hline 0.274229 & 0.0150713 & 0.285527 & 0.0182795 & 0.269264 & 0.0158174 & 0.318617 \\
\hline 0.282176 & 0.0217481 & 0.287448 & 0.0195663 & 0.273643 & 0.0114633 & 0.329573 \\
\hline 0.277188 & 0.0182436 & 0.288939 & 0.0200272 & 0.278145 & 0.0145442 & 0.329574 \\
\hline 0.283395 & 0.029438 & 0.290449 & 0.0193032 & 0.278011 & 0.0139262 & 0.327839 \\
\hline 0.293447 & 0.02147 & 0.293782 & 0.0192664 & 0.284767 & 0.0118785 & 0.333681 \\
\hline 0.291957 & 0.0242524 & 0.295712 & 0.0223447 & 0.282282 & 0.011905 & 0.325146 \\
\hline 0.288238 & 0.0201036 & 0.300121 & 0.0206755 & 0.285985 & 0.0137477 & 0.342654 \\
\hline 0.297085 & 0.0198579 & 0.303414 & 0.0194929 & 0.281008 & 0.0175788 & 0.338996 \\
\hline 0.292414 & 0.0222423 & 0.304867 & 0.0205022 & 0.292217 & 0.0148348 & 0.346029 \\
\hline 0.298343 & 0.0215823 & 0.307159 & 0.0182492 & 0.303584 & 0.0158216 & 0.346311 \\
\hline 0.302435 & 0.0193748 & 0.311678 & 0.0165537 & 0.2971 & 0.0128455 & 0.351917 \\
\hline 0.305318 & 0.0186204 & 0.31077 & 0.0210675 & 0.302513 & 0.0149744 & 0.345724 \\
\hline 0.307052 & 0.0172961 & 0.312151 & 0.0202877 & 0.307319 & 0.018011 & 0.344396 \\
\hline 0.315578 & 0.0246177 & 0.32455 & 0.0162842 & 0.311035 & 0.0104974 & 0.355931 \\
\hline 0.309535 & 0.0160914 & 0.324464 & 0.0193158 & 0.313614 & 0.0129968 & 0.365586 \\
\hline
\end{tabular}




\begin{tabular}{|c|c|c|c|c|c|c|}
\hline 0.3195 & 0.0205146 & 0.328647 & 0.019343 & 0.319728 & 0.0146505 & 0.344061 \\
\hline 0.327723 & 0.0227885 & 0.334587 & 0.017437 & 0.327856 & 0.0139217 & 0.3666 \\
\hline 0.325154 & 0.0228474 & 0.341166 & 0.0193546 & 0.326911 & 0.0145971 & 0.368865 \\
\hline 0.337107 & 0.02517 & 0.334387 & 0.0219895 & 0.334797 & 0.0148965 & 0.3727 \\
\hline 0.340481 & 0.0240806 & 0.344126 & 0.0169604 & 0.341305 & 0.0153693 & 0.36944 \\
\hline 0.344691 & 0.0226591 & 0.351596 & 0.0253277 & 0.343831 & 0.0155535 & 0.37076 \\
\hline 0.345723 & 0.0215362 & 0.362356 & 0.0202448 & 0.359843 & 0.0161063 & 0.392019 \\
\hline 0.351097 & 0.0225799 & 0.368949 & 0.021168 & 0.358847 & 0.0241575 & 0.389321 \\
\hline 0.359662 & 0.0228517 & 0.376103 & 0.0249576 & 0.374329 & 0.0184786 & 0.391758 \\
\hline 0.373022 & 0.0228105 & 0.373629 & 0.0168554 & 0.378843 & 0.016309 & 0.382292 \\
\hline 0.382661 & 0.0192277 & 0.3821 & 0.0190488 & 0.382375 & 0.016032 & 0.390263 \\
\hline 0.384454 & 0.0237667 & 0.393662 & 0.0270993 & 0.391795 & 0.0131222 & 0.405344 \\
\hline 0.401172 & 0.0299731 & 0.399597 & 0.018787 & 0.401832 & 0.0208458 & 0.415503 \\
\hline 0.409669 & 0.0193708 & 0.412057 & 0.0225585 & 0.417209 & 0.0177888 & 0.440509 \\
\hline 0.427833 & 0.0221403 & 0.425042 & 0.0268172 & 0.427746 & 0.0202967 & 0.433404 \\
\hline 0.423884 & 0.0196037 & 0.425175 & 0.0218068 & 0.434242 & 0.0217048 & 0.430772 \\
\hline 0.431286 & 0.0274319 & 0.431904 & 0.0230492 & 0.453694 & 0.018051 & 0.437694 \\
\hline 0.43932 & 0.0374163 & 0.451444 & 0.0257424 & 0.458096 & 0.018933 & 0.448171 \\
\hline 0.460301 & 0.0220143 & 0.45954 & 0.0195778 & 0.47171 & 0.020557 & 0.448803 \\
\hline 0.467498 & 0.0226374 & 0.465541 & 0.0195421 & 0.48069 & 0.0235713 & 0.46984 \\
\hline 0.485763 & 0.0245541 & 0.486913 & 0.0247774 & 0.503254 & 0.0207355 & 0.478013 \\
\hline 0.501215 & 0.0206865 & 0.496331 & 0.0241 & 0.50 & 0.0199169 & 0.491279 \\
\hline 0.517999 & 0.022964 & 0.505245 & 0.0237538 & 0.528877 & 0.018907 & 0.500347 \\
\hline 0.525829 & 0.0304329 & 0.524045 & 0.0227386 & 0.543634 & 0.0200675 & 0.510165 \\
\hline 0.539617 & 0.0311932 & 0.542483 & 0.0245725 & 0.562452 & 0.0222842 & 0.50458 \\
\hline 0.554711 & 0.0293446 & 0.558073 & 0.0225456 & 0.570826 & 0.0165235 & 0.514434 \\
\hline 0.563485 & 0.0282601 & 0.558459 & 0.024943 & 0.591267 & 0.0208055 & 0.551466 \\
\hline 0.584863 & 0.0374057 & 0.591097 & 0.0265439 & 0.605661 & 0.0253969 & 0.53799 \\
\hline 0.597974 & 0.0312914 & 0.594685 & 0.0303151 & 0.622564 & 0.0239715 & 0.541721 \\
\hline 0.621668 & 0.0234541 & 0.617288 & 0.0175908 & 0.643001 & 0.0265125 & 0.579883 \\
\hline 0.630266 & 0.0262221 & 0.639387 & 0.0212707 & 0.654574 & 0.0264548 & 0.58198 \\
\hline 0.651383 & 0.0262129 & 0.644425 & 0.0286708 & 0.672716 & 0.0202292 & 0.598639 \\
\hline 0.664579 & 0.0329937 & 0.665703 & 0.0228046 & 0.692206 & 0.0166971 & 0.596296 \\
\hline 0.679424 & 0.0293892 & 0.6803 & 0.0306381 & 0.707317 & 0.0206571 & 0.620647 \\
\hline 0.702803 & 0.037263 & 0.695472 & 0.0302066 & 0.721745 & 0.0240791 & 0.637122 \\
\hline 0.717543 & 0.0302581 & 0.701715 & 0.0266521 & 0.74038 & 0.0260881 & 0.648112 \\
\hline 0.738762 & 0.0269172 & 0.728148 & 0.0363027 & 0.752107 & 0.0212028 & 0.651535 \\
\hline 0.750317 & 0.0294285 & 0.746214 & 0.0210226 & 0.782806 & 0.0221272 & 0.666351 \\
\hline 0.76257 & 0.0321664 & 0.76443 & 0.0286046 & 0.788835 & 0.0300879 & 0.679241 \\
\hline 0.785038 & 0.0280653 & 0.775273 & 0.0301175 & 0.817467 & 0.0256139 & 0.689324 \\
\hline 0.792314 & 0.0317504 & 0.793387 & 0.0265463 & 0.827224 & 0.0255009 & 0.695261 \\
\hline 0.809248 & 0.0408336 & 0.804558 & 0.0303222 & 0.841719 & 0.0309188 & 0.724238 \\
\hline 0.821245 & 0.0360119 & 0.81668 & 0.0244764 & 0.855422 & 0.0270313 & 0.723409 \\
\hline 0.838519 & 0.0383004 & 0.838846 & 0.0303673 & 0.863762 & 0.0292946 & 0.74356 \\
\hline 0.850703 & 0.0372058 & 0.843953 & 0.0305898 & 0.879883 & 0.0303741 & 0.739605 \\
\hline 0.876087 & 0.0366784 & 0.859676 & 0.027155 & 0.894282 & 0.021986 & 0.742947 \\
\hline 0.881935 & 0.0355927 & 0.874343 & 0.0254948 & 0.911158 & 0.0306164 & 0.766735 \\
\hline
\end{tabular}




\begin{tabular}{|c|c|c|c|c|c|c|}
\hline 0.901103 & 0.0318896 & 0.894189 & 0.0379775 & 0.917468 & 0.024621 & 0.774827 \\
\hline 0.89541 & 0.0478576 & 0.897684 & 0.0394417 & 0.927549 & 0.0216385 & 0.773016 \\
\hline 0.916155 & 0.0409685 & 0.90525 & 0.0284221 & 0.935135 & 0.0267759 & 0.795449 \\
\hline 0.914801 & 0.0343062 & 0.921731 & 0.0401188 & 0.930089 & 0.0282339 & 0.795953 \\
\hline 0.927805 & 0.0376201 & 0.92676 & 0.0311428 & 0.951941 & 0.0291146 & 0.824272 \\
\hline 0.933855 & 0.0371549 & 0.926131 & 0.0401681 & 0.961426 & 0.027854 & 0.826801 \\
\hline 0.944003 & 0.0326804 & 0.940907 & 0.0338074 & 0.959757 & 0.029191 & 0.829475 \\
\hline 0.940753 & 0.0308843 & 0.939835 & 0.0305851 & 0.967559 & 0.0199547 & 0.832529 \\
\hline 0.946405 & 0.0362133 & 0.948476 & 0.0369252 & 0.963502 & 0.0236022 & 0.85217 \\
\hline 0.95059 & 0.0333961 & 0.952949 & 0.036814 & 0.96873 & 0.0244649 & 0.845742 \\
\hline 0.946407 & 0.0356351 & 0.956301 & 0.0311772 & 0.967145 & 0.0250172 & 0.850407 \\
\hline 0.951156 & 0.0344957 & 0.948333 & 0.0406847 & 0.953022 & 0.0319778 & 0.868806 \\
\hline 0.949571 & 0.0386788 & 0.965795 & 0.0272638 & 0.9638 & 0.0262456 & 0.881659 \\
\hline 0.96223 & 0.0307557 & 0.956302 & 0.0336316 & 0.955216 & 0.0288643 & 0.873993 \\
\hline 0.934966 & 0.0353849 & 0.953957 & 0.0332246 & 0.949406 & 0.0272166 & 0.878795 \\
\hline 0.938129 & 0.0375357 & 0.950893 & 0.0328395 & 0.951729 & 0.0271549 & 0.889599 \\
\hline 0.929999 & 0.0445537 & 0.941494 & 0.0276489 & 0.934739 & 0.0272295 & 0.884116 \\
\hline 0.93228 & 0.0345616 & 0.933506 & 0.0318147 & 0.934796 & 0.0283444 & 0.891177 \\
\hline 0.922845 & 0.0465651 & 0.930504 & 0.0297254 & 0.922923 & 0.0307706 & 0.900298 \\
\hline 0.904975 & 0.0380633 & 0.92206 & 0.033863 & 0.908957 & 0.0273958 & 0.905223 \\
\hline 0.910688 & 0.0389107 & 0.910546 & 0.0351635 & 0.895107 & 0.0279129 & 0.90233 \\
\hline 0.883186 & 0.0409587 & 0.898423 & 0.0341396 & 0.876041 & 0.0271332 & 0.91527 \\
\hline 0.867318 & 0.0472885 & 0.886075 & 0.0313758 & 0.864285 & 0.027928 & 0.892902 \\
\hline 0.877362 & 0.0377859 & 0.885484 & 0.0323233 & 0.860319 & 0.0302198 & 0.907426 \\
\hline 0.849909 & 0.0402789 & 0.867651 & 0.0339184 & 0.844861 & 0.0236658 & 0.902206 \\
\hline 0.840253 & 0.0356852 & 0.850575 & 0.0301315 & 0.820688 & 0.0277322 & 0.891177 \\
\hline 0.841596 & 0.0378234 & 0.844478 & 0.0387539 & 0.802419 & 0.021734 & 0.894094 \\
\hline 0.816361 & 0.0312058 & 0.825184 & 0.0297723 & 0.793972 & 0.0267491 & 0.910327 \\
\hline 0.795635 & 0.0394129 & 0.810516 & 0.0285787 & 0.771204 & 0.0260231 & 0.879467 \\
\hline 0.787729 & 0.0297737 & 0.803445 & 0.0251958 & 0.755328 & 0.0261474 & 0.867001 \\
\hline 0.761763 & 0.035504 & 0.776573 & 0.0299291 & 0.740027 & 0.0235432 & 0.871759 \\
\hline 0.740243 & 0.0424939 & 0.773373 & 0.0290228 & 0.719495 & 0.024949 & 0.861737 \\
\hline 0.727485 & 0.0338681 & 0.743431 & 0.0304555 & 0.706509 & 0.0259197 & 0.850744 \\
\hline 0.714827 & 0.0376142 & 0.728435 & 0.024645 & 0.682654 & 0.0218836 & 0.819658 \\
\hline 0.692815 & 0.0308686 & 0.706083 & 0.0290139 & 0.659962 & 0.017507 & 0.836369 \\
\hline 0.679532 & 0.0350642 & 0.694365 & 0.0251753 & 0.649345 & 0.0223821 & 0.813577 \\
\hline 0.665615 & 0.0308921 & 0.673586 & 0.0253742 & 0.629123 & 0.0238431 & 0.808563 \\
\hline 0.652341 & 0.0393036 & 0.655165 & 0.0225838 & 0.610157 & 0.0277509 & 0.788899 \\
\hline 0.623003 & 0.0272862 & 0.642017 & 0.0277181 & 0.59974 & 0.0242178 & 0.76045 \\
\hline 0.606887 & 0.0294067 & 0.631687 & 0.0200558 & 0.578914 & 0.0219269 & 0.740352 \\
\hline 0.595097 & 0.0231564 & 0.597062 & 0.0249929 & 0.56302 & 0.0222141 & 0.705057 \\
\hline 0.584111 & 0.0269978 & 0.579906 & 0.0297153 & 0.541777 & 0.0228326 & 0.697487 \\
\hline 0.55871 & 0.0229015 & 0.574755 & 0.0213591 & 0.527519 & 0.0189671 & 0.66689 \\
\hline 0.552983 & 0.026084 & 0.556904 & 0.0259151 & 0.507079 & 0.0241988 & 0.648434 \\
\hline 0.527301 & 0.03269 & 0.540366 & 0.0276016 & 0.496136 & 0.0177872 & 0.62875 \\
\hline 0.511796 & 0.0218702 & 0.526021 & 0.0266893 & 0.472435 & 0.0196936 & 0.622544 \\
\hline 0.499199 & 0.024619 & 0.514344 & 0.0279486 & 0.468209 & 0.0232798 & 0.588399 \\
\hline
\end{tabular}




\begin{tabular}{|c|c|c|c|c|c|c|}
\hline 0.483743 & 0.025518 & 0.494356 & 0.0270112 & 0.454205 & 0.0187359 & 0.574432 \\
\hline 0.4725 & 0.027152 & 0.48496 & 0.0227396 & 0.437126 & 0.0194244 & 0.558717 \\
\hline 0.45968 & 0.0233471 & 0.476734 & 0.020807 & 0.424165 & 0.0167827 & 0.53179 \\
\hline 0.440072 & 0.0213094 & 0.453007 & 0.0285272 & 0.411657 & 0.0183361 & 0.52463 \\
\hline 0.429148 & 0.0217406 & 0.440991 & 0.0234478 & 0.395801 & 0.0148318 & 0.495064 \\
\hline 0.424296 & 0.0284059 & 0.43437 & 0.0201006 & 0.392233 & 0.0123143 & 0.484339 \\
\hline 0.41029 & 0.025059 & 0.422172 & 0.017471 & 0.378769 & 0.0170953 & 0.475085 \\
\hline 0.398369 & 0.0234502 & 0.409621 & 0.0267353 & 0.365347 & 0.0161878 & 0.447431 \\
\hline 0.38978 & 0.025594 & 0.394251 & 0.0199 & 0.357783 & 0.0168658 & 0.442534 \\
\hline 0.382655 & 0.021233 & 0.389489 & 0.0199475 & 0.345334 & 0.0157748 & 0.434324 \\
\hline 0.365665 & 0.0218366 & 0.380105 & 0.0238093 & 0.338486 & 0.016373 & 0.412674 \\
\hline 0.352491 & 0.0212516 & 0.369911 & 0.0209516 & 0.329135 & 0.0144613 & 0.407943 \\
\hline 0.349241 & 0.0195367 & 0.357356 & 0.0226074 & 0.324084 & 0.0183182 & 0.413909 \\
\hline 0.343536 & 0.0227338 & 0.349036 & 0.0165927 & 0.316217 & 0.016353 & 0.398871 \\
\hline 0.331035 & 0.0167502 & 0.341579 & 0.0234899 & 0.308416 & 0.0133923 & 0.371687 \\
\hline 0.324078 & 0.0231953 & 0.333537 & 0.0181249 & 0.297677 & 0.0134822 & 0.37356 \\
\hline 0.317946 & 0.017791 & 0.327593 & 0.0194635 & 0.298884 & 0.0150595 & 0.369481 \\
\hline 0.310056 & 0.0192998 & 0.314819 & 0.0198322 & 0.284394 & 0.0132749 & 0.350979 \\
\hline 0.301954 & 0.0207472 & 0.316047 & 0.0199201 & 0.284192 & 0.0159167 & 0.358251 \\
\hline 0.303676 & 0.0238232 & 0.298715 & 0.0162566 & 0.272769 & 0.0142717 & 0.35808 \\
\hline 0.294041 & 0.0178128 & 0.290498 & 0.0191574 & 0.267813 & 0.0147484 & 0.340242 \\
\hline 0.286996 & 0.019739 & 0.284665 & 0.01742 & 0.267649 & 0.0116553 & 0.340812 \\
\hline 0.279818 & 0.0207907 & 0.283053 & 0.0145006 & 0.263085 & 0.01183 & 0.328661 \\
\hline 0.281401 & 0.0227153 & 0.279885 & 0.014526 & 0.257159 & 0.012118 & 0.331536 \\
\hline 0.270529 & 0.0246204 & 0.273956 & 0.0223633 & 0.254094 & 0.0144669 & 0.312722 \\
\hline 0.256943 & 0.0173768 & 0.270118 & 0.0234124 & 0.248718 & 0.0125288 & 0.307532 \\
\hline 0.258185 & 0.0232346 & 0.263855 & 0.0149604 & 0.243831 & 0.0148843 & 0.320321 \\
\hline 0.256905 & 0.0208102 & 0.261307 & 0.0167382 & 0.24132 & 0.0102792 & 0.301661 \\
\hline 0.25036 & 0.021373 & 0.25119 & 0.0174653 & 0.240327 & 0.0117913 & 0.30079 \\
\hline 0.248073 & 0.018938 & 0.247881 & 0.015279 & 0.226652 & 0.0080209 & 0.308737 \\
\hline 0.239229 & 0.0160789 & 0.24268 & 0.0171696 & 0.228584 & 0.0139423 & 0.291798 \\
\hline 0.238353 & 0.0185316 & 0.239642 & 0.0169694 & 0.223156 & 0.0135666 & 0.298016 \\
\hline 0.231503 & 0.0213584 & 0.23095 & 0.018758 & 0.220553 & 0.0108394 & 0.290064 \\
\hline 0.22582 & 0.0197257 & 0.229886 & 0.0124592 & 0.21909 & 0.0145042 & 0.294277 \\
\hline 0.226466 & 0.0180866 & 0.229556 & 0.0165456 & 0.215285 & 0.0140299 & 0.281975 \\
\hline 0.21989 & 0.0179634 & 0.220429 & 0.0214143 & 0.20883 & 0.011679 & 0.274241 \\
\hline 0.214064 & 0.013042 & 0.217865 & 0.0140195 & 0.211607 & 0.014491 & 0.282741 \\
\hline 0.216541 & 0.0221379 & 0.218872 & 0.0157955 & 0.203375 & 0.0118938 & 0.284983 \\
\hline 0.211227 & 0.0181608 & 0.211248 & 0.0172965 & 0.20253 & 0.0122299 & 0.270667 \\
\hline 0.204811 & 0.0179078 & 0.212339 & 0.0122309 & 0.200249 & 0.0132644 & 0.289946 \\
\hline 0.201039 & 0.0151144 & 0.207178 & 0.0151919 & 0.196024 & 0.0137027 & 0.289967 \\
\hline 0.202518 & 0.0159277 & 0.201294 & 0.0145458 & 0.190694 & 0.0128149 & 0.281494 \\
\hline 0.194279 & 0.0164434 & 0.198961 & 0.0184978 & 0.187399 & 0.0106001 & 0.294852 \\
\hline 0.197835 & 0.0154831 & 0.197373 & 0.0135829 & 0.183807 & 0.0106938 & 0.277735 \\
\hline 0.195647 & 0.0125549 & 0.196577 & 0.017063 & 0.184258 & 0.0107909 & 0.290293 \\
\hline 0.185034 & 0.0187747 & 0.190536 & 0.0153441 & 0.182349 & 0.00899204 & 0.28485 \\
\hline 0.182264 & 0.0164126 & 0.188772 & 0.0137414 & 0.176579 & 0.0108333 & 0.288522 \\
\hline
\end{tabular}




\begin{tabular}{|c|c|c|c|c|c|c|}
\hline 0.184546 & 0.0162756 & 0.186398 & 0.0137703 & 0.180187 & 0.0119627 & 89413 \\
\hline 0.176161 & 0.0133583 & 0.185369 & 0.0176812 & 0.178047 & 0.0129813 & 0.282079 \\
\hline .178668 & 0.0176569 & 0.18535 & 0.0176004 & 0.176437 & 0.0107589 & .299605 \\
\hline .178399 & 0.0156962 & 0.189512 & 0.0115211 & 0.175167 & 0.00855991 & 0.296005 \\
\hline 0.175592 & 0.0150016 & 0.194854 & 0.0164561 & 0.171708 & 0.0128839 & 0.296044 \\
\hline 0.171834 & 0.0170267 & 0.192042 & 0.0159669 & 0.173535 & 0.0101539 & 0.300791 \\
\hline 0.172957 & 0.0152562 & 0.200598 & 0.0155887 & 0.17028 & 0.0109145 & 0.304163 \\
\hline 0.16968 & 0.0177297 & 0.198472 & 0.0159787 & 0.174616 & 0.0143748 & 0.310073 \\
\hline 0.170593 & 0.0152035 & 0.199134 & 0.019688 & 0.174707 & 0.011485 & 0.320992 \\
\hline 0.176077 & 0.0158216 & 0.211422 & 0.0137863 & 0.181251 & 0.0124467 & 0.330293 \\
\hline 0.168323 & 0.0166852 & 0.215727 & 0.0204123 & 0.180838 & 0.0101474 & 0.339803 \\
\hline 0.172568 & 0.0117677 & 0.226831 & 0.0256388 & 0.189136 & 0.00945993 & 0.353321 \\
\hline 0.173641 & 0.0113944 & 0.236468 & 0.0214887 & 0.192334 & 0.0100439 & 0.379948 \\
\hline 0.18346 & 0.0130802 & 0.246516 & 0.0231266 & 0.203912 & 0.0145602 & 0.385529 \\
\hline 0.185565 & 0.0207585 & 0.255307 & 0.0270988 & 0.206801 & 0.009867 & 0.404385 \\
\hline 0.185791 & 0.014251 & 0.27826 & 0.0214218 & 0.218899 & 0.0132573 & 3588 \\
\hline 0.193425 & 0.0125441 & 0.289123 & 0.0219221 & 0.225116 & 0.0153914 & 0.462164 \\
\hline 0.201144 & 0.0149717 & 0.311595 & 0.0286566 & 0.240791 & 0.0152889 & 0.498664 \\
\hline 0.207621 & 0.016598 & 0.333205 & 0.0 & .249073 & 0.0134413 & 158 \\
\hline 0.22018 & 0.0165287 & 0.349694 & 0.0325025 & 0.263 & 0.01866 & 0.55789 \\
\hline 0.232894 & 0.0163789 & 0.370307 & 0.0373491 & 0.277202 & 0.0175174 & 0.600128 \\
\hline 0.252947 & 0.0216025 & 0.401829 & 0.03 & 0.295493 & 0.0121085 & 042 \\
\hline 0.260909 & 0.0170744 & 0.419372 & 0.0440708 & 0.307301 & 0.0184157 & 0.708125 \\
\hline 0.283519 & 0.0149221 & 0.445215 & 0.0440767 & 0.326556 & 0.0141577 & 0.738874 \\
\hline 0.296743 & 0.0211051 & 0.461542 & 0.0524005 & 0.336524 & 0.021419 & 0.776923 \\
\hline 0.318113 & 0.0213555 & 0.485675 & 0.0568858 & 0.357804 & 0.0157467 & 0.842352 \\
\hline 0.339535 & 0.0215182 & 0.512192 & 0.0564348 & 0.376902 & 0.0195132 & 0.872137 \\
\hline 0.364699 & 0.0174984 & 0.530558 & 0.0588019 & 0.392693 & 0.018354 & 0.914993 \\
\hline 0.385029 & 0.0224131 & 0.552928 & 0.0620198 & 0.41471 & 0.0209146 & 0.956749 \\
\hline 0.413086 & 0.02279 & 0.57377 & 0.0709712 & 0.425071 & 0.0187971 & 1.01615 \\
\hline 0.430027 & 0.0232263 & 0.600875 & 0.061017 & 0.43768 & 0.024792 & 1.07953 \\
\hline 0.459782 & 0.0274964 & 0.625932 & 0.0704187 & 0.456084 & 0.0206363 & 1.09864 \\
\hline 0.487285 & 0.0283681 & 0.637022 & 0.0740886 & 0.471954 & 0.0240183 & 1.13166 \\
\hline 0.51823 & 0.0222783 & 0.645604 & 0.0652037 & 0.482551 & 0.0231195 & 1.19049 \\
\hline 0.524127 & 0.021622 & 0.671366 & 0.0737202 & 0.494653 & 0.017657 & 1.20697 \\
\hline 0.549737 & 0.0294006 & 0.679182 & 0.0843268 & 0.499058 & 0.0191195 & 1.25253 \\
\hline 0.58469 & 0.0302703 & 0.693704 & 0.0762112 & 0.519804 & 0.0180128 & 1.2656 \\
\hline 0.601346 & 0.034337 & 0.693299 & 0.0817989 & 0.523865 & 0.0205641 & 1.31298 \\
\hline 0.614706 & 0.0314508 & 0.689641 & 0.0715775 & 0.530098 & 0.0210934 & 1.28799 \\
\hline 0.638468 & 0.035929 & 0.687451 & 0.0746154 & 0.528843 & 0.0209327 & 1.29374 \\
\hline 0.655274 & 0.0293698 & 0.694993 & 0.0917725 & 0.531904 & 0.0172651 & 1.32219 \\
\hline 0.675482 & 0.0419135 & 0.677514 & 0.0641346 & 0.526589 & 0.02202 & 1.27718 \\
\hline 0.688909 & 0.0401016 & 0.671481 & 0.076633 & 0.525173 & 0.0181738 & 1.28363 \\
\hline 0.682992 & 0.0326616 & 0.670008 & 0.0746842 & 0.515504 & 0.0255059 & 1.26805 \\
\hline 0.69623 & 0.0283086 & 0.635146 & 0.0639704 & 0.499653 & 0.0235768 & 1.23331 \\
\hline 0.697505 & 0.0363935 & 0.613488 & 0.0685828 & 0.491009 & 0.0232158 & 1.18864 \\
\hline 0.696156 & 0.033383 & 0.587578 & 0.0653042 & 0.479914 & 0.0171441 & 1.16398 \\
\hline
\end{tabular}




\begin{tabular}{|c|c|c|c|c|c|c|}
\hline 0.705591 & 0.0330066 & 0.56221 & 0.0566241 & 0.466791 & 2728 & \\
\hline 0.684975 & 0.0376951 & 0.539551 & 0.0656734 & 0.444417 & 0.0267321 & 1.04062 \\
\hline 679611 & 0.0293509 & 0.512363 & 0.0589665 & 0.41812 & 0.0209443 & 1.00589 \\
\hline 0.666364 & 0.0345181 & 0.472536 & 0.0539325 & 0.397403 & 0.0160808 & 0.937743 \\
\hline 0.649087 & 0.030015 & 0.436814 & 0.0518452 & 0.379754 & 0.0161136 & 0.892747 \\
\hline 0.622488 & 0.038419 & 0.405946 & 0.0485934 & 0.352435 & 0.0168641 & 0.807136 \\
\hline 0.597601 & 0.0355082 & 0.380926 & 0.0433355 & 0.328061 & 0.0151431 & 0.753108 \\
\hline 0.570857 & 0.0287481 & 0.350903 & 0.0371334 & 0.311098 & 0.0145673 & 0.699886 \\
\hline 0.543768 & 0.0275989 & 0.313946 & 0.0325805 & 0.282099 & 0.0167015 & 0.651188 \\
\hline 0.517087 & 0.0298766 & 0.294427 & 0.0368234 & 0.265727 & 0.0148407 & 0.581909 \\
\hline 0.485464 & 0.0242198 & 0.268049 & 0.0349519 & 0.239611 & 0.0115253 & 0.524715 \\
\hline 0.453349 & 0.0260756 & 0.24503 & 0.0271148 & 0.223408 & 0.0146582 & 0.460552 \\
\hline 0.410406 & 0.0244614 & 0.229076 & 0.0276106 & 0.211032 & 0.013924 & 0.431263 \\
\hline 0.38467 & 0.02 & 0.204773 & 0.0259694 & 0.19 & 0.0102878 & 0.398806 \\
\hline 0.352581 & 0.0220554 & 0.187881 & 0.0159819 & 0.180399 & 0.0117804 & 0.354746 \\
\hline 0.324813 & 0.026543 & 0.180295 & 0.0254973 & 0.170258 & 0.0112147 & 0.31477 \\
\hline 0.297898 & 0.0189259 & 0.167797 & 0.0160554 & 0.158761 & 0.0107385 & 0.296285 \\
\hline 0.274267 & 0.0207864 & 0.157847 & 0.0181694 & 0.148132 & 0.008828 & 0.266233 \\
\hline 0.248251 & 0.0183081 & 0.144992 & 0.0148585 & 0.139917 & 0.0100364 & 0.251682 \\
\hline 0.227485 & 563 & 0.139349 & 0.0153584 & 0.137897 & 0.00750698 & 0.240008 \\
\hline 0.208268 & 0.0166217 & 0.134025 & 0.0140838 & 0.129973 & 0.0097802 & 0.226888 \\
\hline 0.193768 & 0.016054 & 0.127988 & 0.0124497 & 0.125196 & 0.00928703 & 0.213403 \\
\hline 0.176482 & 0.01787 & 0.126993 & 0.0118024 & 0.119386 & 0.00911401 & 0.201708 \\
\hline 0.16936 & 0.015722 & 0.12502 & 0.0139651 & 0.120359 & 0.0113471 & 0.189462 \\
\hline 0.1562 & 0.01 & 0.122477 & 524 & 71 & 0.00 & 0.1 \\
\hline 0.143865 & 0.0142472 & 0.116421 & 0.0107779 & 0.116245 & 0.00964147 & 0.181853 \\
\hline 0.141479 & 0.0129628 & 0.113045 & 0.0117456 & 0.11227 & 0.00950028 & 0.1787 \\
\hline 0.136995 & 0.0128425 & 0.119499 & 0.0134584 & 0.112786 & 0.00899454 & 0.169678 \\
\hline 0.128589 & 0.010756 & 0.117738 & 0.0124982 & 0.11224 & 0.00985398 & 0.179615 \\
\hline 0.127038 & 0.0154334 & 0.114644 & 0.0104714 & 0.111676 & 0.0112589 & 0.166976 \\
\hline 0.121689 & 0.0136291 & 0.11949 & 0.0123551 & 0.112859 & 0.00898895 & 0.162673 \\
\hline 0.11865 & 0.0121283 & 0.115628 & 0.0123007 & 0.111511 & 0.00853906 & 0.162909 \\
\hline 0.117497 & 0.0121142 & 0.111161 & 0.0115936 & 0.109335 & 0.0105326 & 0.161114 \\
\hline 0.119976 & 0.0131515 & 0.114557 & 0.012062 & 0.11073 & 0.00761779 & 0.156315 \\
\hline 0.116757 & 0.0103795 & 0.11537 & 0.0143539 & 0.110709 & 0.00820042 & 0.149447 \\
\hline 0.11976 & 0.0133118 & 0.113759 & 0.0126852 & 0.111451 & 0.00908741 & 0.159289 \\
\hline 0.116324 & 0.012109 & 0.115878 & 0.0161959 & 0.112363 & 0.0106027 & 0.161113 \\
\hline 0.11407 & 0.0134326 & 0.117864 & 0.0095703 & 0.112533 & 0.0097241 & 0.151005 \\
\hline 0.118929 & 0.013987 & 0.117962 & 0.0130394 & 0.115973 & 0.00742504 & 0.155367 \\
\hline 0.112528 & 0.0142674 & 0.118699 & 0.0147603 & 0.111351 & 0.00801164 & 0.151179 \\
\hline 0.115467 & 0.0116095 & 0.116455 & 0.013001 & 0.112465 & 0.00626227 & 0.15099 \\
\hline 0.115997 & 0.0127482 & 0.117199 & 0.00999868 & 0.115852 & 0.00920016 & 0.148608 \\
\hline 0.117921 & 0.0142658 & 0.118403 & 0.0126529 & 0.116381 & 0.00747057 & 0.153271 \\
\hline 0.116065 & 0.0112226 & 0.116911 & 0.0116672 & 0.114499 & 0.00825664 & 0.148804 \\
\hline 0.11707 & 0.0112436 & 0.115186 & 0.0130972 & 0.116902 & 0.00834933 & 0.155221 \\
\hline 0.121072 & 0.0121146 & 0.116236 & 0.0130681 & 0.116792 & 0.0107288 & 0.149554 \\
\hline 0.116314 & 0.0135722 & 0.121118 & 0.0126381 & 0.11669 & 0.00833264 & 0.15104 \\
\hline
\end{tabular}




$\begin{array}{rrrrrrr}0.119483 & 0.0134712 & 0.119406 & 0.0114989 & 0.118334 & 0.00799637 & 0.142269 \\ 0.127106 & 0.0139928 & 0.121059 & 0.0129529 & 0.120062 & 0.00855003 & 0.147912 \\ 0.119163 & 0.0141353 & 0.12556 & 0.0107001 & 0.119952 & 0.00805147 & 0.150607 \\ 0.125174 & 0.0118564 & 0.12268 & 0.0149585 & 0.120696 & 0.00893863 & 0.150098 \\ 0.124137 & 0.0147327 & 0.125319 & 0.0117904 & 0.121825 & 0.00992392 & 0.145599 \\ 0.126827 & 0.0142199 & 0.131911 & 0.013575 & 0.125968 & 0.0104449 & 0.149271 \\ 0.126994 & 0.0133714 & 0.128954 & 0.011646 & 0.127894 & 0.0111466 & 0.146207 \\ 0.129578 & 0.0153655 & 0.133899 & 0.0150805 & 0.127585 & 0.00892522 & 0.154732 \\ 0.131812 & 0.0117325 & 0.134126 & 0.0119092 & 0.126093 & 0.0106256 & 0.154176 \\ 0.132591 & 0.0149199 & 0.131537 & 0.0109294 & 0.129879 & 0.0106193 & 0.150355 \\ 0.128748 & 0.0141268 & 0.134457 & 0.0119196 & 0.13128 & 0.00853928 & 0.15331 \\ 0.133345 & 0.0133648 & 0.134958 & 0.0152801 & 0.133015 & 0.00898211 & 0.15843 \\ 0.136582 & 0.0133202 & 0.134534 & 0.011851 & 0.135017 & 0.0108925 & 0.154184 \\ 0.138386 & 0.0169455 & 0.135285 & 0.0145822 & 0.137144 & 0.0117821 & 0.153067 \\ 0.140049 & 0.0116715 & 0.140407 & 0.0121281 & 0.140074 & 0.00954814 & 0.152042 \\ 0.138681 & 0.0105405 & 0.137638 & 0.0101792 & 0.138872 & 0.00956613 & 0.155261 \\ 0.138787 & 0.0148804 & 0.14438 & 0.0143844 & 0.141546 & 0.00976785 & 0.158635 \\ 0.145747 & 0.0142234 & 0.144458 & 0.0119797 & 0.13917 & 0.00978675 & 0.159652 \\ 0.147458 & 0.014226 & 0.142396 & 0.0120494 & 0.144723 & 0.00937127 & 0.15631 \\ 0.144941 & 0.0126385 & 0.144791 & 0.0128454 & 0.143478 & 0.00857167 & 0.160901 \\ 0.145555 & 0.0158219 & 0.149694 & 0.0151822 & 0.148972 & 0.00836343 & 0.156739 \\ 0.151266 & 0.0173448 & 0.15198 & 0.0140668 & 0.147402 & 0.0101437 & 0.160881 \\ 0.15358 & 0.0149336 & 0.15065 & 0.0122329 & 0.149141 & 0.0121304 & 0.171541 \\ 0.155817 & 0.0162382 & 0.153143 & 0.0120528 & 0.15354 & 0.0120383 & 0.161166 \\ 0.155209 & 0.0138266 & 0.157278 & 0.0132148 & 0.154635 & 0.0114896 & 0.163658 \\ 0.157689 & 0.0142665 & 0.155228 & 0.0141783 & 0.154523 & 0.00981945 & 0.160297 \\ 0.155235 & 0.0143532 & 0.160557 & 0.0119961 & 0.158626 & 0.0119239 & 0.165805 \\ 0.158778 & 0.0152725 & 0.156118 & 0.0134256 & 0.160641 & 0.00787009 & 0.172904\end{array}$




\begin{tabular}{|c|c|c|c|c|c|c|}
\hline 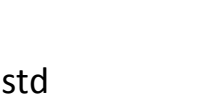 & $\begin{array}{l}\text { SB Ru } \\
\text { mean }\end{array}$ & std & $\begin{array}{l}\text { SB Rc } \\
\text { mean }\end{array}$ & std & $\begin{array}{l}\text { SB Rb } \\
\text { mean }\end{array}$ & std \\
\hline 0.160022 & 0.414779 & 0.0396361 & 0.246999 & 0.0355575 & 0.219046 & 0.0237054 \\
\hline 0.16577 & 0.41935 & 0.0391818 & 0.251183 & 0.0463229 & 0.225464 & 0.0283012 \\
\hline 0.206033 & 0.419251 & 0.0398095 & 0.245345 & 0.0365502 & 0.221423 & 0.0219308 \\
\hline 0.171586 & 0.413871 & 0.0383521 & 0.240785 & 0.0426016 & 0.224599 & 0.0212836 \\
\hline 0.184532 & 0.410389 & 0.0365561 & 0.24347 & 0.0388571 & 0.220209 & 0.0197176 \\
\hline 0.148431 & 0.412056 & 0.0378681 & 0.243633 & 0.0405405 & 0.22251 & 0.0259831 \\
\hline 0.193611 & 0.40759 & 0.0379245 & 0.237773 & 0.0406711 & 0.221521 & 0.0184433 \\
\hline 0.168888 & 0.401381 & 0.0338912 & 0.240675 & 0.038309 & 0.225026 & 0.0203709 \\
\hline 0.176389 & 0.406426 & 0.0372156 & 0.238894 & 0.0358067 & 0.218111 & 0.0201055 \\
\hline 0.172527 & 0.397683 & 0.0326627 & 0.239532 & 0.0384017 & 0.220209 & 0.02481 \\
\hline 0.190425 & 0.405531 & 0.0430814 & 0.233267 & 0.0429858 & 0.219208 & 0.0241948 \\
\hline 0.161995 & 0.403367 & 0.0388538 & 0.23921 & 0.0430773 & 0.213844 & 0.0229495 \\
\hline 0.162602 & 0.395222 & 0.0368999 & 0.236391 & 0.0400993 & 0.216479 & 0.0237501 \\
\hline 0.186156 & 0.398615 & 0.0313438 & 0.236967 & 0.0424654 & 0.217257 & 0.019222 \\
\hline 0.186654 & 0.402177 & 0.0366532 & 0.23957 & 0.0386176 & 0.21929 & 0.0234442 \\
\hline 0.194399 & 0.408318 & 0.0342755 & 0.241328 & 0.0416089 & 0.216931 & 0.0225813 \\
\hline 0.187099 & 0.419174 & 0.0373018 & 0.237714 & 0.0439192 & 0.219767 & 0.0292699 \\
\hline 0.16485 & 0.430185 & 0.0332631 & 0.245069 & 0.0426619 & 0.223269 & 0.0236013 \\
\hline 0.179337 & 0.447275 & 0.036634 & 0.243699 & 0.0383746 & 0.228212 & 0.0186804 \\
\hline 0.159489 & 0.480381 & 0.0358655 & 0.245426 & 0.0437852 & 0.237251 & 0.0235309 \\
\hline 0.165406 & 0.50984 & 0.0422821 & 0.247269 & 0.04035 & 0.243977 & 0.025029 \\
\hline 0.197574 & 0.537378 & 0.0449791 & 0.258171 & 0.0456306 & 0.249737 & 0.0264067 \\
\hline 0.185353 & 0.561891 & 0.0495212 & 0.262633 & 0.0449508 & 0.255356 & 0.0313032 \\
\hline 0.17414 & 0.589192 & 0.0498859 & 0.263091 & 0.0436256 & 0.266288 & 0.0261491 \\
\hline 0.163043 & 0.602898 & 0.0550131 & 0.268014 & 0.0404423 & 0.269325 & 0.0296341 \\
\hline 0.162079 & 0.605333 & 0.0491874 & 0.270719 & 0.0487615 & 0.275542 & 0.0308929 \\
\hline 0.15601 & 0.599334 & 0.046535 & 0.266128 & 0.0443956 & 0.274284 & 0.0255819 \\
\hline 0.174494 & 0.574484 & 0.0504109 & 0.263813 & 0.0455 & 0.266597 & 0.0253243 \\
\hline 0.164734 & 0.54204 & 0.0469827 & 0.262365 & 0.0438652 & 0.260685 & 0.0319286 \\
\hline 0.182823 & 0.506494 & 0.0355557 & 0.258535 & 0.0445171 & 0.258395 & 0.0253376 \\
\hline 0.179726 & 0.479336 & 0.0412564 & 0.251627 & 0.0416743 & 0.247983 & 0.0326018 \\
\hline 0.169899 & 0.450696 & 0.0360121 & 0.248331 & 0.0431184 & 0.246854 & 0.0280415 \\
\hline 0.17532 & 0.428913 & 0.0299521 & 0.246648 & 0.0446053 & 5079 & 0.0270135 \\
\hline 0.185129 & 0.422042 & 0.03451 & 0.243389 & 0.0452872 & 0.241654 & 0.0269365 \\
\hline 0.189456 & 0.408039 & 0.0293579 & 0.24687 & 0.0444906 & 0.249726 & 0.0271943 \\
\hline 0.18209 & 0.407933 & 0.0322783 & 0.245801 & 0.0443404 & 0.259872 & 0.0301359 \\
\hline 0.195429 & 0.414621 & 0.0396938 & 0.250566 & 0.0412233 & 0.277791 & 0.0341378 \\
\hline 0.169443 & 0.438689 & 0.0397684 & 0.257073 & 0.0460656 & 0.301789 & 0.0353424 \\
\hline 0.179955 & 0.476361 & 0.0406175 & 0.275119 & 0.0471082 & 0.352458 & 0.0434241 \\
\hline 0.202413 & 0.522055 & 0.0507679 & 0.292417 & 0.0489617 & 0.4366 & 0.0493406 \\
\hline 0.203411 & 0.599969 & 0.0748351 & 0.330233 & 0.0570488 & 0.561215 & 0.0732286 \\
\hline 0.24186 & 0.698031 & 0.0917319 & 0.374297 & 0.0717831 & 0.742332 & 0.0866274 \\
\hline 0.303656 & 0.836977 & 0.121366 & 0.433196 & 0.0907014 & 0.98571 & 0.134976 \\
\hline 0.31926 & 0.990951 & 0.141433 & 0.502639 & 0.110434 & 1.25159 & 0.182546 \\
\hline 0.394536 & 1.14341 & 0.178969 & 0.587764 & 0.140717 & 1.56761 & 0.222819 \\
\hline
\end{tabular}




\begin{tabular}{|c|c|c|c|c|c|c|}
\hline 0.420632 & 1.28568 & 0.226073 & 0.667085 & 0.157056 & 1.86813 & 0.256165 \\
\hline 0.520988 & 1.40252 & 0.249954 & 0.742437 & 0.175464 & 2.14262 & 0.289038 \\
\hline 0.554873 & 1.48735 & 0.274139 & 0.79659 & 0.191581 & 2.34299 & 0.328902 \\
\hline 0.602498 & 1.50518 & 0.280148 & 0.822916 & 0.205557 & 2.45027 & 0.350094 \\
\hline 0.604933 & 1.46969 & 0.275106 & 0.823344 & 0.201074 & 2.43444 & 0.342894 \\
\hline 0.573879 & 1.38364 & 0.24771 & 0.796944 & 0.196706 & 2.29733 & 0.319785 \\
\hline 0.525416 & 1.25249 & 0.205545 & 0.739779 & 0.183993 & 2.07287 & 0.280669 \\
\hline 0.462485 & 1.12347 & 0.17336 & 0.678028 & 0.160496 & 1.8072 & 0.247623 \\
\hline 0.391206 & 0.99204 & 0.14253 & 0.605555 & 0.142253 & 1.52015 & 0.197365 \\
\hline 0.336138 & 0.870748 & 0.117453 & 0.527486 & 0.113274 & 1.22294 & 0.149434 \\
\hline 0.306971 & 0.782109 & 0.0910478 & 0.466042 & 0.0956164 & 0.980306 & 0.132817 \\
\hline 0.286734 & 0.72466 & 0.078848 & 0.419074 & 0.0818846 & 0.783347 & 0.108273 \\
\hline 0.238991 & 0.709886 & 0.0653528 & 0.390516 & 0.0716093 & 0.640875 & 0.0836078 \\
\hline 0.194521 & 0.738804 & 0.0686709 & 0.377867 & 0.0725376 & 0.559247 & 0.0646695 \\
\hline 0.190783 & 0.853126 & 0.0828101 & 0.382455 & 0.070806 & 0.521708 & 0.0562581 \\
\hline 0.217264 & 1.10376 & 0.129987 & 0.414189 & 0.0756218 & 0.554832 & 0.0652626 \\
\hline 0.203042 & 1.55542 & 0.209171 & 0.500352 & 0.10054 & 0.677424 & 0.086533 \\
\hline 0.253781 & 2.34215 & 0.345329 & 0.66796 & 0.135826 & 0.923079 & 0.119677 \\
\hline 0.317715 & 3.64376 & 0.572464 & 0.937576 & 0.199493 & 1.34633 & 0.184922 \\
\hline 0.383592 & 5.62537 & 0.926661 & 1.37171 & 0.313344 & 2.04834 & 0.280934 \\
\hline 0.542937 & 8.44253 & 1.40865 & 2.01437 & 0.450914 & 3.06845 & 0.447785 \\
\hline 0.750873 & 12.2101 & 2.04905 & 2.92299 & 0.661356 & 4.50316 & 0.660096 \\
\hline 1.06537 & 16.8529 & 2.87702 & 4.131 & 0.950293 & 6.36087 & 0.935287 \\
\hline 1.38095 & 22.1276 & 3.79694 & 5.59828 & 1.30941 & 8.61156 & 1.2528 \\
\hline 1.78915 & 27.6748 & 4.74677 & 7.25256 & 1.70152 & 11.1332 & 1.63472 \\
\hline 2.17842 & 32.803 & 5.67446 & 8.93467 & 2.13338 & 13.7215 & 2.0616 \\
\hline 2.53349 & 36.8195 & 6.43405 & 10.4855 & 2.51131 & 15.9906 & 2.38812 \\
\hline 2.90862 & 39.2923 & 6.87822 & 11.7283 & 2.79938 & 17.711 & 2.6499 \\
\hline 3.05502 & 39.784 & 6.93486 & 12.4307 & 2.97403 & 18.6586 & 2.72887 \\
\hline 3.02898 & 38.2154 & 6.61746 & 12.5689 & 3.00386 & 18.7991 & 2.77821 \\
\hline 2.94936 & 34.884 & 6.02732 & 12.1025 & 2.89787 & 17.9197 & 2.65871 \\
\hline 2.73256 & 30.31 & 5.26192 & 11.0648 & 2.64949 & 16.2397 & 2.39585 \\
\hline 2.43959 & 25.0975 & 4.37385 & 9.63293 & 2.29325 & 14.0038 & 2.06507 \\
\hline 2.08148 & 19.9156 & 3.4448 & 8.01363 & 1.91122 & 11.6049 & 1.70604 \\
\hline 1.69195 & 15.2515 & 2.60462 & 6.41742 & 1.51561 & 9.18915 & 1.3531 \\
\hline 1.3427 & 11.3054 & 1.90058 & 4.96812 & 1.18166 & 7.02319 & 1.03108 \\
\hline 1.01744 & 8.21728 & 1.34788 & 3.73584 & 0.872079 & 5.20804 & 0.758673 \\
\hline 0.81116 & 5.97628 & 0.958682 & 2.79073 & 0.639089 & 3.78015 & 0.571447 \\
\hline 0.640996 & 4.35746 & 0.684798 & 2.07037 & 0.456461 & 2.73882 & 0.392251 \\
\hline 0.500362 & 3.18835 & 0.486381 & 1.54169 & 0.334216 & 1.99315 & 0.292056 \\
\hline 0.396618 & 2.35431 & 0.334799 & 1.15663 & 0.247063 & 1.44732 & 0.204247 \\
\hline 0.304363 & 1.71533 & 0.229184 & 0.891641 & 0.187674 & 1.06649 & 0.151268 \\
\hline 0.270885 & 1.2448 & 0.163976 & 0.711917 & 0.145544 & 0.810432 & 0.110011 \\
\hline 0.238037 & 0.899874 & 0.113025 & 0.575207 & 0.109165 & 0.616842 & 0.0809196 \\
\hline 0.227721 & 0.67836 & 0.0847103 & 0.462546 & 0.0813608 & 0.480845 & 0.0573667 \\
\hline 0.200637 & 0.553018 & 0.0745453 & 0.394225 & 0.0726806 & 0.374114 & 0.048181 \\
\hline 0.202568 & 0.519776 & 0.0460902 & 0.335109 & 0.0618823 & 0.301669 & 0.0419586 \\
\hline
\end{tabular}




\begin{tabular}{|c|c|c|c|c|c|c|}
\hline 0.201751 & 0.564538 & 0.0558998 & 0.308778 & 0.059213 & 0.251713 & 314285 \\
\hline 0.178575 & .652739 & 0.0792542 & 0.296745 & 0.0584095 & 0.221957 & 0.0219746 \\
\hline 0.154082 & 0.748911 & 0.0692138 & 0.290096 & 0.0541415 & 0.196997 & 0.0230328 \\
\hline 0.160857 & .836583 & 0.0823285 & 0.289686 & 0.0512471 & 0.188548 & 0.0191509 \\
\hline 0.172921 & 0.909331 & 0.0913932 & 0.284501 & 0.0517361 & 0.18424 & 0.0232631 \\
\hline 0.190444 & 0.946955 & 0.082618 & 0.284743 & 0.0553594 & 0.188152 & 0.0245834 \\
\hline 0.182423 & 0.948749 & 0.0801244 & 0.284315 & 0.0601538 & 0.188173 & 0.0251011 \\
\hline 0.174235 & 0.92243 & 0.0838813 & 0.302104 & 0.0555074 & 0.195288 & 0.0244242 \\
\hline 0.19396 & 0.882207 & 0.0803486 & 0.295961 & 0.0512778 & 0.200075 & 0.0224616 \\
\hline 0.189818 & 0.82019 & 0.0688446 & 0.298639 & 0.0538295 & 0.202626 & 0.021926 \\
\hline 0.19456 & 0.750233 & 0.0724076 & 0.29363 & 0.0594645 & 0.201318 & 0.0204065 \\
\hline 0.179661 & 0.686684 & 0.0668843 & 0.286089 & 0.0564176 & 0.20144 & 0.0263339 \\
\hline 0.188216 & 0.630613 & 0.0597287 & 0.276777 & 0.0602158 & 0.197753 & 0.0275165 \\
\hline 0.165452 & 0.583034 & 0.0517372 & 0.274425 & 0.054987 & 0.190334 & 0.0316435 \\
\hline 0.172059 & 0.539362 & 0.0503024 & 0.262882 & 0.0483742 & 0.186336 & 0.0300205 \\
\hline 0.168784 & 0.506829 & 0.0425248 & 0.253696 & 0.0545172 & 0.178588 & 0.0281629 \\
\hline 0.167617 & 0.493019 & 0.0464127 & 0.247937 & 0.0506259 & 0.176021 & 0.0261969 \\
\hline 0.163091 & 0.480785 & 0.0498256 & 0.238044 & 0.0445792 & 0.169505 & 0.0240613 \\
\hline 0.1701 & 0.468171 & 0.0500392 & 0.238755 & 0.0444248 & 0.168879 & 0.0265156 \\
\hline 0.155226 & 0.460166 & 0.0442455 & 0.235559 & 0.0426678 & 0.166745 & 0.0286308 \\
\hline 0.16378 & 0.463103 & 0.044424 & 0.229565 & 0.0416682 & 0.160602 & 0.0215446 \\
\hline 0.157392 & 0.463384 & 0.0421288 & 0.229104 & 0.0427841 & 0.161322 & 2841 \\
\hline 0.167363 & 0.465225 & 0.0441868 & 0.228669 & 0.0433415 & 0.159296 & 0.0187902 \\
\hline 0.159707 & 0.472918 & 0.0429315 & 0.230049 & 0.0445107 & 0.155979 & 0.0190128 \\
\hline 0.176953 & 0.4846 & 0.0576437 & 0.234786 & 0.040864 & 0.155041 & 0.0186474 \\
\hline 0.176211 & 0.500134 & 0.0579359 & 0.237706 & 0.043393 & 0.155096 & 0.0159533 \\
\hline 0.162876 & 0.548624 & 0.0417728 & 0.24953 & 0.0446532 & 0.156999 & 0.0186099 \\
\hline 0.150336 & 0.621829 & 0.0578389 & 0.263707 & 0.0492235 & 0.15732 & 0.018254 \\
\hline 0.154159 & 0.733151 & 0.0755131 & 0.289869 & 0.053361 & 0.159332 & 0.0190356 \\
\hline 0.165447 & 0.901361 & 0.0945223 & 0.328578 & 0.0633153 & 0.172497 & 4035 \\
\hline 0.167305 & 1.10062 & 0.120343 & 0.384146 & 0.0750933 & 0.189605 & 0.0225377 \\
\hline 0.173985 & 1.36621 & 0.167179 & 0.455441 & 0.0899772 & 0.208135 & 0.0249998 \\
\hline 0.210932 & 1.65545 & 0.215623 & 0.538877 & 0.104358 & 0.227542 & 0.0282125 \\
\hline 0.232875 & 1.95597 & 0.253286 & 0.6445 & 0.131263 & 0.247282 & 0.0275734 \\
\hline 0.267814 & 2.23596 & 0.286937 & 0.753832 & 0.160839 & 0.274658 & 0.0316162 \\
\hline 0.315838 & 2.49923 & 0.34824 & 0.853796 & 0.186434 & 0.302387 & 0.0380221 \\
\hline 0.356849 & 2.66634 & 0.360185 & 0.946176 & 0.20335 & 0.319498 & 0.0414578 \\
\hline 0.369412 & 2.73463 & 0.357234 & 1.01835 & 0.222931 & 0.339123 & 0.0430333 \\
\hline 0.379433 & 2.69635 & 0.355114 & 1.05405 & 0.231485 & 0.348079 & 0.0434706 \\
\hline 0.390821 & 2.56921 & 0.342125 & 1.05275 & 0.22621 & 0.348633 & 0.0438207 \\
\hline 0.378106 & 2.34373 & 0.327446 & 1.02065 & 0.227367 & 0.339182 & 0.043324 \\
\hline 0.346855 & 2.0774 & 0.266492 & 0.954176 & 0.204884 & 0.317827 & 0.0394036 \\
\hline 0.316508 & 1.79716 & 0.211083 & 0.863824 & 0.179553 & 0.293644 & 0.0393016 \\
\hline 0.288181 & 1.50629 & 0.183903 & 0.759076 & 0.163811 & 0.27412 & 0.0367155 \\
\hline 0.249607 & 1.24398 & 0.148201 & 0.649748 & 0.132611 & 0.251693 & 0.0296308 \\
\hline 0.231684 & 1.0316 & 0.10605 & 0.553196 & 0.105403 & 0.220747 & 0.0287586 \\
\hline 0.211538 & 0.869455 & 0.0817224 & 0.477328 & 0.094372 & 0.201049 & 0.0234934 \\
\hline
\end{tabular}




\begin{tabular}{|c|c|c|c|c|c|c|}
\hline 0.208586 & 0.748016 & 0.0815606 & 0.410089 & 0.081813 & 0.187498 & 0.0201103 \\
\hline 0.195352 & 0.657011 & 0.0618082 & 0.357823 & 0.072386 & 0.175309 & 0.0197475 \\
\hline 0.178548 & 0.600478 & 0.0627628 & 0.327543 & 0.0628895 & 0.168888 & 0.022516 \\
\hline 0.168031 & 0.566817 & 0.0577855 & 0.304567 & 0.0550219 & 0.164991 & 0.0229972 \\
\hline 0.154599 & 0.552668 & 0.0524753 & 0.286818 & 0.0539314 & 0.161584 & 0.0209612 \\
\hline 0.161134 & 0.537422 & 0.0458312 & 0.281349 & 0.0519544 & 0.158096 & 0.0186029 \\
\hline 0.157125 & 0.518776 & 0.0529291 & 0.280024 & 0.0534809 & 0.159332 & 0.0186319 \\
\hline 0.15934 & 0.510149 & 0.0544202 & 0.279765 & 0.0532175 & 0.159608 & 0.0193065 \\
\hline 0.165537 & 0.491147 & 0.0503137 & 0.273552 & 0.0519362 & 0.15552 & 0.0160795 \\
\hline 0.171677 & 0.481904 & 0.0469151 & 0.264783 & 0.0452813 & 0.15792 & 0.0165566 \\
\hline 0.153937 & 0.46683 & 0.0459422 & 0.260609 & 0.0455169 & 0.157954 & 0.0214131 \\
\hline 0.147496 & 0.457897 & 0.0440893 & 0.25744 & 0.0481641 & 0.155008 & 0.0190706 \\
\hline 0.145908 & 0.442727 & 0.0399623 & 0.254546 & 0.0464386 & 0.155099 & 0.0166795 \\
\hline 0.164254 & 0.439813 & 0.0444369 & 0.252066 & 0.0465109 & 0.154524 & 0.0178563 \\
\hline 0.176856 & 0.452786 & 0.0434794 & 0.249115 & 0.0459794 & 0.157444 & 0.0157116 \\
\hline 0.17255 & 0.476643 & 0.0449971 & 0.251344 & 0.0486539 & 0.160992 & 0.0130008 \\
\hline 0.18349 & 0.511202 & 0.0545458 & 0.259534 & 0.0484861 & 0.163179 & 0.015409 \\
\hline 0.202083 & 0.557362 & 0.061634 & 0.26881 & 0.050898 & 0.174221 & 0.0177616 \\
\hline 0.263991 & 0.648317 & 0.0732325 & 0.279578 & 0.0525047 & 0.19107 & 0.0188579 \\
\hline 0.345202 & 0.765752 & 0.0776672 & 0.302499 & 0.0521145 & 0.205246 & 0.0223158 \\
\hline 0.435045 & 0.900999 & 0.0979088 & 0.335623 & 0.0578388 & 0.225712 & 0.0246639 \\
\hline 0.610406 & 1.07931 & 0.128565 & 0.37266 & 0.0666694 & 0.262959 & 0.0312059 \\
\hline 0.859032 & 1.29066 & 0.164403 & 0.409914 & 0.0785477 & 0.306288 & 0.0339434 \\
\hline 1.16661 & 1.54737 & 0.198104 & 0.454475 & 0.0885999 & 0.35881 & 0.0408006 \\
\hline 1.50178 & 1.79685 & 0.252396 & 0.512448 & 0.0979466 & 0.423893 & 0.0511654 \\
\hline 1.89809 & 2.0345 & 0.293679 & 0.573939 & 0.109909 & 0.487563 & 0.0609923 \\
\hline 2.36437 & 2.28126 & 0.334839 & 0.63857 & 0.126426 & 0.548578 & 0.0711326 \\
\hline 2.77441 & 2.45143 & 0.351943 & 0.69737 & 0.138002 & 0.612241 & 0.0782725 \\
\hline 3.15346 & 2.57729 & 0.385454 & 0.739495 & 0.144443 & 0.67392 & 0.0726579 \\
\hline 3.46552 & 2.60156 & 0.387041 & 0.775923 & 0.150823 & 0.713847 & 0.0828939 \\
\hline 3.63319 & 2.57066 & 0.371819 & 0.79309 & 0.152345 & 0.734847 & 0.0924278 \\
\hline 3.65226 & 2.47152 & 0.355611 & 0.792396 & 0.149758 & 0.737372 & 0.0841013 \\
\hline 3.47961 & 2.32149 & 0.303208 & 0.779075 & 0.147059 & 0.722181 & 0.0835424 \\
\hline 3.18836 & 2.18482 & 0.259645 & 0.752909 & 0.139801 & 0.68891 & 0.0888289 \\
\hline 2.87428 & 2.06505 & 0.236225 & 0.720224 & 0.13796 & 0.64479 & 0.0874173 \\
\hline 2.55533 & 1.97998 & 0.194747 & 0.693174 & 0.139398 & 0.608436 & 0.078461 \\
\hline 2.20692 & 1.96384 & 0.175529 & 0.679532 & 0.133124 & 0.584037 & 0.0773579 \\
\hline 1.83612 & 1.99468 & 0.164553 & 0.666186 & 0.128026 & 0.56717 & 0.0813974 \\
\hline 1.59583 & 2.07006 & 0.174311 & 0.671707 & 0.132292 & 0.56766 & 0.0839056 \\
\hline 1.4796 & 2.17723 & 0.194131 & 0.693818 & 0.135742 & 0.584783 & 0.0934254 \\
\hline 1.44991 & 2.28556 & 0.195554 & 0.726679 & 0.146817 & 0.606949 & 0.104334 \\
\hline 1.56628 & 2.36676 & 0.215895 & 0.767673 & 0.159611 & 0.6368 & 0.112589 \\
\hline 1.68866 & 2.40594 & 0.209415 & 0.791789 & 0.162727 & 0.670722 & 0.124168 \\
\hline 1.73552 & 2.41933 & 0.208862 & 0.818673 & 0.171734 & 0.690981 & 0.126026 \\
\hline 1.81636 & 2.36217 & 0.211076 & 0.839346 & 0.179995 & 0.693454 & 0.126251 \\
\hline 1.8523 & 2.24518 & 0.203679 & 0.832831 & 0.174747 & 0.686935 & 0.128935 \\
\hline 1.8061 & 2.10134 & 0.186873 & 0.816741 & 0.170884 & 0.669041 & 0.121772 \\
\hline
\end{tabular}




\begin{tabular}{|c|c|c|c|c|c|c|}
\hline 1.70688 & 1.93437 & 0.161422 & 0.788875 & 0.167475 & 0.63417 & 0.112344 \\
\hline 1.55182 & 1.78388 & 0.140806 & 0.744311 & 0.152752 & 0.596299 & 0.103505 \\
\hline 1.37442 & 1.63516 & 0.136441 & 0.696915 & 0.142706 & 0.552556 & 0.087516 \\
\hline 1.21717 & 1.51364 & 0.117082 & 0.653203 & 0.130915 & 0.504783 & 0.0736265 \\
\hline 1.0319 & 1.41792 & 0.130858 & 0.606419 & 0.117693 & 0.4587 & 0.0662973 \\
\hline 0.871395 & 1.35424 & 0.154494 & 0.571357 & 0.111086 & 0.417985 & 0.0577794 \\
\hline 0.738234 & 1.32246 & 0.153872 & 0.539422 & 0.0964943 & 0.389124 & 0.0499428 \\
\hline 0.606248 & 1.29738 & 0.176582 & 0.512652 & 0.0904162 & 0.360146 & 0.0419667 \\
\hline 0.520106 & 1.30093 & 0.205855 & 0.49515 & 0.0863253 & 0.336816 & 0.0367855 \\
\hline 0.432181 & 1.29498 & 0.212551 & 0.478322 & 0.0790497 & 0.318 & 0.0313562 \\
\hline 0.330893 & 1.28183 & 0.226583 & 0.466585 & 0.0802231 & 0.302196 & 0.0281696 \\
\hline 0.294268 & 1.24224 & 0.236544 & 0.452354 & 0.0802325 & 0.285984 & 0.0275213 \\
\hline 0.235627 & 1.20135 & 0.23554 & 0.4412 & 0.0760733 & 0.267445 & 0.0318281 \\
\hline 0.216414 & 1.1731 & 0.220129 & 0.431418 & 0.0755095 & 0.255995 & 0.0331037 \\
\hline 0.227346 & 1.11667 & 0.211236 & 0.416802 & 0.0741999 & 0.244472 & 0.02852 \\
\hline 0.195806 & 1.0483 & 0.187765 & 0.402647 & 0.0708396 & 0.231759 & 0.0282533 \\
\hline 0.179996 & 0.980288 & 0.177256 & 0.387008 & 0.0743008 & 0.220505 & 0.0256265 \\
\hline 0.179312 & 0.918492 & 0.157258 & 0.374476 & 0.0730607 & 0.212796 & 0.0249635 \\
\hline 0.177999 & 0.863146 & 0.134505 & 0.363614 & 0.0696689 & 0.201451 & 0.0216684 \\
\hline 0.165103 & 0.808613 & 0.109214 & 0.357273 & 0.069115 & 0.191564 & 0.0226112 \\
\hline 0.161259 & 0.763783 & 0.0965207 & 0.351541 & 0.0707793 & 0.185493 & 0.0237966 \\
\hline 0.165491 & 0.724996 & 0.085409 & 0.346203 & 0.0713323 & 0.184153 & 0.0229235 \\
\hline 0.160775 & 0.696142 & 0.073047 & 0.33978 & 0.0681469 & 0.186644 & 0.022013 \\
\hline 0.155469 & 0.688602 & 0.057198 & 0.335277 & 0.0656545 & 0.183802 & 0.0239503 \\
\hline 0.15816 & 0.67567 & 0.0657209 & 0.344636 & 0.0734224 & 0.182462 & 0.0222661 \\
\hline 0.167998 & 0.668129 & 0.0633623 & 0.348353 & 0.0751263 & 0.188272 & 0.0213031 \\
\hline 0.147215 & 0.678123 & 0.0638557 & 0.35004 & 0.072992 & 0.194529 & 0.0223557 \\
\hline 0.133963 & 0.695654 & 0.0665904 & 0.362858 & 0.0772024 & 0.204572 & 0.0221784 \\
\hline 0.159267 & 0.707233 & 0.0592568 & 0.37102 & 0.0766269 & 0.219833 & 0.0266239 \\
\hline 0.169077 & 0.728111 & 0.0620194 & 0.383261 & 0.0779693 & 0.240046 & 0.0305457 \\
\hline 0.184832 & 0.757625 & 0.0730086 & 0.398179 & 0.0797105 & 0.276417 & 0.0374056 \\
\hline 0.184231 & 0.808231 & 0.0911439 & 0.42905 & 0.0867582 & 0.334225 & 0.0454802 \\
\hline 0.187061 & 0.888503 & 0.104915 & 0.472992 & 0.104659 & 0.414789 & 0.0582545 \\
\hline 0.203635 & 0.989288 & 0.147048 & 0.535061 & 0.126221 & 0.533476 & 0.0754359 \\
\hline 0.232789 & 1.12715 & 0.197747 & 0.622159 & 0.158303 & 0.707991 & 0.103437 \\
\hline 0.273296 & 1.30614 & 0.250731 & 0.738947 & 0.206158 & 0.952198 & 0.144358 \\
\hline 0.333704 & 1.54525 & 0.327318 & 0.892795 & 0.271183 & 1.26327 & 0.189557 \\
\hline 0.421772 & 1.80915 & 0.428082 & 1.10512 & 0.366338 & 1.64555 & 0.251925 \\
\hline 0.493954 & 2.07187 & 0.525099 & 1.34861 & 0.4697 & 2.09529 & 0.323979 \\
\hline 0.615027 & 2.34832 & 0.6212 & 1.60521 & 0.581613 & 2.60486 & 0.400865 \\
\hline 0.726601 & 2.60798 & 0.708442 & 1.8853 & 0.693239 & 3.14987 & 0.488037 \\
\hline 0.858675 & 2.80599 & 0.783777 & 2.17807 & 0.820622 & 3.67608 & 0.576976 \\
\hline 0.98145 & 2.96101 & 0.834631 & 2.42995 & 0.921284 & 4.11252 & 0.63827 \\
\hline 1.04404 & 3.01803 & 0.867073 & 2.61347 & 1.01394 & 4.43672 & 0.697568 \\
\hline 1.05571 & 2.99844 & 0.868046 & 2.73104 & 1.07224 & 4.64042 & 0.727157 \\
\hline 1.05319 & 2.87266 & 0.813532 & 2.77487 & 1.09063 & 4.66712 & 0.726697 \\
\hline 1.0447 & 2.66509 & 0.730663 & 2.73677 & 1.065 & 4.52328 & 0.714358 \\
\hline
\end{tabular}




\begin{tabular}{|c|c|c|c|c|c|c|}
\hline 1.02438 & 2.41506 & 0.641119 & 2.59692 & 0.998794 & 4.22464 & 0.655836 \\
\hline 0.960508 & 2.13711 & 0.538906 & 2.38771 & 0.914769 & 3.81116 & 0.598718 \\
\hline 0.834119 & 1.86687 & 0.450141 & 2.14531 & 0.811562 & 3.30921 & 0.522871 \\
\hline 0.725458 & 1.61422 & 0.35758 & 1.86289 & 0.679517 & 2.77818 & 0.43674 \\
\hline 0.664389 & 1.39522 & 0.281396 & 1.57529 & 0.548794 & 2.25347 & 0.343531 \\
\hline 0.53966 & 1.22077 & 0.190838 & 1.3253 & 0.437114 & 1.77442 & 0.279358 \\
\hline 0.404033 & 1.091 & 0.14244 & 1.10463 & 0.338937 & 1.35896 & 0.215263 \\
\hline 0.322197 & 1.02984 & 0.130927 & 0.916149 & 0.262771 & 1.02998 & 0.154315 \\
\hline 0.26498 & 0.999771 & 0.104177 & 0.777405 & 0.203518 & 0.766878 & 0.117494 \\
\hline 0.200738 & 1.02287 & 0.113112 & 0.680333 & 0.161343 & 0.569959 & 0.0865421 \\
\hline 0.191812 & 1.0728 & 0.10621 & 0.622553 & 0.14084 & 0.440766 & 0.0661403 \\
\hline 0.196447 & 1.16356 & 0.123774 & 0.585168 & 0.123361 & 0.353883 & 0.0472697 \\
\hline 0.185007 & 1.32398 & 0.152098 & 0.577926 & 0.117987 & 0.302526 & 0.0379307 \\
\hline 0.158504 & 1.52816 & 0.164754 & 0.600636 & 0.125148 & 0.285166 & 0.0366403 \\
\hline 0.16518 & 1.83986 & 0.200462 & 0.641628 & 0.14006 & 0.285903 & 0.0339081 \\
\hline 0.165343 & 2.28161 & 0.241063 & 0.711369 & 0.150221 & 0.304102 & 0.032995 \\
\hline 0.176781 & 2.97141 & 0.312235 & 0.815179 & 0.171888 & 0.343121 & 0.0412645 \\
\hline 0.166894 & 4.05505 & 0.440543 & 0.966786 & 0.203758 & 0.394629 & 0.0522589 \\
\hline 0.171295 & 5.74313 & 0.628074 & 1.19566 & 0.251982 & 0.46533 & 0.0567554 \\
\hline 0.204379 & 8.38141 & 0.917134 & 1.54095 & 0.334885 & 0.55987 & 0.0655621 \\
\hline 0.233865 & 12.4056 & 1.33707 & 2.05498 & 0.459993 & 0.678172 & 0.0839173 \\
\hline 0.268688 & 18.4923 & 1.97796 & 2.83072 & 0.620686 & 0.804052 & 0.103555 \\
\hline 0.276409 & 27.298 & 2.95891 & 4.02139 & 0.881526 & 0.972153 & 0.126149 \\
\hline 0.306787 & 39.7666 & 4.34894 & 5.73171 & 1.26589 & 1.19256 & 0.155451 \\
\hline 0.387316 & 56.6119 & 6.22428 & 8.16561 & 1.81362 & 1.46168 & 0.188494 \\
\hline 0.475231 & 78.3704 & 8.6131 & 11.5597 & 2.59571 & 1.81243 & 0.23374 \\
\hline 0.575863 & 105.103 & 11.6619 & 16.0411 & 3.62427 & 2.23488 & 0.281961 \\
\hline 0.706292 & 136.663 & 15.0444 & 21.7353 & 4.9341 & 2.73948 & 0.354119 \\
\hline 0.873879 & 171.932 & 18.8688 & 28.7174 & 6.52448 & 3.34365 & 0.43364 \\
\hline 1.06766 & 208.599 & 22.9286 & 36.7636 & 8.36496 & 4.02534 & 0.514927 \\
\hline 1.28853 & 244.311 & 26.762 & 45.6023 & 10.41 & 4.75021 & 0.61474 \\
\hline 1.48078 & 276.027 & 30.1598 & 54.7337 & 12.4443 & 5.4706 & 0.708985 \\
\hline 1.7154 & 300.771 & 33.0485 & 63.4784 & 14.4096 & 6.17501 & 0.794177 \\
\hline 1.91459 & 315.942 & 34.5749 & 71.1436 & 16.1931 & 6.76637 & 0.889364 \\
\hline 2.121 & 319.66 & 34.904 & 76.9843 & 17.549 & 7.16651 & 0.958337 \\
\hline 2.21995 & 311.934 & 34.1207 & 80.3603 & 18.2882 & 7.36714 & 0.981657 \\
\hline 2.1869 & 293.258 & 31.9574 & 80.8958 & 18.4044 & 7.31548 & 0.968255 \\
\hline 2.13807 & 265.994 & 29.1223 & 78.6998 & 17.9533 & 7.00791 & 0.933576 \\
\hline 1.98354 & 232.175 & 25.4698 & 73.871 & 16.7763 & 6.48629 & 0.866342 \\
\hline 1.81087 & 195.567 & 21.5322 & 66.8446 & 15.1272 & 5.83097 & 0.764528 \\
\hline 1.63395 & 158.707 & 17.3056 & 58.3529 & 13.2221 & 5.07112 & 0.660088 \\
\hline 1.38569 & 124.291 & 13.6039 & 49.1925 & 11.1685 & 4.26023 & 0.555368 \\
\hline 1.14481 & 94.0059 & 10.2653 & 40.0522 & 9.11193 & 3.46254 & 0.44974 \\
\hline 0.939361 & 68.5629 & 7.55858 & 31.5075 & 7.16621 & 2.72086 & 0.35341 \\
\hline 0.7321 & 48.3875 & 5.21479 & 23.9645 & 5.44275 & 2.07515 & 0.27 \\
\hline 0.585496 & 33.1661 & 3.61575 & 17.6288 & 3.98897 & 1.53274 & 0.197927 \\
\hline 0.476776 & 22.1363 & 2.4503 & 12.5612 & 2.85286 & 1.12479 & 0.144973 \\
\hline
\end{tabular}




\begin{tabular}{|c|c|c|c|c|c|c|}
\hline 0.36452 & 14.5889 & 1.59693 & 8.68895 & 1.97818 & 0.815594 & 0.108366 \\
\hline 0.295287 & 9.6333 & 1.05062 & 5.8865 & 1.33105 & 0.586025 & 0.0806417 \\
\hline 0.251891 & 6.59916 & 0.701319 & 3.93434 & 0.895154 & 0.436818 & 0.0551451 \\
\hline 0.233237 & 4.90536 & 0.518745 & 2.65832 & 0.606203 & 0.339204 & 0.0381174 \\
\hline 0.202658 & 4.15864 & 0.459353 & 1.86528 & 0.421588 & 0.283039 & 0.0331798 \\
\hline 0.195601 & 4.12452 & 0.436774 & 1.42217 & 0.31767 & 0.248812 & 0.0323331 \\
\hline 0.195085 & 4.66599 & 0.507012 & 1.2266 & 0.274567 & 0.235329 & 0.030931 \\
\hline 0.179603 & 5.76921 & 0.640019 & 1.21364 & 0.270153 & 0.240601 & 0.0302998 \\
\hline 0.184354 & 7.4146 & 0.846357 & 1.37688 & 0.301387 & 0.257355 & 0.0273158 \\
\hline 0.222827 & 9.58861 & 1.06609 & 1.66927 & 0.36385 & 0.291364 & 0.0324125 \\
\hline 0.234451 & 12.3926 & 1.38606 & 2.09677 & 0.443474 & 0.332689 & 0.0378496 \\
\hline 0.222675 & 15.8511 & 1.76991 & 2.68469 & 0.572698 & 0.387243 & 0.045733 \\
\hline 0.225669 & 19.8973 & 2.1997 & 3.43709 & 0.743404 & 0.460696 & 0.0552112 \\
\hline 0.23536 & 24.4516 & 2.76818 & 4.35069 & 0.931736 & 0.544223 & 0.0668222 \\
\hline 0.257291 & 29.0733 & 3.26394 & 5.39723 & 1.15401 & 0.650716 & 0.0824423 \\
\hline 0.273345 & 33.6665 & 3.76085 & 6.56132 & 1.4188 & 0.763744 & 0.0952968 \\
\hline 0.314878 & 37.8128 & 4.22402 & 7.77408 & 1.68735 & 0.86667 & 0.11285 \\
\hline 0.350744 & 41.1351 & 4.61502 & 8.93062 & 1.92661 & 0.964188 & 0.123027 \\
\hline 0.380592 & 43.2526 & 4.77553 & 9.97394 & 2.15403 & 1.05924 & 0.127202 \\
\hline 0.414932 & 43.9461 & 4.91366 & 10.7978 & 2.33133 & 1.1283 & 0.136751 \\
\hline 0.408291 & 43.3091 & 4.86113 & 11.3379 & 2.41757 & 1.16492 & 0.141304 \\
\hline 0.402337 & 41.1487 & 4.51635 & 11.5492 & 2.46503 & 1.18096 & 0.143656 \\
\hline 0.422955 & 37.8252 & 4.18775 & 11.3711 & 2.45167 & 1.15592 & 0.143971 \\
\hline 0.402062 & 33.6124 & 3.73391 & 10.8373 & 2.33584 & 1.09509 & 0.13961 \\
\hline 0.378027 & 28.9356 & 3.17754 & 9.98093 & 2.14808 & 1.01996 & 0.121146 \\
\hline 0.391215 & 23.9887 & 2.67446 & 8.88796 & 1.90285 & 0.916281 & 0.108973 \\
\hline 0.35582 & 19.2363 & 2.12334 & 7.68011 & 1.63662 & 0.8016 & 0.106865 \\
\hline 0.302892 & 14.9006 & 1.62061 & 6.42992 & 1.38391 & 0.694175 & 0.0911108 \\
\hline 0.269537 & 11.2031 & 1.24825 & 5.20791 & 1.11443 & 0.585694 & 0.0683918 \\
\hline 0.25632 & 8.16731 & 0.897816 & 4.08978 & 0.876545 & 0.494386 & 0.055018 \\
\hline 0.234812 & 5.75848 & 0.622969 & 3.11307 & 0.66669 & 0.412923 & 0.0468616 \\
\hline 0.215638 & 3.96282 & 0.425562 & 2.29899 & 0.490785 & 0.338997 & 0.0406233 \\
\hline 0.20956 & 2.65207 & 0.28083 & 1.64461 & 0.339924 & 0.285035 & 0.03493 \\
\hline 0.193767 & 1.75287 & 0.176816 & 1.15582 & 0.236008 & 0.246511 & 0.0301776 \\
\hline 0.18463 & 1.15255 & 0.11926 & 0.804753 & 0.167934 & 0.216011 & 0.0277268 \\
\hline 0.196863 & 0.75222 & 0.0616321 & 0.551646 & 0.114126 & 0.192769 & 0.0266002 \\
\hline 0.169753 & 0.506909 & 0.0481993 & 0.382109 & 0.0792216 & 0.180502 & 0.0253503 \\
\hline 0.167208 & 0.362724 & 0.0337335 & 0.269737 & 0.0553222 & 0.173193 & 0.0240138 \\
\hline 0.183412 & 0.281363 & 0.0238308 & 0.197681 & 0.0390885 & 0.167262 & 0.0221116 \\
\hline 0.18248 & 0.234124 & 0.0166445 & 0.15415 & 0.0289387 & 0.16438 & 0.0211213 \\
\hline 0.182121 & 0.20701 & 0.0192809 & 0.129995 & 0.0261451 & 0.166708 & 0.023508 \\
\hline 0.185889 & 0.197873 & 0.0172684 & 0.113788 & 0.0228414 & 0.170361 & 0.023664 \\
\hline 0.211282 & 0.191767 & 0.0154614 & 0.103413 & 0.0202582 & 0.169486 & 0.0227189 \\
\hline 0.200126 & 0.186963 & 0.0156058 & 0.0996979 & 0.0218506 & 0.168319 & 0.0253497 \\
\hline 0.199387 & 0.190523 & 0.0201605 & 0.0997846 & 0.0224863 & 0.16863 & 0.0231887 \\
\hline 0.209777 & 0.188627 & 0.0161383 & 0.0988547 & 0.0219518 & 0.171468 & 0.0245505 \\
\hline 0.194355 & 0.190322 & 0.0141799 & 0.0994506 & 0.0228482 & 0.173218 & 0.0282257 \\
\hline
\end{tabular}




\begin{tabular}{|c|c|c|c|c|c|c|}
\hline 0.198765 & 0.192102 & 0.0171661 & 0.0985683 & 0.0242527 & 0.177917 & 0.0261348 \\
\hline 0.204147 & 0.199308 & 0.0200549 & 0.0970648 & 0.0234554 & 0.183889 & 0.028003 \\
\hline .195397 & 0.199326 & 0.0204313 & 0.0992521 & 0.0214846 & 0.188298 & 0.0297735 \\
\hline .193201 & 0.205697 & 0.0238199 & 0.1025 & 0.0193513 & 0.194302 & 0.0296095 \\
\hline 209717 & 0.208873 & 0.0252635 & 0.103163 & 0.02199 & 0.203488 & 0.0335941 \\
\hline 0.206325 & 0.212258 & 0.0230074 & 0.103118 & 0.0237582 & 0.216905 & 0.0395995 \\
\hline 0.218796 & 0.218726 & 0.0204418 & 0.107149 & 0.0239229 & 0.237668 & 0.045287 \\
\hline 0.218917 & 0.229298 & 0.0258348 & 0.112688 & 0.0242674 & 0.271404 & 0.0595414 \\
\hline 0.234838 & 0.242397 & 0.0297456 & 0.118982 & 0.0269296 & 0.318512 & 0.0814513 \\
\hline 0.259214 & 0.261053 & 0.0334557 & 0.131209 & 0.0304205 & 0.387853 & 0.113254 \\
\hline 0.261253 & 0.284598 & 0.0426304 & 0.143373 & 0.0326001 & 0.485415 & 0.157354 \\
\hline 0.252833 & 0.315305 & 0.0495054 & 0.158145 & 0.0356143 & 0.620356 & 0.217699 \\
\hline 0.260103 & 0.353489 & 0.070448 & 0.183308 & 0.0423039 & 0.804346 & 0.300584 \\
\hline 0.290524 & 0.400307 & 0.0768463 & 0.209256 & 0.0494222 & 1.0354 & 0.413638 \\
\hline 0.332115 & 0.445493 & 0.0915985 & 0.242893 & 0.0599416 & 1.32885 & 0.544966 \\
\hline 0.374999 & 0.497081 & 0.115894 & 0.29083 & 0.0735528 & 1.68536 & 0.711647 \\
\hline 0.4306 & 0.553543 & 0.138522 & 0.334566 & 0.085441 & 2.09264 & 0.908915 \\
\hline .486546 & 0.607212 & 0.160115 & 0.383068 & 0.100335 & 2.53779 & 1.11493 \\
\hline 0.585383 & 0.654231 & 0.175781 & 0.436075 & 0.11 & 986 & 1.35028 \\
\hline 0.651267 & 0.700043 & 0.198575 & 0.489876 & 0.131949 & 3.45608 & 1.58117 \\
\hline 0.674209 & 0.733227 & 0.220587 & 0.537192 & 0.15018 & 3.85858 & 1.78246 \\
\hline 0.710393 & 0.741809 & 0.223575 & 0.5788 & 0.15 & 671 & 1.96204 \\
\hline 0.785517 & 0.735195 & 0.21643 & 0.602719 & 0.164185 & 4.43583 & 2.05594 \\
\hline 0.832314 & 0.721508 & 0.208029 & 0.615999 & 0.163966 & 4.5412 & 2.1197 \\
\hline 0.797494 & 0.691028 & 0.210182 & 0.617558 & 0.163707 & 4.50802 & 2.12386 \\
\hline 0.801526 & 0.650417 & 0.188036 & 0.600692 & 0.162793 & 4.33348 & 2.02995 \\
\hline 0.767366 & 0.59752 & 0.151934 & 0.568573 & 0.155573 & 4.05291 & 1.89721 \\
\hline 0.712771 & 0.548644 & 0.14319 & 0.531557 & 0.144132 & 3.70079 & 1.72652 \\
\hline 0.684434 & 0.489929 & 0.120367 & 0.486213 & 0.132595 & 3.25931 & 1.52366 \\
\hline 0.601243 & 0.435851 & 0.0950606 & 0.433719 & 0.111881 & 2.79441 & 1.2984 \\
\hline 0.559631 & 0.392 & 0.0759368 & 0.379602 & 0.0941799 & 2.33878 & 1.06292 \\
\hline 0.511091 & 0.358017 & 0.0599978 & 0.327227 & 0.0825611 & 1.9031 & 0.836695 \\
\hline 0.491934 & 0.327066 & 0.050012 & 0.278693 & 0.0688717 & 1.52923 & 0.663687 \\
\hline 0.484041 & 0.300183 & 0.044633 & 0.241794 & 0.0561971 & 1.20018 & 0.486578 \\
\hline 0.494985 & 0.287084 & 0.0325455 & 0.211209 & 0.0436469 & 0.92906 & 0.363366 \\
\hline 0.488875 & 0.277279 & 0.0230789 & 0.185504 & 0.0367456 & 0.719742 & 0.260875 \\
\hline 0.434322 & 0.273386 & 0.0256938 & 0.168382 & 0.0363774 & 0.574505 & 0.180483 \\
\hline 0.433709 & 0.267594 & 0.0245257 & 0.152881 & 0.0319647 & 0.46586 & 0.132756 \\
\hline 0.462747 & 0.26476 & 0.0251188 & 0.146346 & 0.0301913 & 0.394361 & 0.0901936 \\
\hline 0.454565 & 0.266132 & 0.0237251 & 0.140275 & 0.0281151 & 0.347223 & 0.0711231 \\
\hline 0.459256 & 0.264302 & 0.0227372 & 0.134861 & 0.0268965 & 0.308523 & 0.0570991 \\
\hline 0.469886 & 0.265198 & 0.0203939 & 0.134541 & 0.0289634 & 0.286747 & 0.0523803 \\
\hline 0.503883 & 0.26629 & 0.0192212 & 0.130591 & 0.0286315 & 0.276006 & 0.0436421 \\
\hline 0.467901 & 0.270475 & 0.0209615 & 0.135186 & 0.0276782 & 0.269635 & 0.0372518 \\
\hline 0.444926 & 0.272652 & 0.0223048 & 0.135444 & 0.0272935 & 0.266712 & 0.0328994 \\
\hline 0.480923 & 0.280089 & 0.0214074 & 0.133073 & 0.0294522 & 0.269315 & 0.0322772 \\
\hline 0.512567 & 0.281561 & 0.0202344 & 0.136929 & 0.0282287 & 0.276721 & 0.034798 \\
\hline
\end{tabular}




\begin{tabular}{|c|c|c|c|c|c|c|}
\hline 0.479486 & 0.285391 & 0.024223 & 0.141224 & 0.0306677 & 0.281628 & 0.0364699 \\
\hline 0.480046 & 0.292868 & 0.0222008 & 0.142641 & 0.0302105 & 0.296273 & 0.0425639 \\
\hline 0.448061 & 0.305886 & 0.0241197 & 0.147336 & 0.03441 & 0.317892 & 0.043517 \\
\hline .435445 & 0.310749 & 0.02553 & 0.153447 & 0.0357153 & 0.334809 & 0.045883 \\
\hline 0.440175 & 0.320321 & 0.0295099 & 0.163906 & 0.0352219 & 0.355043 & 0.0526614 \\
\hline 0.426905 & 0.331099 & 0.0237998 & 0.168019 & 0.0371316 & 0.384026 & 0.0593045 \\
\hline 0.424421 & 0.342707 & 0.0290757 & 0.174767 & 0.0410644 & 0.424184 & 0.0644552 \\
\hline 0.438124 & 0.361073 & 0.0314123 & 0.181982 & 0.0403164 & 0.467323 & 0.0768737 \\
\hline 0.411337 & 0.367519 & 0.0350936 & 0.193686 & 0.0476184 & 0.503158 & 0.0980425 \\
\hline 0.400695 & 0.381707 & 0.0388362 & 0.200576 & 0.0453254 & 0.553966 & 0.10603 \\
\hline 0.404137 & 0.399431 & 0.0391835 & 0.211658 & 0.051959 & 0.610267 & 0.128802 \\
\hline 0.379301 & 0.412799 & 0.048803 & 0.218419 & 0.0543121 & 0.679604 & 0.157047 \\
\hline 0.380649 & 0.429057 & 0.0531026 & 0.23048 & 0.0556503 & 0.740739 & 0.176684 \\
\hline 0.37955 & 0.448407 & 0.0580534 & 0.238617 & 0.0569567 & 0.808468 & 0.213261 \\
\hline 0.379863 & 0.460932 & 0.0627101 & 0.248458 & 0.0553872 & 0.888143 & 0.249111 \\
\hline 0.392551 & 0.473176 & 0.0689127 & 0.256474 & 0.0540786 & 0.959492 & 0.272503 \\
\hline 0.367919 & 0.483464 & 0.06043 & 0.268824 & 0.0535183 & 1.02434 & 0.303942 \\
\hline 0.394116 & 0.489441 & 0.0649455 & 0.269968 & 0.0531424 & 1.05911 & 0.317291 \\
\hline 0.384328 & 0.488788 & 0.067 & 0.27 & 0.0584762 & .08693 & 0.320969 \\
\hline 0.413025 & 0.492238 & 0.0731597 & 0.279351 & 0.0577313 & 1.09184 & 0.328278 \\
\hline 0.374756 & 0.490807 & 0.0672521 & 0.278822 & 0.0585405 & 1.07889 & 0.321111 \\
\hline 0.363063 & 0.483555 & 0.07 & 683 & 0.0533982 & 578 & 1555 \\
\hline 0.336077 & 0.475834 & 0.0714451 & 0.276643 & 0.057282 & 0.992051 & 0.284055 \\
\hline 0.358512 & 0.463601 & 0.0687355 & 0.269569 & 0.0539627 & 0.935675 & 0.264271 \\
\hline 0.353552 & 0.452255 & 0.0597076 & 0.259222 & 0.0483981 & 0.861229 & 0.231908 \\
\hline 0.362549 & 0.441546 & 0.0619708 & 0.250953 & 0.0436082 & 0.787663 & 0.208747 \\
\hline 0.355375 & 0.427858 & 0.0573399 & 0.23777 & 0.0462286 & 0.710921 & 0.173381 \\
\hline 0.306924 & 0.417899 & 0.059459 & 0.231577 & 0.0424421 & 0.644127 & 0.150495 \\
\hline 0.291642 & 0.411414 & 0.0600052 & 0.223293 & 0.0349385 & 0.586939 & 0.120758 \\
\hline 0.307576 & 0.399316 & 0.0543517 & 0.21192 & 0.0361263 & 0.532873 & 0.0980453 \\
\hline 0.310326 & 0.392742 & 0.0513102 & 0.205719 & 0.0373933 & 0.486785 & 0.0966524 \\
\hline 0.295856 & 0.385896 & 0.0493079 & 0.202022 & 0.0336453 & 0.451033 & 0.0837427 \\
\hline 0.291507 & 0.38234 & 0.0484484 & 0.198845 & 0.0340082 & 0.426003 & 0.0807738 \\
\hline 0.281027 & 0.376008 & 0.0526076 & 0.196344 & 0.0307973 & 0.40632 & 0.0821446 \\
\hline 0.275311 & 0.377478 & 0.0499922 & 0.195863 & 0.0325632 & 0.395132 & 0.0824815 \\
\hline 0.271227 & 0.376876 & 0.0471855 & 0.194074 & 0.0312663 & 0.382976 & 0.0781067 \\
\hline 0.275428 & 0.367235 & 0.0454157 & 0.190635 & 0.0306002 & 0.378464 & 0.0737101 \\
\hline 0.282193 & 0.364271 & 0.0456539 & 0.19569 & 0.0314604 & 0.380566 & 0.0756953 \\
\hline 0.274227 & 0.3582 & 0.0446466 & 0.194642 & 0.0307029 & 0.379737 & 0.0687589 \\
\hline 0.29159 & 0.35379 & 0.0419382 & 0.193704 & 0.0344392 & 0.377141 & 0.0701277 \\
\hline 0.269596 & 0.35258 & 0.0390377 & 0.200869 & 0.0369015 & 0.378963 & 0.0772261 \\
\hline 0.286023 & 0.347298 & 0.0441226 & 0.194122 & 0.0340023 & 0.374017 & 0.0741249 \\
\hline 0.283282 & 0.34595 & 0.0359601 & 0.198759 & 0.0336537 & 0.372275 & 0.0729559 \\
\hline 0.290009 & 0.34843 & 0.0333665 & 0.19851 & 0.0332483 & 0.377857 & 0.0700233 \\
\hline 0.317937 & 0.34726 & 0.0364751 & 0.192075 & 0.0364655 & 0.371953 & 0.0645912 \\
\hline 0.285453 & 0.341279 & 0.0360724 & 0.191477 & 0.0343068 & 0.362607 & 0.0690339 \\
\hline 0.28932 & 0.340011 & 0.0370476 & 0.195132 & 0.032419 & 0.367726 & 0.0596109 \\
\hline
\end{tabular}




\begin{tabular}{|c|c|c|c|c|c|c|}
\hline 0.283624 & 0.338374 & 0.0347176 & 0.194649 & 0.0315497 & 0.370765 & 0.0578565 \\
\hline 0.293823 & 0.339693 & 0.0385408 & 0.19054 & 0.0328529 & 0.366004 & 0.056336 \\
\hline 0.271934 & 0.338343 & 0.0312017 & 0.189129 & 0.0312662 & 0.364576 & 0.0541807 \\
\hline 0.29637 & 0.343967 & 0.0281184 & 0.189004 & 0.0365332 & 0.362606 & 0.0486983 \\
\hline 0.318947 & 0.349372 & 0.0315589 & 0.191492 & 0.0330416 & 0.361262 & 0.0508518 \\
\hline 0.296444 & 0.365015 & 0.0387823 & 0.195344 & 0.0294612 & 0.365807 & 0.0456158 \\
\hline 0.3435 & 0.375971 & 0.0431871 & 0.201712 & 0.0330712 & 0.365161 & 0.0409401 \\
\hline 0.329053 & 0.39779 & 0.0512116 & 0.207746 & 0.035293 & 0.379882 & 0.0469186 \\
\hline 0.397206 & 0.42352 & 0.0608825 & 0.217739 & 0.0442171 & 0.39297 & 0.0538143 \\
\hline 0.448652 & 0.453331 & 0.0727065 & 0.225836 & 0.0421401 & 0.422651 & 0.0514414 \\
\hline 0.428543 & 0.488401 & 0.0968175 & 0.246945 & 0.0519135 & 0.437036 & 0.0565618 \\
\hline 0.534778 & 0.53496 & 0.122317 & 0.267123 & 0.0618256 & 0.46998 & 0.0637013 \\
\hline 0.591345 & 0.571774 & 0.141024 & 0.292266 & 0.07088 & 0.510222 & 0.074709 \\
\hline 0.703508 & 0.608121 & 0.163888 & 0.322651 & 0.101284 & 0.54978 & 0.0862812 \\
\hline 0.794583 & 0.644719 & 0.185703 & 0.348094 & 0.110957 & 0.595116 & 0.0997288 \\
\hline 0.893829 & 0.673271 & 0.210287 & 0.384455 & 0.131852 & 0.637067 & 0.118732 \\
\hline 1.04419 & 0.692244 & 0.219829 & 0.423048 & 0.151405 & 0.678052 & 0.129087 \\
\hline 1.1206 & 0.712531 & 0.219171 & 0.442555 & 0.169149 & 0.710746 & 0.133774 \\
\hline 1.18026 & 0.725947 & 0.212831 & 0.459251 & 0.173803 & 0.740785 & 0.147253 \\
\hline 1.2682 & 0.723694 & 0.218333 & 0.480028 & 0.184704 & 0.752311 & 0.156063 \\
\hline 1.28299 & 0.702966 & 0.203847 & 0.486495 & 0.184246 & 0.769001 & 0.156362 \\
\hline 1.3078 & 0.681779 & 0.188145 & 0.492925 & 0.189847 & 0.765853 & 0.140092 \\
\hline 1.3373 & 0.654712 & 0.173303 & 0.480469 & 0.188491 & 0.744358 & 0.153085 \\
\hline 1.21149 & 0.62065 & 0.142377 & 0.461392 & 0.17055 & 0.715924 & 0.135296 \\
\hline 1.12294 & 0.596443 & 0.124155 & 0.453711 & 0.16779 & 0.691281 & 0.128685 \\
\hline 1.06681 & 0.568649 & 0.110463 & 0.417285 & 0.138445 & 0.652741 & 0.121729 \\
\hline 0.907683 & 0.53244 & 0.0886932 & 0.394842 & 0.128029 & 0.599265 & 0.10161 \\
\hline 0.831469 & 0.506824 & 0.0768048 & 0.366112 & 0.117147 & 0.567747 & 0.0833955 \\
\hline 0.692919 & 0.487438 & 0.0611439 & 0.3403 & 0.100132 & 0.537114 & 0.0810465 \\
\hline 0.625329 & 0.465248 & 0.048636 & 0.312692 & 0.0796104 & 0.497884 & 0.0721645 \\
\hline 0.548307 & 0.4484 & 0.043142 & 0.293246 & 0.0706107 & 0.4696 & 0.0634993 \\
\hline 0.460787 & 0.442317 & 0.0391011 & 0.278959 & 0.0658632 & 0.451119 & 0.0549394 \\
\hline 0.470242 & 0.436441 & 0.0373051 & 0.261205 & 0.0584194 & 0.430741 & 0.0601912 \\
\hline 0.396925 & 0.435844 & 0.0366166 & 0.251172 & 0.0560811 & 0.419792 & 0.0548619 \\
\hline 0.380844 & 0.429451 & 0.0346282 & 0.244436 & 0.0510566 & 0.413373 & 0.047838 \\
\hline 0.393427 & 0.429707 & 0.0349235 & 0.243172 & 0.0575648 & 0.405737 & 0.0469284 \\
\hline 0.378897 & 0.432711 & 0.0346092 & 0.232356 & 0.0479726 & 0.401545 & 0.0513337 \\
\hline 0.414616 & 0.440352 & 0.0416407 & 0.234629 & 0.0518663 & 0.401063 & 0.0463017 \\
\hline 0.381466 & 0.445277 & 0.0368202 & 0.232933 & 0.0567834 & 0.399191 & 0.044605 \\
\hline 0.330583 & 0.45152 & 0.0468856 & 0.231184 & 0.0551096 & 0.397759 & 0.0457713 \\
\hline 0.32476 & 0.456506 & 0.0457967 & 0.233435 & 0.0500724 & 0.390367 & 0.0445316 \\
\hline 0.353219 & 0.464406 & 0.0477797 & 0.235431 & 0.0587181 & 0.404338 & 0.0466189 \\
\hline 0.403963 & 0.464667 & 0.0454788 & 0.234016 & 0.0541465 & 0.396389 & 0.0473597 \\
\hline 0.340748 & 0.469363 & 0.0489783 & 0.238847 & 0.0561782 & 0.402124 & 0.0568162 \\
\hline 0.390254 & 0.480917 & 0.0526268 & 0.23891 & 0.0577274 & 0.412607 & 0.0532457 \\
\hline 0.361019 & 0.491975 & 0.0477667 & 0.239514 & 0.0542661 & 0.415621 & 0.0602198 \\
\hline 0.366686 & 0.500803 & 0.0508172 & 0.241133 & 0.0577705 & 0.416954 & 0.0590726 \\
\hline
\end{tabular}




\begin{tabular}{|c|c|c|c|c|c|c|}
\hline 0.334409 & 0.512036 & 0.057327 & 0.245288 & 0.0584729 & 0.415861 & 0.0583236 \\
\hline 0.334572 & 0.52361 & 0.0586562 & 0.253826 & 0.0572301 & 0.433366 & 0.0761684 \\
\hline 0.354397 & 0.534575 & 0.0611116 & 0.251648 & 0.0564633 & 0.440863 & 0.0859735 \\
\hline .404465 & 0.552252 & 0.05731 & 0.265902 & 0.0541382 & 0.448147 & 0.092416 \\
\hline 0.353417 & 0.571522 & 0.0627667 & 0.265077 & 0.0561717 & 0.462679 & 0.113081 \\
\hline 0.378221 & 0.592655 & 0.0619286 & 0.277657 & 0.057807 & 0.483372 & 0.123212 \\
\hline 0.385356 & 0.618338 & 0.0630686 & 0.286193 & 0.0621336 & 0.492613 & 0.136886 \\
\hline 0.423764 & 0.648172 & 0.0600547 & 0.300416 & 0.0563486 & 0.523379 & 0.153678 \\
\hline 0.466223 & 0.6902 & 0.0672015 & 0.316725 & 0.0555505 & 0.554373 & 0.163932 \\
\hline 0.462513 & 0.735999 & 0.0793012 & 0.340048 & 0.0582307 & 0.586894 & 0.172219 \\
\hline 0.492188 & 0.797919 & 0.0749817 & 0.364728 & 0.0570977 & 0.630933 & 0.176971 \\
\hline 0.539728 & 0.854376 & 0.083675 & 0.39714 & 0.0698721 & 0.684925 & 0.189104 \\
\hline 0.593269 & 0.921618 & 0.0919713 & 0.442137 & 0.074501 & 0.750045 & 0.177215 \\
\hline 0.681703 & 0.9799 & 0.102314 & 0.494166 & 0.0845099 & 0.822689 & 0.186468 \\
\hline 0.711132 & 1.04959 & 0.100289 & 0.547035 & 0.0953475 & 0.915648 & 0.185285 \\
\hline 0.806525 & 1.11515 & 0.111439 & 0.601289 & 0.116908 & 0.98759 & 0.196725 \\
\hline 0.935542 & 1.1589 & 0.121583 & 0.66275 & 0.13189 & 1.06946 & 0.19213 \\
\hline 1.03953 & 1.20217 & 0.126275 & 0.722212 & 0.13706 & 1.15412 & 0.208856 \\
\hline 1.10596 & 1.22799 & 0.115905 & 0.771166 & 0.1417 & 1.21542 & 0.185757 \\
\hline 1.19798 & 1.24342 & 0.125696 & 0.82157 & 0.156401 & 1.27468 & 0.181972 \\
\hline 1.25335 & 1.23796 & 0.131843 & 0.860598 & 0.170507 & 1.32341 & 0.194486 \\
\hline 1.26674 & 1.21076 & 0.129443 & & 0.172768 & 1.35676 & 0.180684 \\
\hline 1.39346 & 1.17744 & 0.128508 & 0.877073 & 0.174673 & 1.35358 & 0.170946 \\
\hline 1.28953 & 1.13822 & 0.121773 & 0.874015 & 0.169088 & 1.33169 & 0.175599 \\
\hline 1.2724 & 1.08245 & 0.107369 & 0.848094 & 0.159512 & 1.28133 & 0.170977 \\
\hline 1.18677 & 1.01939 & 0.0923853 & 0.816556 & 0.149724 & 1.2281 & 0.145281 \\
\hline 1.14523 & 0.959515 & 0.0843269 & 0.768536 & 0.139898 & 1.15411 & 0.145809 \\
\hline 1.05906 & 0.908932 & 0.0851578 & 0.721166 & 0.134635 & 1.0934 & 0.132757 \\
\hline 1.04951 & 0.848055 & 0.0716519 & 0.673565 & 0.11972 & 0.999141 & 0.116658 \\
\hline 0.914169 & 0.79847 & 0.0640948 & 0.607834 & 0.106081 & 0.933809 & 0.120068 \\
\hline 0.862519 & 0.756643 & 0.058822 & 0.557092 & 0.0911928 & 0.856746 & 0.0939447 \\
\hline 0.833132 & 0.722452 & 0.056677 & 0.513532 & 0.0893557 & 0.791585 & 0.0954774 \\
\hline 0.766016 & 0.692784 & 0.0554346 & 0.468541 & 0.0813546 & 0.735071 & 0.0811952 \\
\hline 0.697188 & 0.669286 & 0.0595939 & 0.445982 & 0.0788157 & 0.702996 & 0.0703068 \\
\hline 0.720437 & 0.644298 & 0.0543259 & 0.407812 & 0.0688536 & 0.654694 & 0.0655283 \\
\hline 0.680983 & 0.627374 & 0.0498818 & 0.394423 & 0.0693227 & 0.638622 & 0.0612402 \\
\hline 0.699999 & 0.614612 & 0.0522305 & 0.373747 & 0.0640568 & 0.61315 & 0.0634182 \\
\hline 0.65339 & 0.602556 & 0.0523941 & 0.360642 & 0.064085 & 0.606194 & 0.0584741 \\
\hline 0.676062 & 0.600437 & 0.0520977 & 0.357729 & 0.0717773 & 0.606317 & 0.0499105 \\
\hline 0.705047 & 0.599846 & 0.0501272 & 0.348788 & 0.065604 & 0.602476 & 0.0502971 \\
\hline 0.745155 & 0.588492 & 0.0527778 & 0.34706 & 0.0654982 & 0.602196 & 0.0581046 \\
\hline 0.771886 & 0.586565 & 0.0471047 & 0.346056 & 0.0611277 & 0.614942 & 0.0462526 \\
\hline 0.7218 & 0.585814 & 0.0557068 & 0.347696 & 0.0703055 & 0.632585 & 0.0546557 \\
\hline 0.860991 & 0.587189 & 0.0597443 & 0.349467 & 0.0689336 & 0.625461 & 0.0593916 \\
\hline 0.798548 & 0.597569 & 0.0606842 & 0.349246 & 0.0687127 & 0.659119 & 0.061241 \\
\hline 0.870843 & 0.607401 & 0.0625854 & 0.361075 & 0.0754383 & 0.670905 & 0.0648628 \\
\hline 0.861564 & 0.626962 & 0.0681339 & 0.365762 & 0.0732358 & 0.706396 & 0.058183 \\
\hline
\end{tabular}




\begin{tabular}{|c|c|c|c|c|c|c|}
\hline 1.07279 & 0.657798 & 0.078679 & 0.368591 & 0.073338 & 0.748654 & 0.0642073 \\
\hline 1.1039 & 0.697703 & 0.0905234 & 0.385495 & 0.0864582 & 0.802743 & 0.0766527 \\
\hline 1.2166 & 0.756659 & 0.101686 & 0.408111 & 0.0888236 & 0.871327 & 0.0713357 \\
\hline 1.3809 & 0.866712 & 0.141175 & 0.437551 & 0.0976833 & 0.985489 & 0.100265 \\
\hline 1.66068 & 1.02118 & 0.186568 & 0.474868 & 0.118689 & 1.14465 & 0.099102 \\
\hline 2.05264 & 1.25242 & 0.251674 & 0.536511 & 0.139913 & 1.40254 & 0.132939 \\
\hline 2.53178 & 1.58237 & 0.360383 & 0.64647 & 0.186417 & 1.75713 & 0.160997 \\
\hline 3.53636 & 2.03715 & 0.492013 & 0.795657 & 0.229813 & 2.29496 & 0.214554 \\
\hline 4.46693 & 2.66385 & 0.697632 & 1.00131 & 0.337886 & 3.08297 & 0.280945 \\
\hline 6.3201 & 3.50103 & 0.957707 & 1.30326 & 0.436778 & 4.15416 & 0.363372 \\
\hline 8.80965 & 4.57599 & 1.29247 & 1.73502 & 0.619932 & 5.66729 & 0.504527 \\
\hline 12.1371 & 5.93178 & 1.7426 & 2.30481 & 0.84928 & 7.68128 & 0.665466 \\
\hline 16.3524 & 7.55976 & 2.22774 & 3.0801 & 1.15952 & 10.3583 & 0.921693 \\
\hline 21.7905 & 9.47848 & 2.81905 & 4.07925 & 1.56075 & 13.7564 & 1.28922 \\
\hline 28.8871 & 11.6463 & 3.47515 & 5.2999 & 2.05533 & 17.9028 & 1.58111 \\
\hline 36.9856 & 13.9727 & 4.21035 & 6.81219 & 2.64751 & 22.7908 & 2.07478 \\
\hline 47.2077 & 16.4544 & 4.95215 & 8.53117 & 3.32292 & 28.4736 & 2.62301 \\
\hline 59.1444 & 18.8764 & 5.77182 & 10.5238 & 4.13052 & 34.8362 & 3.23421 \\
\hline 71.3179 & 21.1195 & 6.48406 & 12.6163 & 4.96955 & 41.4284 & 3.76185 \\
\hline 83.9831 & 23.0558 & 7.064 & 14.8104 & 5.78641 & 48.3172 & 4.49601 \\
\hline 97.4547 & 24.5471 & 7.51067 & 16.9769 & 6.66282 & 54.8546 & 5.16745 \\
\hline 110.626 & 25.4989 & 7.75338 & 19.0446 & 7.53979 & 60.7473 & 5.60481 \\
\hline 120.904 & 25.8543 & 7.91146 & 20.8463 & 8.20873 & 65.7786 & 6.08034 \\
\hline 130.133 & 25.5682 & 7.81083 & 22.1464 & 8.72621 & 69.165 & 6.51586 \\
\hline 136.315 & 24.5991 & 7.49717 & 22.9565 & 9.09012 & 71.0412 & 6.65515 \\
\hline 138.065 & 23.1701 & 7.03853 & 23.3481 & 9.23555 & 71.1657 & 6.75469 \\
\hline 138.966 & 21.2308 & 6.40434 & 23.0778 & 9.09575 & 69.5385 & 6.66104 \\
\hline 134.992 & 19.0353 & 5.73417 & 22.2221 & 8.78167 & 66.1697 & 6.33425 \\
\hline 126.999 & 16.6273 & 4.9526 & 20.8848 & 8.20776 & 61.3628 & 5.88699 \\
\hline 116.651 & 14.2027 & 4.21297 & 19.1821 & 7.53238 & 55.5451 & 5.30132 \\
\hline 105.279 & 11.8697 & 3.50348 & 17.1643 & 6.73402 & 49.1532 & 4.79761 \\
\hline 93.0746 & 9.72116 & 2.83011 & 14.9821 & 5.89349 & 42.3293 & 4.09695 \\
\hline 80.1195 & 7.78789 & 2.24175 & 12.7585 & 4.91494 & 35.5958 & 3.54082 \\
\hline 67.1034 & 6.13921 & 1.72404 & 10.6271 & 4.12104 & 29.159 & 2.85697 \\
\hline 55.4222 & 4.79814 & 1.30303 & 8.67006 & 3.34135 & 23.3846 & 2.34296 \\
\hline 44.3065 & 3.72744 & 0.952541 & 6.88162 & 2.61987 & 18.3032 & 1.80481 \\
\hline 34.868 & 2.90462 & 0.690578 & 5.3681 & 2.00318 & 14.0027 & 1.36465 \\
\hline 26.2886 & 2.27251 & 0.488293 & 4.139 & 1.52361 & 10.4951 & 1.04319 \\
\hline 20.0783 & 1.81366 & 0.338936 & 3.11617 & 1.14233 & 7.71 & 0.761811 \\
\hline 14.6221 & 1.48735 & 0.239591 & 2.32424 & 0.793567 & 5.58116 & 0.536254 \\
\hline 10.5904 & 1.27544 & 0.173582 & 1.73918 & 0.570606 & 4.0226 & 0.408542 \\
\hline 7.66741 & 1.12414 & 0.13498 & 1.30439 & 0.401079 & 2.89151 & 0.293672 \\
\hline 5.49714 & 1.03835 & 0.108238 & 1.00042 & 0.279016 & 2.09028 & 0.193954 \\
\hline 3.94747 & 0.976321 & 0.0901172 & 0.781852 & 0.200022 & 1.54 & 0.149505 \\
\hline 2.80515 & 0.937873 & 0.083513 & 0.63751 & 0.157571 & 1.16088 & 0.114471 \\
\hline 2.17489 & 0.916148 & 0.0765444 & 0.539808 & 0.114717 & 0.927858 & 0.0851394 \\
\hline 1.649 & 0.904194 & 0.0569866 & 0.477916 & 0.0948945 & 0.78089 & 0.076989 \\
\hline
\end{tabular}




\begin{tabular}{|c|c|c|c|c|c|c|}
\hline 1.29427 & 0.894505 & 0.0642197 & 0.441993 & 0.0711823 & 0.694851 & 0.0628987 \\
\hline 1.00349 & 0.897566 & 0.0621366 & 0.418793 & 0.0710184 & 0.620651 & 0.0593832 \\
\hline 0.895768 & 0.900136 & 0.0602234 & 0.402385 & 0.0677591 & 0.591155 & 0.0583691 \\
\hline 0.87032 & 0.900759 & 0.0661016 & 0.392083 & 0.0629876 & 0.568316 & 0.0613838 \\
\hline 0.799799 & 0.903414 & 0.0649131 & 0.400862 & 0.0656671 & 0.554124 & 0.0594834 \\
\hline 0.678094 & 0.901297 & 0.0616461 & 0.391317 & 0.0631244 & 0.554327 & 0.0584392 \\
\hline 0.73876 & 0.895774 & 0.0636004 & 0.39223 & 0.0586558 & 0.558583 & 0.0616487 \\
\hline 0.639922 & 0.904017 & 0.0560937 & 0.395822 & 0.0622388 & 0.556957 & 0.0537569 \\
\hline 0.660904 & 0.901494 & 0.0541111 & 0.397833 & 0.0560699 & 0.565074 & 0.0651751 \\
\hline 0.655779 & 0.902366 & 0.0586835 & 0.399483 & 0.0538525 & 0.556466 & 0.0638433 \\
\hline 0.688612 & 0.900071 & 0.0569755 & 0.395695 & 0.0524721 & 0.565074 & 0.0561913 \\
\hline 0.650905 & 0.909005 & 0.0545434 & 0.402432 & 0.0516957 & 0.564914 & 0.0497883 \\
\hline 0.68014 & 0.913881 & 0.0647529 & 0.406857 & 0.0597635 & 0.582316 & 0.0595777 \\
\hline 0.658876 & 0.919766 & 0.0629884 & 0.408013 & 0.0484858 & 0.587877 & 0.0623491 \\
\hline 0.712528 & 0.925793 & 0.0574715 & 0.413483 & 0.0513401 & 0.594169 & 0.0585457 \\
\hline 0.683407 & 0.930172 & 0.0558305 & 0.413092 & 0.053007 & 0.608881 & 0.0565344 \\
\hline 0.662523 & 0.931478 & 0.0548824 & 0.422527 & 0.0581017 & 0.622013 & 0.0665531 \\
\hline 0.678849 & 0.94037 & 0.0568321 & 0.425647 & 0.0575544 & 0.636475 & 0.054245 \\
\hline 0.72128 & 0.95215 & 0.0566033 & 0.431182 & 0.0624174 & 0.654052 & 0.0611873 \\
\hline 0.6796 & 0.963955 & 0.0585311 & 0.432918 & 0.061006 & 0.674666 & 0.0536096 \\
\hline 0.692431 & 0.979753 & 0.0621035 & 0.452505 & 0.0629322 & 0.701624 & 0.0616244 \\
\hline 0.699198 & 1.00602 & 0.0662468 & 0.464769 & 0.0674404 & 0.741003 & 0.0590997 \\
\hline 0.821349 & 1.04252 & 0.070192 & 0.478107 & 0.0627917 & 0.780586 & 0.0652913 \\
\hline 0.883137 & 1.09222 & 0.084643 & 0.494707 & 0.0787425 & 0.847045 & 0.0583353 \\
\hline 0.925422 & 1.14833 & 0.0974181 & 0.519052 & 0.0897336 & 0.93253 & 0.066181 \\
\hline 1.02588 & 1.22942 & 0.127416 & 0.543535 & 0.0961227 & 1.0329 & 0.0780081 \\
\hline 1.24048 & 1.33472 & 0.163615 & 0.583569 & 0.110882 & 1.17995 & 0.1021 \\
\hline 1.49935 & 1.46551 & 0.194428 & 0.635088 & 0.132187 & 1.36194 & 0.100436 \\
\hline 1.71006 & 1.63386 & 0.255027 & 0.715766 & 0.160044 & 1.61191 & 0.136446 \\
\hline 2.01291 & 1.83938 & 0.309244 & 0.809912 & 0.182438 & 1.91788 & 0.148003 \\
\hline 2.60598 & 2.08404 & 0.364174 & 0.915296 & 0.235449 & 2.31792 & 0.185028 \\
\hline 3.19752 & 2.36493 & 0.451597 & 1.0694 & 0.306281 & 2.83008 & 0.22104 \\
\hline 3.93239 & 2.66594 & 0.545963 & 1.26351 & 0.375878 & 3.40534 & 0.276986 \\
\hline 4.91553 & 3.02033 & 0.644584 & 1.47093 & 0.464496 & 4.12168 & 0.318378 \\
\hline 5.78639 & 3.34411 & 0.764117 & 1.72691 & 0.55219 & 4.94186 & 0.416006 \\
\hline 7.06943 & 3.70127 & 0.84405 & 2.00945 & 0.663663 & 5.81256 & 0.467385 \\
\hline 8.31663 & 4.03312 & 0.954305 & 2.3183 & 0.787632 & 6.7619 & 0.558242 \\
\hline 9.74184 & 4.31326 & 1.02293 & 2.63361 & 0.910561 & 7.76362 & 0.645602 \\
\hline 11.0604 & 4.52578 & 1.10188 & 2.9633 & 1.03108 & 8.71861 & 0.699105 \\
\hline 12.7199 & 4.71424 & 1.14075 & 3.254 & 1.18213 & 9.57618 & 0.802721 \\
\hline 13.4995 & 4.80004 & 1.15543 & 3.53592 & 1.29794 & 10.3446 & 0.842044 \\
\hline 15.0982 & 4.77845 & 1.16559 & 3.76439 & 1.34158 & 10.9441 & 0.910437 \\
\hline 15.7835 & 4.6669 & 1.1298 & 3.90017 & 1.40019 & 11.3124 & 0.968173 \\
\hline 16.0888 & 4.46015 & 1.05861 & 4.01099 & 1.43491 & 11.4354 & 0.954964 \\
\hline 16.4338 & 4.1955 & 0.981635 & 4.0052 & 1.46994 & 11.3345 & 0.998023 \\
\hline 16.1014 & 3.89265 & 0.894877 & 3.90518 & 1.42075 & 10.9486 & 0.936274 \\
\hline 15.4019 & 3.56098 & 0.802957 & 3.77826 & 1.36024 & 10.3709 & 0.919485 \\
\hline
\end{tabular}




\begin{tabular}{|c|c|c|c|c|c|c|}
\hline 14.6984 & 3.20116 & 0.699196 & 3.53551 & 1.27609 & 9.61624 & 0.807514 \\
\hline 13.4855 & 2.84631 & 0.584551 & 3.2669 & 1.15037 & 8.69913 & 0.759821 \\
\hline 12.2525 & 2.51239 & 0.492747 & 2.97313 & 1.03343 & 7.71246 & 0.707605 \\
\hline 10.6962 & 2.19546 & 0.397253 & 2.63698 & 0.906695 & 6.73259 & 0.573564 \\
\hline 9.43463 & 1.92101 & 0.312251 & 2.29946 & 0.771784 & 5.73654 & 0.507073 \\
\hline 7.91218 & 1.67941 & 0.239867 & 1.98439 & 0.657942 & 4.7837 & 0.422057 \\
\hline 6.50159 & 1.49125 & 0.180481 & 1.6825 & 0.515246 & 3.9269 & 0.358698 \\
\hline 5.34666 & 1.34136 & 0.142546 & 1.42162 & 0.420169 & 3.17792 & 0.271479 \\
\hline 4.11664 & 1.21589 & 0.113558 & 1.20454 & 0.339327 & 2.54614 & 0.2147 \\
\hline 3.33296 & 1.12884 & 0.0819461 & 0.995894 & 0.25411 & 2.00722 & 0.171975 \\
\hline 2.52625 & 1.06885 & 0.0730541 & 0.855646 & 0.201135 & 1.59158 & 0.142571 \\
\hline 1.95669 & 1.02131 & 0.0728794 & 0.712081 & 0.145533 & 1.2642 & 0.110309 \\
\hline 1.4565 & 0.997908 & 0.0645688 & 0.629883 & 0.111501 & 1.00531 & 0.0844182 \\
\hline 1.11662 & 0.982817 & 0.0511274 & 0.563451 & 0.091212 & 0.821789 & 0.068581 \\
\hline 0.824799 & 0.987425 & 0.0510307 & 0.509201 & 0.0789942 & 0.677769 & 0.0486258 \\
\hline 0.624661 & 0.998873 & 0.0539311 & 0.474253 & 0.0586258 & 0.590268 & 0.0528097 \\
\hline 0.485432 & 1.0114 & 0.0639671 & 0.448888 & 0.052324 & 0.514516 & 0.0419877 \\
\hline 0.360759 & 1.03813 & 0.0641015 & 0.439285 & 0.0619266 & 0.470005 & 0.0337875 \\
\hline 0.295291 & 1.06783 & 0.0669633 & 0.435349 & 0.0497591 & 0.440696 & 0.0389016 \\
\hline 0.231438 & 1.09326 & 0.0741798 & 0.436853 & 0.0579241 & 0.424193 & 0.0387495 \\
\hline 0.219043 & 1.12441 & 0.0787399 & 0.431503 & 0.0536226 & 0.415522 & 0.0332388 \\
\hline 0.178611 & 1.15247 & 0.0737908 & 0.446256 & 0.0580101 & 0.408429 & 0.032897 \\
\hline 0.179137 & 1.18329 & 0.0852458 & 0.45702 & 0.0619345 & 0.401088 & 0.0321218 \\
\hline 0.181837 & 1.19932 & 0.0893441 & 0.465619 & 0.0653782 & 0.394602 & 0.037129 \\
\hline 0.163251 & 1.21654 & 0.0886371 & 0.472622 & 0.0658093 & 0.404341 & 0.0328114 \\
\hline 0.154999 & 1.22481 & 0.089394 & 0.479139 & 0.0757418 & 0.408361 & 0.0323227 \\
\hline 0.17943 & 1.2238 & 0.090029 & 0.490562 & 0.0705054 & 0.418966 & 0.03505 \\
\hline 0.177925 & 1.22328 & 0.0906855 & 0.498674 & 0.0774615 & 0.42114 & 0.0343264 \\
\hline 0.210087 & 1.20405 & 0.0879674 & 0.515604 & 0.0752039 & 0.435291 & 0.0375256 \\
\hline 0.21379 & 1.18783 & 0.0734355 & 0.51103 & 0.0859726 & 0.447773 & 0.0351021 \\
\hline 0.246172 & 1.18295 & 0.0763366 & 0.518058 & 0.0775389 & 0.460986 & 0.0427404 \\
\hline 0.259319 & 1.16188 & 0.0732436 & 0.513151 & 0.0778294 & 0.473506 & 0.0346694 \\
\hline 0.286466 & 1.13895 & 0.066399 & 0.520118 & 0.064821 & 0.481906 & 0.0419616 \\
\hline 0.300574 & 1.11047 & 0.0648459 & 0.517772 & 0.0721642 & 0.498333 & 0.0409041 \\
\hline 0.32333 & 1.08857 & 0.0608382 & 0.515713 & 0.0769016 & 0.507576 & 0.0449691 \\
\hline 0.336652 & 1.06631 & 0.0536377 & 0.510321 & 0.0686841 & 0.511766 & 0.0368668 \\
\hline 0.386705 & 1.03951 & 0.0562582 & 0.512242 & 0.0708935 & 0.529377 & 0.0327129 \\
\hline 0.406718 & 1.02039 & 0.0625783 & 0.503202 & 0.0722087 & 0.530136 & 0.0475164 \\
\hline 0.419256 & 1.01097 & 0.0535226 & 0.500363 & 0.0633363 & 0.536228 & 0.0396697 \\
\hline 0.371615 & 0.988862 & 0.0550076 & 0.494616 & 0.0617128 & 0.536842 & 0.0517814 \\
\hline 0.375552 & 0.970465 & 0.0592776 & 0.48856 & 0.0641572 & 0.541704 & 0.0426934 \\
\hline 0.380761 & 0.96191 & 0.0579486 & 0.483249 & 0.0679359 & 0.541783 & 0.0446009 \\
\hline 0.366281 & 0.959218 & 0.0624579 & 0.468943 & 0.0617193 & 0.529335 & 0.049887 \\
\hline 0.310866 & 0.958338 & 0.0625162 & 0.467741 & 0.0528721 & 0.526645 & 0.0553571 \\
\hline 0.293787 & 0.949807 & 0.0628559 & 0.466197 & 0.0646975 & 0.51284 & 0.0495861 \\
\hline 0.265387 & 0.947241 & 0.0619349 & 0.454895 & 0.0667171 & 0.518314 & 0.0635861 \\
\hline 0.263664 & 0.949563 & 0.0597253 & 0.455649 & 0.0590531 & 0.510994 & 0.0600625 \\
\hline
\end{tabular}




\begin{tabular}{|c|c|c|c|c|c|c|}
\hline 0.239323 & 0.949586 & 0.0601531 & 0.446184 & 0.0529012 & 0.501009 & 0.0676795 \\
\hline 0.20151 & 0.952886 & 0.0523668 & 0.438833 & 0.0605439 & 0.494602 & 0.0704392 \\
\hline 0.182293 & 0.958173 & 0.0491987 & 0.448904 & 0.0682066 & 0.483002 & 0.0837324 \\
\hline 0.177616 & 0.96749 & 0.0616882 & 0.452047 & 0.0673244 & 0.490085 & 0.0813358 \\
\hline 0.154393 & 0.969837 & 0.0636359 & 0.448615 & 0.0609147 & 0.487985 & 0.0845174 \\
\hline 0.154072 & 0.97998 & 0.0649936 & 0.448209 & 0.0579059 & 0.482122 & 0.0796312 \\
\hline 0.114836 & 0.974893 & 0.0555938 & 0.447372 & 0.0600655 & 0.481405 & 0.0875627 \\
\hline 0.119183 & 0.977131 & 0.0562559 & 0.460188 & 0.0577207 & 0.48212 & 0.088649 \\
\hline 0.124637 & 0.970829 & 0.0569205 & 0.455004 & 0.0604347 & 0.490327 & 0.08562 \\
\hline 0.0990258 & 0.973648 & 0.0530231 & 0.46204 & 0.057163 & 0.481442 & 0.0833675 \\
\hline 0.105388 & 0.973507 & 0.0510112 & 0.459646 & 0.0633873 & 0.478934 & 0.0706467 \\
\hline 0.107512 & 0.955779 & 0.0536249 & 0.459404 & 0.0709501 & 0.486386 & 0.0715792 \\
\hline 0.114997 & 0.950234 & 0.0594816 & 0.465048 & 0.0635551 & 0.492191 & 0.0722369 \\
\hline 0.119884 & 0.946964 & 0.0632066 & 0.464595 & 0.0576193 & 0.488654 & 0.0706703 \\
\hline 0.107877 & 0.945337 & 0.0545257 & 0.458018 & 0.0587977 & 0.480985 & 0.0767619 \\
\hline 0.112351 & 0.947586 & 0.0517848 & 0.463312 & 0.0647178 & 0.489826 & 0.0618618 \\
\hline 0.106332 & 0.942309 & 0.0543078 & .466665 & 0.0677396 & 0.489605 & 9927 \\
\hline 0.11404 & 0.943077 & 0.0473838 & 0.4647 & 0.0706412 & 0.48942 & 0.0530158 \\
\hline 0.105791 & 0.946353 & 0.0541303 & 0.465523 & 0.0623014 & 0.489369 & 0.0664286 \\
\hline 0.116616 & 0.928581 & 7196 & 494 & 478 & 0.485123 & 0.04 \\
\hline 0.107601 & 0.926796 & 0.0625468 & 0.459797 & 0.0618275 & 0.482991 & 0.0516537 \\
\hline 0.104479 & 0.928612 & 0.0510837 & 0.460955 & 0.0600459 & 0.48634 & 0.0484477 \\
\hline 0.110972 & 0.917202 & 0.052172 & 0.458044 & 0.0594642 & 0.477237 & 0.0427297 \\
\hline 0.0856878 & 0.910616 & 0.0553309 & 0.457272 & 0.0659642 & 0.48239 & 0.0440347 \\
\hline 0.0966248 & 0.913599 & 0.0599032 & 0.461546 & 0.066204 & 0.489949 & 0.043745 \\
\hline 0.109693 & 0.903571 & 0.0536792 & 0.453005 & 0.058198 & 0.494954 & 0.0375638 \\
\hline 0.105158 & 0.891733 & 0.0574151 & 0.444533 & 0.0576705 & 0.48172 & 0.0390412 \\
\hline 0.111329 & 0.887036 & 0.0518489 & 0.441326 & 0.0599543 & 0.490259 & 0.0428678 \\
\hline 0.103886 & 0.884104 & 0.0459089 & 0.451184 & 0.0635569 & 0.483441 & 0.0366457 \\
\hline 0.107036 & 0.891436 & 0.0570807 & 0.444151 & 0.0642872 & 0.494367 & 0.0386191 \\
\hline 0.0975881 & 0.89357 & 0.0508015 & 0.450207 & 0.0605744 & 0.484012 & 0.0363178 \\
\hline 0.0956286 & 0.893497 & 0.0529496 & 0.442195 & 0.0577774 & 0.496061 & 0.0394199 \\
\hline 0.114313 & 0.889824 & 0.0552349 & 0.445829 & 0.0605803 & 0.492909 & 0.0344918 \\
\hline 0.140166 & 0.891016 & 0.0523136 & 0.447144 & 0.0488557 & 0.489592 & 0.0379214 \\
\hline 0.121139 & 0.899487 & 0.0506571 & 0.45543 & 0.0543712 & 0.490277 & 0.0364788 \\
\hline 0.100607 & 0.904651 & 0.0577001 & 0.446332 & 0.0534447 & 0.493947 & 0.040404 \\
\hline 0.140177 & 0.902841 & 0.0433651 & 0.45942 & 0.0464325 & 0.507118 & 0.0405272 \\
\hline 0.139595 & 0.903527 & 0.0520583 & 0.452295 & 0.0469341 & 0.509601 & 0.0269612 \\
\hline 0.130291 & 0.908797 & 0.0487619 & 0.457698 & 0.0573051 & 0.517932 & 0.0372214 \\
\hline 0.141934 & 0.923197 & 0.0436261 & 0.466695 & 0.0467316 & 0.529321 & 0.0449686 \\
\hline 0.168186 & 0.92241 & 0.0556714 & 0.465329 & 0.0478154 & 0.536827 & 0.0488036 \\
\hline 0.161584 & 0.925891 & 0.0477818 & 0.474809 & 0.0530765 & 0.546774 & 0.0400659 \\
\hline 0.183394 & 0.935068 & 0.0483851 & 0.475908 & 0.0536258 & 0.554946 & 0.0431185 \\
\hline 0.185237 & 0.942821 & 0.0468763 & 0.475854 & 0.0470964 & 0.576167 & 0.0407528 \\
\hline 0.211069 & 0.93519 & 0.0479648 & 0.483879 & 0.0546683 & 0.58023 & 0.040752 \\
\hline 0.212701 & 0.938114 & 0.0465401 & 0.489424 & 0.054454 & 0.579291 & 0.0462271 \\
\hline 0.211772 & 0.942098 & 0.0480756 & 0.493763 & 0.0599562 & 0.588157 & 0.0479928 \\
\hline
\end{tabular}




\begin{tabular}{|c|c|c|c|c|c|c|}
\hline 0.21523 & 0.944767 & 0.0452953 & 0.501153 & 0.0558547 & 0.59655 & 477 \\
\hline 0.267783 & 0.951486 & 0.0429054 & 0.493337 & 0.059477 & 0.606089 & 0.0512384 \\
\hline 0.217568 & 0.944481 & 0.0395433 & 0.494171 & 0.0573739 & 0.606887 & 0.0473102 \\
\hline 0.263352 & 0.953397 & 0.0535906 & 0.495938 & 0.0601603 & 0.599401 & 0.0465667 \\
\hline 0.241577 & .955098 & 0.0523374 & 0.496272 & 0.0606185 & 0.599404 & 0.0428862 \\
\hline 0.240196 & 0.950016 & 0.0439409 & 0.499254 & 0.0640947 & 0.600043 & 0.044774 \\
\hline 0.267886 & 0.949683 & 0.0435456 & 0.49497 & 0.0612241 & 0.592889 & 0.0445035 \\
\hline 0.248756 & 0.949833 & 0.0516228 & 0.488585 & 0.0661872 & 0.590607 & 0.0488613 \\
\hline 0.232097 & 0.944505 & 0.0519528 & 0.485656 & 0.0554956 & 0.576749 & 0.0370544 \\
\hline 0.227129 & 0.948604 & 0.0514881 & 0.482907 & 0.0573227 & 0.581052 & 0.0300366 \\
\hline 0.231706 & 0.951507 & 0.0494571 & 0.478094 & 0.0502083 & 0.563997 & 0.0406183 \\
\hline 0.226277 & 0.956868 & 0.0479285 & 0.483469 & 0.0547166 & 0.564382 & 0.0426423 \\
\hline 0.227101 & 0.965098 & 0.0479768 & 0.477143 & 0.0430273 & 0.563623 & 0.0360813 \\
\hline 0.198007 & 0.971804 & 9043 & 0.482603 & 0.057 & 0.556934 & 0.0355999 \\
\hline 0.195566 & 0.975183 & 0.0477251 & 0.475274 & 0.058077 & 0.552871 & 0.0316167 \\
\hline 0.174351 & 0.976961 & 0.0472091 & 0.477585 & 0.0549095 & 0.548699 & 0.0385979 \\
\hline 0.184482 & 0.990757 & 0.0517089 & 0.474406 & 0.0541867 & 0.553451 & 0.0410961 \\
\hline 0.177815 & 0.997215 & 0.0470506 & 0.482161 & 0.0569496 & 0.557962 & 0.0464594 \\
\hline 0.172905 & 1.00435 & 0.0533798 & 0.475933 & 0.050881 & 0.55086 & 0.0422359 \\
\hline 0.177777 & 1.02075 & 0.049827 & 0.487059 & 0.0539117 & 0.553285 & 0.0366171 \\
\hline 0.157701 & 1.02977 & 0.0560179 & 0.482865 & 0.050365 & 0.556171 & 0.0463488 \\
\hline 0.153105 & 1.02999 & 0.0580947 & 0.480144 & 0.0524789 & 0.555309 & 0.0401321 \\
\hline 0.149825 & 1.04374 & 0.0604038 & 0.486208 & 0.0510979 & 0.569942 & 0.0328322 \\
\hline 0.149388 & 1.0555 & 0.0604657 & 0.490277 & 0.056 & 0.558293 & 0.0452767 \\
\hline 0.100961 & 1.06701 & 0.04 & 849 & 48 & 966 & 881 \\
\hline 0.139985 & 1.07781 & 0.0631206 & 0.492121 & 0.0500057 & 0.575338 & 0.0484218 \\
\hline 0.125408 & 1.08914 & 0.0645734 & 0.502941 & 0.0560674 & 0.573061 & 0.0462462 \\
\hline 0.115112 & 1.08802 & 0.0582639 & 0.506949 & 0.0615067 & 0.585705 & 0.0463827 \\
\hline 0.136573 & 1.10534 & 0.0625755 & 0.507747 & 0.0545955 & 0.583235 & 0.0474614 \\
\hline 0.116914 & 1.1255 & 0.0659912 & 0.508599 & 0.0519391 & 0.585858 & 0.0443639 \\
\hline 0.11968 & 1.13141 & 0.0731253 & 0.508392 & 0.056983 & 0.597228 & 0.04645 \\
\hline 0.0953681 & 1.15162 & 0.0750047 & 0.521514 & 0.0588211 & 0.589419 & 0.0525044 \\
\hline 0.0993864 & 1.15232 & 0.0746848 & 0.518647 & 0.0597634 & 0.606957 & 0.0450545 \\
\hline 0.113893 & 1.1551 & 0.080002 & 0.519956 & 0.0590579 & 0.60267 & 0.0430324 \\
\hline 0.102234 & 1.16983 & 0.0894533 & 0.528839 & 0.0651164 & 0.613281 & 0.0546689 \\
\hline 0.100125 & 1.1893 & 0.0852721 & 0.53174 & 0.0630769 & 0.614482 & 0.0416395 \\
\hline 0.104779 & 1.20651 & 0.0823271 & 0.541744 & 0.0714383 & 0.623381 & 0.0502625 \\
\hline 0.113182 & 1.22277 & 0.0951548 & 0.544519 & 0.0574875 & 0.611587 & 0.0518808 \\
\hline 0.120008 & 1.22776 & 0.0909103 & 0.549907 & 0.0615972 & 0.638781 & 0.0475032 \\
\hline 0.101536 & 1.23816 & 0.0878654 & 0.557596 & 0.0746422 & 0.629826 & 0.0489991 \\
\hline 0.106209 & 1.25158 & 0.0949434 & 0.554834 & 0.0691966 & 0.642675 & 0.0435933 \\
\hline 0.101772 & 1.26659 & 0.0989945 & 0.563054 & 0.0640514 & 0.634114 & 0.0480808 \\
\hline 0.111063 & 1.28313 & 0.0966303 & 0.579214 & 0.0812915 & 0.65394 & 0.0486887 \\
\hline 0.106265 & 1.29427 & 0.0978902 & 0.565969 & 0.064758 & 0.659959 & 0.0510101 \\
\hline 0.112765 & 1.31188 & 0.0906657 & 0.58448 & 0.0767114 & 0.668679 & 0.0457487 \\
\hline 0.109546 & 1.33123 & 0.0944289 & 0.585387 & 0.0714786 & 0.683328 & 0.0597014 \\
\hline 0.0959754 & 1.35646 & 0.0914292 & 0.600997 & 0.0757611 & 0.696263 & 0.0587796 \\
\hline
\end{tabular}




\begin{tabular}{|c|c|c|c|c|c|c|}
\hline 0.0986272 & 1.37365 & 0.109913 & 0.605784 & 0.0797692 & 0.716389 & 0.0434225 \\
\hline 0.111244 & 1.3956 & 0.108055 & 0.616379 & 0.0752908 & 0.733399 & 0.0556829 \\
\hline 0.109576 & 1.40873 & 0.0998991 & 0.630181 & 0.0825142 & 0.762234 & 0.0530671 \\
\hline 0.124514 & 1.42707 & 0.0931185 & 0.637595 & 0.0775188 & 0.778369 & 0.0474859 \\
\hline 0.132466 & 1.4497 & 0.105576 & 0.648717 & 0.0797071 & 0.814543 & 0.0453475 \\
\hline 0.107465 & 1.46131 & 0.104742 & 0.66565 & 0.0855513 & 0.83898 & 0.0536224 \\
\hline 0.121345 & 1.48402 & 0.0865545 & 0.672157 & 0.0800394 & 0.869035 & 0.0500474 \\
\hline 0.121525 & 1.50007 & 0.0974198 & 0.685074 & 0.075741 & 0.902035 & 0.0468661 \\
\hline 0.0974662 & 1.51212 & 0.0981638 & 0.699254 & 0.0932091 & 0.931838 & 0.0694084 \\
\hline 0.0997277 & 1.50593 & 0.0932069 & 0.717821 & 0.088717 & 0.951119 & 0.0699575 \\
\hline 0.106369 & 1.51957 & 0.0903157 & 0.736347 & 0.0786742 & 0.975344 & 0.062439 \\
\hline 0.125371 & 1.53356 & 0.082873 & 0.738861 & 0.0776036 & 0.978991 & 0.0607615 \\
\hline 0.107215 & 1.52073 & 0.0870462 & 0.745118 & 0.0820754 & 1.00295 & 0.0673997 \\
\hline 0.102887 & 1.51985 & 0.087795 & 0.745417 & 0.0790102 & 0.997341 & 0.058588 \\
\hline 0.116002 & 1.51104 & 0.078524 & 0.754793 & 0.0895043 & 0.99607 & 0.0716464 \\
\hline 0.119942 & 1.4991 & 0.0797169 & 0.745091 & 0.087418 & 0.981326 & 0.0670539 \\
\hline 0.110054 & 1.50127 & 0.0802391 & 0.746429 & 0.0861003 & 0.977253 & 0.0702543 \\
\hline 0.121189 & 1.48776 & 0.0764272 & 0.752824 & 0.088283 & 0.946736 & 0.0594436 \\
\hline 0.127786 & 1.49568 & 0.0691331 & 0.737617 & 0.0884952 & 0.93141 & 0.0547967 \\
\hline 0.10163 & 1.47986 & 0.0685322 & 0.736386 & 0.0845716 & 0.910098 & 0.066991 \\
\hline 0.131457 & 1.46264 & 0.0782369 & 0.725901 & 0.078551 & 0.87532 & 0.06174 \\
\hline 0.129522 & 1.46254 & 0.077536 & 0.727076 & 0.0765138 & 0.856714 & 0.0592957 \\
\hline 0.116715 & 1.46593 & 0.0791023 & 0.707497 & 0.0745259 & 0.828916 & 0.0607172 \\
\hline 0.119186 & 1.45548 & 0.0806012 & 0.707354 & 0.0748536 & 0.810036 & 0.0602629 \\
\hline 0.119706 & 1.45712 & 0.0780898 & 0.698242 & 0.0738303 & 0.805187 & 0.0619163 \\
\hline 0.127488 & 1.45464 & 0.0839931 & 0.692975 & 0.0834936 & 0.788724 & 0.0522901 \\
\hline 0.12951 & 1.44791 & 0.0791638 & 0.676699 & 0.0740966 & 0.769893 & 0.0577744 \\
\hline 0.111333 & 1.45126 & 0.0806122 & 0.687694 & 0.0766131 & 0.759177 & 0.0496366 \\
\hline 0.115274 & 1.4638 & 0.0789038 & 0.682268 & 0.06378 & 0.749593 & 0.057582 \\
\hline 0.130557 & 1.45484 & 0.0757527 & 0.67995 & 0.0740189 & 0.744677 & 0.0576745 \\
\hline 0.130787 & 1.46027 & 0.0947016 & 0.681228 & 0.0748137 & 0.730918 & 0.0543545 \\
\hline 0.115063 & 1.45879 & 0.0848792 & 0.673913 & 0.0688411 & 0.728116 & 0.0540607 \\
\hline 0.129937 & 1.45405 & 0.0709876 & 0.678709 & 0.0565981 & 0.741078 & 0.0573711 \\
\hline 0.133075 & 1.46412 & 0.0946729 & 0.686596 & 0.0662865 & 0.735098 & 0.0564154 \\
\hline 0.122555 & 1.46872 & 0.0932926 & 0.687669 & 0.0627953 & 0.741219 & 0.0485724 \\
\hline 0.140359 & 1.4625 & 0.0834404 & 0.682345 & 0.0592416 & 0.733568 & 0.057408 \\
\hline 0.110717 & 1.46182 & 0.0977664 & 0.676928 & 0.0611871 & 0.726604 & 0.050198 \\
\hline 0.103571 & 1.47381 & 0.0893253 & 0.692047 & 0.0664222 & 0.724592 & 0.0580498 \\
\hline 0.129624 & 1.45664 & 0.0811133 & 0.685647 & 0.0565706 & 0.732702 & 0.0477554 \\
\hline 0.144031 & 1.4662 & 0.0858918 & 0.688029 & 0.0534326 & 0.731253 & 0.0488882 \\
\hline 0.112192 & 1.45196 & 0.0859922 & 0.684497 & 0.0489543 & 0.7269 & 0.0529937 \\
\hline 0.12518 & 1.45174 & 0.0820036 & 0.691247 & 0.0594911 & 0.738753 & 0.0366997 \\
\hline 0.130511 & 1.46747 & 0.0992565 & 0.680406 & 0.0654076 & 0.73133 & 0.0530785 \\
\hline 0.118723 & 1.45933 & 0.0762015 & 0.678487 & 0.0612776 & 0.729637 & 0.0426805 \\
\hline 0.11636 & 1.46283 & 0.0781547 & 0.67693 & 0.0555937 & 0.723904 & 0.0498202 \\
\hline 0.131096 & 1.44634 & 0.082724 & 0.681393 & 0.0522278 & 0.723105 & 0.0517714 \\
\hline 0.143549 & 1.44201 & 0.0858348 & 0.687733 & 0.0526539 & 0.714817 & 0.0467929 \\
\hline
\end{tabular}




\begin{tabular}{|c|c|c|c|c|c|c|}
\hline 0.136276 & 1.45242 & 0.0873006 & 0.689451 & 0.0521975 & 0.731521 & 185 \\
\hline 0.126007 & 1.44528 & 0.084596 & 0.690703 & 0.0574705 & 0.715024 & 0.0420244 \\
\hline 0.130741 & 1.43918 & 0.0824084 & 0.687913 & 0.0530957 & 0.72331 & 0.044195 \\
\hline 0.139856 & 1.43417 & 0.0926384 & 0.677398 & 0.0538715 & 0.715612 & 0.0427187 \\
\hline 0.126269 & 1.43607 & 0.0948336 & 0.674555 & 0.0594002 & 0.706387 & 0.0529919 \\
\hline 0.149448 & 1.43897 & 0.0835318 & 0.687769 & 0.0597779 & 0.69702 & 0.0447088 \\
\hline 0.148618 & 1.43942 & 0.0840978 & 0.682086 & 0.0590175 & 0.707963 & 0.0405342 \\
\hline 0.176727 & 1.44247 & 0.0878757 & 0.680799 & 0.049923 & 0.704263 & 0.0417453 \\
\hline 0.157729 & 1.43168 & 0.0868608 & 0.673746 & 0.0647753 & 0.705942 & 0.0396809 \\
\hline 0.140273 & 1.42993 & 0.0837346 & 0.67168 & 0.0566203 & 0.716826 & 0.0362717 \\
\hline 0.145458 & 1.43225 & 0.0846835 & 0.680466 & 0.0568194 & 0.708044 & 0.0383841 \\
\hline 0.157558 & 1.42493 & 0.0844184 & 0.675313 & 0.058188 & 0.702459 & 0.0487179 \\
\hline 0.149854 & 1.43644 & 0.0897241 & 0.680962 & 0.0565477 & 0.697253 & 0.0385034 \\
\hline 0.177615 & 1.42284 & 0.0805134 & 0.674271 & 0.0538832 & 0.715939 & 0.0350569 \\
\hline 0.161862 & 1.428 & 0.0791623 & 0.676304 & 0.0578812 & 0.706813 & 0.0392494 \\
\hline 0.148426 & 1.4341 & 0.0826446 & 0.680857 & 0.0633094 & 0.706298 & 0.0384029 \\
\hline 0.178968 & 1.42731 & 0.0904582 & 0.675371 & 0.049972 & 0.723015 & 0.0455593 \\
\hline 0.175119 & 1.42123 & 0.0813506 & 0.671137 & 0.0564534 & 0.720855 & 0.0403153 \\
\hline 0.169396 & 1.42402 & 0.0761734 & 0.679774 & 0.0531446 & 0.722385 & 0.0356037 \\
\hline 0.178347 & 1.43277 & 0.080 & 0.666996 & 0.0553267 & 0.724746 & 0.0381882 \\
\hline 0.156199 & 1.43029 & 0.0776472 & 0.674625 & 0.0606538 & 0.722913 & 0.0371799 \\
\hline 0.149483 & 1.43258 & 0.0740146 & 0.679693 & 0.0592796 & 0.726121 & 0.0412048 \\
\hline 0.153729 & 1.42644 & 0.0760987 & 0.683367 & 0.064441 & 0.745147 & 0.0393016 \\
\hline 0.148997 & 1.42871 & 0.0804492 & 0.678533 & 0.0530732 & 0.738521 & 0.0434697 \\
\hline 0.147587 & 1.43095 & 0.0898775 & 0.683492 & 0.0607816 & 0.749332 & 9964 \\
\hline 0.153141 & 1.42735 & 0.0733113 & 0.672703 & 0.0527252 & 0.74742 & 0.0384829 \\
\hline 0.13044 & 1.43407 & 0.0822363 & 0.666256 & 0.0661105 & 0.758117 & 0.0426639 \\
\hline 0.1327 & 1.43051 & 0.0703865 & 0.678221 & 0.0566225 & 0.763687 & 0.0439412 \\
\hline 0.143774 & 1.42891 & 0.0738019 & 0.68928 & 0.0569503 & 0.75394 & 0.0444991 \\
\hline 0.160439 & 1.42771 & 0.0750869 & 0.683008 & 0.0570962 & 0.755614 & 0.0322368 \\
\hline 0.140228 & 1.42443 & 0.0743853 & 0.6862 & 0.0564705 & 0.742444 & 0.0479825 \\
\hline 0.128434 & 1.421 & 0.064614 & 0.674476 & 0.0592512 & 0.75518 & 0.0360921 \\
\hline 0.132323 & 1.41954 & 0.0602367 & 0.677305 & 0.0547401 & 0.753817 & 0.0426651 \\
\hline 0.139347 & 1.42117 & 0.0639833 & 0.679967 & 0.0559991 & 0.748533 & 0.0390675 \\
\hline 0.151528 & 1.43177 & 0.0715197 & 0.682718 & 0.0623105 & 0.745884 & 0.037312 \\
\hline 0.137552 & 1.42573 & 0.0761374 & 0.683741 & 0.0482168 & 0.752362 & 0.0338552 \\
\hline 0.148017 & 1.42565 & 0.0755555 & 0.675258 & 0.0495927 & 0.739711 & 0.0445456 \\
\hline 0.126374 & 1.42398 & 0.0627133 & 0.677397 & 0.0512878 & 0.74545 & 0.0421443 \\
\hline 0.131907 & 1.42435 & 0.0747298 & 0.684295 & 0.0660261 & 0.737237 & 0.0358137 \\
\hline 0.124387 & 1.4239 & 0.0723194 & 0.682904 & 0.047258 & 0.736862 & 0.0375312 \\
\hline 0.113901 & 1.43056 & 0.0707082 & 0.68522 & 0.0495224 & 0.728491 & 0.0494707 \\
\hline 0.106511 & 1.43785 & 0.0728798 & 0.681095 & 0.0590283 & 0.735471 & 0.0364231 \\
\hline 0.100003 & 1.43852 & 0.0726106 & 0.691886 & 0.0540961 & 0.744929 & 0.0349661 \\
\hline 0.113532 & 1.43773 & 0.0664133 & 0.689904 & 0.0525229 & 0.738242 & 0.0434046 \\
\hline 0.115479 & 1.44136 & 0.0591987 & 0.688596 & 0.0486318 & 0.745958 & 0.0407946 \\
\hline 0.0973816 & 1.43503 & 0.066186 & 0.688698 & 0.0573378 & 0.74937 & 0.0488894 \\
\hline 0.116596 & 1.44782 & 0.0673478 & 0.693072 & 0.0527958 & 0.742546 & 0.0436018 \\
\hline
\end{tabular}




\begin{tabular}{|c|c|c|c|c|c|c|}
\hline 0.12733 & 1.46201 & 0.064085 & 0.701945 & 0.0493321 & 0.752577 & 0.0503197 \\
\hline 0.103567 & 1.45823 & 0.073576 & 0.70256 & 0.0553229 & 0.759288 & 0.0411441 \\
\hline 0.114731 & .46066 & 0.0518151 & 0.699135 & 0.0527816 & 0.76338 & 0.0416992 \\
\hline 0.0864103 & 1.46554 & 0.0577179 & 0.706642 & 0.0553796 & 0.755107 & 0.0366157 \\
\hline 0.102652 & 1.47524 & 0.0619933 & 0.70869 & 0.0532072 & 0.771793 & 0.0483243 \\
\hline 0.110633 & 1.49206 & 0.0765503 & 0.710195 & 0.0557015 & 0.777158 & 0.0490705 \\
\hline 0.108219 & 1.50115 & 0.0740523 & 0.724573 & 0.0514681 & 0.790668 & 0.0249848 \\
\hline 0.0929882 & 1.49985 & 0.0736347 & 0.721568 & 0.0577958 & 0.789826 & 0.0467696 \\
\hline 0.107978 & 1.50052 & 0.0613065 & 0.709895 & 0.0503488 & 0.795873 & 0.042744 \\
\hline 0.0921147 & 1.5048 & 0.0565175 & 0.725835 & 0.059349 & 0.797704 & 0.0501656 \\
\hline 0.109468 & 1.5107 & 0.0673994 & 0.728825 & 0.0576603 & 0.793442 & 0.0486732 \\
\hline 0.0997048 & 1.5126 & 0.0724425 & 0.728089 & 0.0557355 & 0.815747 & 0.0361851 \\
\hline 0.101014 & 1.51108 & 0.0677963 & 0.72819 & 0.0564205 & 0.810566 & 0.0509148 \\
\hline 0.0949478 & 1.52205 & 0.0771082 & 0.724842 & 0.0529003 & 0.807529 & 0.0470599 \\
\hline 0.124819 & 1.52687 & 0.081972 & 0.734444 & 0.056922 & 0.814768 & 0.0392135 \\
\hline 0.114417 & 1.52307 & 0.0782438 & 0.729813 & 0.0568382 & 0.813457 & 0.042182 \\
\hline 0.105116 & 1.53602 & 0.0827066 & 0.747336 & 0.0519527 & 0.814479 & 0.0383847 \\
\hline 0.13312 & 1.53975 & 0.0826963 & 0.748136 & 0.0631964 & 0.810587 & 0.0491775 \\
\hline 0.105803 & 1.54167 & 0.0769634 & 0.739059 & 0.0575539 & 0.812804 & 0.0396101 \\
\hline 0.141253 & 1.54945 & 0.081095 & 0.746509 & 0.0661577 & 0.812312 & 0.0389265 \\
\hline 0.119535 & 1.54151 & 0.0802948 & 0.744183 & 0.0691603 & 0.804545 & 0.0519286 \\
\hline 0.126789 & 1.5529 & 0.0767267 & 0.739053 & 0.0626825 & 0.801772 & 0.042699 \\
\hline 0.127347 & 1.55556 & 0.0695443 & 0.755672 & 0.0631265 & 0.812699 & 0.047273 \\
\hline 0.133267 & 1.55205 & 0.0736161 & 0.743988 & 0.0629309 & 0.81481 & 0.0440766 \\
\hline 0.118152 & 1.54619 & 0.07579 & 0.754696 & 0.0581053 & 0.803433 & 0.0438268 \\
\hline 0.138595 & 1.55032 & 0.0696044 & 0.754305 & 0.0597397 & 0.801877 & 0.0475628 \\
\hline 0.133465 & 1.55694 & 0.0637263 & 0.750389 & 0.0640989 & 0.797165 & 0.0493592 \\
\hline 0.128714 & 1.55885 & 0.0688473 & 0.752321 & 0.063207 & 0.793631 & 0.045193 \\
\hline 0.126074 & 1.55541 & 0.0702426 & 0.749311 & 0.0587598 & 0.801961 & 0.0436202 \\
\hline 0.125741 & 1.55698 & 0.064 & 446 & 0.0554944 & 0.799126 & 0.055086 \\
\hline 0.140655 & 1.55154 & 0.0623909 & 0.752119 & 0.0655757 & 0.801564 & 0.0424261 \\
\hline 0.127346 & 1.56089 & 0.0733978 & 0.749856 & 0.0666222 & 0.800035 & 0.0363693 \\
\hline 0.129572 & 1.56661 & 0.0684013 & 0.759447 & 0.0644025 & 0.801081 & 0.0442136 \\
\hline 0.119106 & 1.56384 & 0.0745823 & 0.759052 & 0.0639786 & 0.791772 & 0.0613219 \\
\hline 0.124878 & 1.56047 & 0.0789593 & 0.76074 & 0.0654077 & 0.796291 & 0.0487655 \\
\hline 0.120441 & 1.56229 & 0.0577207 & 0.756411 & 0.0605393 & 0.790899 & 0.0492933 \\
\hline 0.120574 & 1.56481 & 0.0613023 & 0.75915 & 0.0538275 & 0.800428 & 0.0615938 \\
\hline 0.110327 & 1.57068 & 0.0702538 & 0.761205 & 0.0597949 & 0.800733 & 0.0340748 \\
\hline 0.125751 & 1.57142 & 0.0695562 & 0.763558 & 0.0704736 & 0.806813 & 0.0513196 \\
\hline 0.116677 & 1.57608 & 0.0592985 & 0.757293 & 0.0562213 & 0.805177 & 0.0534771 \\
\hline 0.0997382 & 1.58083 & 0.0669258 & 0.763829 & 0.0583867 & 0.802575 & 0.057773 \\
\hline 0.112855 & 1.5721 & 0.0707836 & 0.766819 & 0.062463 & 0.797343 & 0.0462066 \\
\hline 0.0964331 & 1.57163 & 0.0705061 & 0.768036 & 0.0559786 & 0.807705 & 0.0461591 \\
\hline 0.120216 & 1.57596 & 0.0726533 & 0.75997 & 0.0598332 & 0.798517 & 0.0474374 \\
\hline 0.0954949 & 1.57909 & 0.0770238 & 0.781376 & 0.0550849 & 0.803479 & 0.0445884 \\
\hline 0.11373 & 1.57918 & 0.0786322 & 0.778394 & 0.0620065 & 0.808577 & 0.0468623 \\
\hline 0.0996956 & 1.56716 & 0.0721334 & 0.769866 & 0.0529928 & 0.798053 & 0.043234 \\
\hline
\end{tabular}




\begin{tabular}{|c|c|c|c|c|c|c|}
\hline 0.104135 & 1.56763 & 0.064515 & 0.779272 & 0.0519322 & 0.801878 & 0.0461123 \\
\hline 0.122797 & 1.56109 & 0.0689022 & 0.780185 & 0.0637278 & 0.807569 & 0.0451369 \\
\hline 0.130519 & 1.57561 & 0.0771475 & 0.788644 & 0.0664932 & 0.806562 & 0.0490311 \\
\hline 0.12435 & .57767 & 0.0680973 & 0.777092 & 0.0500322 & 0.803807 & 0.0412602 \\
\hline 0.110387 & 1.57506 & 0.0582271 & 0.786307 & 0.048273 & 0.7974 & 0.0400097 \\
\hline 0.107734 & 1.57339 & 0.0658025 & 0.794014 & 0.0580038 & 0.794778 & 0.0465919 \\
\hline 0.120612 & 1.57794 & 0.0636144 & 0.79036 & 0.0565468 & 0.802918 & 0.0450892 \\
\hline 0.136106 & 1.57751 & 0.0662865 & 0.79721 & 0.0677277 & 0.806416 & 0.0410566 \\
\hline 0.11363 & 1.58694 & 0.0732274 & 0.801145 & 0.053955 & 0.802711 & 0.0465478 \\
\hline 0.123941 & 1.57748 & 0.0717567 & 0.809603 & 0.0646253 & 0.807226 & 0.0501288 \\
\hline 0.142552 & 1.58299 & 0.0706363 & 0.805598 & 0.0612991 & 0.806218 & 0.0409289 \\
\hline 0.118101 & 1.58174 & 0.0585242 & 0.818462 & 0.0719463 & 0.805016 & 0.0496787 \\
\hline 0.15518 & 1.58502 & 0.0587863 & 0.818702 & 0.0603227 & 0.807084 & 0.0386712 \\
\hline 0.160735 & 1.58493 & 0.071372 & 0.830341 & 0.0655625 & 0.808609 & 0.038869 \\
\hline 0.147355 & 1.59009 & 0.0717941 & 0.824689 & 0.069106 & 0.80424 & 0.0401795 \\
\hline 0.136205 & 1.58796 & 0.0698703 & 0.830984 & 0.070482 & 0.808446 & 0.0491096 \\
\hline 0.127383 & 1.57521 & 0.0783181 & 0.824256 & 0.071608 & 0.80423 & 0.039639 \\
\hline 0.140695 & 1.56836 & 0.0732019 & 0.822285 & 0.0707647 & 0.799663 & 0.0424809 \\
\hline 0.154621 & 1.56857 & 0.0690545 & 0.828928 & 0.0536488 & 345 & 0.0494668 \\
\hline 0.145037 & 1.56341 & 0.068504 & 0.831632 & 0.075699 & 0.800727 & 0.0432205 \\
\hline 0.139299 & 1.5549 & 0.0649954 & 0.821443 & 0.065727 & 0.803284 & 0.0463589 \\
\hline 0.156506 & 1.57361 & 0.076402 & 0.829545 & 0.0597551 & 0.802543 & 9754 \\
\hline 0.118228 & 1.56426 & 0.0695018 & 0.814159 & 0.0749875 & 0.805624 & 0.0472321 \\
\hline 0.166047 & 1.56258 & 0.0655978 & 0.807905 & 0.0682965 & 0.790529 & 0.0390317 \\
\hline 0.129375 & 1.55306 & 0.0681627 & 0.805571 & 0.0529738 & 0.794593 & 0.0492651 \\
\hline 0.128036 & 1.55747 & 0.0533595 & 0.802881 & 0.0661912 & 0.798747 & 0.0382495 \\
\hline 0.153714 & 1.55044 & 0.0567079 & 0.78638 & 0.0572224 & 0.803587 & 0.0590674 \\
\hline 0.136165 & 1.55177 & 0.0594334 & 0.788948 & 0.0588802 & 0.792952 & 0.0479382 \\
\hline 0.130034 & 1.5511 & 0.0594069 & 0.799351 & 0.0520464 & 0.803411 & 0.0463048 \\
\hline 0.120909 & 1.54476 & 0.0647086 & 0.780732 & 0.0497616 & 0.797103 & 0.0429976 \\
\hline 0.121381 & 1.54006 & 0.0723961 & 0.796192 & 0.0523929 & 0.805882 & 0.0503047 \\
\hline 0.122273 & 1.54432 & 0.0712991 & 0.781112 & 0.0511034 & 0.800564 & 0.0374798 \\
\hline 0.116813 & 1.53103 & 0.0677252 & 0.781111 & 0.0421346 & 0.795236 & 0.0468855 \\
\hline 0.121112 & 1.53726 & 0.0651253 & 0.767379 & 0.0506091 & 0.790283 & 0.0436326 \\
\hline 0.107763 & 1.53675 & 0.0654749 & 0.767135 & 0.0460797 & 0.791099 & 0.0462539 \\
\hline 0.0930421 & 1.53696 & 0.0667607 & 0.763834 & 0.0415229 & 0.79101 & 0.037477 \\
\hline 0.128549 & 1.53587 & 0.0634537 & 0.779912 & 0.0491864 & 0.78892 & 0.0345875 \\
\hline 0.108321 & 1.53982 & 0.0550708 & 0.781744 & 0.0422293 & 0.776443 & 0.0395642 \\
\hline 0.115789 & 1.53355 & 0.0654397 & 0.764008 & 0.0463258 & 0.799896 & 0.0520658 \\
\hline 0.133302 & 1.52896 & 0.0613919 & 0.777059 & 0.0389091 & 0.797168 & 0.045362 \\
\hline 0.109531 & 1.5335 & 0.0576506 & 0.768997 & 0.0425294 & 0.789252 & 0.0458159 \\
\hline 0.101351 & 1.53498 & 0.0557406 & 0.777057 & 0.0447304 & 0.779545 & 0.0417192 \\
\hline 0.12186 & 1.53502 & 0.0617743 & 0.770315 & 0.0480907 & 0.798378 & 0.0417272 \\
\hline 0.127702 & 1.53488 & 0.0604936 & 0.771572 & 0.0489533 & 0.791945 & 0.0410373 \\
\hline 0.12629 & 1.53853 & 0.0555329 & 0.765965 & 0.0484983 & 0.780523 & 0.0374345 \\
\hline 0.121473 & 1.53337 & 0.0666688 & 0.766403 & 0.0452696 & 0.793591 & 0.0415964 \\
\hline 0.117611 & 1.53945 & 0.0588993 & 0.771906 & 0.0488304 & 0.783305 & 0.0399279 \\
\hline
\end{tabular}




\begin{tabular}{|c|c|c|c|c|c|c|}
\hline 0.134219 & 1.53108 & 0.0667765 & 0.763935 & 0.0523155 & 0.785684 & 0.0445043 \\
\hline 0.134347 & 1.52848 & 0.0701449 & 0.769154 & 0.0435145 & 0.80099 & 0.0461264 \\
\hline 0.125737 & .54123 & 0.0624605 & 0.772706 & 0.0464552 & 0.793613 & 0.0459674 \\
\hline 0.0937242 & 1.5386 & 0.0581081 & 0.778956 & 0.0550613 & 0.811638 & 0.0466851 \\
\hline 0.107025 & 1.53422 & 0.0558564 & 0.778468 & 0.0635104 & 0.802683 & 0.0372577 \\
\hline 0.102598 & 1.53267 & 0.0518984 & 0.777767 & 0.0522141 & 0.811126 & 0.0515974 \\
\hline 0.124164 & 1.532 & 0.0609812 & 0.775592 & 0.0492324 & 0.813369 & 0.0538791 \\
\hline 0.113545 & 1.53864 & 0.0635382 & 0.792342 & 0.0571504 & 0.822516 & 0.050985 \\
\hline 0.0962854 & 1.52811 & 0.0605505 & 0.780608 & 0.0580237 & 0.823679 & 0.0382978 \\
\hline 0.0916729 & 1.5312 & 0.0654952 & 0.782932 & 0.0491395 & 0.820683 & 0.0472139 \\
\hline 0.122175 & 1.52481 & 0.0703936 & 0.782882 & 0.0525794 & 0.834329 & 0.0486966 \\
\hline 0.119316 & 1.53179 & 0.0608043 & 0.787682 & 0.0552238 & 0.838448 & 0.0552162 \\
\hline 0.108993 & 1.53094 & 0.0643771 & 0.791934 & 0.0524261 & 0.836264 & 0.0475845 \\
\hline 0.122923 & 1.53292 & 0.0561144 & 0.793335 & 0.0630411 & 0.83189 & 0.0424029 \\
\hline 0.126043 & 1.53059 & 0.0593528 & 0.791985 & 0.0656933 & 0.840648 & 0.0534698 \\
\hline 0.117316 & 1.52673 & 0.0678479 & 0.797435 & 0.0629854 & 0.841905 & 0.0488257 \\
\hline 0.0966087 & 1.5197 & 0.0673275 & 0.798116 & 0.0680535 & 0.846002 & 0.0545543 \\
\hline 0.111149 & 1.51803 & 0.0700699 & 0.790168 & 0.0661879 & 0.824411 & 0.0635993 \\
\hline 0.117772 & 1.50372 & 0.0607611 & 0.793897 & 0.060715 & 0.849549 & 0.0600047 \\
\hline 0.117138 & 1.50433 & 0.0510343 & 0.796367 & 0.0632465 & 0.836271 & 0.0531544 \\
\hline 0.102703 & 1.50482 & 0.0547119 & 0.782362 & 0.0533161 & 0.829873 & 0.0651703 \\
\hline 0.119977 & 1.5075 & 0.061281 & 0.777732 & 0.0595509 & 0.824838 & 0.0545876 \\
\hline 0.105531 & 1.50015 & 0.0587242 & 0.776193 & 0.0529735 & 0.824902 & 0.0551116 \\
\hline 0.126841 & 1.49402 & 0.0558405 & 0.773407 & 0.0636627 & 0.805467 & 0.056041 \\
\hline 0.116562 & 1.49149 & 0.0517557 & 0.770556 & 0.0578345 & 0.797621 & 0.0499634 \\
\hline 0.111971 & 1.49072 & 0.0596235 & 0.767164 & 0.0475061 & 0.801479 & 0.0592235 \\
\hline 0.109037 & 1.48207 & 0.0596705 & 0.758278 & 0.0498877 & 0.794264 & 0.0599751 \\
\hline 0.104749 & 1.49471 & 0.0559577 & 0.763949 & 0.0481993 & 0.779729 & 0.0542444 \\
\hline 0.109255 & 1.48922 & 0.0521746 & 0.757874 & 0.0414251 & 0.77721 & 0.0485145 \\
\hline 0.11438 & 1.48055 & 0.047977 & 0.753478 & 0.0453979 & 0.781762 & 0.0607356 \\
\hline 0.0945218 & 1.47757 & 0.0621726 & 0.748391 & 0.0484535 & 0.771867 & 0.0477799 \\
\hline 0.10174 & 1.4862 & 0.062023 & 0.75371 & 0.0499029 & 0.761806 & 0.0504145 \\
\hline 0.107972 & 1.47955 & 0.0602048 & 0.744635 & 0.0568326 & 0.764948 & 0.0422283 \\
\hline 0.123813 & 1.46933 & 0.0607988 & 0.752556 & 0.0496103 & 0.756094 & 0.0409968 \\
\hline 0.0952014 & 1.46772 & 0.0541214 & 0.756894 & 0.0556572 & 0.763287 & 0.0471034 \\
\hline 0.135145 & 1.46862 & 0.0547373 & 0.74769 & 0.0519924 & 0.753339 & 0.0411459 \\
\hline 0.103407 & 1.46153 & 0.0588223 & 0.746542 & 0.054731 & 0.753225 & 0.0422874 \\
\hline 0.107641 & 1.47377 & 0.0507262 & 0.739047 & 0.0503094 & 0.760197 & 0.0576463 \\
\hline 0.118156 & 1.48108 & 0.0490915 & 0.736207 & 0.051418 & 0.760412 & 0.0445954 \\
\hline 0.0897647 & 1.46726 & 0.0548435 & 0.744451 & 0.0524598 & 0.760693 & 0.0371283 \\
\hline 0.109571 & 1.44943 & 0.0502261 & 0.741 & 0.0580138 & 0.741443 & 0.0387979 \\
\hline 0.117554 & 1.45583 & 0.0599534 & 0.740248 & 0.0566227 & 0.755355 & 0.0435005 \\
\hline 0.104986 & 1.46923 & 0.059348 & 0.734245 & 0.0505046 & 0.745192 & 0.0413415 \\
\hline 0.119706 & 1.46739 & 0.0600514 & 0.738171 & 0.0551344 & 0.747067 & 0.0438906 \\
\hline 0.100902 & 1.46567 & 0.0590657 & 0.739293 & 0.0547999 & 0.746533 & 0.0405718 \\
\hline 0.116636 & 1.45815 & 0.0585188 & 0.742295 & 0.0559217 & 0.746096 & 0.0447626 \\
\hline 0.116317 & 1.44938 & 0.0560432 & 0.738646 & 0.0473141 & 0.752568 & 0.0391208 \\
\hline
\end{tabular}




\begin{tabular}{|c|c|c|c|c|c|c|}
\hline 0.114914 & 1.45371 & 0.0545629 & 0.732818 & 0.0504899 & 0.745195 & 0.0398837 \\
\hline 0.130613 & 1.45288 & 0.0580172 & 0.737833 & 0.0430191 & 0.745103 & 0.042018 \\
\hline 0.120375 & 1.4571 & 0.0584201 & 0.731133 & 0.0615085 & 0.740647 & 0.037223 \\
\hline 0.117448 & 1.45596 & 0.0553873 & 0.737333 & 0.0544944 & 0.736876 & 0.0447853 \\
\hline 0.120181 & 1.4581 & 0.0629995 & 0.73952 & 0.0488411 & 0.741673 & 0.0396074 \\
\hline 0.13542 & 1.4483 & 0.0562951 & 0.733849 & 0.0502776 & 0.741609 & 0.0463985 \\
\hline 0.123395 & 1.4526 & 0.0526361 & 0.723043 & 0.0559598 & 0.741791 & 0.0439341 \\
\hline 0.109578 & 1.45505 & 0.0588559 & 0.722679 & 0.0531836 & 0.754304 & 0.0444128 \\
\hline 0.116429 & 1.45333 & 0.0628687 & 0.732335 & 0.0530789 & 0.738958 & 0.0388653 \\
\hline 0.127539 & 1.45306 & 0.0606069 & 0.726365 & 0.0531308 & 0.745253 & 0.0372756 \\
\hline 0.141349 & 1.44904 & 0.0611978 & 0.735287 & 0.0441106 & 0.748884 & 0.0383395 \\
\hline 0.110686 & 1.45304 & 0.0702767 & 0.726486 & 0.0423018 & 0.727948 & 0.0312685 \\
\hline 0.119289 & 1.44245 & 0.0610482 & 0.716734 & 0.0449344 & 0.738494 & 0.0375602 \\
\hline 0.12643 & 1.43848 & 0.0584353 & 0.731278 & 0.0486147 & 0.731843 & 0.0337077 \\
\hline 0.133294 & 1.44612 & 0.0598928 & 0.72637 & 0.0554235 & 0.735571 & 0.038458 \\
\hline 0.120555 & 1.44431 & 0.0626223 & 0.722173 & 0.051607 & 0.726496 & 0.0358794 \\
\hline 0.116849 & 1.44583 & 0.0622106 & 0.733304 & 0.048825 & 0.727046 & 0.0330033 \\
\hline 0.127674 & 1.45108 & 0.0608255 & 0.727789 & 0.0406132 & 0.722478 & 0.0422693 \\
\hline 0.1346 & 1.44883 & 0.0625487 & 0.725192 & 0.0427397 & 0.726108 & 0.0300383 \\
\hline 0.118696 & 1.44524 & 0.0556799 & 0.727931 & 0.0503624 & 0.715039 & 0.0359449 \\
\hline 0.119205 & 1.44488 & 0.0528755 & 0.722378 & 0.0402644 & 0.715462 & 0.0388813 \\
\hline 0.127663 & 1.44211 & 0.0530483 & 0.725321 & 0.0457366 & 0.730783 & 0.0328245 \\
\hline 0.113933 & 1.43794 & 0.0603954 & 0.71587 & 0.0477017 & 0.722759 & 0.0314694 \\
\hline 0.133134 & 1.44337 & 0.0675583 & 0.72142 & 0.0503917 & 0.722292 & 0.032909 \\
\hline 0.123874 & 1.44552 & 0.0547399 & 0.717727 & 0.0502287 & 0.727508 & 0.0411293 \\
\hline 0.117315 & 1.44608 & 0.0547304 & 0.724234 & 0.0438371 & 0.726391 & 0.0338682 \\
\hline 0.114393 & 1.44548 & 0.054883 & 0.715634 & 0.0552907 & 0.715543 & 0.0355487 \\
\hline 0.112568 & 1.44961 & 0.0563291 & 0.719113 & 0.0427315 & 0.718196 & 0.0401164 \\
\hline 0.109852 & 1.45263 & 0.0577055 & 0.716042 & 0.0480262 & 0.722044 & 0.0371636 \\
\hline 0.10579 & 1.44504 & 0.055743 & 0.714085 & 0.0525491 & 0.719988 & 0.027324 \\
\hline 0.106494 & 1.44864 & 0.057704 & 0.724451 & 0.0407932 & 0.723548 & 0.0384499 \\
\hline 0.106722 & 1.44982 & 0.0552133 & 0.720981 & 0.0472071 & 0.712051 & 0.0356444 \\
\hline 0.112546 & 1.44288 & 0.0518074 & 0.715406 & 0.0451563 & 0.718495 & 0.0349925 \\
\hline 0.101032 & 1.44106 & 0.0511899 & 0.725979 & 0.0548072 & 0.716048 & 0.0369409 \\
\hline 0.100331 & 1.44964 & 0.0512486 & 0.713557 & 0.0386812 & 0.729548 & 0.0321596 \\
\hline 0.108138 & 1.44274 & 0.0471421 & 0.715368 & 0.0395173 & 0.722097 & 0.0320038 \\
\hline 0.112448 & 1.43545 & 0.0531765 & 0.716685 & 0.0491949 & 0.726867 & 0.0388908 \\
\hline 0.10246 & 1.44043 & 0.0523568 & 0.708658 & 0.0399995 & 0.725204 & 0.0323259 \\
\hline 0.090305 & 1.44907 & 0.0527732 & 0.711863 & 0.0516793 & 0.716204 & 0.0326056 \\
\hline 0.106735 & 1.4467 & 0.0535375 & 0.712235 & 0.0487612 & 0.717183 & 0.0333776 \\
\hline 0.118077 & 1.44343 & 0.0608723 & 0.717225 & 0.0433395 & 0.72088 & 0.041923 \\
\hline 0.096478 & 1.45058 & 0.0523891 & 0.713871 & 0.0460109 & 0.712876 & 0.042581 \\
\hline 0.0832042 & 1.44363 & 0.0508161 & 0.711503 & 0.0473238 & 0.722856 & 0.0366014 \\
\hline 0.117023 & 1.43842 & 0.0532124 & 0.701758 & 0.0463577 & 0.711417 & 0.0455133 \\
\hline 0.0926013 & 1.44248 & 0.0579004 & 0.714115 & 0.0435705 & 0.717366 & 0.0452398 \\
\hline 0.100939 & 1.44724 & 0.0596303 & 0.713043 & 0.0452317 & 0.72077 & 0.0391757 \\
\hline 0.120275 & 1.44093 & 0.0566159 & 0.711543 & 0.0464278 & 0.708272 & 0.0392626 \\
\hline
\end{tabular}




\begin{tabular}{|c|c|c|c|c|c|c|}
\hline 0.0939606 & 1.44359 & 0.0616014 & 0.708812 & 0.0369639 & 0.721427 & 0.03 \\
\hline 0.0842214 & 1.45007 & 0.062405 & 0.70272 & 0.0426486 & 0.708915 & 0.0300002 \\
\hline 0.0999885 & 1.43869 & 0.0611387 & 0.715645 & 0.0480288 & 0.703048 & 0.0322299 \\
\hline 0.110293 & 1.4322 & 0.0607602 & 0.707316 & 0.0478565 & 0.70996 & 0.0336835 \\
\hline 0.0776421 & 1.43642 & 0.056165 & 0.712483 & 0.0442152 & 0.712322 & 0.0482027 \\
\hline 0.0915889 & 1.44318 & 0.0667222 & 0.712262 & 0.0404747 & 0.706357 & 0.0360474 \\
\hline 0.086063 & 1.4446 & 0.0705593 & 0.713571 & 0.0413902 & 0.709612 & 0.0293688 \\
\hline 0.101992 & 1.43383 & 0.0660199 & 0.703638 & 0.0456606 & 0.714095 & 0.0390504 \\
\hline 0.0746534 & 1.43732 & 0.0646394 & 0.70297 & 0.0426774 & 0.713744 & 0.04198 \\
\hline 0.0855542 & 1.44026 & 0.0710845 & 0.712357 & 0.0462822 & 0.720351 & 0.0346563 \\
\hline 0.108161 & 1.44099 & 0.0664617 & 0.706174 & 0.0451315 & 0.707926 & 0.0366404 \\
\hline 0.101956 & 1.44192 & 0.0636711 & 0.70777 & 0.0499448 & 0.708499 & 0.0393154 \\
\hline 0.0910303 & 1.43313 & 0.0686477 & 0.711913 & 0.0403623 & 0.708991 & 0.0369952 \\
\hline 0.0939386 & 1.43238 & 0.0681786 & 0.706604 & 0.039825 & 0.704686 & 0.0365297 \\
\hline 0.0945612 & 1.43492 & 0.0659388 & 0.709312 & 0.0498011 & 0.704779 & 0.0271222 \\
\hline 0.0961785 & 1.43405 & 0.0602211 & 0.704673 & 0.0329015 & 0.711034 & 0.047597 \\
\hline 0.0952835 & 1.43015 & 0.0538639 & 0.709054 & 0.0407793 & 0.715312 & 0.0391871 \\
\hline 0.0975665 & 1.42829 & 0.0679791 & 0.699821 & 0.034378 & 0.707416 & 0.0311132 \\
\hline 0.0833344 & 1.42968 & 0.0701344 & 0.706547 & 0.0406763 & 0.704723 & 0.0428949 \\
\hline 0.0986814 & 1.43243 & 0.0627319 & 0.700816 & 0.0352045 & 0.704986 & 0.0397759 \\
\hline 0.102459 & 1.42978 & 0.063488 & 0.701931 & 0.042045 & 0.709106 & 0.0370273 \\
\hline 0.102086 & 1.43046 & 0.0650735 & 0.698764 & 0.0430619 & 0.708581 & 0.0397009 \\
\hline 0.0907508 & 1.42747 & 0.0607949 & 0.70484 & 0.0438915 & 0.705243 & 0.0355643 \\
\hline 0.084617 & 1.42464 & 0.0593368 & 0.694686 & 0.0365648 & 0.701459 & 0.0341421 \\
\hline 0.0800393 & 1.42835 & 0.055 & 0.71293 & 0.03 & 0.702776 & 0.0411242 \\
\hline 0.0893979 & 1.43225 & 0.0626138 & 0.701373 & 0.0441493 & 0.715643 & 0.0378789 \\
\hline 0.0848255 & 1.43469 & 0.0676252 & 0.708654 & 0.034705 & 0.705705 & 0.0321479 \\
\hline 0.10123 & 1.42808 & 0.0608416 & 0.713249 & 0.0427223 & 0.70646 & 0.0341822 \\
\hline 0.0834103 & 1.43078 & 0.0573655 & 0.70319 & 0.0415474 & 0.713435 & 0.0447263 \\
\hline 0.08442 & 1.43152 & 0.0618994 & 0.707951 & 0.0414091 & 0.706478 & 0.0435314 \\
\hline 0.092184 & 1.43013 & 0.0700764 & 0.698384 & 0.0468413 & 0.711652 & 0.0388516 \\
\hline 0.0847422 & 1.42726 & 0.0681325 & 0.703888 & 0.0408153 & 0.706329 & 0.0365042 \\
\hline 0.10528 & 1.42626 & 0.0660946 & 0.716766 & 0.045921 & 0.710823 & 0.0448864 \\
\hline 0.088319 & 1.43071 & 0.066968 & 0.698231 & 0.0444724 & 0.697274 & 0.040783 \\
\hline 0.0879489 & 1.42432 & 0.0626167 & 0.70237 & 0.040989 & 0.719305 & 0.0316244 \\
\hline 0.0747808 & 1.42808 & 0.0545267 & 0.708323 & 0.044114 & 0.703831 & 0.0297215 \\
\hline 0.107153 & 1.43291 & 0.0533226 & 0.701672 & 0.0456043 & 0.710519 & 0.0349561 \\
\hline 0.0922435 & 1.43258 & 0.0547848 & 0.706459 & 0.0466093 & 0.70611 & 0.0350053 \\
\hline 0.0946685 & 1.43331 & 0.0627637 & 0.70808 & 0.0395232 & 0.709816 & 0.0433641 \\
\hline 0.0831862 & 1.42854 & 0.0692448 & 0.694842 & 0.0477242 & 0.717435 & 0.0387902 \\
\hline 0.0808928 & 1.42404 & 0.067044 & 0.708871 & 0.0436945 & 0.706019 & 0.0503785 \\
\hline 0.0936033 & 1.42579 & 0.0651897 & 0.710633 & 0.0454222 & 0.714552 & 0.0438861 \\
\hline 0.0909978 & 1.4285 & 0.0662643 & 0.701262 & 0.0507984 & 0.702076 & 0.0301835 \\
\hline 0.0899109 & 1.4258 & 0.0579193 & 0.710524 & 0.0468911 & 0.70807 & 0.0410478 \\
\hline 0.0869359 & 1.4187 & 0.0516015 & 0.704519 & 0.0323442 & 0.705759 & 0.0343498 \\
\hline 0.102791 & 1.41786 & 0.0571067 & 0.704325 & 0.038557 & 0.69322 & 0.0435681 \\
\hline 0.103897 & 1.4193 & 0.0615703 & 0.6882 & 0.039008 & 0.70776 & 0.0305519 \\
\hline
\end{tabular}




\begin{tabular}{|c|c|c|c|c|c|c|}
\hline 0.111141 & 1.41779 & 0.0572319 & 0.702602 & 0.0407582 & 0.707954 & 0.0361382 \\
\hline 0.0930845 & 1.42381 & 0.0564567 & 0.710208 & 0.0353545 & 0.69587 & 0.0412692 \\
\hline 0.100337 & 1.42428 & 0.058445 & 0.711408 & 0.0352387 & 0.696859 & 0.041147 \\
\hline 0.0970268 & 1.42523 & 0.0537068 & 0.708877 & 0.0526105 & 0.697988 & 0.0345394 \\
\hline 0.0934533 & 1.42188 & 0.05259 & 0.688264 & 0.0354081 & 0.690446 & 0.0323362 \\
\hline 0.102269 & 1.41116 & 0.0492129 & 0.700696 & 0.0445768 & 0.691817 & 0.0348633 \\
\hline 0.103097 & 1.41164 & 0.0474799 & 0.704712 & 0.0420848 & 0.698726 & 0.0396029 \\
\hline 0.0917362 & 1.41244 & 0.0506337 & 0.704338 & 0.0347312 & 0.698793 & 0.0304982 \\
\hline 0.0870884 & 1.41048 & 0.047564 & 0.710483 & 0.0362231 & 0.694688 & 0.0396707 \\
\hline 0.0914956 & 1.40736 & 0.0410239 & 0.701478 & 0.0327707 & 0.701924 & 0.0396269 \\
\hline 0.0828858 & 1.40345 & 0.0459529 & 0.705594 & 0.0414773 & 0.689736 & 0.0389484 \\
\hline 0.0814288 & 1.40104 & 0.0569702 & 0.698025 & 0.0385372 & 0.68755 & 0.0389658 \\
\hline 0.0839852 & 1.40193 & 0.0569253 & 0.696273 & 0.0389343 & 0.677546 & 0.0379909 \\
\hline 0.0635735 & 1.40638 & 0.0517255 & 0.699004 & 0.0446172 & 0.68621 & 0.0382456 \\
\hline 0.0941396 & 1.40429 & 0.0524744 & 0.697212 & 0.0332009 & 0.689365 & 0.0387463 \\
\hline 0.0809527 & 1.39767 & 0.0545313 & 0.686953 & 0.0435552 & 0.683576 & 0.0422947 \\
\hline 0.066298 & 1.39922 & 0.0539372 & 0.68505 & 0.0411085 & 0.6898 & 0.0387223 \\
\hline 0.085098 & 1.40097 & 0.0496372 & 0.689762 & 0.0285716 & 0.683834 & 0.0367775 \\
\hline 0.0920013 & 1.39478 & 0.0478433 & 0.690435 & 0.03 & 0.681249 & 0.0302107 \\
\hline 0.0750664 & 1.39054 & 0.0516981 & 0.682171 & 0.0425431 & 0.688506 & 0.0430949 \\
\hline 0.075285 & 1.38561 & 0.0553938 & 0.676238 & 0.0315671 & 0.67548 & 0.036499 \\
\hline 0.0833774 & 1.37985 & 0.0547451 & 0.689547 & 0.0 & 0.680883 & 0.0356976 \\
\hline 0.101601 & 1.37685 & 0.0480913 & 0.686722 & 0.0427786 & 0.672187 & 0.0353369 \\
\hline 0.0944045 & 1.377 & 0.0458378 & 0.683823 & 0.036541 & 0.676625 & 0.0382414 \\
\hline 0.0918071 & 1.3787 & 0.047658 & 0.684354 & 0.0384494 & 0.682983 & 0.0410309 \\
\hline 0.0989941 & 1.3807 & 0.0481636 & 0.69172 & 0.0399527 & 0.667443 & 0.0365098 \\
\hline 0.0720013 & 1.3806 & 0.0509647 & 0.686749 & 0.0380663 & 0.678116 & 0.0380251 \\
\hline 0.0953796 & 1.37743 & 0.0477988 & 0.674093 & 0.0368449 & 0.666854 & 0.0437418 \\
\hline 0.0773762 & 1.3812 & 0.0433423 & 0.684769 & 0.0424707 & 0.669187 & 0.033523 \\
\hline 0.0856515 & 1.37822 & 0.049098 & 0.693087 & 1588 & 0.672567 & 0.0362368 \\
\hline 0.0839852 & 1.36886 & 0.0499625 & 0.674342 & 0.0376929 & 0.669445 & 0.0304569 \\
\hline 0.102915 & 1.36196 & 0.048627 & 0.687362 & 0.038992 & 0.670334 & 0.0345338 \\
\hline 0.0890668 & 1.3511 & 0.0485462 & 0.678928 & 0.0396002 & 0.671008 & 0.0357837 \\
\hline 0.100101 & 1.35325 & 0.0461845 & 0.683736 & 0.0288632 & 0.675785 & 0.0338475 \\
\hline 0.100848 & 1.35538 & 0.0447136 & 0.680473 & 0.0370245 & 0.676351 & 0.0387953 \\
\hline 0.0958809 & 1.34795 & 0.0417582 & 0.679801 & 0.0389532 & 0.663388 & 0.0336504 \\
\hline 0.0869473 & 1.34859 & 0.0397262 & 0.674091 & 0.0345715 & 0.668891 & 0.03388 \\
\hline 0.0900176 & 1.35188 & 0.0453627 & 0.683908 & 0.0347493 & 0.665998 & 0.0431302 \\
\hline 0.0965016 & 1.34831 & 0.0438496 & 0.677492 & 0.0345978 & 0.667345 & 0.0403247 \\
\hline 0.111044 & 1.34279 & 0.0431634 & 0.678682 & 0.0417489 & 0.659188 & 0.0260266 \\
\hline 0.102288 & 1.34259 & 0.0501032 & 0.668356 & 0.0322185 & 0.649725 & 0.0395195 \\
\hline 0.115255 & 1.34077 & 0.0511664 & 0.667002 & 0.0371394 & 0.664454 & 0.0306287 \\
\hline 0.111153 & 1.33221 & 0.0490672 & 0.676557 & 0.0310664 & 0.654895 & 0.0343323 \\
\hline 0.115234 & 1.32616 & 0.0439368 & 0.670007 & 0.043694 & 0.658756 & 0.0397719 \\
\hline 0.116095 & 1.3326 & 0.0443122 & 0.675206 & 0.0325685 & 0.648842 & 0.0350391 \\
\hline 0.117553 & 1.32808 & 0.0458225 & 0.666336 & 0.0370152 & 0.660341 & 0.0389992 \\
\hline 0.110593 & 1.32214 & 0.0471637 & 0.662234 & 0.0393488 & 0.655538 & 0.033655 \\
\hline
\end{tabular}




\begin{tabular}{|c|c|c|c|c|c|c|}
\hline 0.121764 & 1.32455 & 0.0474255 & 0.662765 & 0.0386788 & 0.651129 & 5824 \\
\hline 0.13066 & 1.31344 & 0.0465202 & 0.662807 & 0.0352626 & 0.658852 & 0.0350145 \\
\hline 0.124806 & 1.31236 & 0.0548358 & 0.660699 & 0.0387252 & 0.648503 & 0.0451047 \\
\hline 0.116169 & 1.31701 & 0.0440938 & 0.664908 & 0.0303001 & 0.645317 & 0.0342461 \\
\hline 0.132494 & 1.30741 & 0.0370866 & 0.657695 & 0.034679 & 0.646222 & 0.0332672 \\
\hline 0.12832 & 1.29996 & 0.0426088 & 0.659454 & 0.0404995 & 0.65101 & 0.0331829 \\
\hline 0.100938 & 1.29832 & 0.0400102 & 0.64918 & 0.0397684 & 0.655495 & 0.0382063 \\
\hline 0.114661 & 1.29391 & 0.0422849 & 0.650616 & 0.0367764 & 0.640721 & 0.0362784 \\
\hline 0.142009 & 1.29404 & 0.0455336 & 0.657215 & 0.0303267 & 0.639142 & 0.0329983 \\
\hline 0.102742 & 1.29112 & 0.0514845 & 0.656295 & 0.0369011 & 0.640073 & 0.0234456 \\
\hline 0.102448 & 1.28606 & 0.0429055 & 0.648343 & 0.0374234 & 0.640201 & 0.0316082 \\
\hline 0.112877 & 1.2886 & 0.0368601 & 0.645493 & 0.0417361 & 0.628811 & 0.0371566 \\
\hline 0.120204 & 1.29091 & 0.0439749 & 0.649132 & 0.0356741 & 0.637475 & 0.0361977 \\
\hline 0.0937429 & 1.29 & 0.0448191 & 0.653296 & 0.0358702 & 0.639892 & 0.0324678 \\
\hline 0.132092 & 1.28949 & 0.0463644 & 0.645241 & 0.0392723 & 0.632864 & 0.0388697 \\
\hline 0.105841 & 1.28256 & 0.0475854 & 0.645778 & 0.0430778 & 0.633163 & 0.0311302 \\
\hline 0.109382 & 1.27832 & 0.0485041 & 0.643876 & 0.0305927 & 0.623358 & 0.0297944 \\
\hline 0.0986863 & 1.27303 & 0.0477047 & 0.649457 & 0.0434509 & 0.641232 & 0.0300353 \\
\hline 0.0848537 & 1.26334 & 0.0446719 & 0.630073 & 0.0262246 & 0.623468 & 0.0278704 \\
\hline 0.107542 & 1.26619 & 0.0495323 & 0.634629 & 0.0387835 & 0.620549 & 0.0265695 \\
\hline 0.10933 & 1.27555 & 0.0463816 & 0.632629 & 0.0366656 & 0.628093 & 0.034993 \\
\hline 0.0767579 & 1.27203 & 0.0474896 & 0.636568 & 0.0336887 & 0.623285 & 0.0316096 \\
\hline 0.123485 & 1.26655 & 0.0452769 & 0.634687 & 0.0343284 & 0.630536 & 0.0317789 \\
\hline 0.101539 & 1.26389 & 0.0453247 & 0.638589 & 0.0289661 & 0.621599 & 0.0355444 \\
\hline 0.120757 & 1.26292 & 345 & 129 & 0.03 & 0.626974 & 0.02 \\
\hline 0.121374 & 1.26179 & 0.0491146 & 0.634949 & 0.0406403 & 0.628314 & 0.0285436 \\
\hline 0.139132 & 1.26725 & 0.0462614 & 0.63457 & 0.0361106 & 0.621101 & 0.0290496 \\
\hline 0.14786 & 1.26181 & 0.0423614 & 0.632954 & 0.0388922 & 0.632231 & 0.0355163 \\
\hline 0.171164 & 1.25182 & 0.0397695 & 0.63022 & 0.038676 & 0.631681 & 0.0373888 \\
\hline 0.193526 & 1.2578 & 0.0410193 & 0.627513 & 0.0356612 & 0.646315 & 0.0312616 \\
\hline 0.247582 & 1.25569 & 0.0404354 & 0.637804 & 0.0337322 & 0.63748 & 0.0302564 \\
\hline 0.263696 & 1.2572 & 0.0432561 & 0.635656 & 0.0329598 & 0.634947 & 0.0400308 \\
\hline 0.301069 & 1.26035 & 0.0485941 & 0.632457 & 0.0323479 & 0.636765 & 0.0330026 \\
\hline 0.367921 & 1.25187 & 0.0477282 & 0.620578 & 0.0443002 & 0.642379 & 0.0297983 \\
\hline 0.457773 & 1.24381 & 0.0462035 & 0.62931 & 0.036889 & 0.644444 & 0.0301814 \\
\hline 0.544134 & 1.23931 & 0.0447766 & 0.626925 & 0.0383305 & 0.645415 & 0.0239017 \\
\hline 0.61111 & 1.23966 & 0.0479479 & 0.637331 & 0.0379938 & 0.643675 & 0.0293549 \\
\hline 0.775703 & 1.23323 & 0.0394006 & 0.633383 & 0.037907 & 0.647648 & 0.0247844 \\
\hline 0.905482 & 1.22799 & 0.0444086 & 0.631661 & 0.0344462 & 0.649411 & 0.0333066 \\
\hline 1.03042 & 1.22743 & 0.0482201 & 0.623174 & 0.0399218 & 0.642735 & 0.0403567 \\
\hline 1.25979 & 1.22959 & 0.043825 & 0.630183 & 0.0394075 & 0.644019 & 0.0336604 \\
\hline 1.45157 & 1.22922 & 0.049796 & 0.621751 & 0.0327154 & 0.654031 & 0.0309568 \\
\hline 1.64597 & 1.22865 & 0.042481 & 0.623846 & 0.0332633 & 0.648715 & 0.0330369 \\
\hline 1.8613 & 1.22818 & 0.0372315 & 0.623411 & 0.0290942 & 0.64805 & 0.0314094 \\
\hline 2.05954 & 1.22641 & 0.0446238 & 0.622175 & 0.0322717 & 0.650809 & 0.0302424 \\
\hline 2.30303 & 1.22634 & 0.0430132 & 0.61582 & 0.0371494 & 0.647098 & 0.0301503 \\
\hline 2.52664 & 1.22403 & 0.0382491 & 0.618534 & 0.0414334 & 0.646057 & 0.037371 \\
\hline
\end{tabular}




\begin{tabular}{|c|c|c|c|c|c|c|}
\hline 2.64708 & 1.21732 & 0.0387648 & 0.619539 & 0.0381463 & 0.647917 & 0.0427563 \\
\hline 2.85552 & 1.21144 & 0.0426931 & 0.615855 & 0.0314918 & 0.648256 & 0.0295209 \\
\hline 2.9436 & 1.21871 & 0.0484709 & 0.615472 & 0.0280206 & 0.65567 & 0.0374932 \\
\hline 3.13415 & 1.21172 & 0.0442949 & 0.618943 & 0.0351935 & 0.652338 & 0.0358214 \\
\hline 3.18473 & .20188 & 0.038266 & 0.615694 & 0.0382387 & 0.645687 & 0.0320862 \\
\hline 3.27805 & 1.20438 & 0.0406414 & 0.621583 & 0.0295541 & 0.645814 & 0.0329423 \\
\hline 3.1794 & 1.1977 & 0.0452878 & 0.621093 & 0.0400778 & 0.63877 & 0.0351166 \\
\hline 3.2049 & 1.19896 & 0.0412365 & 0.612823 & 0.031728 & 0.638136 & 0.0407829 \\
\hline 3.15581 & 1.2016 & 0.0433144 & 0.614414 & 0.0375489 & 0.649966 & 0.0302774 \\
\hline 2.92544 & 1.19239 & 0.0420737 & 0.606068 & 0.0338892 & 0.640013 & 0.0346894 \\
\hline 2.81208 & 1.18456 & 0.0387743 & 0.615071 & 0.0338894 & 0.62722 & 0.0347962 \\
\hline 2.62761 & 1.18989 & 0.0387616 & 0.60626 & 0.0335584 & 0.636043 & 0.0233067 \\
\hline 2.42302 & 1.18603 & 0.0414131 & 0.605911 & 0.0358204 & 0.635739 & 0.0424043 \\
\hline 2.20952 & 1.18289 & 0.0399852 & 0.608238 & 0.0375094 & 0.627804 & 0.0275227 \\
\hline 2.06231 & 1.18746 & 0.0394933 & 0.604042 & 0.0369318 & 0.608782 & 0.0269517 \\
\hline 1.70888 & 1.18411 & 0.0347292 & 0.600434 & 0.0302122 & 0.613611 & 0.0269963 \\
\hline 1.55105 & 1.18499 & 0.0350125 & 0.600592 & 0.0424811 & 0.610043 & 0.0363631 \\
\hline 1.32821 & 1.18179 & 0.0376538 & 0.598649 & 0.0296819 & 0.60587 & 0.0358204 \\
\hline 1.13807 & 1.18205 & 0.0403792 & 0.586968 & 0.0266326 & 0.596864 & 0.0221463 \\
\hline 0.983309 & 1.17823 & 0.0416415 & 0.6007 & 0.0302499 & 0.596658 & 0.0335061 \\
\hline 0.815623 & 1.17295 & 0.0428417 & 0.601306 & 0.0373753 & 0.588801 & 0.0290933 \\
\hline 0.737745 & 1.16431 & 0.0408649 & 0.585992 & 0.0377624 & 1407 & 4479 \\
\hline 0.568641 & 1.15245 & 0.0398811 & 0.597308 & 0.0292843 & 0.589551 & 0.0369605 \\
\hline 0.458828 & 1.14778 & 0.0367321 & 0.59298 & 0.0343232 & 0.589792 & 0.0229101 \\
\hline 0.3762 & 1.14771 & 0.0402464 & 0.59546 & 0.0287628 & 0.594297 & 0.0333934 \\
\hline 0.295295 & 1.14143 & 0.0390267 & 0.586606 & 0.0308155 & 0.577949 & 0.026002 \\
\hline 0.251799 & 1.14904 & 0.0454263 & 0.588334 & 0.0332392 & 0.584366 & 0.037819 \\
\hline 0.218094 & 1.14721 & 0.047414 & 0.58236 & 0.0276027 & 0.573505 & 0.0282392 \\
\hline 0.169754 & 1.13532 & 0.0437373 & 0.575531 & 0.0245452 & 0.573374 & 0.0279674 \\
\hline 0.130181 & 1.13318 & 0.0474821 & 0.579485 & 0.0274258 & 0.567476 & 0.0300739 \\
\hline 0.144582 & 1.13011 & 0.0371114 & 0.584674 & 0.037816 & 0.568268 & 0.03263 \\
\hline 0.109767 & 1.12587 & 0.0425994 & 0.57808 & 0.0386217 & 0.569458 & 0.0260791 \\
\hline 0.0917397 & 1.12134 & 0.041478 & 0.57527 & 0.0334841 & 0.567356 & 0.0242645 \\
\hline 0.0849886 & 1.12323 & 0.0388567 & 0.569083 & 0.0271563 & 0.567197 & 0.0278374 \\
\hline 0.0962389 & 1.11955 & 0.0342347 & 0.568567 & 0.0321331 & 0.568997 & 0.0255178 \\
\hline 0.0976248 & 1.10813 & 0.0374481 & 0.571145 & 0.0418494 & 0.560981 & 0.0322487 \\
\hline 0.0667772 & 1.10662 & 0.0398175 & 0.568055 & 0.0306499 & 0.564754 & 0.0271755 \\
\hline 0.0793177 & 1.10932 & 0.0379554 & 0.565448 & 0.0302939 & 0.564424 & 0.0267492 \\
\hline 0.0932561 & 1.11169 & 0.0327562 & 0.562594 & 0.0307871 & 0.551263 & 0.0351726 \\
\hline 0.0847462 & 1.11206 & 0.0368962 & 0.562348 & 0.0268158 & 0.562397 & 0.0304819 \\
\hline 0.0808763 & 1.10958 & 0.0355342 & 0.560285 & 0.027625 & 0.562745 & 0.0268183 \\
\hline 0.0972219 & 1.10407 & 0.033239 & 0.561679 & 0.0358705 & 0.567264 & 0.0272288 \\
\hline 0.0730407 & 1.10054 & 0.0385399 & 0.560585 & 0.0209071 & 0.561813 & 0.0243766 \\
\hline 0.0943243 & 1.09297 & 0.0427998 & 0.561157 & 0.0311355 & 0.561021 & 0.0254645 \\
\hline 0.0894272 & 1.09562 & 0.0425609 & 0.554678 & 0.035197 & 0.554965 & 0.0245457 \\
\hline 0.0748405 & 1.09804 & 0.0437915 & 0.563027 & 0.0318454 & 0.56858 & 0.0321969 \\
\hline 0.0810419 & 1.09429 & 0.0409617 & 0.555545 & 0.0252018 & 0.56722 & 0.0212145 \\
\hline
\end{tabular}




\begin{tabular}{|c|c|c|c|c|c|c|}
\hline 0.0886521 & 1.09041 & 0.035411 & 0.561254 & 0.0273942 & 0.581944 & 0.0278186 \\
\hline 0.1251 & 1.09362 & 0.0340454 & 0.559634 & 0.0290692 & 0.59107 & 0.0289373 \\
\hline 0.119815 & 1.09859 & 0.0456682 & 0.565122 & 0.0245927 & 0.593245 & 0.0372034 \\
\hline 0.142889 & 1.0939 & 0.0464831 & 0.575789 & 0.0344069 & 0.608365 & 0.0313305 \\
\hline 0.189147 & 1.08507 & 0.0384128 & 0.57068 & 0.0285438 & 0.618517 & 0.0361685 \\
\hline 0.18405 & 1.08552 & 0.0321804 & 0.566301 & 0.0375976 & 0.619547 & 0.0320458 \\
\hline 0.17315 & 1.09018 & 0.0410703 & 0.57592 & 0.0319609 & 0.650298 & 0.0309435 \\
\hline 0.254773 & 1.09175 & 0.0460985 & 0.57747 & 0.0296528 & 0.66052 & 0.0365374 \\
\hline 0.254246 & 1.09243 & 0.0424833 & 0.568147 & 0.023575 & 0.663748 & 0.0330116 \\
\hline 0.301366 & 1.09582 & 0.0425474 & 0.578589 & 0.0310268 & 0.682468 & 0.0331501 \\
\hline 0.354547 & 1.09666 & 0.0413641 & 0.583165 & 0.032244 & 0.690537 & 0.0363073 \\
\hline 0.40947 & 1.09994 & 0.0409002 & 0.589926 & 0.029986 & 0.703173 & 0.0393528 \\
\hline 0.425118 & 1.0998 & 0.0472461 & 0.591497 & 0.0315321 & 0.71835 & 0.0286891 \\
\hline 0.497074 & 1.09428 & 0.0410081 & 0.595081 & 0.0376407 & 0.728671 & 0.0283762 \\
\hline 0.508554 & 1.0834 & 0.0400868 & 0.599664 & 0.0279214 & 0.727396 & 0.0305934 \\
\hline 0.589033 & 1.08354 & 0.0466132 & 0.592441 & 0.0291183 & 0.738715 & 0.0352586 \\
\hline 0.6463 & 1.08885 & 0.047766 & 0.601189 & 0.0317899 & 0.73387 & 0.027196 \\
\hline 0.679752 & 1.08136 & 0.0445354 & 0.603122 & 0.0302052 & 0.748683 & 0.0306015 \\
\hline 0.739567 & 1.0754 & 0.0397121 & 0.60104 & 0.0291126 & 0.738722 & 0.0426365 \\
\hline 0.784587 & 1.06367 & 0.0407103 & 0.603086 & 0.033343 & 0.741363 & 0.0389861 \\
\hline 0.822 & 1.07028 & 0.0426148 & 0.601036 & 0.0321403 & 0.72634 & 0.0395776 \\
\hline 0.859581 & 1.06924 & 0.0398985 & 0.585429 & 3096 & 0.713327 & 0.036719 \\
\hline 0.856775 & 1.06153 & 0.0385777 & 0.58271 & 0.0328563 & 0.698326 & 0.0322881 \\
\hline 0.891109 & 1.05494 & 0.0348802 & 0.5849 & 0.0283292 & 0.689774 & 0.0380274 \\
\hline 0.896958 & 1.04964 & 0.0384269 & 0.576596 & 0.0333576 & 0.67875 & 0.0413512 \\
\hline 0.884145 & 1.04544 & 0.0346073 & 0.572757 & 0.0335762 & 0.669127 & 0.0297553 \\
\hline 0.828954 & 1.04268 & 0.0327783 & 0.568982 & 0.0310892 & 0.656739 & 0.023745 \\
\hline 0.827294 & 1.0379 & 0.033478 & 0.563003 & 0.0281782 & 0.632083 & 0.0335872 \\
\hline 0.785922 & 1.03699 & 0.0308571 & 0.563215 & 0.0261628 & 0.622463 & 0.0260879 \\
\hline 0.775906 & 1.03101 & 0.0275941 & 0.552125 & 0.0273525 & 0.612715 & 0.0342167 \\
\hline 0.691692 & 1.02365 & 0.0334749 & 0.545786 & 0.0328932 & 0.602831 & 0.0282029 \\
\hline 0.630661 & 1.02108 & 0.0382108 & 0.541412 & 0.0260563 & 0.58583 & 0.024058 \\
\hline 0.565208 & 1.02505 & 0.0424249 & 0.542036 & 0.0267737 & 0.574448 & 0.0330356 \\
\hline 0.546689 & 1.021 & 0.0369895 & 0.539039 & 0.0306272 & 0.572475 & 0.0365318 \\
\hline 0.4416 & 1.01809 & 0.0320007 & 0.540047 & 0.0322354 & 0.553166 & 0.0274394 \\
\hline 0.425172 & 1.02446 & 0.0334486 & 0.533162 & 0.0270018 & 0.545719 & 0.0341688 \\
\hline 0.368043 & 1.02003 & 0.0371281 & 0.519094 & 0.0285073 & 0.534192 & 0.0309347 \\
\hline 0.324495 & 1.01101 & 0.032105 & 0.529307 & 0.0320441 & 0.531175 & 0.0273708 \\
\hline 0.262367 & 1.00743 & 0.0314257 & 0.528213 & 0.0305746 & 0.532945 & 0.0237351 \\
\hline 0.204157 & 1.00376 & 0.0343616 & 0.523665 & 0.0287829 & 0.519223 & 0.0287663 \\
\hline 0.192404 & 1.00383 & 0.0391575 & 0.522634 & 0.029289 & 0.521777 & 0.0276957 \\
\hline 0.198821 & 1.00416 & 0.0410451 & 0.517844 & 0.0293813 & 0.518049 & 0.0297293 \\
\hline 0.146839 & 0.999259 & 0.0382439 & 0.519167 & 0.0285114 & 0.525199 & 0.029378 \\
\hline 0.129758 & 1.00184 & 0.0307479 & 0.524983 & 0.0261598 & 0.521158 & 0.0336887 \\
\hline 0.119906 & 1.00225 & 0.0322748 & 0.522673 & 0.0248386 & 0.512147 & 0.0333258 \\
\hline 0.0950385 & 1.00283 & 0.0331524 & 0.513186 & 0.0331692 & 0.522159 & 0.0293272 \\
\hline 0.0936402 & 0.997656 & 0.030806 & 0.511912 & 0.0275873 & 0.50957 & 0.0336619 \\
\hline
\end{tabular}




\begin{tabular}{|c|c|c|c|c|c|c|}
\hline 0.0777605 & 0.995641 & 0.0248131 & 0.507538 & 0.0314702 & 0.510631 & 0.0306905 \\
\hline 0.0868432 & 1.00018 & 0.0274932 & 0.506082 & 0.0331467 & 0.517521 & 0.0280041 \\
\hline 0.0673065 & 1.00103 & 0.0285663 & 0.513611 & 0.0284657 & 0.518715 & 0.0227337 \\
\hline 0.0839607 & 0.994646 & 0.0337611 & 0.508998 & 0.0303382 & 0.513087 & 0.0225682 \\
\hline 0.0576869 & 0.989575 & 0.0268119 & 0.510376 & 0.0254544 & 0.500917 & 0.0311938 \\
\hline 0.0557535 & 0.994026 & 0.0292375 & 0.508439 & 0.0308078 & 0.511113 & 0.0306995 \\
\hline 0.0669254 & 0.986697 & 0.0312665 & 0.511828 & 0.0257597 & 0.509965 & 0.0238229 \\
\hline 0.0534328 & 0.985704 & 0.0378245 & 0.502898 & 0.0306536 & 0.500635 & 0.0256 \\
\hline 0.0574546 & 0.986157 & 0.0369664 & 0.498732 & 0.0275059 & 0.507395 & 0.0352704 \\
\hline 0.06157 & 0.985242 & 0.0355686 & 0.511228 & 0.0283829 & 0.499903 & 0.0237093 \\
\hline 0.0618464 & 0.986021 & 0.0314934 & 0.500227 & 0.0240717 & 0.498078 & 0.0220636 \\
\hline 0.0669563 & 0.984 & 0.0292699 & 0.502894 & 0.0269589 & 0.50103 & 0.0341259 \\
\hline 0.0550048 & 0.983025 & 0.0302248 & 0.504987 & 0.0344568 & 0.492419 & 0.0321105 \\
\hline 0.0623739 & 0.97959 & 0.0342939 & 0.49372 & 0.0258949 & 0.498776 & 0.020413 \\
\hline 0.0594516 & 0.989415 & 0.0348635 & 0.501125 & 0.0299033 & 0.500619 & 0.0264417 \\
\hline 0.0656972 & 0.985847 & 0.0339773 & 0.504234 & 0.026278 & 0.501326 & 0.0238557 \\
\hline 0.0807908 & 0.97992 & 0.0308538 & 0.507019 & 0.0251673 & 0.49496 & 0.0247089 \\
\hline 0.0745334 & 0.985873 & 0.0341175 & 0.495599 & 0.0321173 & 0.498029 & 0.0255838 \\
\hline 0.0605111 & 0.985581 & 0.028 & 0.49852 & 0.026 & 0.502293 & 0.0262693 \\
\hline 0.0736016 & 0.985735 & 0.0296775 & 0.500766 & 0.0283596 & 0.502263 & 0.0273452 \\
\hline 0.0610092 & 0.995959 & 0.0314054 & 0.498589 & 0.0316555 & 0.509547 & 0.0288777 \\
\hline 0.0596503 & 1.00212 & 0.02 & 0.506157 & 281 & 0.49874 & 0.0289097 \\
\hline 0.0690135 & 1.01507 & 0.0357827 & 0.506052 & 0.0277745 & 0.496133 & 0.0292669 \\
\hline 0.0754769 & 1.03533 & 0.0288714 & 0.51268 & 0.0285148 & 0.499395 & 0.0275495 \\
\hline 0.0585721 & 1.03935 & 0.0350804 & 0.509427 & 0.0247627 & 0.510475 & 0.0297807 \\
\hline 0.0789442 & 1.05267 & 0.036399 & 0.517518 & 0.0266684 & 0.508793 & 0.0252758 \\
\hline 0.0791659 & 1.0735 & 0.0386439 & 0.513086 & 0.0252351 & 0.513902 & 0.0204485 \\
\hline 0.0659972 & 1.09941 & 0.0370176 & 0.523538 & 0.0265218 & 0.521085 & 0.0347628 \\
\hline 0.0815162 & 1.11994 & 0.040708 & 0.537775 & 0.0251959 & 0.528297 & 0.0227271 \\
\hline 0.0758593 & 1.15043 & 0.0463005 & 344 & 0.025859 & 0.526328 & 0.0237673 \\
\hline 0.0755625 & 1.177 & 0.0367798 & 0.560166 & 0.0243233 & 0.547126 & 0.0307846 \\
\hline 0.0812186 & 1.21044 & 0.0388332 & 0.574344 & 0.029582 & 0.547777 & 0.0300401 \\
\hline 0.0747864 & 1.2479 & 0.0394834 & 0.587802 & 0.0308331 & 0.569014 & 0.0342412 \\
\hline 0.108779 & 1.27617 & 0.0433167 & 0.602877 & 0.0322254 & 0.580434 & 0.0305591 \\
\hline 0.100156 & 1.30305 & 0.0483299 & 0.619771 & 0.0355966 & 0.59682 & 0.0337179 \\
\hline 0.112132 & 1.33558 & 0.0497461 & 0.64098 & 0.0359484 & 0.601878 & 0.0323842 \\
\hline 0.117632 & 1.35695 & 0.0557929 & 0.665854 & 0.0340825 & 0.624447 & 0.0399849 \\
\hline 0.132728 & 1.38296 & 0.0552849 & 0.693647 & 0.0444446 & 0.635083 & 0.0280732 \\
\hline 0.12888 & 1.40543 & 0.0590002 & 0.714338 & 0.0350941 & 0.660092 & 0.036398 \\
\hline 0.153683 & 1.42928 & 0.0590506 & 0.746032 & 0.0444739 & 0.668935 & 0.038271 \\
\hline 0.12878 & 1.43847 & 0.062565 & 0.756033 & 0.04026 & 0.678959 & 0.0380035 \\
\hline 0.131847 & 1.44415 & 0.0612564 & 0.786891 & 0.0496505 & 0.702156 & 0.0384283 \\
\hline 0.132497 & 1.4448 & 0.0663833 & 0.805832 & 0.0480736 & 0.710537 & 0.0456257 \\
\hline 0.161418 & 1.43298 & 0.0709873 & 0.817973 & 0.0330012 & 0.726272 & 0.0357718 \\
\hline 0.13577 & 1.42346 & 0.0647483 & 0.823334 & 0.0420481 & 0.731921 & 0.0483982 \\
\hline 0.149659 & 1.40159 & 0.0575754 & 0.844574 & 0.0444265 & 0.734734 & 0.0389093 \\
\hline 0.141784 & 1.38089 & 0.0555056 & 0.849158 & 0.0538167 & 0.727876 & 0.0345122 \\
\hline
\end{tabular}




\begin{tabular}{|c|c|c|c|c|c|c|}
\hline 0.131101 & 1.35566 & 0.0553103 & 0.84465 & 0.0457149 & 0.730547 & \\
\hline 0.122191 & 1.31954 & 0.053833 & 0.847461 & 0.0470761 & 0.726661 & 0.0342682 \\
\hline 0.137941 & 1.28145 & 0.0551468 & 0.839858 & 0.0438848 & 0.709411 & 0.0393938 \\
\hline 0.134816 & 1.2427 & 0.0524113 & 0.823188 & 0.0488769 & 0.705479 & 0.0375913 \\
\hline 0.154089 & 1.2091 & 0.0463008 & 0.814578 & 0.0520033 & 0.69312 & 0.0385905 \\
\hline 0.129251 & 1.17891 & 0.0434291 & 0.792843 & 0.040854 & 0.686248 & 0.0389527 \\
\hline 0.105734 & 1.14885 & 0.0347058 & 0.765612 & 0.0420046 & 0.657828 & 0.0355056 \\
\hline 0.114535 & 1.09424 & 0.0401009 & 0.747202 & 0.0418846 & 0.642468 & 0.0334102 \\
\hline 0.110393 & 1.05661 & 0.0381485 & 0.71959 & 0.0422306 & 0.616448 & 0.035555 \\
\hline 0.0856758 & 1.03515 & 0.0331474 & 0.699745 & 0.0351849 & 0.613105 & 0.0355483 \\
\hline 0.0961957 & 1.01895 & 0.0269604 & 0.666268 & 0.0278828 & 0.583526 & 0.0373329 \\
\hline 0.0945416 & 0.989303 & 0.0314493 & 0.64715 & 0.0343187 & 0.567516 & 0.0276147 \\
\hline 0.0664684 & 0.956434 & 0.0331016 & 0.620265 & 0.0390466 & 0.551923 & 0.0357574 \\
\hline 0.0845601 & 0.946384 & 0.0300704 & 0.590936 & 0.0301783 & 0.539808 & 0.0241573 \\
\hline 0.0646871 & 0.925975 & 0.0281772 & 0.564204 & 0.0355494 & 0.517163 & 0.0287582 \\
\hline 0.0654316 & 0.917137 & 0.0311034 & 0.549923 & 0.0262974 & 0.512381 & 0.0291403 \\
\hline 0.0630177 & 0.902863 & 0.0322811 & 0.534135 & 0.0263536 & 0.502427 & 0.0242057 \\
\hline 0.0629234 & 0.890641 & 0.0286527 & 0.524515 & 0.0213768 & 0.492158 & 0.028645 \\
\hline 0.0609184 & 0.878122 & 0.0287167 & 0.507819 & 0.0274654 & 0.472716 & 0.0226257 \\
\hline 0.0532949 & 0.876492 & 0.0272135 & 0.495379 & 0.0233792 & 0.470441 & 0.0268364 \\
\hline 0.0556345 & 0.877079 & 0.0250134 & 0.483165 & 0.027791 & 0.46301 & 0.0244757 \\
\hline 0.0487454 & 0.870332 & 0.0280173 & 0.476948 & 0.0272756 & 0.45858 & 0.0216473 \\
\hline 0.0627396 & 0.86488 & 0.0306825 & 0.469131 & 0.0289171 & 0.45979 & 0.0249595 \\
\hline 0.0479176 & 0.860683 & 0.0325882 & 0.466403 & 0.0233735 & 0.450767 & 0.0273768 \\
\hline 0.0588351 & 0.86 & 0.03 & 0.45 & 257 & 924 & 8231 \\
\hline 0.056295 & 0.855877 & 0.0265924 & 0.455665 & 0.0277762 & 0.445027 & 0.0213628 \\
\hline 0.0500858 & 0.840883 & 0.0266218 & 0.449565 & 0.0263953 & 0.440817 & 0.0241374 \\
\hline 0.0563991 & 0.846881 & 0.0298911 & 0.452392 & 0.019406 & 0.441068 & 0.0250988 \\
\hline 0.047789 & 0.844805 & 0.0302588 & 0.447492 & 0.0231088 & 0.429166 & 0.0272465 \\
\hline 0.0508902 & 0.839044 & 0.0318025 & 0.452697 & 0.0291124 & 0.442328 & 0.021821 \\
\hline 0.0492149 & 0.835386 & 0.0287696 & 0.433888 & 0.0250138 & 0.438667 & 0.0266548 \\
\hline 0.0560203 & 0.835678 & 0.0243192 & 0.442506 & 0.0224293 & 0.438416 & 0.0188464 \\
\hline 0.0681233 & 0.827435 & 0.0244899 & 0.437472 & 0.0200283 & 0.438193 & 0.0257513 \\
\hline 0.0635961 & 0.824146 & 0.02689 & 0.436375 & 0.0278457 & 0.429288 & 0.0218017 \\
\hline 0.0624461 & 0.825567 & 0.0238017 & 0.440658 & 0.0259922 & 0.431649 & 0.0283204 \\
\hline 0.0521204 & 0.826646 & 0.0273617 & 0.434044 & 0.0240818 & 0.429854 & 0.0227922 \\
\hline 0.0637521 & 0.824646 & 0.0277782 & 0.431192 & 0.0224394 & 0.426282 & 0.0257832 \\
\hline 0.054732 & 0.816918 & 0.0281824 & 0.427055 & 0.0208279 & 0.420004 & 0.0249756 \\
\hline 0.060663 & 0.811372 & 0.0306241 & 0.434 & 0.0171725 & 0.419686 & 0.0278194 \\
\hline 0.0543202 & 0.812777 & 0.026341 & 0.429447 & 0.0190491 & 0.422033 & 0.0250487 \\
\hline 0.0481495 & 0.812797 & 0.0286626 & 0.426129 & 0.0193778 & 0.42269 & 0.0242191 \\
\hline 0.0475975 & 0.81037 & 0.03043 & 0.428854 & 0.0244361 & 0.419975 & 0.0230774 \\
\hline 0.0571003 & 0.807278 & 0.0250027 & 0.429965 & 0.0326343 & 0.417403 & 0.0208025 \\
\hline 0.0519781 & 0.807162 & 0.0301895 & 0.429104 & 0.022067 & 0.42036 & 0.0229904 \\
\hline 0.045744 & 0.805998 & 0.0297676 & 0.425639 & 0.0192912 & 0.427794 & 0.0191488 \\
\hline 0.0381278 & 0.800611 & 0.0291384 & 0.41818 & 0.0231802 & 0.418376 & 0.0252245 \\
\hline 0.0472112 & 0.798446 & 0.0272907 & 0.425943 & 0.0228411 & 0.411109 & 0.018867 \\
\hline
\end{tabular}




\begin{tabular}{|c|c|c|c|c|c|c|}
\hline 0.0585236 & 0.799559 & 0.0252802 & 0.416174 & 0.0276892 & 0.420487 & 289776 \\
\hline 0.0525688 & 0.79406 & 0.0223841 & 0.425549 & 0.020934 & 0.40255 & 0.0242846 \\
\hline 0.0520464 & 0.793785 & 0.0223561 & 0.420998 & 0.0209242 & 0.412752 & 0.0213645 \\
\hline .0466211 & 0.793083 & 0.0242945 & 0.414535 & 0.0235736 & 0.401182 & 0.0186313 \\
\hline 0.051882 & 0.790297 & 0.0271956 & 0.418547 & 0.0229766 & 0.4127 & 0.0246903 \\
\hline 0.0426328 & 0.784639 & 0.0286496 & 0.414075 & 0.0255316 & 0.406895 & 0.025058 \\
\hline 0.0501727 & 0.781718 & 0.0250923 & 0.417973 & 0.0197513 & 0.406731 & 0.0220611 \\
\hline 0.0460051 & 0.782267 & 0.0252504 & 0.408558 & 0.0266879 & 0.411543 & 0.0211635 \\
\hline 0.0479068 & 0.778265 & 0.0304413 & 0.415969 & 0.0227603 & 0.41771 & 0.0268133 \\
\hline 0.0478654 & 0.773204 & 0.0204881 & 0.404638 & 0.0195327 & 0.412348 & 0.0216291 \\
\hline 0.0497992 & 0.775517 & 0.0212796 & 0.409613 & 0.0256083 & 0.41648 & 0.0264609 \\
\hline 0.0444827 & 0.774037 & 0.0271788 & 0.412966 & 0.0229141 & 0.411026 & 0.0217219 \\
\hline 0.0466107 & 0.771369 & 0.0311748 & 0.41488 & 0.0218931 & 0.410093 & 0.0203103 \\
\hline 0.0517436 & 0.769335 & 0.0242462 & 0.403637 & 0.0194502 & 0.408929 & 0.0266093 \\
\hline 0.0426326 & 0.766253 & 0.0288659 & 0.409942 & 0.027525 & 0.417264 & 0.0234312 \\
\hline 0.0481029 & 0.765391 & 0.0350609 & 0.412239 & 0.0270171 & 0.422269 & 1257 \\
\hline 0.0551079 & 0.763987 & 0.0276808 & 0.406511 & 0.0274668 & 0.416145 & 0.021439 \\
\hline 0.0546802 & 0.767928 & 0.0229508 & 0.409568 & 0.0197776 & 0.419414 & 0.0270722 \\
\hline 0.0496825 & 0.766906 & 323 & 0.405215 & 0.02 & 0.4 & 0.02 \\
\hline 0.0495973 & 0.763863 & 0.0249923 & 0.414932 & 0.0203115 & 0.425453 & 0.0247 \\
\hline 0.0380337 & 0.767488 & 0.0214457 & 0.409172 & 0.0205701 & 0.425839 & 0.022281 \\
\hline 0.0493943 & 0.759622 & 0.02 & 5054 & 5398 & 353 & 939 \\
\hline 0.0500854 & 0.761974 & 0.0172957 & 0.40667 & 0.0225893 & 0.428895 & 0.0289793 \\
\hline 0.0501358 & 0.754699 & 0.0208951 & 0.414075 & 0.0214057 & 0.438935 & 0.0268533 \\
\hline 0.0429537 & 0.759601 & 0.0225604 & 0.413302 & 0.0261318 & 0.436167 & 0.0294979 \\
\hline 0.0396657 & 0.760155 & 0.024064 & 0.410504 & 0.0204087 & 0.446774 & 0.0231608 \\
\hline 0.0425759 & 0.751605 & 0.0280242 & 0.414179 & 0.0160888 & 0.453986 & 0.0263629 \\
\hline 0.0558062 & 0.755217 & 0.0308293 & 0.408594 & 0.0222962 & 267 & 0.0269307 \\
\hline 0.0440352 & 0.754813 & 0.0269256 & 0.410436 & 0.0238468 & 0.461518 & 0.0274097 \\
\hline 0.0434042 & 365 & & 557 & 5391 & 331 & 708 \\
\hline 0.049587 & 0.745734 & 0.0243574 & 0.415566 & 0.0243846 & 0.4 & 0.0248163 \\
\hline 0.0508302 & 0.736656 & 0.0246841 & 0.416518 & 0.0245228 & 0.456758 & 0.0298259 \\
\hline 0.0526076 & 0.737246 & 0.0307926 & 0.415878 & 0.0244511 & 323 & 0.0236086 \\
\hline 0.0447252 & 0.739708 & 0.0289651 & 0.413791 & 0.0252899 & 0.4 & 0.0313497 \\
\hline 0.0468624 & 0.740267 & 0.0280587 & 0.401214 & 0.026158 & 0.458756 & 0.0275176 \\
\hline 0.0477446 & 0.732437 & 0.02851 & 0.409297 & 0.0145159 & 0.447736 & 0.0221352 \\
\hline 0.0396583 & 0.724488 & 0.0290959 & 0.407344 & 0.0223179 & 0.443875 & 0.0281479 \\
\hline 0.0321133 & 0.730549 & 0.0267629 & 0.400575 & 0.020534 & 0.442666 & 0.0247246 \\
\hline 0.0497316 & 0.730013 & 0.0244461 & 0.400748 & 0.0292758 & 0.43308 & 0.0321437 \\
\hline 0.0510215 & 0.732742 & 0.0278276 & 0.399314 & 0.0166532 & 0.427346 & 0.0309577 \\
\hline 0.0465795 & 0.722781 & 0.0267416 & 0.401081 & 0.0253415 & 0.422558 & 0.0242924 \\
\hline 0.0423502 & 0.719619 & 0.0229066 & 0.394278 & 0.0220089 & 0.425 & 0.0285972 \\
\hline 0.0539785 & 0.715668 & 0.0262214 & 0.395829 & 0.0262688 & 0.417226 & 0.0255041 \\
\hline 0.0423958 & 0.715107 & 0.0293698 & 0.385969 & 0.0224635 & 0.406818 & 0.0257446 \\
\hline 0.0670519 & 0.712548 & 0.0249735 & 0.389074 & 0.0249674 & 0.399683 & 0.0215209 \\
\hline 0.0432747 & 0.710616 & 0.0169691 & 0.382327 & 0.0206576 & 0.397186 & 0.0237525 \\
\hline 0.0572399 & 0.704753 & 0.0292499 & 0.385885 & 0.0192884 & 0.386924 & 0.0256159 \\
\hline
\end{tabular}




\begin{tabular}{|c|c|c|c|c|c|c|}
\hline 0.0600781 & 0.695981 & 0.0271685 & 0.373345 & 0.0162088 & 0.380427 & 0.019958 \\
\hline 0.037758 & 0.692845 & 0.0232895 & 0.373387 & 0.0203095 & 0.380548 & 0.0292195 \\
\hline 0.0530108 & 0.692121 & 0.0213035 & 0.38155 & 0.0269735 & 0.378579 & 0.0252618 \\
\hline 0.0467394 & .689781 & 0.0283921 & 0.380379 & 0.0170963 & 0.377401 & 0.0236705 \\
\hline 0.0448662 & 0.686648 & 0.0281109 & 0.369529 & 0.0189686 & 0.373727 & 0.0264051 \\
\hline 0.0473737 & 0.685629 & 0.0263065 & 0.366321 & 0.0247872 & 0.372295 & 0.0235615 \\
\hline 0.0586769 & 0.68352 & 0.0256219 & 0.362965 & 0.0190437 & 0.358029 & 0.0195621 \\
\hline 0.0486992 & 0.683685 & 0.0257512 & 0.3662 & 0.0210297 & 0.363197 & 0.0240371 \\
\hline 0.038133 & 0.67838 & 0.0250555 & 0.364216 & 0.0172361 & 0.366463 & 0.0209879 \\
\hline 0.0452548 & 0.681411 & 0.0227036 & 0.35796 & 0.0130407 & 0.357891 & 0.0218827 \\
\hline 0.0451016 & 0.673937 & 0.0215311 & 0.358705 & 0.0144574 & 0.355772 & 0.0238566 \\
\hline 0.0474529 & 0.672542 & 0.0217196 & 0.368693 & 0.0185423 & 0.35376 & 0.0225251 \\
\hline 0.0407141 & 0.671245 & 0.0196844 & 0.361625 & 0.0158067 & 0.347441 & 0.0205858 \\
\hline 0.0428905 & 0.676854 & 0.0226516 & 0.353863 & 0.0207267 & 0.356192 & 0.0189762 \\
\hline 0.0455852 & 0.667599 & 0.0264728 & 0.355378 & 0.0213734 & 0.354444 & 0.0202569 \\
\hline 0.0376696 & 0.67301 & 0.0273741 & 0.354422 & 0.0216122 & 18959 & 0.0257788 \\
\hline 0.039481 & 0.670157 & 0.0237428 & 0.353786 & 0.0177418 & 0.355766 & 0.0197548 \\
\hline 0.0439456 & 0.661641 & 0.0238591 & 0.354316 & 0.0183332 & 0.346011 & 0.0207166 \\
\hline 0.0411927 & 0.658701 & 929 & 008 & 0.0159219 & 0062 & 0.022082 \\
\hline 0.0462417 & 0.659992 & 0.0261251 & 0.350929 & 0.0148095 & 0.34856 & 0.0193733 \\
\hline 0.056527 & 0.660849 & 0.0198343 & 0.346138 & 0.0190564 & 0.342703 & 0.0240187 \\
\hline 0.0340806 & 0.661062 & 0.02 & 145 & 411 & 008 & 5929 \\
\hline 0.0497663 & 0.654721 & 0.0212547 & 0.343284 & 0.0154633 & 0.340916 & 0.0220572 \\
\hline 0.0391072 & 0.659094 & 0.0197492 & 0.344713 & 0.0206748 & 0.340908 & 0.0219941 \\
\hline 0.043448 & 0.656345 & 0.0222248 & 0.346898 & 0.019487 & 0.344208 & 0.0264802 \\
\hline 0.0349256 & 0.653124 & 0.0212379 & 0.339777 & 0.0200909 & 0.331576 & 0.0190112 \\
\hline 0.0381083 & 0.644772 & 0.0285652 & 0.342723 & 0.0209833 & 0.332668 & 0.0184463 \\
\hline 0.0444462 & 0.644196 & 0.0209477 & 0.337164 & 0.0187544 & 0.340051 & 0.0232909 \\
\hline 0.0375173 & 0.646986 & 0.0236757 & 0.342121 & 0.017516 & 0.334679 & 0.0175857 \\
\hline 0.0353466 & 0.65278 & 0.0252559 & 0.340733 & 0.014122 & 0.336133 & 0.0234568 \\
\hline 0.0513267 & 0.641094 & 0.0217831 & 0.337174 & 0.0232763 & 0.337137 & 0.026862 \\
\hline 0.0379722 & 0.638855 & 0.022666 & 0.340955 & 0.0275116 & 0.337196 & 0.0195013 \\
\hline 0.0502899 & 0.640201 & 0.0218142 & 0.335805 & 0.0219982 & 0.331789 & 0.019015 \\
\hline 0.0441164 & 0.640256 & 0.0227464 & 0.340434 & 0.0214446 & 0.336536 & 0.0207594 \\
\hline 0.0445084 & 0.647253 & 0.0237528 & 0.336574 & 0.0231635 & 0.334589 & 0.023015 \\
\hline 0.0513135 & 0.642715 & 0.0270494 & 0.340623 & 0.0174381 & 0.329531 & 0.0229888 \\
\hline 0.0394538 & 0.648174 & 0.0197681 & 0.340641 & 0.0197299 & 0.339213 & 0.0219334 \\
\hline 0.0494087 & 0.64538 & 0.0219327 & 0.340812 & 0.0171191 & 0.342088 & 0.0203416 \\
\hline 0.0422088 & 0.645536 & 0.0253367 & 0.341921 & 0.0182839 & 0.338069 & 0.0176739 \\
\hline 0.052899 & 0.644112 & 0.0232682 & 0.340677 & 0.0230709 & 0.345479 & 0.0214831 \\
\hline 0.0404605 & 0.645435 & 0.0190802 & 0.338562 & 0.019938 & 0.346402 & 0.0247856 \\
\hline 0.0424381 & 0.650987 & 0.0256138 & 0.336275 & 0.0186467 & 0.353182 & 0.0245818 \\
\hline 0.0431262 & 0.660208 & 0.0284975 & 0.338627 & 0.0171146 & 0.364503 & 0.0247813 \\
\hline 0.0418752 & 0.6576 & 0.0265624 & 0.343672 & 0.0197344 & 0.369209 & 0.0322257 \\
\hline 0.0469988 & 0.660765 & 0.0217493 & 0.347242 & 0.0193345 & 0.38537 & 0.030603 \\
\hline 0.0366562 & 0.663251 & 0.0188219 & 0.346609 & 0.0232601 & 0.391893 & 0.0348606 \\
\hline 0.0437251 & 0.676303 & 0.02516 & 0.348854 & 0.0214186 & 0.397409 & 0.0281381 \\
\hline
\end{tabular}




\begin{tabular}{|c|c|c|c|c|c|c|}
\hline 0.0449088 & 0.677483 & 0.0243285 & 0.356654 & 0.0156115 & 0.415237 & 0.0356679 \\
\hline 0.0499905 & 0.688757 & 0.0197285 & 0.365711 & 0.0181508 & 0.43848 & 0.0448374 \\
\hline 0.0349152 & 0.699272 & 0.0170322 & 0.368957 & 0.0216061 & 0.457067 & 0.0418102 \\
\hline .0564789 & .712325 & 0.0270482 & .370526 & 0.0196264 & 0.483391 & 0.0599672 \\
\hline 0.0486349 & 0.727796 & 0.0296314 & 0.376651 & 0.0206413 & 0.49885 & 0.0571215 \\
\hline 0.0485971 & 0.722466 & 0.0254026 & 0.395938 & 0.0251583 & 0.524004 & 0.0601528 \\
\hline 0.0472588 & 0.73706 & 0.0231146 & 0.404614 & 0.0207165 & 0.557225 & 0.0687583 \\
\hline 0.0524757 & 0.747916 & 0.0205102 & 0.411753 & 0.0239632 & 0.579563 & 0.0807572 \\
\hline 0.0559703 & 0.762385 & 0.0256252 & 0.420148 & 0.0240425 & 0.606909 & 0.0877522 \\
\hline 0.042621 & 0.77201 & 0.0260746 & 0.431654 & 0.0195671 & 0.635732 & 0.0952725 \\
\hline 0.0549267 & 0.780269 & 0.0215486 & 0.44473 & 0.0211101 & 0.667017 & 0.0998635 \\
\hline 0.0469485 & 0.791484 & 0.0318056 & 0.456858 & 0.0216308 & 0.705199 & 0.0944304 \\
\hline 0.0442162 & 0.797776 & 0.0327868 & 0.480029 & 0.0221039 & 0.727811 & 0.127768 \\
\hline 0.0508555 & 0.809909 & 0.027085 & 0.482453 & 0.0316144 & 0.749183 & 0.127622 \\
\hline 0.0626759 & 0.813176 & 0.0290726 & 0.502075 & 0.0257446 & 0.769638 & 0.124448 \\
\hline 0.0550165 & 0.811581 & 0.0308201 & 0.508342 & 0.0248248 & 0.7 & 0.135094 \\
\hline 0.0532766 & 0.812008 & 0.029293 & 0.509957 & 0.0285781 & 0.814163 & 0.141253 \\
\hline 0.0613202 & 0.815067 & 0.0228122 & 0.511584 & 0.0254675 & 0.83 & 0.134367 \\
\hline 0.0508464 & 0.81 & 0.0259702 & 0.53078 & 0.0321322 & 0.8 & 0.146021 \\
\hline 0.0707803 & 0.798804 & 0.026084 & 0.531086 & 0.0311215 & 0.833574 & 0.14123 \\
\hline 0.0537283 & 0.794804 & 0.02633 & 0.538802 & 0.0394089 & 0.834374 & 0.135727 \\
\hline 0.0651188 & 0.7 & 0.022 & 0.540488 & 5965 & 286 & 35173 \\
\hline 0.057123 & 0.775008 & 0.0303802 & 0.538413 & 0.028645 & 0.807028 & 0.135549 \\
\hline 0.0607703 & 0.767637 & 0.0223655 & 0.532334 & 0.0369477 & 0.771113 & 0.126266 \\
\hline 0.071286 & 0.755825 & 0.0180923 & 0.526797 & 0.0240686 & 0.769833 & 0.113973 \\
\hline 0.0576698 & 0.747913 & 0.0217213 & 0.525714 & 0.0295341 & 0.737492 & 0.123207 \\
\hline 0.0606999 & 0.727245 & 0.02121 & 0.51483 & 0.0302727 & 0.71335 & 0.113353 \\
\hline 0.0642087 & 0.705739 & 0.02423 & 0.503305 & 0.0284523 & 0.67 & 0.0974304 \\
\hline 0.0527581 & 0.692136 & 0.0253577 & 0.503775 & 0.0293333 & 0.6 & 0.085624 \\
\hline 0.0557089 & 0.68 & 0.02 & 0.481711 & 0.0285453 & & 132 \\
\hline 0.0589636 & 0.673245 & 0.0193362 & 0.468009 & 0.0268178 & 0.5 & 0.0741484 \\
\hline 0.0530875 & 0.658519 & 0.0220925 & 0.451696 & 0.0294765 & 0.542476 & 0.0673739 \\
\hline 0.0583146 & 0.647089 & 0.0217116 & 0.43574 & 0.0230254 & 0.513141 & 0.0635922 \\
\hline 0.0620079 & 0.637638 & 0.014804 & 0.421981 & 0.0232822 & 0.48993 & 0.0476258 \\
\hline 0.0533696 & 0.624485 & 0.0217441 & 0.413334 & 0.0178441 & 0.462913 & 0.0519121 \\
\hline 0.0540233 & 0.616813 & 0.0214898 & 0.397397 & 0.0243879 & 0.435431 & 0.0379729 \\
\hline 0.04195 & 0.602195 & 0.0186244 & 0.389743 & 0.0181314 & 0.405328 & 0.0302479 \\
\hline 0.0392748 & 0.593813 & 0.0187413 & 0.36742 & 0.0169124 & 0.388454 & 0.0347714 \\
\hline 0.0410138 & 0.588403 & 0.0214799 & 0.357884 & 0.0222937 & 0.37357 & 0.0356044 \\
\hline 0.0402857 & 0.590264 & 0.0232297 & 0.353341 & 0.0178316 & 0.365094 & 0.0264946 \\
\hline 0.0382588 & 0.587836 & 0.0290248 & 0.340797 & 0.0173109 & 0.351892 & 0.024761 \\
\hline 0.0411116 & 0.574135 & 0.0223212 & 0.338911 & 0.0167027 & 0.333162 & 0.0200853 \\
\hline 0.046213 & 0.570394 & 0.0179717 & 0.331315 & 0.0208092 & 0.323021 & 0.0213067 \\
\hline 0.040953 & 0.571483 & 0.0293355 & 0.326713 & 0.0211033 & 0.320284 & 0.0267759 \\
\hline 0.0341542 & 0.572715 & 0.0252861 & 0.319795 & 0.0187405 & 0.320408 & 0.0260058 \\
\hline 0.036261 & 0.561584 & 0.0204817 & 0.32 & 0.022593 & 0.305116 & 0.0220761 \\
\hline 0.0418148 & 0.557058 & 0.0253809 & 0.309518 & 0.0194989 & 0.304268 & 0.0227584 \\
\hline
\end{tabular}




\begin{tabular}{|c|c|c|c|c|c|c|}
\hline 0.0448648 & 0.557769 & 0.02605 & 0.310902 & 0.0141982 & 0.300295 & 0.0147141 \\
\hline 0.0419151 & .555902 & 0.0165157 & 0.312904 & 0.0156141 & 0.302312 & 0.019235 \\
\hline 0.0404016 & 0.558905 & 0.0244151 & .304847 & 0.0182399 & 0.299267 & 0.0240413 \\
\hline 0399947 & .547085 & 0.0223886 & 0.30958 & 0.0160625 & 0.300842 & 0.0214179 \\
\hline 0.0352383 & 0.556616 & .0167739 & 0.297802 & 0.0157153 & 0.298879 & 0.0151275 \\
\hline 0.0444747 & 0.549672 & 0.02116 & 0.304247 & 0.019214 & 0.294814 & 0.0203473 \\
\hline 0.0384344 & 0.543581 & 0.0204405 & 0.308084 & 0.0161646 & 0.290172 & 0.0241629 \\
\hline 0.0355205 & 0.538018 & 0.0247455 & 0.299853 & 0.021649 & 0.295095 & 0.0187486 \\
\hline 0.0395421 & 0.536289 & 0.0218367 & 0.291377 & 0.015218 & 0.29595 & 0.0178175 \\
\hline 0.0357673 & 0.544097 & 0.0216103 & 0.297038 & 0.0173939 & 0.29236 & 0.0204468 \\
\hline 0.0469588 & 0.534984 & 0.0180879 & 0.298412 & 0.0198015 & 0.293354 & 0.0185378 \\
\hline 0.0432845 & 0.536754 & 0.0230375 & 0.296714 & 0.0172905 & 0.28703 & 0.0205477 \\
\hline 0.0486171 & 0.53656 & 0.021383 & 0.288338 & 0.0196591 & 0.289979 & 0.0186529 \\
\hline 0.0386798 & 0.528944 & 0.0218459 & 0.288332 & 0.0134976 & 0.285249 & 0.0205026 \\
\hline 0.0431221 & 0.528747 & 0.029477 & 0.293629 & 0.0176751 & 0.288418 & 0.020304 \\
\hline 0.0459433 & 0.531676 & 0.0214465 & 0.287515 & 0.0185891 & 0.282906 & 0.0230515 \\
\hline 0.0368743 & 0.520433 & 0.0183491 & 0.285997 & 0.0187387 & 0.286876 & 0.0192381 \\
\hline 0.0380321 & 0.532344 & 0.0212389 & 0.290984 & 0.0180403 & 0.279697 & 0.016542 \\
\hline 0.0365383 & 0.520787 & 0.0217893 & 0.287692 & 0.018 & 0.2831 & 0.01 \\
\hline 0.0383428 & 0.525189 & 0.0202209 & 0.283285 & 0.0140184 & 0.274744 & 0.0178223 \\
\hline 0.0294809 & 0.515541 & 0.022715 & 0.286854 & 0.0208952 & 0.278566 & 0.022182 \\
\hline 0.0419079 & 0.52 & 0.020392 & 0.2843 & 45 & 283437 & 458 \\
\hline 0.039431 & 0.511983 & 0.0204906 & 0.282969 & 0.0131457 & 0.273953 & 0.0168841 \\
\hline 0.0420719 & 0.504531 & 0.0225559 & 0.284537 & 0.0233943 & 0.276833 & 0.0155869 \\
\hline 0.039911 & 0.507283 & 0.0259529 & 0.278227 & 0.0144627 & 0.276987 & 0.0205825 \\
\hline 0.0354551 & 0.514708 & 0.0198623 & 0.277837 & 0.0131031 & 0.273524 & 0.0190459 \\
\hline 0.0381465 & 0.50871 & 0.0225048 & 0.276856 & 0.0177332 & 0.275546 & 0.0209097 \\
\hline 0.0341122 & 0.50433 & 0.0229726 & 0.273921 & 0.015517 & 0.275223 & 0.0201422 \\
\hline 0.0440397 & 0.503455 & 0.0233934 & 0.272743 & 0.0180769 & 0.270936 & 0.0189398 \\
\hline 0.0350447 & 0.511 & 0.021 & 0.276356 & 0.016 & 0.275417 & 982 \\
\hline 0.0404944 & 0.51034 & 0.0213928 & 0.26727 & 0.0154132 & 0.274247 & 0.0178481 \\
\hline 0.0443656 & 0.508658 & 0.0222969 & 0.268205 & 0.0164777 & 0.269555 & 0.0213876 \\
\hline 0.0359424 & 0.49691 & 0.0216432 & 0.27318 & 0.0144613 & 0.271647 & 0.0146854 \\
\hline 0.0281955 & 0.497804 & 0.0224531 & 0.276225 & 0.017228 & 0.274439 & 0.0190167 \\
\hline 0.0338622 & 0.498626 & 0.0215172 & 0.271548 & 0.0144541 & 0.270423 & 0.0207952 \\
\hline 0.0365744 & 0.49779 & 0.0249147 & 0.269326 & 0.0147475 & 0.269207 & 0.0177413 \\
\hline 0.0206119 & 0.500024 & 0.0242046 & 0.268599 & 0.0166966 & 0.268105 & 0.0179647 \\
\hline 0.0312019 & 0.496932 & 0.0169653 & 0.268487 & 0.0147575 & 0.270618 & 0.0163692 \\
\hline 0.0438715 & 0.49261 & 0.0198098 & 0.270028 & 0.0181813 & 0.26529 & 0.0201737 \\
\hline 0.0394494 & 0.489863 & 0.0222472 & 0.268349 & 0.0157069 & 0.277597 & 0.016808 \\
\hline 0.0377763 & 0.48683 & 0.0270252 & 0.264748 & 0.0176709 & 0.268625 & 0.0183295 \\
\hline 0.0346687 & 0.490114 & 0.0227698 & 0.271276 & 0.0163439 & 0.273049 & 0.0209648 \\
\hline 0.0391935 & 0.49612 & 0.0183822 & 0.26672 & 0.0144621 & 0.269919 & 0.0184228 \\
\hline 0.0496102 & 0.489784 & 0.020022 & 0.272499 & 0.016983 & 0.272763 & 0.0192906 \\
\hline 0.0356873 & 0.485948 & 0.0243208 & 0.272982 & 0.0196362 & 0.269692 & 0.0173109 \\
\hline 0.0415323 & 0.492964 & 0.0245783 & 0.26959 & 0.017414 & 0.280325 & 0.0263023 \\
\hline 0.0360291 & 0.477085 & 0.0221343 & 0.258469 & 0.0194164 & 0.286673 & 0.0207159 \\
\hline
\end{tabular}




\begin{tabular}{|c|c|c|c|c|c|c|}
\hline 0.0399994 & 0.482793 & 0.0179749 & 0.257018 & 0.0145516 & 0.271959 & 0.0167625 \\
\hline 0.0382244 & 0.477815 & 0.0207709 & 0.267558 & 0.0194075 & 0.271413 & 0.0231129 \\
\hline 0.0318982 & 0.476233 & 0.0261687 & 0.261895 & 0.0116931 & 0.278919 & 0.017669 \\
\hline 0372994 & .481425 & 0.020569 & .266175 & 0.0131232 & 0.276942 & 0.01909 \\
\hline 0.0379482 & 0.480211 & 0.0203949 & 0.261854 & 0.0174585 & 0.281627 & 0.0171868 \\
\hline 0.0403691 & 0.481538 & 0.0190014 & 0.270081 & 0.0163304 & 0.279327 & 0.0200482 \\
\hline 0.0336139 & 0.479563 & 0.019813 & 0.263765 & 0.0168103 & 0.277725 & 0.018891 \\
\hline 0.034002 & 0.472193 & 0.0174757 & 0.270792 & 0.0182967 & 0.281172 & 0.0182884 \\
\hline 0.0325003 & 0.475423 & 0.0262877 & 0.258205 & 0.0173405 & 0.278743 & 0.018992 \\
\hline 0.0324401 & 0.474708 & 0.0201931 & 0.263103 & 0.0163969 & 0.278186 & 0.0176481 \\
\hline 0.0349006 & 0.473325 & 0.0231739 & 0.264415 & 0.0136093 & 0.279324 & 0.0183133 \\
\hline 0.0392216 & 0.474827 & 0.0157827 & 0.257946 & 0.0139462 & 0.278761 & 0.0227503 \\
\hline 0.0318716 & 0.468484 & 0.0219865 & 0.260655 & 0.0176079 & 0.277522 & 0.0221045 \\
\hline 0.0453572 & 0.473354 & 0.0239037 & 0.265357 & 0.0122671 & 0.276146 & 0.0186435 \\
\hline 0.0344242 & 0.470872 & 0.0170311 & 0.261012 & 0.0150994 & 0.280223 & 0.0189235 \\
\hline 0.0345738 & 0.467145 & 0.0240564 & 0.258438 & 0.0153863 & 0.283218 & 0.0155841 \\
\hline 0.0366106 & 0.471403 & 0.0227685 & 0.265287 & 0.0173219 & 0.268833 & 0.0186138 \\
\hline 0.0290035 & 0.465356 & 0.0167021 & 0.262326 & 0.0155179 & 0.270531 & 0.0218325 \\
\hline 0.0355494 & 0.454191 & 0.0190121 & 0.2 & 0.01 & 52899 & 0.0183797 \\
\hline 0.0434815 & 0.461317 & 0.0176881 & 0.257661 & 0.0183184 & 0.265688 & 0.0177869 \\
\hline 0.0363845 & 0.459885 & 0.0181255 & 5961 & 0.0156811 & 0.260898 & 0.0145303 \\
\hline 0.0347338 & 0.4 & 304 & 03 & 0.01 & 649 & 393 \\
\hline 0.0389378 & 0.462774 & 0.0240838 & 0.257313 & 0.0132644 & 0.270725 & 0.0220085 \\
\hline 0.0327146 & 0.46286 & 0.0170108 & 0.258223 & 0.0161087 & 0.259827 & 0.0187211 \\
\hline 0.0360397 & 0.459155 & 0.0191234 & 0.256421 & 0.018986 & 0.261021 & 0.016711 \\
\hline 0.0408513 & 0.454208 & 0.0150299 & 0.253605 & 0.015782 & 0.261881 & 0.0169508 \\
\hline 0.0491888 & 0.447552 & 0.0199944 & 0.255981 & 0.0154621 & 0.260825 & 0.0207438 \\
\hline 0.0349903 & 0.452745 & 0.0218852 & 0.25401 & 0.0158756 & 0.259459 & 0.0245501 \\
\hline 0.0390549 & 0.450426 & 0.0218848 & 0.254488 & 0.0101616 & 0.256774 & 0.0175321 \\
\hline 0.0346003 & 0.447074 & 0.0151857 & 077 & 771 & 0.262632 & 0.018387 \\
\hline 0.0436333 & 0.451604 & 0.0154663 & 0.249205 & 0.016532 & 0.254295 & 0.01697 \\
\hline 0.0324173 & 0.447869 & 0.0221176 & 0.25264 & 0.0147766 & 0.249357 & 0.0153469 \\
\hline 0.036047 & 0.445657 & 0.0168379 & 0.248768 & 0.0157841 & 0.253934 & 0.019646 \\
\hline 0.0439519 & 0.443887 & 0.0197189 & 0.248142 & 0.0175681 & 0.249508 & 0.0138252 \\
\hline 0.0363799 & 0.449644 & 0.0183612 & 0.254785 & 0.0149886 & 0.247946 & 0.0158805 \\
\hline 0.0338196 & 0.445566 & 0.0208859 & 0.247596 & 0.017711 & 0.251211 & 0.0206203 \\
\hline 0.0314052 & 0.439542 & 0.0166092 & 0.246462 & 0.00994866 & 0.242652 & 0.0182082 \\
\hline 0.0438828 & 0.446119 & 0.0208477 & 0.246378 & 0.0129158 & 0.245739 & 0.0169437 \\
\hline 0.0342599 & 0.436403 & 0.0172917 & 0.246179 & 0.0164997 & 0.243385 & 0.0167754 \\
\hline 0.0342557 & 0.442726 & 0.0220037 & 0.251691 & 0.0192403 & 0.245278 & 0.0159876 \\
\hline 0.0425152 & 0.439183 & 0.0200439 & 0.245721 & 0.0145341 & 0.246308 & 0.0155484 \\
\hline 0.0285654 & 0.440211 & 0.0135956 & 0.245767 & 0.0146846 & 0.243722 & 0.0182558 \\
\hline 0.0355198 & 0.435953 & 0.0146302 & 0.248512 & 0.0177029 & 0.251354 & 0.017443 \\
\hline 0.0351915 & 0.441405 & 0.0158365 & 0.250501 & 0.0149875 & 0.245208 & 0.0198635 \\
\hline 0.0270796 & 0.436094 & 0.0189741 & 0.248537 & 0.0143796 & 0.241318 & 0.0195417 \\
\hline 0.0455328 & 0.435273 & 0.0168509 & 0.24368 & 0.014441 & 0.246712 & 0.0164668 \\
\hline 0.0389961 & 0.432771 & 0.0174915 & 0.248786 & 0.0129048 & 0.239188 & 0.0220268 \\
\hline
\end{tabular}




\begin{tabular}{|c|c|c|c|c|c|c|}
\hline 0.0416297 & 0.433166 & 0.0179146 & 0.240254 & 0.0131614 & 0.243194 & 0.0157493 \\
\hline 0.0373225 & 0.430728 & 0.0175184 & 0.245782 & 0.0152361 & 0.246277 & 0.0167773 \\
\hline 0.0274715 & 0.429069 & 0.0195657 & 0.245068 & 0.0135705 & 0.252021 & 0.019115 \\
\hline 0.0326819 & .437419 & 0.0193088 & 0.243448 & 0.0146671 & 0.242717 & 0.0201787 \\
\hline 0.038475 & 0.428302 & 0.0200783 & 0.239429 & 0.0156483 & 0.240162 & 0.0170537 \\
\hline 0.033838 & 0.427432 & 0.0179746 & 0.241428 & 0.0140709 & 0.239273 & 0.0124491 \\
\hline 0.0386222 & 0.433987 & 0.0115112 & 0.236101 & 0.0137512 & 0.238551 & 0.0168564 \\
\hline 0.0472658 & 0.429551 & 0.0174449 & 0.24119 & 0.0128769 & 0.240909 & 0.0177575 \\
\hline 0.0298682 & 0.427399 & 0.0179721 & 0.244653 & 0.0193901 & 0.240026 & 0.0162068 \\
\hline 0.0369498 & 0.424716 & 0.0166305 & 0.23566 & 0.017091 & 0.237969 & 0.0179128 \\
\hline 0.0423897 & 0.423655 & 0.0172403 & 0.241492 & 0.0142742 & 0.241434 & 0.0184477 \\
\hline 0.0346627 & 0.422788 & 0.0260153 & 0.24092 & 0.0160347 & 0.24213 & 0.0186156 \\
\hline 0.0434039 & 0.426262 & 0.0194732 & 0.240367 & 0.0172558 & 0.2373 & 0.0147779 \\
\hline 0.0316453 & 0.422864 & 0.0206504 & 0.235397 & 0.0145531 & 0.243111 & 0.020314 \\
\hline 0.0313298 & 0.416766 & 0.013355 & 0.244711 & 0.0148191 & 0.241291 & 0.0168781 \\
\hline 0.0397436 & 0.418768 & 0.0179042 & 0.233287 & 0.0140818 & 0.236018 & 0.0164308 \\
\hline 0.0348478 & 0.424208 & 0.0124565 & 0.238637 & 0.0213324 & 0.235243 & 0.0178082 \\
\hline 0.0322181 & 0.42154 & 0.0181256 & 0.241361 & 0.0224114 & 0.24453 & 0.01 \\
\hline 0.03 & 0.419797 & 0.0171931 & 081 & 0.0185817 & 114 & 502 \\
\hline 0.0338597 & 0.414411 & 0.0122228 & 0.236947 & 0.0177449 & 0.235501 & 0.0179089 \\
\hline 0.0427373 & 0.416822 & 0.0142669 & 0.241412 & 0.0149486 & 0.234452 & 0.0194229 \\
\hline 0.033264 & 0.422664 & 0.01 & 442 & 0.0155749 & 748 & 739 \\
\hline 0.0344603 & 0.416331 & 0.0194614 & 0.238081 & 0.0168544 & 0.235255 & 0.0169395 \\
\hline 0.0394936 & 0.419402 & 0.0189437 & 0.236341 & 0.0160139 & 0.23881 & 0.0175817 \\
\hline 0.0385123 & 0.417415 & 0.0191749 & 0.239817 & 0.014286 & 0.237645 & 0.0179959 \\
\hline 0.0359626 & 0.412125 & 0.0152211 & 0.233679 & 0.0115277 & 0.235661 & 0.0160781 \\
\hline 0.0313752 & 0.419755 & 0.0160282 & 0.235001 & 0.0131759 & 0.243434 & 0.0232771 \\
\hline 0.0297175 & 0.421599 & 0.0189616 & 0.237909 & 0.0197348 & 0.231824 & 0.0174158 \\
\hline 0.0315153 & 0.42267 & 0.0223296 & 0.243036 & 0.0159872 & 0.236164 & 0.0181435 \\
\hline 0.0352495 & 0.416733 & 0.0175163 & & 0.01 & & 893 \\
\hline 0.0433127 & 0.416327 & 0.0177315 & 0.235454 & 0.0114623 & 0.239167 & 0.017945 \\
\hline 0.0354092 & 0.417398 & 0.0193892 & 0.236777 & 0.0132609 & 0.241086 & 0.0180503 \\
\hline 0.0270859 & 0.423137 & 0.0140097 & 0.238967 & 0.0107688 & 0.244887 & 0.0196256 \\
\hline 0.0367421 & 0.416384 & 0.0139708 & 0.24475 & 0.0156334 & 0.241105 & 0.0188978 \\
\hline 0.0346756 & 0.416706 & 0.0203707 & 0.244787 & 0.0149402 & 0.237215 & 0.0142422 \\
\hline 0.0387486 & 0.416952 & 0.0187537 & 0.246291 & 0.0174945 & 0.234962 & 0.0170258 \\
\hline 0.0411288 & 0.41525 & 0.0159114 & 0.246176 & 0.015149 & 0.249713 & 0.0182447 \\
\hline 0.0344498 & 0.417493 & 0.019311 & 0.242911 & 0.0125316 & 0.243127 & 0.017051 \\
\hline 0.0362179 & 0.419527 & 0.0166981 & 0.239863 & 0.0157595 & 0.246516 & 0.0171495 \\
\hline 0.0295133 & 0.419275 & 0.0196602 & 0.245013 & 0.014245 & 0.244413 & 0.0193676 \\
\hline 0.0287029 & 0.424448 & 0.0123149 & 0.246666 & 0.0175636 & 0.244709 & 0.0172198 \\
\hline 0.0397375 & 0.42318 & 0.0136604 & 0.245794 & 0.0205397 & 0.245031 & 0.0147768 \\
\hline 0.0376683 & 0.419833 & 0.0158042 & 0.249554 & 0.0115385 & 0.246643 & 0.0212603 \\
\hline 0.0296935 & 0.421532 & 0.0172049 & 0.24718 & 0.0139153 & 0.248771 & 0.0168964 \\
\hline 0.0346275 & 0.419851 & 0.0146768 & 0.246465 & 0.0177814 & 0.256126 & 0.0207353 \\
\hline 0.0389252 & 0.415456 & 0.0159521 & 0.249726 & 0.0147597 & 0.2492 & 0.0189501 \\
\hline 0.0345616 & 0.412278 & 0.0170378 & 0.245706 & 0.0142602 & 0.250508 & 0.0201534 \\
\hline
\end{tabular}




\begin{tabular}{|c|c|c|c|c|c|c|}
\hline 0.0366429 & 0.41553 & 0.0165187 & 0.254095 & 0.0149798 & 0.25509 & 0.0203739 \\
\hline 0.0414786 & 0.417036 & 0.0159911 & 0.259145 & 0.0165377 & 0.255337 & 0.0160383 \\
\hline 0.0346457 & 0.416898 & 0.0154961 & 0.255001 & 0.0188547 & 0.259704 & 0.0186095 \\
\hline 0461404 & 0.41838 & 0.0175184 & 0.251447 & 0.0159876 & 0.263762 & 0.0222539 \\
\hline 0.0321862 & 0.418841 & 0.0143975 & 0.251598 & 0.0133169 & 0.262454 & 0.018963 \\
\hline 0.0300424 & 0.420267 & 0.0155532 & 0.25341 & 0.0153976 & 0.263948 & 0.0195644 \\
\hline 0.041793 & 0.415252 & 0.018078 & 0.256249 & 0.0140649 & 0.273994 & 0.0187149 \\
\hline 0.0370777 & 0.419197 & 0.013021 & 0.252839 & 0.0173876 & 0.272726 & 0.0216376 \\
\hline 0.0330266 & 0.420535 & 0.0144428 & 0.251188 & 0.0146294 & 0.269874 & 0.021699 \\
\hline 0.0262478 & 0.420393 & 0.0162391 & 0.259402 & 0.0156704 & 0.27934 & 0.0248237 \\
\hline 0.0424888 & 0.416072 & 0.0163563 & 0.256676 & 0.0153439 & 0.280738 & 0.0217083 \\
\hline 0.0354099 & 0.412331 & 0.0147233 & 0.256174 & 0.0138372 & 0.292204 & 0.0247353 \\
\hline 0.0389657 & 0.417238 & 0.0179344 & 0.255878 & 0.0155225 & 0.292495 & 0.0252661 \\
\hline 0.0341831 & 0.414896 & 0.0164641 & 0.256793 & 0.0134732 & 0.29322 & 0.0294909 \\
\hline 0.0330551 & 0.423781 & 0.0186681 & 0.259334 & 0.0166146 & 0.297913 & 0.0235727 \\
\hline 0.0357706 & 0.421632 & 0.0180672 & 0.259344 & 0.0144451 & 0.302368 & 0.0240532 \\
\hline 0.0361021 & 0.416106 & 0.0152429 & 0.270644 & 0.0177375 & 0.310848 & 0.0282386 \\
\hline 0.0381735 & 0.416957 & 0.0185382 & 0.25617 & 0.0151141 & 0.314342 & 0.0188869 \\
\hline 0.0334017 & 0.415034 & 0.0144833 & 327 & 0.0 & 89 & 0.0300223 \\
\hline 0.0283496 & 0.412605 & 0.0129988 & 0.263001 & 0.0174547 & 0.313103 & 0.0337054 \\
\hline 0.0457688 & 0.412453 & 0.0175376 & 0.260936 & 0.0129992 & 0.310941 & 0.0307936 \\
\hline 0.0398106 & 0.41 & 347 & 526 & 0.01 & 64 & 738 \\
\hline 0.0449681 & 0.413886 & 0.012134 & 0.262811 & 0.0178237 & 0.314604 & 0.0288104 \\
\hline 0.0428704 & 0.408409 & 0.0125936 & 0.263617 & 0.0167188 & 0.317571 & 0.0326987 \\
\hline 0.0393857 & 0.408273 & 0.0145721 & 0.264979 & 0.015477 & 0.310722 & 0.0256027 \\
\hline 0.037476 & 0.407172 & 0.0151952 & 0.262813 & 0.0173756 & 0.312554 & 0.0355438 \\
\hline 0.0358508 & 0.407262 & 0.0197233 & 0.260321 & 0.0167814 & 0.303266 & 0.0256318 \\
\hline 0.0364007 & 0.403747 & 0.013149 & 0.261773 & 0.0141852 & 0.300111 & 0.0252843 \\
\hline 0.035492 & 0.401483 & 0.0151225 & 0.256079 & 0.0163401 & 0.299576 & 0.0242542 \\
\hline 0.0369888 & 0.402863 & 0.0170897 & 0.260762 & 0.0170128 & 0.296168 & 0.0252723 \\
\hline 0.0307645 & 0.402401 & 0.0194179 & 0.262478 & 0.0172493 & 0.292766 & 0.0199751 \\
\hline 0.0395873 & 0.401665 & 0.016253 & 0.257007 & 0.016432 & 0.290819 & 0.0316359 \\
\hline 0.0420003 & 0.398453 & 0.0154712 & 0.254034 & 0.0123125 & 0.282391 & 0.0223119 \\
\hline 0.0277698 & 0.390333 & 0.0138211 & 0.249715 & 0.0152955 & 0.276785 & 0.0249936 \\
\hline 0.0307725 & 0.393466 & 0.0128197 & 0.247372 & 0.0185381 & 0.267664 & 0.0220174 \\
\hline 0.0319938 & 0.394308 & 0.0151051 & 0.246247 & 0.0150065 & 0.268985 & 0.024277 \\
\hline 0.0330772 & 0.392272 & 0.013335 & 0.25404 & 0.0174201 & 0.271682 & 0.0189224 \\
\hline 0.0359896 & 0.391373 & 0.0133689 & 0.252699 & 0.0171021 & 0.265604 & 0.0164757 \\
\hline 0.0361928 & 0.39216 & 0.0103582 & 0.252019 & 0.0145797 & 0.262246 & 0.0178199 \\
\hline 0.0377474 & 0.390431 & 0.0130987 & 0.252723 & 0.0146483 & 0.258747 & 0.0162847 \\
\hline 0.0381546 & 0.391202 & 0.0163059 & 0.253397 & 0.0161954 & 0.24736 & 0.0168355 \\
\hline 0.0341811 & 0.389374 & 0.0160497 & 0.247749 & 0.0125935 & 0.254858 & 0.0187629 \\
\hline 0.0287212 & 0.391476 & 0.0175517 & 0.246892 & 0.017383 & 0.260245 & 0.0245282 \\
\hline 0.0346388 & 0.399185 & 0.0107967 & 0.242999 & 0.0155415 & 0.260838 & 0.0206307 \\
\hline 0.035162 & 0.3874 & 0.012002 & 0.242197 & 0.0150966 & 0.261973 & 0.0176447 \\
\hline 0.0261867 & 0.388429 & 0.0134618 & 0.247891 & 0.0140523 & 0.257529 & 0.0181638 \\
\hline 0.0361893 & 0.388309 & 0.0121326 & 0.249143 & 0.014907 & 0.256182 & 0.0230698 \\
\hline
\end{tabular}




\begin{tabular}{|c|c|c|c|c|c|c|}
\hline 0.0347512 & 0.389598 & 0.015028 & 0.252357 & 0.0164369 & 0.260373 & 0.0218091 \\
\hline 0.041671 & .390695 & 0.0147185 & 0.247623 & 0.011059 & 0.26149 & 0.0169612 \\
\hline 0.0371317 & 0.3924 & 0.0135137 & 0.251607 & 0.0173483 & 0.259441 & 0.0190671 \\
\hline 0.0292654 & .390996 & 0.0123391 & 0.248925 & 0.017209 & 0.261915 & 0.0213596 \\
\hline 0.0324212 & 389741 & 0.010756 & 0.24903 & 0.0162515 & 0.258016 & 0.0179402 \\
\hline 0.0485707 & 0.393148 & 0.017555 & 0.253955 & 0.0161479 & 0.256512 & 0.0181962 \\
\hline 0.0329451 & 0.389643 & 0.0181227 & 0.256247 & 0.0165983 & 0.26519 & 0.0220422 \\
\hline 0.0340001 & 0.387549 & 0.0150368 & 0.250628 & 0.0143536 & 0.264771 & 0.0193533 \\
\hline 0.0406995 & 0.390098 & 0.0165614 & 0.250244 & 0.0182757 & 0.263623 & 0.0168542 \\
\hline 0.0419439 & 0.391317 & 0.018234 & 0.248506 & 0.0141684 & 0.261164 & 0.0173376 \\
\hline 0.0356507 & 0.391253 & 0.0114351 & 0.253695 & 0.0113784 & 0.269301 & 0.0195349 \\
\hline 0.0348849 & 0.395612 & 0.0155457 & 0.260104 & 0.0125173 & 0.265301 & 0.0161759 \\
\hline 0.0323531 & 0.395965 & 0.0148675 & 0.25632 & 0.017904 & 0.268364 & 0.0194802 \\
\hline 0.0367166 & 0.393924 & 0.0142721 & 0.255489 & 0.0125374 & 0.264683 & 0.0251788 \\
\hline 0.0286205 & 0.393323 & 0.0117365 & 0.255014 & 0.0192305 & 0.263516 & 0.0165632 \\
\hline 0.0337605 & 0.392257 & 0.0158998 & 0.259916 & 0.0133176 & 0.272508 & 0.0221598 \\
\hline 0.0411414 & 0.39805 & 0.0184206 & 0.258963 & 0.0139807 & 0.263056 & 0.0160776 \\
\hline 0.0319093 & 0.399477 & 0.0138977 & 0.260356 & 0.0131017 & 0.258639 & 0.0179728 \\
\hline 0.033852 & 0.393985 & 0.0136109 & 24 & 0.0124227 & 0.27 & 4964 \\
\hline 0.0327059 & 0.396113 & 0.015441 & 0.261164 & 0.0117176 & 0.267759 & 0.0212574 \\
\hline 0.0329393 & 0.401479 & 0.0173921 & 0.269363 & 0.0171357 & 0.268982 & 0.0200847 \\
\hline 0.028204 & 40246 & 0.0141212 & 59 & 0.015 & 92 & 3944 \\
\hline 0.0355349 & 0.396777 & 0.015397 & 0.263926 & 0.0154669 & 0.265845 & 0.0194711 \\
\hline 0.0235923 & 0.402671 & 0.0185724 & 0.270886 & 0.0173917 & 0.279744 & 0.0202696 \\
\hline 0.0388001 & 0.403811 & 0.0143848 & 0.266135 & 0.0136081 & 0.272299 & 0.0193847 \\
\hline 0.0342074 & 0.406873 & 0.0140398 & 0.265204 & 0.0160464 & 0.277598 & 0.0168999 \\
\hline 0.0308666 & 0.404387 & 0.020345 & 0.270961 & 0.0178563 & 0.277412 & 0.0195002 \\
\hline 0.0312072 & 0.408715 & 0.014211 & 0.272486 & 0.0138924 & 0.281272 & 0.0180937 \\
\hline 0.0445887 & 0.413484 & 0.0173323 & 0.276873 & 0.019943 & 0.2747 & 0.0175223 \\
\hline 0.0389423 & 0.416411 & 0.0137442 & 0.279181 & 0.0159222 & 921 & 0.0177627 \\
\hline 0.0444246 & 0.416918 & 0.0143402 & 0.275219 & 0.0161122 & 0.285841 & 0.0196293 \\
\hline 0.0240386 & 0.417583 & 0.0146087 & 0.275876 & 0.0181036 & 0.293176 & 0.0176219 \\
\hline 0.0439062 & 0.417305 & 0.0178338 & 0.276442 & 0.0188613 & 0.285674 & 0.0202713 \\
\hline 0.0346897 & 0.421159 & 0.0193026 & 0.285536 & 0.0172213 & 0.2912 & 0.0205852 \\
\hline 0.0382366 & 0.425309 & 0.0146197 & 0.287387 & 0.0118997 & 0.287358 & 0.0178015 \\
\hline 0.0326898 & 0.423145 & 0.0145366 & 0.2891 & 0.0118113 & 0.297056 & 0.0214003 \\
\hline 0.040071 & 0.425518 & 0.0136378 & 0.283936 & 0.0146948 & 0.297397 & 0.0222132 \\
\hline 0.0345933 & 0.426004 & 0.0164048 & 0.29168 & 0.0185203 & 0.304643 & 0.0208435 \\
\hline 0.0363562 & 0.430601 & 0.0176897 & 0.292157 & 0.0160708 & 0.305203 & 0.0239154 \\
\hline 0.0369077 & 0.437366 & 0.0141739 & 0.298291 & 0.01646 & 0.309319 & 0.0194727 \\
\hline 0.0381477 & 0.440396 & 0.0167175 & 0.294288 & 0.017727 & 0.312765 & 0.0201476 \\
\hline 0.0364585 & 0.444573 & 0.0189691 & 0.311851 & 0.0152371 & 0.307971 & 0.020459 \\
\hline 0.0425807 & 0.448332 & 0.0176173 & 0.308453 & 0.0153966 & 0.319724 & 0.0196662 \\
\hline 0.0344847 & 0.454416 & 0.0155608 & 0.31468 & 0.0188084 & 0.323874 & 0.0245532 \\
\hline 0.0385776 & 0.460929 & 0.0153041 & 0.312455 & 0.0243675 & 0.334231 & 0.0262079 \\
\hline 0.0369052 & 0.465225 & 0.0143314 & 0.314506 & 0.0172435 & 0.345327 & 0.0255198 \\
\hline 0.0352727 & 0.470955 & 0.0174713 & 0.322637 & 0.0153639 & 0.335443 & 0.0224756 \\
\hline
\end{tabular}




\begin{tabular}{|c|c|c|c|c|c|c|}
\hline 0.0396855 & 0.471109 & 0.0161128 & 0.324539 & 0.017481 & 0.341925 & 0.0202469 \\
\hline 0.0459344 & 0.479158 & 0.014111 & 0.331857 & 0.0193889 & 0.35012 & 0.0193457 \\
\hline 0.0383598 & 0.484454 & 0.0179686 & 0.337397 & 0.0167927 & 0.353456 & 0.0228626 \\
\hline 0.0421719 & .489257 & 0.0167941 & 0.339259 & 0.0190265 & 0.358608 & 0.0180711 \\
\hline 0.03751 & 0.498705 & 0.0172208 & 0.34538 & 0.0253614 & 0.365806 & 0.0195915 \\
\hline 0.044368 & 0.508893 & 0.013581 & 0.344521 & 0.0163891 & 0.365603 & 0.0242904 \\
\hline 0.048557 & 0.518629 & 0.0157543 & 0.350572 & 0.0179242 & 0.381997 & 0.0281026 \\
\hline 0.0374921 & 0.523263 & 0.0166316 & 0.36561 & 0.019196 & 0.387923 & 0.0301186 \\
\hline 0.0505527 & 0.530342 & 0.02019 & 0.36597 & 0.0196331 & 0.392311 & 0.0193866 \\
\hline 0.0388099 & 0.53919 & 0.0176756 & 0.37659 & 0.0179669 & 0.404557 & 0.0203034 \\
\hline 0.0390637 & 0.547247 & 0.015956 & 0.381241 & 0.0181212 & 0.418569 & 0.0282249 \\
\hline 0.0397629 & 0.55789 & 0.0186256 & 0.390789 & 0.0169445 & 0.426584 & 0.0230505 \\
\hline 0.047739 & 0.572542 & 0.0191619 & 0.403404 & 0.0227726 & 0.427528 & 0.0238725 \\
\hline 0.0408826 & 0.577282 & 0.0178193 & 0.410035 & 0.0180107 & 0.440122 & 0.0221292 \\
\hline 0.0419429 & 0.593078 & 0.0190404 & 0.41881 & 0.0208276 & 0.448514 & 0.0267911 \\
\hline 0.0407602 & 0.610323 & 0.0187357 & 0.425859 & 0.0187285 & 0.452033 & 0.0267201 \\
\hline 0.0445503 & 0.615465 & 0.0210464 & 0.432829 & 0.0187172 & 0.467269 & 0.0266842 \\
\hline 0.0446157 & 0.625962 & 0.0230385 & 0.45063 & 0.025 & 0.478266 & 0.0250656 \\
\hline 0.0531105 & 834 & 0.0229899 & 0.45 & 0.02 & 0.493231 & 0.032 \\
\hline 0.0435292 & 0.651341 & 0.0167815 & 0.47026 & 0.0188701 & 0.508898 & 0.0240535 \\
\hline 0.0518816 & 0.666004 & 0.0187523 & 0.480667 & 0.0224866 & 0.51834 & 0.0264792 \\
\hline 0.0616761 & 43 & 0.0208961 & 17 & 0.02 & 0.524782 & 185 \\
\hline 0.0486968 & 0.700541 & 0.0212243 & 0.507863 & 0.0233678 & 0.54801 & 0.0339522 \\
\hline 0.0501058 & 0.712904 & 0.0269648 & 0.513147 & 0.0274151 & 0.555662 & 0.027912 \\
\hline 0.0517564 & 0.728546 & 0.0193262 & 0.524424 & 0.0281981 & 0.580081 & 0.0313744 \\
\hline 0.0407645 & 0.746362 & 0.0202056 & 0.554655 & 0.0266867 & 0.572858 & 0.0269076 \\
\hline 0.0551209 & 0.755085 & 0.0203742 & 0.565292 & 0.0229142 & 0.59868 & 0.031674 \\
\hline 0.0560542 & 0.766122 & 0.0234326 & 0.578651 & 0.0280626 & 0.626534 & 0.0304952 \\
\hline 0.0490676 & 0.782113 & 0.0263525 & 0.586925 & 0.0252837 & 0.633774 & 0.0308626 \\
\hline 0.0548789 & & 0.0269 & & 0.02 & 446 & 0.0422688 \\
\hline 0.0492161 & 0.809389 & 0.0256855 & 0.625527 & 0.0259288 & 0.662398 & 0.0279641 \\
\hline 0.036757 & 0.824901 & 0.0195607 & 0.641372 & 0.0267596 & 0.677676 & 0.0308556 \\
\hline 0.0535237 & 0.843292 & 0.0231534 & 0.657285 & 0.0266259 & 0.70034 & 0.0338806 \\
\hline 0.0514744 & 0.854862 & 0.0192724 & 0.685383 & 0.0271961 & 0.71128 & 0.0281962 \\
\hline 0.0613098 & 0.867851 & 0.0202639 & 0.686731 & 0.0233361 & 0.736713 & 0.0351319 \\
\hline 0.0630335 & 0.876502 & 0.0221038 & 0.722028 & 0.0242996 & 0.74652 & 0.03479 \\
\hline 0.0620185 & 0.886373 & 0.0230033 & 0.719338 & 0.0326287 & 0.753571 & 0.0376029 \\
\hline 0.0631364 & 0.904726 & 0.0207525 & 0.730561 & 0.0274392 & 0.773043 & 0.0377463 \\
\hline 0.0530376 & 0.913157 & 0.0227166 & 0.759976 & 0.0297125 & 0.792554 & 0.0333439 \\
\hline 0.046779 & 0.923297 & 0.0223315 & 0.773144 & 0.0290197 & 0.805731 & 0.0382373 \\
\hline 0.0524637 & 0.925443 & 0.0226282 & 0.792968 & 0.0268663 & 0.815342 & 0.0325052 \\
\hline 0.0609754 & 0.938672 & 0.0213403 & 0.802554 & 0.0301176 & 0.835288 & 0.032294 \\
\hline 0.0679108 & 0.951694 & 0.0267806 & 0.812878 & 0.0196902 & 0.850343 & 0.0390061 \\
\hline 0.0567089 & 0.956644 & 0.0216653 & 0.831944 & 0.026491 & 0.85567 & 0.0381103 \\
\hline 0.0742667 & 0.95806 & 0.0169288 & 0.848887 & 0.0353537 & 0.866599 & 0.0321775 \\
\hline 0.0638075 & 0.968851 & 0.0198667 & 0.855637 & 0.0295533 & 0.876796 & 0.0401276 \\
\hline 0.0689664 & 0.974993 & 0.015953 & 0.869656 & 0.0272496 & 0.892621 & 0.0447493 \\
\hline
\end{tabular}




\begin{tabular}{|c|c|c|c|c|c|c|}
\hline 0.0628893 & 0.975755 & 0.0169561 & 0.884752 & 0.0265174 & 0.914257 & 0.0451445 \\
\hline 0.0597283 & .972147 & 0.0200548 & 0.89988 & 0.0259811 & 0.902435 & 0.0364524 \\
\hline 0.0629695 & 0.971667 & 0.0202804 & 0.907697 & 0.0243486 & 0.929067 & 0.032946 \\
\hline .0496599 & .968315 & 0.0199656 & 0.910855 & 0.0286247 & 0.92174 & 0.0400309 \\
\hline .0580825 & .971818 & 0.0207719 & 0.926247 & 0.025498 & 0.917314 & 0.0327214 \\
\hline 0.05814 & 0.968725 & 0.0246105 & 0.936096 & 0.0296694 & 0.943165 & 0.0464089 \\
\hline 0.0648555 & 0.966367 & 0.0266052 & 0.94382 & 0.031541 & 0.949431 & 0.0353945 \\
\hline 0.0588387 & 0.967878 & 0.0218142 & 0.938864 & 0.0305535 & 0.943408 & 0.035188 \\
\hline 0.0548317 & 0.957383 & 0.020135 & 0.949195 & 0.0301632 & 0.939789 & 0.0363271 \\
\hline 0.0802022 & 0.948779 & 0.0239552 & 0.94917 & 0.0289116 & 0.947006 & 0.0325766 \\
\hline 0.0472783 & 0.940516 & 0.021491 & 0.956894 & 0.0325299 & 0.944213 & 0.0316117 \\
\hline 0.0576431 & 0.933683 & 0.021626 & 0.953866 & 0.0338575 & 0.947977 & 0.0411948 \\
\hline 0.083169 & 0.933143 & 0.0235243 & 0.953535 & 0.0304989 & 0.939525 & 0.0333223 \\
\hline 0.0585421 & 0.924452 & 0.0234001 & 0.949436 & 0.0261882 & 0.932703 & 0.040192 \\
\hline 0.0601066 & 0.912761 & 0.0270636 & 0.934665 & 0.0348068 & 0.933639 & 0.0412737 \\
\hline 0.0680318 & 0.900055 & 0.0240331 & 0.943739 & 0.0320082 & 0.925112 & 0.0404035 \\
\hline 0.0599696 & 0.890455 & 0.0195784 & 0.946967 & 0.0333088 & 0.921067 & 0.0401594 \\
\hline 0.0536944 & 0.873049 & 0.0199216 & 0.939268 & 0.032976 & 0.902638 & 0.0387432 \\
\hline 0.055433 & 0.86 & 0.017567 & 0.931257 & 0.0282739 & 0.90 & 0.0456721 \\
\hline 0.0571688 & 0.848362 & 0.0239047 & 0.923999 & 0.0286709 & 0.894608 & 0.0461311 \\
\hline 0.0595534 & 0.83491 & 0.0209001 & 0.907968 & 0.0263509 & 0.887993 & 0.0435281 \\
\hline 0.0662731 & 0.82 & 0.0237178 & 0.89 & 0.0342898 & 331 & 749 \\
\hline 0.0639529 & 0.810684 & 0.0238247 & 0.893278 & 0.0308382 & 0.859483 & 0.0418677 \\
\hline 0.0431788 & 0.791541 & 0.0258565 & 0.881777 & 0.0358725 & 0.841776 & 0.0264098 \\
\hline 0.0567606 & 0.776719 & 0.0272842 & 0.863987 & 0.0299888 & 0.832202 & 0.024421 \\
\hline 0.0502742 & 0.766121 & 0.0198046 & 0.85004 & 0.0250093 & 0.819982 & 0.0293084 \\
\hline 0.0721674 & 0.751121 & 0.0158127 & 0.835149 & 0.0321872 & 0.805622 & 0.0327737 \\
\hline 0.0606053 & 0.735851 & 0.0195358 & 0.819828 & 0.0202368 & 0.793141 & 0.0303243 \\
\hline 0.063327 & 0.71986 & 0.0222635 & 0.810727 & 0.026333 & 0.764662 & 0.0284328 \\
\hline 0.0693294 & 0.702482 & 0.0182763 & 0.790815 & 0.0353506 & 0.757591 & 0.0373221 \\
\hline 0.0673099 & 0.684332 & 0.0178206 & 0.782704 & 0.0292132 & 0.736426 & 0.0277104 \\
\hline 0.0724869 & 0.667626 & 0.0212963 & 0.758544 & 0.0269977 & 0.724528 & 0.0324112 \\
\hline 0.0678885 & 0.653423 & 0.0233952 & 0.752536 & 0.0285852 & 0.712149 & 0.0401421 \\
\hline 0.0582169 & 0.638065 & 0.0227133 & 0.731379 & 0.0268082 & 0.698303 & 0.0306327 \\
\hline 0.0553115 & 0.618278 & 0.0201146 & 0.71607 & 0.0259126 & 0.679432 & 0.0295201 \\
\hline 0.0765005 & 0.604878 & 0.0166912 & 0.696106 & 0.0215413 & 0.656409 & 0.0327598 \\
\hline 0.0658757 & 0.592379 & 0.0188488 & 0.688606 & 0.0261793 & 0.63387 & 0.0232882 \\
\hline 0.0723515 & 0.580488 & 0.0229229 & 0.663628 & 0.0308037 & 0.619202 & 0.0290145 \\
\hline 0.0521904 & 0.566796 & 0.0231919 & 0.646129 & 0.0264734 & 0.611352 & 0.0320327 \\
\hline 0.0681785 & 0.554433 & 0.0219174 & 0.626391 & 0.0254521 & 0.587574 & 0.030118 \\
\hline 0.0587321 & 0.539603 & 0.018659 & 0.620975 & 0.0296443 & 0.577475 & 0.0331435 \\
\hline 0.0546033 & 0.529373 & 0.0166572 & 0.592926 & 0.0175485 & 0.569084 & 0.0233754 \\
\hline 0.0653275 & 0.523387 & 0.0156105 & 0.573961 & 0.0204951 & 0.542699 & 0.0236894 \\
\hline 0.0458256 & 0.505531 & 0.0170759 & 0.557676 & 0.0238973 & 0.530406 & 0.0288133 \\
\hline 0.0483486 & 0.490913 & 0.0175536 & 0.547483 & 0.0229567 & 0.51951 & 0.0267812 \\
\hline 0.055513 & 0.484832 & 0.0169598 & 0.5286 & 0.0216894 & 0.499925 & 0.0232159 \\
\hline 0.0463638 & 0.475742 & 0.018815 & 0.515107 & 0.024656 & 0.488914 & 0.0221391 \\
\hline
\end{tabular}




\begin{tabular}{|c|c|c|c|c|c|c|}
\hline 0.0546257 & 0.464394 & 0.0184341 & 0.502353 & 0.0195272 & 0.469276 & 0.0287758 \\
\hline 0.0592901 & 0.453569 & 0.0160815 & 0.482675 & 0.0256678 & 0.468766 & 0.0280096 \\
\hline 0.0387106 & 0.444288 & 0.0126846 & .477908 & 0.0195175 & 0.448609 & 0.0252795 \\
\hline 0533253 & 0.432365 & 0.0145221 & .464131 & 0.0231387 & 0.436 & 0.02719 \\
\hline 0.0458121 & 0.41953 & 0.0148983 & 0.449414 & 0.0209002 & 0.428887 & 0.0215529 \\
\hline 0.0583217 & 0.414076 & 0.0124657 & 0.438765 & 0.0293498 & 0.413865 & 0.0225472 \\
\hline 0.0542186 & 0.405982 & 0.0133523 & 0.420779 & 0.0210994 & 0.402422 & 0.0333723 \\
\hline 0.0453495 & 0.396132 & 0.0159635 & 0.411186 & 0.0164323 & 0.402276 & 0.0246522 \\
\hline 0.041335 & 0.39163 & 0.0134076 & 0.404861 & 0.0179353 & 0.38989 & 0.0240087 \\
\hline 0.0517301 & 0.386767 & 0.0115846 & 0.398061 & 0.0188258 & 0.380071 & 0.0179707 \\
\hline 0.0539654 & 0.378732 & 0.0101309 & 0.382772 & 0.0190948 & 0.365584 & 0.0237639 \\
\hline 0.0370652 & 0.375071 & 0.0103774 & 0.374549 & 0.0231174 & 0.349952 & 0.0198644 \\
\hline 0.0388154 & 0.370044 & 0.0106426 & 0.355247 & 0.0193805 & 0.345211 & 0.0192353 \\
\hline 0.0407964 & 0.360159 & 0.0105497 & 0.357702 & 0.0163879 & 0.33516 & 0.0214502 \\
\hline 0.0365464 & 0.35249 & 0.0149426 & 0.341413 & 0.0154753 & 0.33309 & 0.0175944 \\
\hline 0.0333821 & 0.348444 & 0.0117632 & 0.337006 & 0.0130727 & 0.323752 & 0.0155544 \\
\hline 0.0411668 & 0.344128 & 0.0109243 & 0.330269 & 0.0148596 & 0.315594 & 0.0276043 \\
\hline 0.0395034 & 0.338532 & 0.0115371 & 0.319989 & 0.0161723 & 0.316969 & 0.0231928 \\
\hline 0.0411823 & 0.33 & 0.015158 & 44 & 0.0134432 & & 5106 \\
\hline 0.0335574 & 0.330677 & 0.0102832 & 0.313477 & 0.0184671 & 0.28999 & 0.0176235 \\
\hline 0.0460177 & 0.324907 & 0.0112658 & 0.294548 & 0.018357 & 0.292934 & 0.022905 \\
\hline 0.036022 & 0.31 & 0.0162722 & 95 & 0.015 & 57 & 452 \\
\hline 0.03925 & 0.309706 & 0.0137571 & 0.286219 & 0.0162968 & 0.276215 & 0.0140843 \\
\hline 0.0322716 & 0.308114 & 0.0107267 & 0.288027 & 0.0104409 & 0.276982 & 0.0229223 \\
\hline 0.0346414 & 0.305957 & 0.0101654 & 0.272664 & 0.0132386 & 0.268775 & 0.0200901 \\
\hline 0.0411858 & 0.301387 & 0.0131925 & 0.270745 & 0.0129937 & 0.263251 & 0.0215282 \\
\hline 0.0306591 & 0.295343 & 0.0142065 & 0.260436 & 0.0178948 & 0.260384 & 0.0204678 \\
\hline 0.0268385 & 0.294584 & 0.0101172 & 0.259895 & 0.012901 & 0.258867 & 0.019892 \\
\hline 0.0381088 & 0.290623 & 0.00818192 & 0.260199 & 0.0172787 & 0.246676 & 0.0210143 \\
\hline 0.0348602 & 0.284869 & 0.0114583 & & 0.0171978 & 24311 & 0.0172687 \\
\hline 0.0361705 & 0.283231 & 0.0116043 & 0.24 & 0.0167303 & 0.239289 & 0.0185594 \\
\hline 0.0378473 & 0.276476 & 0.0127805 & 0.239952 & 0.0140873 & 0.237602 & 0.0154251 \\
\hline 0.0347298 & 0.272525 & 0.0139374 & 0.232611 & 0.0140705 & 0.227968 & 0.0157703 \\
\hline 0.0306058 & 0.269772 & 0.0107791 & 0.231221 & 0.0105265 & 0.229418 & 0.0125239 \\
\hline 0.0382161 & 0.267669 & 0.0107551 & 0.225468 & 0.0136278 & 0.221845 & 0.0180972 \\
\hline 0.0271581 & 0.265296 & 0.0109784 & 0.223545 & 0.0145706 & 0.221429 & 0.0155388 \\
\hline 0.0356827 & 0.261597 & 0.010477 & 0.216211 & 0.0155012 & 0.216135 & 0.0153627 \\
\hline 0.0326509 & 0.261201 & 0.0132117 & 0.213982 & 0.014128 & 0.212964 & 0.0146097 \\
\hline 0.0322201 & 0.259833 & 0.010473 & 0.210096 & 0.010217 & 0.207638 & 0.0196462 \\
\hline 0.0311154 & 0.255473 & 0.00976098 & 0.210227 & 0.0112513 & 0.209606 & 0.0124068 \\
\hline 0.0378099 & 0.25492 & 0.0101239 & 0.204755 & 0.015626 & 0.200679 & 0.0162127 \\
\hline 0.0338507 & 0.251372 & 0.010012 & 0.199423 & 0.0131597 & 0.197333 & 0.0179001 \\
\hline 0.0339479 & 0.247581 & 0.0109653 & 0.199042 & 0.012334 & 0.192417 & 0.0152791 \\
\hline 0.0354896 & 0.248974 & 0.0116044 & 0.193134 & 0.0126111 & 0.188635 & 0.0184498 \\
\hline 0.0383387 & 0.249254 & 0.0119215 & 0.190233 & 0.0131945 & 0.196382 & 0.0132816 \\
\hline 0.0396512 & 0.250715 & 0.00938004 & 0.189643 & 0.0131971 & 0.18804 & 0.0118942 \\
\hline 0.0381752 & 0.250464 & 0.00967716 & 0.190023 & 0.0123089 & 0.187883 & 0.0167628 \\
\hline
\end{tabular}




\begin{tabular}{|c|c|c|c|c|c|c|}
\hline 0.0316589 & .25298 & 0.010526 & 0.189343 & 0.0148985 & 0.183809 & 0.0128855 \\
\hline 0.0339491 & 0.256699 & 0.0100508 & 0.184591 & 0.0102186 & 0.188164 & 0.0149639 \\
\hline 0.0327655 & 0.261576 & 0.00850483 & 0.184643 & 0.0106205 & 0.188796 & 0.0164008 \\
\hline 0320728 & 0.26698 & 0.00916157 & .183584 & 0.0124738 & 0.182353 & 0.01404 \\
\hline 0.0301792 & 0.273797 & 0.0110121 & 0.182528 & 0.0145553 & 0.19107 & 0.015013 \\
\hline 0.0384695 & 0.280203 & 0.0120425 & 0.183721 & 0.0160807 & 0.189401 & 0.017355 \\
\hline 0.0415115 & 0.284996 & 0.0124784 & 0.186081 & 0.0148133 & 0.192529 & 0.0148511 \\
\hline 0.0368676 & 0.295719 & 0.012366 & 0.195317 & 0.0119537 & 0.198702 & 0.0194626 \\
\hline 0.0339903 & 0.310272 & 0.0116014 & 0.196748 & 0.013842 & 0.204635 & 0.0133582 \\
\hline 0.0411568 & 0.323902 & 0.0114976 & 0.197633 & 0.0142698 & 0.210276 & 0.0168589 \\
\hline 0.0458891 & 0.336717 & 0.0109787 & 0.207135 & 0.0141042 & 0.215411 & 0.0121909 \\
\hline 0.0326953 & 0.350819 & 0.0111387 & 0.212722 & 0.0132352 & 0.230429 & 0.0143525 \\
\hline 0.0401049 & 0.366509 & 0.0119812 & 0.226473 & 0.013906 & 0.233228 & 0.0184979 \\
\hline 0.0515572 & 0.387037 & 0.0144152 & 0.234862 & 0.013526 & 0.245426 & 0.0221248 \\
\hline 0.0394696 & 0.405807 & 0.0137833 & 0.246761 & 0.0155626 & 0.259552 & 0.0165705 \\
\hline 0.0523867 & 0.425861 & 0.0134653 & 0.256068 & 0.0172037 & 0.272059 & 0.0208442 \\
\hline 0.0336263 & 0.446545 & 0.0101592 & 0.27182 & 0.0144898 & 0.285752 & 0.0233213 \\
\hline 0.0560612 & 0.465416 & 0.010617 & 0.292741 & 0.0162033 & 0.30561 & 0.0156332 \\
\hline 0.0490591 & 0.48622 & 0.0121822 & 932 & 0.01 & 42 & 0.02 \\
\hline 0.0603253 & 0.505935 & 0.0140123 & 0.322045 & 0.0118688 & 0.343841 & 0.0185248 \\
\hline 0.0574051 & 0.529698 & 0.0151862 & 0.339704 & 0.0171032 & 0.349795 & 0.0172956 \\
\hline 0.0539122 & 0.54 & 0.0168355 & 546 & 0.01 & 575 & 5811 \\
\hline 0.0656639 & 0.564156 & 0.0168158 & 0.388838 & 0.0201768 & 0.395477 & 0.0228594 \\
\hline 0.0732415 & 0.586118 & 0.0158174 & 0.410568 & 0.0207992 & 0.411866 & 0.0273884 \\
\hline 0.0602273 & 0.604491 & 0.014134 & 0.436697 & 0.02 & 0.437621 & 0.0261478 \\
\hline 0.0542664 & 0.622502 & 0.0176964 & 0.455886 & 0.0234878 & 0.458697 & 0.0260232 \\
\hline 0.0782791 & 0.635551 & 0.023812 & 0.472058 & 0.0259701 & 0.491165 & 0.028345 \\
\hline 0.0755266 & 0.64784 & 0.0172335 & 0.501802 & 0.0267867 & 0.494893 & 0.0238509 \\
\hline 0.067301 & 0.665585 & 0.0212245 & 0.519384 & 0.0192007 & 0.518857 & 0.025514 \\
\hline 0.0741438 & 0.677589 & 0.0197984 & 0.541693 & 0.0197367 & 0.537361 & 0.0279279 \\
\hline 0.0749942 & 0.680898 & 0.0201059 & 0.558471 & 0.025 & 0.557485 & 0.0241026 \\
\hline 0.0879594 & 0.683094 & 0.0202966 & 0.581921 & 0.0234809 & 0.571465 & 0.0235049 \\
\hline 0.0925311 & 0.687328 & 0.0189221 & 0.601203 & 0.0246205 & 0.589893 & 0.0349122 \\
\hline 0.0967296 & 0.692113 & 0.020981 & 0.609388 & 0.0256675 & 0.592655 & 0.030601 \\
\hline 0.079853 & 0.686562 & 0.0251264 & 0.634672 & 0.0303463 & 0.608836 & 0.0273712 \\
\hline 0.0849879 & 0.672171 & 0.021696 & 0.639239 & 0.0322624 & 0.616039 & 0.0262889 \\
\hline 0.102612 & 0.659537 & 0.0173055 & 0.652833 & 0.0354838 & 0.610406 & 0.026554 \\
\hline 0.0854993 & 0.646843 & 0.0139434 & 0.659701 & 0.0274104 & 0.616983 & 0.0347793 \\
\hline 0.101276 & 0.633347 & 0.015193 & 0.65317 & 0.0321107 & 0.619344 & 0.0287841 \\
\hline 0.0929962 & 0.61498 & 0.0195891 & 0.662145 & 0.0325084 & 0.616628 & 0.0272638 \\
\hline 0.073405 & 0.592149 & 0.0170789 & 0.664338 & 0.0288474 & 0.604736 & 0.0268609 \\
\hline 0.106723 & 0.568012 & 0.015572 & 0.664166 & 0.0253508 & 0.594829 & 0.0286354 \\
\hline 0.120978 & 0.542192 & 0.0141073 & 0.644667 & 0.0324011 & 0.58672 & 0.0336446 \\
\hline 0.0922195 & 0.513 & 0.0153968 & 0.64113 & 0.0249493 & 0.57005 & 0.0281693 \\
\hline 0.099384 & 0.482993 & 0.0120556 & 0.609956 & 0.0319298 & 0.556207 & 0.0306544 \\
\hline 0.0939418 & 0.45482 & 0.0122267 & 0.598443 & 0.0240237 & 0.541707 & 0.0280303 \\
\hline 0.0997095 & 0.427684 & 0.0144778 & 0.579055 & 0.0200652 & 0.517206 & 0.028462 \\
\hline
\end{tabular}




\begin{tabular}{|c|c|c|c|c|c|c|}
\hline 0.0885118 & 0.4036 & 0.0131102 & 0.550964 & 0.030309 & 0.491369 & 5071 \\
\hline 0.0784901 & 0.377438 & 0.0136907 & 0.525346 & 0.0272267 & 0.453994 & 0.0262647 \\
\hline 0.0737988 & 0.349235 & 0.0144286 & .502879 & 0.0218817 & 0.429826 & 0.0278824 \\
\hline 0786694 & .327918 & 0.0130222 & 0.47282 & 0.020437 & 0.405457 & 0.0268368 \\
\hline 0.0832691 & 0.303406 & 0.0102286 & 0.440703 & 0.0251306 & 0.373194 & 0.0203622 \\
\hline 0.0612264 & 0.281539 & 0.0117688 & 0.408029 & 0.0162226 & 0.345709 & 0.0169579 \\
\hline 0.0578752 & 0.269357 & 0.012716 & 0.378165 & 0.0183529 & 0.320792 & 0.0185395 \\
\hline 0.0674407 & 0.25369 & 0.0113593 & 0.349063 & 0.0138694 & 0.294327 & 0.0192807 \\
\hline 0.051268 & 0.235403 & 0.010396 & 0.323796 & 0.0187361 & 0.266897 & 0.0131805 \\
\hline 0.0470863 & 0.224187 & 0.0100283 & 0.296916 & 0.0151954 & 0.25239 & 0.01772 \\
\hline 0.0540734 & 0.213877 & 0.00893492 & 0.272312 & 0.0159684 & 0.22935 & 0.0150071 \\
\hline 0.0415996 & 0.205153 & 0.0107251 & 0.254477 & 0.0193958 & 0.215886 & 0.0176982 \\
\hline 0.047576 & 0.200263 & 0.00783811 & 0.229368 & 0.0126385 & 0.192531 & 0.0182062 \\
\hline 0.0477401 & 0.194241 & 0.00692413 & 0.205016 & 0.0136874 & 0.179318 & 0.015408 \\
\hline 0.0300142 & 0.188731 & 0.00861725 & 0.193949 & 0.0137532 & 0.167808 & 0.0148396 \\
\hline 0.0365469 & 0.184174 & 0.00867567 & 0.178955 & 0.0138361 & 0.1548 & 9584 \\
\hline 0.028323 & 0.182258 & 0.00820797 & 0.169769 & 0.0140171 & 0.153115 & 0.0160662 \\
\hline 0.035907 & 0.179604 & 0.00773793 & 0.155363 & 0.0104546 & 0.140002 & 1528 \\
\hline 0.0323528 & 0.176644 & 0.007 & 262 & 037 & 13 & 473 \\
\hline 0.0299665 & 0.175718 & 0.00876643 & 0.140681 & 0.0130642 & 0.129518 & 0.0141955 \\
\hline 0.0270815 & 0.17281 & 0.00836613 & 0.137292 & 0.0136263 & 0.126734 & 0.0115328 \\
\hline 0.031839 & 646 & 0.00 & 839 & 0.00 & & 359 \\
\hline 0.023076 & 0.172019 & 0.00778423 & 0.124343 & 0.0100539 & 0.123188 & 0.0125388 \\
\hline 0.0252878 & 0.170635 & 0.00790476 & 0.12606 & 0.0121374 & 0.120441 & 0.0132091 \\
\hline 0.0364125 & 0.168426 & 0.00921928 & 0.11696 & 0.00789095 & 0.115536 & 0.0120762 \\
\hline 0.0260675 & 0.168203 & 0.0104183 & 0.117971 & 0.0100183 & 0.114628 & 0.0125982 \\
\hline 0.0322828 & 0.168011 & 0.00751165 & 0.118163 & 0.0104184 & 0.116286 & 0.0124621 \\
\hline 0.0224529 & 0.169347 & 0.00895898 & 0.118144 & 0.0114755 & 0.112013 & 0.0111058 \\
\hline 0.0256496 & 0.170642 & 0.0095648 & 0.116209 & 0.00815836 & 0.112089 & 0.0118751 \\
\hline 0.0286439 & 0.169194 & 0.010 & 0.113818 & 0.010363 & 0.115911 & 2722 \\
\hline 0.0210231 & 0.167986 & 0.00741552 & 0.112355 & 0.0107474 & 0.112942 & 0.0133818 \\
\hline 0.0259321 & 0.16953 & 0.00855666 & 0.116629 & 0.0101421 & 0.1117 & 0.010972 \\
\hline 0.0207879 & 0.172645 & 0.00872184 & 0.112854 & 0.0108656 & 0.117062 & 0.0110926 \\
\hline 0.0220968 & 0.171524 & 0.00807526 & 0.114237 & 0.00634563 & 0.119274 & 0.0122442 \\
\hline 0.0207714 & 0.168712 & 0.00744397 & 0.115609 & 0.0107075 & 0.118575 & 0.0144215 \\
\hline 0.0251081 & 0.169957 & 0.00732525 & 0.117696 & 0.0122816 & 0.117445 & 0.0119207 \\
\hline 0.0198871 & 0.171598 & 0.0108847 & 0.117639 & 0.00963507 & 0.1132 & 0.0147284 \\
\hline 0.0304519 & 0.173181 & 0.0113088 & 0.116846 & 0.0108413 & 0.112352 & 0.00919493 \\
\hline 0.0254495 & 0.173956 & 0.00972527 & 0.114391 & 0.0111164 & 0.115713 & 0.01146 \\
\hline 0.0226373 & 0.174948 & 0.00819171 & 0.11273 & 0.0119975 & 0.11606 & 0.0115266 \\
\hline 0.0216702 & 0.176199 & 0.00781007 & 0.118237 & 0.011209 & 0.116497 & 0.0108618 \\
\hline 0.0261412 & 0.175196 & 0.00759933 & 0.120065 & 0.0122582 & 0.118386 & 0.0103616 \\
\hline 0.0235591 & 0.177419 & 0.0072629 & 0.119782 & 0.0105779 & 0.117551 & 0.0112159 \\
\hline 0.0252912 & 0.179955 & 0.00914775 & 0.118832 & 0.0106206 & 0.118355 & 0.0104166 \\
\hline 0.0252539 & 0.178117 & 0.0101948 & 0.120608 & 0.0122131 & 0.119774 & 0.0106866 \\
\hline 0.0286642 & 0.174614 & 0.00852516 & 0.120549 & 0.0124759 & 0.11982 & 0.0125921 \\
\hline 0.0198433 & 0.173785 & 0.00748054 & 0.122196 & 0.0106185 & 0.125282 & 0.0118697 \\
\hline
\end{tabular}




$\begin{array}{rrrrrrr}0.0267957 & 0.176744 & 0.00869528 & 0.121443 & 0.0094337 & 0.126649 & 0.0143971 \\ 0.0205817 & 0.180199 & 0.0102275 & 0.119844 & 0.0101525 & 0.123433 & 0.0138334 \\ 0.0237775 & 0.179986 & 0.0126081 & 0.124366 & 0.0126281 & 0.12195 & 0.0120719 \\ 0.0275517 & 0.180411 & 0.00982481 & 0.123528 & 0.0089807 & 0.128604 & 0.0139932 \\ 0.0238738 & 0.182535 & 0.0102076 & 0.126564 & 0.0126246 & 0.129054 & 0.0117067 \\ 0.0248718 & 0.185136 & 0.00951424 & 0.12547 & 0.00966249 & 0.130736 & 0.0132092 \\ 0.0230128 & 0.187087 & 0.00862778 & 0.130993 & 0.00813954 & 0.126265 & 0.00929173 \\ 0.0272171 & 0.18818 & 0.010425 & 0.128428 & 0.00887737 & 0.133585 & 0.0131707 \\ 0.0195466 & 0.189007 & 0.0108048 & 0.127718 & 0.0109008 & 0.132981 & 0.015578 \\ 0.023979 & 0.18987 & 0.00858879 & 0.130624 & 0.00973808 & 0.136269 & 0.0120752 \\ 0.0260836 & 0.190732 & 0.00810961 & 0.132461 & 0.0118196 & 0.136967 & 0.0143256 \\ 0.0305166 & 0.189754 & 0.0110841 & 0.132284 & 0.0112092 & 0.136613 & 0.0116072 \\ 0.0246101 & 0.193048 & 0.0104957 & 0.133125 & 0.011096 & 0.138814 & 0.0158401 \\ 0.0211813 & 0.196322 & 0.0114637 & 0.139623 & 0.0115045 & 0.137149 & 0.013881 \\ 0.0290172 & 0.195233 & 0.0102278 & 0.140044 & 0.0108475 & 0.133634 & 0.0120355 \\ 0.0222481 & 0.193596 & 0.00885864 & 0.143092 & 0.0131492 & 0.140449 & 0.0143364 \\ 0.02394 & 0.197949 & 0.00890883 & 0.139486 & 0.00843923 & 0.143957 & 0.00897351 \\ 0.0311431 & 0.197629 & 0.00898524 & 0.143378 & 0.0100056 & 0.142836 & 0.0169299 \\ 0.0232894 & 0.194099 & 0.00905429 & 0.144162 & 0.0110193 & 0.147553 & 0.0125955 \\ 0.0252591 & 0.197444 & 0.0107786 & 0.145446 & 0.0118549 & 0.150248 & 0.0106334 \\ 0.0261131 & 0.199907 & 0.00967535 & 0.146529 & 0.014243 & 0.154051 & 0.0135478 \\ 0.0288547 & 0.20141 & 0.00861808 & 0.152456 & 0.0106938 & 0.150096 & 0.0140619 \\ 0.0235059 & 0.201863 & 0.00996916 & 0.149859 & 0.0151307 & 0.151975 & 0.0146699 \\ 0.0255689 & 0.20092 & 0.00988273 & 0.153107 & 0.010649 & 0.156639 & 0.0171358 \\ 0.0271253 & 0.204356 & 0.00790213 & 0.153167 & 0.00707604 & 0.157039 & 0.0139374 \\ 0.026893 & 0.201933 & 0.00898684 & 0.153682 & 0.0104289 & 0.156597 & 0.0107228 \\ 0.0321093 & 0.203049 & 0.00975137 & 0.159159 & 0.0151188 & 0.156721 & 0.0151804 \\ 0.0241523 & 0.208082 & 0.0093383 & 0.158896 & 0.0106804 & 0.161528 & 0.0131388\end{array}$




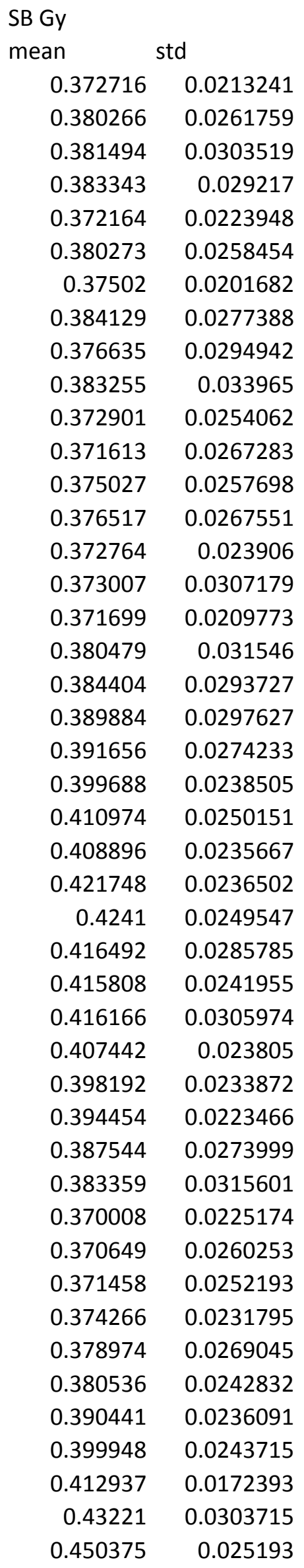




\begin{tabular}{|c|c|}
\hline 0.472457 & 0.0277405 \\
\hline 0.498 & 0.0292648 \\
\hline 0.512533 & 0.0328701 \\
\hline 0.515413 & 0.0293187 \\
\hline 0.530284 & 0.0355724 \\
\hline 0.520182 & 0.03097 \\
\hline 0.511319 & 0.0291712 \\
\hline 0.495357 & 0.0286304 \\
\hline 0.476559 & 0.0297605 \\
\hline 0.455196 & 0.0236857 \\
\hline 0.431886 & 0.0298639 \\
\hline 0.425728 & 0.0258066 \\
\hline 0.405181 & 0.0233254 \\
\hline 0.393977 & 0.0290388 \\
\hline 0.395433 & 0.0249935 \\
\hline 0.397644 & 0.0212563 \\
\hline 0.396738 & 0.0225985 \\
\hline 0.41182 & 0.0234825 \\
\hline 0.426622 & 0.023352 \\
\hline 0.473306 & 0.0280582 \\
\hline 0.546224 & 0.0327075 \\
\hline 0.632605 & 0.0390847 \\
\hline 0.751081 & 0.0510928 \\
\hline 0.903525 & 0.0600441 \\
\hline 1.10555 & 0.0808396 \\
\hline 1.30088 & 0.105435 \\
\hline 1.51293 & 0.128142 \\
\hline 1.69474 & 0.124461 \\
\hline 1.82472 & 0.14738 \\
\hline 1.89005 & 0.139062 \\
\hline 1.89167 & 0.147937 \\
\hline 1.82637 & 0.1431 \\
\hline 1.68839 & 0.129044 \\
\hline 1.54518 & 0.101766 \\
\hline 1.34241 & 0.0978442 \\
\hline 1.17637 & 0.0739933 \\
\hline 1.048 & 0.0608868 \\
\hline 0.929764 & 0.0550786 \\
\hline 0.844002 & 0.0460938 \\
\hline 0.798807 & 0.042529 \\
\hline 0.759484 & 0.0497701 \\
\hline 0.739432 & 0.0408895 \\
\hline 0.722478 & 0.0376147 \\
\hline 0.711702 & 0.036852 \\
\hline 0.713068 & 0.0344655 \\
\hline 0.715768 & 0.0485585 \\
\hline 0.707745 & 0.0404225 \\
\hline
\end{tabular}




\begin{tabular}{rr}
0.70294 & 0.0483059 \\
0.69537 & 0.0524448 \\
0.658041 & 0.0426863 \\
0.619547 & 0.054873 \\
0.596756 & 0.0603676 \\
0.571603 & 0.0478929 \\
0.554683 & 0.0407247 \\
0.560185 & 0.0433066 \\
0.56399 & 0.0399822 \\
0.570893 & 0.0320076 \\
0.593637 & 0.0300065 \\
0.617765 & 0.0384793 \\
0.638362 & 0.0392277 \\
0.654538 & 0.0403441 \\
0.663806 & 0.0299902 \\
0.672772 & 0.0273933 \\
0.673502 & 0.0333002 \\
0.682997 & 0.0307671 \\
0.700228 & 0.0397011 \\
0.705575 & 0.0356558 \\
0.735114 & 0.0378579 \\
0.767705 & 0.0382508 \\
0.84213 & 0.0382054 \\
0.963136 & 0.0428276 \\
1.15721 & 0.054753 \\
1.49855 & 0.068892 \\
2.05752 & 0.0840323 \\
2.92723 & 0.119234 \\
4.27618 & 0.156162 \\
6.28791 & 0.231019 \\
9.13014 & 0.310094 \\
12.9534 & 0.451718 \\
17.8921 & 0.650147 \\
23.8371 & 0.826144 \\
30.6409 & 1.0168 \\
37.7573 & 1.32311 \\
44.5817 & 1.5909 \\
50.4373 & 1.81811 \\
54.5841 & 1.91153 \\
56.4233 & 1.97923 \\
55.7988 & 1.94306 \\
52.7842 & 1.81533 \\
47.7514 & 1.65581 \\
41.3805 & 1.43442 \\
\hline & 0.939717 \\
\hline
\end{tabular}




\begin{tabular}{rr} 
& \\
15.7949 & 0.558569 \\
11.6894 & 0.411496 \\
8.75696 & 0.310487 \\
6.78892 & 0.229867 \\
5.58353 & 0.192327 \\
4.90036 & 0.176586 \\
4.54975 & 0.171108 \\
4.34428 & 0.160519 \\
4.16705 & 0.143103 \\
3.91412 & 0.138431 \\
3.59086 & 0.140698 \\
3.1958 & 0.111948 \\
2.75212 & 0.0897652 \\
2.2936 & 0.0769859 \\
1.88007 & 0.071502 \\
1.49248 & 0.0614355 \\
1.1772 & 0.0450162 \\
0.941556 & 0.0349625 \\
0.769069 & 0.0357297 \\
0.655884 & 0.0295008 \\
0.601404 & 0.0281233 \\
0.595001 & 0.0322782 \\
0.621957 & 0.0407109 \\
0.687865 & 0.0452526 \\
0.786704 & 0.0632332 \\
0.910689 & 0.0808785 \\
1.05246 & 0.0953228 \\
1.19908 & 0.124151 \\
1.33325 & 0.14497 \\
1.44697 & 0.15292 \\
1.53845 & 0.17251 \\
1.58605 & 0.187399 \\
1.57439 & 0.183575 \\
1.51674 & 0.181831 \\
1.43153 & 0.152791 \\
1.32344 & 0.140409 \\
1.1962 & 0.131814 \\
1.0775 & 0.110467 \\
0.976032 & 0.0815855 \\
0.886271 & 0.0589615 \\
0.834327 & 0.053636 \\
0.804654 & 0.0393803 \\
\hline & 0.0354921 \\
\hline & 0.0426232 \\
\hline & 0.0401601 \\
\hline
\end{tabular}




\begin{tabular}{|c|c|}
\hline 79 & 20 \\
\hline 0.755934 & 0.0271153 \\
\hline 0.738013 & .0314278 \\
\hline 0.718921 & 0.0326773 \\
\hline 0.703013 & 0.0336959 \\
\hline 0.697636 & 0.0343313 \\
\hline 0.69787 & 0.0314584 \\
\hline 0.688885 & 0.030187 \\
\hline 0.679076 & 0.0364028 \\
\hline 0.678533 & 0.0334932 \\
\hline 0.678414 & 0.030876 \\
\hline 0.670694 & 0.0337615 \\
\hline 0.661744 & 0.0384873 \\
\hline 0.650916 & 0.0377832 \\
\hline 0.638708 & 0.0352241 \\
\hline 0.626833 & 0.0333965 \\
\hline 0.602146 & 0.0349021 \\
\hline 0.577522 & 0.0303192 \\
\hline 0.559 & 5785 \\
\hline 0.536042 & 0.0271029 \\
\hline 0.51879 & 0.0226814 \\
\hline 0.505612 & 0.0245369 \\
\hline 0.498301 & 0.0284991 \\
\hline 0.495228 & 0.0276086 \\
\hline 0.490933 & 0.0229089 \\
\hline 0.489591 & 0.0224953 \\
\hline 0.48 & 0.0231697 \\
\hline 0.487348 & 0.0263137 \\
\hline 0.493624 & 0.0259613 \\
\hline 0.505962 & 0.0293031 \\
\hline 0.524971 & 0.0298974 \\
\hline 0.526903 & 0.0262914 \\
\hline 0.533581 & 0.0270116 \\
\hline 0.546232 & 0.0264137 \\
\hline 0.564335 & 0.0262866 \\
\hline 0.579431 & 0.0323719 \\
\hline 0.593735 & 0.0338645 \\
\hline 0.607313 & 0.0266893 \\
\hline 0.636288 & 0.029684 \\
\hline 0.665248 & 0.0321439 \\
\hline 0.694318 & 0.0242754 \\
\hline 0.72288 & 0.0294067 \\
\hline 0.766697 & 0.0365183 \\
\hline 0.801274 & 0.0439138 \\
\hline 0.818638 & 0.049581 \\
\hline 0.831094 & 0.0442562 \\
\hline 0.832441 & 0.0470938 \\
\hline
\end{tabular}




\begin{tabular}{rr}
0.829957 & 0.0516645 \\
0.821808 & 0.0501677 \\
0.799577 & 0.0422057 \\
0.768415 & 0.0347857 \\
0.747946 & 0.0336694 \\
0.726036 & 0.0316346 \\
0.70718 & 0.0323375 \\
0.694931 & 0.0337009 \\
0.684584 & 0.0325681 \\
0.690149 & 0.0376781 \\
0.702845 & 0.0354651 \\
0.723058 & 0.0328066 \\
0.762223 & 0.0336426 \\
0.807597 & 0.0337152 \\
0.865142 & 0.0291717 \\
0.950853 & 0.0315156 \\
1.05422 & 0.0436613 \\
1.20483 & 0.0552122 \\
1.43591 & 0.0651619 \\
1.77145 & 0.0719451 \\
2.27874 & 0.0847984 \\
3.07655 & 0.114858 \\
4.30867 & 0.167359 \\
6.18951 & 0.225492 \\
8.98532 & 0.318582 \\
13.0242 & 0.468534 \\
18.686 & 0.651179 \\
26.2776 & 0.945931 \\
36.0684 & 1.27473 \\
48.0681 & 1.66116 \\
62.1404 & 2.1598 \\
77.8349 & 2.68989 \\
94.196 & 3.26809 \\
110.173 & 3.79421 \\
124.549 & 4.37613 \\
135.845 & 4.77371 \\
143.108 & 5.02775 \\
145.455 & 5.11556 \\
142.602 & 5.03641 \\
134.904 & 4.82674 \\
123.152 & 4.30528 \\
\hline 34.198 .47 & 3.72035 \\
\hline 5.85969 & 3.15397 \\
\hline & 2.5538 \\
\hline & 1.0304 \\
\hline
\end{tabular}




\begin{tabular}{rr}
24.4163 & 0.869565 \\
17.0133 & 0.608204 \\
11.5521 & 0.379906 \\
7.70807 & 0.23998 \\
5.14452 & 0.177302 \\
3.52825 & 0.126587 \\
2.58904 & 0.0830851 \\
2.14404 & 0.0747842 \\
2.05985 & 0.0754324 \\
2.25991 & 0.0801706 \\
2.70744 & 0.0867646 \\
3.40804 & 0.112464 \\
4.36658 & 0.156567 \\
5.63177 & 0.195426 \\
7.19252 & 0.251477 \\
9.01366 & 0.323583 \\
11.0824 & 0.409603 \\
13.2466 & 0.471928 \\
15.3667 & 0.538853 \\
17.3661 & 0.619849 \\
19.0481 & 0.674242 \\
20.208 & 0.726503 \\
20.7467 & 0.714494 \\
20.6332 & 0.686782 \\
19.8924 & 0.670902 \\
18.5386 & 0.62738 \\
16.723 & 0.583533 \\
14.5656 & 0.501739 \\
12.2713 & 0.412997 \\
10.0426 & 0.349927 \\
7.93488 & 0.266115 \\
6.06653 & 0.220508 \\
4.51484 & 0.180521 \\
3.27056 & 0.126859 \\
2.29831 & 0.0883192 \\
1.57484 & 0.0710781 \\
1.05527 & 0.0494537 \\
0.703673 & 0.0314026 \\
0.476854 & 0.0253823 \\
0.329256 & 0.0185118 \\
0.231128 & 0.0146133 \\
0.172857 & 0.0115835 \\
\hline & 0.00800486 \\
0.0101061 \\
\hline
\end{tabular}




\begin{tabular}{|c|c|}
\hline 087 & 0.00770 \\
\hline 0952662 & 0.00924388 \\
\hline 924062 & 0.0105983 \\
\hline 0917546 & 0.00793704 \\
\hline 0921165 & 0.00549585 \\
\hline 0898673 & 0.00722141 \\
\hline 0878878 & 0.0070874 \\
\hline 0897843 & 0.00506456 \\
\hline .0898014 & 0.0074409 \\
\hline 0892425 & 0.00756517 \\
\hline .0902533 & 0.00888054 \\
\hline .0947633 & 0.00872779 \\
\hline 0.100709 & 0056 \\
\hline 0.108737 & 0.0141541 \\
\hline 0.11304 & 1639 \\
\hline 0.121754 & 0825 \\
\hline 0.137492 & 0.0262649 \\
\hline 0.150986 & 0.0340488 \\
\hline 3956 & 9616 \\
\hline 0.181431 & 0.0517584 \\
\hline 0.199876 & 0.0584596 \\
\hline 0.213217 & 0.0687115 \\
\hline 0.225729 & 0.0734272 \\
\hline 0.237254 & 8782 \\
\hline 0.245219 & 0.089259 \\
\hline 0.251795 & 0.0957245 \\
\hline 0.255958 & \\
\hline 0.247662 & 0.0827964 \\
\hline 0.23464 & 0.0813667 \\
\hline 0.229318 & 0.0808938 \\
\hline 0.220743 & 0.0665006 \\
\hline 0.207048 & 0.0522694 \\
\hline 0.194389 & 0.0370583 \\
\hline 0.181991 & 0.0376021 \\
\hline 0.170625 & 0.0297795 \\
\hline 0.160793 & 0.026302 \\
\hline 0.153482 & 0.0204461 \\
\hline 0.149322 & 0.0161264 \\
\hline 0.139231 & 0.0132671 \\
\hline 0.137264 & 0.0105368 \\
\hline 0.132414 & 0.0118896 \\
\hline 0.130831 & 0.0140143 \\
\hline 0.131222 & 0.0108052 \\
\hline 0.125637 & 0.00946786 \\
\hline 0.124988 & 0.0118189 \\
\hline 0.123967 & 0.0127261 \\
\hline 0.12186 & 0.0104646 \\
\hline
\end{tabular}




\begin{tabular}{rr} 
& \\
0.123058 & 0.0108462 \\
0.12637 & 0.0121867 \\
0.125766 & 0.0112009 \\
0.128551 & 0.0140629 \\
0.130737 & 0.0112497 \\
0.133933 & 0.0111301 \\
0.130692 & 0.0129497 \\
0.133694 & 0.0118204 \\
0.137587 & 0.0115689 \\
0.138529 & 0.0133954 \\
0.142767 & 0.0145518 \\
0.14467 & 0.0132928 \\
0.142328 & 0.0114496 \\
0.143778 & 0.013685 \\
0.144424 & 0.0127217 \\
0.152002 & 0.0135893 \\
0.150444 & 0.0141152 \\
0.152471 & 0.0163489 \\
0.155092 & 0.017643 \\
0.159695 & 0.021127 \\
0.162607 & 0.0204253 \\
0.163466 & 0.0185693 \\
0.165249 & 0.0185024 \\
0.165146 & 0.0179473 \\
0.162119 & 0.0196238 \\
0.162968 & 0.0163457 \\
0.156878 & 0.0146794 \\
0.15257 & 0.0152804 \\
0.148613 & 0.0139917 \\
0.149875 & 0.0147743 \\
0.150155 & 0.0141225 \\
0.145958 & 0.0110783 \\
0.142247 & 0.0106191 \\
0.143534 & 0.0128977 \\
0.147571 & 0.0145961 \\
0.145757 & 0.0149061 \\
0.143223 & 0.0119269 \\
0.14271 & 0.0109012 \\
0.148662 & 0.0121301 \\
0.146175 & 0.0142393 \\
0.141352 & 0.0123073 \\
0.145824 & 0.00935231 \\
\hline & 0.012859 \\
\hline & 0.0145125 \\
\hline & 0.0135608 \\
\hline
\end{tabular}




\begin{tabular}{|c|c|}
\hline 0.149046 & 0.0151232 \\
\hline 0.145052 & 0.0120739 \\
\hline 0.148941 & 0.0173923 \\
\hline 0.148862 & 0.0163482 \\
\hline 0.150637 & 0.0123972 \\
\hline 0.151626 & 0.0160471 \\
\hline 0.154435 & 0.0144171 \\
\hline 0.152992 & 0.0148324 \\
\hline 0.154502 & 0.01426 \\
\hline 0.15881 & 0.0130098 \\
\hline 0.161974 & 0.015563 \\
\hline 0.167055 & 0.0147217 \\
\hline 0.171432 & 0.0124857 \\
\hline 0.176481 & 0.0151044 \\
\hline 0.184784 & 0.0134373 \\
\hline 0.192962 & 0.0148093 \\
\hline 0.197919 & 0.0163869 \\
\hline 0.201462 & 0.0122642 \\
\hline 0.209317 & 0.0193193 \\
\hline 0.213598 & 0.0175184 \\
\hline 0.218603 & 0.0203201 \\
\hline 0.228703 & 0.0170431 \\
\hline 0.230293 & 0.0188532 \\
\hline 0.229489 & 0.0173821 \\
\hline 0.233927 & 0.0188456 \\
\hline 0.228963 & 0.0150076 \\
\hline 0.230005 & 0.0216778 \\
\hline 0.232828 & 0.021338 \\
\hline 0.229174 & 0.0175924 \\
\hline 0.221265 & 0.0160429 \\
\hline 0.219507 & 0.0195085 \\
\hline 0.228013 & 0.0179282 \\
\hline 0.222046 & 0.0166245 \\
\hline 0.221653 & 0.0144172 \\
\hline 0.220926 & 0.0182145 \\
\hline 0.221841 & 0.0163525 \\
\hline 0.21921 & 0.0186856 \\
\hline 0.220505 & 0.0152808 \\
\hline 0.215325 & 0.0176884 \\
\hline 0.216778 & 0.0143858 \\
\hline 0.214662 & 0.0181113 \\
\hline 0.215885 & 0.0139064 \\
\hline 0.211902 & 0.0200428 \\
\hline 0.214447 & 0.0199218 \\
\hline 0.212056 & 0.0163734 \\
\hline 0.206556 & 0.0202983 \\
\hline 0.207812 & 0.0174127 \\
\hline
\end{tabular}




\begin{tabular}{rr}
0.216472 & 0.0152578 \\
0.211488 & 0.0161351 \\
0.213214 & 0.0156818 \\
0.211663 & 0.0179413 \\
0.214777 & 0.0150893 \\
0.221949 & 0.0150355 \\
0.22571 & 0.0182687 \\
0.224718 & 0.0205449 \\
0.227831 & 0.0202068 \\
0.239124 & 0.017129 \\
0.238537 & 0.0217245 \\
0.251113 & 0.0176954 \\
0.256171 & 0.0214945 \\
0.271015 & 0.0184257 \\
0.275798 & 0.0222717 \\
0.295402 & 0.0223111 \\
0.315952 & 0.0268241 \\
0.327325 & 0.0238547 \\
0.342119 & 0.0193821 \\
0.369209 & 0.028592 \\
0.373247 & 0.0239276 \\
0.392341 & 0.0215759 \\
0.410861 & 0.0253978 \\
0.42209 & 0.0323473 \\
0.438091 & 0.0238554 \\
0.449086 & 0.0348606 \\
0.457838 & 0.0342788 \\
0.464444 & 0.0262884 \\
0.473033 & 0.0295967 \\
0.469797 & 0.026727 \\
0.464054 & 0.0281947 \\
0.46515 & 0.0270696 \\
0.283959 \\
0.275868 & 0.019359 \\
0.456843 & 0.0251147 \\
0.442797 & 0.0195384 \\
0.422219 & 0.0277837 \\
0.416284 & 0.0312156 \\
0.397437 & 0.0305841 \\
0.385937 & 0.0216929 \\
0.356066 & 0.0284117 \\
0.345045 & 0.0246834 \\
0.026019 & 0.0180854 \\
\hline
\end{tabular}




\begin{tabular}{rr}
0.272521 & 0.0171116 \\
0.269378 & 0.0227351 \\
0.272195 & 0.0167459 \\
0.274476 & 0.0157399 \\
0.272904 & 0.0190724 \\
0.271792 & 0.0181558 \\
0.284012 & 0.018211 \\
0.291995 & 0.0205171 \\
0.302678 & 0.0243775 \\
0.335683 & 0.0260234 \\
0.355731 & 0.0235856 \\
0.405823 & 0.0274803 \\
0.451694 & 0.0346639 \\
0.536835 & 0.0434415 \\
0.629114 & 0.0379553 \\
0.765389 & 0.0469514 \\
0.925909 & 0.07662 \\
1.1057 & 0.0844052 \\
1.33524 & 0.09238 \\
1.57232 & 0.127661 \\
1.85819 & 0.132348 \\
2.15061 & 0.165043 \\
2.42904 & 0.183177 \\
2.68121 & 0.201296 \\
2.9117 & 0.225226 \\
3.08435 & 0.213983 \\
3.19734 & 0.231403 \\
3.26731 & 0.233598 \\
3.24591 & 0.232052 \\
3.14064 & 0.26008 \\
2.99782 & 0.196674 \\
2.74969 & 0.201956 \\
2.5149 & 0.190806 \\
2.23372 & 0.160146 \\
1.94913 & 0.146123 \\
1.68283 & 0.126273 \\
1.44562 & 0.106121 \\
1.20703 & 0.0797906 \\
1.02663 & 0.0812693 \\
0.846431 & 0.04934 \\
0.729146 & 0.0418396 \\
0.560745 & 0.0375157 \\
\hline & 0.0394308 \\
\hline & 0.02358671 \\
\hline
\end{tabular}




\begin{tabular}{|c|c|}
\hline 0.402467 & 0.0335621 \\
\hline 0.394832 & 0.0266795 \\
\hline 0.382728 & 0.0240049 \\
\hline 0.386637 & 0.0252377 \\
\hline 0.391509 & 0.0312597 \\
\hline 0.391369 & 0.0255311 \\
\hline 0.390883 & 0.0278155 \\
\hline 0.392718 & 0.0245059 \\
\hline 0.400349 & 0.0263058 \\
\hline 0.402413 & 0.0265483 \\
\hline 0.4025 & 0.0308385 \\
\hline 0.398191 & 0.0234157 \\
\hline 0.406334 & 0.0325259 \\
\hline 0.406229 & 0.0267422 \\
\hline 0.417607 & 0.0333537 \\
\hline 0.399099 & 0.0274648 \\
\hline 0.419612 & 0.0267127 \\
\hline 0.410395 & 0.0211471 \\
\hline 0.418447 & 0.0267329 \\
\hline 0.412196 & 0.0303997 \\
\hline 0.41821 & 0.0250826 \\
\hline 0.418667 & 0.0217823 \\
\hline 0.427949 & 0.0257889 \\
\hline 0.434326 & 0.0250777 \\
\hline 0.426772 & 0.0268691 \\
\hline 0.429214 & 0.0234067 \\
\hline 0.439701 & 0.0380626 \\
\hline 0.442922 & 0.0244442 \\
\hline 0.44718 & 0.0247349 \\
\hline 0.446651 & 0.0297062 \\
\hline 0.459183 & 0.031136 \\
\hline 0.467402 & 0.0211286 \\
\hline 0.485948 & 0.0247192 \\
\hline 0.511451 & 0.0284367 \\
\hline 0.524214 & 0.0358143 \\
\hline 0.558387 & 0.0329121 \\
\hline 0.574362 & 0.0316822 \\
\hline 0.602703 & 0.0379904 \\
\hline 0.642011 & 0.0426871 \\
\hline 0.692209 & 0.0379365 \\
\hline 0.723016 & 0.044246 \\
\hline 0.77547 & 0.0421708 \\
\hline 0.801192 & 0.0512674 \\
\hline 0.829224 & 0.0459896 \\
\hline 0.859626 & 0.0533253 \\
\hline 0.851537 & 0.0530327 \\
\hline 0.867506 & 0.0575767 \\
\hline
\end{tabular}




\begin{tabular}{|c|c|}
\hline 0.862619 & 0.0542283 \\
\hline 0.834795 & 0.0449096 \\
\hline 0.814521 & 0.0525104 \\
\hline 0.770355 & 0.0444869 \\
\hline 0.752082 & 0.0476568 \\
\hline 0.693114 & 0.035964 \\
\hline 0.667821 & 0.0468528 \\
\hline 0.616414 & 0.0259887 \\
\hline 0.583336 & 0.0351381 \\
\hline 0.544108 & 0.0259237 \\
\hline 0.513457 & 0.0320141 \\
\hline 0.496591 & 0.0348952 \\
\hline 0.469278 & 0.0243022 \\
\hline 0.459781 & 0.0229088 \\
\hline 0.447451 & 0.0263791 \\
\hline 0.442388 & 0.0270518 \\
\hline 0.448034 & 0.0286166 \\
\hline 0.444267 & 0.0303522 \\
\hline 0.448898 & 0.0246633 \\
\hline 0.461794 & 0.0303523 \\
\hline 0.466309 & 0.0255527 \\
\hline 0.48029 & 0.0315092 \\
\hline 0.485308 & 0.0319769 \\
\hline 0.510351 & 0.0366843 \\
\hline 0.536309 & 0.0334227 \\
\hline 0.544772 & 0.0306367 \\
\hline 0.563274 & 0.0281968 \\
\hline 0.591913 & 0.0354163 \\
\hline 0.603271 & 0.0338848 \\
\hline 0.612546 & 0.0457993 \\
\hline 0.620041 & 0.034554 \\
\hline 0.624411 & 0.0382925 \\
\hline 0.637539 & 0.0355504 \\
\hline 0.630633 & 0.0300967 \\
\hline 0.622165 & 0.0399371 \\
\hline 0.620751 & 0.0396978 \\
\hline 0.612024 & 0.0277623 \\
\hline 0.595935 & 0.0398092 \\
\hline 0.579034 & 0.0376433 \\
\hline 0.564589 & 0.0324687 \\
\hline 0.550919 & 0.0409481 \\
\hline 0.549309 & 0.0304739 \\
\hline 0.527049 & 0.0320083 \\
\hline 0.516527 & 0.0246431 \\
\hline 0.506816 & 0.034279 \\
\hline 0.514774 & 0.030142 \\
\hline 0.500613 & 0.0264534 \\
\hline
\end{tabular}




\begin{tabular}{rr} 
& \\
0.494271 & 0.0367363 \\
0.483743 & 0.0288246 \\
0.489656 & 0.0326344 \\
0.488864 & 0.0301915 \\
0.487944 & 0.02942 \\
0.49832 & 0.0318196 \\
0.482086 & 0.0341074 \\
0.488836 & 0.0341462 \\
0.492362 & 0.0274356 \\
0.496203 & 0.0244791 \\
0.512617 & 0.0280552 \\
0.519124 & 0.0321731 \\
0.516768 & 0.0332242 \\
0.527857 & 0.0257864 \\
0.52979 & 0.0347802 \\
0.536778 & 0.0296195 \\
0.540895 & 0.0306319 \\
0.531995 & 0.0315901 \\
0.535237 & 0.0252574 \\
0.536276 & 0.0237866 \\
0.531516 & 0.0303314 \\
0.526396 & 0.0300142 \\
0.52519 & 0.031379 \\
0.514254 & 0.0379234 \\
0.519105 & 0.0299345 \\
0.508814 & 0.0327403 \\
0.508996 & 0.0302509 \\
0.511892 & 0.0273709 \\
0.506185 & 0.0282694 \\
0.497407 & 0.0327149 \\
0.500387 & 0.035269 \\
0.499985 & 0.0297676 \\
0.500343 & 0.0290652 \\
0.498261 & 0.031389 \\
0.497765 & 0.0350265 \\
0.502759 & 0.0253394 \\
0.508809 & 0.0309231 \\
0.507504 & 0.03 \\
0.517793 & 0.0317664 \\
0.498252 & 0.031584 \\
0.506567 & 0.0314694 \\
0.508302 & 0.0300906 \\
\hline & 0.033949439 \\
\hline
\end{tabular}




\begin{tabular}{rr}
0.531632 & 0.029309 \\
0.535063 & 0.0317246 \\
0.536896 & 0.0292475 \\
0.552434 & 0.0300414 \\
0.537546 & 0.0317409 \\
0.545144 & 0.0341375 \\
0.542429 & 0.033526 \\
0.54085 & 0.0383404 \\
0.536669 & 0.0328047 \\
0.551657 & 0.0363924 \\
0.54173 & 0.0286275 \\
0.538144 & 0.0308595 \\
0.541285 & 0.0329498 \\
0.532176 & 0.0326602 \\
0.540369 & 0.0314973 \\
0.536917 & 0.0341104 \\
0.534042 & 0.0275518 \\
0.535944 & 0.0321645 \\
0.537269 & 0.0380972 \\
0.541942 & 0.0354047 \\
0.544956 & 0.0357864 \\
0.553937 & 0.0246584 \\
0.545372 & 0.0356501 \\
0.544355 & 0.0357503 \\
0.560736 & 0.0349845 \\
0.549312 & 0.0366083 \\
0.558819 & 0.0351244 \\
0.574146 & 0.0316236 \\
0.580255 & 0.0338467 \\
0.572242 & 0.0336161 \\
0.582454 & 0.0395294 \\
0.578188 & 0.0377369 \\
0.65952 & 0.0361818 \\
0.601039 & 0.0394392 \\
0.594709 & 0.0404258 \\
0.609546 & 0.0347107 \\
0.613087 & 0.0374328 \\
0.615333 & 0.0324741 \\
0.620346 & 0.0405127 \\
0.622352 & 0.0408533 \\
0.628185 & 0.0437528 \\
0.632571 & 0.0307099 \\
\hline & 0.0401639 \\
0.0345 & 0.0386255 \\
\hline
\end{tabular}




\begin{tabular}{|c|c|}
\hline 0.670306 & 0.0361895 \\
\hline 0.674703 & 0.0536382 \\
\hline 0.70241 & 0.0475 \\
\hline 0.709017 & 0.0359953 \\
\hline 0.727361 & 0.0410253 \\
\hline 0.737523 & 0.04234 \\
\hline 0.757445 & 0.0409128 \\
\hline 0.78509 & 0.0448646 \\
\hline 0.791242 & 0.0440612 \\
\hline 0.823496 & 0.0372528 \\
\hline 0.838171 & 0.0347606 \\
\hline 0.873773 & 0.0504667 \\
\hline 0.898378 & 0.0516233 \\
\hline 0.9108 & 0.0362479 \\
\hline 0.922713 & 0.0385786 \\
\hline 0.931869 & 0.0478539 \\
\hline 0.940912 & 0.0421535 \\
\hline 0.935832 & 0.0477847 \\
\hline 0.943566 & 0.0378 \\
\hline 0.913593 & 0.0441624 \\
\hline 0.909733 & 0.0398984 \\
\hline 0.8905 & 0.0394057 \\
\hline 0.891554 & 0.046985 \\
\hline 0.862648 & 0.0398647 \\
\hline 0.8515 & 0.0427416 \\
\hline 0.829702 & 0.0408523 \\
\hline 0.815555 & 0.0521006 \\
\hline 0.808203 & 0.0413252 \\
\hline 0.793894 & 0.0433545 \\
\hline 0.776222 & 0.0411668 \\
\hline 0.781374 & 0.0404124 \\
\hline 0.761058 & 0.0446235 \\
\hline 0.778096 & 0.0411386 \\
\hline 0.781627 & 0.0482708 \\
\hline 0.770627 & 0.0375871 \\
\hline 0.765513 & 0.042357 \\
\hline 0.762918 & 0.0385652 \\
\hline 0.779373 & 0.0404215 \\
\hline 0.774678 & 0.032388 \\
\hline 0.766351 & 0.0465723 \\
\hline 0.78033 & 0.0351847 \\
\hline 0.780188 & 0.0421092 \\
\hline 0.791442 & 0.0535511 \\
\hline 0.785988 & 0.0328184 \\
\hline 0.78693 & 0.0429107 \\
\hline 0.785935 & 0.0389105 \\
\hline 0.793247 & 0.0388883 \\
\hline
\end{tabular}




\begin{tabular}{|c|c|}
\hline 0.787463 & 0.0359528 \\
\hline 0.784345 & 0.0370571 \\
\hline 0.783924 & 0.047076 \\
\hline 0.790671 & 0.0467898 \\
\hline 0.780129 & 0.0458087 \\
\hline 0.785858 & 0.0442374 \\
\hline 0.786615 & 0.0338482 \\
\hline 0.78751 & 0.0358372 \\
\hline 0.783053 & 0.0321582 \\
\hline 0.799136 & 0.0349048 \\
\hline 0.785444 & 0.0363052 \\
\hline 0.787049 & 0.0422475 \\
\hline 0.800235 & 0.0369471 \\
\hline 0.781372 & 0.0387392 \\
\hline 0.780209 & 0.0353501 \\
\hline 0.775177 & 0.0364375 \\
\hline 0.785519 & 0.0388733 \\
\hline 0.788284 & 0.0400767 \\
\hline 0.790441 & 0.0421395 \\
\hline 0.795409 & 0.0249923 \\
\hline 0.788191 & 0.0381458 \\
\hline 0.780685 & 0.0390506 \\
\hline 0.788784 & 0.0357649 \\
\hline 0.781709 & 0.0338451 \\
\hline 0.783956 & 0.0382216 \\
\hline 0.786749 & 0.0400844 \\
\hline 0.789212 & 0.0456323 \\
\hline 0.791909 & 0.0374232 \\
\hline 0.792617 & 0.0435274 \\
\hline 0.777178 & 0.0378316 \\
\hline 0.790279 & 0.0342244 \\
\hline 0.775678 & 0.0384353 \\
\hline 0.779416 & 0.044976 \\
\hline 0.778754 & 0.0357754 \\
\hline 0.78316 & 0.0332347 \\
\hline 0.78055 & 0.0292006 \\
\hline 0.778849 & 0.0452984 \\
\hline 0.787727 & 0.0401288 \\
\hline 0.77356 & 0.0366987 \\
\hline 0.776739 & 0.0406859 \\
\hline 0.777949 & 0.0417168 \\
\hline 0.774702 & 0.0334462 \\
\hline 0.789741 & 0.0340262 \\
\hline 0.785639 & 0.0387791 \\
\hline 0.778517 & 0.0460602 \\
\hline 0.808252 & 0.0466859 \\
\hline 0.800784 & 0.0478725 \\
\hline
\end{tabular}




\begin{tabular}{|c|c|}
\hline 0.783904 & 0.0436964 \\
\hline 0.794798 & 0.0393305 \\
\hline 0.809032 & 0.0449165 \\
\hline 0.802052 & 0.0517099 \\
\hline 0.811904 & 0.042012 \\
\hline 0.812943 & 0.0401844 \\
\hline 0.812696 & 0.0410657 \\
\hline 0.833888 & 0.0494377 \\
\hline 0.836063 & 0.0459244 \\
\hline 0.835401 & 0.0384831 \\
\hline 0.838328 & 0.0404735 \\
\hline 0.847189 & 0.0421177 \\
\hline 0.851799 & 0.0486422 \\
\hline 0.857238 & 0.0393893 \\
\hline 0.852991 & 0.0350676 \\
\hline 0.850693 & 0.047416 \\
\hline 0.865483 & 0.044718 \\
\hline 0.858223 & 0.0389013 \\
\hline 0.8593 & 0.0420967 \\
\hline 0.853605 & 0.0439302 \\
\hline 0.867295 & 0.038949 \\
\hline 0.865055 & 0.0396619 \\
\hline 0.861049 & 0.0372425 \\
\hline 0.867804 & 0.0433166 \\
\hline 0.862898 & 0.0494286 \\
\hline 0.854558 & 0.0502689 \\
\hline 0.855722 & 0.0438907 \\
\hline 0.839339 & 0.042 \\
\hline 0.856157 & 0.0472226 \\
\hline 0.855652 & 0.0457681 \\
\hline 0.849431 & 0.0429653 \\
\hline 0.850104 & 0.0419436 \\
\hline 0.842301 & 0.0420128 \\
\hline 0.846107 & 0.0490515 \\
\hline 0.84826 & 0.0352851 \\
\hline 0.852145 & 0.0398839 \\
\hline 0.854201 & 0.0492003 \\
\hline 0.844532 & 0.044169 \\
\hline 0.853946 & 0.0476224 \\
\hline 0.849007 & 0.0331306 \\
\hline 0.849937 & 0.0399491 \\
\hline 0.867199 & 0.0457264 \\
\hline 0.853516 & 0.0452766 \\
\hline 0.845464 & 0.0363502 \\
\hline 0.853684 & 0.0398429 \\
\hline 0.84905 & 0.0426153 \\
\hline 0.846502 & 0.0362238 \\
\hline
\end{tabular}




\begin{tabular}{|c|c|}
\hline 0.86067 & 0.0529792 \\
\hline 0.853421 & 0.0345412 \\
\hline 0.847347 & 0.0358793 \\
\hline 0.858604 & 0.0415698 \\
\hline 0.872815 & 0.0411475 \\
\hline 0.853107 & 0.0423984 \\
\hline 0.847318 & 0.0404679 \\
\hline 0.858323 & 0.0458025 \\
\hline 0.876023 & 0.0543616 \\
\hline 0.868542 & 0.0553168 \\
\hline 0.870829 & 0.0405221 \\
\hline 0.857045 & 0.0462058 \\
\hline 0.868709 & 0.0441931 \\
\hline 0.871892 & 0.0408822 \\
\hline 0.866368 & 0.0429572 \\
\hline 0.869632 & 0.0444799 \\
\hline 0.865805 & 0.049376 \\
\hline 0.853362 & 0.042648 \\
\hline 0.871804 & 0.0440495 \\
\hline 0.863925 & 0.0504499 \\
\hline 0.865308 & 0.039761 \\
\hline 0.871377 & 0.042618 \\
\hline 0.849912 & 0.044299 \\
\hline 0.858324 & 0.0338172 \\
\hline 0.855068 & 0.0335291 \\
\hline 0.864126 & 0.046805 \\
\hline 0.861802 & 0.0479044 \\
\hline 0.86135 & 0.0392057 \\
\hline 0.858117 & 0.0383222 \\
\hline 0.853455 & 0.0517437 \\
\hline 0.856188 & 0.0446416 \\
\hline 0.85703 & 0.0426268 \\
\hline 0.858527 & 0.0408017 \\
\hline 0.853067 & 0.0357398 \\
\hline 0.854128 & 0.0495628 \\
\hline 0.843801 & 0.043209 \\
\hline 0.853948 & 0.0464292 \\
\hline 0.843348 & 0.0419318 \\
\hline 0.844394 & 0.0415323 \\
\hline 0.845652 & 0.0423525 \\
\hline 0.850051 & 0.0486715 \\
\hline 0.843894 & 0.0417067 \\
\hline 0.844557 & 0.0447975 \\
\hline 0.842529 & 0.0496598 \\
\hline 0.838313 & 0.0419638 \\
\hline 0.848315 & 0.0417945 \\
\hline 0.838973 & 0.046321 \\
\hline
\end{tabular}




\begin{tabular}{|c|c|}
\hline 0.838761 & 0.0405873 \\
\hline 0.83377 & 0.0407967 \\
\hline 0.831158 & 0.0371386 \\
\hline 0.843229 & 0.0345951 \\
\hline 0.834201 & 0.0473336 \\
\hline 0.843699 & 0.0420765 \\
\hline 0.840037 & 0.0391193 \\
\hline 0.842435 & 0.0368118 \\
\hline 0.850208 & 0.0395694 \\
\hline 0.85989 & 0.0454629 \\
\hline 0.861339 & 0.0386847 \\
\hline 0.865213 & 0.0440706 \\
\hline 0.853685 & 0.0369935 \\
\hline 0.881142 & 0.0492657 \\
\hline 0.879144 & 0.0625007 \\
\hline 0.885408 & 0.0420382 \\
\hline 0.880438 & 0.0462595 \\
\hline 0.891436 & 0.0437047 \\
\hline 0.903551 & 0.0396681 \\
\hline 0.893358 & 0.048967 \\
\hline 0.886757 & 0.0368487 \\
\hline 0.892582 & 0.0481696 \\
\hline 0.891804 & 0.0444942 \\
\hline 0.888349 & 0.0309159 \\
\hline 0.894446 & 0.0410535 \\
\hline 0.878732 & 0.0393413 \\
\hline 0.882313 & 0.0483119 \\
\hline 0.866698 & 0.0351767 \\
\hline 0.869799 & 0.0465492 \\
\hline 0.867358 & 0.0395226 \\
\hline 0.859306 & 0.0373821 \\
\hline 0.842867 & 0.0369896 \\
\hline 0.856837 & 0.0509439 \\
\hline 0.837738 & 0.0371347 \\
\hline 0.83811 & 0.0351286 \\
\hline 0.838832 & 0.0374277 \\
\hline 0.848751 & 0.0459633 \\
\hline 0.846058 & 0.0388449 \\
\hline 0.844326 & 0.0351475 \\
\hline 0.839198 & 0.0451275 \\
\hline 0.839707 & 0.0402739 \\
\hline 0.840237 & 0.0499812 \\
\hline 0.851748 & 0.0436735 \\
\hline 0.849307 & 0.039688 \\
\hline 0.845985 & 0.0446901 \\
\hline 0.849418 & 0.0425667 \\
\hline 0.846145 & 0.0390052 \\
\hline
\end{tabular}




\begin{tabular}{rr}
0.848405 & 0.0486996 \\
0.847176 & 0.0405875 \\
0.843671 & 0.0335678 \\
0.847196 & 0.0346854 \\
0.847905 & 0.0429883 \\
0.846509 & 0.036298 \\
0.858672 & 0.0421144 \\
0.857593 & 0.0378118 \\
0.853528 & 0.033279 \\
0.869 & 0.0469867 \\
0.860515 & 0.0455446 \\
0.85465 & 0.0447772 \\
0.860897 & 0.0417809 \\
0.855308 & 0.0460532 \\
0.86099 & 0.0476998 \\
0.862828 & 0.0399831 \\
0.873284 & 0.048336 \\
0.864503 & 0.0461755 \\
0.866981 & 0.0332354 \\
0.8529 & 0.0410277 \\
0.870564 & 0.0374355 \\
0.856012 & 0.0463113 \\
0.867813 & 0.0355794 \\
0.874754 & 0.0388627 \\
0.861233 & 0.039427 \\
0.854731 & 0.046543 \\
0.85619 & 0.045326 \\
0.867438 & 0.0408561 \\
0.864015 & 0.0438959 \\
0.849034 & 0.0427056 \\
0.86661 & 0.0383892 \\
0.859966 & 0.0380701 \\
0.865129 & 0.0434513 \\
0.862237 & 0.0432781 \\
0.844717 & 0.0438368 \\
0.842625 & 0.0456076 \\
0.857397 & 0.0460697 \\
0.851467 & 0.0365942 \\
0.863979 & 0.0406875 \\
0.851104 & 0.0410449 \\
0.838598 & 0.0380401 \\
0.854898 & 0.0439535 \\
\hline & 0.034565959 \\
\hline
\end{tabular}




\begin{tabular}{|c|c|}
\hline 0.850698 & 0.039333 \\
\hline 0.840479 & 0.0396731 \\
\hline 0.843469 & 0.0359548 \\
\hline 0.846546 & 0.039098 \\
\hline 0.831275 & 0.0428439 \\
\hline 0.833879 & 0.0401753 \\
\hline 0.842216 & 0.048601 \\
\hline 0.834308 & 0.0489264 \\
\hline 0.848004 & 0.0345427 \\
\hline 0.832748 & 0.0374267 \\
\hline 0.834295 & 0.0359662 \\
\hline 0.8285 & 0.0434347 \\
\hline 0.83892 & 0.0444121 \\
\hline 0.834213 & 0.0368092 \\
\hline 0.848231 & 0.0481967 \\
\hline 0.840758 & 0.0333417 \\
\hline 0.836375 & 0.0401974 \\
\hline 0.837 & 0.0491307 \\
\hline 0.831435 & 0.0455257 \\
\hline 0.823336 & 0.0392367 \\
\hline 0.844217 & 0.0428871 \\
\hline 0.834626 & 0.0339781 \\
\hline 0.820977 & 0.0456489 \\
\hline 0.832424 & 0.0494099 \\
\hline 0.835103 & 0.0405389 \\
\hline 0.841842 & 0.0490687 \\
\hline 0.834478 & 0.0321205 \\
\hline 0.831536 & 0.0409308 \\
\hline 0.829607 & 0.0469951 \\
\hline 0.839029 & 0.0457984 \\
\hline 0.833083 & 0.0386086 \\
\hline 0.828297 & 0.0341597 \\
\hline 0.833078 & 0.0361229 \\
\hline 0.8285 & 0.0342609 \\
\hline 0.842399 & 0.0380671 \\
\hline 0.839311 & 0.0342687 \\
\hline 0.843657 & 0.0384446 \\
\hline 0.833385 & 0.0336941 \\
\hline 0.82016 & 0.0308444 \\
\hline 0.841726 & 0.046134 \\
\hline 0.842455 & 0.0413516 \\
\hline 0.827631 & 0.0335236 \\
\hline 0.824714 & 0.0391878 \\
\hline 0.830326 & 0.0373922 \\
\hline 0.828435 & 0.0404531 \\
\hline 0.82206 & 0.0451394 \\
\hline 0.825563 & 0.0407062 \\
\hline
\end{tabular}




\begin{tabular}{rr} 
& \\
0.825658 & 0.0386597 \\
0.824935 & 0.0356302 \\
0.817907 & 0.0395812 \\
0.805257 & 0.0335708 \\
0.816671 & 0.0295689 \\
0.815968 & 0.042772 \\
0.809455 & 0.0346701 \\
0.812395 & 0.037794 \\
0.809081 & 0.039483 \\
0.816266 & 0.0410126 \\
0.806826 & 0.0447412 \\
0.80421 & 0.0419473 \\
0.802999 & 0.0340117 \\
0.796246 & 0.0412301 \\
0.80549 & 0.0365467 \\
0.803771 & 0.0461712 \\
0.800426 & 0.0332329 \\
0.797057 & 0.0308849 \\
0.79206 & 0.0370994 \\
0.785618 & 0.0376571 \\
0.785065 & 0.0338744 \\
0.795263 & 0.0414314 \\
0.793077 & 0.0377466 \\
0.794192 & 0.0421001 \\
0.783169 & 0.028723 \\
0.78685 & 0.0301117 \\
0.778919 & 0.045914 \\
0.787884 & 0.030787 \\
0.773412 & 0.0426539 \\
0.785745 & 0.0506185 \\
0.781002 & 0.0505028 \\
0.757036 & 0.0415234 \\
0.773511 & 0.0469891 \\
0.770256 & 0.032475 \\
0.767002 & 0.0327726 \\
0.768627 & 0.0425142 \\
0.764625 & 0.0396065 \\
0.762762 & 0.0376237 \\
0.763434 & 0.0479703 \\
0.766899 & 0.0389631 \\
0.753749 & 0.0400127 \\
0.751424 & 0.0437233 \\
\hline & 0.0417551 \\
\hline & 0.0328176 \\
\hline
\end{tabular}




\begin{tabular}{rr} 
& \\
0.747686 & 0.0348459 \\
0.750112 & 0.0327794 \\
0.744809 & 0.0394794 \\
0.746438 & 0.0346799 \\
0.732498 & 0.0431975 \\
0.736583 & 0.0394093 \\
0.734938 & 0.0380376 \\
0.733256 & 0.0360894 \\
0.733258 & 0.0379431 \\
0.730751 & 0.0328783 \\
0.739689 & 0.0355194 \\
0.747122 & 0.0420051 \\
0.738511 & 0.0301019 \\
0.735766 & 0.0424107 \\
0.719198 & 0.0322756 \\
0.728386 & 0.0339125 \\
0.723433 & 0.037033 \\
0.729049 & 0.0341901 \\
0.714672 & 0.0294171 \\
0.73132 & 0.0391078 \\
0.730561 & 0.0337336 \\
0.721762 & 0.0359656 \\
0.710792 & 0.037101 \\
0.718703 & 0.0346457 \\
0.729363 & 0.0377169 \\
0.710576 & 0.0406256 \\
0.724276 & 0.0346723 \\
0.725914 & 0.0364443 \\
0.726804 & 0.0311733 \\
0.73818 & 0.0334408 \\
0.735685 & 0.0449901 \\
0.732133 & 0.0382046 \\
0.739028 & 0.0442798 \\
0.750783 & 0.0415977 \\
0.739918 & 0.0343087 \\
0.743519 & 0.0387593 \\
0.744245 & 0.0405671 \\
0.753565953 & 0.0296239 \\
0.7353 & 0.0383868 \\
\hline
\end{tabular}




\begin{tabular}{|c|c|}
\hline 0.724592 & 0.0406875 \\
\hline 0.713781 & 0.0276943 \\
\hline 0.699677 & 0.0386789 \\
\hline 0.703071 & 0.0356178 \\
\hline 0.704591 & 0.0387725 \\
\hline 0.690454 & 0.0260051 \\
\hline 0.682903 & 0.0394224 \\
\hline 0.688745 & 0.0310815 \\
\hline 0.680676 & 0.0356054 \\
\hline 0.67357 & 0.0396407 \\
\hline 0.679237 & 0.0360753 \\
\hline 0.667754 & 0.042197 \\
\hline 0.665785 & 0.0337319 \\
\hline 0.663807 & 0.0331811 \\
\hline 0.667833 & 0.0343418 \\
\hline 0.670583 & 0.0285583 \\
\hline 0.66528 & 0.0287997 \\
\hline 0.650799 & 0.0415258 \\
\hline 0.664775 & 0.0356457 \\
\hline 0.661938 & 0.0261695 \\
\hline 0.664496 & 0.0409443 \\
\hline 0.663474 & 0.0364521 \\
\hline 0.654036 & 0.0366044 \\
\hline 0.654275 & 0.0357727 \\
\hline 0.64794 & 0.0331241 \\
\hline 0.648437 & 0.0330404 \\
\hline 0.64517 & 0.0295818 \\
\hline 0.639766 & 0.0319626 \\
\hline 0.644024 & 0.0388813 \\
\hline 0.648865 & 0.029835 \\
\hline 0.639032 & 0.0378634 \\
\hline 0.642343 & 0.0358957 \\
\hline 0.645051 & 0.0320855 \\
\hline 0.640542 & 0.0328498 \\
\hline 0.633393 & 0.0313291 \\
\hline 0.62859 & 0.034828 \\
\hline 0.635022 & 0.0365342 \\
\hline 0.625513 & 0.034567 \\
\hline 0.633564 & 0.0424712 \\
\hline 0.628083 & 0.038744 \\
\hline 0.624813 & 0.0324154 \\
\hline 0.619539 & 0.0343992 \\
\hline 0.606679 & 0.0299114 \\
\hline 0.614601 & 0.0374819 \\
\hline 0.625925 & 0.0317952 \\
\hline 0.622233 & 0.0292699 \\
\hline 0.621663 & 0.029896 \\
\hline
\end{tabular}




\begin{tabular}{|c|c|}
\hline 0.620508 & 0.039744 \\
\hline 0.614682 & 0.0334725 \\
\hline 0.609719 & 0.025528 \\
\hline 0.613734 & 0.0296645 \\
\hline 0.59804 & 0.0348238 \\
\hline 0.618982 & 0.0294414 \\
\hline 0.602109 & 0.0292139 \\
\hline 0.607041 & 0.0342343 \\
\hline 0.60642 & 0.0335709 \\
\hline 0.606957 & 0.0298983 \\
\hline 0.613392 & 0.0307011 \\
\hline 0.60149 & 0.0306966 \\
\hline 0.603475 & 0.0393442 \\
\hline 0.611593 & 0.0296038 \\
\hline 0.61281 & 0.0297517 \\
\hline 0.614398 & 0.0310258 \\
\hline 0.593953 & 0.0301143 \\
\hline 0.609395 & 0.0327554 \\
\hline 0.608117 & 0.0324191 \\
\hline 0.61434 & 0.0283861 \\
\hline 0.605251 & 0.039904 \\
\hline 0.609052 & 0.0264609 \\
\hline 0.609565 & 0.0261956 \\
\hline 0.59914 & 0.0351034 \\
\hline 0.600031 & 0.0340094 \\
\hline 0.597082 & 0.0289319 \\
\hline 0.59975 & 0.0371901 \\
\hline 0.59224 & 0.0309263 \\
\hline 0.597002 & 0.0304517 \\
\hline 0.608273 & 0.0305447 \\
\hline 0.60161 & 0.028748 \\
\hline 0.588488 & 0.0331903 \\
\hline 0.597193 & 0.0282843 \\
\hline 0.594522 & 0.0427784 \\
\hline 0.593506 & 0.0324615 \\
\hline 0.592461 & 0.0319687 \\
\hline 0.58575 & 0.0314932 \\
\hline 0.587034 & 0.0360401 \\
\hline 0.58191 & 0.0306646 \\
\hline 0.5894 & 0.029097 \\
\hline 0.584979 & 0.0356675 \\
\hline 0.578005 & 0.0240229 \\
\hline 0.587613 & 0.0299813 \\
\hline 0.574736 & 0.0330834 \\
\hline 0.580404 & 0.0252783 \\
\hline 0.579819 & 0.0291071 \\
\hline 0.581624 & 0.0315587 \\
\hline
\end{tabular}




\begin{tabular}{rr}
0.589563 & 0.0253373 \\
0.584381 & 0.0320799 \\
0.569007 & 0.0275484 \\
0.573777 & 0.0308751 \\
0.569933 & 0.0381553 \\
0.571889 & 0.027839 \\
0.569986 & 0.0246392 \\
0.57006 & 0.0299981 \\
0.566935 & 0.0291506 \\
0.571812 & 0.0373532 \\
0.56695 & 0.0341429 \\
0.574429 & 0.037602 \\
0.568419 & 0.0308518 \\
0.565168 & 0.0362642 \\
0.572088 & 0.0308017 \\
0.572505 & 0.0247762 \\
0.575143 & 0.036004 \\
0.568843 & 0.0284158 \\
0.570164 & 0.0264156 \\
0.570641 & 0.0311307 \\
0.567125 & 0.0322773 \\
0.579777 & 0.0284526 \\
0.584805 & 0.0296736 \\
0.593259 & 0.0269557 \\
0.596197 & 0.0293207 \\
0.609112 & 0.0428777 \\
0.620558 & 0.0300785 \\
0.642015 & 0.0398168 \\
0.667338 & 0.0307963 \\
0.689825 & 0.0375692 \\
0.754784 & 0.0469969 \\
0.794788 & 0.0574424 \\
0.845547 & 0.0570102 \\
0.931309 & 0.0678012 \\
1.02711 & 0.0738474 \\
1.11775 & 0.0870677 \\
1.24148 & 0.0885916 \\
1.37005 & 0.104781 \\
\hline .51808 & 0.118082 \\
\hline .69648 & 0.139804 \\
\hline & 0.158274 \\
\hline & 0.179235 \\
\hline & 0.196784 \\
\hline
\end{tabular}




\begin{tabular}{rr} 
& \\
3.08097 & 0.273188 \\
3.20692 & 0.285975 \\
3.33998 & 0.305627 \\
3.3747 & 0.31093 \\
3.44085 & 0.331503 \\
3.43042 & 0.31145 \\
3.36867 & 0.331777 \\
3.32149 & 0.322542 \\
3.21567 & 0.294367 \\
3.08892 & 0.266367 \\
2.94177 & 0.269756 \\
2.78912 & 0.267491 \\
2.58594 & 0.226465 \\
2.39791 & 0.220079 \\
2.23152 & 0.199131 \\
2.01156 & 0.173751 \\
1.83504 & 0.160404 \\
1.6425 & 0.159776 \\
1.47125 & 0.115277 \\
1.30639 & 0.0863896 \\
1.18545 & 0.0879692 \\
1.07001 & 0.0754804 \\
0.96032 & 0.0553705 \\
0.866156 & 0.0558392 \\
0.791763 & 0.0448238 \\
0.708699 & 0.036652 \\
0.665438 & 0.0376188 \\
0.629585 & 0.0417429 \\
0.596897 & 0.0328971 \\
0.573365 & 0.0277303 \\
0.537057 & 0.0353449 \\
0.525711 & 0.0301985 \\
0.509527 & 0.0298111 \\
0.506275 & 0.0291974 \\
0.496285 & 0.035569 \\
0.48673 & 0.026952 \\
0.491151 & 0.0220831 \\
0.495678 & 0.0324598 \\
0.480039 & 0.0227731 \\
0.477471 & 0.0241979 \\
0.478648 & 0.0287182 \\
0.031295 & 0.0255929 \\
\hline & 0.026936 \\
\hline & 0.02676999 \\
\hline
\end{tabular}




\begin{tabular}{|c|c|}
\hline 0.464268 & 0.0283781 \\
\hline 0.467135 & 0.026792 \\
\hline 0.457713 & 0.0275041 \\
\hline 0.46725 & 0.0250168 \\
\hline 0.459912 & 0.0247551 \\
\hline 0.449109 & 0.0261545 \\
\hline 0.459394 & 0.0288714 \\
\hline 0.461332 & 0.0276065 \\
\hline 0.454717 & 0.0230333 \\
\hline 0.460029 & 0.0298299 \\
\hline 0.455058 & 0.0258198 \\
\hline 0.455738 & 0.0324506 \\
\hline 0.458927 & 0.024397 \\
\hline 0.46657 & 0.0273751 \\
\hline 0.460982 & 0.0245355 \\
\hline 0.45933 & 0.0300135 \\
\hline 0.457775 & 0.0274367 \\
\hline 0.455296 & 0.0235804 \\
\hline 0.454438 & 0.0274683 \\
\hline 0.454822 & 0.0306505 \\
\hline 0.447171 & 0.0223377 \\
\hline 0.450297 & 0.0230173 \\
\hline 0.447978 & 0.0234843 \\
\hline 0.44849 & 0.0191251 \\
\hline 0.439888 & 0.0284899 \\
\hline 0.444822 & 0.0291303 \\
\hline 0.444286 & 0.0232663 \\
\hline 0.448896 & 0.025368 \\
\hline 0.446733 & 0.0262391 \\
\hline 0.448952 & 0.0316397 \\
\hline 0.438856 & 0.0256272 \\
\hline 0.430536 & 0.0234954 \\
\hline 0.433829 & 0.0220303 \\
\hline 0.446647 & 0.0299802 \\
\hline 0.428597 & 0.0298484 \\
\hline 0.43277 & 0.0308257 \\
\hline 0.431558 & 0.0233478 \\
\hline 0.429473 & 0.0268805 \\
\hline 0.430543 & 0.0262861 \\
\hline 0.429612 & 0.0299126 \\
\hline 0.432457 & 0.0221065 \\
\hline 0.42918 & 0.0247558 \\
\hline 0.433287 & 0.0211033 \\
\hline 0.422265 & 0.0291305 \\
\hline 0.426941 & 0.0249578 \\
\hline 0.421887 & 0.0206214 \\
\hline 0.426264 & 0.0257914 \\
\hline
\end{tabular}




\begin{tabular}{|c|c|}
\hline 0.41676 & 0.0268354 \\
\hline 0.421209 & 0.020349 \\
\hline 0.417547 & 0.0273405 \\
\hline 0.421017 & 0.0258252 \\
\hline 0.416177 & 0.0242499 \\
\hline 0.411402 & 0.032982 \\
\hline 0.412767 & 0.0320025 \\
\hline 0.414777 & 0.0273483 \\
\hline 0.411709 & 0.0206306 \\
\hline 0.415038 & 0.027779 \\
\hline 0.403879 & 0.0324922 \\
\hline 0.405351 & 0.0269945 \\
\hline 0.406875 & 0.0245078 \\
\hline 0.407713 & 0.021255 \\
\hline 0.400086 & 0.0241934 \\
\hline 0.399691 & 0.0237797 \\
\hline 0.411241 & 0.0279025 \\
\hline 0.400062 & 0.0235072 \\
\hline 0.399545 & 0.0298336 \\
\hline 0.402075 & 0.0193604 \\
\hline 0.395129 & 0.0294175 \\
\hline 0.392696 & 0.023487 \\
\hline 0.388081 & 0.0201332 \\
\hline 0.383301 & 0.0234168 \\
\hline 0.384514 & 0.0259593 \\
\hline 0.383478 & 0.0243364 \\
\hline 0.390956 & 0.0201808 \\
\hline 0.38649 & 0.028489 \\
\hline 0.383669 & 0.0228458 \\
\hline 0.386343 & 0.024239 \\
\hline 0.380068 & 0.0222764 \\
\hline 0.377566 & 0.0268287 \\
\hline 0.380191 & 0.0248201 \\
\hline 0.378618 & 0.0205123 \\
\hline 0.381004 & 0.0180105 \\
\hline 0.378779 & 0.020012 \\
\hline 0.36375 & 0.0186736 \\
\hline 0.374134 & 0.0216623 \\
\hline 0.370352 & 0.0260606 \\
\hline 0.371657 & 0.026431 \\
\hline 0.361418 & 0.023488 \\
\hline 0.36631 & 0.0250857 \\
\hline 0.368611 & 0.022338 \\
\hline 0.372983 & 0.0247618 \\
\hline 0.373695 & 0.0203167 \\
\hline 0.372565 & 0.0238763 \\
\hline 0.367507 & 0.0252263 \\
\hline
\end{tabular}




\begin{tabular}{|c|c|}
\hline 0.373101 & 0.0273198 \\
\hline 0.372747 & 0.0227219 \\
\hline 0.374305 & 0.0237585 \\
\hline 0.385034 & 0.028005 \\
\hline 0.382458 & 0.026142 \\
\hline 0.387572 & 0.022275 \\
\hline 0.390933 & 0.0248179 \\
\hline 0.393666 & 0.0231348 \\
\hline 0.403038 & 0.0248605 \\
\hline 0.412109 & 0.0293871 \\
\hline 0.422185 & 0.0243386 \\
\hline 0.438379 & 0.0236905 \\
\hline 0.44605 & 0.0311423 \\
\hline 0.457509 & 0.030931 \\
\hline 0.482695 & 0.0248145 \\
\hline 0.492618 & 0.0331723 \\
\hline 0.517868 & 0.0280956 \\
\hline 0.550447 & 0.0301159 \\
\hline 0.567068 & 0.0416313 \\
\hline 0.599097 & 0.0285495 \\
\hline 0.634362 & 0.0391401 \\
\hline 0.667313 & 0.0428992 \\
\hline 0.691694 & 0.0380604 \\
\hline 0.739457 & 0.0502417 \\
\hline 0.758465 & 0.0459265 \\
\hline 0.787311 & 0.0473008 \\
\hline 0.814967 & 0.0406562 \\
\hline 0.846948 & 0.0578279 \\
\hline 0.865456 & 0.0513862 \\
\hline 0.879251 & 0.0628924 \\
\hline 0.894939 & 0.0611724 \\
\hline 0.903477 & 0.0581665 \\
\hline 0.896992 & 0.0692615 \\
\hline 0.893701 & 0.0633693 \\
\hline 0.878145 & 0.0576213 \\
\hline 0.860963 & 0.0551546 \\
\hline 0.842802 & 0.0663398 \\
\hline 0.811444 & 0.0547667 \\
\hline 0.78513 & 0.0595718 \\
\hline 0.750425 & 0.0570436 \\
\hline 0.713574 & 0.0514614 \\
\hline 0.683845 & 0.0378127 \\
\hline 0.634551 & 0.0507087 \\
\hline 0.601395 & 0.039391 \\
\hline 0.585071 & 0.0389024 \\
\hline 0.54545 & 0.0333785 \\
\hline 0.518376 & 0.0328267 \\
\hline
\end{tabular}




\begin{tabular}{|c|c|}
\hline 0.495429 & 0.0273562 \\
\hline 0.470301 & 0.0297434 \\
\hline 0.444803 & 0.0247156 \\
\hline 0.433332 & 0.036724 \\
\hline 0.420973 & 0.0250667 \\
\hline 0.407953 & 0.034263 \\
\hline 0.406882 & 0.0233342 \\
\hline 0.399343 & 0.0295255 \\
\hline 0.390484 & 0.0243817 \\
\hline 0.379143 & 0.0262167 \\
\hline 0.383608 & 0.0248558 \\
\hline 0.379453 & 0.0244322 \\
\hline 0.370782 & 0.0258836 \\
\hline 0.372391 & 0.026099 \\
\hline 0.364336 & 0.0270242 \\
\hline 0.351734 & 0.024795 \\
\hline 0.352457 & 0.0249939 \\
\hline 0.353244 & 0.022364 \\
\hline 0.341526 & 0.0243206 \\
\hline 0.341346 & 0.0234739 \\
\hline 0.344861 & 0.0213764 \\
\hline 0.329023 & 0.0245757 \\
\hline 0.327122 & 0.0235436 \\
\hline 0.313202 & 0.0197215 \\
\hline 0.317661 & 0.0191942 \\
\hline 0.312922 & 0.0261367 \\
\hline 0.306693 & 0.0220535 \\
\hline 0.306369 & 0.0243737 \\
\hline 0.304682 & 0.0215716 \\
\hline 0.295589 & 0.0186517 \\
\hline 0.287162 & 0.0258775 \\
\hline 0.296714 & 0.0209079 \\
\hline 0.294479 & 0.0192243 \\
\hline 0.288366 & 0.0208972 \\
\hline 0.292331 & 0.0235089 \\
\hline 0.293592 & 0.021106 \\
\hline 0.286132 & 0.0147861 \\
\hline 0.287881 & 0.0229284 \\
\hline 0.283464 & 0.0210654 \\
\hline 0.28629 & 0.0254145 \\
\hline 0.2804 & 0.0190923 \\
\hline 0.28157 & 0.0230214 \\
\hline 0.283389 & 0.020536 \\
\hline 0.286848 & 0.0219463 \\
\hline 0.276703 & 0.0203844 \\
\hline 0.279708 & 0.0231304 \\
\hline 0.285129 & 0.0159699 \\
\hline
\end{tabular}




\begin{tabular}{|c|c|}
\hline 0.277747 & 0.014617 \\
\hline 0.277063 & 0.0238026 \\
\hline 0.274781 & 0.0159088 \\
\hline 0.280104 & 0.0215283 \\
\hline 0.276817 & 0.0146052 \\
\hline 0.271715 & 0.0211279 \\
\hline 0.272281 & 0.0191874 \\
\hline 0.264315 & 0.0190926 \\
\hline 0.27503 & 0.0219475 \\
\hline 0.272855 & 0.024648 \\
\hline 0.26698 & 0.0200084 \\
\hline 0.268665 & 0.0191836 \\
\hline 0.274406 & 0.025276 \\
\hline 0.264842 & 0.0164092 \\
\hline 0.266152 & 0.0147587 \\
\hline 0.265782 & 0.019789 \\
\hline 0.269076 & 0.0192794 \\
\hline 0.265818 & 0.0196514 \\
\hline 0.265564 & 0.0175797 \\
\hline 0.266491 & 0.0193402 \\
\hline 0.263352 & 0.019464 \\
\hline 0.265606 & 0.0195495 \\
\hline 0.262767 & 0.0185545 \\
\hline 0.266319 & 0.017548 \\
\hline 0.264803 & 0.0175329 \\
\hline 0.263926 & 0.021087 \\
\hline 0.259475 & 0.0190185 \\
\hline 0.262233 & 0.0176373 \\
\hline 0.264868 & 0.0187316 \\
\hline 0.260026 & 0.0191576 \\
\hline 0.259806 & 0.017218 \\
\hline 0.259026 & 0.0191524 \\
\hline 0.256145 & 0.0225233 \\
\hline 0.255501 & 0.0185535 \\
\hline 0.257449 & 0.0178059 \\
\hline 0.25981 & 0.0170009 \\
\hline 0.256918 & 0.0205417 \\
\hline 0.253793 & 0.0196498 \\
\hline 0.257019 & 0.019751 \\
\hline 0.252662 & 0.0188534 \\
\hline 0.254022 & 0.0193541 \\
\hline 0.258615 & 0.0182826 \\
\hline 0.248169 & 0.0179587 \\
\hline 0.248547 & 0.0173315 \\
\hline 0.252594 & 0.0214461 \\
\hline 0.257026 & 0.0181573 \\
\hline 0.246938 & 0.01977 \\
\hline
\end{tabular}




\begin{tabular}{|c|c|}
\hline 0.247233 & 0.0189572 \\
\hline 0.251283 & 0.0188273 \\
\hline 0.253949 & 0.0204754 \\
\hline 0.251024 & 0.0180801 \\
\hline 0.250638 & 0.0206616 \\
\hline 0.24842 & 0.0177291 \\
\hline 0.248078 & 0.0201888 \\
\hline 0.245072 & 0.021382 \\
\hline 0.246583 & 0.0188218 \\
\hline 0.250689 & 0.0138213 \\
\hline 0.253925 & 0.0182461 \\
\hline 0.245077 & 0.0174209 \\
\hline 0.246931 & 0.0213814 \\
\hline 0.249779 & 0.0198636 \\
\hline 0.255666 & 0.0205387 \\
\hline 0.250918 & 0.0175241 \\
\hline 0.245217 & 0.0187661 \\
\hline 0.244498 & 0.0197807 \\
\hline 0.245013 & 0.0212035 \\
\hline 0.245348 & 0.0216577 \\
\hline 0.241634 & 0.017676 \\
\hline 0.246731 & 0.0208495 \\
\hline 0.247196 & 0.0213043 \\
\hline 0.244297 & 0.0217774 \\
\hline 0.243253 & 0.0209588 \\
\hline 0.24023 & 0.0197353 \\
\hline 0.246558 & 0.0163443 \\
\hline 0.244721 & 0.0193173 \\
\hline 0.242825 & 0.0164349 \\
\hline 0.241185 & 0.0196896 \\
\hline 0.242918 & 0.0176806 \\
\hline 0.243438 & 0.0130118 \\
\hline 0.241478 & 0.0184988 \\
\hline 0.241188 & 0.0179706 \\
\hline 0.24426 & 0.0193528 \\
\hline 0.245041 & 0.0206107 \\
\hline 0.238916 & 0.0192538 \\
\hline 0.238169 & 0.0168474 \\
\hline 0.245847 & 0.0213944 \\
\hline 0.244371 & 0.019353 \\
\hline 0.245136 & 0.0221091 \\
\hline 0.243365 & 0.0190316 \\
\hline 0.243906 & 0.0151155 \\
\hline 0.236897 & 0.0164737 \\
\hline 0.239821 & 0.0166612 \\
\hline 0.241067 & 0.0201339 \\
\hline 0.236623 & 0.0163735 \\
\hline
\end{tabular}




\begin{tabular}{|c|c|}
\hline 0.238258 & 0.0174438 \\
\hline 0.236096 & 0.0174141 \\
\hline 0.243527 & 0.0202658 \\
\hline 0.235115 & 0.0248477 \\
\hline 0.240025 & 0.0201476 \\
\hline 0.238301 & 0.0167876 \\
\hline 0.239849 & 0.01664 \\
\hline 0.240216 & 0.0204838 \\
\hline 0.246191 & 0.0185599 \\
\hline 0.247733 & 0.0157628 \\
\hline 0.242771 & 0.0205872 \\
\hline 0.251116 & 0.0176693 \\
\hline 0.242566 & 0.0211194 \\
\hline 0.241041 & 0.0153999 \\
\hline 0.245332 & 0.0193527 \\
\hline 0.249698 & 0.0209161 \\
\hline 0.246361 & 0.0187447 \\
\hline 0.255628 & 0.0170824 \\
\hline 0.248338 & 0.0215798 \\
\hline 0.250683 & 0.0190933 \\
\hline 0.251378 & 0.017501 \\
\hline 0.250708 & 0.0181034 \\
\hline 0.263537 & 0.0201218 \\
\hline 0.258382 & 0.0224865 \\
\hline 0.258724 & 0.0173847 \\
\hline 0.253556 & 0.0144528 \\
\hline 0.256851 & 0.0160574 \\
\hline 0.261341 & 0.0136159 \\
\hline 0.263329 & 0.0173735 \\
\hline 0.259604 & 0.0187586 \\
\hline 0.265189 & 0.0166286 \\
\hline 0.266129 & 0.0209993 \\
\hline 0.256983 & 0.0212161 \\
\hline 0.263538 & 0.0221196 \\
\hline 0.261732 & 0.0191258 \\
\hline 0.263179 & 0.0173206 \\
\hline 0.25676 & 0.0197114 \\
\hline 0.261455 & 0.0213859 \\
\hline 0.267623 & 0.0200966 \\
\hline 0.262211 & 0.0183186 \\
\hline 0.259937 & 0.0206268 \\
\hline 0.260841 & 0.0194416 \\
\hline 0.256609 & 0.022605 \\
\hline 0.255887 & 0.0183815 \\
\hline 0.256306 & 0.0210167 \\
\hline 0.264449 & 0.0219444 \\
\hline 0.257322 & 0.0206406 \\
\hline
\end{tabular}




\begin{tabular}{rr}
0.253595 & 0.0208055 \\
0.255076 & 0.017184 \\
0.253853 & 0.0218653 \\
0.256118 & 0.0196864 \\
0.256559 & 0.0199679 \\
0.253577 & 0.0193584 \\
0.259815 & 0.0197162 \\
0.258973 & 0.0180138 \\
0.255449 & 0.0201288 \\
0.254879 & 0.0157095 \\
0.261572 & 0.0169001 \\
0.253651 & 0.0135474 \\
0.25704 & 0.0152767 \\
0.256059 & 0.0157284 \\
0.25869 & 0.0208011 \\
0.257611 & 0.0234462 \\
0.259557 & 0.0152981 \\
0.265486 & 0.0175017 \\
0.259496 & 0.0186388 \\
0.268564 & 0.0170582 \\
0.264503 & 0.01785 \\
0.269809 & 0.0210246 \\
0.27401 & 0.0193395 \\
0.268753 & 0.0165677 \\
0.264448 & 0.0184198 \\
0.27084 & 0.0181668 \\
0.26634 & 0.0201062 \\
0.269314 & 0.0249999 \\
0.27931 & 0.0208432 \\
0.274173 & 0.0178583 \\
0.31557 \\
0.275497 & 0.0168754 \\
0.274046 & 0.0199992 \\
0.274229 & 0.0150713 \\
0.282176 & 0.0217481 \\
0.277188 & 0.0182436 \\
0.283395 & 0.029438 \\
0.293447 & 0.02147 \\
0.291957 & 0.0242524 \\
0.288238 & 0.0201036 \\
0.297085 & 0.0198579 \\
0.292414 & 0.0222423 \\
\hline & 0.0215823 \\
\hline & 0.0193748 \\
\hline
\end{tabular}




\begin{tabular}{|c|c|}
\hline 0.3195 & 0.0205146 \\
\hline 0.327723 & 0.0227885 \\
\hline 0.325154 & 0.0228474 \\
\hline 0.337107 & 0.02517 \\
\hline 0.340481 & 0.0240806 \\
\hline 0.344691 & 0.0226591 \\
\hline 0.345723 & 0.0215362 \\
\hline 0.351097 & 0.0225799 \\
\hline 0.359662 & 0.0228517 \\
\hline 0.373022 & 0.0228105 \\
\hline 0.382661 & 0.0192277 \\
\hline 0.384454 & 0.0237667 \\
\hline 0.401172 & 0.0299731 \\
\hline 0.409669 & 0.0193708 \\
\hline 0.427833 & 0.0221403 \\
\hline 0.423884 & 0.0196037 \\
\hline 0.431286 & 0.0274319 \\
\hline 0.43932 & 0.0374163 \\
\hline 0.460301 & 0.0220143 \\
\hline 0.467498 & 0.0226374 \\
\hline 0.485763 & 0.0245541 \\
\hline 0.501215 & 0.0206865 \\
\hline 0.517999 & 0.022964 \\
\hline 0.525829 & 0.0304329 \\
\hline 0.539617 & 0.0311932 \\
\hline 0.554711 & 0.0293446 \\
\hline 0.563485 & 0.0282601 \\
\hline 0.584863 & 0.0374057 \\
\hline 0.597974 & 0.0312914 \\
\hline 0.621668 & 0.0234541 \\
\hline 0.630266 & 0.0262221 \\
\hline 0.651383 & 0.0262129 \\
\hline 0.664579 & 0.0329937 \\
\hline 0.679424 & 0.0293892 \\
\hline 0.702803 & 0.037263 \\
\hline 0.717543 & 0.0302581 \\
\hline 0.738762 & 0.0269172 \\
\hline 0.750317 & 0.0294285 \\
\hline 0.76257 & 0.0321664 \\
\hline 0.785038 & 0.0280653 \\
\hline 0.792314 & 0.0317504 \\
\hline 0.809248 & 0.0408336 \\
\hline 0.821245 & 0.0360119 \\
\hline 0.838519 & 0.0383004 \\
\hline 0.850703 & 0.0372058 \\
\hline 0.876087 & 0.0366784 \\
\hline 0.881935 & 0.0355927 \\
\hline
\end{tabular}




\begin{tabular}{rr} 
& \\
0.901103 & 0.0318896 \\
0.89541 & 0.0478576 \\
0.916155 & 0.0409685 \\
0.914801 & 0.0343062 \\
0.927805 & 0.0376201 \\
0.933855 & 0.0371549 \\
0.944003 & 0.0326804 \\
0.940753 & 0.0308843 \\
0.946405 & 0.0362133 \\
0.95059 & 0.0333961 \\
0.946407 & 0.0356351 \\
0.951156 & 0.0344957 \\
0.949571 & 0.0386788 \\
0.96223 & 0.0307557 \\
0.934966 & 0.0353849 \\
0.938129 & 0.0375357 \\
0.929999 & 0.0445537 \\
0.93228 & 0.0345616 \\
0.922845 & 0.0465651 \\
0.904975 & 0.0380633 \\
0.910688 & 0.0389107 \\
0.883186 & 0.0409587 \\
0.867318 & 0.0472885 \\
0.877362 & 0.0377859 \\
0.849909 & 0.0402789 \\
0.840253 & 0.0356852 \\
0.841596 & 0.0378234 \\
0.816361 & 0.0312058 \\
0.795635 & 0.0394129 \\
0.787729 & 0.0297737 \\
0.761763 & 0.035504 \\
0.740243 & 0.0424939 \\
0.727485 & 0.0338681 \\
0.714827 & 0.0376142 \\
0.692815 & 0.0308686 \\
0.679532 & 0.0350642 \\
0.665615 & 0.0308921 \\
0.652341 & 0.0393036 \\
0.60683003 & 0.0272862 \\
0.595097 & 0.0294067 \\
\hline & 0.0231564 \\
\hline & 0.0269978 \\
\hline
\end{tabular}




\begin{tabular}{|c|c|}
\hline 0.483743 & 0.025518 \\
\hline 0.4725 & 0.027152 \\
\hline 0.45968 & 0.0233471 \\
\hline 0.440072 & 0.0213094 \\
\hline 0.429148 & 0.0217406 \\
\hline 0.424296 & 0.0284059 \\
\hline 0.41029 & 0.025059 \\
\hline 0.398369 & 0.0234502 \\
\hline 0.38978 & 0.025594 \\
\hline 0.382655 & 0.021233 \\
\hline 0.365665 & 0.0218366 \\
\hline 0.352491 & 0.0212516 \\
\hline 0.349241 & 0.0195367 \\
\hline 0.343536 & 0.0227338 \\
\hline 0.331035 & 0.0167502 \\
\hline 0.324078 & 0.0231953 \\
\hline 0.317946 & 0.017791 \\
\hline 0.310056 & 0.0192998 \\
\hline 0.301954 & 0.0207472 \\
\hline 0.303676 & 0.0238232 \\
\hline 0.294041 & 0.0178128 \\
\hline 0.286996 & 0.019739 \\
\hline 0.279818 & 0.0207907 \\
\hline 0.281401 & 0.0227153 \\
\hline 0.270529 & 0.0246204 \\
\hline 0.256943 & 0.0173768 \\
\hline 0.258185 & 0.0232346 \\
\hline 0.256905 & 0.0208102 \\
\hline 0.25036 & 0.021373 \\
\hline 0.248073 & 0.018938 \\
\hline 0.239229 & 0.0160789 \\
\hline 0.238353 & 0.0185316 \\
\hline 0.231503 & 0.0213584 \\
\hline 0.22582 & 0.0197257 \\
\hline 0.226466 & 0.0180866 \\
\hline 0.21989 & 0.0179634 \\
\hline 0.214064 & 0.013042 \\
\hline 0.216541 & 0.0221379 \\
\hline 0.211227 & 0.0181608 \\
\hline 0.204811 & 0.0179078 \\
\hline 0.201039 & 0.0151144 \\
\hline 0.202518 & 0.0159277 \\
\hline 0.194279 & 0.0164434 \\
\hline 0.197835 & 0.0154831 \\
\hline 0.195647 & 0.0125549 \\
\hline 0.185034 & 0.0187747 \\
\hline 0.182264 & 0.0164126 \\
\hline
\end{tabular}




\begin{tabular}{|c|c|}
\hline 0.184546 & 0.0162756 \\
\hline 0.176161 & 0.0133583 \\
\hline 0.178668 & 0.0176569 \\
\hline 0.178399 & 0.0156962 \\
\hline 0.175592 & 0.0150016 \\
\hline 0.171834 & 0.0170267 \\
\hline 0.172957 & 0.0152562 \\
\hline 0.16968 & 0.0177297 \\
\hline 0.170593 & 0.0152035 \\
\hline 0.176077 & 0.0158216 \\
\hline 0.168323 & 0.0166852 \\
\hline 0.172568 & 0.0117677 \\
\hline 0.173641 & 0.0113944 \\
\hline 0.18346 & 0.0130802 \\
\hline 0.185565 & 0.0207585 \\
\hline 0.185791 & 0.014251 \\
\hline 0.193425 & 0.0125441 \\
\hline 0.201144 & 0.0149717 \\
\hline 0.207621 & 0.016598 \\
\hline 0.22018 & 0.0165287 \\
\hline 0.232894 & 0.0163789 \\
\hline 0.252947 & 0.0216025 \\
\hline 0.260909 & 0.0170744 \\
\hline 0.283519 & 0.0149221 \\
\hline 0.296743 & 0.0211051 \\
\hline 0.318113 & 0.0213555 \\
\hline 0.339535 & 0.0215182 \\
\hline 0.364699 & 0.0174984 \\
\hline 0.385029 & 0.0224131 \\
\hline 0.413086 & 0.02279 \\
\hline 0.430027 & 0.0232263 \\
\hline 0.459782 & 0.0274964 \\
\hline 0.487285 & 0.0283681 \\
\hline 0.51823 & 0.0222783 \\
\hline 0.524127 & 0.021622 \\
\hline 0.549737 & 0.0294006 \\
\hline 0.58469 & 0.0302703 \\
\hline 0.601346 & 0.034337 \\
\hline 0.614706 & 0.0314508 \\
\hline 0.638468 & 0.035929 \\
\hline 0.655274 & 0.0293698 \\
\hline 0.675482 & 0.0419135 \\
\hline 0.688909 & 0.0401016 \\
\hline 0.682992 & 0.0326616 \\
\hline 0.69623 & 0.0283086 \\
\hline 0.697505 & 0.0363935 \\
\hline 0.696156 & 0.033383 \\
\hline
\end{tabular}




\begin{tabular}{|c|c|}
\hline 0.705591 & 0.0330066 \\
\hline 0.684975 & 0.0376951 \\
\hline 0.679611 & 0.0293509 \\
\hline 0.666364 & 0.0345181 \\
\hline 0.649087 & 0.030015 \\
\hline 0.622488 & 0.038419 \\
\hline 0.597601 & 0.0355082 \\
\hline 0.570857 & 0.0287481 \\
\hline 0.543768 & 0.0275989 \\
\hline 0.517087 & 0.0298766 \\
\hline 0.485464 & 0.0242198 \\
\hline 0.453349 & 0.0260756 \\
\hline 0.410406 & 0.0244614 \\
\hline 0.38467 & 0.0239561 \\
\hline 0.352581 & 0.0220554 \\
\hline 0.324813 & 0.026543 \\
\hline 0.297898 & 0.0189259 \\
\hline 0.274267 & 0.0207864 \\
\hline 0.248251 & 0.0183081 \\
\hline 0.227485 & 0.0153563 \\
\hline 0.208268 & 0.0166217 \\
\hline 0.193768 & 0.016054 \\
\hline 0.176482 & 0.01787 \\
\hline 0.16936 & 0.015722 \\
\hline 0.1562 & 0.0153729 \\
\hline 0.143865 & 0.0142472 \\
\hline 0.141479 & 0.0129628 \\
\hline 0.136995 & 0.0128425 \\
\hline 0.128589 & 0.010756 \\
\hline 0.127038 & 0.0154334 \\
\hline 0.121689 & 0.0136291 \\
\hline 0.11865 & 0.0121283 \\
\hline 0.117497 & 0.0121142 \\
\hline 0.119976 & 0.0131515 \\
\hline 0.116757 & 0.0103795 \\
\hline 0.11976 & 0.0133118 \\
\hline 0.116324 & 0.012109 \\
\hline 0.11407 & 0.0134326 \\
\hline 0.118929 & 0.013987 \\
\hline 0.112528 & 0.0142674 \\
\hline 0.115467 & 0.0116095 \\
\hline 0.115997 & 0.0127482 \\
\hline 0.117921 & 0.0142658 \\
\hline 0.116065 & 0.0112226 \\
\hline 0.11707 & 0.0112436 \\
\hline 0.121072 & 0.0121146 \\
\hline 0.116314 & 0.0135722 \\
\hline
\end{tabular}




$\begin{array}{rr}0.119483 & 0.0134712 \\ 0.127106 & 0.0139928 \\ 0.119163 & 0.0141353 \\ 0.125174 & 0.0118564 \\ 0.124137 & 0.0147327 \\ 0.126827 & 0.0142199 \\ 0.126994 & 0.0133714 \\ 0.129578 & 0.0153655 \\ 0.131812 & 0.0117325 \\ 0.132591 & 0.0149199 \\ 0.128748 & 0.0141268 \\ 0.133345 & 0.0133648 \\ 0.136582 & 0.0133202 \\ 0.138386 & 0.0169455 \\ 0.140049 & 0.0116715 \\ 0.138681 & 0.0105405 \\ 0.138787 & 0.0148804 \\ 0.145747 & 0.0142234 \\ 0.147458 & 0.014226 \\ 0.144941 & 0.0126385 \\ 0.145555 & 0.0158219 \\ 0.151266 & 0.0173448 \\ 0.15358 & 0.0149336 \\ 0.155817 & 0.0162382 \\ 0.155209 & 0.0138266 \\ 0.157689 & 0.0142665 \\ 0.155235 & 0.0143532 \\ 0.158778 & 0.0152725\end{array}$

